Final Report

FHWA/IN/JTRP-2006/27

\title{
FREIGHT FLOWS OF INDIANA
}

\author{
By \\ William R. Black, Ph.D. \\ Professor of Geography and \\ Public and Environmental Affairs \\ Indiana University \\ Bloomington, Indiana 47405
Joint Transportation Research Program
Project Number: C-36-73Z
File Number: 3-4-26
SPR-2860 \\ Prepared in Cooperation with the \\ Indiana Department of Transportation and the \\ U. S. Department of Transportation \\ Federal Highway Administration
}

The contents of this report reflect the views of the authors, who are responsible for the facts and the accuracy of the data presented herein. The contents do not necessarily reflect the official views and policies pf the Indiana Department of Transportation or Federal Highway Administration at the time of publication. The report does not constitute a standard, specification or regulation.

Purdue University

West Lafayette, IN 47907

August 2006 


\section{Table of Contents}

\section{Item}

Table of Figures

Table of Tables

Preface

Chapter 1 Introduction, Study Area and Networks

Chapter 2 Commodities Examined and Data Sources

Chapter 3 Goods Examined and Transport Facilities

Chapter 4 The Generation of Commodity Traffic

Chapter 5 The Distribution of Commodity Traffic

Chapter 6 The Modes in the Analysis

Chapter 7 Assignment of Traffic and Evaluation

Chapter 8 Forecasts for 2015 and 2030

Chapter 9 Implementation

Appendix A $\quad$ Productions and Attractions 1997

Appendix B Computer Programs Developed

Appendix C Distance Decay Curves

Appendix D Mode Choice/Distance Tables

Appendix E $\quad$ Productions and Attractions 2015

Appendix F $\quad$ Productions and Attractions 2030 


\section{Preface}

Why examine freight flows? It is a reasonable question to ask. Freight is not something that immediately comes to mind when most people think of transportation. It is not like congestion, or potholes, or any of those topics that people discuss around the water fountain soon after arriving at work. It is far more important than most of these topics and to paraphrase a scholar from several decades ago, people don't talk about freight transport because in most cases it works.

You may take any size area you wish from the smallest town to the largest country. These will be economically viable if the value of the products or services they sell in the aggregate to the areas beyond their borders exceed the value of the products they buy from these same areas. It is the exporting of goods that brings new income to the state. Selling goods only to ourselves circulates wealth, and perhaps some regions or counties may benefit from this, but it brings little new wealth to the state in the aggregate.

For the most part we will not concern ourselves with services since this report concerns itself primarily with freight. But one should not dismiss these flows since they often do involve selling something of value to those outside of Indiana. Perhaps one of the best examples of this is the university education sold to a student (or his or her parents) from outside Indiana or in some cases outside the United States. The tuition, lodging, and fees paid by these students represent contributions to the economic base of Indiana and the country. Consulting firms, accounting firms, law firms, and many others often sell their services beyond the borders of the state and are very important economically to the state. Nevertheless, we will focus our concern here on the movement of goods or freight transport.

We will examine freight flows in order to determine what is moving within the state of Indiana and where it is moving. In some cases we want to know this to make sure that the routes being used for these movements are well-maintained. In other cases we want to make sure that many of these same routes are kept in working order so that our manufacturers can get the raw materials that they need to carry out their production processes, which are so important to the state economy.

The routes of primary concern here are made up of rail lines or highway segments, and these receive the lion's share of our attention. We are aware of the use of water modes and the existence of air freight flows, but the former become important when the freight arrives at Indiana ports on the northern (Lake Michigan) and southern (Ohio River) borders, and the latter 
become important when the air freight destined for Indiana arrives at an airport within or near the state. This view of these modes is not a statement that we view them as unimportant, but rather the recognition that the water routes are maintained by others and the movement of aircraft, freight or otherwise, is not influenced by the state. From the lake and river ports or airports these flows become highway freight movements in most cases and at least the latter are treated as such here. The same is also true in the reverse case when goods are leaving Indiana. Air freight shipments are treated as highway moves until they reach the point when they are moved by an alternate mode.

The freight that we will examine here includes all of the freight that has an origin and a destination within the United States. Exports are generally treated as goods shipped to the point of export; we do not know their final destination outside the country. Imports are also treated as goods arriving at the point where they enter the country in most cases. Once again for these flows we do not know the foreign point of origin. The foreign origins and destinations of goods are discussed here, but they are not a subject of analysis or modeling.

The approach that is taken here in analyzing freight flows of Indiana is a typical 4-step transport planning process: traffic generation, traffic distribution, modal split and route assignment. We begin with a discussion of the areas used (Chapter 1 ) followed by a discussion of the rail and highway networks (Chapter 2). The commodities examined and their importance nationally and to the state of Indiana as well as the source of these data appear next (Chapter 3).

It is not always apparent why we develop models that enable us to predict data that we already know. The reason for this is that we want to use the models to predict future flows. The primary assumption made is that future flows will be predictable based on the same relationships observed in the first analysis. For example, let us say that each employee in an industry is found to produce 2,000 tons of a commodity that is shipped according to current data. At some point in the future we want to know how many tons of a commodity will be produced in an area that has 100 employees in that industry. The answer would be 200,000 tons. We would use this as our prediction of future flow from that area for that particular industry. However, for many industries we find there are changes in the level of productivity anticipated. If we assume that workers in 2015 will become more productive by a factor of 2 per cent, then each worker will produce 2040 tons of the commodity, and the resulting level of future flow from our area would be 204,000 tons. We will use productivity changes and expected growth factors to estimate flows produced. The methods used for flows produced as well the manner in which flows attracted are handled will be discussed in Chapter 4.

Distributing the flows between origins and destinations will be discussed in Chapter 5. A fully-constrained gravity model and a production-constrained gravity model were evaluated as part of the project. The former model was used in the earlier 1997 study and it was thought that a more realistic replication of flows could be achieved with the production-attraction constrained model. This will be discussed in more detail in Chapter 5 . 
Modal split analysis attempts to estimate the amount of the estimated flows that will be shipped by different modes moving between an origin and destination. In general it is believed that this is a function of the costs of the different modes, but the type of detailed cost data we would need for this approach is generally not available. Therefore, we will use historical patterns in part. Such an approach would look at the length of the shipment and look at the modes that have been used historically for assigning such traffic. This was the approach used in the previous (1997) study.

Once the modes are known we can proceed with assigning the current and future flows to the modal networks. The principal modal networks of concern here are those of the highway and railroad and the methods used for assignment are discussed in Chapter 7.

Forecasts of future flows are discussed in Chapter 8. For the most part these forecasts are based on procedures derived elsewhere by and for the State.

Chapter 9 discusses implementation of the projections and forecasts derived here. Aside from its value to the state in identifying priority corridors, we know of numerous metropolitan planning organizations that have an interest in the findings derived and the modeling used here. It is for this reason that much of the production and attraction data are included in appendices of this report. This chapter provides a guide as to how different agencies can use these and other data found here. 


\section{Chapter 1}

\section{INTRODUCTION, STUDY AREA, AND NETWORKS}

The primary objective of this project is the forecasting of freight flows for the state of Indiana for 2015 and 2030. The manner in which this is done is to follow a classical transportation planning process. This involves an inventory of facilities, an analysis of what is being moved, the development of models to replicate freight traffic generation, the modeling of flows between places, the separation of traffic between the various modes, and the assignment of that traffic to existing highway and rail networks. In this chapter we will examine the study area and networks used in the study.

The primary study area for this examination of freight flows is the state of Indiana and its ninety-two counties. While the flows to, from, and between each county are of interest, the analysis would be incomplete if it focused solely on intrastate flows. A significant amount of commodity traffic in Indiana has neither origin nor destination within the state's boundaries; instead it represents goods passing through the state. As the state slogan proclaims, Indiana is the "Crossroads of America." The consequence of this overhead traffic on the state's economy is questionable; however its impact on urban traffic congestion, air pollution, highway wear and tear, and rail traffic is decidedly significant. Therefore, the study area goes beyond the state's borders.

A transportation network consists of nodes and route segments. There are 145 nodes of origin and destination. As well as the 92 counties of Indiana, there are 53 major terntinals for the other 47 contiguous states (excluding Indiana) and the District of Columbia. All states are represented by one node, with the exceptions of Illinois, Kentucky, and Michigan, which are represented by two nodes, and Ohio, which is represented by three. There were also five nodes added later in the study to represent the five major airports serving the state. For reference a map of Indiana and its counties appears as Figure 1-1.

There are four major route transport networks serving Indiana: the highway system, the railway system, the air transport system, and the waterway network. This study is primarily concerned with the highway and the rail route segments. Flows on the other networks are considered implicitly if motor carriers or rail are used in part of the movement. 


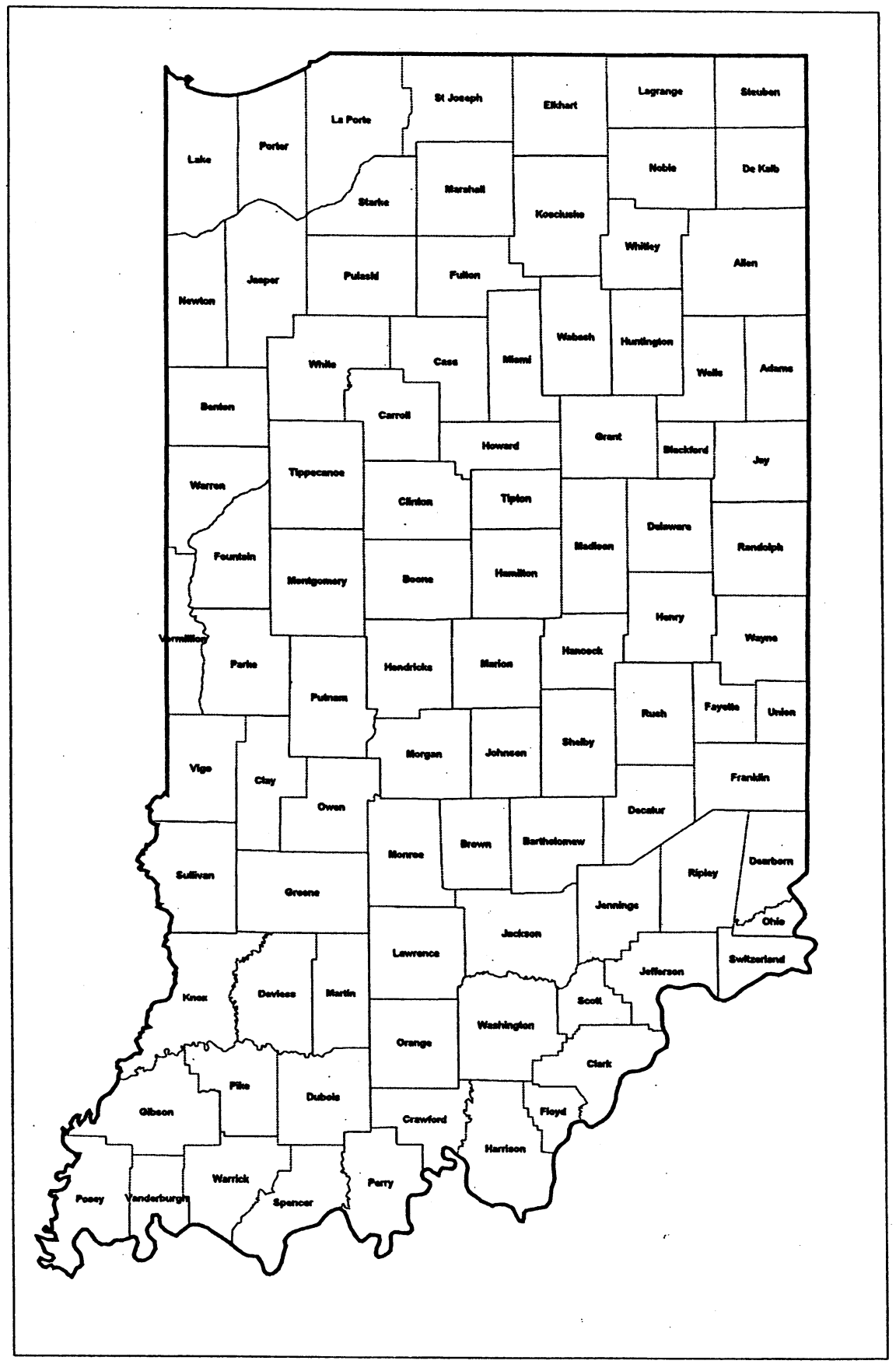

Figure 1-1. The Counties of Indiana 


\section{The Highway Network}

The highway network used in this study is an integrated network consisting of the highway network of Indiana as used by the Indiana travel demand model and the U.S. Interstate Highway network beyond the area covered by the former network. The network is not merely a visual image, but is a connected network to allow for traffic assignment of flows from and to all parts of the nation from locations within Indiana.

In terms of size the network consists of 73,346 segments covering 86,596 miles of highways and roads (see Figure 1-2). The average length of a segment of highway is 1.18 miles. The network is much denser in Indiana than it is through the remainder of the country since the primary concern here are the highway flows within the state (see Figure 1-3). The interconnections between the two networks are shown in Figure 1-4. It will be noted that the network is also dense just beyond the boundary of the state. This is to allow for flows to find their natural path into and out of the state. Confining the dense network only to the area within the boundary of Indiana would result in illogical paths being used by traffic assignment algorithms later in the study.

For purposes of analysis the state's counties are represented by 92 nodes, one for each county. Outside of Indiana each state is also represented by a node, except that contiguous states are represented by two nodes (in the case of Michigan, Kentucky, and Illinois), or three nodes (in the case of Ohio). The District of Columbia is also included and this yields a total of 145 nodes (see Table 1-1).

\section{The Railway Network}

The railway network for this study will be similar to the network used for the 1997 flow study. There have been minimal changes in rail line additions and closures and these changes have been incorporated in the network used here based on information supplied by the Indiana Department of Transportation's Rail Division.

The network used consists of 12, 815 line segments covering 148,996 route miles, not track miles. Track miles also include the length of industrial sidings and classification yards. The network used appears as Figure 1-5, with the Indiana portion shown in more detail as Figure 1-6.

As is true for the highway network, the rail network is also represented with network nodes, referred to here as stations. The Indiana nodes appear in Figure 1-7 and all nodes are identified in Table 1-2.

\section{Other Networks}

The approach taken here to the water transport and air transport networks remains the same as that used in the 1997 study: they were deemed superfluous and left out of the analysis as 


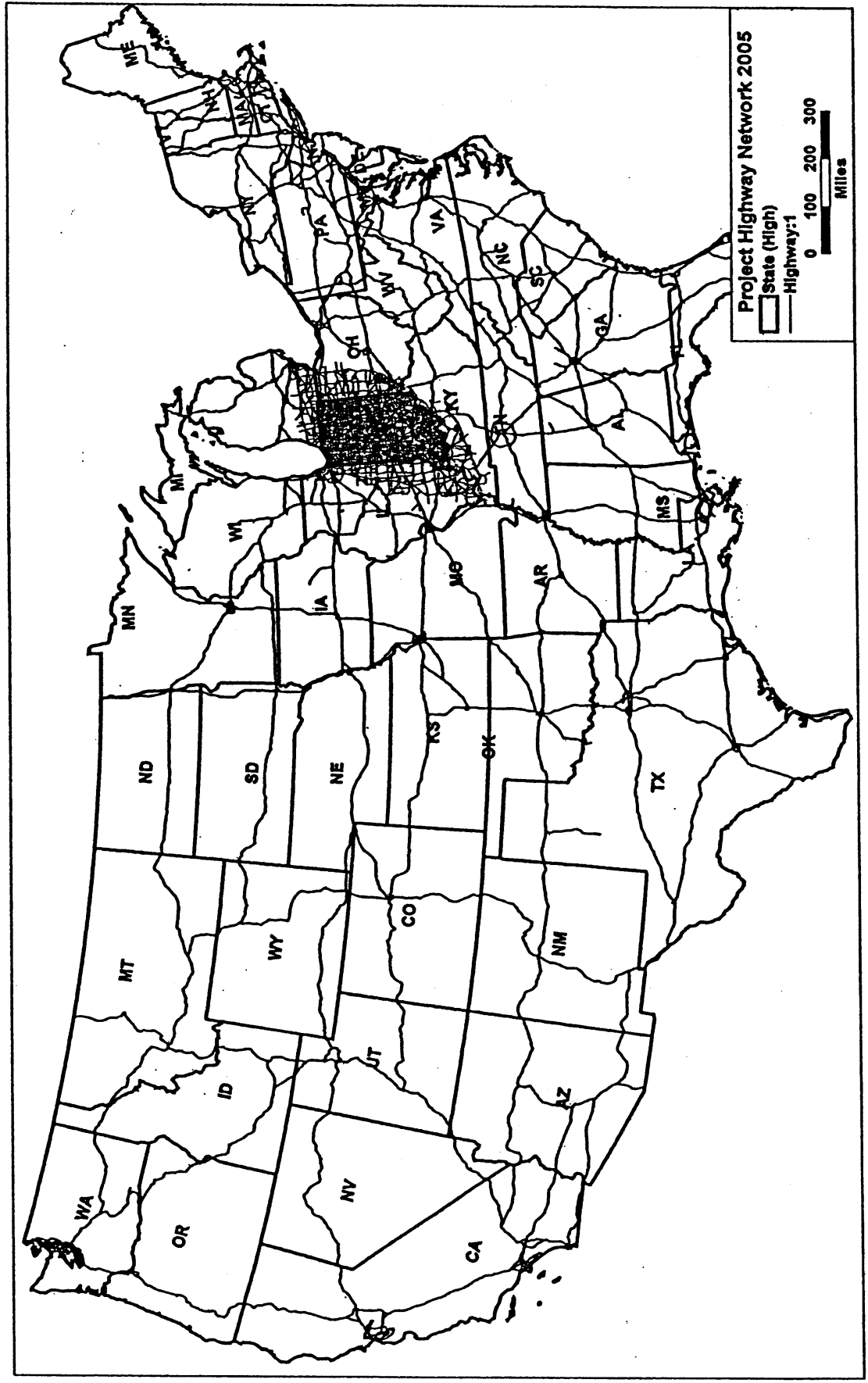

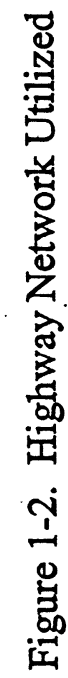




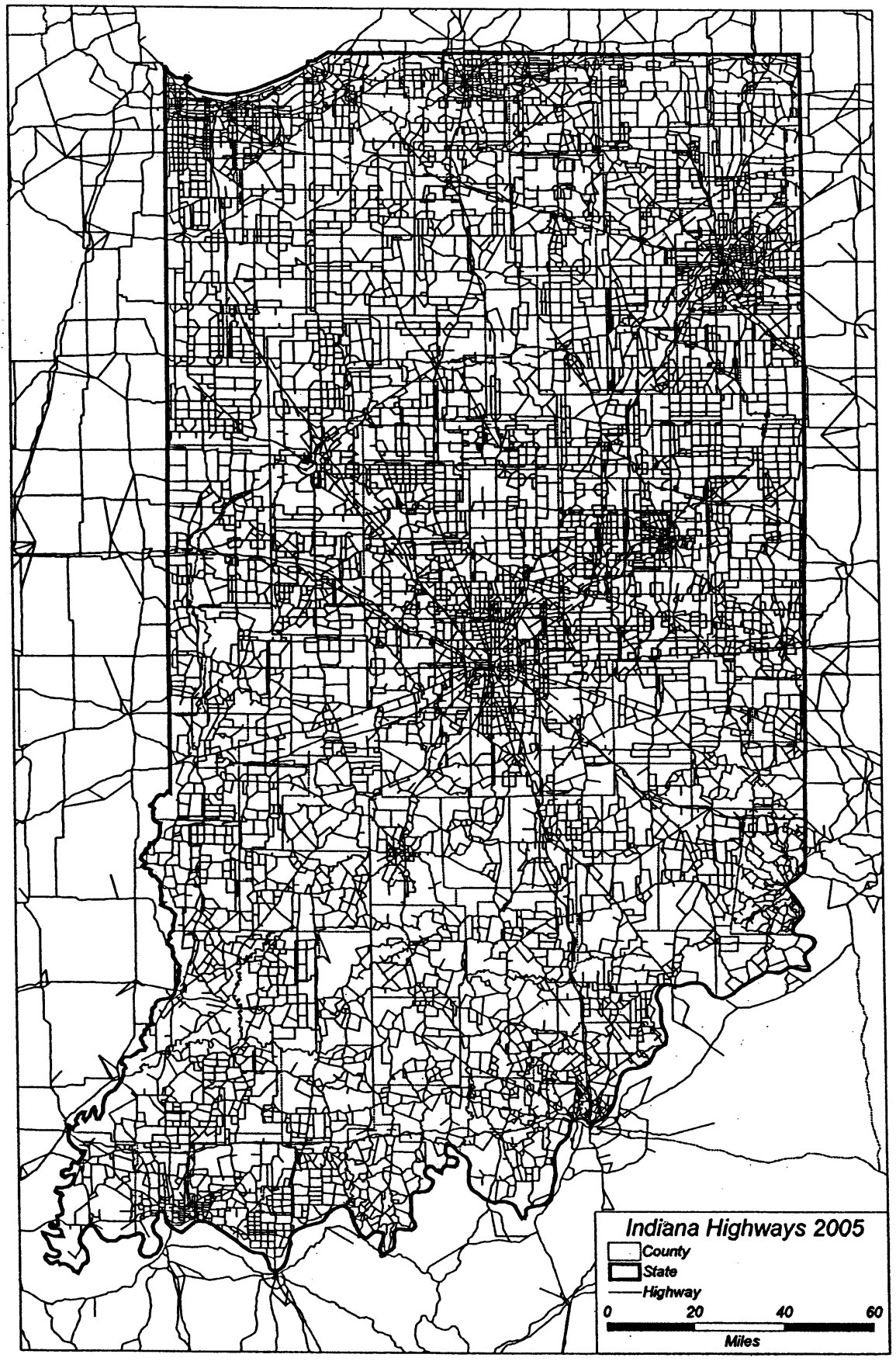

Figure 1-3. Indiana Portion of the Highway Network 


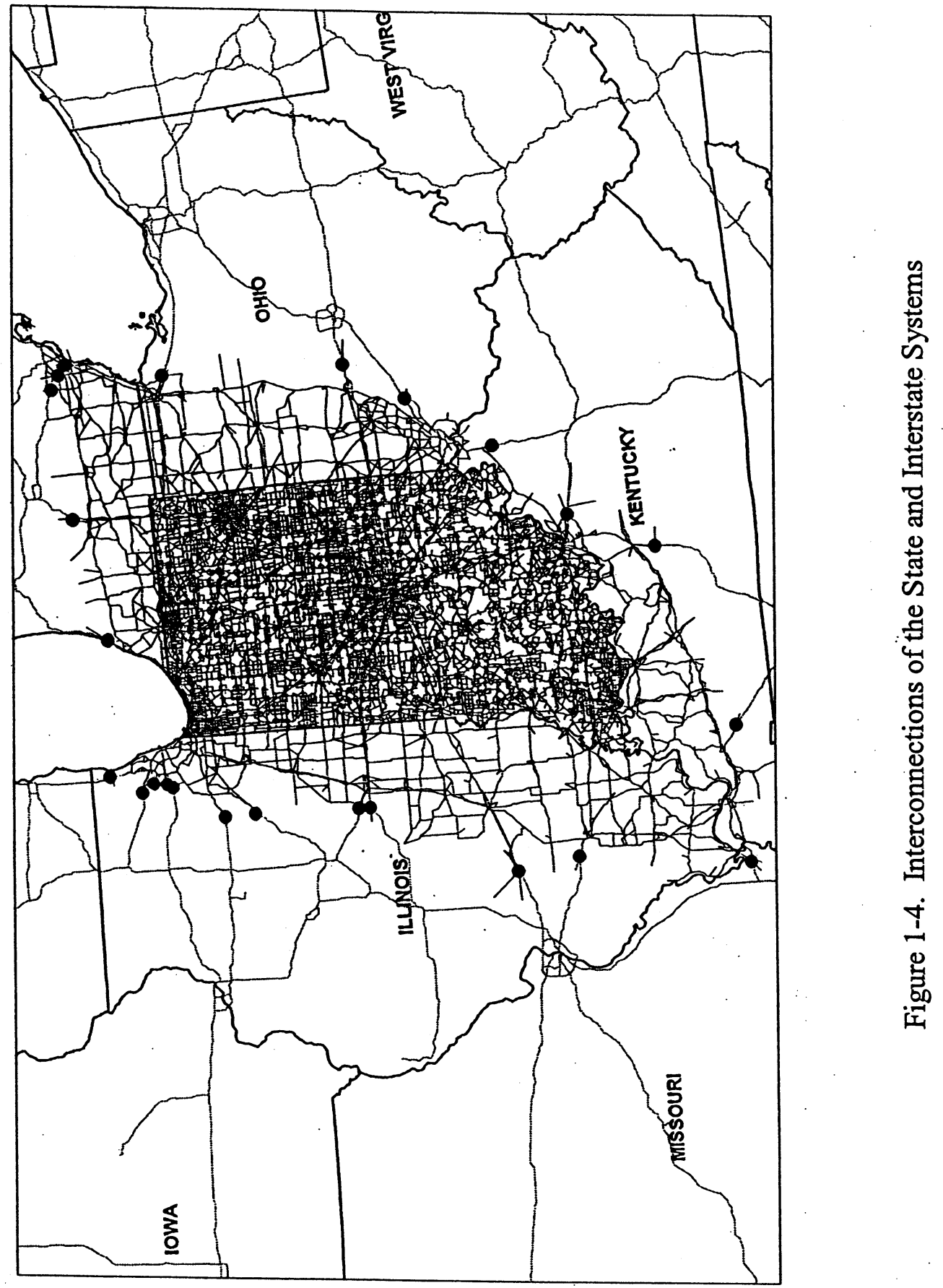


Table 1-1. Nodes of the Highway Network and Coordinates

\begin{tabular}{|c|c|c|c|c|c|}
\hline & ID & Longitude & Latitude & State & County \\
\hline 1 & 230218 & -86820037 & 33443494 & Alabama & \\
\hline 2 & 231636 & -111966957 & 33388622 & Arizona & \\
\hline 3 & 243103 & -92307369 & 34799568 & Arkansas & \\
\hline 4 & 232956 & -120104507 & 36079524 & California & \\
\hline 5 & 237253 & -105002769 & 39762338 & Colorado & \\
\hline 6 & 257459 & -72814362 & 41549751 & Connecticut & \\
\hline 7 & 254517 & -75740625 & 39647189 & Delaware & \\
\hline 8 & 254013 & -77170502 & 38894955 & District of & \\
\hline 9 & 241443 & -81654805 & 28228774 & Florida & \\
\hline 10 & 248164 & -84332879 & 33833346 & Georgia & \\
\hline 11 & 236567 & -112406986 & 42833123 & Idaho & \\
\hline 12 & 200286 & -87920168 & 41933007 & Illinois-n & \\
\hline 13 & 246094 & -89644442 & 39732689 & IIllinois-s & \\
\hline 14 & 224368 & -84947973 & 40744878 & Indiana & Adams \\
\hline 15 & 226025 & -85142641 & 41079783 & Indiana & Allen \\
\hline 16 & 219760 & -85877816 & 39210716 & Indiana & Bartholomew \\
\hline 17 & 212421 & -87318552 & 40613678 & Indiana & Benton \\
\hline 18 & 222304 & -85313342 & 40471636 & Indiana & Blackford \\
\hline 19 & 215222 & -86467969 & 40046700 & Indiana & Boone \\
\hline 20 & 209588 & -86251592 & 39221586 & Indiana & Brown \\
\hline 21 & 214043 & -86544369 & 40608494 & Indiana & Carroll \\
\hline 22 & 215531 & -86373778 & 40767474 & Indiana & Cass \\
\hline 23 & 10025 & -85723950 & 38440461 & Indiana & Clark \\
\hline 24 & 11012 & -87096569 & 39410856 & Indiana & Clay \\
\hline 25 & 213693 & -86487276 & 40290288 & Indiana & Clinton \\
\hline 26 & 208686 & -86471936 & 38299722 & Indiana & Crawford \\
\hline 27 & 226746 & -87061024 & 38727220 & Indiana & Daviess \\
\hline 28 & 218354 & -84945292 & 39142283 & Indiana & Dearborn \\
\hline 29 & 228865 & -85458207 & 39342636 & Indiana & Decatur \\
\hline 30 & 229559 & -85023674 & 41381355 & Indiana & Dekalb \\
\hline 31 & 223824 & -85391868 & 40200351 & Indiana & Delaware \\
\hline 32 & 227360 & -86892542 & 38355588 & Indiana & Dubois \\
\hline 33 & 225493 & -85834268 & 41584907 & Indiana & Elkhart \\
\hline 34 & 220269 & -85136700 & 39640325 & Indiana & Fayette \\
\hline 35 & 202300 & -85890756 & 38303455 & Indiana & Floyd \\
\hline 36 & 212199 & -87243085 & 40113480 & Indiana & Fountain \\
\hline 37 & 228990 & -85049733 & 39435160 & Indiana & Franklin \\
\hline 38 & 211455 & -86240069 & 41068697 & Indiana & Fulton \\
\hline 39 & 207494 & -87574085 & 38341066 & Indiana & Gibson \\
\hline 40 & 223104 & -85662870 & 40522666 & Indiana & Grant \\
\hline 41 & 205992 & -86986146 & 39029143 & Indiana & Greene \\
\hline 42 & 202425 & -86037180 & 40054579 & Indiana & Hamilton \\
\hline 43 & 219460 & -85769890 & 39814609 & Indiana & Hancock \\
\hline 44 & 31006 & -86105365 & 38182729 & Indiana & Harrison \\
\hline 45 & 209955 & -86542622 & 39760453 & Indiana & Hendricks \\
\hline 46 & 223405 & -85381826 & 39929155 & Indiana & Henry \\
\hline 47 & 215623 & -86102831 & 40476802 & Indiana & Howard \\
\hline 48 & 220796 & -85507496 & 40894927 & Indiana & Huntington \\
\hline 49 & 205480 & -86033541 & 38896113 & Indiana & Jackson \\
\hline 50 & 37019 & -87101896 & 41059717 & Indiana & Jasper \\
\hline 51 & 221075 & -84965623 & 40432540 & Indiana & Jay \\
\hline
\end{tabular}




\begin{tabular}{|c|c|c|c|c|c|}
\hline & ID & Longitude & Latitude & State & County \\
\hline 52 & 39019 & -85366337 & 38808533 & Indiana & Jefferson \\
\hline 53 & 219865 & -85617348 & 39005641 & Indiana & Jennings \\
\hline 54 & 205059 & -86059000 & 39471207 & Indiana & Johnson \\
\hline 55 & 207138 & -87364406 & 38700598 & Indiana & Knox . \\
\hline 56 & 225255 & -85852539 & 41246104 & Indiana & Kosciusko \\
\hline 57 & 226186 & -85416623 & 41633346 & Indiana & Lagrange \\
\hline 58 & 213198 & -87364804 & 41485895 & Indiana & Lake \\
\hline 59 & 216782 & -86714144 & 41608253 & Indiana & LaPorte \\
\hline 60 & 227804 & -86494835 & 38846907 & Indiana & Lawrence \\
\hline 61 & 222379 & -85696775 & 40135183 & Indiana & Madison \\
\hline 62 & 210529 & -86171203 & 39764490 & Indiana & Marion \\
\hline 63 & 216308 & -86276287 & 41343320 & Indiana & Marshall \\
\hline 64 & 208835 & -86808774 & 38706101 & Indiana & Martin \\
\hline 65 & 215676 & -86037418 & 40753536 & Indiana & Miami \\
\hline 66 & 209318 & -86533606 & 39166608 & Indiana & Monroe \\
\hline 67 & 203873 & -86901407 & 40047364 & Indiana & Montgomery \\
\hline 68 & 209685 & -86444002 & 39490714 & Indiana & Morgan \\
\hline 69 & 212654 & -87392576 & 40940527 & Indiana & Newton \\
\hline 70 & 57015 & -85379061 & 41421966 & Indiana & Noble \\
\hline 71 & 217716 & -84958239 & 38952353 & Indiana & Ohio \\
\hline 72 & 208722 & -86466330 & 38536551 & Indiana & Orange \\
\hline 73 & 227771 & -86833473 & 39304481 & Indiana & Owen \\
\hline 74 & 203122 & -87236075 & 39768930 & Indiana & Parke \\
\hline 75 & 205910 & -86610269 & 38109730 & Indiana & Perry \\
\hline 76 & 207896 & -87220492 & 38395125 & Indiana & Pike \\
\hline 77 & 229840 & -87082796 & 41461388 & Indiana & Porter \\
\hline 78 & 207464 & -87832984 & 38063140 & Indiana & Posey \\
\hline 79 & 216102 & -86699613 & 41055440 & Indiana & Pulaski \\
\hline 80 & 209927 & -86865922 & 39653139 & Indiana & Putnam \\
\hline 81 & 222181 & -84974353 & 40172563 & Indiana & Randolph \\
\hline 82 & 219315 & -85279495 & 39104668 & Indiana & Ripley \\
\hline 83 & 220340 & -85446370 & 39608326 & Indiana & Rush \\
\hline 84 & 217078 & -86251572 & 41669097 & Indiana & Saint Joseph \\
\hline 85 & 72004 & -85747395 & 38667739 & Indiana & Scott \\
\hline 86 & 219955 & -85783475 & 39540059 & Indiana & Shelby \\
\hline 87 & 206448 & -87016255 & 38002497 & Indiana & Spenser \\
\hline 88 & 215938 & -86639971 & 41317257 & Indiana & Starke \\
\hline 89 & 220829 & -85015205 & 41635117 & Indiana & Steuben \\
\hline 90 & 208148 & -87418958 & 39082115 & Indiana & Sullivan \\
\hline 91 & 217743 & -85065498 & 38815197 & Indiana & Switzerland \\
\hline 92 & 214830 & -86899538 & 40444761 & Indiana & Tippecanoe \\
\hline 93 & 202535 & -86079381 & 40303494 & Indiana & Tipton \\
\hline 94 & 203499 & -84942383 & 39611086 & Indiana & Union \\
\hline 95 & 226866 & -87581795 & 38022070 & Indiana & Vanderburgh \\
\hline 96 & 211948 & -87446011 & 39893355 & Indiana & Vermillion \\
\hline 97 & 207987 & -87413575 & 39450070 & Indiana & Vigo \\
\hline 98 & 221038 & -85818840 & 40797090 & Indiana & Wabash \\
\hline 99 & 212033 & -87347490 & 40351615 & Indiana & Warren \\
\hline 100 & 207758 & -87273908 & 38060337 & Indiana & Warrick \\
\hline 101 & 209312 & -86094090 & 38609618 & Indiana & Washington \\
\hline 102 & 227273 & -84995466 & 39876434 & Indiana & Wayne \\
\hline
\end{tabular}




\begin{tabular}{|c|c|c|c|c|c|}
\hline & ID & Longitude & Latitude & State & County \\
\hline 103 & 224062 & -85179024 & 40733401 & Indiana & Wells \\
\hline 104 & 91021 & -86844227 & 40776750 & Indiana & White \\
\hline 105 & 225362 & -85485436 & 41156939 & Indiana & Whitley \\
\hline 106 & 245132 & -93625795 & 41649499 & lowa & \\
\hline 107 & 244005 & -97618105 & 38737601 & Kansas & \\
\hline 108 & 248286 & -84461509 & 38083662 & Kentucky-e & \\
\hline 109 & 100828 & -88366722 & 36833610 & Kentucky-w & \\
\hline 110 & 238151 & -92435321 & 31074228 & Louisianna & \\
\hline 111 & 258516 & -69476868 & 44684250 & Maine & \\
\hline 112 & 254478 & -76641972 & 39451506 & Maryland & \\
\hline 113 & 257590 & -71474012 & 42299699 & Massachusett & \\
\hline 114 & 249843 & -83619247 & 42885796 & Michigan-e & \\
\hline 115 & 249123 & -85707501 & 42960929 & Michigan-w & \\
\hline 116 & 245934 & -93285426 & 45067452 & Minnesota & \\
\hline 117 & 240372 & -90151194 & 32359372 & Mississippi & \\
\hline 118 & 243046 & -92737076 & 38936512 & Missouri & \\
\hline 119 & 236582 & -112628318 & 46008908 & Montana & \\
\hline 120 & 230184 & -99416267 & 40692745 & Nebraska & \\
\hline 121 & 231953 & -116904617 & 40619709 & Nevada & \\
\hline 122 & 258333 & -71533895 & 43211126 & NewHampshire & \\
\hline 123 & 256124 & -74428631 & 40161577 & NewJersey & \\
\hline 124 & 237070 & -106728378 & 35094742 & NewMexico & \\
\hline 125 & 249550 & -75128105 & 43086742 & NewYork & \\
\hline 126 & 253109 & -78983956 & 35915326 & NorthCarolin & \\
\hline 127 & 230541 & -100294382 & 46836312 & NorthDakota & \\
\hline 128 & 252487 & -81757324 & 41312634 & Ohio-n & \\
\hline 129 & 251974 & -82944556 & 39951533 & Ohio-m & \\
\hline 130 & 218162 & -84441939 & 39289760 & Ohio-s & \\
\hline 131 & 243665 & -97527200 & 35464360 & Oklahoma & \\
\hline 132 & 234350 & -123004569 & 43990692 & Oregon & \\
\hline 133 & 254597 & -77530757 & 41029711 & Pennsylvania & \\
\hline 134 & 257526 & -71603735 & 41650606 & Rhodelsland & \\
\hline 135 & 251082 & -81046326 & 34062847 & SouthCarolin & \\
\hline 136 & 245402 & -100051782 & 43898533 & SouthDakota & \\
\hline 137 & 247850 & -86827942 & 36155684 & Tennessee & \\
\hline 138 & 238642 & -97250637 & 31297776 & Texas & \\
\hline 139 & 235264 & -111841802 & 39679488 & Utah & \\
\hline 140 & 257878 & -72610994 & 44110530 & Vermont & \\
\hline 141 & 253295 & -77427674 & 37552263 & \begin{tabular}{|l} 
Virginia \\
\end{tabular} & \\
\hline 142 & 234657 & -122025454 & 47532033 & Washington & \\
\hline 143 & 252067 & -81588158 & 38391693 & West Virgini & \\
\hline 144 & 248646 & -89527193 & 43517451 & Wisconsin & \\
\hline 145 & 237765 & -106282325 & 42855627 & Wyoming & \\
\hline 146 & 200440 & -87763098 & 41811527 & Chicago-O'Ha & \\
\hline 147 & 218652 & -85729640 & 38190492 & Louisville A & \\
\hline 148 & 217953 & -84647423 & 39001326 & Cincinnati A & \\
\hline 149 & 225647 & -85207397 & 41074554 & Fort Wayne A & \\
\hline 150 & 210243 & -86269683 & 39729598 & Indianapolis & \\
\hline
\end{tabular}




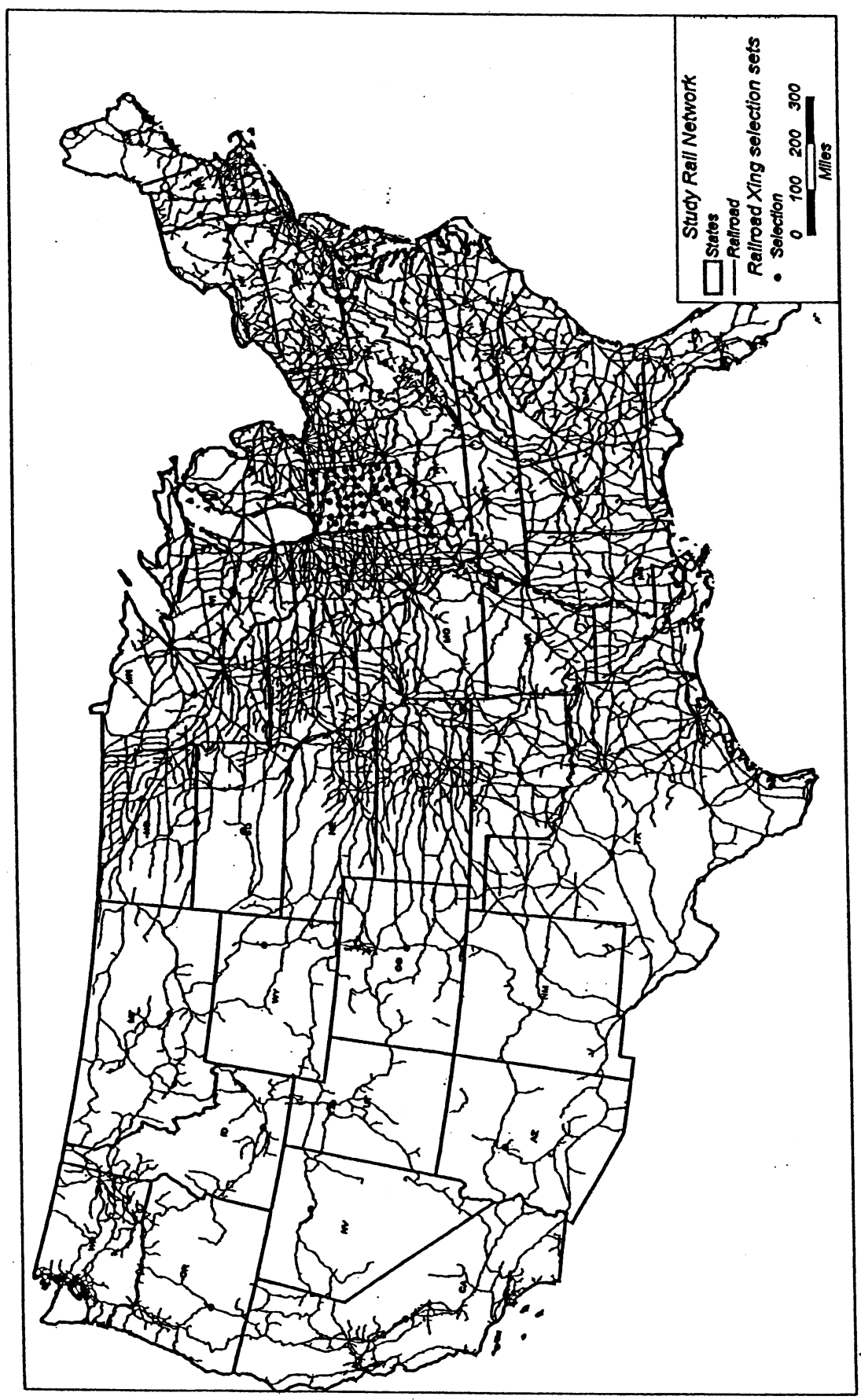

芦 


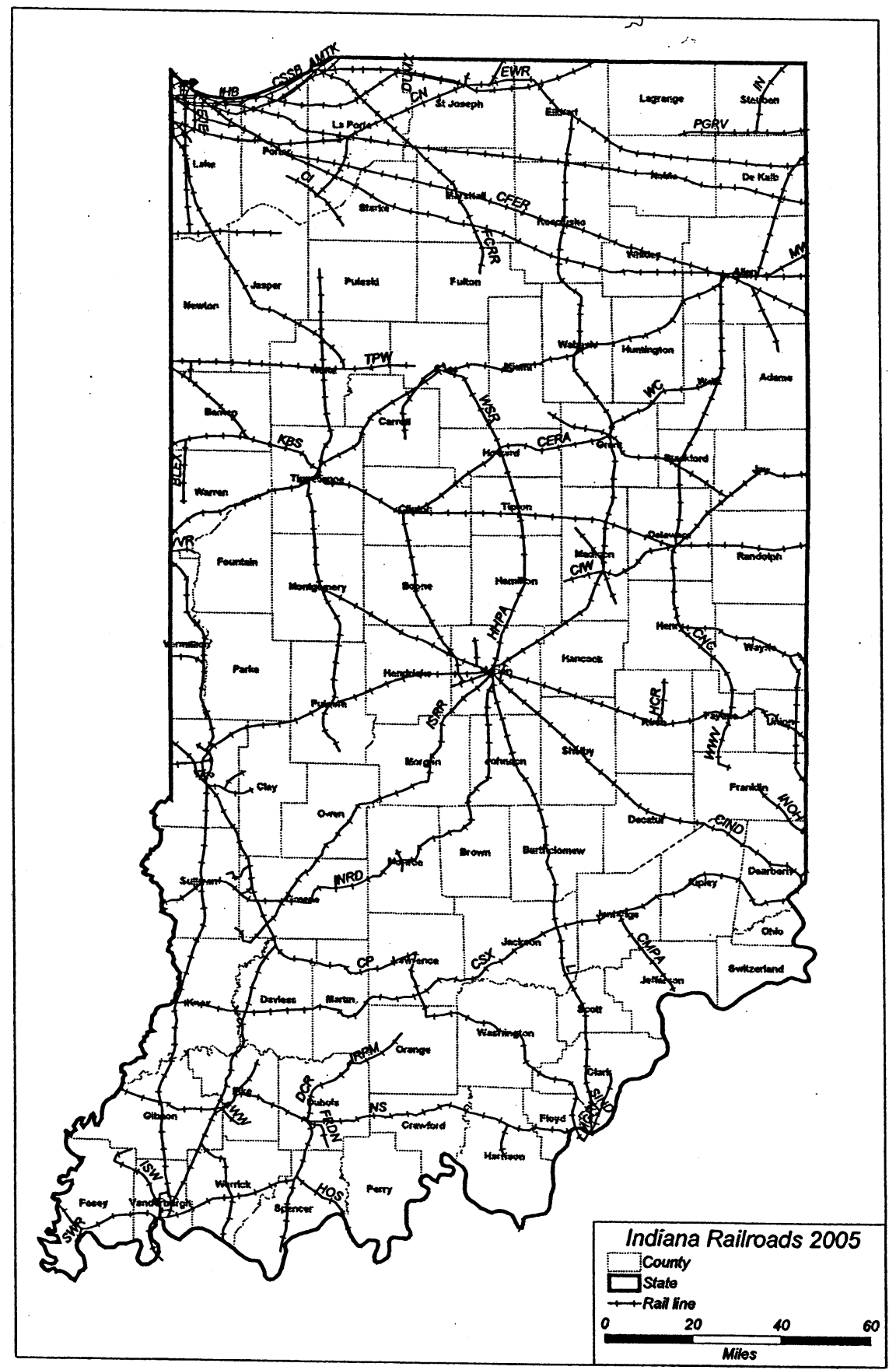

Figure 1-6. Indiana Portion of the Rail Network 
Table 1-2. Nodes of the Rail Network and Coordinates

\begin{tabular}{|c|c|c|c|c|c|}
\hline & ID & Longitude & Latitude & State & County \\
\hline 1 & 832454 & -86754144 & 33129406 & Alabama & \\
\hline 2 & 287311 & -112372278 & 34989207 & \begin{tabular}{|l} 
Arizona \\
\end{tabular} & \\
\hline 3 & 1154620 & -92259754 & 34775464 & Arkansas & \\
\hline 4 & 105441 & -120015879 & 36974614 & California & \\
\hline 5 & 486522 & -104809612 & 38835637 & Colorado & \\
\hline 6 & 2549093 & -72631570 & 41656868 & Connecticut & \\
\hline 7 & 2391830 & -75575965 & 38928557 & Delaware & \\
\hline 8 & 2382821 & -77003398 & 38902120 & District of $\mathrm{Col}$ & \\
\hline 9 & 912119 & -82430773 & 29832155 & Florida & \\
\hline 10 & 929464 & -83557487 & 32849301 & Georgia & \\
\hline 11 & 328414 & -113478614 & 42751205 & Idaho & \\
\hline 12 & 1592088 & -89083508 & 39388413 & Illinois- $n$ & \\
\hline 13 & 1775449 & -87774828 & 41804784 & Illinois-s & \\
\hline 14 & 1846978 & -84926327 & 40827592 & Indiana & Adams \\
\hline 15 & 1852740 & -85161050 & 41083426 & Indiana & Allen \\
\hline 16 & 1814690 & -85916090 & 39197325 & Indiana & Bartholomew \\
\hline 17 & 1762637 & -87193923 & 40517290 & Indiana & Benton \\
\hline 18 & 1842508 & -85356333 & 40444524 & Indiana & Blackford \\
\hline 19 & 1790152 & -86477502 & 40057303 & Indiana & Boone \\
\hline 20 & 1814690 & -85916090 & 39197325 & Indiana & Brown \\
\hline 21 & 1790208 & -86518057 & 40523962 & Indiana & Carroll \\
\hline 22 & 1842548 & -85368271 & 39958153 & Indiana & Cass \\
\hline 23 & 1814578 & -85751879 & 38409852 & Indiana & Clark \\
\hline 24 & 1790144 & -86528055 & 40285352 & Indiana & Clinton \\
\hline 25 & 1744271 & -86348011 & 38360123 & Indiana & Crawford \\
\hline 26 & 1732068 & -87215582 & 38658987 & Indiana & Daviess \\
\hline 27 & 1814802 & -85067132 & 39072894 & Indiana & Dearborn \\
\hline 28 & 2644057 & -85484267 & 39324882 & Indiana & Decatur \\
\hline 29 & 1869007 & -84888626 & 41381615 & Indiana & DeKalb \\
\hline 30 & 1838582 & -85369661 & 40188147 & Indiana & Delaware \\
\hline 31 & 1735768 & -86947774 & 38298435 & Indiana & Dubois \\
\hline 32 & 1852556 & -85813317 & 41585929 & Indiana & Elkhart \\
\hline 33 & 1821269 & -85135760 & 39641207 & Indiana & Fayette \\
\hline 34 & 1744343 & -85809374 & 38290139 & Indiana & Floyd \\
\hline 35 & 1762565 & -87425856 & 40146187 & Indiana & Fountain \\
\hline 36 & 1821293 & -85014921 & 39404550 & Indiana & Franklin \\
\hline 37 & 1806118 & -86205002 & 41071754 & Indiana & Fulton \\
\hline 38 & 1731868 & -87580879 & 38354813 & Indiana & Gibson \\
\hline 39 & 1842468 & -85651611 & 40545641 & Indiana & Grant \\
\hline 40 & 1735960 & -87230034 & 39197582 & Indiana & Greene \\
\hline 41 & 1790264 & -86013863 & 40045904 & Indiana & Hamilton \\
\hline 42 & 1756508 & -86131639 & 39806194 & Indiana & Hancock \\
\hline 43 & 1744327 & -86097445 & 38295961 & Indiana & Harrison \\
\hline 44 & 1756508 & -86131639 & 39806194 & Indiana & Hendricks \\
\hline 45 & 1796347 & -86385559 & 40758139 & Indiana & Henry \\
\hline 46 & 1796339 & -86122764 & 40498966 & Indiana & Howard \\
\hline 47 & 1842524 & -85474669 & 40885090 & Indiana & Huntington \\
\hline 48 & 1751092 & -85885231 & 38952889 & Indiana & Jackson \\
\hline 49 & 1781053 & -87147545 & 40948135 & Indiana & Jasper \\
\hline 50 & 1846930 & -84978270 & 40432590 & Indiana & Jay \\
\hline 51 & 1814714 & -85386745 & 38746116 & Indiana & Jefferson \\
\hline
\end{tabular}




\begin{tabular}{|c|c|c|c|c|c|}
\hline & ID & Longitude & Latitude & State & County \\
\hline 52 & 1814602 & -85623831 & 39005108 & Indiana & Jennings \\
\hline 53 & 1751148 & -86062471 & 39487599 & Indiana & Johnson \\
\hline 54 & 1731900 & -87513090 & 38684812 & Indiana & Knox \\
\hline 55 & 1806198 & -85848876 & 41230090 & Indiana & Kosciusko \\
\hline 56 & 1852628 & -85354402 & 41524819 & Indiana & Lagrange \\
\hline 57 & 1785363 & -87369836 & 41606245 & Indiana & Lake \\
\hline 58 & 1800242 & -86780325 & 41416473 & Indiana & Laporte \\
\hline 59 & 1744335 & -86480813 & 38866762 & Indiana & Lawrence \\
\hline 60 & 1838486 & -85682204 & 40103210 & Indiana & \begin{tabular}{|l} 
Madison \\
\end{tabular} \\
\hline 61 & 1756508 & -86131639 & 39806194 & Indiana & Marion \\
\hline 62 & 1806142 & -86305013 & 41337591 & Indiana & Marshall \\
\hline 63 & 1744263 & -86770551 & 38648165 & Indiana & Martin \\
\hline 64 & 1796403 & -86075264 & 40752033 & Indiana & Miami \\
\hline 65 & 1750956 & -86533887 & 39165372 & Indiana & Monroe \\
\hline 66 & 1762605 & -86872512 & 40032304 & Indiana & Montgomery \\
\hline 67 & 1751044 & -86424708 & 39419542 & Indiana & Morgan \\
\hline 68 & 1766481 & -87440874 & 40770637 & Indiana & Newton \\
\hline 69 & 1852636 & -85261061 & 41448990 & Indiana & \begin{tabular}{|l} 
Noble \\
\end{tabular} \\
\hline 70. & 1814802 & -85067132 & 39072894 & Indiana & Ohio \\
\hline 71 & 2644056 & -86490857 & 38596124 & Indiana & Orange \\
\hline 72 & 1744391 & -86659450 & 39348423 & Indiana & Owen \\
\hline 73 & 1744103 & -87388079 & 39786465 & Indiana & Parke \\
\hline 74 & 1744239 & -86734705 & 37919284 & Indiana & Perry \\
\hline 75 & 1735736 & -87247239 & 38284821 & Indiana & Pike \\
\hline 76 & 1788113 & -87057044 & 41462716 & Indiana & Porter \\
\hline 77 & 1724066 & -87895573 & 37937319 & Indiana & Posey \\
\hline 78 & 1796451 & -86880870 & 41079248 & Indiana & Pulaski \\
\hline 79 & 1744175 & -86837236 & 39661762 & Indiana & Putnam \\
\hline 80 & 1846906 & -84977154 & 40181201 & Indiana & Randolph \\
\hline 81 & 1814722 & -85341034 & 39083996 & Indiana & Ripley \\
\hline 82 & 1814770 & -85448549 & 39610093 & Indiana & Rush \\
\hline 83 & 1751092 & -85885231 & 38952889 & Indiana & Scott \\
\hline 84 & 1814730 & -85773569 & 39513149 & Indiana & Shelby \\
\hline 85 & 1744391 & -86659450 & 39348423 & Indiana & Spenser \\
\hline 86 & 1806254 & -86261405 & 41675658 & Indiana & St. Joseph \\
\hline 87 & 1800274 & -86626982 & 41301476 & Indiana & Starke \\
\hline 88 & 1856325 & -85015156 & 41537697 & Indiana & Steuben \\
\hline 89 & 1735928 & -87399753 & 39082863 & Indiana & Sullivan \\
\hline 90 & 1814714 & -85386745 & 38746116 & Indiana & Switzerland \\
\hline 91 & 1762621 & -86882249 & 40418130 & Indiana & Tippecanoe \\
\hline 92 & 1790272 & -86034693 & 40286747 & Indiana & Tipton \\
\hline 93 & 1821301 & -84859081 & 39594270 & Indiana & Union \\
\hline 94 & 1729329 & -87539512 & 38003872 & Indiana & Vanderburgh \\
\hline 95 & 1762517 & -87459748 & 39952576 & Indiana & Vermillion \\
\hline 96 & 1741705 & -87368100 & 39504245 & Indiana & Vigo \\
\hline 97 & 1842492 & -85806076 & 40807866 & Indiana & Wabash \\
\hline 98 & 1762597 & -87248545 & 40303088 & Indiana & Warren \\
\hline 99 & 1731972 & -87274451 & 38047592 & Indiana & Warrick \\
\hline 100 & 1744343 & -85809374 & 38290139 & Indiana & Washington \\
\hline 101 & 1831417 & -84883253 & 39835657 & Indiana & \begin{tabular}{|l} 
Wayne \\
\end{tabular} \\
\hline 102 & 1846946 & -85175767 & 40744542 & Indiana & Wells \\
\hline
\end{tabular}




\begin{tabular}{|c|c|c|c|c|c|}
\hline & ID & Longitude & Latitude & State & County \\
\hline 103 & 1790176 & -86865866 & 40753686 & Indiana & White \\
\hline 104 & 1847034 & -85628018 & 41081761 & Indiana & Whitely \\
\hline 105 & 1250660 & -93592647 & 41660809 & lowa & \\
\hline 106 & 1053150 & -97657012 & 38376683 & Kansas & \\
\hline 107 & 1680662 & -87490279 & 37327872 & Kenetuck-e & \\
\hline 108 & 1821453 & -84180417 & 37985160 & Kentucky-w & \\
\hline 109 & 1814682 & -85044895 & 38739294 & Kentucky & \\
\hline 110 & 710367 & -92434166 & 31349811 & Louisianna & \\
\hline 111 & 2630042 & -69257594 & 44837910 & Maine & \\
\hline 112 & 2391702 & -76641607 & 39487994 & Maryland & \\
\hline 113 & 2594729 & -71688479 & 42426854 & Massachusetts & \\
\hline 114 & 2558307 & -70902019 & 41871596 & Massachusetts & \\
\hline 115 & 2442298 & -83242918 & 42378966 & Michigan-e & \\
\hline 116 & 1979398 & -85792580 & 43038938 & Michigan-w & \\
\hline 117 & 1415491 & -94364172 & 45976601 & Minnesota & \\
\hline 118 & 1235763 & -92290911 & 39233408 & Missouri & \\
\hline 119 & 396739 & -109059215 & 46306866 & Montana & \\
\hline 120 & 1083504 & -97986504 & 41125308 & Nebraska & \\
\hline 121 & 277265 & -116536567 & 40705654 & Nevada & \\
\hline 122 & 2601835 & -71582932 & 43452103 & New Hampshire & \\
\hline 123 & 2516725 & -74445580 & 40357995 & New Jersey & \\
\hline 124 & 406547 & -105214583 & 34610832 & New Mexico & \\
\hline 125 & 2560517 & -74993092 & 43036527 & New York & \\
\hline 126 & 791241 & -89851279 & 33088870 & Nississippi & \\
\hline 127 & 2285896 & -78782512 & 35791132 & North Carolina & \\
\hline 128 & 1298188 & -99128569 & 47674448 & North Dakota & \\
\hline 129 & 2188395 & -81747490 & 41479822 & Ohio-n & \\
\hline 130 & 2154193 & -82947863 & 39977347 & Ohio-m & \\
\hline 131 & 1831193 & -84482502 & 39181742 & Ohio-s & \\
\hline 132 & 987867 & -97507297 & 35467070 & Oklahoma & \\
\hline 133 & 169375 & -121780050 & 43215263 & Oregon & \\
\hline 134 & 2400417 & -77580267 & 40634032 & Pennsylvania & \\
\hline 135 & 2558123 & -71424558 & 41830201 & Rhode Island & \\
\hline 136 & 2079666 & -81049086 & 33973367 & South Carolina & \\
\hline 137 & 553174 & -100349715 & 44371072 & South Dakota & \\
\hline 138 & 1680798 & -86790833 & 36026512 & Tennessee & \\
\hline 139 & 309972 & -111864865 & 40593786 & Utah & \\
\hline 140 & 2585658 & -72964379 & 43601245 & Vermont & \\
\hline 141 & 2301196 & -78338342 & 37736959 & Virginia & \\
\hline 142 & 230374 & -120306809 & 47426637 & Washington & \\
\hline 143 & 2196653 & -80720195 & 38660199 & West Virginia & \\
\hline 144 & 1940198 & -89754693 & 44583260 & Wisconsin & \\
\hline 145 & 530576 & -105348600 & 43470478 & Wyoming & \\
\hline
\end{tabular}




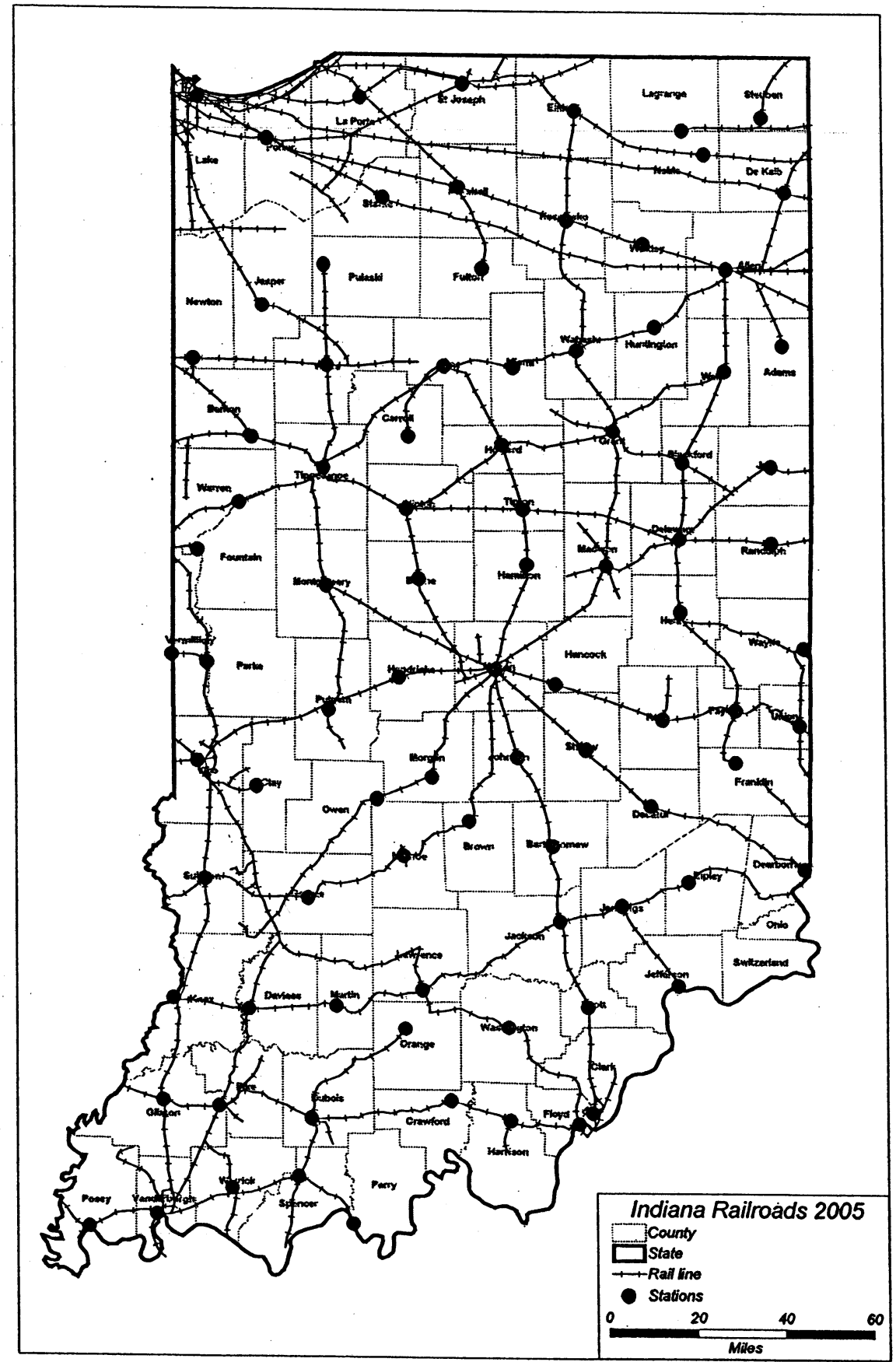

Figure 1-7. Nodes of the Indiana Rail Network 
networks (Black, 1997). The great majority of the goods being transported on the water and air networks reached those networks via a transfer from the highway or the railway network.

The goods transported via the waterway network are generally bulk quantities of raw materials whose destination is outside the state of Indiana. With existing data we are unable to assert whether water flows are moving via the Great Lakes or the Ohio River. Even if we could make this distinction it would be hard to know what river ports are used for these flows. As a result we have supplied information on future water flows on $C D$ as part of this research effort, but we have not assigned these to a water network.

The air transport network, though involved in the movement of some freight and express deliveries, is predominantly a passenger transport system. Intercity air travel was not included in the study. Most freight moving by air reached the air transport nodes via the highway network. This is discussed in more detail in later chapters of this report. It will be noted there that both parcel shipments and air freight are handled in a very explicit way. The study design of the project, therefore, has already captured the movement of those goods on the highway network.

Pipeline movement of freight is included in the freight transported. These flows are not examined in any further detail here, but they are included as part of the modal breakdown of freight traffic on the $\mathrm{CD}$.

\section{Summary}

This chapter has introduced the objectives of the study, the approach taken in reaching this objective and the networks used for the highway and rail transport sectors. It was noted that the water network was not used as such. In addition to the data problems related to this mode, the waterway network is not maintained by the State of Indiana, but by the U.S. Army Corps of Engineers. The pipeline network is also not examined here. Even though these networks were not examined, there are forecasts of the movement of freight by water and pipeline provided here. In addition, although the air network was not included as such, air freight and parcel movements were viewed as "special" variations of the highway network as will be described later in the report. 


\section{References Cited}

Black, William R. (1997), Transport Flows in the State of Indiana: Commodity Database Development and Traffic Assignment: Phase 2. Transportation Research Center and Department of Geography, Indiana University, Bloomington, IN. 


\section{Chapter 2}

\section{COMMODITIES EXAMINED AND DATA SOURCES}

The 1997 state study sought to have the most detailed commodity grouping possible. For that research it was determined that the two-digit Standard Transportation Commodity Code (STCC) of commodity data provided by the 1993 Commodity Flow Survey (U.S. Bureau of Census, 1996) was the most suitable level of specificity. However, since that time the adopted classification methodology for freight movement in the US has evolved to a new system, the Standard Classification of Transported Goods (SCTG).

The level of specification for this research is the two-digit level for the SCTG as presented in the 1997 Commodity Flow Survey (U.S. Bureau of Census, 1999). It can be argued, as there are forty-one categories in this study compared to nineteen in the previous, that this study is an increase in specificity. This study, however, runs into the same problem as the 1997 work when trying to look at a higher level of commodity detail: a considerable amount of information is lost at higher levels of detail. To keep from revealing confidential aspects of individual firms operations (salaries, production volume, market areas), flags are placed in the data that can represent an insufficient sample size or simply a notice that data could not be disclosed. In these cases such values were excluded from the modeling and the subsequent models developed were used to replace the missing values. The table on the following page (Table 2-1) describes the commodities used in this study.

Employment and population data were also utilized in the modeling of the commodities. These data are coded by the North American Industrial Classification System, or NAICS, and appear in County Business Patterns (CBP) (U.S. Bureau of Census, 2004). The year of analysis for the $C B P$ was for the year closest to 1997 commodity data, 1998. A three-digit NAICS level was used. However, there is no natural alignment between any level of NAICS data and SCTG data. However, there is a guide for determining the proportion of NAICS data to SCTG data. Symmetry was reached between the two data sets by determining the proportions of each NAICS commodity (the more specific level) within each SCTG commodity (the less specific level).

The study and the modeling here are based primarily on the 1997 Commodity Flow Survey (CFS). This was the latest version of the $C F S$ with data available when the project began; the 2002 CFS appeared in 2005 and this was used for commodity discussions and model evaluation (U.S. Bureau of Census, 2005). 
Table 2-1 Major Commodity Groups Included in the Study

\begin{tabular}{|c|c|}
\hline SCTG & Commodity \\
\hline 01 & Live Animals and Fish \\
\hline 02 & Cereal Grains \\
\hline 03 & Agricult Products Exc. Live Animals, Cereal Grains, and Forage Products \\
\hline 04 & Animal Feed and Products of Animal Origin \\
\hline 05 & Meat, Fish, Seafood, and Preparations \\
\hline 06 & Milled Grain Products and Preparations, and Bakery Products \\
\hline 07 & Prepared Foodstuffs, Fats, and Oils \\
\hline 08 & Alcoholic Beverages \\
\hline 09 & Tobacco Products \\
\hline 10 & Monumental or Building Stone \\
\hline 11 & Natural Sands \\
\hline 12 & Gravel and Crushed Stone \\
\hline 13 & Non-metallic Minerals \\
\hline 14 & Metallic Ores \\
\hline 15 & Coal \\
\hline 17 & Gasoline and Aviation Turbine Fuel \\
\hline 18 & Fuel Oils \\
\hline 19 & Products of Petroleum Refining and Coal Products \\
\hline 20 & Basic Chemicals \\
\hline 21 & Pharmaceutical Products \\
\hline 22 & Fertilizers and Fertilizer Materials \\
\hline 23 & Chemical Products and Preparations \\
\hline 24 & Plastics and Rubber \\
\hline 25 & Logs and Other Wood in the Rough \\
\hline 26 & Wood Products \\
\hline 27 & Pulp, Newspaper, Print, and Paperboard \\
\hline 28 & Paper or Paperboard Articles \\
\hline 29 & Printed Products \\
\hline 30 & Textiles, Leather, and Articles \\
\hline 31 & Non-metallic Mineral Products \\
\hline 32 & Base Metal in Primary or Semi-finished Forms and in Basic Shapes \\
\hline 33 & Articles of Base Metal \\
\hline 34 & Machinery \\
\hline 35 & Electronic and Other Elect. Equipment/Components; Office Equipment \\
\hline 36 & Motorized Vehicles \\
\hline 37 & Transportation Equipment \\
\hline 38 & Precision Instruments and Apparatus \\
\hline 39 & Furniture, Mattresses, Lamps, Lighting Fittings, and Illuminated Signs \\
\hline 40 & Miscellaneous Manufactured Products \\
\hline 41 & Waste and Scrap \\
\hline 43 & Mixed Freight \\
\hline
\end{tabular}




\section{References}

U.S. Census Bureau (1996), 1992 Census of Transportation, Communication, and Utilities “1993 Commodity Flow Survey.” Washington, D.C.: U.S. Department of Commerce.

U.S. Census Bureau (1999), Economic Census, Transportation: 1997 Commodity Flow Survey, Washington, DC: U.S. Department of Commerce.

U.S. Census Bureau (2004), 1998 County Business Patterns, Washington, DC: U.S. Department of Commerce. Web page, http://www.census.gov/epcd/cbp/view/cbpview.html, various access dates.

U.S. Census Bureau (2005), 2002 Commodity Flow Survey, Washington, DC: U.S. Department of Commerce. CD C1-E02-ECFS-00-US1 


\section{Chapter 3}

\section{GOODS EXAMINED AND TRANSPORT FACILITIES}

\section{All Commodities}

A discussion of the existing flows of commodities is limited by the nature of freight data collection in the US. The latest available data for comparison are from the 1997 Commodity Flow Survey (CFS) of the Department of Commerce, released in 1999 and the 2002 Commodity Flow Survey released by the same agency in 2005. The project which led to the preparation of this report was initiated with the idea that it would use 1997 data for all modeling. The appearance of the 2002 census led to certain revisions in the study design so as to make use of these newer data. For one thing the discussion of commodities in this chapter makes use of both the 1997 and the 2002 census databases. In addition the 2002 census was used as a target date for modeling the flows using 1997 data. In other words, the models derived were used to estimate values for 2002. This will be described in a later chapter where we evaluate the models.

With each of the last three CFSs, the nationwide sample size has shrunk. The original CFS included a sample of the shipping practices of 200,000 shippers. In 1997 the CFS involved only 100,000 shippers. The most recent CFS surveyed the shipping of only 50,000 firms. This means that the quality of the data, and thus the quality of subsequent analyses, is most likely declining in accuracy and specificity. While the precursor to this research, the 1997 Indiana flow study (involving data from 1993), was able to describe the breakdown of shipments to and from individual states with some confidence, the 1997 data had far more missing values that needed to be estimated.

Another limitation has arisen with the change from Standard Transportation Commodity Classification (STCC) codes to the Standard Classification of Transported Goods (SCTG) codes for classification of commodities. This was a consequence of the North American Free Trade Agreement, also known as NAFTA, which necessitated a bridge between Canadian shipment codes and US shipment codes. The result has been a new set of commodity groups that is largely incompatible with the previous set of these commodity groups. While even some names at the 2digit level have remained the same, the individual subcategories have been drastically altered. Any comparison to flows from the previous study, therefore, would be biased by changes in the subcategories making up each classification code. As mentioned earlier, further specification (at 
the 3 or 4-digit level) is undesirable due to the smaller sample and impossible due to the reporting restrictions for these data.

Summary tables of the commodities, tons, and values originating in Indiana in 1997 and 2002 appear on the following pages as tables 3-1 and 3-2.

The discussion of the commodities below includes value, tonnage, and average shipment length of goods originating in and attracted to Indiana. This is for the 41 major classes of commodities summarized in the tables on the following pages.

\section{Individual Commodities}

\section{SCTG 01: Live Animals and Fish}

Most of the information on SCTG 01 is flagged due to sampling and other error in the 1997 CFS. The only data available indicate that 926,000 tons of Live Animals and Fish were shipped in 1997. Shipments leaving Indiana averaged 506 miles per shipment. Travel on trucks averaged 197 miles per shipment, while shipments on multiple modes averaged 787 miles. Other and unknown modes averaged 47 miles per shipment.

By 2002 the transport of live animals and live fish had dropped to nothing according to the CFS. Although there was a slight drop at the national level, it is more likely that the sampling design simply missed the sector in Indiana. This does not mean that the industry is gone in an absolute sense. It is likely that in the Indiana case there may have been shippers moving cattle, chickens or turkeys to market, and these were simply not sampled in 2002. It should be noted that the data were not witheld due to disclosure requirements or statistically unreliability; the industry is simply not reported and this suggests it may have been missed by the sampling system used in the CFS.

The attraction of live animals to Indiana in 1997 was 320,000 tons valued at $\$ 348$ million. Looking at this sector in 2002 the attractions are not reported primarily due to potential unreliability of the sample size. The problems with this sector are undoubtedly related to the decrease in overall sample size of the CFS and this a demonstration of the inherent problems in decreasing the size of the sample.

\section{SCTG 02: Cereal Grains}

In 1997 Indiana shipped 12.32 million tons of cereal grains at a value of $\$ 1.36$ billion dollars; these moved an average of 89 miles. Almost all of these shipments were by single modes. Trucks accounted for 5 million tons (40.6\%) of shipments and $\$ 528$ million of value (38.8\%), while distance shipped averaged 52 miles per shipment. Rail accounted for a significant load of cereal grain traffic, with 6.17 million tons (50.1\%) worth $\$ 705$ million 
Table 3-1 Value and Tons of Commodities for Indiana, 1997

\begin{tabular}{|c|c|c|c|}
\hline $\begin{array}{l}\text { SCTG } \\
\text { Code }\end{array}$ & Commodity Group & $\begin{array}{r}\text { Value } \\
\text { (millions) }\end{array}$ & $\begin{array}{c}\text { Tons } \\
\text { (thousands) }\end{array}$ \\
\hline 01 & Live Animals and Fish & N/A & 926 \\
\hline 02 & Cereal Grains & $\$ 1,362$ & 12,316 \\
\hline 03 & $\begin{array}{l}\text { Agricultural Products Except Live Animals, Cereal Grains, and } \\
\text { Forage products }\end{array}$ & 2,323 & 7,318 \\
\hline 04 & Animal Feed and Products of Animal Origin & 2,443 & 6,759 \\
\hline 05 & Meat, Fish, Seafood, and Preparations & 2,172 & 1,018 \\
\hline 06 & Milled Grain Products and Preparations, and Bakery Products & 4,746 & 3,664 \\
\hline 07 & Prepared Foodstuffs, Fats, and Oils & 7,725 & 12,856 \\
\hline 08 & Alcoholic Beverages & 1,348 & 1,315 \\
\hline 09 & Tobacco Products & 561 & 31 \\
\hline 10 & Monumental or Building Stone & 102 & 535 \\
\hline 11 & Natural Sands & 27 & 6,772 \\
\hline 12 & Gravel and Crushed Stone & 456 & 80,944 \\
\hline 13 & Non-metallic Minerals & 204 & 7,559 \\
\hline 14 & Metallic Ores & N/A & N/A \\
\hline 15 & Coal & 552 & 24,817 \\
\hline 17 & Gasoline and Aviation Turbine Fuel & 4,620 & 20,031 \\
\hline 18 & Fuel Oils & 2,706 & 14,141 \\
\hline 19 & Products of Petroleum Refining and Coal Products & 3,273 & 26,530 \\
\hline 20 & Basic Chemicals & 1,740 & 7,653 \\
\hline 21 & Pharmaceutical Products & N/A & 83 \\
\hline 22 & Fertilizers and Fertilizer Materials & 626 & 2,738 \\
\hline 23 & Chemical Products and Preparations & 2,816 & 1,367 \\
\hline 24 & Plastics and Rubber & 7,732 & 2,310 \\
\hline 25 & Logs and Other Wood in the Rough & 101 & N/A \\
\hline 26 & Wood Products & 2,911 & 3,212 \\
\hline 27 & Pulp, Newspaper, Print, and Paperboard & 1,113 & 1,363 \\
\hline 28 & Paper or Paperboard Articles & 1,997 & 1,553 \\
\hline 29 & Printed Products & 10,893 & 2,527 \\
\hline 30 & Textiles, Leather, and Articles & 6,216 & 329 \\
\hline 31 & Non-metallic Mineral Products & 3,510 & 18,975 \\
\hline 32 & Base Metal in Primary or Semi-finished Forms and in Basic Shapes & 23,929 & 38,952 \\
\hline 33 & Articles of Base Metal & 6,630 & 3,077 \\
\hline 34 & Machinery & 17,486 & 2,540 \\
\hline 35 & $\begin{array}{l}\text { Electronic and Other Electrical Equipment and Components; Office } \\
\text { Equipment }\end{array}$ & 17,989 & 2,062 \\
\hline 36 & Vehicles & 34,975 & 8,370 \\
\hline 37 & Transportation Equipment & 2,364 & N/A \\
\hline 38 & Precision Instruments and Apparatus & 3,117 & 62 \\
\hline 39 & $\begin{array}{l}\text { Furniture, Mattresses and Mattress Supports, Lamps, Lighting } \\
\text { Fittings, and Illuminated Signs }\end{array}$ & 3,817 & 676 \\
\hline 40 & Miscellaneous Manufactured Products & 12,838 & 3,182 \\
\hline 41 & Waste and Scrap & 1,512 & 8,426 \\
\hline 43 & Mixed Freight & 1,356 & 481 \\
\hline
\end{tabular}


Table 3-2 Value and Tons of Commodities for Indiana, 2002

\begin{tabular}{|c|c|c|c|}
\hline $\begin{array}{l}\text { SCTG } \\
\text { Code }\end{array}$ & Commodity Group & $\begin{array}{c}\text { Value } \\
\text { (millions) }\end{array}$ & $\begin{array}{c}\text { Tons } \\
\text { (thousands) }\end{array}$ \\
\hline 01 & Live Animals and Fish & N/A & N/A \\
\hline 02 & Cereal Grains & $\$ 1,948$ & 12,316 \\
\hline 03 & $\begin{array}{l}\text { Agricultural Products Except Live Animals, Cereal Grains, and } \\
\text { Forage products }\end{array}$ & 1,911 & 7,318 \\
\hline 04 & Animal Feed and Products of Animal Origin & 811 & 6,759 \\
\hline 05 & Meat, Fish, Seafood, and Preparations & 1,774 & 1,018 \\
\hline 06 & Milled Grain Products and Preparations, and Bakery Products & N/A & 3,664 \\
\hline 07 & Prepared Foodstuffs, Fats, and Oils & 12,356 & 12,856 \\
\hline 08 & Alcoholic Beverages & 276 & 1,315 \\
\hline 09 & Tobacco Products & 1,108 & 31 \\
\hline 10 & Monumental or Building Stone & N/A & 535 \\
\hline 11 & Natural Sands & 62 & 6,772 \\
\hline 12 & Gravel and Crushed Stone & 402 & 80,944 \\
\hline 13 & Non-metallic Minerals & 415 & 7,559 \\
\hline 14 & Metallic Ores & 73 & N/A \\
\hline 15 & Coal & 477 & 24,817 \\
\hline 17 & Gasoline and Aviation Turbine Fuel & 8,180 & 20,031 \\
\hline 18 & Fuel Oils & 2,055 & 14,141 \\
\hline 19 & Products of Petroleum Refining and Coal Products & 3,406 & 26,530 \\
\hline 20 & Basic Chemicals & 2,354 & 7,653 \\
\hline 21 & Pharmaceutical Products & 6,063 & 83 \\
\hline 22 & Fertilizers and Fertilizer Materials & 1,065 & 2,738 \\
\hline 23 & Chemical Products and Preparations & 7,351 & 1,367 \\
\hline 24 & Plastics and Rubber & 11,835 & 2,310 \\
\hline 25 & Logs and Other Wood in the Rough & N/A & N/A \\
\hline 26 & Wood Products & 3,877 & 3,212 \\
\hline 27 & Pulp, Newspaper, Print, and Paperboard & N/A & 1,363 \\
\hline 28 & Paper or Paperboard Articles & 2,857 & 1,553 \\
\hline 29 & Printed Products & 3,211 & 2,527 \\
\hline 30 & Textiles, Leather, and Articles & 10,962 & 329 \\
\hline 31 & Non-metallic Mineral Products & 3,369 & 18,975 \\
\hline 32 & Base Metal in Primary or Semi-finished Forms and in Basic Shapes & 23,253 & 38,952 \\
\hline 33 & Articles of Base Metal & 8,328 & 3,077 \\
\hline 34 & Machinery & 30,097 & 2,540 \\
\hline 35 & $\begin{array}{l}\text { Electronic and Other Electrical Equipment and Components; Office } \\
\text { Equipment }\end{array}$ & 23,158 & 2,062 \\
\hline 36 & Vehicles & 56,621 & 8,370 \\
\hline 37 & Transportation Equipment & N/A & N/A \\
\hline 38 & Precision Instruments and Apparatus & 4,145 & 62 \\
\hline 39 & $\begin{array}{l}\text { Furniture, Mattresses and Mattress Supports, Lamps, Lighting } \\
\text { Fittings, and Illuminated Signs }\end{array}$ & 4,678 & 676 \\
\hline 40 & Miscellaneous Manufactured Products & 12,558 & 3,182 \\
\hline 41 & Waste and Scrap & 1,483 & 9,883 \\
\hline 43 & Mixed Freight & 29,361 & 8,782 \\
\hline
\end{tabular}


(51.7\%) traveling an average of 577 miles. Water shipments (entirely in shallow draft) accounted for 1.15 million tons (9.3\%) and \$129 million (9.4\%) in goods, average 835 miles per shipment.

Shipments increased in 2002 to 23.85 million tons, nearly twice the 1997 volume, but the value of these shipments only increased to $\$ 1.94$ billion (an increase of $43 \%$ ). One could attribute part of this to an export market, however since the shipments in the CFS only indicate the domestic destinations, i.e., the point of export, we do not know where the shipments were going outside the U.S. This suggests that the drop in value per unit of weight was most likely related to an increase in production, or possibly a substantial negotiated trade agreement. The length of shipments was up substantially to 354 miles with $81 \%$ of the shipments moving by rail and $12 \%$ moving by water.

The attraction of cereal grains to Indiana destinations in 1997 was valued at \$817 million and represented 7,790,000 tons. This was moved an average of 62 miles suggesting that the grains also originated in Indiana for the most part.

The value of these grain shipments in 2002 was significantly lower with the value dropping to $\$ 430$ million and the tonnage dropping to 4,325,000 tons, slightly more than half of its 1997 level. Shipment length was 242 miles, representing a significant jump from 1997.

\section{SCTG 03: Other Agricultural Products}

There were 7.32 million tons of other agricultural products with a value of $\$ 2.3$ billion dollars shipped from Indiana in 1997. These products include crops such as soya beans, dried fruit, potatoes, vegetables, and flowers. The average length of these shipments was 149 miles. Among these products, $88.4 \%$ of tons (6.5 million) and $89.4 \%$ ( $\$ 2.1$ billion) of the value was shipped an average of 101 miles by single modes of travel. Truck shipments were 3.15 million tons (43\%) with a value of $\$ 1.25$ billion (53.8\%) and averaged 99 miles per shipment. Rail modes accounted for about 2 million tons (27.2\%) and \$483 million (20.8\%), and averaged 538 miles in shipment length. Water shipments also occurred, most along shallow draft, which accounted for 1.33 million tons (18.2\%) and \$344 million (14.8\%), averaging 817 miles. Multiple mode shipments averaged 763 miles, while unknown modes averaged 226 miles.

These shipments were down slightly for Indiana in 2002. They amounted to 7.17 million tons valued at $\$ 1.9$ billion, which were shipped an average of 158 miles. Truck modal share had increased to $58.9 \%$ of the tonnage and $73.3 \%$ of the value, while rail shares had dropped to $23.5 \%$ and $15 \%$, respectively. Transfers by water also dropped in an absolute and share sense between 1997 and 2002. There were no shipments by multiple modes in 2002 based on the CFS of that year. 
The attraction of other agricultural products to Indiana locations in 1997 was at 3,884,000 tons and this was valued at $\$ 1.574$ million. Contrary to the grain case, other agricultural products saw a significant jump in tonnage and value at the time of the 2002 CFS. The value had risen to 2.019 million dollars on 5.252 million tons of products. The 207 mile shipment length would suggest that much of this originated outside of Indiana in 1997, but this was to increase even more in 2002 to 388 miles.

\section{SCTG 04: Animal Feed and Products of Animal Origin}

There were 6.76 million tons of animal feeds and similar products, valued at $\$ 2.44$ billion, shipped an average of 105 miles from Indiana in 1997. This commodity group includes products such as feed for cattle, bird seed, and cat and dog food. Over $99 \%$ of this group moved as single mode shipments an average distance of 50 miles. Trucks accounted for 5.56 million tons (82.2\%) worth $\$ 2.2$ billion (90.3\%), and moved an average of 43 miles. Rail modes accounted for 1.2 million tons (17.6\%), \$228 million (9.3\%) in value and moved an average of 688 miles. What few multiple mode shipments there were averaged 1,074 miles, while other/unknown modes averaged 835 miles per shipment. By 2002 production had dropped to 3.91 million tons and $\$ 805$ million. The truck modal share had dropped to 59.9\%, and rail had increased to $34.3 \%$.

The attraction of goods from this sector to Indiana destinations experienced a drop between the samples for1997 to 2002. In the former year the tonnage was 5.278 million tons compared to 3.997 million tons in 2002. The value of these shipments also dropped as would be expected; it was \$2.1 billion in 1997 and \$1.0 billion in 2002. The average shipping distance of 51 miles would suggest most origins were in Indiana.

\section{SCTG 05: Meat, Fish, Seafood, and Their Preparations}

A total of 1.1 million tons of meats and poultry, valued at $\$ 2.17$ billion dollars, originated in Indiana in 1997. Almost all (99.5\% of value and tonnage) were single mode shipments. Furthermore, as could be expected, almost all (99\% of value and tonnage) was by truck. Shipments moved an average distance of 101 miles.

Shipments from this sector also dropped in 2002. Tonnage had decreased to 871 thousand tons, valued at $\$ 1.77$ million. All of these shipments moved by motor carrier. The average length of haul was 142 miles.

The goods in this group sent to Indiana destinations increased significantly in value from 1997 to 2002. The values were $\$ 2.9$ billion and $\$ 4.2$ billion, respectively. The tonnage changed from 1.1 billion to 1.6 billion tons. The length of these moves increased over the 1997 to 2002 period from 136 miles to 188 miles. 


\section{SCTG 06: Milled Grain Products and Preparations, and Bakery Products}

There were 3.66 million tons of this commodity group, worth $\$ 4.75$ billion, shipped from Indiana origins in 1997. The group included products such as baked goods, wheat flour, malt, and pasta, which was shipped an average distance of 134 miles. Over $98 \%$ of the tonnage and value moved by single modes averaging 119 miles per shipment. Three million tons (82\%) and $\$ 4.53$ billion (95.5\%) in value traveled an average of 116 miles by truck. Rail shipments moved an average of 662 miles and included 452,000 tons (12.3\%) that were worth \$116 million (2.4\%). The minimal amount of water shipments (no exact data available) averaged 1,140 miles per shipment, while multiple mode shipments averaged 708. Changes in tonnages and values in 2002 were not very significant.

The attraction of milled grain products and other goods in this sector to Indiana nearly doubled in value and tonnage between the two CFS years. The value of shipments received was $\$ 2.4$ billion dollars in 1997 and this had increased to $\$ 4.8$ billion in 2002. Tonnage increased from 2.3 million tons to 4.4 million tons in 2002.

\section{SCTG 07: Other Prepared Foodstuffs and Fats and Oils}

This sector includes milk, cheeses, ice creams, juices, fats and oils, among a broad list of other foodstuffs. A total of 12.9 million tons of this group, valued at $\$ 7.73$ billion, moved an average of 75 miles per shipment. Slightly less than $97 \%$ of this tonnage and $99 \%$ of the value traveled via single modes an average of 72 miles per shipment. Trucks accounted for 10.4 million tons (81\%) and $\$ 7$ billion (90\%) of the group's value. These shipments moved an average of 69 miles. Two million tons (16\%), valued at \$607 million (8\%), moved an average of 829 miles via rail. An undisclosed amount of air shipments traveled 700 miles per load, while $1.0 \%$ ( $\$ 78$ million) of the goods total value was shipped by multiple modes an average of 575 miles.

The volume and value of this commodity group were up in 2002 to 16.1 million tons and $\$ 12.36$ billion, respectively. The increase was picked up primarily by motor carriers, which increased their modal share to $87.1 \%$ of the tonnage and $95.9 \%$ of the value. Rail was responsible for most of the remainder.

Shipments of these goods to Indiana destinations were probably to a large extent from Indiana origins since the average shipping distance was only 79 miles. The value of such terminating shipments increased in value only slightly from $\$ 8.2$ billion to $\$ 9.1$ billion. The tonnage was 9.5 million tons in 1997 compared with 12.6 million tons in 2002. This suggests a significant drop in the value per ton. 


\section{SCTG 08: Alcoholic Beverages}

Beers, wine and other alcoholic beverages amounted to 1.3 million tons worth $\$ 1.35$ billion in 1997. These were shipped an average of 48 miles per shipment. Over $98 \%$ of value and tonnage traveled in single mode journeys, averaging 47 miles per shipment. Almost all of this commodity group (1.29 million tons and $\$ 1.3$ billion of its value) moved 47 miles by truck per shipment. The undisclosed amount traveling on rail moved 1,006 miles per shipment. \$16 million (1.2\%) traveled 847 miles per shipment via multiple modes.

The volume and value of this commodity group dropped significantly in 2002 according to the CFS for that year. Tons were down to 303 thousand and value was down to $\$ 276$ million, representing decreases of $77 \%$ and $80 \%$, respectively, from their 1997 levels. Motor carriers moved this commodity group an average of 64 miles per shipment in 2002.

Shipments of alcoholic beverages to Indiana destinations dropped between 1997 and 2002, both in value and tonnage. In 1997 the value was $\$ 1.7$ billion and by 2002 this had dropped to $\$ 867$ million, a drop of nearly 50\%. The tonnage of this product also dropped from 1.8 million tons in 1997 to 1.2 million tons in 2002. These shipments moved an average of 61 miles suggesting numerous origins for these shipments in Indiana. On the other hand this distance increased to 241 miles in 2002.

\section{SCTG 09: Tobacco Products}

Only 31,000 tons, valued at $\$ 561$ million, of tobacco products were shipped from Indiana origins an average of 45 miles per shipment in 1997. Over 99\% of the value and tonnage were by single modes an average of 40 miles, almost exclusively by truck. Multiple mode shipments averaged 415 miles, almost all reportedly occurring by mail service.

By 2002 motor carriers had captured all of this traffic. Traffic production was up in 2002 to $\$ 1.1$ billion, while tonnage had dropped to 16,000 tons. Average length of shipment was nearly the same (39 miles).

Tobacco product shipments to Indiana destinations in 1997 were 48,000 tons valued at $\$ 948$ million. These shipments have dropped significantly to 22,000 tons, but the value of shipments has actually increased to $\$ 1.1$ billion. The average length of shipment was 179 miles suggesting out of state origins for most of the shipments. This distance value increased to 304 miles in 2002.

\section{SCTG 10: Monumental or Building Stone}

There was 535,000 tons of monumental and building stone worth \$561 million dollars shipped an average of 45 miles per shipment in 1997. Over 99\% of the value and tonnage moved 
by single modes, and averaged 40 miles per shipment. Almost all of these shipments were by truck. An undisclosed amount of multiple mode shipments averaged 145 miles per shipment.

By 2002 shipments had dropped significantly. In fact the shipments were so low that nothing was reported in 2002 except for the average shipment length of seven miles. We do know that truck remained the dominant mode, but the data collected was considered too unreliable for publication. This probably indicates a loss of market share for this sector of Indiana's economy. It has been under significant pressure from various concrete manufacturers.

The value of monumental and building stone shipped to Indiana destinations was suppressed in 1997 and 2002. We do know that the weight of these shipments was 476 million tons, but once again the smaller sampling rate misses this industry for 2002. The industry continues to be viable for specialized construction needs.

\section{SCTG 11: Natural Sands}

A total of 6.7 million tons of natural sands valued at $\$ 27$ million was shipped an average of 24 miles from Indiana origins in 1997. This would suggest that most of the traffic also terminated in Indiana. No tonnages are disclosed, but $\$ 22$ million worth moved by single modes for 25 miles per shipment. Of this amount $76.8 \%$ (\$20.8 million) was shipped by truck an average distance of 25 miles. Water shipments, meanwhile, traveled 237 miles, while multiple modes traveled 474 miles per shipment.

This sector saw some growth and by 2002 the tonnages had increased to 14.7 million tons valued at $\$ 62$ million. Motor carriers moved 100\% of this traffic and average of 15 miles per shipment.

The shipment of natural sands to Indiana destinations nearly doubled in value and tonnage for the two CFS years. The value increased from $\$ 57$ million in 1997 to $\$ 103$ million in 2002, on tonnages of 8 million tons and 16 million tons, respectively. Average length of these shipments was 59 miles suggesting mostly in-state origins.

\section{SCTG 12: Gravel and Crushed Stone}

Almost 81 million tons of gravel and crushed stone with a value of $\$ 456$ million was shipped from Indiana origins in 1997. The average shipment length was 24 miles so this is also a product group usually destined for Indiana. More than $98 \%$ of the tonnage (80 million tons) and $97 \%$ of the value (\$446 million) were shipped by single modes an average distance of 22 miles. Most of this is by truck (77.5 million tons or $95.7 \%$ and $\$ 432$ million or $94.8 \%$ ) an average distance of 22 miles per shipment. Shallow draft water modes accounted for $2.6 \%$ of the tonnage and $3.1 \%$ of the value shipped, averaging 359 miles per shipment. Multiple mode shipments averaged 447 miles. 
Both the tonnage and value of gravel and crushed stone shipments dropped in 2002. The new values were 73 million tons and $\$ 402$ million. Trucking continued as the dominant shipping mode with $94.3 \%$ of the tonnage.

Shipments of this low-valued product to Indiana destinations dropped between 1997 and 2002 from 82 million tons to 71 million tons, with decreases in total value from $\$ 466$ million to $\$ 418$ million. Shipment length was about 34 miles which is consistent with the low value of the product. This distance dropped to 20 miles in 2002 CFS.

\section{SCTG 13: Nonmetallic Minerals}

In 1997 nonmetallic minerals, which includes limestone, clays, and salt and other such minerals, accounted for 7.56 million tons with a value of $\$ 204$ million. Of this $98 \%$ of the tonnage and value were shipped by single modes an average of 57 miles. Of the total 7.4 million tons (98.2\%) worth $\$ 189$ million (92.5\%) were shipped by truck, with an average distance of 56 miles. Undisclosed rail shipments averaged 1,667 miles per shipment, while multimodal shipments averaged 310 miles.

Although the value of these shipments was up in 2002 to $\$ 415$ million, other data on tonnages and mode shares were considered too unstable statistically to report.

Shipments of non-metallic minerals to Indiana destinations amounted to 10 million tons in 1997, valued at $\$ 295$ million. These shipments were valued at $\$ 411$ million in 2002, but the tonnage value was withheld. It is reasonable to infer that the tonnage of these shipments increased in 2002. Most of these shipments would be between Indiana locations as the average shipping distance was about 98 miles.

\section{SCTG 14: Metallic Ores and Concentrates}

There is very limited information about the shipment characteristics for metallic ores and concentrates (usually iron ores in Indiana's case) for 1997. We only know that average travel distance shipped by for-hire truck is 396 miles, while multiple modes and other and unknown modes averaged shipping distances of 166 mile and 276 miles respectively. This may very well indicate shipments of foreign origin being counted as originating at an Indiana Great Lakes port.

In 2002 the tonnage shipped was 66 thousand tons, valued at $\$ 73$ million. Motor carriers control most of this traffic with a $99 \%$ share of both tonnage and value. The average length of these shipments was 318 miles.

Shipments of these ores to Indiana destinations come for the most part from outside of 
the state, except for the port case above. The state really has no indigenous metallic ores except for those it receives from other areas and then may transship from one Indiana location to another. The value of these shipments was \$295 million in 1997 and this increased to \$558 in 2002. The values also increased from 13.5 million tons in the former year to 17.6 million tons in the latter year. Shipping distances increased substantially from 263 miles to 446 miles.

\section{SCTG 15: Coal}

There was 24.19 million tons of coal valued at \$552,000 million shipped from Indiana origins in 1997. The average shipment length was 33 miles. Approximately $85 \%$ of the tonnage and $87 \%$ of the value was shipped by single modes, averaging 33 miles per shipment. There were 3.6 million tons (15\%) and $\$ 62$ million (11.3\%) shipped by truck and average of 30 miles per shipment. Coal is a major product moved by rail in the U.S. and in the case of Indiana it accounted for 16.86 million tons (69.7\%) worth $\$ 416$ million (75.3\%) for which the average shipping distance was 109 miles. The multiple mode shipments averaged 120 miles, with no disclosed value and tonnage data available.

For 2002 the total coal moved is nearly the same with differences being accounted for by changes in energy demand for heating or cooling. Total tonnage moved was 23.45 million tons valued at $\$ 477$ million. Rail lost some modal share by 2002. It moved about $52 \%$ of the tonnage and trucks picked up 36.5\% of this traffic. Average length of shipments was 82 miles by truck and 60 miles by rail, which is counter to the general tendency for these modes.

Shipments of coal to Indiana destinations averaged 76 miles in 1997 (and 94 miles in 2002), most likely representing shipments from the southwestern part of the state to other state destinations. The tonnages of 50.2 million tons in 1997 and 62.2 million tons in 2002 reflect increasing demand in the state. The value of these shipments increased from 1 billion dollars in 1997 to 2.6 billion dollars in 2002.

\section{SCTG 17: Gasoline and Aviation Turbine Fuel}

A total of 20 million tons, valued at $\$ 4.6$ billion dollars, originated in Indiana, with an average shipping distance of 40 miles. Almost all (99.9\% of the value and tonnage) were single mode shipments. Of the total, $86.2 \%$ of tonnage ( $\$ 17.27$ million tons) and $87.1 \%$ of value ( $\$ 4$ billion dollars) were shipped by truck an average of 40 miles. No further data were offered.

By 2002 the tonnage had increased to 30.8 million tons with a value of $\$ 8.2$ billion. Approximately $43 \%$ of the tonnage moved by motor carriers and the remainder moved by pipeline. Average length of shipments in both cases was 29 miles.

There was a substantial increase in the value of these shipments to Indiana destinations between 1997 to 2002 of $\$ 5.8$ billion to $\$ 10.1$ billion. Tonnages also increased from 35.4 
million tons to 39.5 million tons, but this increase is low compared to the doubling of value reflecting the higher price of these goods in 2002. Shipping distances increased from 29 to 39 miles.

\section{SCTG 18: Fuel Oils}

A total of 14.14 million tons of fuel oil, valued at $\$ 2.7$ billion, originated in Indiana in 1997. Almost all (99.9\% of the value and tonnage) were single mode shipments. $68.1 \%$ of the tonnage (9.6 million tones) and $70.3 \%$ of the value ( $\$ 1.9$ billion dollars) were shipped by truck. The average shipment length by water and air are 2 and 543 miles respectively, and there is no further information available for 1997.

Motor carriers increased their modal share in 2002, but they lost traffic. Tonnages dropped in 2002 to 8.6 million tons and $\$ 2.1$ billion in total. Trucks picked up $85.8 \%$ of this traffic with rail and pipelines sharing the rest.

Shipments of fuel oil to Indiana destinations actually dropped between the two CFS years. These were 16.4 million tons in 1997 and 11.3 million tons in 2002. The value of these shipments also decreased from \$3 billion in 1997 to 2.6 billion in 2002. Shipment length of 19 miles would suggest these were mostly movements from pipelines in Indiana to Indiana distributors. The value remained low at 26 miles in 2002.

\section{SCTG 19: Coal and Petroleum Products}

Indiana shipped 26.5 million tons of coal and petroleum products, valued at $\$ 3.3$ billion dollars in 1997. This commodity group includes lubricating oils, liquified natural gas, asphalt, and similar products. The shipments for this group averaged 46 miles. Almost all of the value and tonnage (99.6\% of value and $99.7 \%$ of tonnage) were single mode shipments with an average shipment distance of 45 miles. Among the total shipments of this commodity, 17 million tons (6.9\%) and $\$ 1.95$ billion dollars (59.7\%) were shipped by truck. No other number is available for the rail, air or pipeline shipments except the average distance of 157 miles per air shipment.

In 2002 the tonnages were up. A total of 30.8 million tons valued at $\$ 3.4$ billion was shipped. Trucks handled $63.5 \%$ of the tonnage, while rail handled $8.2 \%$. The residual was picked up by air, pipeline, water and multiple modes in small enough amounts not to be reported.

Shipments of coal and petroleum products to Indiana destinations dropped only slightly between the two years. These were valued at \$3.7 billion in 1997 and this dropped to \$3 billion in 2002. Tonnages dropped slightly from 27.5 million tons to 25.1 million tons. Shipping distances averaged 53 miles and this would suggest primarily internal movement of these products within the state. The increase in this distance in 2002 to 81 miles does not change this 
conclusion.

\section{SCTG 20: Basic Chemicals}

Almost 7.65 million tons of basic chemicals with a value of $\$ 1.74$ billion were shipped from Indiana in 1997. This commodity group includes a host of chemicals and chemical compounds including among others bases, acids, phenols, and industrial gases. More than $99 \%$ of the tonnage (7.6 million tons) and $97.6 \%$ of the value ( $\$ 1.7$ billion) were shipped by single modes. About 3.8 million tons (50\%) and $\$ 1.35$ billion dollars (77.4\%) were shipped by truck, with an average distance of 43 miles. Another $\$ 241,000$ (13.8\%) was shipped by rail with tonnages and average shipping distances unavailable, but it is know that the average distance for air shipments was 1,180 miles. About $\$ 32$ million (1.9\%) of value was shipped by multiple modes, with undisclosed tonnage or average shipping distance.

Tonnages had dropped significantly by 2002. In that year the total tonnage shipped was 4.3 million tons valued at $\$ 2.4$ billion. Of these tons, approximately $46 \%$ moved by rail, and most of the remainder moved by truck except for a small amount moved by air. Truck shipping distances were an average of 272 miles in comparison to the air average of 1,886 miles.

Shipments of chemicals to Indiana destinations decreased over the two years examined here. Tonnages were 7,505,000 in 1997 and 3,469,000 in 2002; the value of these in 1997 was $\$ 2.3$ billion and $\$ 1.8$ billion in 2002. The average shipping distance in 1997 was 189 miles which would suggest that these are coming primarily from out of state. By 2002 this was more evident with an average shipping distance of 482 miles.

\section{SCTG 21: Pharmaceutical Products}

Indiana shipped 83,000 tons of pharmaceuticals in 1997 with an average shipping distance of 392 miles per shipment. Shipments going by single mode totaled 67,000 tons or $81 \%$ of the total weight shipped, with those shipments averaging 113 miles. Most of these were truck shipments (66,000 tons, $79.6 \%)$. The only data available for value reveal 1.35 billion dollars in private truck pharmaceutical shipments. There was also 297 million dollars ( $2.7 \%$ of all value) shipped by air, traveling an average distance of 424 miles per shipment. In addition, 14,000 tons (17.3\%), with a value of 965 million dollars, were shipped by multiple modes an average distance of 595 miles.

Traffic in pharmaceuticals was up in 2002. Shipments totaling 235,000 tons, representing a three-fold increase, were reported. Value of these shipments was reported at $\$ 6.1$ million. Motor carriers remained the dominant carrier with $91.4 \%$ of the tonnage moving an average of 323 miles. The remaining tonnage was moved by parcel post or couriers and averaged 363 miles. 
The shipments having destinations in Indiana increased substantially between 1997 and 2002. These were 205 thousand tons in 1997 and 751 million tons in 2002. These are low tonnages, but one should bear in mind that we are talking about pharmaceuticals which have low weight. The value on the other hand increased from $\$ 4.9$ million to $\$ 10.2$ million. These shipments reflect a national pattern in that the average length of shipments is 728 miles. This distance had dropped substantially by 2002 and was 406 miles.

\section{SCTG 22: Fertilizers}

There were 2.74 million tons of fertilizers moved an average shipment length of 447 miles in 1997; its value was $\$ 626$ million. Single modes accounted for 2.29 million tons, 83.5\% of all shipments; these were valued at $\$ 562$ million, nearly $90 \%$ of the total, and averaged 20 miles per shipment. Almost all single mode shipments (82\%) were by truck. No further data is available with the exception of shipments made on shallow draft water, which averaged 1,140 miles. This accounts for the multiple mode average of 971 miles, though only 3,000 tons were shipped this way - a tenth of a percent of all shipments.

Tonnages more than doubled by 2002, when 5.86 million tons of fertilizers worth $\$ 1.1$ billion originated in Indiana. Motor carriers moved $90.6 \%$ of this with lesser amounts moved by rail and water.

Shipments of fertilizers to Indiana destinations increased from 5.2 million tons in 1997 to 7.8 million tons in 2002. The value of these also increased from $\$ 1$ billion to $\$ 1.4$ billion in 2002. These shipments most likely went from distribution centers to retail outlets in that the average length of these shipments was 61 miles.

\section{SCTG 23: Chemical Products and Preparations}

A total of 1.37 million tons of chemical products and preparations, valued at $\$ 2.8$ billion, originated in Indiana and moved an average of 271 miles in 1997. This commodity group includes products such as paints and varnishes, soaps, pesticides, perfumes and cosmetics, among others. Approximately 1.3 million tons (95.8\%) valued at $\$ 2.36$ billion (83.7\%) traveled via single modes an average distance of 174 miles. Almost all of those shipments $(94.1 \%$ of the total) moved by truck. What little rail traffic there was (values and tons were flagged), moved 2,314 miles per shipment. There were also some air shipments; these averaged 1,119 miles per shipment. There were also shipments involving multiple modes; these shipments averaged 376 miles.

This sector saw significant growth between 1997 and 2002 with the latter year having traffic production of 2.19 million tons, valued at $\$ 7.4$ billion. Of this amount motor carriers moved $94.2 \%$ with lesser amounts moved by rail and some courier services. Average shipping distances overall were 199 miles. 
Plastics and rubber shipments to Indiana destinations were 2.7 million tons in 1997 and represented $\$ 4.3$ billion. In 2002 these numbers were 2.5 million tons and $\$ 5.4$ billion suggesting an increase in the value of the product, but this could be due to a different mix of chemical products in the category. Shipping distances changed from 261 miles in 1997 to 255 miles in 2002 suggesting a rather stable situation.

\section{SCTG 24: Plastics and Rubber}

In 1997 the total amount shipped of the plastics and rubber commodity group (which includes tires) was 2.3 million tons valued at $\$ 7.7$ billion dollars; its average shipping distance was 344 miles. For this group 2.14 million tons (92.8\%) and $\$ 6.5$ billion (84.3\%) of its value were shipped in single modes, which averaged 172 miles. Almost all of the total single mode shipments were carried by truck; a total of 2.11 million tons and $\$ 6.47$ billion, averaging 164 miles, were shipped by truck. Rail shipments averaged 899 miles. There was a paucity of air shipments, with 3,000 tons ( $0.1 \%$ ) valued at $\$ 28$ million (0.4\%) moving an average of 735 miles per shipment. There were 90,000 tons (3.9\%) and $\$ 982$ million (12.7\%) of these products with an average shipping distance of 543 miles shipped via multiple modes, while unknown modes accounted for $3 \%$ of the goods shipped and averaged 55 miles in shipment length.

By 2002 the shipments of this commodity group had increased to 3.78 million tons with a value of $\$ 11.1$ billion. The average length of haul for this commodity was 311 miles with 232 miles being the average length of motor carrier shipments. The latter mode handled $91.9 \%$ of the tonnage and $92.8 \%$ of the value of these shipments. Additional traffic moved by rail and air, as well as by multiple modes.

Shipments of plastics and rubber to Indiana destinations were fairly stable for the two years examined here. The value of these increased from $\$ 9.6$ billion to $\$ 9.75$ billion and the tonnages changed from 3.7 million tons to 3.8 million tons. The average length of shipments was 308 miles suggesting mostly out-of-state origins. The 2002 distance value was 376 miles.

\section{SCTG 25: Logs and Other Wood in the Rough}

Total tonnages and shipment lengths are not available for Indiana shipments even though these were valued at $\$ 101$ million. Most of these shipments (in value at least) were by single mode trips (98.9\%) with trucks accounting for $\$ 94$ million (93.6\%) of shipments. There were 19,000 tons (4.3\%) of shipments with a value of $\$ 5$ million (5.3\%) that moved an average of 878 miles per shipment by rail. There are no further data available for 1997.

The sector appears in the 2002 CFS, but except for some shipping distances there are no other data, i.e., there are no data for tonnages, value, or mode use for Indiana, due to the data collected being statistically unreliable. 
The shipment of logs and other wood to Indiana destinations is not very significant and the comparative values for shipments are not available. It seems relatively stable based on available information.

\section{SCTG 26: Wood Products}

Over 3.2 million tons of wood products, worth $\$ 2.9$ billion, originated in Indiana and were shipped an average of 242 miles in 1997 . Of these totals $97 \%$ of the tonnage and $95 \%$ of the value traveled by single modes an average distance of 179 miles. Trucks dominated the modal choice; $91 \%$ of the value ( $\$ 2.66$ billion) and $94 \%$ of the tonnage (3 million tons) moved an average distance of 173 miles by that mode. Rail accounted for 98,000 tons (3\% of the total tonnage) and $\$ 62$ million (2.1\% of the value) with those shipments averaging 1,442 miles. There were some air shipments of products and these averaged 935 miles per shipment. A small amount (22,000 tons and $\$ 83$ million in value) averaged 731 miles per shipment via multiple shipment modes.

For 2002 both tonnages and values were higher: tonnages were 6.34 million tons and value was at $\$ 3.88$ billion. Motor carrier shipments averaged 106 miles and carried $97.1 \%$ of the tonnage. A small amount of traffic moves by the multiple modes of rail and truck.

Shipment of wood products to Indiana destinations is significant with values in the range of $\$ 3.5$ billion in 1997 and $\$ 4.0$ billion in 2002. Tonnages for these years were 5.1 million tons and 7.0 million tons, respectively. The average shipping distance of 160 miles suggests a significant amount of out-of-state flow to Indiana destinations. This distance was 162 miles in 2002 suggesting once again a stable situation.

\section{SCTG 27: Pulp, Newsprint, Paper, and Paperboard}

A total of 1.36 million tons of this paper area, valued at $\$ 1.11$ billion was shipped from Indiana origins an average of 529 miles in 1997. About 93\% of the value ( $\$ 1.03$ billion) and tonnage (1.27 million tons) averaged 81 miles per shipment on single modes in Indiana. Also, 1.22 million tons (89\%) and $\$ 977$ million (88\%) averaged 78 miles per shipment on truck modes. A small proportion of traffic moved by rail; this was 3.7\% of the tonnage (51,000 tons) and $\$ 55$ million (5\%) of the value and it averaged 499 miles per shipment. Air shipments averaged 1,337 miles traveled, while multi-modal shipments (4,000 tons, $0.3 \%$ and $\$ 62$ million, $5.5 \%)$ moved 870 miles per shipment.

Data from the 2002 CFS are withheld in most cases due to their unreliable nature, statistically. We only know that these products moved by truck, rail, and air, but more detailed data for the state is not available. 
Shipments of pulp, newsprint and other items in this group to Indiana destinations represented \$2.3 billion in 1997 and \$2.3 billion as well in 2002. The tonnages for these two years were 3.3 million tons compared to the later value of 3.3 million tons. A fairly stable situation, but the average distances shipped increased from 164 miles to 258 miles.

\section{SCTG 28: Paper or Paperboard Articles}

Total tonnage of paper and paperboard articles was 1.55 million tons, valued at \$2 billion, with an average shipment length of 253 miles in 1997. Of the tonnage $97 \%$ with a value of $\$ 1.89$ billion) was transported on single modes an average of 117 miles per shipment. The vast majority of this group (96\% of tons and $92 \%$ of the value) was shipped by truck an average distance of 111 miles. Undisclosed amounts of rail shipments averaged 2,442 miles and air shipments 665 miles. Just 7,000 tons (0.5\%), valued at \$39 million (2\%) were shipped an average of 736 miles by multiple modes.

In 2002 the value of shipments increased to approximately $\$ 2.9$ billion. Tonnages were withheld, but we do know that motor carriers accounted for $95.9 \%$ of the value transported. Additional traffic was moved by rail, air and multiple modes.

Shipments of paper and paperboard products to Indiana destinations increased slightly from $\$ 2.4$ billion to $\$ 2.9$ billion. Tonnage values were suppressed for 2002, but the value for 1997 was 1.5 million tons. Even the length of these shipments was rather stable at 168 and 142 miles.

\section{SCTG 29: Printed Products}

There were 2.53 million tons of printed products with a value of $\$ 10.9$ billion dollars that originated in Indiana in 1997; these averaged 601 miles per shipment. Of this total 2.35 million tons (93\%) valued at $\$ 9$ billion (83\%) was transported by single modes an average of 590 miles. Motor carriers transported 92\% (2.33 million tons) of this category valued at $\$ 8.47$ billion (77.7\%) an average of 377 miles per shipment. Undisclosed amounts of air shipments averaged 1,263 miles. There were $4.7 \%$ (119,000 tons) and $\$ 1.36$ billion (12.5\%) shipped 651 miles by multiple modes, and $4.5 \%$ of the value ( $\$ 494$ million), shipped by unknown modes.

For 2002 originating flows dropped to 1.02 million tons valued at $\$ 3.2$ billion. Of this amount, $91.4 \%$ moved by truck, a share similar to 1997, but with substantially less tons than the former year. Average shipping distances overall were 717 miles and for motor carriers 308 miles.

Shipments of printed products to Indiana dropped in value and tonnage between 1997 and 2002. The value for the former was $\$ 6.4$ billion and this had dropped to $\$ 3.2$ billion in 2002 . The tonnages were 2.2 million in 1997 and 1 billion in 2002. It is difficult to assess whether this 
may be due to a difference in the manner in which these shipments were identified. The average length of these shipments was 358 miles in 1997 and 554 in 2002 suggesting a distribution of national origins.

\section{SCTG 30: Textiles, Leather, and Articles of Textiles or Leather}

SCTG 30 includes all of the goods in the title above, which includes textiles, yarns, carpets, luggage, footwear and some clothing. For this group 329,000 tons worth $\$ 6.2$ billion originated in Indiana, and averaged 701 miles per shipment. Of the total 251,000 tons (76\%) and $\$ 3.3$ billion based on value (53\%) moved as single mode shipments an average of 393 miles. Almost all of this was exclusively by truck, with an average distance of 373 miles per shipment. Undisclosed amounts of rail shipments averaged 1,119 miles, with air shipments averaging 699 miles. Multimodal shipments represented 68,000 tons (21\%) and $\$ 2.75$ billion (44\%) of shipments averaging 723 miles, all of which appears to be by mail.

Tonnages increased for total traffic produced for this group in 2002. Total tons increased to 649,000 tons valued at $\$ 10.96$ billion. Trucks carried $81.2 \%$ of this traffic and air and multiple modes (parcel, USPS, and courier) were responsible for the remainder. Average length of shipments for trucks was 187 miles, for air 1,790 miles and for parcel and related traffic it was 875 miles.

Shipments with destinations in Indiana were valued at $\$ 5.5$ billion in 1997 and these increased to $\$ 8.9$ billion in 2002. The tonnages involved also increased from 422,000 tons to 664,000 tons between these two years. The average length of shipments of 577 miles suggests that this is an industry moving goods from import locations. By 2002 this distance had increased to 595 miles.

\section{SCTG 31: Nonmetallic Mineral Products}

There were 19 million tons of nonmetallic mineral products (e.g., cements, glass products, and ceramic products) worth $\$ 3.5$ billion dollars that originated in Indiana in 1997. It averaged 500 miles per shipment. There were18.9 million tons (99.6\%) and \$3.3 billion dollars (94\%) of this group that moved by single modes, with an average shipment distance of 144 miles. Motor carriers transported $94.4 \%$ of the tonnage (18 million tons) and $92.8 \%$ of the value ( $\$ 3.26$ billion) an average of 141 miles. Shipments by rail represented $5.2 \%$ of the tonnage $(990,000$ tons) and $\$ 50$ million of the value (1.4\%). Undisclosed amounts of air shipments averaged 1,045 miles. There were also 58,000 tons $(0.3 \%)$ valued at $\$ 172$ million dollars $(4.9 \%)$ transported by multiple modes with an average travel distance of 809 miles.

For 2002 tonnages had increased to nearly 32 million tons valued at $\$ 3.4$ billion. Of these shipments $98 \%$ of the tons were handled by trucks, $1.8 \%$ by rail, and the remainder moved by air or multiple modes. 
Non-metallic mineral shipments with destinations in Indiana increased from 17.4 million tons in 1997 to 30.8 million tons in 2002. In spite of this significant increase in tonnage the value of these shipments only increased from $\$ 3.0$ billion to $\$ 3.1$ billion. The average length of shipments was 243 miles in 1997 and 206 miles in 2002.

\section{SCTG 32: Base Metal in Primary or Semi-finished Forms and in Finished Basic Shapes}

There were 39 million tons transported from Indiana origins in 1997. This commodity group, which includes iron, steel, copper, and aluminum forms, was valued at $\$ 24$ billion, and was shipped an average of 275 miles. Approximately 92\% (35.8 million tons) of the commodity valued at $\$ 22.4$ billion (93.7\%) were shipped on single modes that averaged 227 miles. Of these shipments $65.5 \%$ or 25.5 million tons woth $\$ 17.5$ billion dollars (73.2\%) were by truck and had an average shipment length of 203 miles per shipment. Rail hauled 10.3 million tons (26.3\%) worth $\$ 4.8$ billion (20.2\%) an average of 626 miles. An undisclosed amount of air shipments moved an average of 1,271 miles per shipment, while multiple modes averaged 690 miles per shipment.

By 2002 shipments had increased to 42.5 million tons, but the value had dropped to $\$ 23.3$ billion. These commodities moved an average of 232 miles. Of this total tonnage, 69\% moved by truck, 22.3\% moved by rail, with air, water, and multiple modes picking up the remainder. Shipments by truck averaged 191 miles, by rail 692 miles, by water 1,360 miles, and by air 784 miles.

Base metal forms and shapes shipments to Indiana decreased in value between 1997 and 2002; these values were $\$ 15.2$ billion in the earlier year and $\$ 13$ billion in the latter year. Tonnages also decreased. It dropped from 22.6 million tons to 19 million tons. Shipment length was 225 miles in 1997 and dropped to 202 miles in 2002.

\section{SCTG 33: Articles of Base Metal}

Three million tons of base metal articles worth $\$ 6.6$ billion moved an average shipment length of 226 miles in 1997. This commodity group includes everything from nuts and bolts to pipes and hand tools. Approximately $96 \%$ of the tonnage, valued at $\$ 6$ billion (90.4\%) was shipped by single modes an average of 230 miles, and $94 \%$ of the tonnage (2.9 million tons) with a value of nearly $\$ 6$ billion (89.4\%) was shipped an average of 217 miles by truck. Nondisclosed rail tonnages moved an average of 1,773 miles per shipment, and air modes averaged 988 miles per shipment. Multiple modes accounted for only 23,000 tons (0.7\%), but $\$ 437$ million (6.6\%) of shipments and averaged 319 miles per shipment. Truck and rail mode shipments averaged 2,135 miles.

By 2002 this group had increased only slightly to 3.1 million tons valued at $\$ 8.3$ billion. 
Of the total tonnage, 91.4\% moved by rail, with air, rail, and multiple modes picking up the remainder. Average shipment length by truck was 384 miles per shipment. Shipments by rail were shorter than in 1997 while shipments by air had increased in length.

Base metal articles with destinations in Indiana increased slightly in value and weight between the two census years. The shipments increased from 3.2 million tons to 3.5 million tons and the values increased from $\$ 6.4$ billion to $\$ 7$ billion. Shipping distances were high with an average of 280 miles in 1997 increasing to 341 miles in 2002.

\section{SCTG 34: Machinery}

This commodity group includes all types of machinery ranging from refrigerating equipment and air conditioners, to dishwashers and power tools, and boilers as well as turbines. There were 2.5 million tons of these goods shipped in 1997 with a value of $\$ 17.5$ billion; average shipping distance was 461 miles per shipment. Among those, 2.25 million tons (88.6\%) with a value of $\$ 14.25$ billion (81.5\%) were transported via single modes, with an average shipping distance of 383 miles. Trucks shipped $88.1 \%$ of the tonnage ( 2.23 million tons) and $\$ 14$ billion ( $80 \%)$ of the value on average 151 miles. Undisclosed rail shipments averaged 1,331 miles, and multiple modes averaged 585 miles in shipment length. There were 7,000 tons (worth \$245 million) shipped by air freight an average of 1,475 miles.

Shipments of this commodity group had increased to 3.5 million tons, valued at $\$ 30$ billion by 2002 . Of this tonnage $84.2 \%$ moved by truck, $2.1 \%$ by rail, and $3.6 \%$ moved by multiple modes. The length of shipments increased for trucks to 249 miles, and for rail it dropped to 829 miles.

Shipments of machinery ending in Indiana increased in value from $\$ 14$ billion to $\$ 18$ billion and the tonnages changed from 2 million tons to 2.1 million tons. Shipping distances were about 208 miles in 1997 and 264 miles in 2002 suggesting a Midwest origin for most of these Indiana bound shipments.

\section{SCTG 35: Electronic and other Electrical Equipment and Components and Office Equipment}

Two million tons of various types of electronics and electronic equipment worth $\$ 18$ billion were transported an average of 268 miles per shipment in 1997. Of this 90.7\% (1.9 million tons) with a value of $\$ 14$ billion (78.7\%) were transported an average of 150 miles by single modes. Most of transport was by truck, with 1.8 million tons (88.4\%) valued at $\$ 13$ billion (73\%) moving an average distance of 129 miles per shipment. Another 30,000 tons (1.5\%) were shipped by rail an average of 1,144 miles, while 14,000 tons $(0.8 \%)$ worth $\$ 837$ million (4.7\%) were shipped on average 1,197 miles by air. Multiple mode shipments accounted for 7.5\% (157,000 tons) valued at \$3.3 billion (18.2\%) with an average shipment length of 406 
miles.

Shipment tonnages had dropped to 1.6 million tons as its worth increased to $\$ 23.2$ billion in 2002. Of the various modes, trucks were dominant with $79.1 \%$ of the tonnage and $53.2 \%$ of the value. Multiple modes were also important with parcel, USPS and courier moving 10.1\% of the tonnage and truck and rail moving $9.2 \%$ of tonnage.

Electronics and electrical equipment shipments that had a destination in Indiana increased from 1.564 million tons in 1997 to 1.569 million tons in 2002, practically no change at all. On the other hand the value of these shipments increased substantially from \$15.8 billion to \$24.8 billion. Shipment lengths decreased from 347 miles to 265 miles suggesting more local origins for these goods in the Midwest.

\section{SCTG 36: Motorized and Other Vehicles}

Indiana shipped nearly 8.4 millions tons of motorized and other vehicles valued at \$35 billion an average of 278 miles per shipment in 1997. This commodity group includes roadbased motor vehicles ranging from bicycles to tractors, including all cars, trucks, and even combat vehicles. Most of the tonnage (85\%) and the value (73\%) were shipped by single modes an average of 173 miles. Trucks accounted for 6.1 million tons (73\%) and $\$ 24.2$ billion (73.3\%) of shipments. These moved an average of 129 miles. Rail shipped $12 \%$ of the tonnage but 3.6\% of the total value. These moved 603 miles per shipment. Multiple mode shipments occurred for $4 \%$ of the tonnage but nearly $10 \%$ of the value, while $11 \%$ of the tonnage and $17 \%$ of the value were shipped by unknown modes an average of 62 miles.

Traffic production increased for this sector in 2002 with 12.2 million tons valued at \$56.6 billion. Trucks moved the majority of this tonnage (73.8\%) and value (67.1\%), but rail was also significant with $11.9 \%$ of the tonnage and $5.9 \%$ of the value. Average shipment distances for these two modes were 207 and 604 miles, respectively. Multiple modes were important in 4.1\% of the moves by tonnage and $9.6 \%$ of the moves by value. Truck and rail multiple moves were the most significant of these.

Motor vehicles and the parts for these that were shipped to destinations in Indiana represented $\$ 24.6$ billion in value in 1997 and $\$ 38.2$ billion in 2002. The tonnages changed from 4.8 million tons to 6.8 million tons. The length of shipments changed from 162 miles to 186 miles, which is not a significant change.

\section{SCTG 37: Transportation Equipment}

This commodity group includes aircraft, spacecraft, and locomotives, as well as parts for these. There was $\$ 2.35$ billion in transport equipment shipped from Indiana an average of 528 miles in 1997 . Single modes handled $62.8 \%$ of that value, shipments averaging 528 miles, while 
the remaining $37.2 \%$ was handled by multiple modes and averaged 954 miles per shipment. No data are available on tonnages for 1997.

Data were not available on this group for Indiana in 2002 due to small sample size and statistical unreliability.

Shipments into Indiana are available and these increased in value from $\$ 734$ million for 1997 to $\$ 2$ billion in 2002. Tonnage in 1997 was 98,000 tons, but the 2002 value was not reported. The average length of these shipments changed from 503 miles to 724 miles suggesting some of these may be coming from port areas.

\section{SCTG 38: Precision Instruments and Apparatus}

The precision instruments included in this commodity group include cameras, photocopy machines, X-ray machines, and scientific measuring equipment. Only 62,000 tons of these instruments accounted for $\$ 3.1$ billion in freight flow originating in Indiana in 1997. These were shipped an average of 692 miles per shipment. A third of the value traveled 338 miles by single mode, while nearly $\$ 2$ billion (61.6\%) weighing 10,000 tons (16.8\%) traveled 780 miles in multiple mode shipments. An undisclosed amount of air shipments averaged 1,007 miles per shipment.

By 2002 the tonnage had dropped to 34,000 tons, but the value had increased to $\$ 4.1$ billion. Average shipment distance remained about the same at 741 miles. Approximately $95 \%$ of the shipments were by motor carrier or parcel, USPS, or courier.

Precision instruments shipped to Indiana destinations were down in terms of tonnage dropping from 123,000 tons to 107,000 tons between 1997 and 2002, but the value of these shipment increased from $\$ 3.2$ billion to $\$ 7.2$ billion. Shipping distances increased from 627 miles to 938 miles suggesting shipments from more distant locations, possibly port areas.

\section{SCTG 39: Furniture, Mattresses and Mattress Supports, Lamps, Lighting Fittings, and Illuminated Signs}

The title of this group is fairly descriptive of its content. In 1997, $\$ 3.8$ billion of furniture commodities weighing 676,000 tons originated in Indiana and were transported an average of 409 miles per shipment. Approximately $87 \%$ of this tonnage and $92 \%$ of this value were in single mode shipments averaging 366 miles. Trucks were used for most of this transport, with $\$ 3.5$ billion and 586,000 tons ( $91.8 \%$ and $86.7 \%$, respectively) being transported 352 miles per shipment. Multiple mode shipments accounted for $5 \%$ of the value and $2.5 \%$ of the tonnage, averaging 869 miles in shipment length.

Shipped tonnage remained nearly the same in 2002 at 660,000 tons. The value of these 
increased to $\$ 4.6$ billion. Motor carriers increased their modal share to $98.6 \%$ of the tonnage and $97.4 \%$ of the value in 2002 . The little remaining tonnage moved by rail, air, and multiple modes.

Shipments of these goods to Indiana destinations increased from about \$2 billion to \$3.4 billion. Tonnages increased from 247 miles to 425 miles, which may reflect a further decrease in the furniture industry within Indiana. Tonnages increased from 481,000 tons to nearly 746,000 tons.

\section{SCTG 40: Miscellaneous Manufactured Products}

This group of products that doesn't seem to fit anyplace else includes arms, munitions, toys, sporting equipment, clocks and prefabricated buildings. These products accounted for 3.2 million tons worth $\$ 12.8$ billion and averaged 522 miles per shipment. Of the total tonnage $95 \%$ and $83 \%$ of the value were transported 345 miles in single mode shipments. Trucks accounted for 2.85 million tons (89.5\%) and $\$ 9.9$ billion (76.8\%) of shipments, averaging 324 miles per load. An undisclosed amount of rail shipments averaged 952 miles per shipment, while multiple mode shipments averaging 612 miles accounted for only $2 \%$ of the tonnage but $14 \%$ ( $\$ 1.8$ billion) of the value.

In 2002 the tonnage was a little less, slightly more than 2.8 million tons, valued at $\$ 12.6$ billion. Of these totals, $95.5 \%$ of the tons and $91.7 \%$ of the value was transported by truck. The remainder of this traffic was carried by multiple modes. Average shipping distances were 387 miles by truck and 667 miles by multiple modes.

Shipments of this catch-all group with destinations in Indiana decreased from 2.4 million tons to 1.4 million tons; the value of shipments did not change. The length of the shipments increased slightly from 637 mile to 706 miles.

\section{SCTG 41: Waste and Scrap}

Waste and scrap includes slag, ash, sawdust and paper. In 1997 there were 8.4 million tons of this valued at $\$ 1.5$ billion that originated in Indiana. Of this amount $42.5 \%$ of the tonnage moved by truck and $57.5 \%$ moved by rail. In terms of value this situation was reversed with trucks handling $61.8 \%$ and rail handling $38.1 \%$. Average shipping distances for the two modes were 117 miles for trucks and 130 miles for rail.

This traffic increased in 2002 to 9.9 million tons, but its value remained the same at $\$ 1.5$ billion. Trucks increased their modal share handling $51.6 \%$ of the tonnage and $72.1 \%$ of the value transported. Rail continued as the second mode in this area with $38.9 \%$ of the tonnage and $21.5 \%$ of the value shipped. Average shipping distances were about 120 miles by the two modes combined. 
Shipments of waste and scrap destined for Indiana locations increased in value from \$2.5 billion to $\$ 4.7$ billion. Tonnage in 1997 was 10.5 million tons, but not reported in 2002 . The average shipping distance dropped from 134 miles to 70 miles.

\section{SCTG 43: Mixed Freight}

In 1997 mixed freight was moved almost entirely by motor carriers. It represented 478,000 tons by weight and $\$ 1.4$ billion in terms of value. They handled $99.2 \%$ of the tonnage and $98.0 \%$ of the value of these shipments. Shipments by parcel, USPS, and courier picked up most of the remainder. The Truck moves were 66 miles in length on the average and the parcel moves were 246 miles in length.

In 2002 the tonnage had increased to 8.7 million tons, 18 times its value in 1997. Value had increased to $\$ 29.4$ billion, 21 times its value in 1997. Most of this continued to be moved by truck transport which accounted for $98.7 \%$ of the tonnage and $97.1 \%$ of the value. Once again parcel, USPS and courier accounted for most of the remainder. Shipments averaged 468 miles in length for this latter group and 78 miles for truck.

Shipments of mixed freight having a destination in Indiana in 1997 represented 1.5 million tons and $\$ 2.6$ billion. In 2002 these were 7.3 million tons and $\$ 20$ billion. This is a phenomenal increase by any standard. The length of these shipments dropped from 238 to 208 miles.

\section{Intermodal Facilities in Indiana}

\section{The Ports}

Indiana has a total of six maritime ports; two along the Ohio River which eventually leads to the Gulf of Mexico by way of the Inland Waterway System, and four on Lake Michigan which gives access not only to the Inland Waterway System, but also to the rest of the Great Lakes, as well as to the Atlantic Ocean by way of the St. Lawrence Seaway. Only three of these ports are intermodal facilities, and these will be examined further in this section. Maritime ports are vital to Indiana's economy in that they are a major mode of transportation for heavy industrial goods, agricultural products, and stone and other minerals.

The Clark Maritime Center in Jeffersonville, Indiana is located on the north shore of the Ohio River across from Louisville, Kentucky. Built in 1985, it is reportedly the fastest growing port on the Inland Waterway System.

This port offers year round access to the Gulf of Mexico via the Inland Waterway System, service for many different general cargo products, and on-site storage for up to 1.6 million bushels of grain. This port is also a site of intermodal transportation with direct linkages 
to rail and highways, and two airports within fifteen miles. Additionally, on-site switching services for cargo on boats and rail are offered here.

The Clark Maritime Center has 962-acres with 20 tenants, 3,200 linear feet of riverfront access, and is classified as a Foreign Trade Zone. The goods most often handled at this port are steel, iron, grain, fertilizer, salt, and asphalt.

The Southwind Maritime Center in Mount Vernon, Indiana is located on the north shore of the Ohio River fifteen miles west of Evansville, Indiana. It is a top port in throughput tonnage, handling an average of more than 2 million tons of cargo each year. This port also offers year round access to the Gulf of Mexico via the Inland Waterway System, including the Mississippi and Tennessee-Tombigbee Waterway Systems.

This port offers storage facilities for general cargo, bulk commodities, and specialized agricultural commodities including a 4.75 million bushel capacity grain elevator, three milliongallon liquid fertilizer storage tanks, and a covered storage facility with the capacity to hold up to 85,000 tons of dry fertilizer. The Southwind Maritime Center also offers advanced material handling equipment such as a 60-ton 760 -foot bridge overhead crane, container handling equipment, and a 50-inch 5-ton electromagnet. Additionally, stevedoring, handling, tug, towing, fleeting, and switching services are offered on site.

Finally, the Southwind Maritime Center also has 745 acres with eight tenants, 7,500 feet of riverfront access, and is classified as a Foreign Trade Zone. The goods most often handled at this port are grain, soybean products, coal, fertilizer, cement, and minerals.

Burns Harbor in Portage, Indiana is located on the south shore of Lake Michigan, just 18 nautical miles from Chicago. This port has a 27 -foot draft with a special design that allows ships to dock and turn around at the port, and it is capable of simultaneously berthing up to ten ships.

Access to the Gulf of Mexico via the Inland Waterway System and to the Atlantic Ocean via the St. Lawrence Seaway is offered at Burns Harbor. This port also offers twelve modern shipping berths, as well as services for cargo and ships. These services include the capability of handling heavy-lift project cargo, metals, grain, chemicals, fertilizers, and coal. Tenant ship services include tug, barge, fleeting, railroad switching, waste disposal, sanitation, security, and fire protection. Burns Harbor is capable of handling Great Lakes bulk carriers up to 1000 feet in length, as well as saltwater vessels that are capable of transiting the locks on the Great Lakes and St. Lawrence Seaway system.

This port has 530 spaces with more than 30 tenants, and is classified as a Foreign Trade Zone. It is also an intermodal transportation site with service by the Indiana Harbor Belt Railroad, and close proximity to several interstate highways and airports. The goods most 
commonly handled at Burns Harbor are iron, steel, grain, chemicals, fertilizers, limestone, coal, and heavy-lift project cargo.

\section{Intermodal Rail Facilities}

Intermodal rail facilities seem to be very efficient, but there is significant ambiguity in identifying their use and effectiveness due to unclear data. It has been speculated that more of these sites do not exist in Indiana because the majority of the cargo traveling through Indiana originates or terminates in the adjacent states of Michigan, Ohio, Kentucky, and Illinois. This means that traditional intermodal rail facilities are put at a service and cost disadvantage because of the short line-haul distances to adjacent states. However, new bimodal technologies have presented a low-cost structure, and certain Indiana intermodal rail facilities are reaping the benefits.

The Clark Maritime Center in Jeffersonville and Burns Harbor in Portage, as mentioned above, are both sites of intermodal transportation which are capable of handling cargo sent by boat, rail, and truck. Both of these facilities are near airports with cargo handling and shipping capabilities as well.

The Clark Maritime Center provides rail service through the Louisville Indiana Railroad Co. and CSX with interchanges to several other major rail lines, including Canadian Pacific, Norfolk Southern, CN, KCS, BNSF, and Union Pacific. This facility also has an intraport short line capable of allowing on-site switching through M.G. Rail.

Burns Harbor provides rail service through the Indiana Harbor Belt Railroad, Norfolk Southern, CSX, and South Shore Railroad. Indiana Harbor Belt Railroad is the largest switch carrier in the nation. At Burns Harbor, Indiana Harbor Belt Railroad owns 54 miles of main track, as well as 266 miles of yard and siding track.

Several other intermodal rail facilities exist in Indiana which have the capability of handling and transferring cargo between rail and truck only. These sites are the Conrail's Avon Yard west of Indianapolis, the Howell Yard in Evansville, Norfolk Southern Triple Crown Facility in Fort Wayne, the General Motors Roanoke Facility near Fort Wayne, and the Toledo, Peoria and Western Hoosier Lift at Remington. For the most part, these facilities handle containerized bulk cargo. At the GM Roanoke Facility and the Hoosier Lift Facility, intermodal services are generally carried out by contracted third-party companies, such as the Hub Group, Inc.

The intermodal rail facility at Avon Yard west of Indianapolis occupies 25 acres of land with room for possible expansion. It has two eastbound and two westbound intermodal trains. Eastbound service dominates, going to Boston and Springfield, MA; Philadelphia, PA; and Syracuse, NY. Westbound service to East St. Louis, however, is minimal. This site offers a total 
of ten origin-destination pairs, including those just mentioned as well as four in Canada. Avon Yard performed 24,000 lifts in 2001 with its main customer being United Parcel Service sending significant volumes to its Worcester, MA and Little Ferry, NJ sort centers. The equipment split is even at 50 percent containers and 50 percent trailers.

The Howell Yard intermodal rail facility in Evansville occupies 17 acres of land with no room available for expansion. It has one northbound and one southbound intermodal train operating between Chicago, IL; Nashville, TN; Atlanta, GA; and Jacksonville, FL. In 2001, Howell Yard performed 23,000 lifts, and the terminal is currently operating at 75 percent capacity. Howell Yard's major inbound customer is water-rail container traffic to Toyota, Inc, and its major outbound customer is Whirlpool, Inc., to the southeast and west coast. The equipment split is 65 percent containers and 35 percent trailers. This site offers many more origin-destination pairs than the intermodal rail facility in Avon, serving a total of 39 origindestination pairs. CSXI offers service between Evansville and Bedford Park in Chicago, the largest rail hub in the United States, with a line haul of under 300 miles, and no direct Interstate Highway alternative.

The Norfolk Southern Triple Crown Facility in Fort Wayne uses a new carless, bimodal trailer technology called RoadRailer ${ }^{\circledR}$. RoadRailer ${ }^{\circledR}$ combines truck and rail line haul movement. The Triple Crown Service has a fleet that consists of 5,500 trailers that are all 53 feet long and 102 inches wide with slack-free coupling for movement. Typical train size is 73 units, but the Federal Railroad Administration has authorized the operation of trains of up to 155 units. There are a total of eleven origin-destination pairs from Fort Wayne, including sites in Canada and Mexico. The principal commodity market is automotive parts, and the highest to Ft. Wayne is to and from Atlanta, GA; Kansas City, MO; and Harrisburg, PA. Other commodities served by TCS include appliances, paper, and food.

The Toledo, Peoria and Western Railway operates the Hoosier Lift intermodal rail facility in Remington, Indiana. In 2001, Hoosier Lift performed only 5,000 lifts with an annual capacity of 35,000 lifts. This intermodal rail facility is underused mostly because it competes with Chicago's 26 rail hubs.

Intermodal rail service expansion in Indiana is possible in the future if the facilities were to use technologies such as RoadRailer ${ }^{\circledR}$ by Norfolk Southern's Triple Crown Services Inc. or Expressway by Canadian Pacific which have a significantly higher cost and service advantage than conventional intermodal rail services. It remains to be seen whether Indiana will upgrade its intermodal rail facilities to accommodate these new services. 


\section{Air Freight Facilities}

Indianapolis International Airport, Indianapolis, Indiana

Indianapolis International Airport was built in 1931. Currently, it covers 7,700 acres and has one terminal with 33 gates. It is owned by the Indianapolis Airport Authority and managed by BAA, the world's largest airport management company. Indianapolis International Airport is the largest privately operated airport in the United States. In 2003, the airport ranked $8^{\text {th }}$ in U.S. all-cargo landed weight, handling over 2.2 million tons of cargo, and ranked $88^{\text {th }}$ in U.S. freight gateway handling of international merchandise valued over \$1 billion, trading over \$2.6 million in freight. A major user of this airport is FedEx, which has its own terminals on the site.

Fort Wayne International Airport, Ft. Wayne, Indiana

Baer Field Air Base was built in Fort Wayne in 1941 and was used as a World War II military base. After having its name changed several times, in 1991, the Airport Authority changed its name to Fort Wayne International Airport. It is currently owned and operated by the Fort Wayne-Allen County Airport Authority. Fort Wayne International Airport is considered a medium sized airport, and between 2001 and 2003, it handled an average of 360 million tons of cargo. The Air Trade Center at Fort Wayne International Airport offers 450 acres of industrial space. It also has ten T-hangars available to small single or light twin engine planes. In 2003, this airport was ranked $36^{\text {th }}$ in the US in all-cargo landed weight, handling over 374,000 tons of cargo.

South Bend Regional Airport, South Bend, Indiana

South Bend Regional Airport was built in 1929. It is currently operated by the St. Joseph County Airport Authority. In 2003, South Bend Regional Airport handled 7,431 tons of cargo. The majority of the freight traffic handled at the airport is from FedEx, Airborne Express and UPS. Currently, South Bend Regional Airport is working to complete a project to lengthen its runways to allow for increased commercial traffic. It recently acquired 250 acres for this expansion project, which will be finished by the end of 2006.

O’Hare International Airport, Chicago, Illinois

Originally, O'Hare International Airport was a military base named Orchard Field. In 1946, the Chicago Municipal Airport (Midway) bought Orchard Field from the U.S. government and renamed it in honor of naval pilot Butch O'Hare who was killed in World War II. O'Hare Airport was used predominantly by the military until the mid 1950's when it first opened its doors to domestic commercial flights. Today, O’Hare International Airport covers 7,700 acres, has four terminals with 178 gates and 7 runways. It is operated by the Chicago City Department of Aviation. In 2004, Chicago O’Hare International Airport handled nearly 457,000 tons of 
domestic freight and over 957,000 tons of international freight, totaling over 1.4 million tons of freight. Goods are shipped to and from more than 140 domestic and 30 international non-stop destinations, serviced by 22 airlines. O'Hare is an important hub for Delta and American Airlines, as well as shipping companies. The airport also has a large capacity for storing cargo. The Southwest Cargo Facility dubbed "Cargo City" covers 240 acres and offers 1.2 million square feet of enclosed storage space. The O'Hare Express Center was the first private development on O'Hare property; it covers 50.2 acres and includes five buildings offering a total of 850,000 square feet of storage space. O'Hare International Airport ranked $7^{\text {th }}$ in 2003 for allcargo landed weight, handling over 2.35 million tons of cargo, making it the seventh busiest cargo moving airport in the United States.

\section{Cincinnati/Northern Kentucky International Airport in Covington, Ohio}

Cincinnati/Northern Kentucky International Airport was built in 1943 as an alternative to Lunken Airfield which was prone to flooding. Currently, it covers 7000 acres with three concourses and three terminals, totaling over 100 different flight gates. It is operated by the Kenton County Airport Board. Delta and Comair are the two main passenger airlines servicing the airport. Both of these airlines have over 50 gates each, making Cincinnati/Northern Kentucky International Airport the largest facility of its kind in the world. Not only does this airport handle large amounts of passengers, but it also handles large amounts of freight: over 2 billion tons 2003. In 1984, DHL opened its package-sorting hub at Cincinnati/Northern Kentucky International Airport. Following a series of expansions, DHL opened a new hub on the airfield in 2003 that covers 150 acres. This allows DHL to handle up to two million pounds of cargo nightly with enough ramp space to park more than 60 aircraft. The Bureau of Transportation Statistics ranked Cincinnati/Northern Kentucky International Airport $16^{\text {th }}$ in U.S. all-cargo landed weight, handling nearly 1.1 million tons of cargo in 2003.

\section{Cincinnati Municipal Airport in Cincinnati, Ohio}

Originally named Lunken Airfield, Cincinnati Municipal Airport was built in 1925. It currently covers 2,000 acres in Cincinnati near the Little Miami River, and has been prone to flooding in the past. It is owned by the City of Cincinnati and is operated by the Aviation Division of the Department of Transportation and Engineering in conjunction with PB Aviation. Freight traffic to this airport is light, as it serves mostly as a general and corporate aviation facility and as a reliever airport to the Cincinnati/Northern Kentucky International Airport. In 2002, Cincinnati Municipal Airport handled over 500 tons of freight. This freight is primarily comprised of cancelled checks and small packages, flown by AirNet and OnFlight Express. Cincinnati Municipal Airport also offers aircraft engine service and repair, aircraft interior services, aircraft sales, leasing, charter and courier services. The airport also has plans for future expansion to accommodate more passenger and cargo traffic. 
Louisville International Airport, Louisville, Kentucky

Standiford Field, a World War II air base, was built in 1941. In 1947 it was sold to its current operator, the Louisville Air Board, and was immediately made into a commercial passenger airport called Louisville Airport. United Parcel Service, the largest private employer in Kentucky, makes up the majority of the airport's cargo. Louisville International Airport has many professional facilities and amenities to handle massive amounts of specialized cargo, including mechanical handling, heated storage facilities, the ability to handle hazardous and dangerous goods. The airport also boasts an Express Courier Centre, and has a limited amount of parking space for large transient aircraft. The Airport Authority began plans for expansion in 1988, including new east and west parallel runways, a new Kentucky Air National Guard Base, a new United States Postal Service air mail facility, new corporate hangars, a new fixed-based operator, a four-level parking garage, and a new control tower. In 2003, Louisville International Airport ranked third in all-cargo landed weight, handling over 4.1 million tons of cargo; four times the amount of cargo handled by Indianapolis International Airport, the country's $8^{\text {th }}$ busiest cargo handling airport.

\section{Intermodal Flows}

We have not focused on intermodal flows in the report that follows. It may be a significant proportion of total flows in the United States, but it is not that significant when it comes to Indiana. Let us look at this briefly here.

According to the 1997 Commodity Flow Survey (CFS) the shipments leaving Indiana that moved by multiple modes represented $10.7 \%$ of the value of all goods. This makes it sound significant, but when we look at the tonnages involved it is about $2.2 \%$. This includes all parcel, courier, and postal moves, truck/rail moves, truck/water moves, rail/water moves, and unknown multiple modes. For the $2.2 \%$ of multiple mode moves we know that $0.2 \%$ is parcel moves, but the samples drawn of other modal pairs were so small that the data were viewed as unreliable. If we move to flows of specific commodities, there is nothing reported in most cases.

As for 1997 shipments arriving in Indiana we know that $10.9 \%$ of the value of the shipments is via multiple modes, but only $6.3 \%$ of the tonnage. More specifically the truck and rail moves represent $1.0 \%$ of the value and an undisclosed amount of the tonnage, probably something around $0.5 \%$ of the value.

In the 2002 CFS shipments by multiple modes represented a total of $11.1 \%$ of the value of Indiana shipments, but $8.6 \%$ of this value was for parcel and similar moves. Truck and rail flows represented $2.2 \%$ of the value. The tonnage moving by multiple modes dropped in 2002 from its $2.2 \%$ in 1997 to $1.6 \%$ of the tonnage in 2002. 
Again the 2002 picture is actually less clear. We know $11 \%$ of the value of the shipments received arrives by multiple modes, and about $5.3 \%$ of the tonnage. The sampling of this area is undoubtedly something that could be improved upon, because the value of the intermodal rail/truck moves is $0.1 \%$ of the total. The tonnages, actual or percentage, are not revealed and probably come out significantly less than that.

In effect, we have not looked at the intermodal rail/truck flows originating in Indiana because the sector seems of minor importance based on the data available from the 1997 and 2002 CFS. Of course there is always the possibility that the sample used by the CFS is not picking up the volumes correctly.

There may be anecdotal evidence that a significant amount of intermodal rail/truck traffic arrives in Indiana. Even if the CFS is significantly understating the value and tonnages, we have no way to improve this. The CFS is the best data source available. If we were able to work with the general multipliers developed here and discussed later, we would not have sufficient data to work with once this was distributed across 41 different commodity groups.

We suspect that we are picking up some of the intermodal flows in the form of simply rail traffic, we have no way of knowing this for sure and as a result this type of flow has not been treated any differently than rail flows here.

\section{Exports and Imports Related to Freight Flows}

Although it is beyond the scope of the present study and clearly beyond the data published by the Commodity Flow Survey (CFS) on which this study is based, there is a natural interest in the extent to which exports and imports play a role in the flow of goods to, from, and within the state of Indiana. The way in which such flows are treated in the CFS, is worth noting here.

Freight that is being shipped to a port or other site for export appears in the CFS as a flow from the state of origin to the state of export. Therefore export flows leaving Indiana by rail and destined for the port of Norfolk, Virginia, and further transshipment to a nation in Europe are treated as flows from Indiana to Virginia. Flows leaving by aircraft from Indianapolis that are destined for a nation in Africa are treated as flows beginning and ending in the state of Indiana. In effect, this is an internal or intrastate shipment. Likewise a container of merchandise from Beijing arriving at the port of Los Angeles-Long Beach in California prior to a West Coast warehouse, followed by its shipment by motor carrier to Indianapolis is treated as a flow from California to Indiana. All of this is rather unsatisfactory if you really care about origins and destinations and Indiana's role in international trade. It is unlikely that we can satisfy all the readers of this document in this matter, but we will try to improve on the situation somewhat. The reader should bear in mind that we will be using estimates for much of what follows. 


\section{Export Flows}

Of the total goods exported from the United States, Indiana was responsible for $2.4 \%$ of the value. This amounted to $\$ 21.5$ billion dollars in goods in 2005 and this was a $12.4 \%$ increase over 2004. Of these exports the dominant commodities were transportation related components and pharmaceuticals and medical-related supplies. The former would most likely move by rail or truck, while the latter might very well be shipped as air freight. These two broad classes of goods accounted for more than $49 \%$ of the value of exports.

The major destinations for exports from the state of Indiana are not unexpectedly Canada (44.5\%) and Mexico (12.2\%) in terms of value. These shipments would be primarily surface moves and would most likely appear in the CFS as shipments to Michigan in the former case and shipments to Texas in the latter case.

Other export destinations that comprise the top ten destinations are the United Kingdom (7.1\%), France (6.8\%), Japan (3.6\%), Germany (3.2\%), Netherlands (2.0\%) and China (1.9\%). These countries along with Canada and Mexico represent more than $80 \%$ of the destinations for Indiana exports in terms of value.

The heavier exports to European destinations would most likely move from the ports of New York or Norfolk, Virginia, to Rotterdam, Bremerhaven, or Antwerp in container ships. Of course higher value goods (such as pharmaceuticals) would probably be shipped by air.

Those exports destined for Asia would most likely pass through the port of Los Angeles and Long Beach, since they handle $70 \%$ of the West Coast transpacific traffic. The other choices are Oakland and Seattle/Tacoma, but these handle only about $20 \%$ of the traffic. The receiving port on the Asian side is determined by the final destination of the exports. Hong Kong continues to be very dominant for China, Pusan for South Korea, and Kobe for Japan.

\section{Import Flows}

While the export picture is rather clear, the import picture is not. We can speak of the estimated value of goods being imported, but even this requires certain assumptions. If we assume that Indiana consumes goods as a straight proportion of its population to the total U.S. population then we can make some estimates. This is not an all an unreasonable assumption since the state is proportionally quite similar to the U.S. as a whole and it is primarily consumer goods that are being imported in many cases.

In this case the leading source of imports for Indiana would be Canada with $17.2 \%$ of the value. China is second with nearly $14.6 \%$ of the total value. This would be followed by Mexico with $10.2 \%$ of the value, Japan with $8.3 \%$, Germany with 5\%, the United Kingdom with 3.1\% 
and Korea with 2.6\%. Let us look briefly at what is being imported from these areas to the U.S.

Our best estimate of the value of these imports (in billions of U.S. dollars) arriving in Indiana (based on 2005 data) is as follows: Canada (6.1), China (5.1), Mexico (3.6), Japan (2.9), Germany (1.8), the U.K. (1.1) and Korea (.9). The increasing dominance of China as an import source is the major change over the last decade.

For Canada the major U.S. imports are transportation equipment (26.9\%), energy-related products (23.0\%), forest products (9.8\%), and minerals and metals (8.9\%). China's picture is quite different with electronics accounting for the major share of its exports to the U.S. (35.7\%). Miscellaneous products of manufacturing are the second most common import from China (19.1\%), followed by textiles (11.1\%) and machinery (8.8\%). Mexico's leading export to the U.S. is also electronics (23.7\%), followed by transportation equipment (20.3\%), energy-related products $(14.8 \%)$, and machinery $(11.9 \%)$. Japan's exports to the U.S. are dominated by transportation equipment (45.2\%), followed by electronics (22.9\%), and machinery (13.5\%).

Most of above imports would move by motor carrier or possibly rail. Goods coming in from Europe are not very significant in comparison to the Asian trade. The impact of the latter trade on West Coast ports is quite significant and this can impact firms in Indiana in terms of congestion delays preventing ships from being unloaded. The major Asian ports involved in shipments to the U.S. would continue to be the same as those receiving shipments.

\section{Summary}

In this chapter we have summarized the attributes and trends related to the forty-one commodity groups examined in this study using a combination of data from both the 1997 and the 2002 Commodity Flow Surveys. We can not examine longer term trends since the data for 1993 are not comparable with the newer classification systems.

Also summarized here are the major transportation facilities of the state of Indiana including its port facilities, airports, and intermodal facilities and terminals.

We also examined the intermodal (rail/truck) flows in Indiana commodity productions and attractions. It was noted that in terms of tonnages, in other words traffic, this represents a very small amount of the total volume of goods leaving or entering the state of Indiana. It was also noted that we have no way of improving this situation and as a result this type of flow has not been examined here specifically. Instead this portion of the shipments has been combined with rail shipments.

We closed this chapter with some discussion of the role of exports and imports originating and terminating in Indiana. We have focused primarily on goods based on their value. In the export case Indiana leads with transportation related equipment and 
pharmaceutical-related products. Imports are being dominated by consumer goods from Asia.

Indiana DOT planners should carefully monitor the development of bridge tables being prepared that will enable one to translate STCC data into SCTG data. If this were available then it would be possible to work with the STCC data of the Carload Waybill Sample and treat it as SCTG data. At present such tables are crude at best and will not provide reliable estimates. 


\section{References}

Bureau of Transport Statistics, U.S. Department of Transportation www.bts.gov

Chicago Airports:

www.airwise.com/airports/us/ORD/ORD_07.html

www.chicagotraveler.com/attractions/ohare-international-airport.html

Cincinnati (Municipal) Airport:

www.cincinnati-oh.gov/transeng/downloads/transeng_pdf7216.pdf

Cincinnati NKY:

www.cincinnati-transit.net/cvg.html

Federal Aviation Administration, U.S. Department of Transportation www.faa.gov

Fort Wayne:

www.globalsecurity.org/military/facility/ft-wayne.htm

Louisville International:

www.azworldairports.com/airports/p2740sdf.htm

South Bend:

www.southbendtribune.com/discover2005/page28.html

www.sbnair.com/Airport/About.php

www.macog.com/PDFs/MPO/D05stfnl.pdf

U.S. Census Bureau (2005), Foreign Trade Statistics

www.census.gov/foreign-trade/www/

U.S. Census Bureau (1999), Economic Census, Transportation: 1997 Commodity Flow Survey, Washington, DC: US Department of Commerce.

U.S. Census Bureau (2004), 1998 County Business Patterns, Washington, DC: U.S. Department of Commerce. Web page, http://www.census.gov/epcd/cbp/view/cbpview.html, various access dates.

U.S. Census Bureau (2005), 2002 Commodity Flow Survey, Washington, DC: US Department of Commerce. CD C1-E02-ECFS-00-US1 


\section{Chapter 4}

\section{THE GENERATION OF COMMODITY TRAFFIC}

As noted in Chapter 1, one of the major efforts in the analysis of commodity flows is the identification of variables that influence the production and attraction of this traffic from and to the various state and county origins and destinations. These productions and attractions are referred to collectively as traffic generation. The traffic generation phase of this project was also negatively impacted by the manner in which commodity traffic and employment within industries is now categorized. These problems are worth some discussion at the outset.

\section{Traffic Production}

Commodity traffic production, the shipments that emanate from a given area for a given industry, is obviously a function of the amount of production or manufacturing that takes place in that industry within the area of interest. It is of primary interest to determine those factors that are related to this productivity and to model this process so that estimates can be made of future traffic. The usual way of approaching this type of modeling is to relate flow production for a given commodity to employment in that particular industry for the areas of interest.

In the previous flow study commodity traffic was available for the STCC (Standard Transportation Commodity Classification) system. Employment data in that earlier study was available for the SIC (Standard Industrial Classification) system. There were clear linkages between these two systems so that one could be relatively sure that the flows and employees were for the same sectors to a large extent for any given area. The changes in classification systems that occurred in the 1990s weakened this linkage. Flows are now available by SCTG (Standard Classification of Transported Goods) and employment is available for NAICS (North American Industrial Classification System) categories. The linkage between flows and employees is still present to some extent, but it is not as strong as it once was.

One might suggest that we revert to the previous approach and use the older data, but there have been far too many changes in the last twelve years to assume stability in the relationships identified at that time. In addition the models can not be revised because the necessary data are no longer published in the earlier format. If this were not enough, the SIC and NAICS systems are so different that it is not possible to set up a translation of one system into 
the other at the level at which data are available for planning purposes. One could make such a translation at the five-digit level, but this level of detail is not released to researchers or planners. As a result the project team was required to start from the very beginning and develop entirely new traffic production models for the 41 SCTG commodity flow groups.

The traffic production models developed appear on the following page of this report (see Table 4-1 and Table 4-1a). The data in these two tables as well as the data used in the attraction models are essentially the same. The second table in each case uses acronyms in place of the SCTG codes and the NAICS codes.

These models were derived by correlating each flow group with employment in each of the NAICS industrial groups examined. In addition, population was also examined as were all of the other flow sectors. In the case of population this indicates production occurring in response to consumer demand in the production area. The relationship to other flows would occur when an industrial complex is present and the commodities being shipped are representative of the magnitude of that operation's other related sectors.

The NAICS industrial sectors for which employment was used in the modeling appear as Table 4-2.

It should be noted that the flows used to develop the models are expressed in thousands of tons. Therefore, if one wishes to derive actual tons the decimal point for the regression coefficient should be shifted three places to the right. The $\mathrm{N}$ value in the table represents the number of states with data for both the dependent and independent variables that were used to develop the models. These vary from 12 to 46 .

The models derived vary significantly in terms of accuracy and in several cases these are hardly what we would want in a research study, but the intent here is not perfectly accurate models. The objective is to get general relationships rather than focusing on the unique aspects of the relationship.

The relationships represented by the models in the table have been forced to have an intercept that runs through the origin. In other words when there are no employees in the industry noted there is no flow for the commodity of interest. This seems like a reasonable assumption. Nevertheless, several of the models are very poor and for this reason some of these were replaced by simply the ratio of employees to thousands of tons produced.

\section{Traffic Attraction}

The 1997 Commodity Flow Survey published the amount of traffic produced for each commodity in each state for that year. This enabled the project team to develop the traffic production models discussed in the previous section. Similar data were compiled for the traffic 
Table 4-1 Production Models Developed

\begin{tabular}{|c|c|c|c|}
\hline SCTG & Equation & $\mathbf{N}$ & $\mathrm{R}^{2}$ \\
\hline 01 & $0.003(331)+.007(337)$ & 22 & 0.498 \\
\hline 02 & $0.256(311)$ & 36 & 0.337 \\
\hline 03 & $0.135(311)$ & 34 & 0.647 \\
\hline 04 & $0.149(311)$ & 41 & 0.772 \\
\hline 05 & $0.054(311)$ & 42 & 0.880 \\
\hline 06 & $0.045(311)+0.027(333)$ & 43 & 0.853 \\
\hline 07 & $0.000748($ Pop $)+0.141(335)+0.083(311)$ & 46 & 0.964 \\
\hline 08 & $0.0002188(\mathrm{Pop})+0.013(334)$ & 46 & 0.882 \\
\hline 09 & $0.009(313)+0.005(337)$ & 19 & 0.690 \\
\hline 10 & $0.016(422)+0.0001118($ Pop $)+0.005(331)$ & 22 & 0.919 \\
\hline 11 & $0.087(421)$ & 28 & 0.839 \\
\hline 12 & $0.835(326)+1.145(314)+0.443(311)$ & 40 & 0.940 \\
\hline 13 & $0.226(325)$ & 29 & 0.507 \\
\hline 14 & modeled using employment growth and productivity only & & \\
\hline 15 & $7.34(212)$ & 30 & 0.604 \\
\hline 17 & $7.812(324)$ & 44 & 0.873 \\
\hline 18 & $4.017(324)$ & 45 & 0.939 \\
\hline 19 & $3.388(324)+0.142(325)$ & 41 & 0.918 \\
\hline 20 & $3.151(324)$ & 43 & 0.782 \\
\hline 21 & $0.011(337)+0.007(313)$ & 35 & 0.793 \\
\hline 22 & 0.00081 (Pop) & 35 & 0.304 \\
\hline 23 & $0.025(332)+0.017(325)$ & 44 & 0.790 \\
\hline 24 & $0.912(324)$ & 46 & 0.709 \\
\hline 25 & $0.667(321)$ & 21 & 0.518 \\
\hline 26 & $0.544(321)$ & 44 & 0.826 \\
\hline 27 & $0.225(322)+0.058(324)$ & 44 & 0.810 \\
\hline 28 & $0.029(311)+0.015(334)+0.053(314)$ & 45 & 0.931 \\
\hline 29 & $0.024(422)+0.040(322)$ & 43 & 0.946 \\
\hline 30 & $0.101(314)+0.051(313)+0.058(324)$ & 44 & 0.970 \\
\hline 31 & 0.002 (Pop) $+0.248(311)$ & 45 & 0.909 \\
\hline 32 & $0.356(331)+0.080(336)$ & 45 & 0.911 \\
\hline 33 & $0.030(332)+0.266(324)+0.033(327)$ & 45 & 0.949 \\
\hline 34 & $0.019(333)+0.026(326)$ & 47 & 0.897 \\
\hline 35 & $0.017(332)+0.074(324)$ & 46 & 0.913 \\
\hline 36 & $0.061(336)$ & 44 & 0.798 \\
\hline 37 & $0.008(331)$ & 33 & 0.620 \\
\hline 38 & $0.001(421)$ & 39 & 0.826 \\
\hline 39 & $0.020(337)+0.004(336)$ & 45 & 0.918 \\
\hline 40 & $0.000183($ Pop $)+0.066(314)+0.022(311)$ & 39 & 0.946 \\
\hline 41 & $0.099(332)$ & 37 & 0.931 \\
\hline 43 & 0.0004 (Pop) & 38 & 0.905 \\
\hline
\end{tabular}


Table 4-1a Production Models Developed

\begin{tabular}{|c|c|c|c|}
\hline SCTG & Equation & $\mathrm{N}$ & $\mathrm{R}^{2}$ \\
\hline (Animals) & 0.003 (MetProd) +.007 (Furniture) & 22 & 0.498 \\
\hline (Cereals) & 0.256 (Food) & 36 & 0.337 \\
\hline (AgProd) & 0.135 (Food) & 34 & 0.647 \\
\hline (Food) & 0.149 (Food) & 41 & 0.772 \\
\hline (Meat) & 0.054 (Food) & 42 & 0.880 \\
\hline (BakedGds) & 0.045 (Food) +0.027 (Machinery) & 43 & 0.853 \\
\hline (Foodstuffs) & 0.000748 (Pop) +0.141 (Appliances) +0.083 (Food) & 46 & 0.964 \\
\hline (Alcohol) & $0.0002188(\mathrm{Pop})+0.013$ (Electronics) & 46 & 0.882 \\
\hline (Tobacco) & 0.009 (Textiles) +0.005 (Furniture) & 19 & 0.690 \\
\hline (Stone) & $0.016($ WholesaleN $)+0.0001118$ (Pop) +0.005 (MetalProd)) & 22 & 0.919 \\
\hline (Sand) & 0.087 (WholesaleD) & 28 & 0.839 \\
\hline (Gravel) & 0.835 (Plastics) +1.145 (TexProd) +0.443 (Food) & 40 & 0.940 \\
\hline (Minerals) & 0.226 (Chemicals) & 29 & 0.507 \\
\hline (Ores) & see Table 4-1 & & \\
\hline (Coal) & 7.34 (MinOres) & 30 & 0.604 \\
\hline (Gas) & 7.812 (Print) & 44 & 0.873 \\
\hline (Oils) & 4.017 (Print) & 45 & 0.939 \\
\hline (OilProd) & 3.388 (Print) +0.142 (Chemicals) & 41 & 0.918 \\
\hline (Chemicals) & 3.151 (Print) & 43 & 0.782 \\
\hline (Drugs) & 0.011 (Furniture) +0.007 (Textiles) & 35 & 0.793 \\
\hline (Fertilizer) & 0.00081 (Pop) & 35 & 0.304 \\
\hline (ChemProd) & 0.025 (FabMetal) +0.017 (Chemicals) & 44 & 0.790 \\
\hline (Plastics) & 0.912 (Print)) & 46 & 0.709 \\
\hline (Wood) & 0.667 (WoodProd)) & 21 & 0.518 \\
\hline (WoodProd) & 0.544 (WoodProd)) & 44 & 0.826 \\
\hline (Newsprint) & 0.225 (Paper) +0.058 (Print) & 44 & 0.810 \\
\hline (Paper) & 0.029 (Food) +0.015 (Electronics) +0.053 (TexProd) & 45 & 0.931 \\
\hline (Print) & 0.024 (WholesaleN) +0.040 (Paper) & 43 & 0.946 \\
\hline (Textiles) & 0.101 (TexProd) +0.051 (Textiles) +0.058 (Print) & 44 & 0.970 \\
\hline (MinProd) & 0.002 (Pop) +0.248 (Food) & 45 & 0.909 \\
\hline (BaseMetal) & 0.356 (MetProd) +0.080 (TranEquip) & 45 & 0.911 \\
\hline (MetArticles) & 0.030 (FabMet) +0.266 (Print) +0.033 (Minerals) & 45 & 0.949 \\
\hline (Machinery) & 0.019 (Machinery) +0.026 (Plastics) & 47 & 0.897 \\
\hline (Electronics) & 0.017 (FabMet) +0.074 (Print) & 46 & 0.913 \\
\hline (Vehicles) & 0.061 (TranEquip) & 44 & 0.798 \\
\hline (TranEquip) & 0.008 (MetProd) & 33 & 0.620 \\
\hline (Instrument) & 0.001 (WholesaleD) & 39 & 0.826 \\
\hline (Furniture) & 0.020 (Furniture) +0.004 (TranEquip) & 45 & 0.918 \\
\hline (MiscMan) & $0.000183($ Pop $)+0.066(314$ TexProd $)+0.022$ (Food)) & 39 & 0.946 \\
\hline (Waste) & 0.099 (FabMet) & 37 & 0.931 \\
\hline (Mixed) & 0.0004 (Pop) & 38 & 0.905 \\
\hline
\end{tabular}


Table 4-2 Employment Variables Used in Modeling

\begin{tabular}{|l|l|}
\hline NAICS & Description \\
\hline 212 & Minerals and Ores \\
\hline 311 & Food Manufacturing \\
\hline 312 & Beverages and Tobacco Products \\
\hline 313 & Textiles and Fabrics \\
\hline 314 & Textile Mill Products \\
\hline 315 & Apparel and Accessories \\
\hline 321 & Wood Products \\
\hline 322 & Paper \\
\hline 324 & Printing, Publishing and Similar Products \\
\hline 325 & Chemicals \\
\hline 326 & Plastics and Rubber Products \\
\hline 327 & Nonmetallic Mineral Products \\
\hline 331 & Primary Metal Products \\
\hline 332 & Fabricated Metal Products \\
\hline 333 & Machinery, Except Electrical \\
\hline 334 & Computer and Electronic Parts \\
\hline 335 & Electrical Equipment, Appliances, and Components \\
\hline 336 & Transportation Equipment \\
\hline 337 & Furniture and Fixtures \\
\hline 421 & Wholesale trade, durable goods \\
\hline 422 & Wholesale trade, nondurable goods \\
\hline
\end{tabular}


attracted. While the shipments out from an area are obviously related to industrial activity for that commodity in the origin area, the attraction of traffic is handled somewhat differently.

We know that commodities are shipped in response to demand by markets and that these markets have essentially two components. One of these is a consumer market that is often represented by population. The other market is an industrial market, and this is often represented by related industries. In other words, automobile parts are often sent to an area involved in the assembly of automobiles. Or they are sent to parts distributers who in turn supply these to local dealers. It is reasonable to expect that the models developed should be related to both the industrial market (represented once again by NAIAS employment sectors) and the consumer market as represented by population.

It is also understood that total productions are highly related to total attractions. This is reasonably well represented by the diagram below. This diagram is based on the total flow into each state and the total flow out of each state in tons for 1997. Unfortunately, as nice as this relationship is at the aggregate level, it does not hold with desegregation. For example, the states that are the largest shippers of coal are not the greatest importers of coal. Therefore, we will revert to the use of the earlier relationships between shipments and the industrial and consumer markets.

The models derived for traffic attraction appear in Table 4-3 and Table 4-3a. The estimates derived for all 41 SCAG groups for each of the 145 states and counties of the study can be found in Appendix A.

One technical point worth some discussion is related to the value of the coefficient of determination in the models derived. The models as noted used a zero intercept value in the model developed. This was done for a very practical reason. The value of the intercept could turn out to be negative and this would give an area a negative flow value in some cases. This is something very difficult to explain in a real world situation. Alternatively, the value of the intercept could be extremely large and when we are trying to estimate county flows, this would yield in most cases an unlikely value. So this is the reason for the zero intercept.

Returning to the interpretation of $R^{2}$ in these cases, it should be noted that the value of $R^{2}$ is not the ratio of an explained variance to total variances in the SASS regression routine. Rather it is a measure of variation about the origin, a notion that has little or no practical meaning. As a result a somewhat different approach was taken in this study. We have measured total variation and then measured how much of this can be explained by our model. This is not an OLS (ordinary least squares) model and therefore it is not a true $\mathrm{R}^{2}$ value, but it can be interpreted as such. There is one possible flaw with this approach and that occurs when the model derived may be worse than simply using the mean value of the data series. The ramifications of this are that the $R^{2}$ value may exceed the normal range of -1 to +1 . For the most part this was not a problem here. The modified values appear in Table 4-4 and 4-5. 
Figure 4-1 Relationship of Total Productions and Total Attractions

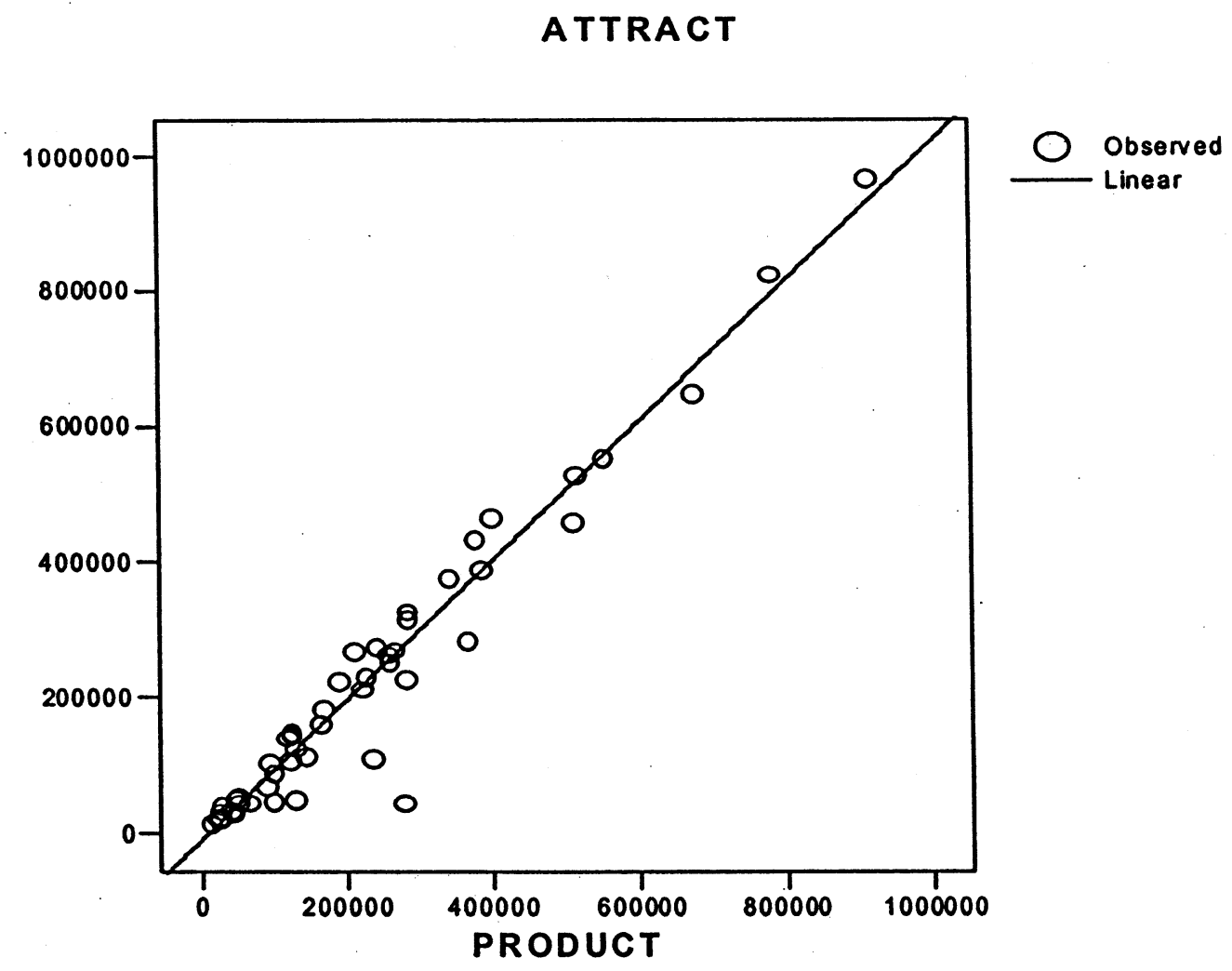




\section{Models Derived Discussion}

The production and attraction models derived are not uniformly as good as might be desired. Looking over the production models one notes that the level of explanation on any criterion is not particularly good for those SCTG groups up through 15 . These are agricultural or natural resource-based types of goods that are generally produced in states with good agricultural land or other natural resource endowments. Exceptions to this would be for foods and alcoholic beverages which may be for local markets. SCTG groups beyond 15 are often local marketoriented or industrial complex related. The worse models tend to be those where employment is not the best indicator of production or shipping capacity. The best example of this is the fertilizer industry (SCTG 22).

In general the attraction models tend to be much better that are related to consumer or industrial market sectors. Even here the models are not very good at the lower levels of the SCTG groups $(1,2$, and 3, or live animals and fish, cereal grains, and agricultural products). Most of the other industrial or commodity sectors do much better, but fertilizer consumption is again low.

There is a clear need for some substantial research on the traffic generation models used in freight analysis. The models used in the earlier study tended to be much better. We suspect this was due to the close tie between the STCC and the SIC systems, which does not seem to be present between the SCTG and the NAICS systems. However, there are other potential sources of error. One of these may be due to the significant increase in the number of SCTG groups as compared to the STCC sectors. We now have nearly three times as many SCTG groups in comparison to the 15 or so STCC groups. This will tend to increase the possible sources of variance as well as error.

In addition to these points it should be apparent that some of the models don't make much sense on the surface. In particular the presence of employment in Print industries (NAICS 324) may bother some. We do not believe that this is a causal relationship so much as it is an associational variable. What this means is that in some cases the flows are clearly a causal function as is the case when we look at the generation of textiles flows as a function of employment in that industry and in the textile products industry. We do not believe the production of gasoline flows are causally determined by employment in the print industry, but we do believe that the Print variable may also be related to some higher level economic functions in an area and this is the basis for the association observed.

It might be desirable to not use such relationships, but that would be difficult to do since we have included all of the data we know of that is available on a county basis in our analysis. In other words there are no better economic variables available for use.Of course if those using these models find them completely unacceptable they could simply work with the data as published in 2002 and apply forecasted growth and expected increases in productivity to those values. This may be seen as being more effective than the approach we have used here although it would not yield estimates at the county level. 
Table 4-3 Attraction Models Developed

\begin{tabular}{|c|c|c|c|}
\hline SCTG & Equation & $\mathrm{N}$ & $\mathrm{R}^{2}$ \\
\hline 01 & $0.004(311)$ & 18 & 0.488 \\
\hline 02 & $2.724(324)$ & 37 & 0.399 \\
\hline 03 & $1.196(324)$ & 45 & 0.504 \\
\hline 04 & $0.148(311)$ & 45 & 0.839 \\
\hline 05 & $0.030(311)+0.00015($ Pop $)+0.0004(336)$ & 48 & 0.971 \\
\hline 06 & $0.00018($ Pop $)+0.025(311)+0.022(325)$ & 47 & 0.980 \\
\hline 07 & 0.000903 (Pop) $+0.068(311)+0.104(322)$ & 48 & 0.986 \\
\hline 08 & $0.000250($ Pop $)+0.008(334)+0.023(315)+0.078(312)$ & 47 & 0.984 \\
\hline 09 & $0.008(313)+0.004(337)$ & 44 & 0.732 \\
\hline 10 & $0.015(325)$ & 22 & 0.688 \\
\hline 11 & 0.00121 (Pop) & 30 & 0.899 \\
\hline 12 & $0.395(311)+1.237(314)+0.903(331)+2.003(312)$ & 41 & 0.966 \\
\hline 13 & $0.338(322)$ & 37 & 0.628 \\
\hline 14 & $0.172(331)$ & 29 & 0.651 \\
\hline 15 & $3.472(212)+0.727(311)$ & 42 & 0.847 \\
\hline 17 & $4.60(324)+0.00169(\mathrm{Pop})$ & 44 & 0.912 \\
\hline 18 & $3.237(324)+0.110(325)$ & 47 & 0.943 \\
\hline 19 & $2.936(324)+0.199(325)$ & 44 & 0.899 \\
\hline 20 & $3.218(324)+0.050(334)$ & 46 & 0.865 \\
\hline 21 & $0.006(325)+0.002(422)$ & 48 & 0.866 \\
\hline 22 & 0.000653 (Pop) & 40 & 0.372 \\
\hline 23 & $0.000104(\mathrm{Pop})+0.208(324)+0.067(314)+0.026(326)$ & 47 & 0.965 \\
\hline 24 & $0.041(325)+0.295(324)+0.027(333)+0.062(314)$ & 45 & 0.931 \\
\hline 25 & $0.683(321)$ & 33 & 0.555 \\
\hline 26 & $0.494(321)+0.391(324)$ & 47 & 0.908 \\
\hline 27 & $0.043(311)+0.123(322)+0.122(324)$ & 47 & 0.970 \\
\hline 28 & $.00007030($ Pop $)+0.017(334)+0.021(311)$ & 48 & 0.951 \\
\hline 29 & 0.000295 (Pop) & 45 & 0.964 \\
\hline 30 & $0.000041($ Pop $)+0.079(314)+0.032(313)+0.058(324)$ & 47 & 0.983 \\
\hline 31 & 0.001777 (Pop) $+0.227(311)$ & 47 & 0.918 \\
\hline 32 & $0.315(311)+0.079(336)$ & 47 & 0.911 \\
\hline 33 & $0.428(324)+0.035(333)$ & 46 & 0.927 \\
\hline 34 & $0.015(333)+0.009(336)+0.013(325)$ & 47 & 0.939 \\
\hline 35 & $0.000076($ Pop) $+0.076(324)+0.011(326)$ & 48 & 0.957 \\
\hline 36 & $0.053(336)$ & 48 & 0.860 \\
\hline 37 & $0.035(324)$ & 39 & 0.723 \\
\hline 38 & $0.000415(421)+0.001848(314)+0.000442(422)$ & 48 & 0.959 \\
\hline 39 & 0.000068 (Pop) & 48 & 0.899 \\
\hline 40 & 0.000235 (Pop) $+0.031(321)+0.014(313)$ & 44 & 0.946 \\
\hline 41 & $0.051(332)+0.066(331)+0.037(311)$ & 40 & 0.941 \\
\hline 43 & 0.000356 (Pop) $+0.036(314)$ & 46 & 0.924 \\
\hline
\end{tabular}


Table 4-3a Attraction Models Developed

\begin{tabular}{|c|c|c|c|}
\hline SCTG & Equation & $\mathbf{N}$ & $\mathrm{R}^{2}$ \\
\hline (Animals) & 0.004 (Food) & 18 & 0.488 \\
\hline (Cereals) & 2.724 (Print) & 37 & 0.399 \\
\hline (AgProd) & 1.196 (Print) & 45 & 0.504 \\
\hline (Food) & 0.148 (Food) & 45 & 0.839 \\
\hline (Meat) & 0.030 (Food) +0.00015 (Pop) +0.0004 (TransEquip) & 48 & 0.971 \\
\hline (BakedGds) & $0.00018($ Pop $)+0.025$ (Food) +0.022 (Chemicals) & 47 & 0.980 \\
\hline (Foodstuffs) & 0.000903 (Pop) +0.068 (Food) +0.104 (Paper) & 48 & 0.986 \\
\hline (Alcohol) & 0.000250 (Pop) +0.008 (Electronics) +0.023 (Apparel) +0.078 (BevTob) & 47 & 0.984 \\
\hline (Tobacco) & 0.008 (Textiles) +0.004 (Furniture) & 44 & 0.732 \\
\hline (Stone) & 0.015 (Chemicals) & 22 & 0.688 \\
\hline (Sand) & 0.00121 (Pop) & 30 & 0.899 \\
\hline (Gravel) & 0.395 (Food) +1.237 (TexProd) +0.903 (MetProd) +2.003 (BevTob) & 41 & 0.966 \\
\hline (Minerals) & 0.338 (Paper) & 37 & 0.628 \\
\hline (Ores) & 0.172(MetProd) & 29 & 0.651 \\
\hline (Coal) & 3.472 (MinOres)+ 0.727 (Food) & 42 & 0.847 \\
\hline (Gas) & 4.60 (Print) +0.00169 (Pop) & 44 & 0.912 \\
\hline (Oils) & 3.237 (Print) +0.110 (Chemicals) & 47 & 0.943 \\
\hline (OilProd) & 2.936 (Print) +0.199 (Chemicals) & 44 & 0.899 \\
\hline (Chemicals) & 3.218 (Print) +0.050 (Electronics) & 46 & 0.865 \\
\hline (Drugs) & 0.006 (Chemicals) +0.002 (WholesaleN) & 48 & 0.866 \\
\hline (Fertilizer) & 0.000653 (Pop) & 40 & 0.372 \\
\hline (ChemProd) & 0.000104 (Pop) +0.208 (Print) +0.067 (TexProd) +0.026 (Plastics) & 47 & 0.965 \\
\hline (Plastics) & 0.041 (Chemicals) +0.295 (Print) + 0.027 (Machinery) +0.062 (TexProd) & 45 & 0.931 \\
\hline (Wood) & 0.683 (Print) & 33 & 0.555 \\
\hline (WoodProd) & 0.494 (WoodProd) +0.391 (Print) & 47 & 0.908 \\
\hline (Newsprint) & 0.043 (Food) +0.123 (Paper) +0.122 (Print) & 47 & 0.970 \\
\hline (Paper) & .00007030 (Pop) +0.017 (Electronics) +0.021 (Food) & 48 & 0.951 \\
\hline (Print) & 0.000295 (Pop) & 45 & 0.964 \\
\hline (Textiles) & 0.000041 (Pop) +0.079 (TexProd) +0.032 (Textiles) +0.058 (Print) & 47 & 0.983 \\
\hline (MinProd) & 0.001777 (Pop) +0.227 (Food) & 47 & 0.918 \\
\hline (BaseMetal) & 0.315 (Food) +0.079 (TransEquip) & 47 & 0.911 \\
\hline (MetArticles) & 0.428 (Print) +0.035 (Machinery) & 46 & 0.927 \\
\hline (Machinery) & 0.015 (Machinery) +0.009 (TransEquip) +0.013 (Chemicals) & 47 & 0.939 \\
\hline (Electronics) & 0.000076 (Pop) +0.076 (Print) +0.011 (Plastics) & 48 & 0.957 \\
\hline (Vehicles) & 0.053 (Plastics) & 48 & 0.860 \\
\hline (TranEquip) & 0.035 (Print) & 39 & 0.723 \\
\hline (Instrument) & 0.000415 (WholesaleD) +0.001848 (TexProd) +0.000442 (WholesaleN) & 48 & 0.959 \\
\hline (Furniture) & 0.000068 (Pop) & 48 & 0.899 \\
\hline (MiscMan) & 0.000235 (Pop) +0.031 (WoodProd) +0.014 (Textiles) & 44 & 0.946 \\
\hline (Waste) & 0.051 (FabMet) +0.066 (MetProd) +0.037 (Food) & 40 & 0.941 \\
\hline (Mixed) & 0.000356 (Pop) +0.036 (TexProd) & 46 & 0.924 \\
\hline
\end{tabular}


Table 4-4. Adjusted Explanation for Zero Intercept - Production

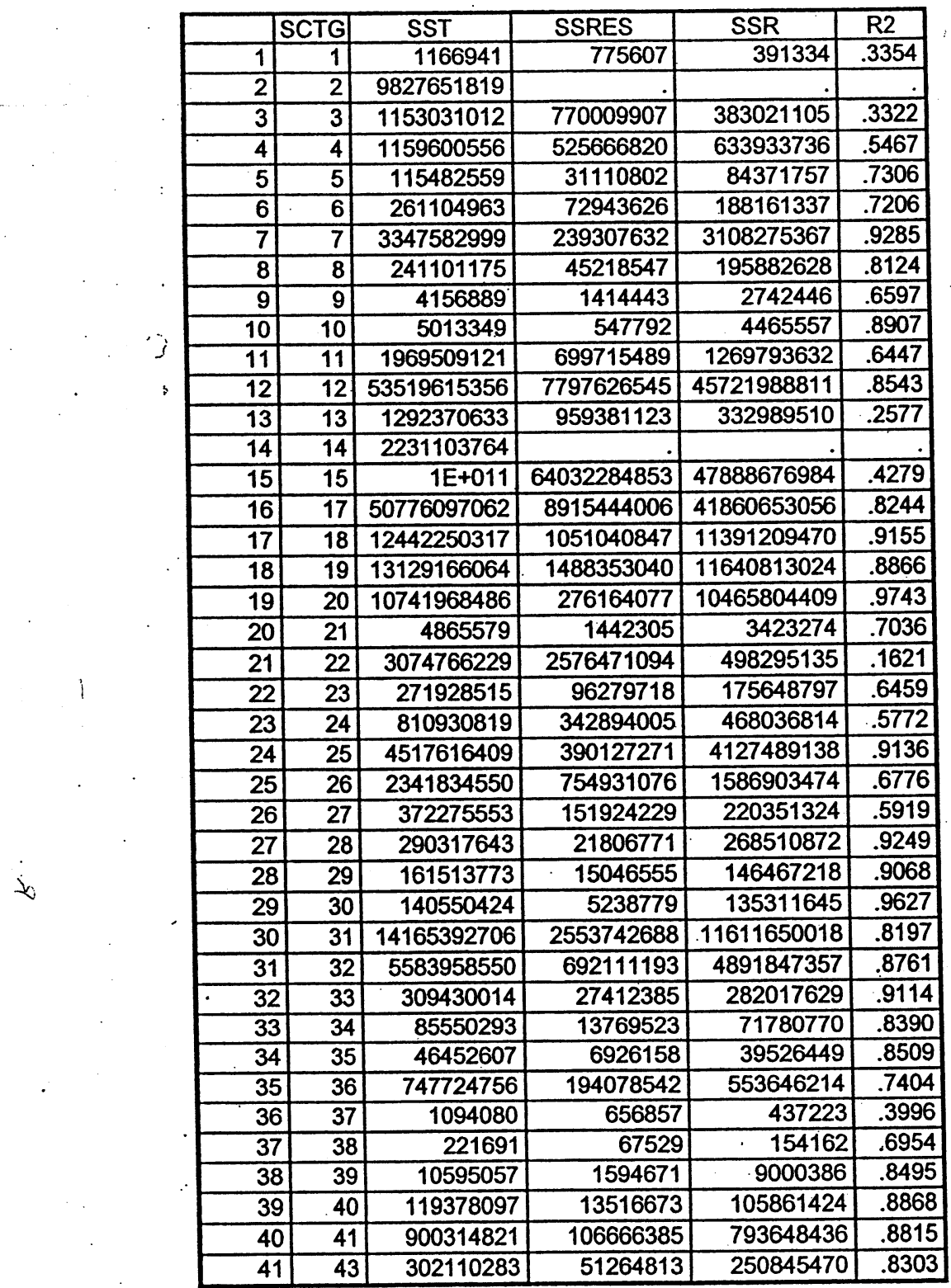


Table 4-5. Adjusted Explanation for Zero Intercept - Attraction

\begin{tabular}{|r|r|r|r|r|r|}
\hline & SCTG & \multicolumn{1}{|c|}{ SST } & \multicolumn{1}{c|}{ SSRES } & \multicolumn{1}{c|}{ SSR } & \multicolumn{1}{c|}{ R2 } \\
\hline 1 & 1 & & & & \\
\hline 2 & 2 & 121564576 & 111177105 & 10387471 & .0854 \\
\hline 3 & 3 & 197409801 & 141518894 & 55890907 & .2831 \\
\hline 4 & 4 & 1017483392 & 334379850 & 683103542 & .6714 \\
\hline 5 & 5 & 144569195 & 7922086 & 136647109 & .9452 \\
\hline 6 & 6 & 224918809 & 9082367 & 215836442 & .9596 \\
\hline 7 & 7 & 3154176610 & 93355445 & 3060821165 & .9704 \\
\hline 8 & 8 & 162735625 & 4954435 & 157781190 & .9696 \\
\hline 9 & 9 & 3050215 & 467194 & 2583021 & .8468 \\
\hline 10 & 10 & 4659047 & 2050195 & 2608852 & .5600 \\
\hline 11 & 11 & 2030884335 & 456419418 & 1574464917 & .7753 \\
\hline 12 & 12 & 52208285031 & 4266973688 & 47941311343 & .9183 \\
\hline 13 & 13 & 1134574594 & 686750896 & 447823698 & .3947 \\
\hline 14 & 14 & 583546684 & 273289209 & 310257475 & .5317 \\
\hline 15 & 15 & 30692834582 & 10030481693 & 20662352889 & .6732 \\
\hline 16 & 17 & 36503841716 & 4943518707 & 31560323009 & .8646 \\
\hline 17 & 18 & 10423849516 & 854355944 & 9569493572 & .9180 \\
\hline 18 & 19 & 12420089498 & 1732775018 & 10687314480 & .8605 \\
\hline 19 & 20 & 6889080187 & 1186847134 & 5702233053 & .8277 \\
\hline 20 & 21 & 2962599 & 670852 & 2291747 & .7736 \\
\hline 21 & 22 & 2655657653 & 2076398982 & 579258671 & .2181 \\
\hline 22 & 23 & 214571306 & 13708176 & 200863130 & .9361 \\
\hline 23 & 24 & 374735007 & 47608072 & 327126935 & .8730 \\
\hline 24 & 25 & 6183589921 & 4172934398 & 2010655523 & .3252 \\
\hline 25 & 26 & 2154010191 & 406623416 & 1747386775 & .8112 \\
\hline 26 & 27 & 375229029 & 23913761 & 351315268 & .9363 \\
\hline 27 & 28 & 270994045 & 15697566 & 255296479 & .9421 \\
\hline 28 & 29 & 158941322 & 10371117 & 148570205 & .9347 \\
\hline 29 & 30 & 85196165 & 2171640 & 83024525 & .9745 \\
\hline 30 & 31 & 13360778680 & 2205674433 & 11155104247 & .8349 \\
\hline 31 & 32 & 4378079421 & 594821297 & 3783258124 & .8641 \\
\hline 32 & 33 & 293609823 & 39464420 & 254145403 & .8656 \\
\hline 33 & 34 & 64782949 & 7250362 & 57532587 & .8881 \\
\hline 34 & 35 & 42257428 & 3252821 & 39004607 & .9230 \\
\hline 35 & 36 & 482172434 & 96246183 & 385926251 & .8004 \\
\hline 36 & 37 & 1077648 & 471241 & 606407 & .5627 \\
\hline 37 & 38 & 221533 & 16181 & 205352 & .9270 \\
\hline 38 & 39 & 8206145 & 1664656 & 6541489 & .7971 \\
\hline 39 & 40 & 137111674 & 15872566 & 121239108 & .8842 \\
\hline 40 & 41 & 832359522 & 63284863 & 769074659 & .9240 \\
\hline 41 & 43 & 274913997 & 31460707 & 243453290 & .8856 \\
\hline
\end{tabular}




\section{Evaluation of the Models Developed}

During the course of the study the results of the 2002 Commodity Flow Survey were released. Although it was beyond the scope of the project we were asked to take the models developed to that point using 1997 data and use these to estimate the value of the flows for the 2002 data and then to evaluate how close these were. This task was undertaken using the production models developed since these were the only ones available at the time.

The results of that analysis are displayed in Figure 4-2. The values along the vertical axis run from approximately .55 to nearly 1.0 and these reflect the values of the correlations obtained in the initial modeling, i.e., the cases where the models were fitted statistically to the 1997 data. In that case the values for the various NAICS employment variables were used to estimate the flows for the different SCTG groups. There were 36 models evaluated, and sample sizes were too small in the other cases.

The values along the horizontal axis represent the fits obtained when these exact same models were used to estimate the values for 2002. In other words the models derived from analysis of the 1997 data were applied to NAICS data for 2002 to yield estimates of the 2002 flows that were known from the newly released 2002 Commodity Flow Survey.

As one can see the values corresponding to the horizontal axis are not as large and several are quite small. On the other hand the clustering of values in the upper right quadrant of the diagram suggests the models are generally high. We would never expect the estimates to be better than the original fitted model although it does happen in one case here. This involves the estimate of gasoline flows which was .934 in the fitted model and .961 in the estimation of the 2002 data. This is a chance occurrence and should not be expected to occur.

\section{Estimates at the Sub-County Level}

The Indiana Travel Demand Model is actually used at the traffic analysis zone level (TAZ). It should be apparent to the reader that the entire analysis of freight traffic generation here was based on models that were derived using state-level data. These models once derived were then used to estimate the tonnages produced and attracted at the county level. We are still some way from the TAZ level since these are considerably smaller than the county level and this raises the question of how the state planners and their consultants can move from the county level to the TAZ level.

During the previous freight planning work in the 1990s the state made use of addressspecific Dun \& Bradstreet employment data. These data specify exactly what kind of industry is carried out at different locations. The employment in that industry can then be used to allocate a portion of the county's flows to the TAZ where that employment is located. Similarly on the attraction side the allocation can go to that TAZ that has employment in the sector that attracts the 


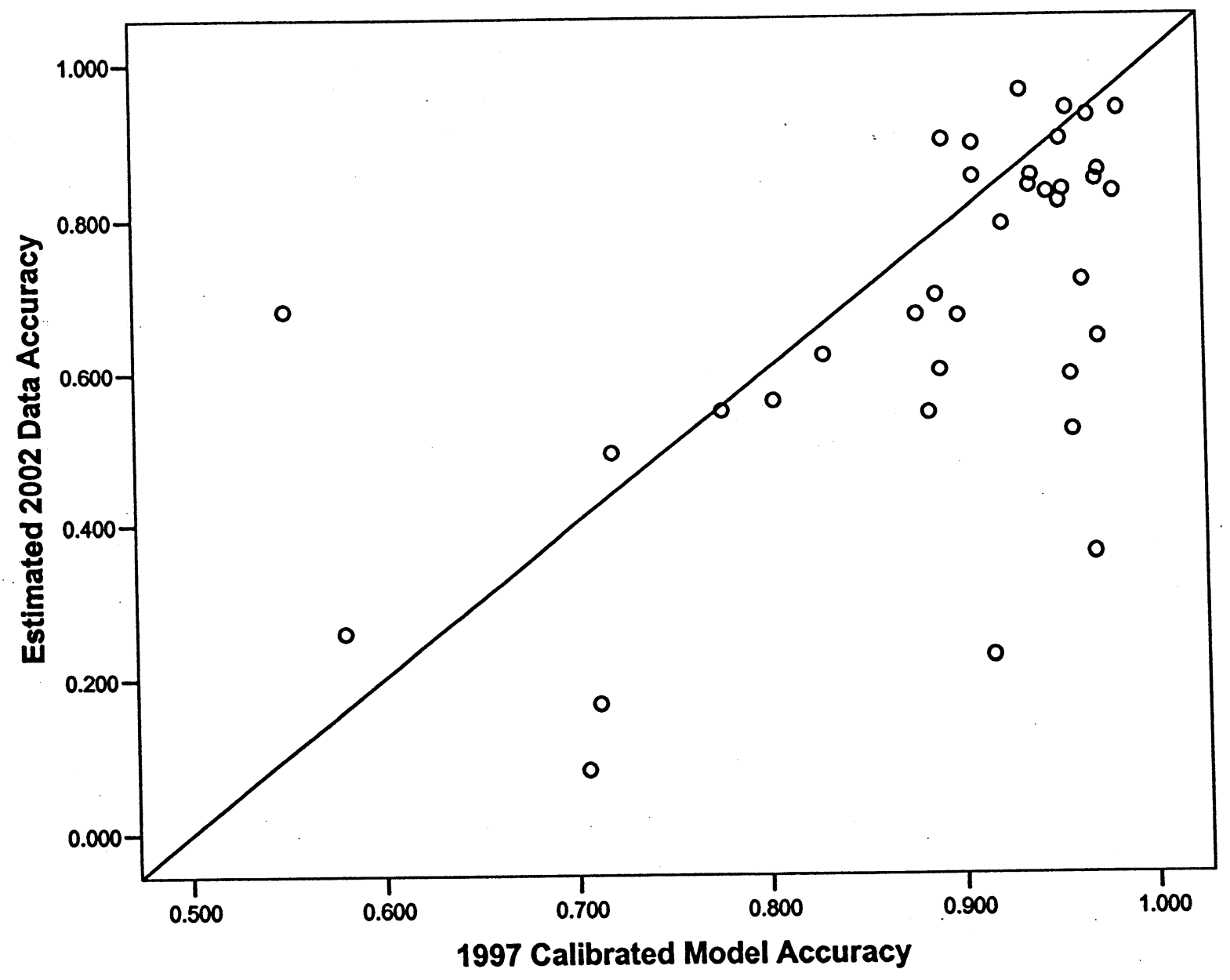

Figure 4-2. Evaluation of the 1997 Models with 2002 Estimates 
flows. If the flows are consumer-oriented then the population of the TAZ can also be used to allocate attracted flows to a county to its sub-county TAZ units.

This approach seems to have worked reasonably well in the past so there is no reason to believe that it will fail to work now. Fortunately, those that did this portion of the analysis in the past are still available to the state.

\section{Summary}

This chapter has discussed the models developed for the freight traffic generation portion of this study. Production and attraction models developed for predicting the future volume of freight traffic originating or terminating were presented for all of the SCTG classes examined in this study. The independent variables from the NAICS groups were also identified. Attributes of the models and an evaluation of the models for 2002 were also discussed here. The chapter concludes by reviewing the basic manner in which the modeling could be used to estimate traffic production and attraction at the sub-county level. 


\section{Chapter 5}

\section{THE DISTRIBUTION OF COMMODITY TRAFFIC}

Given the level of shipments emanating from the origin or production areas of interest and the level of flow attracted to the different destination or market areas, the next step is to model the volume of flow taking place between all origins and destinations. This modeling takes place for several reasons in this study, but in most studies we model current flows so that we can insert forecasted values into the model for some future time and estimate the volume of flows that should take place between the areas of interest at that point in the future.

This study took the known flows produced by the states of the US and the known flows attracted to a set of states and developed estimates for the remaining states for which data were not released. It also used these same models to estimate the traffic produced and attracted to the counties of Indiana. After addition of several nodes in neighboring states there was a possible $145 \times 145$ flow matrix to fill with flow estimates. There are different models that can be used for this purpose.

The models that can be used for distributing flows between origins and destinations today are almost exclusively models based on the gravity model. In general, that model states that the level of interaction between two areas is directly proportional to the product of their masses and inversely proportional to the square of the distance between them. Transport planners use the volume of flow produced and the volume of flow attracted as measures of the mass, and usually Euclidian distance as a measure of the distance or spatial separation between the areas. Rather than using the square of the distance, a power function, or in some cases an exponential function, is allowed to vary and the value selected is usually the one that yields the best fit to the data at hand.

In the 1997 study the flow model utilized was a fully-constrained gravity model. This model constrains the estimated flows in such a way that the total flows produced do not exceed the observed flows produced, and the estimated flows arriving at any given destination do not exceed the observed flows received at these locations. In addition, the average length of shipments generated by the model is constrained to be the same as the average length of the observed shipments. 
The 1997 study evaluated the model results by looking at selected locations in the state to see if the volume of trucks at those locations were similar to the number of trucks estimated by the modeling and traffic assignment of such vehicles. This is at the very end of the modeling process at a point where the entire flow modeling and traffic assignment process would have to be repeated if it was found to have a large level of error.

At the outset of the modeling process in the present case it was not apparent that similar "actual" flows would be available for comparison with the modeled flows and this necessitated some consideration of how the model could be evaluated. In the urban transportation planning process the actual flows between places are known based on an expanded sample of data received by roadside interviews and home interview surveys. In that case correlation could be used, but it is not. Instead the modeling process is evaluated immediately after the flows are estimated by comparing the trip length frequency distribution of these estimated flows with the trip length frequency distribution of the flows from the expanded sample. This seemed as though it would be a reasonable approach since the trip length frequency distribution of shipments is know for each commodity. As a result it was decided at the outset of the project that a similar approach to evaluation would be utilized here, but if other data became available it would be used as well.

\section{The Initial Model}

The first model used in the present study was the fully-constrained gravity model noted above. This was the model used in the 1997 study. The production and attraction of commodities were derived as explained in the previous chapter. The average length of shipments was known from the 1997 Commodity Flow Survey. These distance values for different commodities were used in the modeling.

The model used took the form:

$$
S_{j k}=A_{j} B_{k} O_{j} D_{k} \exp \left(-\beta c_{j k}\right)
$$

where $S_{j k}=$ the amount of a given commodity shipped from origin area $j$ to destination area k;

$\mathrm{O}_{\mathrm{j}}=$ the amount of a given commodity available for shipment at origin $j$;

$\mathrm{D}_{\mathrm{k}}=$ the amount of a given commodity demanded by destination $k$,

$\mathrm{c}_{\mathrm{jk}}=\mathrm{a}$ measure of the cost or impedance of moving from $j$ to $k$.

In addition, 


$$
A_{j}=\left[\Sigma B_{k} D_{k} \exp \left(-\beta c_{j k}\right)\right]^{-1}
$$

and

$$
B_{j}=\left[\Sigma A_{j} D_{j} \exp \left(-\beta c_{j k}\right)\right]^{-1}
$$

The flows of all 41 commodity groups were estimated using this model. This was done even though some of these commodities are not very significant to Indiana. They do nevertheless often move through the state on their way from origins to destinations north, south, east or west of the state.

The next step in the analysis was to evaluate how closely frequency distributions of the generated flows resembled the frequency distributions of the actual flows as published in the volumes of the 1997 Commodity Flow Survey (CFS). In order to create the model frequency distributions the 21,025 flow estimates for each commodity were examined, along with their distances, and these values were placed into distance classes identical to those in the CFS. This was accomplished using a program written for this purpose.

It is an understatement to say that the results were not very satisfactory. The primary problem appears to be that the estimated flows failed to display the expected distance decay in commodity shipments similar to what is found in the actual flows. In part this may be due to the model trying to satisfy the various constraints of this form of the gravity model. In addition there is definitely a problem with the possibility of short range flows (flows up to 250 miles in length), since the only possibility of truly short moves in the database are those between counties in Indiana and between a few small states on the East Coast. The situation is illustrated below for SCTG 01. The actual flows histogram reflects the flow values as published in the CFS and the modeled flows are those from the fully constrained model used here.
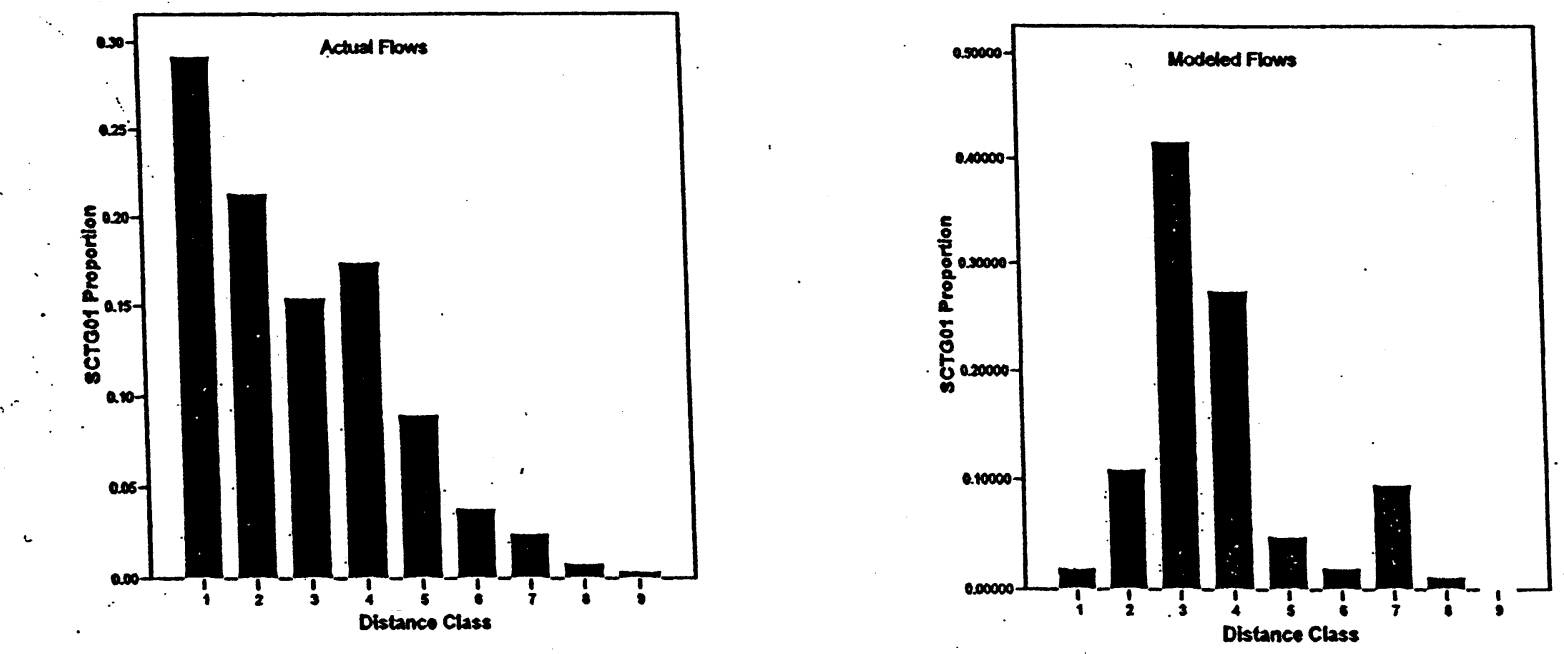
One possible way of correcting this bias against short trips was to look again at the intrastate distances used as input to the gravity model. The average length of a trip beginning and ending in the same state has traditionally been calculated as one-half the square root of the state's area. This may tend to make the intrastate distances too long in many cases. One way of correcting this figure was to use the average length of an intrastate shipment for all commodities as published in the 1997 Commodity Flow Survey report. The new distance values were considerably shorter than the previous values.

The fully-constrained model was rerun, but the new values did not have an appreciable impact on the accuracy of the estimates. A decision was made at this point to try the productionconstrained gravity model. One of its major attributes is a tendency for shipments to drop off rather quickly following departure from the origin for nearly every commodity group and it was thought that this would improve the overall quality of the modeled flows.

The production-constrained model used had the form

$$
S_{i j}=O_{i} D_{j} F_{i j} G_{j}
$$

where $\quad F_{i j}=1 / c_{i j}{ }^{\lambda}$

and

$$
G_{j}=1 /\left[\Sigma D_{j} F_{i j}\right]
$$

The only problem with this formulation is that we need some way of determining the value of the parameter lamda ( $\lambda)$. The value can not be calculated directly, but it can be determined iteratively by setting the value equal to 0.00 and incrementing it by .01 after each model iteration. Also after each iteration the average length of the shipments for each commodity between the $145 \times 145$ areas in this study is calculated. The iterative process continues until the average length of these shipments for each commodity as published by the CFS is achieved by the model. The corresponding lamda value becomes the value used in the model for that commodity. This solution method could be called a computer intensive solution. The lamda values obtained are in Table 5-1.

This particular model resulted in estimates that tend to have a clear distance decay, i.e., the largest flows are of the shortest length for nearly every commodity examined and they drop off significantly with increasing distance. This is reflected in the lamda value obtained in several cases. For example, several of the most extreme values of lamda in the model are obtained for commodities: SCTG 10, Monumental and Building Stone, SCTG11 Natural Sands, and SCTG 13 Gravel and Crushed Stone. These are nearly ubiquitous materials and it makes very little sense to transport them long distances since they are heavy, of low value relative to their weight, and as noted found 
Table 5-1. Production Constrained Model Attributes for 40 Commodities

\begin{tabular}{|c|c|c|c|c|c|c|}
\hline & SCTG & MeanDist & Modelled & Tons & Tonmiles & Exponent \\
\hline 1 & 1 & 253 & 254 & 5922001 & 1503523710 & 1.52 \\
\hline 2 & 2 & 410 & 411 & 489693024 & 201222472000 & 1.26 \\
\hline 3 & 3 & 400 & 400 & 201660960 & 80581140500 & 1.16 \\
\hline 4 & 4 & 213 & 212 & 219698944 & 46605869100 & 1.65 \\
\hline 5 & 5 & 458 & 456 & 79485000 & 36217925600 & 1.02 \\
\hline 6 & 6 & 472 & 471 & 102721008 & 48370352100 & .96 \\
\hline 7 & 7 & 313 & 312 & 396882112 & 123923538000 & 1.29 \\
\hline 8 & 8 & 343 & 343 & 81079008 & 27782098900 & 1.23 \\
\hline 9 & 9 & 245 & 245 & 4128001 & 1012662780 & 1.34 \\
\hline 10 & 10 & 93 & 94 & 15893002 & 1486681730 & 2.24 \\
\hline 11 & 11 & 58 & 59 & 442509120 & 26052460500 & 3.11 \\
\hline 12 & 12 & 51 & 52 & 1814761090 & 94263500800 & 3.40 \\
\hline 13 & 13 & 222 & 221 & 235731008 & 51991052300 & 1.60 \\
\hline 14 & 14 & 526 & 526 & 90705000 & 47667941400 & 1.03 \\
\hline 15 & 15 & 446 & 444 & 1217037950 & 540288123000 & 1.07 \\
\hline 16 & 17 & 142 & 141 & 962814912 & 136201953000 & 1.79 \\
\hline 17 & 18 & 106 & 107. & 481681952 & 51370250200 & 2.01 \\
\hline 18 & 19 & 172 & 172 & 475105088 & 81946148900 & 1.63 \\
\hline 19 & 20 & 462 & 459 & 296055968 & 135883530000 & 1.03 \\
\hline 20 & 21 & 564 & 562 & 9896999 & 5558334460 & .71 \\
\hline 21 & 22 & 243 & 241 & 179056032 & 43098857500 & 1.58 \\
\hline 22 & 23 & 489 & 490 & 92034000 & 45072269300 & .89 \\
\hline 23 & 24 & 530 & 528 & 130410992 & 68808646700 & .79 \\
\hline 24 & 25 & 76 & 77 & 370686112 & 28383195100 & 2.53 \\
\hline 25 & 26 & 294 & 291 & 329118944 & 95821635600 & 1.40 \\
\hline 26 & 27 & 549 & 545 & 152290000 & 82961449000 & .83 \\
\hline 27 & 28 & 299 & 299 & 73512992 & 21994102800 & 1.29 \\
\hline 28 & 29 & 292 & 292 & 78052992 & 22764777500 & 1.28 \\
\hline 29 & 30 & 538 & 536 & 45872000 & 24567932900 & .63 \\
\hline 30 & 31 & 100 & 100 & 910133184 & 91337072600 & 2.20 \\
\hline 31 & 32 & 350 & 348 & 335878048 & 116840858000 & 1.09 \\
\hline 32 & 33 & 457 & 453 & 106518992 & 48282214400 & .97 \\
\hline 33 & 34 & 542 & 539 & 49914996 & 26907611100 & .72 \\
\hline 34 & 35 & 683 & 678 & 39612000 & 26869575700 & .58 \\
\hline 35 & 36 & 464 & 463 & 98073984 & 45397053400 & .81 \\
\hline 36 & 37 & 686 & 686 & 5477000 & 3759114750 & .55 \\
\hline 37 & 38 & 738 & 736 & 2938997 & 2161702400 & .57 \\
\hline 38 & 39 & 581 & 579 & 19909998 & 11524856800 & .72 \\
\hline 39 & 40 & 354 & 352 & 112491976 & 39547281400 & 1.13 \\
\hline 40 & 41 & 225 & 225 & 177823952 & 40061886500 & 1.48 \\
\hline
\end{tabular}


nearly everywhere. The lamda obtained for these commodities were $2.24,3.11$, and 3.40 , respectively. The lowest value of lamda obtained, .55 , was for motorized and other vehicles. This is as it should be. Automobiles are manufactured nearly all over the country, but usually not the same models. In effect, the vehicles shipped are a response to a national market. Also, the buyer of the vehicle usually bears the cost of shipping the vehicle from the plant to the dealership, and within certain limits there is no reason for the shipper to try to minimize this cost.

The frequency distributions obtained using the production-constrained gravity model appear to be much more realistic than they were with the fully-constrained gravity model. We have not subjected these to any statistical analysis because the degrees of freedom for such a test, e.g., the Kolmogorov-Smirnoff (KS) test for comparison of frequency distributions, base their degrees of freedom on the total number of possible observations. In this case with $145 \times 145$ observations, it would be silly to evaluate the results by testing. This number of observations will nearly always result in a finding that the distributions are significantly different. One must bear in mind that these and similar tests were all developed for much smaller data samples and they become inappropriate here.

The actual and estimated frequency distributions were examined individually and it is believed that the production-constrained model does a much better job of capturing the general pattern of these flows (in terms of frequency distributions) at the national level. Perfect replication was not expected. If this did result there is every likelihood that the model would have over-fitted the data.

Although these frequency distributions are of interest the major test of accuracy would come with a comparison of the generated flow volumes on the highways with previously obtained counts from the Indiana road inventory database. When this comparison was undertaken the results were very poor. More specifically, the correlation between the observed and estimated flows was approximately .40. This meant that the modeled flows were only accounting for about $16 \%$ of the variation in the actual flows. This was viewed by the project staff as unacceptable.

\section{Restarting the Analysis}

At this point a complete reexamination of the distribution modeling approach was undertaken. Several basic changes were made in the analysis. An estimation procedure for the modal shift analysis developed earlier in the project was discarded. Instead the staff relied more on the published data and taking care to estimate the missing values in that data. A similar approach was taken with the data on attraction. It was known that the fully-constrained gravity model had worked much better in the earlier project so the decision was made to rely on that model in a complete repetition of the distributional modeling.

At the same time it is imperative that we note a couple of general observations about that 
model. The first of these is that the fully-constrained model does not do a very good job of replicating the published shipment length frequency distributions. The reasons for this seem obvious in retrospect. The actual frequency distributions are based on what the shippers responded in the Commodity Flow Survey. Perhaps some of these shipments only go a relatively short distance and this would be recorded as the shipment length. The data available to the project staff would merely state the shipment's destination. It was not possible to replicate short shipping distances because there are far too few of these possible at the scale of the analysis being undertaken here. Even though we know most shipments are short in length, the only possibility of such short shipments would be between some small states in the Northeast, or between some counties in Indiana.

The second point is that although the shipment length frequency distributions are of interest, the primary goal here is to replicate the flows to, from, through and within the state of Indiana. It was known that the fully-constrained model had done this before so it was assumed that this model would work better. If data were available so that the modeling could be between all the counties in the 48 contiguous states, we suspect that the generated frequency distributions would be very similar to the values published in the CFS. It is not possible to get that level of accuracy here.

One final point is that the level of disaggregation is much higher than it has been in previous studies. As noted above this study examines 41 different commodity groups in comparison to earlier studies that examined 15 to 18 groups. This is bound to have an impact on the level of accuracy since the methods used here tend to work better at the highly aggregated level of the Standard Industrial Classification (SIC) system and the Standard Transportation Commodity Classification (STCC).

The fully-constrained model was run for a second time, disregarding the national picture and focusing more on the Indiana results. The exponential form of this model yields values that are considerably smaller (see Table 5-2) than those from the production constrained model's power function as reflected in Table 5-1. The actual length of shipments was very close to the input values as reflected in Table 5.3.

\section{Modifying the Distributed Flows}

The modeled flows were calculated from the gravity model as simply thousands of tons. A subsequent computer program took these flows and converted them to tons, dollars of product value, and truck loads. The first of these was accomplished quite simply by converting the thousands of tons carried to three decimal places to simply total tons. The original production and attraction data are only published in thousands of tons so this may appear to be going beyond the validity of the data, but it was important to get to a level of detail that would have meaning at the level of inter-county flows in Indiana. 
Table 5-2. Fully-Constrained Gravity Model Exponents for Indiana, 1997

\begin{tabular}{|c|c|c|}
\hline $\begin{array}{l}\text { SCTG } \\
\text { Code }\end{array}$ & Commodity Group & Exponent \\
\hline 01 & Live Animals and Fish & -.0510 \\
\hline 02 & Cereal Grains & -.0031 \\
\hline 03 & Agricultural Products Except Live Animals, Cereal Grains, and Forage products & -.0030 \\
\hline 04 & Animal Feed and Products of Animal Origin & -.0070 \\
\hline 05 & Meat, Fish, Seafood, and Preparations & -.0024 \\
\hline 06 & Milled Grain Products and Preparations, and Bakery Products & -.0023 \\
\hline 07 & Prepared Foodstuffs, Fats, and Oils & -.0039 \\
\hline 08 & Alcoholic Beverages & -.0035 \\
\hline 09 & Tobacco Products & -.0054 \\
\hline 10 & Monumental or Building Stone & -.0199 \\
\hline 11 & Natural Sands & -.0146 \\
\hline 12 & Gravel and Crushed Stone & -.0140 \\
\hline 13 & Non-metallic Minerals & -.0067 \\
\hline 14 & Metallic Ores & -.0016 \\
\hline 15 & Coal & -.0031 \\
\hline 17 & Gasoline and Aviation Turbine Fuel & -.0100 \\
\hline 18 & Fuel Oils & -.0136 \\
\hline 19 & Products of Petroleum Refining and Coal Products & -.0069 \\
\hline 20 & Basic Chemicals & -.0022 \\
\hline 21 & Pharmaceutical Products & -.0014 \\
\hline 22 & Fertilizers and Fertilizer Materials & -.0050 \\
\hline 23 & Chemical Products and Preparations & -.0021 \\
\hline 24 & Plastics and Rubber & -.0018 \\
\hline 25 & Logs and Other Wood in the Rough & -.0104 \\
\hline 26 & Wood Products & -.0043 \\
\hline 27 & Pulp, Newspaper, Print, and Paperboard & -.0016 \\
\hline 28 & Paper or Paperboard Articles & -.0038 \\
\hline 29 & Printed Products & -.0040 \\
\hline 30 & Textiles, Leather, and Articles & -.0013 \\
\hline 31 & Non-metallic Mineral Products & -.0410 \\
\hline 32 & Base Metal in Primary or Semi-finished Forms and in Basic Shapes & -.0031 \\
\hline 33 & Articles of Base Metal & -.0023 \\
\hline 34 & Machinery & -.0015 \\
\hline 35 & Electronic and Other Electrical Equipment and Components; Office Equipment & -.0010 \\
\hline 36 & Vehicles & -.0017 \\
\hline 37 & Transportation Equipment & -.0010 \\
\hline 38 & Precision Instruments and Apparatus & -.0009 \\
\hline 39 & $\begin{array}{l}\text { Furniture, Mattresses and Mattress Supports, Lamps, Lighting Fittings, and Illuminated } \\
\text { Signs }\end{array}$ & -.0015 \\
\hline 40 & Miscellaneous Manufactured Products & -.0318 \\
\hline 41 & Waste and Scrap & -.0067 \\
\hline 43 & Mixed Freight & -.0185 \\
\hline
\end{tabular}


The conversion to dollars was relatively simple. The value per ton per commodity was used as a multiplier against the tons of commodities to yield the dollar value of shipments by commodity.

\section{Density}

One of the very important topics in estimating the volume of motor carrier and rail traffic after the volume of interaction (in tons) and the modal assignment have been determined is the density of the commodity. In other words, how much of the commodity can fit into a rail car or motor carrier? In the earlier (1997) commodity flow study these density factors were determined by examining flows of rail traffic categorized by the STCC system. These traffic data came from the Carload Waybill Sample, a sample of railroad commodity flow data that presents information on both the number of carloads and the tonnage of each commodity.

Changes in the commodity classification system have rendered the Carload Waybill Sample useless for the present purposes because that sample has continued to use the STCC system and the current study is using the Standard Commodity Transportation Group (SCTG) system. Although these two systems are similar they are not the same and this necessitated a search for new density factors.

It is fortunate that although the United States has not begun to publish data both on tonnage and carriers by SCTG commodity, Canada has. Their monthly Railway Carloadings (Transport Canada, 2004) reports give data both on the number of rail cars and the metric tonnes moved, and these are all given in terms of the SCTG system. From the reports consulted there is relatively good coverage in terms of the SCTG sectors included in the present study. There are some exceptions in terms of rail traffic and these are worth a brief discussion.

SCTG 01 Live Animals and Live Fish are not included in the Canadian rail data. As a substitute for this we have included weight estimates based on the recommended number of live animals per railcar derived by the Association of American Railroads. That data is based on the weights of hogs, cattle or sheep. The value of 9.77 tons per railcar was derived for this group.

SCTG 09 Tobacco Products average about 18.30 tons per truck. Rail traffic was estimated as larger by a multiplier of 2.5. Therefore, railcars were viewed as having a density of 45.75 tons.

SCTG 10 Monumental and Building Stone is also not in the Canadian data. It was estimated here as being 100 tons.

Also missing from the Railway Carloadings reports were data for SCTG 21, 
Apparatus. In the first case and the last class these were excluded primarily because rail is not used to move these goods in part because of their higher value. Printed matter simply put is time sensitive and for the most part the railroads are not reliable for this purpose.

The movement of goods by motor carrier in the earlier (1997) study was set at $40 \%$ of the railcar tonnage and in nearly every case this proved to underestimate the actual volume moving, and underestimate the number of vehicles involved. An alternative was searched for and found in a Strategic Freight Transportation Analysis (SFTA) report for the State of Washington (Petersen, et al, 2004). The SFTA report derived density figures for motor carriers of freight based on interviews of motor carrier drivers during a survey. It is compiled by SCTG system and gives empirical rather than theoretical values for density. For the most part we have gone with the SFTA values for motor carriers. Exceptions to this statement do exist and these are as follows:

SCTG 01 Live Animals and Live Fish: the empirical data suggest a value of 22.9 tons per truck. This is more than twice the AAR recommended density for a railcar and probably several times what would be recommended for motor carriers.

SCTG 10 Monumental and Building Stone was set at 25.4 tons, instead of $40 \%$ of the of the rail car maximum density. It may not be representative of most traffic.

SCTG 15 Coal is used for the thermal generation of electricity and by some manufacturing firms. Although the 100 ton hopper car can be taken as a given, the motor carrier level was less obvious. As a result the rather large trucks that are used to move coal have an average capacity of about 22 tons and this is the value used.

All other values are essentially derived from the SFTA report. The commodity, density and mode of traffic appear on the following two pages as Table 5-4.

\section{Density vs. Payload Factors}

During the initial review of a draft of this document a question was raised as to the merits of the density factors discussed above and used in assigning tonnages to trucks and railcars. The earlier study had used density factors derived from STCC data in the railroad Carload Waybill Sample followed by some assumptions regarding the volume that would fit into a semi-tractor trailer. This approach would not work in the present study because the waybill sample continues to use the STCC system. All of this is explained above.

The question raised during the review was to what extent the density factors used in this volume approximate the payload factors derived by Cambridge Systematics (CS) based on their use of the Vehicle Inventory and Use Survey. CS supplied these factors to the IU research team 
Table 5-4. Commodity Density Values for Railcars and Trucks

\begin{tabular}{|l|l|r|r|}
\hline SCTG & Commodity & \multicolumn{1}{l|}{$\begin{array}{l}\text { Railcar } \\
\text { density }\end{array}$} & \multicolumn{2}{l|}{$\begin{array}{l}\text { Motor carrier } \\
\text { density }\end{array}$} \\
\hline 01 & Live animals and live fish & 9.77 & 3.91 \\
\hline 02 & Cereal grains & 96.63 & 30.1 \\
\hline 03 & Other agricultural products & 86.79 & 22.3 \\
\hline 04 & Animal feed and products of animal origin, n.e.c. & 88.28 & 25.3 \\
\hline 05 & Meat, fish, seafood, and their preparations & 74.41 & 18.6 \\
\hline 06 & Milled grain products and preparations and bakery products & 85.50 & 21.4 \\
\hline 07 & Other prepared foodstuffs and fats and oils & 87.02 & 21.0 \\
\hline 08 & Alcoholic beverages & 87.31 & 21.0 \\
\hline 09 & Tobacco products & 45.75 & 18.3 \\
\hline 10 & Monumental and building stone & 100.00 & 25.4 \\
\hline 11 & Natural sands & 97.97 & 25.4 \\
\hline 12 & Gravel and crushed stone & 97.97 & 24.1 \\
\hline 13 & Nonmetallic minerals, n.e.c. & 100.44 & 23.4 \\
\hline 14 & Metallic ores and concentrates & 95.91 & 21.4 \\
\hline 15 & Coal & 109.36 & 22.0 \\
\hline 17 & Gasoline and aviation turbine fuel & 84.04 & 28.2 \\
\hline 18 & Fuel oils & 88.22 & 20.0 \\
\hline 19 & Coal and petroleum products, n.e.c. & 73.66 & 23.5 \\
\hline 20 & Basic chemicals & 98.66 & 17.5 \\
\hline 21 & Pharmaceutical products & & 13.2 \\
\hline 22 & Fertilizers & 93.96 & 27.4 \\
\hline 23 & Chemical products and preparations, n.e.c. & 94.30 & 20.1 \\
\hline 24 & Plastics and rubber & 64.11 & 29.2 \\
\hline 25 & Logs and other wood in rough & & 13.3 \\
\hline & & & \\
\hline
\end{tabular}




\begin{tabular}{|c|c|c|c|}
\hline 26 & Wood products & 82.41 & 24.2 \\
\hline 27 & Pulp, newsprint, paper and paperboard & 82.75 & 23.5 \\
\hline 28 & Paper or paperboard products & 70.90 & 17.2 \\
\hline 29 & Printed products & & 15.1 \\
\hline 30 & Textiles, leather and articles of textiles or leather & 14.17 & 13.3 \\
\hline 31 & Nonmetallic mineral products & 98.64 & 21.2 \\
\hline 32 & $\begin{array}{l}\text { Base metal in primary or semifinished forms and in finished } \\
\text { basic shapes }\end{array}$ & 91.47 & 18.4 \\
\hline 33 & Articles of base metal & 79.66 & 12.2 \\
\hline 34 & Machinery & 49.77 & 13.8 \\
\hline 35 & $\begin{array}{l}\text { Electronic and other electrical equipment and components } \\
\text { and office equipment }\end{array}$ & 16.69 & 12.7 \\
\hline 36 & Motorized and other vehicles (including parts) & 21.73 & 13.3 \\
\hline 37 & Transportation equipment, n.e.c. & 41.36 & 12.1 \\
\hline 38 & Precision instruments and apparatus & & 9.0 \\
\hline 39 & $\begin{array}{l}\text { Furniture, mattresses and mattress supports, lamps, lighting } \\
\text { fittings, and illuminated signs }\end{array}$ & 15.00 & 10.7 \\
\hline 40 & Miscellaneous manufactured goods & 65.22 & 14.0 \\
\hline 41 & Waste and scrap & 79.86 & 20.0 \\
\hline 43 & Mixed freight & 32.45 & 14.2 \\
\hline
\end{tabular}

Sources: See text. 
so that a comparison of the two systems could be made. The data used for this test appears as Table 5.5.

There is very little relationship between the two systems and the CS estimates are only able to explain about $14 \%$ of the variation in the detailed Indiana density factors. We see no reason to adopt the less precise CS values for the analysis here, although there is sufficient detail here for CS to use their system of payload factors in future applications of the results of this study.

\section{Summary}

This chapter has discussed the efforts made to identify the best distribution model for the problem at hand. Two models were evaluated for this purpose. A fully-constrained model was selected over a production-constrained model. Traffic density, the amount of a given product that will fit into a tractor trailer or railcar was also discussed here. The approach selected is commodity specific and based on actual measures used in the U.S. and Canada. 
Table 5.5 IU Density Factors vs. Cambridge Payload Factors

\begin{tabular}{|c|c|c|c|}
\hline & SCTG & Density & Payload \\
\hline 1 & 1 & 3.91 & \\
\hline 2 & 2 & 30.10 & 19.48 \\
\hline 3 & 3 & 22.30 & 19.48 \\
\hline 4 & 4 & 25.30 & 19.48 \\
\hline 5 & 5 & 18.60 & 18.35 \\
\hline 6 & 6 & 21.40 & 18.35 \\
\hline 7 & 7 & 21.00 & 18.35 \\
\hline 8 & 8 & 21.00 & 18.35 \\
\hline 9 & 9 & 18.30 & 19.20 \\
\hline 10 & 10 & 25.40 & 21.40 \\
\hline 11 & 11 & 25.40 & 21.00 \\
\hline 12 & 12 & 24.10 & 21.40 \\
\hline 13 & 13 & 23.40 & 21.40 \\
\hline 14 & 14 & 21.40 & 21.40 \\
\hline 15 & 15 & 22.00 & 21.40 \\
\hline 16 & 17 & 28.20 & 20.61 \\
\hline 17 & 18 & 20.00 & 23.03 \\
\hline 18 & 19 & 23.50 & 23.03 \\
\hline 19 & 20 & 17.50 & 20.61 \\
\hline 20 & 21 & 13.20 & \\
\hline 21 & 22 & 27.40 & 20.61 \\
\hline 22 & 23 & 20.10 & 20.61 \\
\hline 23 & 24 & 13.30 & 14.95 \\
\hline 24 & 25 & 29.20 & 18.99 \\
\hline 25 & 26 & 24.20 & 15.82 \\
\hline 26 & 27 & 23.50 & 17.77 \\
\hline 27 & 28 & 17.20 & 17.77 \\
\hline 28 & 29 & 15.10 & 17.77 \\
\hline 29 & 30 & 13.30 & 17.80 \\
\hline 30 & 31 & 21.20 & 18.42 \\
\hline 31 & 32 & 18.40 & 25.42 \\
\hline 32 & 33 & 12.20 & 18.42 \\
\hline 33 & 34 & 13.80 & 17.11 \\
\hline 34 & 35 & 12.70 & 17.11 \\
\hline 35 & 36 & 13.30 & 20.39 \\
\hline 36 & 37 & 12.10 & 20.39 \\
\hline 37 & 38 & 9.00 & 16.40 \\
\hline 38 & 39 & 10.70 & 17.19 \\
\hline 39 & 40 & 14.00 & 20.27 \\
\hline 40 & 41 & 20.00 & 19.05 \\
\hline 41 & 43 & 14.20 & 19.05 \\
\hline
\end{tabular}




\section{References}

Petersen, Steven K., Eric L. Jessup, and Kenneth L. Casavant (2004), Freight Movements on Washington State Highways: Results of the 2003-2004 Origin and Destination Survey, Strategic Freight Transportation Analysis Report \#10, Pullman, WA: Washington State University.

Transport Canada (2004), Railway Carloadings, Ottawa, Canada: Transport Canada. Various issues.

U.S. Bureau of Census (2002), Vehicle Inventory and Use Survey, Washington, D.C.: U.S. Department of Commerce. 


\section{Chapter 6}

\section{THE MODES IN THE ANALYSIS}

The Commodity Flow Survey reports flow movements in several modal categories. This chapter simply notes the manner in which these are treated here. It is not possible to work with all of the various modal categories since flow data are not published for a number of them. It is for this reason that we have aggregated some of these categories.

\section{Motor carriers}

Motor carrier flows are often available as for-hire trucks or as private trucks. The former would be represented by a number of trucking companies, while the latter would be represented by those large companies that own a fleet of trucks. If proprietary data would be revealed on either category then the data available would be reported as trucks or would not appear in the data.

\section{Rail}

Rail is treated as a single modal category and flows are reported in this manner for the study. As we noted in Chapter 3, the Commodity Flow Survey also reports data as moving by the multiple modes of truck and rail and rail and water. As was also discussed in Chapter 3 truck and rail flows represent such a small proportion of the tonnage moving that we view these as primarily as rail moves and have categorized them in this manner. Rail and water moves were treated as water moves since this would be the manner in which they would tend to arrive in Indiana.

\section{Water}

In addition to the rail and water category the CFS recognizes water moves as shallow draft, Great Lakes, or deep draft. We have simply grouped all of these as well as the previously mentioned rail and water moves as water moves. Once again the rationale is that the data are simply not very good at these final modal categories once one leaves the national level. It is true that we could infer the flows that are coming into Indiana through Lake Michigan (the Great Lakes category) or the Ohio River (shallow draft). That is not the problem. The problem is that the data are simply not reported in a sufficient number of cases to merit breaking out the flows in 
this manner. To use national level estimates of what these should be would be very misleading.

Therefore, the water data are simply all grouped into simply water. We do not break the data down any further than to infer what the origin and destination county of the traffic is. The data are only reported in this manner and are not later assigned.

Air

Air transport of freight even though it may and usually does involve another mode (usually a motor carrier) is often treated as only an air move. Indiana has little to do with air transport infrastructure and as a result it is the motor carrier portion of these moves that are of primary interest here.

Air freight shipments from outside of Indiana were treated as motor carrier moves generated from the nearest large airport to the county that was the final destination of these flows based on the distribution modeling. In a similar manner air freight flows generated by counties in Indiana were considered as motor carrier flows to the airport from which the flows would leave the state. Figure 6-1 is a representation of how these assignments were made for air freight.

\section{Parcels}

At one time one could almost disregard the previous air freight category as well as the shipment of parcels in a study such as this. This is no longer the case and moves by various express companies become major components for some shipments between different origins and destinations. There are some counties in Indiana that receive little or no commodity flows as such on a daily basis, but they all receive some goods via FedEx, UPS, the US Postal Service (USPS), and more recently DHL.

Since these are private for-profit operations in most cases (the exception is the USPS), data are not published on the companies involved in the flows. Research by the project team suggests that the market for such parcel shipments are broken down into proportions as follows:

$\begin{array}{ll}\text { FedEx } & .27 \\ \text { UPS } & .53 \\ \text { USPS } & .13 \\ \text { DHL } & .07\end{array}$

Therefore, total parcel shipments were divided among the various companies using the proportions reported above.

Further research revealed the primary airports used by these different parcel handlers and these were used in a manner similar to what was done with the air freight category above. That 
is, flows going out of state or coming in state were treated as flows arriving at the nearest airport used by that parcel handler. Flows originating and terminating in the state were treated as ground moves of that carrier. The manner in which these flows were assigned to airports is reflected in Figures 6-2 through 6-5.

\section{Pipelines}

Pipelines were the final category reported in the $C F S$ and set aside here. These flows were not included in any analysis simply because the state has no responsibility for the infrastructure used by this mode.

\section{A Comment}

The modes examined were selected because of their volumes. In a like manner some categories were collapsed into broader categories because their was a lack of sufficient data to do much more with them. We would note that the manner in which air and parcel traffic were treated is a bit more complicated than its volumes would indicate. This was done as much for research reasons as for planning reasons. We wanted to see if this approach has merit. It does and perhaps it might make sense to try and improve on some of the other modal flows $n$ this manner.

\section{Summary}

This study has looked at flows by motor carriers, rail, water, pipelines, air freight, and parcels. These six modes were used as the basis for constructing tables of modal use by distance. These in turn were used to allocate the generated flows to such modes. Tables for this purpose appear in Appendix D. We have not assigned any flows to their respective networks beyond the rail and highway case. 


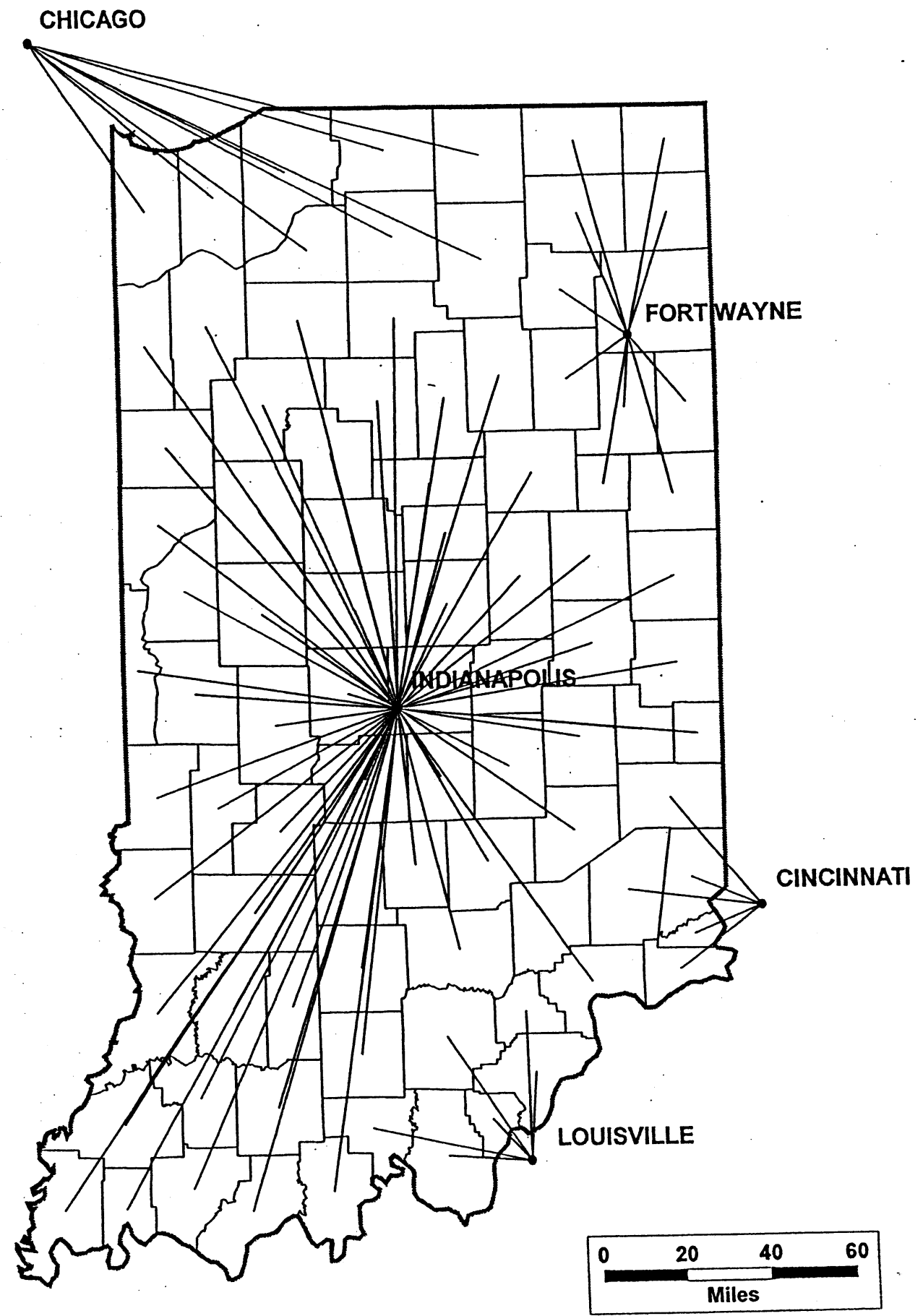

Figure 6-1. Market Area for General Air Freight in Indiana 


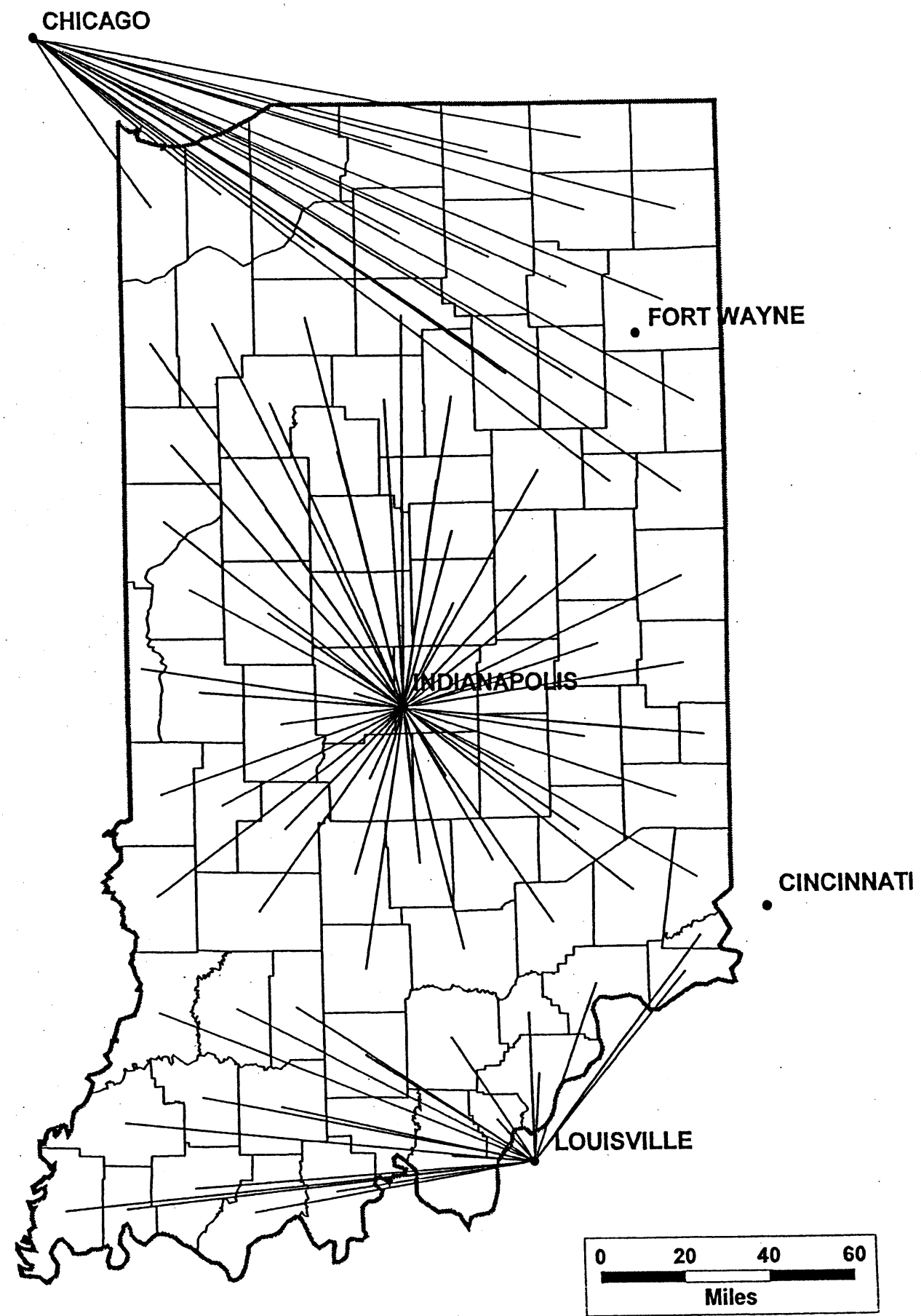

Figure 6-2. Market Areas for FedEx Parcels in Indiana 


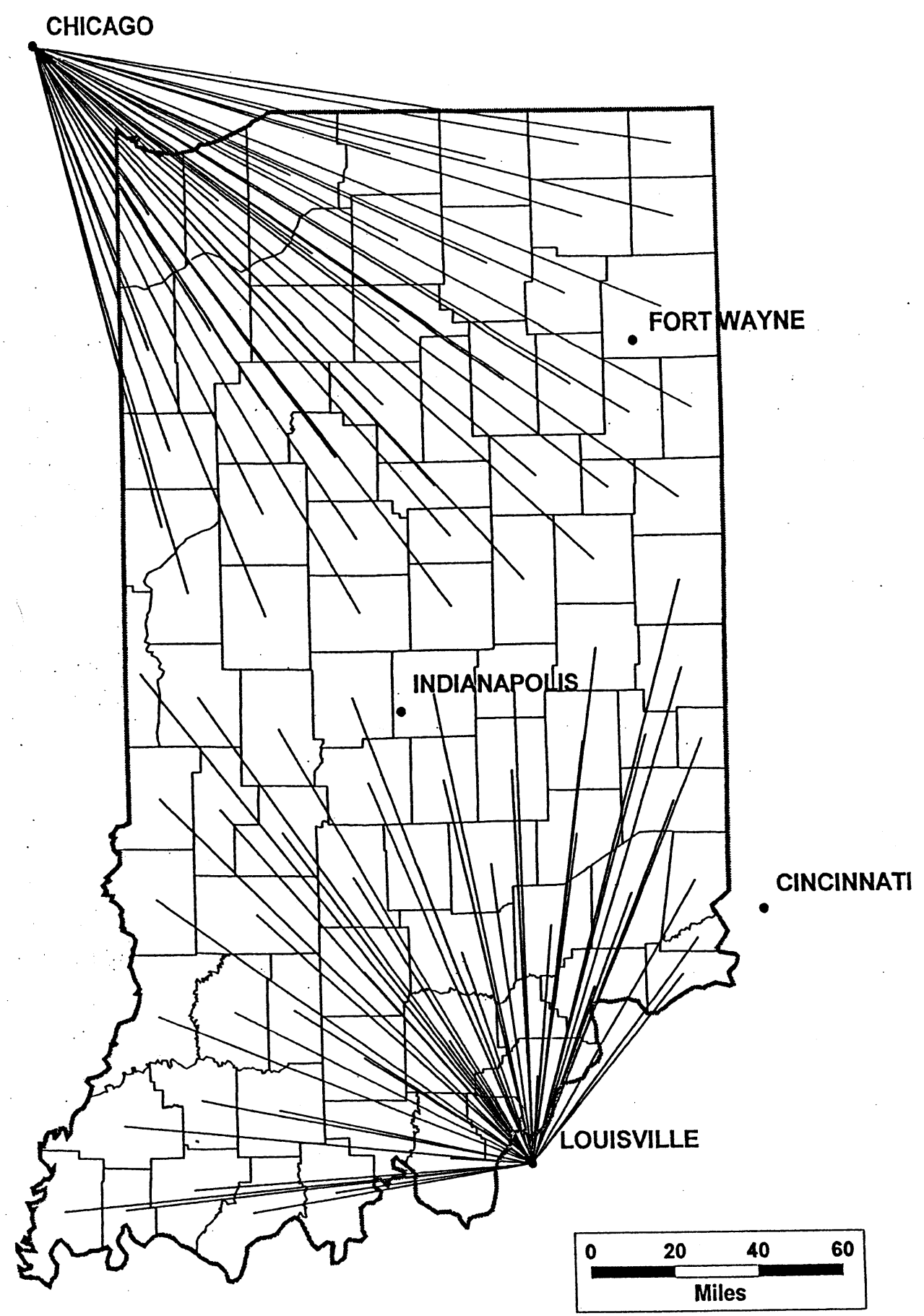

Figure 6-3. Market Areas for UPS Parcels in Indiana 


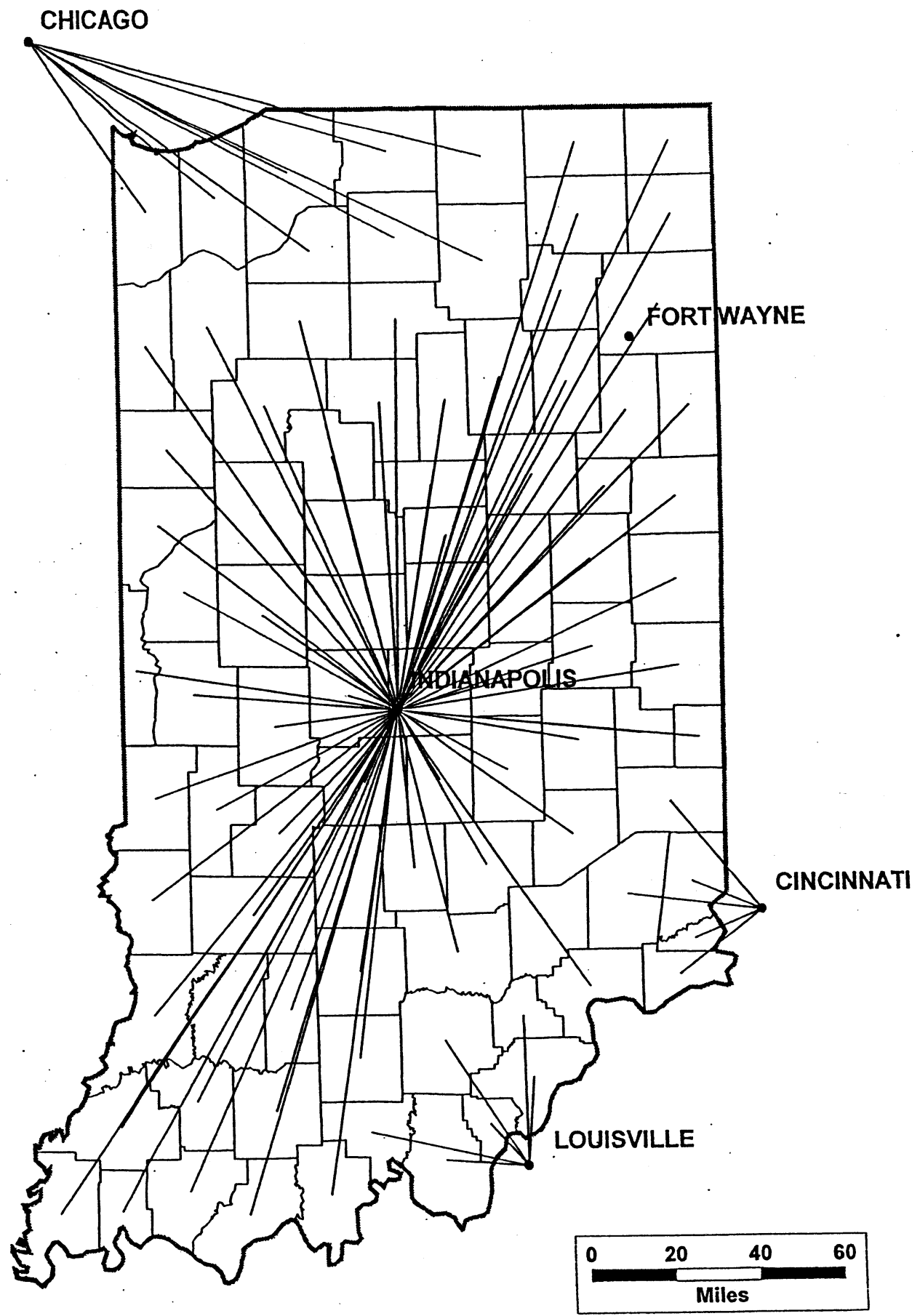

Figure 6-4. Market Areas for the USPS Parcels in Indiana 


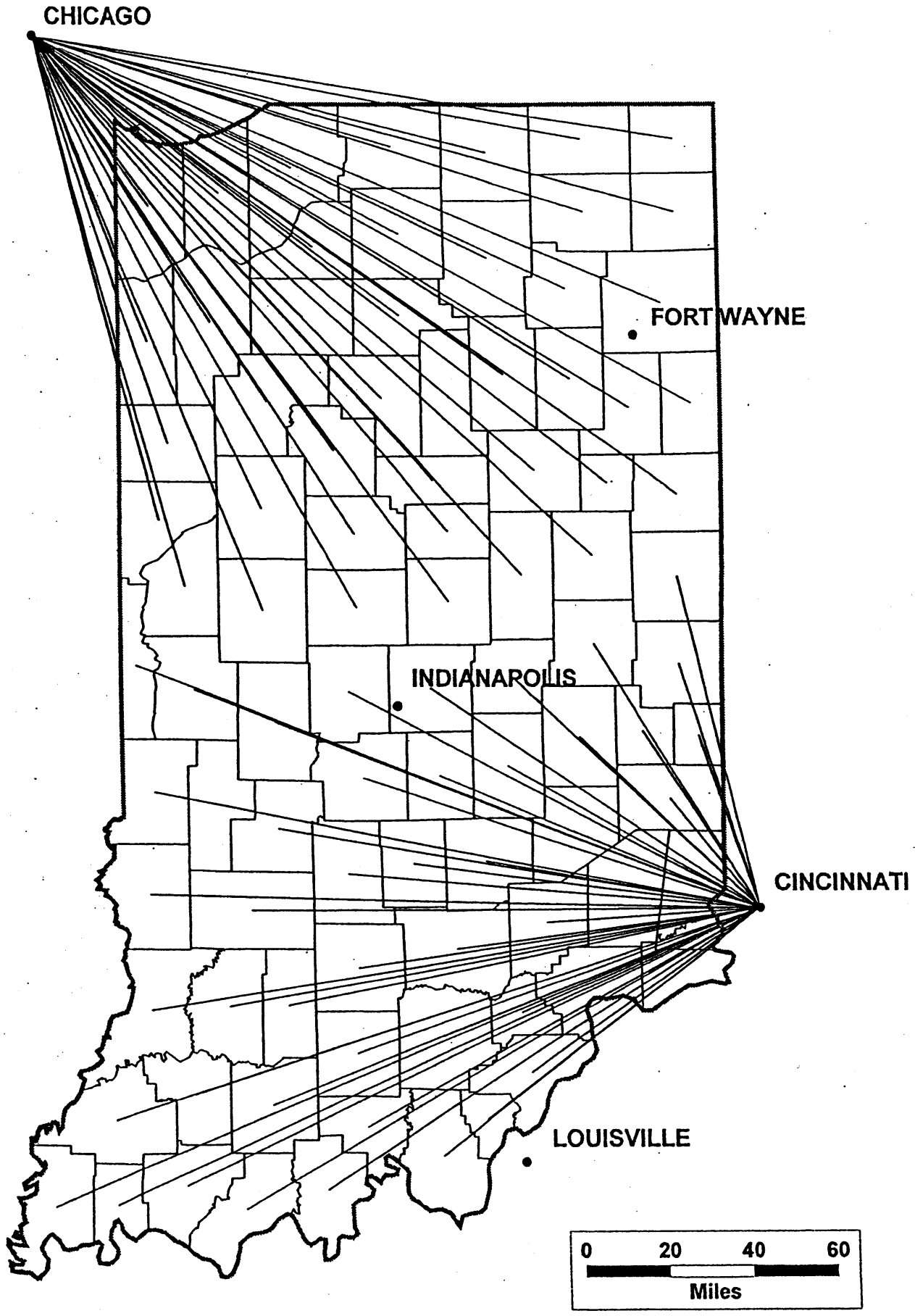

Figure 6-5. Market Areas for DHL Parcels in Indiana 


\section{Chapter 7}

\section{ASSIGNMENT OF TRAFFIC AND EVALUATION}

This chapter describes the procedures that were used for traffic assignment in this study. To place this in the proper context, we have distributed flows to a series of origins and destinations. These are gross estimates of interaction between states and counties without any clear indication of the paths, routes, or highways that these flows would take. Put another way, the flows from our gravity model lack geographic detail. It is the purpose of traffic assignment to provide the geographic detail so that we know those portions of the network over which these flows take place.

There are numerous analytical methods for assigning traffic to a network. The simplest of these methods is referred to as "all or nothing" assignment. This approach assumes that all of the traffic going between any origin-destination pair will travel by the shortest (time, distance, or other metric) path over the network between the two places. Now if we increase this to include all origins and all destinations, and follow the same procedure, then we have assigned our traffic using the "all or nothing" approach to traffic assignment.

This method of traffic assignment has its share of critics. Most of these are concerned with the application of this approach in urban areas where a multitude of possible routes are available. It should be apparent that whatever we use as a metric of spatial separation, e.g., cost, distance, or travel time, this approach will select the route for all flows between an origin and destination for which this is a minimum. Obviously, reality is different and the number of routes that will see some use is a function of the total number of such routes that are available. This is the basis for part of the criticism, although one could also attack the methodology on the grounds that not everyone is attempting to minimize their "friction." Some may want a scenic route, others may want a rural route with less traffic, while others may prefer a bypass that is longer. From a modeling standpoint we can't incorporate the motives of all travelers in our modeling.

When the modeling pertains only to motor carriers functioning over a large area, it is apparent that the "all or nothing" assignment procedure tends to reflect the behavior of those carriers the best. It would be possible to add congestion and capacity effects to the modeling, but the reality here is that these vehicles tend to try to minimize travel time. Even if we added more variables to the assignment modeling, these carriers would still tend to use the Interstate 
Highway System whenever they can. If their shipments are originating or terminating off the Interstate, they will try to stay on that system until they are off of it for the least amount of time.

\section{Transport Costs}

Traffic assignment requires the construction of a network over which shipments must move. This network connects all origins on the network to all destinations and includes the "cost" of movement over the links or segments of the network. In general, cost studies over the years have tended to use distance, travel time, or traffic flow functions related to distance or travel time for this cost measure. This project used travel time as its initial measure of travel cost. For shipments over very large areas, such as the United States, travel time is rarely known. Instead it is approximated by the use of the following:

$$
\text { Travel time }=(\text { link length }) /(\text { posted speed })
$$

Here the length is in miles and the speed is in miles per hour. This results in travel time being measured in hours or parts of hours.

As was true in the earlier study, this study made use of a conversion of the speeds in an attempt to get at user perception of speeds better than the above formulation allows. In other words, drivers generally exceed the speed limit. We therefore modified the previously obtained speeds by taking that value and modifying it as follows:

$$
\text { NewSpeed }=(\text { Posted Speed }+(2 * \sqrt{ }(70-\text { Posted Speed })
$$

This results in the following changes:

$\begin{array}{cc}\text { Old Speed } & \text { New Speed } \\ 70.00 & 70.00 \\ 65.00 & 69.47 \\ 60.00 & 66.32 \\ 55.00 & 62.75 \\ 50.00 & 58.94 \\ 45.00 & 55.00 \\ 40.00 & 50.95 \\ 35.00 & 46.83\end{array}$

Travel time was redefined as:

$$
\text { Travel time }=(\text { Link length } / \text { New Speed }) * 60
$$

This yields an estimate of travel time in minutes for all links of the network analyzed, and for 
those links of the interstate and for those in the study area that had no such value in the database. Although travel times are available for segments of the Indiana Road Inventory, the above approach was also used for these segments for consistency.

\section{Highway Assignment Results}

The assignment procedure used was "All or Nothing," without any constraint in the form of link capacity. This resulted in the bulk of the traffic being assigned to the Interstate Highway System and only traffic moving toward the counties off of that system using the other highways, in most cases. There are of course exceptions to this statement.

Perhaps the easiest way to discuss this is to refer the reader to the figures on the following two pages. The first of these figures shows the volume of traffic on an annual basis for the entire United States in 1997 (see Figure 7-1). There is a heavy concentration of flows out of Texas reflecting primarily heavy volumes of petroleum and petrochemicals. The heavy volumes between Wisconsin and Illinois reflect not only movement between those states, but also the role of O'Hare Airport in the airfreight and parcel flows in that region. Some of this could also be the result of the modeling attempting to generate the overall short shipping distances that are used as constraints on the flow model.

The distribution of flows within Indiana for 1997 is more clearly displayed in Figure 7-2. As one would expect the flows are heaviest on the Interstate Highway System within the state. The heaviest volumes here are on I- 65 between Chicago and Indianapolis. The lowest interstate volume is across the I-64 corridor in Southern Indiana. Volumes are lower than expected on I-69 between Fort Wayne and Indianapolis; this is particularly true for the southern end of that corridor. This may be attributable to the fact that commodity traffic coming into the state from Canada may not be included in the data used here, i.e., only domestic shippers are surveyed.

The accuracy of the generated truck flows is an issue here. The flows in the Indiana map were compared with truck counts for 1997 based on data in the Indiana Road Inventory. The relationship across all segments for which data were available is .65 , which means that the models capture about $42 \%$ of the variation in the count data. While not as high as might be desirable it should be noted that some of the vehicles counted near urban areas might not be commodity flows as such, e.g., local lumber, stone, brick, and block moves, garbage truck flows, and similar large heavy vehicles would be in any count data.

The Indiana DOT also supplied the project team with vehicle counts for 2002 in a GIS point format. The data were not complete, i.e., only some of the counties have values. A sample of 59 segments appears as Table 7-1 and a map of the sample locations follows that (Figure 7.3). As can be seen the segments range from high volume Interstate links to low volume state highways, and are distributed around the state proportionally to the sampling completed to date. 


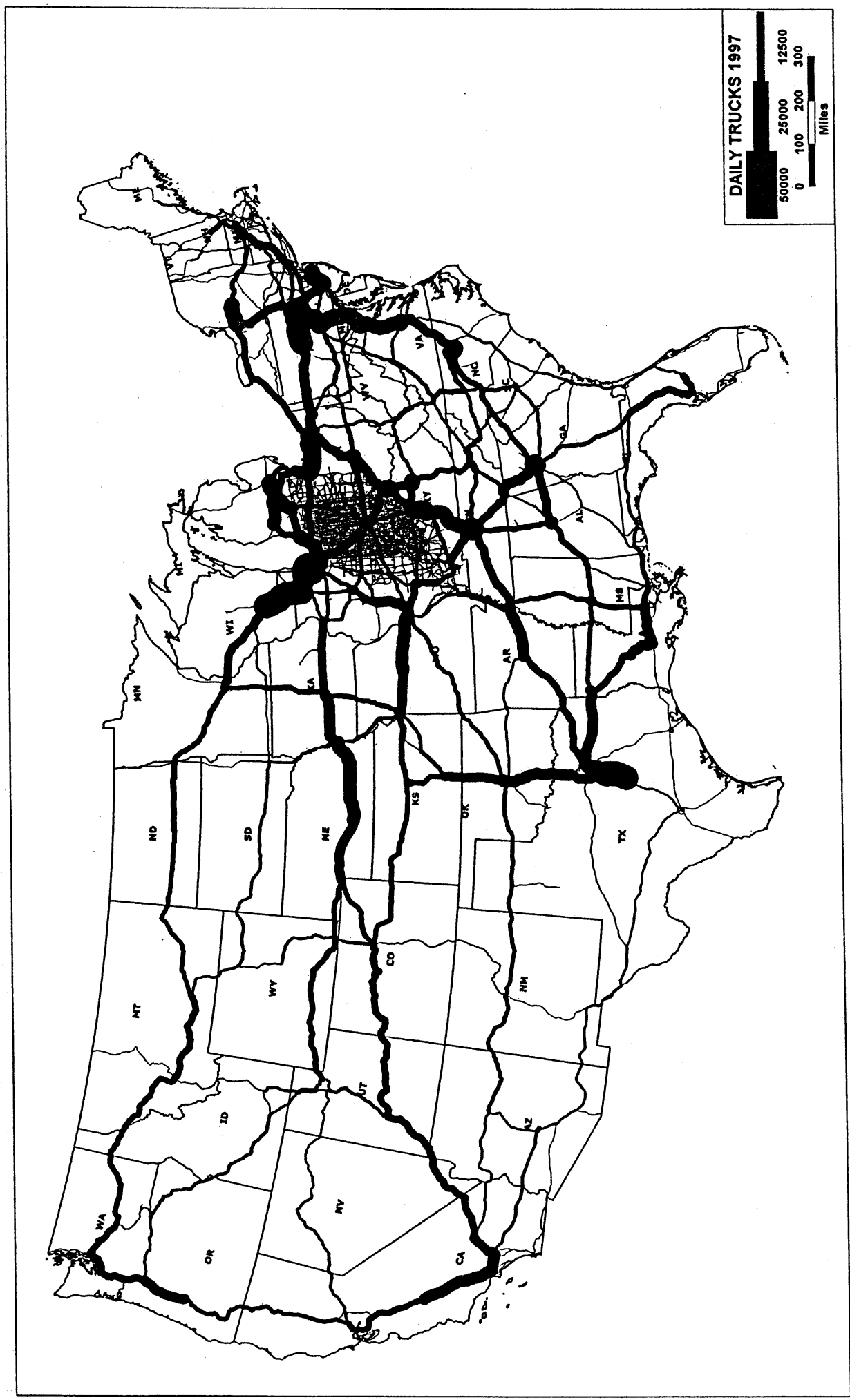

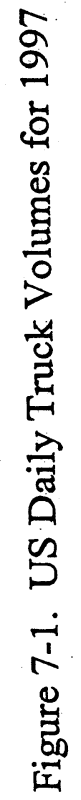




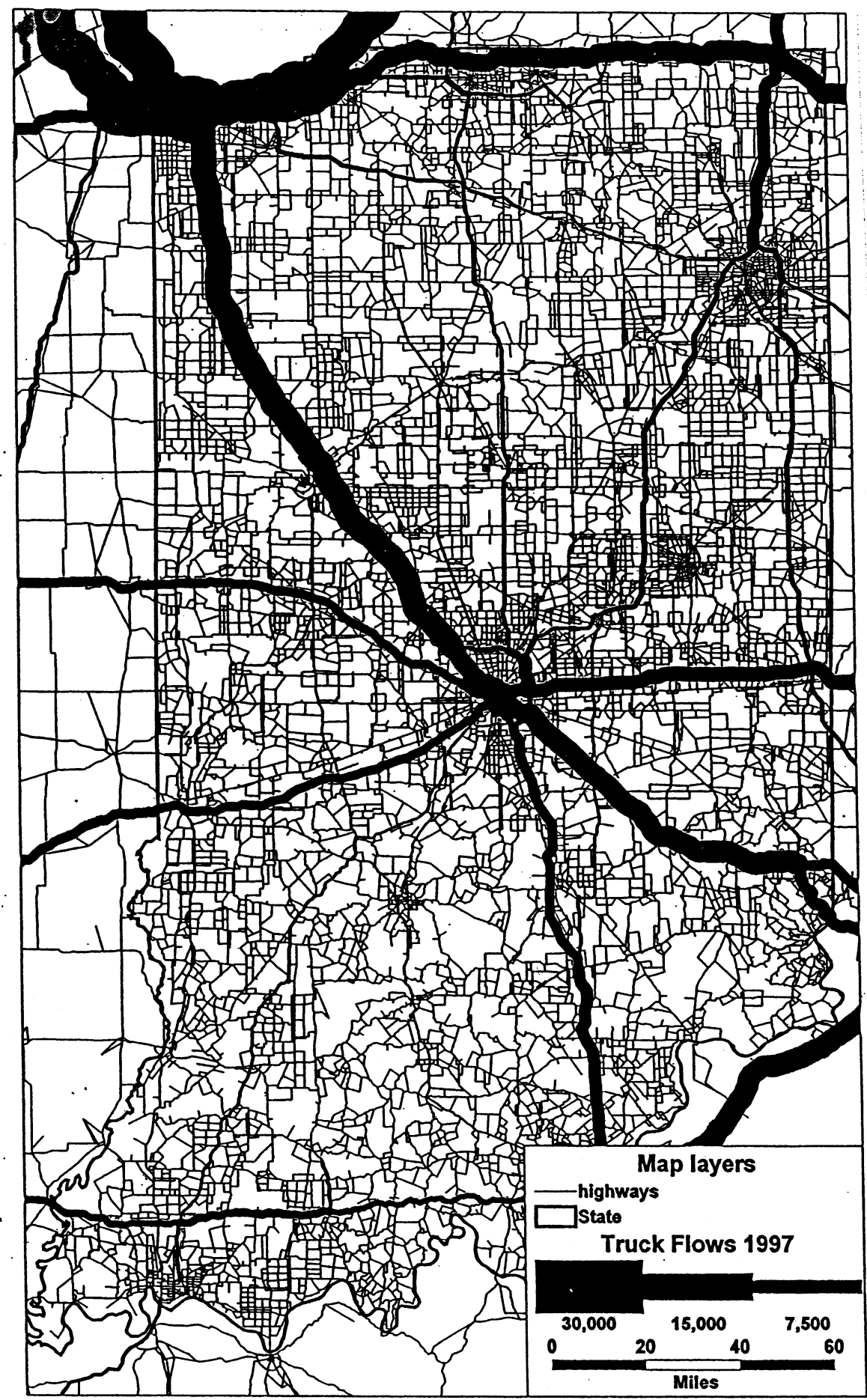

Figure 7-2. Indiana Daily Truck Volumes for 1997 
Taking the count data as the dependent variable, the estimated flow volumes based the fully-constrained gravity model followed by an "all-or-nothing" assignment served as an independent variable. The level of statistical explanation was considerably higher than the research team expected. This was slightly in excess of 89 percent (with an $r^{2}$ value of .893 ). The relationship is illustrated in the Figure 7-4.

It is interesting to note that the model developed was of the form:

$$
2002 \text { Counts }=57.8+1.125 \text { (1997 Model estimates) }
$$

In effect what the model is suggesting is that the values for 2002 have increased roughly $12.5 \%$ since 1997. This is a reasonable level of increase given the downturn in the economy in the late 1990s and the subsequent recovery. This difference may also be attributable to empty trucks, which are not incorporated here.

Of course in the sampling process an attempt was made to exclude links with no flow based on the modeling. But there were very few cases where zero flow estimates occurred. In such cases the counts were also very low as represented by some of the segments included here. The major source of variation here is on the Interstate links and for these the level of relationship is reasonably high.

Illustrations of the flow volumes for 2015 and 2030 appear in the following chapter. Although there are substantially higher flow volumes for those future years, the dominant corridors found in the 1997 analysis continue to be the same for the forecast years. This will be discussed in more detail in Chapter 8.

\section{The Rail Flows}

The basic network used in the study was described in Chapter 1 . It is the 1:2,000,000 rail network prepared by the Federal Railroad Administration as revised to take into account changes due to abandonments and some new construction. It consists of 12,815 segments representing a national network of 148,996 route miles, not track miles.

We used travel time as a measure of the cost of transport in the highway assignment procedure. This is reasonable in that most users of the highway system are interested in minimizing this metric. That is not the case in the rail sector since, for the most part, the railroads are more interested in maximizing their profits. Although this would also usually involve minimizing some measure of transport cost, this is not done unless the traffic is moving entirely on one rail company's lines. In all other cases the railroads involved in the moves must "divide" the total revenue obtained from the move. These divisions, as they are called, are 
Table 7-1. Sampled Locations for Model Evaluation

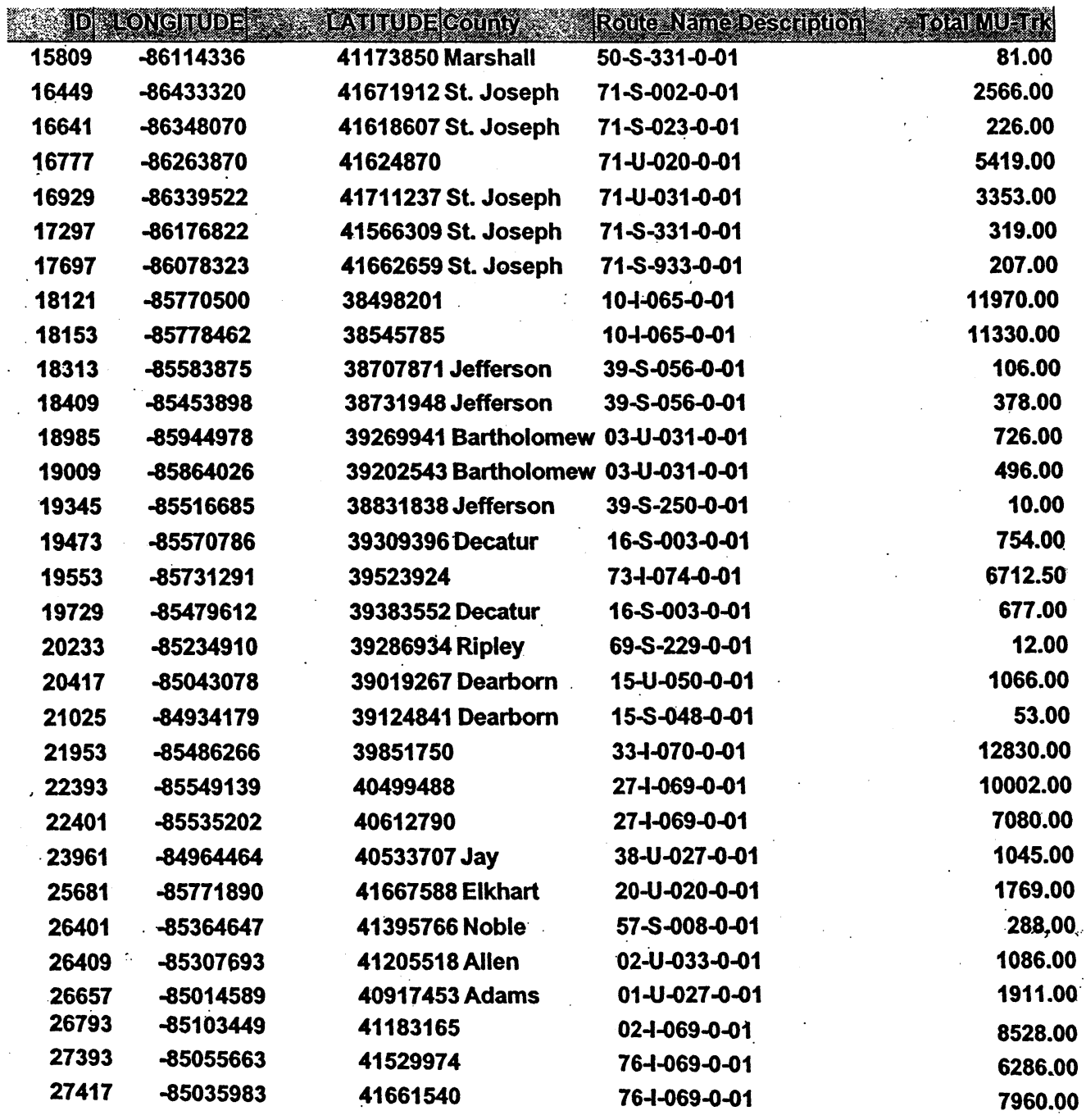




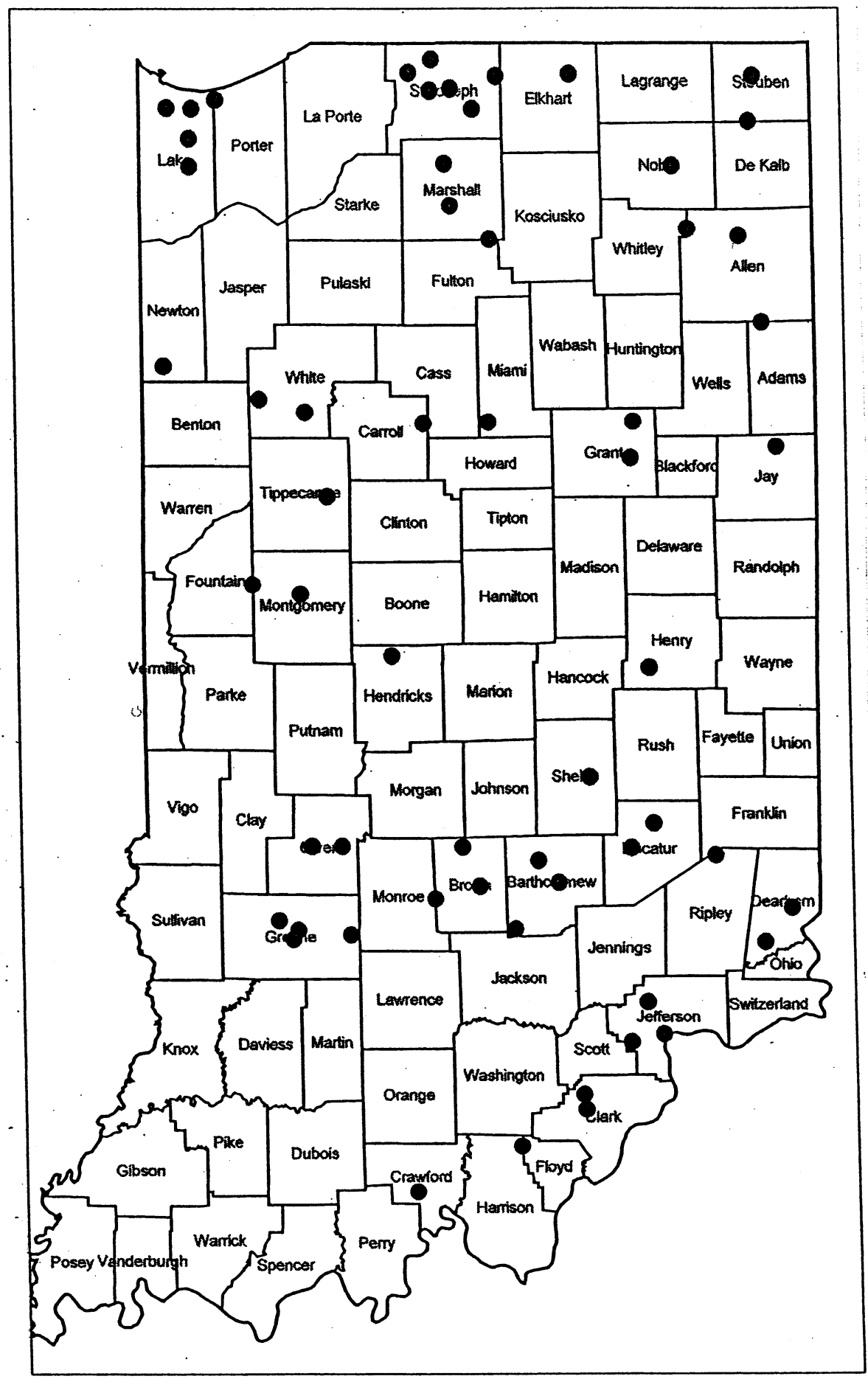

Figure 7-3. Map of Sampled Locations 
Truck Counts 2002

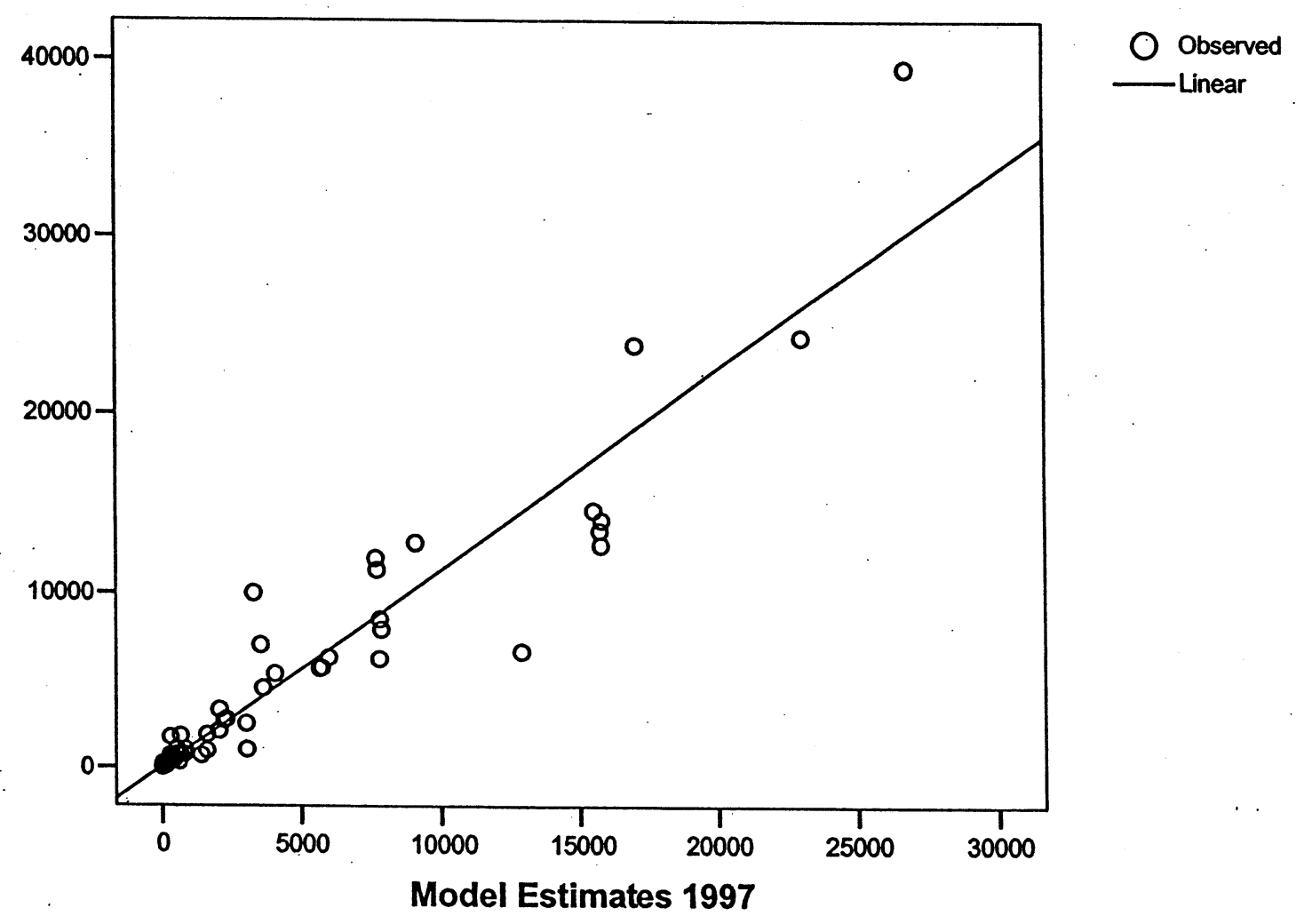

Figure 7-4. Relationship of Counts to Model Estimates 
usually based on the respective miles that the traffic is on the various rail carriers' lines. As a result it is to a railroad's advantage to keep the traffic on their system as long as they can accruing more and more miles before transferring it to another rail carrier. One exception to this involves that portion of the traffic that is originating or terminating on short line railroads. In this latter case the short line railroad gets a set fee whether the traffic originated one mile from a junction with a receiving carrier or 50 miles from that location. It is not immediately apparent what type of metric can be used to capture this situation.

It should be apparent that railroad flows would not necessarily follow a minimal path routing and some alternative approach is necessary. In the 1997 report some consideration was given to a procedure that would keep traffic on a rail company's lines as long as possible before passing it off to another carrier. This approach used turning penalties in the assignment algorithm to ensure this type of routing, but problems with the network in the form of duplicate digital lines resulted in failure of this approach.

Instead of that approach the earlier study noted the tendency of railroads to use the same main lines. Although there is some desire on the part of rail carriers to minimize the length of haul, this is minor in comparison to their desire to use mainline trackage even though secondary lines may be more direct. The question was how to represent this tendency with the rail data available on the digital network. Track condition plays a part in such decisions, but this is a very dynamic variable that would change more frequently than the database available. It seemed a new measure of spatial separation was necessary. The new measure would still incorporate an attempt to minimize length of haul, but would also pick those routes that the railroads tend to use.

Short line or regional railroads that originate or terminate traffic are not important in this methodology, since the origin and destination of shipments must be reached. In other words these moves can be replicated by any methodology regardless of the cost attached to it simply because the end nodes of these moves are automatically selected, i.e., there is no alternative.

The measure finally used in the earlier study had the form

$$
\mathrm{I}=(\mathrm{L}(1 /(\mathrm{D}+1)))
$$

where $I=$ the index of spatial separation;

$\mathrm{L}=$ the length of the line segment of the network; and,

$\mathrm{D}=$ the traffic density of the line in millions of gross ton-miles per year.

The measure diminishes the length of the line segments by dividing the segment by its traffic density, i.e., by gross ton-miles per mile. Typical traffic density values vary from 0 to about six million gross-ton miles per mile of line. 
If we have five route segments of 100 miles in length each with traffic density ranging from 0 to 1 to 2 to 3 to 4 , the index of spatial separation would be $100,50,33,25$, and 20 . When used on lines with high traffic density these routes "become shorter" and are always selected. Lines of low traffic density, do not become "longer" since their traffic density always has a unit value added to it. Lines of 0 traffic density would become lines of 0 length, if it were not for the correction factor.

The transport cost matrix used for assigning the rail traffic was defined using the lengthdensity index described above. In the earlier project, it was noted that there was a need for a major research project that would evaluate a broad array of indices and methods for assigning rail traffic to a rail network. Such a study would require the existence of a set of actual flows, referred to in the highway case as target flows, but these are not generally available in the rail case. The carload waybill sample is not available for SCTG commodities so that even if the STCC could be assigned they would not provide a comparable data set for evaluating the accuracy of the rail flow assignments here.

\section{Selection of the Rail Assignment Approach}

There is a certain intuitive appeal to the earlier routing method that would keep traffic on a railroad's lines longer in order to maximize their share of the divisions from various moves. On the other hand, the tendency for railroads to use main lines with high volumes (the high density lines) also has some logical appeal.

It has been noted in another context here that we should utilize methods that are flexible to changes in circumstances. If we use the first approach then there is a reliance on the current set of rail carriers. Had we used this approach in the earlier study, the breakup of Conrail would have all but destroyed the flow assignments. We see no reason to assume that the current set of carriers will continue, but more than likely the heavier volume lines will continue to be used even if certain carriers fall out of the system. As a result the assignment process used here is the second approach, i.e., the length-density approach.

A map of the rail flows for the United States as generated by the model here appears as Figure 7-5 and an enlargement of these flows for Indiana appears in Figure 7-6. As is true in the highway case, the major corridors remain the same into the future but the volumes increase. This will be more apparent in Chapter 8 .

Flows were also generated for rail and water for the 145 nodes of the original analysis; the five airports included were excluded from this part of the analysis. The flows were generated for rail flows and water flows for all forty-one commodity groups examined here. Digital versions of these are included as part of the deliverables from the project. These files yield the origin, destination and volume of flow for each of these modes by commodity group. 
Water flows are not as easy to deal with. Our best estimates are for the tonnage volume moving to Indiana counties by water primarily. In most cases these are most significant when we begin to consider them as highway flows. Unfortunately the data are not so refined as to give the modelers a clear idea of whether these are coming into Indiana via Lake Michigan and the state's ports on that shore, or whether they are coming into the state via the Ohio River ports noted in Chapters 1 and 6 . Nevertheless, the estimated 1997 commodity flows to Indiana counties is being provided to the project sponsors in digital format and the forecasted flows for 2015 and 2030 are also being provided. It may be possible for those interested in these flows to infer the Indiana port through which they entered the state.

Pipeline data were collected and treated as one of the modes of interest here, but the State has very little impact on this sector and as a result the data were not treated separately.

\section{A Precautionary Note}

It should be noted that while our approach to modal split analysis and assignment discussed in Chapters 6 and 7 have been used in the past, some believe this limits the utility of the models developed. In other words the approach used assumes that modal shares will not change a great deal from historical trends. As a result if the State of Indiana initiates some type of major fuel tax with the objective of diverting truck traffic onto the railroads, the present type of analysis will not enable us to examine such questions. The modal shares are independent of the cost of transport and therefore changes in these costs will not have any impact on the modal shares.

In order to develop an approach to modal split that could evaluate such a question a cost based logistic model would have to be used and this was beyond the scope of the study.

The approach used here would enable state planners to examine the role of changes in employment on traffic, so it still has utility in that regard, although it would not result in a shift of traffic from one mode to another.

The approach used here also has value in examining the impacts of routing as a result of constructing a new highway, or improving an existing highway. It can also be used for various types of economic and policy analysis. 


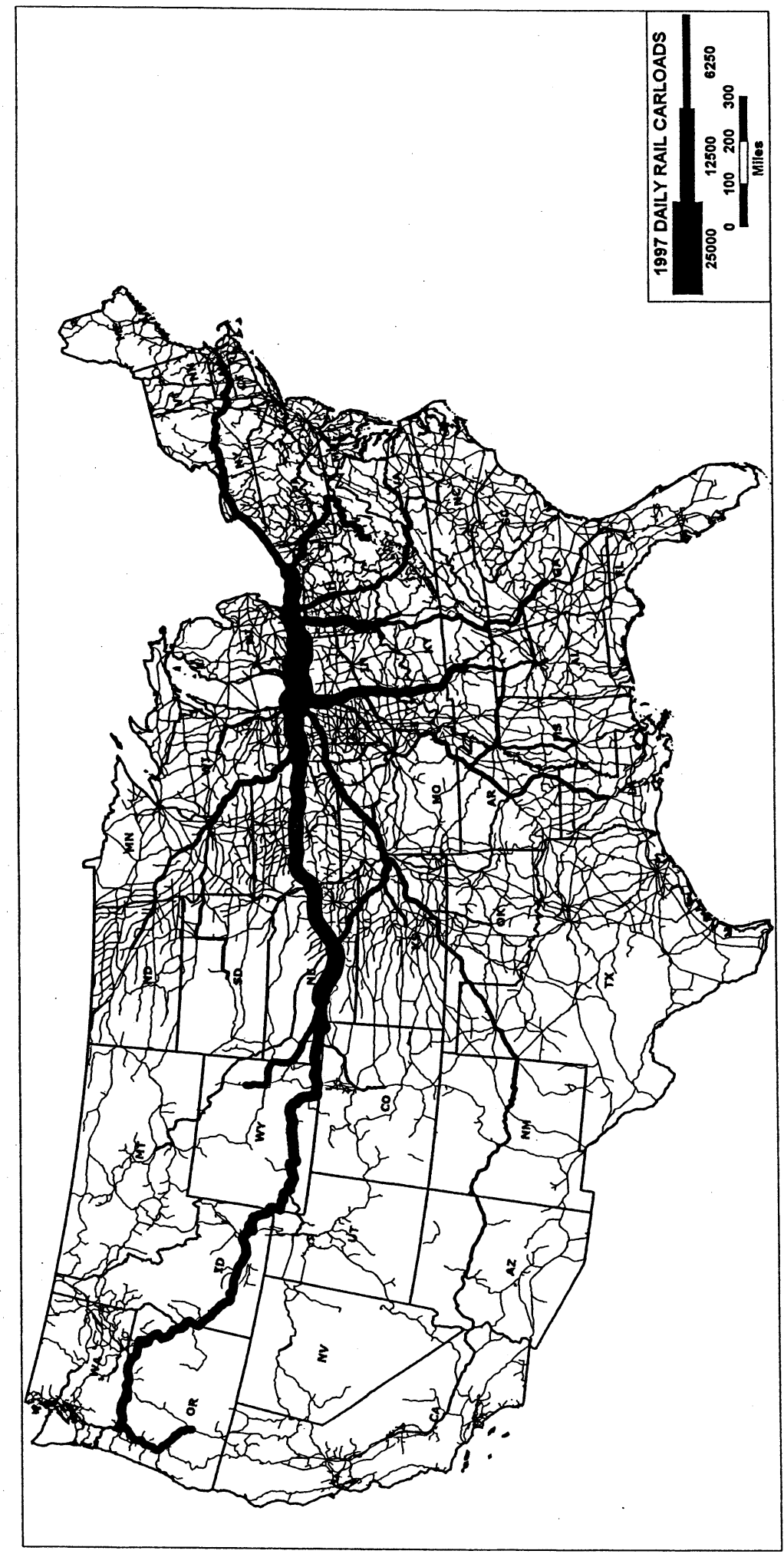

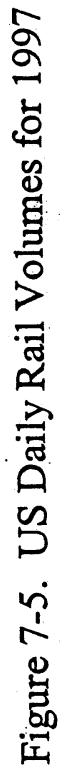




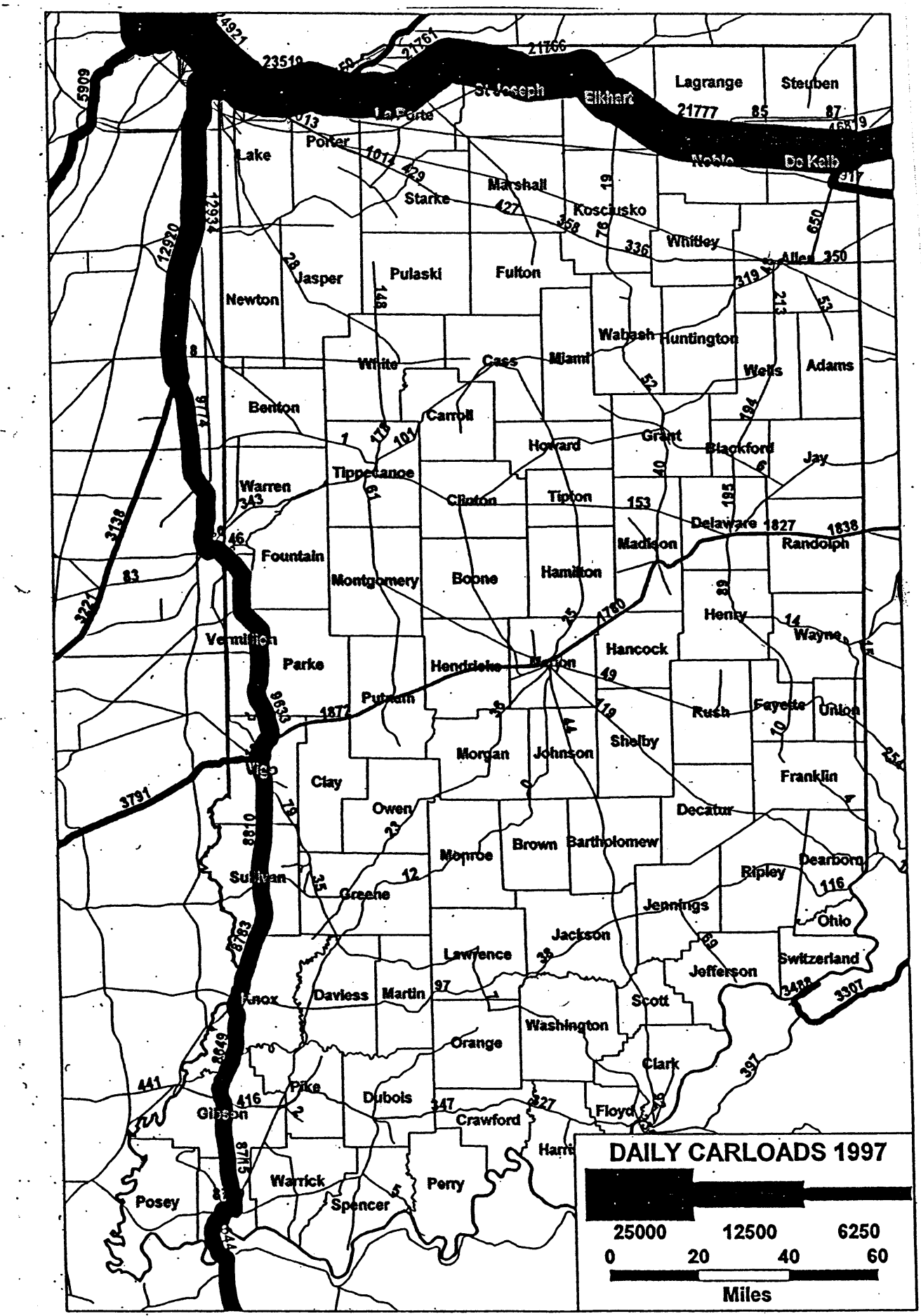

Figure 7-6. Indiana Rail Flows 1997 (modeled) 


\section{Chapter 8}

\section{FORECASTS FOR 2015 AND 2030}

A primary objective of this study was the forecasting of traffic levels for 2015 and 2030. There are numerous ways that this can be accomplished. A standard way of doing short term forecasts involves simply trend extrapolation. This might better be referred to as a projection since one literally projects the trend line into some future time. The projection is sometimes done with a ruler, or more elegantly with a regression model.

In the earlier study that was undertaken we made use of employment projections. In the simplest case let us say that we have found each ton of some commodity shipped appears to be related to .5 employees in some related industrial sector. We project employment using some method and find that the industry at some future date will have 1000 workers in some area of interest. Since we know that one employee will produce 2 tons per year we assume that the 1000 workers will produce shipments of 2,000 tons.

The major problem inherent in the above formulation should be apparent: it assumes that there will be no changes in the productivity of employees over the projection period. Whatever an employee was producing in the base year is the same as what she will produce in the forecast year. This is viewed as unacceptable by many researchers and practitioners in the modeling field. You can look at any one of a number of industries and it is apparent that mechanization, robotics, and computerization have significantly changed worker productivity, so the approach described is unacceptable since it does not incorporate such productivity changes.

In order for this project to incorporate the role of changes in employment the Indiana Department of Transportation supplied the study team with gross county-level employment projections for the year 2015 and the year 2030. By gross we simply mean that the forecasts are not industry specific, but are forecasts of total employment in the county. One could obviously raise some questions regarding the validity of the assumption that employment will increase uniformly across all industries, but this is what we have assumed here. The primary argument against this is due to globalization. We have seen substantial changes in employment levels in the manufacturing sectors and there is no indication that this trend will change in the near future.

Changes in employment for areas beyond the borders of Indiana were not made available. We have no other source for these and we have made the assumption that employment beyond 
Indiana will increase or decrease at the same level as the population will change. The source of the population forecasts is the U.S. Bureau of Census.

\section{Future Traffic Production}

Table 8-1 on the following page gives the estimates of the change in employment used for 2015 and 2030. For Adams County we are assuming that employment will increase by .1213 or $12.13 \%$ between 2000 and 2015 . It is expected that the county's employment will increase by $10.81 \%$ during the period from 2015 to 2030 .

This does not get us around the problem of changes in productivity. For this we have made use of labor productivity changes derived from Indiana's REMI model. That model gives growth factors for labor productivity changes in several employment sectors of Indiana. The values derived cover numerous employment sectors that are not of interest in this study, e.g., the service industries are also included. Therefore the values of interest here appear in Table 8-2.

It may be instructive and clarify the use of the above if an example is provided of the use of these values. Let us assume that we have an industry and SCTG sector for which the relationship between tons moved and employment is as follows:

$$
\text { Tons }=2 * \text { employment }
$$

We will assume that employment is 1000 in the area that we are looking at. This would suggest that the current level of this SCTG from this area is 2000 tons (assuming the model is perfect). Now we expect an increase in employment of $20 \%$ between now and 2030 or a growth factor 1.20 for this employment sector. So the 1000 employees will be 1200 employees by 2030 and the tonnage shipped will increase to 2400 tons. But we have not yet considered any changes in labor productivity. Let us assume that productivity will increase by $91 \%$, or by a growth factor of 1.91. This means that the expected tons will actually increase from the 2400 tons to 4584 tons.

For the year 2015 we would assume half of the increase in employment to 1100 employees with a tonnage of 2200 without the productivity increase. The total increase of 2,184 tons due to productivity is divided proportionally based on employment and this suggests that 1048 tons accompanied the growth to $2015(48 \%)$ and the remaining 1136 tons $(52 \%)$ came during the next fifteen years. Therefore, the tonnage of the SCTG for 2015 would be 2200 plus the 1048 or a total of 3248 tons. This should make it apparent that changes in productivity are quite significant in terms of the total amount of a commodity that will be moved at some future point in time. The forecasts of future commodity traffic production for 2015 and 2030 appear in the appendices $\mathrm{E}$ and $\mathrm{F}$. 
Table 8-1 Proportional Increases in Employment by Area

\begin{tabular}{|l|r|r|}
\hline Locale & 2000-2015 & 2015-2030 \\
\hline Alabama & 1.0486 & 1.0453 \\
\hline Arizona & 1.4609 & 1.4292 \\
\hline Arkansas & 1.1105 & 1.0914 \\
\hline California & 1.1846 & 1.1576 \\
\hline Colorado & 1.1740 & 1.1471 \\
\hline Connecticut & 1.0675 & 1.0146 \\
\hline Delaware & 1.1835 & 1.0919 \\
\hline District of Colombia & -0.1149 & -0.1440 \\
\hline Florida & 1.3267 & 1.3528 \\
\hline Georgia & 1.2497 & 1.1747 \\
\hline Idaho & 1.2597 & 1.2083 \\
\hline Illinois-Chicago & 1.0546 & 1.0256 \\
\hline Illinois- Springfield & 1.0546 & 1.0256 \\
\hline Indiana - Adams & 1.1213 & 1.1081 \\
\hline Indiana - Allen & 1.1152 & 1.1033 \\
\hline Indiana - Bartholomew & 1.1119 & 1.1006 \\
\hline Indiana - Benton & -0.0316 & -0.0329 \\
\hline Indiana - Blackford & -0.0803 & -0.0873 \\
\hline Indiana - Boone & 1.3460 & 1.2570 \\
\hline Indiana - Brown & 1.0258 & 1.0251 \\
\hline Indiana - Carroll & 1.0680 & 1.0636 \\
\hline Indiana - Cass & -0.0054 & -0.0055 \\
\hline Indiana - Clark & 1.1264 & 1.1122 \\
\hline Indiana - Clay & 1.0302 & 1.0293 \\
\hline & & \\
\hline & & \\
\hline
\end{tabular}


Table 8-1, Continued

\begin{tabular}{|c|c|c|}
\hline Indiana - Clinton & 1.0638 & 1.0600 \\
\hline Indiana - Crawford & 1.1917 & 1.1606 \\
\hline Indiana - Daviess & 1.0904 & 1.0828 \\
\hline Indiana - Dearborn & 1.2626 & 1.2080 \\
\hline Indiana - Decatur & 1.0441 & 1.0422 \\
\hline Indiana - DeKalb & 1.1507 & 1.1310 \\
\hline Indiana -Delaware & 1.0596 & 1.0563 \\
\hline Indiana - Dubois & 1.0798 & 1.0739 \\
\hline Indiana - Elkhart & 1.1170 & 0.1047 \\
\hline Indiana - Fayette & -0.0481 & -0.0506 \\
\hline Indiana - Floyd & 1.1130 & 1.1015 \\
\hline Indiana -Fountain & 1.0157 & 1.0154 \\
\hline Indiana - Franklin & 1.1356 & 1.1193 \\
\hline Indiana - Fulton & 1.0282 & 1.0274 \\
\hline Indiana - Gibson & 1.1114 & 1.1002 \\
\hline Indiana - Grant & -0.0934 & -0.1031 \\
\hline Indiana - Greene & 1.0000 & 1.0000 \\
\hline Indiana - Hamilton & 1.3766 & 1.2736 \\
\hline Indiana - Hancock & 1.3311 & 1.2487 \\
\hline Indiana -Harrison & 1.2538 & 1.2024 \\
\hline Indiana - Hendricks & 1.5767 & 1.3658 \\
\hline Indiana - Henry & -0.0588 & -0.0625 \\
\hline Indiana - Howard & 1.0241 & 1.0236 \\
\hline Indiana - Huntington & 1.1117 & 1.1004 \\
\hline Indiana - Jackson & 1.0376 & 1.0362 \\
\hline Indiana - Jasper & 1.0667 & 1.0625 \\
\hline
\end{tabular}


Table 8-1, Continued

\begin{tabular}{|c|c|c|}
\hline Indiana - Jay & -0.0277 & -0.0286 \\
\hline Indiana- Jefferson & 1.0790 & 1.0732 \\
\hline Indiana - Jennings & 1.1263 & 1.1122 \\
\hline Indiana - Johnson & 1.3637 & 1.2667 \\
\hline Indiana - Knox & -0.0501 & -0.0528 \\
\hline Indiana - Kosciusko & 1.0670 & 1.0628 \\
\hline Indiana - LaGrange & 1.1358 & 1.1195 \\
\hline Indiana - Lake & 1.0189 & 1.0185 \\
\hline Indiana - LaPorte & 1.0127 & 1.0126 \\
\hline Indiana - Lawrence & -0.0146 & -0.0149 \\
\hline Indiana - Madison & 1.0338 & 1.0327 \\
\hline Indiana - Marion & 1.0492 & 1.0469 \\
\hline Indiana - Marshall & 1.1088 & 1.0981 \\
\hline Indiana - Martin & -0.0470 & -0.0494 \\
\hline Indiana - Miami & -0.0486 & -0.0511 \\
\hline Indiana - Monroe & 1.1422 & 1.1244 \\
\hline Indiana - Montgomery & 1.0557 & 1.0527 \\
\hline Indiana - Morgan & 1.2179 & 1.1789 \\
\hline Indiana - Newton & 1.0149 & 1.0147 \\
\hline Indiana - Noble & 1.0842 & 1.0777 \\
\hline Indiana - Ohio & 1.1028 & 1.0932 \\
\hline Indiana - Orange & -0.0020 & -0.0020 \\
\hline Indiana - Owen & 1.0856 & 1.0788 \\
\hline Indiana - Parke & 1.0082 & 1.0081 \\
\hline Indiana - Perry & -0.0413 & -0.0431 \\
\hline
\end{tabular}


Table 8-1, Continued

\begin{tabular}{|c|c|c|}
\hline Indiana - Pike & 1.0541 & 1.0510 \\
\hline Indiana - Porter & 1.0574 & 1.0543 \\
\hline Indiana - Posey & 1.0657 & 1.0615 \\
\hline Indiana - Pulaski & 1.0526 & 1.0499 \\
\hline Indiana - Putnam & 1.0589 & 1.0556 \\
\hline Indiana - Randolph & -0.0282 & -0.0291 \\
\hline Indiana - Ripley & 1.0770 & 1.0715 \\
\hline Indiana - Rush & -0.0417 & -0.0435 \\
\hline Indiana - St. Joseph & 1.0545 & 1.0516 \\
\hline Indiana - Scott & 1.0559 & 1.0530 \\
\hline Indiana - Shelby & 1.0186 & 1.0182 \\
\hline Indiana - Spencer & -0.0062 & -0.0064 \\
\hline Indiana -Starke & -0.0165 & -0.0168 \\
\hline Indiana - Steuben & 1.0527 & 1.0501 \\
\hline Indiana - Sullivan & 1.0522 & 1.0495 \\
\hline Indiana - Switzerland & 1.2387 & 1.1927 \\
\hline Indiana - Tippecanoe & 1.1927 & 1.1616 \\
\hline Indiana - Tipton & -0.0049 & -0.0049 \\
\hline Indiana - Union & 1.0363 & 1.0346 \\
\hline Indiana - Vanderburgh & 1.0817 & 1.0755 \\
\hline Indiana - Vermillion & -0.0457 & -0.0481 \\
\hline Indiana - Vigo & 1.0351 & 1.0339 \\
\hline Indiana - Wabash & -0.0294 & -0.0304 \\
\hline Indiana - Warren & 1.0560 & 1.0531 \\
\hline Indiana - Warrick & 1.1217 & 1.1085 \\
\hline Indiana - Washington & 1.0879 & 1.0808 \\
\hline
\end{tabular}


Table 8-1, continued

\begin{tabular}{|c|c|c|}
\hline Indiana - Wayne & -0.0601 & -0.0640 \\
\hline Indiana - Wells & 1.1172 & 1.1049 \\
\hline Indiana - White & 1.0214 & 1.0208 \\
\hline Indiana - Whitley & 1.1150 & 1.1031 \\
\hline Iowa & 1.0342 & -0.0235 \\
\hline Kansas & 1.0611 & 1.0306 \\
\hline Kentucky - Louisville & 1.0766 & 1.0468 \\
\hline Kentucky - Lexington & 1.0766 & 1.0468 \\
\hline Louisiana & 1.0458 & 1.0276 \\
\hline Maine & 1.0894 & 1.0160 \\
\hline Maryland & 1.0722 & 1.1311 \\
\hline Massachusetts & 1.0645 & 1.0375 \\
\hline Michigan - Detroit & 1.0665 & 1.0090 \\
\hline Michigan - Grand Rapids & 1.0665 & 1.0090 \\
\hline Minnesota & 1.1522 & 1.1125 \\
\hline Mississippi & 1.0597 & 1.0259 \\
\hline Missouri & 1.0848 & 1.0594 \\
\hline Montana & 1.1078 & 1.0454 \\
\hline Nebraska & 1.0451 & 1.0177 \\
\hline Nevada & 1.5304 & 1.4002 \\
\hline New Hampshire & 1.1787 & 1.1303 \\
\hline New Jersey & 1.1000 & 1.0591 \\
\hline New Mexico & 1.1223 & 1.0285 \\
\hline New York & 1.0300 & -0.0035 \\
\hline North Carolina & 1.2437 & 1.2215 \\
\hline North Dakota & -0.0110 & -0.0450 \\
\hline
\end{tabular}


Table 8-1, continued

\begin{tabular}{|l|r|r|}
\hline Ohio - Cleveland & 1.0249 & -0.0073 \\
\hline Ohio - Columbus & 1.0249 & -0.0073 \\
\hline Ohio - Cincinnati & 1.0249 & -0.0073 \\
\hline Oklahoma & 1.0612 & 1.0687 \\
\hline Oregon & 1.1729 & 1.2046 \\
\hline Pennsylvania & 1.0350 & 1.0045 \\
\hline Rhode Island & 1.0870 & 1.0118 \\
\hline South Carolina & 1.1571 & 1.1091 \\
\hline South Dakota & 1.0558 & 1.0044 \\
\hline Tennessee & 1.1429 & 1.1351 \\
\hline Texas & 1.2750 & 1.2532 \\
\hline Utah & 1.2462 & 1.2524 \\
\hline Vermont & 1.1057 & 1.0575 \\
\hline Virginia & 1.1961 & 1.1604 \\
\hline Washington & 1.1792 & 1.2409 \\
\hline West Virginia & 1.0080 & -0.0564 \\
\hline Wisconsin & 1.0968 & 1.0456 \\
\hline Wyoming & 1.0693 & -0.0095 \\
\hline
\end{tabular}

Source: Indiana Department of Transportation 
Table 8.2 Labor Productivity Growth Factors for Indiana, 2002-2030

\begin{tabular}{|c|c|c|c|}
\hline $\begin{array}{l}\text { SCTG } \\
\text { Code }\end{array}$ & Commodity Group & $\begin{array}{r}\text { Growth } \\
2000-2015 \\
\end{array}$ & $\begin{array}{r}\text { Growth } \\
2000-2030 \\
\end{array}$ \\
\hline 01 & Live Animals and Fish & 1.27 & 1.54 \\
\hline 02 & Cereal Grains & 1.27 & 1.54 \\
\hline 03 & $\begin{array}{l}\text { Agricultural Products Except Live Animals, Cereal Grains, } \\
\text { and Forage products }\end{array}$ & 1.27 & 1.54 \\
\hline 04 & Animal Feed and Products of Animal Origin & 1.27 & 1.54 \\
\hline 05 & Meat, Fish, Seafood, and Preparations & 1.31 & 1.62 \\
\hline 06 & Milled Grain Products and Preparations, and Bakery Products & 1.31 & 1.62 \\
\hline 07 & Prepared Foodstuffs, Fats, and Oils & 1.31 & 1.62 \\
\hline 08 & Alcoholic Beverages & 1.48 & 1.96 \\
\hline 09 & Tobacco Products & 1.48 & 1.96 \\
\hline 10 & Monumental or Building Stone & 1.02 & 1.03 \\
\hline 11 & Natural Sands & 1.02 & 1.03 \\
\hline 12 & Gravel and Crushed Stone & 1.02 & 1.03 \\
\hline 13 & Non-metallic Minerals & 1.34 & 1.68 \\
\hline 14 & Metallic Ores & 1.46 & 1.91 \\
\hline 15 & Coal & 1.11 & 1.22 \\
\hline 17 & Gasoline and Aviation Turbine Fuel & 1.60 & 2.19 \\
\hline 18 & Fuel Oils & 1.60 & 2.19 \\
\hline 19 & Products of Petroleum Refining and Coal Products & 1.60 & 2.19 \\
\hline 20 & Basic Chemicals & 1.35 & 1.71 \\
\hline 21 & Pharmaceutical Products & 1.35 & 1.71 \\
\hline 22 & Fertilizers and Fertilizer Materials & 1.35 & 1.71 \\
\hline 23 & Chemical Products and Preparations & 1.35 & 1.71 \\
\hline 24 & Plastics and Rubber & 1.64 & 2.29 \\
\hline 25 & Logs and Other Wood in the Rough & 1.30 & 1.60 \\
\hline 26 & Wood Products & 1.30 & 1.60 \\
\hline 27 & Pulp, Newspaper, Print, and Paperboard & 1.34 & 1.68 \\
\hline 28 & Paper or Paperboard Articles & 1.34 & 1.68 \\
\hline 29 & Printed Products & 1.10 & 1.20 \\
\hline $30^{\circ}$ & Textiles, Leather, and Articles & 1.55 & 2.10 \\
\hline 31 & Non-metallic Mineral Products & 1.34 & 1.68 \\
\hline 32 & $\begin{array}{l}\text { Base Metal in Primary or Semi-finished Forms and in Basic } \\
\text { Shapes }\end{array}$ & 1.63 & 2.27 \\
\hline 33 & Articles of Base Metal & 1.63 & 2.27 \\
\hline 34 & Machinery & 1.82 & 2.64 \\
\hline 35 & $\begin{array}{l}\text { Electronic and Other Electrical Equipment and Components; } \\
\text { Office Equipment }\end{array}$ & 7.95 & 14.95 \\
\hline 36 & Motorized Vehicles & 2.13 & 3.26 \\
\hline 37 & Transportation Equipment & 1.58 & 2.16 \\
\hline 38 & Precision Instruments and Apparatus & 1.43 & 1.97 \\
\hline 39 & $\begin{array}{l}\text { Furniture, Mattresses and Mattress Supports, Lamps, } \\
\text { Lighting Fittings, and Illuminated Signs }\end{array}$ & 1.58 & 2.17 \\
\hline 40 & Miscellaneous Manufactured Products & 1.43 & 1.97 \\
\hline 41 & Waste and Scrap & 1.43 & 1.97 \\
\hline 43 & Mixed Freight & 1.43 & 1.97 \\
\hline
\end{tabular}




\section{Future Attractions}

The same employment increase factors and productivity increases were assumed to influence the consumption side of the analysis. That is it was generally assumed that the increases in shipments produced would be met with an increase in the shipments received. There is nothing seriously wrong with such an assumption except that some of the increased production may very well be exported. We have no way of knowing this with any certainly and therefore we are assuming that the total increase in production is consumed by the domestic market.

Values for productions and attractions for the forecast years of 2015 and 2030 appear in appendices $\mathrm{E}$ and $\mathrm{F}$ of this report.

\section{The Future Flows}

Using the models developed for productions and attractions we next inserted estimates of the employment variables assuming the growth in employment and productivity noted earlier for 2015 and 2030. This was followed by the use of the calibrated fully-constrained gravity model assuming the parameters from the earlier study held. This gave us the distributed total traffic for the two forecast years. The historical patterns of modal choice were again used and the traffic was divided among modes once again for each year.

Maps of the forecasted truck traffic for Indiana in 2015 and 2030 appear as Figures 8-1 and 8-2. Similar maps appear for forecasted rail traffic for Indian in 2015 and 2030 appear as Figures 8-3 and 8-4.

Examining the maps should reveal that the major corridors in 2015 and 2030 in both the truck and the rail case are very similar. This is to be expected since we have done nothing to change the major corridors in a relative sense. Speeds and travel times are assumed to be the same in the truck case and density per unit length is also assumed to be the same in the rail case. In reality the flows are changing more in the highway case than in the rail case as can be seen by comparing the legends on the map. Fine detail can be achieved by using some of the methods discussed in Chapter 9 on Implementation.

We have some confidence in the highway forecasts since the model used has been evaluated on the 1997 data and found to be very good. We have not performed a similar evaluation on any of the rail modeling since we have no actual data set to use for such a test. Therefore, we offer the rail forecasts with this caveat. 


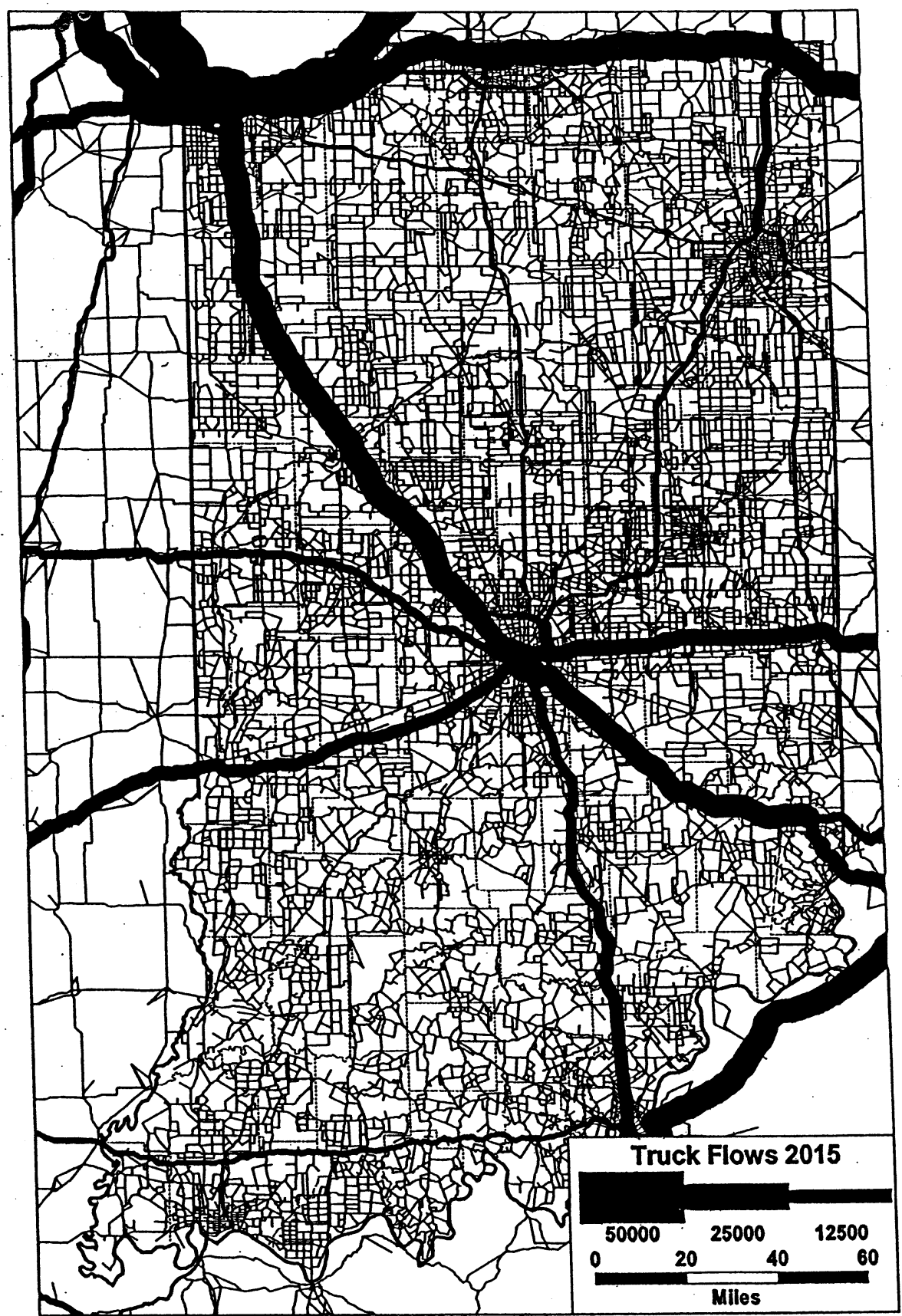

Figure 8-1. Indiana Daily Truckload Freight Volumes for 2015 


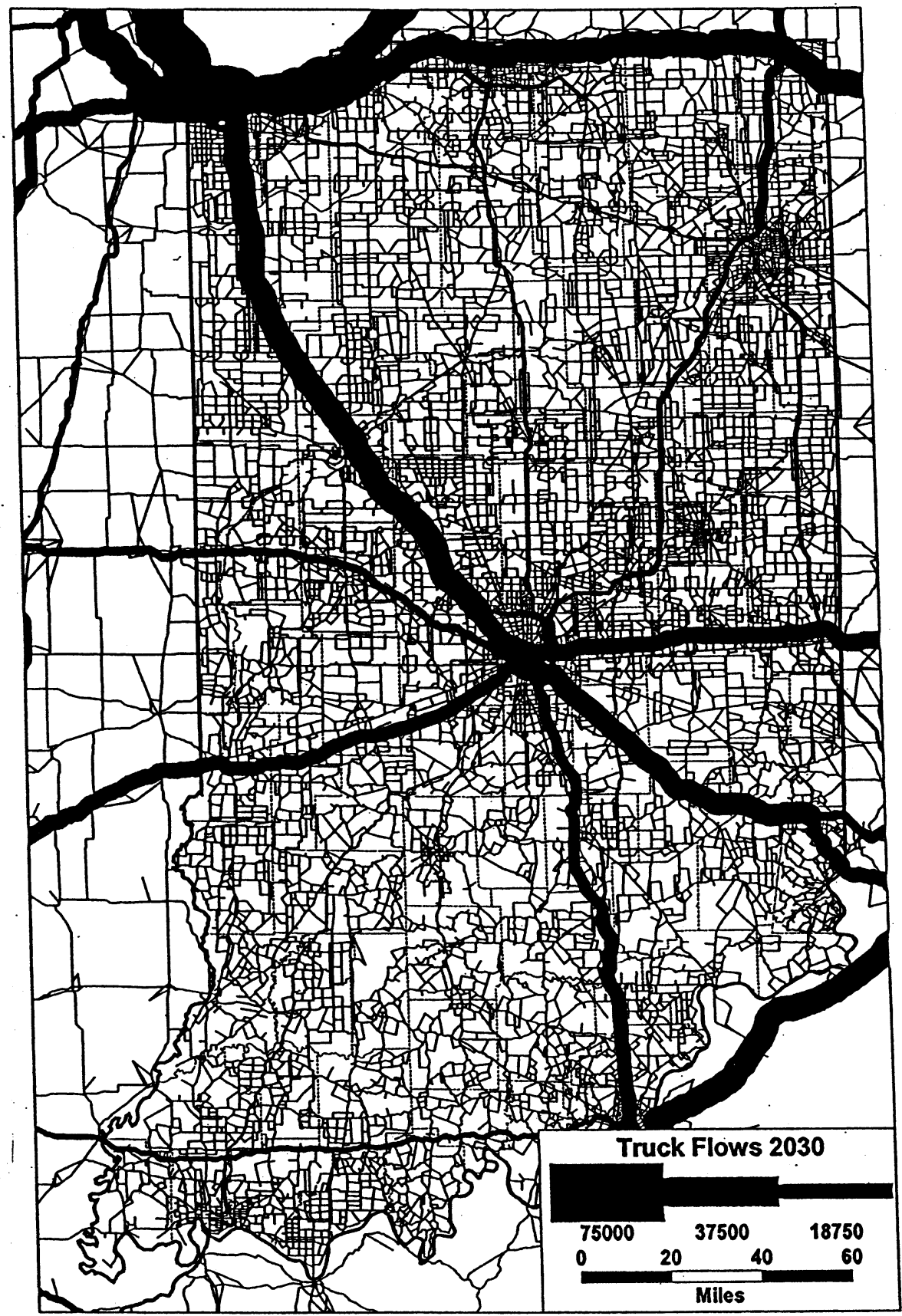

Figure 8-2. Indiana Daily Truckload Freight Volumes for 2030 


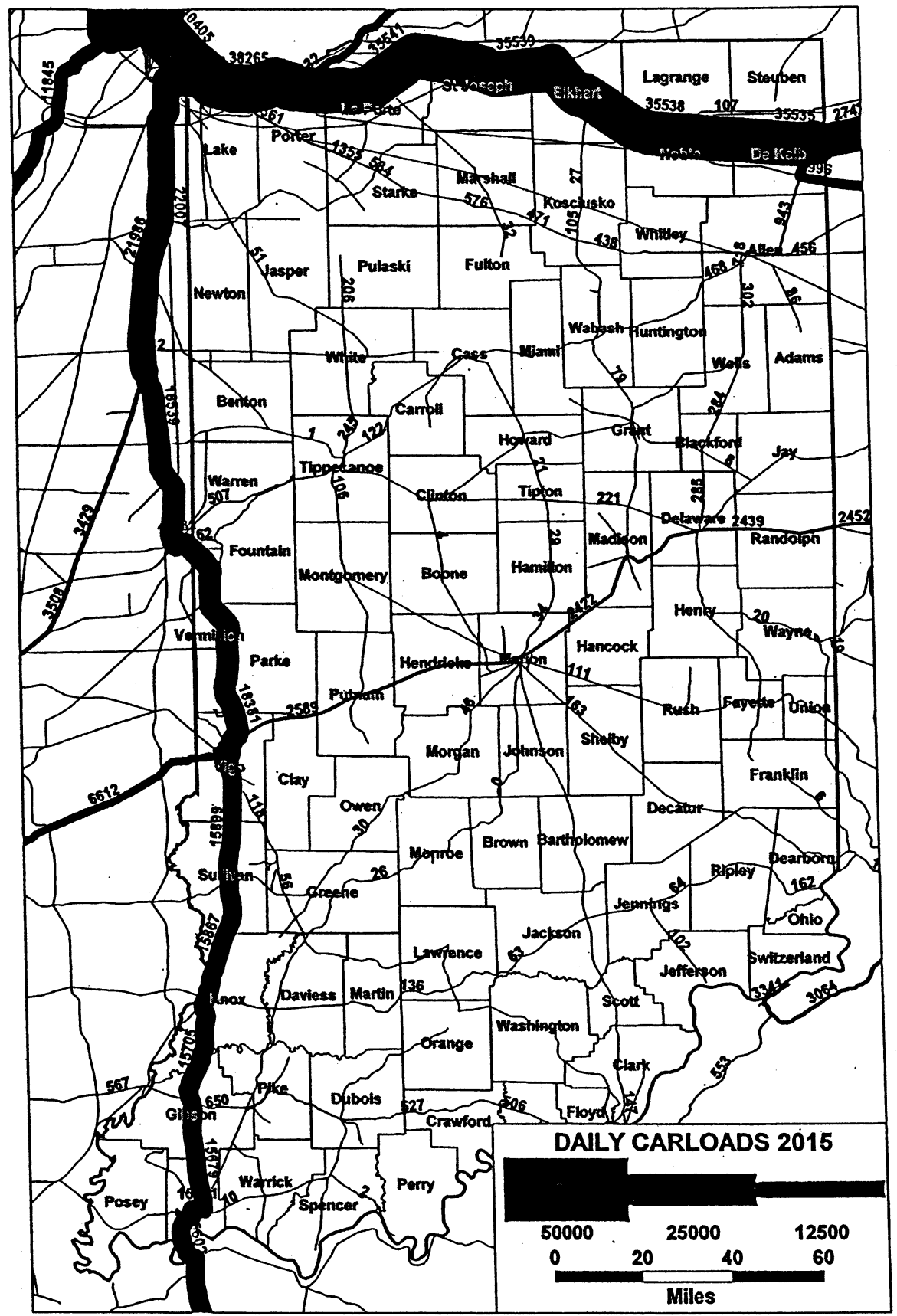

Figure 8-3. Indiana Daily Railcar Freight Volumes for 2015 


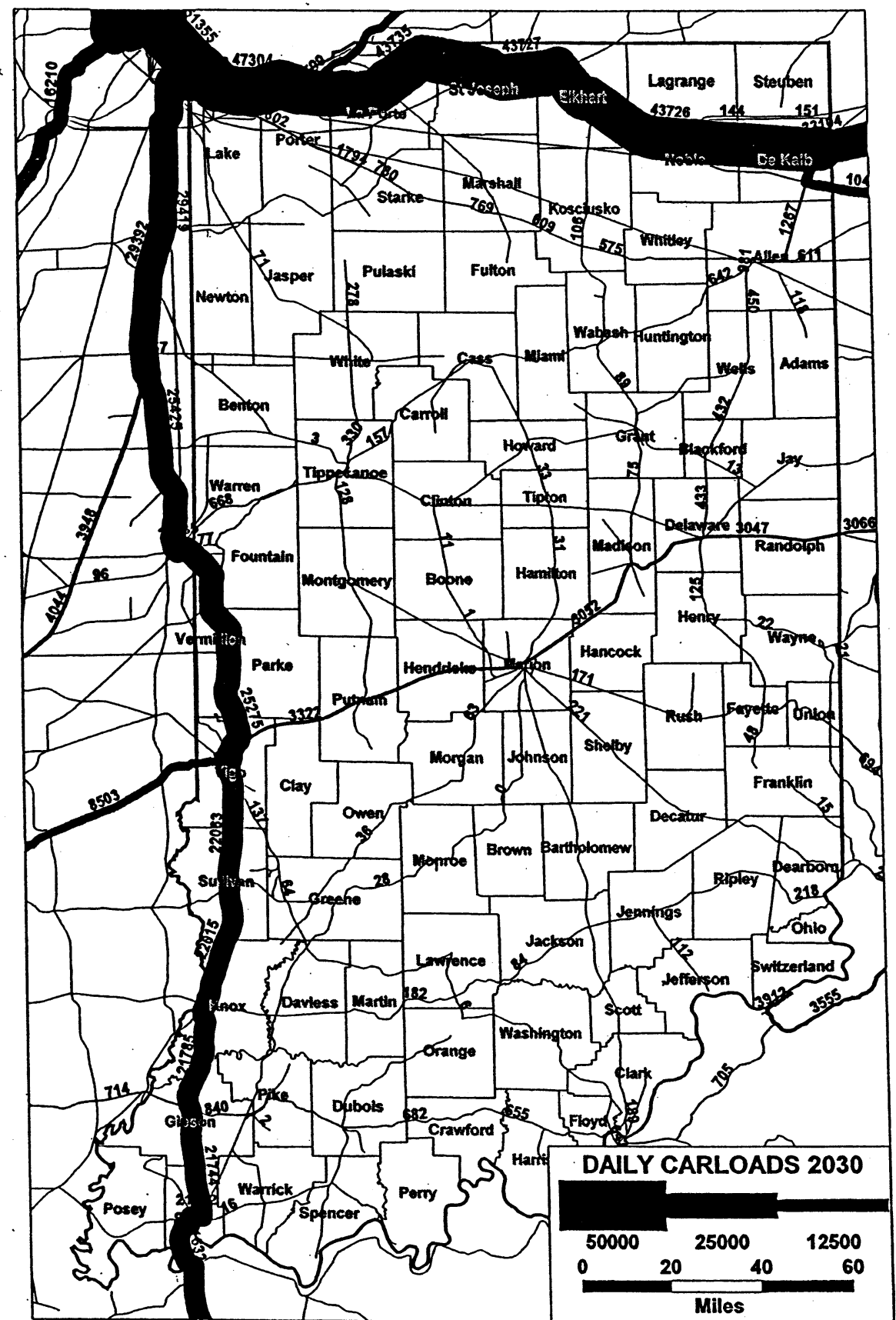

Figure 8-4. Indiana Daily Railcar Freight Volumes for 2030 


\section{Possible Improvements}

One possible area where improvement is possible in the forecasting area is in the use of more refined estimates of employment growth. As noted above the values used here were county-level specific, but they were not industry-level specific. The assumption is that all of the industries in the county will grow at exactly the same rate. This is perhaps unrealistic. The state may wish to refine these in the future, perhaps using one of the economic models that they already use.

The values used here were supplied by the state and the research team was requested to use these for estimates of employment growth. 


\section{Chapter 9}

\section{IMPLEMENTATION}

This study was undertaken as a planning and analysis project. It should be apparent to the reader that the study also has many of the attributes of a research project. Many of the problems encountered as well as the models used are often found in research papers. We have also evaluated the different models as we went along. The end result is a study that should have value for economic analysis, transport analysis, transport policy formation, and for planning studies conducted by the state or subareas of the state such as metropolitan areas.

In this chapter we will briefly suggest some possible applications for the findings of this study in the areas noted. We will also identify the various deliverables from this project. Beyond this report these consist of computer files that will enable the state to replicate and utilize the models developed here.

\section{Use of the Results for Economic Analysis}

The appendices of this report include several project generated databases that should be of use to organizations interested in the flow of goods into, through, and out of counties and urban areas of the state. MPOs (metropolitan planning organizations) have shown considerable interest in the results of this study for such purposes. This would enable these areas to identify which of the myriad of highway projects facing it may have the highest value in terms of local industrial production activities. If an area has an interest in examining its economic base this study will give such areas some information on those economic activities that may wield the greatest impact in terms of the value of commodity flows. This can be done by taking the values from goods for Indiana in 1997 or 2002 (see tables 3-1 and 3-2), and multiplying the tonnage by these values. In this case it should be noted that the values in several of the tables are in thousands of tons.

Using the same procedure a county or metropolitan area can assess to what extent it is an importer or exporter of individual commodity groups. One should bear in mind that the categories or commodity groups used here are indeed groups. That is, these are not individual goods. It may be that a county is importing exactly the same amount of a commodity group as it is exporting of that group. All that this means is that one good of the group may be imported, while another good in the same group may be exported at the same time. Because of these 
possible idiosyncrasies it might be best to conduct all such analyses in terms of dollars, rather than tons.

\section{Use of Results for Transport Analysis}

The type of transport analysis that can be undertaken using the results of this study are probably primarily at the regional corridor level. In that case we might be talking about a major highway link between Indianapolis and Evansville and the concern might be to what extent such a highway link would facilitate the movement of commodities to or from the state. It would be possible to use the results of this study to estimate the net transport cost savings for the state's manufacturers if such a highway were built. Such an analysis needs some reasonably good estimates of the volume of goods currently moving and this study could provide the same.

Use of the study for such a problem would necessitate the use of a transport network similar to the one that was utilized in this study.

We are also reminded of the fact that the oft cited 1997 freight study was used for a couple of major studies after its completion. One of these involved a statewide analysis of intermodal facilities (Booz-Allen \& Hamilton, et al., 1997) and another looked at the Indianapolis region in terms of freight flows (Cambridge Systematics, Inc., et al.,1998).

For county level studies it might be desirable to try and disaggregate the county level estimates provided here to a lower level of geographic detail. This has been done by the state in the past using data from Dun \& Bradstreet to estimate the locations of production and attraction of flows.

\section{Use of the Results for Policy Analysis}

The state of Indiana could also use the results of this project to evaluate different policy decisions of the state. For example, the state recently decided to increase the speed limit on some of its highways to $70 \mathrm{mph}$. This was done without any analysis in terms of evaluating what the impact of such a change might do for the state's commodity flows. While there is no reason to believe this decision would result in alternate route selection, such an evaluation could be undertaken here. The reason why little change would be expected is that the leading routes in terms of previous speed limits are for the most part the same ones that received the increase. There is nevertheless the possibility that a $70 \mathrm{mph}$ speed limit corridor could combine with a 50 $\mathrm{mph}$ speed limit corridor and completely pull traffic off of an urban 55 mph corridor. By simply changing the speed limits of the network database, such policy decisions could be examined $a$ priori the change.

\section{Databases and Deliverables}

Aside from this report and the various flow estimates and forecasts, this project will also 
supply the sponsor with production and attraction vectors developed for the 41 commodity groups examined here. These are all being supplied electronically as well as in published form in the appendices of this report. The values for 1997 appear as appendix A. The forecasts over these same set of commodities for 2015 appear as appendix E and the forecasts for 2030 appear as appendix F.

The last deliverable supplied includes the computer programs used here. One of these was used in the 1997 study, but the others are new. They appear here as Fortran 77 code, but they have also been run on a Fortan 95 compiler. They have not been supplied as executable files. They can easily be made computer-ready by running them through any Fortan compiler, e.g., Microsoft, Lahey, Salford, and so forth. The programs used appear here as appendix B.

There are distance decay curves created from data in the 1997 CFS reports that appear in appendix $\mathrm{C}$, and illustrate the manner in which traffic falls with increasing distance from an origin.

A second set of databases are the proportions of each of the commodity groups used in the modal assignments. These are being supplied in electronic form as well as in appendix D of this document.

The project will also provide the sponsor with master data files generated by the project. These will include files for all highway, rail, water, pipeline and air freight traffic. Interaction matrices for 1997, 2015, and 2030 will be provided as well. In order for the state analysts to duplicate the findings here and to use the models derived for evaluating projects and alternatives, we will also supply the state with networks used for highways and rail, the density variable for the railroad traffic assignment, as well as the railroad *.net file. Similarly, the same will be provided for the highway sector, along with any files necessary to run the analyses.

\section{A Guide to Using the Data Files}

There are numerous uses for the data supplied here and on the CD ROM that will be supplied to the sponsor. As indicated elsewhere we see the users of these as the Indiana DOT and the MPOs of the state of Indiana. We can envision MPOs being interested in the tonnages of goods produced by and attracted to counties within their jurisdiction. This information can easily be obtained by using the appropriate tables of appendix A, E, and F, for 1997, 2015 and 2030, respectively. Appropriate conversions of the data to trucks can be obtained by using the density figures of Table 5-4 in the text of this report. Dividing the tonnages by the truck density will yield the trucks moving these goods in the local area. Conversion of the tonnages to 1997 dollars requires the use of a measure of value for the SCTG groups. These values appear on appendix page B40. The data on that page consists of three columns. The first is the two-digit SCTG code; the second in the motor carrier density, e.g., 9.77, 96.63, and so forth; and the third is the value per ton in 1997 dollars, e.g., \$1,042.38. 
The other uses of the data supplied here are for evaluating the use of different routes for the movement of goods. This is not nearly so easily done unless the user is skilled in the use of TransCAD, the GIS system used here (Caliper, 2000). We will discuss how this can be done if the user is somewhat familiar with the GIS software.

There are two broad problem areas that the user might want to work on with the data here. The first of these is concerned with the volume of traffic on the local roads of an MPO or other areal unit. In effect the maps prepared here may all be enlarged to reveal such information while the map is resident on the computer, but you lose this ability when the maps appear in print as they do here. So the first thing the user may want to do is create an assignment for the flows distributed. We will call this simply the Assignment Problem below. The second problem seeks to evaluate what the volume of trucks would be on different roads and streets if traffic were prevented from taking some existing link segments in the area under analysis. We will refer to this as the Alternate Routing problem.

\section{The Assignment Problem}

We begin by starting TransCAD, and going to File, and then Open. We next find the flow data of interest that we want to assign to the network. In our case the data is on a CD in the D drive entitled February 14, 2006, and it is entitled TRUCK_97.ASC. In order for TransCAD

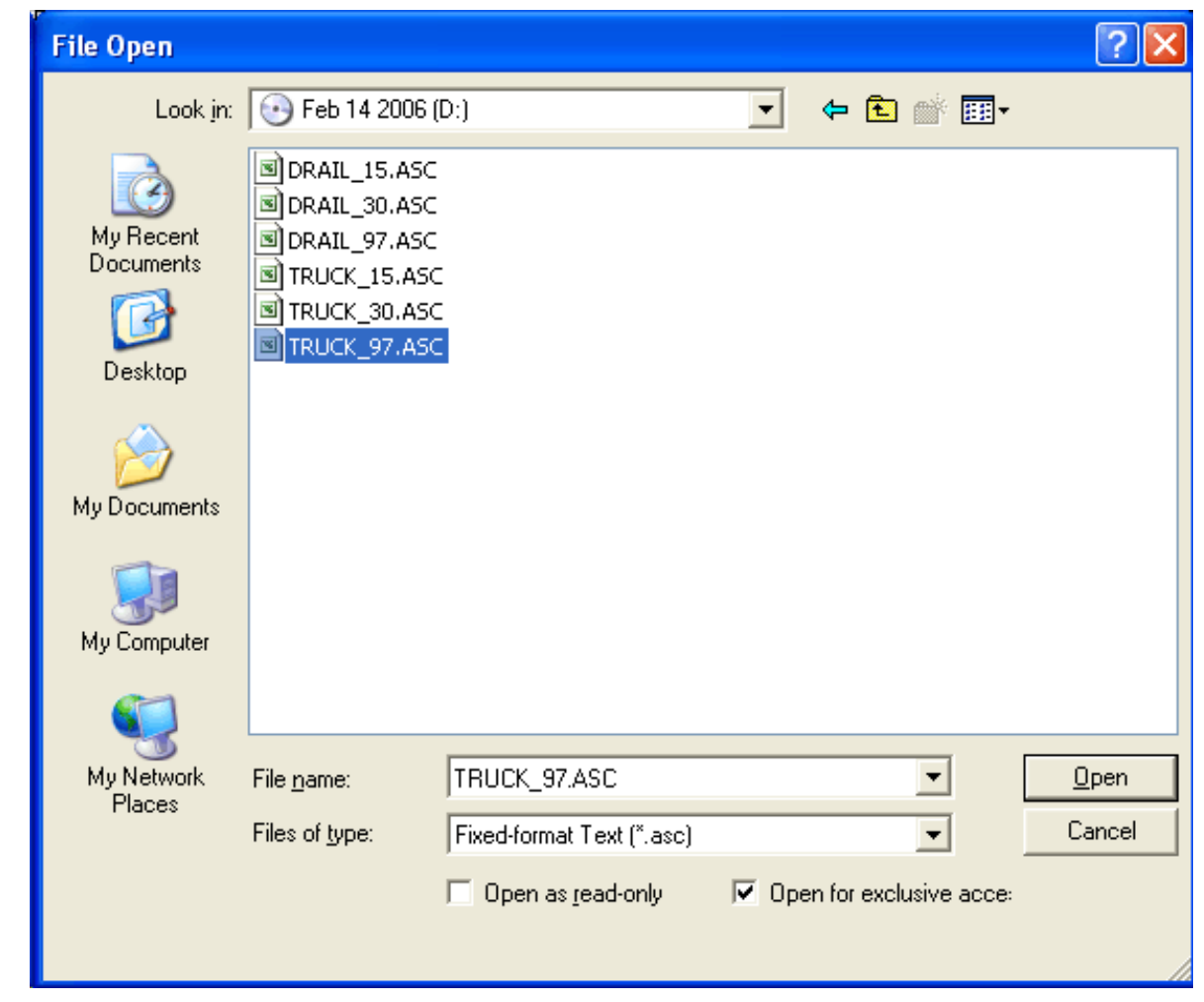


to recognize the data there must also be a dictionary file in the same directory. These files have the extension of .DCT. This file in the case of our flow data has the following form:

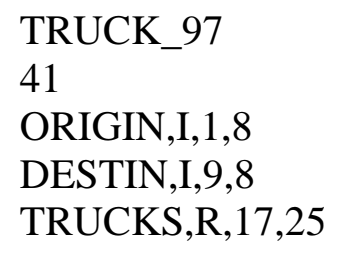

The name of the DCT file must have the same name as the beginning of the flow data file, so the above five line file is named TRUCK_97.DCT. These files are being supplied with the flow data, but the user may have occasion to create a different flow file (e.g., by converting the trucks to dollars). In such a case the first line is a name for identification purposes, the second line gives the total length of data, line three the name of the rows, the type of data (I for integer here), the column where the data begins, and its length. The next line is the same for the columns. The final line identifies what is being assigned, the type of data ( $\mathrm{R}$ for real or decimal data here), the column where the variable begins, and the length of the variable. Consult the TransCAD manual for further information on this if necessary.

We next want to create an origin-destination matrix from this data. We go to Matrix on the horizontal TransCAD toolbar at the top of the page. Go down the matrix toolbar to IMPORT and press this. This results in the following screen:

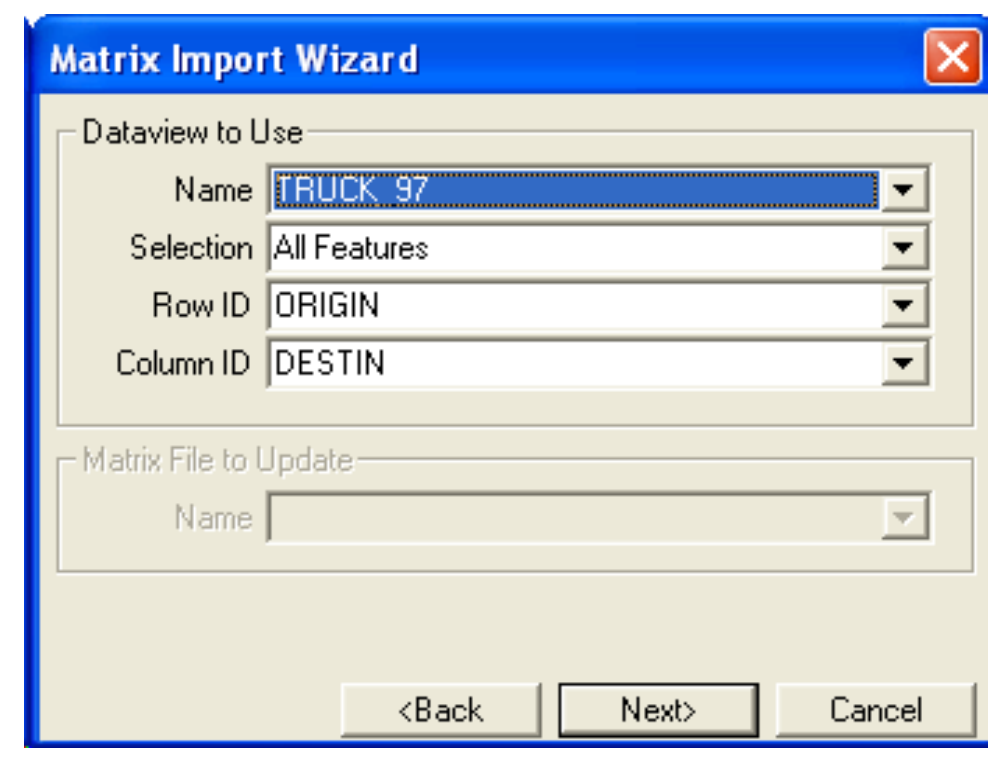

Clicking on Next yields: 


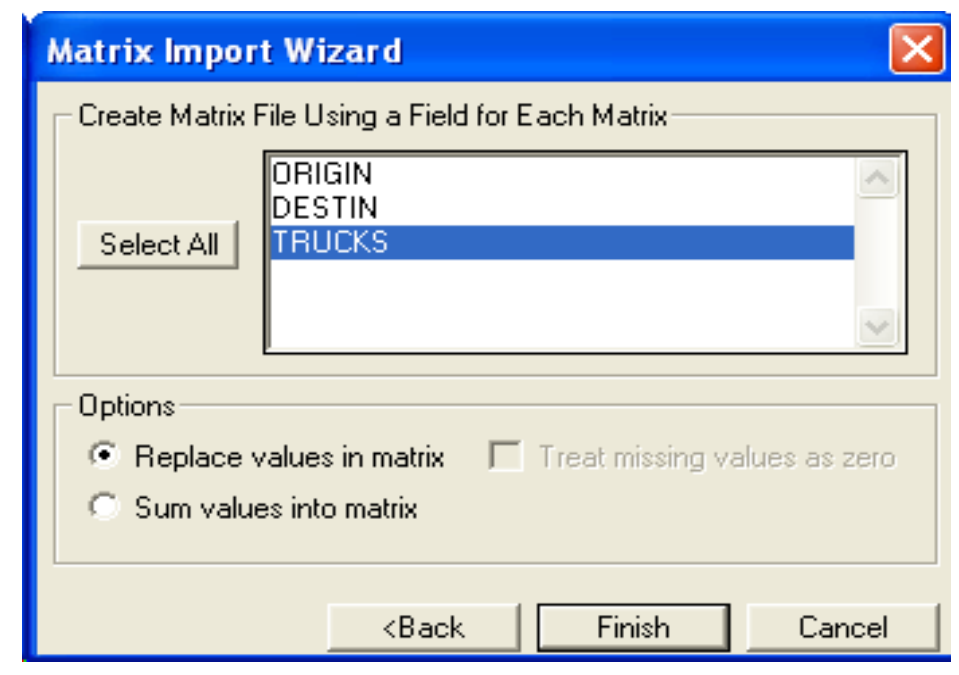

and clicking on Finish yields a screen requesting that you type in the name of the matrix file you are creating. Here we have called it TRUCK_97.MTX. Click on Save and your matrix will appear. Minimize the matrix.

Now that we have the flow matrix, we need to assign these flows to a network. If you have not yet created a network you must do this first. Go to your boundary file directory. This is labeled

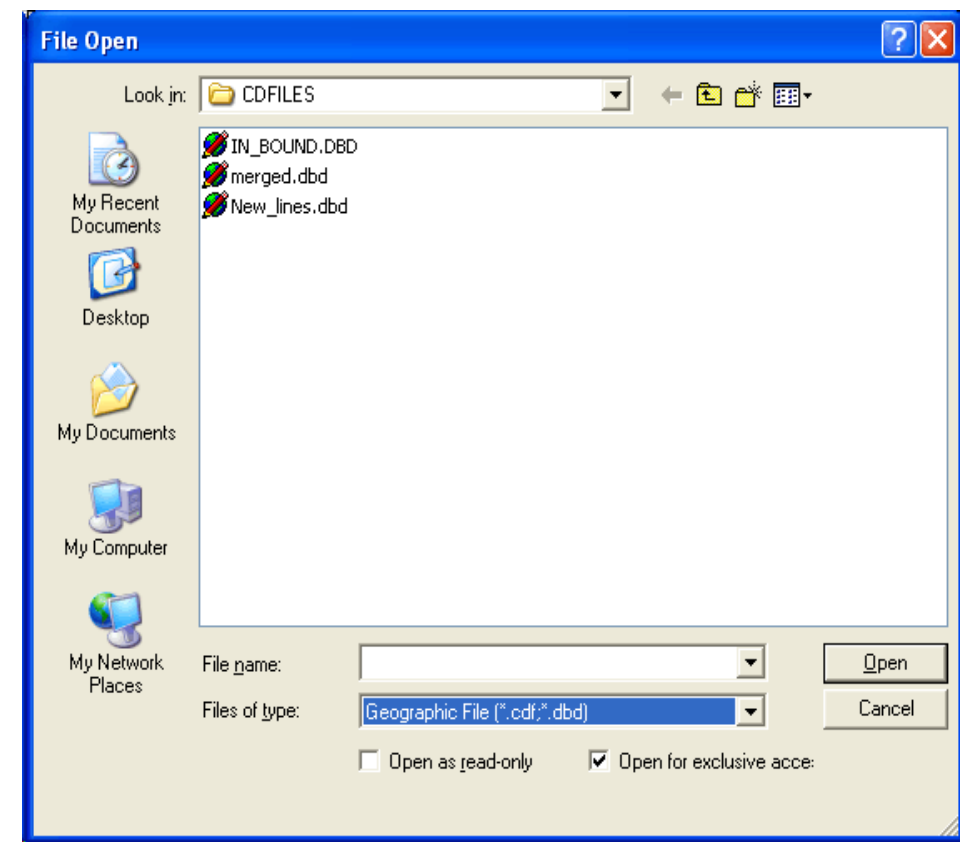


CDFILES here. Make sure the Geographic Files appears and load the IN_BOUND.DBD. This will create a map of Indiana on the screen with counties and the state outlined. Next go to the toolbar at the top of the TransCAD screen. Click on the layers icon. Click on Add Layer and add the merged.DBD. This will yield the display below and create a highway network for the state. Clicking on Close will produce the network on the screen.

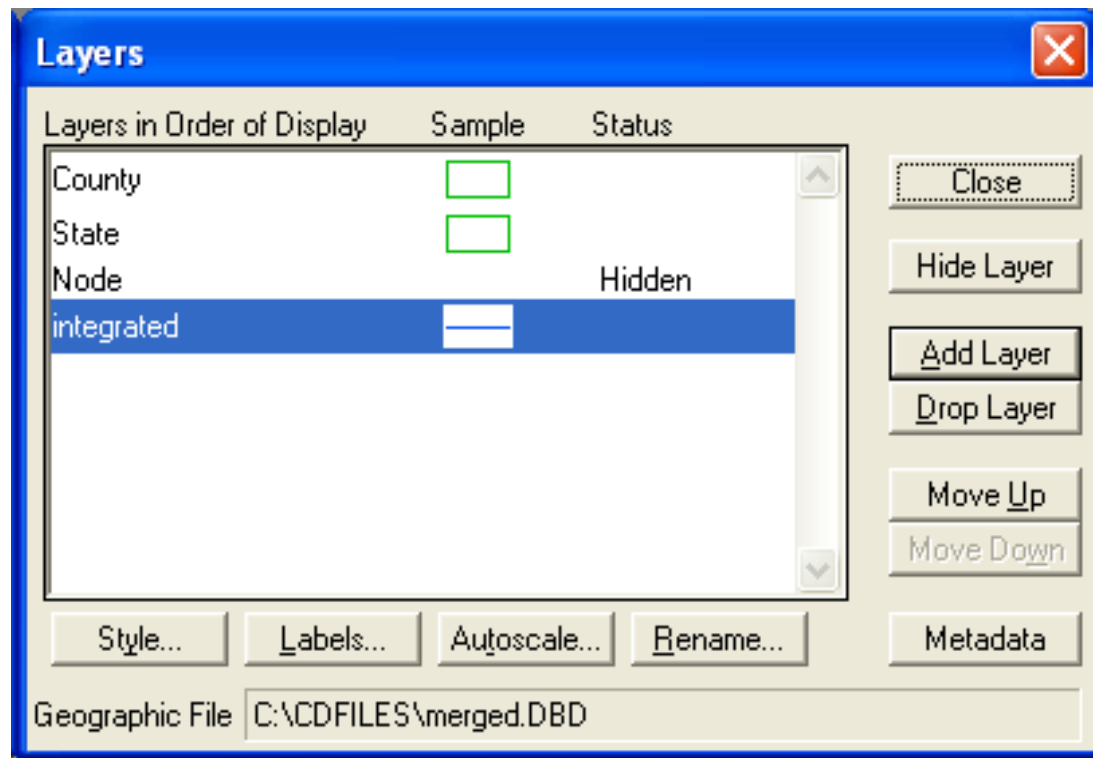

To create a network for assigning flows you next go to the horizontal toolbar at the top of the TransCAD screen. Make sure the active layer is the network (called integrated here). Now go to the Network/Paths icon and click it. From the toolbar produced click Create. The screen below appears.

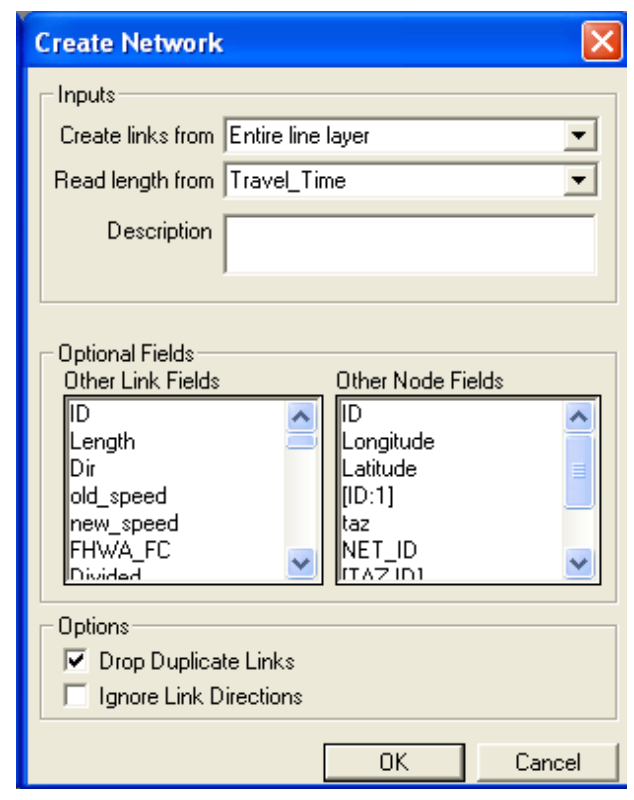


The travel time we will use is listed as Travel_Time. Select it so that it is displayed in the window. Click on OK. You will be asked for a name for the network file. We have called ours merged.net.

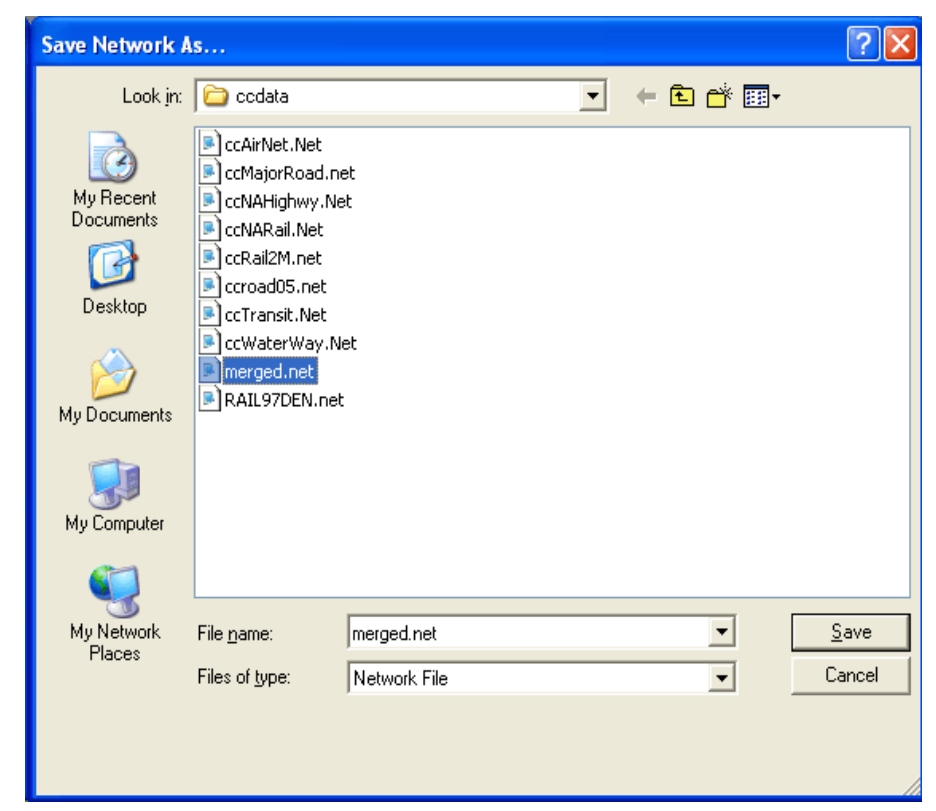

Make sure your flow matrix is active and click on Planning on the horizontal toolbar. Then click on Traffic Assignment. Since we are dealing only with trucks we use All or Nothing as noted in this report. Insert Travel_Time if it does not appear. It is found below.

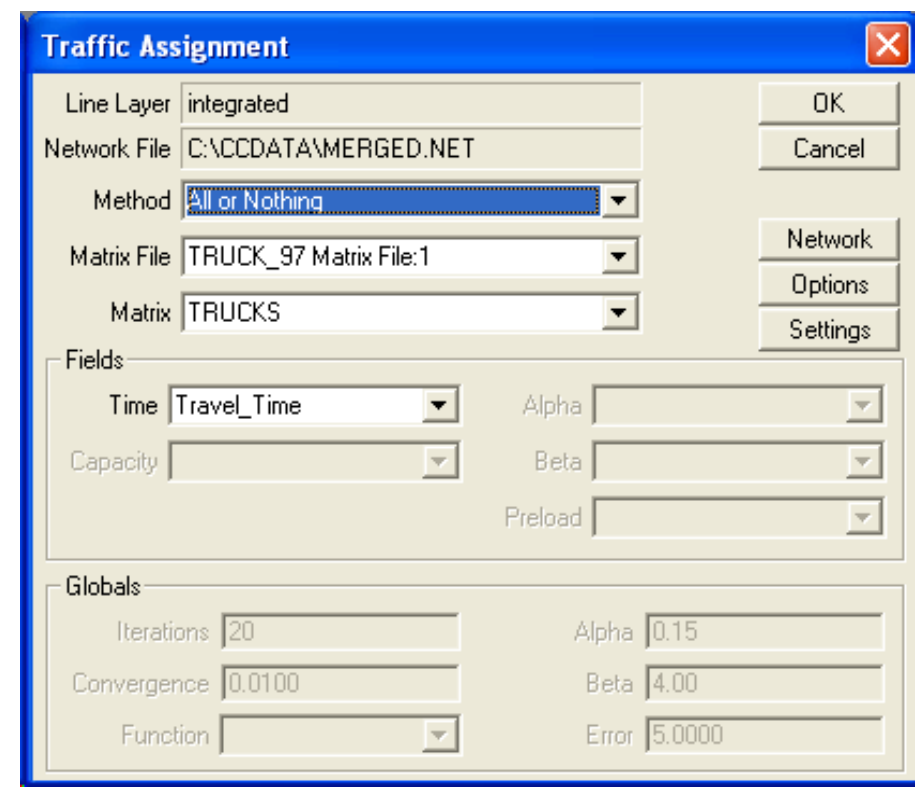


Make sure the TRUCK_97 and merged.net are active. If they do not appear in the above window, try again. As noted above we have selected the "All or Nothing" assignment routine. Other assignment algorithms require a capacity value which is not in the database. Click OK.

We are next asked for a name for the assignment flows. We have used TRUCK97_LinkFlow.bin to store the assigned flows. Click Save.

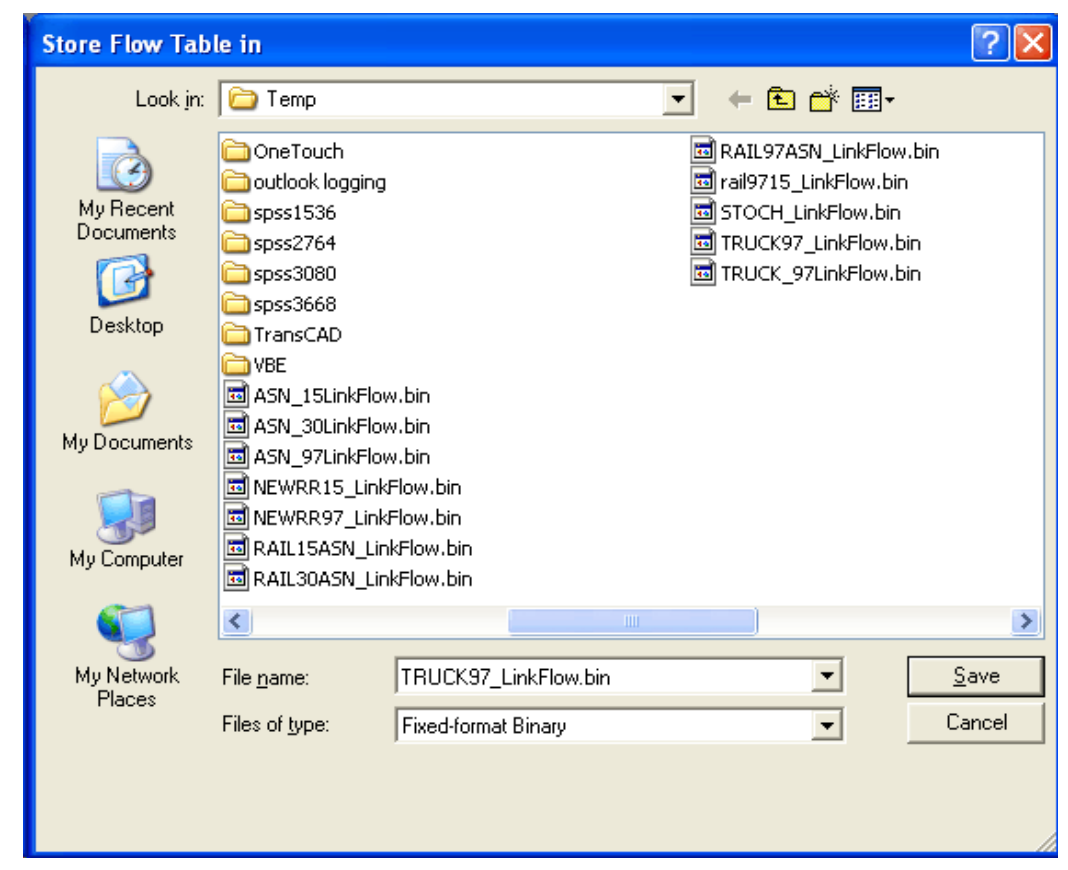

The assignment is executed and a small window appears telling you whether this has been successful.

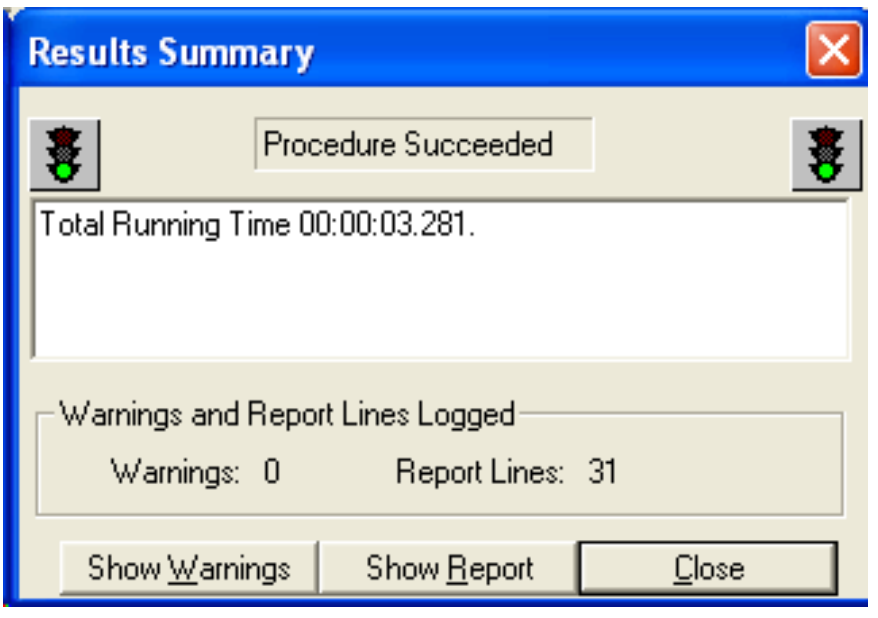


A new window appears behind the above and this is a dataview that combined the integrated database and the assignments.

If we go back to our Indiana map and click on the TOT_FLOW and the proportional scaling icon (the one with the stars) we will then get a map of the Indiana highway system that displays the volume of traffic on all links Such as the one below:

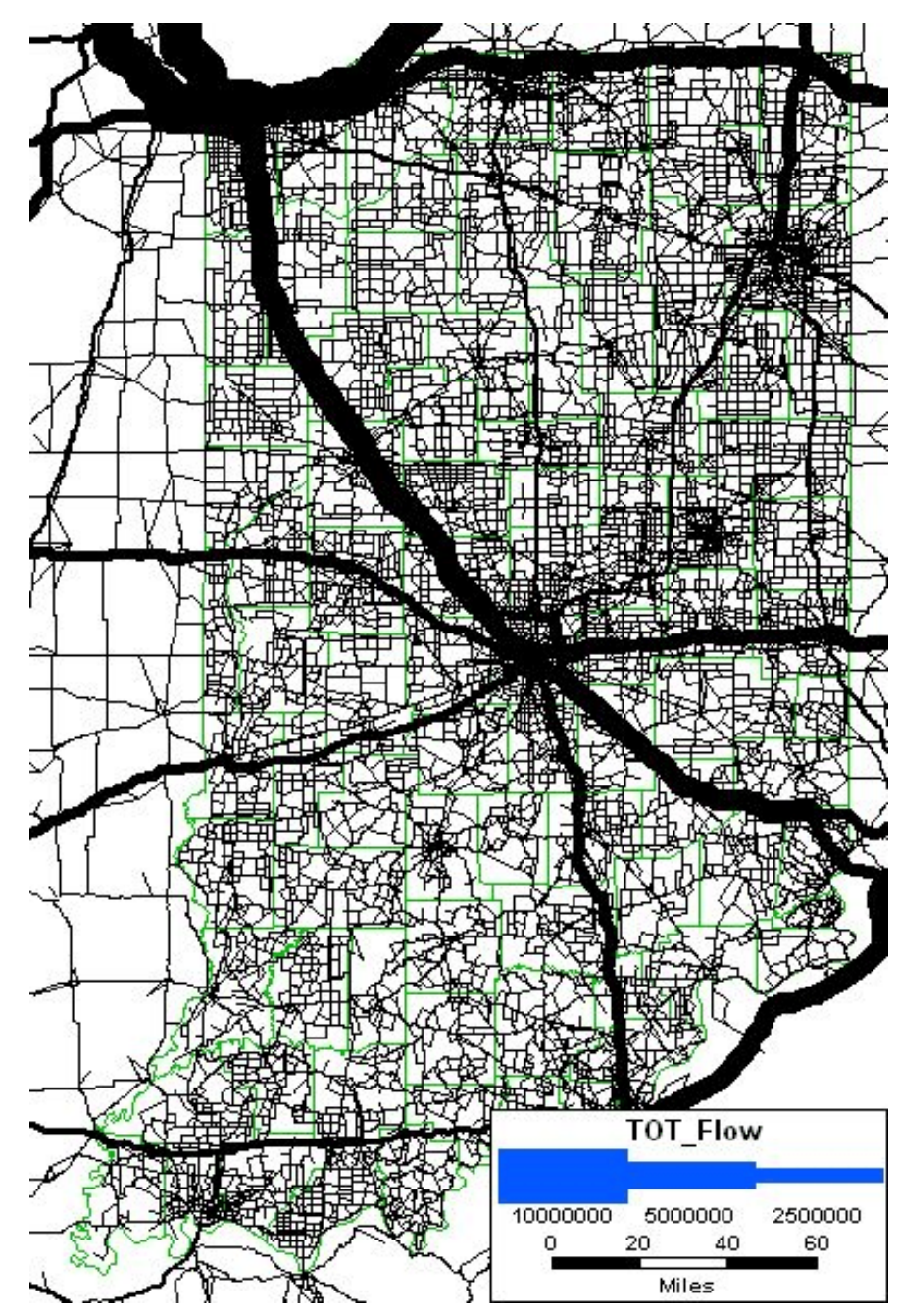

While in TransCAD you can focus on any part of this network and identify flow volumes for metropolitan area highways, counties, and so forth. 


\section{The Alternate Routing Problem}

When a road or highway is closed due to an accident, you may want to assess the most likely way that traffic can be diverted to alternate routes; or you may want to evaluate the impacts of building new roads, e.g., a bypass, on truck traffic on local roads; or you may want to evaluate alternate routings if you don't want trucks going through the center of town. All of these are alternate routing problems. Depending on the complexity of the problem at hand, we can evaluate it by dropping one of more links from the network.

At the outset the primary thing you want to do is back up the network file before you begin anything else. You may be tempted to simply go into the network layer and delete links of the system. That is one approach, but I would rather not mess up the network in that way. My preference is to use the side toolbar on the right side of the TransCAD window.

In this approach you enlarge the highway network map and identify the links that you do not want used. Click on the large $\mathbf{i}$. Note the link number and make sure this is the link you do not want used in the routing. Go to the Travel_Time variable, and change the travel time to 1000 or any large number. When you run the assignment routine, or the shortest path routine between any two nodes, this is read as a very high cost and the link is not selected. Follow this approach for any other links of interest. You can go back and edit the travel time values for all links changed in the same manner, assuming you have kept a record of the initial values which you changed, or you can click on Edit on the horizontal bar, click on Fill, and have the travel time variable replaced with the initial values as calculated by:

$$
\text { Travel_time }=(\text { Length/new_speed }) * 60
$$

This will replace any changes with the original values.

\section{Summary}

This chapter has summarized the various ways in which this study could be used by the state and by metropolitan planning organization (MPOs). It was noted that the study could be used for transport analysis, economic analysis and for policy analysis. It is important to emphasize that because of the way in which the modal split analysis was carried out here, it would not be possible to examine some of the questions related to this area. In other words, we have used historical patterns to allocate future splits of traffic between the various modes. Such an approach is not sensitive to changes in prices, tolls and the like and this does limit its utility.

In addition this chapter has also provided a guide as to how this report and its databases could be used by planners at different scales (state level and MPO level). We have outlined the various steps that are necessary to use this approach to assign the traffic generated. We have not dwelt upon the various commodities that could be assigned, but if the state and MPOs have an 
interest in this they can certainly undertake such analyses. The appropriate files are provided here. We have also provided some direction on how the data could be examined at the county level in terms of productions and attractions. Finally we have suggested the manner in which this study could be used to examine the problem of alternative routes could be examined. This would be very useful in the event that planners wanted to examine a number of different transport questions. 


\section{References}

Booz-Allen Hamilton, Inc., Bill Black (Indiana University), Professional Data Dimensions, Caliper Corporation and GIS/Trans, Ltd. (1997), Intermodal Management System, (San Francisco: Booz-Allen Hamilton, Inc.), October.

Caliper (2000), TransCAD: Transportation GIS Software, User's Guide, Newton, MA: Caliper Corporation.

Cambridge Systematics, Inc., Dyer Environmental Services. URS Greiner, Inc., and William R. Black (1998), Indianapolis Intermodal Freight System Plan, Final Report, prepared for the Indianapolis Department of Metropolitan Development, (Cambridge, Mass.: Cambridge Systematics, Inc.), June. 


\begin{abstract}
APPENDICES
APPENDIX A - PRODUCTIONS AND ATTRACTIONS, 1997

APPENDIX B - COMPUTER PROGRAMS DEVELOPED

APPENDIX C - DISTANCE DECAY CURVES

APPENDIX D - MODE CHOICE/DISTANCE TABLES

APPENDIX E - PRODUCTIONS AND ATTRACTIONS 2015

APPENDIX F - PRODUCTIONS AND ATTRACTIONS 2030
\end{abstract}


APPENDIX A

PRODUCTIONS AND ATTRACTIONS 1997 


\section{Table Notes}

The tables in this appendix give the estimated volume of traffic produced and attracted by areas included in this study for each commodity. The area appears under the column label State and includes the 145 different areas included in the freight study. From line 14 to line 105 we have the counties of Indiana. States represented by two or more nodes are evident here as well.

The data columns labeled $\mathrm{P}$ are productions and those labeled $\mathrm{A}$ are attractions. The next two digits represent the commodity groups SCTG 1, SCTG 2, and so forth. The 97 represents the year of the data, 1997.

The data in some cases are taken directly from the Commodity Flow Survey. Where data could not be released due to sample size or disclosure considerations, the values have been estimated here using regression analysis. Controls on estimates were taken from the US totals as adjusted for Hawaii and Alaska, which are not part of the study. For the counties of Indiana these values were estimated using the regression models described in the text of the report. The controls on these estimates were the commodity totals for the state of Indiana.

The data are actually given here to three decimal places. This is because the data as published is in thousands of tons. In many cases this would completely eliminate flows into Indiana counties of several goods. It is for this reason that three decimal places were used. Programs used later would multiply flow estimates by 1,000 to yield tons of commodities. The reader should judge the reliability of the data given these facts. 


\begin{tabular}{|c|c|c|c|}
\hline & State & P01 97 & A01 97 \\
\hline 1 & Alabama & 125.000 & 44.011 \\
\hline 2 & Arizona & .000 & 37.009 \\
\hline 3 & Arkansas & 96.692 & 213.051 \\
\hline 4 & California & 536.836 & 616.149 \\
\hline 5 & Colorado & .000 & 76.018 \\
\hline 6 & Connecticut & .000 & 37.009 \\
\hline 7 & Delaware & 5.331 & 39.009 \\
\hline 8 & $D C$ & .000 & .000 \\
\hline 9 & Florida & 147.569 & 13.003 \\
\hline 10 & Georgia & 117.756 & 250.060 \\
\hline 11 & Idaho & 14.048 & 67.016 \\
\hline 12 & Illinois_N & 112.000 & 174.042 \\
\hline 13 & Illinois_S & 48.000 & 75.018 \\
\hline 14 & Adams & 8.839 & 3.204 \\
\hline 15 & Allen & 33.455 & 15.402 \\
\hline 16 & Bartholomew & 25.016 & 11.976 \\
\hline 17 & Benton & 2.001 & .000 \\
\hline 18 & Blackford & .334 & .554 \\
\hline 19 & Boone & 2.001 & .092 \\
\hline 20 & Brown & .000 & .092 \\
\hline 21 & Carroll & 12.508 & 16.159 \\
\hline 22 & Cass & .334 & 16.159 \\
\hline 23 & Clark & 37.324 & 2.659 \\
\hline 24 & Clay & .334 & .092 \\
\hline 25 & Clinton & 2.001 & 19.290 \\
\hline 26 & Crawford & .000 & .000 \\
\hline 27 & Daviess & 2.001 & 9.382 \\
\hline 28 & Dearborn & .000 & .092 \\
\hline 29 & Decatur & .334 & 3.417 \\
\hline 30 & DeKalb & .334 & .554 \\
\hline 31 & Delaware & 4.703 & 1.154 \\
\hline 32 & Dubois & 219.809 & 7.369 \\
\hline 33 & Elkhart & 128.550 & 5.993 \\
\hline 34 & Fayette & 2.001 & .000 \\
\hline 35 & Floyd & 14.776 & 6.695 \\
\hline 36 & Fountain & .000 & .554 \\
\hline 37 & Franklin & .000 & .092 \\
\hline 38 & Fulton & .000 & 1.431 \\
\hline 39 & Gibson & .000 & 3.463 \\
\hline 40 & Grant & 5.837 & 2.447 \\
\hline 41 & Greene & .334 & 3.463 \\
\hline 42 & Hamilton & 8.706 & 1.228 \\
\hline 43 & Hancock & 2.001 & .092 \\
\hline 44 & Harrison & 21.647 & 6.695 \\
\hline 45 & Hendricks & .334 & 4.257 \\
\hline 46 & Henry & 5.837 & .554 \\
\hline 47 & Howard & 12.508 & 1.468 \\
\hline 48 & Huntington & .000 & 3.463 \\
\hline 49 & Jackson & 2.001 & .332 \\
\hline 50 & Jasper & .334 & 2.078 \\
\hline 51 & Jay & 2.001 & 4.100 \\
\hline
\end{tabular}




\begin{tabular}{|c|c|c|c|}
\hline & \begin{tabular}{|l} 
State \\
\end{tabular} & P01 97 & A01 97 \\
\hline 52 & Jefferson & .334 & .185 \\
\hline 53 & Jennings & .000 & .000 \\
\hline 54 & Johnson & 2.902 & .065 \\
\hline 55 & Knox & .334 & 1.219 \\
\hline 56 & Kosciusko & 10.207 & 8.458 \\
\hline 57 & LaGrange & 10.040 & 1.644 \\
\hline 58 & Lake & 8.439 & 10.425 \\
\hline 59 & La Porte & 5.837 & 5.180 \\
\hline 60 & Lawrence & .334 & .554 \\
\hline 61 & Madison & 13.709 & 6.925 \\
\hline 62 & Marion & 42.227 & 35.634 \\
\hline 63 & Marshall & 2.001 & 3.943 \\
\hline 64 & Martin & .000 & .554 \\
\hline 65 & Miami & 12.508 & .554 \\
\hline 66 & Monroe & 2.335 & .896 \\
\hline 67 & Montgomery & .000 & 3.463 \\
\hline 68 & Morgan & .334 & .092 \\
\hline 69 & Newton & 2.001 & .092 \\
\hline 70 & Noble & 2.001 & 6.925 \\
\hline 71 & Ohio & .000 & .000 \\
\hline 72 & Orange & 58.371 & .554 \\
\hline 73 & Owen & .000 & .092 \\
\hline 74 & Parke & .334 & 1.616 \\
\hline 75 & Perry & 9.773 & .092 \\
\hline 76 & Pike & .000 & .000 \\
\hline 77 & Porter & 1.701 & 2.973 \\
\hline 78 & Posey & .000 & .554 \\
\hline 79 & Pulaski & .334 & .637 \\
\hline 80 & Putnam & .000 & .092 \\
\hline 81 & Randolph & .334 & .711 \\
\hline 82 & Ripley & 5.837 & .092 \\
\hline 83 & Rush & .000 & .240 \\
\hline 84 & St. Joseph & 5.604 & 6.408 \\
\hline 85 & Scott & 2.001 & 3.463 \\
\hline 86 & Shelby & 11.074 & 1.616 \\
\hline 87 & Spencer & 25.016 & .554 \\
\hline 88 & Starke & .334 & .000 \\
\hline 89 & Steuben & .334 & 2.327 \\
\hline 90 & Sullivan & .000 & .000 \\
\hline 91 & Switzerland & .334 & .000 \\
\hline$\overline{92}$ & Tippecanoe & 25.016 & 5.947 \\
\hline 93 & Tipton & .000 & 1.616 \\
\hline$\overline{94}$ & Union & .000 & .000 \\
\hline 95 & Vanderburgh & 13.375 & 32.347 \\
\hline 96 & Vermillion & .000 & .074 \\
\hline 97 & Vigo & 2.001 & 2.290 \\
\hline 98 & Wabash & .334 & .554 \\
\hline 99 & Warren & .000 & .092 \\
\hline 100 & Warrick & .334 & .000 \\
\hline 101 & Washington & 58.371 & .554 \\
\hline 102 & Wayne & 23.815 & 6.510 \\
\hline
\end{tabular}




\begin{tabular}{|c|c|c|c|}
\hline & State & P01 97 & A01 97 \\
\hline 103 & Wells & .334 & 4.728 \\
\hline 104 & White & 5.837 & .388 \\
\hline 105 & Whitley & 1.468 & .092 \\
\hline 106 & lowa & 653.000 & 762.184 \\
\hline 107 & Kansas & 135.000 & 257.062 \\
\hline 108 & Kentucky_E & 48.000 & 34.008 \\
\hline 109 & Kentucky_W & 32.000 & 23.006 \\
\hline 110 & Louisiana & 9.967 & 73.018 \\
\hline 111 & Maine & 1.000 & 1.000 \\
\hline 112 & Maryland & 33.404 & 71.017 \\
\hline 113 & Massachusetts & .000 & 90.022 \\
\hline 114 & Michigan_E & 141.000 & 68.016 \\
\hline 115 & Michigan_W & 141.000 & 68.016 \\
\hline$\overline{116}$ & Minnesota & 92.001 & 179.043 \\
\hline 117 & Missisippi & 229.469 & 111.027 \\
\hline 118 & Missouri & 96.779 & 15.004 \\
\hline 119 & Montana & 6.391 & 9.002 \\
\hline 120 & Nebraska & 24.513 & 143.034 \\
\hline 121 & Nevada & 2.000 & 13.003 \\
\hline 122 & New Hampshire & 10.892 & 9.002 \\
\hline 123 & New Jersey & 17.000 & 124.030 \\
\hline 124 & New Mexico & 7.791 & 21.005 \\
\hline 125 & New York & 166.806 & 203.049 \\
\hline 126 & North Carolina & 615.453 & 219.053 \\
\hline 127 & North Dakota & .000 & 23.006 \\
\hline 128 & Ohio_N & 92.000 & 35.008 \\
\hline 129 & Ohio M & 92.000 & 35.008 \\
\hline 130 & Ohio_s & 91.000 & 34.008 \\
\hline 131 & Oklahoma & 26.973 & 133.032 \\
\hline 132 & Oregon & 47.444 & 82.020 \\
\hline 133 & Pennsylvania & 81.000 & 65.016 \\
\hline 134 & Rhode Island & .000 & 12.003 \\
\hline 135 & South Carolina & 45.095 & 66.016 \\
\hline 136 & South Dakota & 13.012 & 14.003 \\
\hline 137 & Tennessee & 198.675 & 151.036 \\
\hline 138 & Texas & 245.000 & 388.094 \\
\hline 139 & Utah & .000 & 50.012 \\
\hline 140 & Vermont & 22.409 & 16.004 \\
\hline$\overline{141}$ & Virginia & 181.645 & 27.007 \\
\hline 142 & Washington & 58.953 & 140.034 \\
\hline 143 & West Virginia & .000 & 22.005 \\
\hline 144 & Wisconsin & 135.522 & 175.042 \\
\hline 145 & Wyoming & .000 & .000 \\
\hline
\end{tabular}




\begin{tabular}{|c|c|c|c|}
\hline & State & P02 97 & $\mathrm{~A} 0297$ \\
\hline 1 & Alabama & 3047.189 & 2912.000 \\
\hline 2 & Arizona & 786.836 & 410.000 \\
\hline 3 & Arkansas & 2051.906 & 10474.000 \\
\hline 4 & California & 1953.959 & 10511.000 \\
\hline 5 & Colorado & 7726.882 & 9764.000 \\
\hline 6 & Connecticut & 780.428 & 509.000 \\
\hline 7 & Delaware & 1302.306 & 1463.000 \\
\hline 8 & $\mathrm{DC}$ & .000 & .000 \\
\hline 9 & Florida & 3227.396 & 1916.000 \\
\hline 10 & Georgia & 5275.241 & 5832.000 \\
\hline 11 & Idaho & 2988.407 & 1944.000 \\
\hline 12 & Illinois $\mathbf{N}$ & 47316.780 & 34398.000 \\
\hline 13 & Illinois_S & 20279.191 & 14757.000 \\
\hline 14 & Adams & 123.226 & 23.281 \\
\hline 15 & Allen & 592.336 & 144.345 \\
\hline 16 & Bartholomew & 460.587 & .000 \\
\hline 17 & Benton & .000 & .000 \\
\hline 18 & Blackford & 21.307 & .000 \\
\hline 19 & Boone & 3.551 & .000 \\
\hline 20 & Brown & 3.551 & .000 \\
\hline 21 & Carroll & 621.456 & .000 \\
\hline 22 & Cass & 621.456 & .000 \\
\hline 23 & Clark & 102.274 & 235.143 \\
\hline 24 & Clay & 3.551 & .000 \\
\hline 25 & Clinton & 741.840 & .000 \\
\hline 26 & Crawford & .000 & .000 \\
\hline 27 & Daviess & 360.799 & 23.281 \\
\hline 28 & Dearborn & 3.551 & 23.281 \\
\hline 29 & Decatur & 131.393 & .000 \\
\hline 30 & DeKalb & 21.307 & .000 \\
\hline 31 & Delaware & 44.390 & .000 \\
\hline 32 & Dubois & 283.384 & .000 \\
\hline 33 & Elkhart & 230.471 & 23.281 \\
\hline 34 & Fayette & .000 & .000 \\
\hline 35 & Floyd & 257.460 & .000 \\
\hline 36 & Fountain & 21.307 & .000 \\
\hline 37 & Franklin & 3.551 & 139.689 \\
\hline 38 & Fulton & 55.043 & .000 \\
\hline 39 & Gibson & 133.169 & .000 \\
\hline 40 & Grant & 94.106 & .000 \\
\hline 41 & Greene & 133.169 & .000 \\
\hline 42 & Hamilton & 47.231 & 23.281 \\
\hline 43 & Hancock & 3.551 & .000 \\
\hline 44 & Harrison & 257.460 & 139.689 \\
\hline 45 & Hendricks & 163.709 & .000 \\
\hline 46 & Henry & 21.307 & .000 \\
\hline 47 & Howard & 56.464 & .000 \\
\hline 48 & Huntington & 133.169 & 23.281 \\
\hline 49 & Jackson & 12.784 & .000 \\
\hline 50 & Jasper & 79.901 & .000 \\
\hline 51 & Jay & 157.672 & 23.281 \\
\hline
\end{tabular}




\begin{tabular}{|c|c|c|c|}
\hline & State & P02 97 & $\mathrm{~A} 02 \quad 97$ \\
\hline 52 & Jefferson & 7.102 & .000 \\
\hline 53 & Jennings & .000 & 139.689 \\
\hline 54 & Johnson & 2.486 & .000 \\
\hline 55 & Knox & 46.876 & 23.281 \\
\hline 56 & Kosciusko & 325.288 & 23.281 \\
\hline 57 & LaGrange & 63.211 & .000 \\
\hline 58 & Lake & 400.928 & 5026.472 \\
\hline 59 & La Porte & 199.221 & 23.281 \\
\hline 60 & Lawrence & 21.307 & 139.689 \\
\hline 61 & Madison & 266.338 & .000 \\
\hline 62 & Marion & 1370.398 & 277.050 \\
\hline 63 & Marshall & 151.635 & 139.689 \\
\hline 64 & Martin & 21.307 & .000 \\
\hline 65 & Miami & 21.307 & .000 \\
\hline 66 & Monroe & 34.446 & 139.689 \\
\hline 67 & Montgomery & 133.169 & .000 \\
\hline 68 & Morgan & 3.551 & .000 \\
\hline 69 & Newton & 3.551 & .000 \\
\hline 70 & Noble & 266.338 & .000 \\
\hline 71 & \begin{tabular}{|l} 
Ohio \\
\end{tabular} & .000 & .000 \\
\hline 72 & Orange & 21.307 & .000 \\
\hline 73 & Owen & 3.551 & .000 \\
\hline 74 & Parke & 62.146 & .000 \\
\hline 75 & Perry & 3.551 & .000 \\
\hline 76 & Pike & .000 & .000 \\
\hline 77 & Porter & 114.348 & .000 \\
\hline 78 & Posey & 21.307 & 873.056 \\
\hline 79 & Pulaski & 24.503 & .000 \\
\hline 80 & Putnam & 3.551 & .000 \\
\hline 81 & Randolph & 27.344 & 23.281 \\
\hline 82 & Ripley & 3.551 & 23.281 \\
\hline 83 & Rush & 9.233 & .000 \\
\hline 84 & St. Joseph & 246.452 & 23.281 \\
\hline 85 & Scott & 133.169 & 23.281 \\
\hline 86 & Shelby & 62.146 & .000 \\
\hline 87 & Spencer & 21.307 & .000 \\
\hline 88 & Starke & .000 & .000 \\
\hline 89 & Steuben & 89.490 & 23.281 \\
\hline 90 & Sullivan & .000 & .000 \\
\hline 91 & Switzerland & .000 & .000 \\
\hline 92 & Tippecanoe & 228.696 & 23.281 \\
\hline 93 & Tipton & 62.146 & .000 \\
\hline 94 & Union & .000 & .000 \\
\hline 95 & Vanderburgh & 1243.977 & .000 \\
\hline 96 & Vermillion & 2.841 & .000 \\
\hline 97 & Vigo & 88.069 & .000 \\
\hline 98 & Wabash & 21.307 & 23.281 \\
\hline 99 & Warren & 3.551 & .000 \\
\hline 100 & Warrick & .000 & .000 \\
\hline 101 & Washington & 21.307 & .000 \\
\hline 102 & Wayne & 250.358 & .000 \\
\hline
\end{tabular}




\begin{tabular}{|c|c|c|c|}
\hline & State & P02 97 & A02 97 \\
\hline 103 & Wells & 181.820 & .000 \\
\hline 104 & White & 14.915 & .000 \\
\hline 105 & Whitley & 3.551 & .000 \\
\hline 106 & lowa & 31921.986 & 24068.000 \\
\hline 107 & Kansas & 41168.057 & 26762.000 \\
\hline 108 & Kentucky_E & 3509.130 & 1843.000 \\
\hline 109 & Kentucky_W & 2338.753 & 1228.000 \\
\hline 110 & Louisiana & 40132.609 & 90987.000 \\
\hline 111 & Maine & 8.995 & 197.000 \\
\hline 112 & Maryland & 1182.370 & 1211.000 \\
\hline 113 & Massachusetts & 1895.024 & 1180.000 \\
\hline 114 & Michigan_E & 2611.608 & 1369.000 \\
\hline 115 & Michigan W & 2611.608 & 1369.000 \\
\hline 116 & Minnesota & 37414.058 & 27634.000 \\
\hline 117 & Missisippi & 2344.444 & 2023.000 \\
\hline 118 & Missouri & 13516.796 & 11645.000 \\
\hline 119 & Montana & 4362.675 & 913.000 \\
\hline 120 & Nebraska & 38598.427 & 21319.000 \\
\hline 121 & Nevada & 267.633 & 67.000 \\
\hline 122 & New Hampshire & .000 & 173.000 \\
\hline 123 & New Jersey & 5.997 & 100.000 \\
\hline 124 & New Mexico & 357.429 & 872.000 \\
\hline 125 & New York & 760.595 & 3136.000 \\
\hline 126 & North Carolina & 2103.879 & 10835.000 \\
\hline 127 & North Dakota & 27469.359 & 6543.000 \\
\hline 128 & Ohio_N & 8350.549 & 3352.000 \\
\hline 129 & Ohio_M & 8349.550 & 3352.000 \\
\hline 130 & Ohio_s & 8349.550 & 3351.000 \\
\hline 131 & Oklahoma & 4736.475 & 5017.000 \\
\hline 132 & Oregon & 18588.093 & 28974.000 \\
\hline 133 & Pennsylvania & 1490.206 & 3012.000 \\
\hline 134 & Rhode Island & .000 & 11.000 \\
\hline 135 & South Carolina & 271.855 & 924.000 \\
\hline 136 & South Dakota & 706.695 & 3039.000 \\
\hline 137 & Tennessee & 1665.112 & 6710.000 \\
\hline 138 & Texas & 23203.632 & 31802.000 \\
\hline 139 & Utah & 1055.610 & 715.000 \\
\hline 140 & Vermont & 331.359 & 109.000 \\
\hline 141 & Virginia & 2268.791 & 2563.000 \\
\hline 142 & Washington & 38235.620 & 49543.000 \\
\hline 143 & West Virginia & .000 & 135.000 \\
\hline 144 & Wisconsin & 8136.663 & 7730.000 \\
\hline 145 & Wyoming & 65.833 & 940.000 \\
\hline
\end{tabular}




\begin{tabular}{|c|c|c|c|}
\hline & State & P03 97 & A03 97 \\
\hline 1 & Alabama & 1682.000 & 3020.820 \\
\hline 2 & Arizona & 827.131 & 1355.817 \\
\hline 3 & Arkansas & 2171.000 & 2934.769 \\
\hline 4 & California & 16275.000 & 14203.559 \\
\hline 5 & Colorado & 1676.684 & 502.303 \\
\hline 6 & Connecticut & 820.395 & 407.245 \\
\hline 7 & Delaware & 481.000 & 670.404 \\
\hline 8 & DC & .000 & .000 \\
\hline 9 & Florida & 5351.000 & 5132.093 \\
\hline 10 & Georgia & 2641.000 & 2806.691 \\
\hline$\overline{11}$ & Idaho & 1909.000 & 779.470 \\
\hline 12 & Illinois_N & 17375.000 & 13189.949 \\
\hline 13 & Illinois_S & 7447.000 & 5653.407 \\
\hline 14 & Adams & 73.258 & 11.615 \\
\hline 15 & Allen & 352.146 & 72.012 \\
\hline 16 & Bartholomew & 273.821 & .000 \\
\hline 17 & Benton & .000 & .000 \\
\hline 18 & Blackford & 12.667 & .000 \\
\hline 19 & Boone & 2.111 & .000 \\
\hline 20 & Brown & 2.111 & .000 \\
\hline 21 & Carroll & 369.457 & .000 \\
\hline 22 & Cass & 369.457 & .000 \\
\hline 23 & Clark & 60.802 & 117.310 \\
\hline 24 & Clay & 2.111 & .000 \\
\hline 25 & Clinton & 441.027 & .000 \\
\hline 26 & Crawford & .000 & .000 \\
\hline 27 & Daviess & 214.496 & 11.615 \\
\hline 28 & Dearborn & 2.111 & 11.615 \\
\hline 29 & Decatur & 78.114 & .000 \\
\hline 30 & DeKalb & 12.667 & .000 \\
\hline 31 & Delaware & 26.390 & .000 \\
\hline 32 & Dubois & 168.473 & .000 \\
\hline$\overline{33}$ & Elkhart & 137.016 & 11.615 \\
\hline 34 & Fayette & .000 & .000 \\
\hline 35 & Floyd & 153.061 & .000 \\
\hline 36 & Fountain & 12.667 & .000 \\
\hline 37 & Franklin & 2.111 & 69.689 \\
\hline 38 & Fulton & 32.723 & .000 \\
\hline 39 & Gibson & 79.169 & .000 \\
\hline 40 & Grant & 55.946 & .000 \\
\hline 41 & Greene & 79.169 & .000 \\
\hline 42 & Hamilton & 28.079 & 11.615 \\
\hline 43 & Hancock & 2.111 & .000 \\
\hline 44 & Harrison & 153.061 & 69.689 \\
\hline 45 & Hendricks & 97.326 & .000 \\
\hline 46 & Henry & 12.667 & .000 \\
\hline 47 & Howard & 33.568 & .000 \\
\hline 48 & Huntington & 79.169 & 11.615 \\
\hline 49 & Jackson & 7.600 & .000 \\
\hline 50 & Jasper & 47.502 & .000 \\
\hline 51 & Jay & 93.737 & 11.615 \\
\hline
\end{tabular}




\begin{tabular}{|c|c|c|c|}
\hline & State & P03 97 & A03 97 \\
\hline 52 & Jefferson & 4.222 & .000 \\
\hline 53 & Jennings & .000 & 69.689 \\
\hline 54 & Johnson & 1.478 & .000 \\
\hline 55 & Knox & 27.868 & 11.615 \\
\hline 56 & Kosciusko & 193.385 & 11.615 \\
\hline 57 & LaGrange & 37.579 & .000 \\
\hline 58 & Lake & 238.353 & 2507.656 \\
\hline 59 & La Porte & 118.437 & 11.615 \\
\hline 60 & \begin{tabular}{|l|} 
Lawrence \\
\end{tabular} & 12.667 & 69.689 \\
\hline 61 & Madison & 158.339 & .000 \\
\hline 62 & Marion & 814.706 & 138.217 \\
\hline 63 & Marshall & 90.148 & 69.689 \\
\hline 64 & Martin & 12.667 & .000 \\
\hline 65 & Miami & 12.667 & .000 \\
\hline 66 & Monroe & 20.478 & 69.689 \\
\hline 67 & Montgomery & 79.169 & .000 \\
\hline 68 & Morgan & 2.111 & .000 \\
\hline 69 & Newton & 2.111 & .000 \\
\hline 70 & Noble & 158.339 & .000 \\
\hline 71 & Ohio & .000 & .000 \\
\hline 72 & Orange & 12.667 & .000 \\
\hline 73 & Owen & 2.111 & .000 \\
\hline 74 & Parke & 36.946 & .000 \\
\hline 75 & Perry & 2.111 & .000 \\
\hline 76 & Pike & .000 & .000 \\
\hline 77 & Porter & 67.980 & .000 \\
\hline 78 & Posey & 12.667 & 435.559 \\
\hline 79 & Pulaski & 14.567 & .000 \\
\hline 80 & Putnam & 2.111 & .000 \\
\hline 81 & Randolph & 16.256 & 11.615 \\
\hline 82 & Ripley & 2.111 & 11.615 \\
\hline 83 & Rush & 5.489 & .000 \\
\hline 84 & St. Joseph & 146.516 & 11.615 \\
\hline 85 & Scott & 79.169 & 11.615 \\
\hline 86 & Shelby & 36.946 & .000 \\
\hline 87 & Spencer & 12.667 & .000 \\
\hline 88 & Starke & .000 & .000 \\
\hline 89 & Steuben & 53.202 & 11.615 \\
\hline 90 & Sullivan & .000 & .000 \\
\hline 91 & Switzerland & .000 & .000 \\
\hline 92 & Tippecanoe & 135.960 & 11.615 \\
\hline 93 & Tipton & 36.946 & .000 \\
\hline 94 & Union & .000 & .000 \\
\hline 95 & Vanderburgh & 739.548 & .000 \\
\hline 96 & Vermillion & 1.689 & .000 \\
\hline 97 & Vigo & 52.357 & .000 \\
\hline 98 & Wabash & 12.667 & 11.615 \\
\hline 99 & Warren & 2.111 & .000 \\
\hline 100 & Warrick & .000 & .000 \\
\hline 101 & Washington & 12.667 & .000 \\
\hline 102 & Wayne & 148.839 & .000 \\
\hline
\end{tabular}




\begin{tabular}{|c|c|c|c|}
\hline & State & P03 97 & A03 97 \\
\hline$\overline{103}$ & Wells & 108.093 & .000 \\
\hline 104 & White & 8.867 & .000 \\
\hline 105 & Whitley & 2.111 & .000 \\
\hline 106 & lowa & 19127.000 & 16395.881 \\
\hline 107 & Kansas & 1990.000 & 1432.863 \\
\hline 108 & Kentucky_E & 1462.000 & 1393.840 \\
\hline 109 & Kentucky_W & 975.000 & 928.560 \\
\hline 110 & Louisiana & 14288.000 & 38375.126 \\
\hline 111 & Maine & 584.454 & 492.297 \\
\hline 112 & Maryland & 1572.785 & 1874.129 \\
\hline 113 & Massachusetts & 1992.071 & 2239.349 \\
\hline 114 & Michigan_E & 1968.000 & 1980.193 \\
\hline 115 & Michigan_W & 1968.000 & 1980.193 \\
\hline 116 & Minnesota & 13925.000 & 8862.341 \\
\hline 117 & Missisippi & 2464.506 & 795.479 \\
\hline 118 & Missouri & 5923.000 & 5342.219 \\
\hline 119 & Montana & 203.645 & 544.328 \\
\hline 120 & Nebraska & 6159.000 & 5340.218 \\
\hline 121 & Nevada & 281.339 & 160.096 \\
\hline 122 & New Hampshire & 203.922 & 67.040 \\
\hline 123 & New Jersey & 930.000 & 3591.164 \\
\hline 124 & New Mexico & 145.000 & 556.335 \\
\hline 125 & New York & 1990.000 & 3887.343 \\
\hline 126 & North Carolina & 3547.000 & 4952.985 \\
\hline 127 & North Dakota & 3651.000 & 2738.650 \\
\hline 128 & Ohio N & 3521.000 & 2686.619 \\
\hline 129 & Ohio $M$ & 3521.000 & 2686.619 \\
\hline 130 & Ohio_s & 3521.000 & 2686.619 \\
\hline 131 & Oklahoma & 536.000 & 618.373 \\
\hline 132 & Oregon & 3273.000 & 1167.704 \\
\hline 133 & Pennsylvania & 7331.000 & 5171.116 \\
\hline 134 & Rhode Island & 260.024 & 181.109 \\
\hline 135 & South Carolina & 1022.000 & 1520.917 \\
\hline 136 & South Dakota & 2064.000 & 448.270 \\
\hline 137 & Tennessee & 3348.015 & 1133.683 \\
\hline 138 & Texas & 8821.000 & 10095.084 \\
\hline 139 & Utah & 535.000 & 734.443 \\
\hline 140 & Vermont & 1086.000 & 438.264 \\
\hline$\overline{141}$ & Virginia & 2767.000 & 2658.602 \\
\hline 142 & Washington & 5410.000 & 7346.427 \\
\hline 143 & West Virginia & 485.353 & 1110.669 \\
\hline 144 & Wisconsin & 2766.000 & 2495.504 \\
\hline 145 & Wyoming & 69.204 & 8.005 \\
\hline
\end{tabular}




\begin{tabular}{|c|c|c|c|}
\hline & State & P04 97 & A04 97 \\
\hline 1 & Alabama & 7211.606 & 7817.000 \\
\hline 2 & Arizona & 579.415 & 1125.000 \\
\hline 3 & Arkansas & 11990.272 & 11917.000 \\
\hline 4 & California & 17284.196 & 20003.000 \\
\hline 5 & Colorado & 3295.044 & 3085.000 \\
\hline 6 & Connecticut & 574.402 & 1116.000 \\
\hline 7 & Delaware & 1029.513 & 1359.000 \\
\hline 8 & $\mathrm{DC}$ & .000 & .000 \\
\hline 9 & Florida & 3876.464 & 4471.000 \\
\hline 10 & Georgia & 10255.036 & 9825.000 \\
\hline 11 & Idaho & 2635.434 & 2210.000 \\
\hline 12 & Illinois_N & 9495.181 & 4916.000 \\
\hline 13 & Illinois_S & 4069.936 & 2107.000 \\
\hline 14 & Adams & 67.828 & 52.836 \\
\hline 15 & Allen & 326.042 & 253.980 \\
\hline 16 & Bartholomew & 253.523 & 197.489 \\
\hline 17 & Benton & .000 & .000 \\
\hline 18 & Blackford & 11.728 & 9.136 \\
\hline 19 & Boone & 1.955 & 1.523 \\
\hline 20 & Brown & 1.955 & 1.523 \\
\hline 21 & Carroll & 342.071 & 266.466 \\
\hline 22 & Cass & 342.071 & 266.466 \\
\hline 23 & Clark & 56.295 & 43.853 \\
\hline 24 & Clay & 1.955 & 1.523 \\
\hline 25 & Clinton & 408.335 & 318.084 \\
\hline 26 & Crawford & .000 & .000 \\
\hline 27 & Daviess & 198.597 & 154.702 \\
\hline 28 & Dearborn & 1.955 & 1.523 \\
\hline 29 & Decatur & 72.324 & 56.338 \\
\hline 30 & DeKalb & 11.728 & 9.136 \\
\hline 31 & Delaware & 24.434 & 19.033 \\
\hline 32 & Dubois & 155.984 & 121.508 \\
\hline 33 & Elkhart & 126.859 & 98.821 \\
\hline 34 & Fayette & .000 & .000 \\
\hline 35 & Floyd & 141.715 & 110.393 \\
\hline 36 & Fountain & 11.728 & 9.136 \\
\hline 37 & Franklin & 1.955 & 1.523 \\
\hline 38 & Fulton & 30.298 & 23.601 \\
\hline 39 & Gibson & 73.301 & 57.100 \\
\hline 40 & Grant & 51.799 & 40.351 \\
\hline 41 & Greene & 73.301 & 57.100 \\
\hline 42 & Hamilton & 25.997 & 20.251 \\
\hline 43 & Hancock & 1.955 & 1.523 \\
\hline 44 & Harrison & 141.715 & 110.393 \\
\hline 45 & Hendricks & 90.111 & 70.195 \\
\hline 46 & Henry & 11.728 & 9.136 \\
\hline 47 & Howard & 31.080 & 24.210 \\
\hline 48 & Huntington & 73.301 & 57.100 \\
\hline 49 & Jackson & 7.037 & 5.482 \\
\hline 50 & Jasper & 43.981 & 34.260 \\
\hline 51 & Jay & 86.788 & 67.606 \\
\hline
\end{tabular}




\begin{tabular}{|c|c|c|c|}
\hline & State & P04 97 & A04 97 \\
\hline 52 & Jefferson & 3.909 & 3.045 \\
\hline 53 & Jennings & .000 & .000 \\
\hline 54 & Johnson & 1.368 & 1.066 \\
\hline 55 & Knox & 25.802 & 20.099 \\
\hline 56 & Kosciusko & 179.050 & 139.476 \\
\hline 57 & LaGrange & 34.793 & 27.103 \\
\hline 58 & Lake & 220.685 & 171.908 \\
\hline 59 & La Porte & 109.658 & 85.421 \\
\hline 60 & Lawrence & 11.728 & 9.136 \\
\hline 61 & Madison & 146.602 & 114.200 \\
\hline 62 & Marion & 754.315 & 587.595 \\
\hline 63 & Marshall & 83.465 & 65.018 \\
\hline 64 & Martin & 11.728 & 9.136 \\
\hline 65 & Miami & 11.728 & 9.136 \\
\hline 66 & Monroe & 18.960 & 14.770 \\
\hline 67 & Montgomery & 73.301 & 57.100 \\
\hline 68 & Morgan & 1.955 & 1.523 \\
\hline 69 & Newton & 1.955 & 1.523 \\
\hline 70 & Noble & 146.602 & 114.200 \\
\hline$\overline{71}$ & Ohio & .000 & .000 \\
\hline 72 & Orange & 11.728 & 9.136 \\
\hline 73 & Owen & 1.955 & 1.523 \\
\hline 74 & Parke & 34.207 & 26.647 \\
\hline 75 & Perry & 1.955 & 1.523 \\
\hline 76 & Pike & .000 & .000 \\
\hline 77 & Porter & 62.941 & 49.030 \\
\hline 78 & Posey & 11.728 & 9.136 \\
\hline 79 & Pulaski & 13.487 & 10.506 \\
\hline 80 & Putnam & 1.955 & 1.523 \\
\hline 81 & Randolph & 15.051 & 11.724 \\
\hline 82 & Ripley & 1.955 & 1.523 \\
\hline 83 & Rush & 5.082 & 3.959 \\
\hline 84 & St. Joseph & 135.656 & 105.673 \\
\hline 85 & Scott & 73.301 & 57.100 \\
\hline 86 & Shelby & 34.207 & 26.647 \\
\hline 87 & Spencer & 11.728 & 9.136 \\
\hline 88 & Starke & .000 & .000 \\
\hline 89 & Steuben & 49.258 & 38.371 \\
\hline 90 & Sullivan & .000 & .000 \\
\hline 91 & Switzerland & .000 & .000 \\
\hline 92 & Tippecanoe & 125.882 & 98.059 \\
\hline 93 & Tipton & 34.207 & 26.647 \\
\hline 94 & Union & .000 & .000 \\
\hline 95 & Vanderburgh & 684.728 & 533.388 \\
\hline 96 & Vermillion & 1.564 & 1.218 \\
\hline 97 & Vigo & 48.476 & 37.762 \\
\hline 98 & Wabash & 11.728 & 9.136 \\
\hline 99 & Warren & 1.955 & 1.523 \\
\hline 100 & Warrick & .000 & .000 \\
\hline 101 & Washington & 11.728 & 9.136 \\
\hline 102 & Wayne & 137.806 & 107.348 \\
\hline
\end{tabular}




\begin{tabular}{|c|c|c|c|}
\hline & State & P04_97 & A04 97 \\
\hline 103 & Wells & 100.080 & 77.960 \\
\hline 104 & White & 8.210 & 6.395 \\
\hline 105 & Whitley & 1.955 & 1.523 \\
\hline 106 & lowa & 24406.584 & 18386.000 \\
\hline 107 & Kansas & 6591.091 & 4432.000 \\
\hline 108 & Kentucky_E & 1294.159 & 1268.000 \\
\hline 109 & Kentucky_W & 863.107 & 846.000 \\
\hline 110 & Louisiana & 3974.704 & 8121.000 \\
\hline 111 & Maine & 408.998 & 211.000 \\
\hline 112 & Maryland & 1855.530 & 1975.000 \\
\hline 113 & Massachusetts & 1395.407 & 1309.000 \\
\hline 114 & Michigan_E & 1544.771 & 1843.000 \\
\hline 115 & Michigan_W & 1544.771 & 1843.000 \\
\hline 116 & Minnesota & 7002.094 & 7368.000 \\
\hline 117 & Missisippi & 4110.034 & 3075.000 \\
\hline 118 & Missouri & 11014.891 & 9673.000 \\
\hline 119 & Montana & 313.766 & 430.000 \\
\hline 120 & Nebraska & 7560.458 & 6247.000 \\
\hline 121 & Nevada & 196.480 & 206.000 \\
\hline 122 & New Hampshire & .000 & 277.000 \\
\hline 123 & New Jersey & 175.428 & 987.000 \\
\hline 124 & New Mexico & 97.237 & 997.000 \\
\hline 125 & New York & 6199.134 & 5954.000 \\
\hline 126 & North Carolina & 7552.438 & 8169.000 \\
\hline 127 & North Dakota & 978.389 & 373.000 \\
\hline 128 & Ohio_N & 2815.874 & 2088.000 \\
\hline 129 & Ohio_M & 2815.874 & 2088.000 \\
\hline 130 & Ohio_s & 2815.874 & 2888.000 \\
\hline 131 & Oklahoma & 2515.140 & 4757.000 \\
\hline 132 & Oregon & 1609.930 & 1752.000 \\
\hline 133 & Pennsylvania & 3817.319 & 7247.000 \\
\hline 134 & Rhode Island & 182.445 & 44.000 \\
\hline 135 & South Carolina & 868.119 & 2000.000 \\
\hline 136 & South Dakota & 950.320 & 1083.000 \\
\hline 137 & Tennessee & 2926.144 & 3060.000 \\
\hline 138 & Texas & 16690.748 & 17967.000 \\
\hline 139 & Utah & 1425.480 & 1201.000 \\
\hline 140 & Vermont & 525.282 & 749.000 \\
\hline 141 & Virginia & 3478.492 & 4202.000 \\
\hline 142 & Washington & 2679.542 & 3539.000 \\
\hline 143 & West Virginia & 468.143 & 792.000 \\
\hline 144 & Wisconsin & 5452.311 & 5445.000 \\
\hline 145 & Wyoming & 182.445 & 250.000 \\
\hline
\end{tabular}




\begin{tabular}{|c|c|c|c|}
\hline & State & P05 97 & $\mathrm{~A} 05 \quad 97$ \\
\hline 1 & Alabama & 1810.777 & 1232.000 \\
\hline 2 & Arizona & 344.205 & 826.000 \\
\hline 3 & Arkansas & 3626.484 & 2364.000 \\
\hline 4 & California & 7819.083 & 10065.000 \\
\hline 5 & Colorado & 1395.560 & 904.000 \\
\hline 6 & Connecticut & 97.640 & 314.000 \\
\hline$\overline{7}$ & DC & .000 & .000 \\
\hline$\overline{8}$ & Delaware & 581.894 & 540.000 \\
\hline 9 & Florida & 1909.403 & 3473.000 \\
\hline 10 & Georgia & 2967.662 & 3150.000 \\
\hline 11 & Idaho & 542.444 & 186.000 \\
\hline 12 & Illinois_N & 2152.023 & 2448.000 \\
\hline 13 & Illinois_s & 922.155 & 1049.000 \\
\hline 14 & Adams & 10.051 & 9.204 \\
\hline 15 & Allen & 48.314 & 57.643 \\
\hline 16 & Bartholomew & 37.568 & 28.666 \\
\hline 17 & Benton & .000 & .811 \\
\hline 18 & Blackford & 1.738 & 2.220 \\
\hline 19 & Boone & .290 & 3.973 \\
\hline 20 & Brown & .290 & 1.429 \\
\hline 21 & Carroll & 50.689 & 31.305 \\
\hline 22 & Cass & 50.689 & 33.704 \\
\hline 23 & Clark & 8.342 & 12.886 \\
\hline 24 & Clay & .290 & 2.796 \\
\hline 25 & Clinton & 60.508 & 38.311 \\
\hline 26 & Crawford & .000 & .886 \\
\hline 27 & Daviess & 29.428 & 19.708 \\
\hline 28 & Dearborn & .290 & 3.979 \\
\hline 29 & Decatur & 10.717 & 8.656 \\
\hline 30 & DeKalb & 1.738 & 4.593 \\
\hline 31. & Delaware & 3.621 & 12.961 \\
\hline 32 & Dubois & 23.114 & 17.210 \\
\hline 33 & Elkhart & 18.798 & 30.936 \\
\hline 34 & Fayette & .000 & 3.035 \\
\hline 35 & Floyd & 21.000 & 18.353 \\
\hline 36 & Fountain & 1.738 & 2.707 \\
\hline 37 & Franklin & .290 & 2.086 \\
\hline 38 & Fulton & 4.490 & 4.350 \\
\hline 39 & Gibson & 10.862 & 9.096 \\
\hline 40 & Grant & 7.676 & 11.468 \\
\hline 41 & Greene & 10.862 & 9.165 \\
\hline 42 & Hamilton & 3.852 & 16.472 \\
\hline$\overline{43}$ & Hancock & .290 & 5.044 \\
\hline$\overline{44}$ & Harrison & 21.000 & 15.256 \\
\hline 45 & Hendricks & 13.353 & 16.053 \\
\hline 46 & Henry & 1.738 & 5.534 \\
\hline 47 & Howard & 4.605 & 13.768 \\
\hline 48 & Huntington & 10.862 & 10.024 \\
\hline 49 & Jackson & 1.043 & 4.617 \\
\hline 50 & Jasper & 6.517 & 6.308 \\
\hline 51 & Jay & 12.860 & 9.435 \\
\hline
\end{tabular}




\begin{tabular}{|c|c|c|c|}
\hline & State & P05 97 & A05 97 \\
\hline 52 & Jefferson & .579 & 3.360 \\
\hline 53 & Jennings & .000 & 2.447 \\
\hline 54 & Johnson & .203 & 9.681 \\
\hline 55 & Knox & 3.823 & 5.593 \\
\hline 56 & Kosciusko & 26.532 & 22.056 \\
\hline 57 & LaGrange & 5.156 & 6.178 \\
\hline 58 & Lake & 32.701 & 60.541 \\
\hline 59 & La Porte & 16.249 & 18.954 \\
\hline 60 & Lawrence & 1.738 & 5.284 \\
\hline 61 & Madison & 21.724 & 25.505 \\
\hline 62 & Marion & 111.776 & 140.975 \\
\hline 63 & Marshall & 12.368 & 11.420 \\
\hline 64 & Martin & 1.738 & 1.901 \\
\hline 65 & Miami & 1.738 & 3.998 \\
\hline 66 & Monroe & 2.810 & 11.768 \\
\hline 67 & Montgomery & 10.862 & 9.809 \\
\hline 68 & Morgan & .290 & 5.694 \\
\hline 69 & Newton & .290 & 1.443 \\
\hline 70 & Noble & 21.724 & 17.150 \\
\hline 71 & Ohio & .000 & .470 \\
\hline 72 & Orange & 1.738 & 2.634 \\
\hline 73 & Owen & .290 & 1.963 \\
\hline 74 & Parke & 5.069 & 4.400 \\
\hline 75 & Perry & .290 & 1.787 \\
\hline 76 & Pike & .000 & 1.085 \\
\hline 77 & Porter & 9.327 & 17.668 \\
\hline 78 & Posey & 1.738 & 3.298 \\
\hline 79 & Pulaski & 1.999 & 2.325 \\
\hline 80 & Putnam & .290 & 3.305 \\
\hline 81 & Randolph & 2.230 & 3.795 \\
\hline 82 & Ripley & .290 & 2.377 \\
\hline 83 & Rush & .753 & 2.006 \\
\hline 84 & St. Joseph & 20.102 & 34.979 \\
\hline 85 & Scott & 10.862 & 8.254 \\
\hline 86 & Shelby & 5.069 & 6.777 \\
\hline 87 & Spencer & 1.738 & 2.771 \\
\hline 88 & Starke & .000 & 2.082 \\
\hline 89 & Steuben & 7.299 & 7.600 \\
\hline 90 & Sullivan & .000 & 1.858 \\
\hline 91 & Switzerland & .000 & .745 \\
\hline 92 & Tippecanoe & 18.653 & 24.575 \\
\hline 93 & Tipton & 5.069 & 4.447 \\
\hline 94 & Union & .000 & .615 \\
\hline 95 & Vanderburgh & 101.464 & 73.770 \\
\hline 96 & Vermillion & .232 & 1.556 \\
\hline 97 & Vigo & 7.183 & 13.286 \\
\hline 98 & Wabash & 1.738 & 4.063 \\
\hline 99 & Warren & .290 & .885 \\
\hline 100 & Warrick & .000 & 4.361 \\
\hline 101 & Washington & 1.738 & 3.285 \\
\hline 102 & Wayne & 20.420 & 18.269 \\
\hline
\end{tabular}




\begin{tabular}{|c|c|c|c|}
\hline & State & P05 97 & A05 97 \\
\hline 103 & Wells & 14.830 & 11.069 \\
\hline 104 & White & 1.217 & 3.233 \\
\hline 105 & Whitley & .290 & 3.004 \\
\hline 106 & lowa & 4280.376 & 2860.000 \\
\hline 107 & Kansas & 3076.150 & 1746.000 \\
\hline 108 & Kentucky_E & 338.288 & 623.000 \\
\hline 109 & Kentucky W & 224.868 & 415.000 \\
\hline 110 & Louisiana & 595.702 & 917.000 \\
\hline 111 & Maine & 233.744 & 320.000 \\
\hline 112 & Maryland & 1572.101 & 1360.000 \\
\hline$\overline{113}$ & Massachusetts & 1266.360 & 1536.000 \\
\hline 114 & Michigan_E & 1077.984 & 1264.000 \\
\hline$\overline{115}$ & Michigan_W & 1077.984 & 1264.000 \\
\hline$\overline{116}$ & Minnesota & 2127.367 & 1635.000 \\
\hline 117 & Missisippi & 1568.156 & 1537.000 \\
\hline 118 & Missouri & 1599.717 & 2126.000 \\
\hline 119 & Montana & 161.747 & 146.000 \\
\hline 120 & Nebraska & 5277.487 & 995.000 \\
\hline 121 & Nevada & 140.049 & 324.000 \\
\hline 122 & New Hampshire & 199.225 & 216.000 \\
\hline 123 & New Jersey & 769.284 & 1488.000 \\
\hline 124 & New Mexico & 183.445 & 251.000 \\
\hline 125 & New York & 2202.323 & 3580.000 \\
\hline 126 & North Carolina & 2782.244 & 2424.000 \\
\hline 127 & North Dakota & 211.060 & 105.000 \\
\hline 128 & Ohio_N & 616.414 & 926.000 \\
\hline 129 & Ohio M & 616.414 & 926.000 \\
\hline 130 & Ohio_S & 615.427 & 926.000 \\
\hline 131 & Oklahoma & 891.581 & 1264.000 \\
\hline 132 & Oregon & 421.134 & 651.000 \\
\hline 133 & Pennsylvania & 2407.465 & 3085.000 \\
\hline 134 & Rhode Island & 193.307 & 120.000 \\
\hline 135 & South Carolina & 1319.618 & 1052.000 \\
\hline 136 & South Dakota & 746.600 & 162.000 \\
\hline 137 & Tennessee & 1745.683 & 1709.000 \\
\hline 138 & Texas & 5884.038 & 6215.000 \\
\hline 139 & Utah & 388.587 & 398.000 \\
\hline 140 & Vermont & 165.692 & 45.000 \\
\hline 141 & Virginia & 2252.622 & 2019.000 \\
\hline 142 & Washington & 1746.670 & 1838.000 \\
\hline 143 & West Virginia & 255.442 & 245.000 \\
\hline 144 & Wisconsin & 1988.304 & 1955.000 \\
\hline 145 & Wyoming & .000 & 48.000 \\
\hline
\end{tabular}




\begin{tabular}{|c|c|c|c|}
\hline & State & P06 97 & A06 97 \\
\hline 1 & Alabama & 385.586 & 1402.000 \\
\hline 2 & Arizona & 624.329 & 1341.000 \\
\hline 3 & Arkansas & 1422.471 & 1560.000 \\
\hline 4 & California & 9968.288 & 11082.000 \\
\hline 5 & Colorado & 946.982 & 1429.000 \\
\hline 6 & Connecticut & 782.160 & 738.000 \\
\hline 7 & DC & .000 & .000 \\
\hline 8 & Delaware & 363.609 & 135.000 \\
\hline 9 & Florida & 2246.586 & 3575.000 \\
\hline 10 & Georgia & 1891.967 & 3222.000 \\
\hline 11 & Idaho & 102.889 & 263.000 \\
\hline 12 & Illinois_N & 6828.662 & 4206.000 \\
\hline 13 & Illinois S & 2926.855 & 1802.000 \\
\hline 14 & Adams & 57.193 & 16.838 \\
\hline 15 & Allen & 281.157 & 98.640 \\
\hline 16 & Bartholomew & 122.911 & 44.463 \\
\hline 17 & Benton & .000 & 1.564 \\
\hline 18 & Blackford & 4.306 & 3.668 \\
\hline 19 & Boone & 17.940 & 7.452 \\
\hline 20 & Brown & 1.148 & 2.860 \\
\hline 21 & Carroll & 125.581 & 43.043 \\
\hline 22 & Cass & 141.727 & 46.375 \\
\hline 23 & Clark & 24.542 & 35.600 \\
\hline 24 & Clay & 1.148 & 5.745 \\
\hline 25 & Clinton & 182.200 & 56.496 \\
\hline 26 & Crawford & .000 & 1.714 \\
\hline 27 & Daviess & 72.909 & 29.135 \\
\hline 28 & Dearborn & 1.148 & 7.798 \\
\hline 29 & Decatur & 26.551 & 12.427 \\
\hline 30 & DeKalb & 16.749 & 12.283 \\
\hline 31 & Delaware & 9.401 & 24.087 \\
\hline 32 & Dubois & 89.557 & 25.753 \\
\hline 33 & Elkhart & 142.157 & 78.834 \\
\hline 34 & Fayette & .000 & 4.236 \\
\hline 35 & Floyd & 78.204 & 30.064 \\
\hline 36 & Fountain & 6.889 & 4.510 \\
\hline 37 & Franklin & .718 & 3.771 \\
\hline 38 & Fulton & 11.553 & 7.040 \\
\hline 39 & Gibson & 36.469 & 13.771 \\
\hline 40 & Grant & 94.365 & 20.010 \\
\hline 41 & Greene & 29.494 & 13.956 \\
\hline 42 & Hamilton & 18.328 & 31.316 \\
\hline 43 & Hancock & 1.148 & 9.027 \\
\hline$\overline{44}$ & Harrison & 52.026 & 22.920 \\
\hline 45 & Hendricks & 40.616 & 26.548 \\
\hline 46 & Henry & 6.803 & 9.343 \\
\hline 47 & Howard & 14.295 & 17.413 \\
\hline 48 & Huntington & 92.657 & 15.911 \\
\hline 49 & Jackson & 2.583 & 11.001 \\
\hline 50 & Jasper & 23.681 & 9.879 \\
\hline 51 & Jay & 31.862 & 13.651 \\
\hline
\end{tabular}




\begin{tabular}{|c|c|c|c|}
\hline & State & P06 97 & A06 97 \\
\hline 52 & Jefferson & 1.435 & 6.780 \\
\hline 53 & Jennings & .000 & 4.608 \\
\hline 54 & Johnson & 25.260 & 19.305 \\
\hline 55 & Knox & 12.960 & 9.496 \\
\hline 56 & Kosciusko & 70.899 & 39.356 \\
\hline 57 & LaGrange & 15.357 & 9.823 \\
\hline 58 & Lake & 88.423 & 134.802 \\
\hline 59 & La Porte & 43.358 & 37.351 \\
\hline 60 & Lawrence & 6.889 & 8.860 \\
\hline 61 & Madison & 54.251 & 46.329 \\
\hline 62 & Marion & 357.094 & 348.439 \\
\hline 63 & Marshall & 31.072 & 19.581 \\
\hline 64 & Martin & 4.306 & 3.077 \\
\hline 65 & Miami & 4.736 & 8.307 \\
\hline 66 & Monroe & 50.620 & 24.980 \\
\hline 67 & Montgomery & 26.910 & 18.126 \\
\hline 68 & Morgan & 76.066 & 18.337 \\
\hline 69 & Newton & 8.252 & 2.815 \\
\hline 70 & Noble & 56.404 & 28.683 \\
\hline 71 & Ohio & .000 & .909 \\
\hline 72 & Orange & 4.306 & 4.496 \\
\hline 73 & Owen & .718 & 3.698 \\
\hline 74 & Parke & 12.558 & 6.762 \\
\hline 75 & Perry & 1.148 & 3.358 \\
\hline 76 & Pike & .000 & 2.098 \\
\hline 77 & Porter & 27.886 & 40.215 \\
\hline 78 & Posey & 4.306 & 40.769 \\
\hline 79 & Pulaski & 4.951 & 3.804 \\
\hline 80 & Putnam & 8.252 & 5.967 \\
\hline 81 & Randolph & 8.109 & 6.244 \\
\hline 82 & Ripley & 3.301 & 5.693 \\
\hline 83 & Rush & 2.296 & 3.595 \\
\hline 84 & St. Joseph & 89.327 & 76.124 \\
\hline 85 & Scott & 26.910 & 15.693 \\
\hline 86 & Shelby & 12.558 & 14.842 \\
\hline 87 & Spencer & 4.306 & 4.884 \\
\hline 88 & Starke & .000 & 5.099 \\
\hline 89 & Steuben & 20.667 & 11.026 \\
\hline 90 & Sullivan & .000 & 3.719 \\
\hline 91 & Switzerland & .000 & 1.442 \\
\hline 92 & Tippecanoe & 97.537 & 71.375 \\
\hline 93 & Tipton & 12.558 & 6.890 \\
\hline 94 & Union & .000 & 2.050 \\
\hline 95 & Vanderburgh & 251.807 & 154.528 \\
\hline 96 & Vermillion & .574 & 37.919 \\
\hline 97 & Vigo & 93.145 & 43.655 \\
\hline 98 & Wabash & 27.039 & 14.573 \\
\hline 99 & Warren & .718 & 1.586 \\
\hline 100 & Warrick & .000 & 9.960 \\
\hline 101 & Washington & 4.306 & 5.751 \\
\hline 102 & Wayne & 90.590 & 30.503 \\
\hline
\end{tabular}




\begin{tabular}{|c|c|c|c|}
\hline & State & P06 97 & A06 97 \\
\hline$\overline{103}$ & Wells & 37.172 & 16.131 \\
\hline 104 & White & 10.549 & 5.072 \\
\hline 105 & Whitley & 8.166 & 5.370 \\
\hline 106 & lowa & 7094.377 & 2204.000 \\
\hline 107 & Kansas & 4443.225 & 1915.000 \\
\hline 108 & Kentucky_E & 662.288 & 842.000 \\
\hline 109 & Kentucky_W & 441.526 & 561.000 \\
\hline 110 & Louisiana & 812.127 & 2536.000 \\
\hline 111 & Maine & 269.710 & 531.000 \\
\hline 112 & Maryland & 580.376 & 1182.000 \\
\hline$\overline{113}$ & Massachusetts & 1536.349 & 1851.000 \\
\hline 114 & Michigan_E & 1093.825 & 1697.000 \\
\hline 115 & Michigan_W & 1093.825 & 1697.000 \\
\hline 116 & Minnesota & 3691.034 & 1839.000 \\
\hline 117 & Missisippi & 820.119 & 774.000 \\
\hline 118 & Missouri & 5551.035 & 2803.000 \\
\hline 119 & Montana & 425.543 & 196.000 \\
\hline 120 & Nebraska & 2506.307 & 1051.000 \\
\hline 121 & Nevada & 41.955 & 303.000 \\
\hline 122 & New Hampshire & .000 & 342.000 \\
\hline 123 & New Jersey & 2259.572 & 3843.000 \\
\hline 124 & New Mexico & 276.703 & 549.000 \\
\hline 125 & New York & 5495.095 & 5895.000 \\
\hline 126 & North Carolina & 1569.314 & 2733.000 \\
\hline 127 & North Dakota & 840.097 & 284.000 \\
\hline 128 & Ohio_N & 1998.852 & 1887.000 \\
\hline 129 & Ohio M & 1998.852 & 1886.000 \\
\hline 130 & Ohio_s & 1997.853 & 1886.000 \\
\hline 131 & Oklahoma & 862.074 & 952.000 \\
\hline 132 & Oregon & 1549.335 & 1916.000 \\
\hline 133 & Pennsylvania & 3467.274 & 5342.000 \\
\hline 134 & Rhode Island & 176.810 & 317.000 \\
\hline 135 & South Carolina & 870.065 & 1019.000 \\
\hline 136 & South Dakota & 117.873 & 132.000 \\
\hline 137 & Tennessee & 3071.699 & 2979.000 \\
\hline 138 & Texas & 5476.116 & 7745.000 \\
\hline 139 & Utah & 572.385 & 483.000 \\
\hline 140 & Vermont & 29.968 & 245.000 \\
\hline 141 & Virginia & 1912.944 & 1934.000 \\
\hline 142 & Washington & 2141.699 & 2453.000 \\
\hline 143 & West Virginia & 13.985 & 164.000 \\
\hline 144 & Wisconsin & 2411.409 & 2322.000 \\
\hline 145 & Wyoming & 42.954 & 48.000 \\
\hline
\end{tabular}




\begin{tabular}{|c|c|c|c|}
\hline & State & P07 97 & A07 97 \\
\hline 1 & Alabama & 4405.448 & 5548.000 \\
\hline 2 & Arizona & 6435.272 & 7120.000 \\
\hline 3 & Arkansas & 6051.494 & 5219.000 \\
\hline 4 & California & 41801.783 & 42226.000 \\
\hline 5 & Colorado & 5435.851 & 6474.000 \\
\hline 6 & Connecticut & 1981.852 & 2906.000 \\
\hline 7 & DC & .000 & .000 \\
\hline 8 & Delaware & 471.727 & 1334.000 \\
\hline 9 & Florida & 19127.918 & 18561.000 \\
\hline 10 & Georgia & 12029.031 & 16057.000 \\
\hline 11 & Idaho & 3358.055 & 1522.000 \\
\hline 12 & Illinois_N & 18947.023 & 15173.000 \\
\hline 13 & Illinois_s & 8120.296 & 6503.000 \\
\hline 14 & Adams & 117.258 & 55.109 \\
\hline 15 & Allen & 643.314 & 506.542 \\
\hline 16 & Bartholomew & 158.337 & 227.643 \\
\hline 17 & Benton & 17.880 & 27.534 \\
\hline 18 & Blackford & 27.599 & 62.810 \\
\hline 19 & Boone & 88.974 & 42.688 \\
\hline 20 & Brown & 28.374 & 14.478 \\
\hline 21 & Carroll & 73.839 & 140.697 \\
\hline 22 & Cass & 111.935 & 159.572 \\
\hline 23 & Clark & 183.305 & 114.182 \\
\hline 24 & Clay & 49.579 & 25.159 \\
\hline 25 & Clinton & 106.621 & 183.510 \\
\hline 26 & Crawford & 19.597 & 9.709 \\
\hline 27 & Daviess & 76.549 & 98.372 \\
\hline 28 & Dearborn & 84.471 & 42.447 \\
\hline 29 & Decatur & 53.994 & 48.590 \\
\hline 30 & DeKalb & 109.046 & 59.121 \\
\hline 31 & Delaware & 252.502 & 168.209 \\
\hline 32 & Dubois & 89.984 & 114.895 \\
\hline 33 & Elkhart & 377.860 & 290.140 \\
\hline 34 & Fayette & 48.425 & 23.992 \\
\hline 35 & Floyd & 149.092 & 134.698 \\
\hline 36 & Fountain & 41.104 & 20.878 \\
\hline 37 & Franklin & 40.724 & 20.772 \\
\hline 38 & Fulton & 47.317 & 36.011 \\
\hline 39 & Gibson & 94.253 & 55.909 \\
\hline 40 & Grant & 156.924 & 131.358 \\
\hline 41 & Greene & 76.095 & 56.955 \\
\hline 42 & Hamilton & 319.873 & 162.746 \\
\hline 43 & Hancock & 100.803 & 69.854 \\
\hline 44 & Harrison & 83.720 & 81.556 \\
\hline 45 & Hendricks & 191.037 & 122.077 \\
\hline 46 & Henry & 92.825 & 55.789 \\
\hline 47 & Howard & 167.233 & 89.260 \\
\hline 48 & Huntington & 91.779 & 79.908 \\
\hline 49 & Jackson & 79.275 & 91.060 \\
\hline 50 & Jasper & 59.164 & 42.699 \\
\hline 51 & Jay & 49.946 & 57.564 \\
\hline
\end{tabular}




\begin{tabular}{|c|c|c|c|}
\hline & State & P07 97 & A07 97 \\
\hline 52 & Jefferson & 72.231 & 30.427 \\
\hline 53 & Jennings & 50.390 & 31.369 \\
\hline 54 & Johnson & 218.533 & 120.816 \\
\hline 55 & Knox & 77.011 & 52.411 \\
\hline 56 & Kosciusko & 173.563 & 132.421 \\
\hline 57 & LaGrange & 67.476 & 44.021 \\
\hline 58 & Lake & 934.276 & 571.806 \\
\hline 59 & La Porte & 223.437 & 192.992 \\
\hline 60 & Lawrence & 87.301 & 46.649 \\
\hline 61 & Madison & 265.030 & 194.431 \\
\hline 62 & Marion & 1691.067 & 1360.080 \\
\hline 63 & Marshall & 99.421 & 120.438 \\
\hline 64 & Martin & 20.838 & 13.894 \\
\hline 65 & Miami & 93.357 & 76.742 \\
\hline 66 & Monroe & 357.328 & 135.989 \\
\hline 67 & Montgomery & 104.040 & 79.433 \\
\hline 68 & Morgan & 121.522 & 60.804 \\
\hline 69 & Newton & 38.395 & 20.624 \\
\hline 70 & Noble & 134.830 & 112.492 \\
\hline 71 & Ohio & 10.390 & 5.148 \\
\hline 72 & Orange & 63.501 & 21.932 \\
\hline 73 & Owen & 39.889 & 20.358 \\
\hline 74 & Parke & 41.654 & 27.993 \\
\hline 75 & Perry & 49.215 & 18.429 \\
\hline 76 & Pike & 23.986 & 11.884 \\
\hline 77 & Porter & 280.162 & 156.376 \\
\hline 78 & Posey & 51.740 & 29.205 \\
\hline 79 & Pulaski & 40.221 & 18.550 \\
\hline 80 & Putnam & 65.830 & 33.211 \\
\hline 81 & Randolph & 52.985 & 30.833 \\
\hline 82 & Ripley & 48.984 & 43.539 \\
\hline 83 & Rush & 34.886 & 25.234 \\
\hline 84 & St. Joseph & 541.381 & 352.972 \\
\hline 85 & Scott & 51.894 & 46.973 \\
\hline 86 & Shelby & 86.083 & 53.081 \\
\hline 87 & Spencer & 39.212 & 22.998 \\
\hline 88 & Starke & 44.576 & 28.488 \\
\hline 89 & Steuben & 82.469 & 54.011 \\
\hline 90 & Sullivan & 40.224 & 19.929 \\
\hline 91 & Switzerland & 16.479 & 9.232 \\
\hline 92 & Tippecanoe & 288.852 & 186.782 \\
\hline 93 & Tipton & 34.657 & 33.985 \\
\hline 94 & Union & 13.958 & 6.741 \\
\hline 95 & Vanderburgh & 525.825 & 470.233 \\
\hline 96 & Vermillion & 31.578 & 34.797 \\
\hline 97 & Vigo & 206.486 & 171.823 \\
\hline 98 & Wabash & 68.661 & 76.558 \\
\hline 99 & Warren & 15.744 & 8.396 \\
\hline 100 & Warrick & 95.925 & 48.419 \\
\hline 101 & Washington & 53.520 & 29.039 \\
\hline 102 & Wayne & 174.888 & 140.891 \\
\hline
\end{tabular}




\begin{tabular}{|c|c|c|c|}
\hline & State & P07 97 & A07 97 \\
\hline 103 & Wells & 88.486 & 67.605 \\
\hline 104 & White & 74.383 & 32.656 \\
\hline 105 & Whitley & 79.172 & 47.371 \\
\hline 106 & lowa & 14785.434 & 6603.000 \\
\hline 107 & Kansas & 4274.524 & 4412.000 \\
\hline 108 & Kentucky_E & 3796.800 & 3884.000 \\
\hline 109 & Kentucky_W & 2531.533 & 2589.000 \\
\hline 110 & Louisiana & 6837.039 & 9727.000 \\
\hline 111 & Maine & 857.503 & 1220.000 \\
\hline 112 & Maryland & 7579.609 & 6371.000 \\
\hline 113 & Massachusetts & 4768.238 & 6221.000 \\
\hline 114 & Michigan_E & 6257.375 & 6518.000 \\
\hline 115 & Michigan_W & 6257.375 & 6518.000 \\
\hline 116 & Minnesota & 13187.360 & 10292.000 \\
\hline 117 & Missisippi & 3356.056 & 4336.000 \\
\hline 118 & Missouri & 8071.324 & 9630.000 \\
\hline 119 & Montana & 1004.418 & 1139.000 \\
\hline 120 & Nebraska & 3705.853 & 3208.000 \\
\hline 121 & Nevada & 1624.059 & 2089.000 \\
\hline 122 & New Hampshire & 1352.217 & 1184.000 \\
\hline 123 & New Jersey & 11930.088 & 13513.000 \\
\hline 124 & New Mexico & 538.688 & 824.000 \\
\hline 125 & New York & 21784.379 & 21844.000 \\
\hline 126 & North Carolina & 11752.191 & 14276.000 \\
\hline 127 & North Dakota & 2089.789 & 1104.000 \\
\hline 128 & Ohio $\mathbf{N}$ & 6972.960 & 5669.000 \\
\hline 129 & Ohio_M & 6972.960 & 5669.000 \\
\hline 130 & Ohio_s & 6972.960 & 5668.000 \\
\hline 131 & Oklahoma & 919.467 & 2952.000 \\
\hline 132 & Oregon & 4886.169 & 4735.000 \\
\hline 133 & Pennsylvania & 18364.361 & 20026.000 \\
\hline 134 & Rhode Island & 303.824 & 989.000 \\
\hline 135 & South Carolina & 3358.055 & 4696.000 \\
\hline 136 & South Dakota & 1260.270 & 1454.000 \\
\hline 137 & Tennessee & 12358.840 & 10844.000 \\
\hline 138 & Texas & 24064.059 & 25807.000 \\
\hline 139 & Utah & 1611.067 & 2467.000 \\
\hline 140 & Vermont & 455.736 & 632.000 \\
\hline 141 & Virginia & 5611.749 & 7013.000 \\
\hline 142 & Washington & 5789.646 & 5712.000 \\
\hline 143 & West Virginia & 390.774 & 1648.000 \\
\hline 144 & Wisconsin & 15078.265 & 13244.000 \\
\hline 145 & Wyoming & 539.687 & 389.000 \\
\hline
\end{tabular}




\begin{tabular}{|c|c|c|c|}
\hline & State & P08 97 & A08 97 \\
\hline 1 & Alabama & 482.989 & 690.000 \\
\hline 2 & Arizona & 1092.236 & 2095.000 \\
\hline 3 & Arkansas & 338.693 & 514.000 \\
\hline 4 & California & 12672.946 & 10838.000 \\
\hline 5 & Colorado & 6544.399 & 1424.000 \\
\hline 6 & Connecticut & 496.016 & 992.000 \\
\hline 7 & DC & .000 & .000 \\
\hline 8 & Delaware & 182.373 & 300.000 \\
\hline 9 & Florida & 4557.330 & 4886.000 \\
\hline 10 & Georgia & 1221.501 & 1886.000 \\
\hline 11 & Idaho & 228.468 & 409.000 \\
\hline 12 & Illinois $\mathbf{N}$ & 2498.114 & 3037.000 \\
\hline 13 & Illinois_S & 1071.193 & 1302.000 \\
\hline 14 & Adams & 13.478 & 17.001 \\
\hline 15 & Allen & 94.856 & 104.301 \\
\hline 16 & Bartholomew & 19.281 & 21.221 \\
\hline 17 & Benton & 1.654 & 2.123 \\
\hline 18 & Blackford & 2.438 & 3.129 \\
\hline 19 & Boone & 11.753 & 12.853 \\
\hline 20 & Brown & 2.676 & 4.599 \\
\hline 21 & Carroll & 3.471 & 4.455 \\
\hline 22 & Cass & 10.850 & 13.562 \\
\hline 23 & Clark & 17.297 & 21.857 \\
\hline 24 & Clay & 4.670 & 5.933 \\
\hline 25 & Clinton & 13.562 & 16.998 \\
\hline 26 & Crawford & 1.813 & 2.327 \\
\hline 27 & \begin{tabular}{|l} 
Daviess \\
\end{tabular} & 5.131 & 10.161 \\
\hline 28 & Dearborn & 7.898 & 60.302 \\
\hline 29 & Decatur & 4.252 & 5.457 \\
\hline 30 & DeKalb & 9.741 & 10.743 \\
\hline 31 & Delaware & 20.776 & 27.297 \\
\hline 32 & Dubois & 14.502 & 14.251 \\
\hline 33 & Elkhart & 53.594 & 55.477 \\
\hline 34 & Fayette & 4.480 & 5.750 \\
\hline 35 & Floyd & 18.468 & 21.227 \\
\hline 36 & Fountain & 3.733 & 5.652 \\
\hline 37 & Franklin & 3.748 & 4.811 \\
\hline 38 & Fulton & 3.612 & 12.928 \\
\hline 39 & Gibson & 7.835 & 8.910 \\
\hline 40 & Grant & 30.773 & 28.844 \\
\hline 41 & Greene & 6.366 & 7.805 \\
\hline 42 & Hamilton & 30.751 & 38.431 \\
\hline 43 & Hancock & 9.409 & 15.590 \\
\hline$\overline{44}$ & Harrison & 5.782 & 7.422 \\
\hline 45 & Hendricks & 18.586 & 22.994 \\
\hline 46 & Henry & 9.036 & 11.244 \\
\hline 47 & Howard & 15.283 & 23.569 \\
\hline 48 & Huntington & 22.244 & 19.254 \\
\hline 49 & Jackson & 7.069 & 9.073 \\
\hline 50 & Jasper & 6.840 & 7.714 \\
\hline 51 & Jay & 3.768 & 8.993 \\
\hline
\end{tabular}




\begin{tabular}{|c|c|c|c|}
\hline & State & P08 97 & A08 97 \\
\hline 52 & Jefferson & 5.421 & 7.650 \\
\hline 53 & Jennings & 4.662 & 5.983 \\
\hline 54 & Johnson & 24.890 & 29.672 \\
\hline 55 & Knox & 7.703 & 9.394 \\
\hline 56 & Kosciusko & 13.826 & 20.589 \\
\hline 57 & LaGrange & 6.517 & 7.999 \\
\hline 58 & Lake & 85.655 & 136.093 \\
\hline 59 & La Porte & 19.762 & 40.940 \\
\hline 60 & Lawrence & 8.545 & 14.759 \\
\hline 61 & Madison & 23.148 & 29.649 \\
\hline 62 & Marion & 166.902 & 294.744 \\
\hline 63 & Marshall & 7.802 & 22.281 \\
\hline 64 & Martin & 1.813 & 2.326 \\
\hline 65 & Miami & 6.178 & 8.359 \\
\hline 66 & Monroe & 31.063 & 38.262 \\
\hline 67 & Montgomery & 6.459 & 8.494 \\
\hline 68 & Morgan & 29.211 & 26.838 \\
\hline 69 & Newton & 4.324 & 4.484 \\
\hline 70 & Noble & 8.363 & 10.368 \\
\hline 71 & Ohio & .961 & 1.234 \\
\hline 72 & Orange & 3.313 & 4.945 \\
\hline 73 & Owen & 3.671 & 4.712 \\
\hline 74 & Parke & 2.947 & 4.475 \\
\hline 75 & Perry & 3.414 & 4.320 \\
\hline 76 & Pike & 2.219 & 2.848 \\
\hline 77 & Porter & 26.144 & 33.083 \\
\hline 78 & Posey & 4.671 & 5.995 \\
\hline 79 & Pulaski & 2.365 & 3.036 \\
\hline 80 & Putnam & 7.869 & 9.035 \\
\hline 81 & Randolph & 5.370 & 6.732 \\
\hline 82 & Ripley & 5.129 & 8.136 \\
\hline 83 & Rush & 3.280 & 4.353 \\
\hline 84 & St. Joseph & 55.082 & 66.005 \\
\hline 85 & Scott & 3.885 & 9.143 \\
\hline 86 & Shelby & 7.432 & 9.539 \\
\hline 87 & Spencer & 3.512 & 4.508 \\
\hline 88 & Starke & 4.124 & 5.293 \\
\hline 89 & Steuben & 6.223 & 7.622 \\
\hline 90 & Sullivan & 3.721 & 6.002 \\
\hline 91 & Switzerland & 1.524 & 1.957 \\
\hline 92 & Tippecanoe & 37.542 & 41.132 \\
\hline 93 & Tipton & 2.870 & 3.684 \\
\hline 94 & Union & 1.259 & 1.615 \\
\hline 95 & Vanderburgh & 29.792 & 51.526 \\
\hline 96 & Vermillion & 2.906 & 3.730 \\
\hline 97 & Vigo & 36.418 & 36.293 \\
\hline 98 & Wabash & 11.468 & 11.505 \\
\hline 99 & Warren & 1.437 & 1.845 \\
\hline 100 & Warrick & 8.841 & 11.348 \\
\hline 101 & Washington & 4.640 & 7.874 \\
\hline 102 & Wayne & 21.929 & 23.387 \\
\hline
\end{tabular}




\begin{tabular}{|c|c|c|c|}
\hline & State & P08 97 & A08 97 \\
\hline 103 & Wells & 4.860 & 6.177 \\
\hline 104 & White & 6.153 & 6.833 \\
\hline 105 & Whitley & 7.006 & 7.939 \\
\hline 106 & lowa & 2108.316 & 917.000 \\
\hline 107 & Kansas & 547.120 & 583.000 \\
\hline 108 & Kentucky_E & 552.130 & 665.000 \\
\hline 109 & Kentucky_W & 367.753 & 445.000 \\
\hline 110 & Louisiana & 465.954 & 1308.000 \\
\hline 111 & Maine & 167.343 & 297.000 \\
\hline 112 & Maryland & 855.752 & 1206.000 \\
\hline 113 & Massachusetts & 1417.903 & 2455.000 \\
\hline 114 & Michigan_E & 662.356 & 1299.000 \\
\hline 115 & Michigan W & 662.356 & 1299.000 \\
\hline 116 & Minnesota & 1076.203 & 1486.000 \\
\hline 117 & Missisippi & 369.757 & 523.000 \\
\hline 118 & Missouri & 3770.720 & 1687.000 \\
\hline 119 & Montana & 170.349 & 266.000 \\
\hline 120 & Nebraska & 337.691 & 360.000 \\
\hline 121 & Nevada & 468.960 & 820.000 \\
\hline 122 & New Hampshire & 705.444 & 273.000 \\
\hline 123 & New Jersey & 2865.867 & 2421.000 \\
\hline 124 & New Mexico & 316.648 & 604.000 \\
\hline 125 & New York & 5911.102 & 3507.000 \\
\hline 126 & North Carolina & 1698.477 & 1691.000 \\
\hline 127 & North Dakota & 105.215 & 189.000 \\
\hline 128 & Ohio_N & 966.980 & 1142.000 \\
\hline 129 & Ohio M & 966.980 & 1142.000 \\
\hline 130 & Ohio_s & 966.980 & 1142.000 \\
\hline 131 & Oklahoma & 544.114 & 818.000 \\
\hline 132 & Oregon & 1056.162 & 1638.000 \\
\hline 133 & Pennsylvania & 2519.158 & 3161.000 \\
\hline 134 & Rhode Island & 362.743 & 861.000 \\
\hline 135 & South Carolina & 737.510 & 1215.000 \\
\hline 136 & South Dakota & 388.796 & 448.000 \\
\hline 137 & Tennessee & 1477.024 & 2098.000 \\
\hline 138 & Texas & 6044.375 & 5940.000 \\
\hline 139 & Utah & 227.466 & 324.000 \\
\hline 140 & Vermont & 68.140 & 224.000 \\
\hline 141 & Virginia & 3316.791 & 2963.000 \\
\hline 142 & Washington & 1329.722 & 1090.000 \\
\hline 143 & West Virginia & 351.720 & 591.000 \\
\hline 144 & Wisconsin & 1317.698 & 1676.000 \\
\hline 145 & Wyoming & 131.269 & 123.000 \\
\hline
\end{tabular}




\begin{tabular}{|c|c|c|c|}
\hline & State & P09 97 & A09 97 \\
\hline 1 & Alabama & 50.765 & 71.000 \\
\hline 2 & Arizona & 15.926 & 28.000 \\
\hline 3 & Arkansas & 27.871 & 22.000 \\
\hline 4 & California & 138.360 & 196.000 \\
\hline 5 & Colorado & 9.954 & 9.000 \\
\hline$\overline{6}$ & Connecticut & 25.880 & 25.000 \\
\hline$\overline{7}$ & $\mathrm{DC}$ & .000 & .000 \\
\hline 8 & Delaware & 5.972 & 3.000 \\
\hline$\overline{9}$ & Florida & 43.797 & 78.000 \\
\hline 10 & Georgia & 10.949 & 55.000 \\
\hline 11 & Idaho & .995 & 3.000 \\
\hline 12 & Illinois_N & 86.599 & 57.000 \\
\hline 13 & Illinois_S & 37.825 & 24.000 \\
\hline 14 & Adams & .285 & .442 \\
\hline 15 & Allen & 1.100 & 1.708 \\
\hline 16 & Bartholomew & .808 & 1.252 \\
\hline 17 & Benton & .065 & .100 \\
\hline 18 & Blackford & .011 & .017 \\
\hline$\overline{19}$ & Boone & .065 & .100 \\
\hline 20 & Brown & .000 & .000 \\
\hline 21 & Carroll & .404 & .626 \\
\hline 22 & Cass & .011 & .017 \\
\hline 23 & Clark & 1.205 & 1.868 \\
\hline 24 & Clay & .011 & .017 \\
\hline 25 & Clinton & .065 & .100 \\
\hline 26 & Crawford & .000 & .000 \\
\hline 27 & Daviess & .065 & .100 \\
\hline 28 & Dearborn & .000 & .000 \\
\hline 29 & Decatur & .011 & .017 \\
\hline 30 & DeKalb & .011 & .017 \\
\hline 31 & Delaware & .152 & .235 \\
\hline 32 & Dubois & 7.097 & 11.002 \\
\hline 33 & Elkhart & 4.170 & 6.467 \\
\hline 34 & Fayette & .065 & .100 \\
\hline 35 & Floyd & .477 & .740 \\
\hline 36 & Fountain & .000 & .000 \\
\hline 37 & Franklin & .000 & .000 \\
\hline 38 & Fulton & .000 & .000 \\
\hline 39 & Gibson & .000 & .000 \\
\hline 40 & Grant & .188 & .292 \\
\hline 41 & Greene & .011 & .017 \\
\hline 42 & Hamilton & .300 & .469 \\
\hline 43 & Hancock & .065 & .100 \\
\hline 44 & Harrison & .699 & 1.083 \\
\hline 45 & Hendricks & .011 & .017 \\
\hline 46 & Henry & .188 & .292 \\
\hline 47 & Howard & .404 & .626 \\
\hline 48 & Huntington & .000 & .000 \\
\hline 49 & Jackson & .065 & .100 \\
\hline 50 & Jasper & .011 & .017 \\
\hline 51 & Jay & .065 & .100 \\
\hline
\end{tabular}




\begin{tabular}{|c|c|c|c|}
\hline & State & P09 97 & A09 97 \\
\hline 52 & Jefferson & .011 & .017 \\
\hline 53 & Jennings & .000 & .000 \\
\hline 54 & Johnson & .094 & .145 \\
\hline 55 & Knox & .011 & .017 \\
\hline 56 & Kosciusko & .349 & .544 \\
\hline 57 & LaGrange & .324 & .503 \\
\hline 58 & Lake & .272 & .422 \\
\hline 59 & La Porte & .305 & .492 \\
\hline 60 & Lawrence & .011 & .017 \\
\hline 61 & Madison & .462 & .720 \\
\hline 62 & Marion & 1.480 & 2.314 \\
\hline 63 & Marshall & .065 & .100 \\
\hline 64 & Martin & .000 & .000 \\
\hline 65 & Miami & .404 & .626 \\
\hline 66 & Monroe & .075 & .117 \\
\hline 67 & Montgomery & .000 & .000 \\
\hline 68 & Morgan & .011 & .017 \\
\hline 69 & Newton & .065 & .100 \\
\hline 70 & Noble & .065 & .100 \\
\hline 71 & Ohio & .000 & .000 \\
\hline 72 & Orange & 1.885 & 2.922 \\
\hline 73 & Owen & .000 & .000 \\
\hline 74 & Parke & .011 & .017 \\
\hline 75 & Perry & .316 & .489 \\
\hline 76 & Pike & .000 & .000 \\
\hline 77 & Porter & .171 & .285 \\
\hline 78 & Posey & .000 & .000 \\
\hline 79 & Pulaski & .011 & .017 \\
\hline 80 & Putnam & .000 & .000 \\
\hline 81 & Randolph & .127 & .217 \\
\hline 82 & Ripley & .188 & .292 \\
\hline 83 & Rush & .000 & .000 \\
\hline 84 & St. Joseph & .200 & .314 \\
\hline 85 & Scott & .065 & .100 \\
\hline 86 & Shelby & .358 & .554 \\
\hline 87 & Spencer & .808 & 1.252 \\
\hline 88 & Starke & .011 & .017 \\
\hline 89 & Steuben & .011 & .017 \\
\hline 90 & Sullivan & .000 & .000 \\
\hline 91 & Switzerland & .011 & .017 \\
\hline 92 & Tippecanoe & .808 & 1.252 \\
\hline 93 & Tipton & .000 & .000 \\
\hline 94 & Union & .000 & .000 \\
\hline 95 & Vanderburgh & .451 & .703 \\
\hline 96 & Vermillion & .000 & .000 \\
\hline 97 & Vigo & .065 & .100 \\
\hline 98 & Wabash & .011 & .017 \\
\hline 99 & Warren & .000 & .000 \\
\hline 100 & Warrick & .011 & .017 \\
\hline 101 & Washington & 1.885 & 2.922 \\
\hline 102 & Wayne & .769 & 1.192 \\
\hline
\end{tabular}




\begin{tabular}{|c|c|c|c|}
\hline & State & P09 97 & A09 97 \\
\hline 103 & Wells & .030 & .050 \\
\hline 104 & White & .528 & .876 \\
\hline 105 & Whitley & .047 & .073 \\
\hline 106 & lowa & 23.890 & 35.000 \\
\hline 107 & Kansas & 7.963 & 29.000 \\
\hline 108 & Kentucky_E & 150.305 & 83.000 \\
\hline 109 & |Kentucky_W & 99.540 & 56.000 \\
\hline 110 & Louisiana & 2.986 & 63.000 \\
\hline 111 & Maine & 17.917 & 35.000 \\
\hline 112 & Maryland & 9.954 & 12.000 \\
\hline 113 & Massachusetts & 64.701 & 54.000 \\
\hline 114 & Michigan_E & 31.853 & 29.000 \\
\hline 115 & Michigan_W & 31.853 & 29.000 \\
\hline 116 & Minnesota & 37.825 & 43.000 \\
\hline 117 & Missisippi & 6.968 & 21.000 \\
\hline 118 & Missouri & 111.484 & 136.000 \\
\hline 119 & Montana & 1.991 & 6.000 \\
\hline 120 & Nebraska & 12.940 & 13.000 \\
\hline 121 & Nevada & 12.940 & 14.000 \\
\hline 122 & New Hampshire & 10.949 & 15.000 \\
\hline 123 & New Jersey & 49.770 & 111.000 \\
\hline 124 & New Mexico & .995 & 2.000 \\
\hline 125 & New York & 50.765 & 103.000 \\
\hline 126 & North Carolina & 2041.558 & 1788.000 \\
\hline 127 & North Dakota & 2.986 & 7.000 \\
\hline 128 & Ohio_N & 16.922 & 25.000 \\
\hline 129 & Ohio_M & 16.922 & 25.000 \\
\hline 130 & Ohio_s & 16.922 & 25.000 \\
\hline 131 & Oklahoma & 1.991 & 33.000 \\
\hline 132 & Oregon & 11.945 & 12.000 \\
\hline 133 & Pennsylvania & 153.291 & 129.000 \\
\hline 134 & Rhode Island & .000 & 9.000 \\
\hline 135 & South Carolina & 4.977 & 12.000 \\
\hline 136 & South Dakota & .995 & 2.000 \\
\hline 137 & Tennessee & 114.471 & 89.000 \\
\hline 138 & Texas & 28.866 & 73.000 \\
\hline 139 & Utah & .000 & 10.000 \\
\hline 140 & Vermont & 2.986 & 13.000 \\
\hline 141 & Virginia & 426.030 & 243.000 \\
\hline 142 & Washington & 7.963 & 46.000 \\
\hline 143 & West Virginia & 18.913 & 19.000 \\
\hline 144 & Wisconsin & 14.931 & 37.000 \\
\hline 145 & Wyoming & 1.991 & 3.000 \\
\hline
\end{tabular}




\begin{tabular}{|c|c|c|c|}
\hline & State & P10 97 & A10 97 \\
\hline 1 & Alabama & 259.000 & 308.278 \\
\hline 2 & Arizona & 21.000 & 148.580 \\
\hline 3 & Arkansas & 35.000 & 5.054 \\
\hline 4 & California & 2021.000 & 1425.155 \\
\hline 5 & Colorado & 90.000 & 110.172 \\
\hline 6 & Connecticut & 211.000 & 352.751 \\
\hline 7 & Delaware & .000 & 159.698 \\
\hline 8 & $\mathrm{DC}$ & .000 & .000 \\
\hline 9 & Florida & 850.000 & 440.686 \\
\hline 10 & Georgia & 333.000 & 240.558 \\
\hline 11 & Idaho & 75.000 & 1.011 \\
\hline 12 & Illinois_N & 607.000 & 835.889 \\
\hline 13 & Illinois_S & 260.000 & 357.805 \\
\hline 14 & Adams & 4.544 & 3.332 \\
\hline 15 & Allen & 39.038 & 7.140 \\
\hline 16 & Bartholomew & 5.304 & 3.332 \\
\hline 17 & Benton & 1.075 & .000 \\
\hline 18 & Blackford & 1.666 & .000 \\
\hline 19 & Boone & 2.749 & .000 \\
\hline 20 & Brown & .598 & .190 \\
\hline 21 & Carroll & 1.481 & .000 \\
\hline 22 & Cass & 4.091 & .000 \\
\hline 23 & Clark & 6.135 & 12.929 \\
\hline 24 & Clay & 1.700 & 1.142 \\
\hline 25 & Clinton & 2.305 & 3.332 \\
\hline 26 & Crawford & .391 & .000 \\
\hline 27 & Daviess & 1.919 & 1.142 \\
\hline 28 & Dearborn & 2.222 & .190 \\
\hline 29 & Decatur & 3.133 & .000 \\
\hline 30 & DeKalb & 7.096 & 4.303 \\
\hline 31 & Delaware & 7.064 & 1.619 \\
\hline 32 & Dubois & 5.616 & 1.142 \\
\hline 33 & Elkhart & 24.614 & 33.322 \\
\hline 34 & Fayette & 1.639 & .000 \\
\hline 35 & Floyd & 7.990 & 1.942 \\
\hline 36 & Fountain & 1.111 & .190 \\
\hline 37 & Franklin & 1.485 & .000 \\
\hline 38 & Fulton & 1.604 & .190 \\
\hline 39 & Gibson & 2.487 & .000 \\
\hline 40 & Grant & 5.126 & 1.809 \\
\hline 41 & Greene & 1.581 & .000 \\
\hline 42 & Hamilton & 15.484 & 1.142 \\
\hline 43 & Hancock & 3.702 & .000 \\
\hline 44 & Harrison & 2.030 & .933 \\
\hline 45 & Hendricks & 4.958 & .190 \\
\hline 46 & Henry & 3.637 & .000 \\
\hline 47 & Howard & 5.118 & .000 \\
\hline 48 & Huntington & 3.209 & 1.142 \\
\hline 49 & Jackson & 3.863 & 3.332 \\
\hline 50 & Jasper & 1.772 & .000 \\
\hline 51 & Jay & 2.029 & .000 \\
\hline
\end{tabular}




\begin{tabular}{|c|c|c|c|}
\hline & State & P10 97 & A10 97 \\
\hline 52 & Jefferson & 1.816 & 1.142 \\
\hline 53 & Jennings & 2.935 & .190 \\
\hline 54 & Johnson & 6.843 & 1.142 \\
\hline 55 & Knox & 3.246 & .000 \\
\hline 56 & Kosciusko & 6.193 & 6.322 \\
\hline 57 & LaGrange & 4.203 & .190 \\
\hline 58 & Lake & 27.666 & 28.410 \\
\hline 59 & La Porte & 9.243 & 6.303 \\
\hline 60 & Lawrence & 2.568 & .000 \\
\hline 61 & Madison & 7.562 & 7.140 \\
\hline 62 & Marion & 83.410 & 115.276 \\
\hline 63 & Marshall & 8.239 & 2.475 \\
\hline 64 & Martin & .651 & .000 \\
\hline 65 & Miami & 4.045 & 1.142 \\
\hline 66 & Monroe & 9.438 & 3.104 \\
\hline 67 & Montgomery & 3.669 & 3.332 \\
\hline 68 & Morgan & 2.935 & 7.140 \\
\hline 69 & Newton & 2.047 & .190 \\
\hline 70 & Noble & 7.378 & 4.113 \\
\hline 71 & Ohio & .207 & .000 \\
\hline 72 & Orange & 1.378 & .000 \\
\hline 73 & Owen & .859 & .000 \\
\hline 74 & Parke & 1.312 & .000 \\
\hline 75 & Perry & .902 & .000 \\
\hline 76 & Pike & .658 & .000 \\
\hline 77 & Porter & 9.595 & 8.816 \\
\hline 78 & Posey & 2.512 & 33.322 \\
\hline 79 & Pulaski & 1.599 & .000 \\
\hline 80 & Putnam & 4.360 & .000 \\
\hline 81 & Randolph & 1.421 & .000 \\
\hline 82 & Ripley & 1.742 & 1.142 \\
\hline 83 & Rush & 1.174 & .000 \\
\hline 84 & St. Joseph & 28.150 & 16.375 \\
\hline 85 & Scott & 3.441 & 3.332 \\
\hline 86 & Shelby & 7.051 & 3.656 \\
\hline 87 & Spencer & 2.659 & .190 \\
\hline 88 & Starke & 1.502 & 1.142 \\
\hline 89 & Steuben & 2.844 & .000 \\
\hline 90 & Sullivan & 1.840 & .190 \\
\hline 91 & Switzerland & .792 & .000 \\
\hline 92 & Tippecanoe & 9.562 & 31.266 \\
\hline 93 & \begin{tabular}{|l} 
Tipton \\
\end{tabular} & 1.232 & .190 \\
\hline 94 & Union & .527 & .819 \\
\hline 95 & Vanderburgh & 20.705 & 44.633 \\
\hline 96 & Vermillion & .955 & 33.322 \\
\hline 97 & Vigo & 11.112 & 19.613 \\
\hline 98 & Wabash & 2.082 & 7.140 \\
\hline 99 & Warren & .589 & .000 \\
\hline 100 & Warrick & 2.723 & 1.523 \\
\hline 101 & Washington & 1.582 & .000 \\
\hline 102 & Wayne & 7.247 & 2.647 \\
\hline
\end{tabular}




\begin{tabular}{|c|c|c|c|}
\hline & State & P10 97 & A10 97 \\
\hline 103 & Wells & 9.275 & .000 \\
\hline 104 & White & 3.133 & .000 \\
\hline 105 & Whitley & 2.625 & .190 \\
\hline 106 & lowa & 209.000 & 154.644 \\
\hline 107 & Kansas & 182.000 & 146.558 \\
\hline 108 & Kentucky_E & 148.000 & 18.193 \\
\hline 109 & Kentucky_W & 99.000 & 12.129 \\
\hline 110 & Louisiana & .000 & 18.193 \\
\hline 111 & Maine & 74.000 & 38.408 \\
\hline 112 & Maryland & 65.000 & 312.321 \\
\hline 113 & Massachusetts & 405.000 & 267.848 \\
\hline 114 & Michigan_E & 224.000 & 254.708 \\
\hline 115 & Michigan_W & 224.000 & 254.708 \\
\hline 116 & Minnesota & 346.000 & 321.418 \\
\hline 117 & Missisippi & .000 & 17.183 \\
\hline 118 & \begin{tabular}{|l} 
Missouri \\
\end{tabular} & 367.000 & 491.223 \\
\hline 119 & Montana & .000 & 11.118 \\
\hline 120 & Nebraska & 120.000 & 61.656 \\
\hline 121 & Nevada & .000 & 28.301 \\
\hline 122 & New Hampshire & 72.000 & 59.634 \\
\hline 123 & New Jersey & 2140.000 & 2110.442 \\
\hline 124 & New Mexico & 15.000 & 7.075 \\
\hline 125 & New York & 1054.000 & 1041.070 \\
\hline 126 & North Carolina & 501.000 & 967.286 \\
\hline 127 & North Dakota & 47.000 & 1.011 \\
\hline 128 & Ohio $\mathrm{N}$ & 264.000 & 352.751 \\
\hline 129 & Ohio_M & 264.000 & 352.751 \\
\hline 130 & Ohio_s & 263.000 & 352.751 \\
\hline 131 & Oklahoma & 198.000 & 83.892 \\
\hline 132 & Oregon & 208.000 & 98.043 \\
\hline 133 & Pennsylvania & 724.000 & 290.085 \\
\hline 134 & Rhode Island & 60.000 & 5.054 \\
\hline 135 & South Carolina & .000 & 833.867 \\
\hline 136 & South Dakota & 49.000 & 16.172 \\
\hline 137 & Tennessee & 277.000 & 144.537 \\
\hline 138 & Texas & 448.000 & 540.750 \\
\hline 139 & Utah & 120.000 & 19.204 \\
\hline 140 & Vermont & 285.000 & 175.870 \\
\hline 141 & Virginia & 364.000 & 432.600 \\
\hline 142 & Washington & 342.000 & 117.247 \\
\hline 143 & West Virginia & 82.000 & 307.267 \\
\hline 144 & Wisconsin & 356.000 & 280.988 \\
\hline 145 & Wyoming & .000 & 27.290 \\
\hline
\end{tabular}




\begin{tabular}{|c|c|c|c|}
\hline & State & P11 97 & A11 97 \\
\hline 1 & Alabama & 11337.540 & 11328.000 \\
\hline 2 & Arizona & 8427.198 & 8612.000 \\
\hline 3 & Arkansas & 5327.964 & 6746.000 \\
\hline 4 & California & 38670.965 & 39163.000 \\
\hline 5 & Colorado & 4754.291 & 5058.000 \\
\hline 6 & Connecticut & 2573.534 & 4286.000 \\
\hline 7 & Delaware & 1608.084 & 1118.000 \\
\hline 8 & DC & .000 & .000 \\
\hline 9 & Florida & 24852.839 & 24741.000 \\
\hline 10 & Georgia & 4350.521 & 4869.000 \\
\hline 11 & Idaho & 2723.448 & 3186.000 \\
\hline 12 & Illinois_N & 33608.849 & 22123.000 \\
\hline 13 & Illinois_s & 14403.793 & 9481.000 \\
\hline 14 & Adams & 12.543 & 44.756 \\
\hline 15 & Allen & 721.387 & 436.863 \\
\hline 16 & Bartholomew & 62.990 & 94.321 \\
\hline 17 & Benton & 2.490 & 12.833 \\
\hline 18 & Blackford & 6.179 & 18.914 \\
\hline 19 & Boone & 34.123 & 59.280 \\
\hline 20 & Brown & .922 & 19.962 \\
\hline 21 & Carroll & 3.966 & 26.931 \\
\hline 22 & Cass & 25.915 & 54.272 \\
\hline 23 & Clark & 103.754 & 127.015 \\
\hline 24 & Clay & 2.582 & 35.433 \\
\hline 25 & Clinton & 13.557 & 45.410 \\
\hline 26 & Crawford & .922 & 14.065 \\
\hline 27 & Daviess & 8.946 & 39.808 \\
\hline 28 & Dearborn & 14.941 & 60.476 \\
\hline 29 & Decatur & 10.975 & 32.988 \\
\hline 30 & DeKalb & 27.668 & 52.523 \\
\hline 31 & Delaware & 94.715 & 160.383 \\
\hline 32 & Dubois & 107.074 & 52.696 \\
\hline 33 & Elkhart & 403.302 & 238.752 \\
\hline 34 & Fayette & 7.747 & 34.754 \\
\hline 35 & Floyd & 55.981 & 94.788 \\
\hline 36 & Fountain & 2.306 & 24.179 \\
\hline 37 & Franklin & 5.534 & 29.078 \\
\hline 38 & Fulton & 4.335 & 27.223 \\
\hline 39 & Gibson & 9.130 & 43.085 \\
\hline 40 & Grant & 44.729 & 99.189 \\
\hline 41 & Greene & 7.931 & 44.600 \\
\hline 42 & Hamilton & 292.262 & 222.303 \\
\hline 43 & Hancock & 15.955 & 72.197 \\
\hline 44 & Harrison & 16.877 & 44.861 \\
\hline 45 & Hendricks & 76.086 & 130.240 \\
\hline 46 & Henry & 19.828 & 65.474 \\
\hline 47 & Howard & 56.903 & 113.227 \\
\hline 48 & Huntington & 20.658 & 50.797 \\
\hline 49 & Jackson & 23.425 & 54.841 \\
\hline 50 & Jasper & 11.251 & 39.111 \\
\hline 51 & Jay & 5.626 & 29.234 \\
\hline
\end{tabular}




\begin{tabular}{|c|c|c|c|}
\hline & State & P11_97 & A11 97 \\
\hline 52 & Jefferson & 14.848 & 42.054 \\
\hline 53 & Jennings & 6.456 & 36.165 \\
\hline 54 & Johnson & 53.399 & 147.248 \\
\hline 55 & Knox & 33.662 & 53.304 \\
\hline 56 & Kosciusko & 46.943 & 97.691 \\
\hline 57 & LaGrange & 22.319 & 45.776 \\
\hline 58 & Lake & 480.125 & 650.802 \\
\hline 59 & La Porte & 76.455 & 147.577 \\
\hline 60 & Lawrence & 14.756 & 61.509 \\
\hline 61 & Madison & 52.937 & 178.788 \\
\hline 62 & Marion & 2023.425 & 1146.358 \\
\hline 63 & Marshall & 45.098 & 59.732 \\
\hline 64 & Martin & 2.029 & 14.062 \\
\hline 65 & Miami & 17.984 & 47.133 \\
\hline 66 & Monroe & 49.986 & 160.132 \\
\hline 67 & Montgomery & 14.756 & 50.109 \\
\hline 68 & Morgan & 29.973 & 87.067 \\
\hline 69 & Newton & 2.767 & 19.589 \\
\hline 70 & Noble & 18.722 & 60.094 \\
\hline 71 & Ohio & .000 & 7.457 \\
\hline 72 & Orange & 4.150 & 25.705 \\
\hline 73 & Owen & 5.534 & 28.480 \\
\hline 74 & Parke & 5.534 & 22.861 \\
\hline 75 & Perry & 5.349 & 25.685 \\
\hline 76 & Pike & 7.378 & 17.215 \\
\hline 77 & Porter & 125.150 & 193.974 \\
\hline 78 & Posey & 21.027 & 36.240 \\
\hline 79 & Pulaski & 9.407 & 18.352 \\
\hline 80 & Putnam & 4.611 & 47.097 \\
\hline 81 & Randolph & 10.053 & 36.880 \\
\hline 82 & Ripley & 34.585 & 35.007 \\
\hline 83 & Rush & 10.606 & 24.650 \\
\hline 84 & St. Joseph & 412.893 & 354.126 \\
\hline 85 & Scott & 5.810 & 30.141 \\
\hline 86 & Shelby & 65.664 & 57.657 \\
\hline 87 & Spencer & 6.456 & 27.249 \\
\hline 88 & Starke & 3.873 & 31.992 \\
\hline 89 & Steuben & 18.998 & 43.493 \\
\hline 90 & Sullivan & 5.257 & 28.869 \\
\hline 91 & Switzerland & .922 & 11.827 \\
\hline 92 & Tippecanoe & 63.820 & 196.199 \\
\hline 93 & Tipton & 6.640 & 22.267 \\
\hline 94 & Union & 5.534 & 9.764 \\
\hline 95 & Vanderburgh & 325.002 & 230.336 \\
\hline 96 & Vermillion & 1.014 & 22.544 \\
\hline 97 & Vigo & 85.862 & 142.983 \\
\hline 98 & Wabash & 14.941 & 46.866 \\
\hline 99 & Warren & 5.534 & 11.151 \\
\hline 100 & Warrick & 12.082 & 68.592 \\
\hline 101 & Washington & 7.562 & 36.000 \\
\hline 102 & Wayne & 96.376 & 96.042 \\
\hline
\end{tabular}




\begin{tabular}{|c|c|c|c|}
\hline & State & P11_97 & A11 97 \\
\hline 103 & Wells & 9.499 & 36.906 \\
\hline 104 & White & 12.543 & 33.784 \\
\hline 105 & Whitley & 16.324 & 40.556 \\
\hline 106 & lowa & 11155.643 & 11873.000 \\
\hline 107 & Kansas & 13952.050 & 9939.000 \\
\hline 108 & Kentucky_E & 7068.972 & 5020.000 \\
\hline 109 & Kentucky_W & 4712.315 & 3347.000 \\
\hline 110 & Louisiana & 11038.710 & 11466.000 \\
\hline 111 & Maine & 2414.624 & 3254.000 \\
\hline 112 & Maryland & 13069.553 & 13376.000 \\
\hline 113 & Massachusetts & 8477.170 & 8261.000 \\
\hline 114 & Michigan_E & 8531.139 & 7483.000 \\
\hline 115 & Michigan W & 8531.139 & 7483.000 \\
\hline 116 & Minnesota & 12051.133 & 12079.000 \\
\hline 117 & Missisippi & 4734.302 & 7202.000 \\
\hline 118 & Missouri & 2497.577 & 5968.000 \\
\hline 119 & Montana & 1874.932 & 53.000 \\
\hline 120 & Nebraska & 4970.168 & 4374.000 \\
\hline 121 & Nevada & 4389.499 & 4575.000 \\
\hline 122 & New Hampshire & 815.535 & 369.000 \\
\hline 123 & New Jersey & 33886.691 & 21316.000 \\
\hline 124 & New Mexico & 177.899 & 4603.000 \\
\hline 125 & New York & 5046.125 & 9076.000 \\
\hline 126 & North Carolina & 12749.735 & 12113.000 \\
\hline 127 & North Dakota & 1956.885 & 1685.000 \\
\hline 128 & Ohio_N & 1244.291 & 9750.000 \\
\hline 129 & Ohio_M & 1243.292 & 9749.000 \\
\hline 130 & Ohio_S & 1243.292 & 9749.000 \\
\hline 131 & Oklahoma & 8525.142 & 8748.000 \\
\hline 132 & Oregon & 3241.153 & 3285.000 \\
\hline 133 & Pennsylvania & 11087.682 & 15928.000 \\
\hline 134 & Rhode Island & 2880.359 & 2659.000 \\
\hline 135 & South Carolina & 5904.635 & 4865.000 \\
\hline 136 & South Dakota & 1937.896 & 1930.000 \\
\hline 137 & \begin{tabular}{|l|} 
Tennessee \\
\end{tabular} & 16966.332 & 5852.000 \\
\hline 138 & Texas & 23585.561 & 25280.000 \\
\hline 139 & Utah & 6531.278 & 5498.000 \\
\hline 140 & Vermont & 1137.352 & 348.000 \\
\hline 141 & Virginia & 8745.017 & 9253.000 \\
\hline 142 & Washington & 4928.192 & 4851.000 \\
\hline 143 & West Virginia & 792.548 & 1036.000 \\
\hline 144 & Wisconsin & 8752.013 & 8528.000 \\
\hline 145 & Wyoming & .000 & 1269.000 \\
\hline
\end{tabular}




\begin{tabular}{|c|c|c|c|}
\hline & State & P12 97 & A12 97 \\
\hline 1 & Alabama & 36191.948 & 33018.000 \\
\hline 2 & Arizona & 17410.835 & 22365.000 \\
\hline 3 & Arkansas & 19596.684 & 16020.000 \\
\hline 4 & California & 158887.361 & 159098.000 \\
\hline 5 & Colorado & 13651.814 & 14502.000 \\
\hline 6 & Connecticut & 9898.789 & 10876.000 \\
\hline 7 & Delaware & 11781.798 & 4262.000 \\
\hline 8 & DC & .000 & .000 \\
\hline 9 & Florida & 65627.453 & 66685.000 \\
\hline 10 & Georgia & 110374.898 & 112201.000 \\
\hline 11 & Idaho & 9173.171 & 9129.000 \\
\hline 12 & Illinois_N & 48963.226 & 52726.000 \\
\hline 13 & lllinois_S & 20983.954 & 22597.000 \\
\hline 14 & Adams & 1257.523 & 306.533 \\
\hline 15 & Allen & 5758.001 & 3318.648 \\
\hline 16 & Bartholomew & 1490.093 & 1157.045 \\
\hline 17 & Benton & 62.095 & 52.704 \\
\hline 18 & Blackford & 421.037 & 23.054 \\
\hline 19 & Boone & 15.840 & 3.842 \\
\hline 20 & Brown & 5.491 & 3.842 \\
\hline 21 & Carroll & 960.860 & 672.414 \\
\hline 22 & Cass & 1751.238 & 703.931 \\
\hline 23 & Clark & 627.232 & 296.171 \\
\hline 24 & Clay & 186.601 & 12.626 \\
\hline 25 & Clinton & 1328.103 & 919.576 \\
\hline 26 & Crawford & .000 & .000 \\
\hline 27 & Daviess & 619.943 & 390.384 \\
\hline 28 & Dearborn & 67.586 & 1416.447 \\
\hline 29 & Decatur & 591.247 & 150.952 \\
\hline 30 & DeKalb & 2224.894 & 2116.267 \\
\hline 31 & Delaware & 335.344 & 709.355 \\
\hline 32 & Dubois & 1499.678 & 318.654 \\
\hline 33 & Elkhart & 7137.863 & 1994.686 \\
\hline 34 & Fayette & .000 & 52.704 \\
\hline 35 & Floyd & 1902.837 & 287.356 \\
\hline 36 & Fountain & 32.944 & 681.850 \\
\hline 37 & Franklin & 186.601 & 3.842 \\
\hline 38 & Fulton & 229.993 & 408.439 \\
\hline 39 & Gibson & 593.992 & 144.089 \\
\hline 40 & Grant & 786.399 & 327.739 \\
\hline 41 & Greene & 220.090 & 309.841 \\
\hline 42 & Hamilton & 1502.212 & 662.227 \\
\hline 43 & Hancock & 24.119 & 12.626 \\
\hline 44 & Harrison & 579.181 & 278.572 \\
\hline 45 & Hendricks & 329.404 & 342.885 \\
\hline 46 & Henry & 299.202 & 424.650 \\
\hline 47 & Howard & 561.292 & 836.794 \\
\hline 48 & Huntington & 550.228 & 304.570 \\
\hline 49 & Jackson & 505.879 & 460.671 \\
\hline 50 & Jasper & 185.634 & 86.453 \\
\hline 51 & Jay & 424.894 & 287.506 \\
\hline
\end{tabular}




\begin{tabular}{|c|c|c|c|}
\hline & \begin{tabular}{|l} 
State \\
\end{tabular} & P12 97 & A12 97 \\
\hline 52 & Jefferson & 206.283 & 47.986 \\
\hline 53 & Jennings & 1249.372 & 503.939 \\
\hline 54 & Johnson & 810.780 & 278.241 \\
\hline 55 & Knox & 82.825 & 380.117 \\
\hline 56 & Kosciusko & 1671.882 & 1968.568 \\
\hline 57 & LaGrange & 888.111 & 89.211 \\
\hline 58 & Lake & 1285.628 & 19624.083 \\
\hline 59 & La Porte & 1142.903 & 1640.207 \\
\hline 60 & Lawrence & 284.131 & 1689.182 \\
\hline 61 & \begin{tabular}{|l} 
Madison \\
\end{tabular} & 783.772 & 515.152 \\
\hline 62 & Marion & 4326.798 & 7699.159 \\
\hline 63 & Marshall & 2767.173 & 912.787 \\
\hline 64 & Martin & 32.944 & 176.773 \\
\hline 65 & Miami & 435.228 & 35.087 \\
\hline 66 & Monroe & 421.974 & 226.374 \\
\hline 67 & Montgomery & 982.085 & 802.884 \\
\hline 68 & Morgan & 113.859 & 68.579 \\
\hline 69 & Newton & 471.203 & 157.561 \\
\hline 70 & Noble & 2681.335 & 1154.914 \\
\hline 71 & Ohio & .000 & .000 \\
\hline 72 & Orange & 214.054 & 42.538 \\
\hline 73 & Owen & 100.988 & 76.040 \\
\hline 74 & Parke & 277.196 & 86.726 \\
\hline 75 & Perry & 90.639 & 405.438 \\
\hline 76 & Pike & .000 & 52.704 \\
\hline 77 & Porter & 511.813 & 7294.669 \\
\hline 78 & Posey & 373.716 & 95.252 \\
\hline 79 & Pulaski & 37.885 & 180.231 \\
\hline 80 & Putnam & 1221.516 & 3.842 \\
\hline 81 & Randolph & 104.373 & 297.496 \\
\hline 82 & Ripley & 15.840 & 23.327 \\
\hline 83 & Rush & 90.562 & 351.421 \\
\hline 84 & St. Joseph & 3544.755 & 2030.397 \\
\hline 85 & Scott & 1556.432 & 480.354 \\
\hline 86 & Shelby & 2183.510 & 537.182 \\
\hline 87 & Spencer & 421.037 & 176.773 \\
\hline 88 & Starke & 181.110 & .000 \\
\hline 89 & Steuben & 901.382 & 609.101 \\
\hline 90 & Sullivan & 181.110 & .000 \\
\hline 91 & Switzerland & 181.110 & .000 \\
\hline 92 & Tippecanoe & 1064.868 & 1856.836 \\
\hline 93 & Tipton & 96.086 & 119.945 \\
\hline 94 & Union & .000 & .000 \\
\hline 95 & Vanderburgh & 5698.968 & 2291.908 \\
\hline 96 & Vermillion & 4.393 & 3.074 \\
\hline 97 & Vigo & 1893.156 & 436.721 \\
\hline 98 & Wabash & 228.245 & 693.883 \\
\hline 99 & Warren & 15.840 & 3.842 \\
\hline 100 & Warrick & 24.541 & 3306.011 \\
\hline 101 & Washington & 264.765 & 51.322 \\
\hline 102 & Wayne & 1569.998 & 1095.858 \\
\hline
\end{tabular}




\begin{tabular}{|c|c|c|c|}
\hline & State & P12 97 & A12 97 \\
\hline 103 & Wells & 1071.498 & 362.481 \\
\hline 104 & White & 406.717 & 80.875 \\
\hline 105 & Whitley & 479.482 & 537.028 \\
\hline 106 & lowa. & 38559.702 & 39757.000 \\
\hline 107 & Kansas & 12223.565 & 13631.000 \\
\hline 108 & Kentucky_E & 33080.586 & 30929.000 \\
\hline 109 & Kentucky_W & 22054.390 & 20619.000 \\
\hline 110 & Louisiana & 15856.653 & 24995.000 \\
\hline 111 & Maine & 1007.470 & 1378.000 \\
\hline 112 & Maryland & 23614.569 & 25334.000 \\
\hline 113 & Massachusetts & 18521.250 & 17176.000 \\
\hline 114 & Michigan_E & 40895.472 & 32694.000 \\
\hline 115 & Michigan_W & 40895.472 & 32694.000 \\
\hline 116 & Minnesota & 33125.563 & 32594.000 \\
\hline 117 & Missisippi & 7727.932 & 14418.000 \\
\hline 118 & Missouri & 59839.500 & 45981.000 \\
\hline 119 & Montana & 1875.013 & 4469.000 \\
\hline 120 & Nebraska & 19138.925 & 19831.000 \\
\hline 121 & Nevada & 2864.492 & 2750.000 \\
\hline 122 & New Hampshire & 11323.040 & 11026.000 \\
\hline 123 & New Jersey & 35520.302 & 37292.000 \\
\hline 124 & New Mexico & 1846.028 & 1934.000 \\
\hline 125 & New York & 70769.747 & 71165.000 \\
\hline 126 & North Carolina & 77931.976 & 73143.000 \\
\hline 127 & North Dakota & 4778.485 & 4491.000 \\
\hline 128 & Ohio_N & 33127.562 & 33160.000 \\
\hline 129 & Ohio_M & 33126.562 & 33160.000 \\
\hline 130 & Ohio_s & 33126.562 & 33159.000 \\
\hline 131 & Oklahoma & 24167.278 & 22583.000 \\
\hline 132 & Oregon & 18941.029 & 25899.000 \\
\hline 133 & Pennsylvania & 114752.594 & 106516.000 \\
\hline 134 & Rhode Island & 10554.444 & 9977.000 \\
\hline 135 & South Carolina & 23573.591 & 23991.000 \\
\hline 136 & South Dakota & 8171.698 & 7805.000 \\
\hline 137 & Tennessee & 70347.969 & 78081.000 \\
\hline 138 & Texas & 71808.200 & 77290.000 \\
\hline 139 & Utah & 14112.571 & 20862.000 \\
\hline 140 & Vermont & 2192.846 & 4176.000 \\
\hline 141 & Virginia & 63177.743 & 57398.000 \\
\hline 142 & Washington & 34448.866 & 27563.000 \\
\hline 143 & West Virginia & 5664.018 & 11282.000 \\
\hline 144 & Wisconsin & 71263.487 & 72378.000 \\
\hline 145 & Wyoming & 941.504 & 1298.000 \\
\hline
\end{tabular}




\begin{tabular}{|c|c|c|c|}
\hline & State & P13 97 & A13 97 \\
\hline 1 & Alabama & 2909.486 & 2903.000 \\
\hline 2 & Arizona & 69.107 & 2305.000 \\
\hline 3 & Arkansas & 1897.926 & 8989.000 \\
\hline 4 & California & 3479.364 & 4127.000 \\
\hline 5 & Colorado & 916.413 & 1004.000 \\
\hline 6 & Connecticut & 786.212 & 735.000 \\
\hline 7 & Delaware & .000 & 1205.000 \\
\hline 8 & $\mathrm{DC}$ & .000 & .000 \\
\hline 9 & Florida & 16386.264 & 15851.000 \\
\hline 10 & Georgia & 22344.450 & 16134.000 \\
\hline 11 & Idaho & 812.252 & 1341.000 \\
\hline 12 & Illinois_N & 9915.287 & 13907.000 \\
\hline 13 & Illinois_s & 4249.552 & 5960.000 \\
\hline 14 & Adams & 52.435 & 55.586 \\
\hline 15 & Allen & 112.360 & 417.522 \\
\hline 16 & Bartholomew & 52.435 & 145.828 \\
\hline 17 & Benton & .000 & 10.119 \\
\hline 18 & Blackford & .000 & 89.548 \\
\hline 19 & Boone & .000 & 42.140 \\
\hline 20 & Brown & 2.996 & 8.317 \\
\hline 21 & Carroll & .000 & 59.884 \\
\hline 22 & Cass & .000 & 98.558 \\
\hline 23 & Clark & 203.447 & 149.293 \\
\hline 24 & Clay & 17.978 & 15.248 \\
\hline 25 & Clinton & 52.435 & 35.071 \\
\hline 26 & Crawford & .000 & .000 \\
\hline 27 & Daviess & 17.978 & 21.625 \\
\hline 28 & Dearborn & 2.996 & 30.912 \\
\hline 29 & Decatur & .000 & 395.204 \\
\hline 30 & DeKalb & 67.716 & 446.771 \\
\hline 31 & Delaware & 25.468 & 260.050 \\
\hline 32 & Dubois & 17.978 & 21.070 \\
\hline 33 & Elkhart & 524.347 & 866.095 \\
\hline 34 & Fayette & .000 & 51.982 \\
\hline 35 & Floyd & 30.562 & 86.776 \\
\hline 36 & Fountain & 2.996 & 8.317 \\
\hline 37 & Franklin & .000 & 1.386 \\
\hline 38 & Fulton & 2.996 & 117.688 \\
\hline 39 & Gibson & .000 & 18.298 \\
\hline 40 & Grant & 28.465 & 23.981 \\
\hline 41 & Greene & .000 & 12.892 \\
\hline 42 & Hamilton & 17.978 & 163.571 \\
\hline 43 & Hancock & .000 & 63.626 \\
\hline 44 & Harrison & 14.682 & 10.258 \\
\hline 45 & Hendricks & 2.996 & 27.863 \\
\hline 46 & Henry & .000 & 48.517 \\
\hline 47 & Howard & .000 & 28.971 \\
\hline 48 & Huntington & 17.978 & 138.758 \\
\hline 49 & Jackson & 52.435 & 95.786 \\
\hline 50 & Jasper & .000 & 93.568 \\
\hline 51 & Jay & .000 & 131.273 \\
\hline
\end{tabular}




\begin{tabular}{|c|c|c|c|}
\hline & State & P13 97 & A13 97 \\
\hline 52 & Jefferson & 17.978 & 19.268 \\
\hline 53 & Jennings & 2.996 & 88.439 \\
\hline 54 & Johnson & 17.978 & 149.432 \\
\hline 55 & Knox & .000 & 16.218 \\
\hline 56 & Kosciusko & 99.476 & 199.058 \\
\hline 57 & LaGrange & 2.996 & 29.526 \\
\hline 58 & Lake & 447.043 & 350.569 \\
\hline 59 & La Porte & 99.177 & 302.468 \\
\hline 60 & Lawrence & .000 & 79.706 \\
\hline 61 & Madison & 112.360 & 145.828 \\
\hline 62 & Marion & 1813.942 & 1399.364 \\
\hline 63 & Marshall & 38.952 & 142.085 \\
\hline 64 & Martin & .000 & 1.386 \\
\hline 65 & Miami & 17.978 & 36.596 \\
\hline 66 & Monroe & 48.839 & 53.646 \\
\hline 67 & Montgomery & 52.435 & 88.994 \\
\hline 68 & Morgan & 112.360 & 44.913 \\
\hline 69 & Newton & 2.996 & 8.317 \\
\hline 70 & Noble & 64.719 & 193.929 \\
\hline 71 & Ohio & .000 & 1.386 \\
\hline 72 & Orange & .000 & 8.317 \\
\hline 73 & Owen & .000 & 1.802 \\
\hline 74 & Parke & .000 & 1.386 \\
\hline 75 & Perry & .000 & 8.317 \\
\hline 76 & Pike & .000 & 8.317 \\
\hline 77 & Porter & 138.727 & 214.722 \\
\hline 78 & Posey & 524.347 & 8.317 \\
\hline 79 & Pulaski & .000 & 24.258 \\
\hline 80 & Putnam & .000 & 1.386 \\
\hline 81 & Randolph & .000 & 15.387 \\
\hline 82 & Ripley & 17.978 & 29.110 \\
\hline 83 & Rush & .000 & 8.317 \\
\hline 84 & St. Joseph & 257.679 & 609.510 \\
\hline 85 & Scott & 52.435 & 12.199 \\
\hline 86 & Shelby & 57.528 & 50.873 \\
\hline 87 & Spencer & 2.996 & 8.317 \\
\hline 88 & Starke & 17.978 & .000 \\
\hline 89 & Steuben & .000 & 187.968 \\
\hline 90 & Sullivan & 2.996 & 1.386 \\
\hline 91 & Switzerland & .000 & .000 \\
\hline 92 & Tippecanoe & 491.987 & 84.419 \\
\hline 93 & Tipton & 2.996 & 17.189 \\
\hline 94 & Union & 12.884 & 1.386 \\
\hline 95 & Vanderburgh & 702.326 & 266.565 \\
\hline 96 & Vermillion & 524.347 & 24.258 \\
\hline 97 & Vigo & 308.616 & 92.875 \\
\hline 98 & Wabash & 112.360 & 140.699 \\
\hline 99 & Warren & .000 & 17.189 \\
\hline 100 & Warrick & 23.970 & 32.298 \\
\hline 101 & Washington & .000 & 84.419 \\
\hline 102 & Wayne & 41.648 & 88.162 \\
\hline
\end{tabular}




\begin{tabular}{|c|c|c|c|}
\hline & State & P13 97 & A13 97 \\
\hline 103 & Wells & .000 & 84.697 \\
\hline 104 & White & .000 & 130.302 \\
\hline 105 & Whitley & 2.996 & 97.172 \\
\hline 106 & lowa & 4677.211 & 6802.000 \\
\hline 107 & Kansas & 3771.815 & 1161.000 \\
\hline 108 & Kentucky_E & 2244.460 & 4687.000 \\
\hline 109 & Kentucky_W & 1496.307 & 2884.000 \\
\hline 110 & Louisiana & 7259.192 & 2709.000 \\
\hline 111 & Maine & 456.704 & 1604.000 \\
\hline 112 & Maryland & 2994.617 & 3059.000 \\
\hline 113 & Massachusetts & 394.608 & 1332.000 \\
\hline 114 & Michigan_E & 4618.120 & 3289.000 \\
\hline 115 & Michigan W & 4618.120 & 3289.000 \\
\hline 116 & Minnesota & 2327.589 & 3972.000 \\
\hline 117 & Missisippi & 1319.034 & 2475.000 \\
\hline 118 & Missouri & 5827.985 & 1735.000 \\
\hline 119 & Montana & 773.192 & 669.000 \\
\hline 120 & Nebraska & 736.135 & 1015.000 \\
\hline 121 & Nevada & 1093.686 & 161.000 \\
\hline 122 & New Hampshire & 1408.171 & 316.000 \\
\hline 123 & New Jersey & 17835.498 & 4362.000 \\
\hline 124 & New Mexico & 358.553 & 399.000 \\
\hline 125 & New York & 24618.957 & 20171.000 \\
\hline 126 & North Carolina & 1970.037 & 1573.000 \\
\hline 127 & North Dakota & 436.673 & 432.000 \\
\hline 128 & Ohio_N & 5912.115 & 5913.000 \\
\hline 129 & Ohio_M & 5911.114 & 5913.000 \\
\hline 130 & Ohio_s & 5911.114 & 5913.000 \\
\hline 131 & Oklahoma & 1000.543 & 2649.000 \\
\hline 132 & Oregon & 1162.793 & 5472.000 \\
\hline 133 & Pennsylvania & 8842.633 & 16380.000 \\
\hline 134 & Rhode Island & 682.052 & 1360.000 \\
\hline 135 & South Carolina & 971.498 & 1249.000 \\
\hline 136 & South Dakota & 1545.383 & 221.000 \\
\hline 137 & Tennessee & 554.855 & 3912.000 \\
\hline 138 & Texas & 20430.499 & 14532.000 \\
\hline 139 & Utah & 1481.284 & 516.000 \\
\hline 140 & Vermont & 143.221 & 101.000 \\
\hline 141 & Virginia & 5136.920 & 10442.000 \\
\hline 142 & Washington & 848.308 & 1291.000 \\
\hline 143 & West Virginia & 3651.630 & 819.000 \\
\hline 144 & Wisconsin & 4729.291 & 6180.000 \\
\hline 145 & Wyoming & 4604.098 & 668.000 \\
\hline
\end{tabular}




\begin{tabular}{|c|c|c|c|}
\hline & State & P14 97 & A14 97 \\
\hline 1 & Alabama & 423.968 & 2003.000 \\
\hline 2 & Arizona & 9120.331 & 8188.000 \\
\hline 3 & Arkansas & 423.968 & 208.000 \\
\hline 4 & California & 77.359 & 673.000 \\
\hline 5 & Colorado & 68.317 & 32.000 \\
\hline 6 & Connecticut & 423.968 & 505.000 \\
\hline 7 & Delaware & 423.968 & 67.000 \\
\hline 8 & DC & .000 & .000 \\
\hline 9 & Florida & 305.418 & 212.000 \\
\hline 10 & Georgia & 423.968 & 574.000 \\
\hline 11 & Idaho & 192.895 & 16.000 \\
\hline 12 & Illinois $\mathbf{N}$ & 174.811 & 2358.000 \\
\hline 13 & Illinois_s & 75.350 & 1010.000 \\
\hline 14 & Adams & .000 & 35.969 \\
\hline 15 & Allen & .000 & 509.118 \\
\hline 16 & Bartholomew & .000 & 146.754 \\
\hline 17 & Benton & .000 & 12.332 \\
\hline 18 & Blackford & .000 & .000 \\
\hline 19 & Boone & .000 & .000 \\
\hline 20 & Brown & .000 & .000 \\
\hline 21 & Carroll & .000 & .000 \\
\hline 22 & Cass & .000 & .000 \\
\hline 23 & Clark & .000 & 26.514 \\
\hline 24 & Clay & .000 & 2.055 \\
\hline 25 & Clinton & .000 & .000 \\
\hline 26 & Crawford & .000 & .000 \\
\hline 27 & Daviess & .000 & .000 \\
\hline 28 & Dearborn & .000 & .000 \\
\hline 29 & Decatur & .000 & 2.055 \\
\hline 30 & DeKalb & .000 & 489.798 \\
\hline 31 & Delaware & .000 & 147.371 \\
\hline 32 & \begin{tabular}{|l} 
Dubois \\
\end{tabular} & .000 & .000 \\
\hline 33 & Elkhart & .000 & 247.057 \\
\hline 34 & Fayette & .000 & 12.332 \\
\hline 35 & Floyd & .000 & 2.055 \\
\hline 36 & Fountain & .000 & 154.154 \\
\hline 37 & Franklin & .000 & .000 \\
\hline 38 & Fulton & .000 & 77.077 \\
\hline 39 & Gibson & .000 & .000 \\
\hline 40 & Grant & .000 & 35.969 \\
\hline 41 & Greene & .000 & 35.969 \\
\hline 42 & Hamilton & .000 & 93.725 \\
\hline 43 & Hancock & .000 & 2.055 \\
\hline 44 & Harrison & .000 & .000 \\
\hline 45 & Hendricks & .000 & 35.969 \\
\hline 46 & Henry & .000 & 77.077 \\
\hline 47 & Howard & .000 & 154.154 \\
\hline 48 & Huntington & .000 & 34.736 \\
\hline 49 & Jackson & .000 & 101.741 \\
\hline 50 & Jasper & .000 & .000 \\
\hline 51 & Jay & .000 & .000 \\
\hline
\end{tabular}




\begin{tabular}{|c|c|c|c|}
\hline & State & P14 97 & A14 97 \\
\hline 52 & Jefferson & .000 & 2.055 \\
\hline 53 & Jennings & .000 & 12.332 \\
\hline 54 & Johnson & .000 & 61.661 \\
\hline 55 & Knox & .000 & 77.077 \\
\hline 56 & Kosciusko & .000 & 359.692 \\
\hline 57 & LaGrange & .000 & 2.055 \\
\hline 58 & Lake & .000 & 4302.533 \\
\hline 59 & La Porte & .000 & 325.984 \\
\hline 60 & Lawrence & .000 & 359.692 \\
\hline 61 & Madison & .000 & 47.479 \\
\hline 62 & Marion & .000 & 750.215 \\
\hline 63 & Marshall & .000 & 78.516 \\
\hline 64 & Martin & .000 & 35.969 \\
\hline 65 & Miami & .000 & .000 \\
\hline 66 & Monroe & .000 & .000 \\
\hline 67 & Montgomery & .000 & 154.154 \\
\hline 68 & Morgan & .000 & 12.332 \\
\hline 69 & Newton & .000 & 35.969 \\
\hline 70 & Noble & .000 & 153.537 \\
\hline 71 & Ohio & .000 & .000 \\
\hline 72 & Orange & .000 & .000 \\
\hline 73 & Owen & .000 & .000 \\
\hline 74 & Parke & .000 & .000 \\
\hline 75 & Perry & .000 & 77.077 \\
\hline 76 & Pike & .000 & 12.332 \\
\hline 77 & Porter & .000 & 1675.137 \\
\hline 78 & Posey & .000 & .000 \\
\hline 79 & Pulaski & .000 & 35.969 \\
\hline 80 & Putnam & .000 & .000 \\
\hline 81 & Randolph & .000 & 62.689 \\
\hline 82 & Ripley & .000 & .000 \\
\hline 83 & Rush & .000 & 77.077 \\
\hline 84 & St. Joseph & .000 & 358.870 \\
\hline 85 & Scott & .000 & 2.055 \\
\hline 86 & Shelby & .000 & 109.963 \\
\hline 87 & Spencer & .000 & 35.969 \\
\hline 88 & Starke & .000 & .000 \\
\hline 89 & Steuben & .000 & 102.975 \\
\hline 90 & Sullivan & .000 & .000 \\
\hline 91 & Switzerland & .000 & .000 \\
\hline 92 & Tippecanoe & .000 & 359.692 \\
\hline 93 & Tipton & .000 & 12.332 \\
\hline 94 & Union & .000 & .000 \\
\hline 95 & Vanderburgh & .000 & 35.969 \\
\hline 96 & Vermillion & .000 & .000 \\
\hline 97 & Vigo & .000 & 77.077 \\
\hline 98 & Wabash & .000 & 154.154 \\
\hline 99 & Warren & .000 & .000 \\
\hline 100 & Warrick & .000 & 770.769 \\
\hline 101 & Washington & .000 & 2.055 \\
\hline 102 & Wayne & .000 & 188.479 \\
\hline
\end{tabular}




\begin{tabular}{|c|c|c|c|}
\hline & State & P14 97 & A14 97 \\
\hline 103 & Wells & .000 & 35.969 \\
\hline 104 & White & .000 & 12.332 \\
\hline 105 & Whitley & .000 & 124.762 \\
\hline 106 & lowa & 423.968 & 856.000 \\
\hline 107 & Kansas & 423.968 & 240.000 \\
\hline 108 & Kentucky_E & 254.180 & 805.000 \\
\hline 109 & Kentucky_W & 169.788 & 536.000 \\
\hline 110 & Louisiana & 423.968 & 339.000 \\
\hline 111 & Maine & 423.968 & 65.000 \\
\hline 112 & Maryland & 423.968 & 14.000 \\
\hline 113 & Massachusetts & 423.968 & 58.000 \\
\hline 114 & Michigan_E & 7432.497 & 8080.000 \\
\hline 115 & Michigan_W & 7432.497 & 8080.000 \\
\hline 116 & Minnesota & 47607.966 & 8023.000 \\
\hline 117 & Missisippi & 423.968 & 245.000 \\
\hline 118 & Missouri & 2092.713 & 2021.000 \\
\hline 119 & Montana & 491.280 & 445.000 \\
\hline 120 & Nebraska & 423.968 & 102.000 \\
\hline 121 & Nevada & 55.256 & 34.000 \\
\hline 122 & New Hampshire & .000 & 14.000 \\
\hline 123 & New Jersey & 423.968 & 79.000 \\
\hline 124 & New Mexico & 492.285 & 152.000 \\
\hline 125 & New York & 1362.323 & 1025.000 \\
\hline 126 & North Carolina & 423.968 & 76.000 \\
\hline 127 & North Dakota & 423.968 & 11.000 \\
\hline 128 & Ohio_N & 141.657 & 4188.000 \\
\hline 129 & Ohio_M & 141.657 & 4188.000 \\
\hline 130 & Ohio_s & 140.653 & 4187.000 \\
\hline 131 & Oklahoma & 423.968 & 10.000 \\
\hline 132 & Oregon & 423.968 & 42.000 \\
\hline 133 & Pennsylvania & 723.357 & 5193.000 \\
\hline 134 & Rhode Island & 423.968 & 297.000 \\
\hline 135 & South Carolina & 140.653 & 102.000 \\
\hline 136 & South Dakota & .000 & 53.000 \\
\hline 137 & Tennessee & 423.968 & 1867.000 \\
\hline 138 & Texas & 423.968 & 2529.000 \\
\hline 139 & Utah & 957.444 & 3706.000 \\
\hline 140 & Vermont & 423.968 & 23.000 \\
\hline 141 & Virginia & 31.145 & 102.000 \\
\hline 142 & Washington & 423.968 & 232.000 \\
\hline 143 & West Virginia & 423.968 & 978.000 \\
\hline 144 & Wisconsin & 423.968 & 2331.000 \\
\hline 145 & Wyoming & 423.968 & .000 \\
\hline
\end{tabular}




\begin{tabular}{|c|c|c|c|}
\hline & State & P15 97 & A15 97 \\
\hline 1 & Alabama & 30993.076 & 49921.000 \\
\hline 2 & Arizona & 13010.032 & 23776.000 \\
\hline 3 & Arkansas & 409.001 & 12332.000 \\
\hline 4 & California & 2454.006 & 5556.000 \\
\hline 5 & Colorado & 37648.093 & 26073.000 \\
\hline 6 & Connecticut & .000 & 1739.000 \\
\hline 7 & Delaware & .000 & 357.000 \\
\hline 8 & DC & .000 & .000 \\
\hline 9 & Florida & .000 & 27426.000 \\
\hline 10 & Georgia & 2099.005 & 35553.000 \\
\hline 11 & Idaho & 791.002 & 3828.000 \\
\hline 12 & Illinois_N & 52725.130 & 54265.000 \\
\hline 13 & Illinois_S & 22597.056 & 23257.000 \\
\hline 14 & Adams & 226.683 & 487.618 \\
\hline 15 & Allen & 321.134 & 1596.237 \\
\hline 16 & Bartholomew & .000 & 998.253 \\
\hline 17 & Benton & .000 & .000 \\
\hline 18 & Blackford & 37.780 & 82.937 \\
\hline 19 & Boone & 37.780 & 44.454 \\
\hline 20 & Brown & .000 & 7.697 \\
\hline 21 & Carroll & 226.683 & 1567.456 \\
\hline 22 & Cass & 226.683 & 1567.456 \\
\hline 23 & Clark & 661.158 & 864.919 \\
\hline 24 & Clay & 226.683 & 228.242 \\
\hline 25 & Clinton & .000 & 1607.827 \\
\hline 26 & Crawford & 661.158 & 643.256 \\
\hline 27 & Daviess & 1416.768 & 2160.384 \\
\hline 28 & Dearborn & 226.683 & 228.242 \\
\hline 29 & Decatur & 226.683 & 505.320 \\
\hline 30 & DeKalb & 173.790 & 215.264 \\
\hline 31 & Delaware & 1416.768 & 1474.614 \\
\hline 32 & Dubois & 226.683 & 834.736 \\
\hline 33 & Elkhart & 226.683 & 720.057 \\
\hline 34 & Fayette & 37.780 & 36.758 \\
\hline 35 & Floyd & .000 & 558.006 \\
\hline 36 & Fountain & .000 & 46.180 \\
\hline 37 & Franklin & 37.780 & 44.454 \\
\hline 38 & Fulton & 37.780 & 156.055 \\
\hline 39 & Gibson & 1594.336 & 1839.791 \\
\hline 40 & Grant & 226.683 & 424.506 \\
\hline 41 & Greene & 661.158 & 931.880 \\
\hline 42 & Hamilton & 661.158 & 745.622 \\
\hline 43 & Hancock & 37.780 & 44.454 \\
\hline 44 & Harrison & 298.466 & 848.390 \\
\hline 45 & Hendricks & 226.683 & 575.360 \\
\hline 46 & Henry & 37.780 & 82.937 \\
\hline 47 & Howard & 226.683 & 342.922 \\
\hline 48 & Huntington & 283.354 & 564.305 \\
\hline 49 & Jackson & 37.780 & 64.465 \\
\hline 50 & Jasper & 37.780 & 209.932 \\
\hline 51 & Jay & 226.683 & 562.276 \\
\hline
\end{tabular}




\begin{tabular}{|c|c|c|c|}
\hline & State & P15 97 & A15 97 \\
\hline 52 & Jefferson & .000 & 15.393 \\
\hline 53 & Jennings & 226.683 & 220.545 \\
\hline 54 & Johnson & 37.780 & 42.145 \\
\hline 55 & Knox & 1783.238 & 1836.550 \\
\hline 56 & Kosciusko & 37.780 & 741.769 \\
\hline 57 & LaGrange & 37.780 & 173.758 \\
\hline 58 & Lake & 226.683 & 1089.495 \\
\hline 59 & La Porte & 102.007 & 531.026 \\
\hline 60 & Lawrence & 238.017 & 277.752 \\
\hline 61 & Madison & 226.683 & 797.793 \\
\hline 62 & Marion & 661.158 & 3613.387 \\
\hline 63 & Marshall & 37.780 & 365.404 \\
\hline 64 & Martin & .000 & 46.180 \\
\hline 65 & Miami & 226.683 & 266.725 \\
\hline 66 & Monroe & 525.149 & 585.587 \\
\hline 67 & Montgomery & 37.780 & 325.381 \\
\hline 68 & Morgan & 226.683 & 228.242 \\
\hline 69 & Newton & 226.683 & 228.242 \\
\hline 70 & Noble & 37.780 & 614.005 \\
\hline 71 & Ohio & .000 & .000 \\
\hline 72 & Orange & 207.793 & 248.346 \\
\hline 73 & Owen & 37.780 & 44.454 \\
\hline 74 & Parke & .000 & 134.691 \\
\hline 75 & Perry & .000 & 7.697 \\
\hline 76 & Pike & 226.683 & 220.545 \\
\hline 77 & Porter & 37.780 & 284.589 \\
\hline 78 & Posey & 37.780 & 82.937 \\
\hline 79 & Pulaski & 226.683 & 273.652 \\
\hline 80 & Putnam & 234.239 & 235.593 \\
\hline 81 & Randolph & 37.780 & 96.022 \\
\hline 82 & Ripley & 226.683 & 228.242 \\
\hline 83 & Rush & 37.780 & 56.769 \\
\hline 84 & St. Joseph & 226.683 & 754.691 \\
\hline 85 & Scott & 37.780 & 325.381 \\
\hline 86 & Shelby & 423.141 & 546.375 \\
\hline 87 & Spencer & 226.683 & 266.725 \\
\hline 88 & Starke & .000 & .000 \\
\hline 89 & Steuben & 37.780 & 230.713 \\
\hline 90 & Sullivan & 1416.768 & 1378.407 \\
\hline 91 & Switzerland & 226.683 & 220.545 \\
\hline 92 & Tippecanoe & 226.683 & 716.208 \\
\hline 93 & Tipton & .000 & 134.691 \\
\hline 94 & Union & .000 & .000 \\
\hline 95 & Vanderburgh & 661.158 & 3339.387 \\
\hline 96 & Vermillion & 37.780 & 42.915 \\
\hline 97 & Vigo & 1416.768 & 1569.283 \\
\hline 98 & Wabash & 37.780 & 82.937 \\
\hline 99 & Warren & 226.683 & 228.242 \\
\hline 100 & Warrick & 1416.768 & 1378.407 \\
\hline 101 & Washington & 37.780 & 82.937 \\
\hline 102 & Wayne & 37.780 & 579.370 \\
\hline
\end{tabular}




\begin{tabular}{|c|c|c|c|}
\hline & State & P15 97 & A15 97 \\
\hline 103 & Wells & 37.780 & 430.825 \\
\hline 104 & White & 37.780 & 69.083 \\
\hline 105 & Whitley & .000 & 7.697 \\
\hline 106 & lowa & 586.001 & 19010.000 \\
\hline 107 & Kansas & 432.001 & 8486.000 \\
\hline 108 & Kentucky_E & 114689.283 & 65842.000 \\
\hline 109 & Kentucky_W & 76459.188 & 43895.000 \\
\hline 110 & Louisiana & 3418.008 & 13916.000 \\
\hline 111 & Maine & 20.000 & 923.000 \\
\hline 112 & Maryland & 4610.011 & 23226.000 \\
\hline 113 & Massachusetts & 586.001 & 4175.000 \\
\hline 114 & Michigan_E & 673.002 & 9694.000 \\
\hline 115 & Michigan_W & 673.002 & 9694.000 \\
\hline 116 & Minnesota & 401.001 & 15838.000 \\
\hline 117 & Missisippi & 353.001 & 9347.000 \\
\hline 118 & Missouri & 1418.003 & 37875.000 \\
\hline 119 & Montana & 54569.134 & 12696.000 \\
\hline 120 & Nebraska & 218.001 & 11221.000 \\
\hline 121 & Nevada & .000 & 6462.000 \\
\hline 122 & New Hampshire & .000 & 1369.000 \\
\hline 123 & New Jersey & 586.001 & 1929.000 \\
\hline 124 & New Mexico & 31085.077 & 14454.000 \\
\hline 125 & New York & 1064.003 & 23542.000 \\
\hline 126 & North Carolina & 1107.003 & 22668.000 \\
\hline 127 & North Dakota & 34075.084 & 34785.000 \\
\hline 128 & Ohio_N & 13192.033 & 26386.000 \\
\hline 129 & Ohio M & 13192.033 & 26386.000 \\
\hline 130 & Ohio_s & 13192.033 & 26386.000 \\
\hline 131 & Oklahoma & 1007.002 & 18197.000 \\
\hline 132 & Oregon & .000 & 4055.000 \\
\hline 133 & Pennsylvania & 103683.255 & 98261.000 \\
\hline 134 & Rhode Island & .000 & .000 \\
\hline 135 & South Carolina & .000 & 3362.000 \\
\hline 136 & South Dakota & 530.001 & 2176.000 \\
\hline 137 & Tennessee & 1221.003 & 22900.000 \\
\hline 138 & Texas & 49543.122 & 81949.000 \\
\hline 139 & Utah & 21171.052 & 10433.000 \\
\hline 140 & Vermont & .000 & .000 \\
\hline 141 & Virginia & 57611.142 & 63502.000 \\
\hline 142 & Washington & 975.002 & 6101.000 \\
\hline 143 & West Virginia & 187835.463 & 56029.000 \\
\hline 144 & Wisconsin & 586.001 & 35973.000 \\
\hline 145 & Wyoming & 239363.590 & 29602.000 \\
\hline
\end{tabular}




\begin{tabular}{|c|c|c|c|}
\hline & State & P1797 & A1797 \\
\hline 1 & Alabama & 12748.143 & 14015.000 \\
\hline 2 & Arizona & 5654.540 & 6238.000 \\
\hline 3 & Arkansas & 6808.610 & 6632.000 \\
\hline 4 & California & 122646.626 & 124851.000 \\
\hline 5 & Colorado & 5369.547 & 8378.000 \\
\hline 6 & Connecticut & 3201.386 & 3593.000 \\
\hline 7 & Delaware & 10794.482 & 6615.000 \\
\hline 8 & DC & .000 & .000 \\
\hline 9 & Florida & 35280.706 & 63957.000 \\
\hline 10 & Georgia & 38412.606 & 44337.000 \\
\hline 11 & Idaho & 1476.323 & 1942.000 \\
\hline 12 & Illinois_N & 34676.481 & 28380.000 \\
\hline 13 & Illinois_S & 14860.917 & 12163.000 \\
\hline 14 & Adams & 60.287 & 141.927 \\
\hline 15 & Allen & 373.780 & 1158.208 \\
\hline 16 & Bartholomew & .000 & 164.681 \\
\hline 17 & Benton & .000 & 22.405 \\
\hline 18 & Blackford & .000 & 33.023 \\
\hline 19 & Boone & .000 & 103.500 \\
\hline 20 & Brown & .000 & 34.853 \\
\hline 21 & Carroll & .000 & 47.020 \\
\hline 22 & Cass & .000 & 94.757 \\
\hline 23 & Clark & 608.900 & 865.986 \\
\hline 24 & Clay & .000 & 61.865 \\
\hline 25 & Clinton & .000 & 79.284 \\
\hline 26 & Crawford & .000 & 24.556 \\
\hline 27 & Daviess & 60.287 & 133.287 \\
\hline 28 & Dearborn & 60.287 & 169.373 \\
\hline 29 & Decatur & .000 & 57.596 \\
\hline 30 & DeKalb & .000 & 91.704 \\
\hline 31 & Delaware & .000 & 280.023 \\
\hline 32 & Dubois & .000 & 92.006 \\
\hline 33 & Elkhart & 60.287 & 480.637 \\
\hline 34 & Fayette & .000 & 60.680 \\
\hline 35 & Floyd & .000 & 165.497 \\
\hline 36 & Fountain & .000 & 42.216 \\
\hline 37 & Franklin & 361.723 & 433.476 \\
\hline 38 & Fulton & .000 & 47.531 \\
\hline 39 & Gibson & .000 & 75.225 \\
\hline 40 & Grant & .000 & 173.181 \\
\hline 41 & Greene & .000 & 77.871 \\
\hline 42 & Hamilton & 60.287 & 451.917 \\
\hline 43 & Hancock & .000 & 126.053 \\
\hline 44 & Harrison & 361.723 & 461.032 \\
\hline 45 & Hendricks & .000 & 227.395 \\
\hline 46 & Henry & .000 & 114.315 \\
\hline 47 & Howard & .000 & 197.690 \\
\hline 48 & Huntington & 60.287 & 152.474 \\
\hline 49 & Jackson & .000 & 95.751 \\
\hline 50 & Jasper & .000 & 68.286 \\
\hline 51 & Jay & 60.287 & 114.826 \\
\hline
\end{tabular}




\begin{tabular}{|c|c|c|c|}
\hline & State & P17 97 & A17 97 \\
\hline 52 & Jefferson & .000 & 73.425 \\
\hline 53 & Jennings & 361.723 & 445.849 \\
\hline 54 & Johnson & .000 & 257.090 \\
\hline 55 & Knox & 60.287 & 156.852 \\
\hline 56 & Kosciusko & 60.287 & 234.350 \\
\hline 57 & LaGrange & .000 & 79.923 \\
\hline 58 & Lake & 13015.987 & 14907.324 \\
\hline 59 & La Porte & 60.287 & 321.449 \\
\hline 60 & Lawrence & 361.723 & 490.099 \\
\hline 61 & Madison & .000 & 312.157 \\
\hline 62 & Marion & 717.417 & 2760.531 \\
\hline 63 & Marshall & 361.723 & 486.996 \\
\hline 64 & Martin & .000 & 24.552 \\
\hline 65 & Miami & .000 & 82.293 \\
\hline 66 & Monroe & 361.723 & 662.291 \\
\hline 67 & Montgomery & .000 & 87.488 \\
\hline 68 & Morgan & .000 & 152.015 \\
\hline 69 & Newton & .000 & 34.202 \\
\hline 70 & Noble & .000 & 104.923 \\
\hline 71 & Ohio & .000 & 13.020 \\
\hline 72 & Orange & .000 & 44.880 \\
\hline 73 & Owen & .000 & 49.724 \\
\hline 74 & Parke & .000 & 39.915 \\
\hline 75 & Perry & .000 & 44.845 \\
\hline 76 & Pike & .000 & 30.056 \\
\hline 77 & Porter & .000 & 338.670 \\
\hline 78 & Posey & 2260.767 & 2455.188 \\
\hline 79 & Pulaski & .000 & 32.041 \\
\hline 80 & Putnam & .000 & 82.229 \\
\hline 81 & Randolph & 60.287 & 128.176 \\
\hline 82 & Ripley & 60.287 & 124.904 \\
\hline 83 & Rush & .000 & 43.039 \\
\hline 84 & St. Joseph & 60.287 & 682.074 \\
\hline 85 & Scott & 60.287 & 116.410 \\
\hline 86 & Shelby & .000 & 100.667 \\
\hline 87 & Spencer & .000 & 47.575 \\
\hline 88 & Starke & .000 & 55.857 \\
\hline 89 & Steuben & 60.287 & 139.722 \\
\hline 90 & Sullivan & .000 & 50.404 \\
\hline 91 & Switzerland & .000 & 20.650 \\
\hline 92 & Tippecanoe & 60.287 & 406.340 \\
\hline 93 & Tipton & .000 & 38.877 \\
\hline 94 & Union & .000 & 17.048 \\
\hline 95 & Vanderburgh & .000 & 402.157 \\
\hline 96 & \begin{tabular}{|l|} 
Vermillion \\
\end{tabular} & .000 & 39.362 \\
\hline 97 & Vigo & .000 & 249.643 \\
\hline 98 & Wabash & 60.287 & 145.611 \\
\hline 99 & Warren & .000 & 19.469 \\
\hline 100 & Warrick & .000 & 119.759 \\
\hline 101 & Washington & .000 & 62.854 \\
\hline 102 & Wayne & .000 & 167.685 \\
\hline
\end{tabular}




\begin{tabular}{|c|c|c|c|}
\hline & State & P17 97 & A17 97 \\
\hline 103 & Wells & .000 & 64.436 \\
\hline 104 & White & .000 & 58.985 \\
\hline 105 & Whitley & .000 & 70.810 \\
\hline 106 & lowa & 7762.279 & 9033.000 \\
\hline 107 & Kansas & 7061.378 & 4564.000 \\
\hline 108 & Kentucky E & 9024.102 & 11507.000 \\
\hline 109 & Kentucky_W & 6016.068 & 7672.000 \\
\hline 110 & Louisiana & 157986.747 & 91822.000 \\
\hline 111 & Maine & 1015.098 & 3780.000 \\
\hline 112 & Maryland & 7081.519 & 8596.000 \\
\hline 113 & Massachusetts & 6971.751 & 8106.000 \\
\hline 114 & Michigan_E & 18967.634 & 20701.000 \\
\hline 115 & Michigan_W & 18967.634 & 20701.000 \\
\hline 116 & Minnesota & 13348.340 & 15355.000 \\
\hline 117 & Missisippi & 15846.811 & 17902.000 \\
\hline 118 & Missouri & 5748.195 & 6735.000 \\
\hline 119 & Montana & 5338.329 & 5105.000 \\
\hline 120 & Nebraska & 2953.654 & 3470.000 \\
\hline 121 & Nevada & 1854.971 & 2299.000 \\
\hline 122 & New Hampshire & 1985.887 & 3512.000 \\
\hline 123 & New Jersey & 23275.759 & 24141.000 \\
\hline 124 & New Mexico & 3489.400 & 3339.000 \\
\hline 125 & New York & 14450.044 & 14180.000 \\
\hline 126 & North Carolina & 12913.298 & 13421.000 \\
\hline 127 & North Dakota & 3878.118 & 2098.000 \\
\hline 128 & Ohio_N & 11996.890 & 12414.000 \\
\hline 129 & Ohio $M$ & 11996.890 & 12414.000 \\
\hline 130 & Ohio_s & 11995.883 & 12414.000 \\
\hline 131 & Oklahoma & 11700.820 & 13706.000 \\
\hline 132 & Oregon & 6656.547 & 6329.000 \\
\hline 133 & Pennsylvania & 30598.968 & 31163.000 \\
\hline 134 & Rhode Island & 1910.358 & 1569.000 \\
\hline 135 & South Carolina & 6339.329 & 8466.000 \\
\hline 136 & South Dakota & 340.380 & 448.000 \\
\hline 137 & Tennessee & 12641.397 & 14799.000 \\
\hline 138 & Texas & 137066.459 & 125473.000 \\
\hline 139 & Utah & 7235.596 & 7060.000 \\
\hline 140 & Vermont & 1470.281 & 3594.000 \\
\hline 141 & Virginia & 14148.939 & 11000.000 \\
\hline 142 & Washington & 19868.937 & 19034.000 \\
\hline 143 & West Virginia & 5094.625 & 5957.000 \\
\hline 144 & Wisconsin & 5390.695 & 13982.000 \\
\hline 145 & Wyoming & 935.542 & 1067.000 \\
\hline
\end{tabular}




\begin{tabular}{|c|c|c|c|}
\hline & State & P18 97 & A18 97 \\
\hline 1 & Alabama & 3633.378 & 5100.000 \\
\hline 2 & Arizona & 2425.948 & 4305.000 \\
\hline 3 & Arkansas & 4150.416 & 4440.000 \\
\hline 4 & California & 39491.443 & 36234.000 \\
\hline 5 & Colorado & 2718.230 & 3847.000 \\
\hline 6 & Connecticut & 2498.514 & 3986.000 \\
\hline 7 & Delaware & 3988.149 & 12028.000 \\
\hline 8 & DC & .000 & .000 \\
\hline 9 & Florida & 11922.116 & 15959.000 \\
\hline 10 & Georgia & 12921.925 & 10853.000 \\
\hline 11 & Idaho & 642.014 & 757.000 \\
\hline 12 & Illinois_N & 18379.551 & 16775.000 \\
\hline 13 & Illinois_S & 7876.519 & 7190.000 \\
\hline 14 & Adams & 42.595 & 62.102 \\
\hline 15 & Allen & 264.090 & 291.071 \\
\hline 16 & Bartholomew & .000 & 23.159 \\
\hline 17 & Benton & .000 & .000 \\
\hline 18 & Blackford & .000 & .000 \\
\hline 19 & Boone & .000 & .000 \\
\hline 20 & Brown & .000 & 1.323 \\
\hline 21 & Carroll & .000 & .000 \\
\hline 22 & Cass & .000 & .000 \\
\hline 23 & Clark & 430.210 & 483.179 \\
\hline 24 & Clay & .000 & 7.940 \\
\hline 25 & Clinton & .000 & 23.159 \\
\hline 26 & Crawford & .000 & .000 \\
\hline 27 & Daviess & 42.595 & 46.883 \\
\hline 28 & Dearborn & 42.595 & 40.266 \\
\hline 29 & Decatur & .000 & .000 \\
\hline 30 & DeKalb & .000 & 29.908 \\
\hline 31 & Delaware & .000 & 11.249 \\
\hline 32 & Dubois & .000 & 7.940 \\
\hline 33 & Elkhart & 42.595 & 270.531 \\
\hline 34 & Fayette & .000 & .000 \\
\hline 35 & Floyd & .000 & 13.498 \\
\hline 36 & Fountain & .000 & 1.323 \\
\hline 37 & Franklin & 255.571 & 233.657 \\
\hline 38 & Fulton & .000 & 1.323 \\
\hline 39 & Gibson & .000 & .000 \\
\hline 40 & Grant & .000 & 12.572 \\
\hline 41 & Greene & .000 & .000 \\
\hline 42 & Hamilton & 42.595 & 46.883 \\
\hline 43 & Hancock & .000 & .000 \\
\hline 44 & Harrison & 255.571 & 240.141 \\
\hline 45 & Hendricks & .000 & 1.323 \\
\hline 46 & Henry & .000 & .000 \\
\hline 47 & Howard & .000 & .000 \\
\hline 48 & Huntington & 42.595 & 46.883 \\
\hline 49 & Jackson & .000 & 23.159 \\
\hline 50 & Jasper & .000 & .000 \\
\hline 51 & Jay & 42.595 & 38.943 \\
\hline
\end{tabular}




\begin{tabular}{|c|c|c|c|}
\hline & State & P18 97 & A18 97 \\
\hline 52 & Jefferson & .000 & 7.940 \\
\hline 53 & Jennings & 255.571 & 234.980 \\
\hline 54 & Johnson & .000 & 7.940 \\
\hline 55 & Knox & 42.595 & 38.943 \\
\hline 56 & Kosciusko & 42.595 & 82.878 \\
\hline 57 & LaGrange & .000 & 1.323 \\
\hline 58 & Lake & 9196.282 & 8605.201 \\
\hline 59 & La Porte & 42.595 & 82.746 \\
\hline 60 & Lawrence & 255.571 & 233.657 \\
\hline 61 & Madison & .000 & 49.626 \\
\hline 62 & Marion & 506.882 & 1264.581 \\
\hline 63 & Marshall & 255.571 & 250.861 \\
\hline 64 & Martin & .000 & .000 \\
\hline 65 & Miami & .000 & 7.940 \\
\hline 66 & Monroe & 255.571 & 255.228 \\
\hline 67 & Montgomery & .000 & 23.159 \\
\hline 68 & Morgan & .000 & 49.626 \\
\hline 69 & Newton & .000 & 1.323 \\
\hline 70 & Noble & .000 & 28.585 \\
\hline 71 & Ohio & .000 & .000 \\
\hline 72 & Orange & .000 & .000 \\
\hline 73 & Owen & .000 & .000 \\
\hline 74 & Parke & .000 & .000 \\
\hline 75 & Perry & .000 & .000 \\
\hline 76 & Pike & .000 & .000 \\
\hline 77 & Porter & .000 & 61.271 \\
\hline 78 & Posey & 1597.316 & 1691.944 \\
\hline 79 & Pulaski & .000 & .000 \\
\hline 80 & Putnam & .000 & .000 \\
\hline 81 & Randolph & 42.595 & 38.943 \\
\hline 82 & Ripley & 42.595 & 46.883 \\
\hline 83 & Rush & .000 & .000 \\
\hline 84 & St. Joseph & 42.595 & 152.752 \\
\hline 85 & Scott & 42.595 & 62.102 \\
\hline 86 & Shelby & .000 & 25.408 \\
\hline 87 & Spencer & .000 & 1.323 \\
\hline 88 & Starke & .000 & 7.940 \\
\hline 89 & Steuben & 42.595 & 38.943 \\
\hline 90 & Sullivan & .000 & 1.323 \\
\hline 91 & Switzerland & .000 & .000 \\
\hline 92 & Tippecanoe & 42.595 & 256.238 \\
\hline 93 & Tipton & .000 & 1.323 \\
\hline 94 & Union & .000 & 5.690 \\
\hline 95 & Vanderburgh & .000 & 310.195 \\
\hline 96 & Vermillion & .000 & 231.588 \\
\hline 97 & Vigo & .000 & 136.306 \\
\hline 98 & Wabash & 42.595 & 88.569 \\
\hline 99 & Warren & .000 & .000 \\
\hline 100 & Warrick & .000 & 10.587 \\
\hline 101 & Washington & .000 & .000 \\
\hline 102 & Wayne & .000 & 18.395 \\
\hline
\end{tabular}




\begin{tabular}{|c|c|c|c|}
\hline & $\begin{array}{r}\text { State } \\
\end{array}$ & P18 97 & A18 97 \\
\hline 103 & Wells & .000 & .000 \\
\hline 104 & White & .000 & .000 \\
\hline 105 & Whitley & .000 & 1.323 \\
\hline 106 & lowa & 2910.734 & 3011.000 \\
\hline 107 & Kansas & 1678.107 & 1766.000 \\
\hline 108 & Kentucky_E & 5914.192 & 5078.000 \\
\hline 109 & Kentucky_W & 3942.795 & 3386.000 \\
\hline 110 & Louisiana & 55534.747 & 37879.000 \\
\hline 111 & Maine & 3530.575 & 3667.000 \\
\hline 112 & Maryland & 5165.343 & 3183.000 \\
\hline 113 & Massachusetts & 5003.076 & 4360.000 \\
\hline 114 & Michigan_E & 5109.910 & 5124.000 \\
\hline 115 & Michigan_W & 5109.910 & 5124.000 \\
\hline 116 & Minnesota & 7459.260 & 7626.000 \\
\hline 117 & Missisippi & 5472.744 & 11719.000 \\
\hline 118 & Missouri & 3980.086 & 6966.000 \\
\hline 119 & Montana & 3299.772 & 3100.000 \\
\hline 120 & Nebraska & 2485.412 & 2168.000 \\
\hline 121 & Nevada & 1030.045 & 1558.000 \\
\hline 122 & New Hampshire & 1554.138 & 1553.000 \\
\hline 123 & New Jersey & 25677.551 & 20078.000 \\
\hline 124 & New Mexico & 2691.018 & 2168.000 \\
\hline 125 & New York & 8076.077 & 11194.000 \\
\hline 126 & North Carolina & 9724.956 & 9112.000 \\
\hline 127 & North Dakota & 2884.529 & 2254.000 \\
\hline 128 & Ohio_N & 4605.974 & 4766.000 \\
\hline 129 & Ohio_M & 4605.974 & 4766.000 \\
\hline 130 & Ohio_s & 4605.974 & 4765.000 \\
\hline 131 & Oklahoma & 3626.323 & 3962.000 \\
\hline 132 & Oregon & 2612.404 & 4100.000 \\
\hline 133 & Pennsylvania & 21992.772 & 25992.000 \\
\hline 134 & Rhode Island & 1183.242 & 802.000 \\
\hline 135 & South Carolina & 2308.027 & 2570.000 \\
\hline 136 & South Dakota & 47.370 & 383.000 \\
\hline 137 & Tennessee & 4656.368 & 7218.000 \\
\hline 138 & Texas & 96829.273 & 93369.000 \\
\hline 139 & Utah & 3208.056 & 2999.000 \\
\hline 140 & Vermont & 366.865 & 739.000 \\
\hline 141 & Virginia & 7013.780 & 7442.000 \\
\hline 142 & Washington & 13227.310 & 10516.000 \\
\hline 143 & West Virginia & 971.588 & 2813.000 \\
\hline 144 & Wisconsin & 9420.578 & 8198.000 \\
\hline 145 & Wyoming & 2639.616 & 1735.000 \\
\hline
\end{tabular}




\begin{tabular}{|c|c|c|c|}
\hline & \begin{tabular}{|l|} 
State \\
\end{tabular} & P19 97 & A19 97 \\
\hline 1 & Alabama & 4671.000 & 2818.438 \\
\hline 2 & Arizona & 1340.000 & 1981.259 \\
\hline 3 & Arkansas & 3722.000 & 3805.546 \\
\hline 4 & California & 47339.000 & 49871.568 \\
\hline 5 & Colorado & 2912.000 & 3481.541 \\
\hline 6 & Connecticut & 3485.000 & 4589.396 \\
\hline 7 & Delaware & 3599.000 & 1091.755 \\
\hline 8 & DC & .000 & .000 \\
\hline 9 & Florida & 3090.000 & 6887.615 \\
\hline 10 & Georgia & 3624.000 & 4121.501 \\
\hline 11 & Idaho & 743.000 & 2503.490 \\
\hline 12 & Illinois $\mathbf{N}$ & 12233.000 & 9108.356 \\
\hline 13 & Illinois_S & 5243.000 & 3904.156 \\
\hline 14 & Adams & 104.402 & 119.629 \\
\hline 15 & Allen & 468.067 & 478.360 \\
\hline 16 & Bartholomew & 44.175 & 64.907 \\
\hline 17 & Benton & .000 & .000 \\
\hline 18 & Blackford & .000 & .000 \\
\hline 19 & Boone & .000 & .000 \\
\hline 20 & Brown & 2.524 & 3.709 \\
\hline 21 & Carroll & .000 & .000 \\
\hline 22 & Cass & .000 & .000 \\
\hline 23 & Clark & 779.690 & 804.526 \\
\hline 24 & Clay & 15.146 & 22.254 \\
\hline 25 & Clinton & 44.175 & 64.907 \\
\hline 26 & Crawford & .000 & .000 \\
\hline 27 & Daviess & 75.373 & 76.975 \\
\hline 28 & Dearborn & 62.751 & 58.430 \\
\hline 29 & Decatur & .000 & .000 \\
\hline 30 & DeKalb & 57.048 & 83.823 \\
\hline 31 & Delaware & 21.456 & 31.526 \\
\hline 32 & Dubois & 15.146 & 22.254 \\
\hline 33 & Elkhart & 501.974 & 703.793 \\
\hline 34 & Fayette & .000 & .000 \\
\hline 35 & Floyd & 25.748 & 37.832 \\
\hline 36 & Fountain & 2.524 & 3.709 \\
\hline 37 & Franklin & 361.362 & 328.329 \\
\hline 38 & Fulton & 2.524 & 3.709 \\
\hline 39 & Gibson & .000 & .000 \\
\hline 40 & Grant & 23.981 & 35.235 \\
\hline 41 & Greene & .000 & .000 \\
\hline 42 & Hamilton & 75.373 & 76.975 \\
\hline 43 & Hancock & .000 & .000 \\
\hline 44 & Harrison & 373.730 & 346.503 \\
\hline 45 & Hendricks & 2.524 & 3.709 \\
\hline 46 & Henry & .000 & .000 \\
\hline 47 & Howard & .000 & .000 \\
\hline 48 & Huntington & 75.373 & 76.975 \\
\hline 49 & Jackson & 44.175 & 64.907 \\
\hline 50 & Jasper & .000 & .000 \\
\hline 51 & Jay & 60.227 & 54.721 \\
\hline
\end{tabular}




\begin{tabular}{|c|c|c|c|}
\hline & \begin{tabular}{|l} 
State \\
\end{tabular} & P19 97 & A19 97 \\
\hline 52 & Jefferson & 15.146 & 22.254 \\
\hline 53 & Jennings & 363.886 & 332.038 \\
\hline 54 & Johnson & 15.146 & 22.254 \\
\hline 55 & Knox & 60.227 & 54.721 \\
\hline 56 & Kosciusko & 144.033 & 177.860 \\
\hline 57 & LaGrange & 2.524 & 3.709 \\
\hline 58 & Lake & 13379.612 & 12367.736 \\
\hline 59 & La Porte & 143.780 & 177.489 \\
\hline 60 & Lawrence & 361.362 & 328.329 \\
\hline 61 & Madison & 94.660 & 139.087 \\
\hline 62 & Marion & 2244.893 & 2896.601 \\
\hline 63 & Marshall & 394.177 & 376.545 \\
\hline 64 & Martin & .000 & .000 \\
\hline 65 & Miami & 15.146 & 22.254 \\
\hline 66 & Monroe & 402.507 & 388.785 \\
\hline 67 & Montgomery & 44.175 & 64.907 \\
\hline 68 & Morgan & 94.660 & 139.087 \\
\hline 69 & Newton & 2.524 & 3.709 \\
\hline 70 & Noble & 54.524 & 80.114 \\
\hline 71 & Ohio & .000 & .000 \\
\hline 72 & Orange & .000 & .000 \\
\hline 73 & Owen & .000 & .000 \\
\hline 74 & Parke & .000 & .000 \\
\hline 75 & Perry & .000 & .000 \\
\hline 76 & Pike & .000 & .000 \\
\hline 77 & Porter & 116.874 & 171.726 \\
\hline 78 & Posey & 2700.256 & 2701.125 \\
\hline 79 & Pulaski & .000 & .000 \\
\hline 80 & Putnam & .000 & .000 \\
\hline 81 & Randolph & 60.227 & 54.721 \\
\hline 82 & Ripley & 75.373 & 76.975 \\
\hline 83 & Rush & .000 & .000 \\
\hline 84 & St. Joseph & 277.314 & 373.694 \\
\hline 85 & Scott & 104.402 & 119.629 \\
\hline 86 & Shelby & 48.466 & 71.212 \\
\hline 87 & Spencer & 2.524 & 3.709 \\
\hline 88 & Starke & 15.146 & 22.254 \\
\hline 89 & Steuben & 60.227 & 54.721 \\
\hline 90 & Sullivan & 2.524 & 3.709 \\
\hline 91 & Switzerland & .000 & .000 \\
\hline 92 & Tippecanoe & 474.712 & 663.736 \\
\hline 93 & Tipton & 2.524 & 3.709 \\
\hline 94 & Union & 10.854 & 15.949 \\
\hline 95 & Vanderburgh & 591.689 & 869.385 \\
\hline 96 & Vermillion & 441.747 & 649.071 \\
\hline 97 & Vigo & 260.000 & 382.025 \\
\hline 98 & Wabash & 154.887 & 193.808 \\
\hline 99 & Warren & .000 & .000 \\
\hline 100 & Warrick & 20.194 & 29.672 \\
\hline 101 & Washington & .000 & .000 \\
\hline 102 & Wayne & 35.087 & 51.555 \\
\hline
\end{tabular}




\begin{tabular}{|c|c|c|c|}
\hline & State & P19 97 & A19 97 \\
\hline 103 & Wells & .000 & .000 \\
\hline 104 & White & .000 & .000 \\
\hline 105 & Whitley & 2.524 & 3.709 \\
\hline 106 & lowa & 2996.000 & 5859.253 \\
\hline 107 & Kansas & 8549.000 & 3758.253 \\
\hline 108 & Kentucky_E & 1643.000 & 2599.081 \\
\hline 109 & Kentucky_W & 1096.000 & 1732.721 \\
\hline 110 & Louisiana & 27134.000 & 19818.623 \\
\hline 111 & Maine & 1503.000 & 1486.195 \\
\hline 112 & Maryland & 6435.000 & 6090.685 \\
\hline 113 & Massachusetts & 6470.000 & 6851.391 \\
\hline 114 & Michigan_E & 5822.000 & 6247.656 \\
\hline 115 & Michigan_W & 5822.000 & 6247.656 \\
\hline 116 & Minnesota & 15177.000 & 11214.386 \\
\hline 117 & Missisippi & 4445.000 & 5182.063 \\
\hline 118 & Missouri & 9796.000 & 7621.154 \\
\hline 119 & Montana & 4729.000 & 1135.023 \\
\hline 120 & Nebraska & 1140.000 & 3073.014 \\
\hline 121 & Nevada & 264.000 & 416.577 \\
\hline 122 & New Hampshire & 377.000 & 389.409 \\
\hline 123 & New Jersey & 25568.000 & 24116.213 \\
\hline 124 & New Mexico & 1774.000 & 1707.565 \\
\hline 125 & New York & 26265.000 & 31360.033 \\
\hline 126 & North Carolina & 5578.000 & 6936.921 \\
\hline 127 & North Dakota & 3049.000 & 533.300 \\
\hline 128 & Ohio_N & 5928.000 & 6910.759 \\
\hline 129 & Ohio_M & 2928.000 & 6910.759 \\
\hline 130 & Ohio_s & 5927.000 & 6909.752 \\
\hline 131 & Oklahoma & 4655.000 & 7284.068 \\
\hline 132 & Oregon & 2432.000 & 3046.852 \\
\hline 133 & Pennsylvania & 30481.000 & 29642.405 \\
\hline 134 & Rhode Island & 453.000 & 335.073 \\
\hline 135 & South Carolina & 1308.000 & 1787.057 \\
\hline 136 & South Dakota & 278.000 & 1672.347 \\
\hline 137 & Tennessee & 2551.000 & 7763.032 \\
\hline 138 & Texas & 103426.000 & 99122.295 \\
\hline 139 & Utah & 3776.000 & 5704.294 \\
\hline 140 & Vermont & 204.000 & 88.548 \\
\hline 141 & Virginia & 4090.000 & 3933.337 \\
\hline 142 & Washington & 3412.000 & 4263.379 \\
\hline 143 & West Virginia & 3335.000 & 1396.641 \\
\hline 144 & Wisconsin & 4256.000 & 5879.377 \\
\hline 145 & Wyoming & 8011.000 & 2740.959 \\
\hline
\end{tabular}




\begin{tabular}{|c|c|c|c|}
\hline & State & P20 97 & A20 97 \\
\hline 1 & Alabama & 7460.000 & 5755.478 \\
\hline 2 & Arizona & 5020.000 & 5189.332 \\
\hline 3 & Arkansas & 1254.000 & 3759.965 \\
\hline 4 & California & 6170.000 & 12739.271 \\
\hline 5 & Colorado & 886.000 & 1563.401 \\
\hline 6 & Connecticut & 349.000 & 636.163 \\
\hline 7 & Delaware & 2446.000 & 1087.279 \\
\hline 8 & DC & .000 & .000 \\
\hline 9 & Florida & 3222.000 & 8140.090 \\
\hline 10 & Georgia & 3396.000 & 5790.487 \\
\hline 11 & Idaho & 583.000 & 861.221 \\
\hline 12 & Illinois_N & 5346.000 & 9046.323 \\
\hline 13 & Illinois_s & 2291.000 & 3876.995 \\
\hline 14 & Adams & 22.872 & 43.126 \\
\hline 15 & Allen & 141.807 & 239.530 \\
\hline 16 & Bartholomew & .000 & 21.445 \\
\hline 17 & Benton & .000 & .000 \\
\hline 18 & Blackford & .000 & .000 \\
\hline 19 & Boone & .000 & 12.378 \\
\hline 20 & Brown & .000 & .309 \\
\hline 21 & Carroll & .000 & .000 \\
\hline 22 & Cass & .000 & 11.605 \\
\hline 23 & Clark & 231.008 & 203.944 \\
\hline 24 & Clay & .000 & .309 \\
\hline 25 & Clinton & .000 & 23.209 \\
\hline 26 & Crawford & .000 & .000 \\
\hline 27 & Daviess & 22.872 & 19.917 \\
\hline 28 & Dearborn & 22.872 & 20.226 \\
\hline 29 & Decatur & .000 & .000 \\
\hline 30 & DeKalb & .000 & 8.943 \\
\hline 31 & Delaware & .000 & .309 \\
\hline 32 & Dubois & .000 & 23.209 \\
\hline 33 & Elkhart & 22.872 & 88.616 \\
\hline 34 & Fayette & .000 & .000 \\
\hline 35 & Floyd & .000 & 18.815 \\
\hline 36 & Fountain & .000 & 1.857 \\
\hline 37 & Franklin & 137.233 & 119.500 \\
\hline 38 & Fulton & .000 & .309 \\
\hline 39 & Gibson & .000 & 6.870 \\
\hline 40 & Grant & .000 & 54.155 \\
\hline 41 & Greene & .000 & 1.857 \\
\hline 42 & Hamilton & 22.872 & 26.230 \\
\hline 43 & Hancock & .000 & .309 \\
\hline 44 & Harrison & 137.233 & 119.500 \\
\hline 45 & Hendricks & .000 & 5.416 \\
\hline 46 & Henry & .000 & 1.795 \\
\hline 47 & Howard & .000 & 2.073 \\
\hline 48 & Huntington & 22.872 & 67.171 \\
\hline 49 & Jackson & .000 & .000 \\
\hline 50 & Jasper & .000 & 5.416 \\
\hline 51 & Jay & 22.872 & 19.917 \\
\hline
\end{tabular}




\begin{tabular}{|c|c|c|c|}
\hline & State & P20 97 & A20 97 \\
\hline 52 & Jefferson & .000 & .000 \\
\hline 53 & Jennings & 137.233 & 119.500 \\
\hline 54 & Johnson & .000 & 17.794 \\
\hline 55 & Knox & 22.872 & 22.423 \\
\hline 56 & Kosciusko & 22.872 & 23.630 \\
\hline 57 & LaGrange & .000 & 1.857 \\
\hline 58 & Lake & 4938.087 & 4305.334 \\
\hline 59 & La Porte & 22.872 & 22.145 \\
\hline 60 & Lawrence & 137.233 & 121.357 \\
\hline 61 & Madison & .000 & .309 \\
\hline 62 & Marion & 272.178 & 294.629 \\
\hline 63 & Marshall & 137.233 & 119.810 \\
\hline 64 & Martin & .000 & .000 \\
\hline 65 & Miami & .000 & .309 \\
\hline 66 & Monroe & 137.233 & 150.879 \\
\hline 67 & Montgomery & .000 & .000 \\
\hline 68 & Morgan & .000 & 54.155 \\
\hline 69 & Newton & .000 & 5.416 \\
\hline 70 & Noble & .000 & 1.857 \\
\hline 71 & Ohio & .000 & .000 \\
\hline 72 & Orange & .000 & .000 \\
\hline 73 & Owen & .000 & .000 \\
\hline 74 & Parke & .000 & .000 \\
\hline 75 & Perry & .000 & .309 \\
\hline 76 & Pike & .000 & .000 \\
\hline 77 & Porter & .000 & 3.435 \\
\hline 78 & Posey & 857.704 & 746.876 \\
\hline 79 & Pulaski & .000 & .000 \\
\hline 80 & Putnam & .000 & 5.416 \\
\hline 81 & Randolph & 22.872 & 21.773 \\
\hline 82 & Ripley & 22.872 & 21.773 \\
\hline 83 & Rush & .000 & .309 \\
\hline 84 & St. Joseph & 22.872 & 48.325 \\
\hline 85 & Scott & 22.872 & 19.917 \\
\hline 86 & Shelby & .000 & .000 \\
\hline 87 & Spencer & .000 & .000 \\
\hline 88 & Starke & .000 & .000 \\
\hline 89 & Steuben & 22.872 & 21.773 \\
\hline 90 & Sullivan & .000 & .000 \\
\hline 91 & Switzerland & .000 & .000 \\
\hline 92 & Tippecanoe & 22.872 & 56.804 \\
\hline 93 & Tipton & .000 & .000 \\
\hline 94 & Union & .000 & .000 \\
\hline 95 & Vanderburgh & .000 & .309 \\
\hline 96 & Vermillion & .000 & .000 \\
\hline 97 & Vigo & .000 & 54.155 \\
\hline 98 & Wabash & 22.872 & 36.256 \\
\hline 99 & Warren & .000 & .000 \\
\hline 100 & Warrick & .000 & .000 \\
\hline 101 & Washington & .000 & .000 \\
\hline 102 & Wayne & .000 & 28.749 \\
\hline
\end{tabular}




\begin{tabular}{|c|c|c|c|}
\hline & State & P20 97 & A20 97 \\
\hline 103 & Wells & .000 & .309 \\
\hline 104 & White & .000 & 5.416 \\
\hline 105 & Whitley & .000 & 5.354 \\
\hline 106 & lowa & 626.000 & 1894.486 \\
\hline 107 & Kansas & 7344.000 & 1990.511 \\
\hline 108 & Kentucky_E & 1936.000 & 2232.573 \\
\hline 109 & Kentucky_W & 1291.000 & 1488.382 \\
\hline 110 & Louisiana & 56702.000 & 31318.041 \\
\hline 111 & Maine & 724.000 & 562.144 \\
\hline 112 & Maryland & 1000.000 & 1102.283 \\
\hline 113 & Massachusetts & 371.000 & 1266.325 \\
\hline 114 & Michigan_E & 1977.000 & 5960.530 \\
\hline 115 & Michigan W & 1977.000 & 5960.530 \\
\hline 116 & Minnesota & 3609.000 & 1651.424 \\
\hline 117 & Missisippi & 2532.000 & 4659.196 \\
\hline 118 & Missouri & 3270.000 & 3185.818 \\
\hline 119 & Montana & 572.000 & 339.087 \\
\hline 120 & Nebraska & 348.000 & 392.101 \\
\hline 121 & Nevada & 681.000 & 778.200 \\
\hline 122 & New Hampshire & 288.000 & 247.063 \\
\hline 123 & New Jersey & 4314.000 & 6179.587 \\
\hline 124 & New Mexico & 4775.000 & 3928.009 \\
\hline 125 & New York & 5221.000 & 4761.222 \\
\hline 126 & North Carolina & 3489.000 & 10299.644 \\
\hline 127 & North Dakota & 48.000 & 547.140 \\
\hline 128 & Ohio_N & 3278.000 & 3618.929 \\
\hline 129 & Ohio M & 3278.000 & 3618.929 \\
\hline 130 & Ohio_s & 3278.000 & 3619.929 \\
\hline 131 & Oklahoma & 4758.000 & 1807.464 \\
\hline 132 & Oregon & 1341.000 & 6421.649 \\
\hline 133 & Pennsylvania & 6186.000 & 7445.912 \\
\hline 134 & Rhode Island & 195.000 & 213.055 \\
\hline 135 & South Carolina & 3739.000 & 6664.711 \\
\hline 136 & South Dakota & 32.000 & 119.031 \\
\hline 137 & Tennessee & 3773.000 & 6575.688 \\
\hline 138 & Texas & 92964.000 & 80805.747 \\
\hline 139 & Utah & 1639.000 & 2035.523 \\
\hline 140 & Vermont & 112.000 & 587.151 \\
\hline 141 & Virginia & 1304.000 & 4139.063 \\
\hline 142 & Washington & 2860.000 & 2897.744 \\
\hline 143 & West Virginia & 5152.000 & 4087.049 \\
\hline 144 & Wisconsin & 1387.000 & 3144.807 \\
\hline 145 & Wyoming & 11571.000 & 1518.390 \\
\hline
\end{tabular}




\begin{tabular}{|c|c|c|c|}
\hline & State & P21 97 & A21 97 \\
\hline 1 & Alabama & 32.843 & 102.000 \\
\hline 2 & Arizona & 90.568 & 38.000 \\
\hline 3 & Arkansas & 26.872 & 39.000 \\
\hline 4 & California & 671.794 & 963.000 \\
\hline 5 & Colorado & 44.786 & 72.000 \\
\hline 6 & Connecticut & 111.468 & 48.000 \\
\hline 7 & Delaware & 18.910 & 9.000 \\
\hline 8 & $\mathrm{DC}$ & .000 & .000 \\
\hline 9 & Florida & 334.404 & 635.000 \\
\hline 10 & Georgia & 273.694 & 298.000 \\
\hline 11 & Idaho & 29.858 & 31.000 \\
\hline 12 & Illinois_ $\mathbf{N}$ & 302.556 & 311.000 \\
\hline 13 & Illinois_S & 130.378 & 133.000 \\
\hline 14 & Adams & .780 & 1.247 \\
\hline 15 & Allen & 2.970 & 8.773 \\
\hline 16 & Bartholomew & 2.207 & 1.282 \\
\hline 17 & Benton & .177 & .234 \\
\hline 18 & Blackford & .029 & .097 \\
\hline 19 & Boone & .177 & .439 \\
\hline 20 & Brown & .000 & .069 \\
\hline 21 & Carroll & 1.103 & .299 \\
\hline 22 & Cass & .029 & .318 \\
\hline 23 & Clark & 3.292 & 4.236 \\
\hline 24 & Clay & .029 & .432 \\
\hline 25 & Clinton & .177 & 1.165 \\
\hline 26 & Crawford & .000 & .000 \\
\hline 27 & Daviess & .177 & .585 \\
\hline 28 & Dearborn & .000 & .214 \\
\hline 29 & Decatur & .029 & .537 \\
\hline 30 & DeKalb & .029 & 1.400 \\
\hline 31 & Delaware & .415 & 1.266 \\
\hline 32 & \begin{tabular}{|l|} 
Dubois \\
\end{tabular} & 19.389 & 1.014 \\
\hline 33 & Elkhart & 11.358 & 10.798 \\
\hline 34 & Fayette & .177 & .274 \\
\hline 35 & Floyd & 1.303 & 1.290 \\
\hline 36 & Fountain & .000 & .231 \\
\hline 37 & Franklin & .000 & .104 \\
\hline 38 & Fulton & .000 & .261 \\
\hline 39 & Gibson & .000 & .158 \\
\hline 40 & Grant & .515 & .934 \\
\hline 41 & Greene & .029 & .139 \\
\hline 42 & Hamilton & .787 & 2.991 \\
\hline 43 & Hancock & .177 & .674 \\
\hline 44 & Harrison & 1.909 & .403 \\
\hline 45 & Hendricks & .029 & .538 \\
\hline 46 & Henry & .515 & .570 \\
\hline 47 & Howard & 1.103 & .354 \\
\hline 48 & Huntington & .000 & .733 \\
\hline 49 & Jackson & .177 & 1.419 \\
\hline 50 & Jasper & .029 & .221 \\
\hline 51 & Jay & .177 & .325 \\
\hline
\end{tabular}




\begin{tabular}{|c|c|c|c|}
\hline & State & P21 97 & A21 97 \\
\hline 52 & Jefferson & .029 & .405 \\
\hline 53 & Jennings & .000 & .160 \\
\hline 54 & Johnson & .256 & .683 \\
\hline 55 & Knox & .029 & .710 \\
\hline 56 & Kosciusko & .919 & 2.129 \\
\hline 57 & LaGrange & .886 & .512 \\
\hline 58 & Lake & .744 & 11.128 \\
\hline 59 & La Porte & .627 & 3.045 \\
\hline 60 & Lawrence & .029 & .125 \\
\hline 61 & Madison & 1.228 & 2.685 \\
\hline 62 & Marion & 3.837 & 51.041 \\
\hline 63 & Marshall & .177 & 1.042 \\
\hline 64 & Martin & .000 & .106 \\
\hline 65 & Miami & 1.103 & 1.061 \\
\hline 66 & Monroe & .206 & 2.616 \\
\hline 67 & Montgomery & .000 & 1.105 \\
\hline 68 & Morgan & .029 & 2.074 \\
\hline 69 & Newton & .177 & .224 \\
\hline 70 & Noble & .177 & 1.539 \\
\hline 71 & Ohio & .000 & .000 \\
\hline 72 & Orange & 5.149 & .099 \\
\hline 73 & Owen & .000 & .017 \\
\hline 74 & Parke & .029 & .104 \\
\hline 75 & Perry & .862 & .076 \\
\hline 76 & Pike & .000 & .073 \\
\hline 77 & Porter & .262 & 3.823 \\
\hline 78 & Posey & .000 & 9.490 \\
\hline 79 & Pulaski & .029 & .445 \\
\hline 80 & Putnam & .000 & .092 \\
\hline 81 & Randolph & .142 & .102 \\
\hline 82 & Ripley & .515 & .617 \\
\hline 83 & Rush & .000 & .141 \\
\hline 84 & St. Joseph & .513 & 9.189 \\
\hline 85 & Scott & .177 & .929 \\
\hline 86 & Shelby & .977 & 1.245 \\
\hline 87 & Spencer & 2.207 & .460 \\
\hline 88 & Starke & .029 & .391 \\
\hline 89 & Steuben & .029 & .024 \\
\hline 90 & Sullivan & .000 & .304 \\
\hline 91 & Switzerland & .029 & .017 \\
\hline 92 & Tippecanoe & 2.207 & 9.637 \\
\hline 93 & Tipton & .000 & .302 \\
\hline 94 & Union & .000 & .328 \\
\hline 95 & Vanderburgh & 1.199 & 14.976 \\
\hline 96 & Vermillion & .000 & 9.252 \\
\hline 97 & Vigo & .177 & 6.628 \\
\hline 98 & Wabash & .029 & 2.100 \\
\hline 99 & Warren & .000 & .104 \\
\hline 100 & Warrick & .029 & .740 \\
\hline 101 & Washington & 5.149 & .017 \\
\hline 102 & Wayne & 2.101 & 1.471 \\
\hline
\end{tabular}




\begin{tabular}{|c|c|c|c|}
\hline & State & P21 97 & A21 97 \\
\hline 103 & Wells & .048 & 2.633 \\
\hline 104 & White & .843 & .545 \\
\hline 105 & Whitley & .129 & .214 \\
\hline$\overline{106}$ & lowa & 78.625 & 182.000 \\
\hline 107 & Kansas & 79.620 & 66.000 \\
\hline 108 & Kentucky_E & 34.834 & 51.000 \\
\hline 109 & Kentucky_W & 23.886 & 34.000 \\
\hline 110 & Louisiana & 35.829 & 68.000 \\
\hline 111 & Maine & 11.943 & 18.000 \\
\hline 112 & Maryland & 86.587 & 317.000 \\
\hline 113 & Massachusetts & 210.993 & 173.000 \\
\hline 114 & Michigan_E & 132.368 & 191.000 \\
\hline 115 & Michigan_W & 132.368 & 191.000 \\
\hline 116 & Minnesota & 154.264 & 172.000 \\
\hline 117 & Missisippi & 160.235 & 42.000 \\
\hline 118 & Missouri & 451.844 & 194.000 \\
\hline 119 & Montana & 13.934 & 14.000 \\
\hline 120 & Nebraska & 89.573 & 57.000 \\
\hline 121 & Nevada & 11.943 & 34.000 \\
\hline 122 & New Hampshire & 49.763 & 16.000 \\
\hline 123 & New Jersey & 916.626 & 921.000 \\
\hline 124 & New Mexico & 10.948 & 8.000 \\
\hline 125 & New York & 383.172 & 548.000 \\
\hline 126 & North Carolina & 2035.288 & 472.000 \\
\hline 127 & North Dakota & .000 & 10.000 \\
\hline 128 & Ohio_N & 77.630 & 126.000 \\
\hline 129 & Ohio M & 76.634 & 126.000 \\
\hline 130 & Ohio_S & 76.634 & 125.000 \\
\hline 131 & Oklahoma & 26.872 & 46.000 \\
\hline 132 & Oregon & 52.748 & 128.000 \\
\hline 133 & Pennsylvania & 316.490 & 569.000 \\
\hline 134 & Rhode Island & 96.539 & 12.000 \\
\hline 135 & South Carolina & 201.041 & 176.000 \\
\hline 136 & South Dakota & 34.834 & 13.000 \\
\hline 137 & Tennessee & 532.459 & 808.000 \\
\hline 138 & Texas & 631.984 & 589.000 \\
\hline 139 & Utah & 97.535 & 68.000 \\
\hline 140 & Vermont & 47.772 & 9.000 \\
\hline 141 & Virginia & 163.221 & 187.000 \\
\hline 142 & Washington & 91.563 & 99.000 \\
\hline 143 & West Virginia & 21.896 & 47.000 \\
\hline 144 & Wisconsin & 44.786 & 55.000 \\
\hline 145 & Wyoming & 3.981 & 4.000 \\
\hline
\end{tabular}




\begin{tabular}{|c|c|c|c|}
\hline & State & P22 97 & A22 97 \\
\hline 1 & Alabama & 2380.696 & 1736.000 \\
\hline 2 & Arizona & 425.767 & 1089.000 \\
\hline 3 & Arkansas & 3225.233 & 2094.000 \\
\hline 4 & California & 11942.458 & 12521.000 \\
\hline 5 & Colorado & 1477.191 & 762.000 \\
\hline 6 & Connecticut & 1231.325 & 1671.000 \\
\hline 7 & Delaware & 275.849 & 375.000 \\
\hline 8 & $\mathrm{DC}$ & .000 & .000 \\
\hline 9 & Florida & 57248.639 & 52299.000 \\
\hline 10 & Georgia & 2982.366 & 3038.000 \\
\hline 11 & Idaho & 2584.584 & 1899.000 \\
\hline 12 & Illinois_N & 4707.421 & 6095.000 \\
\hline 13 & Illinois_S & 2017.895 & 2612.000 \\
\hline 14 & Adams & 15.212 & 28.968 \\
\hline 15 & Allen & 148.488 & 282.759 \\
\hline 16 & Bartholomew & 32.059 & 61.049 \\
\hline 17 & Benton & 4.362 & 8.306 \\
\hline 18 & Blackford & 6.429 & 12.242 \\
\hline 19 & Boone & 20.149 & 38.369 \\
\hline 20 & Brown & 6.785 & 12.920 \\
\hline 21 & Carroll & 9.154 & 17.431 \\
\hline 22 & Cass & 18.447 & 35.128 \\
\hline 23 & Clark & 43.172 & 82.210 \\
\hline 24 & Clay & 12.044 & 22.934 \\
\hline 25 & Clinton & 15.435 & 29.391 \\
\hline 26 & Crawford & 4.781 & 9.103 \\
\hline 27 & Daviess & 13.530 & 25.765 \\
\hline 28 & Dearborn & 20.555 & 39.143 \\
\hline 29 & Decatur & 11.212 & 21.351 \\
\hline 30 & DeKalb & 17.852 & 33.996 \\
\hline 31 & Delaware & 54.513 & 103.808 \\
\hline 32 & Dubois & 17.911 & 34.108 \\
\hline 33 & Elkhart & 81.151 & 154.532 \\
\hline 34 & Fayette & 11.813 & 22.495 \\
\hline 35 & Floyd & 32.218 & 61.352 \\
\hline 36 & Fountain & 8.218 & 15.650 \\
\hline 37 & Franklin & 9.884 & 18.821 \\
\hline 38 & Fulton & 9.253 & 17.620 \\
\hline 39 & Gibson & 14.644 & 27.887 \\
\hline 40 & Grant & 33.714 & 64.200 \\
\hline 41 & Greene & 15.159 & 28.868 \\
\hline 42 & Hamilton & 75.560 & 143.885 \\
\hline 43 & Hancock & 24.539 & 46.729 \\
\hline 44 & Harrison & 15.248 & 29.036 \\
\hline 45 & Hendricks & 44.268 & 84.298 \\
\hline 46 & Henry & 22.254 & 42.378 \\
\hline 47 & Howard & 38.485 & 73.286 \\
\hline 48 & Huntington & 17.266 & 32.878 \\
\hline 49 & Jackson & 18.640 & 35.496 \\
\hline 50 & Jasper & 13.294 & 25.315 \\
\hline 51 & Jay & 9.936 & 18.922 \\
\hline
\end{tabular}




\begin{tabular}{|c|c|c|c|}
\hline & State & P22 97 & A22 97 \\
\hline 52 & Jefferson & 14.294 & 27.220 \\
\hline 53 & Jennings & 12.292 & 23.408 \\
\hline 54 & Johnson & 50.049 & 95.306 \\
\hline 55 & Knox & 18.118 & 34.501 \\
\hline 56 & Kosciusko & 33.205 & 63.231 \\
\hline 57 & LaGrange & 15.559 & 29.629 \\
\hline 58 & Lake & 221.205 & 421.231 \\
\hline 59 & La Porte & 50.161 & 95.519 \\
\hline 60 & Lawrence & 20.907 & 39.812 \\
\hline 61 & Madison & 60.769 & 115.720 \\
\hline 62 & Marion & 389.642 & 741.979 \\
\hline 63 & Marshall & 20.303 & 38.662 \\
\hline 64 & Martin & 4.780 & 9.102 \\
\hline 65 & Miami & 16.020 & 30.507 \\
\hline 66 & Monroe & 54.428 & 103.645 \\
\hline 67 & Montgomery & 17.032 & 32.433 \\
\hline 68 & Morgan & 29.594 & 56.354 \\
\hline 69 & Newton & 6.658 & 12.679 \\
\hline 70 & Noble & 20.426 & 38.896 \\
\hline 71 & Ohio & 2.535 & 4.827 \\
\hline 72 & Orange & 8.737 & 16.638 \\
\hline 73 & Owen & 9.680 & 18.433 \\
\hline 74 & Parke & 7.770 & 14.797 \\
\hline 75 & Perry & 8.730 & 16.625 \\
\hline 76 & Pike & 5.851 & 11.142 \\
\hline 77 & Porter & 65.931 & 125.549 \\
\hline 78 & Posey & 12.318 & 23.456 \\
\hline 79 & Pulaski & 6.238 & 11.878 \\
\hline 80 & Putnam & 16.008 & 30.483 \\
\hline 81 & Randolph & 12.535 & 23.871 \\
\hline 82 & Ripley & 11.899 & 22.658 \\
\hline 83 & Rush & 8.379 & 15.955 \\
\hline 84 & St. Joseph & 120.366 & 229.207 \\
\hline 85 & Scott & 10.245 & 19.509 \\
\hline 86 & Shelby & 19.597 & 37.318 \\
\hline 87 & Spencer & 9.262 & 17.637 \\
\hline 88 & Starke & 10.874 & 20.707 \\
\hline 89 & Steuben & 14.783 & 28.151 \\
\hline 90 & Sullivan & 9.812 & 18.685 \\
\hline 91 & Switzerland & 4.020 & 7.655 \\
\hline 92 & Tippecanoe & 66.687 & 126.989 \\
\hline 93 & Tipton & 7.568 & 14.412 \\
\hline 94 & Union & 3.319 & 6.320 \\
\hline 95 & Vanderburgh & 78.290 & 149.085 \\
\hline 96 & Vermillion & 7.663 & 14.592 \\
\hline 97 & Vigo & 48.599 & 92.546 \\
\hline 98 & Wabash & 15.930 & 30.334 \\
\hline 99 & Warren & 3.790 & 7.217 \\
\hline 100 & Warrick & 23.314 & 44.396 \\
\hline 101 & Washington & 12.236 & 23.301 \\
\hline 102 & Wayne & 32.644 & 62.163 \\
\hline
\end{tabular}




\begin{tabular}{|c|c|c|c|}
\hline & State & P22 97 & A22 97 \\
\hline 103 & Wells & 12.544 & 23.887 \\
\hline 104 & White & 11.483 & 21.867 \\
\hline 105 & Whitley & 13.785 & 26.250 \\
\hline 106 & lowa & 3824.905 & 4385.000 \\
\hline 107 & Kansas & 2743.497 & 3598.000 \\
\hline 108 & Kentucky_E & 425.767 & 913.000 \\
\hline 109 & Kentucky_W & 283.845 & 608.000 \\
\hline 110 & Louisiana & 12210.311 & 4591.000 \\
\hline 111 & Maine & 461.747 & 626.000 \\
\hline 112 & Maryland & 1895.961 & 935.000 \\
\hline 113 & Massachusetts & 2288.746 & 3107.000 \\
\hline 114 & Michigan_E & 684.625 & 1746.000 \\
\hline 115 & Michigan_W & 684.625 & 1746.000 \\
\hline 116 & Minnesota & 2589.581 & 2832.000 \\
\hline 117 & Missisippi & 2174.809 & 2104.000 \\
\hline 118 & Missouri & 2491.635 & 3716.000 \\
\hline 119 & Montana & 694.619 & 1083.000 \\
\hline 120 & Nebraska & 3529.067 & 4192.000 \\
\hline 121 & Nevada & 94.948 & 316.000 \\
\hline 122 & New Hampshire & .000 & 594.000 \\
\hline 123 & New Jersey & 3021.345 & 570.000 \\
\hline 124 & New Mexico & 3663.993 & 315.000 \\
\hline 125 & New York & 347.809 & 1823.000 \\
\hline 126 & North Carolina & 5316.088 & 5147.000 \\
\hline 127 & North Dakota & 377.793 & 736.000 \\
\hline 128 & Ohio_N & 1382.243 & 1876.000 \\
\hline 129 & Ohio M & 1382.243 & 1876.000 \\
\hline 130 & Ohio_s & 1381.243 & 1876.000 \\
\hline 131 & Oklahoma & 4954.286 & 1549.000 \\
\hline 132 & Oregon & 956.476 & 1442.000 \\
\hline 133 & Pennsylvania & 4495.537 & 6102.000 \\
\hline 134 & Rhode Island & 376.794 & 10.000 \\
\hline 135 & South Carolina & 1286.295 & 2284.000 \\
\hline 136 & South Dakota & 273.850 & 1074.000 \\
\hline 137 & Tennessee & 1333.270 & 1406.000 \\
\hline 138 & Texas & 5092.210 & 9862.000 \\
\hline 139 & Utah & 1486.186 & 810.000 \\
\hline 140 & Vermont & 219.880 & 298.000 \\
\hline 141 & Virginia & 2938.390 & 2932.000 \\
\hline 142 & Washington & 4116.745 & 3909.000 \\
\hline 143 & West Virginia & 668.634 & 825.000 \\
\hline 144 & Wisconsin & 2864.431 & 3411.000 \\
\hline 145 & Wyoming & 641.648 & 265.000 \\
\hline
\end{tabular}




\begin{tabular}{|c|c|c|c|}
\hline & $\begin{array}{r}\text { State } \\
\end{array}$ & P23 97 & A23 97 \\
\hline 1 & Alabama & 1268.347 & 1479.000 \\
\hline 2 & Arizona & 759.412 & 1228.000 \\
\hline 3 & Arkansas & 553.842 & 1016.000 \\
\hline 4 & California & 6991.378 & 9734.000 \\
\hline 5 & Colorado & 330.309 & 712.000 \\
\hline 6 & Connecticut & 701.533 & 438.000 \\
\hline 7 & Delaware & 584.777 & 283.000 \\
\hline$\overline{8}$ & DC & .000 & .000 \\
\hline 9 & Florida & 2566.632 & 3318.000 \\
\hline 10 & Georgia & 7369.586 & 5606.000 \\
\hline 11 & Idaho & 100.789 & 234.000 \\
\hline 12 & Illinois $\mathbf{N}$ & 5914.630 & 3646.000 \\
\hline 13 & Illinois_S & 2534.699 & 1563.000 \\
\hline$\overline{14}$ & Adams & 7.963 & 29.404 \\
\hline 15 & Allen & 49.387 & 162.160 \\
\hline 16 & Bartholomew & 17.738 & 24.690 \\
\hline 17 & Benton & 1.096 & 2.321 \\
\hline 18 & Blackford & 9.700 & 10.193 \\
\hline 19 & Boone & 4.565 & 4.411 \\
\hline 20 & Brown & 1.012 & 1.406 \\
\hline 21 & Carroll & 6.487 & 1.897 \\
\hline 22 & Cass & 10.676 & 22.153 \\
\hline 23 & Clark & 23.707 & 40.457 \\
\hline 24 & Clay & 2.318 & 6.630 \\
\hline 25 & Clinton & 5.741 & 7.333 \\
\hline 26 & Crawford & .000 & .990 \\
\hline 27 & Daviess & 3.008 & 6.111 \\
\hline 28 & Dearborn & 3.459 & 7.567 \\
\hline 29 & Decatur & 42.808 & 11.184 \\
\hline 30 & DeKalb & 50.902 & 53.746 \\
\hline 31 & Delaware & 29.112 & 17.669 \\
\hline 32 & Dubois & 2.948 & 28.233 \\
\hline 33 & Elkhart & 113.236 & 189.866 \\
\hline 34 & Fayette & 5.631 & 2.447 \\
\hline 35 & Floyd & 10.531 & 41.033 \\
\hline 36 & Fountain & 1.012 & 1.703 \\
\hline 37 & Franklin & .150 & 17.525 \\
\hline 38 & Fulton & 12.859 & 5.225 \\
\hline 39 & Gibson & 1.982 & 11.895 \\
\hline 40 & Grant & 3.652 & 23.328 \\
\hline 41 & Greene & 1.396 & 3.750 \\
\hline 42 & Hamilton & 18.384 & 55.163 \\
\hline 43 & Hancock & 6.892 & 5.510 \\
\hline 44 & Harrison & 1.655 & 18.637 \\
\hline 45 & Hendricks & 3.129 & 11.198 \\
\hline 46 & Henry & 5.255 & 12.399 \\
\hline 47 & Howard & 3.138 & 18.796 \\
\hline 48 & Huntington & 15.696 & 13.614 \\
\hline 49 & Jackson & 12.318 & 15.246 \\
\hline 50 & Jasper & 10.135 & 4.172 \\
\hline 51 & Jay & 14.219 & 8.084 \\
\hline
\end{tabular}




\begin{tabular}{|c|c|c|c|}
\hline & State & P23 97 & A23 97 \\
\hline 52 & Jefferson & 2.753 & 7.706 \\
\hline 53 & Jennings & 9.691 & 53.099 \\
\hline 54 & Johnson & 16.852 & 29.079 \\
\hline 55 & Knox & 1.757 & 5.880 \\
\hline 56 & Kosciusko & 25.246 & 37.340 \\
\hline 57 & LaGrange & 3.309 & 21.555 \\
\hline 58 & Lake & 54.532 & 470.870 \\
\hline 59 & La Porte & 36.436 & 31.630 \\
\hline 60 & Lawrence & 8.634 & 21.694 \\
\hline 61 & Madison & 19.958 & 21.653 \\
\hline 62 & Marion & 218.766 & 165.261 \\
\hline 63 & Marshall & 16.833 & 75.086 \\
\hline 64 & Martin & .150 & .990 \\
\hline 65 & Miami & 4.630 & 12.789 \\
\hline 66 & Monroe & 7.620 & 32.747 \\
\hline 67 & Montgomery & 11.582 & 21.251 \\
\hline 68 & Morgan & 9.027 & 8.891 \\
\hline 69 & Newton & 1.012 & 12.013 \\
\hline 70 & Noble & 23.403 & 61.037 \\
\hline 71 & Ohio & .150 & .525 \\
\hline 72 & Orange & .901 & 5.945 \\
\hline 73 & Owen & .195 & 5.895 \\
\hline 74 & Parke & .150 & 5.745 \\
\hline 75 & Perry & .901 & 5.462 \\
\hline 76 & Pike & .901 & 1.212 \\
\hline 77 & Porter & 28.397 & 21.594 \\
\hline 78 & Posey & 20.323 & 82.931 \\
\hline 79 & Pulaski & 2.628 & 1.292 \\
\hline 80 & Putnam & .150 & 31.081 \\
\hline 81 & Randolph & 1.667 & 5.905 \\
\hline 82 & Ripley & 3.819 & 4.592 \\
\hline 83 & Rush & .901 & 3.763 \\
\hline 84 & St. Joseph & 75.566 & 104.049 \\
\hline 85 & Scott & 3.264 & 39.834 \\
\hline 86 & Shelby & 7.641 & 51.721 \\
\hline 87 & Spencer & 1.012 & 10.780 \\
\hline 88 & Starke & .666 & 6.388 \\
\hline 89 & Steuben & 20.360 & 24.084 \\
\hline 90 & Sullivan & .261 & 6.168 \\
\hline 91 & Switzerland & .000 & 4.968 \\
\hline 92 & Tippecanoe & 27.367 & 33.656 \\
\hline 93 & Tipton & 1.973 & 1.568 \\
\hline 94 & Union & .627 & .688 \\
\hline 95 & Vanderburgh & 54.888 & 113.110 \\
\hline 96 & Vermillion & 22.049 & 1.588 \\
\hline 97 & Vigo & 21.491 & 50.470 \\
\hline 98 & Wabash & 19.402 & 9.935 \\
\hline 99 & Warren & 1.862 & 1.022 \\
\hline 100 & Warrick & 4.386 & 5.676 \\
\hline 101 & Washington & 9.144 & 7.828 \\
\hline 102 & Wayne & 11.092 & 33.772 \\
\hline
\end{tabular}




\begin{tabular}{|c|c|c|c|}
\hline & State & P23 97 & A23 97 \\
\hline 103 & Wells & 9.174 & 20.930 \\
\hline 104 & White & 14.114 & 11.424 \\
\hline 105 & Whitley & 10.637 & 13.678 \\
\hline 106 & lowa & 1228.431 & 1157.000 \\
\hline 107 & Kansas & 1015.875 & 629.000 \\
\hline 108 & Kentucky_E & 763.403 & 902.000 \\
\hline 109 & Kentucky_W & 508.936 & 601.000 \\
\hline 110 & Louisiana & 4527.531 & 2796.000 \\
\hline 111 & Maine & 70.852 & 210.000 \\
\hline 112 & Maryland & 1730.381 & 1109.000 \\
\hline 113 & Massachusetts & 1093.712 & 1074.000 \\
\hline 114 & Michigan E & 1801.233 & 1921.000 \\
\hline 115 & Michigan_W & 1801.233 & 1921.000 \\
\hline 116 & Minnesota & 1314.251 & 1444.000 \\
\hline 117 & Missisippi & 956.001 & 1003.000 \\
\hline 118 & Missouri & 5018.504 & 4198.000 \\
\hline 119 & Montana & 48.898 & 596.000 \\
\hline 120 & Nebraska & 273.428 & 442.000 \\
\hline 121 & Nevada & 105.779 & 492.000 \\
\hline 122 & New Hampshire & 55.883 & 158.000 \\
\hline 123 & New Jersey & 3863.919 & 3153.000 \\
\hline 124 & New Mexico & 64.864 & 213.000 \\
\hline 125 & New York & 2040.732 & 2782.000 \\
\hline 126 & North Carolina & 1614.623 & 2906.000 \\
\hline 127 & North Dakota & 56.881 & 164.000 \\
\hline 128 & Ohio_N & 3018.686 & 1646.000 \\
\hline 129 & Ohio_M & 3018.686 & 1646.000 \\
\hline 130 & Ohio_S & 3018.686 & 1645.000 \\
\hline 131 & Oklahoma & 316.338 & 655.000 \\
\hline 132 & Oregon & 840.243 & 957.000 \\
\hline 133 & Pennsylvania & 1843.145 & 4083.000 \\
\hline 134 & Rhode Island & 69.854 & 135.000 \\
\hline 135 & South Carolina & 1808.218 & 1824.000 \\
\hline 136 & South Dakota & 50.894 & 110.000 \\
\hline 137 & Tennessee & 1139.616 & 1780.000 \\
\hline 138 & Texas & 8959.262 & 9150.000 \\
\hline 139 & Utah & 413.136 & 539.000 \\
\hline 140 & Vermont & 109.770 & 192.000 \\
\hline 141 & Virginia & 760.410 & 1456.000 \\
\hline 142 & Washington & 1372.130 & 1483.000 \\
\hline 143 & West Virginia & 944.026 & 698.000 \\
\hline 144 & Wisconsin & 1852.126 & 1886.000 \\
\hline 145 & Wyoming & 1269.345 & 69.000 \\
\hline
\end{tabular}




\begin{tabular}{|c|c|c|c|}
\hline & State & P24 97 & A24 97 \\
\hline 1 & Alabama & 1584.769 & 1765.000 \\
\hline 2 & Arizona & 453.934 & 884.000 \\
\hline 3 & Arkansas & 1228.821 & 1613.000 \\
\hline 4 & California & 5763.160 & 9196.000 \\
\hline 5 & Colorado & 422.938 & 844.000 \\
\hline 6 & Connecticut & 609.911 & 818.000 \\
\hline 7 & Delaware & 441.936 & 5058.000 \\
\hline 8 & DC & .000 & .000 \\
\hline 9 & Florida & 2205.678 & 3439.000 \\
\hline 10 & Georgia & 3749.453 & 5145.000 \\
\hline 11 & Idaho & 148.978 & 260.000 \\
\hline 12 & Illinois $\mathbf{N}$ & 6220.093 & 4697.000 \\
\hline 13 & Illinois_S & 2665.611 & 2013.000 \\
\hline 14 & Adams & 6.903 & 23.179 \\
\hline 15 & Allen & 42.797 & 194.461 \\
\hline 16 & Bartholomew & .000 & 102.717 \\
\hline 17 & Benton & .000 & 1.623 \\
\hline 18 & Blackford & .000 & 1.623 \\
\hline 19 & Boone & .000 & 12.036 \\
\hline 20 & Brown & .000 & .411 \\
\hline 21 & Carroll & .000 & 1.623 \\
\hline 22 & Cass & .000 & 5.895 \\
\hline 23 & Clark & 69.718 & 83.586 \\
\hline 24 & Clay & .000 & 5.034 \\
\hline 25 & Clinton & .000 & 12.976 \\
\hline 26 & Crawford & .000 & .000 \\
\hline 27 & Daviess & 6.903 & 10.153 \\
\hline 28 & Dearborn & 6.903 & 6.639 \\
\hline 29 & Decatur & .000 & 12.117 \\
\hline 30 & DeKalb & .000 & 26.565 \\
\hline 31 & Delaware & .000 & 32.241 \\
\hline 32 & Dubois & .000 & 6.277 \\
\hline 33 & Elkhart & 6.903 & 149.827 \\
\hline 34 & Fayette & .000 & 18.473 \\
\hline 35 & Floyd & .000 & 20.986 \\
\hline 36 & Fountain & .000 & .411 \\
\hline 37 & Franklin & 41.417 & 19.354 \\
\hline 38 & Fulton & .000 & 9.985 \\
\hline 39 & Gibson & .000 & 4.679 \\
\hline 40 & Grant & .000 & 24.884 \\
\hline 41 & Greene & .000 & .621 \\
\hline 42 & Hamilton & 6.903 & 23.808 \\
\hline 43 & Hancock & .000 & 6.302 \\
\hline 44 & Harrison & 41.417 & 21.366 \\
\hline 45 & Hendricks & .000 & 7.361 \\
\hline 46 & Henry & .000 & 13.869 \\
\hline 47 & Howard & .000 & 9.467 \\
\hline 48 & Huntington & 6.903 & 17.563 \\
\hline 49 & Jackson & .000 & 26.336 \\
\hline 50 & Jasper & .000 & .270 \\
\hline 51 & Jay & 6.903 & $\overline{8.446}$ \\
\hline
\end{tabular}




\begin{tabular}{|c|c|c|c|}
\hline & State & P24 97 & A24 97 \\
\hline 52 & Jefferson & .000 & 23.181 \\
\hline 53 & Jennings & 41.417 & 49.330 \\
\hline 54 & Johnson & .000 & 21.613 \\
\hline 55 & Knox & 6.903 & 5.308 \\
\hline 56 & Kosciusko & 6.903 & 44.411 \\
\hline 57 & LaGrange & .000 & 15.854 \\
\hline 58 & Lake & 1490.306 & 739.214 \\
\hline 59 & La Porte & 6.903 & 68.939 \\
\hline 60 & Lawrence & 41.417 & 28.982 \\
\hline 61 & Madison & .000 & 27.490 \\
\hline 62 & Marion & 82.143 & 537.704 \\
\hline 63 & Marshall & 41.417 & 30.962 \\
\hline 64 & Martin & .000 & .270 \\
\hline 65 & Miami & .000 & 8.576 \\
\hline 66 & Monroe & 41.417 & 75.485 \\
\hline 67 & Montgomery & .000 & 11.948 \\
\hline 68 & Morgan & .000 & 23.542 \\
\hline 69 & Newton & .000 & 2.656 \\
\hline 70 & Noble & .000 & 32.182 \\
\hline 71 & Ohio & .000 & .000 \\
\hline 72 & Orange & .000 & .270 \\
\hline 73 & Owen & .000 & 3.997 \\
\hline 74 & Parke & .000 & 1.623 \\
\hline 75 & Perry & .000 & 8.460 \\
\hline 76 & Pike & .000 & .000 \\
\hline 77 & Porter & .000 & 33.215 \\
\hline 78 & Posey & 258.853 & 188.043 \\
\hline 79 & Pulaski & .000 & 12.983 \\
\hline 80 & Putnam & .000 & 4.733 \\
\hline 81 & Randolph & 6.903 & 17.615 \\
\hline 82 & Ripley & 6.903 & 5.419 \\
\hline 83 & Rush & .000 & 14.659 \\
\hline 84 & St. Joseph & 6.903 & 91.150 \\
\hline 85 & Scott & 6.903 & 22.635 \\
\hline 86 & Shelby & .000 & 18.515 \\
\hline 87 & Spencer & .000 & 5.144 \\
\hline 88 & Starke & .000 & 12.607 \\
\hline 89 & Steuben & 6.903 & 20.692 \\
\hline 90 & Sullivan & .000 & .411 \\
\hline 91 & Switzerland & .000 & .000 \\
\hline 92 & Tippecanoe & 6.903 & 146.824 \\
\hline 93 & Tipton & .000 & 5.144 \\
\hline 94 & Union & .000 & 2.037 \\
\hline 95 & Vanderburgh & .000 & 146.962 \\
\hline 96 & Vermillion & .000 & 71.875 \\
\hline 97 & Vigo & .000 & 54.853 \\
\hline 98 & Wabash & 6.903 & 31.988 \\
\hline 99 & Warren & .000 & 4.733 \\
\hline 100 & Warrick & .000 & 5.530 \\
\hline 101 & Washington & .000 & 20.285 \\
\hline 102 & Wayne & .000 & 15.581 \\
\hline
\end{tabular}




\begin{tabular}{|c|c|c|c|}
\hline & State & P24 97 & A24 97 \\
\hline 103 & Wells & .000 & 8.654 \\
\hline 104 & White & .000 & 8.708 \\
\hline 105 & Whitley & .000 & 3.846 \\
\hline 106 & lowa & 1827.734 & 1334.000 \\
\hline 107 & Kansas & 1256.817 & 1115.000 \\
\hline 108 & Kentucky_E & 984.856 & 1575.000 \\
\hline 109 & Kentucky_W & 655.904 & 1050.000 \\
\hline 110 & Louisiana & 7582.895 & 1612.000 \\
\hline$\overline{111}$ & Maine & 173.975 & 2587.000 \\
\hline 112 & Maryland & 535.922 & 1368.000 \\
\hline$\overline{113}$ & Massachusetts & 2035.703 & 5564.000 \\
\hline 114 & Michigan_E & 1530.777 & 2784.000 \\
\hline 115 & Michigan_W & 1530.777 & 2784.000 \\
\hline 116 & Minnesota & 1226.821 & 1971.000 \\
\hline 117 & Missisippi & 1716.750 & 1104.000 \\
\hline 118 & Missouri & 1728.748 & 2421.000 \\
\hline 119 & Montana & 33.995 & 194.000 \\
\hline 120 & Nebraska & 350.949 & 601.000 \\
\hline 121 & Nevada & 210.969 & 373.000 \\
\hline 122 & New Hampshire & 14245.923 & 3839.000 \\
\hline 123 & New Jersey & 5256.234 & 4081.000 \\
\hline 124 & New Mexico & 101.985 & 196.000 \\
\hline 125 & New York & 2991.564 & 4500.000 \\
\hline 126 & North Carolina & 4652.322 & 4766.000 \\
\hline 127 & North Dakota & 50.993 & 265.000 \\
\hline 128 & Ohio $\mathbf{N}$ & 2526.632 & 2270.000 \\
\hline 129 & Ohio_M & 2526.632 & 2270.000 \\
\hline 130 & Ohio_s & 2526.632 & 2270.000 \\
\hline$\overline{131}$ & Oklahoma & 1118.837 & 1010.000 \\
\hline 132 & Oregon & 764.888 & 1092.000 \\
\hline 133 & Pennsylvania & 5585.186 & 4577.000 \\
\hline 134 & Rhode Island & 193.972 & 366.000 \\
\hline 135 & South Carolina & 2661.612 & 2680.000 \\
\hline 136 & South Dakota & 97.986 & 153.000 \\
\hline 137 & Tennessee & 2885.579 & 2944.000 \\
\hline 138 & Texas & 23914.514 & 16010.000 \\
\hline 139 & Utah & 308.955 & 503.000 \\
\hline 140 & Vermont & 16.998 & 1416.000 \\
\hline 141 & Virginia & 1789.739 & 2238.000 \\
\hline 142 & Washington & 1053.846 & 1625.000 \\
\hline 143 & West Virginia & 1315.808 & 1015.000 \\
\hline 144 & Wisconsin & 2313.663 & 2305.000 \\
\hline 145 & Wyoming & 237.965 & 107.000 \\
\hline
\end{tabular}




\begin{tabular}{|c|c|c|c|}
\hline & State & P25 97 & A25 97 \\
\hline 1 & Alabama & 40817.000 & 37663.543 \\
\hline 2 & Arizona & 4221.000 & 4500.583 \\
\hline 3 & Arkansas & 20514.000 & 8132.578 \\
\hline 4 & California & 9419.000 & 9619.200 \\
\hline 5 & Colorado & 31.000 & 58.463 \\
\hline 6 & Connecticut & 752.000 & 801.774 \\
\hline 7 & Delaware & .000 & 596.111 \\
\hline 8 & DC & .000 & .000 \\
\hline 9 & Florida & 8050.000 & 20158.144 \\
\hline 10 & Georgia & 14157.000 & 41215.151 \\
\hline 11 & Idaho & 4478.000 & 4775.149 \\
\hline 12 & Illinois $\mathbf{N}$ & 3181.000 & 128.409 \\
\hline 13 & Illinois_S & 1363.000 & 55.331 \\
\hline 14 & Adams & 386.828 & 54.295 \\
\hline 15 & Allen & 126.256 & 17.721 \\
\hline 16 & Bartholomew & 40.295 & 5.656 \\
\hline 17 & Benton & 117.526 & 16.496 \\
\hline 18 & Blackford & 52.383 & 7.352 \\
\hline 19 & Boone & .000 & .000 \\
\hline 20 & Brown & 18.133 & 2.545 \\
\hline 21 & Carroll & .000 & .000 \\
\hline 22 & Cass & 112.825 & 15.836 \\
\hline 23 & Clark & 447.941 & 62.873 \\
\hline 24 & Clay & 40.295 & 5.656 \\
\hline 25 & Clinton & 22.162 & 3.111 \\
\hline 26 & Crawford & 40.295 & 5.656 \\
\hline 27 & Daviess & 151.105 & 21.209 \\
\hline 28 & Dearborn & 6.716 & .943 \\
\hline 29 & Decatur & 17.461 & 2.451 \\
\hline 30 & DeKalb & 448.613 & 62.968 \\
\hline 31 & Delaware & 40.295 & 5.656 \\
\hline 32 & Dubois & 1152.425 & 161.755 \\
\hline 33 & Elkhart & 4284.656 & 601.396 \\
\hline 34 & Fayette & .000 & .000 \\
\hline 35 & Floyd & 397.573 & 55.804 \\
\hline 36 & Fountain & 6.716 & .943 \\
\hline 37 & Franklin & 26.191 & 3.676 \\
\hline 38 & Fulton & 176.625 & 24.791 \\
\hline 39 & Gibson & .000 & .000 \\
\hline 40 & Grant & 40.295 & 5.656 \\
\hline 41 & Greene & 40.295 & 5.656 \\
\hline 42 & Hamilton & 84.619 & 11.877 \\
\hline 43 & Hancock & 18.804 & 2.639 \\
\hline 44 & Harrison & 114.168 & 16.025 \\
\hline 45 & Hendricks & 40.295 & 5.656 \\
\hline 46 & Henry & 40.295 & 5.656 \\
\hline 47 & Howard & 40.295 & 5.656 \\
\hline 48 & Huntington & 57.084 & 8.012 \\
\hline 49 & Jackson & 197.443 & 27.713 \\
\hline 50 & Jasper & 46.339 & 6.504 \\
\hline 51 & Jay & 40.295 & 5.656 \\
\hline
\end{tabular}




\begin{tabular}{|c|c|c|c|}
\hline & State & P25 97 & A25 97 \\
\hline 52 & Jefferson & 49.025 & 6.881 \\
\hline 53 & Jennings & 47.010 & 6.598 \\
\hline 54 & Johnson & 685.679 & 96.242 \\
\hline 55 & Knox & 51.040 & 7.164 \\
\hline 56 & Kosciusko & 209.532 & 29.410 \\
\hline 57 & LaGrange & 1262.563 & 177.214 \\
\hline 58 & Lake & 159.164 & 22.340 \\
\hline 59 & La Porte & 140.359 & 19.701 \\
\hline 60 & Lawrence & 77.903 & 10.934 \\
\hline 61 & Madison & 120.212 & 16.873 \\
\hline 62 & Marion & 477.491 & 67.021 \\
\hline 63 & Marshall & 432.495 & 60.705 \\
\hline 64 & Martin & 6.716 & .943 \\
\hline 65 & Miami & 90.663 & 12.725 \\
\hline 66 & Monroe & 40.295 & 5.656 \\
\hline 67 & Montgomery & 28.206 & 3.959 \\
\hline 68 & Morgan & 122.898 & 17.250 \\
\hline 69 & Newton & .000 & .000 \\
\hline 70 & Noble & 40.295 & 5.656 \\
\hline 71 & Ohio & .000 & .000 \\
\hline 72 & Orange & 306.910 & 43.078 \\
\hline 73 & Owen & 83.275 & 11.689 \\
\hline 74 & Parke & 117.526 & 16.496 \\
\hline 75 & Perry & 40.295 & 5.656 \\
\hline 76 & Pike & 40.295 & 5.656 \\
\hline 77 & Porter & 72.530 & 10.180 \\
\hline 78 & Posey & 6.716 & .943 \\
\hline 79 & Pulaski & 6.716 & .943 \\
\hline 80 & Putnam & 40.295 & 5.656 \\
\hline 81 & Randolph & 253.184 & 35.537 \\
\hline 82 & Ripley & 81.932 & 11.500 \\
\hline 83 & Rush & 40.295 & 5.656 \\
\hline 84 & St. Joseph & 342.504 & 48.074 \\
\hline 85 & Scott & 40.295 & 5.656 \\
\hline 86 & Shelby & .000 & .000 \\
\hline 87 & Spencer & 101.408 & 14.234 \\
\hline 88 & Starke & 216.919 & 30.447 \\
\hline 89 & Steuben & 32.907 & 4.619 \\
\hline 90 & Sullivan & 59.770 & 8.389 \\
\hline 91 & Switzerland & .000 & .000 \\
\hline 92 & Tippecanoe & 34.250 & 4.807 \\
\hline 93 & Tipton & 40.295 & 5.656 \\
\hline 94 & Union & 6.716 & .943 \\
\hline 95 & Vanderburgh & 162.521 & 22.812 \\
\hline 96 & Vermillion & .000 & .000 \\
\hline 97 & Vigo & 78.574 & 11.029 \\
\hline 98 & Wabash & 117.526 & 16.496 \\
\hline 99 & Warren & 40.295 & 5.656 \\
\hline 100 & Warrick & 84.619 & 11.877 \\
\hline 101 & Washington & 151.776 & 21.303 \\
\hline 102 & Wayne & 40.295 & 5.656 \\
\hline
\end{tabular}




\begin{tabular}{|c|c|c|c|}
\hline & State & P25 97 & A25 97 \\
\hline 103 & Wells & 40.295 & 5.656 \\
\hline 104 & White & 17.461 & 2.451 \\
\hline 105 & Whitley & 117.526 & 16.496 \\
\hline 106 & lowa & 4319.000 & 87.694 \\
\hline 107 & Kansas & 1540.000 & 81.430 \\
\hline 108 & Kentucky_E & 3582.000 & 2348.947 \\
\hline 109 & Kentucky_W & 2388.000 & 939.579 \\
\hline 110 & Louisiana & 19879.000 & 21509.049 \\
\hline 111 & Maine & 4014.000 & 4280.304 \\
\hline 112 & Maryland & 1989.000 & 2121.360 \\
\hline 113 & Massachusetts & 1443.000 & 38.627 \\
\hline 114 & Michigan_E & 1415.000 & 1306.015 \\
\hline 115 & Michigan W & 1415.000 & 1306.015 \\
\hline 116 & Minnesota & 7508.000 & 8005.212 \\
\hline 117 & Missisippi & 13181.000 & 19933.689 \\
\hline 118 & Missouri & 5146.000 & 276.654 \\
\hline 119 & Montana & 2701.000 & 2880.331 \\
\hline 120 & Nebraska & 1271.000 & 135.717 \\
\hline 121 & Nevada & 21.000 & 42.803 \\
\hline 122 & New Hampshire & 1867.000 & 1990.863 \\
\hline 123 & New Jersey & 1287.000 & 129.453 \\
\hline 124 & New Mexico & 772.000 & 822.654 \\
\hline 125 & New York & 1785.000 & 5367.084 \\
\hline 126 & North Carolina & 26405.000 & 26076.447 \\
\hline 127 & North Dakota & 496.000 & 8.352 \\
\hline 128 & Ohio_N & 317.000 & 668.145 \\
\hline 129 & Ohio M & 317.000 & 668.145 \\
\hline 130 & Ohio_s & 317.000 & 668.145 \\
\hline 131 & Oklahoma & 1570.000 & 1674.538 \\
\hline 132 & Oregon & 22395.000 & 24572.077 \\
\hline 133 & Pennsylvania & 1474.000 & 1340.466 \\
\hline 134 & Rhode Island & 256.000 & 272.478 \\
\hline 135 & South Carolina & 8392.000 & 10503.448 \\
\hline 136 & South Dakota & 748.000 & 797.598 \\
\hline 137 & Tennessee & 9529.000 & 2705.987 \\
\hline 138 & Texas & 14452.000 & 18895.976 \\
\hline 139 & Utah & 1113.000 & 29.231 \\
\hline 140 & Vermont & 1668.000 & 1778.936 \\
\hline 141 & Virginia & 17584.000 & 17630.676 \\
\hline 142 & Washington & 59210.000 & 59811.506 \\
\hline$\overline{143}$ & West Virginia & 5627.000 & .000 \\
\hline 144 & Wisconsin & 2283.000 & 3280.174 \\
\hline 145 & Wyoming & 526.000 & 17.748 \\
\hline
\end{tabular}




\begin{tabular}{|c|c|c|c|}
\hline & State & P26 97 & A26 97 \\
\hline 1 & Alabama & 12440.235 & 13803.000 \\
\hline 2 & Arizona & 1525.661 & 3628.000 \\
\hline 3 & Arkansas & 14199.844 & 10141.000 \\
\hline 4 & California & 19551.655 & 23350.000 \\
\hline 5 & Colorado & 2171.517 & 3713.000 \\
\hline 6 & Connecticut & 414.908 & 987.000 \\
\hline$\overline{7}$ & Delaware & 48.989 & 458.000 \\
\hline 8 & $\mathrm{DC}$ & .000 & .000 \\
\hline 9 & Florida & 7275.383 & 9625.000 \\
\hline 10 & Georgia & 23124.861 & 22270.000 \\
\hline$\overline{11}$ & Idaho & 3504.221 & 1628.000 \\
\hline 12 & Illinois_N & 7988.225 & 6338.000 \\
\hline 13 & Illinois_S & 3424.239 & 2716.000 \\
\hline 14 & Adams & 78.966 & 114.712 \\
\hline 15 & Allen & 25.774 & 46.574 \\
\hline 16 & Bartholomew & 8.226 & 11.787 \\
\hline 17 & Benton & 23.991 & 34.379 \\
\hline 18 & Blackford & 10.693 & 15.323 \\
\hline 19 & Boone & .000 & .000 \\
\hline 20 & Brown & 3.702 & 5.304 \\
\hline 21 & Carroll & .000 & .000 \\
\hline 22 & Cass & 23.032 & 33.004 \\
\hline 23 & Clark & 91.442 & 146.739 \\
\hline 24 & Clay & 8.226 & 11.787 \\
\hline 25 & Clinton & 4.524 & 6.483 \\
\hline 26 & Crawford & 8.226 & 11.787 \\
\hline 27 & Daviess & 30.846 & 45.757 \\
\hline 28 & Dearborn & 1.371 & 3.519 \\
\hline 29 & Decatur & 3.564 & 5.108 \\
\hline 30 & DeKalb & 91.579 & 131.231 \\
\hline 31 & Delaware & 8.226 & 11.787 \\
\hline 32 & Dubois & 235.253 & 337.114 \\
\hline 33 & Elkhart & 874.659 & 1254.927 \\
\hline 34 & Fayette & .000 & .000 \\
\hline 35 & Floyd & 81.160 & 116.300 \\
\hline 36 & Fountain & 1.371 & 1.965 \\
\hline 37 & Franklin & 5.347 & 16.991 \\
\hline 38 & Fulton & 36.056 & 51.667 \\
\hline 39 & Gibson & .000 & .000 \\
\hline 40 & Grant & 8.226 & 11.787 \\
\hline 41 & Greene & 8.226 & 11.787 \\
\hline 42 & Hamilton & 17.274 & 26.308 \\
\hline 43 & Hancock & 3.839 & 5.501 \\
\hline 44 & Harrison & 23.306 & 42.727 \\
\hline 45 & Hendricks & 8.226 & 11.787 \\
\hline 46 & Henry & 8.226 & 11.787 \\
\hline 47 & Howard & 8.226 & 11.787 \\
\hline 48 & Huntington & 11.653 & 18.253 \\
\hline 49 & Jackson & 40.306 & 57.757 \\
\hline 50 & Jasper & 9.459 & 13.555 \\
\hline 51 & Jay & 8.226 & 13.342 \\
\hline
\end{tabular}




\begin{tabular}{|c|c|c|c|}
\hline & State & P26 97 & A26 97 \\
\hline 52 & Jefferson & 10.008 & 14.341 \\
\hline 53 & Jennings & 9.597 & 23.081 \\
\hline 54 & Johnson & 139.973 & 200.579 \\
\hline 55 & Knox & 10.419 & 16.485 \\
\hline 56 & Kosciusko & 42.773 & 62.848 \\
\hline 57 & LaGrange & 257.736 & 369.332 \\
\hline 58 & Lake & 32.491 & 382.268 \\
\hline 59 & La Porte & 28.653 & 42.614 \\
\hline 60 & Lawrence & 15.903 & 32.118 \\
\hline 61 & Madison & 24.540 & 35.165 \\
\hline 62 & Marion & 97.474 & 158.182 \\
\hline 63 & Marshall & 88.288 & 135.845 \\
\hline 64 & Martin & 1.371 & 1.965 \\
\hline 65 & Miami & 18.508 & 26.521 \\
\hline 66 & Monroe & 8.226 & 21.117 \\
\hline 67 & Montgomery & 5.758 & 8.251 \\
\hline 68 & Morgan & 25.088 & 35.951 \\
\hline 69 & Newton & .000 & .000 \\
\hline 70 & Noble & 8.226 & 11.787 \\
\hline 71 & Ohio & .000 & .000 \\
\hline 72 & Orange & 62.652 & 89.779 \\
\hline 73 & Owen & 17.000 & 24.360 \\
\hline 74 & Parke & 23.991 & 34.379 \\
\hline 75 & Perry & 8.226 & 11.787 \\
\hline 76 & Pike & 8.226 & 11.787 \\
\hline 77 & Porter & 14.806 & 21.217 \\
\hline 78 & Posey & 1.371 & 60.274 \\
\hline 79 & Pulaski & 1.371 & 1.965 \\
\hline 80 & Putnam & 8.226 & 11.787 \\
\hline 81 & Randolph & 51.684 & 75.618 \\
\hline 82 & Ripley & 16.725 & 25.522 \\
\hline 83 & Rush & 8.226 & 11.787 \\
\hline 84 & St. Joseph & 69.918 & 101.746 \\
\hline 85 & Scott & 8.226 & 13.342 \\
\hline 86 & Shelby & .000 & .000 \\
\hline 87 & Spencer & 20.701 & 29.664 \\
\hline 88 & Starke & 44.281 & 63.454 \\
\hline 89 & Steuben & 6.718 & 11.181 \\
\hline 90 & Sullivan & 12.201 & 17.484 \\
\hline 91 & Switzerland & .000 & .000 \\
\hline 92 & Tippecanoe & 6.992 & 11.574 \\
\hline 93 & Tipton & 8.226 & 11.787 \\
\hline 94 & Union & 1.371 & 1.965 \\
\hline 95 & Vanderburgh & 33.177 & 47.542 \\
\hline 96 & Vermillion & .000 & .000 \\
\hline 97 & Vigo & 16.040 & 22.985 \\
\hline 98 & Wabash & 23.991 & 35.934 \\
\hline 99 & Warren & 8.226 & 11.787 \\
\hline 100 & Warrick & $\overline{17.274}$ & 24.753 \\
\hline 101 & Washington & 30.983 & 44.398 \\
\hline 102 & Wayne & 8.226 & 11.787 \\
\hline
\end{tabular}




\begin{tabular}{|c|c|c|c|}
\hline & State & P26 97 & A26 97 \\
\hline 103 & Wells & 8.226 & 11.787 \\
\hline 104 & White & 3.564 & 5.108 \\
\hline 105 & Whitley & 23.991 & 34.379 \\
\hline 106 & lowa & 1112.753 & 1862.000 \\
\hline 107 & Kansas & 1183.737 & 1709.000 \\
\hline 108 & Kentucky_E & 2541.435 & 2402.000 \\
\hline 109 & Kentucky_W & 1694.623 & 1601.000 \\
\hline 110 & Louisiana & 12151.300 & 13693.000 \\
\hline 111 & Maine & 6889.469 & 5909.000 \\
\hline 112 & Maryland & 2496.445 & 4144.000 \\
\hline 113 & Massachusetts & 11805.376 & 2771.000 \\
\hline 114 & Michigan_E & 2568.429 & 2949.000 \\
\hline 115 & Michigan_W & 2568.429 & 2949.000 \\
\hline 116 & Minnesota & 5797.712 & 5036.000 \\
\hline 117 & Missisippi & 13844.923 & 8748.000 \\
\hline 118 & \begin{tabular}{|l} 
Missouri \\
\end{tabular} & 2719.396 & 3979.000 \\
\hline 119 & Montana & 3421.240 & 1812.000 \\
\hline 120 & Nebraska & 3191.291 & 1120.000 \\
\hline 121 & Nevada & 251.944 & 1480.000 \\
\hline 122 & New Hampshire & 4687.958 & 5361.000 \\
\hline 123 & New Jersey & 1602.644 & 3276.000 \\
\hline 124 & New Mexico & 1233.726 & 968.000 \\
\hline 125 & New York & 4165.074 & 5971.000 \\
\hline 126 & North Carolina & 22830.926 & 18440.000 \\
\hline 127 & North Dakota & 191.957 & 287.000 \\
\hline 128 & Ohio N & 2365.474 & 2740.000 \\
\hline 129 & Ohio_M & 2365.474 & 2740.000 \\
\hline 130 & Ohio_s & 2365.474 & 2740.000 \\
\hline 131 & Oklahoma & 3203.288 & 3062.000 \\
\hline 132 & Oregon & 33472.561 & 25339.000 \\
\hline 133 & Pennsylvania & 7883.248 & 8122.000 \\
\hline 134 & Rhode Island & 346.923 & 342.000 \\
\hline 135 & South Carolina & 7578.316 & 8873.000 \\
\hline 136 & South Dakota & 902.799 & 645.000 \\
\hline 137 & Tennessee & 4937.903 & 6468.000 \\
\hline 138 & Texas & 12074.317 & 25274.000 \\
\hline 139 & Utah & 1327.705 & 2095.000 \\
\hline 140 & Vermont & 990.780 & 670.000 \\
\hline 141 & Virginia & 12906.132 & 14325.000 \\
\hline 142 & Washington & 15663.519 & 14257.000 \\
\hline 143 & West Virginia & 3868.140 & 1910.000 \\
\hline 144 & Wisconsin & 7811.264 & 8410.000 \\
\hline 145 & Wyoming & 587.869 & 225.000 \\
\hline
\end{tabular}




\begin{tabular}{|c|c|c|c|}
\hline & State & P27 97 & A27 97 \\
\hline 1 & Alabama & 8943.887 & 3577.000 \\
\hline 2 & Arizona & 598.658 & 806.000 \\
\hline 3 & Arkansas & 3722.872 & 1791.000 \\
\hline 4 & California & 6251.426 & 13523.000 \\
\hline 5 & Colorado & 207.881 & 1040.000 \\
\hline 6 & Connecticut & 885.494 & 1264.000 \\
\hline 7 & Delaware & 4.997 & 262.000 \\
\hline 8 & DC & .000 & .000 \\
\hline 9 & Florida & 3846.801 & 4906.000 \\
\hline 10 & Georgia & 9674.469 & 7102.000 \\
\hline 11 & Idaho & 321.816 & 443.000 \\
\hline 12 & Illinois_N & 2745.430 & 5858.000 \\
\hline 13 & Illinois_S & 1177.327 & 2511.000 \\
\hline 14 & Adams & .231 & 2.319 \\
\hline 15 & Allen & 75.749 & 157.710 \\
\hline 16 & Bartholomew & 60.440 & 117.270 \\
\hline 17 & Benton & 15.670 & 30.247 \\
\hline 18 & Blackford & 38.234 & 73.802 \\
\hline 19 & Boone & .895 & 1.728 \\
\hline 20 & Brown & .000 & .000 \\
\hline 21 & Carroll & .000 & .000 \\
\hline 22 & Cass & .000 & .604 \\
\hline 23 & Clark & 7.704 & 27.685 \\
\hline 24 & Clay & .000 & .000 \\
\hline 25 & Clinton & 5.372 & 13.996 \\
\hline 26 & Crawford & .000 & .000 \\
\hline 27 & Daviess & .231 & 1.714 \\
\hline 28 & Dearborn & .231 & 45.521 \\
\hline 29 & Decatur & .000 & .000 \\
\hline 30 & DeKalb & 15.670 & 30.247 \\
\hline 31 & Delaware & 40.920 & 79.592 \\
\hline 32 & Dubois & 19.162 & 36.987 \\
\hline 33 & Elkhart & 67.386 & 131.343 \\
\hline 34 & Fayette & .000 & .000 \\
\hline 35 & Floyd & 15.670 & 30.247 \\
\hline 36 & Fountain & .000 & .000 \\
\hline 37 & Franklin & 1.385 & 10.286 \\
\hline 38 & Fulton & 5.372 & 10.975 \\
\hline 39 & Gibson & .000 & .000 \\
\hline 40 & Grant & 37.249 & 71.901 \\
\hline 41 & Greene & .000 & .000 \\
\hline 42 & Hamilton & .231 & 1.714 \\
\hline 43 & Hancock & 16.207 & 31.284 \\
\hline 44 & Harrison & 1.385 & 10.286 \\
\hline 45 & Hendricks & .000 & .000 \\
\hline 46 & Henry & 5.372 & 10.370 \\
\hline 47 & Howard & .000 & 3.625 \\
\hline 48 & Huntington & 15.900 & 31.961 \\
\hline 49 & Jackson & 42.532 & 82.098 \\
\hline 50 & Jasper & .000 & .000 \\
\hline 51 & Jay & 5.603 & 15.710 \\
\hline
\end{tabular}




\begin{tabular}{|c|c|c|c|}
\hline & State & P27 97 & A27 97 \\
\hline 52 & Jefferson & .000 & .604 \\
\hline 53 & Jennings & 6.757 & 20.656 \\
\hline 54 & Johnson & 15.670 & 30.247 \\
\hline 55 & Knox & 5.603 & 12.085 \\
\hline 56 & Kosciusko & 1.126 & 3.443 \\
\hline 57 & LaGrange & .000 & .000 \\
\hline 58 & Lake & 86.544 & 463.648 \\
\hline 59 & La Porte & 43.837 & 86.491 \\
\hline 60 & Lawrence & 1.385 & 13.911 \\
\hline 61 & Madison & 15.670 & 30.247 \\
\hline 62 & Marion & 253.996 & 583.514 \\
\hline 63 & Marshall & 42.842 & 100.884 \\
\hline 64 & Martin & .000 & .000 \\
\hline 65 & Miami & 33.578 & 64.815 \\
\hline 66 & Monroe & 17.054 & 44.158 \\
\hline 67 & Montgomery & 15.670 & 30.247 \\
\hline 68 & Morgan & .000 & .000 \\
\hline 69 & Newton & 5.372 & 10.370 \\
\hline 70 & Noble & 15.670 & 30.247 \\
\hline 71 & Ohio & .000 & .000 \\
\hline 72 & Orange & .000 & .604 \\
\hline 73 & Owen & .000 & .000 \\
\hline 74 & Parke & .000 & .604 \\
\hline 75 & Perry & .000 & .000 \\
\hline 76 & Pike & .000 & .000 \\
\hline 77 & Porter & .000 & .000 \\
\hline 78 & Posey & 8.656 & 64.288 \\
\hline 79 & Pulaski & .895 & 1.728 \\
\hline 80 & Putnam & .000 & .000 \\
\hline 81 & Randolph & .231 & 1.714 \\
\hline 82 & Ripley & 15.900 & 32.565 \\
\hline 83 & Rush & 5.372 & 10.370 \\
\hline 84 & St. Joseph & 50.642 & 99.627 \\
\hline 85 & Scott & .231 & 5.340 \\
\hline 86 & Shelby & .895 & 1.728 \\
\hline 87 & Spencer & .000 & .000 \\
\hline 88 & Starke & 5.372 & 10.370 \\
\hline 89 & Steuben & 5.603 & 12.085 \\
\hline 90 & Sullivan & .000 & .000 \\
\hline 91 & Switzerland & .895 & 1.728 \\
\hline 92 & Tippecanoe & 5.603 & 12.085 \\
\hline 93 & Tipton & 5.372 & 10.370 \\
\hline 94 & Union & .000 & .000 \\
\hline 95 & Vanderburgh & 56.052 & 118.771 \\
\hline 96 & Vermillion & 15.670 & 30.247 \\
\hline 97 & Vigo & 46.829 & 90.395 \\
\hline 98 & Wabash & 33.808 & 66.529 \\
\hline 99 & Warren & .000 & .000 \\
\hline 100 & Warrick & .895 & 1.728 \\
\hline 101 & Washington & .000 & .604 \\
\hline 102 & Wayne & 21.311 & 41.740 \\
\hline
\end{tabular}




\begin{tabular}{|c|c|c|c|}
\hline & State & P27 97 & A27 97 \\
\hline 103 & Wells & 5.372 & 10.370 \\
\hline 104 & White & 5.372 & 10.370 \\
\hline 105 & Whitley & 15.670 & 30.247 \\
\hline 106 & lowa & 899.486 & 1813.000 \\
\hline 107 & Kansas & 569.674 & 1824.000 \\
\hline 108 & Kentucky_E & 1158.338 & 2130.000 \\
\hline 109 & Kentucky_W & 771.559 & 1420.000 \\
\hline 110 & Louisiana & 8981.865 & 3223.000 \\
\hline 111 & Maine & 3616.932 & 851.000 \\
\hline 112 & Maryland & 1319.246 & 1983.000 \\
\hline 113 & Massachusetts & 1570.102 & 2667.000 \\
\hline 114 & Michigan_E & 6981.009 & 7406.000 \\
\hline 115 & Michigan_W & 6981.009 & 7406.000 \\
\hline 116 & Minnesota & 3368.074 & 3480.000 \\
\hline 117 & Missisippi & 4677.326 & 1795.000 \\
\hline 118 & Missouri & 588.663 & 2937.000 \\
\hline 119 & Montana & 684.609 & 114.000 \\
\hline 120 & Nebraska & 143.918 & 635.000 \\
\hline 121 & Nevada & 58.966 & 388.000 \\
\hline 122 & New Hampshire & 777.555 & 459.000 \\
\hline 123 & New Jersey & 4962.163 & 4741.000 \\
\hline 124 & New Mexico & 125.928 & 194.000 \\
\hline 125 & New York & 4755.281 & 5909.000 \\
\hline 126 & North Carolina & 4045.687 & 3763.000 \\
\hline 127 & North Dakota & 22.987 & 109.000 \\
\hline 128 & Ohio N & 1442.175 & 2023.000 \\
\hline 129 & Ohio_M & 1442.175 & 2023.000 \\
\hline 130 & Ohio_s & 1442.175 & 2023.000 \\
\hline 131 & Oklahoma & 1270.274 & 1080.000 \\
\hline 132 & Oregon & 4944.173 & 2585.000 \\
\hline 133 & Pennsylvania & 4881.209 & 7679.000 \\
\hline 134 & Rhode Island & 1412.193 & 279.000 \\
\hline 135 & South Carolina & 5951.597 & 3485.000 \\
\hline 136 & South Dakota & 471.730 & 216.000 \\
\hline 137 & Tennessee & 4864.219 & 4031.000 \\
\hline 138 & Texas & 5692.745 & 8597.000 \\
\hline 139 & Utah & 123.929 & 736.000 \\
\hline 140 & Vermont & 173.901 & 440.000 \\
\hline 141 & Virginia & 4018.702 & 4508.000 \\
\hline 142 & Washington & 5490.861 & 3723.000 \\
\hline 143 & West Virginia & 107.938 & 233.000 \\
\hline$\overline{144}$ & Wisconsin & 11479.437 & 6914.000 \\
\hline 145 & Wyoming & 160.908 & 16.000 \\
\hline
\end{tabular}




\begin{tabular}{|c|c|c|c|}
\hline & State & P28 97 & A28 97 \\
\hline 1 & Alabama & 975.475 & 1232.000 \\
\hline 2 & Arizona & 433.323 & 825.000 \\
\hline 3 & Arkansas & 1842.121 & 1181.000 \\
\hline$\overline{4}$ & California & 12637.250 & 13546.000 \\
\hline 5 & Colorado & 570.109 & 805.000 \\
\hline 6 & Connecticut & 721.872 & 608.000 \\
\hline 7 & Delaware & 353.448 & 223.000 \\
\hline 8 & DC & .000 & .000 \\
\hline 9 & Florida & 2030.826 & 2506.000 \\
\hline 10 & Georgia & 5067.081 & 3136.000 \\
\hline 11 & Idaho & 462.278 & 343.000 \\
\hline 12 & Illinois_N & 2095.725 & 2250.000 \\
\hline 13 & Illinois_S & 897.597 & 964.000 \\
\hline 14 & Adams & 20.517 & 20.851 \\
\hline 15 & Allen & 117.040 & 113.344 \\
\hline 16 & Bartholomew & 46.725 & 40.954 \\
\hline 17 & Benton & .000 & .626 \\
\hline 18 & Blackford & 1.675 & 2.097 \\
\hline 19 & Boone & 6.055 & 9.424 \\
\hline 20 & Brown & .424 & 1.328 \\
\hline 21 & Carroll & 48.854 & 35.552 \\
\hline 22 & Cass & 54.779 & 42.826 \\
\hline 23 & Clark & 12.401 & 13.258 \\
\hline 24 & Clay & .424 & 2.083 \\
\hline 25 & Clinton & 69.147 & 54.965 \\
\hline 26 & Crawford & .000 & .686 \\
\hline 27 & Daviess & 28.363 & 21.820 \\
\hline 28 & Dearborn & .424 & 3.305 \\
\hline 29 & Decatur & 10.329 & 8.849 \\
\hline 30 & DeKalb & 5.848 & 8.314 \\
\hline 31 & Delaware & 4.144 & 10.430 \\
\hline 32 & Dubois & 33.617 & 30.063 \\
\hline 33 & Elkhart & 79.408 & 59.509 \\
\hline 34 & Fayette & .000 & 1.696 \\
\hline 35 & Floyd & 29.019 & 28.439 \\
\hline 36 & Fountain & 2.541 & 3.304 \\
\hline 37 & Franklin & .279 & 1.615 \\
\hline 38 & Fulton & 4.471 & 4.519 \\
\hline 39 & Gibson & 13.674 & 12.955 \\
\hline 40 & Grant & 35.728 & 37.741 \\
\hline 41 & Greene & 11.845 & 10.463 \\
\hline 42 & Hamilton & 15.587 & 16.681 \\
\hline 43 & Hancock & .424 & 3.877 \\
\hline 44 & Harrison & 20.240 & 16.373 \\
\hline 45 & Hendricks & 15.907 & 18.146 \\
\hline 46 & Henry & 5.574 & 5.287 \\
\hline 47 & Howard & 5.406 & 9.697 \\
\hline 48 & Huntington & 33.028 & 34.000 \\
\hline 49 & Jackson & 1.515 & 3.380 \\
\hline 50 & Jasper & 8.808 & 9.082 \\
\hline 51 & Jay & 12.395 & 10.113 \\
\hline
\end{tabular}




\begin{tabular}{|c|c|c|c|}
\hline & State & P28 97 & A28 97 \\
\hline 52 & Jefferson & 1.069 & 2.443 \\
\hline 53 & Jennings & 19.132 & 1.765 \\
\hline 54 & Johnson & 9.008 & 16.429 \\
\hline 55 & Knox & 4.855 & 6.467 \\
\hline 56 & Kosciusko & 30.672 & 24.589 \\
\hline 57 & LaGrange & 6.346 & 6.667 \\
\hline 58 & Lake & 37.063 & 56.570 \\
\hline 59 & La Porte & 17.211 & 19.318 \\
\hline 60 & Lawrence & 3.052 & 5.126 \\
\hline 61 & Madison & 22.102 & 23.556 \\
\hline 62 & Marion & 155.433 & 160.930 \\
\hline 63 & Marshall & 15.126 & 11.427 \\
\hline 64 & Martin & 1.675 & 1.860 \\
\hline 65 & Miami & 2.330 & 3.632 \\
\hline$\overline{66}$ & Monroe & 20.411 & 25.772 \\
\hline 67 & Montgomery & 10.469 & 9.782 \\
\hline 68 & Morgan & 26.059 & 32.161 \\
\hline 69 & Newton & 2.806 & 3.923 \\
\hline 70 & Noble & 30.732 & 18.556 \\
\hline 71 & Ohio & .000 & .364 \\
\hline 72 & Orange & 1.675 & 2.428 \\
\hline 73 & Owen & 3.340 & 1.585 \\
\hline 74 & Parke & 4.885 & 4.539 \\
\hline$\overline{75}$ & Perry & 3.485 & 1.607 \\
\hline 76 & Pike & .000 & .840 \\
\hline$\overline{77}$ & Porter & 11.102 & 17.523 \\
\hline 78 & Posey & 4.736 & 2.942 \\
\hline 79 & Pulaski & 1.926 & 2.245 \\
\hline 80 & Putnam & 2.806 & 5.266 \\
\hline 81 & Randolph & 3.016 & 4.256 \\
\hline 82 & Ripley & 1.146 & 2.854 \\
\hline 83 & Rush & 1.380 & 1.870 \\
\hline 84 & St. Joseph & 41.558 & 45.398 \\
\hline 85 & Scott & 19.397 & 8.808 \\
\hline 86 & Shelby & 4.885 & 6.237 \\
\hline 87 & Spencer & 1.675 & 2.504 \\
\hline 88 & Starke & .000 & 1.561 \\
\hline 89 & Steuben & 10.963 & 8.003 \\
\hline 90 & Sullivan & .000 & 1.409 \\
\hline 91 & Switzerland & .000 & .577 \\
\hline 92 & Tippecanoe & 38.251 & 41.053 \\
\hline 93 & Tipton & 4.885 & 4.510 \\
\hline$\overline{94}$ & Union & .000 & .476 \\
\hline 95 & Vanderburgh & 117.069 & 79.933 \\
\hline 96 & Vermillion & .223 & 1.257 \\
\hline 97 & Vigo & 32.703 & 39.546 \\
\hline$\overline{98}$ & Wabash & 9.809 & 11.823 \\
\hline 99 & Warren & .279 & .740 \\
\hline 100 & Warrick & .510 & 3.347 \\
\hline 101 & Washington & 1.675 & 2.931 \\
\hline 102 & Wayne & 33.096 & 33.193 \\
\hline
\end{tabular}




\begin{tabular}{|c|c|c|c|}
\hline & State & P28 97 & A28 97 \\
\hline 103 & Wells & 14.948 & 11.976 \\
\hline 104 & White & 4.210 & 5.242 \\
\hline 105 & Whitley & 2.777 & 4.915 \\
\hline 106 & lowa & 840.686 & 857.000 \\
\hline 107 & Kansas & 977.472 & 706.000 \\
\hline 108 & Kentucky E & 534.165 & 718.000 \\
\hline 109 & Kentucky_W & 355.444 & 479.000 \\
\hline 110 & Louisiana & 978.471 & 746.000 \\
\hline 111 & Maine & 466.271 & 267.000 \\
\hline 112 & Maryland & 1050.358 & 946.000 \\
\hline 113 & Massachusetts & 1137.223 & 1203.000 \\
\hline 114 & Michigan_E & 709.891 & 875.000 \\
\hline 115 & Michigan_W & 709.891 & 875.000 \\
\hline 116 & Minnesota & 976.474 & 1049.000 \\
\hline 117 & Missisippi & 236.630 & 546.000 \\
\hline 118 & Missouri & 1415.787 & 1650.000 \\
\hline 119 & Montana & 56.911 & 55.000 \\
\hline 120 & Nebraska & 334.477 & 387.000 \\
\hline 121 & Nevada & 57.909 & 168.000 \\
\hline 122 & New Hampshire & 83.869 & 193.000 \\
\hline 123 & New Jersey & 1316.942 & 2235.000 \\
\hline 124 & New Mexico & 61.903 & 117.000 \\
\hline 125 & New York & 3078.189 & 3258.000 \\
\hline 126 & North Carolina & 2163.619 & 2289.000 \\
\hline 127 & North Dakota & 44.930 & 143.000 \\
\hline 128 & Ohio N & 1381.840 & 1363.000 \\
\hline 129 & Ohio M & 1381.840 & 1363.000 \\
\hline 130 & Ohio_s & 1381.840 & 1363.000 \\
\hline 131 & Oklahoma & 515.195 & 529.000 \\
\hline 132 & Oregon & 1346.895 & 1307.000 \\
\hline 133 & Pennsylvania & 4753.571 & 4276.000 \\
\hline 134 & Rhode Island & 52.917 & 115.000 \\
\hline 135 & South Carolina & 1515.631 & 1314.000 \\
\hline 136 & South Dakota & 345.460 & 126.000 \\
\hline 137 & Tennessee & 2040.811 & 2043.000 \\
\hline 138 & Texas & 3629.328 & 4167.000 \\
\hline 139 & Utah & 507.207 & 529.000 \\
\hline 140 & Vermont & 192.699 & 143.000 \\
\hline 141 & Virginia & 1223.088 & 1540.000 \\
\hline 142 & Washington & 2207.550 & 1862.000 \\
\hline 143 & West Virginia & 86.864 & 163.000 \\
\hline 144 & Wisconsin & 3523.493 & 2164.000 \\
\hline 145 & Wyoming & 22.964 & 84.000 \\
\hline
\end{tabular}




\begin{tabular}{|c|c|c|c|}
\hline & State & P29 97 & A29 97 \\
\hline 1 & Alabama & 321.420 & 575.000 \\
\hline 2 & Arizona & 464.273 & 775.000 \\
\hline 3 & Arkansas & 1163.659 & 870.000 \\
\hline$\overline{4}$ & California & 8949.162 & 8930.000 \\
\hline 5 & Colorado & 1130.922 & 1353.000 \\
\hline 6 & Connecticut & 653.752 & 1055.000 \\
\hline 7 & Delaware & 310.507 & 157.000 \\
\hline$\overline{8}$ & DC & .000 & .000 \\
\hline 9 & Florida & 4364.961 & 4846.000 \\
\hline 10 & Georgia & 3376.893 & 2151.000 \\
\hline 11 & Idaho & 148.805 & 152.000 \\
\hline 12 & Illinois_N & 2634.849 & 2747.000 \\
\hline 13 & Illinois_S & 1129.930 & 1178.000 \\
\hline 14 & Adams & 7.314 & 12.480 \\
\hline 15 & Allen & 201.203 & 121.818 \\
\hline 16 & Bartholomew & 50.705 & 26.301 \\
\hline 17 & Benton & 16.169 & 3.578 \\
\hline 18 & Blackford & 29.092 & 5.274 \\
\hline 19 & Boone & 10.219 & 16.530 \\
\hline 20 & Brown & .379 & 5.566 \\
\hline 21 & Carroll & 6.518 & 7.510 \\
\hline 22 & Cass & 6.935 & 15.134 \\
\hline 23 & Clark & 19.024 & 35.418 \\
\hline 24 & Clay & 2.615 & 9.880 \\
\hline 25 & Clinton & 9.322 & 12.662 \\
\hline 26 & Crawford & .000 & 3.922 \\
\hline 27 & Daviess & 5.950 & 11.100 \\
\hline 28 & Dearborn & 3.524 & 16.863 \\
\hline 29 & Decatur & 11.710 & 9.199 \\
\hline 30 & DeKalb & 15.904 & 14.646 \\
\hline 31 & Delaware & 46.827 & 44.722 \\
\hline 32 & Dubois & 28.826 & 14.694 \\
\hline 33 & Elkhart & 84.016 & 66.575 \\
\hline 34 & Fayette & 5.988 & 9.691 \\
\hline 35 & Floyd & 27.614 & 26.431 \\
\hline 36 & Fountain & 3.903 & 6.742 \\
\hline 37 & Franklin & 2.274 & 8.108 \\
\hline 38 & Fulton & 8.337 & 7.591 \\
\hline 39 & Gibson & 3.449 & 12.014 \\
\hline 40 & Grant & 35.862 & 27.659 \\
\hline$\overline{41}$ & Greene & 3.032 & 12.437 \\
\hline 42 & Hamilton & 58.436 & 61.989 \\
\hline 43 & Hancock & 26.136 & 20.132 \\
\hline 44 & Harrison & 3.221 & 12.509 \\
\hline 45 & Hendricks & 10.611 & 36.317 \\
\hline 46 & Henry & 16.220 & 18.257 \\
\hline 47 & Howard & 7.731 & 31.573 \\
\hline 48 & Huntington & 20.224 & 14.165 \\
\hline 49 & Jackson & 41.067 & 15.292 \\
\hline 50 & Jasper & 4.813 & 10.906 \\
\hline 51 & Jay & 10.876 & 8.152 \\
\hline
\end{tabular}




\begin{tabular}{|c|c|c|c|}
\hline & State & P29 97 & A29 97 \\
\hline 52 & Jefferson & 2.008 & 11.727 \\
\hline 53 & Jennings & 6.139 & 10.084 \\
\hline 54 & Johnson & 19.125 & 41.060 \\
\hline 55 & Knox & 19.289 & 14.864 \\
\hline 56 & Kosciusko & 9.348 & 27.241 \\
\hline 57 & LaGrange & 10.042 & 12.765 \\
\hline 58 & Lake & 99.073 & 181.474 \\
\hline 59 & La Porte & 59.560 & 41.152 \\
\hline 60 & Lawrence & 2.729 & 17.152 \\
\hline 61 & Madison & 27.007 & 49.855 \\
\hline 62 & Marion & 602.649 & 319.659 \\
\hline 63 & Marshall & 37.201 & 16.656 \\
\hline 64 & Martin & 2.312 & 3.921 \\
\hline 65 & Miami & 40.018 & 13.143 \\
\hline 66 & Monroe & 49.593 & 44.652 \\
\hline 67 & Montgomery & 15.259 & 13.973 \\
\hline 68 & Morgan & 2.615 & 24.278 \\
\hline 69 & Newton & 7.541 & 5.462 \\
\hline 70 & Noble & 20.072 & 16.757 \\
\hline 71 & Ohio & .000 & 2.079 \\
\hline 72 & Orange & 2.160 & 7.168 \\
\hline 73 & Owen & .379 & 7.941 \\
\hline 74 & Parke & 2.274 & 6.375 \\
\hline 75 & Perry & 1.667 & 7.162 \\
\hline 76 & Pike & 1.592 & 4.800 \\
\hline 77 & Porter & 30.772 & 54.089 \\
\hline 78 & Posey & 8.110 & 10.105 \\
\hline 79 & Pulaski & 10.333 & 5.117 \\
\hline 80 & Putnam & 2.008 & 13.133 \\
\hline 81 & Randolph & 2.236 & 10.284 \\
\hline 82 & Ripley & 17.685 & 9.761 \\
\hline 83 & Rush & 6.859 & 6.874 \\
\hline 84 & St. Joseph & 138.295 & 98.747 \\
\hline 85 & Scott & .379 & 8.405 \\
\hline 86 & Shelby & 5.975 & 16.078 \\
\hline 87 & Spencer & 8.906 & 7.598 \\
\hline 88 & Starke & 5.495 & 8.921 \\
\hline 89 & Steuben & 4.320 & 12.128 \\
\hline 90 & Sullivan & 5.495 & 8.050 \\
\hline 91 & Switzerland & 1.011 & 3.298 \\
\hline 92 & Tippecanoe & 27.399 & 54.710 \\
\hline 93 & Tipton & 9.247 & 6.209 \\
\hline 94 & Union & 2.274 & 2.723 \\
\hline 95 & Vanderburgh & 99.831 & 64.228 \\
\hline 96 & Vermillion & 13.971 & 6.286 \\
\hline 97 & Vigo & 60.545 & 39.870 \\
\hline 98 & Wabash & 26.868 & 13.068 \\
\hline 99 & Warren & 2.274 & 3.109 \\
\hline 100 & Warrick & 7.680 & 19.127 \\
\hline 101 & Washington & .379 & 10.038 \\
\hline 102 & Wayne & 31.327 & 26.781 \\
\hline
\end{tabular}




\begin{tabular}{|c|c|c|c|}
\hline & State & P29_97 & A29 97 \\
\hline 103 & Wells & 61.240 & 10.291 \\
\hline 104 & White & 15.689 & 9.421 \\
\hline 105 & Whitley & 14.577 & 11.309 \\
\hline 106 & lowa & 696.410 & 676.000 \\
\hline 107 & Kansas & 1430.517 & 678.000 \\
\hline 108 & Kentucky_E & 760.892 & 445.000 \\
\hline 109 & Kentucky W & 507.923 & 297.000 \\
\hline 110 & Louisiana & 257.930 & 420.000 \\
\hline 111 & Maine & 276.778 & 519.000 \\
\hline 112 & Maryland & 1071.400 & 2534.000 \\
\hline 113 & Massachusetts & 1974.153 & 2260.000 \\
\hline 114 & Michigan_E & 795.613 & 861.000 \\
\hline 115 & Michigan W & 795.613 & 861.000 \\
\hline 116 & Minnesota & 1707.295 & 1199.000 \\
\hline 117 & Missisippi & 449.393 & 419.000 \\
\hline 118 & Missouri & 1364.050 & 1511.000 \\
\hline 119 & Montana & 68.451 & 368.000 \\
\hline 120 & Nebraska & 327.372 & 403.000 \\
\hline 121 & Nevada & 350.189 & 500.000 \\
\hline 122 & New Hampshire & 195.431 & 321.000 \\
\hline 123 & New Jersey & 4460.197 & 3408.000 \\
\hline 124 & New Mexico & 168.646 & 243.000 \\
\hline 125 & New York & 4765.744 & 5580.000 \\
\hline 126 & North Carolina & 1484.087 & 1397.000 \\
\hline 127 & North Dakota & 22.817 & 86.000 \\
\hline 128 & Ohio_N & 1239.054 & 1280.000 \\
\hline 129 & Ohio M & 1239.054 & 1280.000 \\
\hline 130 & Ohio_s & 1239.054 & 1280.000 \\
\hline 131 & Oklahoma & 566.453 & 553.000 \\
\hline 132 & Oregon & 476.178 & 521.000 \\
\hline 133 & Pennsylvania & 5136.766 & 4316.000 \\
\hline 134 & Rhode Island & 345.229 & 330.000 \\
\hline 135 & South Carolina & 1025.766 & 870.000 \\
\hline 136 & South Dakota & 104.164 & 84.000 \\
\hline 137 & Tennessee & 2298.549 & 2018.000 \\
\hline 138 & Texas & 5623.856 & 6526.000 \\
\hline 139 & Utah & 189.479 & 877.000 \\
\hline 140 & Vermont & 115.076 & 129.000 \\
\hline 141 & Virginia & 1993.994 & 1888.000 \\
\hline 142 & Washington & 826.366 & 997.000 \\
\hline 143 & West Virginia & 260.906 & 339.000 \\
\hline 144 & Wisconsin & 3775.691 & 2219.000 \\
\hline 145 & Wyoming & 67.458 & 83.000 \\
\hline
\end{tabular}




\begin{tabular}{|c|c|c|c|}
\hline & State & P30 97 & A30 97 \\
\hline 1 & Alabama & 2116.670 & 1395.000 \\
\hline 2 & Arizona & 183.711 & 435.000 \\
\hline 3 & Arkansas & 164.741 & 349.000 \\
\hline 4 & California & 3604.330 & 4143.000 \\
\hline 5 & Colorado & 153.758 & 435.000 \\
\hline 6 & Connecticut & 102.838 & 195.000 \\
\hline 7 & Delaware & 353.444 & 95.000 \\
\hline$\overline{8}$ & $D C$ & .000 & .000 \\
\hline 9 & Florida & 785.764 & 1444.000 \\
\hline 10 & Georgia & 7633.991 & 5854.000 \\
\hline 11 & Idaho & 17.972 & 91.000 \\
\hline 12 & Illinois $\mathbf{N}$ & 245.614 & 467.000 \\
\hline$\overline{13}$ & Illinois_S & 104.835 & 200.000 \\
\hline 14 & Adams & .320 & 1.096 \\
\hline 15 & Allen & 20.119 & 23.953 \\
\hline 16 & Bartholomew & .558 & 2.067 \\
\hline 17 & Benton & .000 & .221 \\
\hline 18 & Blackford & .000 & .325 \\
\hline 19 & Boone & .000 & 1.020 \\
\hline 20 & Brown & .000 & .343 \\
\hline 21 & Carroll & .000 & .463 \\
\hline 22 & Cass & .558 & 1.378 \\
\hline 23 & Clark & 6.583 & 8.152 \\
\hline 24 & Clay & .000 & .609 \\
\hline 25 & Clinton & .000 & .781 \\
\hline 26 & Crawford & .000 & .242 \\
\hline 27 & Daviess & .320 & 1.011 \\
\hline 28 & Dearborn & .320 & 1.367 \\
\hline 29 & Decatur & .000 & .567 \\
\hline 30 & DeKalb & .000 & .903 \\
\hline 31 & Delaware & .558 & 3.203 \\
\hline 32 & Dubois & .558 & 1.351 \\
\hline 33 & Elkhart & 32.567 & 30.101 \\
\hline 34 & Fayette & .000 & .598 \\
\hline 35 & Floyd & .000 & 1.630 \\
\hline 36 & Fountain & .000 & .416 \\
\hline 37 & Franklin & 1.922 & 2.460 \\
\hline 38 & Fulton & .000 & .468 \\
\hline 39 & Gibson & .000 & .741 \\
\hline 40 & Grant & 3.347 & 4.375 \\
\hline 41 & Greene & .558 & 1.212 \\
\hline 42 & Hamilton & 10.364 & 12.114 \\
\hline 43 & Hancock & .000 & 1.242 \\
\hline 44 & Harrison & 1.922 & 2.731 \\
\hline 45 & Hendricks & .558 & 2.685 \\
\hline 46 & Henry & 3.347 & 3.795 \\
\hline 47 & Howard & .000 & 1.947 \\
\hline 48 & Huntington & .878 & 1.645 \\
\hline 49 & Jackson & .558 & 1.388 \\
\hline 50 & Jasper & .000 & .673 \\
\hline 51 & Jay & .320 & .829 \\
\hline
\end{tabular}




\begin{tabular}{|c|c|c|c|}
\hline & $\begin{array}{r}\text { State } \\
\end{array}$ & P30 97 & A30 97 \\
\hline 52 & Jefferson & .558 & 1.168 \\
\hline 53 & Jennings & 22.841 & 19.262 \\
\hline 54 & Johnson & .558 & 2.977 \\
\hline 55 & Knox & .320 & 1.243 \\
\hline 56 & Kosciusko & 4.284 & 5.123 \\
\hline 57 & LaGrange & .558 & 1.232 \\
\hline 58 & Lake & 72.510 & 84.369 \\
\hline 59 & La Porte & 2.568 & 4.391 \\
\hline 60 & Lawrence & 2.480 & 3.462 \\
\hline 61 & Madison & 1.397 & 4.145 \\
\hline 62 & Marion & 28.262 & 42.833 \\
\hline 63 & Marshall & 5.269 & 5.656 \\
\hline 64 & Martin & .000 & .242 \\
\hline 65 & Miami & .558 & 1.255 \\
\hline 66 & Monroe & 5.269 & 7.383 \\
\hline 67 & Montgomery & .000 & .862 \\
\hline 68 & Morgan & .558 & 1.942 \\
\hline 69 & Newton & .000 & .337 \\
\hline 70 & Noble & 9.762 & 8.818 \\
\hline 71 & Ohio & .000 & .128 \\
\hline 72 & Orange & .000 & .442 \\
\hline 73 & Owen & 3.347 & 3.159 \\
\hline 74 & Parke & .000 & .393 \\
\hline 75 & Perry & 3.347 & 3.111 \\
\hline 76 & Pike & .000 & .296 \\
\hline 77 & Porter & 2.248 & 4.862 \\
\hline 78 & Posey & 15.360 & 15.539 \\
\hline 79 & Pulaski & .000 & .316 \\
\hline 80 & Putnam & .000 & .810 \\
\hline 81 & Randolph & 2.010 & 2.042 \\
\hline 82 & Ripley & .320 & .929 \\
\hline 83 & Rush & .558 & .869 \\
\hline 84 & St. Joseph & 10.364 & 14.382 \\
\hline 85 & Scott & 10.083 & 8.629 \\
\hline 86 & Shelby & .000 & .992 \\
\hline 87 & Spencer & .000 & .469 \\
\hline 88 & Starke & .000 & .550 \\
\hline 89 & Steuben & 3.667 & 3.744 \\
\hline 90 & Sullivan & .000 & .497 \\
\hline 91 & Switzerland & .000 & .203 \\
\hline 92 & Tippecanoe & 3.667 & 6.370 \\
\hline 93 & Tipton & .000 & .383 \\
\hline 94 & Union & .000 & .168 \\
\hline 95 & Vanderburgh & 21.201 & 20.822 \\
\hline 96 & Vermillion & .000 & .388 \\
\hline 97 & Vigo & .558 & 2.904 \\
\hline 98 & Wabash & .878 & 1.577 \\
\hline 99 & Warren & .000 & .192 \\
\hline 100 & Warrick & .558 & 1.625 \\
\hline 101 & Washington & .000 & .619 \\
\hline 102 & Wayne & .000 & 1.652 \\
\hline
\end{tabular}




\begin{tabular}{|c|c|c|c|}
\hline & State & P30 97 & A30 97 \\
\hline 103 & Wells & .840 & 1.260 \\
\hline 104 & White & 5.487 & 4.179 \\
\hline 105 & Whitley & .000 & .698 \\
\hline 106 & lowa & 78.876 & 270.000 \\
\hline 107 & Kansas & 409.356 & $\overline{170.000}$ \\
\hline 108 & Kentucky_E & 237.626 & 336.000 \\
\hline 109 & Kentucky W & 157.752 & 224.000 \\
\hline 110 & Louisiana & 221.651 & 522.000 \\
\hline 111 & Maine & 208.672 & 193.000 \\
\hline 112 & Maryland & 299.529 & 460.000 \\
\hline 113 & Massachusetts & 605.048 & 768.000 \\
\hline 114 & Michigan_E & 197.689 & 540.000 \\
\hline 115 & Michigan_W & 197.689 & 540.000 \\
\hline 116 & Minnesota & 343.460 & 407.000 \\
\hline 117 & Missisippi & 696.904 & 973.000 \\
\hline 118 & Missouri & 309.513 & 509.000 \\
\hline 119 & Montana & 1.997 & 34.000 \\
\hline 120 & Nebraska & 86.863 & 168.000 \\
\hline 121 & Nevada & 74.882 & 295.000 \\
\hline 122 & New Hampshire & 71.887 & 143.000 \\
\hline 123 & New Jersey & 1352.872 & 1347.000 \\
\hline 124 & New Mexico & 25.959 & 60.000 \\
\hline 125 & New York & 1408.784 & 1846.000 \\
\hline 126 & North Carolina & 7358.424 & 5437.000 \\
\hline 127 & North Dakota & 50.920 & 66.000 \\
\hline 128 & Ohio $N$ & 352.446 & 428.000 \\
\hline 129 & Ohio_M & 352.446 & 428.000 \\
\hline 130 & Ohios & 352.446 & 428.000 \\
\hline 131 & Oklahoma & 129.796 & 270.000 \\
\hline 132 & Oregon & 252.603 & 344.000 \\
\hline 133 & Pennsylvania & 932.533 & 1588.000 \\
\hline 134 & Rhode Island & 84.866 & 130.000 \\
\hline 135 & South Carolina & 5668.083 & 3654.000 \\
\hline 136 & South Dakota & 18.970 & 36.000 \\
\hline 137 & Tennessee & 1626.441 & 968.000 \\
\hline 138 & Texas & 3240.902 & 3582.000 \\
\hline 139 & Utah & 227.642 & 352.000 \\
\hline 140 & Vermont & 12.980 & 116.000 \\
\hline 141 & Virginia & 1562.542 & 1375.000 \\
\hline 142 & Washington & 384.395 & 463.000 \\
\hline 143 & West Virginia & 77.877 & 252.000 \\
\hline 144 & Wisconsin & 271.573 & 504.000 \\
\hline 145 & Wyoming & 26.958 & 17.000 \\
\hline
\end{tabular}




\begin{tabular}{|c|c|c|c|}
\hline & State & P31 97 & A31 97 \\
\hline 1 & Alabama & 14651.294 & 13610.000 \\
\hline 2 & Arizona & 17155.058 & 21138.000 \\
\hline 3 & Arkansas & 5929.131 & 7418.000 \\
\hline 4 & California & 75487.723 & 81947.000 \\
\hline 5 & Colorado & 14609.844 & 17486.000 \\
\hline 6 & Connecticut & 1963.148 & 4036.000 \\
\hline 7 & Delaware & 289.269 & 1223.000 \\
\hline 8 & $\overline{D C}$ & .000 & .000 \\
\hline 9 & Florida & 46616.392 & 54152.000 \\
\hline 10 & Georgia & 21775.423 & 26240.000 \\
\hline 11 & Idaho & 3814.293 & 5039.000 \\
\hline 12 & Illinois_N & 54936.401 & 6415.000 \\
\hline 13 & Illinois_S & 23544.550 & 2750.000 \\
\hline 14 & Adams & 124.124 & 129.780 \\
\hline 15 & Allen & 865.138 & 899.859 \\
\hline 16 & Bartholomew & 375.593 & 394.250 \\
\hline 17 & Benton & 15.539 & 15.975 \\
\hline 18 & Blackford & 34.994 & 36.352 \\
\hline 19 & Boone & 73.796 & 75.931 \\
\hline 20 & Brown & 26.187 & 26.985 \\
\hline 21 & Carroll & 385.283 & 407.045 \\
\hline 22 & Cass & 418.390 & 441.082 \\
\hline 23 & Clark & 211.841 & 219.589 \\
\hline 24 & Clay & 44.921 & 46.245 \\
\hline 25 & Clinton & 475.976 & 502.405 \\
\hline 26 & Crawford & 17.031 & 17.509 \\
\hline 27 & Daviess & 252.954 & 266.410 \\
\hline 28 & Dearborn & 75.244 & 77.419 \\
\hline 29 & Decatur & 114.510 & 120.039 \\
\hline 30 & DeKalb & 75.691 & 78.192 \\
\hline 31 & Delaware & 219.396 & 226.338 \\
\hline 32 & Dubois & 224.628 & 235.926 \\
\hline 33 & Elkhart & 419.893 & 435.741 \\
\hline 34 & Fayette & 42.083 & 43.265 \\
\hline 35 & Floyd & 260.885 & 272.744 \\
\hline 36 & Fountain & 41.370 & 42.907 \\
\hline 37 & Franklin & 37.226 & 38.333 \\
\hline 38 & Fulton & 64.201 & 66.973 \\
\hline 39 & Gibson & 127.744 & 133.676 \\
\hline 40 & Grant & 173.512 & 180.040 \\
\hline 41 & Greene & 129.579 & 135.562 \\
\hline 42 & Hamilton & 295.987 & 305.128 \\
\hline 43 & Hancock & 89.437 & 92.011 \\
\hline 44 & Harrison & 200.429 & 210.590 \\
\hline 45 & Hendricks & 250.610 & 260.530 \\
\hline 46 & Henry & 91.373 & 94.314 \\
\hline 47 & Howard & 169.148 & 174.891 \\
\hline 48 & Huntington & 137.082 & 143.276 \\
\hline 49 & Jackson & 73.661 & 75.955 \\
\hline 50 & Jasper & 92.702 & 96.712 \\
\hline 51 & Jay & 124.877 & 131.160 \\
\hline
\end{tabular}




\begin{tabular}{|c|c|c|c|}
\hline & State & P31 97 & A31 97 \\
\hline 52 & Jefferson & 54.954 & 56.621 \\
\hline 53 & Jennings & 43.792 & 45.021 \\
\hline$\overline{54}$ & Johnson & 179.712 & 184.801 \\
\hline 55 & Knox & 91.147 & 94.532 \\
\hline 56 & Kosciusko & 302.892 & 317.125 \\
\hline 57 & LaGrange & 91.302 & 94.978 \\
\hline 58 & Lake & 1015.572 & 1051.145 \\
\hline 59 & La Porte & 291.756 & 303.456 \\
\hline 60 & Lawrence & 86.572 & 89.378 \\
\hline 61 & Madison & 367.638 & 382.650 \\
\hline 62 & Marion & 2165.803 & 2250.744 \\
\hline 63 & Marshall & 158.381 & 165.498 \\
\hline 64 & Martin & 29.119 & 30.312 \\
\hline 65 & Miami & 69.164 & 71.481 \\
\hline 66 & Monroe & 213.450 & 220.049 \\
\hline 67 & Montgomery & 136.249 & 142.419 \\
\hline 68 & Morgan & 107.443 & 110.522 \\
\hline 69 & Newton & 25.735 & 26.520 \\
\hline 70 & Noble & 223.913 & 234.890 \\
\hline 71 & \begin{tabular}{|l} 
Ohio \\
\end{tabular} & 9.030 & 9.283 \\
\hline 72 & Orange & 43.218 & 44.806 \\
\hline$\overline{73}$ & Owen & 36.501 & 37.588 \\
\hline 74 & Parke & 62.950 & 65.811 \\
\hline 75 & Perry & 33.117 & 34.109 \\
\hline 76 & Pike & 20.845 & 21.430 \\
\hline 77 & Porter & 299.772 & 310.202 \\
\hline 78 & Posey & 55.974 & 57.921 \\
\hline 79 & Pulaski & 36.127 & 37.573 \\
\hline 80 & Putnam & 59.044 & 60.764 \\
\hline 81 & Randolph & 60.175 & 62.346 \\
\hline 82 & Ripley & 44.404 & 45.713 \\
\hline 83 & Rush & 35.088 & 36.236 \\
\hline 84 & St. Joseph & 568.666 & 588.972 \\
\hline 85 & Scott & 112.070 & 117.562 \\
\hline 86 & Shelby & 105.083 & 109.128 \\
\hline 87 & Spencer & 45.087 & 46.728 \\
\hline 88 & Starke & 38.739 & 39.826 \\
\hline 89 & Steuben & 103.450 & 107.931 \\
\hline 90 & Sullivan & 34.957 & 35.938 \\
\hline 91 & Switzerland & 14.321 & 14.723 \\
\hline 92 & Tippecanoe & 367.358 & 381.699 \\
\hline 93 & Tipton & 62.230 & 65.071 \\
\hline 94 & Union & 11.823 & 12.155 \\
\hline 95 & Vanderburgh & 984.860 & 1034.420 \\
\hline 96 & Vermillion & 28.911 & 29.773 \\
\hline 97 & Vigo & 223.115 & 230.930 \\
\hline 98 & Wabash & 68.841 & 71.149 \\
\hline 99 & Warren & 15.518 & 16.016 \\
\hline 100 & Warrick & 83.057 & 85.389 \\
\hline 101 & Washington & 55.683 & 57.622 \\
\hline 102 & Wayne & 258.372 & 270.036 \\
\hline
\end{tabular}




\begin{tabular}{|c|c|c|c|}
\hline & State & P31_97 & A31 97 \\
\hline 103 & Wells & 147.871 & 155.224 \\
\hline 104 & White & 49.373 & 51.021 \\
\hline 105 & Whitley & 51.125 & 52.622 \\
\hline 106 & lowa & 15638.160 & 14336.000 \\
\hline 107 & Kansas & 6072.002 & 7097.000 \\
\hline 108 & Kentucky_E & 8439.068 & 9070.000 \\
\hline 109 & Kentucky_W & 5625.751 & 6046.000 \\
\hline 110 & Louisiana & 22970.422 & 9971.000 \\
\hline$\overline{111}$ & Maine & 888.091 & 1604.000 \\
\hline 112 & Maryland & 17104.789 & 16812.000 \\
\hline 113 & Massachusetts & 10911.083 & 12544.000 \\
\hline 114 & Michigan_E & 11310.591 & 12543.000 \\
\hline 115 & Michigan_W & 11310.591 & 12543.000 \\
\hline 116 & Minnesota & 15415.035 & 16097.000 \\
\hline 117 & Missisippi & 19821.094 & 21441.000 \\
\hline 118 & Missouri & 16412.484 & 18387.000 \\
\hline 119 & Montana & 2190.683 & 2657.000 \\
\hline 120 & Nebraska & 8046.615 & 9667.000 \\
\hline 121 & Nevada & 9550.284 & 11081.000 \\
\hline 122 & New Hampshire & 5101.892 & 1799.000 \\
\hline 123 & New Jersey & 11573.403 & 14220.000 \\
\hline 124 & New Mexico & 4727.959 & 6133.000 \\
\hline 125 & New York & 17569.559 & 21780.000 \\
\hline 126 & North Carolina & 19486.848 & 23870.000 \\
\hline 127 & North Dakota & 2431.446 & 2951.000 \\
\hline 128 & Ohio $\mathbf{N}$ & 7039.465 & 8171.000 \\
\hline 129 & Ohio_M & 7039.465 & 8171.000 \\
\hline 130 & Ohio_s & 7039.465 & 8171.000 \\
\hline 131 & Oklahoma & 12887.460 & 11162.000 \\
\hline 132 & Oregon & 10135.877 & 12807.000 \\
\hline 133 & Pennsylvania & 53335.721 & 54818.000 \\
\hline 134 & Rhode Island & 4793.221 & 798.000 \\
\hline 135 & South Carolina & 12377.711 & 13769.000 \\
\hline 136 & South Dakota & 2143.059 & 2254.000 \\
\hline 137 & Tennessee & 23799.424 & 27119.000 \\
\hline 138 & Texas & 63991.047 & 70957.000 \\
\hline 139 & Utah & 6360.389 & 8139.000 \\
\hline 140 & Vermont & 649.973 & 1329.000 \\
\hline 141 & Virginia & 11975.557 & 15097.000 \\
\hline 142 & Washington & 12613.183 & 14876.000 \\
\hline 143 & West Virginia & 4415.761 & 6346.000 \\
\hline 144 & Wisconsin & 19158.774 & 24315.000 \\
\hline 145 & Wyoming & 1107.688 & 1145.000 \\
\hline
\end{tabular}




\begin{tabular}{|c|c|c|c|}
\hline & State & P32 97 & A32 97 \\
\hline 1 & Alabama & 11212.000 & 8862.138 \\
\hline 2 & Arizona & 3090.000 & 2671.644 \\
\hline 3 & Arkansas & 10219.000 & 7110.715 \\
\hline 4 & California & 13024.000 & 15399.715 \\
\hline 5 & Colorado & 1514.000 & 1943.469 \\
\hline 6 & Connecticut & 2779.000 & 3024.730 \\
\hline 7 & Delaware & 1708.000 & 304.073 \\
\hline 8 & DC & .000 & .000 \\
\hline 9 & Florida & 3234.000 & 5522.332 \\
\hline 10 & Georgia & 7731.000 & 9410.270 \\
\hline 11 & Idaho & 185.000 & 336.081 \\
\hline 12 & Illinois_N & 17037.000 & 21935.292 \\
\hline 13 & Illinois_s & 7302.000 & 9401.268 \\
\hline 14 & Adams & 492.536 & 290.187 \\
\hline 15 & Allen & 2043.365 & 1195.542 \\
\hline 16 & Bartholomew & 1191.423 & 685.227 \\
\hline 17 & Benton & 1.285 & .790 \\
\hline 18 & Blackford & 42.006 & 23.638 \\
\hline 19 & Boone & 45.154 & 27.400 \\
\hline 20 & Brown & 7.001 & 3.940 \\
\hline 21 & Carroll & 1008.091 & 555.937 \\
\hline 22 & Cass & 1393.729 & 793.072 \\
\hline 23 & Clark & 172.342 & 95.451 \\
\hline 24 & Clay & 230.522 & 141.387 \\
\hline 25 & Clinton & 1264.183 & 701.023 \\
\hline 26 & Crawford & .000 & .000 \\
\hline 27 & Daviess & 590.943 & 326.250 \\
\hline 28 & Dearborn & 5.716 & 3.150 \\
\hline 29 & Decatur & 394.180 & 228.866 \\
\hline 30 & DeKalb & 187.552 & 113.136 \\
\hline 31 & Delaware & 494.732 & 299.651 \\
\hline 32 & Dubois & 680.980 & 389.583 \\
\hline 33 & Elkhart & 3173.744 & 1927.873 \\
\hline 34 & Fayette & 481.726 & 296.222 \\
\hline 35 & Floyd & 483.042 & 270.535 \\
\hline 36 & Fountain & 130.644 & 78.143 \\
\hline 37 & Franklin & 53.889 & 32.772 \\
\hline 38 & Fulton & 96.313 & 53.560 \\
\hline 39 & Gibson & 236.848 & 131.937 \\
\hline 40 & Grant & 571.808 & 341.930 \\
\hline 41 & Greene & 222.076 & 122.853 \\
\hline 42 & Hamilton & 200.250 & 118.277 \\
\hline 43 & Hancock & 191.470 & 117.373 \\
\hline 44 & Harrison & 510.790 & 287.598 \\
\hline 45 & Hendricks & 292.819 & 163.211 \\
\hline 46 & Henry & 259.104 & 157.135 \\
\hline 47 & Howard & 2338.945 & 1432.449 \\
\hline 48 & Huntington & 488.759 & 286.842 \\
\hline 49 & Jackson & 335.564 & 205.028 \\
\hline 50 & Jasper & 151.101 & 84.692 \\
\hline 51 & Jay & 301.984 & 169.469 \\
\hline
\end{tabular}




\begin{tabular}{|c|c|c|c|}
\hline & State & P32 97 & A32 97 \\
\hline 52 & Jefferson & 223.649 & 136.795 \\
\hline 53 & Jennings & 96.345 & 59.244 \\
\hline 54 & Johnson & 166.889 & 102.367 \\
\hline 55 & Knox & 76.742 & 42.366 \\
\hline 56 & Kosciusko & 757.684 & 432.437 \\
\hline 57 & LaGrange & 263.485 & 155.516 \\
\hline 58 & Lake & 900.512 & 512.480 \\
\hline 59 & La Porte & 417.040 & 235.943 \\
\hline 60 & Lawrence & 259.104 & 157.135 \\
\hline 61 & Madison & 1316.010 & 781.829 \\
\hline 62 & Marion & 4196.480 & 2439.458 \\
\hline 63 & Marshall & 492.279 & 287.106 \\
\hline 64 & Martin & 34.299 & 18.898 \\
\hline 65 & Miami & 42.006 & 23.638 \\
\hline 66 & Monroe & 77.930 & 44.376 \\
\hline 67 & Montgomery & 391.386 & 226.965 \\
\hline 68 & Morgan & 28.197 & 16.973 \\
\hline 69 & Newton & 28.197 & 16.973 \\
\hline 70 & Noble & 816.429 & 474.627 \\
\hline 71 & Ohio & .000 & .000 \\
\hline 72 & Orange & 34.299 & 18.898 \\
\hline 73 & Owen & 5.716 & 3.150 \\
\hline 74 & Parke & 100.038 & 55.120 \\
\hline 75 & Perry & 5.716 & 3.150 \\
\hline 76 & Pike & .000 & .000 \\
\hline 77 & Porter & 185.355 & 102.210 \\
\hline 78 & Posey & 34.299 & 18.898 \\
\hline 79 & Pulaski & 40.728 & 22.523 \\
\hline 80 & Putnam & 102.062 & 62.394 \\
\hline 81 & Randolph & 140.362 & 83.497 \\
\hline 82 & Ripley & 7.001 & 3.940 \\
\hline 83 & Rush & 22.570 & 12.929 \\
\hline 84 & St. Joseph & 928.163 & 545.381 \\
\hline 85 & Scott & 222.076 & 122.853 \\
\hline 86 & Shelby & 205.247 & 119.815 \\
\hline 87 & Spencer & 56.779 & 32.722 \\
\hline 88 & Starke & 37.767 & 23.224 \\
\hline 89 & Steuben & 484.860 & 288.939 \\
\hline 90 & Sullivan & 22.481 & 13.824 \\
\hline 91 & Switzerland & .000 & .000 \\
\hline 92 & Tippecanoe & 1121.946 & 666.369 \\
\hline 93 & Tipton & 148.211 & 84.742 \\
\hline 94 & Union & .000 & .000 \\
\hline 95 & Vanderburgh & 2012.888 & 1109.738 \\
\hline 96 & Vermillion & 4.573 & 2.520 \\
\hline 97 & Vigo & 189.941 & 107.735 \\
\hline 98 & Wabash & 88.894 & 52.470 \\
\hline 99 & Warren & 13.424 & 7.889 \\
\hline 100 & Warrick & 22.481 & 13.824 \\
\hline 101 & Washington & 35.583 & 19.688 \\
\hline 102 & Wayne & 571.551 & 325.692 \\
\hline
\end{tabular}




\begin{tabular}{|c|c|c|c|}
\hline & State & P32 97 & A32 97 \\
\hline 103 & Wells & 341.499 & 191.282 \\
\hline 104 & White & 248.815 & 151.466 \\
\hline 105 & Whitley & 165.392 & 101.337 \\
\hline 106 & lowa & 3063.000 & 5210.257 \\
\hline 107 & Kansas & 1189.000 & 2413.582 \\
\hline 108 & Kentucky_E & 3371.000 & 5373.296 \\
\hline 109 & Kentucky_W & 2248.000 & 3582.864 \\
\hline 110 & Louisiana & 2656.000 & 2996.723 \\
\hline 111 & Maine & 76.000 & 290.070 \\
\hline 112 & Maryland & 1827.000 & 3190.770 \\
\hline 113 & Massachusetts & 7059.000 & 2558.617 \\
\hline 114 & Michigan_E & 23718.000 & 21117.094 \\
\hline 115 & Michigan W & 23718.000 & 21117.094 \\
\hline 116 & Minnesota & 6528.000 & 5624.357 \\
\hline 117 & Missisippi & 1502.000 & 3316.800 \\
\hline 118 & Missouri & 3911.000 & 5797.399 \\
\hline 119 & Montana & 409.000 & 326.079 \\
\hline 120 & Nebraska & 1093.000 & 2194.529 \\
\hline 121 & Nevada & 139.000 & 737.178 \\
\hline 122 & New Hampshire & 425.000 & 432.104 \\
\hline 123 & New Jersey & 5897.000 & 3783.913 \\
\hline 124 & New Mexico & 546.000 & 304.073 \\
\hline 125 & New York & 3927.000 & 6287.517 \\
\hline 126 & North Carolina & 3320.000 & 6233.504 \\
\hline 127 & North Dakota & 188.000 & 1072.259 \\
\hline 128 & Ohio $\mathbf{N}$ & 14890.000 & 13501.257 \\
\hline 129 & Ohio M & 14890.000 & 13501.257 \\
\hline 130 & Ohio_s & 14890.000 & 13501.257 \\
\hline 131 & Oklahoma & 1626.000 & 3029.731 \\
\hline 132 & Oregon & 4023.000 & 3695.892 \\
\hline 133 & Pennsylvania & 21044.000 & 21203.115 \\
\hline 134 & Rhode Island & 492.000 & 517.125 \\
\hline 135 & South Carolina & 4555.000 & 3347.808 \\
\hline 136 & South Dakota & 167.000 & 508.123 \\
\hline 137 & Tennessee & 6746.000 & 8524.056 \\
\hline 138 & Texas & 16266.000 & 18617.491 \\
\hline 139 & Utah & 2451.000 & 3184.768 \\
\hline 140 & Vermont & 34.000 & 145.035 \\
\hline 141 & Virginia & 2494.000 & 3441.830 \\
\hline 142 & Washington & 4112.000 & 4152.002 \\
\hline 143 & West Virginia & 6306.000 & 3505.846 \\
\hline 144 & Wisconsin & 5985.000 & 8709.101 \\
\hline 145 & Wyoming & 3.000 & 118.028 \\
\hline
\end{tabular}




\begin{tabular}{|c|c|c|c|}
\hline & State & P33 97 & A33 97 \\
\hline 1 & Alabama & 4208.000 & 1693.475 \\
\hline 2 & Arizona & 772.000 & 1268.607 \\
\hline 3 & Arkansas & 1152.000 & 860.733 \\
\hline 4 & California & 8147.000 & 8072.497 \\
\hline 5 & Colorado & 1383.000 & 1353.580 \\
\hline 6 & Connecticut & 1129.000 & 913.717 \\
\hline 7 & Delaware & 113.000 & 367.886 \\
\hline 8 & DC & .000 & .000 \\
\hline 9 & Florida & 2546.000 & 3760.834 \\
\hline 10 & Georgia & 2156.000 & 3083.044 \\
\hline 11 & Idaho & 145.000 & 325.899 \\
\hline 12 & Illinois_ $\mathbf{N}$ & 4130.000 & 3058.052 \\
\hline 13 & Illinois_S & 1770.000 & 1310.594 \\
\hline 14 & Adams & 17.427 & 20.615 \\
\hline 15 & Allen & 101.500 & 203.539 \\
\hline 16 & Bartholomew & 28.135 & 119.711 \\
\hline 17 & Benton & 2.069 & 2.047 \\
\hline 18 & Blackford & 24.204 & 2.047 \\
\hline 19 & Boone & 9.141 & 15.181 \\
\hline 20 & Brown & 1.749 & .000 \\
\hline 21 & Carroll & 11.563 & 2.047 \\
\hline 22 & Cass & 21.903 & 6.653 \\
\hline 23 & Clark & 56.447 & 70.042 \\
\hline 24 & Clay & 4.335 & 3.241 \\
\hline 25 & Clinton & 6.503 & 7.301 \\
\hline 26 & Crawford & .000 & .000 \\
\hline 27 & Daviess & 8.357 & 10.142 \\
\hline 28 & Dearborn & 15.887 & 8.300 \\
\hline 29 & Decatur & 74.970 & 15.284 \\
\hline 30 & DeKalb & 99.713 & 21.800 \\
\hline 31 & Delaware & 46.780 & 35.480 \\
\hline 32 & Dubois & 6.047 & 4.026 \\
\hline 33 & Elkhart & 204.320 & 53.878 \\
\hline 34 & Fayette & 9.508 & 23.301 \\
\hline 35 & Floyd & 20.162 & 21.186 \\
\hline 36 & Fountain & 1.749 & .000 \\
\hline 37 & Franklin & 14.977 & 27.078 \\
\hline 38 & Fulton & 22.024 & 12.077 \\
\hline 39 & Gibson & 4.661 & 5.902 \\
\hline 40 & Grant & 24.583 & 21.766 \\
\hline 41 & Greene & 6.951 & .000 \\
\hline 42 & Hamilton & 38.973 & 13.656 \\
\hline 43 & Hancock & 13.745 & 7.949 \\
\hline 44 & Harrison & 19.669 & 27.078 \\
\hline 45 & Hendricks & 5.222 & 7.983 \\
\hline 46 & Henry & 8.892 & 12.793 \\
\hline 47 & Howard & 12.329 & 11.940 \\
\hline 48 & Huntington & 30.364 & 18.705 \\
\hline 49 & Jackson & 17.482 & 23.369 \\
\hline 50 & Jasper & 18.253 & .341 \\
\hline 51 & Jay & 72.929 & 11.097 \\
\hline
\end{tabular}




\begin{tabular}{|c|c|c|c|}
\hline & State & P33 97 & A33 97 \\
\hline 52 & Jefferson & 3.695 & 25.348 \\
\hline 53 & Jennings & 29.091 & 34.993 \\
\hline 54 & Johnson & 27.611 & 23.369 \\
\hline 55 & Knox & 25.388 & 7.140 \\
\hline 56 & Kosciusko & 57.879 & 34.091 \\
\hline 57 & LaGrange & 5.518 & 18.695 \\
\hline 58 & Lake & 563.242 & 946.345 \\
\hline 59 & La Porte & 60.105 & 69.469 \\
\hline 60 & Lawrence & 43.174 & 38.438 \\
\hline 61 & Madison & 35.370 & 13.680 \\
\hline 62 & Marion & 306.929 & 337.920 \\
\hline 63 & Marshall & 40.439 & 30.285 \\
\hline 64 & Martin & 10.407 & .341 \\
\hline 65 & Miami & 7.072 & 6.925 \\
\hline 66 & Monroe & 32.798 & 84.733 \\
\hline 67 & Montgomery & 17.413 & 6.004 \\
\hline 68 & Morgan & 12.506 & 9.484 \\
\hline 69 & Newton & 1.478 & 2.832 \\
\hline 70 & Noble & 44.622 & 15.693 \\
\hline 71 & Ohio & .246 & .000 \\
\hline 72 & Orange & 1.749 & .341 \\
\hline 73 & Owen & .591 & .341 \\
\hline 74 & Parke & .246 & 2.047 \\
\hline 75 & Perry & 3.944 & 5.970 \\
\hline 76 & Pike & 1.749 & .000 \\
\hline 77 & Porter & 44.930 & 17.126 \\
\hline 78 & Posey & 83.653 & 158.491 \\
\hline 79 & Pulaski & 5.936 & 16.375 \\
\hline 80 & Putnam & 4.988 & 5.970 \\
\hline 81 & Randolph & 15.079 & 22.662 \\
\hline 82 & Ripley & 8.983 & 4.172 \\
\hline 83 & Rush & 3.104 & 17.706 \\
\hline 84 & St. Joseph & 119.978 & 57.153 \\
\hline 85 & Scott & 4.623 & 6.219 \\
\hline 86 & Shelby & 56.458 & 13.407 \\
\hline 87 & Spencer & 6.220 & 5.970 \\
\hline 88 & Starke & .271 & 12.793 \\
\hline 89 & Steuben & 49.839 & 21.844 \\
\hline 90 & Sullivan & 1.872 & .000 \\
\hline 91 & Switzerland & .000 & .000 \\
\hline 92 & Tippecanoe & 20.681 & 95.874 \\
\hline 93 & Tipton & 3.325 & 5.970 \\
\hline 94 & Union & .246 & .341 \\
\hline 95 & Vanderburgh & 64.602 & 34.559 \\
\hline 96 & Vermillion & 5.936 & .000 \\
\hline 97 & Vigo & 18.238 & 15.045 \\
\hline 98 & Wabash & 36.101 & 20.581 \\
\hline 99 & Warren & 3.054 & 5.970 \\
\hline 100 & Warrick & 6.010 & 2.047 \\
\hline 101 & Washington & 15.272 & 25.587 \\
\hline 102 & Wayne & 23.443 & 12.452 \\
\hline
\end{tabular}




\begin{tabular}{|c|c|c|c|}
\hline & State & P33 97 & A33 97 \\
\hline 103 & Wells & 15.051 & 10.132 \\
\hline 104 & White & 24.780 & 10.201 \\
\hline 105 & Whitley & 17.538 & 4.333 \\
\hline 106 & lowa & 1373.000 & 1807.440 \\
\hline 107 & Kansas & 915.000 & 1882.416 \\
\hline 108 & Kentucky_E & 961.000 & 1213.624 \\
\hline 109 & Kentucky_W & 641.000 & 808.749 \\
\hline 110 & Louisiana & 2468.000 & 3226.000 \\
\hline 111 & \begin{tabular}{|l} 
Maine \\
\end{tabular} & 169.000 & 392.878 \\
\hline 112 & Maryland & 675.000 & 1309.594 \\
\hline 113 & Massachusetts & 489.000 & 1115.654 \\
\hline 114 & Michigan_E & 2603.000 & 2537.213 \\
\hline 115 & Michigan_W & 2603.000 & 2537.213 \\
\hline 116 & Minnesota & 1769.000 & 2017.375 \\
\hline 117 & Missisippi & 718.000 & 876.728 \\
\hline 118 & Missouri & 2258.000 & 3078.046 \\
\hline 119 & Montana & 1377.000 & 819.746 \\
\hline 120 & Nebraska & 1857.000 & 1253.611 \\
\hline 121 & Nevada & 131.000 & 583.819 \\
\hline 122 & New Hampshire & 2338.000 & 369.885 \\
\hline 123 & New Jersey & 3040.000 & 2078.356 \\
\hline 124 & New Mexico & 147.000 & 479.851 \\
\hline 125 & New York & 2567.000 & 4098.729 \\
\hline 126 & North Carolina & 2502.000 & 2946.087 \\
\hline 127 & North Dakota & 55.000 & 238.926 \\
\hline 128 & Ohio_N & 3203.000 & 2091.352 \\
\hline 129 & Ohio M & 3203.000 & 2091.352 \\
\hline 130 & Ohio_s & 3203.000 & 2091.352 \\
\hline 131 & Oklahoma & 2171.000 & 1944.397 \\
\hline 132 & Oregon & 1059.000 & 979.696 \\
\hline 133 & Pennsylvania & 7264.000 & 4239.686 \\
\hline 134 & Rhode Island & 252.000 & 381.882 \\
\hline 135 & South Carolina & 1110.000 & 1141.646 \\
\hline 136 & South Dakota & 143.000 & 189.941 \\
\hline 137 & Tennessee & 2321.000 & 2424.248 \\
\hline 138 & Texas & 12377.000 & 15188.291 \\
\hline 139 & Utah & 1044.000 & 1316.592 \\
\hline 140 & Vermont & 46.000 & 179.944 \\
\hline 141 & Virginia & 1250.000 & 1754.456 \\
\hline 142 & Washington & 1113.000 & 2031.370 \\
\hline 143 & West Virginia & 851.000 & 1018.684 \\
\hline 144 & Wisconsin & 2179.000 & 2202.317 \\
\hline 145 & Wyoming & 1266.000 & 265.918 \\
\hline
\end{tabular}




\begin{tabular}{|c|c|c|c|}
\hline & \begin{tabular}{|l|} 
State \\
\end{tabular} & P34 97 & A34 97 \\
\hline 1 & Alabama & 751.537 & 788.000 \\
\hline 2 & Arizona & 380.260 & 452.000 \\
\hline 3 & Arkansas & 804.434 & 466.000 \\
\hline 4 & California & 2561.015 & 2933.000 \\
\hline 5 & Colorado & 423.176 & 504.000 \\
\hline 6 & Connecticut & 246.520 & 266.000 \\
\hline 7 & Delaware & 4.990 & 75.000 \\
\hline 8 & DC & .000 & .000 \\
\hline 9 & Florida & 811.420 & 1821.000 \\
\hline 10 & Georgia & 1243.579 & 1517.000 \\
\hline 11 & Idaho & 97.810 & 130.000 \\
\hline 12 & Illinois_N & 3004.152 & 2542.000 \\
\hline 13 & IIllinois_s & 1287.494 & 1089.000 \\
\hline 14 & Adams & 37.876 & 24.527 \\
\hline 15 & Allen & 220.314 & 129.782 \\
\hline 16 & Bartholomew & 90.424 & 70.398 \\
\hline 17 & Benton & 2.844 & .806 \\
\hline 18 & Blackford & 11.469 & 1.173 \\
\hline 19 & Boone & 9.178 & 7.687 \\
\hline 20 & Brown & .000 & .179 \\
\hline 21 & Carroll & 1.201 & 1.173 \\
\hline 22 & Cass & 24.439 & 24.827 \\
\hline 23 & Clark & 26.527 & 17.622 \\
\hline 24 & Clay & 6.693 & 14.623 \\
\hline 25 & Clinton & 9.074 & 8.462 \\
\hline 26 & Crawford & .000 & .000 \\
\hline 27 & Daviess & 5.145 & 3.352 \\
\hline 28 & Dearborn & 4.064 & 1.584 \\
\hline 29 & Decatur & 19.233 & 15.896 \\
\hline 30 & DeKalb & 70.782 & 18.944 \\
\hline 31 & Delaware & 27.492 & 37.758 \\
\hline 32 & Dubois & 30.072 & 14.904 \\
\hline 33 & Elkhart & 187.068 & 196.254 \\
\hline 34 & Fayette & 13.667 & 35.831 \\
\hline 35 & Floyd & 52.240 & 12.581 \\
\hline 36 & Fountain & .000 & 5.603 \\
\hline 37 & Franklin & 5.993 & 3.482 \\
\hline 38 & Fulton & 10.917 & 4.870 \\
\hline 39 & Gibson & 13.730 & 3.396 \\
\hline 40 & Grant & 27.471 & 32.784 \\
\hline 41 & Greene & .000 & .440 \\
\hline 42 & Hamilton & 36.806 & 11.120 \\
\hline 43 & Hancock & 5.155 & 13.446 \\
\hline 44 & Harrison & 5.993 & 6.749 \\
\hline 45 & Hendricks & 6.325 & 4.636 \\
\hline 46 & Henry & 12.296 & 17.409 \\
\hline 47 & Howard & 19.545 & 132.550 \\
\hline 48 & Huntington & 17.259 & 21.496 \\
\hline 49 & Jackson & 26.193 & 28.194 \\
\hline 50 & Jasper & 1.843 & 1.405 \\
\hline 51 & Jay & 8.854 & 5.229 \\
\hline
\end{tabular}




\begin{tabular}{|c|c|c|c|}
\hline & \begin{tabular}{|l|} 
State \\
\end{tabular} & P34 97 & A34 97 \\
\hline 52 & Jefferson & 19.659 & 21.821 \\
\hline 53 & Jennings & 24.819 & 9.171 \\
\hline 54 & Johnson & 34.682 & 18.298 \\
\hline 55 & Knox & 2.015 & 1.136 \\
\hline 56 & Kosciusko & 45.999 & 27.584 \\
\hline 57 & LaGrange & 31.502 & 16.029 \\
\hline 58 & Lake & 42.135 & 46.700 \\
\hline 59 & La Porte & 60.014 & 32.385 \\
\hline 60 & Lawrence & 14.135 & 17.629 \\
\hline 61 & Madison & 17.115 & 59.497 \\
\hline 62 & Marion & 212.185 & 280.907 \\
\hline 63 & Marshall & 67.841 & 17.419 \\
\hline 64 & Martin & .200 & .122 \\
\hline 65 & Miami & 14.330 & 3.555 \\
\hline 66 & Monroe & 42.520 & 24.388 \\
\hline 67 & Montgomery & 24.058 & 14.104 \\
\hline 68 & Morgan & 8.055 & 8.649 \\
\hline 69 & Newton & 13.983 & 2.403 \\
\hline 70 & Noble & 62.682 & 30.028 \\
\hline 71 & Ohio & .000 & .000 \\
\hline 72 & Orange & 4.992 & .122 \\
\hline 73 & Owen & .474 & .122 \\
\hline 74 & Parke & 5.993 & .733 \\
\hline 75 & Perry & 3.502 & 2.138 \\
\hline 76 & Pike & .000 & .000 \\
\hline 77 & Porter & 18.534 & 11.108 \\
\hline 78 & Posey & 7.964 & 19.262 \\
\hline 79 & Pulaski & 9.605 & 5.937 \\
\hline 80 & Putnam & 35.676 & 7.635 \\
\hline 81 & Randolph & 12.488 & 12.119 \\
\hline 82 & Ripley & .274 & .709 \\
\hline 83 & Rush & 12.028 & 6.780 \\
\hline 84 & St. Joseph & 108.212 & 58.402 \\
\hline 85 & Scott & 30.363 & 3.026 \\
\hline 86 & Shelby & 63.094 & 12.837 \\
\hline 87 & Spencer & 13.770 & 3.527 \\
\hline 88 & Starke & 12.296 & 7.372 \\
\hline 89 & Steuben & 28.301 & 25.775 \\
\hline 90 & Sullivan & 4.792 & 1.389 \\
\hline 91 & Switzerland & 4.792 & .000 \\
\hline 92 & Tippecanoe & 70.354 & 93.236 \\
\hline 93 & Tipton & 3.502 & 4.993 \\
\hline 94 & Union & .200 & .577 \\
\hline 95 & Vanderburgh & 106.086 & 37.787 \\
\hline 96 & Vermillion & .000 & 18.529 \\
\hline$\overline{97}$ & Vigo & 54.936 & 19.042 \\
\hline 98 & Wabash & 14.417 & 12.962 \\
\hline 99 & Warren & 3.776 & 2.578 \\
\hline 100 & Warrick & 1.474 & 2.863 \\
\hline 101 & Washington & 21.141 & 9.236 \\
\hline 102 & Wayne & 38.602 & 15.548 \\
\hline
\end{tabular}




\begin{tabular}{|c|c|c|c|}
\hline & State & P34 97 & A34 97 \\
\hline 103 & Wells & 26.480 & 6.414 \\
\hline 104 & White & 15.759 & 16.480 \\
\hline 105 & Whitley & 15.082 & 10.769 \\
\hline 106 & lowa & 1515.051 & 840.000 \\
\hline 107 & Kansas & 798.446 & 584.000 \\
\hline 108 & Kentucky_E & 532.963 & 551.000 \\
\hline 109 & Kentucky W & 355.308 & 368.000 \\
\hline 110 & Louisiana & 277.460 & 626.000 \\
\hline 111 & Maine & 75.852 & 153.000 \\
\hline 112 & Maryland & 342.334 & 824.000 \\
\hline 113 & Massachusetts & 356.306 & 465.000 \\
\hline 114 & Michigan_E & 2313.497 & 2739.000 \\
\hline 115 & Michigan_W & 2313.497 & 2739.000 \\
\hline 116 & Minnesota & 746.547 & 808.000 \\
\hline 117 & Missisippi & 563.902 & 471.000 \\
\hline 118 & Missouri & 996.061 & 1290.000 \\
\hline 119 & Montana & 50.901 & 233.000 \\
\hline 120 & Nebraska & 512.003 & 347.000 \\
\hline 121 & Nevada & 145.716 & 179.000 \\
\hline 122 & New Hampshire & 102.800 & 101.000 \\
\hline 123 & New Jersey & 711.615 & 1269.000 \\
\hline 124 & New Mexico & 60.881 & 164.000 \\
\hline 125 & New York & 2046.017 & 1898.000 \\
\hline 126 & North Carolina & 1478.123 & 1291.000 \\
\hline 127 & North Dakota & 120.765 & 240.000 \\
\hline 128 & Ohio $\mathbf{N}$ & 2307.508 & 1335.000 \\
\hline 129 & Ohio M & 2307.508 & 1335.000 \\
\hline 130 & Ohio_s & 2307.508 & 1335.000 \\
\hline 131 & Oklahoma & 768.504 & 772.000 \\
\hline 132 & Oregon & 362.295 & 508.000 \\
\hline 133 & Pennsylvania & 1718.654 & 1746.000 \\
\hline 134 & Rhode Island & 34.932 & 110.000 \\
\hline 135 & South Carolina & 788.465 & 748.000 \\
\hline 136 & South Dakota & 118.769 & 130.000 \\
\hline 137 & Tennessee & 2149.815 & 1575.000 \\
\hline 138 & Texas & 2672.797 & 4108.000 \\
\hline 139 & Utah & 157.693 & 250.000 \\
\hline 140 & Vermont & 62.878 & 48.000 \\
\hline 141 & Virginia & 589.852 & 874.000 \\
\hline 142 & Washington & 457.110 & 810.000 \\
\hline 143 & West Virginia & 186.637 & 244.000 \\
\hline 144 & Wisconsin & 2240.638 & 1070.000 \\
\hline 145 & Wyoming & 30.940 & 76.000 \\
\hline
\end{tabular}




\begin{tabular}{|c|c|c|c|}
\hline & State & P35 97 & A35 97 \\
\hline 1 & Alabama & 686.596 & 556.000 \\
\hline 2 & Arizona & 358.268 & 592.000 \\
\hline 3 & Arkansas & 621.729 & 523.000 \\
\hline 4 & California & 5046.683 & 4349.000 \\
\hline 5 & Colorado & 398.186 & 455.000 \\
\hline 6 & Connecticut & 272.443 & 374.000 \\
\hline 7 & Delaware & 176.639 & 130.000 \\
\hline 8 & DC & .000 & .000 \\
\hline 9 & Florida & 927.105 & 1702.000 \\
\hline 10 & Georgia & 1558.813 & 1473.000 \\
\hline 11 & Idaho & 119.755 & 138.000 \\
\hline 12 & Illinois_N & 1668.589 & 1284.000 \\
\hline 13 & Illinois_S & 714.539 & 550.000 \\
\hline 14 & Adams & 10.546 & 17.487 \\
\hline 15 & Allen & 77.858 & 90.914 \\
\hline 16 & Bartholomew & 24.957 & 16.081 \\
\hline 17 & Benton & 1.732 & 1.657 \\
\hline 18 & Blackford & 15.325 & 6.208 \\
\hline 19 & Boone & 7.212 & 4.142 \\
\hline 20 & Brown & 1.423 & 1.351 \\
\hline 21 & Carroll & 10.249 & 1.822 \\
\hline 22 & Cass & 16.867 & 13.529 \\
\hline 23 & Clark & 35.980 & 22.641 \\
\hline 24 & Clay & 2.610 & 4.697 \\
\hline 25 & Clinton & 6.002 & 5.372 \\
\hline 26 & Crawford & .000 & .952 \\
\hline 27 & Daviess & 4.734 & 4.390 \\
\hline 28 & Dearborn & 6.323 & 5.788 \\
\hline 29 & Decatur & 67.636 & 7.161 \\
\hline 30 & DeKalb & 76.461 & 31.391 \\
\hline 31 & Delaware & 44.505 & 14.058 \\
\hline 32 & Dubois & 3.606 & 16.866 \\
\hline 33 & Elkhart & 149.257 & 92.858 \\
\hline 34 & Fayette & 8.896 & 2.351 \\
\hline 35 & Floyd & 14.851 & 25.523 \\
\hline 36 & Fountain & 1.423 & 1.636 \\
\hline 37 & Franklin & 6.433 & 9.716 \\
\hline 38 & Fulton & 20.141 & 3.682 \\
\hline 39 & Gibson & 3.131 & 7.844 \\
\hline 40 & Grant & 4.104 & 13.769 \\
\hline 41 & Greene & 2.206 & 3.018 \\
\hline 42 & Hamilton & 29.026 & 30.945 \\
\hline 43 & Hancock & 10.889 & 5.121 \\
\hline 44 & Harrison & 7.952 & 10.784 \\
\hline 45 & Hendricks & 4.768 & 9.600 \\
\hline 46 & Henry & 8.303 & 6.730 \\
\hline 47 & Howard & 4.958 & 13.680 \\
\hline 48 & Huntington & 24.780 & 8.538 \\
\hline 49 & Jackson & 16.393 & 9.704 \\
\hline 50 & Jasper & 16.013 & 3.435 \\
\hline 51 & Jay & 23.499 & 5.186 \\
\hline
\end{tabular}




\begin{tabular}{|c|c|c|c|}
\hline & State & P35 97 & A35 97 \\
\hline 52 & Jefferson & 3.298 & 5.145 \\
\hline 53 & Jennings & 21.332 & 17.003 \\
\hline 54 & Johnson & 25.574 & 20.030 \\
\hline 55 & Knox & 3.808 & 4.646 \\
\hline 56 & Kosciusko & 35.100 & 21.173 \\
\hline 57 & LaGrange & 5.053 & 12.954 \\
\hline 58 & Lake & 282.949 & 247.458 \\
\hline 59 & La Porte & 52.797 & 21.315 \\
\hline 60 & Lawrence & 19.837 & 12.620 \\
\hline 61 & Madison & 24.957 & 16.460 \\
\hline 62 & Marion & 251.777 & 109.052 \\
\hline 63 & Marshall & 30.513 & 40.573 \\
\hline 64 & Martin & .237 & .951 \\
\hline 65 & Miami & 6.263 & 8.118 \\
\hline 66 & Monroe & 15.377 & 19.884 \\
\hline 67 & Montgomery & 15.230 & 13.248 \\
\hline 68 & Morgan & 7.686 & 7.087 \\
\hline 69 & Newton & 1.423 & 7.240 \\
\hline 70 & Noble & 33.189 & 29.734 \\
\hline 71 & Ohio & .237 & .505 \\
\hline 72 & Orange & 1.423 & 4.039 \\
\hline 73 & Owen & .308 & 2.058 \\
\hline 74 & Parke & .237 & 3.847 \\
\hline 75 & Perry & 1.423 & 1.738 \\
\hline 76 & Pike & 1.423 & 1.165 \\
\hline 77 & Porter & 36.748 & 17.198 \\
\hline 78 & Posey & 40.148 & 39.751 \\
\hline 79 & Pulaski & 4.152 & 1.242 \\
\hline 80 & Putnam & .237 & 18.630 \\
\hline 81 & Randolph & 3.666 & 4.192 \\
\hline 82 & Ripley & 6.015 & 3.408 \\
\hline 83 & Rush & 1.423 & 2.456 \\
\hline 84 & St. Joseph & 105.345 & 61.891 \\
\hline 85 & Scott & 3.120 & 16.945 \\
\hline 86 & Shelby & 8.707 & 30.411 \\
\hline 87 & Spencer & 1.423 & 6.772 \\
\hline 88 & Starke & .000 & 4.465 \\
\hline 89 & Steuben & 33.202 & 12.459 \\
\hline 90 & Sullivan & .237 & 4.253 \\
\hline 91 & Switzerland & .000 & 3.100 \\
\hline 92 & Tippecanoe & 15.480 & 22.134 \\
\hline 93 & Tipton & 2.942 & 1.506 \\
\hline 94 & Union & .237 & .661 \\
\hline 95 & Vanderburgh & 45.620 & 56.774 \\
\hline 96 & Vermillion & 4.152 & 1.525 \\
\hline 97 & Vigo & 15.895 & 31.807 \\
\hline 98 & Wabash & 25.112 & 6.379 \\
\hline 99 & Warren & 2.942 & .886 \\
\hline 100 & Warrick & 5.528 & 4.772 \\
\hline 101 & Washington & 14.448 & 5.380 \\
\hline 102 & Wayne & 15.088 & 21.521 \\
\hline
\end{tabular}




\begin{tabular}{|c|c|c|c|}
\hline & State & P35 97 & A35 97 \\
\hline 103 & Wells & 14.495 & 12.354 \\
\hline 104 & White & 22.300 & 6.978 \\
\hline 105 & Whitley & 16.630 & 8.763 \\
\hline 106 & lowa & 338.308 & 299.000 \\
\hline 107 & Kansas & 675.619 & 294.000 \\
\hline 108 & Kentucky_E & 579.815 & 396.000 \\
\hline 109 & Kentucky_W & 387.208 & 264.000 \\
\hline 110 & Louisiana & 347.290 & 439.000 \\
\hline 111 & Maine & 32.933 & 60.000 \\
\hline 112 & Maryland & 423.135 & 825.000 \\
\hline 113 & Massachusetts & 833.297 & 816.000 \\
\hline 114 & Michigan_E & 511.953 & 869.000 \\
\hline 115 & Michigan W & 511.953 & 869.000 \\
\hline 116 & Minnesota & 728.511 & 736.000 \\
\hline 117 & Missisippi & 970.017 & 591.000 \\
\hline 118 & Missouri & 1033.886 & 804.000 \\
\hline 119 & Montana & 32.933 & 69.000 \\
\hline 120 & Nebraska & 125.743 & 242.000 \\
\hline 121 & Nevada & 78.839 & 308.000 \\
\hline 122 & New Hampshire & 114.765 & 87.000 \\
\hline 123 & New Jersey & 1013.927 & 1272.000 \\
\hline 124 & New Mexico & 88.818 & 187.000 \\
\hline 125 & New York & 1137.674 & 1448.000 \\
\hline 126 & North Carolina & 1631.664 & 912.000 \\
\hline 127 & North Dakota & 32.933 & 64.000 \\
\hline 128 & Ohio_N & 721.525 & 712.000 \\
\hline 129 & Ohio_M & 721.525 & 712.000 \\
\hline 130 & Ohio_s & 721.525 & 712.000 \\
\hline 131 & Oklahoma & 232.525 & 383.000 \\
\hline 132 & Oregon & 395.192 & 397.000 \\
\hline 133 & Pennsylvania & 1783.354 & 1533.000 \\
\hline 134 & Rhode Island & 76.843 & 66.000 \\
\hline 135 & South Carolina & 577.819 & 516.000 \\
\hline 136 & South Dakota & 153.686 & 84.000 \\
\hline 137 & Tennessee & 1498.936 & 1438.000 \\
\hline 138 & Texas & 3980.862 & 4610.000 \\
\hline 139 & Utah & 168.655 & 279.000 \\
\hline 140 & Vermont & 38.920 & 54.000 \\
\hline 141 & Virginia & 441.098 & 759.000 \\
\hline 142 & Washington & 272.443 & 554.000 \\
\hline 143 & West Virginia & 119.755 & 131.000 \\
\hline 144 & Wisconsin & 1238.468 & 962.000 \\
\hline 145 & Wyoming & 103.788 & 39.000 \\
\hline
\end{tabular}




\begin{tabular}{|c|c|c|c|}
\hline & \begin{tabular}{|l} 
State \\
\end{tabular} & P36 97 & A36 97 \\
\hline 1 & Alabama & 956.293 & 831.000 \\
\hline 2 & Arizona & 685.493 & 641.000 \\
\hline 3 & Arkansas & 441.674 & 527.000 \\
\hline 4 & California & 3739.236 & 5590.000 \\
\hline 5 & Colorado & 228.831 & 825.000 \\
\hline 6 & Connecticut & 329.756 & 292.000 \\
\hline 7 & Delaware & 127.905 & 292.000 \\
\hline 8 & DC & .000 & .000 \\
\hline 9 & Florida & 1020.246 & 2650.000 \\
\hline 10 & Georgia & 3158.665 & 3555.000 \\
\hline 11 & Idaho & 106.921 & 136.000 \\
\hline 12 & Illinois_N & 3330.538 & 3025.000 \\
\hline 13 & IIlinois_S & 1426.945 & 1297.000 \\
\hline 14 & Adams & 128.569 & 73.318 \\
\hline 15 & Allen & 476.322 & 271.630 \\
\hline 16 & Bartholomew & 196.671 & 112.155 \\
\hline 17 & Benton & .561 & .320 \\
\hline 18 & Blackford & 3.369 & 1.921 \\
\hline 19 & Boone & 17.236 & 9.829 \\
\hline 20 & Brown & .561 & .320 \\
\hline 21 & Carroll & 3.369 & 1.921 \\
\hline 22 & Cass & 171.912 & 98.035 \\
\hline 23 & Clark & 3.369 & 1.921 \\
\hline 24 & Clay & 98.251 & 56.029 \\
\hline 25 & Clinton & 30.598 & 17.449 \\
\hline 26 & Crawford & .000 & .000 \\
\hline 27 & Daviess & 4.435 & 2.529 \\
\hline 28 & Dearborn & .000 & .000 \\
\hline 29 & Decatur & 79.836 & 45.528 \\
\hline 30 & DeKalb & 66.979 & 38.196 \\
\hline 31 & Delaware & 184.993 & 105.495 \\
\hline 32 & Dubois & 98.251 & 56.029 \\
\hline 33 & Elkhart & 1224.940 & 698.541 \\
\hline 34 & Fayette & 210.538 & 120.063 \\
\hline 35 & Floyd & 29.981 & 17.097 \\
\hline 36 & Fountain & 42.108 & 24.013 \\
\hline 37 & Franklin & 21.054 & 12.006 \\
\hline 38 & Fulton & 3.369 & 1.921 \\
\hline 39 & Gibson & 9.825 & 5.603 \\
\hline 40 & Grant & 183.702 & 104.759 \\
\hline 41 & Greene & 3.369 & 1.921 \\
\hline 42 & Hamilton & 54.291 & 30.960 \\
\hline 43 & Hancock & 81.184 & 46.296 \\
\hline 44 & Harrison & 42.108 & 24.013 \\
\hline 45 & Hendricks & 12.801 & 7.300 \\
\hline 46 & Henry & 98.251 & 56.029 \\
\hline 47 & Howard & 982.512 & 560.293 \\
\hline 48 & Huntington & 119.923 & 68.388 \\
\hline 49 & Jackson & 137.664 & 78.505 \\
\hline 50 & Jasper & 9.825 & 5.603 \\
\hline 51 & Jay & 21.054 & 12.006 \\
\hline
\end{tabular}




\begin{tabular}{|c|c|c|c|}
\hline & State & P36 97 & A36 97 \\
\hline 52 & Jefferson & 92.749 & 52.892 \\
\hline 53 & Jennings & 42.108 & 24.013 \\
\hline 54 & Johnson & 71.190 & 40.597 \\
\hline 55 & Knox & .561 & .320 \\
\hline 56 & Kosciusko & 102.294 & 58.335 \\
\hline 57 & LaGrange & 70.685 & 40.309 \\
\hline 58 & Lake & 111.501 & 63.585 \\
\hline 59 & La Porte & 42.108 & 24.013 \\
\hline 60 & Lawrence & 98.251 & 56.029 \\
\hline 61 & Madison & 387.784 & 221.140 \\
\hline 62 & Marion & 869.944 & 496.099 \\
\hline 63 & Marshall & 108.469 & 61.856 \\
\hline 64 & Martin & .000 & .000 \\
\hline 65 & Miami & 3.369 & 1.921 \\
\hline 66 & Monroe & 9.825 & 5.603 \\
\hline 67 & Montgomery & 77.366 & 44.119 \\
\hline 68 & Morgan & 9.825 & 5.603 \\
\hline 69 & Newton & 9.825 & 5.603 \\
\hline 70 & Noble & 169.441 & 96.627 \\
\hline 71 & Ohio & .000 & .000 \\
\hline 72 & Orange & .000 & .000 \\
\hline 73 & Owen & .000 & .000 \\
\hline 74 & Parke & .000 & .000 \\
\hline 75 & Perry & .000 & .000 \\
\hline 76 & Pike & .000 & .000 \\
\hline 77 & Porter & .561 & .320 \\
\hline 78 & Posey & .000 & .000 \\
\hline 79 & Pulaski & .561 & .320 \\
\hline 80 & Putnam & 42.108 & 24.013 \\
\hline 81 & Randolph & 42.108 & 24.013 \\
\hline 82 & Ripley & .561 & .320 \\
\hline 83 & Rush & 3.369 & 1.921 \\
\hline 84 & St. Joseph & 232.266 & 132.453 \\
\hline 85 & Scott & 3.369 & 1.921 \\
\hline 86 & Shelby & 45.982 & 26.222 \\
\hline 87 & Spencer & 9.825 & 5.603 \\
\hline 88 & Starke & 16.506 & 9.413 \\
\hline 89 & Steuben & 148.949 & 84.940 \\
\hline 90 & Sullivan & 9.825 & 5.603 \\
\hline 91 & Switzerland & .000 & .000 \\
\hline 92 & Tippecanoe & 329.450 & 187.874 \\
\hline 93 & Tipton & 21.054 & 12.006 \\
\hline 94 & Union & .000 & .000 \\
\hline 95 & Vanderburgh & 4.548 & 2.593 \\
\hline 96 & Vermillion & .000 & .000 \\
\hline 97 & Vigo & 21.054 & 12.006 \\
\hline 98 & Wabash & 23.861 & 13.607 \\
\hline 99 & Warren & 3.369 & 1.921 \\
\hline 100 & Warrick & 9.825 & 5.603 \\
\hline 101 & Washington & .561 & .320 \\
\hline 102 & Wayne & 73.660 & 42.006 \\
\hline
\end{tabular}




\begin{tabular}{|c|c|c|c|}
\hline & State & P36 97 & A36 97 \\
\hline 103 & Wells & 21.335 & 12.166 \\
\hline 104 & White & 98.251 & 56.029 \\
\hline 105 & Whitley & 69.786 & 39.797 \\
\hline 106 & lowa & 2063.475 & 1182.000 \\
\hline 107 & Kansas & 495.634 & 842.000 \\
\hline 108 & Kentucky_E & 2831.907 & 2226.000 \\
\hline 109 & Kentucky_W & 1888.604 & 1484.000 \\
\hline 110 & Louisiana & 352.739 & 893.000 \\
\hline 111 & Maine & 102.924 & 183.000 \\
\hline 112 & Maryland & 866.360 & 1928.000 \\
\hline 113 & Massachusetts & 271.799 & 932.000 \\
\hline 114 & Michigan_E & 12165.008 & 9569.000 \\
\hline 115 & Michigan_W & 12165.008 & 9569.000 \\
\hline 116 & Minnesota & 1316.027 & 1478.000 \\
\hline 117 & Missisippi & 666.507 & 509.000 \\
\hline 118 & Missouri & 4300.821 & 3827.000 \\
\hline 119 & Montana & 43.968 & 202.000 \\
\hline 120 & Nebraska & 464.657 & 600.000 \\
\hline 121 & Nevada & 101.925 & 212.000 \\
\hline 122 & New Hampshire & 78.942 & 147.000 \\
\hline 123 & New Jersey & 1851.631 & 2250.000 \\
\hline 124 & New Mexico & 72.946 & 272.000 \\
\hline 125 & New York & 1538.863 & 2952.000 \\
\hline 126 & North Carolina & 1812.660 & 1864.000 \\
\hline 127 & North Dakota & 169.874 & 404.000 \\
\hline 128 & Ohio N & 4170.917 & 3584.000 \\
\hline 129 & Ohio_M & 4170.917 & 3584.000 \\
\hline 130 & Ohio_s & 4170.917 & 3584.000 \\
\hline 131 & Oklahoma & 658.513 & 1320.000 \\
\hline 132 & Oregon & 679.498 & 705.000 \\
\hline 133 & Pennsylvania & 2586.089 & 2505.000 \\
\hline 134 & Rhode Island & 13.990 & 40.000 \\
\hline 135 & South Carolina & 741.452 & 833.000 \\
\hline 136 & South Dakota & 127.905 & 232.000 \\
\hline 137 & Tennessee & 2865.882 & 2633.000 \\
\hline$\overline{138}$ & Texas & 2271.321 & 5195.000 \\
\hline 139 & Utah & 373.724 & 484.000 \\
\hline 140 & Vermont & 49.963 & 66.000 \\
\hline 141 & Virginia & 1277.056 & 1813.000 \\
\hline 142 & Washington & 409.697 & 792.000 \\
\hline 143 & West Virginia & 518.617 & 276.000 \\
\hline 144 & Wisconsin & 3322.544 & 2283.000 \\
\hline 145 & Wyoming & 7.994 & 71.000 \\
\hline
\end{tabular}




\begin{tabular}{|c|c|c|c|}
\hline & State & P37 97 & A37 97 \\
\hline 1 & Alabama & 248.663 & 60.000 \\
\hline 2 & Arizona & 31.702 & 39.000 \\
\hline 3 & Arkansas & 33.683 & 49.000 \\
\hline 4 & California & 128.789 & 190.000 \\
\hline 5 & Colorado & 18.823 & 14.000 \\
\hline 6 & Connecticut & 51.516 & 41.000 \\
\hline 7 & Delaware & 6.935 & 4.000 \\
\hline 8 & DC & .000 & .000 \\
\hline 9 & Florida & 120.864 & 170.000 \\
\hline 10 & Georgia & 456.707 & 211.000 \\
\hline 11 & Idaho & 1.981 & 18.000 \\
\hline 12 & Illinois_N & 437.884 & 473.000 \\
\hline 13 & IIllinois_s & 187.240 & 203.000 \\
\hline 14 & Adams & 1.412 & .293 \\
\hline 15 & Allen & 19.980 & 1.816 \\
\hline 16 & Bartholomew & 5.759 & .000 \\
\hline 17 & Benton & .484 & .000 \\
\hline 18 & Blackford & .000 & .000 \\
\hline 19 & Boone & .000 & .000 \\
\hline 20 & Brown & .000 & .000 \\
\hline 21 & Carroll & .000 & .000 \\
\hline 22 & Cass & .000 & .000 \\
\hline 23 & Clark & 1.041 & 2.958 \\
\hline 24 & Clay & .081 & .000 \\
\hline 25 & Clinton & .000 & .000 \\
\hline 26 & Crawford & .000 & .000 \\
\hline 27 & Daviess & .000 & .293 \\
\hline 28 & Dearborn & .000 & .293 \\
\hline 29 & Decatur & .081 & .000 \\
\hline 30 & DeKalb & 19.222 & .000 \\
\hline 31 & Delaware & 5.783 & .000 \\
\hline 32 & Dubois & .000 & .000 \\
\hline 33 & Elkhart & 9.695 & .293 \\
\hline 34 & Fayette & .484 & .000 \\
\hline 35 & Floyd & .081 & .000 \\
\hline 36 & Fountain & 6.050 & .000 \\
\hline 37 & Franklin & .000 & 1.757 \\
\hline 38 & Fulton & 3.025 & .000 \\
\hline 39 & Gibson & .000 & .000 \\
\hline 40 & Grant & 1.412 & .000 \\
\hline 41 & Greene & 1.412 & .000 \\
\hline 42 & Hamilton & 3.678 & .293 \\
\hline 43 & Hancock & .081 & .000 \\
\hline 44 & Harrison & .000 & 1.757 \\
\hline 45 & Hendricks & 1.412 & .000 \\
\hline 46 & Henry & 3.025 & .000 \\
\hline 47 & Howard & 6.050 & .000 \\
\hline 48 & Huntington & 1.363 & .293 \\
\hline 49 & Jackson & 3.993 & .000 \\
\hline 50 & Jasper & .000 & .000 \\
\hline 51 & Jay & .000 & .293 \\
\hline
\end{tabular}




\begin{tabular}{|c|c|c|c|}
\hline & State & P37 97 & A37 97 \\
\hline 52 & Jefferson & .081 & .000 \\
\hline 53 & Jennings & .484 & 1.757 \\
\hline 54 & Johnson & 2.420 & .000 \\
\hline 55 & Knox & 3.025 & .293 \\
\hline 56 & Kosciusko & 14.116 & .293 \\
\hline 57 & LaGrange & .081 & .000 \\
\hline 58 & Lake & 168.848 & 63.234 \\
\hline 59 & La Porte & 12.793 & .293 \\
\hline 60 & Lawrence & 14.116 & 1.757 \\
\hline 61 & Madison & 1.863 & .000 \\
\hline 62 & Marion & 29.441 & 3.485 \\
\hline 63 & Marshall & 3.081 & 1.757 \\
\hline 64 & Martin & 1.412 & .000 \\
\hline 65 & Miami & .000 & .000 \\
\hline 66 & Monroe & .000 & 1.757 \\
\hline 67 & Montgomery & 6.050 & .000 \\
\hline 68 & Morgan & .484 & .000 \\
\hline 69 & Newton & 1.412 & .000 \\
\hline 70 & Noble & 6.025 & .000 \\
\hline 71 & Ohio & .000 & .000 \\
\hline 72 & Orange & .000 & .000 \\
\hline 73 & Owen & .000 & .000 \\
\hline 74 & Parke & .000 & .000 \\
\hline 75 & Perry & 3.025 & .000 \\
\hline 76 & Pike & .484 & .000 \\
\hline 77 & Porter & 65.739 & .000 \\
\hline 78 & Posey & .000 & 10.983 \\
\hline 79 & Pulaski & 1.412 & .000 \\
\hline 80 & Putnam & .000 & .000 \\
\hline 81 & Randolph & 2.460 & .293 \\
\hline 82 & Ripley & .000 & .293 \\
\hline 83 & Rush & 3.025 & .000 \\
\hline 84 & St. Joseph & 14.083 & .293 \\
\hline 85 & Scott & .081 & .293 \\
\hline 86 & Shelby & 4.315 & .000 \\
\hline 87 & Spencer & 1.412 & .000 \\
\hline 88 & Starke & .000 & .000 \\
\hline 89 & Steuben & 4.041 & .293 \\
\hline 90 & Sullivan & .000 & .000 \\
\hline 91 & Switzerland & .000 & .000 \\
\hline 92 & Tippecanoe & 14.116 & .293 \\
\hline 93 & Tipton & .484 & .000 \\
\hline 94 & Union & .000 & .000 \\
\hline 95 & Vanderburgh & 1.412 & .000 \\
\hline 96 & Vermillion & .000 & .000 \\
\hline 97 & Vigo & 3.025 & .000 \\
\hline 98 & Wabash & 6.050 & .293 \\
\hline 99 & Warren & .000 & .000 \\
\hline 100 & Warrick & 30.248 & .000 \\
\hline 101 & Washington & .081 & .000 \\
\hline 102 & Wayne & 7.397 & .000 \\
\hline
\end{tabular}




\begin{tabular}{|c|c|c|c|}
\hline & State & P37 97 & A37 97 \\
\hline 103 & Wells & 1.412 & .000 \\
\hline 104 & White & .484 & .000 \\
\hline 105 & Whitley & 4.896 & .000 \\
\hline 106 & lowa & 92.134 & 19.000 \\
\hline 107 & Kansas & 22.786 & 109.000 \\
\hline 108 & Kentucky E & 78.264 & 107.000 \\
\hline 109 & Kentucky_W & 51.516 & 72.000 \\
\hline 110 & Louisiana & 73.311 & 463.000 \\
\hline 111 & Maine & 5.944 & 6.000 \\
\hline 112 & Maryland & 33.683 & 26.000 \\
\hline 113 & Massachusetts & 6.935 & 25.000 \\
\hline 114 & Michigan_E & 39.628 & 90.000 \\
\hline 115 & Michigan W & 39.628 & 90.000 \\
\hline 116 & Minnesota & 67.367 & 36.000 \\
\hline 117 & Missisippi & 27.739 & 15.000 \\
\hline 118 & Missouri & 104.022 & 149.000 \\
\hline 119 & Montana & 9.907 & 22.000 \\
\hline 120 & Nebraska & 9.907 & 3.000 \\
\hline 121 & Nevada & .000 & 7.000 \\
\hline 122 & New Hampshire & 29.721 & 8.000 \\
\hline 123 & New Jersey & 85.199 & 62.000 \\
\hline 124 & New Mexico & .000 & 82.000 \\
\hline 125 & New York & 39.628 & 141.000 \\
\hline 126 & North Carolina & 50.525 & 27.000 \\
\hline 127 & North Dakota & .000 & 12.000 \\
\hline 128 & Ohio_N & 76.283 & 42.000 \\
\hline 129 & Ohio M & 76.283 & 42.000 \\
\hline 130 & Ohio_s & 76.283 & 42.000 \\
\hline 131 & Oklahoma & 44.581 & 104.000 \\
\hline 132 & Oregon & 214.979 & 235.000 \\
\hline 133 & Pennsylvania & 799.485 & 358.000 \\
\hline 134 & Rhode Island & 28.730 & 3.000 \\
\hline 135 & South Carolina & 48.544 & 60.000 \\
\hline 136 & South Dakota & 4.953 & 5.000 \\
\hline 137 & Tennessee & 93.125 & 77.000 \\
\hline 138 & Texas & 424.015 & 729.000 \\
\hline 139 & Utah & 57.460 & 162.000 \\
\hline 140 & Vermont & 1.981 & 6.000 \\
\hline 141 & Virginia & 31.702 & 45.000 \\
\hline 142 & Washington & 146.622 & 206.000 \\
\hline 143 & West Virginia & 94.115 & 37.000 \\
\hline 144 & Wisconsin & 82.227 & 44.000 \\
\hline 145 & Wyoming & .991 & 88.000 \\
\hline
\end{tabular}




\begin{tabular}{|c|c|c|c|}
\hline & State & P38 97 & A38 97 \\
\hline 1 & Alabama & 9.847 & 27.000 \\
\hline 2 & Arizona & 26.587 & 64.000 \\
\hline 3 & Arkansas & 4.923 & 12.000 \\
\hline 4 & California & 395.846 & 379.000 \\
\hline 5 & Colorado & 25.602 & 35.000 \\
\hline 6 & Connecticut & 106.347 & 27.000 \\
\hline 7 & Delaware & 12.801 & 4.000 \\
\hline 8 & $\mathrm{DC}$ & .000 & .000 \\
\hline 9 & Florida & 103.393 & 137.000 \\
\hline 10 & Georgia & 211.709 & 163.000 \\
\hline 11 & Idaho & .985 & 7.000 \\
\hline 12 & Illinois $\mathbf{N}$ & 56.127 & 76.000 \\
\hline 13 & IIlinois_s & 23.633 & 33.000 \\
\hline 14 & Adams & .113 & .316 \\
\hline 15 & Allen & 6.507 & 12.442 \\
\hline 16 & Bartholomew & .568 & .884 \\
\hline 17 & Benton & .022 & .158 \\
\hline 18 & Blackford & .056 & .117 \\
\hline 19 & Boone & .308 & .592 \\
\hline 20 & Brown & .008 & .019 \\
\hline 21 & Carroll & .036 & .210 \\
\hline 22 & Cass & .234 & .482 \\
\hline 23 & Clark & .936 & 1.687 \\
\hline 24 & Clay & .023 & .094 \\
\hline 25 & Clinton & .122 & .280 \\
\hline 26 & Crawford & .008 & .009 \\
\hline 27 & Daviess & .081 & .245 \\
\hline 28 & Dearborn & .135 & .242 \\
\hline 29 & Decatur & .099 & .415 \\
\hline 30 & DeKalb & .250 & .404 \\
\hline 31 & Delaware & .854 & 1.461 \\
\hline 32 & Dubois & .966 & 1.516 \\
\hline 33 & Elkhart & 3.638 & 7.370 \\
\hline 34 & Fayette & .070 & .234 \\
\hline 35 & Floyd & .505 & .994 \\
\hline 36 & Fountain & .021 & .125 \\
\hline 37 & Franklin & .050 & .115 \\
\hline 38 & Fulton & .039 & .162 \\
\hline 39 & Gibson & .082 & .182 \\
\hline 40 & Grant & .403 & .947 \\
\hline 41 & Greene & .072 & .200 \\
\hline 42 & Hamilton & 2.636 & 5.180 \\
\hline 43 & Hancock & .144 & .543 \\
\hline 44 & Harrison & .152 & .253 \\
\hline 45 & Hendricks & .686 & 1.082 \\
\hline 46 & Henry & .179 & .770 \\
\hline 47 & Howard & .513 & .773 \\
\hline 48 & Huntington & .186 & .488 \\
\hline 49 & Jackson & .211 & .565 \\
\hline 50 & Jasper & .101 & .238 \\
\hline 51 & Jay & .051 & .241 \\
\hline
\end{tabular}




\begin{tabular}{|c|c|c|c|}
\hline & State & P38 97 & A38 97 \\
\hline 52 & Jefferson & .134 & .243 \\
\hline 53 & Jennings & .058 & 1.673 \\
\hline 54 & Johnson & .482 & .788 \\
\hline 55 & Knox & .304 & .742 \\
\hline 56 & Kosciusko & .423 & .971 \\
\hline 57 & LaGrange & .201 & .527 \\
\hline 58 & Lake & 4.331 & 6.977 \\
\hline 59 & La Porte & .690 & 1.559 \\
\hline 60 & Lawrence & .133 & .261 \\
\hline 61 & Madison & .478 & 1.030 \\
\hline 62 & Marion & 18.252 & 33.090 \\
\hline 63 & Marshall & .407 & .908 \\
\hline 64 & Martin & .018 & .081 \\
\hline 65 & Miami & .162 & .647 \\
\hline 66 & Monroe & .451 & 1.753 \\
\hline 67 & Montgomery & .133 & .258 \\
\hline 68 & Morgan & .270 & .410 \\
\hline 69 & Newton & .025 & .125 \\
\hline 70 & Noble & .169 & 1.145 \\
\hline 71 & Ohio & .000 & .000 \\
\hline 72 & Orange & .037 & .098 \\
\hline 73 & Owen & .050 & .313 \\
\hline 74 & Parke & .050 & .115 \\
\hline 75 & Perry & .048 & .345 \\
\hline 76 & Pike & .067 & .116 \\
\hline 77 & Porter & 1.129 & 2.100 \\
\hline 78 & Posey & .190 & .670 \\
\hline 79 & Pulaski & .085 & .347 \\
\hline 80 & Putnam & .042 & .099 \\
\hline 81 & Randolph & .091 & .159 \\
\hline 82 & Ripley & .312 & .520 \\
\hline 83 & Rush & .096 & .228 \\
\hline 84 & St. Joseph & 3.724 & 7.545 \\
\hline 85 & Scott & .052 & .790 \\
\hline 86 & Shelby & .592 & .799 \\
\hline 87 & Spencer & .058 & .297 \\
\hline 88 & Starke & .035 & .083 \\
\hline 89 & Steuben & .171 & .452 \\
\hline 90 & Sullivan & .047 & .196 \\
\hline 91 & Switzerland & .008 & .019 \\
\hline 92 & Tippecanoe & .576 & 1.504 \\
\hline 93 & Tipton & .060 & .209 \\
\hline 94 & Union & .050 & .115 \\
\hline 95 & Vanderburgh & 2.932 & 6.382 \\
\hline 96 & Vermillion & .009 & .086 \\
\hline 97 & Vigo & .775 & 1.620 \\
\hline 98 & Wabash & .135 & .274 \\
\hline 99 & Warren & .050 & .115 \\
\hline 100 & Warrick & .109 & .346 \\
\hline 101 & Washington & .068 & .086 \\
\hline 102 & Wayne & .869 & 1.393 \\
\hline
\end{tabular}




\begin{tabular}{|c|c|c|c|}
\hline & State & P38 97 & A38 97 \\
\hline 103 & Wells & .086 & 1.633 \\
\hline 104 & White & .113 & .477 \\
\hline 105 & Whitley & .147 & .256 \\
\hline 106 & lowa & 7.878 & 18.000 \\
\hline 107 & Kansas & 14.770 & 18.000 \\
\hline 108 & Kentucky_E & 41.357 & 13.000 \\
\hline 109 & Kentucky_W & 27.571 & 9.000 \\
\hline 110 & Louisiana & 23.633 & 42.000 \\
\hline 111 & Maine & 18.709 & 11.000 \\
\hline 112 & Maryland & 96.500 & 66.000 \\
\hline 113 & Massachusetts & 85.668 & 88.000 \\
\hline 114 & Michigan E & 30.525 & 45.000 \\
\hline 115 & Michigan_W & 30.525 & 45.000 \\
\hline 116 & Minnesota & 124.071 & 87.000 \\
\hline 117 & Missisippi & 36.434 & 15.000 \\
\hline 118 & Missouri & 72.867 & 58.000 \\
\hline 119 & Montana & 1.969 & 7.000 \\
\hline 120 & Nebraska & 139.826 & 34.000 \\
\hline 121 & Nevada & 33.480 & 20.000 \\
\hline 122 & New Hampshire & 16.740 & 5.000 \\
\hline 123 & New Jersey & 82.714 & 155.000 \\
\hline 124 & New Mexico & 2.954 & 22.000 \\
\hline 125 & New York & 164.444 & 157.000 \\
\hline 126 & North Carolina & 35.449 & 142.000 \\
\hline 127 & North Dakota & 14.770 & 4.000 \\
\hline 128 & Ohio $N$ & 25.602 & 28.000 \\
\hline 129 & Ohio M & 25.602 & 28.000 \\
\hline 130 & Ohio_s & 25.602 & 28.000 \\
\hline 131 & Oklahoma & 17.724 & 19.000 \\
\hline 132 & Oregon & 29.541 & 46.000 \\
\hline 133 & Pennsylvania & 92.561 & 90.000 \\
\hline 134 & Rhode Island & 15.755 & 9.000 \\
\hline 135 & South Carolina & 65.974 & 44.000 \\
\hline 136 & South Dakota & 2.954 & 5.000 \\
\hline 137 & Tennessee & 54.158 & 47.000 \\
\hline 138 & Texas & 142.780 & 195.000 \\
\hline 139 & Utah & 25.602 & 23.000 \\
\hline 140 & Vermont & 1.969 & 2.000 \\
\hline 141 & Virginia & 31.510 & 73.000 \\
\hline 142 & Washington & 126.041 & 55.000 \\
\hline 143 & West Virginia & 1.969 & 7.000 \\
\hline 144 & Wisconsin & 60.066 & 42.000 \\
\hline 145 & Wyoming & 6.893 & 3.000 \\
\hline
\end{tabular}




\begin{tabular}{|c|c|c|c|}
\hline & State & P39 37 & A39 97 \\
\hline 1 & Alabama & 501.000 & 250.142 \\
\hline 2 & Arizona & 387.000 & 402.839 \\
\hline 3 & Arkansas & 295.000 & 443.022 \\
\hline 4 & California & 1783.000 & 1514.914 \\
\hline 5 & Colorado & 225.000 & 440.008 \\
\hline 6 & Connecticut & 127.000 & 206.944 \\
\hline 7 & Delaware & 34.000 & 49.225 \\
\hline 8 & DC & .000 & .000 \\
\hline 9 & Florida & 417.000 & 1146.231 \\
\hline 10 & Georgia & 868.000 & 807.686 \\
\hline 11 & Idaho & 25.000 & 65.298 \\
\hline 12 & Illinois $\mathbf{N}$ & 314.000 & 458.091 \\
\hline 13 & Illinois_s & 135.000 & 195.894 \\
\hline 14 & Adams & 8.209 & 2.698 \\
\hline 15 & Allen & 30.700 & 26.338 \\
\hline 16 & Bartholomew & 17.207 & 5.687 \\
\hline 17 & Benton & .830 & .774 \\
\hline 18 & Blackford & .256 & 1.140 \\
\hline 19 & Boone & 1.431 & 3.574 \\
\hline 20 & Brown & .020 & 1.204 \\
\hline 21 & Carroll & 5.181 & 1.624 \\
\hline 22 & Cass & 6.331 & 3.272 \\
\hline 23 & Clark & 15.218 & 7.658 \\
\hline 24 & Clay & 3.676 & 2.136 \\
\hline 25 & Clinton & 1.912 & 2.738 \\
\hline 26 & Crawford & .000 & .848 \\
\hline 27 & Daviess & .969 & 2.400 \\
\hline 28 & Dearborn & .000 & 3.646 \\
\hline 29 & Decatur & 3.013 & 1.989 \\
\hline 30 & DeKalb & 2.549 & 3.167 \\
\hline 31 & Delaware & 8.570 & 9.669 \\
\hline 32 & Dubois & 92.446 & 3.177 \\
\hline 33 & Elkhart & 96.146 & 14.394 \\
\hline 34 & Fayette & 8.398 & 2.095 \\
\hline 35 & Floyd & 7.057 & 5.715 \\
\hline 36 & Fountain & 1.518 & 1.458 \\
\hline 37 & Franklin & .759 & 1.753 \\
\hline 38 & Fulton & .121 & 1.641 \\
\hline 39 & Gibson & .354 & 2.598 \\
\hline 40 & Grant & 8.982 & 5.980 \\
\hline 41 & Greene & .256 & 2.689 \\
\hline 42 & Hamilton & 5.478 & 13.403 \\
\hline 43 & Hancock & 3.736 & 4.353 \\
\hline$\overline{44}$ & Harrison & 10.273 & 2.705 \\
\hline 45 & Hendricks & .596 & 7.852 \\
\hline 46 & Henry & 5.902 & 3.947 \\
\hline 47 & Howard & 40.473 & 6.826 \\
\hline 48 & Huntington & 4.322 & 3.063 \\
\hline 49 & Jackson & 5.771 & 3.306 \\
\hline 50 & Jasper & .489 & 2.358 \\
\hline 51 & Jay & 1.568 & 1.762 \\
\hline
\end{tabular}




\begin{tabular}{|c|c|c|c|}
\hline & State & P39 37 & A39 97 \\
\hline 52 & Jefferson & 3.478 & 2.535 \\
\hline 53 & Jennings & 1.518 & 2.180 \\
\hline 54 & Johnson & 3.740 & 8.878 \\
\hline 55 & Knox & .155 & 3.214 \\
\hline 56 & Kosciusko & 7.815 & 5.890 \\
\hline 57 & LaGrange & 6.609 & 2.760 \\
\hline 58 & Lake & 7.432 & 39.237 \\
\hline 59 & La Porte & 3.879 & 8.897 \\
\hline 60 & Lawrence & 3.676 & 3.708 \\
\hline 61 & Madison & 19.522 & 10.779 \\
\hline 62 & Marion & 48.436 & 69.113 \\
\hline 63 & Marshall & 4.719 & 3.601 \\
\hline 64 & Martin & .000 & .848 \\
\hline 65 & Miami & 5.181 & 2.842 \\
\hline 66 & Monroe & 1.299 & 9.654 \\
\hline 67 & Montgomery & 2.789 & 3.021 \\
\hline 68 & Morgan & .489 & 5.249 \\
\hline 69 & Newton & 1.164 & 1.181 \\
\hline 70 & Noble & 6.917 & 3.623 \\
\hline 71 & Ohio & .000 & .450 \\
\hline 72 & Orange & 23.609 & 1.550 \\
\hline 73 & Owen & .000 & 1.717 \\
\hline 74 & Parke & .135 & 1.378 \\
\hline 75 & Perry & 3.953 & 1.549 \\
\hline 76 & Pike & .000 & 1.038 \\
\hline 77 & Porter & .708 & 11.695 \\
\hline 78 & Posey & .000 & 2.185 \\
\hline 79 & Pulaski & .155 & 1.106 \\
\hline 80 & Putnam & 1.518 & 2.839 \\
\hline 81 & Randolph & 1.653 & 2.223 \\
\hline 82 & Ripley & 2.381 & 2.111 \\
\hline 83 & Rush & .121 & 1.486 \\
\hline 84 & St. Joseph & 10.638 & 21.350 \\
\hline 85 & Scott & .931 & 1.817 \\
\hline 86 & Shelby & 6.136 & 3.476 \\
\hline 87 & Spencer & 10.472 & 1.643 \\
\hline 88 & Starke & .730 & 1.929 \\
\hline 89 & Steuben & 5.504 & 2.622 \\
\hline 90 & Sullivan & .354 & 1.740 \\
\hline 91 & Switzerland & .135 & .713 \\
\hline 92 & Tippecanoe & 21.993 & 11.829 \\
\hline 93 & Tipton & .759 & 1.342 \\
\hline 94 & Union & .000 & .589 \\
\hline 95 & Vanderburgh & 5.574 & 13.887 \\
\hline 96 & Vermillion & .000 & 1.359 \\
\hline 97 & Vigo & 1.568 & 8.620 \\
\hline 98 & Wabash & .995 & 2.826 \\
\hline 99 & Warren & .121 & .672 \\
\hline 100 & Warrick & .489 & 4.135 \\
\hline 101 & Washington & 23.629 & 2.170 \\
\hline 102 & Wayne & 12.288 & 5.790 \\
\hline
\end{tabular}




\begin{tabular}{|c|c|c|c|}
\hline & State & P39 37 & A39 97 \\
\hline 103 & Wells & .904 & 2.225 \\
\hline 104 & White & 5.902 & 2.037 \\
\hline 105 & Whitley & 3.109 & 2.445 \\
\hline 106 & lowa & 359.000 & 159.729 \\
\hline 107 & Kansas & 116.000 & 189.867 \\
\hline 108 & Kentucky_E & 80.000 & 140.642 \\
\hline 109 & Kentucky_W & 53.000 & 94.431 \\
\hline 110 & Louisiana & 38.000 & 203.931 \\
\hline 111 & Maine & 33.000 & 35.160 \\
\hline 112 & Maryland & 167.000 & 448.045 \\
\hline 113 & Massachusetts & 256.000 & 379.733 \\
\hline 114 & Michigan E & 857.000 & 533.435 \\
\hline 115 & Michigan_W & 857.000 & 533.435 \\
\hline 116 & Minnesota & 260.000 & 279.275 \\
\hline 117 & Missisippi & 766.000 & 279.275 \\
\hline 118 & Missouri & 1024.000 & 667.044 \\
\hline 119 & Montana & 13.000 & 29.133 \\
\hline 120 & Nebraska & 112.000 & 96.440 \\
\hline 121 & Nevada & 38.000 & 108.495 \\
\hline 122 & New Hampshire & 12.000 & 58.266 \\
\hline 123 & New Jersey & 164.000 & 601.746 \\
\hline 124 & New Mexico & 8.000 & 69.316 \\
\hline 125 & New York & 644.000 & 1051.800 \\
\hline 126 & North Carolina & 1588.000 & 858.920 \\
\hline 127 & North Dakota & 24.000 & 38.174 \\
\hline 128 & Ohio N & 410.000 & 356.628 \\
\hline 129 & Ohio_M & 410.000 & 356.628 \\
\hline 130 & Ohios & 410.000 & 356.628 \\
\hline 131 & Oklahoma & 46.000 & 121.555 \\
\hline 132 & Oregon & 115.000 & 160.734 \\
\hline 133 & Pennsylvania & 692.000 & 872.984 \\
\hline 134 & Rhode Island & 16.000 & 34.156 \\
\hline 135 & South Carolina & 245.000 & 287.311 \\
\hline 136 & South Dakota & 14.000 & 24.110 \\
\hline 137 & Tennessee & 773.000 & 391.788 \\
\hline 138 & Texas & 1617.000 & 1929.808 \\
\hline 139 & Utah & 123.000 & 185.848 \\
\hline 140 & Vermont & 39.000 & 39.179 \\
\hline 141 & Virginia & 753.000 & 443.022 \\
\hline 142 & Washington & 318.000 & 595.719 \\
\hline 143 & West Virginia & 45.000 & 56.257 \\
\hline 144 & Wisconsin & 616.000 & 340.554 \\
\hline 145 & Wyoming & 2.000 & 25.115 \\
\hline
\end{tabular}




\begin{tabular}{|c|c|c|c|}
\hline & \begin{tabular}{|l} 
State \\
\end{tabular} & P40 97 & A40 97 \\
\hline 1 & Alabama & 2965.000 & 1716.992 \\
\hline 2 & Arizona & 580.000 & 1388.803 \\
\hline 3 & Arkansas & 765.000 & 838.485 \\
\hline 4 & California & 22906.000 & 7018.057 \\
\hline 5 & Colorado & 2787.000 & 1573.910 \\
\hline 6 & Connecticut & 1303.000 & 497.287 \\
\hline 7 & Delaware & 804.000 & 328.190 \\
\hline 8 & DC & .000 & .000 \\
\hline 9 & Florida & 4027.000 & 5355.095 \\
\hline 10 & Georgia & 6226.000 & 4210.434 \\
\hline 11 & Idaho & 176.000 & 362.209 \\
\hline 12 & Illinois $\mathbf{N}$ & 3931.000 & 2429.404 \\
\hline 13 & Illinois_S & 1685.000 & 1041.602 \\
\hline 14 & Adams & 20.758 & 29.094 \\
\hline 15 & Allen & 177.384 & 93.375 \\
\hline 16 & Bartholomew & 63.552 & 20.807 \\
\hline 17 & Benton & 2.644 & 8.688 \\
\hline 18 & Blackford & 5.892 & 6.488 \\
\hline 19 & Boone & 12.547 & 11.753 \\
\hline 20 & Brown & 4.446 & 4.906 \\
\hline 21 & Carroll & 63.730 & 5.340 \\
\hline 22 & Cass & 70.361 & 16.658 \\
\hline 23 & Clark & 41.730 & 48.598 \\
\hline 24 & Clay & 7.633 & 9.132 \\
\hline 25 & Clinton & 78.808 & 10.162 \\
\hline 26 & Crawford & 2.898 & 4.895 \\
\hline 27 & Daviess & 41.980 & 15.791 \\
\hline 28 & Dearborn & 12.793 & 12.342 \\
\hline 29 & Decatur & 19.098 & 7.453 \\
\hline 30 & DeKalb & 12.817 & 33.863 \\
\hline 31 & Delaware & 38.199 & 33.905 \\
\hline 32 & Dubois & 38.386 & 70.687 \\
\hline 33 & Elkhart & 127.920 & 271.461 \\
\hline 34 & Fayette & 7.161 & 6.891 \\
\hline 35 & Floyd & 43.634 & 39.575 \\
\hline 36 & Fountain & 6.977 & 5.145 \\
\hline 37 & Franklin & 6.324 & 7.134 \\
\hline 38 & Fulton & 10.762 & 14.630 \\
\hline 39 & Gibson & 21.345 & 8.542 \\
\hline 40 & Grant & 35.232 & 21.773 \\
\hline 41 & Greene & 22.654 & 10.949 \\
\hline 42 & Hamilton & 67.680 & 48.658 \\
\hline 43 & Hancock & 15.208 & 15.297 \\
\hline 44 & Harrison & 33.347 & 14.862 \\
\hline 45 & Hendricks & 43.159 & 27.929 \\
\hline 46 & Henry & 21.470 & 15.088 \\
\hline 47 & Howard & 28.616 & 24.556 \\
\hline 48 & Huntington & 23.931 & 13.055 \\
\hline 49 & Jackson & 13.494 & 21.194 \\
\hline 50 & Jasper & 15.539 & 10.177 \\
\hline 51 & Jay & 20.785 & 7.902 \\
\hline
\end{tabular}




\begin{tabular}{|c|c|c|c|}
\hline & State & P40 97 & A40 97 \\
\hline 52 & Jefferson & 10.327 & 10.901 \\
\hline 53 & Jennings & 44.853 & 9.628 \\
\hline 54 & Johnson & 31.570 & 65.036 \\
\hline 55 & Knox & 15.372 & 13.237 \\
\hline 56 & Kosciusko & 57.165 & 30.480 \\
\hline 57 & LaGrange & 16.347 & 75.072 \\
\hline 58 & Lake & 177.613 & 137.354 \\
\hline 59 & La Porte & 50.056 & 37.548 \\
\hline 60 & Lawrence & 15.666 & 16.268 \\
\hline 61 & Madison & 63.768 & 41.891 \\
\hline 62 & Marion & 405.191 & 253.199 \\
\hline 63 & Marshall & 32.488 & 34.450 \\
\hline 64 & Martin & 4.892 & 3.139 \\
\hline 65 & Miami & 12.704 & 14.084 \\
\hline 66 & Monroe & 42.203 & 33.856 \\
\hline 67 & Montgomery & 22.792 & 11.409 \\
\hline 68 & Morgan & 19.269 & 23.687 \\
\hline 69 & Newton & 4.369 & 3.884 \\
\hline 70 & Noble & 54.771 & 14.021 \\
\hline 71 & Ohio & 1.536 & 1.479 \\
\hline 72 & Orange & 7.291 & 21.139 \\
\hline 73 & Owen & 12.185 & 10.000 \\
\hline 74 & Parke & 10.528 & 10.676 \\
\hline 75 & Perry & 11.609 & 7.199 \\
\hline 76 & Pike & 3.547 & 5.519 \\
\hline 77 & Porter & 51.670 & 43.202 \\
\hline 78 & Posey & 15.446 & 7.536 \\
\hline 79 & Pulaski & 6.075 & 3.990 \\
\hline 80 & Putnam & 10.036 & 11.444 \\
\hline 81 & Randolph & 10.159 & 21.498 \\
\hline 82 & Ripley & 7.545 & 11.223 \\
\hline 83 & Rush & 6.941 & 6.994 \\
\hline 84 & St. Joseph & 113.493 & 88.274 \\
\hline 85 & Scott & 36.132 & 8.082 \\
\hline 86 & Shelby & 17.698 & 11.432 \\
\hline 87 & Spencer & 7.609 & 10.703 \\
\hline 88 & Starke & 6.592 & 17.682 \\
\hline 89 & Steuben & 23.324 & 10.343 \\
\hline 90 & Sullivan & 5.948 & 8.848 \\
\hline 91 & Switzerland & 2.437 & 2.345 \\
\hline 92 & Tippecanoe & 67.820 & 40.691 \\
\hline 93 & Tipton & 10.406 & 6.521 \\
\hline 94 & Union & 2.012 & 2.287 \\
\hline 95 & Vanderburgh & 201.322 & 54.322 \\
\hline 96 & Vermillion & 4.911 & 4.470 \\
\hline 97 & Vigo & 38.703 & 32.456 \\
\hline 98 & Wabash & 12.649 & 15.435 \\
\hline 99 & Warren & 2.630 & 4.317 \\
\hline 100 & Warrick & 15.130 & 18.023 \\
\hline 101 & Washington & 9.412 & 15.071 \\
\hline 102 & Wayne & 43.227 & 21.148 \\
\hline
\end{tabular}




\begin{tabular}{|c|c|c|c|}
\hline & State & P40 97 & A40 97 \\
\hline 103 & Wells & 25.624 & 9.582 \\
\hline 104 & White & 9.355 & 10.385 \\
\hline 105 & Whitley & 8.689 & 14.184 \\
\hline 106 & lowa & 801.000 & 826.478 \\
\hline 107 & Kansas & 605.000 & 714.413 \\
\hline 108 & Kentucky_E & 1013.000 & 1065.616 \\
\hline 109 & Kentucky W & 675.000 & 710.411 \\
\hline 110 & Louisiana & 416.000 & 844.488 \\
\hline 111 & Maine & 113.000 & 317.183 \\
\hline 112 & Maryland & 2969.000 & 7076.090 \\
\hline 113 & Massachusetts & 1163.000 & 1736.003 \\
\hline 114 & Michigan_E & 1270.000 & 1687.976 \\
\hline 115 & Michigan_W & 1270.000 & 1687.976 \\
\hline 116 & Minnesota & 1708.000 & 1635.946 \\
\hline 117 & Missisippi & 1123.000 & 847.490 \\
\hline 118 & Missouri & 1600.000 & 1928.114 \\
\hline 119 & Montana & 478.000 & 454.263 \\
\hline 120 & Nebraska & 831.000 & 435.252 \\
\hline 121 & Nevada & 894.000 & 1201.695 \\
\hline 122 & New Hampshire & 216.000 & 519.300 \\
\hline 123 & New Jersey & 2731.000 & 2094.210 \\
\hline 124 & New Mexico & 42.000 & 305.176 \\
\hline 125 & New York & 3965.000 & 4408.548 \\
\hline 126 & North Carolina & 3774.000 & 4867.814 \\
\hline 127 & North Dakota & 91.000 & 180.104 \\
\hline 128 & Ohio $N$ & 1167.000 & 1135.656 \\
\hline 129 & Ohio M & 1167.000 & 1135.656 \\
\hline 130 & Ohio_s & 1167.000 & 1135.656 \\
\hline 131 & Oklahoma & 777.000 & 676.391 \\
\hline 132 & Oregon & 723.000 & 1383.800 \\
\hline 133 & Pennsylvania & 9635.000 & 19952.533 \\
\hline 134 & Rhode Island & 711.000 & 1770.023 \\
\hline 135 & South Carolina & 570.000 & 1301.752 \\
\hline 136 & South Dakota & 789.000 & 268.155 \\
\hline 137 & Tennessee & 1784.000 & 2243.297 \\
\hline 138 & Texas & 7154.000 & 7775.494 \\
\hline 139 & Utah & 428.000 & 794.459 \\
\hline 140 & Vermont & 436.000 & 1277.739 \\
\hline 141 & Virginia & 2685.000 & 1965.136 \\
\hline 142 & Washington & 1812.000 & 2569.485 \\
\hline 143 & West Virginia & 134.000 & 408.236 \\
\hline 144 & Wisconsin & 2211.000 & 1741.006 \\
\hline 145 & Wyoming & 19.000 & 188.109 \\
\hline
\end{tabular}




\begin{tabular}{|c|c|c|c|}
\hline & State & P41 97 & A41 97 \\
\hline 1 & Alabama & 2129.856 & 6028.000 \\
\hline 2 & Arizona & 343.977 & 334.000 \\
\hline 3 & Arkansas & 1742.882 & 5476.000 \\
\hline 4 & California & 22071.510 & 21283.000 \\
\hline 5 & Colorado & 1982.866 & 665.000 \\
\hline 6 & Connecticut & 5406.635 & 1618.000 \\
\hline 7 & Delaware & 331.978 & 322.000 \\
\hline 8 & DC & .000 & .000 \\
\hline 9 & Florida & 5850.605 & 4745.000 \\
\hline 10 & Georgia & 4105.723 & 3435.000 \\
\hline 11 & Idaho & 143.990 & 19.000 \\
\hline 12 & Illinois_N & 9090.387 & 8903.000 \\
\hline 13 & lllinois_s & 3895.737 & 3816.000 \\
\hline 14 & Adams & 46.822 & 43.032 \\
\hline 15 & Allen & 351.688 & 417.931 \\
\hline 16 & Bartholomew & 122.834 & 136.491 \\
\hline 17 & Benton & 8.524 & 9.933 \\
\hline 18 & Blackford & 75.428 & 42.881 \\
\hline 19 & Boone & 35.496 & 20.092 \\
\hline 20 & Brown & 7.006 & 4.004 \\
\hline 21 & Carroll & 50.441 & 36.855 \\
\hline 22 & Cass & 83.018 & 55.251 \\
\hline 23 & Clark & 125.753 & 83.397 \\
\hline 24 & Clay & 12.844 & 8.154 \\
\hline 25 & Clinton & 29.541 & 26.674 \\
\hline 26 & Crawford & .000 & .000 \\
\hline 27 & Daviess & 18.215 & 15.146 \\
\hline 28 & Dearborn & 26.038 & 14.751 \\
\hline 29 & Decatur & 332.889 & 190.603 \\
\hline 30 & DeKalb & 376.325 & 416.130 \\
\hline 31 & Delaware & 219.046 & 185.471 \\
\hline 32 & Dubois & 17.748 & 13.839 \\
\hline 33 & Elkhart & 729.530 & 517.629 \\
\hline 34 & Fayette & 43.786 & 29.845 \\
\hline 35 & Floyd & 73.093 & 45.597 \\
\hline 36 & Fountain & 7.006 & 68.239 \\
\hline 37 & Franklin & 1.168 & .707 \\
\hline 38 & Fulton & 99.131 & 88.718 \\
\hline 39 & Gibson & 15.413 & 10.497 \\
\hline 40 & Grant & 20.200 & 27.607 \\
\hline 41 & Greene & 10.859 & 22.858 \\
\hline 42 & Hamilton & 137.779 & 117.349 \\
\hline 43 & Hancock & 53.594 & 31.165 \\
\hline 44 & Harrison & 8.640 & 8.347 \\
\hline 45 & Hendricks & 23.469 & 30.390 \\
\hline 46 & Henry & 40.867 & 55.362 \\
\hline 47 & Howard & 24.403 & 78.537 \\
\hline 48 & Huntington & 116.879 & 82.215 \\
\hline 49 & Jackson & 80.683 & 87.970 \\
\hline 50 & Jasper & 78.815 & 45.582 \\
\hline 51 & Jay & 110.574 & 64.564 \\
\hline
\end{tabular}




\begin{tabular}{|c|c|c|c|}
\hline & \begin{tabular}{|l|} 
State \\
\end{tabular} & P41 97 & A41 97 \\
\hline 52 & Jefferson & 16.230 & 10.114 \\
\hline 53 & Jennings & 74.494 & 47.186 \\
\hline 54 & Johnson & 125.870 & 96.709 \\
\hline 55 & Knox & 13.661 & 40.344 \\
\hline 56 & Kosciusko & 167.671 & 248.387 \\
\hline 57 & LaGrange & 24.870 & 15.749 \\
\hline 58 & Lake & 295.292 & 1958.309 \\
\hline 59 & La Porte & 254.775 & 281.883 \\
\hline 60 & Lawrence & 67.138 & 187.523 \\
\hline 61 & Madison & 122.834 & 92.662 \\
\hline 62 & Marion & 1178.715 & 995.517 \\
\hline 63 & Marshall & 119.681 & 102.221 \\
\hline 64 & Martin & 1.168 & 15.879 \\
\hline 65 & Miami & 30.825 & 17.694 \\
\hline 66 & Monroe & 45.187 & 25.981 \\
\hline 67 & Montgomery & 74.961 & 108.120 \\
\hline 68 & Morgan & 37.831 & 26.530 \\
\hline 69 & Newton & 7.006 & 18.936 \\
\hline 70 & Noble & 163.350 & 159.570 \\
\hline 71 & Ohio & 1.168 & .659 \\
\hline 72 & Orange & 7.006 & 4.243 \\
\hline 73 & Owen & 1.518 & .905 \\
\hline 74 & Parke & 1.168 & 1.496 \\
\hline 75 & Perry & 7.006 & 36.002 \\
\hline 76 & Pike & 7.006 & 9.076 \\
\hline 77 & Porter & 180.865 & 799.092 \\
\hline 78 & Posey & 7.006 & 4.243 \\
\hline 79 & Pulaski & 20.433 & 26.801 \\
\hline 80 & Putnam & 1.168 & .707 \\
\hline 81 & Randolph & 12.961 & 33.712 \\
\hline 82 & Ripley & 24.520 & 13.894 \\
\hline 83 & Rush & 7.006 & 36.078 \\
\hline 84 & St. Joseph & 513.404 & 442.217 \\
\hline 85 & Scott & 10.275 & 8.449 \\
\hline 86 & Shelby & 42.852 & 70.685 \\
\hline 87 & Spencer & 7.006 & 19.175 \\
\hline 88 & Starke & .000 & .000 \\
\hline 89 & Steuben & 158.330 & 133.362 \\
\hline 90 & Sullivan & 1.168 & .659 \\
\hline 91 & Switzerland & .000 & .000 \\
\hline 92 & Tippecanoe & 71.108 & 192.558 \\
\hline 93 & Tipton & 14.479 & 14.133 \\
\hline 94 & Union & 1.168 & .659 \\
\hline 95 & Vanderburgh & 224.534 & 158.482 \\
\hline 96 & Vermillion & 20.433 & 11.577 \\
\hline 97 & Vigo & 78.231 & 77.360 \\
\hline 98 & Wabash & 118.514 & 131.206 \\
\hline 99 & Warren & 14.479 & 8.224 \\
\hline 100 & Warrick & 27.206 & 335.341 \\
\hline 101 & Washington & 71.108 & 41.295 \\
\hline 102 & Wayne & 74.261 & 123.552 \\
\hline
\end{tabular}




\begin{tabular}{|c|c|c|c|}
\hline & State & P41 97 & A41 97 \\
\hline 103 & Wells & 71.342 & 57.668 \\
\hline 104 & White & 109.757 & 67.299 \\
\hline 105 & Whitley & 81.850 & 98.062 \\
\hline 106 & lowa & 2080.860 & 3223.000 \\
\hline 107 & Kansas & 739.950 & 325.000 \\
\hline 108 & Kentucky_E & 1696.885 & 1604.000 \\
\hline 109 & Kentucky_W & 1131.924 & 1069.000 \\
\hline 110 & Louisiana & 1456.902 & 2569.000 \\
\hline 111 & Maine & 966.935 & 701.000 \\
\hline 112 & Maryland & 910.939 & 894.000 \\
\hline 113 & Massachusetts & 5877.603 & 1428.000 \\
\hline 114 & Michigan_E & 5231.647 & 3455.000 \\
\hline 115 & Michigan W & 5231.647 & 3455.000 \\
\hline 116 & Minnesota & 10119.317 & 7194.000 \\
\hline 117 & Missisippi & 533.964 & 318.000 \\
\hline 118 & Missouri & 3780.745 & 2121.000 \\
\hline 119 & Montana & 166.989 & 341.000 \\
\hline 120 & Nebraska & 898.939 & 1103.000 \\
\hline 121 & Nevada & 460.969 & 673.000 \\
\hline 122 & New Hampshire & 535.964 & 161.000 \\
\hline 123 & New Jersey & 1954.868 & 3872.000 \\
\hline 124 & New Mexico & 62.996 & 621.000 \\
\hline 125 & New York & 5504.629 & 6325.000 \\
\hline 126 & North Carolina & 2036.863 & 1930.000 \\
\hline 127 & North Dakota & 242.984 & 457.000 \\
\hline 128 & Ohio $\mathbf{N}$ & 4824.674 & 5009.000 \\
\hline 129 & Ohio M & 4824.674 & 5009.000 \\
\hline 130 & Ohio_s & 4824.674 & 5009.000 \\
\hline 131 & Oklahoma & 1451.902 & 2340.000 \\
\hline 132 & Oregon & 2346.842 & 4510.000 \\
\hline 133 & Pennsylvania & 12568.152 & 12018.000 \\
\hline 134 & Rhode Island & 1323.911 & 1295.000 \\
\hline 135 & South Carolina & 2058.861 & 2452.000 \\
\hline 136 & South Dakota & 136.991 & 35.000 \\
\hline 137 & Tennessee & 6480.563 & 4339.000 \\
\hline 138 & Texas & 7120.519 & 8099.000 \\
\hline 139 & Utah & 1318.911 & 2131.000 \\
\hline 140 & Vermont & 32.998 & 506.000 \\
\hline 141 & Virginia & 3280.779 & 2863.000 \\
\hline 142 & Washington & 2809.810 & 4834.000 \\
\hline 143 & West Virginia & 873.941 & 872.000 \\
\hline 144 & Wisconsin & 4274.712 & 5466.000 \\
\hline 145 & Wyoming & 110.993 & .000 \\
\hline
\end{tabular}




\begin{tabular}{|c|c|c|c|}
\hline & \begin{tabular}{|l} 
State \\
\end{tabular} & P43 97 & A43 97 \\
\hline 11 & Alabama & 2055.852 & 1476.000 \\
\hline 2 & Arizona & 1114.272 & 1662.000 \\
\hline 3 & Arkansas & 1596.369 & 925.000 \\
\hline 4 & California & 15217.415 & 13697.000 \\
\hline 5 & Colorado & 1191.366 & 1070.000 \\
\hline 6 & Connecticut & 2055.852 & 295.000 \\
\hline 7 & Delaware & 461.539 & 387.000 \\
\hline 8 & DC & .000 & .000 \\
\hline 9 & Florida & 7845.130 & 7139.000 \\
\hline 10 & Georgia & 4207.301 & 5289.000 \\
\hline 11 & Idaho & 754.498 & 331.000 \\
\hline 12 & Illinois $\mathbf{N}$ & 2454.687 & 2463.000 \\
\hline 13 & lilinois_s & 1051.568 & 1056.000 \\
\hline 14 & Adams & 2.749 & 6.754 \\
\hline 15 & Allen & 26.829 & 120.465 \\
\hline 16 & Bartholomew & 5.793 & 13.314 \\
\hline 17 & Benton & .788 & .029 \\
\hline 18 & Blackford & 1.162 & .177 \\
\hline 19 & Boone & 3.641 & .905 \\
\hline 20 & Brown & 1.226 & .029 \\
\hline 21 & Carroll & 1.654 & .177 \\
\hline 22 & Cass & 3.333 & 12.014 \\
\hline 23 & Clark & 7.800 & 18.072 \\
\hline 24 & Clay & 2.176 & 5.161 \\
\hline 25 & Clinton & 2.789 & 1.607 \\
\hline 26 & Crawford & .864 & .000 \\
\hline 27 & Daviess & 2.445 & .233 \\
\hline 28 & Dearborn & 3.714 & .000 \\
\hline 29 & Decatur & 2.026 & 4.194 \\
\hline 30 & DeKalb & 3.226 & 3.519 \\
\hline 31 & Delaware & 9.850 & 12.701 \\
\hline 32 & Dubois & 3.236 & 8.144 \\
\hline 33 & Elkhart & 14.662 & 235.251 \\
\hline 34 & Fayette & 2.134 & 11.060 \\
\hline 35 & Floyd & 5.821 & 1.575 \\
\hline 36 & Fountain & 1.485 & 2.212 \\
\hline 37 & Franklin & 1.786 & 1.106 \\
\hline 38 & Fulton & 1.672 & .177 \\
\hline 39 & Gibson & 2.646 & .516 \\
\hline 40 & Grant & 6.091 & 27.546 \\
\hline 41 & Greene & 2.739 & 3.160 \\
\hline 42 & Hamilton & 13.652 & 55.047 \\
\hline 43 & Hancock & 4.434 & 4.265 \\
\hline 44 & Harrison & 2.755 & 2.212 \\
\hline 45 & Hendricks & 7.998 & 3.655 \\
\hline 46 & Henry & 4.021 & 23.057 \\
\hline 47 & Howard & 6.954 & 51.615 \\
\hline 48 & Huntington & 3.120 & 9.283 \\
\hline 49 & Jackson & 3.368 & 10.215 \\
\hline 50 & Jasper & 2.402 & .516 \\
\hline 51 & Jay & 1.795 & 1.106 \\
\hline
\end{tabular}




\begin{tabular}{|c|c|c|c|}
\hline & State & P43 97 & A43 97 \\
\hline 52 & Jefferson & 2.583 & 7.855 \\
\hline 53 & Jennings & 2.221 & 114.058 \\
\hline 54 & Johnson & 9.043 & 6.722 \\
\hline 55 & Knox & 3.274 & .029 \\
\hline 56 & Kosciusko & 5.999 & 25.059 \\
\hline 57 & LaGrange & 2.811 & 6.696 \\
\hline 58 & Lake & 39.967 & 23.753 \\
\hline 59 & La Porte & 9.063 & 5.195 \\
\hline 60 & Lawrence & 3.777 & 8.144 \\
\hline 61 & Madison & 10.980 & 26.337 \\
\hline 62 & Marion & 70.401 & 167.390 \\
\hline 63 & Marshall & 3.668 & 23.594 \\
\hline 64 & Martin & .864 & .000 \\
\hline 65 & Miami & 2.895 & 3.160 \\
\hline 66 & Monroe & 9.834 & 18.412 \\
\hline 67 & Montgomery & 3.077 & 4.064 \\
\hline 68 & Morgan & 5.347 & 3.499 \\
\hline 69 & Newton & 1.203 & .516 \\
\hline 70 & Noble & 3.691 & 61.096 \\
\hline 71 & Ohio & .458 & .000 \\
\hline 72 & Orange & 1.579 & .000 \\
\hline 73 & Owen & 1.749 & 17.895 \\
\hline 74 & Parke & 1.404 & .000 \\
\hline 75 & Perry & 1.577 & 17.895 \\
\hline 76 & Pike & 1.057 & .000 \\
\hline 77 & Porter & 11.912 & 3.012 \\
\hline 78 & Posey & 2.226 & 17.895 \\
\hline 79 & Pulaski & 1.127 & .029 \\
\hline 80 & Putnam & 2.892 & 2.212 \\
\hline 81 & Randolph & 2.265 & 2.212 \\
\hline 82 & Ripley & 2.150 & .029 \\
\hline 83 & Rush & 1.514 & 3.160 \\
\hline 84 & St. Joseph & 21.748 & 64.397 \\
\hline 85 & Scott & 1.851 & 52.372 \\
\hline 86 & Shelby & 3.541 & 2.416 \\
\hline 87 & Spencer & 1.673 & .516 \\
\hline 88 & Starke & 1.965 & .867 \\
\hline 89 & Steuben & 2.671 & 25.720 \\
\hline 90 & Sullivan & 1.773 & .516 \\
\hline 91 & Switzerland & .726 & .000 \\
\hline 92 & Tippecanoe & 12.049 & 35.203 \\
\hline 93 & Tipton & 1.367 & 1.106 \\
\hline 94 & Union & .600 & .000 \\
\hline 95 & Vanderburgh & 14.145 & 112.085 \\
\hline 96 & Vermillion & 1.385 & .000 \\
\hline 97 & Vigo & 8.781 & 4.089 \\
\hline 98 & Wabash & 2.878 & 4.236 \\
\hline 99 & Warren & .685 & .177 \\
\hline 100 & Warrick & 4.212 & 3.499 \\
\hline 101 & Washington & 2.211 & .029 \\
\hline 102 & Wayne & 5.898 & 3.870 \\
\hline
\end{tabular}




\begin{tabular}{|c|c|c|c|}
\hline & State & P43 97 & A43 97 \\
\hline 103 & Wells & 2.266 & 4.103 \\
\hline 104 & White & 2.075 & 8.144 \\
\hline 105 & Whitley & 2.491 & 3.666 \\
\hline 106 & lowa & 1775.228 & 1728.000 \\
\hline 107 & Kansas & 2075.382 & 1299.000 \\
\hline 108 & Kentucky_E & 630.119 & 907.000 \\
\hline 109 & Kentucky_W & 420.422 & 604.000 \\
\hline 110 & Louisiana & 804.866 & 1328.000 \\
\hline 111 & Maine & 420.422 & 408.000 \\
\hline 112 & Maryland & 6705.160 & 5305.000 \\
\hline 113 & Massachusetts & 958.027 & 953.000 \\
\hline 114 & Michigan E & 2639.714 & 2638.000 \\
\hline 115 & Michigan_W & 2639.714 & 2638.000 \\
\hline 116 & Minnesota & 2924.449 & 1807.000 \\
\hline 117 & Missisippi & 2124.723 & 1719.000 \\
\hline 118 & Missouri & 1227.343 & 2357.000 \\
\hline 119 & Montana & 314.545 & 337.000 \\
\hline 120 & Nebraska & 1268.461 & 1171.000 \\
\hline 121 & Nevada & 197.362 & 2668.000 \\
\hline 122 & New Hampshire & 99.709 & 303.000 \\
\hline 123 & New Jersey & 2722.976 & 2770.000 \\
\hline 124 & New Mexico & 1089.601 & 816.000 \\
\hline 125 & New York & 4793.218 & 4161.000 \\
\hline 126 & North Carolina & 2857.634 & 2401.000 \\
\hline 127 & North Dakota & 408.087 & 296.000 \\
\hline 128 & Ohio_N & 966.250 & 1077.000 \\
\hline 129 & Ohio M & 966.250 & 1077.000 \\
\hline 130 & Ohio_s & 966.250 & 1077.000 \\
\hline 131 & Oklahoma & 1175.947 & 1108.000 \\
\hline 132 & Oregon & 2028.098 & 5067.000 \\
\hline 133 & Pennsylvania & 4836.391 & 4177.000 \\
\hline 134 & Rhode Island & 629.091 & 92.000 \\
\hline 135 & South Carolina & 1197.534 & 1359.000 \\
\hline 136 & South Dakota & 101.765 & 364.000 \\
\hline 137 & Tennessee & 1689.910 & 1666.000 \\
\hline 138 & Texas & 8044.548 & 6881.000 \\
\hline 139 & Utah & 369.025 & 348.000 \\
\hline 140 & Vermont & 366.970 & 86.000 \\
\hline 141 & Virginia & 2368.341 & 3348.000 \\
\hline 142 & Washington & 1601.509 & 2607.000 \\
\hline 143 & West Virginia & 436.868 & 1111.000 \\
\hline 144 & Wisconsin & 2453.659 & 2524.000 \\
\hline 145 & Wyoming & 300.154 & 175.000 \\
\hline
\end{tabular}




\section{APPENDIX B}

\section{COMPUTER PROGRAMS DEVELOPED}




\section{Appendix Notes}

The project made extensive use of computer programs due to the extremely large amount of data that had to be manipulated. For each of $\mathbf{4 1}$ different commodity groups and 22,500 flows (150x150) between places there was really no other way to handle such large amounts of data. In this appendix we have reproduced some of the most important programs that were used. In some cases, such as in handling parcel flows for different companies there were programs written for each of the companies involved. In this latter case we have reproduced only one of the programs here since the others follow the same basic logic.

In many cases programs were not written for some of the operations. In these cases the project made use of different spreadsheet programs or statistical programs. The most common of the former was Microsoft Excel and the most common of the latter was SPSS (Statistical Package for the Social Sciences, version 13.0).

The programs that appear in the following pages are followed by their control information. In some cases the programs are documented in other cases they are not. In order for the reader to understand what the programs were used for, the section below documents this information. For those unfamiliar with computer code, all of the programs are written in FORTRAN 77. The programs will not run in their current form, but must first be run through a FORTRAN compiler.

\section{GRAVITY5.FOR}

This is the program that takes productions, attractions, distances, and average shipping distance and uses a fully-constrained gravity model to distribute the flows. None of these data bases are reproduced here except for the productions and attractions which appear in other appendices. All are being supplied to the Indiana DOT.

\section{MODAL05.FOR}

This program takes the output of the GRAVITY5 program and distributes this among modes. The latter data appear in the VEH files. The latter files give information on the flows between 145 places noted in the text of this report for all of the modes of interest here: truck, rail, water, air, pipeline and parcel.

\section{US.FOR}

This is one of five programs (USPS, UPS, DHL, FedEx and Air Freight) used to 
convert the flows into Indiana and out from Indiana into highway flows to appropriate airports. It did this

by taking the parcel flows and assigning these to appropriate carriers based on market share. Air freight flows were treated simply as highway flows to or from airports in Indiana. Parcel flows within Indiana were treated as highway flows.

\section{BIGMAT.FOR}

This program took the highway flows of the parcel and airfreight programs and converted these into a $150 \times 150$ flow matrix. It did this by creating a null (empty) matrix and filling the cells of the $145 \times 145$ matrix with previous flows and incorporating the five additional origins/destinations to represent the airports of interest.

\section{TOTALLY.FOR}

This program took all the highway flows in 1000s of tons and converted these to tons, converted the tons using truck density figures, to truckloads, and totaled these to get the total truck flows between all origins and destinations in the study. The output generated for each commodity as well as for total flows appears in truckloads, tons, and dollars of commodity value.

\section{TRUCKTOT.FOR}

This program summed all truckloads resulting from the TOTALLY.FOR program and added these together for annual truckloads and then converted these to daily truckloads.

\section{TOTALRR.FOR}

This converted thousands of tons of rail traffic to tons, dollars, and carloads for all commodities.

\section{RAILTOT.FOR}

Similar to TRUCKTOT.FOR it converted the total traffic into annual carloads and then converted this to daily carloads assuming a 306 day rail schedule. Multiplying numerical data on maps by $\mathbf{3 0 6}$ will yield annual carloads. 
PROGRAM GRAVITY5

CHARACTER* 15 INFILE

CHARACTER* 15 OUTFILE

COMMON DIST $(156,156), \mathrm{O}(156), \mathrm{A}(156), \mathrm{B}(156), \mathrm{D}(156)$,

$1 \operatorname{OID}(156), \operatorname{DID}(156)$

OPEN ( 6 , FILE= ' CONTROL . CRD', STATUS= ' OLD' $)$

NSETS $=31$

DO $1000 \mathrm{KKK}=1$, NSETS

READ $(6,500)$ INFILE, OUTFILE, COBS, ISCTG

500 FORMAT (2A15, F5 . 0, 5X, I5)

OPEN ( 7, FILE=OUTFILE, STATUS= ' NEW' $)$

OPEN ( 8, FILE=' gmdist.prj', STATUS= ' OLD')

OPEN $(9$, FILE=INFILE, STATUS= ' OLD' $)$

$\mathrm{N}=145$

READ $(9,11) \quad(O(I), D(I), I=1, N)$

11 FORMAT (14X, 2F11.0)

DO $100 I=1, N$

DO $100 \mathrm{~J}=1, \mathrm{~N}$

$100 \operatorname{READ}(8, *) \operatorname{OID}(I), \operatorname{DID}(J), \operatorname{DIST}(I, J)$

BETA $=-1.0 /$ COBS

FLOWS $=0.0$

DO $101 I=1, \mathrm{~N}$

101 FLOWS $=$ FLOWS $+O$ (I)

DO $102 I=1, N$

$O(I)=O(I) /$ FLOWS

$102 D(I)=D(I) /$ FLOWS

CALL ENT5 (N, BETA, COBS, FLOWS)

CALL OUT (N, BETA, COBS, FLOWS)

CLOSE (7)

CLOSE (8)

CLOSE (9)

1000 CONTINUE

STOP

END

SUBROUTINE OUT (N, BETA, COBS, FLOWS)

COMMON DIST $(156,156), \mathrm{O}(156), \mathrm{A}(156), \mathrm{B}(156), \mathrm{D}(156)$, 1OID (156) , DID (156)

DIMENSION X(156)

DIMENSION ITEMP (156)

WRITE $(7,101)$ BETA

101 FORMAT $('$ ' ', BETA $=1, F 8.4 /$ ' CELI ORIGIN FLOWS

VALUE VALUE

DESTINATION FLOWS') 


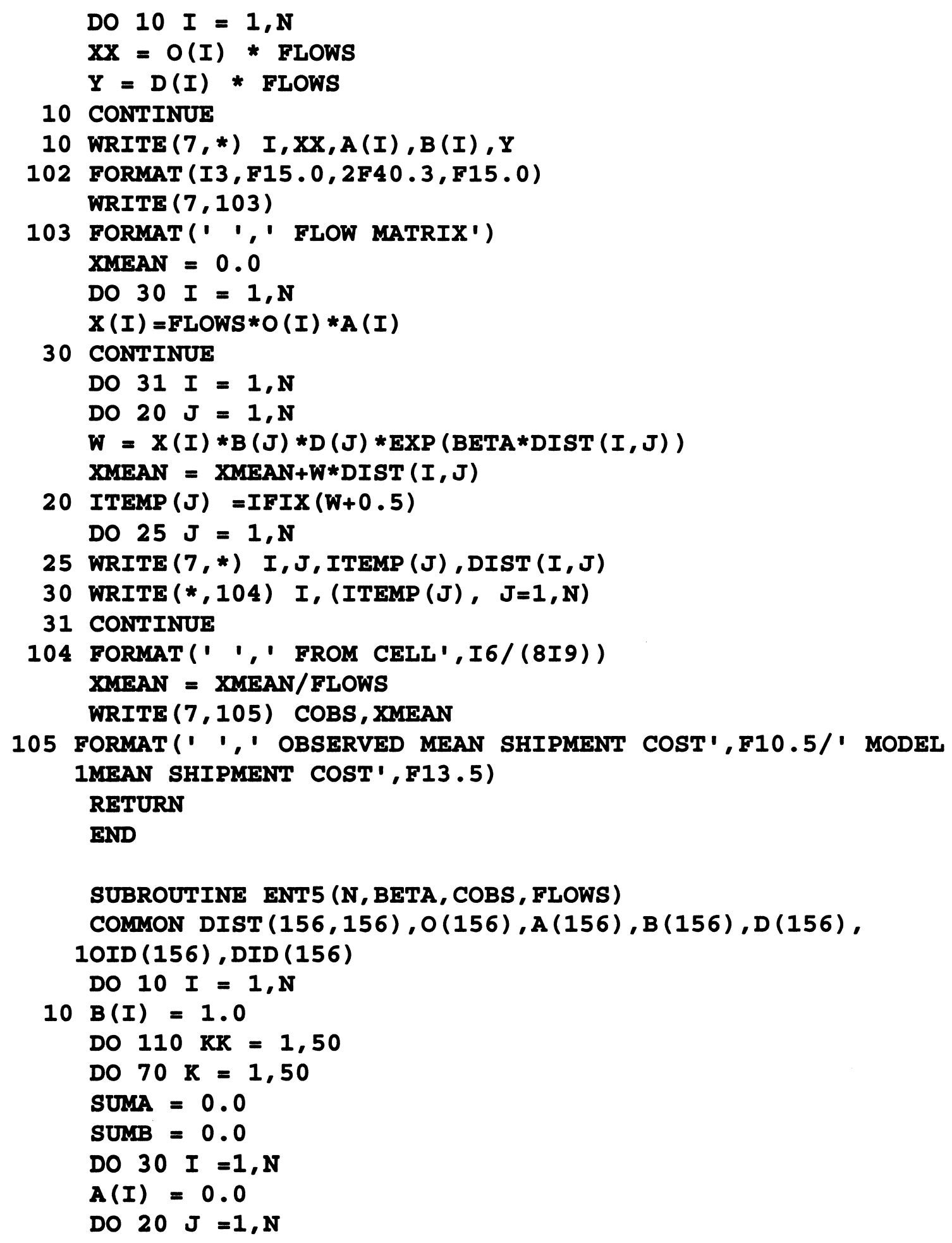




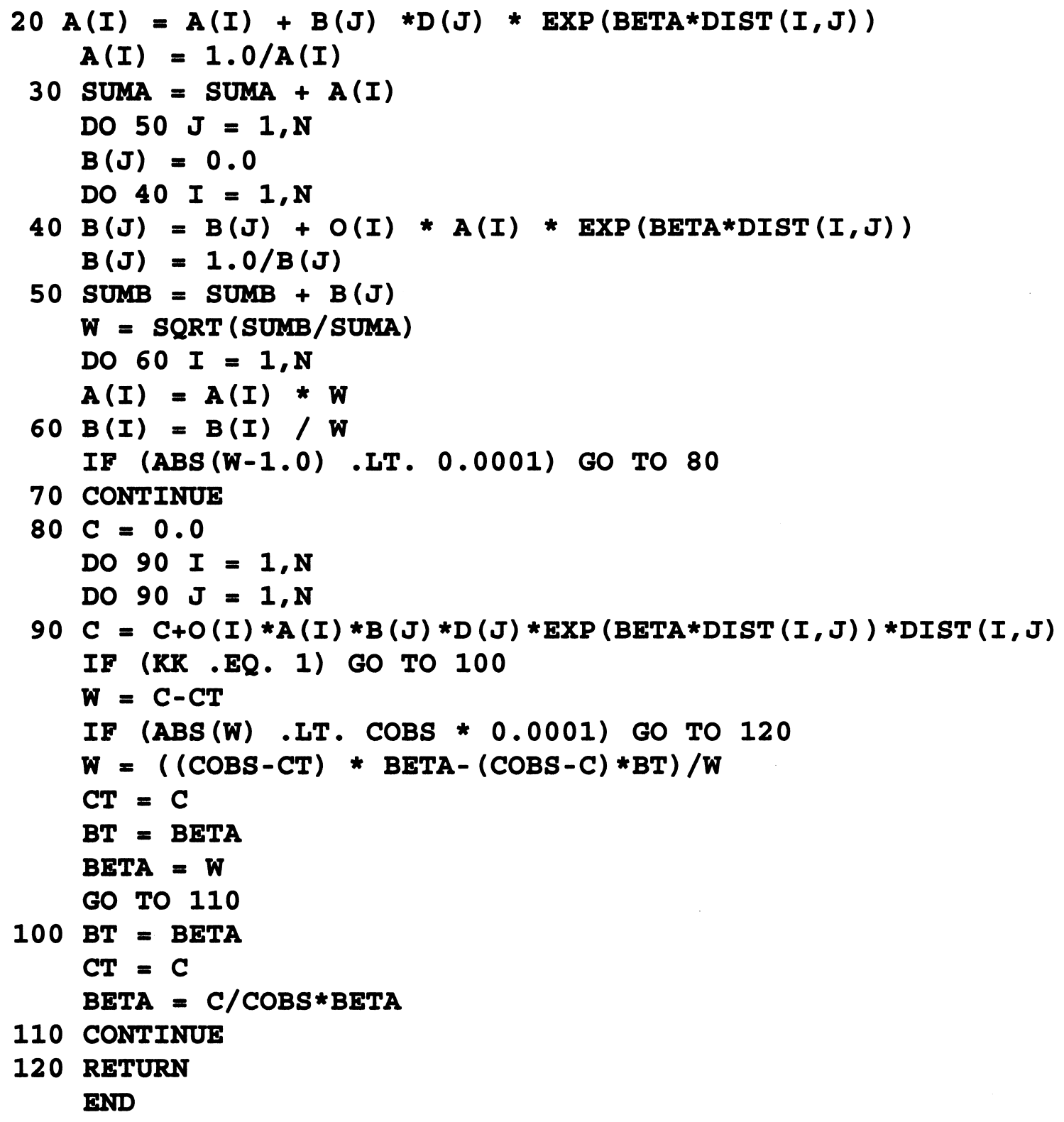




$\begin{array}{lllll}\text { COM_1 } & \text { FUNNY_1 } & 253 & 0.00 & 1 \\ \text { COM_2 } & \text { FUNNY_2 } & 410 & 0.00 & 2 \\ \text { COM_3 } & \text { FUNNY_3 } & 400 & 0.00 & 3 \\ \text { COM_4 } & \text { FUNNY_4 } & 213 & 0.00 & 4 \\ \text { COM_5 } & \text { FUNNY_5 } & 458 & 0.00 & 5 \\ \text { COM_6 } & \text { FUNNY_6 } & 472 & 0.00 & 6 \\ \text { COM_7 } & \text { FUNNY_7 } & 313 & 0.00 & 7 \\ \text { COM_8 } & \text { FUNNY_8 } & 343 & 0.00 & 8 \\ \text { COM_9 } & \text { FUNNY_9 } & 245 & 0.00 & 9 \\ \text { COM_10 } & \text { FUNNY_10 } & 200 & 0.00 & 10 \\ \text { COM_11 } & \text { FUNNY_11 } & 100 & 0.00 & 11 \\ \text { COM_12 } & \text { FUNNY_12 } & 100 & 0.00 & 12 \\ \text { COM_13 } & \text { FUNNY_13 } & 222 & 0.00 & 13 \\ \text { COM_14 } & \text { FUNNY_14 } & 526 & 0.00 & 14 \\ \text { COM_15 } & \text { FUNNY_15 } & 446 & 0.00 & 15 \\ \text { COM_17 } & \text { FUNNY_17 } & 142 & 0.00 & 17 \\ \text { COM_18 } & \text { FUNNY_18 } & 106 & 0.00 & 18 \\ \text { COM_19 } & \text { FUNNY_19 } & 172 & 0.00 & 19 \\ \text { COM_20 } & \text { FUNNY_20 } & 462 & 0.00 & 20 \\ \text { COM_21 } & \text { FUNNY_21 } & 564 & 0.00 & 21 \\ \text { COM_22 } & \text { FUNNY_22 } & 243 & 0.00 & 22 \\ \text { COM_23 } & \text { FUNNY_23 } & 489 & 0.00 & 23 \\ \text { COM_24 } & \text { FUNNY_24 } & 530 & 0.00 & 24 \\ \text { COM_25 } & \text { FUNNY_25 } & 150 & 0.00 & 25 \\ \text { COM_26 } & \text { FUNNY_26 } & 294 & 0.00 & 26 \\ \text { COM_27 } & \text { FUNNY_27 } & 549 & 0.00 & 27 \\ \text { COM_28 } & \text { FUNNY_28 } & 299 & 0.00 & 28 \\ \text { COM_29 } & \text { FUNNY_29 } & 292 & 0.00 & 29 \\ \text { COM_30 } & \text { FUNNY_30 } & 538 & 0.00 & 30 \\ \text { COM_31 } & \text { FUNNY_31 } & 100 & 0.00 & 31 \\ \text { COM_32 } & \text { FUNNY_32 } & 350 & 0.00 & 32 \\ \text { COM_33 } & \text { FUNNY_33 } & 457 & 0.00 & 33 \\ \text { COM_34 } & \text { FUNNY_34 } & 542 & 0.00 & 34 \\ \text { COM_35 } & \text { FUNNY_35 } & 683 & 0.00 & 35 \\ \text { COM_36 } & \text { FUNNY_36 } & 464 & 0.00 & 36 \\ \text { COM_37 } & \text { FUNNY_37 } & 686 & 0.00 & 37 \\ \text { COM_38 } & \text { FUNNY_38 } & 738 & 0.00 & 38 \\ \text { COM_39 } & \text { FUNNY_39 } & 581 & 0.00 & 39 \\ \text { COM_43 } & \text { FUNNY_40 } & 354 & 0.00 & 40 \\ & \text { FUNNY_41 } & 225 & 0.00 & 41 \\ \text { FUNNY_43 } & 156 & 0.00 & 43\end{array}$




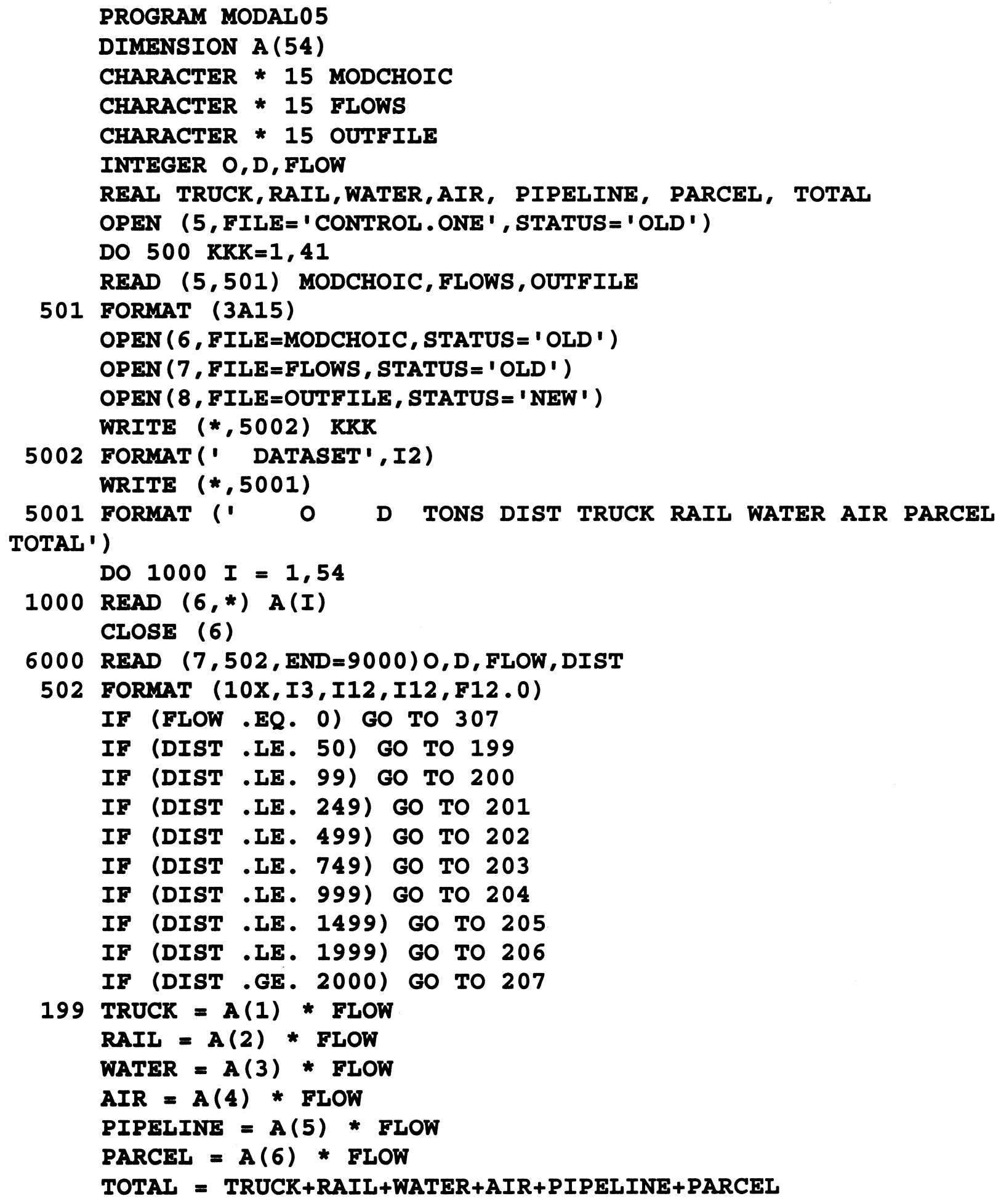




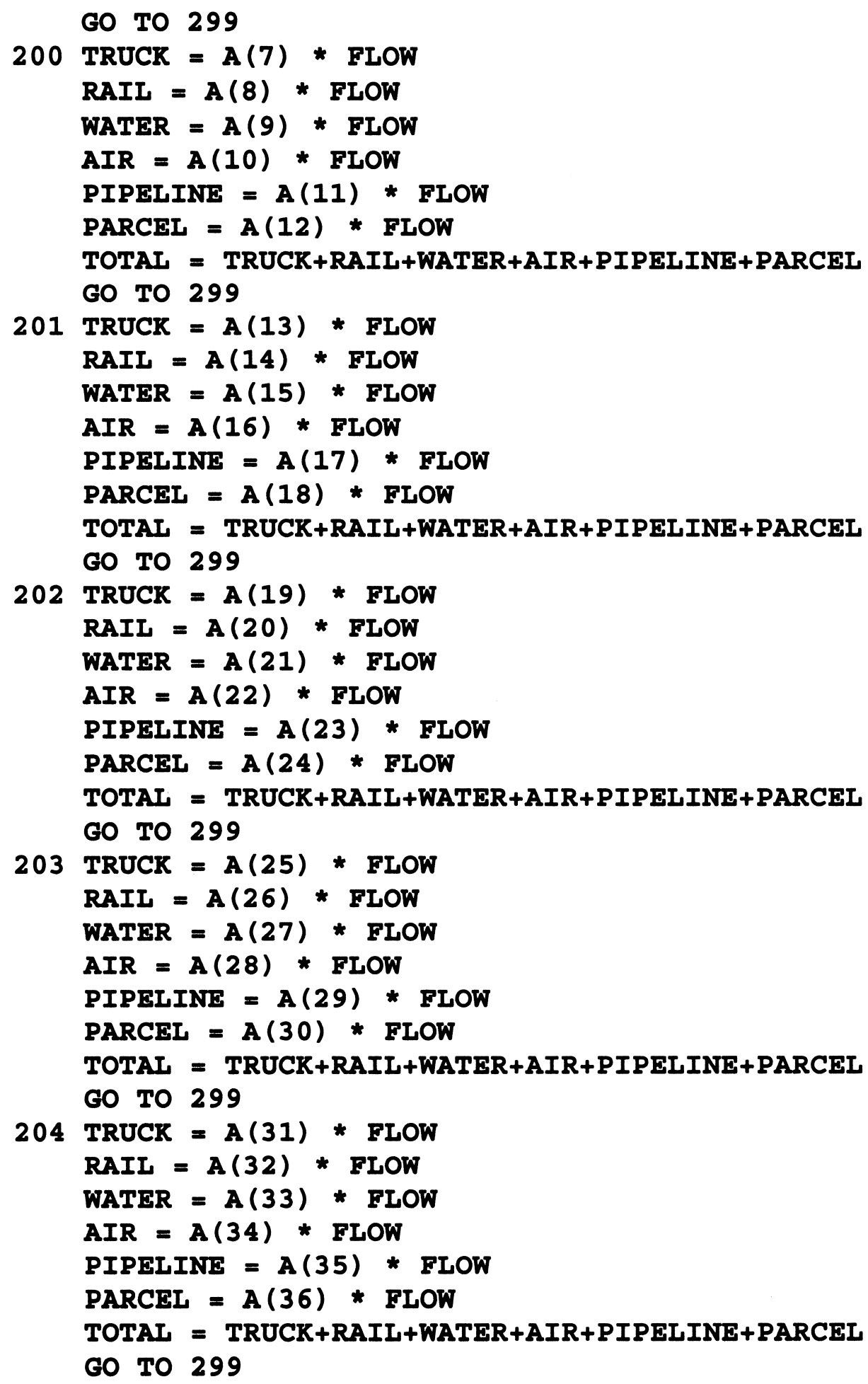




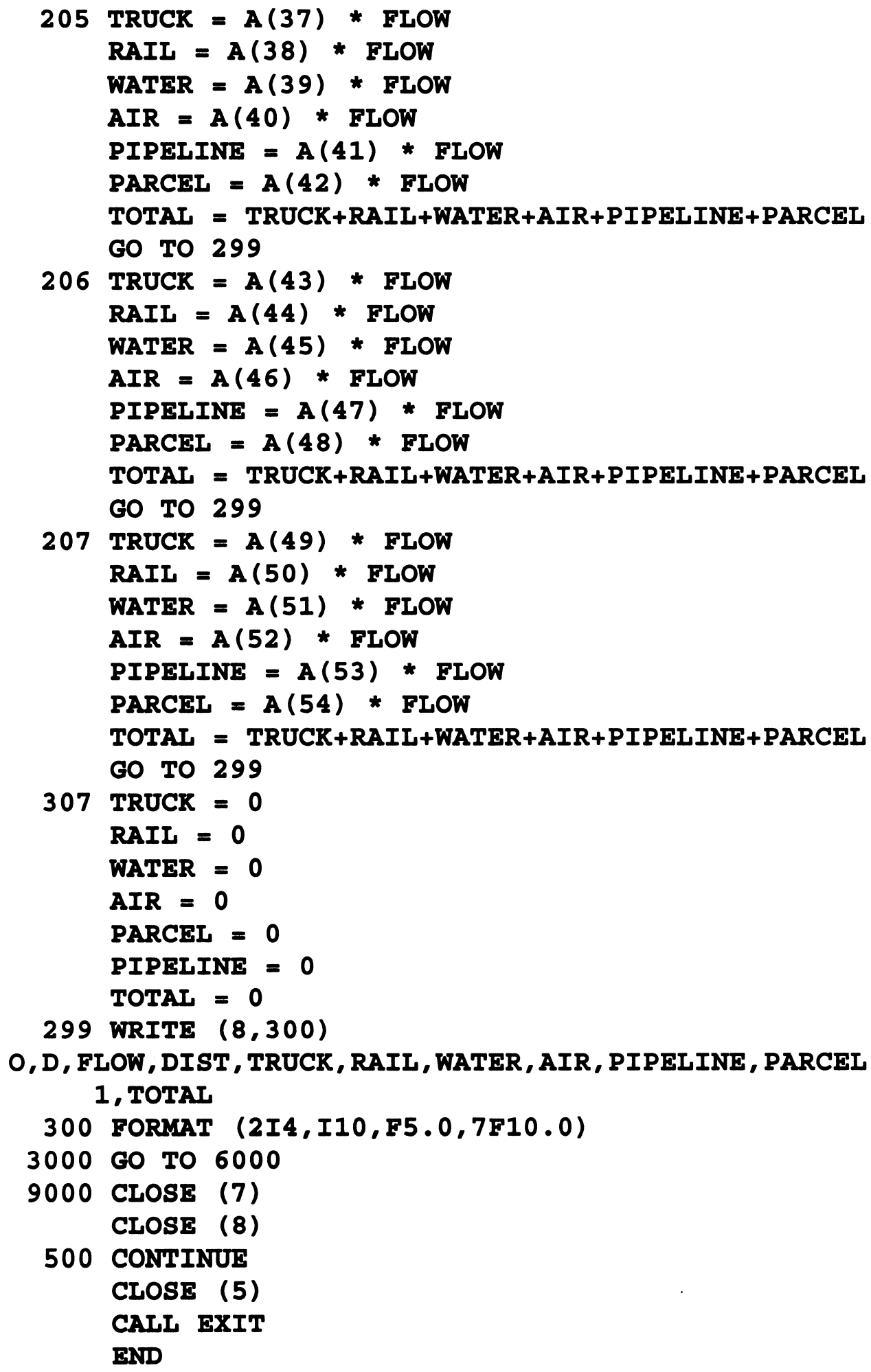




\begin{tabular}{|c|c|c|}
\hline MODE_1 & FUNNY_1 & VEH01 \\
\hline MODE_2 & FUNNY_2 & VEHO2 \\
\hline MODE_3 & FUNNY_3 & VEHO3 \\
\hline MODE_4 & FUNNY_4 & VEH04 \\
\hline MODE_5 & FUNNY_5 & VEH05 \\
\hline MODE_6 & FUNNY_6 & VEHO 6 \\
\hline MODE_7 & FUNNY_7 & VEH07 \\
\hline MODE_8 8 & FUNNY_8 & VEH0 8 \\
\hline MODE_9 & FUNNY_9 & VEHO9 \\
\hline MODE_10 & FUNNY_10 & VEH10 \\
\hline MODE_11 & FUNNY_11 & VEH11 \\
\hline MODE_12 & FUNNY_12 & VEH12 \\
\hline MODE_13 & FUNNY_13 & VEH13 \\
\hline MODE_14 & FUNNY_14 & VEH14 \\
\hline MODE_15 & FUNNY_15 & VEH15 \\
\hline MODE_17 & FUNNY_17 & VEH17 \\
\hline MODE_18 & FUNNY_18 & VEH18 \\
\hline MODE_19 & FUNNY_19 & VEH19 \\
\hline MODE_20 & FUNNY_20 & VFH2 0 \\
\hline MODE_21 & FUNNY_21 & VEH21 \\
\hline MODE_22 & FUNNY_22 & VEH2 2 \\
\hline MODE_23 & FUNNY_23 & VEH2 3 \\
\hline MODE_24 & FUNNY_24 & VEH24 \\
\hline MODE_25 & FUNNY_25 & VEH25 \\
\hline MODE_26 & FUNNY_26 & VEH2 6 \\
\hline MODE_27 & FUNNY_27 & VEH27 \\
\hline MODE_28 & FUNNY_28 & VEH2 8 \\
\hline MODE_29 & FUNNY_29 & VEH2 9 \\
\hline MODE_30 & FUNNY_30 & VEH3O \\
\hline MODE_31 & FUNNY_31 & VEH31 \\
\hline MODE_32 & FUNNIY_32 & VEH32 \\
\hline MODE_33 & FUNNY_33 & VEH3 3 \\
\hline MODE_34 & FUNNY_34 & VEH34 \\
\hline MODE_35 & FUNANY_35 & VEH35 \\
\hline MODE_36 & FUNNY_36 & VEH3 6 \\
\hline MODE_37 & FUNNY_37 & VEH37 \\
\hline MODE_38 & FUNNY_38 & VEH3 8 \\
\hline MODE_39 & FUNNY_39 & VEH39 \\
\hline MODE_40 & FUNNY_40 & VEH4 0 \\
\hline MODE_41 & FUNNTY_41 & VEH41 \\
\hline MODE_43 & FUNNY_43 & VEH43 \\
\hline
\end{tabular}




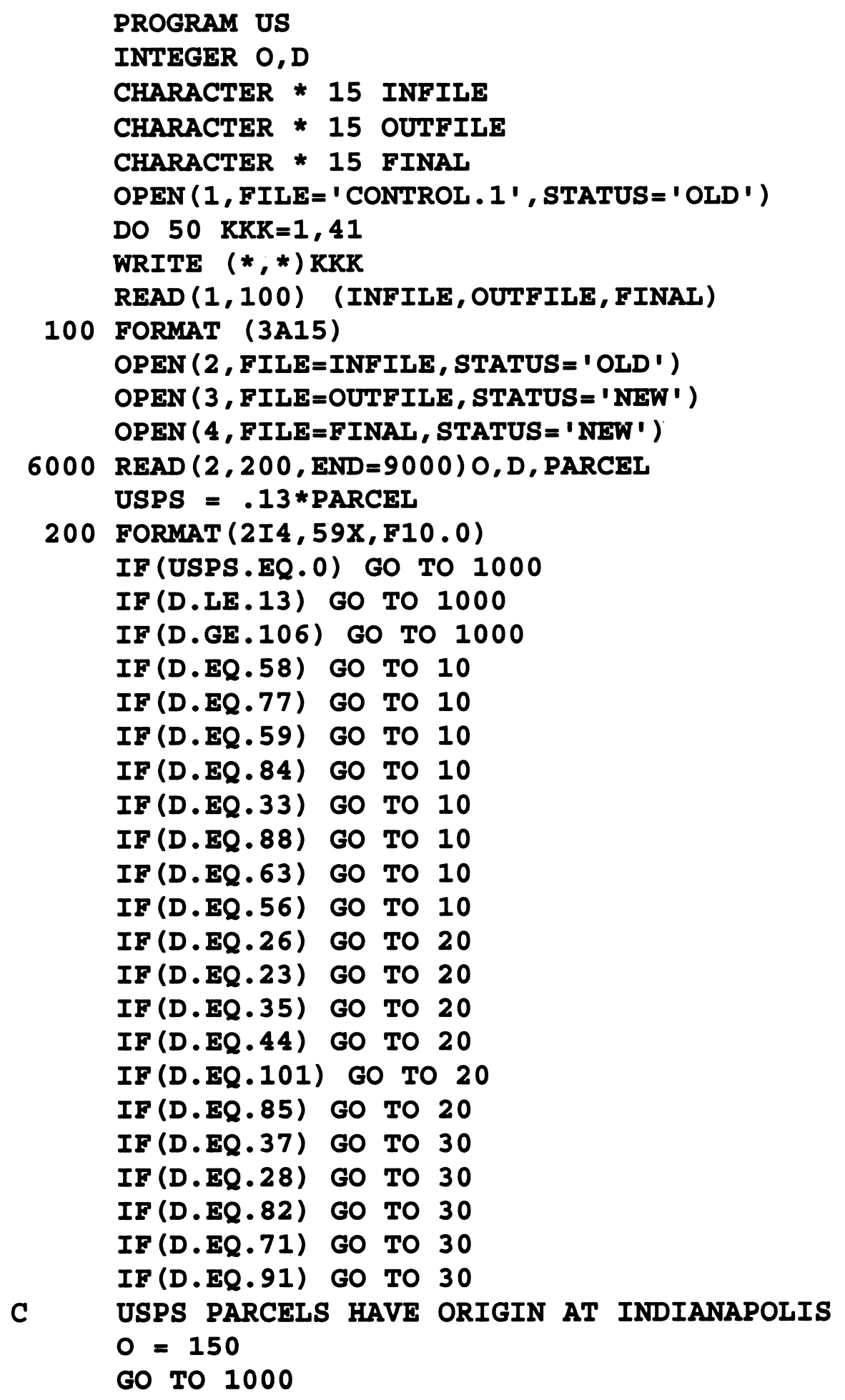




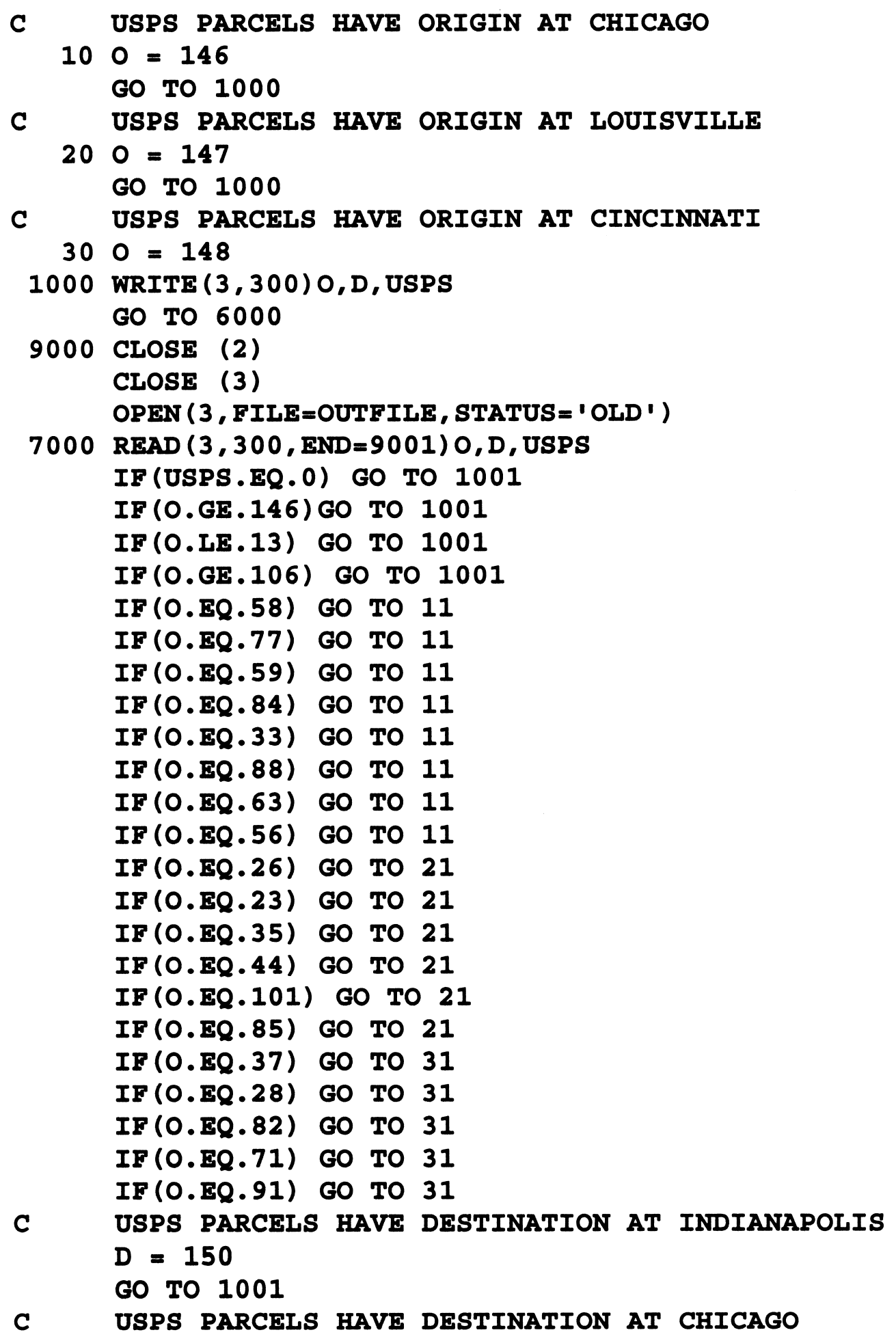


$11 D=146$

GO TO 1001

C USPS PARCELS HAVE DESTINATION AT LOUISVILLE

$21 D=147$

GO TO 1001

C USPS PARCELS HAVE DESTINATION AT CINCINNATI

$31 \mathrm{D}=148$

1001 IF(USPS.LE.(.1)) GO TO 1002

USPS $=$ USPS +1.0

1002 WRITE $(4,300) 0, D, U S P S$

300 FORMAT (2I4, F10.0)

GO TO 7000

9001 CLOSE (3)

CLOSE (4)

50 CONTINUE

CALL EXIT

END 


\begin{tabular}{|c|c|c|}
\hline VEH01 & US01 & USPS01 \\
\hline VEHO2 & US02 & USPSO2 \\
\hline VEH03 & US03 & USPSO3 \\
\hline VEH04 & US04 & USPSO4 \\
\hline VEHO5 & US05 & USPS05 \\
\hline VEHO 6 & US06 & USPS06 \\
\hline VEHO7 & US07 & USPS07 \\
\hline VEH08 & US0 8 & USPS0 8 \\
\hline VEHO9 & USO9 & USPSO9 \\
\hline VEH10 & US10 & USPS10 \\
\hline VEH11 & US11 & USPS11 \\
\hline VEH12 & US12 & USPS12 \\
\hline VEH13 & US13 & USPS13 \\
\hline VEH14 & US14 & USPS14 \\
\hline VEH15 & US15 & USPS15 \\
\hline VEH17 & US17 & USPS17 \\
\hline VEH18 & US18 & USPS18 \\
\hline VEH19 & US19 & USPS19 \\
\hline VEH2 0 & US2 0 & USPS2 0 \\
\hline VEH21 & US21 & USPS21 \\
\hline VEH2 2 & US2 2 & USPS2 2 \\
\hline VEH2 3 & US2 3 & USPS2 3 \\
\hline VEH24 & US24 & USPS24 \\
\hline VEH2 5 & US2 5 & USPS2 5 \\
\hline VEH26 & US2 6 & USPS2 6 \\
\hline VEH27 & US27 & USPS27 \\
\hline VEH2 8 & US2 8 & USPS2 8 \\
\hline VEH29 & US2 9 & USPS29 \\
\hline VEH30 & US30 & USPS30 \\
\hline VEH31 & US31 & USPS31 \\
\hline VEH32 & US32 & USPS32 \\
\hline VEH33 & US33 & USPS33 \\
\hline VEH34 & US34 & USPS34 \\
\hline VEH35 & US35 & USPS 35 \\
\hline VEH36 & US36 & USPS3 6 \\
\hline VEH37 & US37 & USPS37 \\
\hline VEH3 8 & US3 8 & USPS3 8 \\
\hline VEH39 & US39 & USPS39 \\
\hline VEH4 0 & US4 0 & USPS4 0 \\
\hline VEH4 1 & US41 & USPS4 1 \\
\hline VEH4 3 & US4 3 & USPS4 3 \\
\hline
\end{tabular}




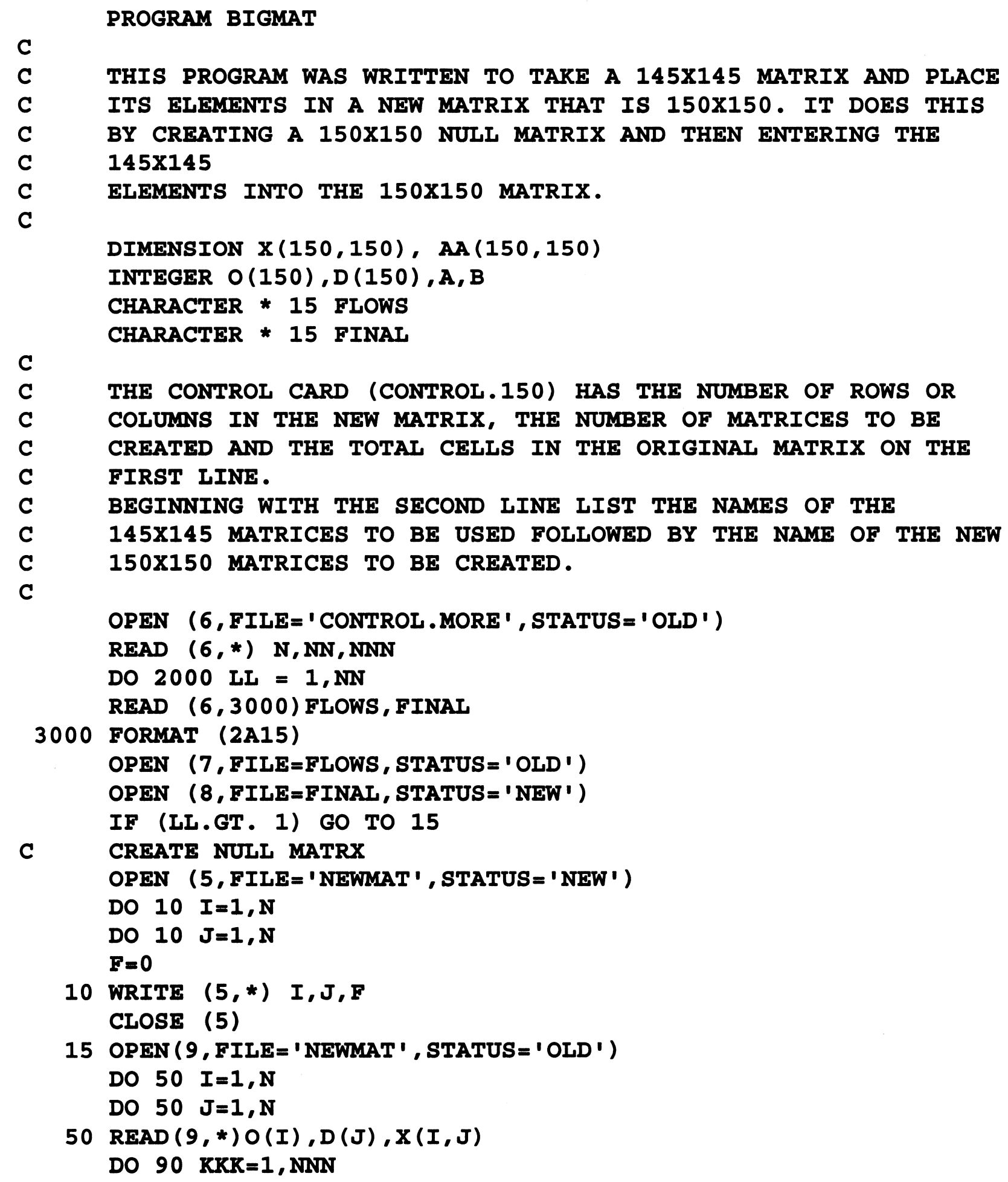




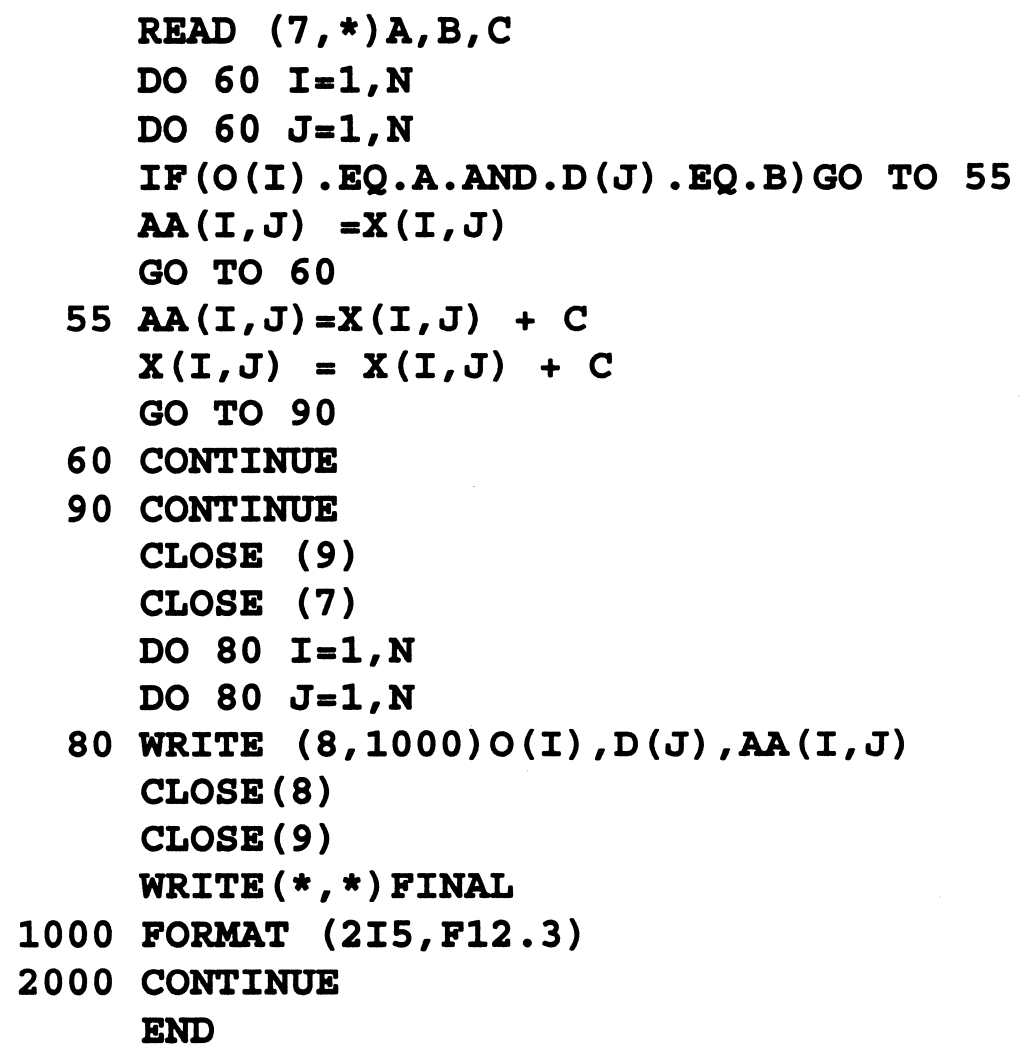




\begin{tabular}{|c|c|}
\hline 150 & 21025 \\
\hline TRUCK1501 & TRUCK1501.1 \\
\hline TRUCK1502 & TRUCK1502.1 \\
\hline TRUCK1503 & TRUCK1503.1 \\
\hline TRUCK1504 & TRUCK1504.1 \\
\hline TRUCK1505 & TRUCK1505.1 \\
\hline TRUCK1506 & TRUCK1506 . 1 \\
\hline TRUCK1507 & TRUCK1507 . 1 \\
\hline TRUCK1508 & TRUCK1508.1 \\
\hline TRUCK1509 & TRUCK1509.1 \\
\hline TRUCK1510 & TRUCK1510.1 \\
\hline TRUCK1511 & TRUCK1511.1 \\
\hline TRUCK1512 & TRUCK1512 .1 \\
\hline TRUCK1513 & TRUCK1513.1 \\
\hline TRUCK1514 & TRUCK1514.1 \\
\hline TRUCK1515 & TRUCK1515.1 \\
\hline TRUCK1517 & TRUCK1517. 1 \\
\hline TRUCK1518 & TRUCK1518.1 \\
\hline TRUCK1519 & TRUCK1519.1 \\
\hline TRUCK1520 & TRUCK1520.1 \\
\hline TRUCK1521 & TRUCK1521.1 \\
\hline TRUCK1522 & TRUCK1522.1 \\
\hline TRUCK1523 & TRUCK1523.1 \\
\hline TRUCK1524 & TRUCK1524.1 \\
\hline TRUCK1525 & TRUCK1525.1 \\
\hline TRUCK1526 & TRUCK1526.1 \\
\hline TRUCK1527 & TRUCK1527 . I \\
\hline TRUCK1528 & TRUCK1528.1 \\
\hline TRUCK1529 & TRUCK1529.1 \\
\hline TRUCK1530 & TRUCK1530.1 \\
\hline TRUCK1531 & TRUCK1531.1 \\
\hline TRUCK1532 & TRUCK1532. 1 \\
\hline TRUCK1533 & TRUCK1533.1 \\
\hline TRUCK1534 & TRUCK1534. 1 \\
\hline TRUCK1535 & TRUCK1535. I \\
\hline TRUCK1536 & TRUCK1536.1 \\
\hline TRUCK1537 & TRUCK1537 15 \\
\hline TRUCK1538 & TRUCK1538. \\
\hline TRUCK1539 & TRUCK1539. \\
\hline TRUCK1540 & TRUCK1540 I \\
\hline TRUCK1541 & TRUCK1541.1 \\
\hline
\end{tabular}




\begin{tabular}{|c|c|}
\hline TRUCK1543 & TRUCK1543. 1 \\
\hline TRUCK3001 & TRUCK3001.1 \\
\hline TRUCK3002 & TRUCK 3002 . 1 \\
\hline TRUCK3003 & TRUCK3003.1 \\
\hline TRUCK3004 & TRUCK3004.1 \\
\hline TRUCK3005 & TRUCK3005.1 \\
\hline TRUCK3006 & TRUCK3006 .1 \\
\hline TRUCK3007 & TRUCK 3007.1 \\
\hline TRUCK3008 & TRUCK3008.1 \\
\hline TRUCK3009 & TRUCK3009.1 \\
\hline TRUCK3010 & TRUCK3010 . 1 \\
\hline TRUCK3011 & TRUCK3011 . 1 \\
\hline TRUCK3012 & TRUCK3012 . 1 \\
\hline TRUCK3013 & TRUCK3013.1 \\
\hline TRUCK3014 & TRUCK3014.1 \\
\hline TRUCK3015 & TRUCK3015.1 \\
\hline TRUCK3017 & TRUCK3017.1 \\
\hline TRUCK3018 & TRUCK3018 . 1 \\
\hline TRUCK3019 & TRUCK3019.1 \\
\hline TRUCK3020 & TRUCK3020.1 \\
\hline TRUCK3021 & TRUCK3021.1 \\
\hline TRUCK3022 & TRUCK3022.1 \\
\hline TRUCK3023 & TRUCK3023.1 \\
\hline TRUCK3024 & TRUCK3024.1 \\
\hline TRUCK3025 & TRUCK3025.1 \\
\hline TRUCK3026 & TRUCK3026.1 \\
\hline TRUCK3027 & TRUCK3027 . I \\
\hline TRUCK3028 & TRUCK3028.1 \\
\hline TRUCK3029 & TRUCK3029.1 \\
\hline TRUCK3030 & TRUCK3030.1 \\
\hline TRUCK3031 & TRUCK3031.1 \\
\hline TRUCK3032 & TRUCK3032 . 1 \\
\hline TRUCK3033 & TRUCK3033.1 \\
\hline TRUCK3034 & TRUCK 3034.1 \\
\hline TRUCK3035 & TRUCK3035.1 \\
\hline TRUCK3036 & TRUCK3036.1 \\
\hline TRUCK3037 & TRUCK3037.1 \\
\hline TRUCK3038 & TRUCK3038.1 \\
\hline TRUCK3039 & TRUCK3039.1 \\
\hline TRUCK3040 & TRUCK3040.1 \\
\hline TRUCK3041 & TRUCK3 041.1 \\
\hline TRUCK3043 & TRUCK3043.1 \\
\hline
\end{tabular}




\section{PROGRAM TOTALLY}

DIMENSION $F(150,150), V(150,150), T(150,150), T O T(150,150)$

DIMENSION DOL $(150,150), \operatorname{TON}(150,150)$

INTEGER NUM(150)

CHARACTER * 15 TRUCK

CHARACTER * 15 DHL

CHARACTER * 15 FEDEX

CHARACTER * 15 UPS

CHARACTER * 15 USPS

CHARACTER * 15 AIR

CHARACTER * 15 TOTAL

OPEN ( 3 , FILE= ' ROADNODE . TXT' , STATUS = ' OLD' )

OPEN (4, FILE= ' CONTROLLER', STATUS = ' OLD' )

OPEN (5, FILE= 'DEN_VALUE.T', STATUS= ' OLD' $)$

$\mathrm{N}=150$

DO $80 I=1, N$

80 READ $(3,1000)$ NUM(I)

DO $90 \mathrm{LL}=1,123$

DO $95 \quad I=1, N$

DO $95 \mathrm{~J}=1, \mathrm{~N}$

$V(I, J)=0.0$

$T(I, J)=0.0$

$95 F(I, J)=0.0$

READ $(4,2000)$ TOTAL, AIR, UPS, USPS , FEDEX, DHL , TRUCK

READ $(5,3000)$ DENSITY, DOLLARS

OPEN ( 7 , FILE=TOTAL, STATUS = ' NEW' $)$

OPEN $(6$, FILE=AIR, STATUS= ' OLD' $)$

DO $5 \quad I=1, N$

DO $5 \mathrm{~J}=1, N$

$\operatorname{TOT}(I, J)=0.0$

$\operatorname{DOL}(I, J)=0.0$

$5 \operatorname{TON}(I, J)=0.0$

DO $10 I=1, N$

DO $10 \mathrm{~J}=1, \mathrm{~N}$

READ $(6,4000) F(I, J)$

$F(I, J)=(F(I, J) * 1000)$

$V(I, J)=(F(I, J) *$ DOLILARS $)$

$T(I, J)=(F(I, J) / D E N S I T Y)$

$\operatorname{IF}(T(I, J)$.EQ. 0.0$)$ GO TO 11

$T(I, J)=T(I, J)+.5$

$11 \operatorname{TOT}(I, J)=\operatorname{TOT}(I, J)+T(I, J)$ 


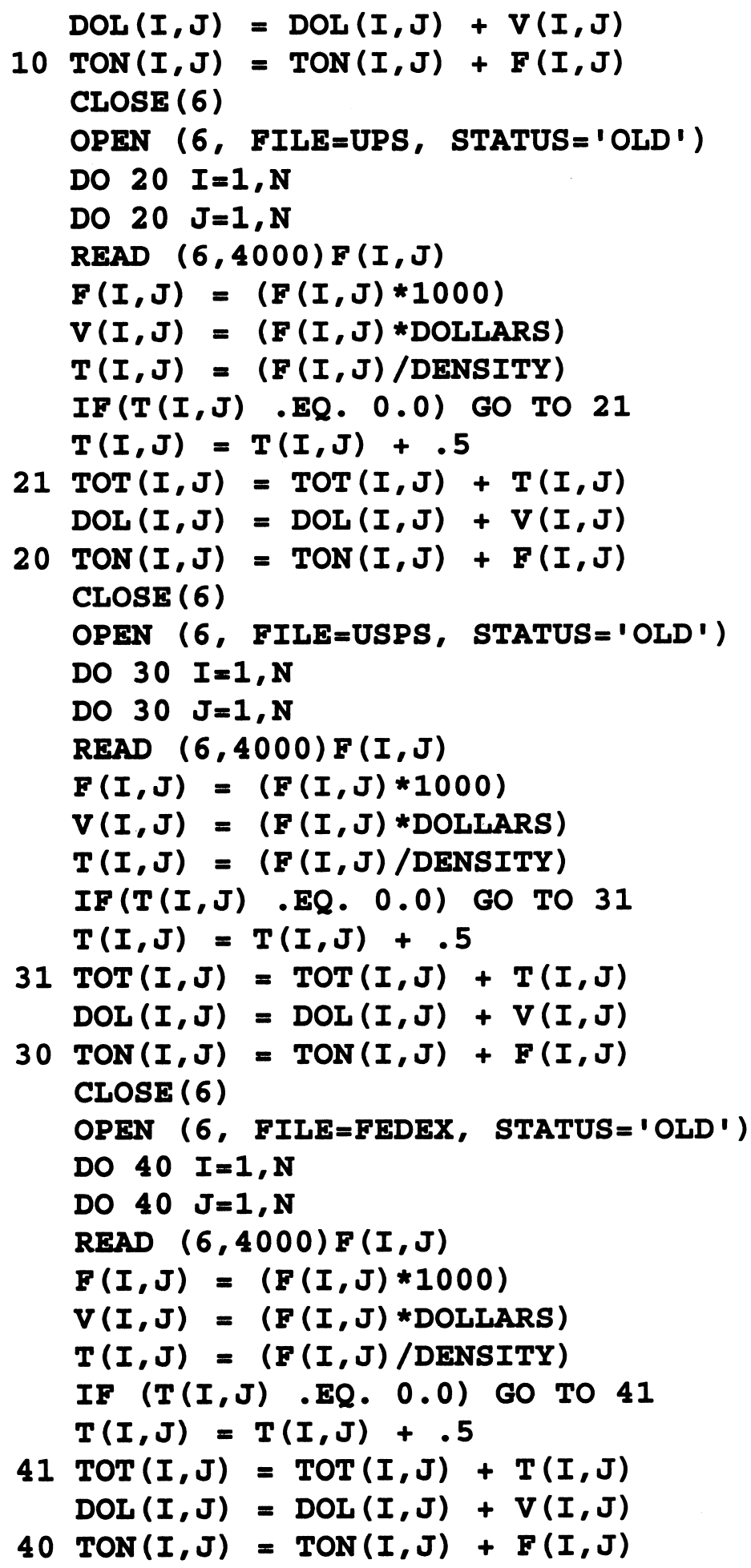




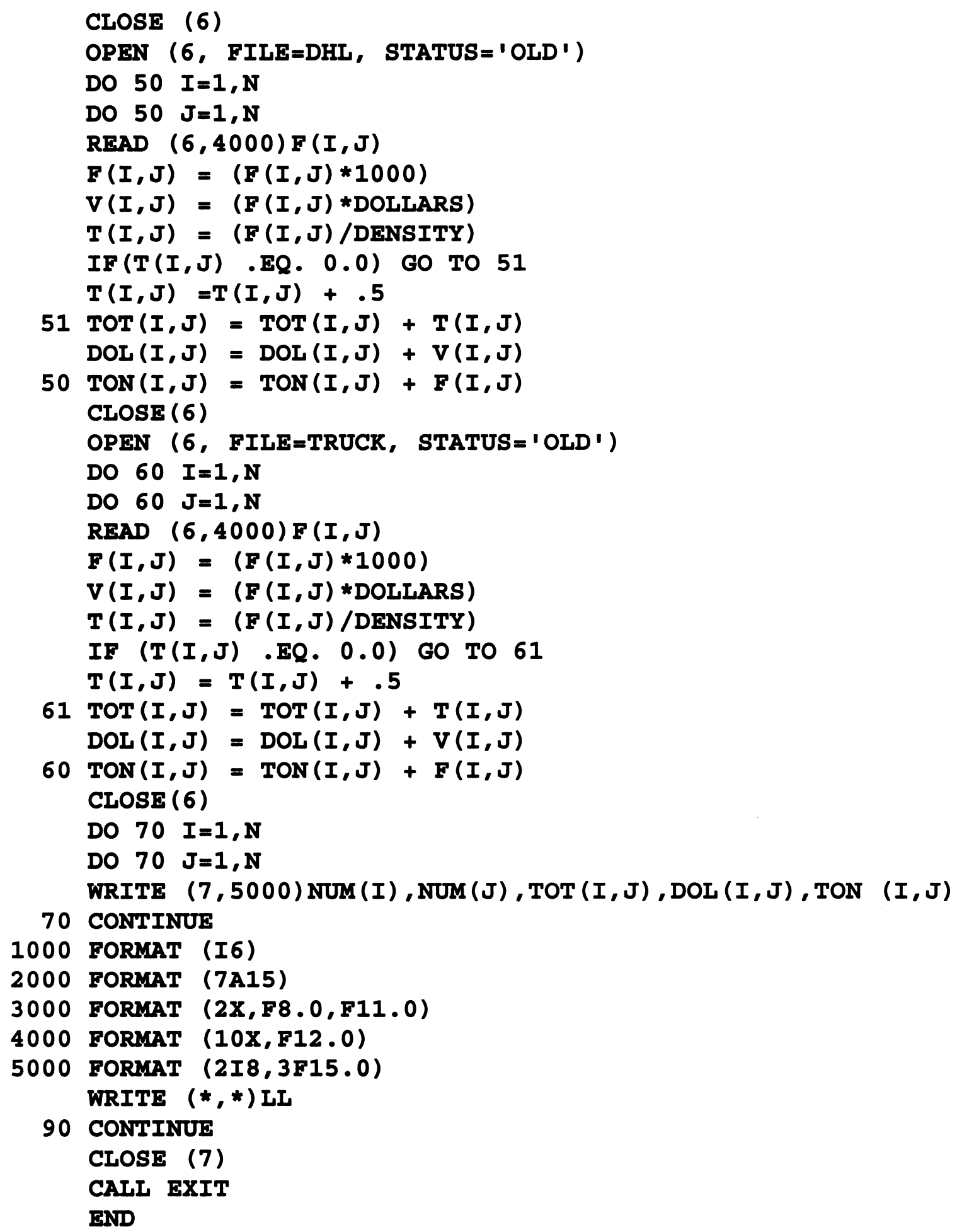


TOTAL9701.1 FEDEX9701.1 TOTAL9702 . 1 FEDEX9702 . 1 TOTAL9703.1 FEDEX9703.1 TOTAL9704.1 FEDEX9704.1 TOTAL9705.1 FEDEX9705.1 TOTAL9706.1 FEDEX9706.1 TOTAL9707.1 FEDEX9707 . 1 TOTAL9708.1 FEDEX9708.1 TOTAL9709.1 FEDEX9709.1 TOTAL9710.1 FEDEX9710.1 TOTAL9711.1 FEDEX9711.1 TOTAL9712.1 FEDEX9712.1 TOTAL9713.1 FEDEX9713.1 TOTAL9714.1 FEDEX9714 . 1 TOTAL9715.1 FEDEX9715.1 TOTAL9717 . 1 FEDEX9717 . 1 TOTAL9718.1 FEDEX9718.1 TOTAL9719.1 FEDEX9719.1 TOTAL9720.1 FEDEX9720.1 TOTAL9721.1 FEDEX9721.1 TOTAL9722.1
AIRF9701.1 DHL9701.1

AIRF9702.1 DHL9702 . 1

AIRF9703.1 DHL9703.1

AIRF9704.1 DHL9704.1

AIRF9705.1 DHL 9705.1

AIRF9706.1 DHL9706.1

AIRF9707.1 DHL9707.1

AIRF9708.1 DHL9708.1

AIRF9709.1 DHL9709.1

AIRF9710.1 DHL9710.1

AIRF9711.1 DHL9711.1

AIRF9712.1 DHL9712.1

AIRF9713.1 DHL9713.1

AIRF9714.1 DHL9714.1

AIRF9715.1 DHL9715.1

AIRF9717.1 DHL9717 . 1

AIRF9718.1 DHL9718.1

AIRF9719.1 DHL 9719.1

AIRF9720.1 DHL9720.1

AIRF9721.1 DHL9721.1 AIRF9722.1
UPS9701.1 USPS9701.1 TRUCK9701.1

UPS9702.1 USPS9702.1 TRUCK9702.1

UPS9703.1 USPS9703.1 TRUCK9703.1

UPS9704.1 USPS9704.1 TRUCK9704.1

UPS9705.1 USPS9705.1 TRUCK9705.1

UPS9706.1 USPS9706.1 TRUCK9706.1

UPS9707.1 USPS9707.1 TRUCK9707.1

UPS9708.1 USPS9708.1 TRUCK9708.1

UPS9709.1 USPS9709.1 TRUCK9709.1

UPS9710.1 USPS9710.1 TRUCK9710.1

UPS9711.1 USPS9711.1 TRUCK9711.1

UPS9712.1 USPS9712.1 TRUCK9712.1

UPS9713.1 USPS9713.1 TRUCK9713.1

UPS9714.1 USPS9714.1 TRUCK9714.1

UPS9715.1 USPS9715.1 TRUCK9715.1

UPS9717.1 USPS9717.1 TRUCK9717.1

UPS9718.1 USPS9718.1 TRUCK9718.1

UPS9719.1 USPS9719.1 TRUCK9719.1

UPS9720.1 USPS9720.1 TRUCK9720.1

UPS9721.1 USPS9721.1 TRUCK9721.1

UPS9722.1 USPS9722.1 
FEDEX9722.1 TOTAL9723.1 FEDEX9723.1 TOTAL9724.1 FEDEX9724.1 TOTAL9725.1 FEDEX9725.1 TOTAL9726.1 FEDEX9726.1 TOTAL9727.1 FEDEX9727.1 TOTAL9728.1 FEDEX9728.1 TOTAL9729.1 FEDEX9729.1 TOTAL9730.1 FEDEX9730.1 TOTAL9731.1 FEDEX9731.1 TOTAL9732.1 FEDEX9732.1 TOTAL9733.1 FEDEX9733.1 TOTAL9734.1 FEDEX9734.1 TOTAL9735.1 FEDEX9735.1 TOTAL9736.1 FEDEX9736.1 TOTAL9737.1 FEDEX9737.1 TOTAL9738.1 FEDEX9738.1 TOTAL9739.1 FEDEX9739.1 TOTAL9740.1 FEDEX9740.1 TOTAL9741.1 FEDEX9741.1 TOTAL9743.1 FEDEX9743.1 TOTAL1501.1
DHL9722. 1

AIRF9723.1

DHL 9723.1

AIRF9724.1

DHL9724.1

AIRF9725.1

DHL9725.1

AIRF9726.1

DHL9726.1

AIRF9727.1

DHL9727.1

AIRF9728.1

DHL9728.1

AIRF9729.1

DHL9729.1

AIRF9730.1

DHL9730.1

AIRF9731.1

DHL9731.1

AIRF9732.1

DHL 9732.1

AIRF9733.1

DHL9733.1

AIRF9734.1

DHL9734.1

AIRF9735.1

DHL9735.1

AIRF9736.1

DHL9736.1

AIRF9737.1

DHL9737.1

AIRF9738.1

DHL9738.1

AIRF9739.1

DHL9739.1

AIRF9740.1

DHL9740.1

AIRF9741.1

DHL9741.1

AIRF9743.1

DHL9743.1

AIRF1501.1
TRUCK9722.1

UPS9723.1 USPS9723.1

TRUCK9723.1

UPS9724.1 USPS9724.1 TRUCK9724.1

UPS9725.1 USPS9725.1 TRUCK9725.1

UPS9726.1 USPS9726.1 TRUCK9726.1

UPS9727.1 USPS9727.1 TRUCK9727 . 1

UPS9728.1 USPS9728.1 TRUCK9728.1

UPS9729.1 USPS9729.1 TRUCK9729.1

UPS9730.1 USPS9730.1 TRUCK9730.1

UPS9731.1 USPS9731.1 TRUCK9731.1

UPS9732.1 USPS9732.1 TRUCK9732.1

UPS9733.1 USPS9733.1 TRUCK9733.1

UPS9734.1 USPS9734.1 TRUCK9734.1

UPS9735.1 USPS9735.1 TRUCK9735.1

UPS9736.1 USPS9736.1 TRUCK9736.1

UPS9737.1 USPS9737.1 TRUCK9737.1

UPS9738.1 USPS9738.1 TRUCK9738.1

UPS9739.1 USPS9739.1 TRUCK9739.1

UPS9740.1 USPS9740.1 TRUCK9740.1

UPS9741.1 USPS9741.1 TRUCK9741.1

UPS9743.1 USPS9743.1 TRUCK9743.1

UPS1501.1 USPS1501.1 
FEDEX1501.1 TOTAL1502 . 1 FEDEX1502 . 1 TOTAL1503.1 FEDEX1503.1 TOTAL1504 .1 FEDEX1504.1 TOTAL1505.1 FEDEX1505.1 TOTAL1506.1 FEDEX 1506.1 TOTAL1507 . 1 FEDEX1507 . 1 TOTAL1508.1 FEDEX1508.1 TOTAL1509.1 FEDEX1509.1 TOTAL1510.1 FEDEX1510.1 TOTAL1511.1 FEDEX1511.1 TOTAL1512.1 FEDEX1512.1 TOTAL1513.1 FEDEX1513.1 TOTAL1514.1 FEDEX1514.1 TOTAL1515.1 FEDEX1515.1 TOTAL1517.1 FEDEX1517 . 1 TOTAL1518.1 FEDEX1518.1 TOTAL1519.1 FEDEX1519.1 TOTAL1520.1 FEDEX1520.1 TOTAL1521.1 FEDEX1521.1 TOTAL1522 . 1 FEDEX1522.1 TOTAL1523.1
DHL1501.1

AIRF1502 . 1 DHL1502 . 1

AIRF1503.1 DHL 1503.1

AIRF1504. 1 DHL1504 . 1

AIRF1505.1 DHL1505.1 AIRF1506.1 DHL1506.1

AIRF1507.1 DHL 1507.1

AIRF1508.1 DHL1508.1

AIRF1509.1 DHL1509.1

AIRF1510.1 DHL1510.1

AIRF1511.1 DHL1511.1

AIRF1512.1 DHL1512.1

AIRF1513.1 DHL1513.1

AIRF 1514.1 DHL1514.1

AIRF1515.1 DHL1515.1

AIRF1517 . 1 DHL1517.1

AIRF1518.1 DHL1518.1

AIRF1519.1 DHL1519.1

AIRF 1520.1 DHL 1520.1

AIRF1521.1 DHL 1521.1

AIRF1522.1 DHL1522.1 AIRF1523.1
TRUCK1501.1

UPS1502.1 USPS1502.1 TRUCK1502.1

UPS1503.1 USPS1503.1 TRUCK1503.1

UPS1504.1 USPS1504.1 TRUCK1504.1

UPS1505.1 USPS1505.1 TRUCK1505.1

UPS1506.1 USPS1506.1 TRUCK1506.1

UPS1507.1 USPS1507.1 TRUCK1507.1

UPS1508.1 USPS1508.1 TRUCK1508.1

UPS1509.1 USPS1509.1 TRUCK1509.1

UPS1510.1 USPS1510.1 TRUCK1510.1

UPS1511.1 USPS1511.1 TRUCK1511.1

UPS1512.1 USPS1512.1 TRUCK1512.1

UPS1513.1 USPS1513.1 TRUCK1513.1

UPS1514.1 USPS1514.1 TRUCK1514.1

UPS1515.1 USPS1515.1 TRUCK1515.1

UPS1517.1 USPS1517.1 TRUCK1517.1

UPS1518.1 USPS1518.1 TRUCK1518.1

UPS1519.1 USPS1519.1 TRUCK1519.1

UPS1520.1 USPS1520.1 TRUCK1520.1

UPS1521.1 USPS1521.1 TRUCK1521.1

UPS1522.1 USPS1522.1 TRUCK1522.1

UPS1523.1 USPS1523.1 
FEDEX1523.1 TOTAL1524.1 FEDEX1524 . 1 TOTAL1525.1 FEDEX1525.1 TOTAL1526.1 FEDEX1526.1 TOTAL1527.1 FEDEX1527.1 TOTAL1528.1 FEDEX1528.1 TOTAL1529.1 FEDEX1529.1 TOTAL1530.1 FEDEX1530.1 TOTAL1531.1 FEDEX1531.1 TOTAL1532.1 FEDEX1532.1 TOTAL1533.1 FEDEX1533.1 TOTAL 1534.1 FEDEX1534 . 1 TOTAL1535.1 FEDEX1535.1 TOTAL1536 . 1 FEDEX1536.1 TOTAL1537.1 FEDEX1537 . 1 TOTAL1538.1 FEDEX1538.1 TOTAL1539.1 FEDEX1539.1 TOTAL1540.1 FEDEX1540.1 TOTAL1541.1 FEDEX1541.1 TOTAL1543.1 FEDEX1543 . 1 TOTAL3001. 1 FEDEX3001.1 TOTAL3002 . 1
DHL1523.1

AIRF1524.1 DHL 1524.1

AIRF1525.1 DHL 1525.1

AIRF1526.1 DHL1526.1

AIRF1527 . 1 DHL1527.1

AIRF1528.1 DHL1528.1

AIRF1529.1 DHL1529.1

AIRF 1530.1 DHL1530.1

AIRF1531.1 DHL1531.1

AIRF1532.1 DHI 1532.1

AIRF 1533.1 DHL1533.1

AIRF1534.1 DHL1534.1

AIRF 1535.1 DHL1535.1

AIRF1536.1 DHL1536.1

AIRF1537.1 DHL1537.1

AIRF1538.1 DHL1538.1

AIRF1539.1 DHL1539.1

AIRF1540.1 DHL1540.1

AIRF1541.1 DHL1541.1

AIRF1543.1 DHL1543.1

AIRF3001.1 DHL 3001.1 AIRF3002 . 1
TRUCK1523.1

UPS1524.1 USPS1524.1 TRUCK1524. 1

UPS1525.1 USPS1525.1 TRUCK1525.1

UPS1526.1 USPS1526.1 TRUCK1526.1

UPS1527.1 USPS1527.1 TRUCK1527 . 1

UPS1528.1 USPS1528.1 TRUCK1528.1

UPS1529.1 USPS1529.1 TRUCK1529.1

UPS1530.1 USPS1530.1 TRUCK1530.1

UPS1531.1 USPS1531.1 TRUCK1531.1

UPS1532.1 USPS1532.1 TRUCK1532.1

UPS1533.1 USPS1533.1 TRUCK1533.1

UPS1534.1 USPS1534.1 TRUCK1534.1

UPS1535.1 USPS1535.1 TRUCK1535.1

UPS1536.1 USPS1536.1 TRUCK1536.1

UPS1537.1 USPS1537.1 TRUCK1537.1

UPS1538.1 USPS1538.1 TRUCK1538.1

UPS1539.1 USPS1539.1 TRUCK1539.1

UPS1540.1 USPS1540.1 TRUCK1540.1

UPS1541.1 USPS1541.1 TRUCK1541.1

UPS1543.1 USPS1543.1 TRUCK1543.1

UPS3001.1 USPS3001.1 TRUCK3001.1

UPS3002.1 USPS3002.1 
FEDEX3002.1 TOTAL 3003.1 FEDEX3003.1 TOTAL 3004.1 FEDEX3004.1 TOTAL3005.1 FEDEX3005.1 TOTAL3006.1 FEDEX3006.1 TOTAL3007.1 FEDEX3007.1 TOTAL3008.1 FEDEX3008.1 TOTAL3009.1 FEDEX3009.1 TOTAL 3010.1 FEDEX3010.1 TOTAL3011.1 FEDEX3011.1 TOTAL3012.1 FEDEX3012.1 TOTAL3013.1 FEDEX3013.1 TOTAL3014.1 FEDEX3 014.1 TOTAL3015.1 FEDEX 3015.1 TOTAL 3017.1 FEDEX3017.1 TOTAL 3018.1 FEDEX3018.1 TOTAL3019.1 FEDEX3019.1 TOTAL 3020.1 FEDEX3020.1 TOTAL 3021.1 FEDEX3021.1 TOTAL 3022.1 FEDEX3022.1 TOTAL3023.1 FEDEX3023.1
DHL 3002.1

AIRF3003.1

DHL 3003.1

AIRF3004.1

DHL 3004.1

AIRF3005.1

DHL3005.1

AIRF3006.1

DHL3006.1

AIRF3007 . 1

DHL3007 . 1

AIRF3008.1 DHL3008.1

AIRF3009.1 DHL3009.1

AIRF3010.1 DHL 3010.1

AIRF3011.1 DHL3011.1

AIRF3012 . 1 DHL 3012.1

AIRF3013.1 DHL 3013.1

AIRF3014.1 DHL 3014.1

AIRF3015.1 DHL3015.1

AIRF3017 . 1 DHL 3017 . 1

AIRF3018 .1 DHL3018.1

AIRF3019.1 DHL3019.1

AIRF 3020.1 DHL3020.1

AIRF3021.1 DHL 3021.1

AIRF3022.1 DHL3022 . 1

AIRF3023.1 DHL 3023.1
TRUCK3002.1

UPS3003 USPS 3003.1 TRUCK3003.1

UPS3004.1 USPS3004.1 TRUCK3004.1

UPS3005.1 USPS3005.1 TRUCK3005.1

UPS3006.1 USPS3006.1 TRUCK3006.1

UPS3007.1 USPS3007.1 TRUCK 3007.1

UPS3008.1 USPS3008.1 TRUCK3008.1

UPS3009.1 USPS3009.1 TRUCK3009.1

UPS3010.1 USPS3010.1 TRUCK3010.1

UPS3011.1 USPS3011.1 TRUCK3011.1

UPS3012.1 USPS3012.1 TRUCK3012.1

UPS3013.1 USPS3013.1 TRUCK3013.1

UPS3014.1 USPS3014.1 TRUCK3014.1

UPS3015.1 USPS3015.1 TRUCK3015.1

UPS3017.1 USPS3017.1 TRUCK3017.1

UPS3018.1 USPS3018.1 TRUCK3018.1

UPS3019.1 USPS3019.1 TRUCK3019.1

UPS3020.1 USPS3020.1 TRUCK3020.1

UPS3021.1 USPS3021.1 TRUCK3021.1

UPS3022.1 USPS3022.1 TRUCK3022.1

UPS3023.1 USPS3023.1 TRUCK3023.1 


\begin{tabular}{|c|c|c|c|}
\hline TOTAL30 & AIRF3024.1 & UPS3024.1 & USPS3024.1 \\
\hline FEDEX3024.1 & DHL 3024.1 & \multicolumn{2}{|c|}{ TRUCK3024.1 } \\
\hline TOTAL3025.1 & AIRF3025.1 & UPS3025.1 & USPS3025.1 \\
\hline FEDEX3025.1 & DHL 3025.1 & \multicolumn{2}{|c|}{ TRUCK3025.1 } \\
\hline TOTAL3026.1 & AIRF3026.1 & UPS3026.1 & USPS3026.1 \\
\hline FEDEX3026.1 & DHL 3026.1 & \multicolumn{2}{|c|}{ TRUCK3026.1 } \\
\hline TOTAL3027. 1 & AIRF3027.1 & UPS3027.1 & USPS3027.1 \\
\hline FEDEX3027.1 & DHL 3027 . 1 & \multicolumn{2}{|c|}{ TRUCK3027.1 } \\
\hline TOTAL3028.1 & AIRF 3028.1 & UPS3028.1 & USPS3028.1 \\
\hline FEDEX3028.1 & DHL 3028.1 & \multicolumn{2}{|c|}{ TRUCK3028.1 } \\
\hline TOTAL3029.1 & AIRF3029.1 & UPS3029.1 & USPS3029.1 \\
\hline FEDEX3029.1 & DHL3029.1 & \multicolumn{2}{|c|}{ TRUCK3029.1 } \\
\hline TOTAL3030.1 & AIRF 3030.1 & UPS3030.1 & USPS3030.1 \\
\hline FEDEX3030.1 & DHL3030.1 & \multicolumn{2}{|c|}{ TRUCK3030.1 } \\
\hline TOTAL3031.1 & AIRF3031.1 & UPS3031.1 & USPS3031.1 \\
\hline FEDEX3031.1 & DHL 3031.1 & \multicolumn{2}{|c|}{ TRUCK3031.1 } \\
\hline TOTAL3032. 1 & AIRF 3032.1 & UPS3032.1 & USPS3032.1 \\
\hline FEDEX3032.1 & DHL 3032.1 & \multicolumn{2}{|c|}{ TRUCK3032.1 } \\
\hline TOTAL3033.1 & AIRF3033.1 & UPS3033.1 & USPS3033.1 \\
\hline FEDEX3033.1 & DHL 3033.1 & \multicolumn{2}{|c|}{ TRUCK3033.1 } \\
\hline TOTAL3034.1 & AIRF 3034.1 & UPS3034.1 & USPS3034.1 \\
\hline FEDEX 3034.1 & DHL 3034.1 & \multicolumn{2}{|c|}{ TRUCK3034.1 } \\
\hline TOTAL3035.1 & AIRF3035.1 & UPS3035.1 & USPS3035.1 \\
\hline FEDEX3035 . 1 & DHL3035. 1 & \multicolumn{2}{|c|}{ TRUCK3035.1 } \\
\hline TOTAL3036.1 & AIRF3036.1 & UPS3036.1 & USPS3036.1 \\
\hline FEDEX3036.1 & DHU3036.1 & \multicolumn{2}{|c|}{ TRUCK3036.1 } \\
\hline TOTAL3037. 1 & AIRF3037.1 & UPS3037.1 & USPS3037.1 \\
\hline FEDEX3037 . 1 & DHL3037.1 & \multicolumn{2}{|c|}{ TRUCK3037.1 } \\
\hline TOTAL3038. 1 & AIRF3038.1 & UPS3038.1 & USPS3038.1 \\
\hline FEDEX3038.1 & DHL 3038.1 & \multicolumn{2}{|c|}{ TRUCK3038.1 } \\
\hline TOTAL3039.1 & AIRF3039.1 & UPS3039.1 & USPS3039.1 \\
\hline FEDEX3039.1 & DHL3039.1 & \multicolumn{2}{|c|}{ TRUCK3039.1 } \\
\hline TOTAL3040.1 & AIRF3040.1 & UPS3040.1 & USPS3040.1 \\
\hline FEDEX 3040.1 & DHL3040.1 & \multicolumn{2}{|c|}{ TRUCK3040.1 } \\
\hline TOTAL3041. 1 & AIRF3041.1 & UPS3041.1 & USPS3041.1 \\
\hline FEDEX3041.1 & DHL3041.1 & \multicolumn{2}{|c|}{ TRUCK3041.1 } \\
\hline TOTAL3043.1 & AIRF3043.1 & UPS3043.1 & USPS3 043.1 \\
\hline FEDEX3043.1 & DHL 3043.1 & \multicolumn{2}{|c|}{ TRUCK3043.1 } \\
\hline
\end{tabular}




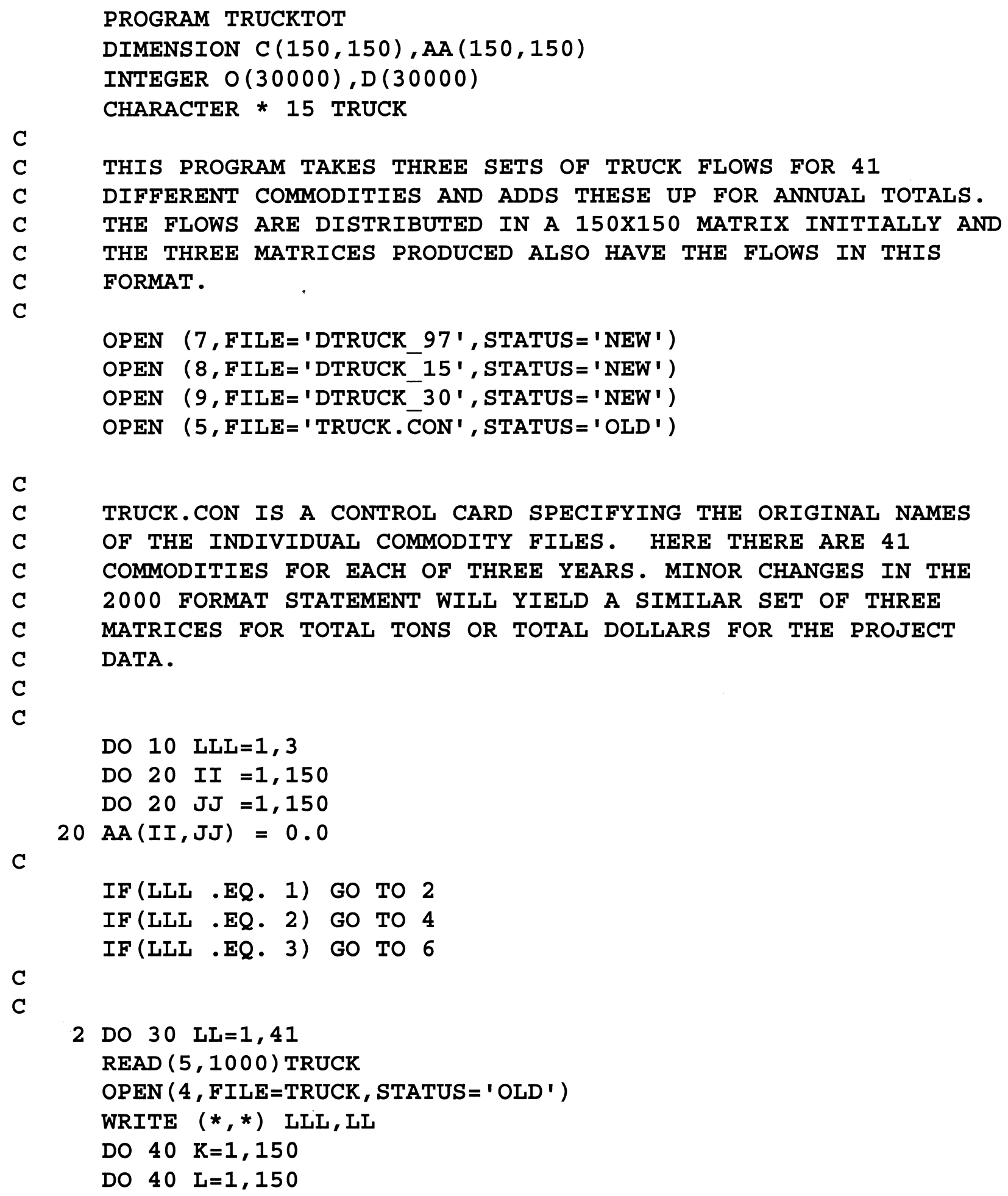

C

C TRUCK.CON IS A CONTROL CARD SPECIFYING THE ORIGINAL NAMES C OF THE INDIVIDUAL COMMODITY FILES. HERE THERE ARE 41 


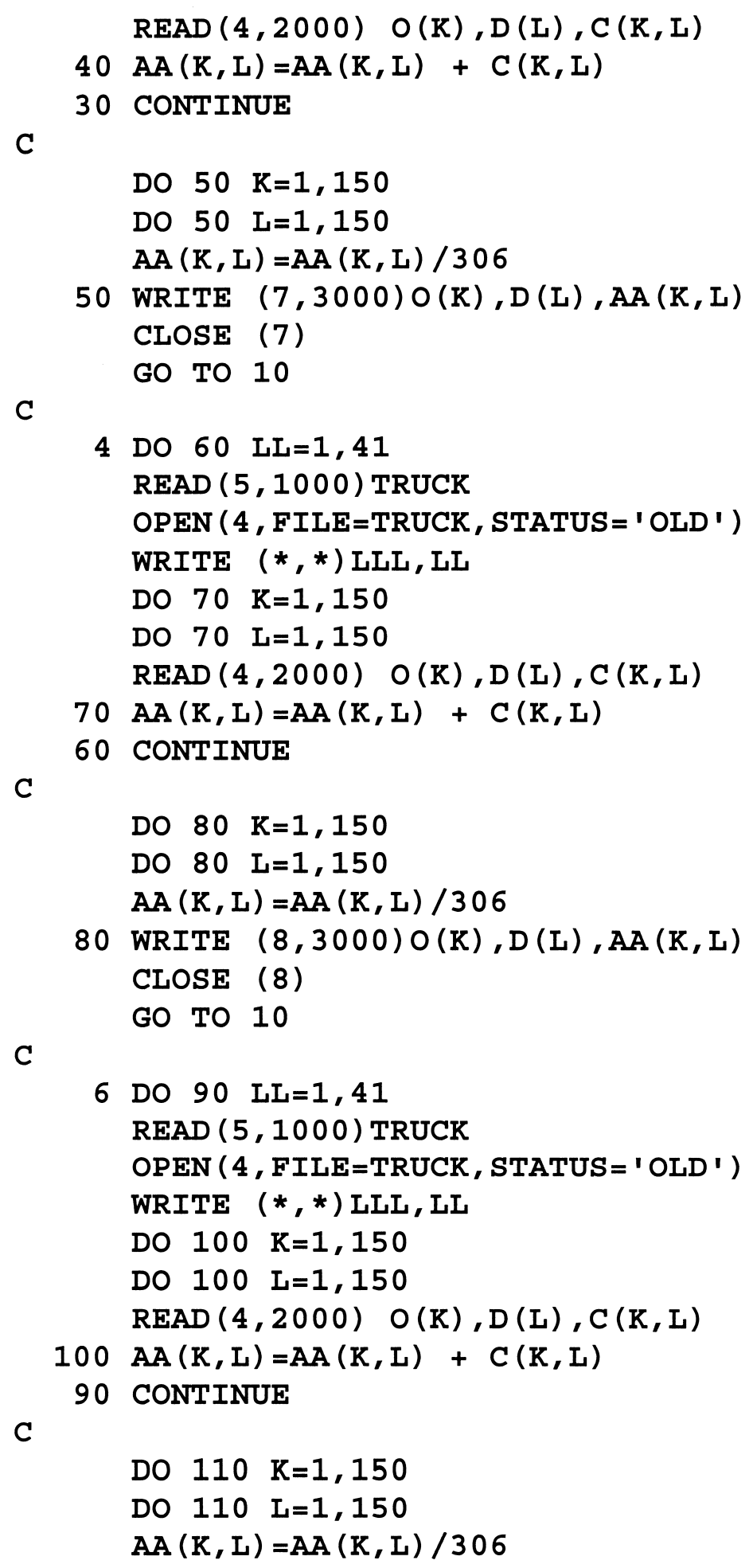




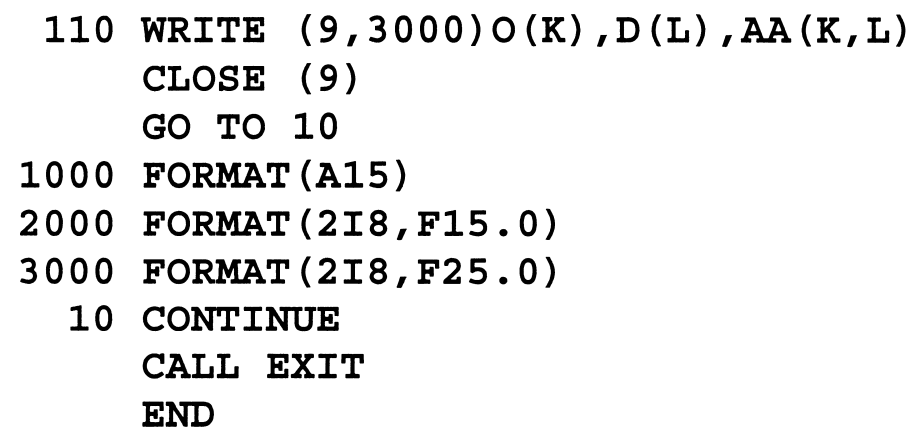




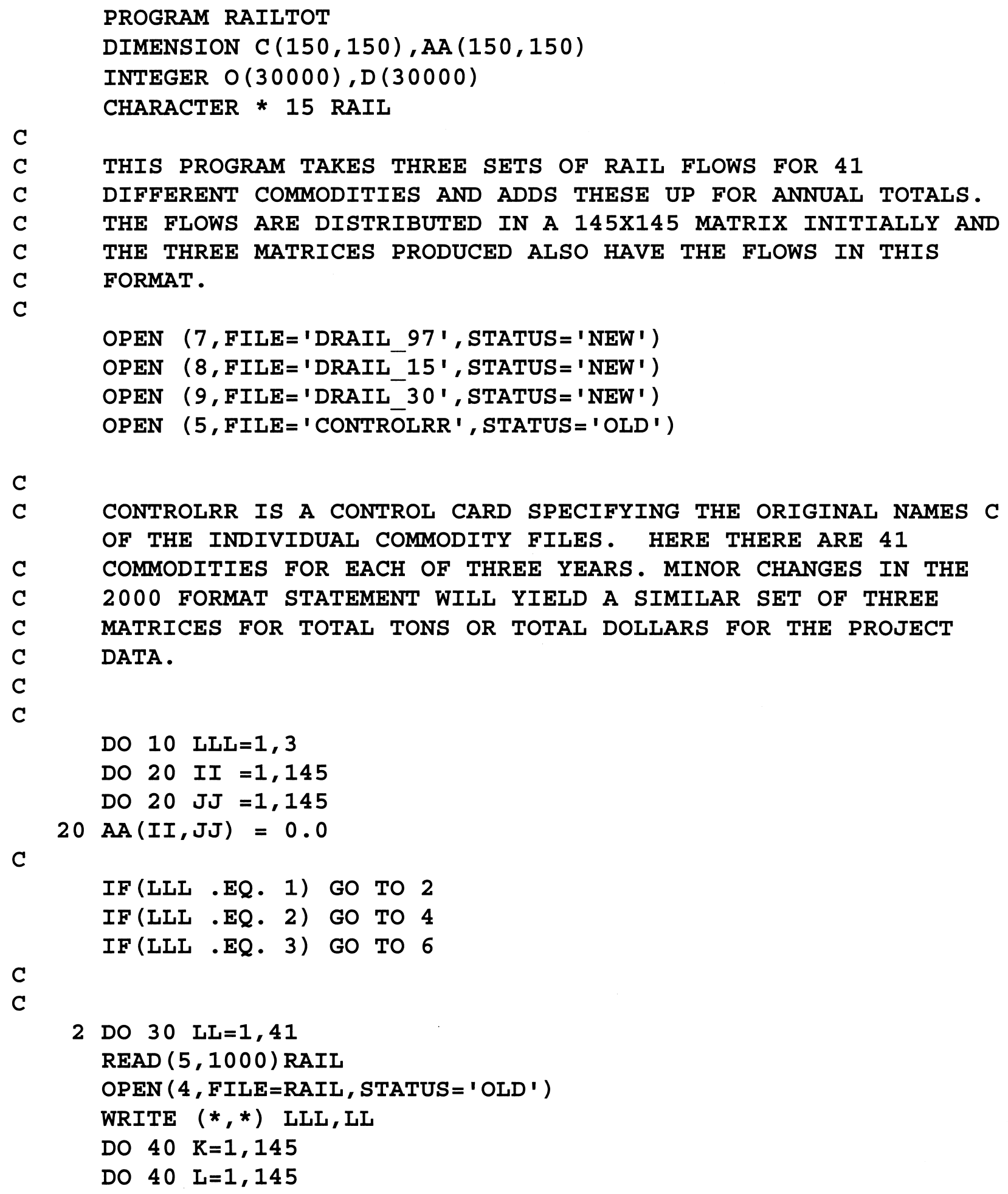




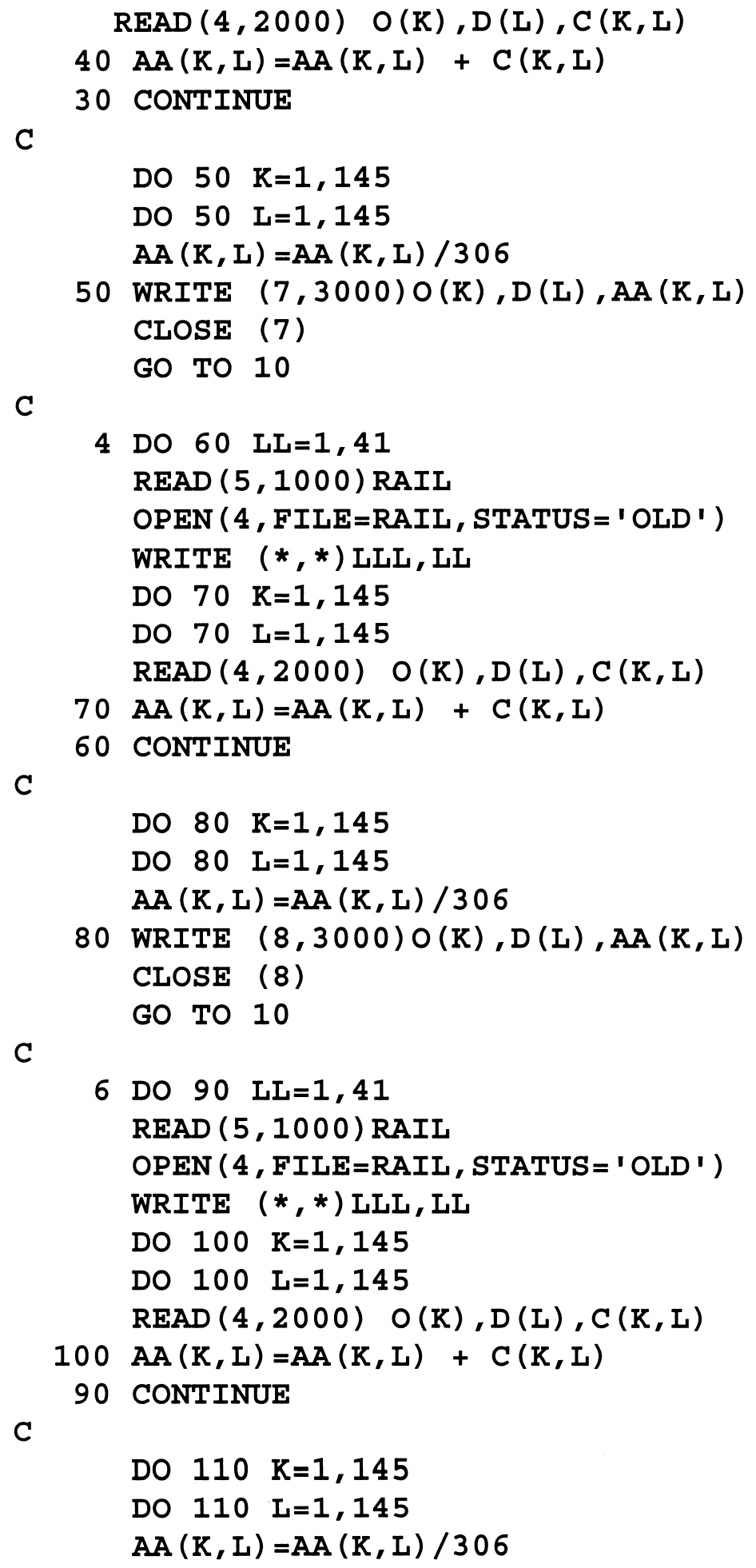




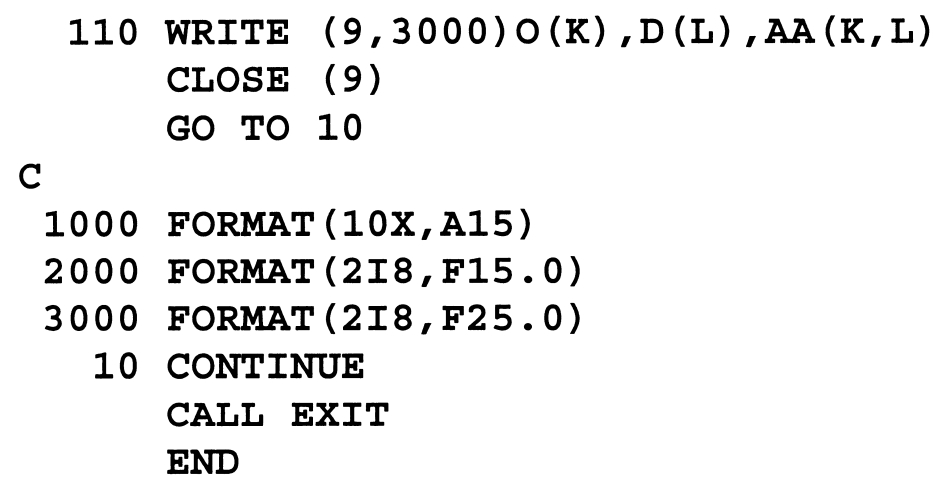




\begin{tabular}{|c|c|}
\hline RAIL9701 & RAIL9701.1 \\
\hline RAIL9702 & RAIL9702.1 \\
\hline RAIL9703 & RAIL9703.1 \\
\hline RAIL9704 & RAIL9704.1 \\
\hline RAIL9705 & RAIL9705.1 \\
\hline RAIL9706 & RAIL9706.1 \\
\hline RAIL9707 & RAIL9707.1 \\
\hline RAIL9708 & RAIL9708.1 \\
\hline RAIL9709 & RAIL9709.1 \\
\hline RAIL9710 & RAIL9710.1 \\
\hline RAIL9711 & RAIL9711.1 \\
\hline RAIL9712 & RAIL9712.1 \\
\hline RAIL9713 & RAIL9713.1 \\
\hline RAIL9714 & RAIL9714.1 \\
\hline RAIL9715 & RAIL9715.1 \\
\hline RAIL9717 & RAIL9717.1 \\
\hline RAIL9718 & RAIL9718.1 \\
\hline RAIL9719 & RAIL9719.1 \\
\hline RAIL9720 & RAIL9720.1 \\
\hline RAIL9721 & RAIL9721.1 \\
\hline RAIL9722 & RAIL9722.1 \\
\hline RAIL9723 & RAIL9723.1 \\
\hline RAIL9724 & RAIL9724.1 \\
\hline RAIL9725 & RAIL9725.1 \\
\hline RAIL9726 & RAIL9726.1 \\
\hline RAIL9727 & RAIL9727.1 \\
\hline RAIL9728 & RAIL9728.1 \\
\hline RAIL9729 & RAIL9729.1 \\
\hline RAIL9730 & RAIL9730.1 \\
\hline RAIL9731 & RAIL9731.1 \\
\hline RAIL9732 & RAIL9732.1 \\
\hline RAIL9733 & RAIL9733.1 \\
\hline RAIL9734 & RAIL9734.1 \\
\hline RAIL9735 & RAIL9735.1 \\
\hline RAIL9736 & RAIL9736.1 \\
\hline RAIL9737 & RAIL9737.1 \\
\hline RAIL9738 & RAIL9738.1 \\
\hline RAIL9739 & RAIL9739.1 \\
\hline RAIL9740 & RAIL9740.1 \\
\hline RAIL9741 & RAIL9741.1 \\
\hline RAIL9743 & RAIL9743.1 \\
\hline
\end{tabular}




\begin{tabular}{|c|c|}
\hline RAIL1501 & RAIL1501.1 \\
\hline RAIL1502 & RAIL1502.1 \\
\hline RAIL1503 & RAIL1503.1 \\
\hline RAIL1504 & RAIL1504.1 \\
\hline RAIL1505 & RAIL1505.1 \\
\hline RAIL1506 & RAIL1506.1 \\
\hline RAIL 1507 & RAIL1507.1 \\
\hline RAIL1508 & RAIL1508.1 \\
\hline RAIL1509 & RAIL1509.1 \\
\hline RAIL1510 & RAIL1510.1 \\
\hline RAIL1511 & RAIL1511.1 \\
\hline RAIL1512 & RAIL1512.1 \\
\hline RAIL1513 & RAIL1513.1 \\
\hline RAIL1514 & RAIL1514.1 \\
\hline RAIL1515 & RAIL1515.1 \\
\hline RAIL1517 & RAIL1517.1 \\
\hline RAIL1518 & RAIL1518.1 \\
\hline RAIL1519 & RAIL1519.1 \\
\hline RAIL1520 & RAIL1520.1 \\
\hline RAIL1521 & RAIL1521.1 \\
\hline RAIL1522 & RAIL1522.1 \\
\hline RAIL1523 & RAIL1523.1 \\
\hline RAIL1524 & RAIL1524.1 \\
\hline RAIL1525 & RAIL1525.1 \\
\hline RAIL1526 & RAIL1526.1 \\
\hline RAIL1527 & RAIL1527.1 \\
\hline RAIL1528 & RAIL1528.1 \\
\hline RAIL1529 & RAIL1529.1 \\
\hline RAIL1530 & RAIL1530.1 \\
\hline RAIL1531 & RAIL1531.1 \\
\hline RAIL1532 & RAIL1532.1 \\
\hline RAIL1533 & RAIL1533.1 \\
\hline RAIL1534 & RAIL1534.1 \\
\hline RAIL1535 & RAIL1535.1 \\
\hline RAIL1536 & RAIL1536.1 \\
\hline RAIL1537 & RAIL1537.1 \\
\hline RAIL1538 & RAIL1538.1 \\
\hline RAIL1539 & RAIL1539.1 \\
\hline RAIL1540 & RAIL1540.1 \\
\hline RAIL 1541 & RAIL1541.1 \\
\hline RAIL1543 & RAIL1543.1 \\
\hline
\end{tabular}




\begin{tabular}{|c|c|}
\hline RAIL3001 & RAIL3001.1 \\
\hline RAIL3002 & RAIL3002.1 \\
\hline RAIL 3003 & RAIL3003.1 \\
\hline RAIL3004 & RAIL3004.1 \\
\hline RAIL3005 & RAIL3005.1 \\
\hline RAIL3006 & RAIL3006.1 \\
\hline RAIL3007 & RAIL3007.1 \\
\hline RAIL3008 & RAIL3008.1 \\
\hline RAIL3009 & RAIL3009.1 \\
\hline RAIL3010 & RAIL3010.1 \\
\hline RAIL3011 & RAIL3011.1 \\
\hline RAIL3012 & RAIL3012.1 \\
\hline RAIL3013 & RAIL3013.1 \\
\hline RAIL3014 & RAIL3 014.1 \\
\hline RAIL3015 & RAIL3015.1 \\
\hline RAIL3017 & RAIL3017.1 \\
\hline RAIL3018 & RAIL3018.1 \\
\hline RAIL3019 & RAIL3019.1 \\
\hline RAIL3020 & RAIL3020.1 \\
\hline RAIL3021 & RAIL 3021.1 \\
\hline RAIL 3022 & RAIL3022.1 \\
\hline RAIL 3023 & RAIL3023.1 \\
\hline RAIL3024 & RAIL3024.1 \\
\hline RAIL3025 & RAIL3025.1 \\
\hline RAIL3026 & RAIL3026.1 \\
\hline RAIL3027 & RAIL3027.1 \\
\hline RAIL3028 & RAIL3028.1 \\
\hline RAIL3029 & RAIL3029.1 \\
\hline RAIL 3030 & RAIL3030.1 \\
\hline RAIL 3031 & RAIL3031.1 \\
\hline RAIL3032 & RAIL3032.1 \\
\hline RAIL 3033 & RAIL3033.1 \\
\hline RAIL3034 & RAIL3034.1 \\
\hline RAIL3035 & RAIL3035.1 \\
\hline RAIL3036 & RAIL3036.1 \\
\hline RAIL 3037 & RAIL3037.1 \\
\hline RAIL3038 & RAIL3038.1 \\
\hline RAIL3039 & RAIL3039.1 \\
\hline RAIL3040 & RAIL3040.1 \\
\hline RAIL 3041 & RAIL3 041.1 \\
\hline RAIL3043 & RAIL3043.1 \\
\hline
\end{tabular}




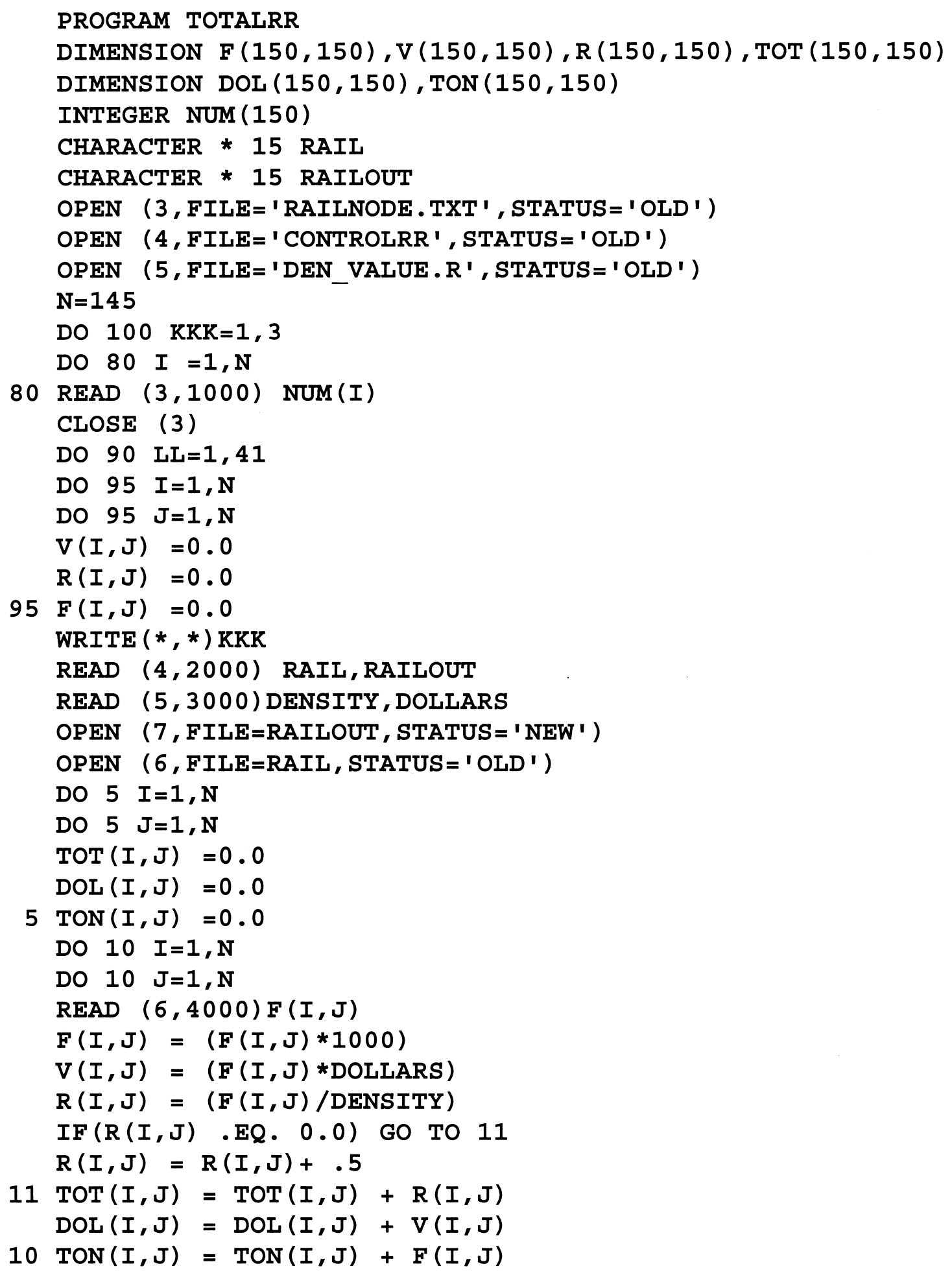




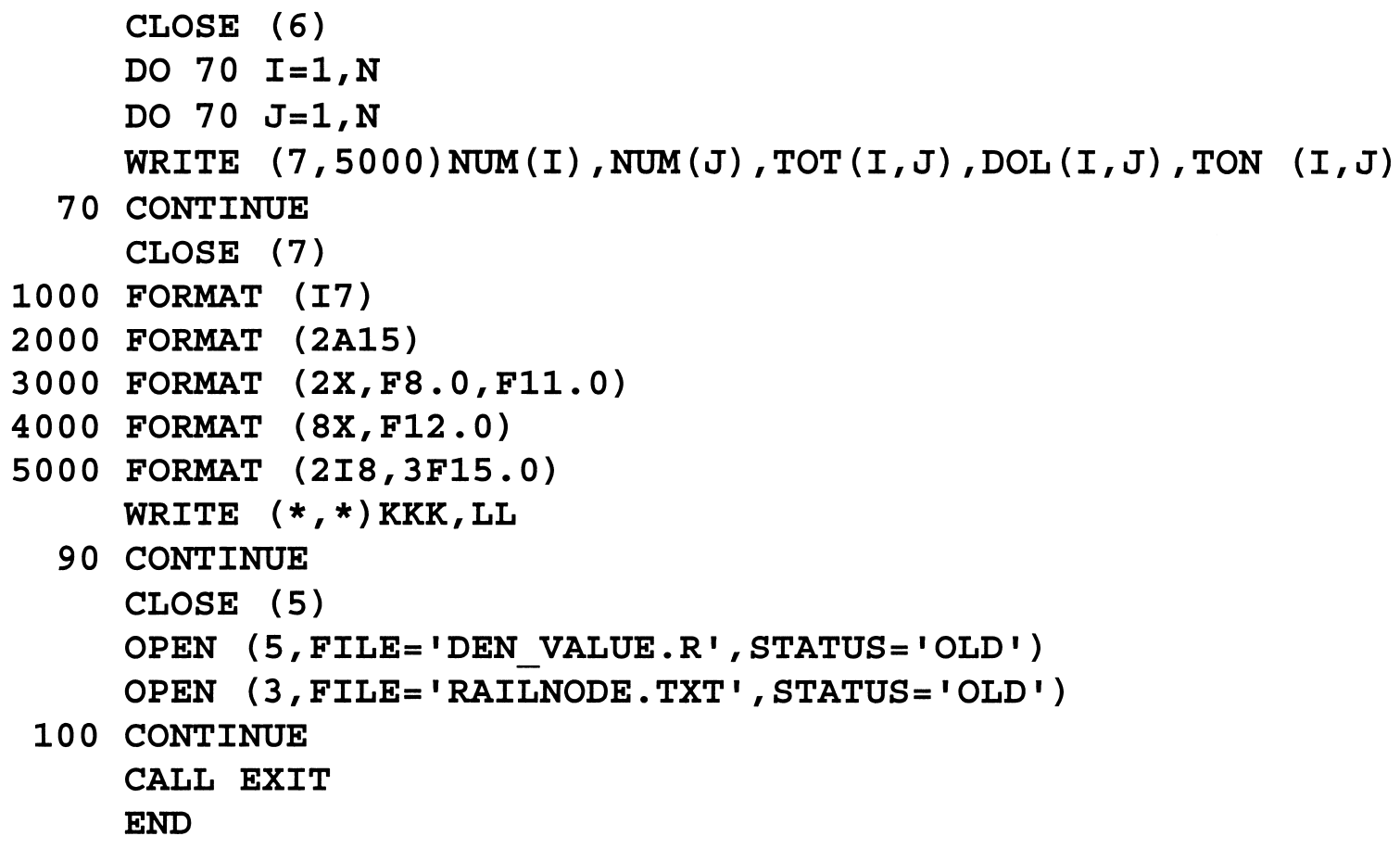




$\begin{array}{rrr}01 & 9.77 & 1042.38 \\ 02 & 96.63 & 121.79 \\ 03 & 86.79 & 507.50 \\ 04 & 88.28 & 304.27 \\ 05 & 74.41 & 2161.68 \\ 06 & 85.50 & 1069.44 \\ 07 & 87.02 & 873.50 \\ 08 & 87.31 & 1084.52 \\ 09 & 45.75 & 13661.34 \\ 10 & 100.00 & 171.52 \\ 11 & 97.97 & 9.66 \\ 12 & 97.97 & 6.34 \\ 13 & 100.44 & 48.06 \\ 14 & 95.91 & 138.97 \\ 15 & 109.36 & 20.94 \\ 17 & 84.04 & 225.43 \\ 18 & 88.22 & 195.79 \\ 19 & 73.66 & 157.65 \\ 20 & 98.66 & 539.16 \\ 21 & 40.00 & 22678.39 \\ 22 & 101.81 & 152.66 \\ 23 & 93.96 & 2276.19 \\ 24 & 94.30 & 2138.10 \\ 25 & 64.11 & 40.81 \\ 26 & 82.41 & 384.13 \\ 27 & 82.75 & 761.84 \\ 28 & 70.90 & 1337.82 \\ 29 & 45.00 & 3335.26 \\ 30 & 14.17 & 8265.63 \\ 31 & 98.64 & 119.96 \\ 32 & 91.47 & 850.58 \\ 33 & 79.66 & 2132.78 \\ 34 & 49.77 & 8356.27 \\ 35 & 16.69 & 21954.84 \\ 36 & 21.73 & 5821.94 \\ 37 & 41.36 & 23586.82 \\ 38 & 18.00 & 53741.41 \\ 39 & 15.00 & 4884.73 \\ 40 & 65.22 & 3741.45 \\ 41 & 79.86 & 183.97 \\ 43 & 32.45 & 2089.53\end{array}$


APPENDIX C

DISTANCE DECAY GRAPHS 


\section{Table Notes}

The graphs that appear on the following pages of this appendix demonstrate the level of distance decay for all of the commodities examined in this project. Commodity groups here are represented by the shorthand of Com1, Com2, and so forth to represent SCTG 1, SCTG 2, and so forth. The ordinate in these graphs represent the total tons (in 1,000s) and the abscissa represents the distance shipped. The graphs are included here to illustrate the extreme level of fall-off of the various shipments with increasing distance. 

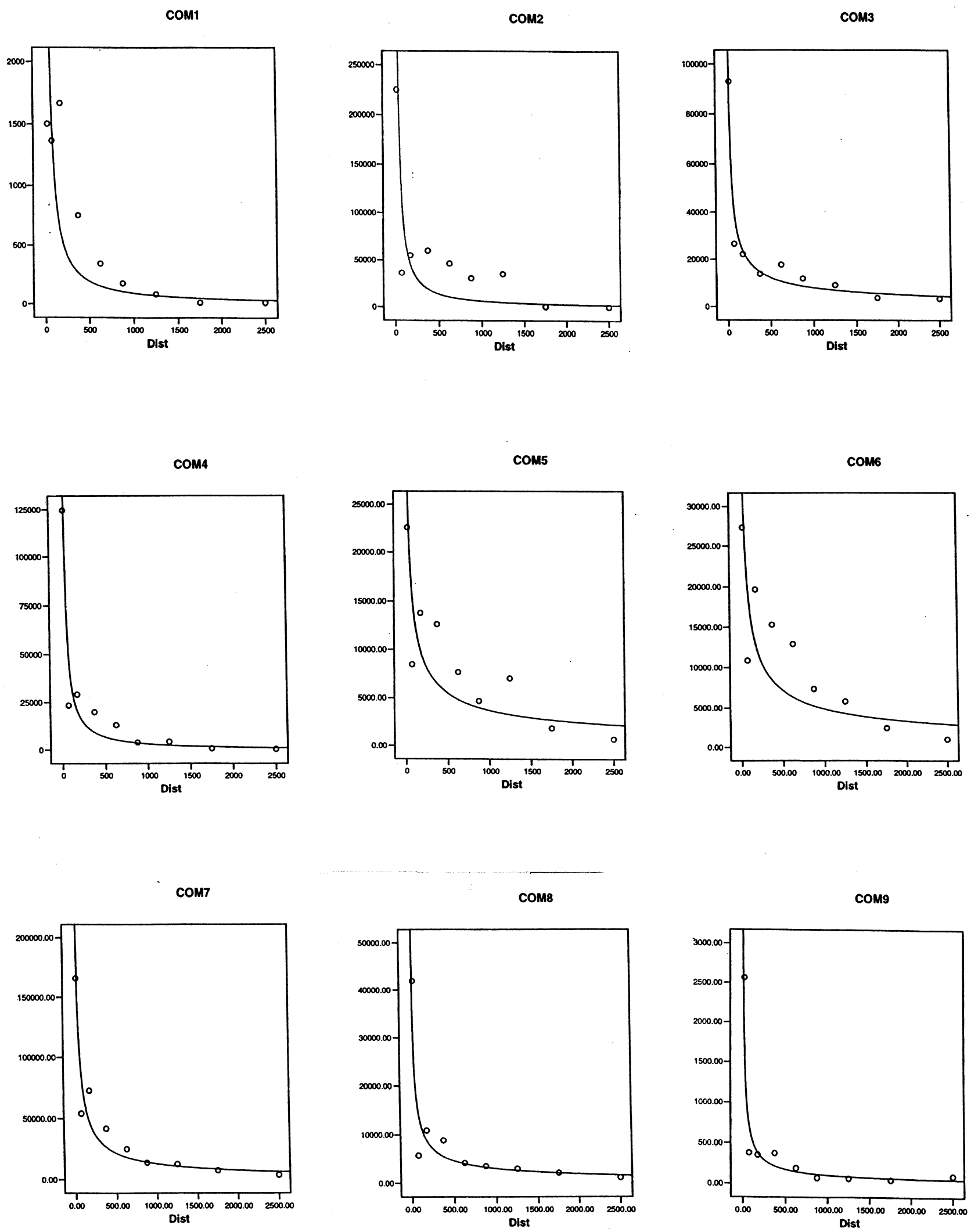

C3 

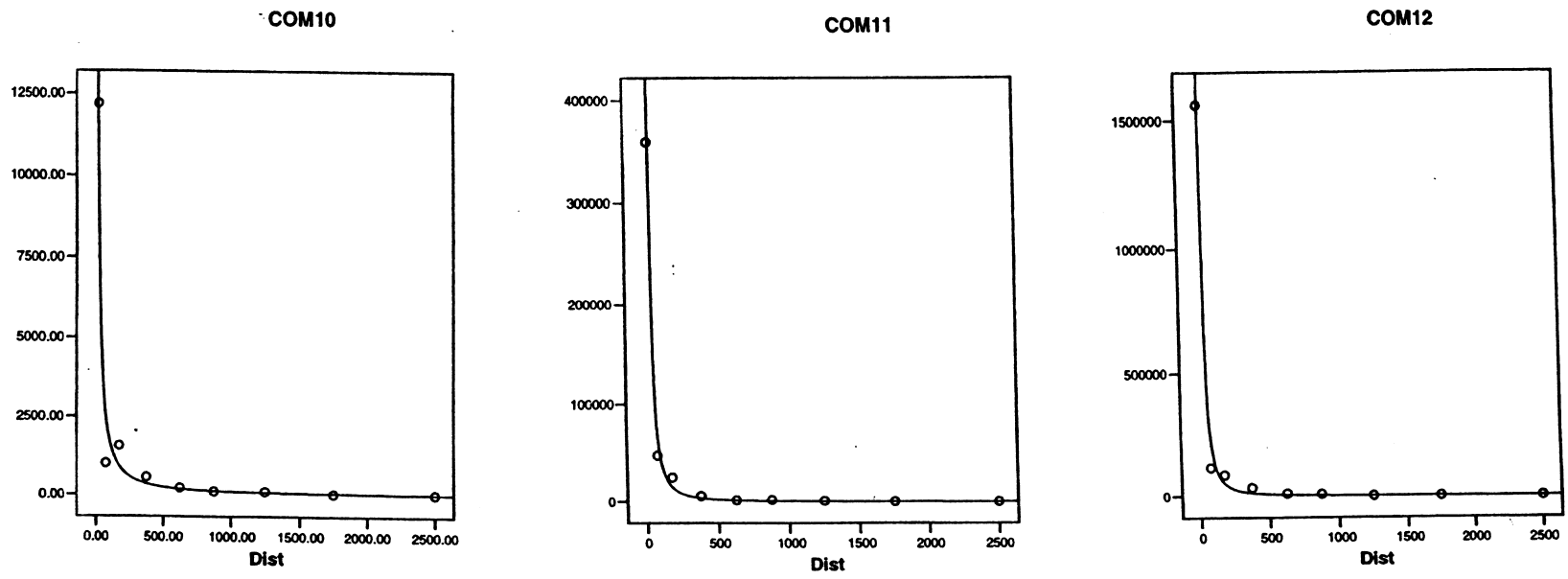

COM13

COM14
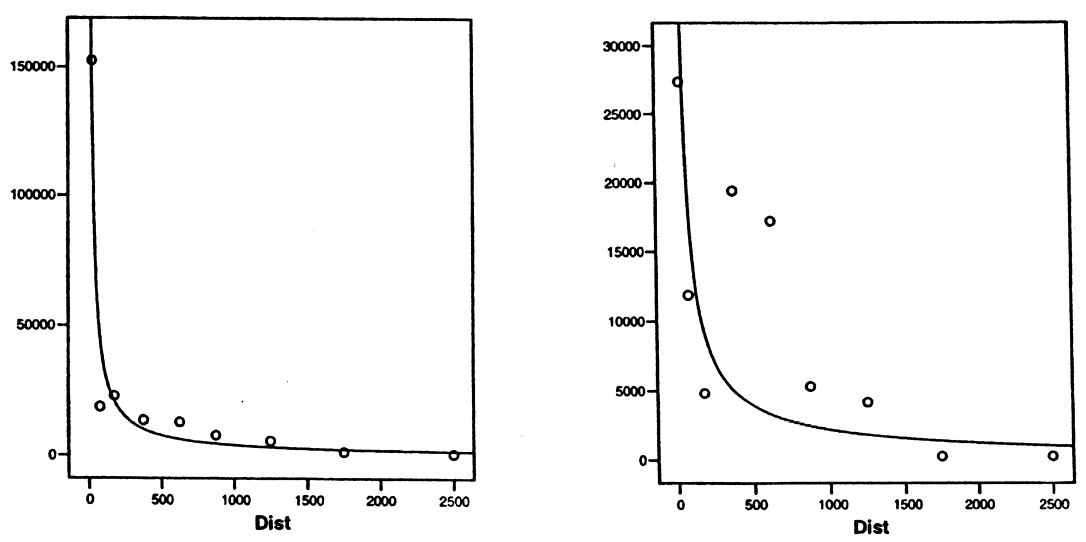

COM15

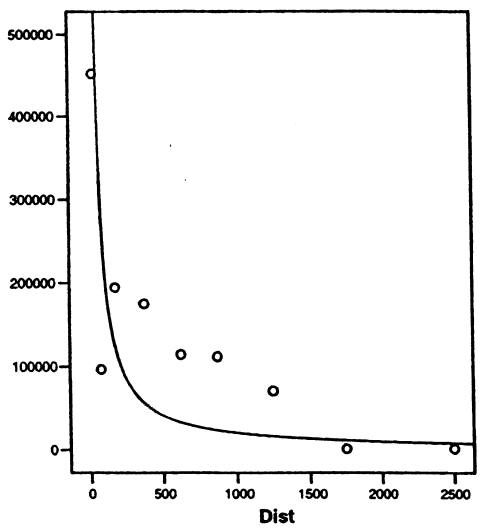

COM17

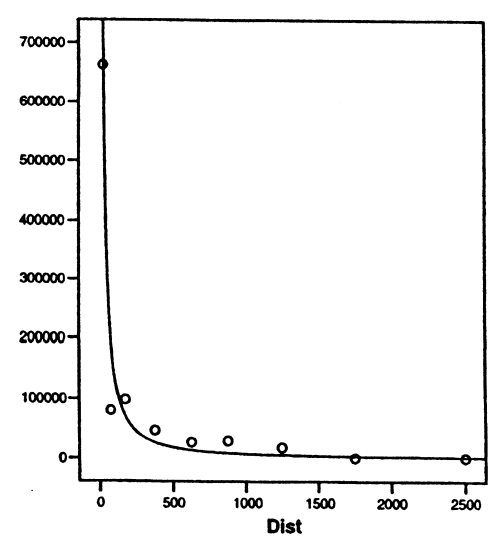

coM18

CoM19
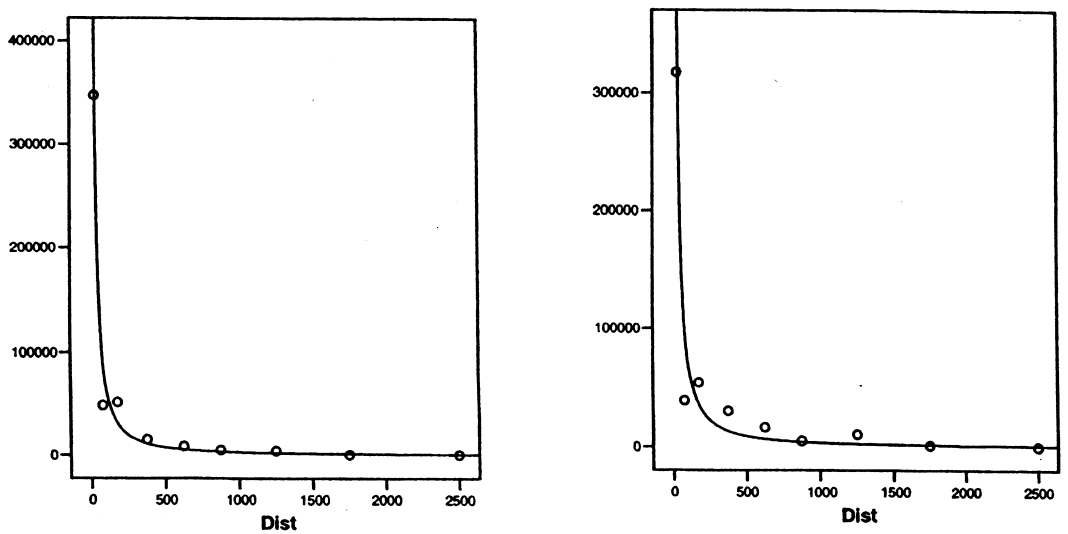
COM20

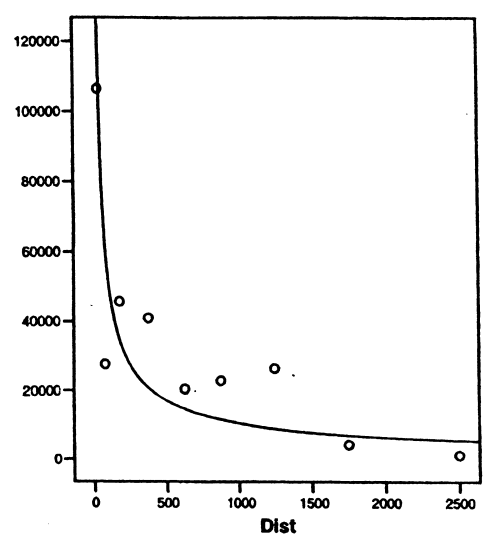

COM23
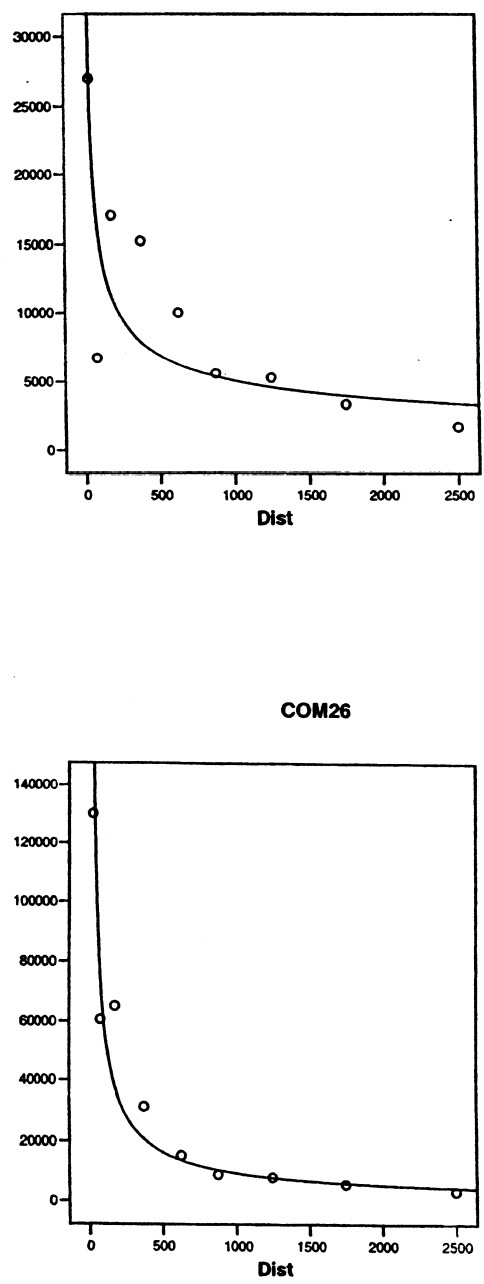

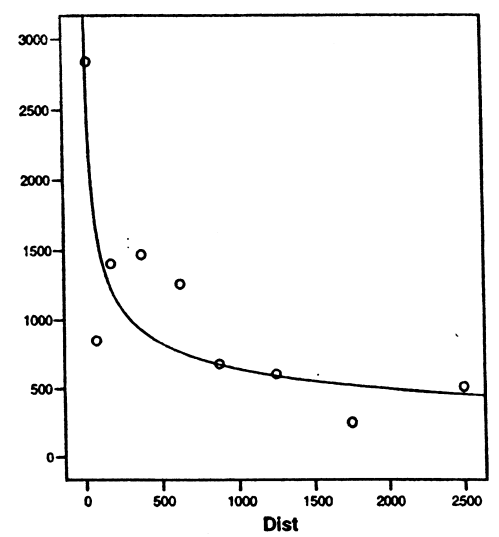

COM24

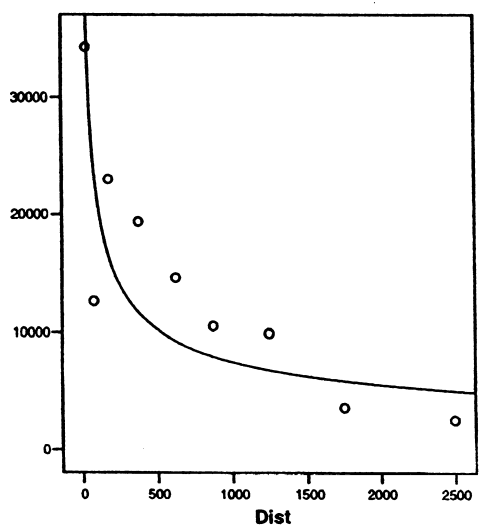

COM27

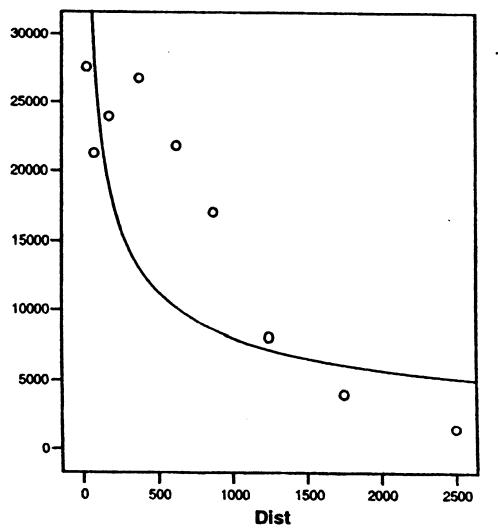

COM22

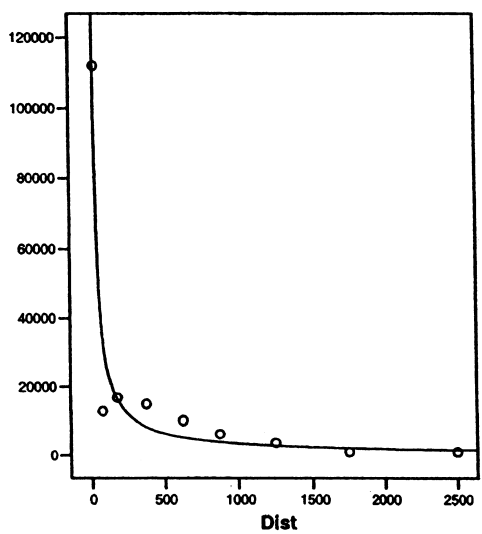

COM25

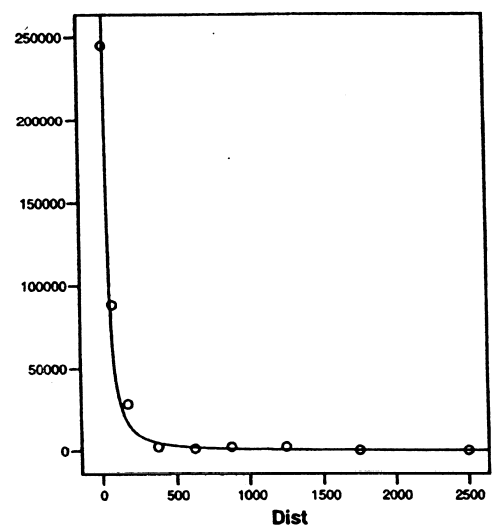

COM28

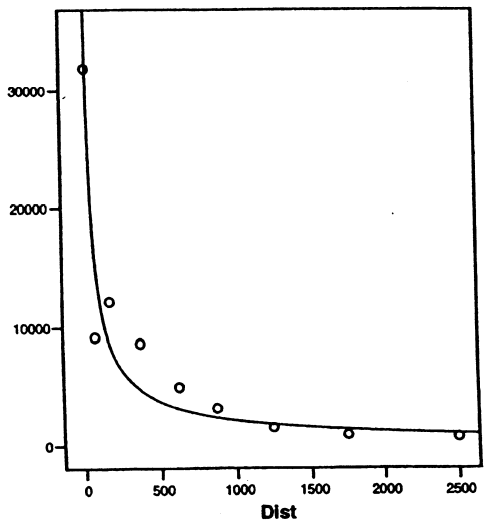



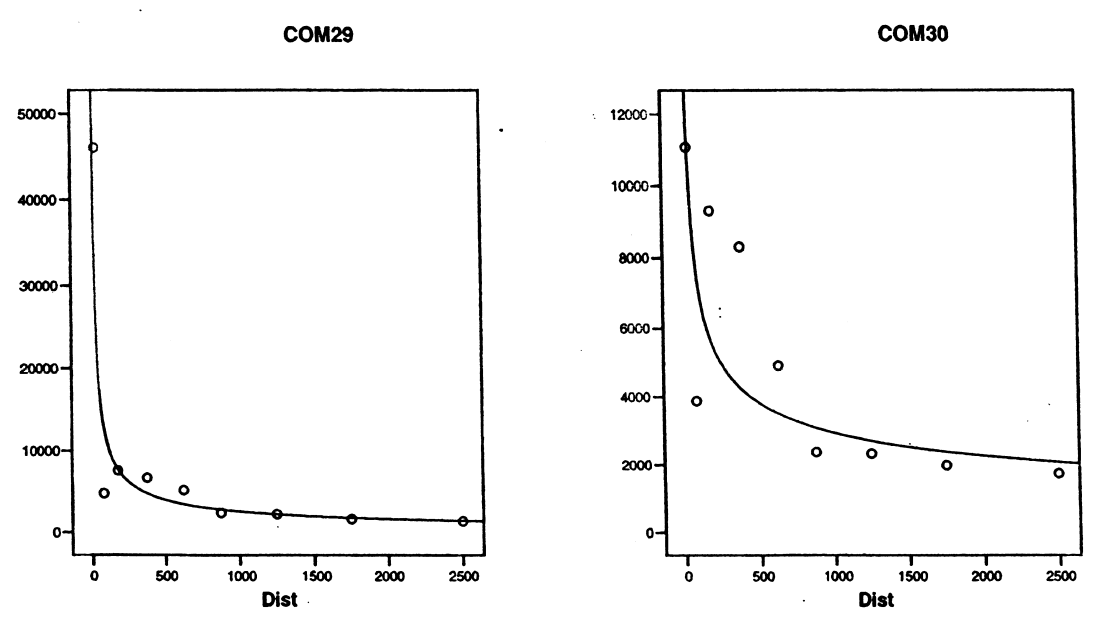

coM31

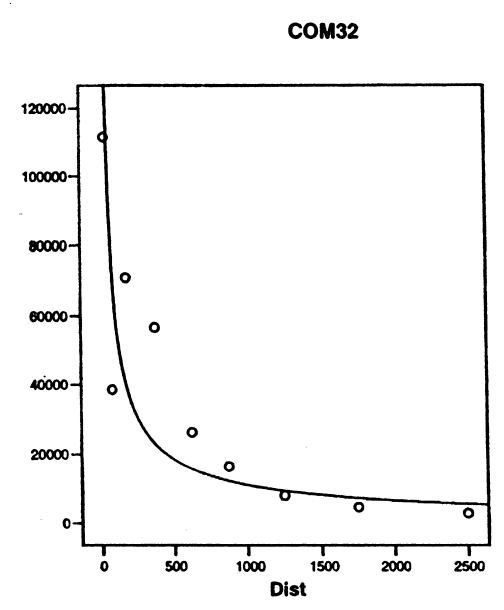

Com33
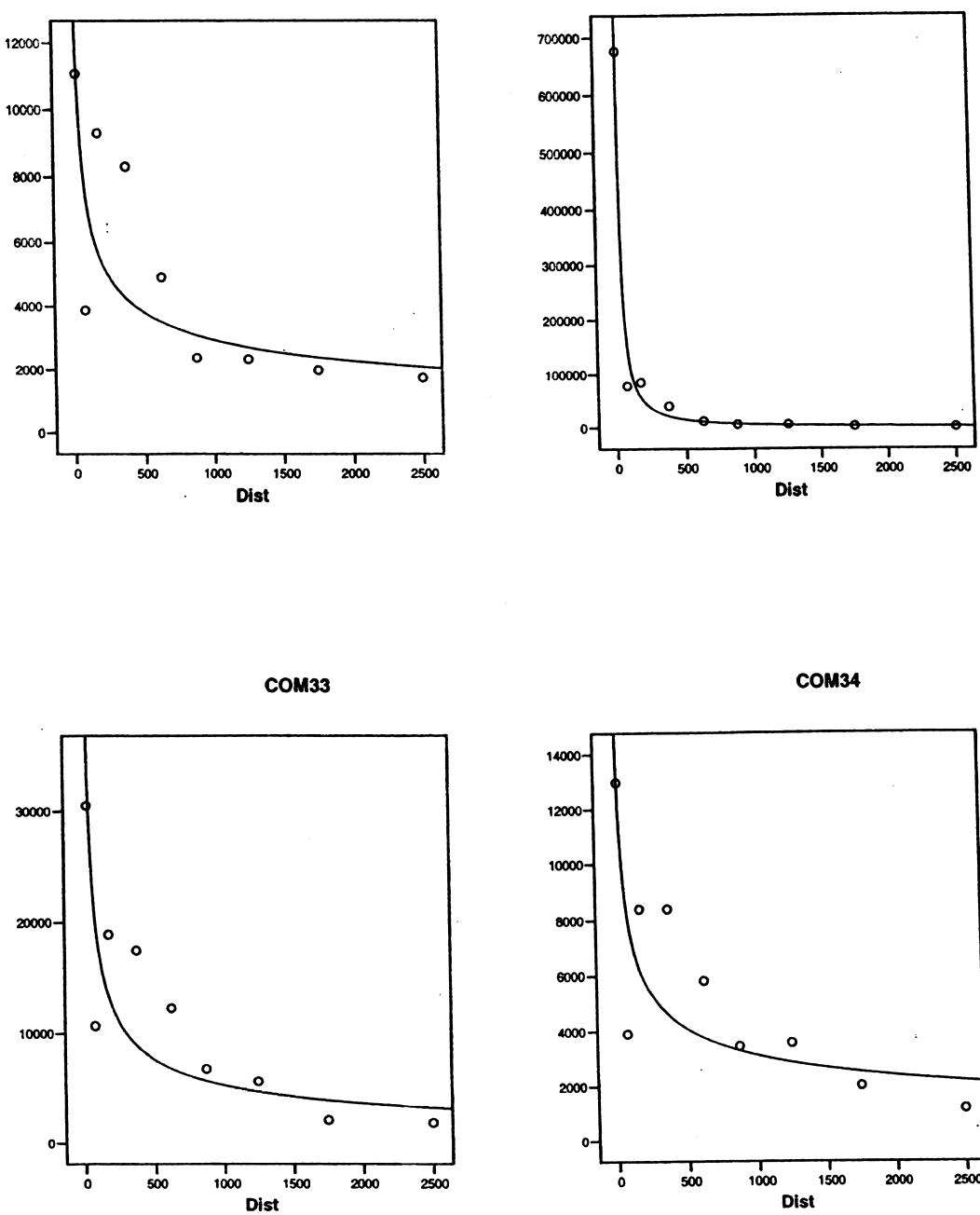

com34
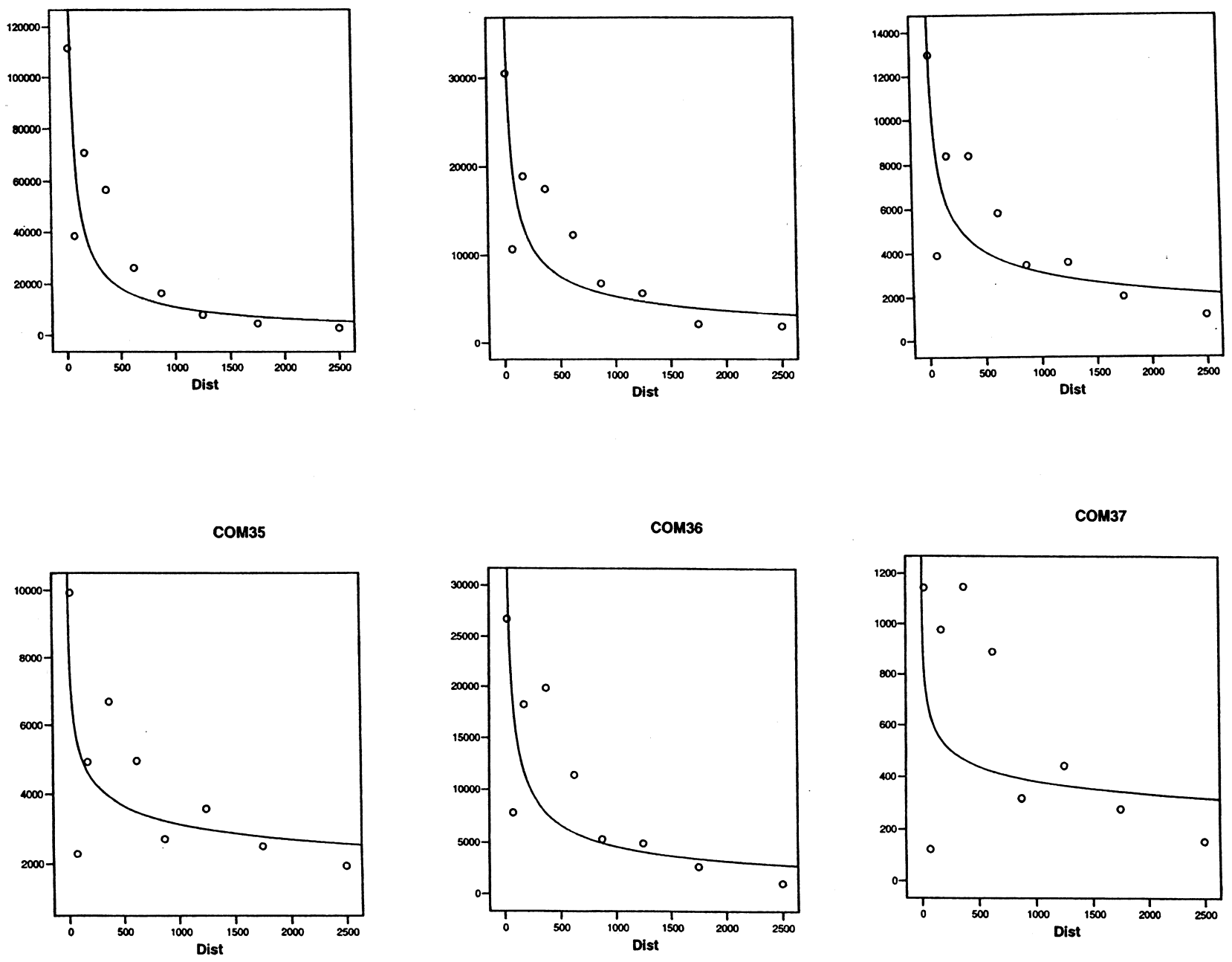

com37

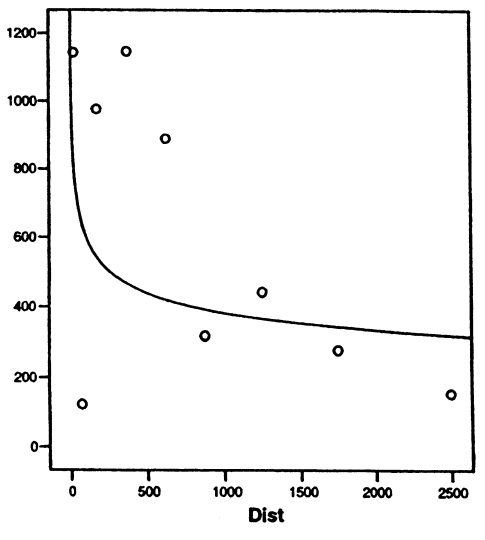


Freight Flows of Indiana

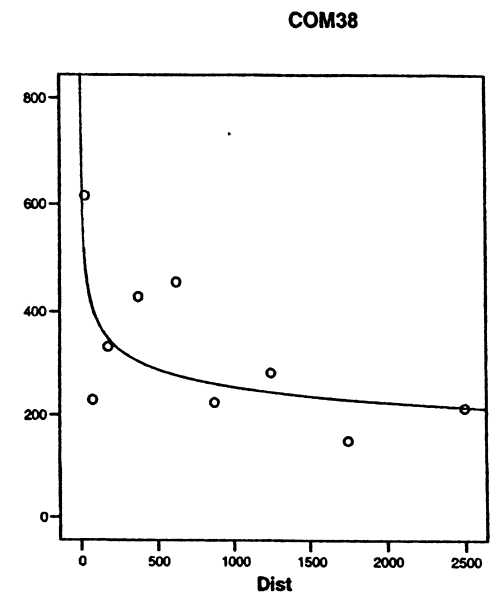

Final Report

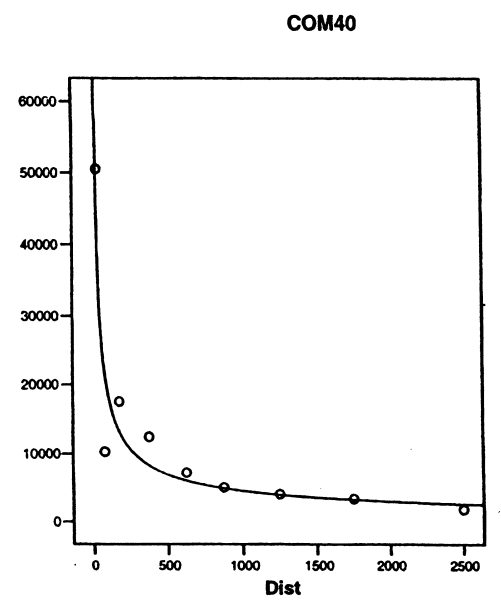

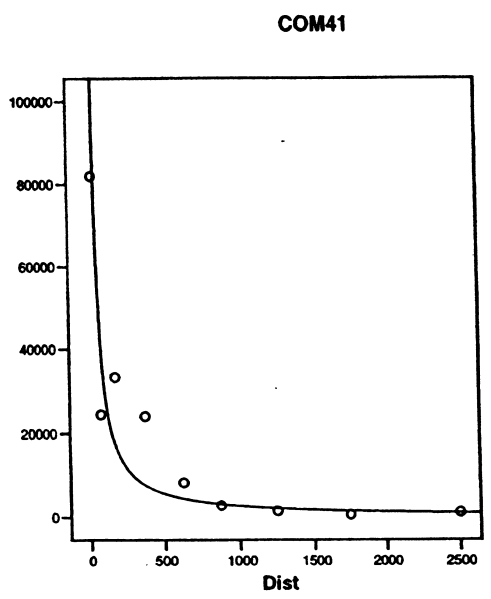

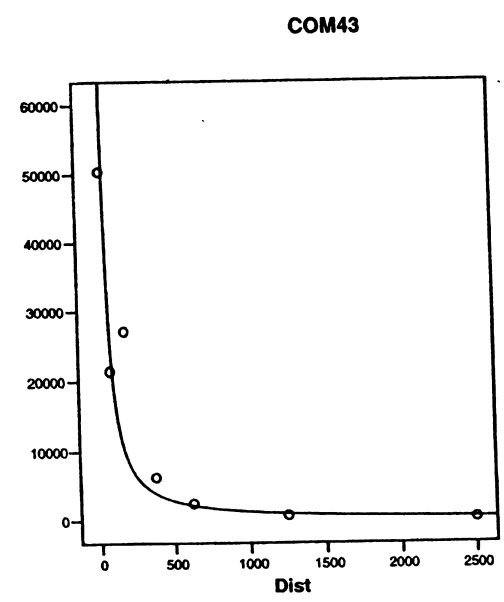


APPENDIX D

MODE CHOICE/DISTANCE TABLES 


\section{Table Notes}

The numbers that occupy the 21 pages of Appendix D give the proportion of traffic by commodity group using the principal modes of transportation of interest in the flow study. The commodities are represented by their individual Standard Commodity Transportation Group (SCTG) number. The commodities represented by these codes are identified in the text at the top of each table.

For each of the distance bands the proportion of traffic using each mode for those distances are given according to the Commodity Flow Survey for 1997. In the SCTG 01 table the value of 1.000 simply means that all movements of live animals and fish occur by truck according to the survey. Multiple modes are used for every other commodity. 

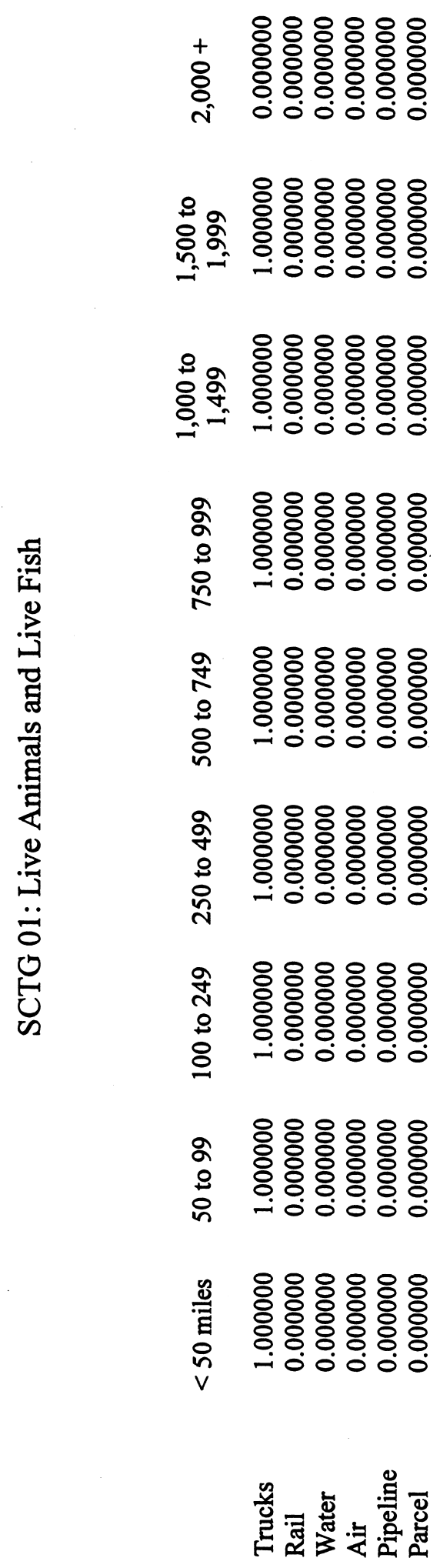

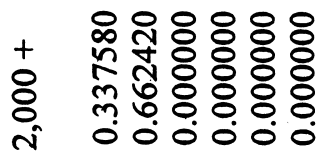

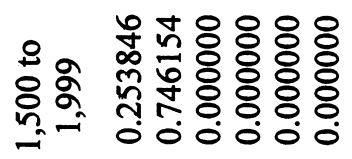

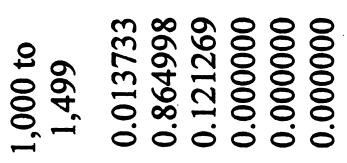

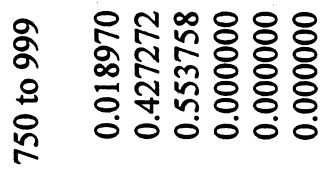

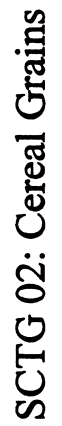

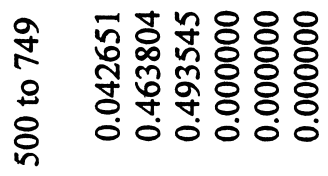

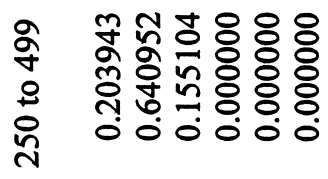

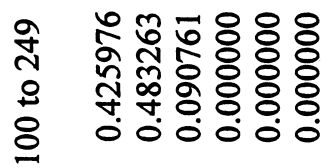

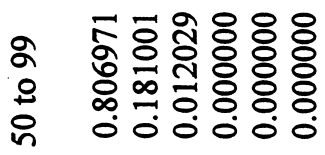

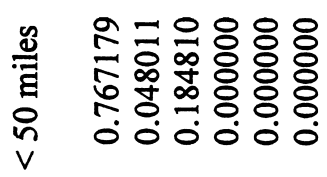

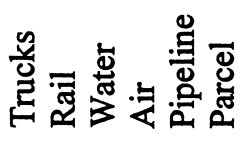




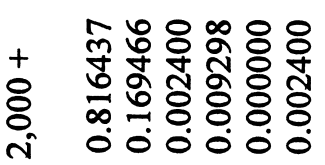

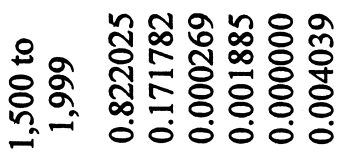

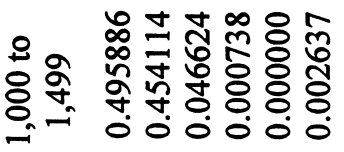

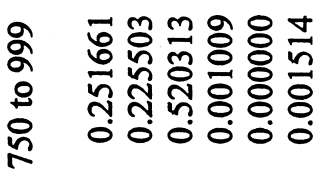

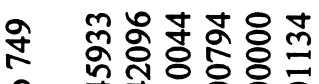

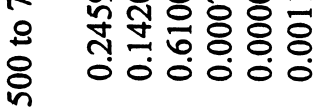

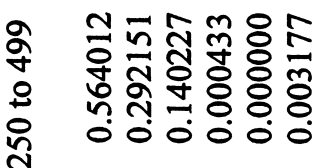

$\ddot{\sigma}$

ర્ટ

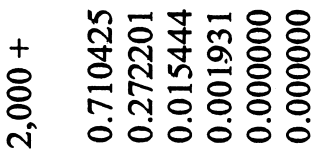

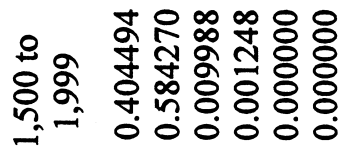

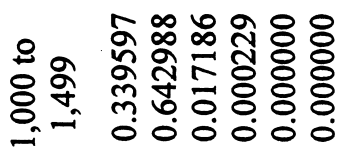

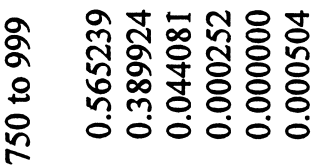

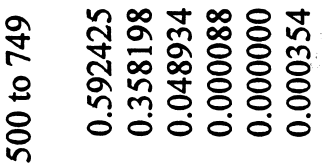

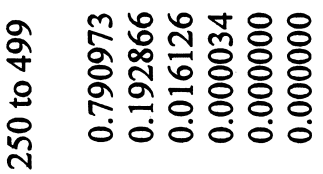

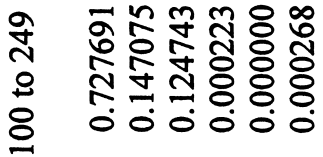

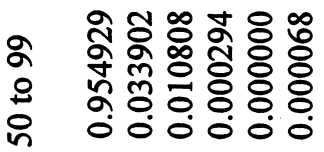

$\dot{\dot{d}}$

.ำ

สี

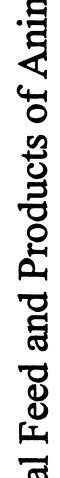

竞

$\ddot{\dot{\delta}}$

巳

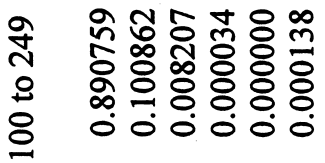

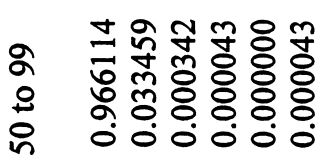

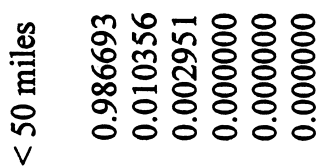

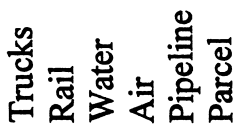




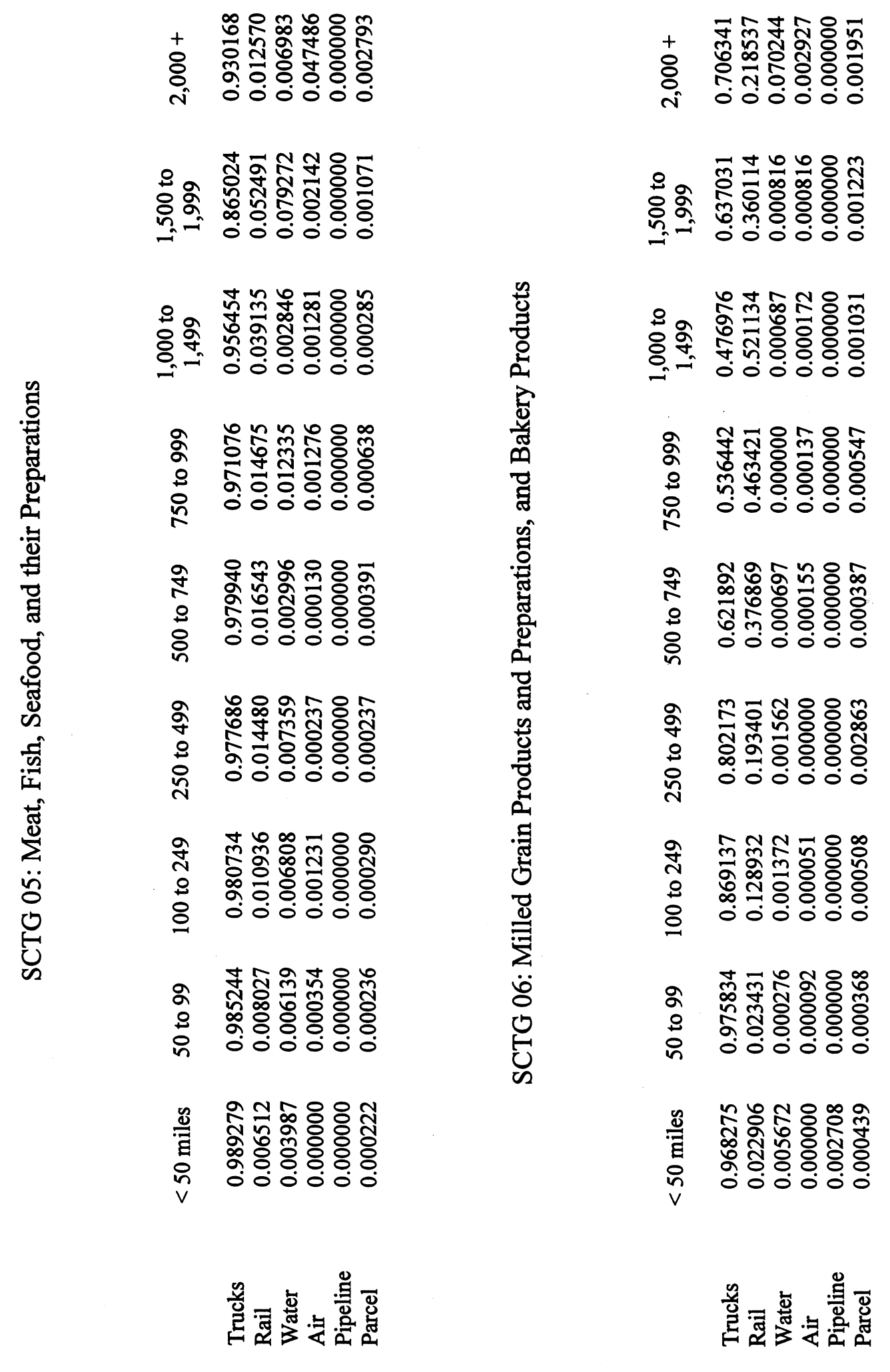




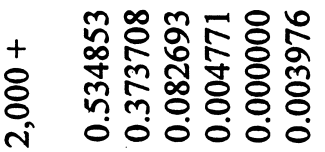

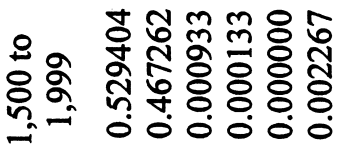

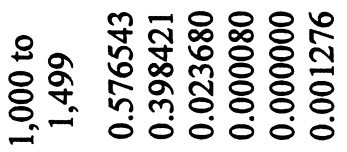

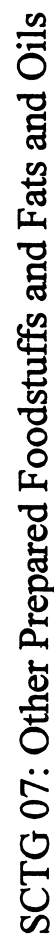

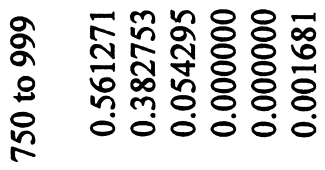

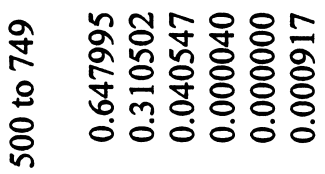

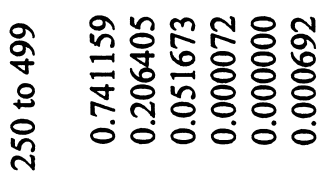

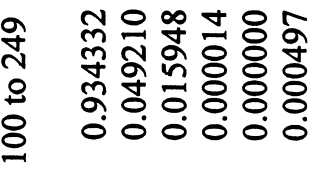

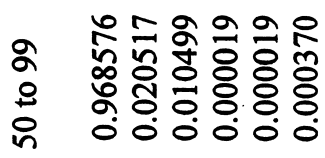

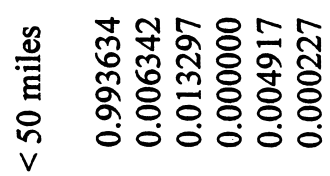

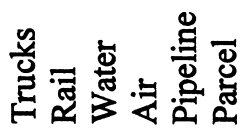

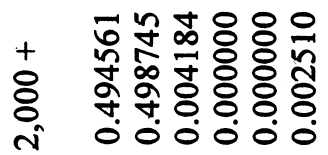

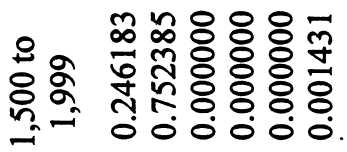

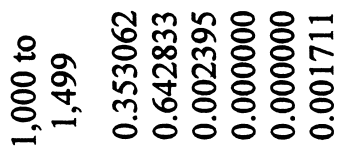

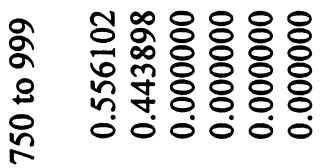

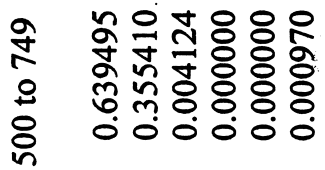

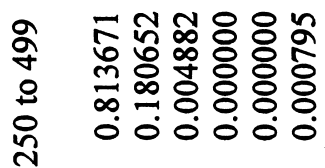

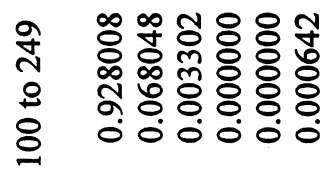

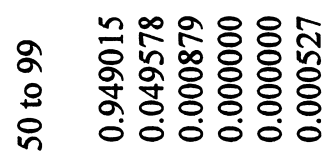

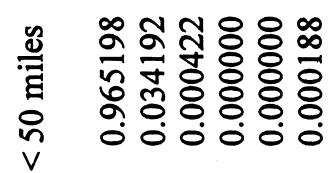

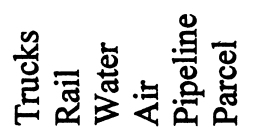




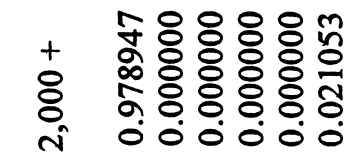

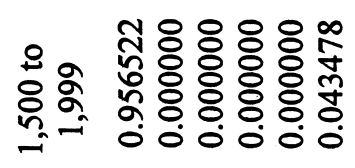

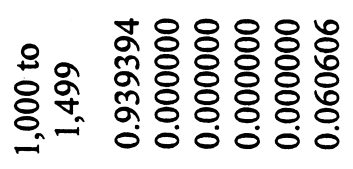

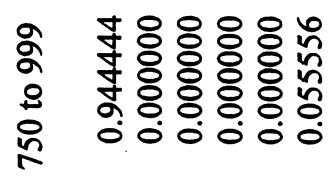

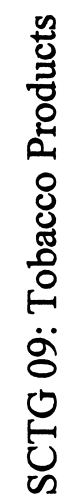

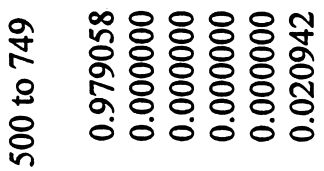

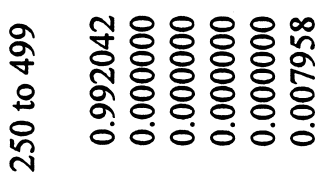

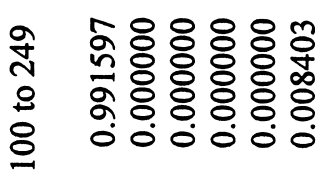

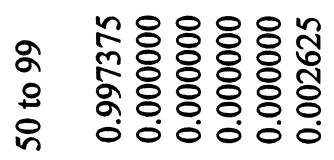

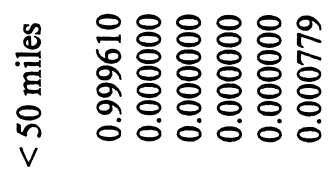

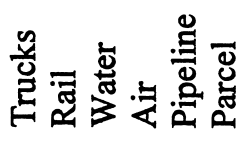

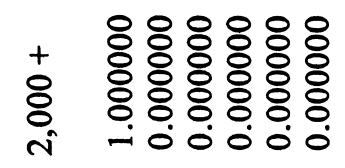

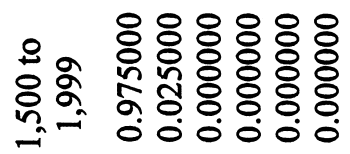

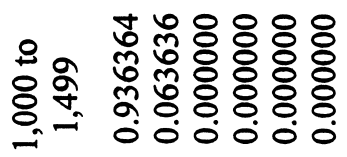

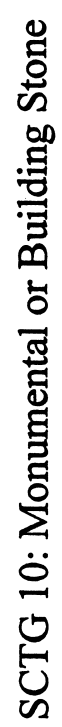

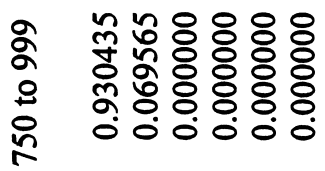

눅 융융융

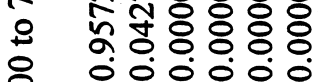

\& 000000

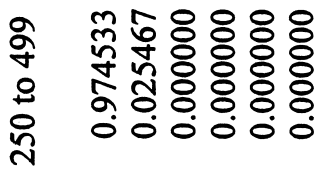

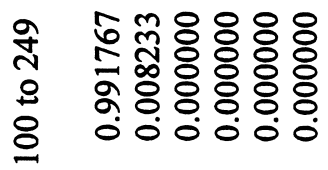

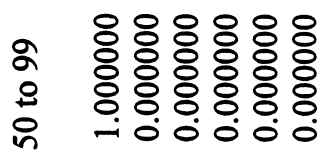

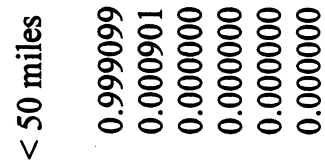

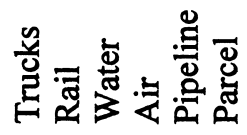




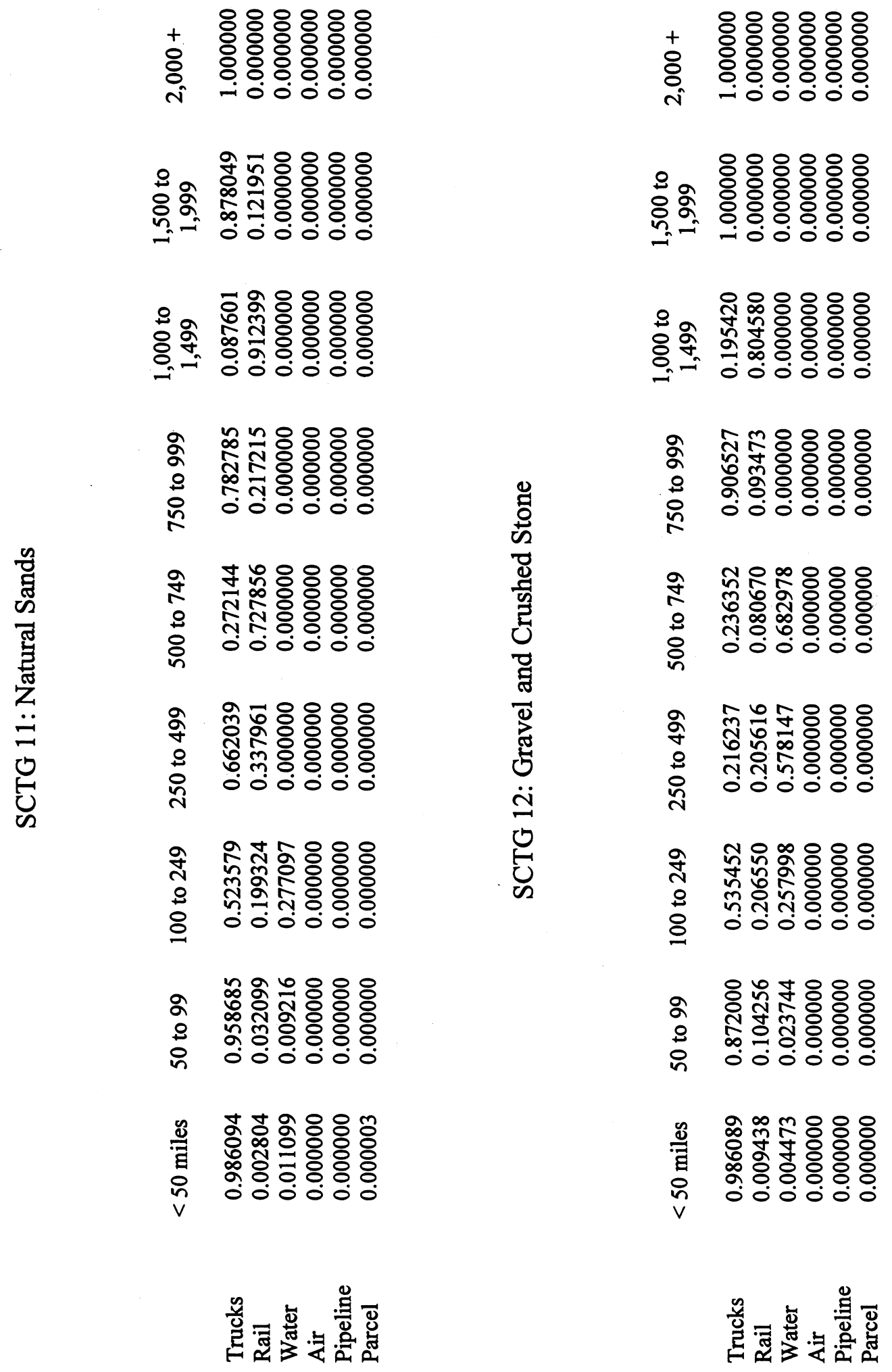




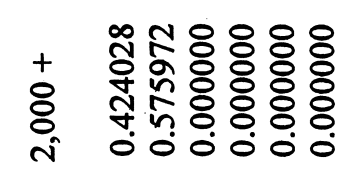

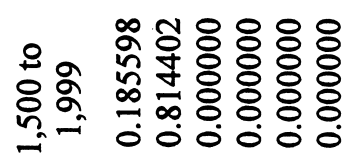

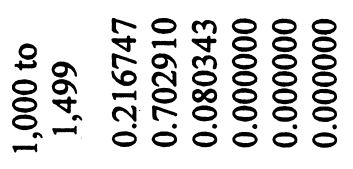

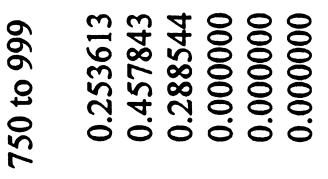

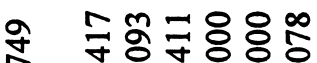

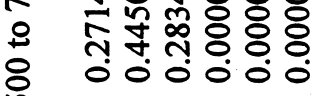

을

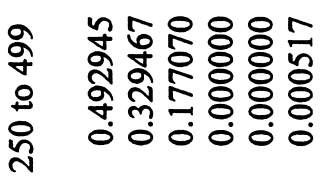

$\ddot{m}$

葛

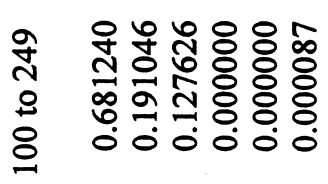
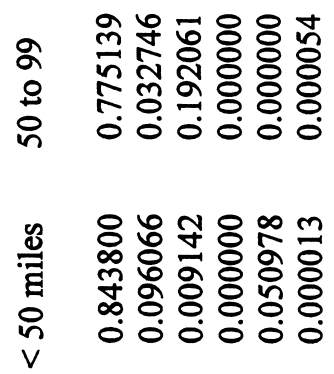

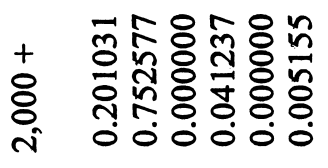

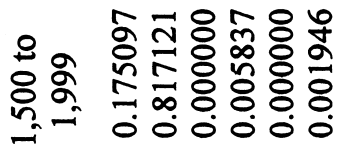

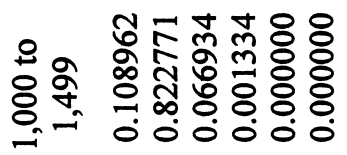

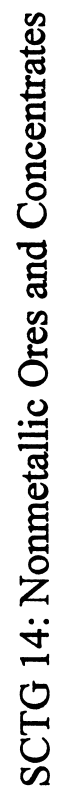

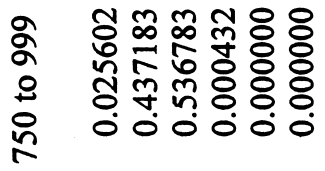

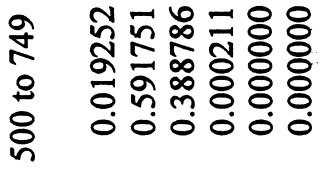

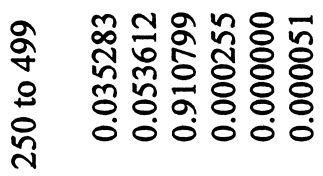

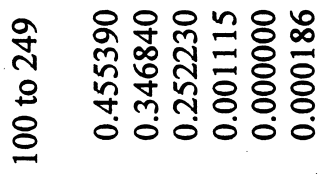

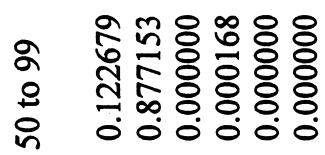

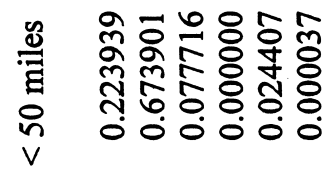

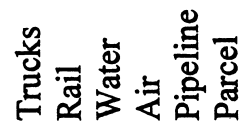




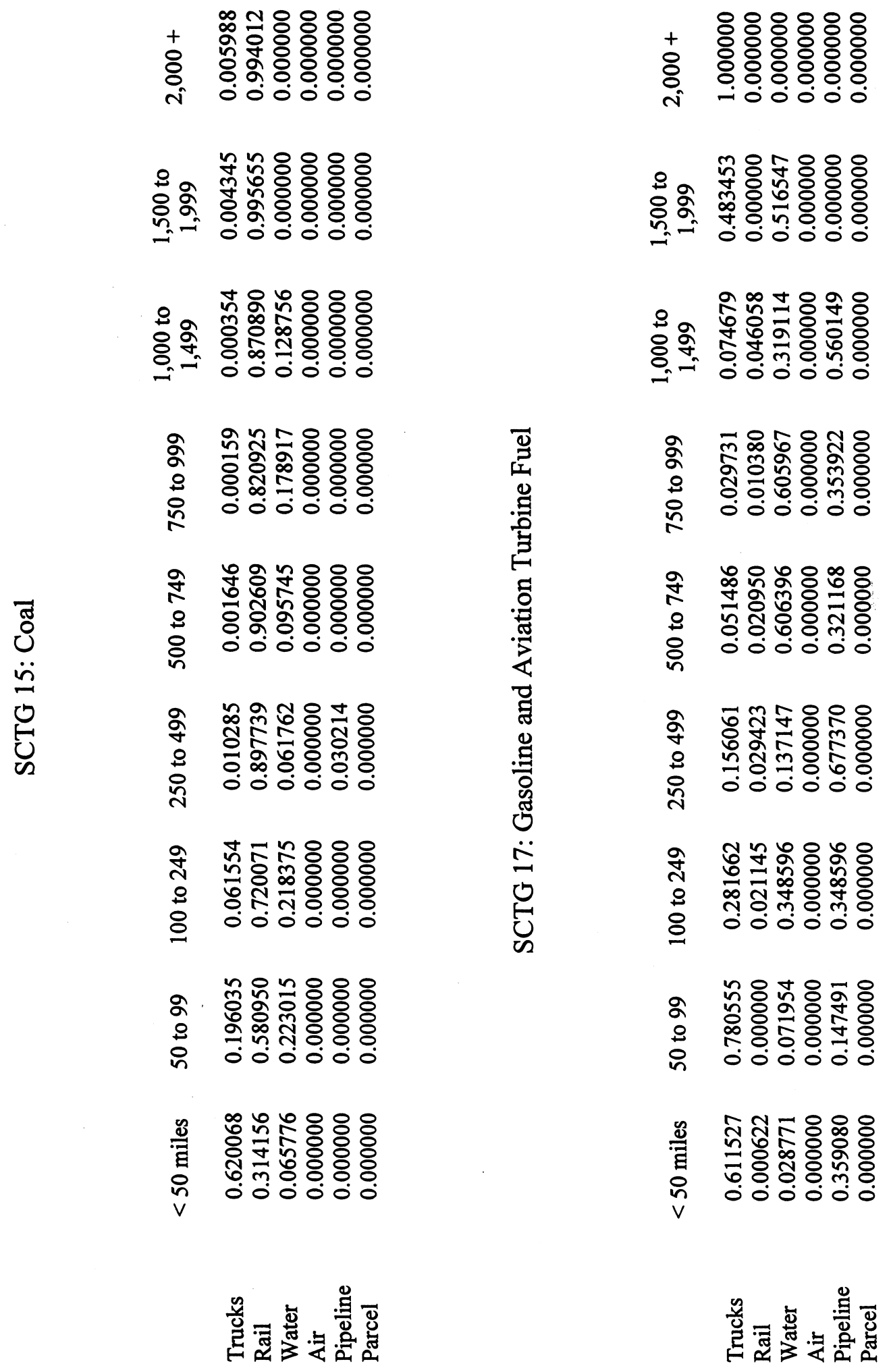




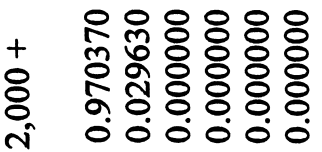

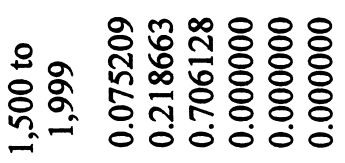

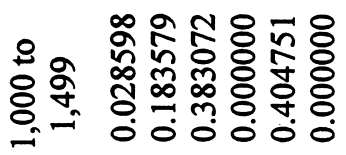

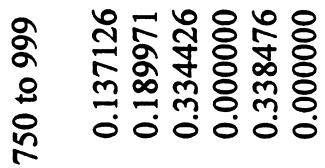

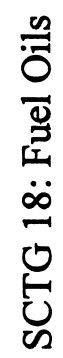

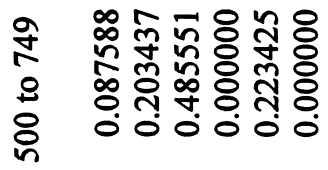

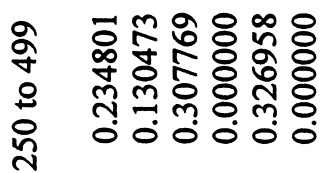

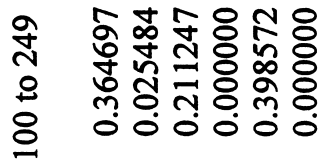

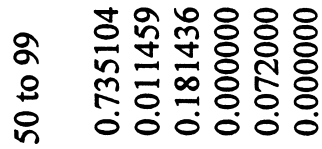

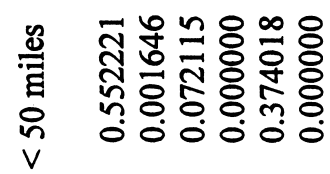

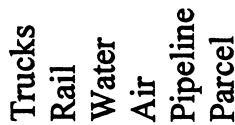

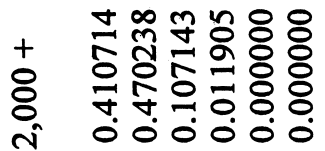

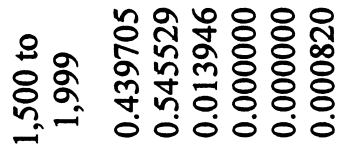

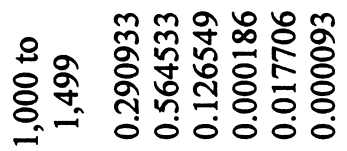

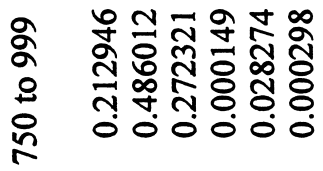

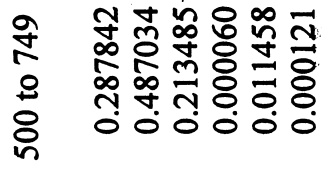

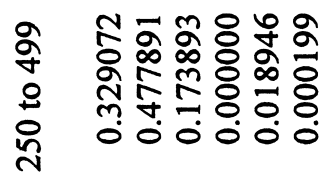

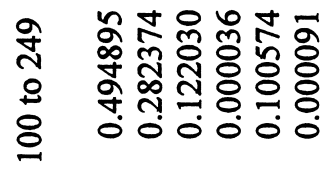

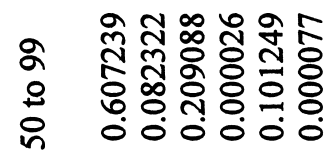

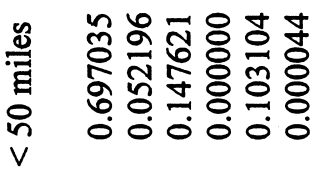

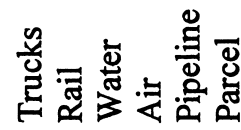




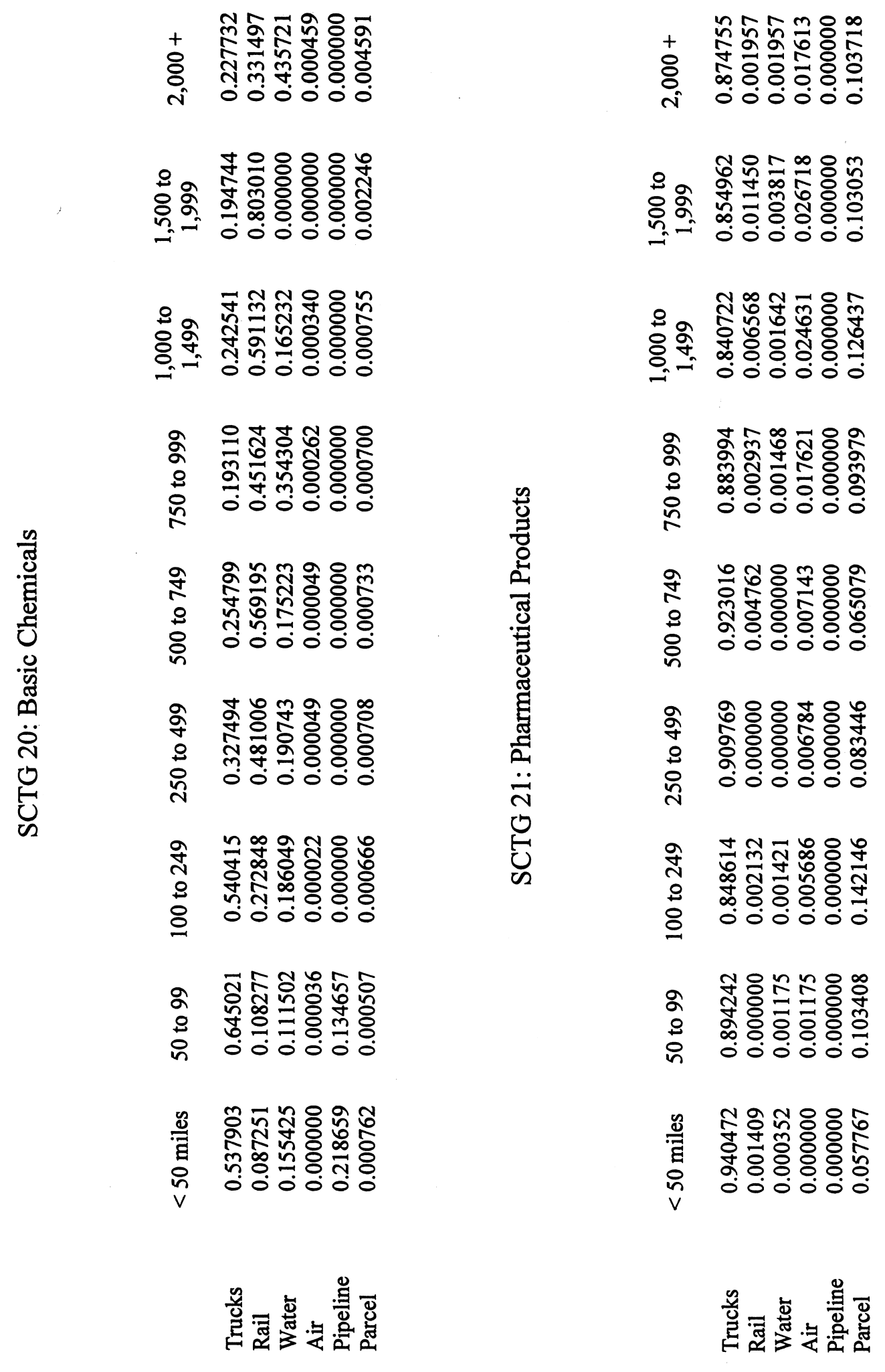

D12 


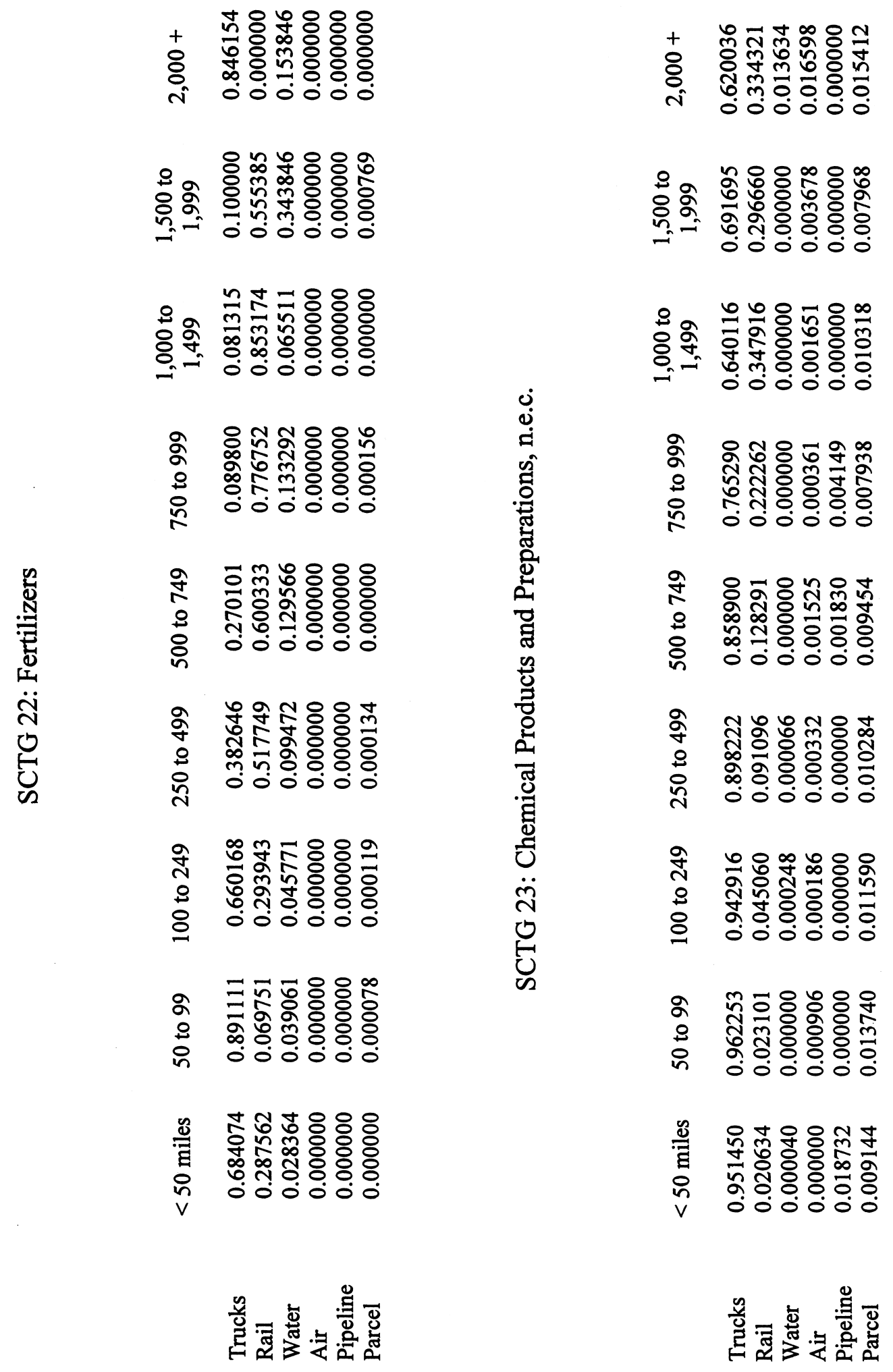



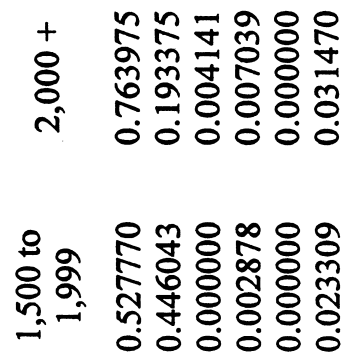

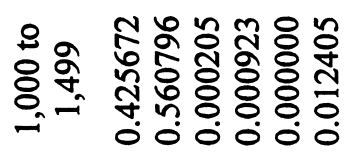

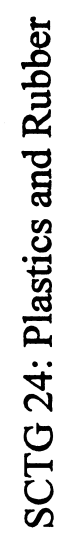

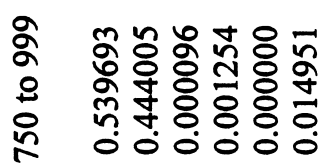

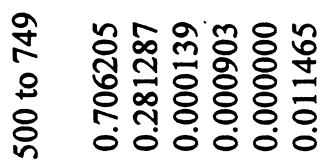

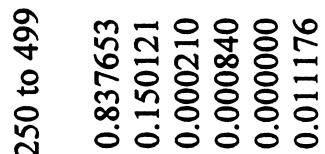

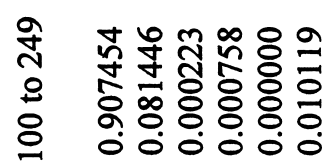

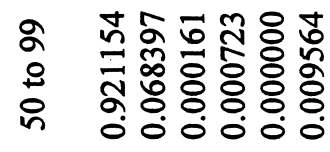

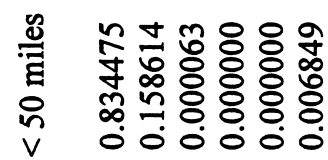

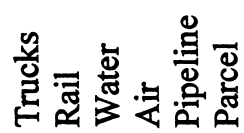

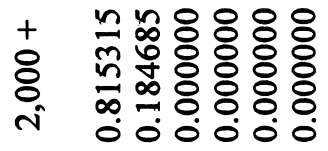

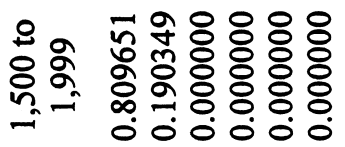

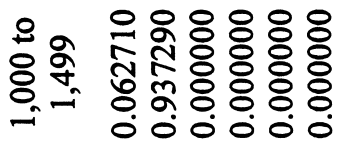

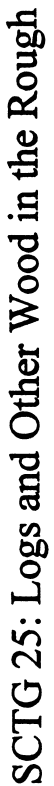

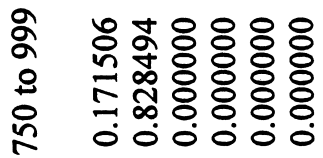

ㅇำ

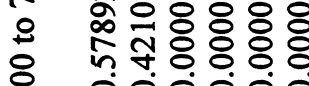

品 000000

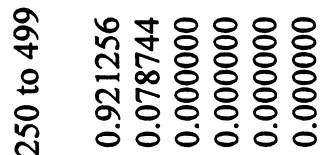

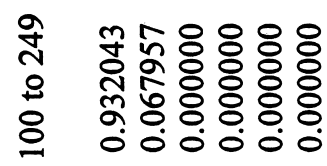

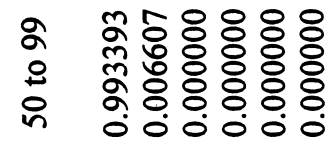

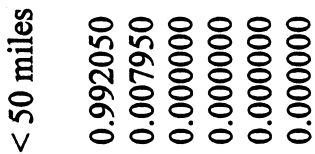

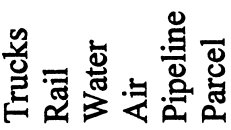



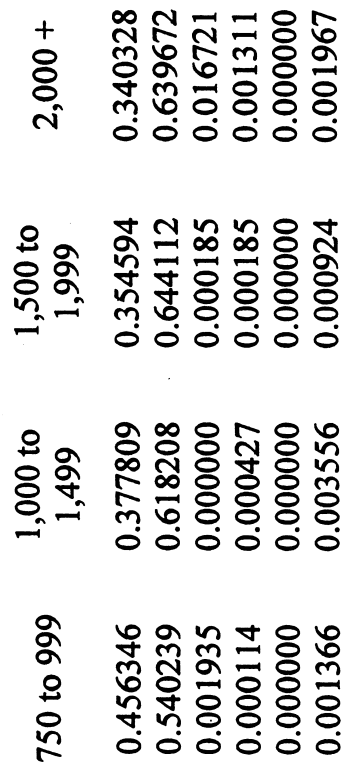

0
0
0
0
0
0
0
0
0
0
0
0
0
0
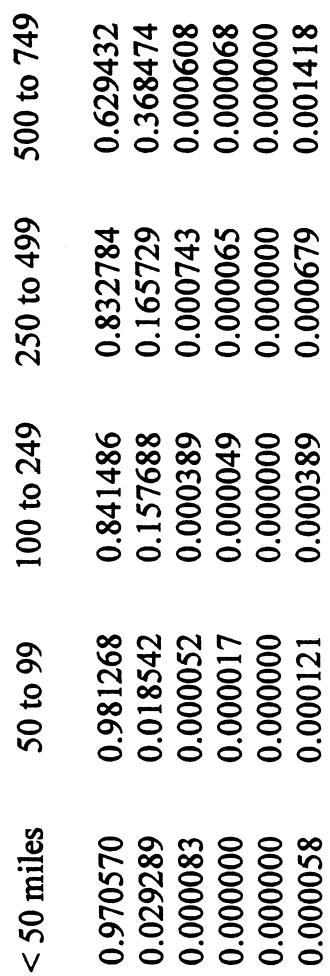

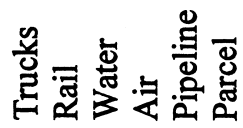

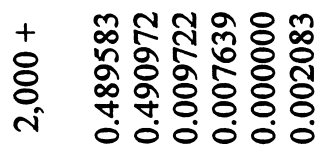

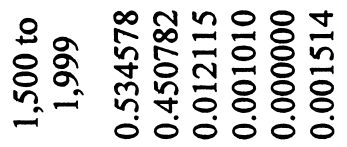

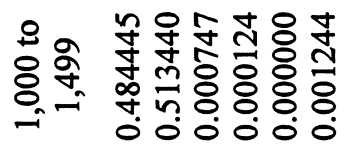

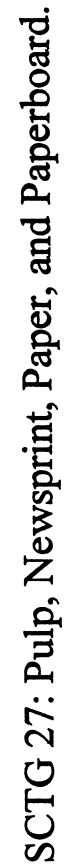

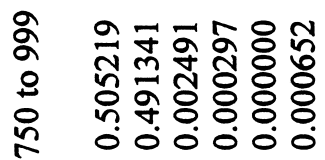

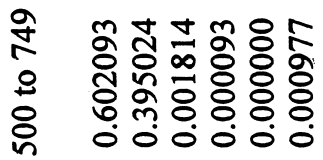

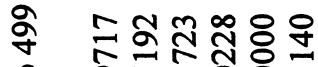

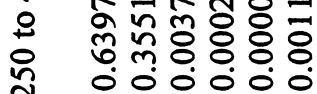

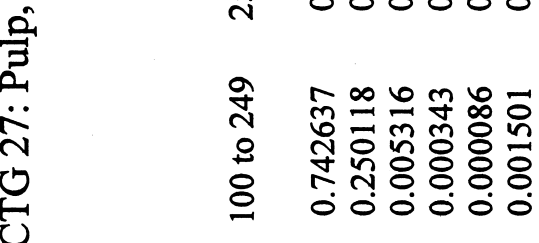

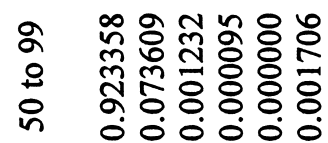

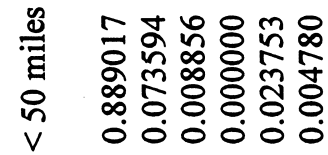

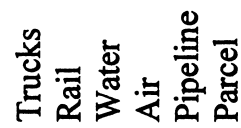




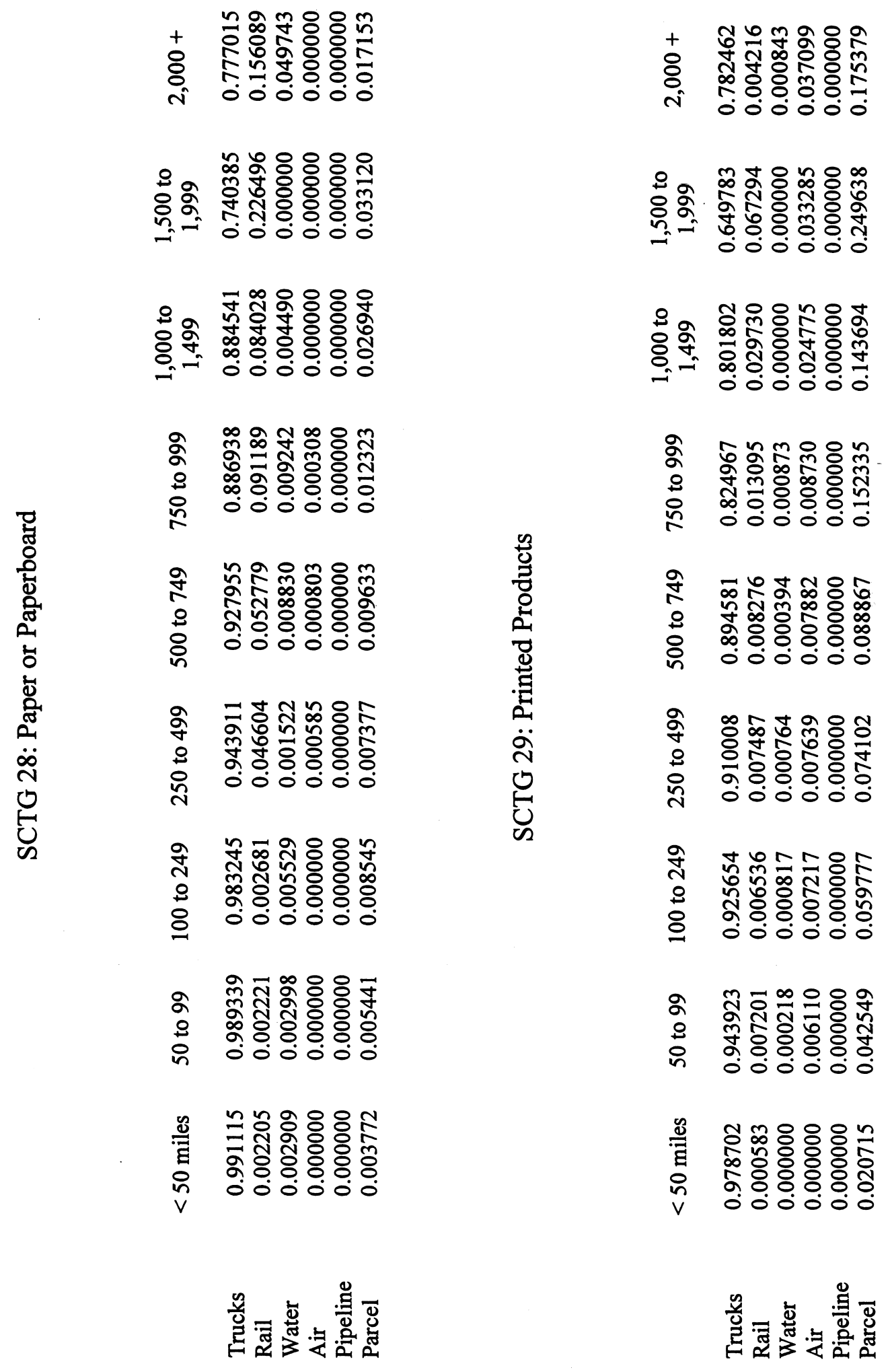

D16 


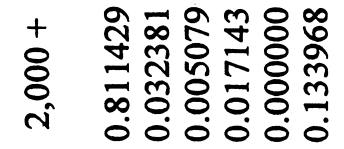

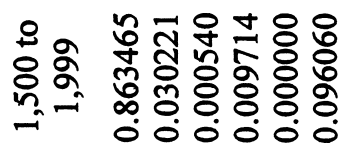

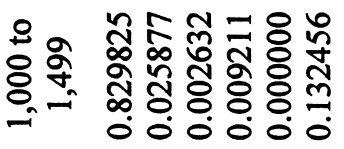

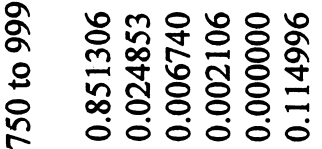

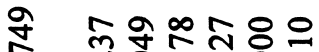

ำㅇํㅇㅇㅇㅇㅇㅇㅇㅇㅇㅇㅇㅇ

웅ㅎㅇ융ㅇㅇ

育

密

莺

官

Ч્ఝ

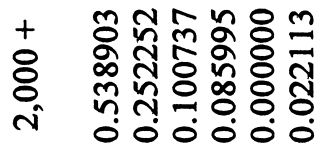

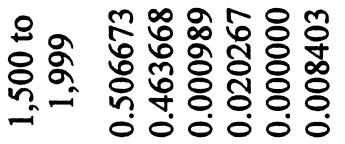

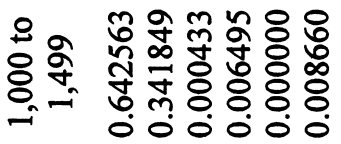

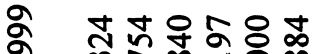

范

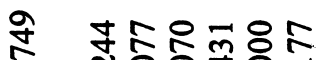

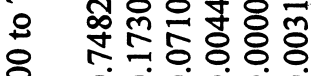

号

号

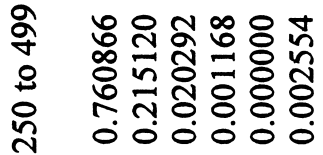

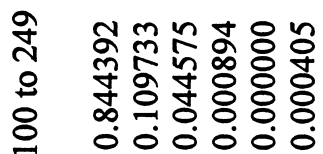

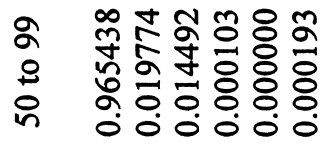

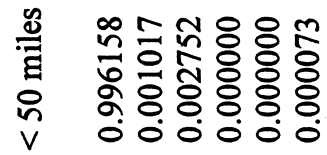

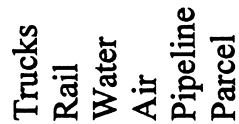




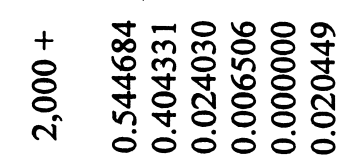

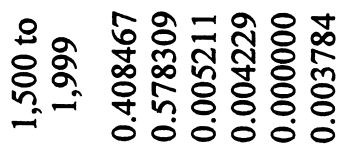

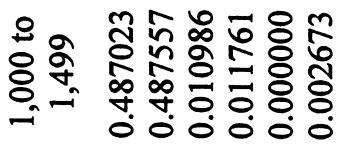

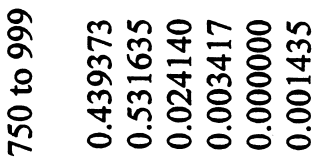

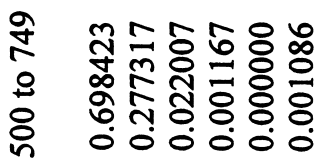

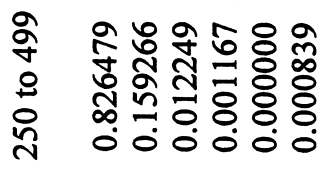

ปิ

\&

丞

离

$\ddot{\text { ஸे }}$

巳ั

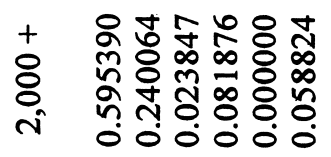

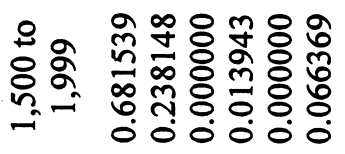

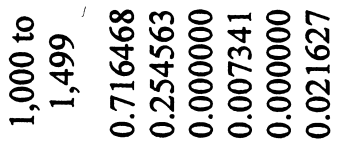

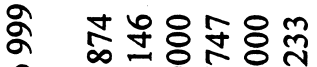

总

퓰

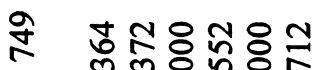

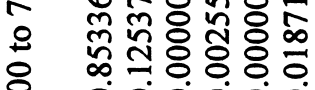

क

总

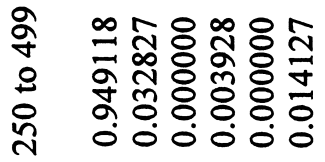

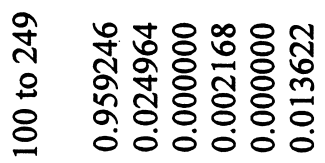

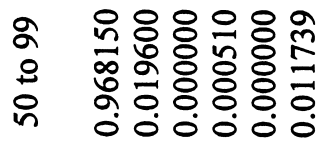

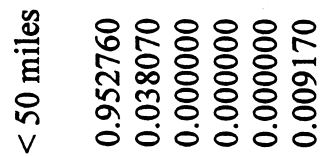

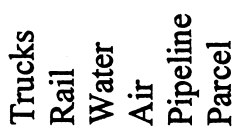




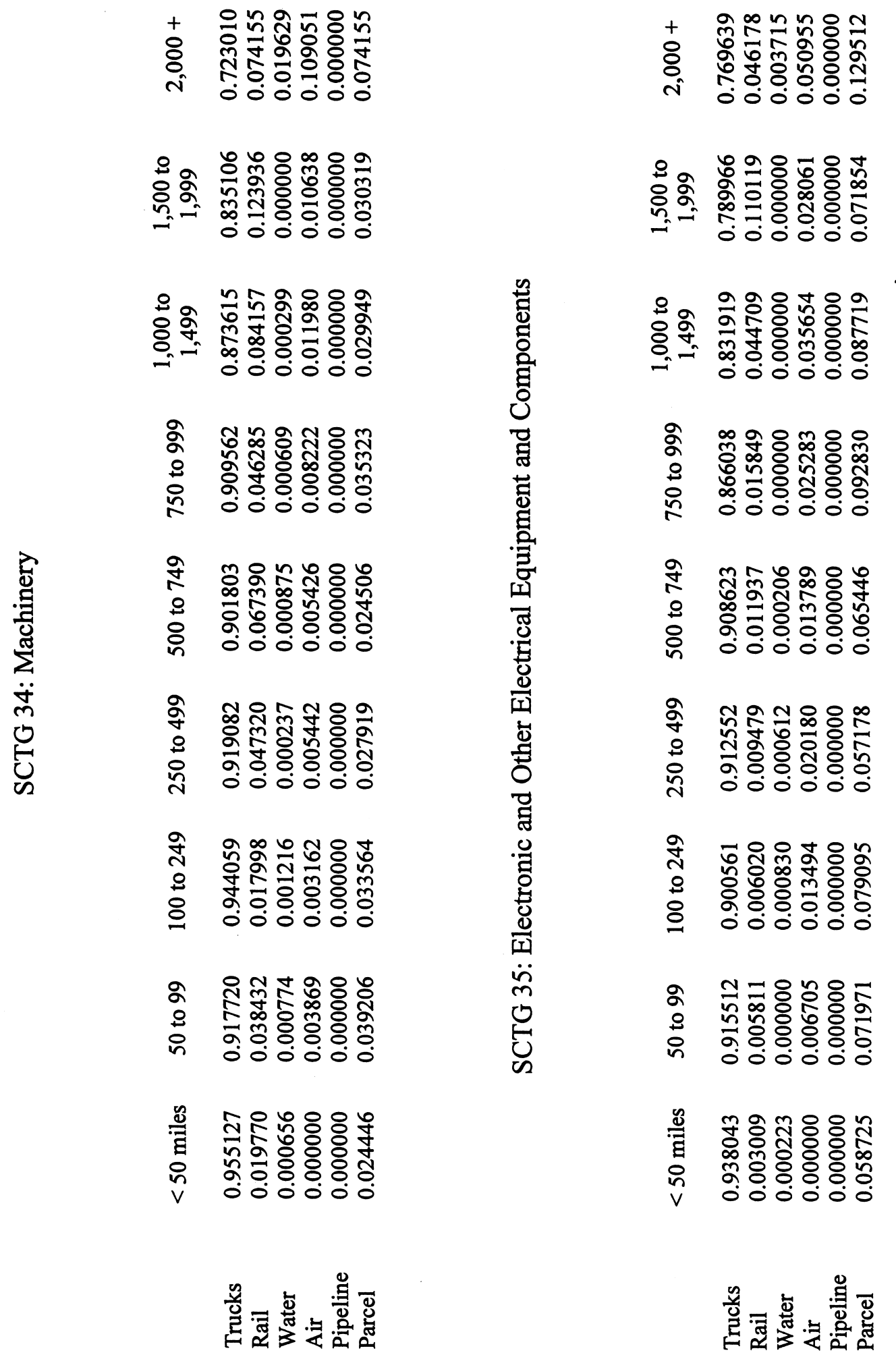




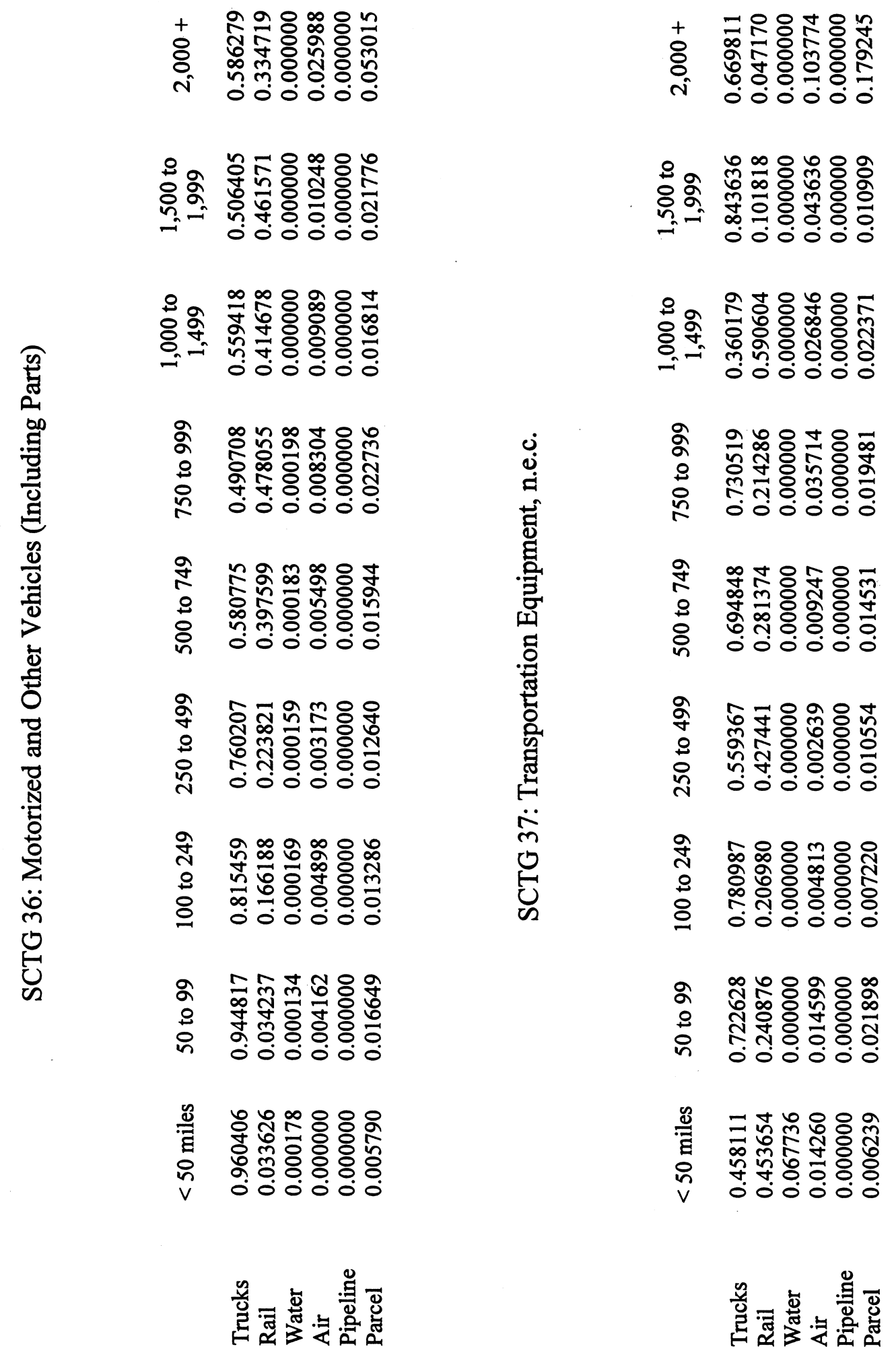




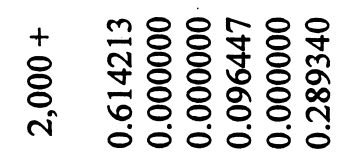

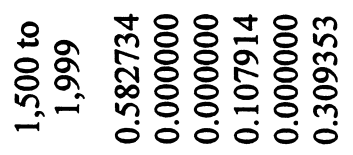

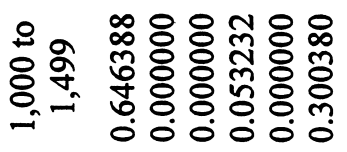

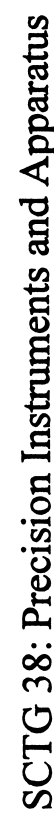

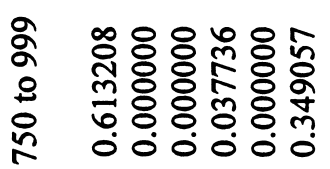

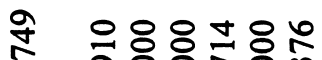

品

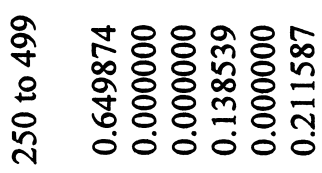

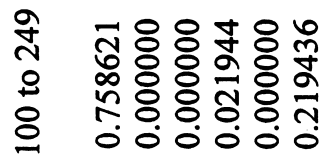

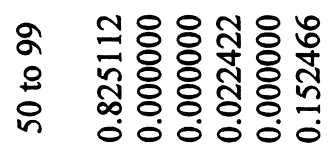

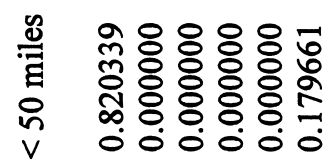

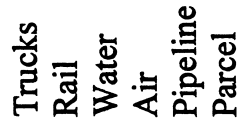

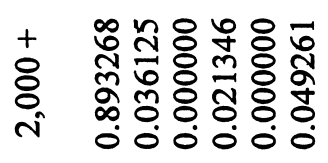

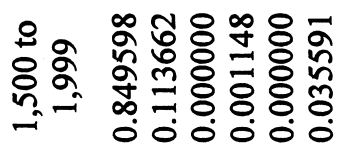

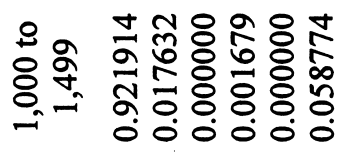

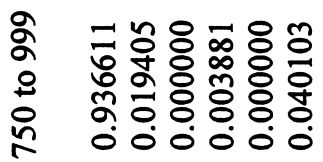

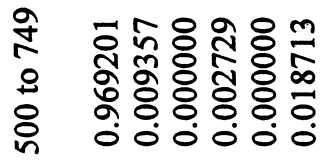

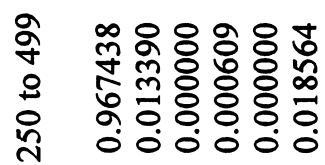

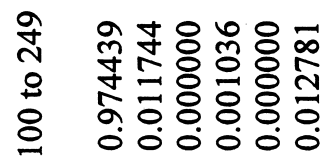

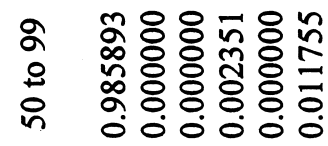

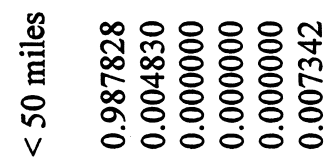

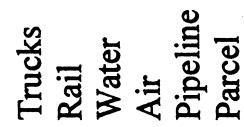



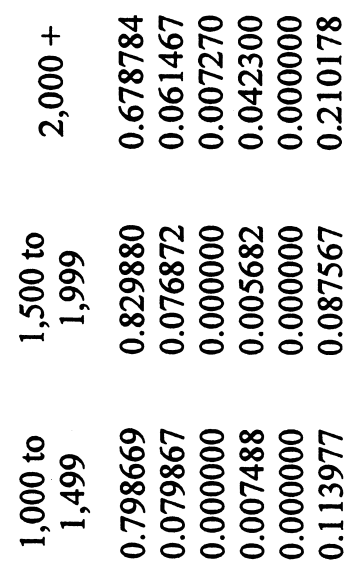

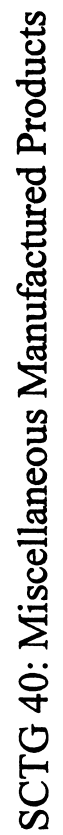

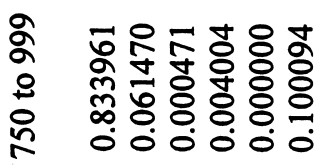

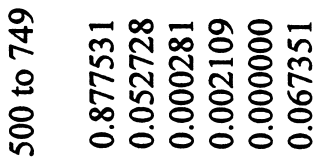

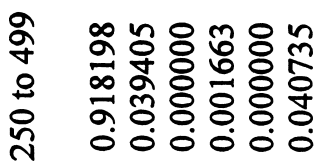
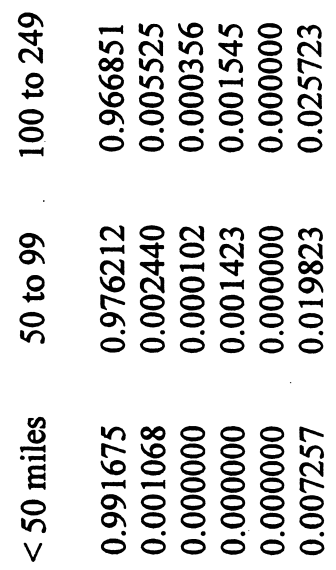

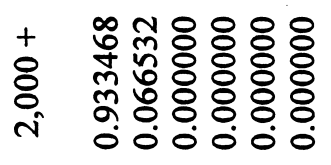

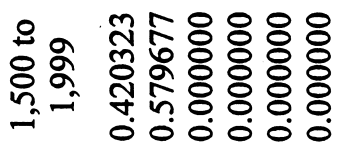

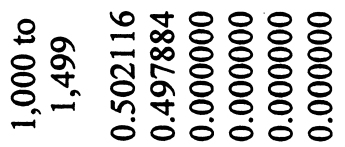

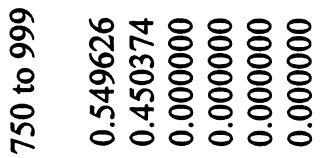

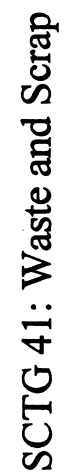

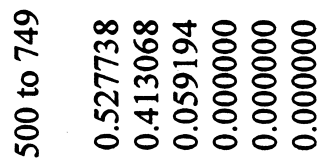

बे $\bar{\sigma} \bar{\circ}$

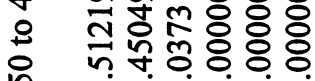

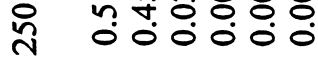

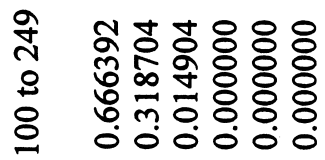

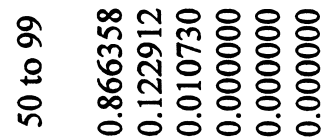

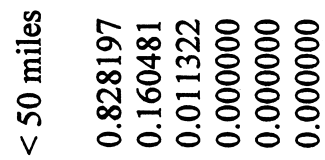

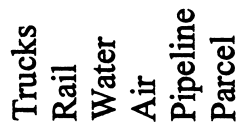




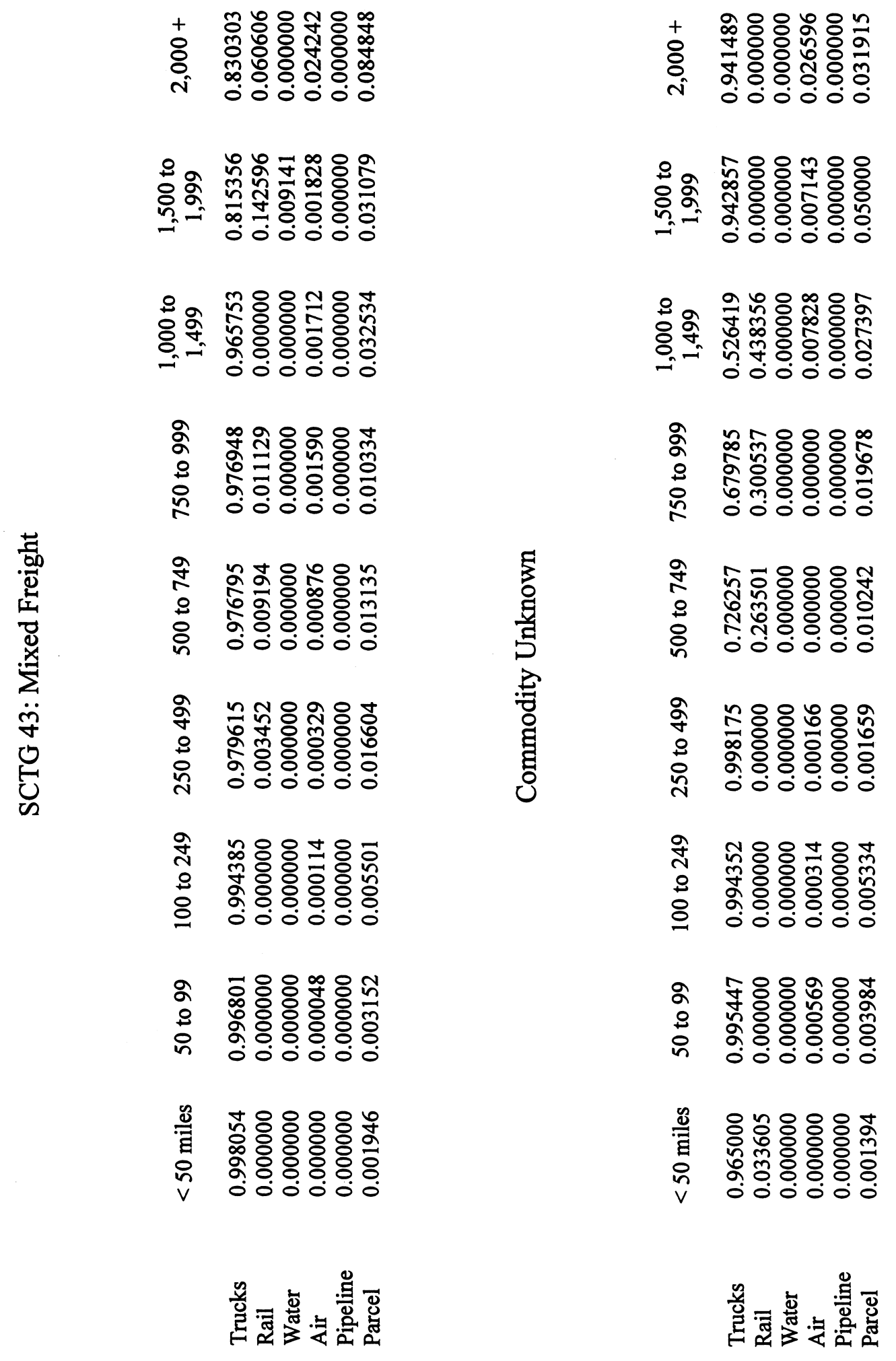


APPENDIX E

PRODUCTIONS AND ATTRACTIONS 2015 


\section{Table Notes}

The tables in this appendix give the projected volume of traffic produced and attracted by areas included in this study for each commodity. The area appears under the column label Locale and includes the 145 different areas included in the freight study. From line 14 to line 105 we have the counties of Indiana. States represented by two or more nodes are evident here as well.

The data columns labeled $\mathrm{P}$ are productions and those labeled $\mathrm{A}$ are attractions. The next two digits represent the commodity groups SCTG 1, SCTG 2, and so forth. The 15 represents the forecast year of the data, 2015.

The data are actually given here to three decimal places. This is because the data as published is in thousands of tons. In many cases this would completely eliminate flows into Indiana counties of several goods. It is for this reason that three decimal places were used. Programs used later would multiply flow estimates by 1,000 to yield tons of commodities. The reader should judge the reliability of the data given these facts. 


\begin{tabular}{|c|c|c|c|}
\hline & Locale & P01 15 & $\mathrm{~A} 01 \quad 15$ \\
\hline 1 & Alabama & 279.990 & 273.215 \\
\hline 2 & Arizona & 207.510 & 98.289 \\
\hline 3 & Arkansas & 241.367 & 426.394 \\
\hline 4 & California & 1429.409 & 1313.489 \\
\hline 5 & Colorado & 106.431 & 160.111 \\
\hline 6 & Connecticut & 57.282 & 71.237 \\
\hline 7 & Delaware & 14.183 & 83.749 \\
\hline$\overline{8}$ & DC & .000 & .000 \\
\hline 9 & Florida & 440.078 & 366.133 \\
\hline 10 & Georgia & 330.783 & 563.707 \\
\hline 11 & Idaho & 39.779 & 152.900 \\
\hline 12 & Illinois_N & 253.183 & 439.435 \\
\hline 13 & Illinois_S & 126.591 & 219.718 \\
\hline 14 & Adams & 5.283 & 2.920 \\
\hline 15 & Allen & 19.887 & 13.961 \\
\hline 16 & Bartholomew & 14.827 & 10.824 \\
\hline 17 & Benton & 1.033 & .000 \\
\hline 18 & Blackford & .164 & .414 \\
\hline 19 & Boone & 1.436 & .101 \\
\hline 20 & Brown & .000 & .077 \\
\hline 21 & Carroll & 7.121 & 14.028 \\
\hline 22 & Cass & .177 & 13.064 \\
\hline 23 & Clark & 22.411 & 2.435 \\
\hline 24 & Clay & .183 & .077 \\
\hline 25 & Clinton & 1.135 & 16.680 \\
\hline 26 & Crawford & .000 & .000 \\
\hline 27 & Daviess & $1: 163$ & 8.315 \\
\hline 28 & Dearborn & .000 & .095 \\
\hline 29 & Decatur & .186 & 2.900 \\
\hline 30 & DeKalb & .205 & .518 \\
\hline 31 & Delaware & 2.657 & .994 \\
\hline 32 & Dubois & 126.521 & 6.468 \\
\hline 33 & Elkhart & 76.541 & 5.441 \\
\hline 34 & Fayette & 1.015 & .000 \\
\hline 35 & Floyd & 8.767 & 6.057 \\
\hline 36 & Fountain & .000 & .457 \\
\hline 37 & Franklin & .000 & .085 \\
\hline 38 & Fulton & .000 & 1.196 \\
\hline 39 & Gibson & .000 & 3.128 \\
\hline 40 & Grant & 2.821 & 1.803 \\
\hline 41 & Greene & .178 & 2.815 \\
\hline 42 & Hamilton & 6.388 & 1.374 \\
\hline 43 & Hancock & 1.420 & .100 \\
\hline 44 & Harrison & 14.467 & 6.822 \\
\hline 45 & Hendricks & .280 & 5.456 \\
\hline 46 & Henry & 2.929 & .424 \\
\hline 47 & Howard & 6.828 & 1.222 \\
\hline 48 & Huntington & .000 & 3.129 \\
\hline 49 & Jackson & 1.107 & .280 \\
\hline 50 & Jasper & .190 & 1.801 \\
\hline 51 & Jay & 1.037 & 3.240 \\
\hline
\end{tabular}




\begin{tabular}{|c|c|c|c|}
\hline & Locale & P01 15 & A01 15 \\
\hline 52 & Jefferson & .192 & .162 \\
\hline 53 & Jennings & .000 & .000 \\
\hline 54 & Johnson & 2.109 & .072 \\
\hline 55 & Knox & .169 & .941 \\
\hline 56 & Kosciusko & 5.805 & 7.336 \\
\hline 57 & LaGrange & 6.079 & 1.518 \\
\hline 58 & Lake & 4.583 & 8.634 \\
\hline 59 & La Porte & 3.151 & 4.264 \\
\hline 60 & Lawrence & .175 & .444 \\
\hline 61 & Madison & 7.555 & 5.820 \\
\hline 62 & Marion & 23.617 & 30.389 \\
\hline 63 & Marshall & 1.183 & 3.554 \\
\hline 64 & Martin & .000 & .429 \\
\hline 65 & Miami & 6.343 & .428 \\
\hline 66 & Monroe & 1.422 & .832 \\
\hline 67 & Montgomery & .000 & 2.971 \\
\hline 68 & Morgan & .217 & .091 \\
\hline 69 & Newton & 1.083 & .076 \\
\hline 70 & Noble & 1.157 & 6.103 \\
\hline 71 & Ohio & .000 & .000 \\
\hline 72 & Orange & 31.054 & .449 \\
\hline 73 & Owen & .000 & .081 \\
\hline 74 & Parke & .179 & 1.324 \\
\hline 75 & Perry & 4.994 & .072 \\
\hline 76 & Pike & .000 & .000 \\
\hline 77 & Porter & .959 & 2.556 \\
\hline 78 & Posey & .000 & .480 \\
\hline 79 & Pulaski & .187 & .545 \\
\hline 80 & Putnam & .000 & .079 \\
\hline 81 & Randolph & .173 & .562 \\
\hline 82 & Ripley & 3.351 & .081 \\
\hline 83 & Rush & .000 & .187 \\
\hline 84 & St. Joseph & 3.150 & 5.493 \\
\hline 85 & Scott & 1.126 & 2.972 \\
\hline 86 & Shelby & 6.013 & 1.338 \\
\hline 87 & Spencer & 13.252 & .448 \\
\hline 88 & Starke & .175 & .000 \\
\hline 89 & Steuben & .187 & 1.991 \\
\hline 90 & Sullivan & .000 & .000 \\
\hline 91 & Switzerland & .220 & .000 \\
\hline 92 & Tippecanoe & 15.905 & 5.765 \\
\hline 93 & Tipton & .000 & 1.307 \\
\hline 94 & Union & .000 & .000 \\
\hline 95 & Vanderburgh & 7.712 & 28.440 \\
\hline 96 & Vermillion & .000 & .057 \\
\hline 97 & Vigo & 1.104 & 1.927 \\
\hline 98 & Wabash & .173 & .437 \\
\hline 99 & Warren & .000 & .079 \\
\hline 100 & Warrick & .199 & .000 \\
\hline 101 & Washington & 33.851 & .490 \\
\hline 102 & Wayne & 11.932 & 4.973 \\
\hline
\end{tabular}




\begin{tabular}{|c|c|c|c|}
\hline & Locale & P01 15 & A01 15 \\
\hline 103 & Wells & .199 & 4.293 \\
\hline 104 & White & 3.178 & .322 \\
\hline 105 & Whitley & .872 & .084 \\
\hline 106 & lowa & 155.599 & 359.394 \\
\hline 107 & Kansas & 89.936 & 244.790 \\
\hline 108 & Kentucky_E & 67.243 & 98.704 \\
\hline 109 & Kentucky_W & 44.828 & 65.802 \\
\hline 110 & Louisiana & 23.429 & 137.226 \\
\hline 111 & Maine & 33.935 & 51.790 \\
\hline 112 & Maryland & 88.012 & 149.961 \\
\hline 113 & Massachusetts & 132.998 & 172.491 \\
\hline 114 & Michigan_E & 321.349 & 130.251 \\
\hline 115 & Michigan_W & 321.349 & 130.251 \\
\hline 116 & Minnesota & 238.273 & 370.723 \\
\hline 117 & Mississippi & 546.577 & 212.432 \\
\hline 118 & Missouri & 235.981 & 311.984 \\
\hline 119 & Montana & 15.916 & 18.351 \\
\hline 120 & Nebraska & 57.587 & 269.081 \\
\hline 121 & Nevada & 45.497 & 35.024 \\
\hline 122 & New Hampshire & 28.859 & 19.552 \\
\hline 123 & New Jersey & 165.089 & 246.176 \\
\hline 124 & New Mexico & 19.655 & 34.301 \\
\hline 125 & New York & 386.212 & 377.384 \\
\hline 126 & North Carolina & 1720.518 & $490: 862$ \\
\hline 127 & North Dakota & 18.903 & 40.174 \\
\hline 128 & Ohio_N & 129.383 & 127.328 \\
\hline 129 & Ohio_M & 129.383 & 127.328 \\
\hline 130 & Ohio_S & 129.383 & 127.328 \\
\hline 131 & Oklahoma & 64.338 & 115.982 \\
\hline 132 & Oregon & 125.082 & 173.751 \\
\hline 133 & Pennsylvania & 451.649 & 594.849 \\
\hline 134 & Rhode Island & 41.592 & 22.992 \\
\hline 135 & South Carolina & 117.284 & 136.842 \\
\hline 136 & South Dakota & 30.880 & 63.799 \\
\hline 137 & Tennessee & 510.376 & 311.240 \\
\hline 138 & Texas & 579.563 & 826.127 \\
\hline 139 & Utah & 130.400 & 112.489 \\
\hline 140 & \begin{tabular}{|l|} 
Vermont \\
\end{tabular} & 55.694 & 31.328 \\
\hline 141 & Virginia & 488.382 & 312.113 \\
\hline 142 & Washington & 156.267 & 297.545 \\
\hline 143 & West Virginia & 20.180 & 39.794 \\
\hline 144 & Wisconsin & 334.106 & 474.224 \\
\hline 145 & Wyoming & 4.145 & 6.019 \\
\hline
\end{tabular}




\begin{tabular}{|c|c|c|c|}
\hline & Locale & P02 15 & $\mathrm{A} 02 \quad 15$ \\
\hline 1 & Alabama & 9578.011 & 9398.901 \\
\hline 2 & Arizona & 3445.686 & 1844.668 \\
\hline 3 & Arkansas & 14947.925 & 4010.928 \\
\hline 4 & California & 46046.475 & 52954.167 \\
\hline 5 & Colorado & 5612.936 & 1957.536 \\
\hline 6 & Connecticut & 2497.322 & 1672.914 \\
\hline 7 & Delaware & 2935.969 & 3426.959 \\
\hline 8 & $D C$ & .000 & .000 \\
\hline 9 & Florida & 12835.370 & 4360.271 \\
\hline 10 & Georgia & 19761.673 & 4829.124 \\
\hline 11 & Idaho & 5360.154 & 762.658 \\
\hline 12 & Illinois_N & 15405.125 & 14347.629 \\
\hline 13 & Illinois_s & 7702.563 & 7173.815 \\
\hline 14 & Adams & 102.379 & 38.791 \\
\hline 15 & Allen & 489.442 & 239.193 \\
\hline 16 & Bartholomew & 379.451 & .000 \\
\hline 17 & Benton & .000 & .000 \\
\hline 18 & Blackford & 14.519 & .000 \\
\hline 19 & Boone & 3.542 & .000 \\
\hline 20 & Brown & 2.699 & .000 \\
\hline 21 & Carroll & 491.757 & .000 \\
\hline 22 & Cass & 457.964 & .000 \\
\hline 23 & Clark & 85.360 & 393.582 \\
\hline 24 & Clay & 2.711 & .000 \\
\hline 25 & Clinton & 584.758 & .000 \\
\hline 26 & Crawford & .000 & .000 \\
\hline 27 & Daviess & 291.494 & 37.721 \\
\hline 28 & Dearborn & 3.322 & 43.681 \\
\hline 29 & Decatur & 101.649 & .000 \\
\hline 30 & DeKalb & 18.166 & .000 \\
\hline 31 & Delaware & 34.852 & .000 \\
\hline 32 & Dubois & 226.730 & .000 \\
\hline 33 & Elkhart & 190.747 & 38.642 \\
\hline 34 & Fayette & .000 & .000 \\
\hline 35 & Floyd & 212.323 & .000 \\
\hline 36 & Fountain & 16.036 & .000 \\
\hline 37 & Franklin & 2.988 & 235.723 \\
\hline 38 & Fulton & 41.933 & .000 \\
\hline 39 & Gibson & 109.664 & .000 \\
\hline 40 & Grant & 63.214 & .000 \\
\hline 41 & Greene & 98.671 & .000 \\
\hline 42 & Hamilton & 48.176 & 47.625 \\
\hline 43 & Hancock & 3.502 & .000 \\
\hline 44 & Harrison & 239.171 & 260.240 \\
\hline 45 & Hendricks & 191.255 & .000 \\
\hline 46 & Henry & 14.859 & .000 \\
\hline 47 & Howard & 42.847 & .000 \\
\hline 48 & Huntington & 109.694 & 38.460 \\
\hline 49 & Jackson & 9.829 & .000 \\
\hline 50 & Jasper & 63.154 & .000 \\
\hline 51 & Jay & 113.590 & 33.636 \\
\hline
\end{tabular}




\begin{tabular}{|c|c|c|c|}
\hline & Locale & P02 15 & $\mathrm{~A} 0215$ \\
\hline 52 & Jefferson & 5.678 & .000 \\
\hline 53 & Jennings & .000 & 233.792 \\
\hline 54 & Johnson & 2.512 & .000 \\
\hline 55 & Knox & 32.993 & 32.862 \\
\hline 56 & Kosciusko & 257.171 & 36.913 \\
\hline 57 & LaGrange & 53.198 & .000 \\
\hline 58 & Lake & 302.676 & 7610.073 \\
\hline 59 & La Porte & 149.493 & 35.036 \\
\hline 60 & Lawrence & 15.556 & 204.531 \\
\hline 61 & Madison & 204.020 & .000 \\
\hline 62 & Marion & 1065.348 & 431.932 \\
\hline 63 & Marshall & 124.578 & 230.153 \\
\hline 64 & Martin & 15.046 & .000 \\
\hline 65 & Miami & 15.020 & .000 \\
\hline 66 & Monroe & 29.151 & 237.075 \\
\hline 67 & Montgomery & 104.167 & .000 \\
\hline 68 & Morgan & 3.205 & .000 \\
\hline 69 & Newton & 2.671 & .000 \\
\hline 70 & Noble & 213.964 & .000 \\
\hline 71 & Ohio & .000 & .000 \\
\hline 72 & Orange & 15.756 & .000 \\
\hline 73 & Owen & 2.856 & .000 \\
\hline 74 & Parke & 46.425 & .000 \\
\hline 75 & Perry & 2.523 & .000 \\
\hline 76 & Pike & .000 & .000 \\
\hline 77 & Porter & 89.591 & .000 \\
\hline 78 & Posey & 16.824 & 1382.495 \\
\hline 79 & Pulaski & 19.110 & .000 \\
\hline 80 & Putnam & 2.786 & .000 \\
\hline 81 & Randolph & 19.689 & 33.619 \\
\hline 82 & Ripley & 2.834 & 37.257 \\
\hline 83 & Rush & 6.556 & .000 \\
\hline 84 & St. Joseph & 192.551 & 36.479 \\
\hline 85 & Scott & 104.188 & 36.529 \\
\hline 86 & Shelby & 46.903 & .000 \\
\hline 87 & Spencer & 15.689 & .000 \\
\hline 88 & Starke & .000 & .000 \\
\hline 89 & Steuben & 69.804 & 36.419 \\
\hline 90 & Sullivan & .000 & .000 \\
\hline 91 & Switzerland & .000 & .000 \\
\hline 92 & Tippecanoe & 202.105 & 41.261 \\
\hline 93 & Tipton & 45.823 & .000 \\
\hline 94 & Union & .000 & .000 \\
\hline 95 & Vanderburgh & 997.004 & .000 \\
\hline 96 & Vermillion & 2.009 & .000 \\
\hline 97 & Vigo & 67.548 & .000 \\
\hline 98 & Wabash & 15.323 & 33.577 \\
\hline 99 & Warren & 2.779 & .000 \\
\hline 100 & Warrick & .000 & .000 \\
\hline 101 & Washington & 17.176 & .000 \\
\hline 102 & Wayne & 174.347 & .000 \\
\hline
\end{tabular}




\begin{tabular}{|c|c|c|c|}
\hline & $\begin{array}{r}\text { Locale } \\
\end{array}$ & P02 15 & $\mathrm{~A} 02 \quad 15$ \\
\hline 103 & Wells & 150.502 & .000 \\
\hline 104 & White & 11.287 & .000 \\
\hline$\overline{105}$ & Whitley & 2.934 & .000 \\
\hline 106 & lowa & 12599.152 & 626.109 \\
\hline 107 & Kansas & 8581.524 & 10171.972 \\
\hline 108 & Kentucky_E & 3460.217 & 2846.87 .2 \\
\hline 109 & Kentucky_W & 2306.811 & 1897.914 \\
\hline 110 & Louisiana & 4810.666 & 44960.578 \\
\hline 111 & Maine & 1815.589 & 659.522 \\
\hline 112 & Maryland & 5257.116 & 4395.734 \\
\hline 113 & Massachusetts & 6046.935 & 3866.728 \\
\hline 114 & Michigan_E & 4566.167 & 4504.826 \\
\hline 115 & Michigan_W & 4566.167 & 3003.217 \\
\hline 116 & Minnesota & 12996.290 & 7692.988 \\
\hline 117 & Mississippi & 7447.132 & 7067.893 \\
\hline 118 & Missouri & 10937.110 & 5291.398 \\
\hline 119 & Montana & 643.338 & 3112.035 \\
\hline 120 & Nebraska & 9433.066 & 36.156 \\
\hline 121 & Nevada & 1227.806 & 317.669 \\
\hline 122 & New Hampshire & 685.444 & 627.989 \\
\hline 123 & New Jersey & 8630.087 & 13410.305 \\
\hline 124 & New Mexico & 1202.489 & 3012.913 \\
\hline 125 & New York & 13229.794 & 5687.246 \\
\hline 126 & North Carolina & 17207.978 & 2968.716 \\
\hline 127 & North Dakota & 1408.353 & 598.747 \\
\hline 128 & Ohio_N & 4463.693 & 6660.818 \\
\hline 129 & Ohio_M & 4463.693 & 6660.818 \\
\hline 130 & Ohio_s & 4463.693 & 6660.818 \\
\hline 131 & Oklahoma & 4065.940 & 9339.176 \\
\hline 132 & Oregon & 6091.127 & 2909.291 \\
\hline 133 & Pennsylvania & 20853.402 & 25887.558 \\
\hline 134 & Rhode Island & 806.005 & 37.605 \\
\hline 135 & South Carolina & 4797.208 & 1501.059 \\
\hline 136 & South Dakota & 2236.584 & 36.525 \\
\hline 137 & Tennessee & 10911.011 & 5309.791 \\
\hline 138 & Texas & 28961.213 & 97169.754 \\
\hline 139 & Utah & 3943.468 & 6604.918 \\
\hline 140 & Vermont & 1098.263 & 229.505 \\
\hline 141 & Virginia & 10941.628 & 2780.741 \\
\hline 142 & Washington & 10430.908 & 8310.090 \\
\hline 143 & West Virginia & 1395.060 & 1377.387 \\
\hline 144 & Wisconsin & 16624.677 & 1586.009 \\
\hline 145 & Wyoming & 211.020 & 3092.572 \\
\hline
\end{tabular}




\begin{tabular}{|c|c|c|c|}
\hline & Locale & P03 15 & A03 15 \\
\hline 1 & Alabama & 6240.990 & 6124.284 \\
\hline$\overline{2}$ & Arizona & 2245.194 & 1201.978 \\
\hline 3 & Arkansas & 9740.003 & 2613.503 \\
\hline 4 & California & 30003.684 & 34504.707 \\
\hline 5 & Colorado & 3657.365 & 1275.522 \\
\hline 6 & Connecticut & 1627.244 & 1090.064 \\
\hline 7 & Delaware & 1913.065 & 2232.992 \\
\hline 8 & DC & .000 & .000 \\
\hline 9 & Florida & 8363.472 & 2841.134 \\
\hline 10 & Georgia & 12876.621 & 3146.637 \\
\hline 11 & Idaho & 3492.653 & 496.945 \\
\hline 12 & Illinois_N & 10037.904 & 9348.853 \\
\hline 13 & Illinois_S & 5018.952 & 4674.427 \\
\hline 14 & Adams & 66.710 & 25.276 \\
\hline 15 & Allen & 318.918 & 155.857 \\
\hline 16 & Bartholomew & 247.248 & .000 \\
\hline 17 & Benton & .000 & .000 \\
\hline 18 & Blackford & 9.461 & .000 \\
\hline 19 & Boone & 2.308 & .000 \\
\hline 20 & Brown & 1.759 & .000 \\
\hline 21 & Carroll & 320.427 & .000 \\
\hline 22 & Cass & 298.407 & .000 \\
\hline 23 & Clark & 55.620 & 256.456 \\
\hline 24 & Clay & 1.766 & .000 \\
\hline 25 & Clinton & 381.026 & .000 \\
\hline 26 & Crawford & .000 & .000 \\
\hline 27 & Daviess & 189.936 & 24.579 \\
\hline 28 & Dearborn & 2.165 & 28.462 \\
\hline 29 & Decatur & 66.234 & .000 \\
\hline 30 & DeKalb & 11.837 & .000 \\
\hline 31 & Delaware & 22.710 & .000 \\
\hline 32 & Dubois & 147.736 & .000 \\
\hline 33 & Elkhart & 124.290 & 25.179 \\
\hline 34 & Fayette & .000 & .000 \\
\hline 35 & Floyd & 138.349 & .000 \\
\hline 36 & Fountain & 10.449 & .000 \\
\hline 37 & Franklin & 1.947 & 153.596 \\
\hline 38 & Fulton & 27.323 & .000 \\
\hline 39 & Gibson & 71.456 & .000 \\
\hline 40 & Grant & 41.190 & .000 \\
\hline 41 & Greene & 64.294 & .000 \\
\hline 42 & Hamilton & 31.391 & 31.032 \\
\hline 43 & Hancock & 2.282 & .000 \\
\hline 44 & Harrison & 155.843 & 169.571 \\
\hline 45 & Hendricks & 124.621 & .000 \\
\hline 46 & Henry & 9.682 & .000 \\
\hline 47 & Howard & 27.919 & .000 \\
\hline 48 & Huntington & 71.476 & 25.060 \\
\hline 49 & Jackson & 6.404 & .000 \\
\hline 50 & Jasper & 41.151 & .000 \\
\hline 51 & Jay & 74.015 & 21.917 \\
\hline
\end{tabular}




\begin{tabular}{|c|c|c|c|}
\hline & Locale & P03 15 & A03 15 \\
\hline 52 & Jefferson & 3.700 & .000 \\
\hline 53 & Jennings & .000 & 152.338 \\
\hline 54 & Johnson & 1.637 & .000 \\
\hline 55 & Knox & 21.498 & 21.413 \\
\hline 56 & Kosciusko & 167.571 & 24.052 \\
\hline 57 & LaGrange & 34.664 & .000 \\
\hline 58 & Lake & 197.223 & 4958.691 \\
\hline 59 & La Porte & 97.409 & 22.829 \\
\hline 60 & Lawrence & 10.136 & 133.272 \\
\hline 61 & Madison & 132.939 & .000 \\
\hline 62 & Marion & 694.176 & 281.445 \\
\hline 63 & Marshall & 81.174 & 149.966 \\
\hline 64 & Martin & 9.804 & .000 \\
\hline 65 & Miami & 9.787 & .000 \\
\hline 66 & Monroe & 18.995 & 154.477 \\
\hline 67 & Montgomery & 67.875 & .000 \\
\hline 68 & Morgan & 2.088 & .000 \\
\hline 69 & Newton & 1.740 & .000 \\
\hline 70 & Noble & 139.418 & .000 \\
\hline 71 & Ohio & .000 & .000 \\
\hline 72 & Orange & 10.267 & .000 \\
\hline 73 & Owen & 1.861 & .000 \\
\hline 74 & Parke & 30.250 & .000 \\
\hline 75 & Perry & 1.644 & .000 \\
\hline 76 & Pike & .000 & .000 \\
\hline 77 & Porter & 58.377 & .000 \\
\hline 78 & Posey & 10.963 & 900.828 \\
\hline 79 & Pulaski & 12.452 & .000 \\
\hline 80 & Putnam & 1.816 & .000 \\
\hline 81 & Randolph & 12.829 & 21.906 \\
\hline 82 & Ripley & 1.846 & 24.276 \\
\hline 83 & Rush & 4.272 & .000 \\
\hline$\overline{84}$ & St. Joseph & 125.466 & 23.769 \\
\hline 85 & Scott & 67.889 & 23.802 \\
\hline 86 & Shelby & 30.562 & .000 \\
\hline 87 & Spencer & 10.223 & .000 \\
\hline 88 & Starke & .000 & .000 \\
\hline 89 & Steuben & 45.484 & 23.731 \\
\hline 90 & Sullivan & .000 & .000 \\
\hline 91 & Switzerland & .000 & .000 \\
\hline 92 & Tippecanoe & 131.691 & 26.886 \\
\hline 93 & Tipton & 29.858 & .000 \\
\hline 94 & Union & .000 & .000 \\
\hline 95 & Vanderburgh & 649.643 & .000 \\
\hline 96 & Vermillion & 1.309 & .000 \\
\hline 97 & Vigo & 44.014 & .000 \\
\hline 98 & Wabash & 9.984 & 21.879 \\
\hline 99 & Warren & 1.811 & .000 \\
\hline 100 & Warrick & .000 & .000 \\
\hline 101 & Washington & 11.192 & .000 \\
\hline 102 & Wayne & 113.604 & .000 \\
\hline
\end{tabular}




\begin{tabular}{|c|c|c|c|}
\hline & Locale & P03 15 & A03 15 \\
\hline 103 & Wells & 98.066 & .000 \\
\hline 104 & White & 7.355 & .000 \\
\hline 105 & Whitley & 1.912 & .000 \\
\hline 106 & lowa & 8209.553 & 407.970 \\
\hline 107 & Kansas & 5591.684 & 6628.013 \\
\hline 108 & Kentucky E & 2254.663 & 1855.009 \\
\hline 109 & Kentucky_W & 1503.108 & 1236.673 \\
\hline 110 & Louisiana & 3134.609 & 29296.118 \\
\hline 111 & Maine & 1183.030 & 429.741 \\
\hline 112 & Maryland & 3425.514 & 2864.241 \\
\hline 113 & Massachusetts & 3940.157 & 2519.543 \\
\hline$\overline{114}$ & Michigan_E & 2975.294 & 2446.104 \\
\hline 115 & Michigan_W & 2975.294 & 2446.104 \\
\hline 116 & Minnesota & 8468.327 & 5012.717 \\
\hline 117 & Mississippi & 4852.519 & 4605.409 \\
\hline 118 & Missouri & 7126.574 & 3447.852 \\
\hline 119 & Montana & 419.196 & 2027.788 \\
\hline 120 & Nebraska & 6146.545 & 23.559 \\
\hline 121 & Nevada & 800.033 & 206.992 \\
\hline 122 & New Hampshire & 446.633 & 409.195 \\
\hline 123 & New Jersey & 5623.327 & 8738.097 \\
\hline 124 & New Mexico & 783.537 & 1963.201 \\
\hline 125 & New York & 8620.477 & 3705.785 \\
\hline 126 & North Carolina & 11212.644 & 1934.402 \\
\hline 127 & North Dakota & 917.677 & 390.141 \\
\hline 128 & Ohio_N & 2908.523 & 4340.161 \\
\hline 129 & Ohio_M & 2908.523 & 4340.161 \\
\hline 130 & Ohio_s & 2908.523 & 4340.161 \\
\hline 131 & Oklahoma & 2649.349 & 6085.367 \\
\hline 132 & Oregon & 3968.952 & 1895.682 \\
\hline 133 & Pennsylvania & 13587.987 & 16868.221 \\
\hline 134 & Rhode Island & 525.189 & 24.503 \\
\hline 135 & South Carolina & 3125.840 & 978.084 \\
\hline 136 & South Dakota & 1457.348 & 23.799 \\
\hline 137 & Tennessee & 7109.568 & 3459.837 \\
\hline 138 & Texas & 18871.002 & 63315.392 \\
\hline 139 & Utah & 2569.547 & 4303.736 \\
\hline 140 & Vermont & 715.624 & 149.545 \\
\hline 141 & Virginia & 7129.518 & 1811.919 \\
\hline 142 & Washington & 6796.735 & 5414.819 \\
\hline 143 & West Virginia & 909.015 & 897.499 \\
\hline 144 & Wisconsin & 10832.568 & 1033.437 \\
\hline 145 & Wyoming & 137.500 & 2015.107 \\
\hline
\end{tabular}




\begin{tabular}{|c|c|c|c|}
\hline & Locale & P04 15 & A04 15 \\
\hline 1 & Alabama & 6841.975 & 6841.975 \\
\hline 2 & Arizona & 2461.398 & 2461.398 \\
\hline 3 & Arkansas & 10677.930 & 10677.930 \\
\hline 4 & California & 32892.928 & 32892.928 \\
\hline 5 & Colorado & 4009.556 & 4009.556 \\
\hline 6 & Connecticut & 1783.942 & 1783.942 \\
\hline 7 & Delaware & 2097.286 & 2097.286 \\
\hline 8 & DC & .000 & .000 \\
\hline$\overline{9}$ & Florida & 9168.843 & 9168.843 \\
\hline 10 & Georgia & 14116.592 & 14116.592 \\
\hline 11 & Idaho & 3828.983 & 3828.983 \\
\hline 12 & Illinois_N & 11004.517 & 11004.517 \\
\hline 13 & Illinois_S & 5502.259 & 5502.259 \\
\hline 14 & Adams & 73.134 & 73.134 \\
\hline 15 & Allen & 349.629 & 349.629 \\
\hline 16 & Bartholomew & 271.057 & 271.057 \\
\hline 17 & Benton & .000 & .000 \\
\hline 18 & Blackford & 10.372 & 10.372 \\
\hline 19 & Boone & 2.530 & 2.530 \\
\hline 20 & Brown & 1.928 & 1.928 \\
\hline 21 & Carroll & 351.283 & 351.283 \\
\hline 22 & Cass & 327.143 & 327.143 \\
\hline 23 & Clark & 60.976 & 60.976 \\
\hline 24 & Clay & 1.936 & $1: 936$ \\
\hline 25 & Clinton & 417.717 & 417.717 \\
\hline 26 & Crawford & .000 & .000 \\
\hline 27 & Daviess & 208.226 & 208.226 \\
\hline 28 & Dearborn & 2.373 & 2.373 \\
\hline 29 & Decatur & 72.612 & 72.612 \\
\hline 30 & DeKalb & 12.977 & 12.977 \\
\hline 31 & Delaware & 24.896 & 24.896 \\
\hline 32 & Dubois & 161.962 & 161.962 \\
\hline 33 & Elkhart & 136.258 & 136.258 \\
\hline 34 & Fayette & .000 & .000 \\
\hline 35 & Floyd & 151.672 & 151.672 \\
\hline 36 & Fountain & 11.455 & 11.455 \\
\hline 37 & Franklin & 2.135 & 2.135 \\
\hline 38 & Fulton & 29.954 & 29.954 \\
\hline 39 & Gibson & 78.337 & 78.337 \\
\hline 40 & Grant & 45.157 & 45.157 \\
\hline 41 & Greene & 70.485 & 70.485 \\
\hline 42 & Hamilton & 34.414 & 34.414 \\
\hline$\overline{43}$ & Hancock & 2.502 & 2.502 \\
\hline 44 & Harrison & 170.850 & 170.850 \\
\hline 45 & Hendricks & 136.622 & 136.622 \\
\hline 46 & Henry & 10.615 & 10.615 \\
\hline 47 & Howard & 30.607 & 30.607 \\
\hline 48 & Huntington & 78.359 & 78.359 \\
\hline 49 & Jackson & 7.021 & 7.021 \\
\hline 50 & Jasper & 45.113 & 45.113 \\
\hline 51 & Jay & 81.142 & 81.142 \\
\hline
\end{tabular}




\begin{tabular}{|c|c|c|c|}
\hline & Locale & P04_15 & $\mathrm{A} 0415$ \\
\hline 52 & Jefferson & 4.056 & 4.056 \\
\hline 53 & Jennings & .000 & .000 \\
\hline 54 & Johnson & 1.794 & 1.794 \\
\hline 55 & Knox & 23.568 & 23.568 \\
\hline 56 & Kosciusko & 183.708 & 183.708 \\
\hline 57 & LaGrange & 38.002 & 38.002 \\
\hline 58 & Lake & 216.214 & 216.214 \\
\hline 59 & La Porte & 106.789 & 106.789 \\
\hline 60 & Lawrence & 11.113 & 11.113 \\
\hline 61 & Madison & 145.740 & 145.740 \\
\hline 62 & Marion & 761.023 & 761.023 \\
\hline 63 & Marshall & 88.991 & 88.991 \\
\hline 64 & Martin & 10.748 & 10.748 \\
\hline 65 & Miami & 10.729 & 10.729 \\
\hline 66 & Monroe & 20.824 & 20.824 \\
\hline 67 & Montgomery & 74.411 & 74.411 \\
\hline 68 & Morgan & 2.289 & 2.289 \\
\hline 69 & Newton & 1.908 & 1.908 \\
\hline 70 & Noble & 152.844 & 152.844 \\
\hline 71 & Ohio & .000 & .000 \\
\hline 72 & Orange & 11.255 & 11.255 \\
\hline 73 & Owen & 2.040 & 2.040 \\
\hline 74 & Parke & 33.163 & 33.163 \\
\hline 75 & Perry & 1.802 & 1.802 \\
\hline 76 & Pike & .000 & .000 \\
\hline 77 & Porter & 63.999 & 63.999 \\
\hline 78 & Posey & 12.018 & 12.018 \\
\hline 79 & Pulaski & 13.651 & 13.651 \\
\hline 80 & Putnam & 1.990 & 1.990 \\
\hline 81. & Randolph & 14.065 & 14.065 \\
\hline 82 & Ripley & 2.024 & 2.024 \\
\hline 83 & Rush & 4.683 & 4.683 \\
\hline 84 & St. Joseph & 137.547 & 137.547 \\
\hline 85 & Scott & 74.426 & 74.426 \\
\hline 86 & Shelby & 33.505 & 33.505 \\
\hline 87 & Spencer & 11.207 & 11.207 \\
\hline 88 & Starke & .000 & .000 \\
\hline 89 & Steuben & 49.864 & 49.864 \\
\hline 90 & Sullivan & .000 & .000 \\
\hline 91 & Switzerland & .000 & .000 \\
\hline 92 & Tippecanoe & 144.372 & 144.372 \\
\hline 93 & Tipton & 32.733 & 32.733 \\
\hline 94 & Union & .000 & .000 \\
\hline 95 & Vanderburgh & 712.202 & 712.202 \\
\hline 96 & Vermillion & 1.435 & 1.435 \\
\hline 97 & Vigo & 48.252 & 48.252 \\
\hline 98 & Wabash & 10.946 & 10.946 \\
\hline 99 & Warren & 1.985 & 1.985 \\
\hline 100 & Warrick & .000 & .000 \\
\hline 101 & Washington & 12.269 & 12.269 \\
\hline 102 & Wayne & 124.543 & 124.543 \\
\hline
\end{tabular}




\begin{tabular}{|c|c|c|c|}
\hline & $\begin{array}{l}\text { Locale } \\
\end{array}$ & P04 15 & A04_15 \\
\hline 103 & Wells & 107.510 & 107.510 \\
\hline 104 & White & 8.063 & 8.063 \\
\hline 105 & Whitley & 2.096 & 2.096 \\
\hline 106 & lowa & 9000.103 & 9000.103 \\
\hline 107 & Kansas & 6130.142 & 6130.142 \\
\hline 108 & Kentucky_E & 2471.778 & 2471.778 \\
\hline 109 & Kentucky W & 1647.852 & 1647.852 \\
\hline 110 & Louisiana & 3436.460 & 3436.460 \\
\hline 111 & Maine & 1296.951 & 1296.951 \\
\hline 112 & Maryland & 3755.379 & 3755.379 \\
\hline 113 & Massachusetts & 4319.579 & 4319.579 \\
\hline 114 & Michigan_E & 3261.804 & 3261.804 \\
\hline 115 & Michigan_W & 3261.804 & 3261.804 \\
\hline 116 & Minnesota & 9283.795 & 9283.795 \\
\hline 117 & Mississippi & 5319.798 & 5319.798 \\
\hline 118 & Missouri & 7812.836 & 7812.836 \\
\hline 119 & Montana & 459.563 & 459.563 \\
\hline 120 & Nebraska & 6738.434 & 6738.434 \\
\hline 121 & Nevada & 877.073 & 877.073 \\
\hline 122 & New Hampshire & 489.642 & 489.642 \\
\hline 123 & New Jersey & 6164.833 & 6164.833 \\
\hline 124 & New Mexico & 858.988 & 858.988 \\
\hline 125 & New York & 9450.597 & 9450.597 \\
\hline 126 & North Carolina & 12292.380 & 12292.380 \\
\hline 127 & North Dakota & 1006.045 & 1006.045 \\
\hline 128 & Ohio_N & 3188.603 & 3188.603 \\
\hline 129 & Ohio_M & 3188.603 & 3188.603 \\
\hline 130 & Ohio_s & 3188.603 & 3188.603 \\
\hline 131 & Oklahoma & 2904.471 & 2904.471 \\
\hline 132 & Oregon & 4351.147 & 4351.147 \\
\hline 133 & Pennsylvania & 14896.459 & 14896.459 \\
\hline 134 & Rhode Island & 575.763 & 575.763 \\
\hline 135 & South Carolina & 3426.847 & 3426.847 \\
\hline 136 & South Dakota & 1597.686 & 1597.686 \\
\hline 137 & Tennessee & 7794.193 & 7794.193 \\
\hline 138 & Texas & 20688.209 & 20688.209 \\
\hline 139 & Utah & 2816.985 & 2816.985 \\
\hline 140 & Vermont & 784.535 & 784.535 \\
\hline 141 & Virginia & 7816.064 & 7816.064 \\
\hline 142 & Washington & 7451.235 & 7451.235 \\
\hline 143 & West Virginia & 996.550 & 996.550 \\
\hline 144 & Wisconsin & 11875.704 & 11875.704 \\
\hline 145 & Wyoming & 150.740 & 150.740 \\
\hline
\end{tabular}




\begin{tabular}{|c|c|c|c|}
\hline & Locale & P06 15 & A06 15 \\
\hline 1 & Alabama & 2852.847 & 2695.418 \\
\hline 2 & Arizona & 1316.959 & 2344.336 \\
\hline 3 & Arkansas & 4133.071 & 2774.362 \\
\hline 4 & California & 15008.745 & 17017.309 \\
\hline 5 & Colorado & 1841.700 & 2029.153 \\
\hline 6 & Connecticut & 1512.388 & 1647.016 \\
\hline 7 & Delaware & 725.402 & 822.642 \\
\hline 8 & DC & .000 & .000 \\
\hline 9 & Florida & 4256.089 & 7114.177 \\
\hline 10 & Georgia & 5747.710 & 5587.065 \\
\hline 11 & Idaho & 1351.809 & 1144.998 \\
\hline 12 & Illinois_N & 6386.968 & 5037.123 \\
\hline 13 & Illinois_S & 3193.484 & 2518.561 \\
\hline 14 & Adams & 42.695 & 27.187 \\
\hline 15 & Allen & 319.889 & 161.887 \\
\hline 16 & Bartholomew & 226.415 & 71.867 \\
\hline 17 & Benton & 2.086 & 2.039 \\
\hline 18 & Blackford & 5.284 & 4.915 \\
\hline 19 & Boone & 22.314 & 14.963 \\
\hline 20 & Brown & .614 & 4.409 \\
\hline 21 & Carroll & 114.156 & 66.096 \\
\hline 22 & Cass & 111.133 & 66.730 \\
\hline 23 & Clark & 52.504 & 58.550 \\
\hline 24 & Clay & $4: 131$ & 8.532 \\
\hline 25 & Clinton & 141.184 & 86.549 \\
\hline 26 & Crawford & .000 & 2.859 \\
\hline 27 & Daviess & 73.155 & 45.525 \\
\hline 28 & Dearborn & 6.242 & 14.745 \\
\hline 29 & Decatur & 39.918 & 18.660 \\
\hline 30 & DeKalb & 30.536 & 20.721 \\
\hline 31 & Delaware & 47.501 & 36.371 \\
\hline 32 & Dubois & 56.147 & 40.253 \\
\hline 33 & Elkhart & 101.830 & 128.938 \\
\hline 34 & Fayette & 23.346 & 5.880 \\
\hline 35 & Floyd & 73.115 & 49.140 \\
\hline 36 & Fountain & 3.647 & 6.486 \\
\hline 37 & Franklin & 3.127 & 6.028 \\
\hline 38 & Fulton & 22.608 & 10.518 \\
\hline 39 & Gibson & 31.849 & 21.540 \\
\hline 40 & Grant & 35.149 & 26.555 \\
\hline 41 & Greene & 22.444 & 20.341 \\
\hline 42 & Hamilton & 24.701 & 78.597 \\
\hline 43 & Hancock & 11.934 & 17.882 \\
\hline 44 & Harrison & 57.103 & 41.531 \\
\hline 45 & Hendricks & 56.752 & 63.062 \\
\hline 46 & Henry & 16.054 & 12.831 \\
\hline 47 & Howard & 22.618 & 25.974 \\
\hline 48 & Huntington & 41.958 & 25.534 \\
\hline 49 & Jackson & 27.759 & 16.552 \\
\hline 50 & Jasper & 14.748 & 15.465 \\
\hline 51 & Jay & 32.925 & 19.106 \\
\hline
\end{tabular}




\begin{tabular}{|c|c|c|c|}
\hline & Locale & P06 15 & A06 15 \\
\hline 52 & Jefferson & 30.080 & 10.688 \\
\hline 53 & Jennings & 11.810 & 7.654 \\
\hline 54 & Johnson & 34.116 & 39.643 \\
\hline 55 & Knox & 10.472 & 13.224 \\
\hline 56 & Kosciusko & 92.099 & 60.896 \\
\hline 57 & LaGrange & 34.452 & 16.835 \\
\hline 58 & Lake & 117.802 & 196.027 \\
\hline 59 & La Porte & 103.611 & 54.284 \\
\hline 60 & Lawrence & 17.445 & 12.815 \\
\hline 61 & Madison & 61.293 & 69.484 \\
\hline 62 & Marion & 560.691 & 529.387 \\
\hline 63 & Marshall & 34.468 & 31.421 \\
\hline 64 & Martin & 3.765 & 4.236 \\
\hline 65 & Miami & 10.352 & 11.732 \\
\hline 66 & Monroe & 78.406 & 41.899 \\
\hline 67 & Montgomery & 30.366 & 27.636 \\
\hline 68 & Morgan & 12.887 & 32.808 \\
\hline 69 & Newton & 3.633 & 4.118 \\
\hline 70 & Noble & 66.578 & 45.170 \\
\hline 71 & Ohio & .000 & 1.352 \\
\hline 72 & Orange & 3.942 & 6.752 \\
\hline 73 & Owen & 1.040 & 5.973 \\
\hline 74 & Parke & 12.732 & 9.817 \\
\hline 75 & Perry & 6.599 & 4.604 \\
\hline 76 & Pike & .000 & 3.166 \\
\hline 77 & Porter & 39.440 & 61.714 \\
\hline 78 & Posey & 6.123 & 62.544 \\
\hline 79 & Pulaski & 22.490 & 5.674 \\
\hline 80 & Putnam & 7.288 & 9.566 \\
\hline 81 & Randolph & 23.393 & 8.733 \\
\hline 82 & Ripley & .645 & 8.951 \\
\hline 83 & Rush & 19.352 & 4.901 \\
\hline 84 & St. Joseph & 102.603 & 118.083 \\
\hline 85 & Scott & 25.974 & 24.162 \\
\hline 86 & Shelby & 25.044 & 22.044 \\
\hline 87 & Spencer & 9.814 & 7.070 \\
\hline 88 & Starke & 13.244 & 7.274 \\
\hline 89 & Steuben & 35.460 & 16.997 \\
\hline 90 & Sullivan & .000 & 5.633 \\
\hline 91 & Switzerland & .000 & 2.411 \\
\hline 92 & Tippecanoe & 161.098 & 123.150 \\
\hline 93 & Tipton & 16.676 & 9.841 \\
\hline 94 & Union & .372 & 3.020 \\
\hline 95 & Vanderburgh & 266.126 & 239.289 \\
\hline 96 & Vermillion & .457 & 52.191 \\
\hline 97 & Vigo & 31.757 & 63.915 \\
\hline 98 & Wabash & 20.250 & 20.595 \\
\hline 99 & Warren & 7.268 & 2.305 \\
\hline 100 & Warrick & 2.417 & 17.150 \\
\hline 101 & Washington & 33.208 & 9.140 \\
\hline 102 & Wayne & 51.976 & 41.358 \\
\hline
\end{tabular}




\begin{tabular}{|c|c|c|c|}
\hline & Locale & P06 15 & A06 15 \\
\hline 103 & Wells & 46.148 & 25.768 \\
\hline 104 & White & 13.534 & 7.464 \\
\hline 105 & Whitley & 5.752 & 8.826 \\
\hline 106 & lowa & 4299.093 & 2482.291 \\
\hline 107 & Kansas & 2776.981 & 1931.336 \\
\hline 108 & Kentucky_E & 1313.990 & 1299.511 \\
\hline 109 & Kentucky_W & 875.993 & 866.340 \\
\hline 110 & Louisiana & 1430.613 & 2527.280 \\
\hline 111 & Maine & 524.738 & 603.244 \\
\hline 112 & Maryland & 1634.808 & 2559.379 \\
\hline 113 & Massachusetts & 2640.942 & 2990.331 \\
\hline 114 & Michigan_E & 3086.634 & 3086.634 \\
\hline 115 & Michigan_W & 3086.634 & 3086.634 \\
\hline 116 & Minnesota & 4665.429 & 3207.925 \\
\hline 117 & Mississippi & 2321.413 & 1845.889 \\
\hline 118 & Missouri & 3825.713 & 3461.323 \\
\hline 119 & Montana & 215.953 & 329.327 \\
\hline 120 & Nebraska & 2597.926 & 1674.618 \\
\hline 121 & Nevada & 366.329 & 846.604 \\
\hline 122 & New Hampshire & 567.558 & 478.455 \\
\hline 123 & New Jersey & 2985.832 & 5372.077 \\
\hline 124 & New Mexico & 324.984 & 663.775 \\
\hline 125 & New York & 5581.535 & 8040.131 \\
\hline 126 & North Carolina & 5839.210 & 5962.654 \\
\hline 127 & North Dakota & 509.104 & 328.520 \\
\hline 128 & Ohio_N & 2360.553 & 1937.769 \\
\hline 129 & Ohio_M & 2360.553 & 1937.769 \\
\hline 130 & Ohio_s & 2360.553 & 1937.769 \\
\hline 131 & Oklahoma & 1864.040 & 1467.333 \\
\hline 132 & Oregon & 1947.218 & 1822.596 \\
\hline 133 & Pennsylvania & 7435.307 & 6590.244 \\
\hline 134 & Rhode Island & 323.195 & 444.998 \\
\hline 135 & South Carolina & 2173.838 & 2913.847 \\
\hline 136 & South Dakota & 702.317 & 485.626 \\
\hline 137 & Tennessee & 4221.984 & 3973.740 \\
\hline 138 & Texas & 10587.258 & 12415.469 \\
\hline 139 & Utah & 1204.068 & 1317.540 \\
\hline 140 & Vermont & 392.351 & 304.382 \\
\hline 141 & Virginia & 3372.282 & 3966.449 \\
\hline 142 & Washington & 2974.990 & 3057.055 \\
\hline 143 & West Virginia & 459.173 & 1012.381 \\
\hline 144 & Wisconsin & 7168.060 & 3835.254 \\
\hline 145 & Wyoming & 76.798 & 188.198 \\
\hline
\end{tabular}




\begin{tabular}{|c|c|c|c|}
\hline & $\begin{array}{r}\text { Locale } \\
\end{array}$ & P06 15 & A06 15 \\
\hline 1 & Alabama & 2852.847 & 2695.418 \\
\hline 2 & Arizona & 1316.959 & 2344.336 \\
\hline 3 & Arkansas & 4133.071 & 2774.362 \\
\hline$\overline{4}$ & California & 15008.745 & 17017.309 \\
\hline 5 & Colorado & 1841.700 & 2029.153 \\
\hline 6 & Connecticut & 1512.388 & 1647.016 \\
\hline 7 & Delaware & 725.402 & 822.642 \\
\hline 8 & DC & .000 & .000 \\
\hline 9 & Florida & 4256.089 & 7114.177 \\
\hline 10 & Georgia & 5747.710 & 5587.065 \\
\hline 11 & Idaho & 3193.484 & 1144.998 \\
\hline 12 & Illinois_N & 3193.484 & 5037.123 \\
\hline 13 & Illinois_S & 3193.484 & 2518.561 \\
\hline 14 & Adams & 42.695 & 27.187 \\
\hline 15 & Allen & 319.889 & 161.887 \\
\hline 16 & Bartholomew & 226.415 & 71.867 \\
\hline 17 & Benton & 2.086 & 2.039 \\
\hline 18 & Blackford & 5.284 & 4.915 \\
\hline 19 & Boone & 22.314 & 14.963 \\
\hline 20 & Brown & .614 & 4.409 \\
\hline 21 & Carroll & 114.156 & 66.096 \\
\hline 22 & Cass & 111.133 & 66.730 \\
\hline 23 & Clark & 52.504 & 58.550 \\
\hline 24 & Clay & 4.131 & 8.532 \\
\hline 25 & Clinton & 141.184 & 86.549 \\
\hline 26 & Crawford & .000 & 2.859 \\
\hline 27 & Daviess & 73.155 & 45.525 \\
\hline 28 & Dearborn & 6.242 & 14.745 \\
\hline 29 & Decatur & 39.918 & 18.660 \\
\hline 30 & DeKalb & 30.536 & 20.721 \\
\hline 31 & Delaware & 47.501 & 36.371 \\
\hline 32 & Dubois & 56.147 & 40.253 \\
\hline 33 & Elkhart & 101.830 & 128.938 \\
\hline 34 & Fayette & 23.346 & 5.880 \\
\hline 35 & Floyd & 73.115 & 49.140 \\
\hline 36 & Fountain & 3.647 & 6.486 \\
\hline 37 & Franklin & 3.127 & 6.028 \\
\hline 38 & Fulton & 22.608 & 10.518 \\
\hline 39 & Gibson & 31.849 & 21.540 \\
\hline 40 & Grant & 35.149 & 26.555 \\
\hline 41 & Greene & 22.444 & 20.341 \\
\hline 42 & Hamilton & 24.701 & 78.597 \\
\hline 43 & Hancock & 11.934 & 17.882 \\
\hline 44 & Harrison & 57.103 & 41.531 \\
\hline 45 & Hendricks & 56.752 & 63.062 \\
\hline 46 & Henry & 16.054 & 12.831 \\
\hline 47 & Howard & 22.618 & 25.974 \\
\hline 48 & Huntington & 41.958 & 25.534 \\
\hline 49 & Jackson & 27.759 & 16.552 \\
\hline 50 & \begin{tabular}{|l} 
Jasper \\
\end{tabular} & 14.748 & 15.465 \\
\hline 51 & Jay & 32.925 & 19.106 \\
\hline
\end{tabular}




\begin{tabular}{|c|c|c|c|}
\hline & Locale & P06 15 & A06 15 \\
\hline 52 & Jefferson & 30.080 & $\quad 10.688$ \\
\hline 53 & Jennings & 11.810 & 7.654 \\
\hline 54 & Johnson & 34.116 & 39.643 \\
\hline 55 & Knox & 10.472 & $\overline{13.224}$ \\
\hline 56 & Kosciusko & 92.099 & 60.896 \\
\hline 57 & LaGrange & 34.452 & 16.835 \\
\hline 58 & Lake & 117.802 & 196.027 \\
\hline 59 & La Porte & 103.611 & 54.284 \\
\hline 60 & Lawrence & 17.445 & 12.815 \\
\hline 61 & Madison & 61.293 & 69.484 \\
\hline 62 & Marion & 560.691 & 529.387 \\
\hline 63 & Marshall & 34.468 & 31.421 \\
\hline 64 & Martin & 3.765 & 4.236 \\
\hline 65 & Miami & 10.352 & 11.732 \\
\hline 66 & Monroe & 78.406 & 41.899 \\
\hline 67 & Montgomery & 30.366 & 27.636 \\
\hline 68 & Morgan & 12.887 & 32.808 \\
\hline 69 & Newton & 3.633 & 4.118 \\
\hline 70 & Noble & 66.578 & 45.170 \\
\hline 71 & Ohio & .000 & 1.352 \\
\hline 72 & Orange & 3.942 & 6.752 \\
\hline$\overline{73}$ & Owen & 1.040 & 5.973 \\
\hline$\overline{74}$ & Parke & 12.732 & 9.817 \\
\hline 75 & Perry & 6.599 & 4.604 \\
\hline 76 & Pike & .000 & 3.166 \\
\hline 77 & Porter & 39.440 & $\overline{61.714}$ \\
\hline 78 & Posey & 6.123 & 62.544 \\
\hline 79 & Pulaski & 22.490 & 5.674 \\
\hline 80 & Putnam & 7.288 & 9.566 \\
\hline 81 & Randolph & 23.393 & 8.733 \\
\hline 82 & Ripley & .645 & 8.951 \\
\hline 83 & Rush & 19.352 & 4.901 \\
\hline 84 & St. Joseph & 102.603 & 118.083 \\
\hline 85 & Scott & 25.974 & 24.162 \\
\hline 86 & Shelby & 25.044 & 22.044 \\
\hline 87 & Spencer & 9.814 & 7.070 \\
\hline 88 & Starke & 13.244 & 7.274 \\
\hline 89 & Steuben & 35.460 & 16.997 \\
\hline 90 & Sullivan & .000 & 5.633 \\
\hline 91 & Switzerland & .000 & 2.411 \\
\hline 92 & Tippecanoe & 161.098 & 123.150 \\
\hline 93 & Tipton & 16.676 & 9.841 \\
\hline$\overline{94}$ & Union & .372 & 3.020 \\
\hline 95 & Vanderburgh & 266.126 & 239.289 \\
\hline 96 & Vermillion & .457 & 52.191 \\
\hline 97 & Vigo & 31.757 & 63.915 \\
\hline 98 & Wabash & 20.250 & 20.595 \\
\hline 99 & Warren & 7.268 & 2.305 \\
\hline 100 & Warrick & 2.417 & 17.150 \\
\hline 101 & Washington & 33.208 & 9.140 \\
\hline 102 & Wayne & 51.976 & 41.358 \\
\hline
\end{tabular}




\begin{tabular}{|c|c|c|c|}
\hline & Locale & P06 15 & A06 15 \\
\hline 103 & Wells & 46.148 & 25.768 \\
\hline 104 & White & 13.534 & 7.464 \\
\hline 105 & Whitley & 5.752 & 8.826 \\
\hline 106 & lowa & 4299.093 & 2482.291 \\
\hline 107 & Kansas & 2776.981 & 1931.336 \\
\hline 108 & Kentucky_E & 1313.990 & 1299.511 \\
\hline 109 & Kentucky_W & 875.993 & 866.340 \\
\hline 110 & Louisiana & 1430.613 & 2527.280 \\
\hline 111 & Maine & 524.738 & 603.244 \\
\hline 112 & Maryland & 1634.808 & 2559.379 \\
\hline 113 & Massachusetts & 2640.942 & 2990.331 \\
\hline 114 & Michigan_E & 3086.634 & 2320.723 \\
\hline 115 & Michigan_W & 3086.634 & 2320.723 \\
\hline 116 & Minnesota & 4665.429 & 3207.925 \\
\hline 117 & Mississippi & 2321.413 & 1845.889 \\
\hline 118 & Missouri & 3825.713 & 3461.323 \\
\hline 119 & Montana & 215.953 & 329.327 \\
\hline 120 & Nebraska & 2597.926 & 1674.618 \\
\hline 121 & Nevada & 366.329 & 846.604 \\
\hline 122 & New Hampshire & 567.558 & 478.455 \\
\hline 123 & New Jersey & 2985.832 & 5372.077 \\
\hline 124 & New Mexico & 324.984 & 663.775 \\
\hline 125 & New York & 5581.535 & 8040.131 \\
\hline 126 & North Carolina & 5839.210 & 5962.654 \\
\hline 127 & North Dakota & 509.104 & 328.520 \\
\hline 128 & Ohio_N & 2360.553 & 1937.769 \\
\hline 129 & Ohio_M & 2360.553 & 1937.769 \\
\hline 130 & Ohio_s & 2360.553 & 1937.769 \\
\hline 131 & Oklahoma & 1864.040 & 1467.333 \\
\hline 132 & Oregon & 1947.218 & 1822.596 \\
\hline 133 & Pennsylvania & 7435.307 & 6590.244 \\
\hline 134 & Rhode Island & 323.195 & 444.998 \\
\hline 135 & South Carolina & 2173.838 & 2913.847 \\
\hline 136 & South Dakota & 702.317 & 485.626 \\
\hline 137 & Tennessee & 4221.984 & 3973.740 \\
\hline 138 & Texas & 10587.258 & 12415.469 \\
\hline 139 & Utah & 1204.068 & 1317.540 \\
\hline 140 & Vermont & 392.351 & 304.382 \\
\hline 141 & Virginia & 3372.282 & 3966.449 \\
\hline 142 & Washington & 2974.990 & 3057.055 \\
\hline 143 & West Virginia & 459.173 & 1012.381 \\
\hline 144 & Wisconsin & 7168.060 & 3835.254 \\
\hline 145 & Wyoming & 76.798 & 188.198 \\
\hline
\end{tabular}




\begin{tabular}{|c|c|c|c|}
\hline & Locale & P07 15 & $\mathrm{A} 07 \mathrm{7}$ \\
\hline 1 & Alabama & 9821.075 & 11327.855 \\
\hline 2 & Arizona & 9340.180 & 10018.967 \\
\hline 3 & Arkansas & 11945.983 & 10453.142 \\
\hline 4 & California & 65136.628 & 66655.181 \\
\hline 5 & Colorado & 7388.569 & 7977.261 \\
\hline 6 & Connecticut & 7269.064 & 5944.618 \\
\hline 7 & Delaware & 2349.375 & 2328.232 \\
\hline 8 & $\overline{D C}$ & .000 & .000 \\
\hline 9 & Florida & 27494.658 & 30398.666 \\
\hline 10 & Georgia & 22067.122 & 22984.943 \\
\hline 11. & Idaho & 3858.434 & 3979.724 \\
\hline 12 & Illinois_N & 21086.014 & 18113.902 \\
\hline 13 & Illinois_s & 10543.007 & 9056.951 \\
\hline 14 & Adams & 354.687 & 78.751 \\
\hline 15 & Allen & 564.790 & 737.865 \\
\hline 16 & Bartholomew & 233.012 & 326.182 \\
\hline 17 & Benton & 8.369 & 33.316 \\
\hline 18 & Blackford & 18.685 & 74.009 \\
\hline 19 & Boone & 104.037 & 75.885 \\
\hline 20 & Brown & 18.478 & 19.862 \\
\hline 21 & Carroll & 220.803 & 191.008 \\
\hline 22 & Cass & 226.878 & 203.850 \\
\hline 23 & Clark & 143.148 & 167.952 \\
\hline 24 & Clay & 27.431 & 33.089 \\
\hline 25 & Clinton & 273.186 & 248.812 \\
\hline 26 & Crawford & 11.735 & 14.341 \\
\hline 27 & Daviess & 149.194 & 135.599 \\
\hline 28 & Dearborn & 58.694 & 71.197 \\
\hline 29 & Decatur & 68.063 & 64.555 \\
\hline 30 & DeKalb & 258.606 & 88.595 \\
\hline 31 & Delaware & 280.074 & 225.449 \\
\hline 32 & Dubois & 134.282 & 159.240 \\
\hline 33 & Elkhart & 461.865 & 423.829 \\
\hline 34 & Fayette & 24.138 & 29.499 \\
\hline 35 & Floyd & 177.846 & 195.945 \\
\hline 36 & Fountain & 56.208 & 26.485 \\
\hline 37 & Franklin & 24.439 & 29.388 \\
\hline 38 & Fulton & 70.482 & 47.696 \\
\hline 39 & Gibson & 229.249 & 76.712 \\
\hline 40 & Grant & 154.358 & 154.079 \\
\hline 41 & Greene & 105.294 & 73.839 \\
\hline 42 & Hamilton & 360.415 & 368.579 \\
\hline 43 & Hancock & 73.047 & 121.533 \\
\hline 44 & Harrison & 178.679 & 131.097 \\
\hline 45 & Hendricks & 237.354 & 259.409 \\
\hline 46 & Henry & 52.863 & 67.816 \\
\hline 47 & Howard & 134.922 & 118.053 \\
\hline 48 & Huntington & 161.722 & 113.599 \\
\hline 49 & Jackson & 56.815 & 121.123 \\
\hline 50 & Jasper & 56.995 & 59.529 \\
\hline 51 & Jay & 66.760 & 71.326 \\
\hline
\end{tabular}




\begin{tabular}{|c|c|c|c|}
\hline & Locale & P07 15 & A07 15 \\
\hline 52 & Jefferson & 109.459 & 42.635 \\
\hline 53 & Jennings & 30.087 & 45.976 \\
\hline 54 & Johnson & 246.103 & 218.840 \\
\hline 55 & Knox & 50.897 & 64.676 \\
\hline 56 & Kosciusko & 283.470 & 182.217 \\
\hline 57 & LaGrange & 62.300 & 67.608 \\
\hline 58 & Lake & 615.127 & 734.004 \\
\hline 59 & La Porte & 200.155 & 248.317 \\
\hline 60 & Lawrence & 52.806 & 59.842 \\
\hline 61 & Madison & 220.292 & 258.854 \\
\hline 62 & Marion & 1386.692 & 1833.965 \\
\hline 63 & Marshall & 141.211 & 171.117 \\
\hline 64 & Martin & 15.844 & 16.951 \\
\hline 65 & Miami & 170.057 & 94.915 \\
\hline 66 & Monroe & 928.478 & 202.177 \\
\hline 67 & Montgomery & 225.358 & 107.325 \\
\hline 68 & Morgan & 80.317 & 97.640 \\
\hline 69 & Newton & 72.652 & 26.723 \\
\hline 70 & Noble & 335.147 & 157.428 \\
\hline 71 & Ohio & 5.551 & 6.784 \\
\hline 72 & Orange & 162.674 & 29.367 \\
\hline$\overline{73}$ & Owen & 24.228 & 29.150 \\
\hline 74 & Parke & 67.722 & 35.977 \\
\hline 75 & Perry & 84.238 & 22.375 \\
\hline 76 & Pike & 12.998 & 15.884 \\
\hline 77 & Porter & 203.769 & 213.205 \\
\hline$\overline{78}$ & Posey & 34.385 & 39.324 \\
\hline 79 & Pulaski & 93.350 & 24.437 \\
\hline 80 & Putnam & 38.982 & 47.192 \\
\hline 81 & Randolph & 33.827 & 38.184 \\
\hline 82 & Ripley & 28.807 & 60.428 \\
\hline 83 & Rush & 19.443 & 30.544 \\
\hline 84 & St. Joseph & 540.828 & 487.110 \\
\hline 85 & Scott & 78.179 & 64.712 \\
\hline 86 & Shelby & 73.688 & 70.294 \\
\hline 87 & Spencer & 26.237 & 29.549 \\
\hline 88 & Starke & 22.880 & 36.001 \\
\hline 89 & Steuben & 153.268 & 73.922 \\
\hline 90 & Sullivan & 21.878 & 26.737 \\
\hline 91 & Switzerland & 9.896 & 13.782 \\
\hline 92 & Tippecanoe & 266.130 & 286.628 \\
\hline 93 & Tipton & 34.513 & 42.967 \\
\hline 94 & Union & 9.018 & 8.710 \\
\hline 95 & Vanderburgh & 1320.017 & 641.110 \\
\hline 96 & Vermillion & 16.474 & 42.562 \\
\hline 97 & Vigo & 140.626 & 220.937 \\
\hline 98 & Wabash & 50.535 & 95.904 \\
\hline 99 & Warren & 9.178 & 10.771 \\
\hline 100 & Warrick & 61.833 & 74.592 \\
\hline 101 & Washington & 47.664 & 40.941 \\
\hline 102 & Wayne & 265.026 & 169.229 \\
\hline
\end{tabular}




\begin{tabular}{|r|l|r|r|}
\hline & \multicolumn{1}{|c|}{ Locale } & \multicolumn{1}{c|}{ P07 15} & \multicolumn{1}{c|}{ A07 15} \\
\hline 103 & Wells & 243.195 & 95.379 \\
\hline 104 & White & 169.248 & 42.564 \\
\hline 105 & Whitley & 164.203 & 68.408 \\
\hline 106 & lowa & 10683.108 & 8592.086 \\
\hline 107 & Kansas & 7209.699 & 6705.209 \\
\hline 108 & Kentucky_E & 6069.782 & 5113.453 \\
\hline 109 & Kentucky_W & 4046.522 & 3408.969 \\
\hline 110 & Louisiana & 6903.260 & 8699.702 \\
\hline 111 & Maine & 2307.547 & 3978.963 \\
\hline 112 & Maryland & 8503.770 & 10026.924 \\
\hline 113 & Massachusetts & 12256.213 & 12676.039 \\
\hline 114 & Michigan_E & 16818.545 & 9115.832 \\
\hline 115 & Michigan_W & 8409.273 & 9115.832 \\
\hline 116 & Minnesota & 12943.816 & 13332.723 \\
\hline 117 & Mississippi & 9046.922 & 7263.316 \\
\hline 118 & Missouri & 14049.054 & 12664.657 \\
\hline 119 & Montana & 1228.979 & 1497.167 \\
\hline 120 & Nebraska & 6528.063 & 5516.874 \\
\hline 121 & Nevada & 3306.357 & 3734.909 \\
\hline 122 & New Hampshire & 2945.218 & 2478.650 \\
\hline 123 & New Jersey & 14361.354 & 16220.069 \\
\hline 124 & New Mexico & 2630.696 & 2848.423 \\
\hline 125 & New York & 27999.403 & 30828.188 \\
\hline 126 & North Carolina & 24094.090 & 20671.276 \\
\hline 127 & North Dakota & 1207.694 & 1244.991 \\
\hline 128 & Ohio_N & 8755.587 & 7466.617 \\
\hline 129 & Ohio_M & 8755.587 & 7466.617 \\
\hline 130 & Ohio_S & 8755.587 & 7466.617 \\
\hline 131 & Oklahoma & 5746.726 & 6166.634 \\
\hline 132 & Oregon & 6598.837 & 7916.742 \\
\hline 133 & Pennsylvania & 26179.999 & 26594.185 \\
\hline 134 & Rhode Island & 2219.484 & 1883.977 \\
\hline 135 & South Carolina & 9581.668 & 9076.029 \\
\hline 136 & South Dakota & 1745.778 & 1781.315 \\
\hline 137 & Tennessee & 15793.673 & 13795.432 \\
\hline 138 & Texas & 41324.497 & 43243.901 \\
\hline 139 & Utah & 13518.129 & 4923.052 \\
\hline 140 & Vermont & 152.354 & 1416.705 \\
\hline 141 & Virginia & & 15838.826 \\
\hline 142 & Washington & 13785.684 \\
\hline 143 & West Virginia & 2726.885 \\
\hline 144 & Wisconsin & Wyoming & 18700.843 \\
\hline 145 & 692.012 \\
\hline
\end{tabular}




\begin{tabular}{|c|c|c|c|}
\hline & Locale & P08 15 & A08 15 \\
\hline 1 & Alabama & 2548.120 & 3509.376 \\
\hline 2 & Arizona & 4849.791 & 3747.554 \\
\hline 3 & Arkansas & 1404.338 & 1515.744 \\
\hline 4 & California & 29059.440 & 29097.086 \\
\hline 5 & Colorado & 3264.401 & 3220.386 \\
\hline 6 & Connecticut & 2213.726 & 1733.902 \\
\hline 7 & Delaware & 455.577 & 397.730 \\
\hline 8 & DC & .000 & .000 \\
\hline 9 & Florida & 11012.671 & 10412.486 \\
\hline 10 & Georgia & 4845.402 & 6119.713 \\
\hline 11 & Idaho & 1245.448 & 877.392 \\
\hline 12 & Illinois_N & 5177.909 & 4362.485 \\
\hline 13 & Illinois_s & 2588.954 & 2181.243 \\
\hline 14 & Adams & 38.285 & 31.722 \\
\hline 15 & Allen & 274.523 & 199.036 \\
\hline 16 & Bartholomew & 55.509 & 40.268 \\
\hline 17 & Benton & 3.794 & 3.199 \\
\hline 18 & Blackford & 5.784 & 4.877 \\
\hline 19 & Boone & 41.065 & 29.620 \\
\hline 20 & Brown & 7.297 & 8.143 \\
\hline 21 & Carroll & 9.098 & 7.671 \\
\hline 22 & Cass & 27.831 & 22.861 \\
\hline 23 & Clark & 50.814 & 42.197 \\
\hline 24 & Clay & 12.204 & 10.184 \\
\hline 25 & Clinton & 36.440 & 29.998 \\
\hline 26 & Crawford & 5.320 & 4.486 \\
\hline 27 & Daviess & 13.695 & 18.042 \\
\hline 28 & Dearborn & 26.325 & 127.742 \\
\hline 29 & Decatur & 11.182 & 9.428 \\
\hline 30 & DeKalb & 29.079 & 21.143 \\
\hline 31 & Delaware & 55.061 & 47.539 \\
\hline 32 & Dubois & 40.036 & 25.908 \\
\hline 33 & Elkhart & 155.592 & 106.409 \\
\hline 34 & Fayette & 10.944 & 9.227 \\
\hline 35 & Floyd & 53.826 & 40.790 \\
\hline 36 & Fountain & 9.401 & 9.382 \\
\hline 37 & Franklin & 10.527 & 8.876 \\
\hline 38 & Fulton & 9.578 & 22.282 \\
\hline 39 & Gibson & 21.119 & 15.681 \\
\hline 40 & Grant & & \\
\hline 41 & Greene & 16.565 & 13.357 \\
\hline 42 & Hamilton & 138.009 & 113.977 \\
\hline 43 & Hancock & 32.817 & 35.463 \\
\hline 44 & Harrison & 18.596 & 15.678 \\
\hline 45 & Hendricks & 79.410 & 64.690 \\
\hline 46 & Henry & 21.861 & 17.878 \\
\hline 47 & Howard & 40.204 & 40.643 \\
\hline 48 & Huntington & 62.796 & 35.725 \\
\hline 49 & Jackson & 18.790 & 15.842 \\
\hline 50 & Jasper & 19.020 & 14.142 \\
\hline 51 & Jay & 9.246 & 14.530 \\
\hline
\end{tabular}




\begin{tabular}{|c|c|c|c|}
\hline & Locale & P08 15 & A08 15 \\
\hline 52 & Jefferson & 15.104 & 13.980 \\
\hline 53 & Jennings & 13.641 & 11.501 \\
\hline 54 & Johnson & 89.254 & 70.083 \\
\hline 55 & Knox & 18.976 & 15.218 \\
\hline 56 & Kosciusko & 38.099 & 37.179 \\
\hline 57 & LaGrange & 20.177 & 16.320 \\
\hline 58 & Lake & 217.745 & 227.995 \\
\hline 59 & La Porte & 50.319 & 68.710 \\
\hline 60 & Lawrence & 21.788 & 24.595 \\
\hline 61 & Madison & 61.532 & 51.774 \\
\hline 62 & Marion & 448.707 & 519.102 \\
\hline 63 & Marshall & 22.177 & 41.362 \\
\hline 64 & Martin & 4.399 & 3.709 \\
\hline 65 & Miami & 15.542 & 13.784 \\
\hline 66 & Monroe & 91.439 & 74.034 \\
\hline 67 & Montgomery & 17.395 & 15.025 \\
\hline 68 & Morgan & 91.394 & 55.436 \\
\hline 69 & Newton & 11.132 & 7.584 \\
\hline 70 & Noble & 23.636 & 19.268 \\
\hline 71 & Ohio & 2.517 & 2.122 \\
\hline 72 & Orange & 8.916 & 8.669 \\
\hline 73 & Owen & 10.456 & 8.815 \\
\hline 74 & Parke & 7.516 & 7.501 \\
\hline 75 & Perry & 8.234 & 6.845 \\
\hline 76 & Pike & 5.893 & 4.968 \\
\hline 77 & Porter & 70.904 & 58.949 \\
\hline 78 & Posey & 12.476 & 10.518 \\
\hline 79 & Pulaski & 6.134 & 5.171 \\
\hline 80 & Putnam & 21.990 & 16.660 \\
\hline 81 & Randolph & 13.213 & 10.879 \\
\hline 82 & Ripley & 14.221 & 14.777 \\
\hline 83 & Rush & 7.852 & 6.849 \\
\hline 84 & St. Joseph & 151.761 & 119.709 \\
\hline 85 & Scott & 10.922 & 16.522 \\
\hline 86 & Shelby & 19.672 & 16.586 \\
\hline 87 & Spencer & 8.992 & 7.581 \\
\hline 88 & Starke & 10.373 & 8.746 \\
\hline 89 & Steuben & 17.111 & 13.786 \\
\hline 90 & Sullivan & 9.919 & 10.512 \\
\hline 91 & Switzerland & 4.487 & 3.783 \\
\hline 92 & Tippecanoe & 114.404 & 82.440 \\
\hline 93 & Tipton & 7.167 & 6.043 \\
\hline 94 & Union & 3.231 & 2.724 \\
\hline 95 & Vanderburgh & 78.680 & 90.288 \\
\hline 96 & Vermillion & 7.097 & 5.984 \\
\hline 97 & Vigo & 93.351 & 60.683 \\
\hline 98 & Wabash & 28.620 & 18.932 \\
\hline 99 & Warren & 3.647 & 3.075 \\
\hline 100 & Warrick & 27.106 & 22.853 \\
\hline 101 & Washington & 13.031 & 14.465 \\
\hline 102 & Wayne & 52.344 & 36.680 \\
\hline
\end{tabular}




\begin{tabular}{|c|c|c|c|}
\hline & Locale & P08 15 & A08 15 \\
\hline 103 & Wells & 13.385 & 11.172 \\
\hline 104 & White & 15.936 & 11.623 \\
\hline 105 & Whitley & 20.177 & 15.055 \\
\hline 106 & lowa & 1747.966 & 1563.537 \\
\hline 107 & Kansas & 1404.565 & 1298.196 \\
\hline 108 & Kentucky_E & 1313.923 & 2021.022 \\
\hline 109 & Kentucky_W & 875.949 & 1347.348 \\
\hline 110 & Louisiana & 2087.918 & 2249.200 \\
\hline 111 & Maine & 781.098 & 747.268 \\
\hline 112 & Maryland & 3392.538 & 2970.209 \\
\hline 113 & Massachusetts & 5659.235 & 4355.236 \\
\hline 114 & Michigan_E & 2653.895 & 2354.822 \\
\hline 115 & Michigan_W & 2653.895 & 2354.822 \\
\hline 116 & Minnesota & 4217.972 & 3135.700 \\
\hline 117 & Mississippi & 1424.282 & 1882.692 \\
\hline 118 & Missouri & 3016.272 & 3335.324 \\
\hline 119 & Montana & 446.496 & 423.725 \\
\hline 120 & Nebraska & 942.202 & 825.406 \\
\hline 121 & Nevada & 1324.207 & 1214.274 \\
\hline 122 & New Hampshire & 1381.317 & 1022.297 \\
\hline 123 & New Jersey & 4975.562 & 5009.001 \\
\hline 124 & New Mexico & 1201.671 & 972.092 \\
\hline 125 & New York & 10696.015 & 11658.982 \\
\hline 126 & North Carolina & 5853.582 & 9282.149 \\
\hline 127 & North Dakota & 320.181 & 279.279 \\
\hline 128 & Ohio_N & 2016.936 & 1873.024 \\
\hline 129 & Ohio_M & 2016.936 & 1873.024 \\
\hline 130 & Ohio_s & 2016.936 & 1873.024 \\
\hline 131 & Oklahoma & 1925.777 & 1903.863 \\
\hline 132 & Oregon & 2925.952 & 2427.854 \\
\hline 133 & Pennsylvania & 7129.334 & 7634.094 \\
\hline 134 & Rhode Island & 679.343 & 573.575 \\
\hline 135 & South Carolina & 2476.084 & 2636.953 \\
\hline 136 & South Dakota & 706.825 & 496.093 \\
\hline 137 & Tennessee & 3162.190 & 4247.257 \\
\hline 138 & Texas & 15678.293 & 14790.898 \\
\hline 139 & Utah & 1681.379 & 1378.877 \\
\hline 140 & Vermont & 572.170 & 461.183 \\
\hline 141 & Virginia & 4553.535 & 6132.610 \\
\hline 142 & Washington & 4404.029 & 3804.660 \\
\hline 143 & West Virginia & 828.464 & 838.277 \\
\hline 144 & Wisconsin & 3193.994 & 2939.385 \\
\hline 145 & Wyoming & 242.173 & 223.106 \\
\hline
\end{tabular}




\begin{tabular}{|c|c|c|c|}
\hline & Locale & P09 15 & A09 15 \\
\hline 1 & Alabama & 363.743 & 370.877 \\
\hline 2 & Arizona & 82.868 & 79.176 \\
\hline 3 & Arkansas & 107.152 & 103.376 \\
\hline 4 & California & 680.387 & 660.226 \\
\hline 5 & Colorado & 42.634 & 40.747 \\
\hline 6 & Connecticut & 56.891 & 57.508 \\
\hline 7 & Delaware & 15.034 & 15.232 \\
\hline 8 & DC & .000 & .000 \\
\hline 9 & Florida & 197.154 & 190.354 \\
\hline 10 & Georgia & 844.728 & 873.113 \\
\hline 11 & Idaho & 14.610 & 13.841 \\
\hline 12 & Illinois_N & 97.930 & 93.273 \\
\hline 13 & Illinois_S & 48.965 & 46.636 \\
\hline 14 & Adams & 1.865 & 1.759 \\
\hline 15 & Allen & 7.146 & 6.754 \\
\hline 16 & Bartholomew & 5.234 & 4.937 \\
\hline 17 & Benton & .365 & .344 \\
\hline 18 & Blackford & .058 & .054 \\
\hline 19 & Boone & .507 & .478 \\
\hline 20 & Brown & .000 & .000 \\
\hline 21 & Carroll & 2.513 & 2.371 \\
\hline 22 & Cass & .062 & .059 \\
\hline 23 & Clark & 7.911 & 7.462 \\
\hline 24 & Clay & .065 & .061 \\
\hline 25 & Clinton & .401 & .378 \\
\hline 26 & Crawford & .000 & .000 \\
\hline 27 & Daviess & .411 & .387 \\
\hline 28 & Dearborn & .000 & .000 \\
\hline 29 & Decatur & .066 & .062 \\
\hline 30 & DeKalb & .072 & .068 \\
\hline 31 & Delaware & .938 & .885 \\
\hline 32 & \begin{tabular}{|l|} 
Dubois \\
\end{tabular} & 44.659 & 42.126 \\
\hline 33 & Elkhart & 27.144 & 25.617 \\
\hline 34 & Fayette & .358 & .338 \\
\hline 35 & Floyd & 3.094 & 2.919 \\
\hline 36 & Fountain & .000 & .000 \\
\hline 37 & Franklin & .000 & .000 \\
\hline 38 & Fulton & .000 & .000 \\
\hline 39 & Gibson & .000 & .000 \\
\hline 40 & Grant & .996 & .939 \\
\hline 41 & Greene & .063 & .059 \\
\hline 42 & Hamilton & 2.411 & 2.290 \\
\hline 43 & Hancock & .501 & .473 \\
\hline 44 & Harrison & 5.107 & 4.817 \\
\hline 45 & Hendricks & .099 & .093 \\
\hline 46 & Henry & 1.034 & .975 \\
\hline 47 & Howard & 2.410 & 2.274 \\
\hline 48 & Huntington & .000 & .000 \\
\hline 49 & Jackson & .391 & .369 \\
\hline 50 & Jasper & .067 & .063 \\
\hline 51 & Jay & .366 & .345 \\
\hline
\end{tabular}




\begin{tabular}{|c|c|c|c|}
\hline & Locale & P09 15 & A09 15 \\
\hline 52 & Jefferson & .068 & .064 \\
\hline 53 & Jennings & .000 & .000 \\
\hline 54 & Johnson & .745 & .702 \\
\hline 55 & Knox & .060 & .056 \\
\hline 56 & Kosciusko & 2.170 & 2.059 \\
\hline 57 & LaGrange & 2.146 & 2.024 \\
\hline 58 & Lake & 1.618 & 1.526 \\
\hline 59 & La Porte & 1.799 & 1.769 \\
\hline 60 & Lawrence & .062 & .058 \\
\hline 61 & Madison & 2.783 & 2.638 \\
\hline 62 & Marion & 9.047 & 8.609 \\
\hline 63 & Marshall & .418 & .394 \\
\hline 64 & Martin & .000 & .000 \\
\hline 65 & Miami & 2.239 & 2.112 \\
\hline 66 & Monroe & .502 & .473 \\
\hline 67 & Montgomery & .000 & .000 \\
\hline 68 & Morgan & .076 & .072 \\
\hline 69 & Newton & .382 & .361 \\
\hline 70 & Noble & .408 & .385 \\
\hline 71 & Ohio & .000 & .000 \\
\hline 72 & Orange & 10.961 & 10.340 \\
\hline 73 & Owen & .000 & .000 \\
\hline 74 & Parke & .063 & .060 \\
\hline 75 & Perry & 1.763 & 1.663 \\
\hline 76 & Pike & .000 & .000 \\
\hline 77 & Porter & 1.055 & 1.070 \\
\hline 78 & Posey & .000 & .000 \\
\hline 79 & Pulaski & .066 & .062 \\
\hline 80 & Putnam & .000 & .000 \\
\hline 81 & Randolph & .720 & .748 \\
\hline 82 & Ripley & 1.183 & 1.116 \\
\hline 83 & Rush & .000 & .000 \\
\hline 84 & St. Joseph & 1.231 & 1.174 \\
\hline 85 & Scott & .398 & .375 \\
\hline 86 & Shelby & 2.122 & 2.002 \\
\hline 87 & Spencer & 4.678 & 4.412 \\
\hline 88 & Starke & .062 & .058 \\
\hline 89 & Steuben & .066 & .062 \\
\hline 90 & Sullivan & .000 & .000 \\
\hline 91 & Switzerland & .078 & .073 \\
\hline 92 & Tippecanoe & 5.614 & 5.296 \\
\hline 93 & Tipton & .000 & .000 \\
\hline 94 & Union & .000 & .000 \\
\hline 95 & Vanderburgh & 2.844 & 2.696 \\
\hline 96 & Vermillion & .000 & .000 \\
\hline 97 & Vigo & .390 & .368 \\
\hline 98 & Wabash & .061 & .057 \\
\hline 99 & Warren & .000 & .000 \\
\hline 100 & Warrick & .070 & .066 \\
\hline 101 & Washington & 11.949 & 11.271 \\
\hline 102 & Wayne & 4.212 & 3.973 \\
\hline
\end{tabular}




\begin{tabular}{|c|c|c|c|}
\hline & Locale & P09 15 & A09 15 \\
\hline 103 & Wells & .196 & .198 \\
\hline 104 & White & 3.141 & 3.174 \\
\hline 105 & Whitley & .308 & .290 \\
\hline 106 & lowa & 56.383 & 53.338 \\
\hline 107 & Kansas & 33.244 & 31.515 \\
\hline 108 & Kentucky_E & 35.513 & 34.733 \\
\hline 109 & Kentucky_W & 23.676 & 23.155 \\
\hline 110 & Louisiana & 9.700 & 9.299 \\
\hline 111 & Maine & 67.751 & 69.754 \\
\hline 112 & Maryland & 53.233 & 52.537 \\
\hline 113 & Massachusetts & 196.565 & 201.097 \\
\hline 114 & Michigan_E & 117.327 & 111.801 \\
\hline 115 & Michigan_W & 117.327 & 111.081 \\
\hline 116 & Minnesota & 93.868 & 89.567 \\
\hline 117 & Mississippi & 225.096 & 215.700 \\
\hline 118 & Missouri & 91.323 & 86.984 \\
\hline 119 & Montana & 6.369 & 6.086 \\
\hline 120 & Nebraska & 24.755 & 23.815 \\
\hline 121 & Nevada & 16.232 & 15.330 \\
\hline 122 & New Hampshire & 45.288 & 46.398 \\
\hline 123 & New Jersey & 172.906 & 175.114 \\
\hline 124 & New Mexico & 7.724 & 7.368 \\
\hline 125 & New York & 284.384 & 283.772 \\
\hline 126 & North Carolina & 2159.161 & 2199.344 \\
\hline 127 & North Dakota & 7.343 & 6.997 \\
\hline 128 & Ohio_N & 59.821 & 57.911 \\
\hline 129 & Ohio_M & 59.821 & 57.911 \\
\hline 130 & Ohio_S & 59.821 & 57.911 \\
\hline 131 & Oklahoma & 27.649 & 26.598 \\
\hline 132 & Oregon & 47.530 & 45.188 \\
\hline 133 & Pennsylvania & 320.038 & 318.720 \\
\hline 134 & Rhode Island & 87.549 & 90.221 \\
\hline 135 & South Carolina & 833.985 & 869.752 \\
\hline 136 & South Dakota & 19.845 & 19.657 \\
\hline 137 & Tennessee & 331.708 & 328.779 \\
\hline 138 & Texas & 290.316 & 282.836 \\
\hline 139 & Utah & 47.788 & 45.262 \\
\hline 140 & Vermont & 21.220 & 20.180 \\
\hline 141 & Virginia & 449.758 & 453.319 \\
\hline 142 & Washington & 65.150 & 62.502 \\
\hline 143 & West Virginia & 11.393 & 11.194 \\
\hline 144 & Wisconsin & 148.139 & 142.903 \\
\hline 145 & Wyoming & 1.584 & 1.507 \\
\hline
\end{tabular}




\begin{tabular}{|c|c|c|c|}
\hline & Locale & P10 15 & A10 15 \\
\hline 1 & Alabama & 244.922 & 273.782 \\
\hline 2 & Arizona & 318.710 & 183.488 \\
\hline 3 & Arkansas & 159.061 & 150.518 \\
\hline 4 & California & 2411.947 & 1430.745 \\
\hline 5 & Colorado & 268.309 & 141.126 \\
\hline 6 & Connecticut & 227.940 & 318.937 \\
\hline 7 & Delaware & 64.415 & 160.078 \\
\hline 8 & DC & .000 & .000 \\
\hline 9 & Florida & 1100.889 & 495.261 \\
\hline 10 & Georgia & 599.313 & 558.035 \\
\hline 11 & Idaho & 100.732 & 73.030 \\
\hline 12 & Illinois_N & 628.845 & 711.181 \\
\hline 13 & Illinois_S & 314.422 & 355.590 \\
\hline 14 & Adams & 1.659 & 3.658 \\
\hline 15 & Allen & 32.225 & 7.795 \\
\hline 16 & Bartholomew & 2.458 & 3.627 \\
\hline 17 & Benton & .935 & .000 \\
\hline 18 & Blackford & .343 & .000 \\
\hline 19 & Boone & 2.268 & .000 \\
\hline 20 & Brown & .068 & .191 \\
\hline 21 & Carroll & 1.223 & .000 \\
\hline 22 & Cass & 1.212 & .000 \\
\hline 23 & Clark & 3.177 & 14.257 \\
\hline 24 & Clay & .485 & 1.152 \\
\hline 25 & Clinton & 1.034 & 3.470 \\
\hline 26 & Crawford & .000 & .000 \\
\hline 27 & Daviess & 1.140 & 1.220 \\
\hline 28 & Dearborn & .782 & .235 \\
\hline 29 & Decatur & 2.160 & .000 \\
\hline 30 & DeKalb & 4.025 & 4.848 \\
\hline 31 & Delaware & 4.188 & 1.679 \\
\hline 32 & Dubois & 2.905 & 1.208 \\
\hline 33 & Elkhart & 8.684 & 36.438 \\
\hline 34 & Fayette & 1.065 & .000 \\
\hline 35 & Floyd & 3.252 & 2.116 \\
\hline 36 & Fountain & 1.542 & .189 \\
\hline 37 & Franklin & .454 & .000 \\
\hline 38 & Fulton & 1.250 & .192 \\
\hline 39 & Gibson & .674 & .000 \\
\hline 40 & Grant & 1.704 & 1.605 \\
\hline 41 & Greene & .727 & .000 \\
\hline 42 & Hamilton & 14.835 & 1.540 \\
\hline 43 & Hancock & 3.454 & .000 \\
\hline 44 & Harrison & .710 & 1.145 \\
\hline 45 & Hendricks & 3.247 & .294 \\
\hline 46 & Henry & 2.448 & .000 \\
\hline 47 & Howard & 2.244 & .000 \\
\hline 48 & Huntington & 2.000 & 1.243 \\
\hline 49 & Jackson & 2.588 & 3.385 \\
\hline 50 & Jasper & .902 & .000 \\
\hline 51 & Jay & 1.211 & .000 \\
\hline
\end{tabular}




\begin{tabular}{|c|c|c|c|}
\hline & $\begin{array}{r}\text { Locale } \\
\end{array}$ & P10 15 & $\mathrm{A} 10 \quad 15$ \\
\hline 52 & Jefferson & .393 & 1.207 \\
\hline 53 & Jennings & .540 & .210 \\
\hline 54 & Johnson & 2.389 & 1.525 \\
\hline 55 & Knox & 2.983 & .000 \\
\hline 56 & Kosciusko & 3.707 & 6.603 \\
\hline 57 & LaGrange & 2.017 & .212 \\
\hline 58 & Lake & 36.778 & 28.337 \\
\hline 59 & La Porte & 6.909 & 6.249 \\
\hline 60 & Lawrence & 2.387 & .000 \\
\hline 61 & Madison & 3.164 & 7.227 \\
\hline 62 & Marion & 82.694 & 118.403 \\
\hline 63 & Marshall & 2.021 & 2.687 \\
\hline 64 & Martin & .572 & .000 \\
\hline 65 & Miami & 2.731 & 1.064 \\
\hline 66 & Monroe & 7.736 & 3.470 \\
\hline 67 & Montgomery & 1.659 & 3.444 \\
\hline 68 & Morgan & .641 & 8.514 \\
\hline 69 & Newton & .866 & .189 \\
\hline 70 & \begin{tabular}{|l|} 
Noble \\
\end{tabular} & 2.618 & 4.366 \\
\hline 71 & Ohio & .000 & .000 \\
\hline 72 & Orange & .379 & .000 \\
\hline 73 & Owen & .072 & .000 \\
\hline 74 & Parke & .403 & .000 \\
\hline 75 & Perry & .680 & .000 \\
\hline 76 & Pike & .365 & .000 \\
\hline 77 & Porter & 15.284 & 9.126 \\
\hline 78 & Posey & 1.519 & 34.763 \\
\hline 79 & Pulaski & 1.999 & .000 \\
\hline 80 & \begin{tabular}{|l} 
Putnam \\
\end{tabular} & .374 & .000 \\
\hline 81 & Randolph & .711 & .000 \\
\hline 82 & Ripley & 1.255 & 1.205 \\
\hline 83 & Rush & .916 & .000 \\
\hline 84 & St. Joseph & 21.082 & 16.904 \\
\hline 85 & Scott & .082 & 3.445 \\
\hline 86 & Shelby & 1.561 & 3.646 \\
\hline 87 & Spencer & 1.748 & .185 \\
\hline 88 & Starke & .295 & 1.100 \\
\hline 89 & Steuben & .684 & .000 \\
\hline 90 & Sullivan & 1.016 & .196 \\
\hline 91 & Switzerland & .082 & .000 \\
\hline 92 & Tippecanoe & 7.266 & 36.506 \\
\hline 93 & Tipton & 1.021 & .185 \\
\hline 94 & Union & .414 & .831 \\
\hline 95 & Vanderburgh & 11.672 & 47.262 \\
\hline 96 & Vermillion & .489 & 31.129 \\
\hline 97 & Vigo & 5.436 & 19.875 \\
\hline 98 & Wabash & 1.351 & 6.785 \\
\hline 99 & Warren & .422 & .000 \\
\hline 100 & Warrick & 6.059 & 1.673 \\
\hline 101 & Washington & .085 & .000 \\
\hline 102 & Wayne & 3.648 & 2.435 \\
\hline
\end{tabular}




\begin{tabular}{|c|c|c|c|}
\hline & \begin{tabular}{|l} 
Locale \\
\end{tabular} & P10 15 & A10 15 \\
\hline 103 & Wells & 11.496 & .000 \\
\hline 104 & White & 2.204 & .000 \\
\hline 105 & Whitley & 1.442 & .208 \\
\hline 106 & lowa & 227.282 & 135.235 \\
\hline 107 & Kansas & 192.196 & 131.851 \\
\hline 108 & Kentucky_E & 150.019 & 172.554 \\
\hline 109 & Kentucky_W & 100.013 & 115.036 \\
\hline 110 & Louisiana & 235.871 & 542.186 \\
\hline 111 & Maine & 78.713 & 35.537 \\
\hline 112 & Maryland & 296.436 & 310.183 \\
\hline 113 & Massachusetts & 432.694 & 436.523 \\
\hline 114 & Michigan_E & 267.768 & 335.701 \\
\hline 115 & Michigan_W & 267.768 & 335.701 \\
\hline 116 & Minnesota & 386.744 & 191.560 \\
\hline 117 & Mississippi & 141.130 & 145.580 \\
\hline 118 & Missouri & 416.731 & 451.453 \\
\hline 119 & Montana & 53.883 & 10.862 \\
\hline 120 & Nebraska & 136.037 & 54.920 \\
\hline 121 & Nevada & 118.403 & 36.973 \\
\hline 122 & New Hampshire & 72.528 & 40.452 \\
\hline 123 & New Jersey & 982.869 & 1401.044 \\
\hline 124 & New Mexico & 70.804 & 28.724 \\
\hline 125 & New York & 1436.067 & 1204.371 \\
\hline 126 & North Carolina & 548.360 & 1018.963 \\
\hline 127 & North Dakota & 57.613 & 1.106 \\
\hline 128 & Ohio N & 249.488 & 306.418 \\
\hline 129 & Ohio_M & 249.488 & 306.418 \\
\hline 130 & Ohio_s & 249.488 & 306.418 \\
\hline 131 & Oklahoma & 183.392 & 75.859 \\
\hline 132 & Oregon & 270.487 & 97.402 \\
\hline 133 & Pennsylvania & 718.334 & 653.555 \\
\hline 134 & Rhode Island & 52.928 & 52.947 \\
\hline 135 & South Carolina & 198.188 & 817.354 \\
\hline 136 & South Dakota & 56.265 & 14.209 \\
\hline 137 & Tennessee & 428.735 & 733.289 \\
\hline 138 & Texas & 1384.412 & 1860.188 \\
\hline 139 & Utah & 151.535 & 131.810 \\
\hline 140 & Vermont & 44.388 & 7.729 \\
\hline 141 & Virginia & 346.544 & 438.800 \\
\hline 142 & Washington & 420.517 & 116.944 \\
\hline 143 & West Virginia & 67.477 & 262.843 \\
\hline 144 & Wisconsin & 379.778 & 261.364 \\
\hline 145 & Wyoming & 16.927 & 24.916 \\
\hline
\end{tabular}




\begin{tabular}{|c|c|c|c|}
\hline & $\begin{array}{r}\text { Locale } \\
\end{array}$ & P11 15 & A11 15 \\
\hline 1 & Alabama & 5038.244 & 5652.760 \\
\hline 2 & Arizona & 7765.671 & 8540.879 \\
\hline 3 & Arkansas & 2507.498 & 3565.122 \\
\hline 4 & California & 55602.684 & 47494.301 \\
\hline 5 & Colorado & 6949.207 & 5822.093 \\
\hline 6 & Connecticut & 4493.983 & 4412.762 \\
\hline 7 & \begin{tabular}{|l} 
Delaware \\
\end{tabular} & 806.355 & 1097.690 \\
\hline 8 & DC & .000 & .000 \\
\hline 9 & Florida & 23246.818 & 24866.619 \\
\hline 10 & Georgia & 15311.021 & 11853.304 \\
\hline 11 & Idaho & 1454.018 & 1910.068 \\
\hline 12 & Illinois $\mathbf{N}$ & 14305.590 & 10573.712 \\
\hline 13 & Illinois_S & 7152.795 & 5286.856 \\
\hline 14 & Adams & 15.288 & 46.001 \\
\hline 15 & Allen & 874.508 & 465.395 \\
\hline 16 & Bartholomew & 76.134 & 99.607 \\
\hline 17 & Benton & 2.621 & 10.671 \\
\hline 18 & Blackford & 6.178 & 16.267 \\
\hline 19 & Boone & 49.928 & 76.009 \\
\hline 20 & Brown & 1.028 & 19.769 \\
\hline 21 & Carroll & 4.604 & 25.587 \\
\hline 22 & Cass & 28.018 & 50.922 \\
\hline 23 & Clark & 127.044 & 135.473 \\
\hline 24 & Clay & 2.892 & 33.565 \\
\hline 25 & Clinton & 15.678 & 43.973 \\
\hline 26 & Crawford & 1.195 & 14.962 \\
\hline 27 & Daviess & 10.603 & 38.514 \\
\hline 28 & Dearborn & 20.507 & 73.109 \\
\hline 29 & Decatur & 12.456 & 31.449 \\
\hline 30 & DeKalb & 34.608 & 57.394 \\
\hline 31 & \begin{tabular}{|l} 
Delaware \\
\end{tabular} & 109.102 & 154.075 \\
\hline 32 & Dubois & 125.683 & 53.209 \\
\hline 33 & Elkhart & 489.703 & 255.741 \\
\hline 34 & Fayette & 8.016 & 30.777 \\
\hline 35 & Floyd & 67.731 & 101.754 \\
\hline 36 & Fountain & 2.546 & 21.969 \\
\hline 37 & Franklin & 6.831 & 29.606 \\
\hline 38 & \begin{tabular}{|l|} 
Fulton \\
\end{tabular} & 4.845 & 26.182 \\
\hline 39 & Gibson & 11.031 & 41.301 \\
\hline 40 & Grant & 44.081 & 84.819 \\
\hline 41 & Greene & 8.622 & 42.186 \\
\hline 42 & Hamilton & 437.366 & 367.537 \\
\hline 43 & Hancock & 23.087 & 91.316 \\
\hline 44 & Harrison & 23.002 & 52.298 \\
\hline 45 & Hendricks & 130.409 & 203.096 \\
\hline 46 & Henry & 20.287 & 57.479 \\
\hline 47 & Howard & 63.350 & 108.036 \\
\hline 48 & Huntington & 24.966 & 52.122 \\
\hline 49 & Jackson & 26.422 & 52.844 \\
\hline 50 & Jasper & 13.047 & 39.802 \\
\hline 51 & Jay & 5.946 & 26.002 \\
\hline
\end{tabular}




\begin{tabular}{|c|c|c|c|}
\hline & Locale & P11 15 & A11_15 \\
\hline 52 & Jefferson & 17.416 & 42.477 \\
\hline 53 & Jennings & 7.904 & 38.363 \\
\hline 54 & Johnson & 79.158 & 193.515 \\
\hline 55 & Knox & 34.760 & 47.724 \\
\hline 56 & Kosciusko & 54.448 & 97.760 \\
\hline 57 & LaGrange & 27.557 & 51.748 \\
\hline 58 & Lake & 531.777 & 599.525 \\
\hline 59 & La Porte & 84.169 & 136.169 \\
\hline 60 & Lawrence & 15.806 & 56.941 \\
\hline 61 & Madison & 59.493 & 172.292 \\
\hline 62 & Marion & 2307.779 & 1118.667 \\
\hline 63 & Marshall & 54.358 & 61.556 \\
\hline 64 & Martin & 2.102 & 12.372 \\
\hline 65 & Miami & 18.599 & 43.011 \\
\hline 66 & Monroe & 62.062 & 172.231 \\
\hline 67 & Montgomery & 16.934 & 48.921 \\
\hline 68 & Morgan & 39.683 & 100.740 \\
\hline 69 & Newton & 3.053 & 18.282 \\
\hline 70 & Noble & 22.066 & 61.704 \\
\hline 71 & Ohio & .000 & 7.078 \\
\hline 72 & Orange & 4.503 & 25.074 \\
\hline 73 & Owen & 6.530 & 29.405 \\
\hline 74 & Parke & 6.065 & 21.138 \\
\hline 75 & Perry & 5.575 & 22.454 \\
\hline 76 & Pike & 8.454 & 16.573 \\
\hline 77 & Porter & 143.857 & 190.800 \\
\hline 78 & Posey & 24.359 & 35.086 \\
\hline 79 & Pulaski & 10.763 & 17.250 \\
\hline 80 & Putnam & 5.308 & 48.254 \\
\hline 81 & Randolph & 10.619 & 32.884 \\
\hline 82 & Ripley & 40.488 & 35.256 \\
\hline 83 & Rush & 11.049 & 21.379 \\
\hline 84 & St. Joseph & 473.276 & 355.824 \\
\hline 85 & Scott & 6.669 & 30.716 \\
\hline 86 & Shelby & 72.708 & 55.325 \\
\hline 87 & Spencer & 6.974 & 25.288 \\
\hline 88 & Starke & 4.141 & 29.173 \\
\hline 89 & Steuben & 21.742 & 43.491 \\
\hline 90 & Sullivan & 6.013 & 27.895 \\
\hline 91 & Switzerland & 1.242 & 12.618 \\
\hline 92 & Tippecanoe & 82.744 & 217.491 \\
\hline 93 & Tipton & 7.183 & 20.157 \\
\hline 94 & Union & 6.233 & 9.087 \\
\hline 95 & Vanderburgh & 382.150 & 220.482 \\
\hline 96 & Vermillion & 1.052 & 19.959 \\
\hline 97 & Vigo & 96.616 & 129.700 \\
\hline 98 & Wabash & 15.763 & 42.912 \\
\hline 99 & Warren & 6.352 & 10.256 \\
\hline 100 & Warrick & 14.732 & 76.230 \\
\hline 101 & Washington & 8.944 & 36.648 \\
\hline 102 & Wayne & 98.465 & 83.184 \\
\hline
\end{tabular}




\begin{tabular}{|c|c|c|c|}
\hline & Locale & P11_15 & A11 15 \\
\hline 103 & Wells & 11.536 & 36.825 \\
\hline 104 & White & 13.926 & 31.712 \\
\hline 105 & Whitley & 19.786 & 42.601 \\
\hline 106 & lowa & 3395.361 & 3690.223 \\
\hline 107 & Kansas & 3959.126 & 3451.215 \\
\hline 108 & Kentucky_E & 2432.797 & 3151.172 \\
\hline 109 & Kentucky_W & 1621.864 & 2100.782 \\
\hline 110 & Louisiana & 4892.568 & 5706.473 \\
\hline 111 & Maine & 1114.862 & 1687.063 \\
\hline 112 & Maryland & 6492.441 & 7461.078 \\
\hline 113 & Massachusetts & 9350.973 & 8179.791 \\
\hline 114 & Michigan_E & 6536.818 & 6455.561 \\
\hline 115 & Michigan_W & 6536.818 & 6455.561 \\
\hline 116 & Minnesota & 9755.333 & 6773.741 \\
\hline 117 & Mississippi & 2126.210 & 3631.902 \\
\hline 118 & Missouri & 7740.374 & 7338.395 \\
\hline 119 & Montana & 880.080 & 1216.711 \\
\hline 120 & Nebraska & 2201.302 & 2175.329 \\
\hline 121 & Nevada & 2847.220 & 3332.137 \\
\hline 122 & New Hampshire & 1686.220 & 1730.387 \\
\hline 123 & New Jersey & 15797.549 & 11157.999 \\
\hline 124 & New Mexico & 1405.437 & 2458.434 \\
\hline 125 & New York & 21116.297 & 23717.837 \\
\hline 126 & North Carolina & 12040.556 & 11752.843 \\
\hline 127 & North Dakota & 820.370 & 793.055 \\
\hline 128 & Ohio_N & 540.139 & 4754.870 \\
\hline 129 & Ohio_M & 540.139 & 4754.870 \\
\hline 130 & Ohio_s & 540.139 & 4754.870 \\
\hline 131 & Oklahoma & 3834.119 & 4417.452 \\
\hline 132 & Oregon & 5256.115 & 4783.255 \\
\hline 133 & Pennsylvania & 14643.124 & 15619.831 \\
\hline 134 & Rhode Island & 1327.021 & 1375.613 \\
\hline 135 & South Carolina & 4200.803 & 5511.812 \\
\hline 136 & South Dakota & 866.882 & 969.761 \\
\hline 137 & Tennessee & 8217.335 & 7756.722 \\
\hline 138 & Texas & 35516.645 & 31063.147 \\
\hline 139 & Utah & 3449.441 & 3260.431 \\
\hline 140 & Vermont & 533.071 & 815.135 \\
\hline 141 & Virginia & 7707.781 & 10081.722 \\
\hline 142 & Washington & 8137.800 & 8259.161 \\
\hline 143 & West Virginia & 1506.797 & 2263.045 \\
\hline 144 & Wisconsin & 7359.903 & 7128.574 \\
\hline 145 & Wyoming & 409.083 & 645.948 \\
\hline
\end{tabular}




\begin{tabular}{|c|c|c|c|}
\hline & Locale & P12 15 & A12 15 \\
\hline 1 & Alabama & 45609.169 & 53289.049 \\
\hline 2 & Arizona & 18167.780 & 21416.751 \\
\hline 3 & Arkansas & 41493.853 & 37161.570 \\
\hline 4 & California & 197961.961 & 199535.734 \\
\hline 5 & Colorado & 18301.052 & 29373.075 \\
\hline 6 & Connecticut & 15851.254 & 12431.448 \\
\hline 7 & Delaware & 10000.044 & 6323.565 \\
\hline 8 & $\mathrm{DC}$ & .000 & .000 \\
\hline 9 & Florida & 53832.342 & 56419.026 \\
\hline 10 & Georgia & 132764.865 & 134548.548 \\
\hline 11 & Idaho & 11250.464 & 9665.199 \\
\hline 12 & Illinois_N & 71270.480 & 57650.106 \\
\hline 13 & Illinois_S & 35635.240 & 28825.053 \\
\hline 14 & Adams & 1155.725 & 360.412 \\
\hline 15 & Allen & 5262.995 & 3880.660 \\
\hline 16 & Bartholomew & 1357.953 & 1348.978 \\
\hline 17 & Benton & 49.285 & 53.516 \\
\hline 18 & Blackford & 317.376 & 22.232 \\
\hline 19 & Boone & 17.474 & 5.423 \\
\hline 20 & Brown & 4.616 & 4.133 \\
\hline 21 & Carroll & 841.064 & 752.989 \\
\hline 22 & Cass & 1427.560 & 734.113 \\
\hline 23 & Clark & 579.090 & 349.819 \\
\hline 24 & Clay & 157.562 & 13.640 \\
\hline 25 & Clinton & 1158.044 & 1025.803 \\
\hline 26 & Crawford & .000 & .000 \\
\hline 27 & Daviess & 554.042 & 446.341 \\
\hline 28 & Dearborn & 69.944 & 1875.336 \\
\hline 29 & Decatur & 505.971 & 165.264 \\
\hline 30 & DeKalb & 2098.370 & 2553.444 \\
\hline 31 & Delaware & 291.250 & 788.176 \\
\hline 32 & Dubois & 1327.270 & 360.797 \\
\hline 33 & Elkhart & 6534.868 & 2336.288 \\
\hline 34 & Fayette & .000 & 52.604 \\
\hline 35 & Floyd & 1735.872 & 335.366 \\
\hline 36 & Fountain & 27.426 & 726.212 \\
\hline 37 & Franklin & 173.688 & 4.575 \\
\hline 38 & Fulton & 193.818 & 440.341 \\
\hline 39 & Gibson & 541.088 & 167.919 \\
\hline 40 & Grant & 584.345 & 311.557 \\
\hline 41 & Greene & 180.391 & 324.890 \\
\hline 42 & Hamilton & 1694.997 & 955.934 \\
\hline 43 & Hancock & 26.314 & 17.623 \\
\hline 44 & Harrison & 595.170 & 366.224 \\
\hline 45 & Hendricks & 425.694 & 566.891 \\
\hline 46 & Henry & 230.815 & 419.095 \\
\hline 47 & Howard & 471.157 & 898.626 \\
\hline 48 & Huntington & 501.362 & 355.042 \\
\hline 49 & Jackson & 430.218 & 501.206 \\
\hline 50 & Jasper & 162.305 & 96.702 \\
\hline 51 & Jay & 338.606 & 293.119 \\
\hline
\end{tabular}




\begin{tabular}{|c|c|c|c|}
\hline & Locale & P12 15 & A12 15 \\
\hline 52 & Jefferson & 182.427 & 54.290 \\
\hline 53 & Jennings & 1153.384 & 595.172 \\
\hline 54 & Johnson & 906.216 & 397.862 \\
\hline 55 & Knox & 64.486 & 378.620 \\
\hline 56 & Kosciusko & 1462.136 & 2202.499 \\
\hline 57 & LaGrange & 826.804 & 106.252 \\
\hline 58 & Lake & 1073.633 & 20965.886 \\
\hline 59 & La Porte & 948.686 & 1741.787 \\
\hline 60 & Lawrence & 229.473 & 1745.312 \\
\hline 61 & Madison & 664.136 & 558.453 \\
\hline 62 & Marion & 3720.825 & 8470.308 \\
\hline 63 & Marshall & 2514.811 & 1061.260 \\
\hline 64 & Martin & 25.733 & 176.652 \\
\hline 65 & Miami & 339.381 & 35.003 \\
\hline 66 & Monroe & 395.026 & 271.112 \\
\hline 67 & Montgomery & 849.776 & 888.774 \\
\hline 68 & Morgan & 113.659 & 87.581 \\
\hline 69 & Newton & 391.983 & 167.684 \\
\hline 70 & Noble & 2382.799 & 1313.014 \\
\hline 71 & Ohio & .000 & .000 \\
\hline 72 & Orange & 175.099 & 44.517 \\
\hline 73 & Owen & 89.856 & 86.557 \\
\hline 74 & Parke & 229.062 & 91.685 \\
\hline 75 & Perry & 71.222 & 407.575 \\
\hline 76 & Pike & .000 & 58.251 \\
\hline 77 & Porter & 443.586 & 8088.251 \\
\hline 78 & Posey & 326.421 & 106.437 \\
\hline 79 & Pulaski & 32.684 & 198.921 \\
\hline 80 & Putnam & 1060.205 & 4.267 \\
\hline 81 & Randolph & 83.134 & 303.149 \\
\hline 82 & Ripley & 13.982 & 26.342 \\
\hline 83 & Rush & 71.135 & 353.143 \\
\hline 84 & St. Joseph & 3063.579 & 2244.952 \\
\hline 85 & Scott & 1347.020 & 531.850 \\
\hline 86 & Shelby & 1822.945 & 573.751 \\
\hline 87 & Spencer & 342.942 & 184.205 \\
\hline 88 & Starke & 145.995 & .000 \\
\hline 89 & Steuben & 777.762 & 672.373 \\
\hline 90 & Sullivan & 156.193 & .000 \\
\hline 91 & Switzerland & 183.879 & .000 \\
\hline 92 & Tippecanoe & 1040.983 & 2322.225 \\
\hline 93 & Tipton & 78.371 & 125.159 \\
\hline 94 & Union & .000 & .000 \\
\hline 95 & Vanderburgh & 5052.530 & 2599.520 \\
\hline 96 & Vermillion & 3.436 & 3.076 \\
\hline 97 & Vigo & 1606.205 & 474.026 \\
\hline 98 & Wabash & 181.571 & 706.178 \\
\hline 99 & Warren & 13.710 & 4.255 \\
\hline 100 & Warrick & 22.562 & 3888.491 \\
\hline 101 & Washington & 236.091 & 58.548 \\
\hline 102 & Wayne & 1209.429 & 1079.989 \\
\hline
\end{tabular}




\begin{tabular}{|c|c|c|c|}
\hline & $\begin{array}{l}\text { Locale } \\
\end{array}$ & P12 15 & $\mathrm{A} 12$ 15 \\
\hline 103 & Wells & 981.113 & 424.616 \\
\hline 104 & White & 340.475 & 86.614 \\
\hline 105 & Whitley & 438.189 & 627.869 \\
\hline 106 & lowa & 37133.087 & 32466.364 \\
\hline 107 & Kansas & 27952.793 & 19685.184 \\
\hline 108 & Kentucky_E & 19728.245 & 27958.120 \\
\hline 109 & Kentucky_W & 13152.163 & 18638.746 \\
\hline 110 & Louisiana & 13991.022 & 17493.856 \\
\hline 111 & Maine & 7945.193 & 7607.266 \\
\hline 112 & Maryland & 17865.590 & 21121.932 \\
\hline 113 & Massachusetts & 37316.418 & 26905.538 \\
\hline 114 & Michigan_E & 39866.190 & 30729.064 \\
\hline 115 & Michigan_W & 39866.190 & 30729.064 \\
\hline 116 & Minnesota & 43754.642 & 33881.593 \\
\hline 117 & Mississippi & 28232.430 & 21789.327 \\
\hline 118 & Missouri & 41708.455 & 41811.303 \\
\hline 119 & Montana & 1497.259 & 3245.300 \\
\hline 120 & Nebraska & 22381.622 & 17233.757 \\
\hline 121 & Nevada & 7678.576 & 6918.664 \\
\hline 122 & New Hampshire & 9490.159 & 8519.343 \\
\hline 123 & New Jersey & 51073.217 & 38247.699 \\
\hline 124 & New Mexico & 2670.942 & 4136.513 \\
\hline 125 & New York & 66535.822 & 64092.875 \\
\hline 126 & North Carolina & 110376.681 & 108321.149 \\
\hline 127 & North Dakota & 3404.873 & 2900.990 \\
\hline 128 & Ohio_N & 36817.743 & 36437.510 \\
\hline 129 & Ohio_M & 36817.743 & 36437.510 \\
\hline 130 & Ohio_s & 36817.743 & 36437.510 \\
\hline 131 & Oklahoma & 20982.821 & 17308.897 \\
\hline 132 & Oregon & 18877.004 & 29082.711 \\
\hline 133 & Pennsylvania & 87640.433 & 116910.907 \\
\hline 134 & Rhode Island & 8165.993 & 7108.689 \\
\hline 135 & South Carolina & 48439.231 & 35271.372 \\
\hline 136 & South Dakota & 6203.396 & 5401.279 \\
\hline 137 & Tennessee & 59981.986 & 50462.750 \\
\hline 138 & Texas & 122163.025 & 121559.962 \\
\hline 139 & Utah & 12622.206 & 17042.119 \\
\hline 140 & Vermont & 3947.744 & 3026.832 \\
\hline 141 & Virginia & 46766.312 & 60516.325 \\
\hline 142 & Washington & 32175.465 & 40856.258 \\
\hline 143 & West Virginia & 4983.853 & 14347.734 \\
\hline 144 & Wisconsin & 62237.094 & 62336.263 \\
\hline 145 & Wyoming & 722.161 & 909.497 \\
\hline
\end{tabular}




\begin{tabular}{|c|c|c|c|}
\hline & Locale & P13 15 & A13 15 \\
\hline 1 & Alabama & 4173.965 & 8867.229 \\
\hline 2 & Arizona & 2797.373 & 2215.244 \\
\hline 3 & Arkansas & 2294.735 & 6567.475 \\
\hline 4 & California & 21812.524 & 18433.549 \\
\hline 5 & Colorado & 2151.539 & 1651.486 \\
\hline 6 & Connecticut & 4862.380 & 2891.262 \\
\hline 7 & Delaware & 2440.477 & 938.063 \\
\hline 8 & DC & .000 & .000 \\
\hline 9 & Florida & 7550.543 & 7379.025 \\
\hline 10 & Georgia & 8507.568 & 16401.905 \\
\hline 11 & Idaho & 1113.387 & 1111.454 \\
\hline 12 & Illinois_N & 10842.354 & 9188.901 \\
\hline 13 & Illinois_s & 5421.177 & 4594.451 \\
\hline 14 & Adams & 55.766 & .000 \\
\hline 15 & Allen & 118.845 & 419.223 \\
\hline 16 & Bartholomew & 55.297 & 339.924 \\
\hline 17 & Benton & .000 & 76.755 \\
\hline 18 & Blackford & .000 & 177.864 \\
\hline 19 & Boone & .000 & 6.096 \\
\hline 20 & Brown & 2.915 & .000 \\
\hline 21 & Carroll & .000 & .000 \\
\hline 22 & Cass & .000 & .000 \\
\hline 23 & Clark & 217.359 & 30.611 \\
\hline 24 & Clay & 17.566 & .000 \\
\hline 25 & Clinton & 52.908 & 28.910 \\
\hline 26 & Crawford & .000 & .000 \\
\hline 27 & \begin{tabular}{|l|} 
Daviess \\
\end{tabular} & 18.592 & .000 \\
\hline 28 & Dearborn & 3.588 & .000 \\
\hline 29 & Decatur & .000 & .000 \\
\hline 30 & DeKalb & 73.904 & 91.205 \\
\hline 31 & Delaware & 25.597 & 219.330 \\
\hline 32 & \begin{tabular}{|l|} 
Dubois \\
\end{tabular} & 18.412 & 104.660 \\
\hline 33 & Elkhart & 555.516 & 379.434 \\
\hline 34 & Fayette & .000 & .000 \\
\hline 35 & Floyd & 32.263 & 88.219 \\
\hline 36 & Fountain & 2.887 & .000 \\
\hline 37 & Franklin & .000 & .000 \\
\hline 38 & \begin{tabular}{|l|} 
Fulton \\
\end{tabular} & 2.922 & 27.941 \\
\hline 39 & Gibson & .000 & .000 \\
\hline 40 & Grant & 24.476 & 170.815 \\
\hline 41 & Greene & .000 & .000 \\
\hline 42 & Hamilton & 23.474 & .000 \\
\hline 43 & Hancock & .000 & 109.122 \\
\hline 44 & Harrison & 17.459 & .000 \\
\hline 45 & Hendricks & 4.481 & .000 \\
\hline 46 & Henry & .000 & 25.577 \\
\hline 47 & Howard & .000 & .000 \\
\hline 48 & Huntington & 18.956 & 88.116 \\
\hline 49 & Jackson & 51.602 & 223.225 \\
\hline 50 & Jasper & .000 & .000 \\
\hline 51 & Jay & .000 & 26.422 \\
\hline
\end{tabular}




\begin{tabular}{|c|c|c|c|}
\hline & Locale & P13 15 & A13 15 \\
\hline 52 & Jefferson & 18.398 & .000 \\
\hline 53 & Jennings & 3.201 & 30.608 \\
\hline 54 & Johnson & 23.252 & 108.087 \\
\hline 55 & Knox & .000 & 25.814 \\
\hline 56 & Kosciusko & 100.672 & 4.833 \\
\hline 57 & LaGrange & 3.228 & .000 \\
\hline 58 & Lake & 432.015 & 189.204 \\
\hline 59 & La Porte & 95.264 & 223.382 \\
\hline 60 & Lawrence & .000 & .000 \\
\hline 61 & Madison & 110.176 & 81.943 \\
\hline 62 & Marion & 1805.114 & 1333.419 \\
\hline 63 & Marshall & 40.964 & 232.518 \\
\hline 64 & Martin & .000 & .000 \\
\hline 65 & Miami & 16.222 & 161.587 \\
\hline 66 & Monroe & 52.907 & 90.528 \\
\hline 67 & Montgomery & 52.503 & 83.676 \\
\hline 68 & Morgan & 129.794 & .000 \\
\hline 69 & Newton & 2.884 & 27.581 \\
\hline 70 & Noble & 66.555 & 85.937 \\
\hline 71 & Ohio & .000 & .000 \\
\hline 72 & Orange & .000 & .000 \\
\hline 73 & Owen & .000 & .000 \\
\hline$\overline{74}$ & Parke & .000 & .000 \\
\hline 75 & Perry & .000 & .000 \\
\hline$\overline{76}$ & Pike & .000 & .000 \\
\hline 77 & Porter & 139.135 & .000 \\
\hline 78 & Posey & 529.986 & .000 \\
\hline 79 & Pulaski & .000 & 4.767 \\
\hline 80 & Putnam & .000 & .000 \\
\hline 81 & Randolph & .000 & .000 \\
\hline 82 & Ripley & 18.363 & 85.360 \\
\hline 83 & Rush & .000 & 26.043 \\
\hline 84 & St. Joseph & 257.710 & 268.879 \\
\hline 85 & Scott & 52.514 & .000 \\
\hline 86 & Shelby & 55.579 & 4.613 \\
\hline 87 & Spencer & 2.824 & .000 \\
\hline 88 & Starke & 16.770 & 26.727 \\
\hline 89 & Steuben & .000 & 28.609 \\
\hline 90 & Sullivan & 2.990 & .000 \\
\hline 91 & Switzerland & .000 & 5.610 \\
\hline 92 & Tippecanoe & 556.558 & 32.412 \\
\hline 93 & Tipton & 2.828 & 27.043 \\
\hline 94 & Union & 12.663 & .000 \\
\hline 95 & Vanderburgh & 720.543 & 306.686 \\
\hline 96 & Vermillion & 474.579 & 75.635 \\
\hline 97 & Vigo & 302.999 & 245.201 \\
\hline 98 & Wabash & 103.435 & 164.847 \\
\hline 99 & Warren & .000 & .000 \\
\hline 100 & Warrick & 25.502 & 5.080 \\
\hline 101 & Washington & .000 & .000 \\
\hline 102 & Wayne & 37.127 & 101.313 \\
\hline
\end{tabular}




\begin{tabular}{|c|c|c|c|}
\hline & $\begin{array}{l}\text { Locale } \\
\end{array}$ & P13 15 & A13 15 \\
\hline 103 & Wells & .000 & 30.359 \\
\hline 104 & White & .000 & 27.756 \\
\hline 105 & Whitley & 3.169 & 88.376 \\
\hline 106 & lowa & 2061.738 & 2625.416 \\
\hline 107 & Kansas & 2010.148 & 1635.946 \\
\hline 108 & Kentucky_E & 2630.686 & 3064.232 \\
\hline 109 & Kentucky_W & 1753.790 & 2042.822 \\
\hline 110 & Louisiana & 8265.932 & 5324.529 \\
\hline 111 & Maine & 541.780 & 5808.832 \\
\hline 112 & Maryland & 4728.922 & 3643.567 \\
\hline 113 & Massachusetts & 6655.049 & 9271.378 \\
\hline 114 & Michigan_E & 5117.950 & 4596.260 \\
\hline 115 & Michigan_W & 5117.950 & 4596.260 \\
\hline 116 & Minnesota & 2920.437 & 8113.253 \\
\hline 117 & Mississippi & 2219.445 & 4192.340 \\
\hline 118 & Missouri & 6882.659 & 6410.707 \\
\hline 119 & Montana & 165.603 & 376.323 \\
\hline 120 & Nebraska & 837.286 & 792.412 \\
\hline 121 & Nevada & 563.667 & 417.283 \\
\hline 122 & New Hampshire & 616.707 & 1955.062 \\
\hline 123 & New Jersey & 21359.717 & 8656.419 \\
\hline 124 & New Mexico & 437.915 & 282.625 \\
\hline 125 & New York & 18361.317 & 12023.417 \\
\hline 126 & North Carolina & 15534.670 & 11904.519 \\
\hline 127 & North Dakota & 16.864 & 26.876 \\
\hline 128 & Ohio_N & 4671.520 & 4647.857 \\
\hline 129 & Ohio_M & 4671.520 & 4647.857 \\
\hline 130 & Ohio_s & 4671.520 & 4647.857 \\
\hline 131 & Oklahoma & 1156.514 & 1848.946 \\
\hline 132 & Oregon & 1484.943 & 4222.178 \\
\hline 133 & Pennsylvania & 9963.826 & 15170.462 \\
\hline 134 & Rhode Island & 807.201 & 972.850 \\
\hline 135 & South Carolina & 12461.030 & 7211.003 \\
\hline 136 & South Dakota & 216.630 & 314.647 \\
\hline 137 & Tennessee & 11179.410 & 8866.334 \\
\hline 138 & Texas & 28359.621 & 12188.599 \\
\hline 139 & Utah & 2009.519 & 1539.233 \\
\hline 140 & Vermont & 117.833 & 876.375 \\
\hline$\overline{141}$ & Virginia & 6689.757 & 8216.779 \\
\hline 142 & Washington & 1782.873 & 7773.341 \\
\hline$\overline{143}$ & West Virginia & 4007.186 & 284.418 \\
\hline 144 & Wisconsin & 3984.651 & 20744.887 \\
\hline 145 & Wyoming & 379.855 & 4.843 \\
\hline
\end{tabular}




\begin{tabular}{|c|c|c|c|}
\hline & $\begin{array}{l}\text { Locale } \\
\end{array}$ & P14 15 & A14 15 \\
\hline 1 & Alabama & 719.258 & 6085.010 \\
\hline 2 & Arizona & 21556.491 & 2529.474 \\
\hline 3 & Arkansas & 761.762 & 2606.676 \\
\hline 4 & California & 148.260 & 7701.755 \\
\hline 5 & Colorado & 129.758 & 654.465 \\
\hline 6 & Connecticut & 732.235 & 1562.304 \\
\hline 7 & Delaware & 811.818 & 230.333 \\
\hline 8 & DC & .000 & .000 \\
\hline 9 & Florida & 655.580 & 1635.178 \\
\hline 10 & Georgia & 857.216 & 2076.885 \\
\hline 11 & Idaho & 393.148 & 59.473 \\
\hline 12 & Illinois_N & 284.553 & 6859.745 \\
\hline 13 & Illinois_S & 142.277 & 3429.872 \\
\hline 14 & Adams & .000 & 49.277 \\
\hline 15 & Allen & .000 & 693.671 \\
\hline 16 & Bartholomew & .000 & 199.359 \\
\hline 17 & Benton & .000 & 14.591 \\
\hline 18 & Blackford & .000 & .000 \\
\hline 19 & Boone & .000 & .000 \\
\hline 20 & Brown & .000 & .000 \\
\hline 21 & Carroll & .000 & .000 \\
\hline 22 & Cass & .000 & .000 \\
\hline 23 & Clark & .000 & 36.490 \\
\hline 24 & Clay & .000 & 2.587 \\
\hline 25 & Clinton & .000 & .000 \\
\hline 26 & Crawford & .000 & .000 \\
\hline 27 & Daviess & .000 & .000 \\
\hline 28 & Dearborn & .000 & .000 \\
\hline 29 & Decatur & .000 & 2.622 \\
\hline 30 & DeKalb & .000 & 688.593 \\
\hline 31 & Delaware & .000 & 190.792 \\
\hline 32 & Dubois & .000 & .000 \\
\hline 33 & Elkhart & .000 & 337.162 \\
\hline 34 & Fayette & .000 & 14.342 \\
\hline 35 & Floyd & .000 & 2.795 \\
\hline 36 & Fountain & .000 & 191.302 \\
\hline 37 & Franklin & .000 & .000 \\
\hline 38 & Fulton & .000 & 96.822 \\
\hline 39 & Gibson & .000 & .000 \\
\hline 40 & Grant & .000 & 39.841 \\
\hline 41 & Greene & .000 & 43.946 \\
\hline 42 & Hamilton & .000 & 157.641 \\
\hline 43 & Hancock & .000 & 3.343 \\
\hline 44 & Harrison & .000 & .000 \\
\hline 45 & Hendricks & .000 & 69.290 \\
\hline 46 & Henry & .000 & 88.633 \\
\hline 47 & Howard & .000 & 192.888 \\
\hline 48 & Huntington & .000 & 47.180 \\
\hline 49 & Jackson & .000 & 128.977 \\
\hline 50 & Jasper & .000 & .000 \\
\hline 51 & Jay & .000 & .000 \\
\hline
\end{tabular}




\begin{tabular}{|c|c|c|c|}
\hline & Locale & P14 15 & A14 15 \\
\hline 52 & Jefferson & .000 & 2.710 \\
\hline 53 & Jennings & .000 & 16.971 \\
\hline 54 & Johnson & .000 & 102.734 \\
\hline 55 & Knox & .000 & 89.454 \\
\hline 56 & Kosciusko & .000 & 468.907 \\
\hline 57 & LaGrange & .000 & 2.852 \\
\hline 58 & Lake & .000 & 5355.970 \\
\hline 59 & La Porte & .000 & 403.350 \\
\hline 60 & Lawrence & .000 & 433.029 \\
\hline 61 & Madison & .000 & 59.972 \\
\hline 62 & Marion & .000 & 961.682 \\
\hline 63 & Marshall & .000 & 106.365 \\
\hline 64 & Martin & .000 & 41.882 \\
\hline 65 & Miami & .000 & .000 \\
\hline 66 & Monroe & .000 & .000 \\
\hline 67 & Montgomery & .000 & 198.830 \\
\hline 68 & Morgan & .000 & 18.351 \\
\hline 69 & Newton & .000 & 44.603 \\
\hline 70 & Noble & .000 & 203.387 \\
\hline 71 & Ohio & .000 & .000 \\
\hline 72 & Orange & .000 & .000 \\
\hline 73 & Owen & .000 & .000 \\
\hline 74 & Parke & .000 & .000 \\
\hline 75 & Perry & .000 & 90.281 \\
\hline 76 & Pike & .000 & 15.882 \\
\hline 77 & Porter & .000 & 2164.160 \\
\hline 78 & Posey & .000 & .000 \\
\hline 79 & Pulaski & .000 & 46.256 \\
\hline 80 & Putnam & .000 & .000 \\
\hline 81 & Randolph & .000 & 74.432 \\
\hline 82 & Ripley & .000 & .000 \\
\hline 83 & Rush & .000 & 90.248 \\
\hline 84 & St. Joseph & .000 & 462.331 \\
\hline 85 & Scott & .000 & 2.652 \\
\hline 86 & Shelby & .000 & 136.848 \\
\hline 87 & Spencer & .000 & 43.672 \\
\hline 88 & Starke & .000 & .000 \\
\hline 89 & Steuben & .000 & 132.447 \\
\hline 90 & Sullivan & .000 & .000 \\
\hline 91 & Switzerland & .000 & .000 \\
\hline 92 & Tippecanoe & .000 & 524.145 \\
\hline 93 & Tipton & .000 & 14.994 \\
\hline 94 & Union & .000 & .000 \\
\hline 95 & Vanderburgh & .000 & 47.535 \\
\hline 96 & Vermillion & .000 & .000 \\
\hline 97 & Vigo & .000 & 97.479 \\
\hline 98 & Wabash & .000 & 182.798 \\
\hline 99 & Warren & .000 & .000 \\
\hline 100 & Warrick & .000 & 1056.309 \\
\hline 101 & Washington & .000 & 2.732 \\
\hline 102 & Wayne & .000 & 216.430 \\
\hline
\end{tabular}




\begin{tabular}{|c|c|c|c|}
\hline & Locale & P14 15 & A14 15 \\
\hline 103 & Wells & .000 & 49.094 \\
\hline 104 & White & .000 & 15.389 \\
\hline 105 & Whitley & .000 & 169.959 \\
\hline 106 & lowa & 709.393 & 2563.560 \\
\hline 107 & Kansas & 727.853 & 736.241 \\
\hline 108 & Kentucky_E & 443.071 & 2509.627 \\
\hline 109 & Kentucky_W & 295.381 & 1673.084 \\
\hline 110 & Louisiana & 717.366 & 1026.601 \\
\hline 111 & Maine & 747.250 & 205.174 \\
\hline 112 & Maryland & 804.039 & 2177.939 \\
\hline 113 & Massachusetts & 730.179 & 1594.005 \\
\hline 114 & Michigan_E & 12824.461 & 4986.558 \\
\hline 115 & Michigan_W & 12824.461 & 4986.558 \\
\hline 116 & Minnesota & 88748.175 & 2190.881 \\
\hline 117 & Mississippi & 726.872 & 873.890 \\
\hline 118 & Missouri & 3672.849 & 3488.200 \\
\hline 119 & Montana & 880.562 & 337.180 \\
\hline 120 & Nebraska & 716.902 & 308.385 \\
\hline 121 & Nevada & 136.820 & 563.799 \\
\hline 122 & New Hampshire & .000 & 1057.929 \\
\hline 123 & New Jersey & 754.532 & 2810.932 \\
\hline 124 & New Mexico & 893.889 & 493.212 \\
\hline 125 & New York & 2270.342 & 4566.234 \\
\hline 126 & North Carolina & 853.089 & 2461.963 \\
\hline 127 & North Dakota & 678.391 & 31.045 \\
\hline 128 & Ohio_N & 234.332 & 6397.648 \\
\hline 129 & Ohio_M & 234.332 & 6397.648 \\
\hline 130 & Ohio_s & 234.332 & 6397.648 \\
\hline 131 & Oklahoma & 727.891 & 1428.324 \\
\hline 132 & Oregon & 1372.660 & 3198.662 \\
\hline 133 & Pennsylvania & 709.950 & 16204.619 \\
\hline 134 & Rhode Island & 247.365 & 935.203 \\
\hline 135 & South Carolina & .000 & 2040.899 \\
\hline 136 & South Dakota & 724.205 & 163.585 \\
\hline 137 & Tennessee & 783.928 & 4897.255 \\
\hline 138 & Texas & 1.975 .022 & 9339.498 \\
\hline 139 & Utah & 854.838 & 2139.660 \\
\hline 140 & Vermont & 55.714 & 74.690 \\
\hline 141 & Virginia & 820.477 & 2008.598 \\
\hline 142 & Washington & 808.890 & 3875.774 \\
\hline 143 & West Virginia & 691.407 & 2855.971 \\
\hline 144 & Wisconsin & 752.323 & 7406.671 \\
\hline 145 & Wyoming & 733.480 & 33.566 \\
\hline
\end{tabular}




\begin{tabular}{|c|c|c|c|}
\hline & Locale & P15 15 & A15 15 \\
\hline 1 & Alabama & 66044.889 & 57961.569 \\
\hline 2 & Arizona & 174637.097 & 86157.460 \\
\hline 3 & Arkansas & 12073.258 & 51069.420 \\
\hline 4 & California & 77331.661 & 174691.678 \\
\hline 5 & Colorado & 46556.196 & 37365.638 \\
\hline 6 & Connecticut & 7128.551 & 10744.533 \\
\hline 7 & Delaware & 1844.107 & 9802.496 \\
\hline 8 & DC & .000 & .000 \\
\hline 9 & Florida & 91395.960 & 78924.487 \\
\hline 10 & Georgia & 69777.676 & 90809.433 \\
\hline 11 & Idaho & 26504.530 & 27911.220 \\
\hline 12 & Illinois_N & 45077.418 & 66757.080 \\
\hline 13 & lllinois_s & 22538.709 & 33378.540 \\
\hline 14 & Adams & 599.032 & 573.271 \\
\hline 15 & Allen & 843.996 & 1866.380 \\
\hline 16 & Bartholomew & .000 & 1163.733 \\
\hline 17 & Benton & .000 & .000 \\
\hline 18 & Blackford & 81.887 & 79.973 \\
\hline 19 & Boone & 119.844 & 62.735 \\
\hline 20 & Brown & .000 & 8.278 \\
\hline 21 & Carroll & 570.532 & 1755.114 \\
\hline 22 & Cass & 531.325 & 1634.504 \\
\hline 23 & Clark & 1755.157 & 1021.492 \\
\hline 24 & Clay & 550.364 & 246.533 \\
\hline 25 & Clinton & .000 & 1793.387 \\
\hline 26 & Crawford & 1856.867 & 803.726 \\
\hline 27 & Daviess & 3640.681 & 2469.811 \\
\hline 28 & Dearborn & 674.538 & 302.156 \\
\hline 29 & Decatur & 557.787 & 553.179 \\
\hline 30 & DeKalb & 471.292 & 259.708 \\
\hline 31 & Delaware & 3538.076 & 1638.309 \\
\hline 32 & Dubois & 576.863 & 945.044 \\
\hline 33 & Elkhart & 596.733 & 843.289 \\
\hline 34 & Fayette & 84.753 & 36.685 \\
\hline 35 & Floyd & .000 & 651.173 \\
\hline 36 & Fountain & .000 & 49.180 \\
\hline 37 & Franklin & 101.115 & 52.931 \\
\hline 38 & Fulton & 91.546 & 168.228 \\
\hline 39 & Gibson & 4175.994 & 2143.863 \\
\hline 40 & Grant & 484.326 & 403.507 \\
\hline 41 & Greene & 1558.165 & 977.050 \\
\hline 42 & Hamilton & 2145.042 & 1076.211 \\
\hline 43 & Hancock & 118.519 & 62.042 \\
\hline 44 & Harrison & 881.889 & 1115.229 \\
\hline 45 & Hendricks & 842.324 & 951.150 \\
\hline 46 & Henry & 83.803 & 81.845 \\
\hline 47 & Howard & 547.127 & 368.225 \\
\hline 48 & Huntington & 742.386 & 657.755 \\
\hline 49 & Jackson & 92.385 & 70.131 \\
\hline 50 & Jasper & 94.980 & 234.797 \\
\hline 51 & Jay & 519.428 & 573.198 \\
\hline
\end{tabular}




\begin{tabular}{|c|c|c|c|}
\hline & Locale & P15 15 & A15 15 \\
\hline 52 & Jefferson & .000 & 17.414 \\
\hline 53 & \begin{tabular}{|l} 
Jennings \\
\end{tabular} & 601.718 & 260.448 \\
\hline 54 & Johnson & 121.420 & 60.258 \\
\hline 55 & Knox & 3992.138 & 1829.143 \\
\hline 56 & Kosciusko & 95.004 & 829.836 \\
\hline 57 & LaGrange & 101.134 & 206.929 \\
\hline 58 & Lake & 544.317 & 1163.877 \\
\hline 59 & La Porte & 243.465 & 563.859 \\
\hline 60 & Lawrence & 552.731 & 286.954 \\
\hline 61 & Madison & 552.305 & 864.767 \\
\hline 62 & Marion & 1634.823 & 3974.921 \\
\hline 63 & Marshall & 98.725 & 424.799 \\
\hline 64 & Martin & .000 & 46.144 \\
\hline 65 & Miami & 508.255 & 266.057 \\
\hline 66 & Monroe & 1413.561 & 701.249 \\
\hline 67 & Montgomery & 93.997 & 360.155 \\
\hline 68 & Morgan & 650.647 & 291.454 \\
\hline 69 & Newton & 542.214 & 242.882 \\
\hline 70 & Noble & 96.538 & 697.991 \\
\hline 71 & Ohio & .000 & .000 \\
\hline 72 & Orange & 488.745 & 259.871 \\
\hline 73 & Owen & 96.658 & 50.598 \\
\hline 74 & Parke & .000 & 142.379 \\
\hline 75 & Perry & .000 & 7.736 \\
\hline 76 & Pike & 563.109 & 243.736 \\
\hline 77 & Porter & 94.151 & 315.519 \\
\hline 78 & Posey & 94.885 & 92.668 \\
\hline 79 & Pulaski & 562.312 & 301.999 \\
\hline 80 & Putnam & 584.578 & 261.574 \\
\hline 81 & Randolph & 86.527 & 97.837 \\
\hline 82 & Ripley & 575.338 & 257.720 \\
\hline 83 & Rush & 85.330 & 57.041 \\
\hline 84 & St. Joseph & 563.319 & 834.360 \\
\hline 85 & Scott & 94.016 & 360.228 \\
\hline 86 & Shelby & 1015.772 & 583.513 \\
\hline 87 & Spencer & 530.899 & 277.911 \\
\hline 88 & Starke & .000 & .000 \\
\hline 89 & Steuben & 93.734 & 254.654 \\
\hline 90 & Sullivan & 3513.265 & 1520.682 \\
\hline 91 & Switzerland & 661.761 & 286.437 \\
\hline 92 & Tippecanoe & 637.175 & 895.629 \\
\hline 93 & Tipton & .000 & 140.533 \\
\hline 94 & Union & .000 & .000 \\
\hline 95 & Vanderburgh & 1685.430 & 3787.222 \\
\hline 96 & Vermillion & 84.965 & 42.937 \\
\hline 97 & Vigo & 3456.254 & 1703.166 \\
\hline 98 & Wabash & 86.418 & 84.399 \\
\hline 99 & Warren & 564.161 & 252.713 \\
\hline 100 & Warrick & 3745.285 & 1621.109 \\
\hline 101 & Washington & 96.868 & 94.604 \\
\hline 102 & Wayne & 83.684 & 570.925 \\
\hline
\end{tabular}




\begin{tabular}{|c|c|c|c|}
\hline & Locale & P15 15 & A15 15 \\
\hline 103 & Wells & 99.469 & 504.627 \\
\hline 104 & White & 90.940 & 73.979 \\
\hline 105 & Whitley & .000 & 8.998 \\
\hline 106 & lowa & 16114.410 & 45615.167 \\
\hline 107 & Kansas & 12187.729 & 31593.921 \\
\hline 108 & Kentucky_E & 109337.170 & 57931.608 \\
\hline 109 & Kentucky_W & 72891.452 & 38621.072 \\
\hline 110 & Louisiana & 15438.837 & 21436.325 \\
\hline 111 & Maine & 581.978 & 5820.113 \\
\hline 112 & Maryland & 19224.552 & 24444.149 \\
\hline 113 & Massachusetts & 16586.580 & 25724.616 \\
\hline 114 & Michigan_E & 19076.853 & 22261.101 \\
\hline 115 & Michigan_W & 19076.853 & 22261.101 \\
\hline 116 & Minnesota & 76941.998 & 73161.759 \\
\hline 117 & Mississippi & 9944.616 & 27143.956 \\
\hline 118 & Missouri & 40933.295 & 51260.473 \\
\hline 119 & Montana & 27234.494 & 13761.225 \\
\hline 120 & Nebraska & 6048.710 & 31548.292 \\
\hline 121 & Nevada & 159090.889 & 72626.448 \\
\hline 122 & New Hampshire & 3935.747 & 3805.730 \\
\hline 123 & New Jersey & 17139.782 & 33886.304 \\
\hline 124 & New Mexico & 55490.287 & 27706.316 \\
\hline 125 & New York & 29146.572 & 53190.116 \\
\hline 126 & North Carolina & 36619.985 & 68625.557 \\
\hline 127 & North Dakota & 13939.609 & 10352.883 \\
\hline 128 & Ohio_N & 23977.981 & 24068.281 \\
\hline 129 & Ohio_M & 23977.981 & 24068.281 \\
\hline 130 & Ohio_s & 23977.981 & 24068.281 \\
\hline 131 & Oklahoma & 19038.424 & 20710.379 \\
\hline 132 & Oregon & 20437.291 & 27526.889 \\
\hline 133 & Pennsylvania & 126721.861 & 118805.366 \\
\hline 134 & Rhode Island & 1054.967 & 2928.559 \\
\hline 135 & South Carolina & 18028.894 & 22516.133 \\
\hline 136 & South Dakota & 14881.005 & 13300.449 \\
\hline 137 & Tennessee & 37131.263 & 49534.761 \\
\hline 138 & Texas & 93701.285 & 129378.500 \\
\hline 139 & Utah & 54559.875 & 35709.877 \\
\hline 140 & Vermont & 7383.576 & 6564.159 \\
\hline 141 & Virginia & 111496.421 & 81816.874 \\
\hline 142 & Washington & 30575.287 & 45224.648 \\
\hline 143 & West Virginia & 150785.088 & 69544.314 \\
\hline 144 & Wisconsin & 17089.605 & 58383.110 \\
\hline 145 & Wyoming & 80242.159 & 35379.191 \\
\hline
\end{tabular}




\begin{tabular}{|c|c|c|c|}
\hline & Locale & P17 15 & A17 15 \\
\hline 1 & Alabama & 38214.104 & 32380.636 \\
\hline 2 & Arizona & 7500.062 & 22636.659 \\
\hline 3 & Arkansas & 16307.652 & 16344.000 \\
\hline 4 & California & 215301.335 & 216714.247 \\
\hline 5 & Colorado & 7958.962 & 16920.216 \\
\hline 6 & Connecticut & 6801.744 & 13226.993 \\
\hline 7 & Delaware & 13933.349 & 9695.734 \\
\hline 8 & DC & .000 & .000 \\
\hline 9 & Florida & 17728.015 & 63756.520 \\
\hline 10 & Georgia & 19634.280 & 36243.220 \\
\hline 11 & Idaho & 3100.820 & 5807.301 \\
\hline 12 & Illinois_N & 58334.666 & 53690.270 \\
\hline 13 & Illinois_s & 29167.333 & 26845.135 \\
\hline 14 & Adams & 157.718 & 183.311 \\
\hline 15 & Allen & 972.512 & 1528.510 \\
\hline 16 & Bartholomew & .000 & 218.229 \\
\hline 17 & Benton & .000 & 23.379 \\
\hline 18 & Blackford & .000 & 35.639 \\
\hline 19 & Boone & .000 & 166.529 \\
\hline 20 & Brown & .000 & 43.313 \\
\hline 21 & Carroll & .000 & 56.059 \\
\hline 22 & Cass & .000 & 111.564 \\
\hline 23 & Clark & 1600.226 & 1134.147 \\
\hline 24 & Clay & .000 & 73.538 \\
\hline 25 & Clinton & .000 & 96.341 \\
\hline 26 & Crawford & .000 & 32.781 \\
\hline 27 & Daviess & 153.368 & 164.633 \\
\hline 28 & Dearborn & 177.598 & 253.105 \\
\hline 29 & Decatur & .000 & 68.901 \\
\hline 30 & DeKalb & .000 & 125.744 \\
\hline 31 & Delaware & .000 & 337.562 \\
\hline 32 & Dubois & .000 & 116.575 \\
\hline 33 & Elkhart & 157.112 & 642.512 \\
\hline 34 & Fayette & .000 & 67.430 \\
\hline 35 & Floyd & .000 & 222.931 \\
\hline 36 & Fountain & .000 & 48.131 \\
\hline 37 & Franklin & 958.405 & 566.362 \\
\hline 38 & Fulton & .000 & 57.363 \\
\hline 39 & Gibson & .000 & 90.487 \\
\hline 40 & Grant & .000 & 185.830 \\
\hline 41 & Greene & .000 & 92.425 \\
\hline 42 & Hamilton & 193.633 & 906.556 \\
\hline 43 & Hancock & .000 & 200.064 \\
\hline 44 & Harrison & 1058.085 & 668.236 \\
\hline 45 & Hendricks & .000 & 444.962 \\
\hline 46 & Henry & .000 & 125.931 \\
\hline 47 & Howard & .000 & 236.695 \\
\hline 48 & Huntington & 156.369 & 196.015 \\
\hline 49 & Jackson & .000 & 115.774 \\
\hline 50 & Jasper & .000 & 87.201 \\
\hline 51 & Jay & 136.759 & 128.529 \\
\hline
\end{tabular}




\begin{tabular}{|c|c|c|c|}
\hline & Locale & P17 15 & A17 15 \\
\hline 52 & Jefferson & .000 & 93.064 \\
\hline 53 & Jennings & 950.551 & 581.437 \\
\hline 54 & Johnson & .000 & 423.971 \\
\hline 55 & Knox & 133.612 & 174.473 \\
\hline 56 & Kosciusko & 150.081 & 292.713 \\
\hline 57 & LaGrange & .000 & 113.373 \\
\hline 58 & Lake & 30941.075 & 17503.829 \\
\hline 59 & La Porte & 142.448 & 372.870 \\
\hline 60 & Lawrence & 831.585 & 559.890 \\
\hline 61 & Madison & .000 & 377.473 \\
\hline 62 & Marion & 1756.151 & 3369.808 \\
\hline 63 & Marshall & 935.756 & 624.509 \\
\hline 64 & Martin & .000 & 27.105 \\
\hline 65 & Miami & .000 & 94.232 \\
\hline 66 & Monroe & 963.903 & 881.716 \\
\hline 67 & Montgomery & .000 & 107.181 \\
\hline 68 & Morgan & .000 & 220.711 \\
\hline 69 & Newton & .000 & 40.054 \\
\hline 70 & Noble & .000 & 135.186 \\
\hline 71 & Ohio & .000 & 15.507 \\
\hline 72 & Orange & .000 & 54.934 \\
\hline 73 & Owen & .000 & 64.423 \\
\hline 74 & Parke & .000 & 46.311 \\
\hline 75 & Perry & .000 & 49.194 \\
\hline 76 & Pike & .000 & 36.309 \\
\hline 77 & Porter & .000 & 418.022 \\
\hline 78 & Posey & 5620.954 & 3018.109 \\
\hline 79 & Pulaski & .000 & 37.794 \\
\hline 80 & Putnam & .000 & 105.718 \\
\hline 81 & Randolph & 136.689 & 143.570 \\
\hline 82 & Ripley & 151.479 & 156.506 \\
\hline 83 & Rush & .000 & 46.839 \\
\hline 84 & St. Joseph & 148.315 & 857.179 \\
\hline 85 & Scott & 148.520 & 145.010 \\
\hline 86 & Shelby & .000 & 121.212 \\
\hline 87 & Spencer & .000 & 55.402 \\
\hline 88 & Starke & .000 & 63.914 \\
\hline 89 & Steuben & 148.075 & 172.766 \\
\hline 90 & Sullivan & .000 & 61.116 \\
\hline 91 & Switzerland & .000 & 27.646 \\
\hline 92 & Tippecanoe & 167.760 & 564.282 \\
\hline 93 & Tipton & .000 & 44.162 \\
\hline 94 & Union & .000 & 19.910 \\
\hline 95 & Vanderburgh & .000 & 483.053 \\
\hline 96 & Vermillion & .000 & 43.729 \\
\hline 97 & Vigo & .000 & 284.158 \\
\hline 98 & Wabash & 136.517 & 165.450 \\
\hline 99 & Warren & .000 & 22.470 \\
\hline 100 & Warrick & .000 & 167.013 \\
\hline 101 & Washington & .000 & 80.293 \\
\hline 102 & Wayne & .000 & 182.247 \\
\hline
\end{tabular}




\begin{tabular}{|c|c|c|c|}
\hline & Locale & P17 15 & A17 15 \\
\hline 103 & Wells & .000 & 80.679 \\
\hline 104 & White & .000 & 69.477 \\
\hline 105 & Whitley & .000 & 93.334 \\
\hline 106 & lowa & 2545.637 & 9416.921 \\
\hline 107 & Kansas & 41357.256 & 29201.984 \\
\hline 108 & Kentucky_E & 11574.825 & 12960.564 \\
\hline 109 & Kentucky_W & 7716.550 & 8640.376 \\
\hline 110 & Louisiana & 182800.957 & 108155.333 \\
\hline 111 & Maine & 2681.486 & 5099.297 \\
\hline 112 & Maryland & 17872.199 & 25698.292 \\
\hline 113 & Massachusetts & 15721.363 & 26147.461 \\
\hline 114 & Michigan_E & 15263.120 & 22130.077 \\
\hline 115 & Michigan_W & 15263.120 & 22130.077 \\
\hline 116 & Minnesota & 31278.190 & 31207.275 \\
\hline 117 & Mississippi & 28736.677 & 22993.962 \\
\hline 118 & Missouri & 21513.792 & 27335.019 \\
\hline 119 & Montana & 12652.928 & 9286.497 \\
\hline 120 & Nebraska & 147.005 & 4842.835 \\
\hline 121 & Nevada & 1291.581 & 7976.193 \\
\hline 122 & New Hampshire & 2553.282 & 5127.131 \\
\hline 123 & New Jersey & 54523.690 & 52976.235 \\
\hline 124 & New Mexico & 12249.916 & 11796.099 \\
\hline 125 & New York & 23123.235 & 64062.784 \\
\hline 126 & North Carolina & 12070.219 & 32065.125 \\
\hline 127 & North Dakota & 2434.388 & 3011.326 \\
\hline 128 & Ohio_N & 27081.591 & 24588.218 \\
\hline 129 & Ohio_M & 27081.591 & 24588.218 \\
\hline 130 & Ohio_S & 27081.591 & 24588.218 \\
\hline 131 & Oklahoma & 37971.272 & 29547.143 \\
\hline 132 & Oregon & 11828.612 & 16669.080 \\
\hline 133 & Pennsylvania & 105253.770 & 89296.828 \\
\hline 134 & Rhode Island & 152.895 & 3093.825 \\
\hline 135 & South Carolina & 6103.015 & 15269.274 \\
\hline 136 & South Dakota & 148.502 & 2202.348 \\
\hline 137 & Tennessee & 21588.575 & 28290.660 \\
\hline 138 & Texas & 395073.304 & 274783.451 \\
\hline 139 & Utah & 26854.312 & 21195.135 \\
\hline 140 & Vermont & 933.123 & 2274.142 \\
\hline 141 & Virginia & 11305.951 & 28003.964 \\
\hline 142 & Washington & 33787.208 & 35774.548 \\
\hline 143 & West Virginia & 5600.186 & 7888.462 \\
\hline 144 & Wisconsin & 6448.406 & 18992.156 \\
\hline 145 & Wyoming & 12573.798 & 7994.612 \\
\hline
\end{tabular}




\begin{tabular}{|c|c|c|c|}
\hline & Locale & P18 15 & A18 15 \\
\hline 1 & Alabama & 18111.504 & 16799.556 \\
\hline 2 & Arizona & 3554.640 & 4522.254 \\
\hline 3 & Arkansas & 7728.982 & 7487.142 \\
\hline 4 & California & 102041.671 & 93594.789 \\
\hline 5 & Colorado & 3772.135 & 4292.980 \\
\hline 6 & Connecticut & 3223.674 & 5541.378 \\
\hline 7 & Delaware & 6603.685 & 6694.228 \\
\hline 8 & DC & .000 & .000 \\
\hline 9 & Florida & 8402.160 & 11270.450 \\
\hline 10 & Georgia & 9305.631 & 12572.222 \\
\hline 11 & Idaho & 1469.628 & 1842.950 \\
\hline 12 & Illinois_N & 27647.608 & 28413.640 \\
\hline 13 & IIllinois_S & 13823.804 & 14206.820 \\
\hline 14 & Adams & 74.750 & 93.203 \\
\hline 15 & Allen & 460.920 & 435.349 \\
\hline 16 & Bartholomew & .000 & 34.246 \\
\hline 17 & Benton & .000 & .000 \\
\hline 18 & Blackford & .000 & .000 \\
\hline 19 & Boone & .000 & .000 \\
\hline 20 & Brown & .000 & 1.805 \\
\hline 21 & Carroll & .000 & .000 \\
\hline 22 & Cass & .000 & .000 \\
\hline 23 & Clark & 758.424 & 729.852 \\
\hline 24 & Clay & .000 & 10.879 \\
\hline 25 & Clinton & .000 & 32.766 \\
\hline 26 & Crawford & .000 & .000 \\
\hline 27 & Daviess & 72.688 & 68.563 \\
\hline 28 & Dearborn & 84.172 & 68.284 \\
\hline 29 & Decatur & .000 & .000 \\
\hline 30 & DeKalb & .000 & 45.770 \\
\hline 31 & Delaware & .000 & 15.852 \\
\hline 32 & Dubois & .000 & 11.403 \\
\hline 33 & Elkhart & 74.463 & 402.478 \\
\hline 34 & Fayette & .000 & .000 \\
\hline 35 & Floyd & .000 & 19.981 \\
\hline 36 & Fountain & .000 & 1.788 \\
\hline 37 & Franklin & 454.234 & 356.500 \\
\hline 38 & Fulton & .000 & 1.810 \\
\hline 39 & Gibson & .000 & .000 \\
\hline 40 & Grant & .000 & 15.158 \\
\hline 41 & Greene & .000 & .000 \\
\hline 42 & Hamilton & 91.772 & 86.564 \\
\hline 43 & Hancock & .000 & .000 \\
\hline 44 & Harrison & 501.477 & 404.390 \\
\hline 45 & Hendricks & .000 & 2.775 \\
\hline 46 & Henry & .000 & .000 \\
\hline 47 & Howard & .000 & .000 \\
\hline 48 & Huntington & 74.111 & 69.905 \\
\hline 49 & Jackson & .000 & 31.958 \\
\hline 50 & Jasper & .000 & .000 \\
\hline 51 & Jay & 64.817 & 50.871 \\
\hline
\end{tabular}




\begin{tabular}{|c|c|c|c|}
\hline & Locale & P18 15 & A18 15 \\
\hline 52 & Jefferson & .000 & 11.394 \\
\hline 53 & \begin{tabular}{|l} 
Jennings \\
\end{tabular} & 450.512 & 355.561 \\
\hline 54 & Johnson & .000 & 14.400 \\
\hline 55 & Knox & 63.325 & 49.700 \\
\hline 56 & Kosciusko & 71.130 & 118.173 \\
\hline 57 & LaGrange & .000 & 1.999 \\
\hline 58 & Lake & 14664.465 & 11776.767 \\
\hline 59 & La Porte & 67.513 & 111.985 \\
\hline 60 & Lawrence & 394.128 & 309.326 \\
\hline 61 & Madison & .000 & 68.233 \\
\hline 62 & Marion & 832.324 & 1771.164 \\
\hline 63 & Marshall & 443.500 & 373.444 \\
\hline 64 & Martin & .000 & .000 \\
\hline 65 & Miami & .000 & 10.047 \\
\hline 66 & Monroe & 456.840 & 391.311 \\
\hline 67 & Montgomery & .000 & 32.516 \\
\hline 68 & Morgan & .000 & 80.383 \\
\hline 69 & Newton & .000 & 1.786 \\
\hline 70 & Noble & .000 & 41.218 \\
\hline 71 & Ohio & .000 & .000 \\
\hline 72 & Orange & .000 & .000 \\
\hline 73 & Owen & .000 & .000 \\
\hline 74 & Parke & .000 & .000 \\
\hline 75 & Perry & .000 & .000 \\
\hline 76 & Pike & .000 & .000 \\
\hline$\overline{77}$ & Porter & .000 & 86.168 \\
\hline 78 & Posey & 2664.041 & 2419.063 \\
\hline 79 & Pulaski & .000 & .000 \\
\hline 80 & Putnam & .000 & .000 \\
\hline 81 & Randolph & 64.783 & 50.844 \\
\hline 82 & Ripley & 71.793 & 67.719 \\
\hline 83 & Rush & .000 & .000 \\
\hline 84 & St. Joseph & 70.294 & 214.771 \\
\hline 85 & Scott & 70.391 & 87.768 \\
\hline 86 & Shelby & .000 & 34.420 \\
\hline 87 & Spencer & .000 & 1.749 \\
\hline 88 & Starke & .000 & 10.386 \\
\hline 89 & Steuben & 70.180 & 55.080 \\
\hline 90 & Sullivan & .000 & 1.852 \\
\hline 91 & Switzerland & .000 & .000 \\
\hline 92 & Tippecanoe & 79.510 & 407.084 \\
\hline 93 & Tipton & .000 & 1.751 \\
\hline 94 & Union & .000 & 7.843 \\
\hline 95 & Vanderburgh & .000 & 446.239 \\
\hline 96 & Vermillion & .000 & 293.912 \\
\hline 97 & Vigo & .000 & 187.650 \\
\hline 98 & Wabash & 64.702 & 114.839 \\
\hline 99 & Warren & .000 & .000 \\
\hline 100 & Warrick & .000 & 15.794 \\
\hline 101 & Washington & .000 & .000 \\
\hline 102 & Wayne & .000 & 22.993 \\
\hline
\end{tabular}




\begin{tabular}{|c|c|c|c|}
\hline & Locale & P18 15 & A18 15 \\
\hline 103 & Wells & .000 & .000 \\
\hline 104 & White & .000 & .000 \\
\hline 105 & Whitley & .000 & 1.962 \\
\hline 106 & lowa & 1206.500 & 2223.760 \\
\hline 107 & Kansas & 19601.195 & 16628.648 \\
\hline 108 & Kentucky_E & 5485.867 & 5934.721 \\
\hline 109 & Kentucky_W & 3657.245 & 3946.480 \\
\hline 110 & Louisiana & 86638.176 & 73116.021 \\
\hline 111 & Maine & 1270.886 & 1332.968 \\
\hline 112 & Maryland & 8470.496 & 9576.626 \\
\hline 113 & Massachusetts & 7451.111 & 9969.444 \\
\hline 114 & Michigan_E & 7233.928 & 8847.050 \\
\hline 115 & Michigan_W & 7233.928 & 8847.050 \\
\hline 116 & Minnesota & 14824.240 & 13443.268 \\
\hline 117 & Mississippi & 13619.695 & 12063.765 \\
\hline 118 & Missouri & 10196.422 & 12265.026 \\
\hline 119 & Montana & 5996.832 & 4809.095 \\
\hline 120 & Nebraska & 69.673 & 573.221 \\
\hline 121 & Nevada & 612.143 & 829.517 \\
\hline 122 & New Hampshire & 1210.123 & 1331.682 \\
\hline 123 & New Jersey & 25841.402 & 33509.568 \\
\hline 124 & New Mexico & 5805.825 & 4827.831 \\
\hline 125 & New York & 10959.214 & 19972.537 \\
\hline 126 & North Carolina & 5720.658 & 14110.555 \\
\hline 127 & North Dakota & 1153.774 & 915.968 \\
\hline 128 & Ohio_N & 12835.270 & 12966.712 \\
\hline 129 & Ohio_M & 12835.270 & 12966.712 \\
\hline 130 & Ohio_s & 12835.270 & 12966.712 \\
\hline 131 & Oklahoma & 17996.414 & 14840.493 \\
\hline 132 & Oregon & 5606.149 & 5319.553 \\
\hline 133 & Pennsylvania & 49884.830 & 45322.156 \\
\hline 134 & Rhode Island & 72.465 & 556.780 \\
\hline 135 & South Carolina & 2892.512 & 9987.387 \\
\hline 136 & South Dakota & 70.382 & 189.400 \\
\hline 137 & Tennessee & 10231.865 & 14953.863 \\
\hline 138 & Texas & 187244.264 & 164519.620 \\
\hline 139 & Utah & 12727.552 & 11233.568 \\
\hline 140 & Vermont & 442.252 & 420.071 \\
\hline 141 & \begin{tabular}{|l} 
Virginia \\
\end{tabular} & 5358.435 & 8348.529 \\
\hline 142 & Washington & 16013.385 & 13672.048 \\
\hline 143 & West Virginia & 2654.198 & 4564.802 \\
\hline 144 & Wisconsin & 3056.210 & 4866.360 \\
\hline 145 & Wyoming & 5959.328 & 4912.349 \\
\hline
\end{tabular}




\begin{tabular}{|c|c|c|c|}
\hline & Locale & P19 15 & A19 15 \\
\hline 1 & Alabama & 18064.491 & 17391.191 \\
\hline 2 & Arizona & 5126.908 & 5623.494 \\
\hline 3 & Arkansas & 8119.463 & 7995.350 \\
\hline 4 & California & 100414.551 & 96079.555 \\
\hline 5 & Colorado & 4787.439 & 5054.740 \\
\hline 6 & Connecticut & 6508.696 & 7698.157 \\
\hline 7 & Delaware & 7320.936 & 7367.403 \\
\hline 8 & DC & .000 & .000 \\
\hline 9 & Florida & 12868.737 & 14340.763 \\
\hline 10 & Georgia & 14368.517 & 16044.954 \\
\hline 11 & \begin{tabular}{|l} 
Idaho \\
\end{tabular} & 2085.163 & 2276.754 \\
\hline 12 & Illinois_N & 31150.046 & 31543.180 \\
\hline 13 & Illinois_S & 15575.023 & 15771.590 \\
\hline 14 & Adams & 105.367 & 114.837 \\
\hline 15 & Allen & 469.815 & 456.692 \\
\hline 16 & Bartholomew & 44.208 & 61.783 \\
\hline 17 & Benton & .000 & .000 \\
\hline 18 & Blackford & .000 & .000 \\
\hline 19 & Boone & .000 & .000 \\
\hline 20 & Brown & 2.331 & 3.257 \\
\hline 21 & Carroll & .000 & .000 \\
\hline 22 & Cass & .000 & .000 \\
\hline 23 & Clark & 790.491 & 775.828 \\
\hline 24 & Clay & 14.044 & 19.627 \\
\hline 25 & Clinton & 42.298 & 59.114 \\
\hline 26 & Crawford & .000 & .000 \\
\hline 27 & Daviess & 73.971 & 71.854 \\
\hline 28 & Dearborn & 71.314 & 63.160 \\
\hline 29 & Decatur & .000 & .000 \\
\hline 30 & DeKalb & 59.085 & 82.574 \\
\hline 31 & Delaware & 20.464 & 28.599 \\
\hline 32 & Dubois & 14.720 & 20.572 \\
\hline 33 & Elkhart & 504.670 & 673.009 \\
\hline 34 & Fayette & .000 & .000 \\
\hline 35 & Floyd & 25.793 & 36.048 \\
\hline 36 & Fountain & 2.308 & 3.225 \\
\hline 37 & Franklin & 369.365 & 319.207 \\
\hline 38 & Fulton & 2.336 & 3.265 \\
\hline 39 & Gibson & .000 & .000 \\
\hline 40 & Grant & 19.568 & 27.347 \\
\hline 41 & Greene & .000 & .000 \\
\hline 42 & Hamilton & 93.392 & 90.719 \\
\hline 43 & Hancock & .000 & .000 \\
\hline 44 & Harrison & 421.738 & 371.913 \\
\hline 45 & Hendricks & 3.582 & 5.006 \\
\hline 46 & Henry & .000 & .000 \\
\hline 47 & Howard & .000 & .000 \\
\hline 48 & Huntington & 75.419 & 73.260 \\
\hline 49 & Jackson & 41.255 & 57.656 \\
\hline 50 & Jasper & .000 & .000 \\
\hline 51 & Jay & 52.706 & 45.549 \\
\hline
\end{tabular}




\begin{tabular}{|c|c|c|c|}
\hline & $\begin{array}{r}\text { Locale } \\
\end{array}$ & P19_15 & A19 15 \\
\hline 52 & Jefferson & 14.709 & 20.556 \\
\hline 53 & Jennings & 368.896 & 320.167 \\
\hline 54 & Johnson & 18.590 & 25.980 \\
\hline 55 & Knox & 51.493 & 44.501 \\
\hline 56 & Kosciusko & 138.325 & 162.468 \\
\hline 57 & LaGrange & 2.581 & 3.607 \\
\hline 58 & Lake & 12269.917 & 10787.931 \\
\hline 59 & La Porte & 131.060 & 153.883 \\
\hline 60 & Lawrence & 320.489 & 276.968 \\
\hline 61 & Madison & 88.083 & 123.101 \\
\hline 62 & Marion & 2119.951 & 2601.769 \\
\hline 63 & Marshall & 393.385 & 357.432 \\
\hline 64 & Martin & .000 & .000 \\
\hline 65 & Miami & 12.969 & 18.125 \\
\hline 66 & Monroe & 413.781 & 380.151 \\
\hline 67 & Montgomery & 41.975 & 58.662 \\
\hline 68 & Morgan & 103.767 & 145.020 \\
\hline 69 & Newton & 2.306 & 3.223 \\
\hline 70 & Noble & 53.209 & 74.362 \\
\hline 71 & \begin{tabular}{|l} 
Ohio \\
\end{tabular} & .000 & .000 \\
\hline 72 & Orange & .000 & .000 \\
\hline 73 & Owen & .000 & .000 \\
\hline 74 & Parke & .000 & .000 \\
\hline 75 & Perry & .000 & .000 \\
\hline 76 & Pike & .000 & .000 \\
\hline 77 & Porter & 111.235 & 155.456 \\
\hline 78 & Posey & 2589.996 & 2464.272 \\
\hline 79 & Pulaski & .000 & .000 \\
\hline 80 & Putnam & .000 & .000 \\
\hline 81 & Randolph & 52.679 & 45.526 \\
\hline 82 & Ripley & 73.060 & 70.969 \\
\hline 83 & Rush & .000 & .000 \\
\hline 84 & St. Joseph & 263.192 & 337.339 \\
\hline 85 & Scott & 99.222 & 108.140 \\
\hline 86 & Shelby & 44.434 & 62.099 \\
\hline 87 & Spencer & 2.258 & 3.155 \\
\hline 88 & Starke & 13.407 & 18.737 \\
\hline 89 & Steuben & 57.067 & 49.318 \\
\hline 90 & Sullivan & 2.391 & 3.341 \\
\hline 91 & Switzerland & .000 & .000 \\
\hline 92 & Tippecanoe & 509.607 & 677.720 \\
\hline 93 & Tipton & 2.261 & 3.160 \\
\hline 94 & Union & 10.124 & 14.149 \\
\hline 95 & Vanderburgh & 576.054 & 805.067 \\
\hline 96 & Vermillion & 379.413 & 530.251 \\
\hline 97. & Vigo & 242.239 & 338.543 \\
\hline 98 & Wabash & 135.306 & 161.036 \\
\hline 99 & Warren & .000 & .000 \\
\hline 100 & Warrick & 20.388 & 28.493 \\
\hline 101 & Washington & .000 & .000 \\
\hline 102 & Wayne & 29.682 & 41.482 \\
\hline
\end{tabular}




\begin{tabular}{|c|c|c|c|}
\hline & Locale & P19 15 & A19 15 \\
\hline 103 & Wells & .000 & .000 \\
\hline 104 & White & .000 & .000 \\
\hline 105 & Whitley & 2.533 & 3.540 \\
\hline 106 & lowa & 2629.379 & 3151.443 \\
\hline 107 & Kansas & 17545.934 & 16020.403 \\
\hline 108 & Kentucky_E & 6564.040 & 6794.394 \\
\hline 109 & Kentucky_W & 4376.026 & 4529.596 \\
\hline 110 & Louisiana & 77058.944 & 70119.284 \\
\hline 111 & Maine & 1466.569 & 1498.430 \\
\hline 112 & Maryland & 10668.497 & 11236.170 \\
\hline 113 & Massachusetts & 11379.462 & 12671.887 \\
\hline 114 & Michigan_E & 9973.988 & 10801.853 \\
\hline 115 & Michigan_W & 9973.988 & 10801.853 \\
\hline 116 & Minnesota & 14389.264 & 13680.540 \\
\hline 117 & Mississippi & 12849.355 & 12050.841 \\
\hline 118 & Missouri & 13793.802 & 14855.423 \\
\hline 119 & Montana & 5008.769 & 4399.215 \\
\hline 120 & Nebraska & 726.042 & 984.467 \\
\hline 121 & Nevada & 948.405 & 1059.963 \\
\hline 122 & New Hampshire & 1477.062 & 1539.447 \\
\hline 123 & New Jersey & 38089.657 & 42025.010 \\
\hline 124 & New Mexico & 5071.156 & 4569.243 \\
\hline 125 & New York & 23590.943 & 28216.640 \\
\hline 126 & North Carolina & 17071.341 & 21377.092 \\
\hline 127 & North Dakota & 951.683 & 829.639 \\
\hline 128 & Ohio_N & 14171.858 & 14239.316 \\
\hline 129 & Ohio_M & 14171.858 & 14239.316 \\
\hline 130 & Ohio_s & 14171.858 & 14239.316 \\
\hline 131 & Oklahoma & 15558.536 & 13938.896 \\
\hline 132 & Oregon & 5745.858 & 5598.775 \\
\hline 133 & Pennsylvania & 48530.071 & 46188.477 \\
\hline 134 & Rhode Island & 704.260 & 952.814 \\
\hline 135 & South Carolina & 12314.321 & 15955.457 \\
\hline 136 & South Dakota & 230.422 & 291.502 \\
\hline 137 & Tennessee & 17257.757 & 19681.118 \\
\hline 138 & Texas & 174931.976 & 163269.539 \\
\hline 139 & Utah & 11956.070 & 11189.348 \\
\hline 140 & Vermont & 453.825 & 442.441 \\
\hline 141 & \begin{tabular}{|l} 
Virginia \\
\end{tabular} & 9705.533 & 11240.069 \\
\hline 142 & Washington & 14446.776 & 13245.186 \\
\hline 143 & West Virginia & 5361.918 & 6342.452 \\
\hline 144 & Wisconsin & 5670.801 & 6599.782 \\
\hline 145 & Wyoming & 5149.561 & 4612.245 \\
\hline
\end{tabular}




\begin{tabular}{|c|c|c|c|}
\hline & Locale & P20 15 & A20 15 \\
\hline 1 & Alabama & 11557.089 & 10386.808 \\
\hline 2 & Arizona & 2268.243 & 5517.389 \\
\hline 3 & Arkansas & 4931.922 & 4243.055 \\
\hline 4 & California & 65113.567 & 77143.894 \\
\hline 5 & Colorado & 2407.028 & 4368.326 \\
\hline 6 & Connecticut & 2057.051 & 2967.185 \\
\hline 7 & Delaware & 4213.862 & 3520.333 \\
\hline 8 & $\mathrm{DC}$ & .000 & .000 \\
\hline 9 & Florida & 5361.482 & 8733.143 \\
\hline 10 & Georgia & 5937.994 & 6058.411 \\
\hline 11 & Idaho & 937.781 & 1907.728 \\
\hline 12 & Illinois_N & 17642.149 & 17071.528 \\
\hline 13 & Illinois_S & 8821.075 & 8535.764 \\
\hline 14 & Adams & 47.699 & 83.024 \\
\hline 15 & Allen & 294.117 & 458.616 \\
\hline 16 & Bartholomew & .000 & 40.939 \\
\hline 17 & Benton & .000 & .000 \\
\hline 18 & Blackford & .000 & .000 \\
\hline 19 & Boone & .000 & 28.605 \\
\hline 20 & Brown & .000 & .545 \\
\hline 21 & Carroll & .000 & .000 \\
\hline 22 & Cass & .000 & 19.816 \\
\hline 23 & Clark & 483.956 & 394.418 \\
\hline 24 & Clay & .000 & .547 \\
\hline 25 & Clinton & .000 & 42.392 \\
\hline 26 & Crawford & .000 & .000 \\
\hline 27 & Daviess & 46.383 & 37.285 \\
\hline 28 & Dearborn & 53.711 & 43.847 \\
\hline 29 & Decatur & .000 & .000 \\
\hline 30 & DeKalb & .000 & 17.669 \\
\hline 31 & Delaware & .000 & .563 \\
\hline 32 & Dubois & .000 & 43.028 \\
\hline 33 & Elkhart & 47.516 & 169.946 \\
\hline 34 & Fayette & .000 & .000 \\
\hline 35 & Floyd & .000 & 35.954 \\
\hline 36 & Fountain & .000 & 3.238 \\
\hline 37 & Franklin & 289.850 & 232.998 \\
\hline 38 & Fulton & .000 & .546 \\
\hline 39 & Gibson & .000 & 13.109 \\
\hline 40 & Grant & .000 & .000 \\
\hline 41 & Greene & .000 & 3.188 \\
\hline 42 & Hamilton & 58.560 & 61.995 \\
\hline 43 & Hancock & .000 & .707 \\
\hline 44 & Harrison & 319.996 & 257.231 \\
\hline 45 & Hendricks & .000 & 14.660 \\
\hline 46 & Henry & .000 & 2.900 \\
\hline 47 & Howard & .000 & 3.646 \\
\hline 48 & Huntington & 47.291 & 128.209 \\
\hline 49 & Jackson & .000 & .000 \\
\hline 50 & Jasper & .000 & 9.918 \\
\hline 51 & Jay & 41.360 & 33.248 \\
\hline
\end{tabular}




\begin{tabular}{|c|c|c|c|}
\hline & Locale & P20 15 & A20 15 \\
\hline 52 & Jefferson & .000 & .000 \\
\hline 53 & Jennings & 287.475 & 231.089 \\
\hline 54 & Johnson & .000 & 41.661 \\
\hline 55 & Knox & 40.408 & 36.571 \\
\hline 56 & Kosciusko & 45.389 & 43.289 \\
\hline 57 & LaGrange & .000 & 3.621 \\
\hline 58 & Lake & 9357.507 & 7531.405 \\
\hline 59 & La Porte & 43.080 & 38.505 \\
\hline 60 & Lawrence & 251.496 & 205.308 \\
\hline 61 & Madison & .000 & .549 \\
\hline 62 & Marion & 531.113 & 530.735 \\
\hline 63 & Marshall & 283.001 & 228.081 \\
\hline 64 & Martin & .000 & .000 \\
\hline 65 & Miami & .000 & .505 \\
\hline 66 & Monroe & 291.513 & 295.868 \\
\hline 67 & Montgomery & .000 & .000 \\
\hline 68 & Morgan & .000 & 113.240 \\
\hline 69 & Newton & .000 & 9.437 \\
\hline 70 & Noble & .000 & 3.456 \\
\hline 71 & Ohio & .000 & .000 \\
\hline 72 & Orange & .000 & .000 \\
\hline 73 & Owen & .000 & .000 \\
\hline 74 & Parke & .000 & .000 \\
\hline 75 & Perry & .000 & .509 \\
\hline 76 & Pike & .000 & .000 \\
\hline 77 & Porter & .000 & 6.236 \\
\hline 78 & Posey & 1699.945 & 1366.512 \\
\hline 79 & Pulaski & .000 & .000 \\
\hline 80 & Putnam & .000 & 9.846 \\
\hline 81 & Randolph & 41.339 & 36.328 \\
\hline 82 & Ripley & 45.812 & 40.259 \\
\hline 83 & Rush & .000 & .509 \\
\hline 84 & St. Joseph & 44.855 & 87.487 \\
\hline 85 & Scott & 44.917 & 36.107 \\
\hline 86 & Shelby & .000 & .000 \\
\hline 87 & Spencer & .000 & .000 \\
\hline 88 & Starke & .000 & .000 \\
\hline 89 & Steuben & 44.782 & 39.354 \\
\hline 90 & Sullivan & .000 & .000 \\
\hline 91 & Switzerland & .000 & .000 \\
\hline 92 & Tippecanoe & 50.736 & 116.320 \\
\hline 93 & Tipton & .000 & .000 \\
\hline 94 & Union & .000 & .000 \\
\hline 95 & Vanderburgh & .000 & .575 \\
\hline 96 & Vermillion & .000 & .000 \\
\hline 97 & Vigo & .000 & 96.246 \\
\hline 98 & Wabash & 41.287 & 60.416 \\
\hline 99 & Warren & .000 & .000 \\
\hline 100 & Warrick & .000 & .000 \\
\hline 101 & Washington & .000 & .000 \\
\hline 102 & Wayne & .000 & 46.390 \\
\hline
\end{tabular}




\begin{tabular}{|c|c|c|c|}
\hline & Locale & P20 15 & A20 15 \\
\hline 103 & Wells & .000 & .594 \\
\hline 104 & White & .000 & 9.496 \\
\hline 105 & Whitley & .000 & 10.249 \\
\hline 106 & lowa & 769.877 & 1506.928 \\
\hline 107 & Kansas & 12507.672 & 10415.864 \\
\hline 108 & Kentucky_E & 3500.573 & 3208.145 \\
\hline 109 & Kentucky_W & 2333.716 & 2138.763 \\
\hline 110 & Louisiana & 55284.480 & 44560.702 \\
\hline 111 & Maine & 810.962 & 1021.168 \\
\hline 112 & Maryland & 5405.088 & 5851.923 \\
\hline 113 & Massachusetts & 4754.611 & 9427.074 \\
\hline 114 & Michigan_E & 4616.025 & 4441.059 \\
\hline 115 & Michigan_W & 4616.025 & 4441.059 \\
\hline 116 & Minnesota & 9459.461 & 11291.024 \\
\hline 117 & Mississippi & 8690.831 & 7256.934 \\
\hline 118 & Missouri & 6506.415 & 6059.417 \\
\hline 119 & Montana & 3826.624 & 3104.310 \\
\hline 120 & Nebraska & 44.459 & 379.518 \\
\hline 121 & Nevada & 390.613 & 598.022 \\
\hline 122 & New Hampshire & 772.189 & 2181.658 \\
\hline 123 & New Jersey & 16489.596 & 15309.386 \\
\hline 124 & New Mexico & 3704.741 & 3645.450 \\
\hline 125 & New York & 6993.158 & 10231.877 \\
\hline 126 & North Carolina & 3650.396 & 6346.635 \\
\hline 127 & North Dakota & 736.232 & 669.645 \\
\hline 128 & Ohio_N & 8190.284 & 7248.560 \\
\hline 129 & Ohio_M & 8190.284 & 7248.560 \\
\hline 130 & Ohio_s & 8190.284 & 7248.560 \\
\hline 131 & Oklahoma & 11483.649 & 9954.676 \\
\hline 132 & Oregon & 3577.326 & 5372.112 \\
\hline 133 & Pennsylvania & 31831.890 & 28798.283 \\
\hline 134 & Rhode Island & 46.240 & 424.757 \\
\hline 135 & South Carolina & 1845.734 & 2535.609 \\
\hline 136 & South Dakota & 44.912 & 773.746 \\
\hline 137 & Tennessee & 6529.031 & 6071.835 \\
\hline 138 & Texas & 119481.991 & 105487.018 \\
\hline 139 & Utah & 8121.548 & 7592.336 \\
\hline 140 & Vermont & 282.204 & 802.147 \\
\hline 141 & Virginia & 3419.258 & 4722.563 \\
\hline 142 & Washington & 10218.263 & 11203.865 \\
\hline 143 & West Virginia & 1693.664 & 1409.929 \\
\hline 144 & Wisconsin & 1950.191 & 2911.029 \\
\hline 145 & Wyoming & 3802.693 & 3082.272 \\
\hline
\end{tabular}




\begin{tabular}{|c|c|c|c|}
\hline & Locale & P21 15 & A21 15 \\
\hline 1 & Alabama & 488.087 & 207.356 \\
\hline 2 & Arizona & 194.357 & 204.405 \\
\hline 3 & Arkansas & 235.717 & 125.218 \\
\hline 4 & California & 1437.020 & 1585.723 \\
\hline 5 & Colorado & 99.803 & 168.925 \\
\hline 6 & Connecticut & 84.145 & 228.198 \\
\hline 7 & Delaware & 21.683 & 95.261 \\
\hline 8 & DC & .000 & .000 \\
\hline 9 & Florida & 431.377 & 658.584 \\
\hline 10 & Georgia & 948.661 & 481.728 \\
\hline 11 & Idaho & 36.115 & 72.465 \\
\hline 12 & Illinois_N & 234.286 & 551.677 \\
\hline 13 & Illinois_s & 117.143 & 275.838 \\
\hline 14 & Adams & 4.729 & 2.174 \\
\hline 15 & Allen & 17.913 & 15.209 \\
\hline 16 & Bartholomew & 13.271 & 2.216 \\
\hline 17 & Benton & .925 & .353 \\
\hline 18 & Blackford & .146 & .139 \\
\hline 19 & Boone & 1.285 & .919 \\
\hline 20 & Brown & .000 & .111 \\
\hline 21 & Carroll & 6.373 & .496 \\
\hline 22 & Cass & .158 & .491 \\
\hline 23 & Clark & 20.059 & 7.418 \\
\hline 24 & Clay & .164 & .693 \\
\hline 25 & Clinton & 1.016 & 1.927 \\
\hline 26 & Crawford & .000 & .000 \\
\hline 27 & Daviess & 1.041 & .992 \\
\hline 28 & Dearborn & .000 & .419 \\
\hline 29 & Decatur & .166 & .871 \\
\hline 30 & DeKalb & .183 & 2.504 \\
\hline 31 & Delaware & 2.378 & 2.086 \\
\hline 32 & Dubois & 113.243 & 1.703 \\
\hline 33 & Elkhart & 68.621 & 18.750 \\
\hline 34 & Fayette & .909 & .406 \\
\hline 35 & Floyd & 7.847 & 2.233 \\
\hline 36 & Fountain & .000 & .365 \\
\hline 37 & Franklin & .000 & .184 \\
\hline 38 & Fulton & .000 & .416 \\
\hline 39 & Gibson & .000 & .273 \\
\hline 40 & Grant & 2.525 & 1.317 \\
\hline 41 & Greene & .159 & .216 \\
\hline 42 & Hamilton & 5.857 & 6.401 \\
\hline 43 & Hancock & 1.271 & 1.394 \\
\hline 44 & Harrison & 12.949 & .785 \\
\hline 45 & Hendricks & .251 & 1.320 \\
\hline 46 & Henry & 2.621 & .834 \\
\hline 47 & Howard & 6.112 & .564 \\
\hline 48 & Huntington & .000 & 1.267 \\
\hline 49 & Jackson & .991 & 2.289 \\
\hline 50 & Jasper & .170 & .366 \\
\hline 51 & Jay & .928 & .491 \\
\hline
\end{tabular}




\begin{tabular}{|c|c|c|c|}
\hline & $\begin{array}{l}\text { Locale } \\
\end{array}$ & P21 15 & A21 15 \\
\hline 52 & Jefferson & .172 & .679 \\
\hline 53 & Jennings & .000 & .280 \\
\hline 54 & Johnson & 1.888 & 1.447 \\
\hline 55 & Knox & .151 & 1.049 \\
\hline 56 & Kosciusko & 5.304 & 3.532 \\
\hline 57 & LaGrange & 5.441 & .905 \\
\hline 58 & Lake & 4.102 & 17.626 \\
\hline 59 & La Porte & 3.436 & 4.793 \\
\hline 60 & Lawrence & .157 & .192 \\
\hline 61 & Madison & 6.867 & 4.315 \\
\hline 62 & Marion & 21.776 & 83.251 \\
\hline 63 & Marshall & 1.059 & 1.796 \\
\hline 64 & Martin & .000 & .157 \\
\hline 65 & Miami & 5.678 & 1.569 \\
\hline 66 & Monroe & 1.272 & 4.644 \\
\hline 67 & Montgomery & .000 & 1.813 \\
\hline 68 & Morgan & .194 & 3.926 \\
\hline 69 & Newton & .969 & .354 \\
\hline 70 & Noble & 1.035 & 2.594 \\
\hline 71 & Ohio & .000 & .000 \\
\hline 72 & Orange & 27.795 & .154 \\
\hline 73 & Owen & .000 & .029 \\
\hline 74 & Parke & .160 & .163 \\
\hline 75 & Perry & 4.470 & .114 \\
\hline 76 & Pike & .000 & .120 \\
\hline 77 & Porter & 1.501 & 6.284 \\
\hline 78 & Posey & .000 & 15.722 \\
\hline 79 & Pulaski & .168 & .728 \\
\hline 80 & Putnam & .000 & .152 \\
\hline 81 & Randolph & .745 & .155 \\
\hline 82 & Ripley & 2.999 & 1.032 \\
\hline 83 & Rush & .000 & .210 \\
\hline 84 & St. Joseph & 2.926 & 15.064 \\
\hline 85 & Scott & 1.008 & 1.525 \\
\hline 86 & Shelby & 5.382 & 1.972 \\
\hline 87 & Spencer & 11.861 & .711 \\
\hline 88 & Starke & .157 & .597 \\
\hline 89 & Steuben & .168 & .040 \\
\hline 90 & Sullivan & .000 & .497 \\
\hline 91 & Switzerland & .197 & .033 \\
\hline 92 & Tippecanoe & 14.235 & 17.869 \\
\hline 93 & Tipton & .000 & .468 \\
\hline 94 & Union & .000 & .529 \\
\hline 95 & Vanderburgh & 7.012 & 25.184 \\
\hline 96 & Vermillion & .000 & 13.725 \\
\hline 97 & Vigo & .988 & 10.665 \\
\hline 98 & Wabash & .154 & 3.168 \\
\hline 99 & Warren & .000 & .171 \\
\hline 100 & Warrick & .179 & 1.290 \\
\hline 101 & Washington & 30.298 & .029 \\
\hline 102 & Wayne & 10.679 & 2.149 \\
\hline
\end{tabular}




\begin{tabular}{|c|c|c|c|}
\hline & Locale & P21 15 & A21 15 \\
\hline 103 & Wells & .291 & 4.573 \\
\hline 104 & White & 4.655 & .866 \\
\hline 105 & Whitley & .781 & .370 \\
\hline 106 & lowa & 140.578 & 146.312 \\
\hline 107 & Kansas & 81.841 & 133.892 \\
\hline 108 & Kentucky_E & 70.744 & 131.302 \\
\hline 109 & Kentucky_W & 47.162 & 87.535 \\
\hline 110 & Louisiana & 22.252 & 329.382 \\
\hline 111 & Maine & 80.371 & 46.985 \\
\hline 112 & Maryland & 98.647 & 251.059 \\
\hline 113 & Massachusetts & 253.167 & 362.245 \\
\hline 114 & Michigan_E & 291.117 & 245.492 \\
\hline 115 & Michigan_W & 291.117 & 245.492 \\
\hline 116 & Minnesota & 222.018 & 236.101 \\
\hline 117 & Mississippi & 518.048 & 118.908 \\
\hline 118 & Missouri & 218.410 & 358.866 \\
\hline 119 & Montana & 14.918 & 25.960 \\
\hline 120 & Nebraska & 55.513 & 78.462 \\
\hline 121 & Nevada & 40.877 & 63.057 \\
\hline 122 & New Hampshire & 57.297 & 45.085 \\
\hline 123 & New Jersey & 250.527 & 1002.225 \\
\hline 124 & New Mexico & 18.297 & 40.302 \\
\hline 125 & New York & 478.407 & 1097.347 \\
\hline 126 & North Carolina & 2931.121 & 660.671 \\
\hline 127 & North Dakota & 17.520 & 23.782 \\
\hline 128 & Ohio_N & 128.491 & 229.495 \\
\hline 129 & Ohio_M & 128.491 & 229.495 \\
\hline 130 & Ohio_s & 128.491 & 229.495 \\
\hline 131 & Oklahoma & 62.013 & 104.752 \\
\hline 132 & Oregon & 114.983 & 146.249 \\
\hline 133 & Pennsylvania & 548.233 & 546.170 \\
\hline 134 & Rhode Island & 102.550 & 42.789 \\
\hline 135 & South Carolina & 815.496 & 431.858 \\
\hline 136 & South Dakota & 35.658 & 28.692 \\
\hline 137 & Tennessee & 592.675 & 483.675 \\
\hline 138 & Texas & 595.601 & 1352.827 \\
\hline 139 & Utah & 118.292 & 114.875 \\
\hline 140 & Vermont & 51.249 & 21.220 \\
\hline 141 & Virginia & 685.776 & 327.565 \\
\hline 142 & Washington & 148.823 & 214.351 \\
\hline 143 & West Virginia & 21.890 & 136.452 \\
\hline 144 & Wisconsin & 326.120 & 254.263 \\
\hline 145 & Wyoming & 3.818 & 17.629 \\
\hline
\end{tabular}




\begin{tabular}{|c|c|c|c|}
\hline & Locale & P22 15 & A22 15 \\
\hline 1 & Alabama & 4037.588 & 4037.588 \\
\hline 2 & Arizona & 6100.480 & 6100.480 \\
\hline 3 & Arkansas & 2546.454 & 2546.454 \\
\hline 4 & California & 33923.676 & 33923.676 \\
\hline 5 & Colorado & 4158.537 & 4158.537 \\
\hline 6 & Connecticut & 3151.896 & 3151.896 \\
\hline 7 & Delaware & 784.045 & 784.045 \\
\hline 8 & DC & .000 & .000 \\
\hline 9 & Florida & 17761.439 & 17761.439 \\
\hline 10 & Georgia & 8466.440 & 8466.440 \\
\hline 11 & Idaho & 1364.301 & 1364.301 \\
\hline 12 & Illinois_N & 7552.468 & 7552.468 \\
\hline 13 & Illinois_S & 3776.234 & 3776.234 \\
\hline 14 & Adams & 32.857 & 32.857 \\
\hline 15 & Allen & 332.417 & 332.417 \\
\hline 16 & Bartholomew & 71.146 & 71.146 \\
\hline 17 & Benton & 7.622 & 7.622 \\
\hline 18 & Blackford & 11.619 & 11.619 \\
\hline 19 & Boone & 54.291 & 54.291 \\
\hline 20 & Brown & 14.121 & 14.121 \\
\hline 21 & Carroll & 18.276 & 18.276 \\
\hline 22 & Cass & 36.372 & 36.372 \\
\hline 23 & Clark & 96.764 & 96.764 \\
\hline 24 & Clay & 23.975 & 23.975 \\
\hline 25 & Clinton & 31.409 & 31.409 \\
\hline 26 & Crawford & 10.687 & 10.687 \\
\hline 27 & Daviess & 27.510 & 27.510 \\
\hline 28 & Dearborn & 52.219 & 52.219 \\
\hline 29 & Decatur & 22.463 & 22.463 \\
\hline 30 & DeKalb & 40.995 & 40.995 \\
\hline 31 & Delaware & 110.051 & 110.051 \\
\hline 32 & Dubois & 38.005 & 38.005 \\
\hline 33 & Elkhart & 182.668 & 182.668 \\
\hline 34 & Fayette & 21.983 & 21.983 \\
\hline 35 & Floyd & 72.679 & 72.679 \\
\hline 36 & Fountain & 15.692 & 15.692 \\
\hline 37 & Franklin & 21.147 & 21.147 \\
\hline 38 & Fulton & 18.701 & 18.701 \\
\hline 39 & Gibson & 29.500 & 29.500 \\
\hline 40 & Grant & 60.584 & 60.584 \\
\hline 41 & Greene & 30.132 & 30.132 \\
\hline 42 & Hamilton & 262.520 & 262.520 \\
\hline 43 & Hancock & 65.224 & 65.224 \\
\hline 44 & Harrison & 37.355 & 37.355 \\
\hline 45 & Hendricks & 145.065 & 145.065 \\
\hline 46 & Henry & 41.056 & 41.056 \\
\hline 47 & Howard & 77.166 & 77.166 \\
\hline 48 & Huntington & 37.229 & 37.229 \\
\hline 49 & Jackson & 37.744 & 37.744 \\
\hline 50 & Jasper & 28.429 & 28.429 \\
\hline 51 & Jay & 18.572 & 18.572 \\
\hline
\end{tabular}




\begin{tabular}{|c|c|c|c|}
\hline & $\begin{array}{l}\text { Locale } \\
\end{array}$ & P22 15 & A22 15 \\
\hline 52 & Jefferson & 30.340 & 30.340 \\
\hline 53 & Jennings & 27.401 & 27.401 \\
\hline 54 & Johnson & 138.222 & 138.222 \\
\hline 55 & Knox & 34.088 & 34.088 \\
\hline 56 & Kosciusko & 69.827 & 69.827 \\
\hline 57 & LaGrange & 36.962 & 36.962 \\
\hline 58 & Lake & 428.222 & 428.222 \\
\hline 59 & La Porte & 97.261 & 97.261 \\
\hline 601 & Lawrence & 40.671 & 40.671 \\
\hline \begin{tabular}{l|l}
61 & \\
\end{tabular} & Madison & 123.063 & 123.063 \\
\hline 62 & Marion & 799.028 & 799.028 \\
\hline 63 & Marshall & 43.967 & 43.967 \\
\hline 64 & Martin & 8.837 & 8.837 \\
\hline 65 & Miami & 30.721 & 30.721 \\
\hline 66 & Monroe & 123.019 & 123.019 \\
\hline 67 & Montgomery & 34.943 & 34.943 \\
\hline 68 & Morgan & 71.956 & 71.956 \\
\hline 69 & Newton & 13.058 & 13.058 \\
\hline 70 & Noble & 44.073 & 44.073 \\
\hline 71 & Ohio & 5.056 & 5.056 \\
\hline 72 & Orange & 17.910 & 17.910 \\
\hline 73 & Owen & 21.003 & 21.003 \\
\hline 74 & Parke & 15.098 & 15.098 \\
\hline 75 & Perry & 16.038 & 16.038 \\
\hline 76 & Pike & 11.837 & 11.837 \\
\hline 77 & Porter & 136.282 & 136.282 \\
\hline 78 & Posey & 25.061 & 25.061 \\
\hline 79 & Pulaski & 12.321 & 12.321 \\
\hline 80 & Putnam & 34.466 & 34.466 \\
\hline 81 & Randolph & 23.488 & 23.488 \\
\hline 82 & Ripley & 25.182 & 25.182 \\
\hline 83 & Rush & 15.270 & 15.270 \\
\hline 84 & St. Joseph & 254.154 & 254.154 \\
\hline 85 & Scott & 21.939 & 21.939 \\
\hline 86 & Shelby & 39.517 & 39.517 \\
\hline 87 & Spencer & 18.062 & 18.062 \\
\hline 88 & Starke & 20.837 & 20.837 \\
\hline 89 & Steuben & 31.064 & 31.064 \\
\hline 90 & Sullivan & 19.925 & 19.925 \\
\hline 91 & Switzerland & 9.013 & 9.013 \\
\hline 92 & Tippecanoe & 155.347 & 155.347 \\
\hline 93 & Tipton & 14.397 & 14.397 \\
\hline 94 & Union & 6.491 & 6.491 \\
\hline 95 & Vanderburgh & 157.484 & 157.484 \\
\hline 96 & Vermillion & 14.256 & 14.256 \\
\hline 97 & Vigo & 92.640 & 92.640 \\
\hline 98 & Wabash & 30.651 & 30.651 \\
\hline 99 & Warren & 7.326 & 7.326 \\
\hline 100 & Warrick & 54.449 & 54.449 \\
\hline 101 & Washington & 26.177 & 26.177 \\
\hline 102 & Wayne & 59.416 & 59.416 \\
\hline
\end{tabular}




\begin{tabular}{|c|c|c|c|}
\hline & $\begin{array}{l}\text { Locale } \\
\end{array}$ & P22 15 & A22 15 \\
\hline 103 & Wells & 26.303 & 26.303 \\
\hline 104 & White & 22.651 & 22.651 \\
\hline 105 & Whitley & 30.428 & 30.428 \\
\hline 106 & lowa & 2635.809 & 2635.809 \\
\hline 107 & Kansas & 2465.094 & 2465.094 \\
\hline 108 & Kentucky_E & 2250.783 & 2250.783 \\
\hline 109 & Kentucky_W & 1500.522 & 1500.522 \\
\hline 110 & Louisiana & 4075.953 & 4075.953 \\
\hline 111 & Maine & 1205.015 & 1205.015 \\
\hline 112 & Maryland & 5329.212 & 5329.212 \\
\hline 113 & Massachusetts & 5842.566 & 5842.566 \\
\hline 114 & Michigan_E & 4611.004 & 4611.004 \\
\hline 115 & Michigan_W & 4611.004 & 4611.004 \\
\hline 116 & Minnesota & 4838.269 & 4838.269 \\
\hline 117 & Mississippi & 2594.153 & 2594.153 \\
\hline 118 & Missouri & 5241.583 & 5241.583 \\
\hline 119 & Montana & 869.058 & 869.058 \\
\hline 120 & Nebraska & 1553.768 & 1553.768 \\
\hline 121 & Nevada & 2380.040 & 2380.040 \\
\hline 122 & New Hampshire & 1235.961 & 1235.961 \\
\hline 123 & New Jersey & 7969.805 & 7969.805 \\
\hline 124 & New Mexico & 1755.981 & 1755.981 \\
\hline 125 & New York & 16940.900 & 16940.900 \\
\hline 126 & North Carolina & 8394.684 & 8394.684 \\
\hline 127 & North Dakota & 566.454 & 566.454 \\
\hline 128 & Ohio_N & 3396.253 & 3396.253 \\
\hline 129 & Ohio_M & 3396.253 & 3396.253 \\
\hline 130 & Ohio_s & 3396.253 & 3396.253 \\
\hline 131 & Oklahoma & 3155.246 & 3155.246 \\
\hline 132 & Oregon & 3416.528 & 3416.528 \\
\hline 133 & Pennsylvania & 11156.751 & 11156.751 \\
\hline 134 & Rhode Island & 982.557 & 982.557 \\
\hline 135 & South Carolina & 3936.913 & 3936.913 \\
\hline 136 & South Dakota & 692.670 & 692.670 \\
\hline 137 & Tennessee & 5540.381 & 5540.381 \\
\hline 138 & Texas & 22187.423 & 22187.423 \\
\hline 139 & Utah & 2328.823 & 2328.823 \\
\hline 140 & Vermont & 582.225 & 582.225 \\
\hline 141 & Virginia & 7201.055 & 7201.055 \\
\hline 142 & Washington & 5899.257 & 5899.257 \\
\hline 143 & West Virginia & 1616.421 & 1616.421 \\
\hline 144 & Wisconsin & 5091.715 & 5091.715 \\
\hline 145 & Wyoming & 461.380 & 461.380 \\
\hline
\end{tabular}




\begin{tabular}{|c|c|c|c|}
\hline & Locale & P23 15 & A23 15 \\
\hline 1 & Alabama & 2203.619 & 2987.761 \\
\hline 2 & Arizona & 2021.553 & 1691.246 \\
\hline 3 & Arkansas & 1618.604 & 1493.095 \\
\hline 4 & California & 14854.393 & 15651.402 \\
\hline 5 & \begin{tabular}{|l} 
Colorado \\
\end{tabular} & 1242.113 & 1291.489 \\
\hline 6 & Connecticut & 3021.151 & 1186.024 \\
\hline 7 & Delaware & 468.372 & 627.825 \\
\hline 8 & DC & .000 & .000 \\
\hline 9 & \begin{tabular}{|l|} 
Florida \\
\end{tabular} & 3727.487 & 4843.787 \\
\hline 10 & Georgia & 3207.109 & 8378.464 \\
\hline 11 & Idaho & 406.489 & 385.369 \\
\hline 12 & Illinois_N & 6219.541 & 4362.691 \\
\hline 13 & Illinois_S & 3109.770 & 2181.345 \\
\hline 14 & Adams & 30.362 & 48.959 \\
\hline 15 & Allen & 189.712 & 270.601 \\
\hline 16 & Bartholomew & 67.797 & 41.139 \\
\hline 17 & Benton & 3.681 & 3.253 \\
\hline 18 & Blackford & 30.936 & 13.956 \\
\hline 19 & Boone & 21.307 & 9.119 \\
\hline 20 & Brown & 3.568 & 2.249 \\
\hline 21 & Carroll & 24.024 & 2.911 \\
\hline 22 & Cass & 36.822 & 32.874 \\
\hline 23 & Clark & 90.253 & 68.139 \\
\hline 24 & Clay & 8.090 & 10.146 \\
\hline 25 & Clinton & 20.607 & 11.537 \\
\hline 26 & Crawford & .000 & 1.702 \\
\hline 27 & Daviess & 11.174 & 9.739 \\
\hline 28 & Dearborn & 15.109 & 14.521 \\
\hline 29 & Decatur & 155.003 & 17.320 \\
\hline 30 & DeKalb & 202.324 & 92.073 \\
\hline 31 & Delaware & 106.702 & 27.561 \\
\hline 32 & Dubois & 10.841 & 45.386 \\
\hline 33 & Elkhart & 432.623 & 316.226 \\
\hline 34 & Fayette & 18.587 & 3.501 \\
\hline 35 & Floyd & 40.300 & 68.378 \\
\hline 36 & Fountain & 3.533 & 2.499 \\
\hline 37 & Franklin & .591 & 29.477 \\
\hline 38 & Fulton & 45.818 & 8.031 \\
\hline 39 & Gibson & 7.639 & 19.327 \\
\hline 40 & Grant & 11.217 & 31.657 \\
\hline 41 & Greene & 4.843 & 5.704 \\
\hline 42 & Hamilton & 87.512 & 122.600 \\
\hline 43 & Hancock & 31.815 & 11.229 \\
\hline 44 & Harrison & 7.006 & 34.774 \\
\hline 45 & Hendricks & 17.061 & 27.850 \\
\hline 46 & Henry & 17.153 & 17.428 \\
\hline 47 & Howard & 11.146 & 28.754 \\
\hline 48 & Huntington & 60.308 & 22.504 \\
\hline 49 & Jackson & 43.763 & 23.557 \\
\hline 50 & Jasper & 37.494 & 6.774 \\
\hline 51 & Jay & 47.946 & 11.660 \\
\hline
\end{tabular}




\begin{tabular}{|c|c|c|c|}
\hline & Locale & P23 15 & A23 15 \\
\hline 52 & Jefferson & 10.102 & 12.436 \\
\hline 53 & \begin{tabular}{|l} 
Jennings \\
\end{tabular} & 37.817 & 88.942 \\
\hline 54 & Johnson & 79.445 & 59.912 \\
\hline 55 & Knox & 5.787 & 8.430 \\
\hline 56 & Kosciusko & 92.328 & 59.399 \\
\hline 57 & LaGrange & 13.000 & 36.815 \\
\hline 58 & Lake & 188.003 & 711.490 \\
\hline 59 & La Porte & 126.937 & 47.439 \\
\hline 60 & Lawrence & 29.503 & 31.891 \\
\hline 61 & Madison & 70.360 & 33.517 \\
\hline 62 & Marion & 776.433 & 259.001 \\
\hline 63 & Marshall & 64.284 & 123.744 \\
\hline 64 & Martin & .496 & 1.407 \\
\hline 65 & Miami & 15.100 & 18.276 \\
\hline 66 & Monroe & 29.608 & 56.019 \\
\hline 67 & Montgomery & 41.834 & 33.356 \\
\hline 68 & Morgan & 36.720 & 16.452 \\
\hline 69 & Newton & 3.530 & 18.111 \\
\hline 70 & Noble & 87.276 & 98.506 \\
\hline 71 & Ohio & .574 & .805 \\
\hline 72 & Orange & 3.118 & 8.983 \\
\hline 73 & Owen & .735 & 9.618 \\
\hline 74 & Parke & .525 & 8.598 \\
\hline 75 & Perry & 2.995 & 7.757 \\
\hline 76 & Pike & 3.293 & 1.885 \\
\hline 77 & Porter & 102.626 & 34.167 \\
\hline 78 & Posey & 69.364 & 131.228 \\
\hline 79 & Pulaski & 9.592 & 1.962 \\
\hline 80 & Putnam & .551 & 49.163 \\
\hline 81 & Randolph & 5.617 & 8.516 \\
\hline 82 & Ripley & 14.064 & 7.413 \\
\hline 83 & Rush & 2.994 & 5.317 \\
\hline 84 & St. Joseph & 273.536 & 164.390 \\
\hline 85 & Scott & 11.381 & 62.645 \\
\hline 86 & Shelby & 26.391 & 78.407 \\
\hline 87 & Spencer & 3.457 & 15.957 \\
\hline 88 & Starke & 2.089 & 9.360 \\
\hline 89 & Steuben & 74.333 & 37.820 \\
\hline 90 & Sullivan & .920 & 9.637 \\
\hline 91 & Switzerland & .000 & 9.044 \\
\hline 92 & Tippecanoe & 107.167 & 59.891 \\
\hline 93 & Tipton & 6.778 & 2.293 \\
\hline 94 & Union & 2.117 & 1.034 \\
\hline 95 & Vanderburgh & 198.089 & 180.759 \\
\hline 96 & Vermillion & 67.826 & 2.271 \\
\hline 97 & Vigo & 73.866 & 76.876 \\
\hline 98 & Wabash & 64.185 & 14.447 \\
\hline 99 & Warren & 6.819 & 1.537 \\
\hline 100 & Warrick & 16.787 & 10.080 \\
\hline 101 & Washington & 34.500 & 12.723 \\
\hline 102 & Wayne & 35.752 & 47.170 \\
\hline
\end{tabular}




\begin{tabular}{|c|c|c|c|}
\hline & Locale & P23 15 & A23 15 \\
\hline 103 & Wells & 35.543 & 34.609 \\
\hline 104 & White & 49.993 & 17.330 \\
\hline 105 & Whitley & 41.095 & 22.771 \\
\hline 106 & \begin{tabular}{|l} 
lowa \\
\end{tabular} & 1627.846 & 1165.385 \\
\hline 107 & Kansas & 1049.473 & 1820.258 \\
\hline 108 & Kentucky_E & 1189.796 & 1281.176 \\
\hline 109 & Kentucky_W & 793.198 & 854.118 \\
\hline 110 & \begin{tabular}{|l} 
Louisiana \\
\end{tabular} & 2156.674 & 4591.092 \\
\hline 111 & \begin{tabular}{|l} 
Maine \\
\end{tabular} & 347.785 & 526.402 \\
\hline 112 & Maryland & 1426.628 & 1607.933 \\
\hline 113 & Massachusetts & 3447.419 & 2578.048 \\
\hline 114 & Michigan_E & 3554.354 & 2427.332 \\
\hline 115 & Michigan_W & 3554.354 & 2427.332 \\
\hline 116 & Minnesota & 3323.628 & 2366.121 \\
\hline 117 & Mississippi & 1100.574 & 1821.545 \\
\hline 118 & Missouri & 3141.730 & 2335.761 \\
\hline 119 & Montana & 98.107 & 413.295 \\
\hline 120 & Nebraska & 654.039 & 526.894 \\
\hline 121 & Nevada & 452.034 & 656.668 \\
\hline 122 & New Hampshire & 872.681 & 652.465 \\
\hline 123 & New Jersey & 4889.641 & 4092.755 \\
\hline 124 & New Mexico & 229.416 & 552.488 \\
\hline 125 & New York & 6077.628 & 5373.269 \\
\hline 126 & North Carolina & 4767.588 & 6106.747 \\
\hline 127 & North Dakota & 102.781 & 189.327 \\
\hline 128 & Ohio $N$ & 3165.781 & 2350.535 \\
\hline 129 & Ohio M & 3165.781 & 2350.535 \\
\hline 130 & Ohio_s & 3165.781 & 2350.535 \\
\hline 131 & Oklahoma & 1664.801 & 1905.107 \\
\hline 132 & Oregon & 1350.373 & 1183.474 \\
\hline 133 & Pennsylvania & 6816.428 & 6366.554 \\
\hline 134 & Rhode Island & 702.827 & 494.454 \\
\hline 135 & South Carolina & 3401.118 & 3045.785 \\
\hline 136 & South Dakota & 214.790 & 245.198 \\
\hline 137 & Tennessee & 4039.144 & 3295.113 \\
\hline 138 & Texas & 11525.312 & 14902.562 \\
\hline 139 & Utah & 938.503 & 1208.763 \\
\hline 140 & Vermont & 222.180 & 202.835 \\
\hline 141 & Virginia & 2169.396 & 2730.350 \\
\hline 142 & Washington & 1608.971 & 2295.180 \\
\hline 143 & West Virginia & 867.945 & 482.070 \\
\hline 144 & Wisconsin & 4932.728 & 2442.691 \\
\hline 145 & Wyoming & 96.828 & 342.164 \\
\hline
\end{tabular}




\begin{tabular}{|c|c|c|c|}
\hline & $\begin{array}{r}\text { Locale } \\
\end{array}$ & P24 15 & A24 15 \\
\hline 1 & Alabama & 4917.022 & 4028.129 \\
\hline 2 & Arizona & 965.035 & 1726.318 \\
\hline 3 & Arkansas & 2098.311 & 2254.923 \\
\hline 4 & California & 27702.895 & 20603.481 \\
\hline 5 & Colorado & 1024.082 & 1744.371 \\
\hline 6 & Connecticut & 875.183 & 2714.836 \\
\hline 7 & Delaware & 1792.809 & 1172.817 \\
\hline 8 & DC & .000 & .000 \\
\hline 9 & Florida & 2281.070 & 4865.904 \\
\hline 10 & Georgia & 2526.349 & 10342.852 \\
\hline 11 & Idaho & 398.984 & 581.617 \\
\hline 12 & Illinois_N & 7505.941 & 8454.420 \\
\hline 13 & Illinois_S & 3752.971 & 4227.210 \\
\hline 14 & Adams & 20.294 & 42.551 \\
\hline 15 & Allen & 125.133 & 355.028 \\
\hline 16 & Bartholomew & .000 & 186.975 \\
\hline 17 & Benton & .000 & 2.573 \\
\hline 18 & Blackford & .000 & 2.443 \\
\hline 19 & Boone & .000 & 26.522 \\
\hline 20 & Brown & .000 & .690 \\
\hline 21 & Carroll & .000 & 2.837 \\
\hline 22 & Cass & .000 & 9.599 \\
\hline 23 & Clark & 205.902 & 154.142 \\
\hline 24 & Clay & .000 & 8.490 \\
\hline 25 & Clinton & .000 & 22.599 \\
\hline 26 & Crawford & .000 & .000 \\
\hline 27 & Daviess & 19.734 & 18.124 \\
\hline 28 & Dearborn & 22.852 & 13.723 \\
\hline 29 & Decatur & .000 & 20.712 \\
\hline 30 & DeKalb & .000 & 50.045 \\
\hline 31 & Delaware & .000 & 55.932 \\
\hline 32 & Dubois & .000 & 11.096 \\
\hline 33 & Elkhart & 20.216 & 273.985 \\
\hline 34 & Fayette & .000 & 28.788 \\
\hline 35 & Floyd & .000 & 38.239 \\
\hline 36 & Fountain & .000 & .683 \\
\hline 37 & Franklin & 123.318 & 35.983 \\
\hline 38 & Fulton & .000 & 16.808 \\
\hline 39 & Gibson & .000 & 8.514 \\
\hline 40 & Grant & .000 & 36.934 \\
\hline 41 & Greene & .000 & 1.017 \\
\hline 42 & Hamilton & 24.915 & 53.656 \\
\hline 43 & Hancock & .000 & 13.733 \\
\hline 44 & Harrison & 136.144 & 43.856 \\
\hline 45 & Hendricks & .000 & 19.001 \\
\hline 46 & Henry & .000 & 21.371 \\
\hline 47 & Howard & .000 & 15.872 \\
\hline 48 & Huntington & 20.120 & 31.965 \\
\hline 49 & Jackson & .000 & 44.736 \\
\hline 50 & Jasper & .000 & .472 \\
\hline 51 & Jay & 17.597 & 13.444 \\
\hline
\end{tabular}




\begin{tabular}{|c|c|c|c|}
\hline & $\begin{array}{l}\text { Locale } \\
\end{array}$ & P24 15 & A24 15 \\
\hline 52 & Jefferson & .000 & 40.948 \\
\hline 53 & Jennings & 122.308 & 90.963 \\
\hline 54 & Johnson & .000 & 48.251 \\
\hline 55 & Knox & 17.192 & 8.255 \\
\hline 56 & Kosciusko & 19.311 & 77.578 \\
\hline 57 & LaGrange & .000 & 29.481 \\
\hline 58 & Lake & 3981.198 & 1233.049 \\
\hline 59 & La Porte & 18.329 & 114.301 \\
\hline 60 & Lawrence & 107.000 & 46.753 \\
\hline 61 & Madison & .000 & 46.528 \\
\hline 62 & Marion & 225.965 & 923.603 \\
\hline 63 & Marshall & 120.404 & 56.204 \\
\hline 64 & Martin & .000 & .422 \\
\hline 65 & Miami & .000 & 13.357 \\
\hline 66 & \begin{tabular}{|l} 
Monroe \\
\end{tabular} & 124.026 & 141.146 \\
\hline 67 & Montgomery & .000 & 20.650 \\
\hline 68 & Morgan & .000 & 46.941 \\
\hline 69 & Newton & .000 & 4.413 \\
\hline 70 & Noble & .000 & 57.124 \\
\hline 71 & \begin{tabular}{|l|} 
Ohio \\
\end{tabular} & .000 & .000 \\
\hline 72 & Orange & .000 & .442 \\
\hline 73 & Owen & .000 & 7.104 \\
\hline 74 & Parke & .000 & 2.679 \\
\hline 75 & Perry & .000 & 13.278 \\
\hline 76 & Pike & .000 & .000 \\
\hline 77 & Porter & .000 & 57.500 \\
\hline 78 & Posey & 723.250 & 328.068 \\
\hline 79 & Pulaski & .000 & 22.372 \\
\hline 80 & Putnam & .000 & 8.206 \\
\hline 81 & Randolph & 17.588 & 28.024 \\
\hline 82 & Ripley & 19.491 & 9.555 \\
\hline 83 & Rush & .000 & 22.999 \\
\hline 84 & St. Joseph & 19.084 & 157.351 \\
\hline 85 & Scott & 19.110 & 39.128 \\
\hline 86 & Shelby & .000 & 30.876 \\
\hline 87 & Spencer & .000 & 8.369 \\
\hline 88 & Starke & .000 & 20.299 \\
\hline 89 & Steuben & 19.053 & 35.663 \\
\hline 90 & Sullivan & .000 & .708 \\
\hline 91 & Switzerland & .000 & .000 \\
\hline 92 & Tippecanoe & 21.586 & 286.692 \\
\hline 93 & \begin{tabular}{|l} 
Tipton \\
\end{tabular} & .000 & 8.380 \\
\hline 94 & Union & .000 & 3.455 \\
\hline 95 & Vanderburgh & .000 & 260.247 \\
\hline 96 & \begin{tabular}{|l|} 
Vermillion \\
\end{tabular} & .000 & 112.288 \\
\hline 97 & Vigo & .000 & 92.957 \\
\hline 98 & Wabash & 17.566 & 50.828 \\
\hline 99 & Warren & .000 & 8.183 \\
\hline 100 & Warrick & .000 & 10.155 \\
\hline 101 & Washington & .000 & 36.130 \\
\hline 102 & Wayne & .000 & 23.975 \\
\hline
\end{tabular}




\begin{tabular}{|c|c|c|c|}
\hline & Locale & P24 15 & A24 15 \\
\hline 103 & Wells & .000 & 15.828 \\
\hline 104 & White & .000 & 14.561 \\
\hline 105 & Whitley & .000 & 7.020 \\
\hline 106 & lowa & 327.548 & 2462.629 \\
\hline 107 & Kansas & 5321.452 & 3042.028 \\
\hline 108 & Kentucky_E & 1489.337 & 1959.952 \\
\hline 109 & Kentucky_W & 992.891 & 1306.635 \\
\hline 110 & Louisiana & 23521.060 & 8788.118 \\
\hline 111 & Maine & 345.028 & 552.080 \\
\hline 112 & Maryland & 2299.622 & 2359.476 \\
\hline 113 & Massachusetts & 2022.873 & 4198.418 \\
\hline 114 & Michigan_E & 1963.911 & 4409.696 \\
\hline 115 & Michigan_W & 1963.911 & 4409.696 \\
\hline 116 & Minnesota & 4024.575 & 4067.616 \\
\hline 117 & Mississippi & 3697.558 & 2752.239 \\
\hline 118 & Missouri & 2768.187 & 4305.720 \\
\hline 119 & Montana & 1628.056 & 574.320 \\
\hline 120 & Nebraska & 18.915 & 804.013 \\
\hline 121 & Nevada & 166.188 & 335.554 \\
\hline 122 & New Hampshire & 328.532 & 886.598 \\
\hline 123 & New Jersey & 7015.581 & 8748.678 \\
\hline 124 & New Mexico & 1576.201 & 595.270 \\
\hline 125 & New York & 2975.274 & 9264.558 \\
\hline 126 & North Carolina & 1553.079 & 9298.069 \\
\hline 127 & North Dakota & 313.234 & 342.806 \\
\hline 128 & Ohio_N & 3484.597 & 3843.090 \\
\hline 129 & Ohio_M & 3484.597 & 3843.090 \\
\hline 130 & Ohio_s & 3484.597 & 3843.090 \\
\hline 131 & Oklahoma & 4885.776 & 2891.678 \\
\hline 132 & Oregon & 1521.991 & 1578.593 \\
\hline 133 & Pennsylvania & 13543.038 & 10108.762 \\
\hline 134 & Rhode Island & 19.673 & 498.578 \\
\hline 135 & South Carolina & 785.277 & 6011.898 \\
\hline 136 & South Dakota & 19.108 & 375.584 \\
\hline 137 & Tennessee & 2777.809 & 6186.743 \\
\hline 138 & Texas & 50834.214 & 26375.600 \\
\hline 139 & Utah & 3455.353 & 1918.464 \\
\hline 140 & Vermont & 120.065 & 249.794 \\
\hline 141 & Virginia & 1454.741 & 3534.376 \\
\hline 142 & Washington & 4347.411 & 2538.042 \\
\hline 143 & West Virginia & 720.578 & 1328.470 \\
\hline 144 & Wisconsin & 829.719 & 5591.047 \\
\hline 145 & Wyoming & 1617.875 & 564.400 \\
\hline
\end{tabular}




\begin{tabular}{|c|c|c|c|}
\hline & Locale & P25 15 & A25 15 \\
\hline 1 & Alabama & 24077.003 & 24077.003 \\
\hline 2 & Arizona & 10664.592 & 10664.592 \\
\hline 3 & Arkansas & 15034.503 & 15034.503 \\
\hline 4 & California & 40294.621 & 40294.621 \\
\hline 5 & Colorado & 4300.786 & 4300.786 \\
\hline 6 & Connecticut & 1387.508 & 1387.508 \\
\hline 7 & Delaware & 1144.239 & 1144.239 \\
\hline 8 & DC & .000 & .000 \\
\hline 9 & \begin{tabular}{|l} 
Florida \\
\end{tabular} & 18470.797 & 18470.797 \\
\hline 10 & Georgia & 30596.853 & 30596.853 \\
\hline 11 & Idaho & 9755.519 & 9755.519 \\
\hline 12 & Illinois_N & 5524.997 & 5524.997 \\
\hline 13 & Illinois_S & 2762.499 & 2762.499 \\
\hline 14 & Adams & 560.035 & 560.035 \\
\hline 15 & Allen & 181.791 & 181.791 \\
\hline 16 & Bartholomew & 57.846 & 57.846 \\
\hline 17 & Benton & 146.945 & 146.945 \\
\hline 18 & Blackford & 62.202 & 62.202 \\
\hline 19 & Boone & .000 & .000 \\
\hline 20 & Brown & 24.016 & 24.016 \\
\hline 21 & Carroll & .000 & .000 \\
\hline 22 & Cass & 144.881 & 144.881 \\
\hline 23 & Clark & 651.475 & 651.475 \\
\hline 24 & Clay & 53.597 & 53.597 \\
\hline 25 & Clinton & 30.441 & 30.441 \\
\hline 26 & Crawford & 61.999 & 61.999 \\
\hline 27 & Daviess & 212.730 & 212.730 \\
\hline 28 & \begin{tabular}{|l|} 
Dearborn \\
\end{tabular} & 10.948 & 10.948 \\
\hline 29 & Decatur & 23.539 & 23.539 \\
\hline 30 & DeKalb & 666.504 & 666.504 \\
\hline 31 & Delaware & 55.129 & 55.129 \\
\hline 32 & Dubois & 1606.691 & 1606.691 \\
\hline 33 & Elkhart & 6179.356 & 6179.356 \\
\hline 34 & Fayette & .000 & .000 \\
\hline 35 & Floyd & 571.336 & 571.336 \\
\hline 36 & Fountain & 8.807 & 8.807 \\
\hline 37 & Franklin & 38.404 & 38.404 \\
\hline 38 & Fulton & 234.471 & 234.471 \\
\hline 39 & Gibson & .000 & .000 \\
\hline 40 & Grant & 47.166 & 47.166 \\
\hline 41 & Greene & 52.026 & 52.026 \\
\hline 42 & Hamilton & 150.405 & 150.405 \\
\hline 43 & Hancock & 32.318 & 32.318 \\
\hline 44 & Harrison & 184.812 & 184.812 \\
\hline 45 & Hendricks & 82.030 & 82.030 \\
\hline 46 & Henry & 48.967 & 48.967 \\
\hline 47 & Howard & 53.282 & 53.282 \\
\hline 48 & Huntington & 81.937 & 81.937 \\
\hline 49 & Jackson & 264.511 & 264.511 \\
\hline 50 & Jasper & 63.823 & 63.823 \\
\hline 51 & Jay & 50.585 & 50.585 \\
\hline
\end{tabular}




\begin{tabular}{|c|c|c|c|}
\hline & $\begin{array}{l}\text { Locale } \\
\end{array}$ & $\mathrm{P} 25 \quad 15$ & A25 15 \\
\hline 52 & Jefferson & 68.297 & 68.297 \\
\hline 53 & Jennings & 68.365 & 68.365 \\
\hline 54 & \begin{tabular}{|l} 
Johnson \\
\end{tabular} & 1207.281 & 1207.281 \\
\hline 55 & Knox & 62.600 & 62.600 \\
\hline 56 & Kosciusko & 288.663 & 288.663 \\
\hline 57 & LaGrange & 1851.599 & 1851.599 \\
\hline 58 & Lake & 209.384 & 209.384 \\
\hline 59 & La Porte & 183.533 & 183.533 \\
\hline 60 & Lawrence & 99.112 & 99.112 \\
\hline 61 & Madison & 160.463 & 160.463 \\
\hline 62 & Marion & 646.839 & 646.839 \\
\hline 63 & Marshall & 619.169 & 619.169 \\
\hline 64 & Martin & 8.264 & 8.264 \\
\hline 65 & Miami & 111.367 & 111.367 \\
\hline 66 & Monroe & 59.422 & 59.422 \\
\hline 67 & Montgomery & 38.447 & 38.447 \\
\hline 68 & Morgan & 193.259 & 193.259 \\
\hline 69 & Newton & .000 & .000 \\
\hline 70 & Noble & 56.408 & 56.408 \\
\hline 71 & Ohio & .000 & .000 \\
\hline 72 & Orange & 395.485 & 395.485 \\
\hline 73 & Owen & 116.723 & 116.723 \\
\hline 74 & Parke & 152.988 & 152.988 \\
\hline 75 & Perry & 49.878 & 49.878 \\
\hline 76 & Pike & 54.839 & 54.839 \\
\hline 77 & Porter & 99.025 & 99.025 \\
\hline 78 & Posey & 9.240 & 9.240 \\
\hline 79 & Pulaski & 9.127 & 9.127 \\
\hline 80 & Putnam & 55.093 & 55.093 \\
\hline 81 & Randolph & 317.677 & 317.677 \\
\hline 82 & Ripley & 113.927 & 113.927 \\
\hline 83 & Rush & 49.859 & 49.859 \\
\hline 84 & St. Joseph & 466.302 & 466.302 \\
\hline 85 & Scott & 54.935 & 54.935 \\
\hline 86 & Shelby & .000 & .000 \\
\hline 87 & Spencer & 130.116 & 130.116 \\
\hline 88 & Starke & 275.456 & 275.456 \\
\hline 89 & Steuben & 44.729 & 44.729 \\
\hline 90 & Sullivan & 81.201 & 81.201 \\
\hline 91 & Switzerland & .000 & .000 \\
\hline 92 & Tippecanoe & 52.744 & 52.744 \\
\hline 93 & Tipton & 51.773 & 51.773 \\
\hline 94 & Union & 8.986 & 8.986 \\
\hline 95 & Vanderburgh & 226.977 & 226.977 \\
\hline 96 & Vermillion & .000 & .000 \\
\hline 97 & Vigo & 105.016 & 105.016 \\
\hline 98 & Wabash & 147.278 & 147.278 \\
\hline 99 & Warren & 54.941 & 54.941 \\
\hline 100 & Warrick & 122.551 & 122.551 \\
\hline 101 & Washington & 213.197 & 213.197 \\
\hline 102 & Wayne & 48.898 & 48.898 \\
\hline
\end{tabular}




\begin{tabular}{|c|c|c|c|}
\hline & Locale & P25 15 & A25 15 \\
\hline 103 & Wells & 58.121 & 58.121 \\
\hline 104 & White & 23.026 & 23.026 \\
\hline 105 & Whitley & 169.193 & 169.193 \\
\hline 106 & lowa & 7725.481 & 7725.481 \\
\hline 107 & \begin{tabular}{|l} 
Kansas \\
\end{tabular} & 2825.575 & 2825.575 \\
\hline 108 & Kentucky_E & 6668.976 & 6668.976 \\
\hline 109 & Kentucky_W & 4445.984 & 4445.984 \\
\hline 110 & Louisiana & 8750.871 & 8750.871 \\
\hline 111 & \begin{tabular}{|l} 
Maine \\
\end{tabular} & 7562.492 & 7562.492 \\
\hline 112 & Maryland & 4033.036 & 4033.036 \\
\hline 113 & Massachusetts & 2656.461 & 2656.461 \\
\hline 114 & Michigan_E & 6371.764 & 6361.764 \\
\hline 115 & Michigan_W & 6371.764 & 6361.764 \\
\hline 116 & Minnesota & 14960.080 & 14960.080 \\
\hline 117 & Mississippi & 17171.338 & 17171.338 \\
\hline 118 & Missouri & 9654.422 & 9654.422 \\
\hline 119 & Montana & 5175.763 & 5175.763 \\
\hline 120 & Nebraska & 2296.413 & 2296.413 \\
\hline 121 & Nevada & 1368.173 & 1368.173 \\
\hline 122 & New Hampshire & 3805.247 & 3805.247 \\
\hline 123 & New Jersey & 2448.426 & 2448.426 \\
\hline 124 & New Mexico & 1497.690 & 1497.690 \\
\hline 125 & New York & 8966.396 & 8966.396 \\
\hline 126 & North Carolina & 37027.770 & 37027.770 \\
\hline 127 & North Dakota & 848.125 & 848.125 \\
\hline 128 & Ohio_N & 6215.889 & 6215.889 \\
\hline 129 & Ohio_M & 6215.889 & 6215.889 \\
\hline 130 & Ohio_s & 6215.889 & 6215.889 \\
\hline 131 & Oklahoma & 2881.851 & 2881.851 \\
\hline 132 & Oregon & 35935.128 & 35935.128 \\
\hline 133 & Pennsylvania & 22129.367 & 22129.367 \\
\hline 134 & Rhode Island & 480.703 & 480.703 \\
\hline 135 & South Carolina & 11239.817 & 11239.817 \\
\hline 136 & South Dakota & 1364.969 & 1364.969 \\
\hline 137 & Tennessee & 18833.352 & 18833.352 \\
\hline 138 & Texas & 32814.677 & 32814.677 \\
\hline 139 & Utah & 2397.863 & 2397.863 \\
\hline 140 & Vermont & 3189.717 & 3189.717 \\
\hline 141 & \begin{tabular}{|l} 
Virginia \\
\end{tabular} & 21481.849 & 21481.849 \\
\hline 142 & Washington & 21893.239 & 21893.239 \\
\hline 143 & West Virginia & 6818.164 & 6818.164 \\
\hline 144 & Wisconsin & 26149.138 & 26149.138 \\
\hline 145 & Wyoming & 972.630 & 972.630 \\
\hline
\end{tabular}




\begin{tabular}{|c|c|c|c|}
\hline & \begin{tabular}{|l} 
Locale \\
\end{tabular} & P26 15 & A26 15 \\
\hline 1 & Alabama & 20482.700 & 19213.120 \\
\hline 2 & Arizona & 9072.543 & 8169.550 \\
\hline 3 & Arkansas & 12790.098 & 11724.323 \\
\hline 4 & California & 34279.293 & 37623.927 \\
\hline 5 & Colorado & 3658.749 & 3472.910 \\
\hline 6 & Connecticut & 1180.376 & 1273.430 \\
\hline 7 & Delaware & 973.423 & 1350.979 \\
\hline 8 & DC & .000 & .000 \\
\hline 9 & Florida & 15713.410 & 14320.674 \\
\hline 10 & Georgia & 26029.243 & 23370.479 \\
\hline 11 & Idaho & 8299.179 & 7337.284 \\
\hline 12 & Illinois_N & 4700.206 & 6200.069 \\
\hline 13 & Illinois_s & 2350.103 & 3100.034 \\
\hline 14 & Adams & 476.431 & 420.478 \\
\hline 15 & Allen & 154.653 & 169.785 \\
\hline 16 & Bartholomew & 49.211 & 42.843 \\
\hline 17 & Benton & 125.009 & 108.832 \\
\hline 18 & Blackford & 52.916 & 46.068 \\
\hline 19 & Boone & .000 & .000 \\
\hline 20 & Brown & 20.430 & 17.787 \\
\hline 21 & Carroll & .000 & .000 \\
\hline 22 & Cass & 123.253 & 107.303 \\
\hline 23 & Clark & 554.220 & 540.330 \\
\hline 24 & Clay & 45.596 & 39.696 \\
\hline 25 & Clinton & 25.897 & 22.546 \\
\hline 26 & Crawford & 52.744 & 45.919 \\
\hline 27 & Daviess & 180.973 & 163.096 \\
\hline 28 & Dearborn & 9.314 & 14.527 \\
\hline 29 & Decatur & 20.025 & 17.434 \\
\hline 30 & DeKalb & 567.006 & 493.633 \\
\hline 31 & Delaware & 46.899 & 40.830 \\
\hline 32 & Dubois & 1366.838 & 1189.963 \\
\hline 33 & Elkhart & 5256.879 & 4582.292 \\
\hline 34 & Fayette & .000 & .000 \\
\hline 35 & Floyd & 486.045 & 423.148 \\
\hline 36 & Fountain & 7.493 & 6.523 \\
\hline 37 & Franklin & 32.671 & 63.078 \\
\hline 38 & Fulton & 199.468 & 173.656 \\
\hline 39 & Gibson & .000 & .000 \\
\hline 40 & Grant & 40.125 & 34.933 \\
\hline 41 & Greene & 44.259 & 38.532 \\
\hline 42 & Hamilton & 127.952 & 118.392 \\
\hline 43 & Hancock & 27.493 & 23.935 \\
\hline 44 & Harrison & 157.222 & 175.114 \\
\hline 45 & Hendricks & 69.784 & 60.754 \\
\hline 46 & Henry & 41.657 & 36.266 \\
\hline 47 & Howard & 45.328 & 39.462 \\
\hline 48 & Huntington & 69.705 & 66.336 \\
\hline 49 & Jackson & 225.024 & 195.905 \\
\hline 50 & Jasper & 54.295 & 47.269 \\
\hline 51 & Jay & 43.033 & 42.407 \\
\hline
\end{tabular}




\begin{tabular}{|c|c|c|c|}
\hline & Locale & P26 15 & A26 15 \\
\hline 52 & Jefferson & 58.101 & 50.583 \\
\hline 53 & Jennings & 58.159 & 84.984 \\
\hline 54 & Johnson & 1027.054 & 894.148 \\
\hline 55 & Knox & 53.254 & 51.192 \\
\hline 56 & Kosciusko & 245.570 & 219.216 \\
\hline 57 & LaGrange & 1575.186 & 1371.349 \\
\hline 58 & Lake & 178.126 & 1273.221 \\
\hline 59 & La Porte & 156.134 & 141.077 \\
\hline 60 & Lawrence & 84.316 & 103.457 \\
\hline 61 & Madison & 136.508 & 118.844 \\
\hline 62 & Marion & 550.277 & 542.532 \\
\hline 63 & Marshall & 526.737 & 492.391 \\
\hline 64 & Martin & 7.030 & 6.120 \\
\hline 65 & Miami & 94.742 & 82.482 \\
\hline 66 & Monroe & 50.551 & 78.843 \\
\hline 67 & Montgomery & 32.707 & 28.475 \\
\hline 68 & Morgan & 164.408 & 143.133 \\
\hline 69 & Newton & .000 & .000 \\
\hline 70 & Noble & 47.987 & 41.778 \\
\hline 71 & Ohio & .000 & .000 \\
\hline 72 & Orange & 336.445 & 292.908 \\
\hline 73 & Owen & 99.298 & 86.448 \\
\hline 74 & Parke & 130.149 & 113.307 \\
\hline 75 & Perry & 42.432 & 36.941 \\
\hline 76 & Pike & 46.652 & 40.615 \\
\hline 77 & Porter & 84.242 & 73.341 \\
\hline 78 & Posey & 7.861 & 209.973 \\
\hline 79 & Pulaski & 7.764 & 6.760 \\
\hline 80 & Putnam & 46.868 & 40.803 \\
\hline 81 & Randolph & 270.253 & 240.221 \\
\hline 82 & Ripley & 96.919 & 89.852 \\
\hline 83 & Rush & 42.416 & 36.927 \\
\hline 84 & St. Joseph & 396.690 & 350.717 \\
\hline 85 & Scott & 46.734 & 46.054 \\
\hline 86 & Shelby & .000 & .000 \\
\hline 87 & Spencer & 110.692 & 96.368 \\
\hline 88 & Starke & 234.335 & 204.011 \\
\hline 89 & Steuben & 38.052 & 38.479 \\
\hline 90 & Sullivan & 69.079 & 60.140 \\
\hline 91 & Switzerland & .000 & .000 \\
\hline 92 & Tippecanoe & 44.870 & 45.126 \\
\hline 93 & Tipton & 44.044 & 38.345 \\
\hline 94 & Union & 7.644 & 6.655 \\
\hline 95 & Vanderburgh & 193.093 & 168.106 \\
\hline 96 & Vermillion & .000 & .000 \\
\hline 97 & Vigo & 89.339 & 77.778 \\
\hline 98 & Wabash & 125.291 & 114.012 \\
\hline 99 & Warren & 46.739 & 40.691 \\
\hline 100 & Warrick & 104.256 & 90.765 \\
\hline 101 & Washington & 181.371 & 157.900 \\
\hline 102 & Wayne & 41.598 & 36.215 \\
\hline
\end{tabular}




\begin{tabular}{|c|c|c|c|}
\hline & Locale & P26 15 & A26 15 \\
\hline 103 & Wells & 49.444 & 43.046 \\
\hline 104 & White & 19.589 & 17.054 \\
\hline 105 & Whitley & 143.935 & 125.309 \\
\hline 106 & lowa. & 6572.193 & 5813.714 \\
\hline 107 & Kansas & 2403.763 & 3587.268 \\
\hline 108 & Kentucky_E & 5673.407 & 5357.531 \\
\hline 109 & Kentucky_W & 3782.271 & 3571.688 \\
\hline 110 & Louisiana & 7444.509 & 13087.194 \\
\hline 111 & Maine & 6433.535 & 5697.909 \\
\hline 112 & Maryland & 3430.970 & 3632.850 \\
\hline 113 & Massachusetts & 2259.895 & 2535.591 \\
\hline 114 & Michigan_E & 5412.057 & 5263.288 \\
\hline 115 & Michigan_W & 5412.057 & 5263.288 \\
\hline 116 & Minnesota & 12726.785 & 12210.207 \\
\hline 117 & Mississippi & 14607.938 & 13756.085 \\
\hline 118 & Missouri & 8213.175 & 7927.815 \\
\hline 119 & Montana & 4403.106 & 4290.574 \\
\hline 120 & Nebraska & 1953.596 & 1706.104 \\
\hline 121 & Nevada & 1163.927 & 1059.984 \\
\hline 122 & New Hampshire & 3237.186 & 2910.549 \\
\hline 123 & New Jersey & 2082.916 & 3783.748 \\
\hline 124 & New Mexico & 1274.109 & 1551.919 \\
\hline 125 & New York & 7627.860 & 7476.404 \\
\hline 126 & North Carolina & 31500.129 & 27860.058 \\
\hline 127 & North Dakota & 721.514 & 716.120 \\
\hline 128 & Ohio_N & 5287.958 & 5582.343 \\
\hline 129 & Ohio_M & 5287.958 & 5582.343 \\
\hline 130 & Ohio_s & 5287.958 & 5582.343 \\
\hline 131 & Oklahoma & 2451.638 & 3506.586 \\
\hline 132 & Oregon & 30570.602 & 27042.084 \\
\hline 133 & Pennsylvania & 18825.815 & 20193.315 \\
\hline 134 & Rhode Island & 408.942 & 361.548 \\
\hline 135 & South Carolina & 9561.896 & 8545.092 \\
\hline 136 & South Dakota & 1161.202 & 1016.303 \\
\hline 137 & Tennessee & 16021.841 & 14728.705 \\
\hline 138 & Texas & 27915.982 & 38580.636 \\
\hline 139 & Utah & 2039.901 & 2746.386 \\
\hline 140 & Vermont & 2713.545 & 2396.120 \\
\hline 141 & \begin{tabular}{|l} 
Virginia \\
\end{tabular} & 18274.960 & 16318.668 \\
\hline 142 & Washington & 18624.936 & 17435.781 \\
\hline 143 & West Virginia & 5800.323 & 5252.113 \\
\hline 144 & Wisconsin & 22245.500 & 19599.860 \\
\hline 145 & Wyoming & 827.432 & 1174.749 \\
\hline
\end{tabular}




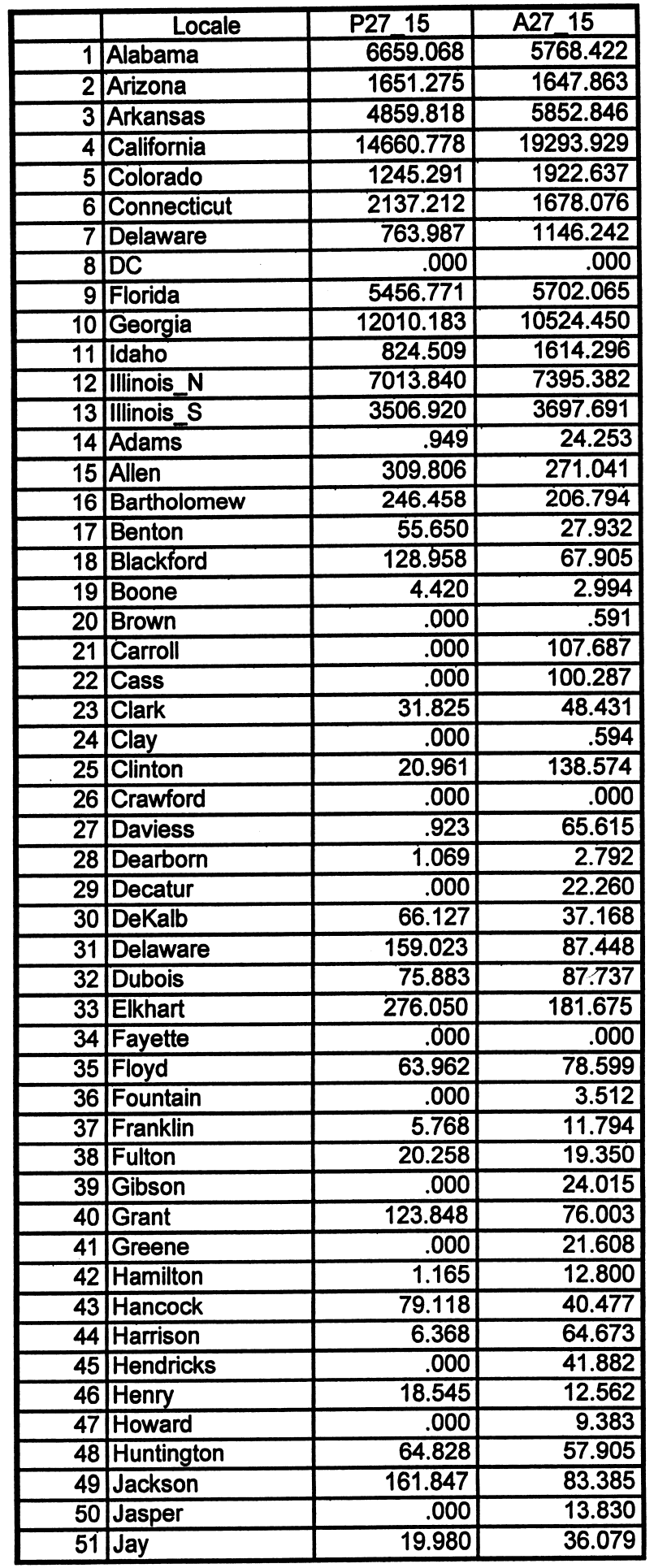




\begin{tabular}{|c|c|c|c|}
\hline & Locale & P27 15 & A27 15 \\
\hline 52 & Jefferson & .000 & 1.243 \\
\hline 53 & Jennings & 27.913 & 22.187 \\
\hline 54 & Johnson & 78.367 & 39.883 \\
\hline 55 & Knox & 19.521 & 18.172 \\
\hline 56 & Kosciusko & 4.407 & 59.819 \\
\hline 57 & LaGrange & .000 & 11.650 \\
\hline 58 & Lake & 323.392 & 494.753 \\
\hline 59 & La Porte & 162.818 & 115.682 \\
\hline 60 & Lawrence & 5.005 & 13.072 \\
\hline 61 & Madison & 59.412 & 74.497 \\
\hline 62 & Marion & 977.349 & 738.944 \\
\hline 63 & Marshall & 174.216 & 122.771 \\
\hline 64 & Martin & .000 & 3.295 \\
\hline 65 & Miami & 117.157 & 62.092 \\
\hline 66 & Monroe & 71.438 & 50.531 \\
\hline 67 & Montgomery & 60.668 & 53.261 \\
\hline 68 & Morgan & .000 & .702 \\
\hline 69 & Newton & 19.998 & 10.622 \\
\hline 70 & Noble & 62.308 & 78.128 \\
\hline 71 & Ohio & .000 & .000 \\
\hline 72 & Orange & .000 & 3.450 \\
\hline 73 & Owen & .000 & .626 \\
\hline 74 & Parke & .000 & 10.166 \\
\hline 75 & Perry & .000 & .552 \\
\hline 76 & Pike & .000 & .000 \\
\hline 77 & Porter & .000 & 19.619 \\
\hline 78 & Posey & 33.828 & 69.015 \\
\hline 79 & Pulaski & 3.456 & 5.920 \\
\hline 80 & Putnam & .000 & .610 \\
\hline 81 & Randolph & .823 & 5.900 \\
\hline 82 & Ripley & 62.801 & 33.444 \\
\hline 83 & Rush & 18.882 & 10.913 \\
\hline 84 & St. Joseph & 195.841 & 141.736 \\
\hline 85 & Scott & .894 & 24.542 \\
\hline 86 & Shelby & 3.345 & 11.950 \\
\hline 87 & Spencer & .000 & 3.436 \\
\hline 88 & Starke & 19.378 & 9.726 \\
\hline 89 & Steuben & 21.633 & 27.418 \\
\hline 90 & Sullivan & .000 & .000 \\
\hline 91 & Switzerland & 4.068 & 2.042 \\
\hline 92 & Tippecanoe & 24.510 & 58.003 \\
\hline 93 & Tipton & 19.607 & 19.876 \\
\hline 94 & Union & .000 & .000 \\
\hline 95 & Vanderburgh & 222.359 & 329.933 \\
\hline 96 & Vermillion & 54.839 & 27.964 \\
\hline 97 & Vigo & 177.780 & 104.022 \\
\hline 98 & Wabash & 120.342 & 64.931 \\
\hline 99 & Warren & .000 & .608 \\
\hline 100 & Warrick & 3.684 & 1.849 \\
\hline 101 & Washington & .000 & 3.761 \\
\hline 102 & Wayne & 73.456 & 75.048 \\
\hline
\end{tabular}




\begin{tabular}{|c|c|c|c|}
\hline & $\begin{array}{l}\text { Locale } \\
\end{array}$ & P27 15 & A27 15 \\
\hline 103 & Wells & 22.011 & 44.005 \\
\hline 104 & White & 20.124 & 12.572 \\
\hline 105 & Whitley & 64.076 & 32.803 \\
\hline 106 & lowa & 1918.849 & 3744.013 \\
\hline 107 & Kansas & 1435.023 & 2955.236 \\
\hline 108 & Kentucky_E & 2291.348 & 2007.356 \\
\hline 109 & Kentucky_W & 1527.565 & 1338.238 \\
\hline 110 & Louisiana & 4960.634 & 5115.729 \\
\hline 111 & Maine & 4227.766 & 2542.617 \\
\hline 112 & Maryland & 2749.287 & 2684.865 \\
\hline 113 & Massachusetts & 6816.724 & 4880.815 \\
\hline 114 & Michigan_E & 3424.325 & 2849.924 \\
\hline 115 & Michigan_W & 3424.325 & 2849.924 \\
\hline 116 & Minnesota & 6070.663 & 6161.983 \\
\hline 117 & Mississippi & 3212.554 & 3490.422 \\
\hline 118 & Missouri & 4777.487 & 4978.000 \\
\hline 119 & Montana & 348.997 & 424.888 \\
\hline 120 & Nebraska & 575.414 & 2355.770 \\
\hline 121 & Nevada & 310.320 & 435.734 \\
\hline 122 & New Hampshire & 1432.862 & 891.235 \\
\hline 123 & New Jersey & 6604.377 & 5673.687 \\
\hline 124 & New Mexico & 278.637 & 508.553 \\
\hline 125 & New York & 8856.606 & 7541.264 \\
\hline 126 & North Carolina & 8703.881 & 8240.692 \\
\hline 127 & North Dakota & 34.137 & 346.482 \\
\hline 128 & Ohio_N & 3532.861 & 2983.622 \\
\hline 129 & Ohio_M & 3532.861 & 2983.622 \\
\hline 130 & Ohio_s & 3532.861 & 2983.622 \\
\hline 131 & Oklahoma & 1569.079 & 2004.549 \\
\hline 132 & Oregon & 3132.431 & 3007.818 \\
\hline 133 & Pennsylvania & 11632.619 & 11310.523 \\
\hline 134 & Rhode Island & 706.274 & 532.305 \\
\hline 135 & South Carolina & 5264.987 & 3745.571 \\
\hline 136 & South Dakota & 229.025 & 606.006 \\
\hline 137 & Tennessee & 6558.362 & 5866.769 \\
\hline 138 & Texas & 11214.861 & 15369.382 \\
\hline 139 & Utah & 1277.620 & 1735.815 \\
\hline 140 & Vermont & 641.021 & 570.266 \\
\hline 141 & Virginia & 6025.526 & 5517.586 \\
\hline 142 & Washington & 5839.315 & 5505.669 \\
\hline 143 & West Virginia & 239.918 & 474.088 \\
\hline 144 & Wisconsin & 15079.657 & 11264.674 \\
\hline 145 & Wyoming & 79.184 & 194.114 \\
\hline
\end{tabular}




\begin{tabular}{|c|c|c|c|}
\hline & Locale & P28 15 & A28 15 \\
\hline 1 & Alabama & 2429.998 & 1925.943 \\
\hline 2 & Arizona & 1990.509 & 2604.237 \\
\hline 3 & Arkansas & 2460.968 & 1990.141 \\
\hline 4 & California & 17713.600 & 19183.501 \\
\hline 5 & Colorado & 1915.371 & 2088.001 \\
\hline 6 & Connecticut & 971.959 & 1167.106 \\
\hline 7 & Delaware & 510.325 & 454.794 \\
\hline 8 & DC & .000 & .000 \\
\hline 9 & Florida & 4101.730 & 5167.174 \\
\hline 10 & Georgia & 7596.144 & 3569.152 \\
\hline 11 & Idaho & 832.794 & 809.180 \\
\hline 12 & Illinois_N & 416.397 & 404.590 \\
\hline 13 & Illinois_S & 5342.365 & 5540.358 \\
\hline 14 & Adams & 31.706 & 33.618 \\
\hline 15 & Allen & 179.883 & 183.130 \\
\hline 16 & Bartholomew & 71.600 & 65.736 \\
\hline 17 & Benton & .000 & .814 \\
\hline 18 & Blackford & 2.123 & 2.794 \\
\hline 19 & Boone & 11.232 & 18.445 \\
\hline 20 & Brown & .599 & 2.031 \\
\hline 21 & Carroll & 71.906 & 54.544 \\
\hline 22 & Cass & 75.086 & 61.360 \\
\hline 23 & Clark & 19.251 & 21.778 \\
\hline 24 & Clay & .601 & 3.086 \\
\hline 25 & Clinton & 101.383 & 84.070 \\
\hline 26 & Crawford & .000 & 1.142 \\
\hline 27 & Daviess & 42.623 & 34.114 \\
\hline 28 & Dearborn & .737 & 6.223 \\
\hline 29 & Decatur & 14.863 & 13.271 \\
\hline 30 & DeKalb & 9.274 & 13.899 \\
\hline 31 & Delaware & 6.052 & 15.729 \\
\hline 32 & Dubois & 50.029 & 46.758 \\
\hline 33 & Elkhart & 122.245 & 96.408 \\
\hline 34 & Fayette & .000 & 2.349 \\
\hline 35 & Floyd & 44.513 & 45.889 \\
\hline 36 & Fountain & 3.558 & 4.780 \\
\hline 37 & Franklin & .437 & 2.579 \\
\hline 38 & Fulton & 6.336 & 6.717 \\
\hline 39 & Gibson & 20.945 & 20.501 \\
\hline 40 & Grant & .000 & .000 \\
\hline 41 & Greene & 16.325 & 15.139 \\
\hline 42 & Hamilton & 29.573 & 39.602 \\
\hline 43 & Hancock & .777 & 7.648 \\
\hline 44 & Harrison & 34.972 & 29.570 \\
\hline 45 & Hendricks & 34.566 & 42.241 \\
\hline 46 & Henry & 7.230 & 7.220 \\
\hline 47 & Howard & 7.631 & 14.391 \\
\hline 48 & Huntington & 50.604 & 54.381 \\
\hline 49 & Jackson & 2.167 & 5.084 \\
\hline 50 & Jasper & 12.950 & 14.045 \\
\hline 51 & Jay & 16.609 & 14.133 \\
\hline
\end{tabular}




\begin{tabular}{|c|c|c|c|}
\hline & Locale & P28 15 & A28 15 \\
\hline 52 & Jefferson & 1.589 & 3.849 \\
\hline 53 & Jennings & 29.699 & 2.928 \\
\hline 54 & Johnson & 16.931 & 32.901 \\
\hline 55 & Knox & 6.356 & 8.924 \\
\hline 56 & Kosciusko & 45.104 & 37.882 \\
\hline 57 & LaGrange & 9.934 & 11.192 \\
\hline 58 & Lake & 52.044 & 82.122 \\
\hline 59 & La Porte & 24.022 & 28.042 \\
\hline 60 & Lawrence & 4.144 & 7.357 \\
\hline 61 & Madison & 31.492 & 35.205 \\
\hline 62 & Marion & 224.756 & 243.822 \\
\hline 63 & Marshall & 23.115 & 18.274 \\
\hline 64 & Martin & 2.200 & 2.553 \\
\hline 65 & Miami & 3.055 & 5.106 \\
\hline 66 & Monroe & 32.129 & 42.646 \\
\hline 67 & Montgomery & 15.232 & 14.874 \\
\hline 68 & Morgan & 43.740 & 56.584 \\
\hline 69 & Newton & 3.925 & 5.727 \\
\hline 70 & Noble & 45.923 & 29.074 \\
\hline 71 & Ohio & .000 & .540 \\
\hline 72 & Orange & 2.304 & 3.599 \\
\hline 73 & Owen & 4.998 & 2.550 \\
\hline 74 & Parke & 6.788 & 6.578 \\
\hline 75 & Perry & 4.604 & 2.202 \\
\hline 76 & Pike & .000 & 1.265 \\
\hline 77 & Porter & 16.180 & 26.818 \\
\hline 78 & Posey & 6.956 & 4.477 \\
\hline 79 & Pulaski & 2.794 & 3.360 \\
\hline 80 & Putnam & 4.095 & 8.203 \\
\hline 81 & Randolph & 4.039 & 5.944 \\
\hline 82 & Ripley & 1.700 & 4.466 \\
\hline 83 & Rush & 1.823 & 2.551 \\
\hline 84 & St. Joseph & 60.394 & 69.802 \\
\hline 85 & Scott & 28.228 & 13.487 \\
\hline 86 & Shelby & 6.858 & 9.239 \\
\hline 87 & Spencer & 2.294 & 3.608 \\
\hline 88 & Starke & .000 & 2.227 \\
\hline 89 & Steuben & 15.905 & 12.224 \\
\hline 90 & Sullivan & .000 & 2.129 \\
\hline 91 & Switzerland & .000 & .963 \\
\hline 92 & Tippecanoe & 62.877 & 70.601 \\
\hline 93 & Tipton & 6.700 & 6.439 \\
\hline 94 & Union & .000 & .694 \\
\hline 95 & Vanderburgh & 174.521 & 123.701 \\
\hline 96 & Vermillion & .294 & 1.738 \\
\hline 97 & Vigo & 46.655 & 58.389 \\
\hline 98 & Wabash & 13.121 & 16.588 \\
\hline 99 & Warren & .406 & 1.080 \\
\hline 100 & Warrick & .789 & 5.818 \\
\hline 101 & Washington & 2.511 & 4.634 \\
\hline 102 & Wayne & 42.869 & 44.885 \\
\hline
\end{tabular}




\begin{tabular}{|c|c|c|c|}
\hline & Locale & P28 15 & A28 15 \\
\hline 103 & Wells & 23.015 & 19.161 \\
\hline 104 & White & 5.926 & 7.699 \\
\hline 105 & Whitley & 4.268 & 7.959 \\
\hline 106 & lowa & 2257.808 & 2009.850 \\
\hline 107 & Kansas & 1477.729 & 1336.169 \\
\hline 108 & Kentucky_E & 853.905 & 779.583 \\
\hline 109 & Kentucky_W & 569.270 & 519.722 \\
\hline 110 & Louisiana & 838.202 & 1001.448 \\
\hline 111 & Maine & 537.844 & 481.264 \\
\hline 112 & Maryland & 1391.609 & 1777.842 \\
\hline 113 & Massachusetts & 3345.001 & 3674.221 \\
\hline 114 & Michigan_E & 1043.892 & 1294.242 \\
\hline 115 & Michigan_W & 1043.892 & 1294.242 \\
\hline 116 & Minnesota & 3414.856 & 3487.725 \\
\hline 117 & Mississippi & 1511.866 & 1189.738 \\
\hline 118 & Missouri & 2108.608 & 2085.314 \\
\hline 119 & Montana & 114.390 & 173.783 \\
\hline 120 & Nebraska & 1537.927 & 1322.261 \\
\hline 121 & Nevada & 320.784 & 507.416 \\
\hline 122 & New Hampshire & 785.344 & 874.635 \\
\hline 123 & New Jersey & 2417.339 & 2655.315 \\
\hline 124 & New Mexico & 430.536 & 602.383 \\
\hline 125 & New York & 4320.381 & 5201.894 \\
\hline 126 & North Carolina & 5753.768 & 4200.392 \\
\hline 127 & North Dakota & 250.519 & 244.515 \\
\hline 128 & Ohio_N & 1003.673 & 1125.310 \\
\hline 129 & Ohio_M & 1003.673 & 1125.310 \\
\hline 130 & Ohio_s & 1003.673 & 1125.310 \\
\hline 131 & Oklahoma & 971.916 & 1082.195 \\
\hline 132 & Oregon & 1914.058 & 2086.878 \\
\hline 133 & Pennsylvania & 4812.754 & 4798.701 \\
\hline 134 & Rhode Island & 352.841 & 357.374 \\
\hline 135 & South Carolina & 2145.644 & 1384.748 \\
\hline 136 & South Dakota & 659.009 & 629.480 \\
\hline 137 & Tennessee & 2355.642 & 2111.982 \\
\hline 138 & Texas & 8562.389 & 9515.941 \\
\hline 139 & Utah & 1072.339 & 1126.694 \\
\hline 140 & Vermont & 385.791 & 426.332 \\
\hline 141 & \begin{tabular}{|l} 
Virginia \\
\end{tabular} & 2666.540 & 2786.014 \\
\hline 142 & Washington & 2791.212 & 3027.848 \\
\hline 143 & West Virginia & 231.002 & 342.706 \\
\hline 144 & Wisconsin & 3107.278 & 2898.012 \\
\hline 145 & Wyoming & 44.901 & 82.783 \\
\hline
\end{tabular}




\begin{tabular}{|c|c|c|c|}
\hline & $\begin{array}{l}\text { Locale } \\
\end{array}$ & P29 15 & A29 15 \\
\hline 1 & Alabama & 1728.765 & 1486.243 \\
\hline 2 & Arizona & 1436.809 & 2245.597 \\
\hline 3 & Arkansas & 1225.045 & 937.354 \\
\hline 4 & California & 11236.806 & 12487.361 \\
\hline 5 & Colorado & 1214.712 & 1530.764 \\
\hline 6 & Connecticut & 1159.125 & 1160.218 \\
\hline 7 & Delaware & 343.046 & 288.608 \\
\hline 8 & DC & .000 & .000 \\
\hline 9 & Florida & 5061.143 & 6538.015 \\
\hline 10 & Georgia & 3939.716 & 3116.510 \\
\hline 11 & Idaho & 507.137 & 502.202 \\
\hline 12 & Illinois_N & 3271.056 & 2780.076 \\
\hline 13 & Illinois_S & 1635.528 & 1390.038 \\
\hline 14 & Adams & 5.724 & 12.095 \\
\hline 15 & Allen & 156.591 & 122.363 \\
\hline 16 & Bartholomew & 39.345 & 26.189 \\
\hline 17 & Benton & 10.927 & 2.806 \\
\hline 18 & Blackford & 18.672 & 4.277 \\
\hline 19 & Boone & 9.600 & 19.985 \\
\hline 20 & Brown & .271 & 5.198 \\
\hline 21 & Carroll & 4.858 & 6.728 \\
\hline 22 & Cass & 4.814 & 13.389 \\
\hline 23 & Clark & 14.955 & 35.619 \\
\hline 24 & Clay & 1.880 & 8.825 \\
\hline 25 & Clinton & 6.921 & 11.562 \\
\hline 26 & Crawford & .000 & 3.934 \\
\hline 27 & Daviess & 4.527 & 10.126 \\
\hline 28 & Dearborn & 3.106 & 19.222 \\
\hline 29 & Decatur & 8.533 & 8.269 \\
\hline 30 & DeKalb & 12.772 & 15.090 \\
\hline 31 & Delaware & 34.629 & 40.510 \\
\hline 32 & Dubois & 21.723 & 13.990 \\
\hline 33 & Elkhart & 65.494 & 67.240 \\
\hline 34 & Fayette & 3.978 & 8.092 \\
\hline 35 & Floyd & 21.449 & 26.753 \\
\hline 36 & Fountain & 2.767 & 5.776 \\
\hline 37 & Franklin & 1.802 & 7.784 \\
\hline 38 & Fulton & 5.982 & 6.884 \\
\hline 39 & Gibson & 2.675 & 10.859 \\
\hline 40 & Grant & 22.690 & 22.301 \\
\hline 41 & Greene & 2.116 & 11.092 \\
\hline 42 & Hamilton & 56.142 & 96.634 \\
\hline 43 & Hancock & 24.279 & 24.009 \\
\hline 44 & Harrison & 2.818 & 13.750 \\
\hline 45 & Hendricks & 11.676 & 53.399 \\
\hline 46 & Henry & 10.654 & 15.113 \\
\hline 47 & Howard & 5.526 & 28.405 \\
\hline 48 & Huntington & 15.691 & 13.704 \\
\hline 49 & Jackson & 29.738 & 13.894 \\
\hline 50 & Jasper & 3.583 & 10.465 \\
\hline 51 & Jay & 7.380 & 6.837 \\
\hline
\end{tabular}




\begin{tabular}{|c|c|c|c|}
\hline & Locale & P29 15 & A29 15 \\
\hline 52 & Jefferson & 1.512 & 11.168 \\
\hline 53 & Jennings & 4.826 & 10.086 \\
\hline 54 & Johnson & 18.201 & 50.880 \\
\hline 55 & Knox & 12.788 & 12.548 \\
\hline 56 & Kosciusko & 6.961 & 25.703 \\
\hline 57 & LaGrange & 7.961 & 13.606 \\
\hline 58 & Lake & 70.448 & 157.629 \\
\hline 59 & La Porte & 42.096 & 35.802 \\
\hline 60 & Lawrence & 1.876 & 14.971 \\
\hline 61 & Madison & 19.486 & 45.300 \\
\hline 62 & Marion & 441.275 & 294.124 \\
\hline 63 & Marshall & 28.787 & 16.184 \\
\hline 64 & Martin & 1.538 & 3.253 \\
\hline 65 & Miami & 26.571 & 11.309 \\
\hline 66 & Monroe & 39.531 & 45.284 \\
\hline 67 & Montgomery & 11.243 & 12.863 \\
\hline 68 & Morgan & 2.223 & 26.487 \\
\hline 69 & Newton & 5.342 & 4.807 \\
\hline 70 & Noble & 15.188 & 16.223 \\
\hline 71 & Ohio & .000 & 1.861 \\
\hline 72 & Orange & 1.505 & 6.593 \\
\hline 73 & Owen & .287 & 7.731 \\
\hline 74 & Parke & 1.600 & 5.558 \\
\hline 75 & Perry & 1.116 & 5.904 \\
\hline 76 & Pike & 1.171 & 4.357 \\
\hline 77 & Porter & 22.708 & 50.166 \\
\hline 78 & Posey & 6.031 & 9.225 \\
\hline 79 & Pulaski & 7.590 & 4.536 \\
\hline 80 & Putnam & 1.484 & 12.687 \\
\hline 81 & Randolph & 1.516 & 8.646 \\
\hline 82 & Ripley & 13.292 & 9.270 \\
\hline 83 & Rush & 4.588 & 5.621 \\
\hline 84 & St. Joseph & 101.771 & 93.554 \\
\hline 85 & Scott & .279 & 8.076 \\
\hline 86 & Shelby & 4.247 & 14.546 \\
\hline 87 & Spencer & 6.176 & $6: 649$ \\
\hline 88 & Starke & 3.772 & 7.670 \\
\hline 89 & Steuben & 3.174 & 11.435 \\
\hline 90 & Sullivan & 4.035 & 7.334 \\
\hline 91 & Switzerland & .874 & 3.318 \\
\hline 92 & Tippecanoe & 22.806 & 57.183 \\
\hline 93 & Tipton & 6.422 & 5.300 \\
\hline 94 & Union & 1.644 & 2.389 \\
\hline 95 & Vanderburgh & 75.362 & 57.970 \\
\hline 96 & Vermillion & 9.304 & 5.248 \\
\hline 97 & Vigo & 43.739 & 34.101 \\
\hline 98 & Wabash & 18.199 & 11.283 \\
\hline 99 & Warren & 1.676 & 2.697 \\
\hline 100 & Warrick & 6.012 & 20.043 \\
\hline 101 & Washington & .288 & 9.636 \\
\hline 102 & Wayne & 20.548 & 21.871 \\
\hline
\end{tabular}




\begin{tabular}{|c|c|c|c|}
\hline & $\begin{array}{r}\text { Locale } \\
\end{array}$ & P29 15 & A29 15 \\
\hline 103 & Wells & 47.746 & 9.682 \\
\hline 104 & White & 11.183 & 8.338 \\
\hline 105 & Whitley & 11.343 & 11.201 \\
\hline 106 & lowa & 1113.065 & 970.246 \\
\hline 107 & Kansas & 909.515 & 907.405 \\
\hline 108 & Kentucky_E & 849.901 & 828.517 \\
\hline 109 & Kentucky_W & 566.601 & 552.344 \\
\hline 110 & Louisiana & 1436.830 & 1500.365 \\
\hline 111 & Maine & 874.296 & 443.568 \\
\hline 112 & Maryland & 1493.537 & 1961.692 \\
\hline 113 & Massachusetts & 2592.585 & 2150.658 \\
\hline 114 & Michigan_E & 1423.112 & 1697.318 \\
\hline 115 & Michigan_W & 1423.112 & 1697.318 \\
\hline 116 & Minnesota & 2286.924 & 1780.975 \\
\hline 117 & Mississippi & 953.102 & 954.912 \\
\hline 118 & Missouri & 2217.542 & 1929.435 \\
\hline 119 & Montana & 244.680 & 319.902 \\
\hline 120 & Nebraska & 611.920 & 571.945 \\
\hline 121 & Nevada & 500.901 & 876.097 \\
\hline 122 & New Hampshire & 459.713 & 454.959 \\
\hline 123 & New Jersey & 4696.158 & 2933.698 \\
\hline 124 & New Mexico & 300.018 & 646.380 \\
\hline 125 & New York & 6792.710 & 6235.973 \\
\hline 126 & North Carolina & 3292.929 & 3090.097 \\
\hline 127 & North Dakota & 230.855 & 208.513 \\
\hline 128 & Ohio_N & 1330.772 & 1250.166 \\
\hline 129 & Ohio_M & 1330.772 & 1250.166 \\
\hline 130 & Ohio_s & 1330.772 & 1250.166 \\
\hline 131 & Oklahoma & 883.135 & 1161.451 \\
\hline 132 & Oregon & 1428.886 & 1257.629 \\
\hline 133 & Pennsylvania & 4044.529 & 4106.818 \\
\hline 134 & Rhode Island & 288.446 & 361.681 \\
\hline 135 & South Carolina & 1452.983 & 1449.184 \\
\hline 136 & South Dakota & 251.184 & 254.973 \\
\hline 137 & Tennessee & 2479.462 & 2039.423 \\
\hline 138 & Texas & 6519.875 & 8167.227 \\
\hline 139 & Utah & 714.000 & 857.243 \\
\hline 140 & Vermont & 260.247 & 214.318 \\
\hline 141 & \begin{tabular}{|l} 
Virginia \\
\end{tabular} & 2140.565 & 2650.720 \\
\hline 142 & Washington & 2358.385 & 2171.526 \\
\hline 143 & West Virginia & 245.504 & 595.007 \\
\hline 144 & Wisconsin & 3397.040 & 1874.268 \\
\hline 145 & Wyoming & 67.100 & 169.835 \\
\hline
\end{tabular}




\begin{tabular}{|c|c|c|c|}
\hline & Locale & P30 15 & $\mathrm{A} 30 \quad 15$ \\
\hline 1 & Alabama & 3381.493 & 2775.960 \\
\hline 2 & Arizona & 335.320 & 709.379 \\
\hline 3 & Arkansas & 621.146 & 681.868 \\
\hline 4 & California & 6198.400 & 7538.027 \\
\hline 5 & Colorado & 480.758 & 691.881 \\
\hline 6 & Connecticut & 540.569 & 631.566 \\
\hline 7 & Delaware & 222.511 & 243.948 \\
\hline 8 & DC & .000 & .000 \\
\hline 9 & Florida & 1625.569 & 2566.670 \\
\hline 10 & Georgia & 14230.549 & 11289.996 \\
\hline 11 & Idaho & 95.111 & 178.515 \\
\hline 12 & Illinois_N & 911.917 & 1349.521 \\
\hline 13 & Illinois_S & 455.959 & 674.760 \\
\hline 14 & Adams & .981 & 3.377 \\
\hline 15 & Allen & 61.259 & 74.430 \\
\hline 16 & Bartholomew & 1.694 & 6.490 \\
\hline 17 & Benton & .000 & .549 \\
\hline 18 & Blackford & .000 & .838 \\
\hline 19 & Boone & .000 & 3.914 \\
\hline 20 & Brown & .000 & 1.018 \\
\hline 21 & Carroll & .000 & 1.318 \\
\hline 22 & Cass & 1.515 & 3.840 \\
\hline 23 & Clark & 20.245 & 25.479 \\
\hline 24 & Clay & .000 & 1.728 \\
\hline 25 & Clinton & .000 & 2.264 \\
\hline 26 & Crawford & .000 & .770 \\
\hline 27 & Daviess & .954 & 2.963 \\
\hline 28 & Dearborn & 1.104 & 4.900 \\
\hline 29 & Decatur & .000 & 1.619 \\
\hline 30 & DeKalb & .000 & 2.955 \\
\hline 31 & Delaware & 1.614 & 9.231 \\
\hline 32 & Dubois & 1.645 & 4.062 \\
\hline 33 & Elkhart & 99.322 & 93.100 \\
\hline 34 & Fayette & .000 & 1.585 \\
\hline 35 & \begin{tabular}{|l} 
Floyd \\
\end{tabular} & .000 & 5.239 \\
\hline 36 & Fountain & .000 & 1.131 \\
\hline 37 & Franklin & 5.960 & 7.650 \\
\hline 38 & Fulton & .000 & 1.348 \\
\hline 39 & Gibson & .000 & 2.127 \\
\hline 40 & Grant & 8.285 & 11.028 \\
\hline 41 & Greene & 1.523 & 3.397 \\
\hline 42 & Hamilton & 38.957 & 50.345 \\
\hline 43 & Hancock & .000 & 4.702 \\
\hline 44 & Harrison & 6.580 & 9.456 \\
\hline 45 & Hendricks & 2.402 & 12.388 \\
\hline 46 & Henry & 8.601 & 9.875 \\
\hline 47 & Howard & .000 & 5.563 \\
\hline 48 & Huntington & 2.666 & 5.045 \\
\hline 49 & Jackson & 1.580 & 3.991 \\
\hline 50 & Jasper & .000 & 2.049 \\
\hline 51 & Jay & .850 & 2.213 \\
\hline
\end{tabular}




\begin{tabular}{|c|c|c|c|}
\hline & Locale & P30 15 & A30 15 \\
\hline 52 & Jefferson & 1.643 & 3.508 \\
\hline 53 & Jennings & 70.244 & 59.771 \\
\hline 54 & Johnson & 2.077 & 11.634 \\
\hline 55 & Knox & .831 & 3.311 \\
\hline 56 & Kosciusko & 12.480 & 15.145 \\
\hline 57 & LaGrange & 1.730 & 4.055 \\
\hline 58 & Lake & 201.718 & 236.115 \\
\hline 59 & La Porte & 7.102 & 12.176 \\
\hline 60 & Lawrence & 6.672 & 9.454 \\
\hline 61 & Madison & 3.944 & 11.916 \\
\hline 62 & Marion & 80.963 & 124.365 \\
\hline 63 & Marshall & 15.952 & 17.297 \\
\hline 64 & Martin & .000 & .637 \\
\hline 65 & Miami & 1.449 & 3.380 \\
\hline 66 & Monroe & 16.432 & 23.421 \\
\hline 67 & Montgomery & .000 & 2.519 \\
\hline 68 & Morgan & 1.855 & 6.679 \\
\hline 69 & Newton & .000 & .941 \\
\hline 70 & Noble & 28.900 & 26.411 \\
\hline 71 & Ohio & .000 & .364 \\
\hline 72 & Orange & .000 & 1.291 \\
\hline 73 & Owen & 9.921 & 9.490 \\
\hline 74 & Parke & .000 & 1.088 \\
\hline 75 & Perry & 8.761 & 8.200 \\
\hline 76 & Pike & .000 & .853 \\
\hline 77 & Porter & 6.490 & 14.266 \\
\hline 78 & Posey & 44.693 & 45.562 \\
\hline 79 & Pulaski & .000 & .888 \\
\hline 80 & Putnam & .000 & 2.485 \\
\hline 81 & Randolph & 5.334 & 5.459 \\
\hline 82 & Ripley & .942 & 2.784 \\
\hline 83 & Rush & 1.460 & 2.274 \\
\hline 84 & St. Joseph & 29.839 & 42.388 \\
\hline 85 & Scott & 29.069 & 25.158 \\
\hline 86 & Shelby & .000 & 2.849 \\
\hline 87 & Spencer & .000 & 1.302 \\
\hline 88 & Starke & .000 & 1.502 \\
\hline 89 & Steuben & 10.542 & 10.920 \\
\hline 90 & Sullivan & .000 & 1.436 \\
\hline 91 & Switzerland & .000 & .650 \\
\hline 92 & Tippecanoe & 11.943 & 21.034 \\
\hline 93 & Tipton & .000 & 1.038 \\
\hline 94 & Union & .000 & .468 \\
\hline 95 & Vanderburgh & 62.614 & 61.559 \\
\hline 96 & Vermillion & .000 & 1.028 \\
\hline 97 & Vigo & 1.577 & 7.946 \\
\hline 98 & Wabash & 2.327 & 4.271 \\
\hline 99 & Warren & .000 & .528 \\
\hline 100 & Warrick & 1.708 & 5.299 \\
\hline 101 & Washington & .000 & 1.887 \\
\hline 102 & Wayne & .000 & 4.283 \\
\hline
\end{tabular}




\begin{tabular}{|c|c|c|c|}
\hline & Locale & P30 15 & A30 15 \\
\hline 103 & Wells & 2.561 & 3.818 \\
\hline 104 & White & 15.302 & 11.749 \\
\hline 105 & Whitley & .000 & 2.194 \\
\hline 106 & lowa & 205.345 & 357.061 \\
\hline 107 & Kansas & 456.960 & 601.030 \\
\hline 108 & Kentucky_E & 586.031 & 636.754 \\
\hline 109 & Kentucky_W & 390.688 & 424.502 \\
\hline 110 & Louisiana & 1341.130 & 1624.968 \\
\hline 111 & Maine & 686.757 & 582.327 \\
\hline 112 & Maryland & 388.635 & 697.498 \\
\hline 113 & Massachusetts & 1899.187 & 1807.909 \\
\hline 114 & Michigan_E & 343.744 & 625.782 \\
\hline 115 & Michigan W & 343.744 & 625.782 \\
\hline 116 & Minnesota & 550.179 & 824.072 \\
\hline 117 & Mississippi & 1094.135 & 1071.810 \\
\hline 118 & Missouri & 619.006 & 896.765 \\
\hline 119 & Montana & 104.886 & 163.774 \\
\hline 120 & Nebraska & 95.688 & 184.347 \\
\hline 121 & Nevada & 84.734 & 241.307 \\
\hline 122 & New Hampshire & 472.451 & 434.471 \\
\hline 123 & New Jersey & 1955.708 & 2098.599 \\
\hline 124 & New Mexico & 91.784 & 216.579 \\
\hline 125 & New York & 2579.340 & 3166.772 \\
\hline 126 & North Carolina & 14884.869 & 10908.289 \\
\hline 127 & North Dakota & 53.143 & 86.222 \\
\hline 128 & Ohio_N & 485.687 & 657.676 \\
\hline 129 & Ohio_M & 485.687 & 657.676 \\
\hline 130 & Ohio_s & 485.687 & 657.676 \\
\hline 131 & Oklahoma & 500.230 & 677.129 \\
\hline 132 & Oregon & 287.888 & 490.548 \\
\hline 133 & Pennsylvania & 2963.608 & 3159.475 \\
\hline 134 & Rhode Island & 691.591 & 548.146 \\
\hline 135 & South Carolina & 7708.853 & 5631.613 \\
\hline 136 & South Dakota & 182.431 & 187.119 \\
\hline 137 & Tennessee & 2144.697 & 1989.571 \\
\hline 138 & Texas & 4755.063 & 5879.442 \\
\hline 139 & Utah & 389.670 & 516.635 \\
\hline 140 & Vermont & 37.484 & 71.715 \\
\hline 141 & Virginia & 2667.574 & 2379.113 \\
\hline 142 & Washington & 594.606 & 939.520 \\
\hline 143 & West Virginia & 83.087 & 186.496 \\
\hline 144 & Wisconsin & 620.953 & 842.538 \\
\hline 145 & Wyoming & 88.784 & 122.012 \\
\hline
\end{tabular}




\begin{tabular}{|c|c|c|c|}
\hline & Locale & P31 15 & A31 15 \\
\hline 1 & Alabama & 21913.396 & 21978.569 \\
\hline 2 & Arizona & 20588.407 & 20461.518 \\
\hline 3 & Arkansas & 23935.479 & 24158.640 \\
\hline 4 & California & 145019.685 & 144863.598 \\
\hline 5 & Colorado & 17741.280 & 17721.499 \\
\hline 6 & Connecticut & 11451.575 & 11400.672 \\
\hline 7 & Delaware & 5477.244 & 5511.893 \\
\hline 8 & DC & .000 & .000 \\
\hline 9 & Florida & 63126.220 & 62814.082 \\
\hline 10 & Georgia & 45584.077 & 45714.149 \\
\hline 11 & Idaho & 9816.254 & 9881.684 \\
\hline 12 & Illinois_N & 38138.462 & 38209.052 \\
\hline 13 & IIllinois_s & 19069.231 & 19104.526 \\
\hline 14 & Adams & 206.076 & 207.106 \\
\hline 15 & Allen & 1464.461 & 1463.713 \\
\hline 16 & Bartholomew & 625.380 & 630.834 \\
\hline 17 & Benton & 20.834 & 20.588 \\
\hline 18 & Blackford & 48.248 & 48.169 \\
\hline 19 & Boone & 152.425 & 150.742 \\
\hline 20 & Brown & 41.664 & 41.262 \\
\hline 21 & Carroll & 608.396 & 617.856 \\
\hline 22 & Cass & 619.484 & 627.668 \\
\hline 23 & Clark & 361.437 & 360.052 \\
\hline 24 & Clay & 68.612 & 67.892 \\
\hline 25 & Clinton & 749.904 & 760.840 \\
\hline 26 & Crawford & 29.213 & 28.867 \\
\hline 27 & Daviess & 406.216 & 411.284 \\
\hline 28 & Dearborn & 146.513 & 144.892 \\
\hline 29 & Decatur & 176.834 & 178.185 \\
\hline 30 & DeKalb & 132.688 & 131.733 \\
\hline 31 & Delaware & 340.399 & 337.552 \\
\hline 32 & Dubois & 361.360 & 364.765 \\
\hline 33 & Elkhart & 715.929 & 713.919 \\
\hline 34 & Fayette & 60.091 & 59.379 \\
\hline 35 & Floyd & 439.781 & 441.770 \\
\hline 36 & Fountain & 61.103 & 60.923 \\
\hline 37 & Franklin & 61.197 & 60.574 \\
\hline 38 & Fulton & 98.738 & 98.990 \\
\hline 39 & Gibson & 205.172 & 206.459 \\
\hline 40 & Grant & 237.390 & 236.722 \\
\hline 41 & Greene & 194.416 & 195.459 \\
\hline 42 & Hamilton & 772.301 & 764.795 \\
\hline 43 & Hancock & 182.266 & 180.228 \\
\hline 44 & Harrison & 373.710 & 377.391 \\
\hline 45 & Hendricks & 613.721 & 612.938 \\
\hline 46 & Henry & 129.098 & 128.074 \\
\hline 47 & Howard & 259.589 & 257.969 \\
\hline 48 & Huntington & 226.332 & 227.370 \\
\hline 49 & Jackson & 114.335 & 113.315 \\
\hline 50 & Jasper & 149.427 & 149.799 \\
\hline 51 & Jay & 179.760 & 181.481 \\
\hline
\end{tabular}




\begin{tabular}{|c|c|c|c|}
\hline & Locale & P31_15 & A31 15 \\
\hline 52 & Jefferson & 89.382 & 88.517 \\
\hline 53 & Jennings & 74.900 & 74.014 \\
\hline 54 & Johnson & 380.678 & 376.258 \\
\hline 55 & Knox & 130.645 & 130.217 \\
\hline 56 & Kosciusko & 482.912 & 485.910 \\
\hline 57 & LaGrange & 161.446 & 161.337 \\
\hline 58 & Lake & 1514.251 & 1506.587 \\
\hline 59 & La Porte & 435.625 & 435.535 \\
\hline 60 & Lawrence & 128.839 & 127.842 \\
\hline 61 & Madison & 568.073 & 568.263 \\
\hline 62 & Marion & 3393.928 & 3389.858 \\
\hline 63 & Marshall & 261.654 & 262.778 \\
\hline 64 & Martin & 41.241 & 41.262 \\
\hline 65 & Miami & 101.032 & 100.345 \\
\hline 66 & Monroe & 369.374 & 365.991 \\
\hline 67 & Montgomery & 213.807 & 214.806 \\
\hline 68 & Morgan & 200.328 & 198.066 \\
\hline 69 & Newton & 38.727 & 38.360 \\
\hline 70 & \begin{tabular}{|l|} 
Noble \\
\end{tabular} & 363.450 & 366.398 \\
\hline 71 & Ohio & 13.820 & 13.656 \\
\hline 72 & Orange & 66.848 & 66.591 \\
\hline 73 & Owen & 60.655 & 60.034 \\
\hline 74 & Parke & 93.990 & 94.451 \\
\hline 75 & Perry & 46.704 & 46.237 \\
\hline 76 & Pike & 32.357 & 31.974 \\
\hline 77 & Porter & 474.264 & 471.687 \\
\hline 78 & Posey & 87.608 & 87.141 \\
\hline 79 & Pulaski & 55.381 & 55.374 \\
\hline 80 & Putnam & 97.376 & 96.318 \\
\hline 81 & Randolph & 86.563 & 86.205 \\
\hline 82 & Ripley & 72.053 & 71.297 \\
\hline 83 & Rush & 49.186 & 48.826 \\
\hline 84 & St. Joseph & 913.382 & 909.098 \\
\hline 85 & Scott & 178.286 & 179.706 \\
\hline 86 & Shelby & 161.282 & 160.963 \\
\hline 87 & Spencer & 67.189 & 66.925 \\
\hline 88 & Starke & 56.958 & 56.284 \\
\hline 89 & Steuben & 164.182 & 164.604 \\
\hline 90 & Sullivan & 54.464 & 53.819 \\
\hline 91 & Switzerland & 24.637 & 24.345 \\
\hline 92 & Tippecanoe & 654.146 & 653.252 \\
\hline 93 & Tipton & 91.391 & 91.862 \\
\hline 94 & Union & 17.743 & 17.533 \\
\hline 95 & Vanderburgh & 1562.672 & 1577.956 \\
\hline 96 & Vermillion & 41.251 & 40.831 \\
\hline 97 & Vigo & 329.937 & 328.321 \\
\hline 98 & Wabash & 101.183 & 100.505 \\
\hline 99 & Warren & 23.180 & 23.000 \\
\hline 100 & Warrick & 148.835 & 147.074 \\
\hline 101 & Washington & 91.058 & 90.563 \\
\hline 102 & Wayne & 360.399 & 362.041 \\
\hline
\end{tabular}




\begin{tabular}{|c|c|c|c|}
\hline & Locale & P31 15 & A31 15 \\
\hline 103 & Wells & 242.807 & 245.033 \\
\hline 104 & White & 74.732 & 74.230 \\
\hline 105 & Whitley & 86.507 & 85.583 \\
\hline 106 & lowa & 21512.470 & 21684.734 \\
\hline 107 & Kansas & 16483.419 & 16579.087 \\
\hline 108 & Kentucky_E & 10081.865 & 10079.789 \\
\hline 109 & Kentucky_W & 6721.244 & 6719.860 \\
\hline 110 & Louisiana & 16604.485 & 16570.977 \\
\hline 111 & Maine & 5355.654 & 5353.788 \\
\hline 112 & Maryland & 20537.223 & 20472.304 \\
\hline 113 & Massachusetts & 22837.377 & 22771.997 \\
\hline 114 & Michigan_E & 17789.377 & 17733.566 \\
\hline 115 & Michigan_W & 17789.377 & 17733.566 \\
\hline 116 & Minnesota & 27983.823 & 28092.966 \\
\hline 117 & Mississippi & 15547.986 & 15616.293 \\
\hline 118 & Missouri & 26747.874 & 26801.884 \\
\hline 119 & Montana & 3106.119 & 3091.159 \\
\hline 120 & Nebraska & 14959.343 & 15101.892 \\
\hline 121 & Nevada & 7900.068 & 7848.183 \\
\hline 122 & New Hampshire & 4156.853 & 4130.887 \\
\hline 123 & New Jersey & 31585.548 & 31504.161 \\
\hline 124 & New Mexico & 6165.470 & 6133.252 \\
\hline 125 & New York & 61331.207 & 61053.670 \\
\hline 126 & North Carolina & 42487.966 & 42568.163 \\
\hline 127 & North Dakota & 3147.709 & 3158.173 \\
\hline 128 & Ohio_N & 1435.521 & 14333.906 \\
\hline 129 & Ohio_M & 1435.521 & 14333.906 \\
\hline 130 & Ohio_s & 1435.521 & 14333.906 \\
\hline 131 & Oklahoma & 13242.049 & 13223.099 \\
\hline 132 & Oregon & 16256.051 & 16270.043 \\
\hline 133 & Pennsylvania & 54177.734 & 54243.103 \\
\hline 134 & Rhode Island & 3601.089 & 3585.782 \\
\hline 135 & South Carolina & 16209.139 & 16179.853 \\
\hline 136 & South Dakota & 4433.251 & 4456.561 \\
\hline 137 & Tennessee & 27534.992 & 27578.804 \\
\hline 138 & Texas & 93536.984 & 93411.289 \\
\hline 139 & Utah & 10843.964 & 10849.241 \\
\hline 140 & Vermont & 2838.680 & 2842.296 \\
\hline 141 & Virginia & 32109.168 & 32099.892 \\
\hline 142 & Washington & 27970.766 & 27993.158 \\
\hline 143 & West Virginia & 6002.670 & 5978.902 \\
\hline 144 & Wisconsin & 32796.995 & 32972.102 \\
\hline 145 & Wyoming & 1500.804 & 1490.194 \\
\hline
\end{tabular}




\begin{tabular}{|c|c|c|c|}
\hline & Locale & P31 15 & A31 15 \\
\hline 1 & Alabama & 21913.396 & 21978.569 \\
\hline 2 & Arizona & 20588.407 & 20461.518 \\
\hline 3 & Arkansas & 23935.479 & 24158.640 \\
\hline 4 & California & 145019.685 & 144863.598 \\
\hline 5 & Colorado & 17741.280 & 17721.499 \\
\hline 6 & Connecticut & 11451.575 & 11400.672 \\
\hline 7 & Delaware & 5477.244 & 5511.893 \\
\hline 8 & DC & .000 & .000 \\
\hline 9 & Florida & 63126.220 & 62814.082 \\
\hline 10 & Georgia & 45584.077 & 45714.149 \\
\hline 11 & Idaho & 9816.254 & 9881.684 \\
\hline 12 & Illinois_N & 38138.462 & 38209.052 \\
\hline 13 & Illinois_S & 19069.231 & 19104.526 \\
\hline 14 & Adams & 206.076 & 207.106 \\
\hline 15 & Allen & 1464.461 & 1463.713 \\
\hline 16 & Bartholomew & 625.380 & 630.834 \\
\hline 17 & Benton & 20.834 & 20.588 \\
\hline 18 & Blackford & 48.248 & 48.169 \\
\hline 19 & Boone & 152.425 & 150.742 \\
\hline 20 & Brown & 41.664 & 41.262 \\
\hline 21 & Carroll & 608.396 & 617.856 \\
\hline 22 & Cass & 619.484 & 627.668 \\
\hline 23 & Clark & 361.437 & 360.052 \\
\hline 24 & Clay & 68.612 & 67.892 \\
\hline 25 & Clinton & 749.904 & 760.840 \\
\hline 26 & Crawford & 29.213 & 28.867 \\
\hline 27 & Daviess & 406.216 & 411.284 \\
\hline 28 & Dearborn & 146.513 & 144.892 \\
\hline 29 & Decatur & 176.834 & 178.185 \\
\hline 30 & DeKalb & 132.688 & 131.733 \\
\hline 31 & Delaware & 340.399 & 337.552 \\
\hline 32 & Dubois & 361.360 & 364.765 \\
\hline 33 & Elkhart & 715.929 & 713.919 \\
\hline 34 & Fayette & 60.091 & 59.379 \\
\hline 35 & Floyd & 439.781 & 441.770 \\
\hline 36 & Fountain & 61.103 & 60.923 \\
\hline 37 & Franklin & 61.197 & 60.574 \\
\hline 38 & Fulton & 98.738 & 98.990 \\
\hline 39 & Gibson & 205.172 & 206.459 \\
\hline 40 & Grant & 237.390 & 236.722 \\
\hline 41 & Greene & 194.416 & 195.459 \\
\hline 42 & Hamilton & 772.301 & 764.795 \\
\hline 43 & Hancock & 182.266 & 180.228 \\
\hline 44 & Harrison & 373.710 & 377.391 \\
\hline 45 & Hendricks & 613.721 & 612.938 \\
\hline 46 & Henry & 129.098 & 128.074 \\
\hline 47 & Howard & 259.589 & 257.969 \\
\hline 48 & Huntington & 226.332 & 227.370 \\
\hline 49 & Jackson & 114.335 & 113.315 \\
\hline 50 & Jasper & 149.427 & 149.799 \\
\hline 51 & Jay & 179.760 & 181.481 \\
\hline
\end{tabular}




\begin{tabular}{|c|c|c|c|}
\hline & Locale & P31_15 & A31 15 \\
\hline 52 & Jefferson & 89.382 & 88.517 \\
\hline 53 & Jennings & 74.900 & 74.014 \\
\hline 54 & Johnson & 380.678 & 376.258 \\
\hline 55 & Knox & 130.645 & 130.217 \\
\hline 56 & Kosciusko & 482.912 & 485.910 \\
\hline 57 & LaGrange & 161.446 & 161.337 \\
\hline 58 & Lake & 1514.251 & 1506.587 \\
\hline 59 & La Porte & 435.625 & 435.535 \\
\hline 60 & Lawrence & 128.839 & 127.842 \\
\hline 61 & Madison & 568.073 & 568.263 \\
\hline 62 & Marion & 3393.928 & 3389.858 \\
\hline 63 & Marshall & 261.654 & 262.778 \\
\hline 64 & Martin & 41.241 & 41.262 \\
\hline 65 & Miami & 101.032 & 100.345 \\
\hline 66 & Monroe & 369.374 & 365.991 \\
\hline 67 & Montgomery & 213.807 & 214.806 \\
\hline 68 & Morgan & 200.328 & 198.066 \\
\hline 69 & Newton & 38.727 & 38.360 \\
\hline 70 & Noble & 363.450 & 366.398 \\
\hline 71 & Ohio & 13.820 & 13.656 \\
\hline 72 & Orange & 66.848 & 66.591 \\
\hline 73 & Owen & 60.655 & 60.034 \\
\hline 74 & Parke & 93.990 & 94.451 \\
\hline 75 & Perry & 46.704 & 46.237 \\
\hline 76 & Pike & 32.357 & 31.974 \\
\hline 77 & Porter & 474.264 & 471.687 \\
\hline 78 & Posey & 87.608 & 87.141 \\
\hline 79 & Pulaski & 55.381 & 55.374 \\
\hline 80 & Putnam & 97.376 & 96.318 \\
\hline 81 & Randolph & 86.563 & 86.205 \\
\hline 82 & Ripley & 72.053 & 71.297 \\
\hline 83 & Rush & 49.186 & 48.826 \\
\hline 84 & St. Joseph & 913.382 & 909.098 \\
\hline 85 & Scott & 178.286 & 179.706 \\
\hline 86 & Shelby & 161.282 & 160.963 \\
\hline 87 & Spencer & 67.189 & 66.925 \\
\hline 88 & Starke & 56.958 & 56.284 \\
\hline 89 & Steuben & 164.182 & 164.604 \\
\hline 90 & Sullivan & 54.464 & 53.819 \\
\hline 91 & Switzerland & 24.637 & 24.345 \\
\hline 92 & Tippecanoe & 654.146 & 653.252 \\
\hline 93 & Tipton & 91.391 & 91.862 \\
\hline 94 & Union & 17.743 & 17.533 \\
\hline 95 & Vanderburgh & 1562.672 & 1577.956 \\
\hline 96 & Vermillion & 41.251 & 40.831 \\
\hline 97 & Vigo & 329.937 & 328.321 \\
\hline 98 & Wabash & 101.183 & 100.505 \\
\hline 99 & Warren & 23.180 & 23.000 \\
\hline 100 & Warrick & 148.835 & 147.074 \\
\hline 101 & Washington & 91.058 & 90.563 \\
\hline 102 & Wayne & 360.399 & 362.041 \\
\hline
\end{tabular}




\begin{tabular}{|c|c|c|c|}
\hline & $\begin{array}{l}\text { Locale } \\
\end{array}$ & P31 15 & A31 15 \\
\hline 103 & Wells & 242.807 & 245.033 \\
\hline 104 & White & 74.732 & 74.230 \\
\hline 105 & Whitley & 86.507 & 85.583 \\
\hline 106 & lowa & 21512.470 & 21684.734 \\
\hline 107 & Kansas & 16483.419 & 16579.087 \\
\hline 108 & Kentucky_E & 10081.865 & 10079.789 \\
\hline 109 & Kentucky_W & 6721.244 & 6719.860 \\
\hline 110 & Louisiana & 16604.485 & 16570.977 \\
\hline 111 & Maine & 5355.654 & 5353.788 \\
\hline 112 & Maryland & 20537.223 & 20472.304 \\
\hline 113 & Massachusetts & 22837.377 & 22771.997 \\
\hline 114 & Michigan_E & 17789.377 & 17733.566 \\
\hline 115 & Michigan_W & 17789.377 & 17733.566 \\
\hline 116 & Minnesota & 27983.823 & 28092.966 \\
\hline 117 & Mississippi & 15547.986 & 15616.293 \\
\hline 118 & Missouri & 26747.874 & 26801.884 \\
\hline 119 & Montana & 3106.119 & 3091.159 \\
\hline 120 & Nebraska & 14959.343 & 15101.892 \\
\hline 121 & Nevada & 7900.068 & 7848.183 \\
\hline 122 & New Hampshire & 4156.853 & 4130.887 \\
\hline 123 & New Jersey & 31585.548 & 31504.161 \\
\hline 124 & New Mexico & 6165.470 & 6133.252 \\
\hline 125 & New York & 61331.207 & 61053.670 \\
\hline 126 & North Carolina & 42487.966 & 42568.163 \\
\hline 127 & North Dakota & 3147.709 & 3158.173 \\
\hline 128 & Ohio N & 14352.522 & 14333.906 \\
\hline 129 & Ohio_M & 14352.522 & 14333.906 \\
\hline 130 & Ohio_s & 14352.522 & 14333.906 \\
\hline 131 & Oklahoma & 13242.049 & 13223.099 \\
\hline 132 & Oregon & 16256.051 & 16270.043 \\
\hline 133 & Pennsylvania & 54177.734 & 54243.103 \\
\hline 134 & Rhode Island & 3601.089 & 3585.782 \\
\hline 135 & South Carolina & 16209.139 & 16179.853 \\
\hline 136 & South Dakota & 4433.251 & 4456.561 \\
\hline 137 & Tennessee & 27534.992 & 27578.804 \\
\hline 138 & Texas & 93536.984 & 93411.289 \\
\hline 139 & Utah & 10843.964 & 10849.241 \\
\hline 140 & Vermont & 2838.680 & 2842.296 \\
\hline 141 & Virginia & 32109.168 & 32099.892 \\
\hline 142 & Washington & 27970.766 & 27993.158 \\
\hline 143 & West Virginia & 6002.670 & 5978.902 \\
\hline 144 & Wisconsin & 32796.995 & 32972.102 \\
\hline 145 & Wyoming & 1500.804 & 1490.194 \\
\hline
\end{tabular}




\begin{tabular}{|c|c|c|c|}
\hline & Locale & P33 15 & A33 15 \\
\hline 1 & Alabama & 8370.787 & 3147.839 \\
\hline 2 & Arizona & 2389.071 & 1293.600 \\
\hline 3 & Arkansas & 2054.446 & 2093.997 \\
\hline 4 & California & 20864.397 & 18976.473 \\
\hline 5 & Colorado & 1758.689 & 1371.326 \\
\hline 6 & Connecticut & 2632.052 & 1949.947 \\
\hline 7 & Delaware & 644.274 & 830.396 \\
\hline 8 & DC & .000 & .000 \\
\hline 9 & Florida & 4708.647 & 3186.187 \\
\hline 10 & Georgia & 3884.452 & 3145.191 \\
\hline 11 & Idaho & 430.388 & 386.716 \\
\hline 12 & Illinois_N & 7069.772 & 7930.173 \\
\hline 13 & Illinois_S & 3534.886 & 3965.087 \\
\hline 14 & Adams & 38.792 & 41.019 \\
\hline 15 & Allen & 224.703 & 402.772 \\
\hline 16 & Bartholomew & 62.102 & 236.187 \\
\hline 17 & Benton & 3.978 & 3.517 \\
\hline 18 & Blackford & 44.190 & 3.340 \\
\hline 19 & Boone & 24.425 & 36.259 \\
\hline 20 & Brown & 3.561 & .000 \\
\hline 21 & Carroll & 24.514 & 3.879 \\
\hline 22 & Cass & 43.246 & 11.740 \\
\hline 23 & Clark & 126.223 & 140.000 \\
\hline 24 & Clay & 8.866 & 5.925 \\
\hline 25 & Clinton & 13.734 & 13.782 \\
\hline 26 & Crawford & .000 & .000 \\
\hline 27 & Daviess & 18.089 & 19.623 \\
\hline 28 & Dearborn & 39.822 & 18.596 \\
\hline 29 & Decatur & 155.390 & 28.316 \\
\hline 30 & DeKalb & 227.775 & 44.512 \\
\hline 31 & Delaware & 98.405 & 66.713 \\
\hline 32 & Dubois & 12.963 & 7.713 \\
\hline 33 & Elkhart & 453.066 & 106.790 \\
\hline 34 & Fayette & 17.967 & 39.357 \\
\hline 35 & Floyd & 44.548 & 41.842 \\
\hline 36 & Fountain & 3.526 & .000 \\
\hline 37 & Franklin & 33.764 & 54.566 \\
\hline 38 & Fulton & 44.953 & 22.034 \\
\hline 39 & Gibson & 10.283 & 11.640 \\
\hline 40 & Grant & 44.244 & 35.015 \\
\hline 41 & Greene & 13.800 & .000 \\
\hline 42 & Hamilton & 106.508 & 33.359 \\
\hline 43 & Hancock & 36.321 & 18.775 \\
\hline 44 & Harrison & 48.955 & 60.241 \\
\hline 45 & Hendricks & 16.345 & 22.335 \\
\hline 46 & Henry & 16.615 & 21.366 \\
\hline 47 & Howard & 25.065 & 21.699 \\
\hline 48 & Huntington & 67.011 & 36.899 \\
\hline 49 & Jackson & 36.009 & 43.026 \\
\hline 50 & Jasper & 38.653 & .646 \\
\hline 51 & Jay & 140.766 & 19.146 \\
\hline
\end{tabular}




\begin{tabular}{|c|c|c|c|}
\hline & $\begin{array}{l}\text { Locale } \\
\end{array}$ & P33 15 & A33 15 \\
\hline 52 & Jefferson & 7.914 & 48.531 \\
\hline 53 & Jennings & 65.047 & 69.938 \\
\hline 54 & Johnson & 74.746 & 56.549 \\
\hline 55 & Knox & 47.876 & 12.035 \\
\hline 56 & Kosciusko & 122.598 & 64.547 \\
\hline 57 & LaGrange & 12.442 & 37.681 \\
\hline 58 & Lake & 1139.246 & 1710.965 \\
\hline 59 & La Porte & 120.839 & 124.840 \\
\hline 60 & Lawrence & 84.453 & 67.209 \\
\hline 61 & Madison & 72.591 & 25.097 \\
\hline 62 & Marion & 639.282 & 629.127 \\
\hline 63 & Marshall & 89.014 & 59.586 \\
\hline 64 & Martin & 19.690 & .577 \\
\hline 65 & Miami & 13.357 & 11.691 \\
\hline 66 & Monroe & 74.366 & 171.729 \\
\hline 67 & Montgomery & 36.493 & 11.248 \\
\hline 68 & Morgan & 30.237 & 20.497 \\
\hline 69 & Newton & 2.978 & 5.100 \\
\hline 70 & Noble & 96.044 & 30.192 \\
\hline 71 & Ohio & .539 & .000 \\
\hline 72 & Orange & 3.465 & .604 \\
\hline 73 & Owen & 1.274 & .657 \\
\hline 74 & Parke & .493 & 3.662 \\
\hline 75 & Perry & 7.506 & 10.156 \\
\hline 76 & Pike & 3.660 & .000 \\
\hline 77 & Porter & 94.316 & 32.135 \\
\hline 78 & Posey & 176.969 & 299.703 \\
\hline 79 & Pulaski & 12.404 & 30.585 \\
\hline 80 & Putnam & 10.486 & 11.218 \\
\hline 81 & Randolph & 29.091 & 39.079 \\
\hline 82 & Ripley & 19.204 & 7.972 \\
\hline 83 & Rush & 5.905 & 30.110 \\
\hline 84 & St. Joseph & 251.145 & 106.938 \\
\hline 85 & Scott & 9.690 & 11.652 \\
\hline 86 & Shelby & 114.163 & 24.233 \\
\hline 87 & Spencer & 12.270 & 10.528 \\
\hline 88 & Starke & .529 & 22.327 \\
\hline 89 & Steuben & 104.156 & 40.805 \\
\hline 90 & Sullivan & 3.910 & .000 \\
\hline 91 & Switzerland & .000 & .000 \\
\hline 92 & Tippecanoe & 48.966 & 202.909 \\
\hline 93 & Tipton & 6.569 & 10.542 \\
\hline 94 & Union & .507 & .627 \\
\hline 95 & Vanderburgh & 138.720 & 66.332 \\
\hline 96 & Vermillion & 11.246 & .000 \\
\hline 97 & Vigo & 37.478 & 27.635 \\
\hline 98 & Wabash & 69.558 & 35.446 \\
\hline 99 & Warren & 6.403 & 11.187 \\
\hline 100 & Warrick & 13.384 & 4.074 \\
\hline 101 & Washington & 32.984 & 49.395 \\
\hline 102 & Wayne & 43.740 & 20.767 \\
\hline
\end{tabular}




\begin{tabular}{|c|c|c|c|}
\hline & Locale & P33 15 & A33 15 \\
\hline 103 & Wells & 33.378 & 20.086 \\
\hline 104 & White & 50.244 & 18.487 \\
\hline 105 & Whitley & 38.821 & 8.572 \\
\hline 106 & lowa & 1717.397 & 2550.208 \\
\hline 107 & Kansas & 2342.391 & 3567.472 \\
\hline 108 & Kentucky_E & 1545.700 & 1497.479 \\
\hline 109 & Kentucky_W & 1030.467 & 998.320 \\
\hline 110 & Louisiana & 6986.127 & 10187.936 \\
\hline 111 & Maine & 419.996 & 329.539 \\
\hline 112 & Maryland & 1692.634 & 1680.724 \\
\hline 113 & Massachusetts & 3408.956 & 2960.797 \\
\hline 114 & Michigan_E & 3772.690 & 4255.821 \\
\hline 115 & Michigan_W & 3772.690 & 4255.821 \\
\hline 116 & Minnesota & 4431.473 & 4527.694 \\
\hline 117 & Mississippi & 1976.311 & 2570.236 \\
\hline 118 & Missouri & 3423.945 & 3387.798 \\
\hline 119 & Montana & 532.391 & 783.291 \\
\hline 120 & Nebraska & 677.446 & 770.193 \\
\hline 121 & Nevada & 723.286 & 214.729 \\
\hline 122 & New Hampshire & 961.953 & 828.329 \\
\hline 123 & New Jersey & 4590.201 & 4593.881 \\
\hline 124 & New Mexico & 699.440 & 731.477 \\
\hline 125 & New York & 5391.107 & 5553.319 \\
\hline 126 & North Carolina & 4559.287 & 3880.551 \\
\hline 127 & North Dakota & 194.273 & 446.334 \\
\hline 128 & Ohio_N & 3959.526 & 3693.106 \\
\hline 129 & Ohio_M & 3959.526 & 3693.106 \\
\hline 130 & Ohio_s & 3959.526 & 3693.106 \\
\hline 131 & Oklahoma & 3036.659 & 3581.736 \\
\hline 132 & Oregon & 1745.366 & 1569.506 \\
\hline 133 & Pennsylvania & 10316.307 & 10077.678 \\
\hline 134 & Rhode Island & 632.030 & 243.824 \\
\hline 135 & South Carolina & 2521.741 & 2146.350 \\
\hline 136 & South Dakota & 238.581 & 334.159 \\
\hline 137 & Tennessee & 4054.581 & 4069.769 \\
\hline 138 & Texas & 22192.503 & 27535.557 \\
\hline 139 & Utah & 1723.585 & 1931.036 \\
\hline 140 & Vermont & 347.570 & 289.404 \\
\hline 141 & Virginia & 2352.205 & 2084.435 \\
\hline 142 & Washington & 2838.770 & 2793.716 \\
\hline 143 & West Virginia & 841.496 & 533.873 \\
\hline 144 & Wisconsin & 4970.191 & 6048.499 \\
\hline 145 & Wyoming & 472.502 & 710.312 \\
\hline
\end{tabular}




\begin{tabular}{|c|c|c|c|}
\hline & Locale & P34 15 & A34 15 \\
\hline 1 & Alabama & 1598.240 & 1323.360 \\
\hline 2 & Arizona & 1031.772 & 1429.260 \\
\hline 3 & Arkansas & 1343.766 & 1035.511 \\
\hline 4 & California & 8845.212 & 8706.285 \\
\hline 5 & Colorado & 804.771 & 861.841 \\
\hline 6 & Connecticut & 1355.710 & 1987.175 \\
\hline 7 & Delaware & 284.702 & 366.069 \\
\hline 8 & DC & .000 & .000 \\
\hline 9 & Florida & 2376.848 & 2531.133 \\
\hline 10 & Georgia & 2645.249 & 2700.542 \\
\hline 11 & Idaho & 199.429 & 235.259 \\
\hline 12 & Illinois_N & 4744.948 & 3786.867 \\
\hline 13 & Illinois_s & 2372.474 & 1893.434 \\
\hline 14 & Adams & 68.647 & 61.458 \\
\hline 15 & Allen & 397.119 & 323.424 \\
\hline 16 & Bartholomew & 162.507 & 174.915 \\
\hline 17 & Benton & 4.451 & 1.745 \\
\hline 18 & Blackford & 17.049 & 2.410 \\
\hline 19 & Boone & 19.968 & 23.120 \\
\hline 20 & Brown & .000 & .411 \\
\hline 21 & Carroll & 2.072 & 2.799 \\
\hline 22 & Cass & 39.287 & 55.178 \\
\hline 23 & Clark & 48.298 & 44.358 \\
\hline 24 & Clay & 11.145 & 33.665 \\
\hline 25 & Clinton & 15.603 & 20.117 \\
\hline 26 & Crawford & .000 & .000 \\
\hline 27 & Daviess & 9.067 & 8.168 \\
\hline 28 & Dearborn & 8.294 & 4.470 \\
\hline 29 & Decatur & 32.458 & 37.089 \\
\hline 30 & DeKalb & 131.648 & 48.712 \\
\hline 31 & Delaware & 47.087 & 89.408 \\
\hline 32 & Dubois & 52.486 & 35.964 \\
\hline 33 & Elkhart & 337.744 & 489.872 \\
\hline 34 & Fayette & 21.027 & 76.218 \\
\hline 35 & Floyd & 93.981 & 31.291 \\
\hline 36 & Fountain & .000 & 12.719 \\
\hline 37 & Franklin & 11.000 & 8.836 \\
\hline 38 & Fulton & 18.143 & 11.190 \\
\hline 39 & Gibson & 24.665 & 8.435 \\
\hline 40 & Grant & 40.255 & 66.417 \\
\hline 41 & Greene & .000 & .983 \\
\hline 42 & Hamilton & 81.898 & 34.208 \\
\hline 43 & Hancock & 11.092 & 39.995 \\
\hline 44 & Harrison & 12.144 & 18.910 \\
\hline 45 & Hendricks & 16.120 & 16.334 \\
\hline 46 & Henry & 18.706 & 36.615 \\
\hline 47 & Howard & 32.354 & 303.357 \\
\hline 48 & Huntington & 31.013 & 53.404 \\
\hline 49 & Jackson & 43.929 & 65.373 \\
\hline 50 & Jasper & 3.178 & 3.349 \\
\hline 51 & Jay & 13.915 & 11.361 \\
\hline
\end{tabular}




\begin{tabular}{|c|c|c|c|}
\hline & Locale & P34 15 & A34 15 \\
\hline 52 & Jefferson & 34.286 & 52.614 \\
\hline 53 & Jennings & 45.184 & 23.082 \\
\hline 54 & Johnson & 76.445 & 55.761 \\
\hline 55 & Knox & 3.093 & 2.412 \\
\hline 56 & Kosciusko & 79.332 & 65.772 \\
\hline 57 & LaGrange & 57.836 & 40.685 \\
\hline 58 & Lake & 69.391 & 106.330 \\
\hline 59 & La Porte & 98.238 & 73.291 \\
\hline 60 & Lawrence & 22.512 & 38.817 \\
\hline 61 & Madison & 28.600 & 137.455 \\
\hline 62 & Marion & 359.837 & 658.614 \\
\hline 63 & Marshall & 121.584 & 43.161 \\
\hline 64 & Martin & .308 & .260 \\
\hline 65 & Miami & 22.037 & 7.558 \\
\hline 66 & Monroe & 78.498 & 62.245 \\
\hline 67 & Montgomery & 41.053 & 33.272 \\
\hline 68 & Morgan & 15.856 & 23.540 \\
\hline 69 & Newton & 22.939 & 5.449 \\
\hline 70 & Noble & 109.850 & 72.755 \\
\hline 71 & \begin{tabular}{|l} 
Ohio \\
\end{tabular} & .000 & .000 \\
\hline 72 & Orange & 8.053 & .272 \\
\hline 73 & Owen & .832 & .296 \\
\hline 74 & Parke & 9.765 & 1.651 \\
\hline 75 & Perry & 5.426 & 4.580 \\
\hline 76 & Pike & .000 & .000 \\
\hline 77 & Porter & 31.677 & 26.248 \\
\hline 78 & Posey & 13.718 & 45.869 \\
\hline 79 & Pulaski & 16.341 & 13.965 \\
\hline 80 & Putnam & 61.064 & 18.068 \\
\hline 81 & Randolph & 19.616 & 26.318 \\
\hline 82 & Ripley & .477 & 1.705 \\
\hline 83 & Rush & 18.632 & 14.520 \\
\hline 84 & St. Joseph & 184.432 & 137.615 \\
\hline 85 & Scott & 51.821 & 7.139 \\
\hline 86 & Shelby & 103.878 & 29.220 \\
\hline 87 & Spencer & 22.119 & 7.831 \\
\hline 88 & Starke & 19.546 & 16.201 \\
\hline 89 & Steuben & 48.156 & 60.635 \\
\hline 90 & Sullivan & 8.150 & 3.265 \\
\hline 91 & Switzerland & 9.594 & .000 \\
\hline 92 & Tippecanoe & 135.629 & 248.500 \\
\hline 93 & Tipton & 5.633 & 11.102 \\
\hline 94 & Union & .335 & 1.337 \\
\hline 95 & Vanderburgh & 185.477 & 91.338 \\
\hline 96 & Vermillion & .000 & 39.511 \\
\hline 97 & Vigo & 91.916 & 44.047 \\
\hline 98 & Wabash & 22.617 & 28.113 \\
\hline 99 & Warren & 6.445 & 6.083 \\
\hline 100 & Warrick & 2.673 & 7.176 \\
\hline 101 & Washington & 37.176 & 22.454 \\
\hline 102 & Wayne & 58.642 & 32.655 \\
\hline
\end{tabular}




\begin{tabular}{|c|c|c|c|}
\hline & Locale & P34 15 & A34 15 \\
\hline 103 & Wells & 47.814 & 16.012 \\
\hline 104 & White & 26.015 & 37.614 \\
\hline 105 & Whitley & 27.182 & 26.831 \\
\hline 106 & lowa & 2032.541 & 1578.307 \\
\hline 107 & Kansas & 1360.235 & 1712.665 \\
\hline 108 & Kentucky_E & 1022.292 & 1104.349 \\
\hline 109 & Kentucky_W & 681.528 & 736.233 \\
\hline 110 & Louisiana & 526.120 & 1364.649 \\
\hline 111 & Maine & 237.499 & 442.345 \\
\hline 112 & Maryland & 808.017 & 927.055 \\
\hline 113 & Massachusetts & 2235.347 & 1744.654 \\
\hline 114 & Michigan_E & 3428.655 & 4272.323 \\
\hline 115 & Michigan_W & 3428.655 & 4272.323 \\
\hline 116 & Minnesota & 2547.498 & 1823.911 \\
\hline 117 & Mississippi & 1096.171 & 1171.782 \\
\hline 118 & Missouri & 2233.714 & 2864.904 \\
\hline 119 & Montana & 75.012 & 80.324 \\
\hline 120 & Nebraska & 707.616 & 550.985 \\
\hline 121 & Nevada & 339.391 & 156.978 \\
\hline 122 & New Hampshire & 718.059 & 420.942 \\
\hline 123 & New Jersey & 2482.767 & 2751.824 \\
\hline 124 & New Mexico & 74.320 & 120.183 \\
\hline 125 & New York & 4039.024 & 4246.638 \\
\hline 126 & North Carolina & 4266.139 & 3445.068 \\
\hline 127 & North Dakota & 208.663 & 193.587 \\
\hline 128 & Ohio_N & 2646.312 & 2381.252 \\
\hline 129 & Ohio_M & 2646.312 & 2381.252 \\
\hline 130 & Ohio_s & 2646.312 & 2381.252 \\
\hline 131 & Oklahoma & 1486.065 & 1177.230 \\
\hline 132 & Oregon & 871.083 & 867.060 \\
\hline 133 & Pennsylvania & 4652.148 & 3784.893 \\
\hline 134 & Rhode Island & 402.606 & 204.691 \\
\hline 135 & South Carolina & 2168.030 & 2330.246 \\
\hline 136 & South Dakota & 250.384 & 205.759 \\
\hline 137 & Tennessee & 3333.392 & 3314.894 \\
\hline 138 & Texas & 6708.948 & 7012.197 \\
\hline 139 & Utah & 499.311 & 718.820 \\
\hline 140 & Vermont & 227.969 & 163.579 \\
\hline 141 & \begin{tabular}{|l} 
Virginia \\
\end{tabular} & 1977.001 & 1976.048 \\
\hline 142 & Washington & 1164.422 & 2464.894 \\
\hline 143 & West Virginia & 255.666 & 500.914 \\
\hline 144 & Wisconsin & 4664.527 & 3688.484 \\
\hline 145 & Wyoming & 40.894 & 60.087 \\
\hline
\end{tabular}




\begin{tabular}{|c|c|c|c|}
\hline & Locale & P35 15 & A35 15 \\
\hline 1 & Alabama & 6223.634 & 6366.842 \\
\hline 2 & Arizona & 4855.416 & 5593.083 \\
\hline 3 & Arkansas & 4318.442 & 3796.409 \\
\hline 4 & California & 42240.714 & 41905.524 \\
\hline 5 & Colorado & 2983.549 & 3776.741 \\
\hline 6 & Connecticut & 6834.133 & 3450.555 \\
\hline 7 & Delaware & 1052.437 & 1595.941 \\
\hline 8 & $\overline{D C}$ & .000 & .000 \\
\hline 9 & Florida & 8315.969 & 15252.688 \\
\hline 10 & Georgia & 6667.880 & 9643.444 \\
\hline 11 & Idaho & 860.000 & 1226.354 \\
\hline 12 & Illinois_N & 15722.152 & 11927.026 \\
\hline 13 & Illinois_s & 7861.076 & 5963.513 \\
\hline 14 & Adams & 70.252 & 130.393 \\
\hline 15 & Allen & 515.826 & 683.104 \\
\hline 16 & Bartholomew & 164.857 & 120.521 \\
\hline 17 & Benton & 9.963 & 10.305 \\
\hline 18 & Blackford & 83.735 & 38.123 \\
\hline 19 & Boone & 57.670 & 38.387 \\
\hline 20 & Brown & 8.675 & 9.678 \\
\hline 21 & Carroll & 65.024 & 12.526 \\
\hline 22 & Cass & 99.664 & 90.160 \\
\hline 23 & Clark & 240.781 & 171.606 \\
\hline 24 & Clay & 15.972 & 32.198 \\
\hline 25 & Clinton & 37.934 & 37.808 \\
\hline 26 & Crawford & .000 & 7.325 \\
\hline 27 & Daviess & 30.663 & 31.164 \\
\hline 28 & Dearborn & 47.431 & 50.044 \\
\hline 29 & Decatur & 419.540 & 49.636 \\
\hline 30 & DeKalb & 522.700 & 241.226 \\
\hline 31 & Delaware & 280.174 & 98.038 \\
\hline 32 & Dubois & 23.133 & 121.610 \\
\hline 33 & Elkhart & 990.476 & 695.277 \\
\hline 34 & Fayette & 50.309 & 15.067 \\
\hline 35 & Floyd & 98.200 & 191.336 \\
\hline 36 & Fountain & 8.589 & 10.755 \\
\hline 37 & Franklin & 43.404 & 73.042 \\
\hline 38 & Fulton & 123.028 & 25.405 \\
\hline 39 & Gibson & 20.677 & 56.666 \\
\hline 40 & Grant & 22.105 & 84.097 \\
\hline 41 & Greene & 13.107 & 20.652 \\
\hline 42 & Hamilton & 237.395 & 325.607 \\
\hline 43 & Hancock & 86.111 & 46.799 \\
\hline 44 & Harrison & 59.227 & 90.241 \\
\hline 45 & Hendricks & 44.667 & 107.698 \\
\hline 46 & Henry & 46.429 & 42.543 \\
\hline 47 & Howard & 30.168 & 93.908 \\
\hline 48 & Huntington & 163.662 & 63.246 \\
\hline 49 & Jackson & 101.051 & 67.246 \\
\hline 50 & Jasper & 101.484 & 25.082 \\
\hline 51 & Jay & 135.738 & 33.484 \\
\hline
\end{tabular}




\begin{tabular}{|c|c|c|c|}
\hline & $\begin{array}{l}\text { Locale } \\
\end{array}$ & P35 15 & A35 15 \\
\hline 52 & Jefferson & 21.138 & 37.307 \\
\hline 53 & Jennings & 142.740 & 127.871 \\
\hline 54 & Johnson & 207.189 & 186.084 \\
\hline 55 & Knox & 21.492 & 29.933 \\
\hline 56 & Kosciusko & 222.497 & 151.254 \\
\hline 57 & LaGrange & 34.098 & 99.830 \\
\hline 58 & Lake & 1712.738 & 1672.585 \\
\hline 59 & La Porte & 317.662 & 143.012 \\
\hline 60 & Lawrence & 116.126 & 83.330 \\
\hline 61 & Madison & 153.286 & 114.361 \\
\hline 62 & Marion & 1569.387 & 767.496 \\
\hline 63 & Marshall & 200.997 & 299.652 \\
\hline 64 & Martin & 1.343 & 6.057 \\
\hline 65 & Miami & 35.399 & 52.255 \\
\hline 66 & Monroe & 104.340 & 153.088 \\
\hline 67 & Montgomery & 95.523 & 93.190 \\
\hline 68 & Morgan & 55.616 & 59.009 \\
\hline 69 & Newton & 8.583 & 48.891 \\
\hline 70 & Noble & 213.783 & 215.382 \\
\hline 71 & Ohio & 1.554 & 3.465 \\
\hline 72 & Orange & 8.440 & 27.549 \\
\hline 73 & Owen & 1.989 & 15.344 \\
\hline 74 & Parke & 1.421 & 25.777 \\
\hline 75 & Perry & 8.107 & 10.992 \\
\hline 76 & Pike & 8.914 & 8.113 \\
\hline 77 & Porter & 230.853 & 122.072 \\
\hline 78 & Posey & 254.183 & 281.648 \\
\hline 79 & Pulaski & 25.961 & 8.445 \\
\hline 80 & Putnam & 1.492 & 132.433 \\
\hline 81 & Randolph & 21.165 & 27.069 \\
\hline 82 & Ripley & 38.482 & 24.708 \\
\hline 83 & Rush & 8.104 & 15.494 \\
\hline 84 & St. Joseph & 659.928 & 440.325 \\
\hline 85 & Scott & 19.574 & 119.758 \\
\hline 86 & Shelby & 52.687 & 206.752 \\
\hline 87 & Spencer & 8.404 & 44.969 \\
\hline 88 & Starke & .000 & 29.333 \\
\hline 89 & Steuben & 207.654 & 87.952 \\
\hline 90 & Sullivan & 1.483 & 29.759 \\
\hline 91 & Switzerland & .000 & 25.134 \\
\hline 92 & Tippecanoe & 109.690 & 176.781 \\
\hline 93 & Tipton & 17.392 & 9.868 \\
\hline 94 & Union & 1.461 & 4.449 \\
\hline 95 & Vanderburgh & 293.164 & 404.390 \\
\hline 96 & Vermillion & 23.536 & 9.771 \\
\hline 97 & Vigo & 97.748 & 215.935 \\
\hline 98 & Wabash & 144.799 & 41.725 \\
\hline 99 & Warren & 18.456 & 5.944 \\
\hline 100 & Warrick & 36.836 & 38.299 \\
\hline 101 & Washington & 93.380 & 39.253 \\
\hline 102 & Wayne & 84.248 & 134.667 \\
\hline
\end{tabular}




\begin{tabular}{|c|c|c|c|}
\hline & \begin{tabular}{|l|} 
Locale \\
\end{tabular} & P35 15 & A35 15 \\
\hline 103 & Wells & 96.203 & 91.299 \\
\hline 104 & White & 135.313 & 47.411 \\
\hline 105 & Whitley & 110.161 & 65.513 \\
\hline 106 & lowa & 3821.768 & 3381.201 \\
\hline 107 & Kansas & 3966.568 & 4685.475 \\
\hline 108 & Kentucky_E & 2838.073 & 3122.033 \\
\hline 109 & Kentucky_W & 1892.049 & 2081.355 \\
\hline 110 & Louisiana & 11023.100 & 11086.892 \\
\hline 111 & Maine & 875.589 & 1211.450 \\
\hline 112 & Maryland & 3046.165 & 5235.506 \\
\hline 113 & Massachusetts & 7772.390 & 6844.349 \\
\hline 114 & Michigan_E & 8560.210 & 6945.822 \\
\hline 115 & Michigan_W & 8560.210 & 6945.822 \\
\hline 116 & Minnesota & 9375.336 & 6651.270 \\
\hline 117 & Mississippi & 3483.819 & 4061.594 \\
\hline 118 & Missouri & 7120.883 & 6549.097 \\
\hline 119 & Montana & 761.583 & 1163.474 \\
\hline 120 & Nebraska & 1494.309 & 1664.503 \\
\hline 121 & Nevada & 1089.748 & 2202.392 \\
\hline 122 & New Hampshire & 2265.444 & 1642.999 \\
\hline 123 & New Jersey & 8409.462 & 10889.845 \\
\hline 124 & New Mexico & 1007.581 & 1784.984 \\
\hline 125 & New York & 11266.518 & 16007.169 \\
\hline 126 & North Carolina & 8191.832 & 11275.454 \\
\hline 127 & North Dakota & 378.687 & 569.179 \\
\hline 128 & Ohio_N & 8174.518 & 6325.786 \\
\hline 129 & Ohio_M & 8174.518 & 6325.786 \\
\hline 130 & Ohio_s & 8174.518 & 6325.786 \\
\hline 131 & Oklahoma & 5772.246 & 5058.488 \\
\hline 132 & Oregon & 3670.160 & 3571.213 \\
\hline 133 & Pennsylvania & 19680.491 & 16569.213 \\
\hline 134 & Rhode Island & 1636.766 & 1226.590 \\
\hline 135 & South Carolina & 5269.534 & 5317.457 \\
\hline 136 & South Dakota & 514.787 & 631.342 \\
\hline 137 & Tennessee & 8104.109 & 8214.138 \\
\hline 138 & Texas & 38863.253 & 38315.590 \\
\hline 139 & Utah & 3033.840 & 3189.762 \\
\hline 140 & Vermont & 602.329 & 635.899 \\
\hline 141 & Virginia & 4108.914 & 7755.129 \\
\hline 142 & Washington & 5227.401 & 6723.350 \\
\hline 143 & West Virginia & 1242.119 & 1601.148 \\
\hline 144 & Wisconsin & 12288.724 & 6956.370 \\
\hline 145 & Wyoming & 682.416 & 885.891 \\
\hline
\end{tabular}




\begin{tabular}{|c|c|c|c|}
\hline & Locale & P36 15 & A36 15 \\
\hline 1 & Alabama & 3192.887 & 3192.887 \\
\hline 2 & Arizona & 5451.554 & 5451.554 \\
\hline 3 & Arkansas & 1979.320 & 1979.320 \\
\hline 4 & California & 23726.158 & 23726.158 \\
\hline 5 & Colorado & 1744.995 & 1744.995 \\
\hline 6 & Connecticut & 5957.613 & 5957.613 \\
\hline 7 & Delaware & 820.879 & 820.879 \\
\hline 8 & DC & .000 & .000 \\
\hline 9 & Florida & 6109.106 & 6109.106 \\
\hline 10 & Georgia & 7166.772 & 7166.772 \\
\hline 11 & Idaho & 287.837 & 287.837 \\
\hline 12 & Illinois_N & 4772.567 & 4772.567 \\
\hline 13 & Illinois_S & 2386.284 & 2386.284 \\
\hline 14 & Adams & 289.877 & 289.877 \\
\hline 15 & Allen & 1068.076 & 1068.076 \\
\hline 16 & Bartholomew & 439.695 & 439.695 \\
\hline 17 & Benton & 1.093 & 1.093 \\
\hline 18 & Blackford & 6.229 & 6.229 \\
\hline 19 & Boone & 46.648 & 46.648 \\
\hline 20 & Brown & 1.158 & 1.158 \\
\hline 21 & Carroll & 7.234 & 7.234 \\
\hline 22 & Cass & 343.791 & 343.791 \\
\hline 23 & Clark & 7.630 & 7.630 \\
\hline 24 & Clay & 203.525 & 203.525 \\
\hline 25 & Clinton & 65.453 & 65.453 \\
\hline 26 & Crawford & .000 & .000 \\
\hline 27 & Daviess & 9.724 & 9.724 \\
\hline 28 & Dearborn & .000 & .000 \\
\hline 29 & Decatur & 167.609 & 167.609 \\
\hline 30 & DeKalb & 154.972 & 154.972 \\
\hline 31 & Delaware & 394.159 & 394.159 \\
\hline 32 & Dubois & 213.324 & 213.324 \\
\hline 33 & Elkhart & 2751.210 & 2751.210 \\
\hline 34 & Fayette & 402.965 & 402.965 \\
\hline 35 & Floyd & 67.096 & 67.096 \\
\hline 36 & Fountain & 85.999 & 85.999 \\
\hline 37 & Franklin & 48.076 & 48.076 \\
\hline 38 & Fulton & 6.964 & 6.964 \\
\hline 39 & Gibson & 21.957 & 21.957 \\
\hline 40 & Grant & 334.873 & 334.873 \\
\hline 41 & Greene & 6.773 & 6.773 \\
\hline 42 & Hamilton & 150.281 & 150.281 \\
\hline 43 & Hancock & 217.289 & 217.289 \\
\hline 44 & Harrison & 106.152 & 106.152 \\
\hline 45 & Hendricks & 40.583 & 40.583 \\
\hline 46 & Henry & 185.942 & 185.942 \\
\hline 47 & Howard & 2023.277 & 2023.277 \\
\hline 48 & Huntington & 268.071 & 268.071 \\
\hline 49 & Jackson & 287.212 & 287.212 \\
\hline 50 & Jasper & 21.074 & 21.074 \\
\hline 51 & Jay & 41.161 & 41.161 \\
\hline
\end{tabular}




\begin{tabular}{|c|c|c|c|}
\hline & Locale & P36 15 & A36 15 \\
\hline 52 & Jefferson & 201.222 & 201.222 \\
\hline 53 & Jennings & 95.364 & 95.364 \\
\hline 54 & Johnson & 195.204 & 195.204 \\
\hline 55 & Knox & 1.072 & 1.072 \\
\hline 56 & Kosciusko & 219.468 & 219.468 \\
\hline 57 & LaGrange & 161.436 & 161.436 \\
\hline 58 & Lake & 228.434 & 228.434 \\
\hline 59 & La Porte & 85.746 & 85.746 \\
\hline 60 & Lawrence & 194.667 & 194.667 \\
\hline 61 & Madison & 806.115 & 806.115 \\
\hline 62 & Marion & 1835.289 & 1835.289 \\
\hline 63 & Marshall & 241.833 & 241.833 \\
\hline 64 & Martin & .000 & .000 \\
\hline 65 & Miami & 6.444 & 6.444 \\
\hline 66 & Monroe & 22.564 & 22.564 \\
\hline 67 & Montgomery & 164.227 & 164.227 \\
\hline 68 & Morgan & 24.061 & 24.061 \\
\hline 69 & Newton & 20.051 & 20.051 \\
\hline 70 & Noble & 369.399 & 369.399 \\
\hline 71 & Ohio & .000 & .000 \\
\hline 72 & Orange & .000 & .000 \\
\hline 73 & Owen & .000 & .000 \\
\hline 74 & Parke & .000 & .000 \\
\hline 75 & Perry & .000 & .000 \\
\hline 76 & Pike & .000 & .000 \\
\hline 77 & Porter & 1.194 & 1.194 \\
\hline 78 & Posey & .000 & .000 \\
\hline 79 & Pulaski & 1.188 & 1.188 \\
\hline 80 & Putnam & 89.659 & 89.659 \\
\hline 81 & Randolph & 82.280 & 82.280 \\
\hline 82 & Ripley & 1.216 & 1.216 \\
\hline 83 & Rush & 6.491 & 6.491 \\
\hline 84 & St. Joseph & 492.457 & 492.457 \\
\hline 85 & Scott & 7.152 & 7.152 \\
\hline 86 & Shelby & 94.176 & 94.176 \\
\hline 87 & Spencer & 19.633 & 19.633 \\
\hline 88 & Starke & 32.642 & 32.642 \\
\hline 89 & Steuben & 315.294 & 315.294 \\
\hline 90 & Sullivan & 20.787 & 20.787 \\
\hline 91 & Switzerland & .000 & .000 \\
\hline 92 & Tippecanoe & 790.092 & 790.092 \\
\hline 93 & Tipton & 42.128 & 42.128 \\
\hline 94 & Union & .000 & .000 \\
\hline 95 & Vanderburgh & 9.891 & 9.891 \\
\hline 96 & Vermillion & .000 & .000 \\
\hline 97 & Vigo & 43.821 & 43.821 \\
\hline 98 & Wabash & 46.567 & 46.567 \\
\hline 99 & Warren & 7.153 & 7.153 \\
\hline 100 & Warrick & 22.160 & 22.160 \\
\hline 101 & Washington & 1.228 & 1.228 \\
\hline 102 & Wayne & 139.205 & 139.205 \\
\hline
\end{tabular}




\begin{tabular}{|c|c|c|c|}
\hline & $\begin{array}{r}\text { Locale } \\
\end{array}$ & P36 15 & A36 15 \\
\hline 103 & Wells & 47.924 & 47.924 \\
\hline 104 & White & 201.777 & 201.777 \\
\hline 105 & Whitley & 156.459 & 156.459 \\
\hline 106 & lowa & 2184.858 & 2184.858 \\
\hline 107 & Kansas & 6327.441 & 6327.441 \\
\hline 108 & Kentucky_E & 3340.802 & 3340.802 \\
\hline 109 & Kentucky_W & 2227.202 & 2227.202 \\
\hline 110 & Louisiana & 2899.721 & 2899.721 \\
\hline 111 & Maine & 2152.156 & 2152.156 \\
\hline$\overline{112}$ & Maryland & 1375.534 & 1375.534 \\
\hline 113 & Massachusetts & 1574.838 & 1574.838 \\
\hline 114 & Michigan_E & 15777.410 & 1577.410 \\
\hline 115 & Michigan_W & 15777.410 & 1577.410 \\
\hline 116 & Minnesota & 1938.717 & 1938.717 \\
\hline 117 & Mississippi & 3514.628 & 3514.628 \\
\hline 118 & Missouri & 8785.314 & 8785.314 \\
\hline 119 & Montana & 93.798 & 93.798 \\
\hline 120 & Nebraska & 947.190 & 947.190 \\
\hline 121 & Nevada & 302.348 & 302.348 \\
\hline 122 & New Hampshire & 390.424 & 390.424 \\
\hline 123 & New Jersey & 1350.320 & 1350.320 \\
\hline 124 & New Mexico & 307.369 & 307.369 \\
\hline 125 & New York & 5254.566 & 5254.566 \\
\hline 126 & North Carolina & 4743.101 & 4743.101 \\
\hline 127 & North Dakota & 335.501 & 335.501 \\
\hline 128 & Ohio_N & 6682.588 & 6682.588 \\
\hline 129 & Ohio_M & 6682.588 & 6682.588 \\
\hline 130 & Ohio_s & 6682.588 & 6682.588 \\
\hline 131 & Oklahoma & 2528.857 & 2528.857 \\
\hline 132 & Oregon & 2180.487 & 2180.487 \\
\hline 133 & Pennsylvania & 6263.410 & 6263.410 \\
\hline 134 & Rhode Island & 214.749 & 214.749 \\
\hline 135 & South Carolina & 3237.297 & 3237.297 \\
\hline 136 & South Dakota & 279.495 & 279.495 \\
\hline 137 & Tennessee & 7313.829 & 7313.830 \\
\hline 138 & Texas & 11098.552 & 11098.552 \\
\hline 139 & Utah & 2192.041 & 2192.041 \\
\hline 140 & Vermont & 312.925 & 312.925 \\
\hline 141 & Virginia & 5151.191 & 5151.191 \\
\hline 142 & Washington & 12808.747 & 12808.747 \\
\hline 143 & West Virginia & 409.757 & 409.757 \\
\hline 144 & Wisconsin & 5390.297 & 5390.297 \\
\hline 145 & Wyoming & 45.268 & 45.268 \\
\hline
\end{tabular}




\begin{tabular}{|c|c|c|c|}
\hline & Locale & P37 15 & A37 15 \\
\hline 1 & Alabama & 246.586 & 150.242 \\
\hline 2 & Arizona & 102.503 & 29.487 \\
\hline 3 & Arkansas & 105.632 & 64.115 \\
\hline 4 & California & 312.102 & 846.476 \\
\hline 5 & Colorado & 26.521 & 31.291 \\
\hline 6 & Connecticut & 63.310 & 26.742 \\
\hline 7 & Delaware & 9.334 & 54.780 \\
\hline 8 & DC & .000 & .000 \\
\hline 9 & Florida & 66.263 & 69.699 \\
\hline 10 & Georgia & 84.163 & 77.194 \\
\hline 11 & Idaho & 2.410 & 12.191 \\
\hline 12 & Illinois_N & 277.981 & 229.348 \\
\hline 13 & Illinois_S & 138.990 & 114.674 \\
\hline 14 & Adams & 1.997 & .620 \\
\hline 15 & Allen & 28.110 & 3.824 \\
\hline 16 & Bartholomew & 8.079 & .000 \\
\hline 17 & Benton & .591 & .000 \\
\hline 18 & Blackford & .000 & .000 \\
\hline 19 & Boone & .000 & .000 \\
\hline 20 & Brown & .000 & .000 \\
\hline 21 & Carroll & .000 & .000 \\
\hline 22 & Cass & .000 & .000 \\
\hline 23 & Clark & 1.479 & 6.291 \\
\hline 24 & Clay & .105 & .000 \\
\hline 25 & Clinton & .000 & .000 \\
\hline 26 & Crawford & .000 & .000 \\
\hline 27 & Daviess & .000 & .603 \\
\hline 28 & Dearborn & .000 & .698 \\
\hline 29 & Decatur & .106 & .000 \\
\hline 30 & DeKalb & 27.904 & .000 \\
\hline 31 & Delaware & 7.732 & .000 \\
\hline 32 & Dubois & .000 & .000 \\
\hline 33 & Elkhart & 13.663 & .618 \\
\hline 34 & Fayette & .581 & .000 \\
\hline 35 & Floyd & .113 & .000 \\
\hline 36 & Fountain & 7.752 & .000 \\
\hline 37 & Franklin & .000 & 3.768 \\
\hline 38 & Fulton & 3.924 & .000 \\
\hline 39 & Gibson & .000 & .000 \\
\hline 40 & Grant & 1.614 & .000 \\
\hline 41 & Greene & 1.781 & .000 \\
\hline 42 & Hamilton & 6.388 & .761 \\
\hline 43 & Hancock & .135 & .000 \\
\hline 44 & Harrison & .000 & 4.160 \\
\hline 45 & Hendricks & 2.808 & .000 \\
\hline 46 & Henry & 3.592 & .000 \\
\hline 47 & Howard & 7.816 & .000 \\
\hline 48 & Huntington & 1.912 & .615 \\
\hline 49 & Jackson & 5.227 & .000 \\
\hline 50 & Jasper & .000 & .000 \\
\hline 51 & Jay & .000 & .538 \\
\hline
\end{tabular}




\begin{tabular}{|c|c|c|c|}
\hline & Locale & P37 15 & A37 15 \\
\hline 52 & Jefferson & .110 & .000 \\
\hline 53 & Jennings & .688 & 3.737 \\
\hline 54 & Johnson & 4.163 & .000 \\
\hline 55 & Knox & 3.625 & .525 \\
\hline 56 & Kosciusko & 19.002 & .590 \\
\hline 57 & LaGrange & .116 & .000 \\
\hline 58 & Lake & 217.043 & 121.647 \\
\hline 59 & La Porte & 16.345 & .560 \\
\hline 60 & Lawrence & 17.548 & 3.269 \\
\hline 61 & Madison & 2.430 & .000 \\
\hline 62 & Marion & 38.971 & 6.904 \\
\hline 63 & Marshall & 4.310 & 3.679 \\
\hline 64 & Martin & 1.697 & .000 \\
\hline 65 & Miami & .000 & .000 \\
\hline 66 & Monroe & .000 & 3.790 \\
\hline 67 & Montgomery & 8.057 & .000 \\
\hline 68 & Morgan & .744 & .000 \\
\hline 69 & Newton & 1.807 & .000 \\
\hline 70 & Noble & 8.242 & .000 \\
\hline 71 & Ohio & .000 & .000 \\
\hline 72 & Orange & .000 & .000 \\
\hline 73 & Owen & .000 & .000 \\
\hline 74 & Parke & .000 & .000 \\
\hline 75 & Perry & 3.659 & .000 \\
\hline 76 & Pike & .644 & .000 \\
\hline 77 & Porter & 87.699 & .000 \\
\hline 78 & Posey & .000 & 22.099 \\
\hline 79 & Pulaski & 1.874 & .000 \\
\hline 80 & Putnam & .000 & .000 \\
\hline 81 & Randolph & 3.016 & .537 \\
\hline 82 & Ripley & .000 & .596 \\
\hline 83 & Rush & 3.657 & .000 \\
\hline 84 & St. Joseph & 18.735 & .583 \\
\hline 85 & Scott & .107 & .584 \\
\hline 86 & Shelby & 5.546 & .000 \\
\hline 87 & Spencer & 1.770 & .000 \\
\hline 88 & Starke & .000 & .000 \\
\hline 89 & Steuben & 5.367 & .582 \\
\hline 90 & Sullivan & .000 & .000 \\
\hline 91 & Switzerland & .000 & .000 \\
\hline 92 & Tippecanoe & 21.240 & .660 \\
\hline 93 & Tipton & .608 & .000 \\
\hline 94 & Union & .000 & .000 \\
\hline 95 & Vanderburgh & 1.926 & .000 \\
\hline 96 & Vermillion & .000 & .000 \\
\hline 97 & Vigo & 3.950 & .000 \\
\hline 98 & Wabash & 7.408 & .537 \\
\hline 99 & Warren & .000 & .000 \\
\hline 100 & Warrick & 42.805 & .000 \\
\hline 101 & Washington & .111 & .000 \\
\hline 102 & Wayne & 8.770 & .000 \\
\hline
\end{tabular}




\begin{tabular}{|c|c|c|c|}
\hline & Locale & P37 15 & A37 15 \\
\hline 103 & Wells & 1.989 & .000 \\
\hline 104 & White & .624 & .000 \\
\hline 105 & Whitley & 6.887 & .000 \\
\hline 106 & lowa & 103.884 & 10.008 \\
\hline 107 & Kansas & 29.835 & 162.600 \\
\hline 108 & Kentucky_E & 101.701 & 45.508 \\
\hline 109 & Kentucky_W & 67.800 & 30.338 \\
\hline 110 & Louisiana & 41.601 & 718.698 \\
\hline 111 & Maine & 8.314 & 10.542 \\
\hline 112 & Maryland & 88.258 & 70.266 \\
\hline 113 & Massachusetts & 64.595 & 61.810 \\
\hline 114 & Michigan_E & 202.073 & 60.009 \\
\hline 115 & Michigan W & 202.073 & 60.009 \\
\hline 116 & Minnesota & 88.782 & 122.973 \\
\hline 117 & Mississippi & 35.413 & 112.981 \\
\hline 118 & Missouri & 141.354 & 84.583 \\
\hline 119 & Montana & 13.664 & 49.746 \\
\hline 120 & Nebraska & 12.497 & .578 \\
\hline 121 & Nevada & 22.847 & 5.078 \\
\hline 122 & New Hampshire & 42.871 & 10.038 \\
\hline 123 & New Jersey & 113.909 & 214.365 \\
\hline 124 & New Mexico & 19.987 & 48.162 \\
\hline 125 & New York & 185.040 & 90.911 \\
\hline 126 & North Carolina & 99.767 & 47.455 \\
\hline 127 & North Dakota & 1.258 & 9.571 \\
\hline 128 & Ohio_N & 259.255 & 106.474 \\
\hline 129 & Ohio_M & 259.255 & 106.474 \\
\hline 130 & Ohio_s & 259.255 & 106.474 \\
\hline 131 & Oklahoma & 57.881 & 149.287 \\
\hline 132 & Oregon & 129.621 & 46.505 \\
\hline 133 & Pennsylvania & 656.668 & 413.814 \\
\hline 134 & Rhode Island & 37.898 & .601 \\
\hline 135 & South Carolina & 82.704 & 23.995 \\
\hline 136 & South Dakota & 6.629 & .584 \\
\hline 137 & Tennessee & 198.454 & 84.877 \\
\hline 138 & Texas & 378.469 & 1553.264 \\
\hline 139 & Utah & 86.706 & 105.580 \\
\hline 140 & Vermont & 3.027 & 3.669 \\
\hline 141 & Virginia & 81.395 & 44.450 \\
\hline 142 & Washington & 157.060 & 132.837 \\
\hline 143 & West Virginia & 115.734 & 22.018 \\
\hline 144 & Wisconsin & 300.144 & 25.352 \\
\hline 145 & Wyoming & 1.360 & 49.435 \\
\hline
\end{tabular}




\begin{tabular}{|c|c|c|c|}
\hline & $\begin{array}{r}\text { Locale } \\
\end{array}$ & P38 15 & A38 15 \\
\hline 1 & Alabama & 61.458 & 73.769 \\
\hline 2 & Arizona & 94.728 & 79.027 \\
\hline 3 & Arkansas & 30.587 & 35.269 \\
\hline 4 & California & 678.261 & 618.355 \\
\hline 5 & Colorado & 84.769 & 73.209 \\
\hline 6 & Connecticut & 54.819 & 51.892 \\
\hline 7 & Delaware & 9.836 & 11.963 \\
\hline 8 & DC & .000 & .000 \\
\hline 9 & Florida & 283.573 & 262.754 \\
\hline 10 & Georgia & 186.769 & 305.433 \\
\hline 11 & Idaho & 17.737 & 19.390 \\
\hline 12 & Illinois_N & 174.505 & 150.000 \\
\hline 13 & Illinois_S & 87.252 & 75.000 \\
\hline 14 & Adams & .186 & .227 \\
\hline 15 & Allen & 10.668 & 8.887 \\
\hline 16 & Bartholomew & .929 & .630 \\
\hline 17 & Benton & .032 & .098 \\
\hline 18 & Blackford & .075 & .069 \\
\hline 19 & Boone & .609 & .511 \\
\hline 20 & Brown & .013 & .013 \\
\hline 21 & Carroll & .056 & .143 \\
\hline 22 & Cass & .342 & .307 \\
\hline 23 & Clark & 1.550 & 1.217 \\
\hline 24 & Clay & .035 & .062 \\
\hline 25 & Clinton & .191 & .191 \\
\hline 26 & Crawford & .015 & .007 \\
\hline 27 & \begin{tabular}{|l|} 
Daviess \\
\end{tabular} & .129 & .171 \\
\hline 28 & Dearborn & .250 & .196 \\
\hline 29 & \begin{tabular}{|l|} 
Decatur \\
\end{tabular} & .152 & .278 \\
\hline 30 & DeKalb & .422 & .298 \\
\hline 31 & Delaware & 1.331 & .991 \\
\hline 32 & Dubois & 1.533 & 1.048 \\
\hline 33 & Elkhart & 5.974 & 5.273 \\
\hline 34 & Fayette & .098 & .143 \\
\hline 35 & Floyd & .826 & .708 \\
\hline 36 & Fountain & .031 & .081 \\
\hline 37 & Franklin & .083 & .084 \\
\hline 38 & Fulton & .059 & .107 \\
\hline 39 & Gibson & .135 & .129 \\
\hline 40 & Grant & .538 & .550 \\
\hline 41 & Greene & .105 & .128 \\
\hline 42 & Hamilton & 5.335 & 4.567 \\
\hline 43 & Hancock & .282 & .463 \\
\hline 44 & Harrison & .281 & .204 \\
\hline 45 & Hendricks & 1.591 & 1.093 \\
\hline 46 & Henry & .247 & .464 \\
\hline 47 & Howard & .773 & .507 \\
\hline 48 & Huntington & .305 & .347 \\
\hline 49 & Jackson & .322 & .375 \\
\hline 50 & Jasper & .159 & .163 \\
\hline 51 & Jay & .073 & .150 \\
\hline
\end{tabular}




\begin{tabular}{|c|c|c|c|}
\hline & \begin{tabular}{|l} 
Locale \\
\end{tabular} & P38 15 & A38 15 \\
\hline 52 & Jefferson & .212 & .168 \\
\hline 53 & Jennings & .096 & 1.207 \\
\hline 54 & \begin{tabular}{|l} 
Johnson \\
\end{tabular} & .966 & .688 \\
\hline 55 & Knox & .424 & .451 \\
\hline 56 & Kosciusko & .664 & .664 \\
\hline 57 & LaGrange & .336 & .383 \\
\hline 58 & Lake & 6.487 & 4.553 \\
\hline 59 & La Porte & 1.027 & 1.011 \\
\hline 60 & Lawrence & .193 & .164 \\
\hline 61 & Madison & .726 & .682 \\
\hline 62 & Marion & 28.151 & 22.237 \\
\hline 63 & Marshall & .663 & .645 \\
\hline 64 & Martin & .026 & .049 \\
\hline 65 & Miami & .227 & .394 \\
\hline 66 & Monroe & .757 & 1.283 \\
\hline 67 & Montgomery & .207 & .174 \\
\hline 68 & Morgan & .484 & .320 \\
\hline 69 & Newton & .037 & .082 \\
\hline 70 & Noble & .269 & .795 \\
\hline 71 & Ohio & .000 & .000 \\
\hline 72 & Orange & .055 & .063 \\
\hline 73 & Owen & .080 & .218 \\
\hline 74 & Parke & .074 & .074 \\
\hline 75 & Perry & .068 & .212 \\
\hline 76 & Pike & .103 & .078 \\
\hline 77 & Porter & 1.755 & 1.422 \\
\hline 78 & Posey & .297 & .457 \\
\hline 79 & Pulaski & .131 & .234 \\
\hline 80 & Putnam & .065 & .067 \\
\hline 81 & Randolph & .130 & .099 \\
\hline 82 & Ripley & .494 & .359 \\
\hline 83 & Rush & .135 & .140 \\
\hline 84 & St. Joseph & 5.773 & 5.096 \\
\hline 85 & Scott & .081 & .534 \\
\hline 86 & Shelby & .887 & .521 \\
\hline 87 & Spencer & .085 & .189 \\
\hline 88 & Starke & .051 & .052 \\
\hline 89 & Steuben & .265 & .305 \\
\hline 90 & Sullivan & .073 & .132 \\
\hline 91 & Switzerland & .015 & .015 \\
\hline 92 & Tippecanoe & 1.009 & 1.149 \\
\hline 937 & Tipton & .088 & .133 \\
\hline 9411 & Union & .076 & .076 \\
\hline 95 & Vanderburgh & 4.662 & 4.422 \\
\hline $96 \mid$ & Vermillion & .013 & .053 \\
\hline 971 & Vigo & 1.179 & 1.074 \\
\hline 981 & Wabash & .192 & .170 \\
\hline 991 & Warren & .077 & .078 \\
\hline 1001 & Warrick & .180 & .249 \\
\hline 1011 & Washington & .109 & .060 \\
\hline 102 & Wayne & 1.201 & .838 \\
\hline
\end{tabular}

E112 


\begin{tabular}{|c|c|c|c|}
\hline & $\begin{array}{l}\text { Locale } \\
\end{array}$ & P38 15 & A38 15 \\
\hline 103 & Wells & .141 & 1.168 \\
\hline 104 & White & .170 & .312 \\
\hline 105 & Whitley & .241 & .183 \\
\hline 106 & lowa & 41.418 & 43.709 \\
\hline 107 & Kansas & 48.295 & 44.657 \\
\hline 108 & Kentucky_E & 29.676 & 35.113 \\
\hline 109 & Kentucky_W & 19.784 & 23.409 \\
\hline 110 & Louisiana & 59.681 & 54.293 \\
\hline 111 & Maine & 13.599 & 19.021 \\
\hline 112 & Maryland & 79.197 & 67.847 \\
\hline 113 & Massachusetts & 114.066 & 109.330 \\
\hline 114 & Michigan_E & 79.739 & 65.872 \\
\hline 115 & Michigan_W & 79.739 & 65.872 \\
\hline 116 & Minnesota & 118.999 & 98.549 \\
\hline 117 & Mississippi & 25.936 & 37.697 \\
\hline 118 & Missouri & 94.420 & 91.376 \\
\hline 119 & Montana & 10.736 & 10.548 \\
\hline 120 & Nebraska & 26.852 & 26.933 \\
\hline 121 & Nevada & 34.731 & 29.165 \\
\hline 122 & New Hampshire & 20.569 & 20.196 \\
\hline 123 & New Jersey & 192.704 & 200.121 \\
\hline 124 & New Mexico & 17.144 & 15.010 \\
\hline 125 & New York & 257.584 & 284.138 \\
\hline 126 & North Carolina & 146.875 & 195.925 \\
\hline 127 & North Dakota & 10.007 & 10.891 \\
\hline 128 & Ohio_N & 6.589 & 28.024 \\
\hline 129 & Ohio_M & 6.589 & 28.024 \\
\hline 130 & Ohio_s & 6.589 & 28.024 \\
\hline 131 & Oklahoma & 46.770 & 43.500 \\
\hline 132 & Oregon & 64.116 & 58.762 \\
\hline 133 & Pennsylvania & 178.622 & 169.145 \\
\hline 134 & Rhode Island & 16.187 & 15.861 \\
\hline 135 & South Carolina & 51.243 & 82.288 \\
\hline 136 & South Dakota & 10.575 & 12.495 \\
\hline 137 & Tennessee & 100.238 & 104.256 \\
\hline 138 & Texas & 433.244 & 367.456 \\
\hline 139 & Utah & 42.077 & 37.558 \\
\hline 140 & Vermont & 6.503 & 7.702 \\
\hline 141 & Virginia & 94.022 & 89.971 \\
\hline 142 & Washington & 99.268 & 91.945 \\
\hline 143 & West Virginia & 18.380 & 14.458 \\
\hline 144 & Wisconsin & 89.779 & 83.012 \\
\hline 145 & Wyoming & 4.990 & 4.183 \\
\hline
\end{tabular}




\begin{tabular}{|c|c|c|c|}
\hline & Locale & P39 15 & A39 15 \\
\hline 1 & Alabama & 676.370 & 525.001 \\
\hline 2 & Arizona & 674.001 & 793.235 \\
\hline 3 & Arkansas & 539.787 & 331.111 \\
\hline 4 & California & 3868.734 & 4411.037 \\
\hline 5 & Colorado & 286.849 & 540.727 \\
\hline 6 & Connecticut & 435.335 & 409.836 \\
\hline 7 & Delaware & 71.163 & 101.948 \\
\hline 8 & DC & .000 & .000 \\
\hline 9 & Florida & 1124.151 & 2309.489 \\
\hline 10 & Georgia & 989.116 & 1100.877 \\
\hline 11 & Idaho & 86.813 & 177.398 \\
\hline 12 & Illinois_N & 717.161 & 982.035 \\
\hline 13 & Illinois_S & 358.581 & 491.017 \\
\hline 14 & Adams & 25.618 & 4.272 \\
\hline 15 & Allen & 95.140 & 43.224 \\
\hline 16 & Bartholomew & 50.967 & 9.251 \\
\hline 17 & Benton & 1.897 & .991 \\
\hline 18 & Blackford & .639 & 1.511 \\
\hline 19 & Boone & 5.164 & 7.059 \\
\hline 20 & Brown & .065 & 1.836 \\
\hline 21 & Carroll & 13.060 & 2.376 \\
\hline 22 & Cass & 19.561 & 4.729 \\
\hline 23 & Clark & 40.258 & 12.582 \\
\hline 24 & Clay & 11.720 & 3.117 \\
\hline 25 & Clinton & 5.681 & 4.084 \\
\hline 26 & Crawford & .000 & 1.390 \\
\hline 27 & Daviess & 2.612 & 3.577 \\
\hline 28 & Dearborn & .000 & 6.790 \\
\hline 29 & Decatur & 9.713 & 2.921 \\
\hline 30 & DeKalb & 9.040 & 5.330 \\
\hline 31 & Delaware & 26.788 & 14.310 \\
\hline 32 & Dubois & 236.806 & 4.942 \\
\hline 33 & Elkhart & 290.058 & 23.752 \\
\hline 34 & Fayette & 24.364 & 2.858 \\
\hline 35 & Floyd & 19.337 & 9.450 \\
\hline 36 & Fountain & 4.815 & 2.040 \\
\hline 37 & Franklin & 2.691 & 2.750 \\
\hline 38 & Fulton & .390 & 2.432 \\
\hline 39 & Gibson & 1.229 & 3.836 \\
\hline 40 & Grant & 23.761 & 7.878 \\
\hline 41 & Greene & .695 & 3.918 \\
\hline 42 & Hamilton & 19.767 & 34.135 \\
\hline 43 & Hancock & 14.688 & 8.481 \\
\hline 44 & Harrison & 31.655 & 4.857 \\
\hline 45 & Hendricks & 2.770 & 18.863 \\
\hline 46 & Henry & 15.615 & 5.338 \\
\hline 47 & Howard & 125.407 & 10.034 \\
\hline 48 & Huntington & 15.008 & 4.841 \\
\hline 49 & Jackson & 18.046 & 4.908 \\
\hline 50 & Jasper & 1.517 & 3.697 \\
\hline 51 & Jay & 4.148 & 2.415 \\
\hline
\end{tabular}




\begin{tabular}{|c|c|c|c|}
\hline & Locale & P39 15 & A39 15 \\
\hline 52 & Jefferson & 11.606 & 3.945 \\
\hline 53 & Jennings & 5.339 & 3.563 \\
\hline 54 & Johnson & 14.677 & 17.973 \\
\hline 55 & Knox & .360 & 4.432 \\
\hline 56 & Kosciusko & 22.604 & 9.079 \\
\hline 57 & LaGrange & 19.842 & 4.806 \\
\hline 58 & Lake & 20.934 & 55.681 \\
\hline 59 & La Porte & 10.401 & 12.647 \\
\hline 60 & Lawrence & 11.210 & 5.288 \\
\hline 61 & Madison & 58.556 & 16.002 \\
\hline 62 & Marion & 144.720 & 103.896 \\
\hline 63 & Marshall & 15.641 & 5.717 \\
\hline 64 & Martin & .000 & 1.149 \\
\hline 65 & Miami & 11.635 & 3.995 \\
\hline 66 & Monroe & 3.790 & 15.996 \\
\hline 67 & Montgomery & 9.194 & 4.544 \\
\hline 68 & Morgan & 1.732 & 9.356 \\
\hline 69 & Newton & 3.047 & 1.698 \\
\hline 70 & \begin{tabular}{|l} 
Noble \\
\end{tabular} & 22.736 & 5.731 \\
\hline 71 & \begin{tabular}{|l} 
Ohio \\
\end{tabular} & .000 & .657 \\
\hline 72 & Orange & 55.191 & 2.329 \\
\hline 73 & Owen & .000 & 2.731 \\
\hline 74 & Parke & .319 & 1.963 \\
\hline 75 & Perry & 8.876 & 2.085 \\
\hline 76 & Pike & .000 & 1.539 \\
\hline 77 & Porter & 1.771 & 17.721 \\
\hline 78 & Posey & .000 & 3.259 \\
\hline 79 & Pulaski & .399 & 1.602 \\
\hline 80 & Putnam & 5.019 & 4.482 \\
\hline 81 & Randolph & 4.913 & 3.054 \\
\hline 82 & Ripley & 6.024 & 3.274 \\
\hline 83 & Rush & .363 & 1.986 \\
\hline 84 & St. Joseph & 33.167 & 33.047 \\
\hline 85 & Scott & 2.402 & 2.853 \\
\hline 86 & Shelby & 15.959 & 5.138 \\
\hline 87 & Spencer & 24.651 & 2.349 \\
\hline 88 & Starke & 2.138 & 2.709 \\
\hline 89 & Steuben & 17.984 & 4.039 \\
\hline 90 & Sullivan & 1.164 & 2.591 \\
\hline 91 & Switzerland & .391 & 1.172 \\
\hline 92 & Tippecanoe & 72.499 & 20.199 \\
\hline 93 & Tipton & 2.358 & 1.872 \\
\hline 94 & Union & .000 & .844 \\
\hline 95 & Vanderburgh & 14.260 & 20.477 \\
\hline 96 & \begin{tabular}{|l|} 
Vermillion \\
\end{tabular} & .000 & 1.854 \\
\hline 97 & Vigo & 4.416 & 12.046 \\
\hline 98 & Wabash & 2.914 & 3.985 \\
\hline 99 & Warren & .400 & .953 \\
\hline 100 & Warrick & 1.595 & 7.080 \\
\hline 101 & Washington & 60.232 & 3.404 \\
\hline 102 & Wayne & 28.999 & 7.726 \\
\hline
\end{tabular}




\begin{tabular}{|c|c|c|c|}
\hline & Locale & P39 15 & A39 15 \\
\hline 103 & Wells & 3.036 & 3.420 \\
\hline 104 & White & 16.944 & 2.945 \\
\hline 105 & Whitley & 10.309 & 3.957 \\
\hline 106 & lowa & 398.858 & 342.730 \\
\hline 107 & Kansas & 514.075 & 320.532 \\
\hline 108 & Kentucky_E & 306.540 & 292.667 \\
\hline 109 & Kentucky_W & 204.360 & 195.110 \\
\hline 110 & Louisiana & 203.977 & 529.989 \\
\hline 111 & Maine & 180.797 & 156.686 \\
\hline 112 & Maryland & 233.430 & 692.948 \\
\hline 113 & Massachusetts & 324.540 & 759.699 \\
\hline 114 & Michigan_E & 2908.806 & 599.561 \\
\hline 115 & Michigan_W & 1454.403 & 599.561 \\
\hline 116 & Minnesota & 532.015 & 629.112 \\
\hline 117 & Mississippi & 1168.182 & 337.313 \\
\hline 118 & Missouri & 911.237 & 681.554 \\
\hline 119 & Montana & 33.537 & 113.002 \\
\hline 120 & Nebraska & 155.376 & 202.034 \\
\hline 121 & Nevada & 97.787 & 309.472 \\
\hline 122 & New Hampshire & 73.148 & 160.710 \\
\hline 123 & New Jersey & 369.005 & 1036.300 \\
\hline 124 & New Mexico & 52.141 & 228.327 \\
\hline 125 & New York & 980.576 & 2202.796 \\
\hline 126 & North Carolina & 3323.374 & 1091.546 \\
\hline 127 & North Dakota & 52.379 & 73.655 \\
\hline 128 & Ohio_N & 604.066 & 441.609 \\
\hline 129 & Ohio_M & $604: 066$ & 441.609 \\
\hline 130 & Ohio_s & 604.066 & 441.609 \\
\hline 131 & Oklahoma & 255.921 & 410.271 \\
\hline 132 & Oregon & 344.378 & 444.245 \\
\hline 133 & Pennsylvania & 1153.355 & 1450.693 \\
\hline 134 & Rhode Island & 85.943 & 127.760 \\
\hline 135 & South Carolina & 389.682 & 511.910 \\
\hline 136 & South Dakota & 70.529 & 90.067 \\
\hline 137 & Tennessee & 1316.535 & 720.406 \\
\hline 138 & Texas & 1651.382 & 2884.992 \\
\hline 139 & Utah & 354.475 & 302.813 \\
\hline 140 & Vermont & 116.502 & 75.706 \\
\hline 141 & Virginia & 1156.373 & 936.341 \\
\hline 142 & Washington & 994.811 & 767.070 \\
\hline 143 & West Virginia & 58.805 & 210.180 \\
\hline 144 & Wisconsin & 895.567 & 662.067 \\
\hline 145 & Wyoming & 9.901 & 59.992 \\
\hline
\end{tabular}




\begin{tabular}{|c|c|c|c|}
\hline & Locale & P40 15 & A40 15 \\
\hline 1 & Alabama & 3174.258 & 3557.862 \\
\hline 2 & Arizona & 2361.211 & 3171.555 \\
\hline 3 & Arkansas & 2772.585 & 1952.989 \\
\hline 4 & California & 17845.220 & 16807.217 \\
\hline 5 & Colorado & 2151.435 & 1992.358 \\
\hline 6 & Connecticut & 1388.283 & 1468.858 \\
\hline 7 & Delaware & 625.668 & 412.012 \\
\hline 8 & DC & .000 & .000 \\
\hline 9 & Florida & 7569.730 & 8554.506 \\
\hline 10 & Georgia & 10547.272 & 6679.386 \\
\hline 11 & Idaho & 1090.461 & 1120.040 \\
\hline 12 & Illinois_N & 4388.028 & 3488.805 \\
\hline 13 & Illinois_S & 2194.014 & 1744.403 \\
\hline 14 & Adams & 21.995 & 45.201 \\
\hline 15 & Allen & 190.878 & 149.622 \\
\hline 16 & Bartholomew & 67.538 & 33.034 \\
\hline 17 & Benton & 2.263 & 11.442 \\
\hline 18 & Blackford & 5.185 & 8.357 \\
\hline 19 & Boone & 16.540 & 22.730 \\
\hline 20 & Brown & 4.514 & 7.260 \\
\hline 21 & Carroll & 64.222 & 7.652 \\
\hline 22 & Cass & 66.492 & 23.362 \\
\hline 23 & Clark & 45.309 & 77.090 \\
\hline 24 & Clay & 7.441 & 13.047 \\
\hline 25 & Clinton & 79.240 & 14.859 \\
\hline 26 & Crawford & 3.172 & 7.955 \\
\hline 27 & Daviess & 43.018 & 23.462 \\
\hline 28 & Dearborn & 15.899 & 22.477 \\
\hline 29 & Decatur & 18.822 & 10.726 \\
\hline 30 & DeKalb & 14.341 & 54.586 \\
\hline 31 & Delaware & 37.836 & 49.169 \\
\hline 32 & Dubois & 39.410 & 106.124 \\
\hline 33 & Elkhart & 137.439 & 423.680 \\
\hline 34 & Fayette & 6.526 & 9.204 \\
\hline 35 & Floyd & 46.961 & 62.507 \\
\hline 36 & Fountain & 6.575 & 7.064 \\
\hline 37 & Franklin & 6.635 & 11.010 \\
\hline 38 & Fulton & 10.565 & 20.995 \\
\hline 39 & Gibson & 21.869 & 12.351 \\
\hline 40 & Grant & 30.676 & 28.012 \\
\hline 41 & Greene & 21.686 & 15.536 \\
\hline 42 & Hamilton & 106.427 & 118.655 \\
\hline 43 & Hancock & 19.781 & 29.121 \\
\hline 44 & Harrison & 39.685 & 26.016 \\
\hline 45 & Hendricks & 67.418 & 65.339 \\
\hline 46 & Henry & 19.294 & 19.938 \\
\hline 47 & Howard & 28.030 & 35.298 \\
\hline 48 & Huntington & 25.216 & 20.187 \\
\hline 49 & Jackson & 13.359 & 30.654 \\
\hline 50 & Jasper & 15.990 & 15.486 \\
\hline 51 & Jay & 19.095 & 10.616 \\
\hline
\end{tabular}




\begin{tabular}{|c|c|c|c|}
\hline & Locale & P40 15 & A40 15 \\
\hline 52 & Jefferson & 10.704 & 16.537 \\
\hline 53 & Jennings & 47.998 & 15.310 \\
\hline 54 & Johnson & 42.619 & 125.654 \\
\hline 55 & Knox & 14.064 & 17.786 \\
\hline 56 & Kosciusko & 58.123 & 45.676 \\
\hline 57 & LaGrange & 18.405 & 119.438 \\
\hline 58 & Lake & 169.077 & 191.036 \\
\hline 59 & La Porte & 47.702 & 52.361 \\
\hline 60 & Lawrence & 14.863 & 22.592 \\
\hline 61 & Madison & 62.876 & 60.758 \\
\hline 62 & Marion & 404.972 & 372.225 \\
\hline 63 & Marshall & 34.226 & 53.172 \\
\hline 64 & Martin & 4.422 & 4.164 \\
\hline 65 & Miami & 11.813 & 19.115 \\
\hline 66 & Monroe & 46.472 & 54.840 \\
\hline 67 & Montgomery & 22.827 & 16.788 \\
\hline 68 & Morgan & 22.893 & 40.976 \\
\hline 69 & Newton & 4.196 & 5.467 \\
\hline 70 & Noble & 56.573 & 21.619 \\
\hline 71 & Ohio & 1.501 & 2.117 \\
\hline 72 & Orange & 7.200 & 29.704 \\
\hline 73 & Owen & 12.724 & 15.347 \\
\hline 74 & Parke & 10.033 & 14.911 \\
\hline 75 & Perry & 10.491 & 9.515 \\
\hline 76 & Pike & 3.514 & 8.035 \\
\hline 77 & Porter & 52.166 & 64.011 \\
\hline 78 & Posey & 15.486 & 11.011 \\
\hline 79 & Pulaski & 5.942 & 5.671 \\
\hline 80 & Putnam & 10.564 & 17.523 \\
\hline 81 & Randolph & 9.327 & 28.952 \\
\hline 82 & Ripley & 7.814 & 16.940 \\
\hline 83 & Rush & 6.221 & 9.193 \\
\hline 84 & St. Joseph & 115.884 & 132.818 \\
\hline 85 & Scott & 36.410 & 12.270 \\
\hline 86 & Shelby & 17.339 & 16.544 \\
\hline 87 & Spencer & 7.238 & 14.868 \\
\hline 88 & Starke & 6.186 & 24.190 \\
\hline 89 & Steuben & 23.529 & 15.517 \\
\hline 90 & Sullivan & 5.915 & 12.901 \\
\hline 91 & Switzerland & 2.676 & 3.773 \\
\hline 92 & Tippecanoe & 77.033 & 67.999 \\
\hline 93 & Tipton & 9.753 & 8.935 \\
\hline 94 & Union & 1.927 & 3.222 \\
\hline 95 & Vanderburgh & 204.238 & 78.914 \\
\hline 96 & Vermillion & 4.472 & 5.969 \\
\hline 97 & Vigo & 36.554 & 44.681 \\
\hline 98 & Wabash & 11.847 & 21.102 \\
\hline 99 & Warren & 2.507 & 6.152 \\
\hline 100 & Warrick & 17.222 & 29.677 \\
\hline 101 & Washington & 9.824 & 22.930 \\
\hline 102 & Wayne & 38.483 & 27.620 \\
\hline
\end{tabular}




\begin{tabular}{|c|c|c|c|}
\hline & Locale & P40 15 & A40 15 \\
\hline 103 & Wells & 26.857 & 14.521 \\
\hline 104 & White & 9.037 & 14.706 \\
\hline 105 & Whitley & 9.384 & 22.239 \\
\hline 106 & lowa & 2400.119 & 1540.123 \\
\hline 107 & Kansas & 1875.280 & 1193.605 \\
\hline 108 & Kentucky_E & 1350.714 & 1339.687 \\
\hline 109 & Kentucky_W & 900.476 & 893.125 \\
\hline 110 & Louisiana & 1905.753 & 2200.569 \\
\hline$\overline{111}$ & Maine & 754.718 & 1037.664 \\
\hline 112 & Maryland & 2288.985 & 2500.724 \\
\hline 113 & Massachusetts & 2942.427 & 2886.417 \\
\hline 114 & Michigan_E & 2052.479 & 2295.233 \\
\hline 115 & Michigan_W & 2052.479 & 2295.233 \\
\hline 116 & Minnesota & 3169.346 & 2884.573 \\
\hline 117 & Mississippi & 2092.038 & 2112.812 \\
\hline 118 & Missouri & 3130.462 & 2752.145 \\
\hline 119 & Montana & 347.971 & 655.911 \\
\hline 120 & Nebraska & 1629.140 & 788.059 \\
\hline 121 & Nevada & 900.120 & 1073.587 \\
\hline 122 & New Hampshire & 583.687 & 799.423 \\
\hline 123 & New Jersey & 3915.854 & 3697.236 \\
\hline 124 & New Mexico & 671.396 & 820.783 \\
\hline 125 & New York & 7495.315 & 7884.111 \\
\hline 126 & North Carolina & 7179.530 & 8613.983 \\
\hline 127 & North Dakota & 357.263 & 286.078 \\
\hline 128 & Ohio_N & 1678.781 & 1798.432 \\
\hline 129 & Ohio_M & 1678.781 & 1798.432 \\
\hline 130 & Ohio_S & 1678.781 & 1798.432 \\
\hline 131 & Oklahoma & 1565.598 & 1492.401 \\
\hline 132 & Oregon & 1861.038 & 3454.628 \\
\hline 133 & Pennsylvania & 6558.454 & 6226.026 \\
\hline 134 & Rhode Island & 508.590 & 580.174 \\
\hline 135 & South Carolina & 3151.849 & 3821.959 \\
\hline 136 & South Dakota & 547.769 & 384.044 \\
\hline 137 & Tennessee & 3555.638 & 3671.975 \\
\hline 138 & Texas & 11111.517 & 11298.369 \\
\hline 139 & Utah & 1293.369 & 1113.048 \\
\hline 140 & Vermont & 317.191 & 425.890 \\
\hline 141 & Virginia & 3885.130 & 4760.816 \\
\hline 142 & Washington & 3194.471 & 3718.496 \\
\hline 143 & West Virginia & 658.528 & 1067.870 \\
\hline 144 & Wisconsin & 3731.687 & 3658.716 \\
\hline 145 & Wyoming & 168.247 & 248.008 \\
\hline
\end{tabular}




\begin{tabular}{|c|c|c|c|}
\hline & Locale & P41 15 & A41 15 \\
\hline 1 & Alabama & 4753.382 & 6570.908 \\
\hline 2 & Arizona & 4723.599 & 3986.720 \\
\hline 3 & Arkansas & 3762.730 & 5852.010 \\
\hline 4 & California & 34266.721 & 29152.102 \\
\hline 5 & Colorado & 2750.114 & 2738.861 \\
\hline 6 & Connecticut & 6819.435 & 4472.177 \\
\hline 7 & Delaware & 463.881 & 907.057 \\
\hline 8 & $\mathrm{DC}$ & .000 & .000 \\
\hline 9 & Florida & 7868.056 & 7098.562 \\
\hline 10 & Georgia & 6062.147 & 7761.509 \\
\hline 11 & Idaho & 756.011 & 1475.221 \\
\hline 12 & Illinois_N & 13746.134 & 12494.746 \\
\hline 13 & Illinois_S & 6873.067 & 6247.373 \\
\hline 14 & Adams & 66.106 & 71.899 \\
\hline 15 & Allen & 493.827 & 604.092 \\
\hline 16 & Bartholomew & 171.967 & 236.534 \\
\hline 17 & Benton & 10.393 & 10.639 \\
\hline 18 & Blackford & 87.346 & 46.248 \\
\hline 19 & Boone & 60.157 & 30.554 \\
\hline 20 & Brown & 9.049 & 5.031 \\
\hline 21 & Carroll & 67.828 & 132.532 \\
\hline 22 & Cass & 103.963 & 143.661 \\
\hline 23 & Clark & 178.358 & 119.355 \\
\hline 24 & Clay & 16.661 & 9.782 \\
\hline 25 & Clinton & 39.571 & 137.215 \\
\hline 26 & Crawford & .000 & .000 \\
\hline 27 & Daviess & 25.008 & 71.020 \\
\hline 28 & Dearborn & 41.396 & 21.203 \\
\hline 29 & Decatur & 437.636 & 238.518 \\
\hline 30 & DeKalb & 545.245 & 532.924 \\
\hline 31 & Delaware & 292.259 & 223.692 \\
\hline 32 & Dubois & 24.130 & 57.562 \\
\hline 33 & Elkhart & 1026.049 & 674.054 \\
\hline 34 & Fayette & 52.479 & 31.423 \\
\hline 35 & Floyd & 102.435 & 94.559 \\
\hline 36 & Fountain & 8.960 & 79.567 \\
\hline 37 & Franklin & 1.670 & 1.429 \\
\hline 38 & Fulton & 128.335 & 108.483 \\
\hline 39 & Gibson & 21.569 & 32.751 \\
\hline 40 & Grant & 23.059 & 39.123 \\
\hline 41 & Greene & 13.673 & 43.140 \\
\hline 42 & Hamilton & 238.824 & 187.405 \\
\hline 43 & Hancock & 89.826 & 46.519 \\
\hline 44 & Harrison & 13.640 & 54.860 \\
\hline 45 & Hendricks & 46.593 & 87.613 \\
\hline 46 & Henry & 48.431 & 60.324 \\
\hline 47 & Howard & 31.469 & 96.720 \\
\hline 48 & Huntington & 163.607 & 120.948 \\
\hline 49 & Jackson & 105.409 & 102.740 \\
\hline 50 & Jasper & 105.861 & 65.212 \\
\hline 51 & Jay & 135.370 & 89.993 \\
\hline
\end{tabular}




\begin{tabular}{|c|c|c|c|}
\hline & Locale & P41 15 & A41 15 \\
\hline 52 & Jefferson & 22.050 & 13.098 \\
\hline 53 & Jennings & 105.648 & 58.786 \\
\hline 54 & Johnson & 216.126 & 146.327 \\
\hline 55 & Knox & 16.340 & 48.360 \\
\hline 56 & Kosciusko & 225.266 & 339.690 \\
\hline 57 & LaGrange & 35.569 & 29.414 \\
\hline 58 & Lake & 378.833 & 2261.754 \\
\hline 59 & La Porte & 324.883 & 342.815 \\
\hline 60 & Lawrence & 83.299 & 207.197 \\
\hline 61 & Madison & 159.898 & 142.883 \\
\hline 62 & Marion & 1557.176 & 1348.109 \\
\hline 63 & Marshall & 167.090 & 147.913 \\
\hline 64 & Martin & 1.401 & 19.461 \\
\hline 65 & Miami & 36.926 & 21.338 \\
\hline 66 & Monroe & 64.984 & 38.098 \\
\hline 67 & Montgomery & 99.643 & 145.103 \\
\hline 68 & Morgan & 58.015 & 36.320 \\
\hline 69 & Newton & 8.953 & 21.742 \\
\hline 70 & Noble & 223.004 & 230.088 \\
\hline 71 & Ohio & 1.621 & .804 \\
\hline 72 & \begin{tabular}{|l} 
Orange \\
\end{tabular} & 8.804 & 7.536 \\
\hline 73 & Owen & 2.075 & 1.604 \\
\hline 74 & Parke & 1.482 & 10.071 \\
\hline 75 & Perry & 8.457 & 38.633 \\
\hline 76 & Pike & 9.298 & 10.581 \\
\hline 77 & Porter & 240.811 & 950.841 \\
\hline 78 & Posey & 9.400 & 8.046 \\
\hline 79 & Pulaski & 27.081 & 34.661 \\
\hline 80 & Putnam & 1.557 & 1.333 \\
\hline 81 & Randolph & 15.859 & 39.800 \\
\hline 82 & Ripley & 33.250 & 17.064 \\
\hline 83 & Rush & 8.454 & 39.430 \\
\hline 84 & St. Joseph & 681.645 & 550.615 \\
\hline 85 & Scott & 13.661 & 28.724 \\
\hline 86 & Shelby & 54.960 & 88.127 \\
\hline 87 & Spencer & 8.766 & 23.917 \\
\hline 88 & Starke & .000 & .000 \\
\hline 89 & Steuben & 209.873 & 167.924 \\
\hline 90 & Sullivan & 1.547 & .767 \\
\hline 91 & Switzerland & .000 & .000 \\
\hline 92 & Tippecanoe & 106.788 & 290.606 \\
\hline 93 & Tipton & 18.142 & 23.849 \\
\hline 94 & Union & 1.524 & .756 \\
\hline 95 & Vanderburgh & 305.809 & 370.047 \\
\hline 96 & Vermillion & 24.551 & 12.583 \\
\hline 97 & Vigo & 101.964 & 100.799 \\
\hline 98 & Wabash & 144.834 & 143.629 \\
\hline 99 & Warren & 19.252 & 10.109 \\
\hline 100 & Warrick & 38.424 & 416.060 \\
\hline 101 & Washington & 97.408 & 52.801 \\
\hline 102 & Wayne & 87.881 & 159.995 \\
\hline
\end{tabular}




\begin{tabular}{|c|c|c|c|}
\hline & Locale & P41 15 & A41 15 \\
\hline 103 & Wells & 100.352 & 98.496 \\
\hline 104 & White & 141.150 & 78.072 \\
\hline 105 & Whitley & 114.912 & 121.470 \\
\hline 106 & lowa & 3870.787 & 5417.106 \\
\hline 107 & Kansas & 2255.953 & 3121.400 \\
\hline 108 & Kentucky_E & 2433.847 & 2846.347 \\
\hline 109 & Kentucky_W & 1622.564 & 1897.564 \\
\hline 110 & Louisiana & 3181.341 & 2931.313 \\
\hline 111 & Maine & 791.351 & 834.754 \\
\hline 112 & Maryland & 2364.391 & 3048.547 \\
\hline 113 & Massachusetts & 7392.330 & 5482.052 \\
\hline 114 & Michigan_E & 8234.981 & 6877.340 \\
\hline 115 & Michigan_W & 8234.981 & 6877.340 \\
\hline 116 & Minnesota & 8356.599 & 7582.120 \\
\hline 117 & Mississippi & 2326.603 & 2980.071 \\
\hline 118 & Missouri & 6449.174 & 6709.437 \\
\hline 119 & Montana & 218.740 & 364.597 \\
\hline 120 & Nebraska & 1552.074 & 2782.663 \\
\hline 121 & Nevada & 1077.986 & 993.532 \\
\hline 122 & New Hampshire & 2246.987 & 1650.076 \\
\hline 123 & New Jersey & 6291.424 & 5912.736 \\
\hline 124 & New Mexico & 493.685 & 672.065 \\
\hline 125 & New York & 10700.393 & 9684.475 \\
\hline 126 & North Carolina & 7995.986 & 8352.023 \\
\hline 127 & North Dakota & 284.260 & 435.875 \\
\hline 128 & Ohio_N & 7294.927 & 6920.752 \\
\hline 129 & Ohio_M & 7294.927 & 6920.752 \\
\hline 130 & Ohio_S & 7294.927 & 6920.752 \\
\hline 131 & Oklahoma & 4293.572 & 3484.273 \\
\hline 132 & Oregon & 3290.276 & 4059.171 \\
\hline 133 & Pennsylvania & 15740.445 & 18091.748 \\
\hline 134 & Rhode Island & 1700.407 & 1357.058 \\
\hline 135 & South Carolina & 5219.143 & 4320.684 \\
\hline 136 & South Dakota & 530.234 & 774.250 \\
\hline 137 & Tennessee & 7471.406 & 7740.850 \\
\hline 138 & Texas & 22564.185 & 20526.910 \\
\hline 139 & Utah & 1942.860 & 2560.903 \\
\hline 140 & Vermont & 585.853 & 539.532 \\
\hline 141 & Virginia & 3771.735 & 4826.094 \\
\hline 142 & Washington & 3915.596 & 5496.512 \\
\hline 143 & West Virginia & 1040.894 & 1870.246 \\
\hline 144 & Wisconsin & 12525.373 & 12339.968 \\
\hline 145 & Wyoming & 139.759 & 124.376 \\
\hline
\end{tabular}




\begin{tabular}{|c|c|c|c|}
\hline & Locale & P43 15 & A43 15 \\
\hline 1 & Alabama & 2524.932 & 2784.643 \\
\hline 2 & Arizona & 3814.975 & 3598.354 \\
\hline 3 & Arkansas & 1592.442 & 1595.680 \\
\hline 4 & California & 21214.394 & 20828.204 \\
\hline 5 & Colorado & 2600.568 & 2535.589 \\
\hline 6 & Connecticut & 1971.059 & 1904.191 \\
\hline 7 & Delaware & 490.308 & 475.620 \\
\hline 8 & $\mathrm{DC}$ & .000 & .000 \\
\hline 9 & Florida & 11107.233 & 10672.870 \\
\hline 10 & Georgia & 5294.544 & 7982.612 \\
\hline 11 & Idaho & 853.175 & 812.180 \\
\hline 12 & Illinois_N & 4722.985 & 4527.331 \\
\hline 13 & Illinois_S & 2361.493 & 2263.666 \\
\hline 14 & Adams & 20.547 & 18.974 \\
\hline 15 & Allen & 207.879 & 210.336 \\
\hline 16 & Bartholomew & 44.492 & 41.658 \\
\hline 17 & Benton & 4.766 & 4.402 \\
\hline 18 & Blackford & 7.266 & 6.710 \\
\hline 19 & Boone & 33.951 & 31.352 \\
\hline 20 & Brown & 8.830 & 8.154 \\
\hline 21 & Carroll & 11.429 & 10.554 \\
\hline 22 & Cass & 22.745 & 21.516 \\
\hline 23 & Clark & 60.512 & 59.359 \\
\hline 24 & Clay & 14.993 & 13.845 \\
\hline 25 & Clinton & 19.642 & 18.138 \\
\hline 26 & Crawford & 6.683 & 6.172 \\
\hline 27 & Daviess & 17.203 & 15.886 \\
\hline 28 & Dearborn & 32.656 & 30.156 \\
\hline 29 & Decatur & 14.047 & 12.972 \\
\hline 30 & DeKalb & 25.636 & 23.674 \\
\hline 31 & Delaware & 68.821 & 64.098 \\
\hline 32 & Dubois & 23.767 & 22.503 \\
\hline 33 & Elkhart & 114.232 & 138.437 \\
\hline 34 & Fayette & 13.747 & 12.695 \\
\hline 35 & Floyd & 45.451 & 41.971 \\
\hline 36 & Fountain & 9.813 & 9.062 \\
\hline 37 & Franklin & 13.224 & 12.212 \\
\hline 38 & Fulton & 11.695 & 10.800 \\
\hline 39 & Gibson & 18.448 & 17.036 \\
\hline 40 & Grant & 37.886 & 37.786 \\
\hline 41 & Greene & 18.843 & 17.916 \\
\hline 42 & Hamilton & 164.169 & 164.003 \\
\hline 43 & Hancock & 40.788 & 37.666 \\
\hline 44 & Harrison & 23.360 & 21.572 \\
\hline 45 & Hendricks & 90.717 & 84.584 \\
\hline 46 & Henry & 25.674 & 26.616 \\
\hline 47 & Howard & 48.257 & 44.562 \\
\hline 48 & Huntington & 23.281 & 22.071 \\
\hline 49 & Jackson & 23.604 & 22.331 \\
\hline 50 & Jasper & 17.778 & 16.417 \\
\hline 51 & Jay & 11.614 & 10.725 \\
\hline
\end{tabular}




\begin{tabular}{|c|c|c|c|}
\hline & Locale & P43 15 & A43 15 \\
\hline 52 & Jefferson & 18.974 & 18.076 \\
\hline 53 & Jennings & 17.136 & 37.568 \\
\hline 54 & Johnson & 86.438 & 80.523 \\
\hline 55 & Knox & 21.317 & 19.685 \\
\hline 56 & Kosciusko & 43.667 & 43.949 \\
\hline 57 & LaGrange & 23.114 & 21.929 \\
\hline 58 & Lake & 267.791 & 250.438 \\
\hline 59 & La Porte & 60.823 & 56.688 \\
\hline 60 & Lawrence & 25.434 & 23.994 \\
\hline 61 & Madison & 76.958 & 72.131 \\
\hline 62 & Marion & 499.677 & 483.462 \\
\hline 63 & Marshall & 27.495 & 28.815 \\
\hline 64 & Martin & 5.526 & 5.103 \\
\hline 65 & Miami & 19.212 & 18.231 \\
\hline 66 & Monroe & 76.931 & 74.569 \\
\hline 67 & Montgomery & 21.852 & 20.179 \\
\hline 68 & Morgan & 44.998 & 42.180 \\
\hline 69 & Newton & 8.166 & 7.541 \\
\hline 70 & Noble & 27.561 & 35.219 \\
\hline 71 & Ohio & 3.162 & 2.920 \\
\hline 72 & Orange & 11.200 & 10.342 \\
\hline 73 & Owen & 13.134 & 15.482 \\
\hline 74 & Parke & 9.442 & 8.719 \\
\hline 75 & Perry & 10.029 & 12.223 \\
\hline 76 & Pike & 7.403 & 6.836 \\
\hline 77 & Porter & 85.225 & 79.245 \\
\hline 78 & Posey & 15.672 & 17.764 \\
\hline 79 & Pulaski & 7.705 & 7.115 \\
\hline 80 & Putnam & 21.554 & 19.904 \\
\hline 81 & Randolph & 14.688 & 13.564 \\
\hline 82 & Ripley & 15.748 & 14.542 \\
\hline 83 & Rush & 9.549 & 9.312 \\
\hline 84 & St. Joseph & 158.937 & 156.269 \\
\hline 85 & Scott & 13.720 & 22.182 \\
\hline 86 & Shelby & 24.712 & 22.821 \\
\hline 87 & Spencer & 11.295 & 10.431 \\
\hline 88 & Starke & 13.031 & 12.033 \\
\hline 89 & Steuben & 19.426 & 21.191 \\
\hline 90 & Sullivan & 12.460 & 11.506 \\
\hline 91 & Switzerland & 5.636 & 5.205 \\
\hline 92 & Tippecanoe & 97.147 & 93.394 \\
\hline 93 & Tipton & 9.004 & 8.314 \\
\hline 94 & Union & 4.059 & 3.748 \\
\hline 95 & Vanderburgh & 98.483 & 111.826 \\
\hline 96 & Vermillion & 8.915 & 8.233 \\
\hline 97 & Vigo & 57.933 & 54.031 \\
\hline 98 & Wabash & 19.168 & 18.200 \\
\hline 99 & Warren & 4.581 & 4.230 \\
\hline 100 & Warrick & 34.050 & 32.021 \\
\hline 101 & Washington & 16.370 & 15.117 \\
\hline 102 & Wayne & 37.156 & 34.311 \\
\hline
\end{tabular}




\begin{tabular}{|c|c|c|c|}
\hline & Locale & P43 15 & A43 15 \\
\hline 103 & Wells & 16.449 & 15.765 \\
\hline 104 & White & 14.165 & 13.606 \\
\hline 105 & Whitley & 19.029 & 17.572 \\
\hline 106 & lowa & 1648.321 & 1582.828 \\
\hline 107 & Kansas & 1541.563 & 1487.625 \\
\hline 108 & Kentucky_E & 1407.542 & 1446.433 \\
\hline 109 & |Kentucky_W & 938.361 & 964.288 \\
\hline 110 & Louisiana & 2548.924 & 2419.584 \\
\hline 111 & Maine & 753.564 & 794.018 \\
\hline 112 & Maryland & 3332.658 & 3120.312 \\
\hline 113 & Massachusetts & 3653.687 & 3638.557 \\
\hline 114 & Michigan_E & 2883.522 & 2737.909 \\
\hline 115 & Michigan_W & 2883.522 & 2737.909 \\
\hline 116 & Minnesota & 3025.643 & 2891.769 \\
\hline 117 & Mississippi & 1622.271 & 1733.471 \\
\hline 118 & Missouri & 3277.859 & 3172.455 \\
\hline 119 & Montana & 543.471 & 508.995 \\
\hline 120 & Nebraska & 971.659 & 919.119 \\
\hline 121 & Nevada & 1488.374 & 1399.959 \\
\hline 122 & New Hampshire & 772.916 & 787.292 \\
\hline 123 & New Jersey & 4983.970 & 4885.056 \\
\hline 124 & New Mexico & 1098.115 & 1017.516 \\
\hline 125 & New York & 10594.104 & 10265.572 \\
\hline 126 & North Carolina & 5249.671 & 6282.386 \\
\hline 127 & North Dakota & 354.236 & 338.420 \\
\hline 128 & Ohio_N & 2123.869 & 2035.951 \\
\hline 129 & Ohio_M & 2123.869 & 2035.951 \\
\hline 130 & Ohio_s & 2123.869 & 2035.951 \\
\hline 131 & Oklahoma & 1973.154 & 1900.001 \\
\hline 132 & Oregon & 2136.548 & 2037.653 \\
\hline 133 & Pennsylvania & 6976.948 & 6853.690 \\
\hline 134 & Rhode Island & 614.448 & 633.162 \\
\hline 135 & South Carolina & 2461.974 & 3042.370 \\
\hline 136 & South Dakota & 433.165 & 440.769 \\
\hline 137 & Tennessee & 3464.714 & 3530.240 \\
\hline 138 & Texas & 13875.051 & 13392.359 \\
\hline 139 & Utah & 1456.345 & 1416.068 \\
\hline 140 & Vermont & 364.098 & 343.340 \\
\hline 141 & Virginia & 4503.227 & 4398.082 \\
\hline 142 & Washington & 3689.139 & 3513.686 \\
\hline 143 & West Virginia & 1010.840 & 939.942 \\
\hline 144 & Wisconsin & 3184.137 & 3067.192 \\
\hline 145 & Wyoming & 288.527 & 269.742 \\
\hline
\end{tabular}




\section{APPENDIX F}

PRODUCTIONS AND ATTRACTIONS 2030 


\section{Table Notes}

The tables in this appendix give the projected volume of traffic produced and attracted by areas included in this study for each commodity. The area appears under the column label Locale and includes the 145 different areas included in the freight study. From line 14 to line 105 we have the counties of Indiana. States represented by two or more nodes are evident here as well.

The data columns labeled $\mathrm{P}$ are productions and those labeled $\mathrm{A}$ are attractions. The next two digits represent the commodity groups SCTG 1, SCTG 2, and so forth. The 30 represents the forecast year of the data, 2030.

The data are actually given here to three decimal places. This is because the data as published is in thousands of tons. In many cases this would completely eliminate flows into Indiana counties of several goods. It is for this reason that three decimal places were used. Programs used later would multiply flow estimates by 1,000 to yield tons of commodities. The reader should judge the reliability of the data given these facts. 


\begin{tabular}{|c|c|c|c|}
\hline & Locale & P01 30 & A01 30 \\
\hline 1 & Alabama & 354.888 & 351.339 \\
\hline 2 & Arizona & 359.631 & 172.821 \\
\hline 3 & Arkansas & 319.427 & 572.501 \\
\hline 4 & California & 2006.389 & 1870.501 \\
\hline 5 & Colorado & 148.045 & 225.953 \\
\hline 6 & Connecticut & 70.476 & 88.921 \\
\hline 7 & Delaware & 18.779 & 112.504 \\
\hline 8 & DC & .000 & .000 \\
\hline 9 & Florida & 721.926 & 609.360 \\
\hline 10 & Georgia & 471.180 & 814.647 \\
\hline 11 & Idaho & 58.285 & 227.290 \\
\hline 12 & Illinois_N & 314.877 & 554.466 \\
\hline 13 & Illinois_s & 157.439 & 277.233 \\
\hline 14 & Adams & 7.099 & 3.981 \\
\hline 15 & Allen & 26.606 & 18.950 \\
\hline 16 & Bartholomew & 19.788 & 14.656 \\
\hline 17 & Benton & 1.212 & .000 \\
\hline 18 & Blackford & .181 & .465 \\
\hline 19 & Boone & 2.189 & .156 \\
\hline 20 & Brown & .000 & .097 \\
\hline 21 & Carroll & 9.184 & 18.355 \\
\hline 22 & Cass & .213 & 15.984 \\
\hline 23 & Clark & 30.225 & 3.332 \\
\hline 24 & Clay & .229 & .098 \\
\hline 25 & Clinton & 1.459 & 21.752 \\
\hline 26 & Crawford & .000 & .000 \\
\hline 27 & Daviess & 1.527 & 11.077 \\
\hline 28 & Dearborn & .000 & .141 \\
\hline 29 & Decatur & .235 & 3.718 \\
\hline 30 & DeKalb & .281 & .721 \\
\hline 31 & Delaware & 3.403 & 1.292 \\
\hline 32 & Dubois & 164.754 & 8.544 \\
\hline 33 & Elkhart & 102.536 & 7.395 \\
\hline 34 & Fayette & 1.169 & .000 \\
\hline 35 & Floyd & 11.710 & 8.208 \\
\hline 36 & Fountain & .000 & .571 \\
\hline 37 & Franklin & .000 & .117 \\
\hline 38 & Fulton & .000 & 1.512 \\
\hline 39 & Gibson & .000 & 4.234 \\
\hline 40 & Grant & 3.068 & 1.990 \\
\hline 41 & Greene & .216 & 3.463 \\
\hline 42 & Hamilton & 9.866 & 2.153 \\
\hline 43 & Hancock & 2.150 & .153 \\
\hline 44 & Harrison & 21.094 & 10.092 \\
\hline 45 & Hendricks & .464 & 9.167 \\
\hline 46 & Henry & 3.329 & .489 \\
\hline 47 & Howard & 8.475 & 1.539 \\
\hline 48 & Huntington & .000 & 4.236 \\
\hline 49 & Jackson & 1.391 & .357 \\
\hline 50 & Jasper & .244 & 2.355 \\
\hline 51 & Jay & 1.222 & 3.872 \\
\hline
\end{tabular}




\begin{tabular}{|c|c|c|c|}
\hline & $\begin{array}{r}\text { Locale } \\
\end{array}$ & P01 30 & $\mathrm{~A} 0130$ \\
\hline 52 & Jefferson & .250 & .214 \\
\hline 53 & Jennings & .000 & .000 \\
\hline 54 & Johnson & 3.240 & .112 \\
\hline 55 & Knox & .194 & 1.097 \\
\hline 56 & Kosciusko & 7.481 & 9.591 \\
\hline 57 & LaGrange & 8.252 & 2.090 \\
\hline 58 & Lake & 5.661 & 10.819 \\
\hline 59 & La Porte & 3.869 & 5.312 \\
\hline 60 & Lawrence & .209 & .538 \\
\hline 61 & Madison & 9.461 & 7.394 \\
\hline 62 & Marion & 29.981 & 39.139 \\
\hline 63 & Marshall & 1.575 & 4.801 \\
\hline 64 & Martin & .000 & .502 \\
\hline 65 & Miami & 7.299 & .500 \\
\hline 66 & Monroe & 1.938 & 1.150 \\
\hline 67 & Montgomery & .000 & 3.848 \\
\hline 68 & Morgan & .310 & .133 \\
\hline 69 & Newton & 1.332 & .095 \\
\hline 70 & Noble & 1.511 & 8.092 \\
\hline 71 & Ohio & .000 & .000 \\
\hline 72 & Orange & 37.581 & .552 \\
\hline 73 & Owen & .000 & .108 \\
\hline 74 & Parke & .219 & 1.642 \\
\hline 75 & Perry & 5.795 & .085 \\
\hline 76 & Pike & .000 & .000 \\
\hline 77 & Porter & 1.226 & 3.315 \\
\hline 78 & Posey & .000 & .627 \\
\hline 79 & Pulaski & .238 & .704 \\
\hline 80 & Putnam & .000 & .103 \\
\hline 81 & Randolph & .203 & .671 \\
\hline 82 & Ripley & 4.354 & .107 \\
\hline 83 & Rush & .000 & .220 \\
\hline 84 & St. Joseph & 4.017 & 7.106 \\
\hline 85 & Scott & 1.438 & 3.850 \\
\hline 86 & Shelby & 7.424 & 1.676 \\
\hline 87 & Spencer & 15.967 & .547 \\
\hline 88 & Starke & .208 & .000 \\
\hline 89 & Steuben & .238 & 2.572 \\
\hline 90 & Sullivan & .000 & .000 \\
\hline 91 & Switzerland & .319 & .000 \\
\hline 92 & Tippecanoe & 22.402 & 8.238 \\
\hline 93 & Tipton & .000 & 1.600 \\
\hline 94 & Union & .000 & .000 \\
\hline 95 & Vanderburgh & 10.058 & 37.629 \\
\hline 96 & Vermillion & .000 & .067 \\
\hline 97 & Vigo & 1.385 & 2.451 \\
\hline 98 & Wabash & .203 & .521 \\
\hline 99 & Warren & .000 & .103 \\
\hline 100 & Warrick & .268 & .000 \\
\hline 101 & Washington & 44.366 & .651 \\
\hline 102 & Wayne & 13.542 & 5.727 \\
\hline
\end{tabular}




\begin{tabular}{|c|c|c|c|}
\hline & Locale & P01 30 & $A 0130$ \\
\hline 103 & Wells & .266 & 5.835 \\
\hline 104 & White & 3.934 & .404 \\
\hline 105 & Whitley & 1.167 & .114 \\
\hline 106 & lowa & 184.239 & 431.738 \\
\hline 107 & Kansas & 112.398 & 310.377 \\
\hline 108 & Kentucky_E & 85.358 & 127.117 \\
\hline 109 & Kentucky_W & 56.905 & 84.745 \\
\hline 110 & Louisiana & 29.194 & 173.477 \\
\hline 111 & Maine & 41.808 & 64.734 \\
\hline 112 & Maryland & 120.714 & 208.672 \\
\hline 113 & Massachusetts & 167.321 & 220.162 \\
\hline 114 & Michigan_E & 393.161 & 161.677 \\
\hline 115 & Michigan_W & 393.161 & 161.677 \\
\hline 116 & Minnesota & 321.447 & 507.406 \\
\hline 117 & Mississippi & 679.929 & 268.104 \\
\hline 118 & Missouri & 303.151 & 406.619 \\
\hline 119 & Montana & 20.176 & 23.602 \\
\hline 120 & Nebraska & 71.070 & 336.909 \\
\hline 121 & Nevada & 77.249 & 60.331 \\
\hline 122 & New Hampshire & 39.554 & 27.188 \\
\hline 123 & New Jersey & 212.010 & 320.743 \\
\hline 124 & New Mexico & 24.513 & 43.401 \\
\hline 125 & New York & 466.660 & 462.627 \\
\hline 126 & North Carolina & 2548.325 & 737.612 \\
\hline 127 & North Dakota & 21.891 & 47.200 \\
\hline 128 & Ohio_N & 155.745 & 155.501 \\
\hline 129 & Ohio_M & 155.745 & 155.501 \\
\hline 130 & Ohio_s & 155.745 & 155.501 \\
\hline 131 & Oklahoma & 83.376 & 152.488 \\
\hline 132 & Oregon & 182.705 & 257.487 \\
\hline 133 & Pennsylvania & 550.136 & 735.103 \\
\hline 134 & Rhode Island & 51.028 & 28.618 \\
\hline 135 & South Carolina & 157.734 & 186.714 \\
\hline 136 & South Dakota & 37.610 & 78.834 \\
\hline 137 & Tennessee & 702.510 & 434.641 \\
\hline 138 & Texas & 880.731 & 1273.686 \\
\hline 139 & Utah & 198.026 & 173.311 \\
\hline 140 & Vermont & 71.417 & 40.757 \\
\hline 141 & Virginia & 687.207 & 445.566 \\
\hline 142 & Washington & 235.131 & 454.222 \\
\hline 143 & West Virginia & 23.090 & 46.196 \\
\hline 144 & Wisconsin & 423.593 & 609.987 \\
\hline 145 & Wyoming & 4.978 & 7.335 \\
\hline
\end{tabular}




\begin{tabular}{|c|c|c|c|}
\hline & Locale & P02 30 & $A 02 \quad 30$ \\
\hline 1 & Alabama & 12218.190 & 11913.120 \\
\hline 2 & Arizona & 6010.036 & 3196.955 \\
\hline 3 & Arkansas & 19909.322 & 5308.078 \\
\hline 4 & California & 65048.651 & 74329.105 \\
\hline 5 & Colorado & 7857.742 & 2722.917 \\
\hline 6 & Connecticut & 3092.331 & 2058.268 \\
\hline 7 & Delaware & 3912.437 & 4537.552 \\
\hline 8 & DC & .000 & .000 \\
\hline 9 & Florida & 21191.151 & 7152.807 \\
\hline 10 & Georgia & 28330.225 & 6878.782 \\
\hline 11 & Idaho & 7904.259 & 1117.457 \\
\hline 12 & Illinois_N & 19282.150 & 17843.810 \\
\hline 13 & Illinois_S & 9641.075 & 8921.905 \\
\hline 14 & Adams & 138.454 & 52.125 \\
\hline 15 & Allen & 659.006 & 320.003 \\
\hline 16 & Bartholomew & 509.666 & .000 \\
\hline 17 & Benton & .000 & .000 \\
\hline 18 & Blackford & 16.172 & .000 \\
\hline 19 & Boone & 5.433 & .000 \\
\hline 20 & Brown & 3.377 & .000 \\
\hline 21 & Carroll & 638.326 & .000 \\
\hline 22 & Cass & 555.844 & .000 \\
\hline 23 & Clark & 115.863 & 530.814 \\
\hline 24 & Clay & 3.405 & .000 \\
\hline 25 & Clinton & 756.462 & .000 \\
\hline 26 & Crawford & .000 & .000 \\
\hline 27 & Daviess & 385.200 & 49.529 \\
\hline 28 & Dearborn & 4.898 & 63.985 \\
\hline 29 & Decatur & 129.285 & .000 \\
\hline 30 & DeKalb & 25.073 & .000 \\
\hline 31 & Delaware & 44.928 & .000 \\
\hline 32 & Dubois & 297.142 & .000 \\
\hline 33 & Elkhart & 257.170 & 51.766 \\
\hline 34 & Fayette & .000 & .000 \\
\hline 35 & Floyd & 285.429 & .000 \\
\hline 36 & Fountain & 19.870 & .000 \\
\hline 37 & Franklin & 4.082 & 319.937 \\
\hline 38 & Fulton & 52.577 & .000 \\
\hline 39 & Gibson & 147.241 & .000 \\
\hline 40 & Grant & 69.196 & .000 \\
\hline 41 & Greene & 120.418 & .000 \\
\hline 42 & Hamilton & 74.880 & 73.550 \\
\hline 43 & Hancock & 5.337 & .000 \\
\hline 44 & Harrison & 350.959 & 379.435 \\
\hline 45 & Hendricks & 318.776 & .000 \\
\hline 46 & Henry & 17.001 & .000 \\
\hline 47 & Howard & 53.522 & .000 \\
\hline 48 & Huntington & 147.317 & 51.320 \\
\hline 49 & Jackson & 12.429 & .000 \\
\hline 50 & Jasper & 81.890 & .000 \\
\hline 51 & Jay & 134.660 & 39.621 \\
\hline
\end{tabular}




\begin{tabular}{|c|c|c|c|}
\hline & Locale & P02 30 & A02 30 \\
\hline 52 & Jefferson & 7.437 & .000 \\
\hline 53 & Jennings & .000 & 315.293 \\
\hline 54 & Johnson & 3.883 & .000 \\
\hline 55 & Knox & 38.140 & 37.746 \\
\hline 56 & Kosciusko & 333.552 & 47.570 \\
\hline 57 & LaGrange & 72.684 & .000 \\
\hline 58 & Lake & 376.230 & 9398.969 \\
\hline 59 & La Porte & 184.735 & 43.018 \\
\hline 60 & Lawrence & 18.702 & 244.320 \\
\hline 61 & Madison & 257.130 & .000 \\
\hline 62 & Marion & 1361.110 & 548.319 \\
\hline 63 & Marshall & 166.953 & 306.468 \\
\hline 64 & Martin & 17.454 & .000 \\
\hline 65 & Miami & 17.393 & .000 \\
\hline 66 & Monroe & 40.003 & 323.254 \\
\hline 67 & Montgomery & 133.826 & .000 \\
\hline 68 & Morgan & 4.611 & .000 \\
\hline 69 & Newton & 3.307 & .000 \\
\hline 70 & Noble & 281.397 & .000 \\
\hline 71 & Ohio & .000 & .000 \\
\hline 72 & Orange & 19.191 & .000 \\
\hline 73 & Owen & 3.761 & .000 \\
\hline 74 & Parke & 57.117 & .000 \\
\hline 75 & Perry & 2.946 & .000 \\
\hline 76 & Pike & .000 & .000 \\
\hline 77 & Porter & 115.273 & .000 \\
\hline 78 & Posey & 21.796 & 1779.591 \\
\hline 79 & Pulaski & 24.486 & .000 \\
\hline 80 & Putnam & 3.590 & .000 \\
\hline 81 & Randolph & 23.329 & 39.579 \\
\hline 82 & Ripley & 3.705 & 48.406 \\
\hline 83 & Rush & 7.653 & .000 \\
\hline 84 & St. Joseph & 247.124 & 46.518 \\
\hline 85 & Scott & 133.884 & 46.641 \\
\hline 86 & Shelby & 58.283 & .000 \\
\hline 87 & Spencer & 19.025 & .000 \\
\hline 88 & Starke & .000 & .000 \\
\hline 89 & Steuben & 89.453 & 46.373 \\
\hline 90 & Sullivan & .000 & .000 \\
\hline 91 & Switzerland & .000 & .000 \\
\hline 92 & Tippecanoe & 286.498 & 58.117 \\
\hline 93 & Tipton & 55.648 & .000 \\
\hline 94 & Union & .000 & .000 \\
\hline 95 & Vanderburgh & 1308.604 & .000 \\
\hline 96 & Vermillion & 2.334 & .000 \\
\hline 97 & Vigo & 85.233 & .000 \\
\hline 98 & Wabash & 18.132 & 39.479 \\
\hline 99 & Warren & 3.571 & .000 \\
\hline 100 & Warrick & .000 & .000 \\
\hline 101 & Washington & 22.655 & .000 \\
\hline 102 & Wayne & 199.154 & .000 \\
\hline
\end{tabular}




\begin{tabular}{|c|c|c|c|}
\hline & Locale & P02 30 & A02 30 \\
\hline 103 & Wells & 202.933 & .000 \\
\hline 104 & White & 14.062 & .000 \\
\hline$\overline{105}$ & Whitley & 3.950 & .000 \\
\hline 106 & lowa & 15014.151 & 741.355 \\
\hline 107 & Kansas & 10793.685 & 12712.393 \\
\hline 108 & Kentucky_E & 4420.627 & 3613.809 \\
\hline$\overline{109}$ & Kentucky_W & 2947.084 & 2409.206 \\
\hline 110 & Louisiana & 6032.842 & 56022.889 \\
\hline$\overline{111}$ & Maine & 2251.181 & 812.529 \\
\hline 112 & Maryland & 7256.797 & 6029.005 \\
\hline 113 & Massachusetts & 7656.362 & 4864.605 \\
\hline$\overline{114}$ & Michigan_E & 5622.496 & 4592.943 \\
\hline 115 & Michigan_W & 5622.496 & 4592.943 \\
\hline 116 & Minnesota & 17645.602 & 10378.365 \\
\hline 117 & Mississippi & 9323.609 & 8792.287 \\
\hline 118 & Missouri & 14140.620 & 6797.561 \\
\hline 119 & Montana & 820.796 & 3945.094 \\
\hline 120 & Nebraska & 11716.353 & 44.621 \\
\hline 121 & Nevada & 2098.082 & 539.367 \\
\hline$\overline{122}$ & New Hampshire & 945.502 & 860.715 \\
\hline 123 & New Jersey & 11154.163 & 17221.753 \\
\hline 124 & New Mexico & 1509.324 & 3757.550 \\
\hline 125 & New York & 16088.347 & 6871.906 \\
\hline$\overline{126}$ & North Carolina & 25651.258 & 4397.079 \\
\hline 127 & North Dakota & 1641.440 & 693.384 \\
\hline 128 & Ohio_N & 5407.708 & 8017.951 \\
\hline$\overline{129}$ & Ohio_M & 5407.708 & 8017.951 \\
\hline 130 & Ohio_S & 5407.708 & 8017.951 \\
\hline 131 & Oklahoma & 5302.941 & 12102.671 \\
\hline 132 & Oregon & 8954.393 & 4249.546 \\
\hline 133 & Pennsylvania & 25563.987 & 31532.588 \\
\hline 134 & Rhode Island & 995.208 & 46.136 \\
\hline 135 & South Carolina & 6493.178 & 2018.754 \\
\hline 136 & South Dakota & 2741.529 & 44.485 \\
\hline 137 & Tennessee & 15115.090 & 7308.697 \\
\hline 138 & Texas & 44293.779 & 147663.766 \\
\hline 139 & Utah & 6027.088 & 10030.291 \\
\hline 140 & Vermont & 1417.364 & 294.296 \\
\hline 141 & Virginia & 15495.048 & 3912.806 \\
\hline 142 & Washington & 15796.047 & 12503.998 \\
\hline$\overline{143}$ & West Virginia & 1606.506 & 1576.021 \\
\hline 144 & Wisconsin & 21212.962 & 2010.809 \\
\hline 145 & Wyoming & 255.076 & 3714.352 \\
\hline
\end{tabular}




\begin{tabular}{|c|c|c|c|}
\hline & Locale & P03 30 & A03 30 \\
\hline 1 & Alabama & 7910.464 & 7712.951 \\
\hline 2 & Arizona & 3891.098 & 2069.815 \\
\hline 3 & Arkansas & 12889.959 & 3436.626 \\
\hline 4 & California & 42114.665 & 48123.139 \\
\hline 5 & Colorado & 5087.364 & 1762.907 \\
\hline 6 & Connecticut & 2002.078 & 1332.591 \\
\hline 7 & Delaware & 2533.042 & 2937.763 \\
\hline 8 & DC & .000 & .000 \\
\hline 9 & Florida & 13719.857 & 4630.966 \\
\hline 10 & Georgia & 18341.932 & 4453.553 \\
\hline 11 & Idaho & 5117.480 & 723.479 \\
\hline 12 & Illinois_N & 12483.906 & 11552.678 \\
\hline 13 & Illinois_S & 6241.953 & 5776.339 \\
\hline 14 & Adams & 89.640 & 33.747 \\
\hline 15 & Allen & 426.663 & 207.180 \\
\hline 16 & Bartholomew & 329.974 & .000 \\
\hline 17 & Benton & .000 & .000 \\
\hline 18 & Blackford & 10.470 & .000 \\
\hline 19 & Boone & 3.518 & .000 \\
\hline 20 & Brown & 2.186 & .000 \\
\hline 21 & Carroll & 413.273 & .000 \\
\hline 22 & Cass & 359.872 & .000 \\
\hline 23 & Clark & 75.014 & 343.667 \\
\hline 24 & Clay & 2.205 & .000 \\
\hline 25 & Clinton & 489.759 & .000 \\
\hline 26 & Crawford & .000 & .000 \\
\hline 27 & Daviess & 249.391 & 32.067 \\
\hline 28 & Dearborn & 3.171 & 41.426 \\
\hline 29 & Decatur & 83.703 & .000 \\
\hline 30 & DeKalb & 16.233 & .000 \\
\hline 31 & Delaware & 29.088 & .000 \\
\hline 32 & Dubois & 192.380 & .000 \\
\hline 33 & Elkhart & 166.500 & 33.515 \\
\hline 34 & Fayette & .000 & .000 \\
\hline 35 & Floyd & 184.796 & .000 \\
\hline 36 & Fountain & 12.865 & .000 \\
\hline 37 & Franklin & 2.643 & 207.138 \\
\hline 38 & Fulton & 34.040 & .000 \\
\hline 39 & Gibson & 95.328 & .000 \\
\hline 40 & Grant & 44.800 & .000 \\
\hline 41 & Greene & 77.963 & .000 \\
\hline 42 & Hamilton & 48.480 & 47.618 \\
\hline 43 & Hancock & 3.456 & .000 \\
\hline 44 & Harrison & 227.222 & 245.659 \\
\hline 45 & Hendricks & 206.386 & .000 \\
\hline 46 & Henry & 11.007 & .000 \\
\hline 47 & Howard & 34.652 & .000 \\
\hline 48 & Huntington & 95.378 & 33.226 \\
\hline 49 & Jackson & 8.047 & .000 \\
\hline 50 & Jasper & 53.018 & .000 \\
\hline 51 & Jay & 87.183 & 25.652 \\
\hline
\end{tabular}




\begin{tabular}{|c|c|c|c|}
\hline & Locale & P03 30 & $\mathrm{~A} 03$ 30 \\
\hline 52 & Jefferson & 4.815 & .000 \\
\hline 53 & Jennings & .000 & 204.131 \\
\hline 54 & Johnson & 2.514 & .000 \\
\hline 55 & Knox & 24.693 & 24.438 \\
\hline 56 & Kosciusko & 215.953 & 30.799 \\
\hline 57 & LaGrange & 47.058 & .000 \\
\hline 58 & Lake & 243.584 & 6085.206 \\
\hline 59 & La Porte & 119.604 & 27.852 \\
\hline 60 & Lawrence & 12.108 & 158.181 \\
\hline 61 & Madison & 166.475 & .000 \\
\hline 62 & Marion & 881.228 & 355.000 \\
\hline 63 & Marshall & 108.091 & 198.417 \\
\hline 64 & Martin & 11.301 & .000 \\
\hline 65 & Miami & 11.261 & .000 \\
\hline 66 & Monroe & 25.899 & 209.285 \\
\hline 67 & Montgomery & 86.644 & .000 \\
\hline 68 & Morgan & 2.985 & .000 \\
\hline 69 & Newton & 2.141 & .000 \\
\hline 70 & Noble & 182.186 & .000 \\
\hline 71 & Ohio & .000 & .000 \\
\hline 72 & Orange & 12.425 & .000 \\
\hline 73 & Owen & 2.435 & .000 \\
\hline 74 & Parke & 36.980 & .000 \\
\hline 75 & Perry & 1.907 & .000 \\
\hline 76 & Pike & .000 & .000 \\
\hline 77 & Porter & 74.632 & .000 \\
\hline 78 & Posey & 14.111 & 1152.167 \\
\hline 79 & Pulaski & 15.853 & .000 \\
\hline 80 & Putnam & 2.324 & .000 \\
\hline 81 & Randolph & 15.104 & 25.625 \\
\hline 82 & Ripley & 2.399 & 31.340 \\
\hline 83 & Rush & 4.955 & .000 \\
\hline 84 & St. Joseph & 159.996 & 30.117 \\
\hline 85 & Scott & 86.681 & 30.197 \\
\hline 86 & Shelby & 37.734 & .000 \\
\hline 87 & Spencer & 12.317 & .000 \\
\hline 88 & Starke & .000 & .000 \\
\hline 89 & Steuben & 57.915 & 30.023 \\
\hline 90 & Sullivan & .000 & .000 \\
\hline 91 & Switzerland & .000 & .000 \\
\hline 92 & Tippecanoe & 185.489 & 37.627 \\
\hline 93 & Tipton & 36.029 & .000 \\
\hline 94 & Union & .000 & .000 \\
\hline 95 & Vanderburgh & 847.234 & .000 \\
\hline 96 & Vermillion & 1.511 & .000 \\
\hline 97 & Vigo & 55.183 & .000 \\
\hline 98 & Wabash & 11.739 & 25.560 \\
\hline 99 & Warren & 2.312 & .000 \\
\hline 100 & Warrick & .000 & .000 \\
\hline 101 & Washington & 14.668 & .000 \\
\hline 102 & Wayne & 128.939 & .000 \\
\hline
\end{tabular}




\begin{tabular}{|c|c|c|c|}
\hline & Locale & P03 30 & $\mathrm{~A} 03 \quad 30$ \\
\hline 103 & Wells & 131.385 & .000 \\
\hline 104 & White & 9.104 & .000 \\
\hline 105 & Whitley & 2.557 & .000 \\
\hline 106 & lowa & 9720.662 & 479.978 \\
\hline 107 & Kansas & 6988.191 & 8230.427 \\
\hline 108 & Kentucky_E & 2862.061 & 2339.701 \\
\hline 109 & Kentucky_W & 1908.041 & 1559.800 \\
\hline 110 & Louisiana & 3905.863 & 36271.085 \\
\hline 111 & Maine & 1457.490 & 526.058 \\
\hline 112 & Maryland & 4698.292 & 3903.379 \\
\hline 113 & Massachusetts & 4956.984 & 3149.508 \\
\hline 114 & Michigan_E & 3640.191 & 2973.624 \\
\hline 115 & Michigan_W & 3640.191 & 2973.624 \\
\hline 116 & Minnesota & 11424.351 & 6719.299 \\
\hline 117 & Mississippi & 6036.416 & 5692.420 \\
\hline 118 & Missouri & 9155.109 & 4400.968 \\
\hline 119 & Montana & 531.411 & 2554.185 \\
\hline 120 & Nebraska & 7585.557 & 28.889 \\
\hline 121 & Nevada & 1358.368 & 349.204 \\
\hline 122 & New Hampshire & 612.150 & 557.256 \\
\hline 123 & New Jersey & 7221.577 & 11149.937 \\
\hline 124 & New Mexico & 977.187 & 2432.763 \\
\hline 125 & New York & 10416.132 & 4449.101 \\
\hline 126 & North Carolina & 16607.479 & 2846.815 \\
\hline 127 & North Dakota & 1062.723 & 448.920 \\
\hline 128 & Ohio_N & 3501.131 & 5191.089 \\
\hline 129 & Ohio_M & 3501.131 & 5191.089 \\
\hline 130 & Ohio_s & 3501.131 & 5191.089 \\
\hline 131 & Oklahoma & 3433.300 & 7835.672 \\
\hline 132 & Oregon & 5797.372 & 2751.298 \\
\hline 133 & Pennsylvania & 16550.977 & 20415.248 \\
\hline 134 & Rhode Island & 644.331 & 29.870 \\
\hline 135 & South Carolina & 4203.900 & 1307.009 \\
\hline 136 & South Dakota & 1774.957 & 28.801 \\
\hline 137 & Tennessee & 9786.013 & 4731.894 \\
\hline 138 & Texas & 28677.269 & 95602.443 \\
\hline 139 & Utah & 3902.138 & 6493.944 \\
\hline 140 & Vermont & 917.649 & 190.537 \\
\hline 141 & Virginia & 10032.010 & 2533.281 \\
\hline 142 & Washington & 10226.888 & 8095.505 \\
\hline 143 & West Virginia & 1040.105 & 1020.368 \\
\hline 144 & Wisconsin & 13733.978 & 1301.864 \\
\hline 145 & Wyoming & 165.145 & 2404.795 \\
\hline
\end{tabular}




\begin{tabular}{|c|c|c|c|}
\hline & $\begin{array}{l}\text { Locale } \\
\end{array}$ & P04 30 & A04 30 \\
\hline 1 & Alabama & 8672.212 & 8672.212 \\
\hline 2 & Arizona & 4265.796 & 4265.796 \\
\hline 3 & Arkansas & 14131.214 & 14131.214 \\
\hline 4 & California & 46170.151 & 46170.151 \\
\hline 5 & Colorado & 5577.259 & 5577.259 \\
\hline 6 & Connecticut & 2194.871 & 2194.871 \\
\hline 7 & Delaware & 2776.965 & 2776.965 \\
\hline 8 & DC & .000 & .000 \\
\hline 9 & Florida & 15041.029 & 15041.029 \\
\hline 10 & Georgia & 20108.192 & 20108.192 \\
\hline 11 & Idaho & 5610.275 & 5610.275 \\
\hline 12 & Illinois_N & 13686.060 & 13686.060 \\
\hline 13 & Illinois_S & 6843.030 & 6843.030 \\
\hline 14 & Adams & 98.272 & 98.272 \\
\hline 15 & Allen & 467.749 & 467.749 \\
\hline 16 & Bartholomew & 361.750 & 361.750 \\
\hline 17 & Benton & .000 & .000 \\
\hline 18 & Blackford & 11.479 & 11.479 \\
\hline 19 & Boone & 3.856 & 3.856 \\
\hline 20 & Brown & 2.397 & 2.397 \\
\hline 21 & Carroll & 453.070 & 453.070 \\
\hline 22 & Cass & 394.526 & 394.526 \\
\hline 23 & Clark & 82.237 & 82.237 \\
\hline 24 & Clay & 2.417 & 2.417 \\
\hline 25 & Clinton & 536.921 & 536.921 \\
\hline 26 & Crawford & .000 & .000 \\
\hline 27 & Daviess & 273.407 & 273.407 \\
\hline 28 & Dearborn & 3.476 & 3.476 \\
\hline 29 & Decatur & 91.763 & 91.763 \\
\hline 30 & DeKalb & 17.797 & 17.797 \\
\hline 31 & Delaware & 31.889 & 31.889 \\
\hline 32 & Dubois & 210.905 & 210.905 \\
\hline 33 & Elkhart & 182.533 & 182.533 \\
\hline 34 & Fayette & .000 & .000 \\
\hline 35 & Floyd & 202.591 & 202.591 \\
\hline 36 & Fountain & 14.104 & 14.104 \\
\hline 37 & Franklin & 2.897 & 2.897 \\
\hline 38 & Fulton & 37.318 & 37.318 \\
\hline 39 & Gibson & 104.508 & 104.508 \\
\hline 40 & Grant & 49.114 & 49.114 \\
\hline 41 & Greene & 85.470 & 85.470 \\
\hline 42 & Hamilton & 53.148 & 53.148 \\
\hline 43 & Hancock & 3.788 & 3.788 \\
\hline 44 & Harrison & 249.103 & 249.103 \\
\hline 45 & Hendricks & 226.261 & 226.261 \\
\hline 46 & Henry & 12.067 & 12.067 \\
\hline 47 & Howard & 37.989 & 37.989 \\
\hline 48 & Huntington & 104.562 & 104.562 \\
\hline 49 & Jackson & 8.822 & 8.822 \\
\hline 50 & Jasper & 58.123 & 58.123 \\
\hline 51 & Jay & 95.579 & 95.579 \\
\hline
\end{tabular}




\begin{tabular}{|c|c|c|c|}
\hline & Locale & P04 30 & A04 30 \\
\hline 52 & Jefferson & 5.278 & 5.278 \\
\hline 53 & Jennings & .000 & .000 \\
\hline 54 & Johnson & 2.756 & 2.756 \\
\hline 55 & Knox & 27.071 & 27.071 \\
\hline 56 & Kosciusko & 236.748 & 236.748 \\
\hline 57 & LaGrange & 51.590 & 51.590 \\
\hline 58 & Lake & 267.040 & 267.040 \\
\hline 59 & La Porte & 131.121 & 131.121 \\
\hline 60 & Lawrence & 13.274 & 13.274 \\
\hline 61 & Madison & 182.505 & 182.505 \\
\hline 62 & Marion & 966.087 & 966.087 \\
\hline 63 & Marshall & 118.499 & 118.499 \\
\hline 64 & Martin & 12.389 & 12.389 \\
\hline 65 & Miami & 12.345 & 12.345 \\
\hline 66 & Monroe & 28.393 & 28.393 \\
\hline 67 & Montgomery & 94.987 & 94.987 \\
\hline 68 & Morgan & 3.273 & 3.273 \\
\hline 69 & Newton & 2.347 & 2.347 \\
\hline 70 & Noble & 199.730 & 199.730 \\
\hline 71 & Ohio & .000 & .000 \\
\hline 72 & Orange & 13.621 & 13.621 \\
\hline 73 & Owen & 2.669 & 2.669 \\
\hline 74 & Parke & 40.541 & 40.541 \\
\hline 75 & Perry & 2.091 & 2.091 \\
\hline 76 & Pike & .000 & .000 \\
\hline 77 & Porter & 81.818 & 81.818 \\
\hline 78 & Posey & 15.470 & 15.470 \\
\hline 79 & Pulaski & 17.380 & 17.380 \\
\hline 80 & Putnam & 2.548 & 2.548 \\
\hline 81 & Randolph & 16.558 & 16.558 \\
\hline 82 & Ripley & 2.630 & 2.630 \\
\hline 83 & Rush & 5.432 & 5.432 \\
\hline 84 & St. Joseph & 175.403 & 175.403 \\
\hline 85 & Scott & 95.028 & 95.028 \\
\hline 86 & Shelby & 41.368 & 41.368 \\
\hline 87 & Spencer & 13.503 & 13.503 \\
\hline 88 & Starke & .000 & .000 \\
\hline 89 & Steuben & 63.492 & 63.492 \\
\hline 90 & Sullivan & .000 & .000 \\
\hline 91 & Switzerland & .000 & .000 \\
\hline 92 & Tippecanoe & 203.351 & 203.351 \\
\hline 93 & Tipton & 39.498 & 39.498 \\
\hline 94 & Union & .000 & .000 \\
\hline 95 & Vanderburgh & 928.819 & 928.819 \\
\hline 96 & Vermillion & 1.656 & 1.656 \\
\hline 97 & Vigo & 60.497 & 60.497 \\
\hline 98 & Wabash & 12.870 & 12.870 \\
\hline 99 & Warren & 2.535 & 2.535 \\
\hline 100 & Warrick & .000 & .000 \\
\hline 101 & Washington & 16.080 & 16.080 \\
\hline 102 & Wayne & 141.355 & 141.355 \\
\hline
\end{tabular}




\begin{tabular}{|c|c|c|c|}
\hline & Locale & P04 30 & A04_30 \\
\hline 103 & Wells & 144.037 & 144.037 \\
\hline 104 & White & 9.981 & 9.981 \\
\hline 105 & Whitley & 2.803 & 2.803 \\
\hline 106 & lowa & 10656.726 & 10656.726 \\
\hline 107 & Kansas & 7661.128 & 7661.128 \\
\hline 108 & Kentucky_E & 3137.667 & 3137.667 \\
\hline 109 & Kentucky_W & 2091.778 & 2091.778 \\
\hline 110 & Louisiana & 4281.983 & 4281.983 \\
\hline 111 & Maine & 1597.840 & 1597.840 \\
\hline 112 & Maryland & 5150.720 & 5150.720 \\
\hline 113 & Massachusetts & 5434.323 & 5434.323 \\
\hline 114 & Michigan_E & 3990.728 & 3990.728 \\
\hline 115 & Michigan_W & 3990.728 & 3990.728 \\
\hline 116 & Minnesota & 12524.473 & 12524.473 \\
\hline 117 & Mississippi & 6617.700 & 6617.700 \\
\hline 118 & Missouri & 10036.712 & 10036.712 \\
\hline 119 & Montana & 582.584 & 582.584 \\
\hline 120 & Nebraska & 8316.018 & 8316.018 \\
\hline 121 & Nevada & 1489.174 & 1489.174 \\
\hline 122 & New Hampshire & 671.097 & 671.097 \\
\hline 123 & New Jersey & 7916.988 & 7916.988 \\
\hline 124 & New Mexico & 1071.286 & 1071.286 \\
\hline 125 & New York & 11419.167 & 11419.167 \\
\hline 126 & North Carolina & 18206.718 & 18206.718 \\
\hline 127 & North Dakota & 1165.059 & 1165.059 \\
\hline 128 & Ohio_N & 3838.276 & 3838.276 \\
\hline 129 & Ohio_M & 3838.276 & 3838.276 \\
\hline 130 & Ohio_s & 3838.276 & 3838.276 \\
\hline 131 & Oklahoma & 3763.915 & 3763.915 \\
\hline 132 & Oregon & 6355.638 & 6355.638 \\
\hline 133 & Pennsylvania & 18144.775 & 18144.775 \\
\hline 134 & Rhode Island & 706.378 & 706.378 \\
\hline 135 & South Carolina & 4608.720 & 4608.720 \\
\hline 136 & South Dakota & 1945.879 & 1945.879 \\
\hline 137 & Tennessee & 10728.370 & 10728.370 \\
\hline 138 & Texas & 31438.784 & 31438.784 \\
\hline 139 & Utah & 4277.899 & 4277.899 \\
\hline 140 & Vermont & 1006.015 & 1006.015 \\
\hline 141 & Virginia & 10998.056 & 10998.056 \\
\hline 142 & Washington & 11211.699 & 11211.699 \\
\hline 143 & West Virginia & 1140.264 & 1140.264 \\
\hline 144 & Wisconsin & 15056.510 & 15056.510 \\
\hline 145 & Wyoming & 181.048 & 181.048 \\
\hline
\end{tabular}




\begin{tabular}{|c|c|c|c|}
\hline & Locale & P05 30 & A05 30 \\
\hline 1 & Alabama & 3651.869 & 3031.713 \\
\hline 2 & Arizona & 1796.327 & 3357.723 \\
\hline 3 & Arkansas & 5950.655 & 3791.711 \\
\hline 4 & California & 19442.254 & 20827.038 \\
\hline 5 & Colorado & 2348.584 & 2515.688 \\
\hline 6 & Connecticut & 924.260 & 1384.257 \\
\hline 7 & Delaware & 1169.380 & 833.276 \\
\hline 8 & DC & .000 & .000 \\
\hline 9 & Florida & 6333.778 & 9878.117 \\
\hline 10 & Georgia & 8467.561 & 7077.537 \\
\hline 11 & Idaho & 2362.487 & 1652.706 \\
\hline 12 & Illinois_N & 5763.201 & 5081.613 \\
\hline 13 & Illinois_s & 2881.601 & 2540.807 \\
\hline 14 & Adams & 41.382 & 32.740 \\
\hline 15 & Allen & 196.969 & 209.158 \\
\hline 16 & Bartholomew & 152.333 & 101.757 \\
\hline 17 & \begin{tabular}{|l} 
Benton \\
\end{tabular} & .000 & 1.925 \\
\hline 18 & Blackford & 4.834 & 5.486 \\
\hline 19 & Boone & 1.624 & 19.883 \\
\hline 20 & Brown & 1.009 & 4.586 \\
\hline 21 & Carroll & 190.788 & 101.824 \\
\hline 22 & Cass & 166.135 & 96.201 \\
\hline 23 & Clark & 34.630 & 47.542 \\
\hline 24 & Clay & 1.018 & 8.483 \\
\hline 25 & Clinton & 226.097 & 124.004 \\
\hline 26 & Crawford & .000 & 3.308 \\
\hline 27 & Daviess & 115.132 & 66.308 \\
\hline 28 & Dearborn & 1.464 & 18.397 \\
\hline 29 & Decatur & 38.642 & 26.998 \\
\hline 30 & DeKalb & 7.494 & 17.623 \\
\hline 31 & Delaware & 13.428 & 40.991 \\
\hline 32 & \begin{tabular}{|l} 
Dubois \\
\end{tabular} & 88.812 & 57.614 \\
\hline 33 & Elkhart & 76.865 & 112.799 \\
\hline 34 & Fayette & .000 & 8.103 \\
\hline 35 & Floyd & 85.311 & 66.157 \\
\hline 36 & Fountain & 5.939 & 7.803 \\
\hline 37 & Franklin & 1.220 & 7.206 \\
\hline 38 & Fulton & 15.714 & 13.336 \\
\hline 39 & Gibson & 44.008 & 30.851 \\
\hline 40 & Grant & 20.682 & 27.771 \\
\hline 41 & Greene & 35.991 & 26.817 \\
\hline 42 & Hamilton & 22.381 & 112.757 \\
\hline 43 & Hancock & 1.595 & 24.791 \\
\hline 44 & Harrison & 104.897 & 66.058 \\
\hline 45 & Hendricks & 95.278 & 103.086 \\
\hline 46 & Henry & 5.081 & 14.434 \\
\hline 47 & Howard & 15.997 & 41.877 \\
\hline 48 & Huntington & 44.031 & 35.287 \\
\hline 49 & Jackson & 3.715 & 14.356 \\
\hline 50 & Jasper & 24.476 & 20.894 \\
\hline 51 & Jay & 40.248 & 25.539 \\
\hline
\end{tabular}




\begin{tabular}{|c|c|c|c|}
\hline & Locale & P05 30 & A05 30 \\
\hline 52 & Jefferson & 2.223 & 11.400 \\
\hline 53 & Jennings & .000 & 9.005 \\
\hline 54 & Johnson & 1.161 & 50.160 \\
\hline 55 & Knox & 11.400 & 15.024 \\
\hline 56 & Kosciusko & 99.695 & 72.319 \\
\hline 57 & LaGrange & 21.724 & 23.932 \\
\hline 58 & Lake & 112.451 & 179.870 \\
\hline 59 & La Porte & 55.215 & 55.901 \\
\hline 60 & Lawrence & 5.590 & 15.192 \\
\hline 61 & Madison & 76.853 & 79.134 \\
\hline 62 & Marion & 406.819 & 448.805 \\
\hline 63 & Marshall & 49.900 & 40.082 \\
\hline 64 & Martin & 5.217 & 5.007 \\
\hline 65 & Miami & 5.199 & 10.846 \\
\hline 66 & Monroe & 11.957 & 44.818 \\
\hline 67 & Montgomery & 39.999 & 31.380 \\
\hline 68 & Morgan & 1.378 & 24.334 \\
\hline 69 & Newton & .988 & 4.165 \\
\hline 70 & Noble & 84.106 & 57.946 \\
\hline 71 & Ohio & .000 & 1.474 \\
\hline 72 & Orange & 5.736 & 8.085 \\
\hline 73 & Owen & 1.124 & 6.858 \\
\hline 74 & Parke & 17.072 & 12.767 \\
\hline 75 & Perry & .881 & 4.863 \\
\hline$\overline{76}$ & Pike & .000 & 3.333 \\
\hline 77 & Porter & 34.454 & 57.343 \\
\hline 78 & Posey & 6.514 & 10.553 \\
\hline 79 & Pulaski & 7.319 & 7.164 \\
\hline 80 & Putnam & 1.073 & 11.334 \\
\hline 81 & Randolph & 6.973 & 10.298 \\
\hline 82 & Ripley & 1.107 & 8.017 \\
\hline 83 & Rush & 2.288 & 5.166 \\
\hline 84 & St. Joseph & 73.862 & 115.857 \\
\hline 85 & Scott & 40.016 & 26.841 \\
\hline 86 & Shelby & 17.420 & 20.610 \\
\hline 87 & Spencer & 5.686 & 7.977 \\
\hline 88 & Starke & .000 & 5.969 \\
\hline 89 & Steuben & 26.736 & 24.502 \\
\hline 90 & Sullivan & .000 & 5.821 \\
\hline 91 & Switzerland & .000 & 2.769 \\
\hline 92 & Tippecanoe & 85.631 & 98.079 \\
\hline 93 & Tipton & 16.633 & 12.576 \\
\hline 94 & Union & .000 & 1.796 \\
\hline 95 & Vanderburgh & 391.126 & 243.161 \\
\hline 96 & Vermillion & .697 & 4.150 \\
\hline 97 & Vigo & 25.475 & 38.539 \\
\hline 98 & Wabash & 5.419 & 11.387 \\
\hline 99 & Warren & 1.067 & 2.575 \\
\hline 100 & Warrick & .000 & 17.449 \\
\hline 101 & Washington & 6.771 & 11.252 \\
\hline 102 & Wayne & 59.525 & 46.382 \\
\hline
\end{tabular}




\begin{tabular}{|c|c|c|c|}
\hline & $\begin{array}{l}\text { Locale } \\
\end{array}$ & P05 30 & $A 05 \quad 30$ \\
\hline 103 & Wells & 60.654 & 38.813 \\
\hline 104 & White & 4.203 & 9.689 \\
\hline 105 & Whitley & 1.181 & 10.889 \\
\hline 106 & lowa & 4487.548 & 2994.076 \\
\hline 107 & Kansas & 3226.102 & 2371.358 \\
\hline 108 & Kentucky_E & 1321.272 & 1338.619 \\
\hline 109 & Kentucky_W & 880.848 & 892.413 \\
\hline 110 & Louisiana & 1803.143 & 2084.692 \\
\hline 111 & Maine & 672.851 & 690.740 \\
\hline 112 & Maryland & 2168.969 & 2768.806 \\
\hline 113 & Massachusetts & 2288.394 & 2839.052 \\
\hline 114 & Michigan_E & 1680.496 & 2669.706 \\
\hline 115 & Michigan_W & 1680.496 & 1779.804 \\
\hline 116 & Minnesota & 5274.057 & 4166.777 \\
\hline 117 & Mississippi & 2786.714 & 2165.390 \\
\hline 118 & Missouri & 4226.460 & 3724.271 \\
\hline 119 & Montana & 245.326 & 375.229 \\
\hline 120 & Nebraska & 3501.876 & 2214.679 \\
\hline 121 & Nevada & 627.091 & 1238.591 \\
\hline 122 & New Hampshire & 282.599 & 530.716 \\
\hline 123 & New Jersey & 3333.844 & 4023.007 \\
\hline 124 & New Mexico & 451.119 & 728.077 \\
\hline 125 & New York & 4808.612 & 7118.219 \\
\hline 126 & North Carolina & 7666.850 & 6741.980 \\
\hline 127 & North Dakota & 490.607 & 399.389 \\
\hline 128 & Ohio_N & 1616.299 & 1785.872 \\
\hline 129 & Ohio_M & 1616.299 & 1785.872 \\
\hline 130 & Ohio_s & 1616.299 & 1785.872 \\
\hline 131 & Oklahoma & 1584.985 & 1747.600 \\
\hline 132 & Oregon & 2676.360 & 2504.750 \\
\hline 133 & Pennsylvania & 7640.766 & 6994.392 \\
\hline$\overline{134}$ & Rhode Island & 297.456 & 425.897 \\
\hline 135 & South Carolina & 1940.732 & 2206.944 \\
\hline 136 & South Dakota & 819.410 & 608.312 \\
\hline 137 & Tennessee & 4517.717 & 4068.877 \\
\hline 138 & Texas & 13238.874 & 14448.260 \\
\hline 139 & Utah & 1801.424 & 1731.888 \\
\hline 140 & Vermont & 423.633 & 386.132 \\
\hline 141 & Virginia & 4631.282 & 4682.840 \\
\hline 142 & Washington & 4721.247 & 4499.756 \\
\hline 143 & West Virginia & 480.165 & 665.800 \\
\hline$\overline{144}$ & Wisconsin & 6340.297 & 4710.368 \\
\hline 145 & Wyoming & 76.239 & 164.832 \\
\hline
\end{tabular}




\begin{tabular}{|c|c|c|c|}
\hline & Locale & P06 30 & A06 30 \\
\hline 1 & Alabama & 3736.085 & 3484.185 \\
\hline 2 & Arizona & 2358.198 & 4143.476 \\
\hline 3 & Arkansas & 5651.388 & 3744.400 \\
\hline 4 & California & 21766.719 & 24359.948 \\
\hline 5 & Colorado & 2646.875 & 2878.499 \\
\hline$\overline{6}$ & Connecticut & 1922.566 & 2066.583 \\
\hline 7 & Delaware & 992.387 & 1110.837 \\
\hline$\overline{8}$ & $D C$ & .000 & .000 \\
\hline 9 & Florida & 7213.790 & 11901.848 \\
\hline 10 & Georgia & 8459.172 & 8116.216 \\
\hline 11 & Idaho & 2046.472 & 1710.929 \\
\hline 12 & Illinois_N & 8207.144 & 6388.762 \\
\hline 13 & Illinois_S & 4103.572 & 3194.381 \\
\hline 14 & Adams & 59.276 & 37.142 \\
\hline 15 & Allen & 442.174 & 222.745 \\
\hline 16 & Bartholomew & 312.206 & 98.124 \\
\hline 17 & Benton & 2.528 & 2.303 \\
\hline 18 & Blackford & 6.042 & 5.646 \\
\hline 19 & Boone & 35.142 & 23.155 \\
\hline 20 & Brown & .789 & 5.682 \\
\hline 21 & Carroll & 152.124 & 86.712 \\
\hline 22 & Cass & 138.474 & 82.240 \\
\hline 23 & Clark & 73.163 & 80.880 \\
\hline 24 & Clay & 5.327 & 10.815 \\
\hline 25 & Clinton & 187.501 & 113.381 \\
\hline 26 & Crawford & .000 & 3.969 \\
\hline 27 & Daviess & 99.245 & 60.646 \\
\hline 28 & Dearborn & 9.447 & 22.348 \\
\hline 29 & Decatur & 52.122 & 24.020 \\
\hline 30 & DeKalb & 43.268 & 29.032 \\
\hline 31 & Delaware & 62.863 & 47.219 \\
\hline 32 & Dubois & 75.543 & 53.549 \\
\hline 33 & Elkhart & 140.943 & 177.117 \\
\hline 34 & Fayette & 27.769 & 7.089 \\
\hline 35 & Floyd & 100.906 & 67.496 \\
\hline 36 & Fountain & 4.640 & 8.027 \\
\hline 37 & Franklin & 4.384 & 8.049 \\
\hline 38 & Fulton & 29.101 & 13.413 \\
\hline 39 & Gibson & 43.900 & 28.684 \\
\hline 40 & Grant & 39.499 & 30.170 \\
\hline 41 & Greene & 28.119 & 25.451 \\
\hline 42 & Hamilton & 39.414 & 133.584 \\
\hline 43 & Hancock & 18.671 & 27.583 \\
\hline 44 & Harrison & 86.023 & 61.547 \\
\hline 45 & Hendricks & 97.109 & 106.399 \\
\hline 46 & Henry & 18.857 & 15.176 \\
\hline 47 & Howard & 29.005 & 33.017 \\
\hline 48 & Huntington & 57.848 & 34.753 \\
\hline 49 & Jackson & 36.037 & 21.194 \\
\hline 50 & Jasper & 19.632 & 20.374 \\
\hline 51 & Jay & 40.071 & 22.899 \\
\hline
\end{tabular}




\begin{tabular}{|c|c|c|c|}
\hline & Locale & P06 30 & A06 30 \\
\hline 52 & Jefferson & 40.444 & 14.256 \\
\hline 53 & Jennings & 16.457 & 10.522 \\
\hline 54 & Johnson & 54.142 & 61.967 \\
\hline 55 & Knox & 12.428 & 15.905 \\
\hline 56 & Kosciusko & 122.632 & 80.084 \\
\hline 57 & LaGrange & 48.325 & 23.894 \\
\hline 58 & Lake & 150.325 & 248.546 \\
\hline 59 & La Porte & 131.444 & 68.328 \\
\hline 60 & Lawrence & 21.530 & 15.872 \\
\hline 61 & Madison & 79.305 & 89.226 \\
\hline 62 & Marion & 735.414 & 686.793 \\
\hline 63 & Marshall & 47.422 & 42.646 \\
\hline 64 & Martin & 4.483 & 5.040 \\
\hline 65 & Miami & 12.307 & 13.939 \\
\hline 66 & Monroe & 110.458 & 58.848 \\
\hline 67 & Montgomery & 40.050 & 35.970 \\
\hline 68 & Morgan & 19.035 & 47.939 \\
\hline 69 & Newton & 4.618 & 5.042 \\
\hline 70 & Noble & 89.891 & 60.172 \\
\hline 71 & \begin{tabular}{|l|} 
Ohio \\
\end{tabular} & .000 & 1.769 \\
\hline 72 & Orange & 4.929 & 8.637 \\
\hline 73 & Owen & 1.405 & 8.021 \\
\hline 74 & Parke & 16.081 & 12.150 \\
\hline 75 & Perry & 7.911 & 5.673 \\
\hline 76 & Pike & .000 & 4.000 \\
\hline 77 & Porter & 52.097 & 80.802 \\
\hline 78 & Posey & 8.143 & 82.010 \\
\hline 79 & Pulaski & 29.584 & 7.229 \\
\hline 80 & Putnam & 9.639 & 12.749 \\
\hline 81 & Randolph & 28.455 & 10.513 \\
\hline 82 & Ripley & .865 & 11.874 \\
\hline 83 & Rush & 23.192 & 5.731 \\
\hline 84 & St. Joseph & 135.186 & 155.736 \\
\hline 85 & Scott & 34.265 & 31.662 \\
\hline 86 & Shelby & 31.948 & 27.934 \\
\hline 87 & Spencer & 12.217 & 8.734 \\
\hline 88 & Starke & 16.315 & 9.009 \\
\hline 89 & Steuben & 46.651 & 22.158 \\
\hline 90 & Sullivan & .000 & 7.229 \\
\hline 91 & Switzerland & .000 & 3.323 \\
\hline 92 & Tippecanoe & 234.445 & 176.550 \\
\hline 93 & Tipton & 20.791 & 12.068 \\
\hline 94 & Union & .482 & 3.798 \\
\hline 95 & Vanderburgh & 358.596 & 316.286 \\
\hline 96 & Vermillion & .545 & 61.505 \\
\hline 97 & Vigo & 41.138 & 80.494 \\
\hline 98 & Wabash & 24.600 & 24.925 \\
\hline 99 & Warren & 9.590 & 2.840 \\
\hline 100 & Warrick & 3.356 & 24.315 \\
\hline 101 & Washington & 44.968 & 12.236 \\
\hline 102 & Wayne & 60.951 & 48.068 \\
\hline
\end{tabular}




\begin{tabular}{|c|c|c|c|}
\hline & $\begin{array}{r}\text { Locale } \\
\end{array}$ & P06 30 & A06 30 \\
\hline 103 & Wells & 63.880 & 34.950 \\
\hline 104 & White & 17.309 & 9.428 \\
\hline 105 & Whitley & 7.950 & 12.097 \\
\hline 106 & lowa & 5259.482 & 2997.476 \\
\hline 107 & Kansas & 3585.791 & 2461.539 \\
\hline 108 & Kentucky_E & 1723.373 & 1682.302 \\
\hline 109 & Kentucky_W & 1148.915 & 1121.534 \\
\hline 110 & Louisiana & 1841.814 & 3211.543 \\
\hline 111 & Maine & 667.947 & 757.931 \\
\hline 112 & Maryland & 2316.705 & 3579.939 \\
\hline 113 & Massachusetts & 3432.833 & 3836.631 \\
\hline 114 & Michigan_E & 3901.838 & 2895.638 \\
\hline 115 & Michigan_W & 3901.838 & 2895.638 \\
\hline 116 & Minnesota & 6503.024 & 4413.517 \\
\hline 117 & Mississippi & 2983.692 & 2341.769 \\
\hline 118 & Missouri & 5077.910 & 4534.731 \\
\hline 119 & Montana & 282.854 & 425.762 \\
\hline 120 & Nebraska & 3312.631 & 2107.651 \\
\hline 121 & Nevada & 642.645 & 1465.942 \\
\hline 122 & New Hampshire & 803.725 & 668.768 \\
\hline 123 & New Jersey & 3961.812 & 7035.705 \\
\hline 124 & New Mexico & 418.764 & 844.240 \\
\hline 125 & New York & 6968.170 & 9907.522 \\
\hline 126 & North Carolina & 8935.926 & 9006.624 \\
\hline 127 & North Dakota & 609.154 & 387.988 \\
\hline 128 & Ohio_N & 2835.888 & 2378.836 \\
\hline 129 & Ohio_M & 2935.888 & 2378.836 \\
\hline 130 & Ohio_s & 2935.888 & 2378.836 \\
\hline 131 & Oklahoma & 2495.846 & 1939.224 \\
\hline 132 & Oregon & 2938.731 & 2715.016 \\
\hline 133 & Pennsylvania & 9357.445 & 8186.471 \\
\hline 134 & Rhode Island & 409.683 & 556.773 \\
\hline 135 & South Carolina & 3020.665 & 3996.493 \\
\hline 136 & South Dakota & 883.787 & 603.188 \\
\hline 137 & Tennessee & 6004.391 & 5578.131 \\
\hline 138 & Texas & 16623.260 & 19241.223 \\
\hline 139 & Utah & 1889.239 & 2040.500 \\
\hline 140 & Vermont & 519.825 & 398.050 \\
\hline 141 & Virginia & 4902.770 & 5691.889 \\
\hline 142 & Washington & 4625.073 & 4691.084 \\
\hline 143 & West Virginia & 542.840 & 1181.345 \\
\hline 144 & Wisconsin & 9389.802 & 4958.904 \\
\hline 145 & Wyoming & 95.302 & 230.518 \\
\hline
\end{tabular}




\begin{tabular}{|c|c|c|c|}
\hline & Locale & P07 30 & A07 30 \\
\hline 1 & Alabama & 12738.885 & 14642.756 \\
\hline 2 & Arizona & 16565.231 & 17707.934 \\
\hline 3 & Arkansas & 16178.502 & 14108.017 \\
\hline 4 & California & 93563.840 & 95415.601 \\
\hline 5 & Colorado & 10517.413 & 11316.316 \\
\hline 6 & Connecticut & 9152.310 & 7458.971 \\
\hline 7 & Delaware & 3183.384 & 3143.878 \\
\hline 8 & DC & .000 & .000 \\
\hline$\overline{9}$ & Florida & 46156.761 & 50856.239 \\
\hline 10 & Georgia & 32167.171 & 33389.762 \\
\hline 11 & Idaho & 5785.432 & 5946.759 \\
\hline 12 & IIllinois_N & 26836.504 & 22974.506 \\
\hline 13 & Illinois_S & 13418.252 & 11487.253 \\
\hline 14 & Adams & 487.260 & 107.343 \\
\hline 15 & Allen & 780.950 & 1016.107 \\
\hline 16 & Bartholomew & 319.510 & 445.502 \\
\hline 17 & Benton & 9.487 & 39.166 \\
\hline 18 & Blackford & 21.569 & 84.027 \\
\hline 19 & Boone & 161.851 & 117.437 \\
\hline 20 & Brown & 23.890 & 25.647 \\
\hline 21 & Carroll & 290.501 & 250.105 \\
\hline 22 & Cass & 280.695 & 251.561 \\
\hline 23 & Clark & 199.006 & 232.755 \\
\hline 24 & Clay & 34.853 & 41.894 \\
\hline 25 & Clinton & 359.042 & 325.789 \\
\hline 26 & Crawford & 16.350 & 19.912 \\
\hline 27 & Daviess & 199.173 & 179.997 \\
\hline 28 & Dearborn & 89.306 & 107.969 \\
\hline 29 & Decatur & 87.901 & 83.052 \\
\hline 30 & DeKalb & 363.143 & 124.164 \\
\hline$\overline{31}$ & Delaware & 365.916 & 293.039 \\
\hline 32 & Dubois & 179.323 & 211.935 \\
\hline 33 & Elkhart & 637.142 & 583.866 \\
\hline 34 & Fayette & 29.200 & 35.562 \\
\hline 35 & Floyd & 245.399 & 269.717 \\
\hline 36 & Fountain & 70.341 & 32.671 \\
\hline 37 & Franklin & 32.731 & 39.199 \\
\hline 38 & Fulton & 90.063 & 60.848 \\
\hline 39 & Gibson & 310.413 & 101.247 \\
\hline 40 & Grant & 174.747 & 174.488 \\
\hline 41 & Greene & 131.883 & 92.800 \\
\hline 42 & Hamilton & 609.960 & 629.650 \\
\hline 43 & Hancock & 113.063 & 187.519 \\
\hline 44 & Harrison & 265.750 & 193.894 \\
\hline 45 & Hendricks & 401.813 & 437.578 \\
\hline 46 & Henry & 62.735 & 80.129 \\
\hline 47 & Howard & 171.947 & 150.131 \\
\hline 48 & Huntington & 220.864 & 154.619 \\
\hline 49 & Jackson & 72.987 & 155.123 \\
\hline 50 & Jasper & 75.370 & 78.490 \\
\hline 51 & Jay & 80.258 & 85.418 \\
\hline
\end{tabular}




\begin{tabular}{|c|c|c|c|}
\hline & Locale & P07 30 & A07 30 \\
\hline 52 & Jefferson & 146.066 & 56.943 \\
\hline 53 & Jennings & 41.502 & 63.208 \\
\hline$\overline{54}$ & Johnson & 386.299 & 342.143 \\
\hline 55 & Knox & 61.532 & 77.839 \\
\hline 56 & Kosciusko & 374.049 & 239.733 \\
\hline 57 & LaGrange & 88.962 & 96.539 \\
\hline 58 & Lake & 784.217 & 932.745 \\
\hline 59 & La Porte & 252.960 & 312.721 \\
\hline 60 & Lawrence & 65.623 & 74.206 \\
\hline 61 & Madison & 284.317 & 333.031 \\
\hline 62 & Marion & 1807.356 & 2381.496 \\
\hline 63 & Marshall & 192.331 & 232.260 \\
\hline 64 & Martin & 18.938 & 20.229 \\
\hline 65 & Miami & 200.950 & 112.240 \\
\hline$\overline{66}$ & Monroe & 1297.954 & 284.069 \\
\hline 67 & Montgomery & 294.360 & 139.680 \\
\hline 68 & Morgan & 117.941 & 142.890 \\
\hline 69 & Newton & 90.965 & 32.903 \\
\hline 70 & Noble & 448.084 & 209.679 \\
\hline 71 & Ohio & 7.285 & 8.872 \\
\hline 72 & Orange & 202.720 & 37.772 \\
\hline 73 & Owen & 32.646 & 39.148 \\
\hline$\overline{74}$ & Parke & 84.356 & 44.409 \\
\hline 75 & Perry & 100.953 & 27.604 \\
\hline$\overline{76}$ & Pike & 16.476 & 20.066 \\
\hline 77 & Porter & 267.987 & 279.676 \\
\hline 78 & Posey & 44.903 & 51.146 \\
\hline 79 & Pulaski & 121.054 & 31.035 \\
\hline 80 & Putnam & 52.142 & 62.920 \\
\hline 81 & Randolph & 40.867 & 45.982 \\
\hline 82 & Ripley & 38.359 & 80.137 \\
\hline 83 & Rush & 22.808 & 35.801 \\
\hline 84 & St. Joseph & 714.711 & 644.369 \\
\hline 85 & Scott & 102.976 & 85.269 \\
\hline 86 & Shelby & 93.834 & 89.399 \\
\hline 87 & Spencer & 32.538 & 36.537 \\
\hline 88 & Starke & 28.592 & 44.597 \\
\hline 89 & Steuben & 200.068 & 96.424 \\
\hline 90 & Sullivan & 28.155 & 34.289 \\
\hline 91 & Switzerland & 13.688 & 19.160 \\
\hline 92 & Tippecanoe & 382.170 & 409.982 \\
\hline 93 & Tipton & 42.442 & 52.660 \\
\hline 94 & Union & 11.304 & 10.811 \\
\hline 95 & Vanderburgh & 1753.579 & 842.792 \\
\hline 96 & Vermillion & 19.734 & 50.437 \\
\hline 97 & Vigo & 175.365 & 276.329 \\
\hline 98 & Wabash & 61.753 & 116.151 \\
\hline 99 & Warren & 11.327 & 13.215 \\
\hline 100 & Warrick & 88.370 & 106.290 \\
\hline 101 & Washington & 64.008 & 54.819 \\
\hline 102 & Wayne & 308.631 & 196.863 \\
\hline
\end{tabular}




\begin{tabular}{|c|c|c|c|}
\hline & Locale & P07 30 & A07 30 \\
\hline 103 & Wells & 332.369 & 129.026 \\
\hline 104 & White & 214.423 & 53.764 \\
\hline 105 & Whitley & 225.013 & 93.608 \\
\hline 106 & lowa & 12944.877 & 10375.323 \\
\hline 107 & Kansas & 9220.688 & 8545.967 \\
\hline 108 & Kentucky_E & 7884.868 & 6619.700 \\
\hline 109 & Kentucky_W & 5256.579 & 4413.134 \\
\hline 110 & Louisiana & 8802.618 & 11055.152 \\
\hline 111 & Maine & 2909.272 & 4999.268 \\
\hline 112 & Maryland & 11935.750 & 14025.188 \\
\hline 113 & Massachusetts & 15779.175 & 16263.510 \\
\hline 114 & Michigan_E & 10528.747 & 11374.106 \\
\hline 115 & Michigan W & 10528.747 & 11374.106 \\
\hline 116 & Minnesota & 17869.823 & 18343.386 \\
\hline 117 & Mississippi & 11516.930 & 9214.538 \\
\hline 118 & Missouri & 18469.444 & 16592.158 \\
\hline 119 & Montana & 1594.342 & 1935.575 \\
\hline 120 & Nebraska & 8244.509 & 6943.465 \\
\hline 121 & Nevada & 5744.911 & 6467.200 \\
\hline 122 & New Hampshire & 4130.938 & 3464.569 \\
\hline 123 & New Jersey & 18873.744 & 21243.110 \\
\hline 124 & New Mexico & 3357.476 & 3622.842 \\
\hline 125 & New York & 34621.667 & 37988.305 \\
\hline 126 & North Carolina & 36519.953 & 31224.082 \\
\hline 127 & North Dakota & 1431.236 & 1470.359 \\
\hline 128 & Ohio_N & 10785.618 & 9166.138 \\
\hline 129 & Ohio_M & 10785.618 & 9166.138 \\
\hline 130 & Ohio_S & 10785.618 & 9166.138 \\
\hline 131 & Oklahoma & 7621.091 & 8149.811 \\
\hline 132 & Oregon & 9863.857 & 11793.116 \\
\hline 133 & Pennsylvania & 32633.388 & 33035.580 \\
\hline 134 & Rhode Island & 2786.566 & 2357.196 \\
\hline 135 & South Carolina & 13187.143 & 12448.246 \\
\hline 136 & South Dakota & 2175.892 & 2212.544 \\
\hline 137 & Tennessee & 22246.910 & 19365.316 \\
\hline 138 & Texas & 64264.985 & 67018.457 \\
\hline 139 & Utah & 7021.481 & 7624.426 \\
\hline 140 & Vermont & 1813.991 & 1852.669 \\
\hline 141 & Virginia & 21529.548 & 22728.853 \\
\hline 142 & Washington & 17753.347 & 21154.278 \\
\hline 143 & West Virginia & 3103.811 & 3181.996 \\
\hline 144 & Wisconsin & 24779.439 & 24179.807 \\
\hline 145 & Wyoming & 730.937 & 847.624 \\
\hline
\end{tabular}




\begin{tabular}{|c|c|c|c|}
\hline & Locale & P08 30 & A08 30 \\
\hline 1 & Alabama & 3524.051 & 4857.979 \\
\hline 2 & Arizona & 9170.966 & 7093.221 \\
\hline 3 & Arkansas & 2027.860 & 2190.764 \\
\hline 4 & California & 44506.131 & 44605.212 \\
\hline 5 & Colorado & 4954.523 & 4892.264 \\
\hline 6 & Connecticut & 2971.843 & 2329.862 \\
\hline 7 & Delaware & 658.185 & 575.146 \\
\hline 8 & DC & .000 & .000 \\
\hline 9 & Florida & 19711.945 & 18654.979 \\
\hline 10 & Georgia & 7530.899 & 9520.322 \\
\hline 11 & Idaho & 1991.132 & 1404.015 \\
\hline 12 & Illinois_N & 7026.445 & 5925.415 \\
\hline 13 & Illinois_S & 3513.223 & 2962.708 \\
\hline 14 & Adams & 55.904 & 46.361 \\
\hline 15 & Allen & 404.461 & 293.958 \\
\hline 16 & Bartholomew & 81.448 & 59.212 \\
\hline 17 & Benton & 4.586 & 3.870 \\
\hline 18 & Blackford & 7.182 & 6.061 \\
\hline 19 & Boone & 68.089 & 49.132 \\
\hline 20 & Brown & 10.083 & 11.212 \\
\hline 21 & Carroll & 12.354 & 10.425 \\
\hline 22 & Cass & 36.959 & 30.395 \\
\hline 23 & Clark & 75.472 & 62.740 \\
\hline 24 & Clay & 16.531 & 13.808 \\
\hline 25 & Clinton & 50.961 & 41.989 \\
\hline 26 & Crawford & 7.904 & 6.670 \\
\hline 27 & Daviess & 18.993 & 25.343 \\
\hline 28 & Dearborn & 42.714 & 204.901 \\
\hline 29 & Decatur & 15.361 & 12.963 \\
\hline 30 & DeKalb & 43.614 & 31.754 \\
\hline 31 & Delaware & 76.375 & 66.013 \\
\hline 32 & Dubois & 57.069 & 37.000 \\
\hline 33 & Elkhart & 229.348 & 157.302 \\
\hline 34 & Fayette & 14.115 & 11.912 \\
\hline 35 & Floyd & 79.559 & 60.442 \\
\hline 36 & Fountain & 12.397 & 12.420 \\
\hline 37 & Franklin & 15.003 & 12.661 \\
\hline 38 & Fulton & 13.119 & 30.401 \\
\hline 39 & Gibson & 29.502 & 21.802 \\
\hline 40 & Grant & .000 & .000 \\
\hline 41 & Greene & 22.507 & 18.188 \\
\hline 42 & Hamilton & 252.064 & 208.702 \\
\hline 43 & Hancock & 54.158 & 58.593 \\
\hline 44 & Harrison & 29.172 & 24.618 \\
\hline 45 & Hendricks & 143.278 & 116.821 \\
\hline 46 & Henry & 27.715 & 22.701 \\
\hline 47 & Howard & 54.725 & 55.327 \\
\hline 48 & Huntington & 91.442 & 52.073 \\
\hline 49 & Jackson & 25.728 & 21.711 \\
\hline 50 & Jasper & 26.846 & 19.991 \\
\hline 51 & Jay & 11.778 & 18.603 \\
\hline
\end{tabular}




\begin{tabular}{|c|c|c|c|}
\hline & Locale & P08 30 & A08 30 \\
\hline 52 & Jefferson & 21.589 & 19.989 \\
\hline 53 & Jennings & 20.062 & 16.930 \\
\hline 54 & Johnson & 149.325 & 117.345 \\
\hline 55 & Knox & 24.607 & 19.786 \\
\hline 56 & Kosciusko & 53.673 & 52.412 \\
\hline 57 & LaGrange & 31.053 & 25.181 \\
\hline 58 & Lake & 296.700 & 310.289 \\
\hline 59 & La Porte & 68.121 & 92.735 \\
\hline 60 & Lawrence & 28.917 & 32.524 \\
\hline 61 & Madison & 85.048 & 71.629 \\
\hline 62 & Marion & 624.375 & 722.105 \\
\hline 63 & Marshall & 32.176 & 60.114 \\
\hline 64 & Martin & 5.653 & 4.770 \\
\hline 65 & Miami & 19.855 & 17.611 \\
\hline 66 & Monroe & 137.202 & 111.229 \\
\hline 67 & Montgomery & 24.213 & 20.934 \\
\hline 68 & Morgan & 142.773 & 86.732 \\
\hline 69 & Newton & 14.695 & 9.980 \\
\hline 70 & Noble & 33.654 & 27.459 \\
\hline 71 & Ohio & 3.522 & 2.972 \\
\hline 72 & Orange & 12.379 & 11.970 \\
\hline 73 & Owen & 15.026 & 12.681 \\
\hline 74 & Parke & 9.849 & 9.866 \\
\hline$\overline{75}$ & Perry & 10.872 & 9.052 \\
\hline 76 & Pike & 7.965 & 6.721 \\
\hline 77 & Porter & 99.582 & 82.877 \\
\hline 78 & Posey & 17.333 & 14.627 \\
\hline 79 & Pulaski & 8.245 & 6.958 \\
\hline 80 & Putnam & 31.234 & 23.731 \\
\hline 81 & Randolph & 17.028 & 14.034 \\
\hline 82 & Ripley & 20.188 & 20.992 \\
\hline 83 & Rush & 9.806 & 8.566 \\
\hline 84 & St. Joseph & 215.482 & 170.365 \\
\hline 85 & Scott & 15.615 & 23.376 \\
\hline 86 & Shelby & 26.855 & 22.663 \\
\hline 87 & Spencer & 11.912 & 10.052 \\
\hline 88 & Starke & 13.822 & 11.664 \\
\hline 89 & Steuben & 23.945 & 19.316 \\
\hline 90 & Sullivan & 13.610 & 14.473 \\
\hline 91 & Switzerland & 6.617 & 5.584 \\
\hline 92 & Tippecanoe & 175.131 & 126.234 \\
\hline 93 & Tipton & 9.351 & 7.891 \\
\hline 94 & Union & 4.291 & 3.621 \\
\hline 95 & Vanderburgh & 108.035 & 125.285 \\
\hline 96 & Vermillion & 9.071 & 7.655 \\
\hline 97 & Vigo & 125.258 & 81.027 \\
\hline 98 & Wabash & 37.176 & 24.697 \\
\hline 99 & Warren & 4.759 & 4.016 \\
\hline 100 & Warrick & 41.357 & 34.901 \\
\hline 101 & Washington & 18.674 & 20.737 \\
\hline 102 & Wayne & 65.214 & 45.797 \\
\hline
\end{tabular}




\begin{tabular}{|c|c|c|c|}
\hline & Locale & P08 30 & A08 30 \\
\hline 103 & Wells & 19.054 & 15.914 \\
\hline 104 & White & 21.536 & $\overline{15.724}$ \\
\hline 105 & Whitley & 29.564 & 22.090 \\
\hline 106 & lowa & 2258.308 & 2021.910 \\
\hline 107 & Kansas & 1915.305 & 1771.902 \\
\hline 108 & Kentucky_E & 1819.874 & 2801.856 \\
\hline 109 & Kentucky_W & 1213.249 & 1867.904 \\
\hline 110 & Louisiana & 2838.709 & 3060.829 \\
\hline$\overline{111}$ & Maine & 1049.999 & 1005.456 \\
\hline 112 & Maryland & 5077.065 & 4449.165 \\
\hline 113 & Massachusetts & 7768.471 & 5984.020 \\
\hline 114 & Michigan_E & 3542.843 & 3146.515 \\
\hline 115 & Michigan_W & 3542.843 & 3146.515 \\
\hline 116 & Minnesota & 6208.854 & 4620.041 \\
\hline 117 & Mississippi & 1933.222 & 2557.812 \\
\hline 118 & Missouri & 4227.923 & 4679.486 \\
\hline 119 & Montana & 617.595 & 586.644 \\
\hline 120 & Nebraska & 1268.744 & 1112.503 \\
\hline 121 & Nevada & 2453.236 & 2251.664 \\
\hline 122 & New Hampshire & 2065.733 & 1530.248 \\
\hline 123 & New Jersey & 6971.957 & 7025.336 \\
\hline 124 & New Mexico & 1635.225 & 1324.045 \\
\hline 125 & New York & 14101.686 & 15385.557 \\
\hline 126 & North Carolina & 9460.002 & 15014.869 \\
\hline 127 & North Dakota & 404.575 & 353.221 \\
\hline 128 & Ohio_N & 2649.120 & 2462.387 \\
\hline 129 & Ohio_M & 2649.120 & 2462.387 \\
\hline 130 & Ohio_s & 2649.120 & 2462.387 \\
\hline 131 & Oklahoma & 2723.030 & 2694.547 \\
\hline 132 & Oregon & 4663.333 & 3873.069 \\
\hline 133 & Pennsylvania & 9475.265 & 10155.549 \\
\hline 134 & Rhode Island & 909.402 & 768.531 \\
\hline 135 & South Carolina & 3633.497 & 3873.159 \\
\hline 136 & South Dakota & 939.313 & 659.880 \\
\hline 137 & Tennessee & 4749.243 & 6384.817 \\
\hline 138 & Texas & 25996.535 & 24547.922 \\
\hline 139 & Utah & 2786.028 & 2286.909 \\
\hline 140 & Vermont & 800.555 & 645.866 \\
\hline 141 & \begin{tabular}{|l} 
Virginia \\
\end{tabular} & 6991.177 & 9424.330 \\
\hline 142 & Washington & 7230.480 & 6252.251 \\
\hline 143 & West Virginia & 1034.317 & 1047.541 \\
\hline 144 & Wisconsin & 4418.480 & 4070.041 \\
\hline 145 & Wyoming & 317.368 & 292.653 \\
\hline
\end{tabular}




\begin{tabular}{|c|c|c|c|}
\hline & $\begin{array}{l}\text { Locale } \\
\end{array}$ & P09 30 & A09 30 \\
\hline 1 & Alabama & 503.724 & 513.399 \\
\hline 2 & Arizona & 156.912 & 149.862 \\
\hline 3 & Arkansas & 154.933 & 149.413 \\
\hline 4 & California & 1043.435 & 1012.112 \\
\hline 5 & Colorado & 64.793 & 61.901 \\
\hline 6 & Connecticut & 76.476 & 77.274 \\
\hline 7 & Delaware & 21.748 & 22.026 \\
\hline 8 & $\mathrm{DC}$ & .000 & .000 \\
\hline 9 & Florida & 353.361 & 341.037 \\
\hline 10 & Georgia & 1314.651 & 1358.286 \\
\hline$\overline{11}$ & Idaho & 23.389 & 22.149 \\
\hline 12 & Illinois_N & 133.068 & 126.689 \\
\hline$\overline{13}$ & Illinois_S & 66.534 & 63.345 \\
\hline 14 & Adams & 2.738 & 2.582 \\
\hline 15 & Allen & 10.445 & 9.868 \\
\hline 16 & Bartholomew & 7.631 & 7.196 \\
\hline 17 & Benton & .467 & .441 \\
\hline 18 & Blackford & .070 & .066 \\
\hline 19 & Boone & .844 & .796 \\
\hline 20 & Brown & .000 & .000 \\
\hline 21 & Carroll & 3.542 & 3.340 \\
\hline 22 & Cass & .082 & .078 \\
\hline 23 & Clark & 11.657 & 10.991 \\
\hline 24 & Clay & .088 & .083 \\
\hline 25 & Clinton & .563 & .530 \\
\hline 26 & Crawford & .000 & .000 \\
\hline 27 & Daviess & .589 & .555 \\
\hline 28 & Dearborn & .000 & .000 \\
\hline 29 & Decatur & .090 & .085 \\
\hline 30 & DeKalb & .108 & .102 \\
\hline 31 & Delaware & 1.312 & 1.237 \\
\hline 32 & Dubois & 63.538 & 59.911 \\
\hline 33 & Elkhart & 39.728 & 37.479 \\
\hline 34 & Fayette & .451 & .425 \\
\hline 35 & Floyd & 4.516 & 4.258 \\
\hline 36 & Fountain & .000 & .000 \\
\hline 37 & Franklin & .000 & .000 \\
\hline 38 & Fulton & .000 & .000 \\
\hline 39 & Gibson & .000 & .000 \\
\hline 40 & Grant & 1.183 & 1.116 \\
\hline 41 & Greene & .083 & .078 \\
\hline 42 & Hamilton & 4.067 & 3.863 \\
\hline 43 & Hancock & .829 & .782 \\
\hline 44 & Harrison & 8.135 & 7.670 \\
\hline 45 & Hendricks & .179 & .169 \\
\hline 46 & Henry & 1.284 & 1.211 \\
\hline 47 & Howard & 3.269 & 3.082 \\
\hline 48 & Huntington & .000 & .000 \\
\hline 49 & Jackson & .536 & .506 \\
\hline 50 & Jasper & .094 & .089 \\
\hline 51 & Jay & .471 & .444 \\
\hline
\end{tabular}




\begin{tabular}{|c|c|c|c|}
\hline & Locale & P09 30 & A09 30 \\
\hline 52 & Jefferson & .096 & .091 \\
\hline 53 & Jennings & .000 & .000 \\
\hline 54 & Johnson & 1.250 & 1.178 \\
\hline 55 & Knox & .075 & .071 \\
\hline 56 & Kosciusko & 3.055 & 2.898 \\
\hline 57 & LaGrange & 3.183 & 3.001 \\
\hline 58 & Lake & 2.183 & 2.058 \\
\hline 59 & La Porte & 2.413 & 2.372 \\
\hline 60 & Lawrence & .081 & .076 \\
\hline 61 & Madison & 3.808 & 3.608 \\
\hline 62 & Marion & 12.549 & 11.935 \\
\hline 63 & Marshall & .607 & .573 \\
\hline 64 & Martin & .000 & .000 \\
\hline 65 & Miami & 2.815 & 2.654 \\
\hline 66 & Monroe & .747 & .705 \\
\hline 67 & Montgomery & .000 & .000 \\
\hline 68 & Morgan & .119 & .113 \\
\hline 69 & Newton & .514 & .484 \\
\hline 70 & Noble & .583 & .550 \\
\hline 71 & Ohio & .000 & .000 \\
\hline 72 & Orange & 14.493 & 13.666 \\
\hline 73 & Owen & .000 & .000 \\
\hline 74 & Parke & .085 & .080 \\
\hline 75 & Perry & 2.235 & 2.107 \\
\hline$\overline{76}$ & Pike & .000 & .000 \\
\hline 77 & Porter & 1.474 & 1.495 \\
\hline 78 & Posey & .000 & .000 \\
\hline 79 & Pulaski & .092 & .087 \\
\hline 80 & Putnam & .000 & .000 \\
\hline 81 & Randolph & .926 & .962 \\
\hline 82 & Ripley & 1.679 & 1.583 \\
\hline 83 & Rush & .000 & .000 \\
\hline 84 & St. Joseph & 1.715 & 1.634 \\
\hline 85 & Scott & .555 & .523 \\
\hline 86 & Shelby & 2.863 & 2.700 \\
\hline 87 & Spencer & 6.158 & 5.806 \\
\hline 88 & Starke & .080 & .076 \\
\hline 89 & Steuben & .092 & .087 \\
\hline 90 & Sullivan & .000 & .000 \\
\hline 91 & Switzerland & .123 & .116 \\
\hline 92 & Tippecanoe & 8.639 & 8.146 \\
\hline 93 & Tipton & .000 & .000 \\
\hline 94 & Union & .000 & .000 \\
\hline 95 & Vanderburgh & 4.053 & 3.840 \\
\hline 96 & Vermillion & .000 & .000 \\
\hline 97 & Vigo & .534 & .503 \\
\hline 98 & Wabash & .078 & .074 \\
\hline 99 & Warren & .000 & .000 \\
\hline 100 & Warrick & .103 & .097 \\
\hline 101 & Washington & 17.110 & 16.133 \\
\hline 102 & Wayne & 5.223 & 4.924 \\
\hline
\end{tabular}




\begin{tabular}{|c|c|c|c|}
\hline & Locale & P09 30 & A09 30 \\
\hline 103 & Wells & .287 & .290 \\
\hline 104 & White & 4.248 & 4.292 \\
\hline 105 & Whitley & .450 & .424 \\
\hline 106 & lowa & 72.942 & 68.975 \\
\hline 107 & Kansas & 45.393 & 43.015 \\
\hline 108 & Kentucky_E & 49.253 & 48.152 \\
\hline 109 & Kentucky_W & 32.835 & 32.101 \\
\hline 110 & Louisiana & 13.205 & 12.655 \\
\hline 111 & Maine & 91.196 & 93.855 \\
\hline 112 & Maryland & 79.771 & 78.697 \\
\hline 113 & Massachusetts & 270.184 & 276.304 \\
\hline 114 & Michigan_E & 156.835 & 148.426 \\
\hline 115 & Michigan_W & 156.835 & 148.426 \\
\hline$\overline{116}$ & Minnesota & 138.357 & 131.965 \\
\hline 117 & Mississippi & 305.936 & 293.048 \\
\hline 118 & Missouri & 128.178 & 122.040 \\
\hline 119 & Montana & 8.821 & 8.426 \\
\hline 120 & Nebraska & 33.378 & 32.098 \\
\hline$\overline{121}$ & Nevada & 30.112 & 28.426 \\
\hline 122 & New Hampshire & 67.817 & 69.452 \\
\hline 123 & New Jersey & 242.605 & 245.605 \\
\hline 124 & New Mexico & 10.525 & 10.036 \\
\hline 125 & New York & 375.431 & 374.474 \\
\hline 126 & North Carolina & 3494.067 & 3557.675 \\
\hline 127 & North Dakota & 9.291 & 8.849 \\
\hline 128 & Ohio_N & 78.676 & 76.134 \\
\hline 129 & Ohio_M & 78.676 & 76.134 \\
\hline 130 & Ohio_s & 78.676 & 76.134 \\
\hline 131 & Oklahoma & 39.147 & 37.645 \\
\hline 132 & Oregon & 75.853 & 72.087 \\
\hline 133 & Pennsylvania & 425.913 & 423.989 \\
\hline 134 & Rhode Island & 117.354 & 120.886 \\
\hline 135 & South Carolina & 1225.446 & 1277.493 \\
\hline 136 & South Dakota & 26.408 & 26.147 \\
\hline 137 & Tennessee & 498.850 & 494.247 \\
\hline 138 & Texas & 482.018 & 469.412 \\
\hline 139 & Utah & 79.290 & 75.068 \\
\hline 140 & Vermont & 29.730 & 28.261 \\
\hline 141 & Virginia & 691.445 & 696.642 \\
\hline 142 & Washington & 107.105 & 102.710 \\
\hline 143 & West Virginia & 14.243 & 13.989 \\
\hline 144 & Wisconsin & 205.204 & 197.871 \\
\hline 145 & Wyoming & 2.078 & 1.976 \\
\hline
\end{tabular}




\begin{tabular}{|c|c|c|c|}
\hline & Locale & P10 30 & A10 30 \\
\hline 1 & Alabama & 231.861 & 263.245 \\
\hline 2 & Arizona & 411.588 & 241.230 \\
\hline 3 & Arkansas & 157.613 & 151.108 \\
\hline 4 & California & 2516.502 & 1523.447 \\
\hline 5 & Colorado & 278.501 & 148.914 \\
\hline 6 & Connecticut & 209.577 & 297.673 \\
\hline 7 & Delaware & 64.586 & 160.787 \\
\hline 8 & $\mathrm{DC}$ & .000 & .000 \\
\hline 9 & Florida & 1342.954 & 616.317 \\
\hline 10 & Georgia & 635.464 & 602.992 \\
\hline 11 & Idaho & 110.875 & 81.173 \\
\hline 12 & Illinois_N & 581.860 & 670.956 \\
\hline 13 & Illinois_S & 290.930 & 335.478 \\
\hline 14 & Adams & 2.876 & 3.729 \\
\hline 15 & Allen & 33.250 & 7.911 \\
\hline 16 & Bartholomew & 3.658 & 3.672 \\
\hline 17 & Benton & 2.035 & .000 \\
\hline 18 & Blackford & 1.502 & .000 \\
\hline 19 & Boone & 3.788 & .000 \\
\hline 20 & Brown & 1.283 & .180 \\
\hline 21 & Carroll & 2.392 & .000 \\
\hline 22 & Cass & 2.306 & .000 \\
\hline 23 & Clark & 4.403 & 14.586 \\
\hline 24 & Clay & 1.670 & 1.091 \\
\hline 25 & Clinton & 2.208 & 3.384 \\
\hline 26 & Crawford & 1.220 & .000 \\
\hline 27 & Daviess & 2.332 & 1.215 \\
\hline 28 & Dearborn & 2.071 & .262 \\
\hline 29 & Decatur & 3.248 & .000 \\
\hline 30 & DeKalb & 5.321 & 5.043 \\
\hline 31 & Delaware & 5.206 & 1.631 \\
\hline 32 & Dubois & 4.031 & 1.193 \\
\hline 33 & Elkhart & 9.863 & 37.029 \\
\hline 34 & Fayette & 2.131 & .000 \\
\hline 35 & Floyd & 4.447 & 2.144 \\
\hline 36 & Fountain & 2.631 & .177 \\
\hline 37 & Franklin & 1.678 & .000 \\
\hline 38 & Fulton & 2.377 & .181 \\
\hline 39 & Gibson & 1.888 & .000 \\
\hline 40 & Grant & 2.597 & 1.325 \\
\hline 41 & Greene & 1.875 & .000 \\
\hline 42 & Hamilton & 18.241 & 1.804 \\
\hline 43 & Hancock & 5.106 & .000 \\
\hline 44 & Harrison & 1.989 & 1.267 \\
\hline 45 & Hendricks & 5.215 & .369 \\
\hline 46 & Henry & 3.287 & .000 \\
\hline 47 & Howard & 3.289 & .000 \\
\hline 48 & Huntington & 3.203 & 1.259 \\
\hline 49 & Jackson & 3.636 & 3.226 \\
\hline 50 & Jasper & 2.084 & .000 \\
\hline 51 & Jay & 2.280 & .000 \\
\hline
\end{tabular}




\begin{tabular}{|c|c|c|c|}
\hline & Locale & P10 30 & A10 30 \\
\hline 52 & Jefferson & 1.600 & 1.191 \\
\hline 53 & Jennings & 1.761 & .215 \\
\hline 54 & Johnson & 3.946 & 1.777 \\
\hline 55 & Knox & 3.766 & .000 \\
\hline 56 & Kosciusko & 4.769 & 6.456 \\
\hline 57 & LaGrange & 3.255 & .218 \\
\hline 58 & Lake & 34.967 & 26.549 \\
\hline 59 & La Porte & 7.523 & 5.820 \\
\hline 60 & Lawrence & 3.338 & .000 \\
\hline 61 & Madison & 4.164 & 6.865 \\
\hline 62 & Marion & 79.213 & 114.021 \\
\hline 63 & Marshall & 3.219 & 2.714 \\
\hline 64 & Martin & 1.710 & .000 \\
\hline 65 & Miami & 3.555 & .929 \\
\hline 66 & Monroe & 9.057 & 3.590 \\
\hline 67 & Montgomery & 2.794 & 3.335 \\
\hline 68 & Morgan & 1.901 & 9.233 \\
\hline 69 & Newton & 2.012 & .177 \\
\hline 70 & Noble & 3.761 & 4.328 \\
\hline 71 & \begin{tabular}{|l|} 
Ohio \\
\end{tabular} & 1.220 & .000 \\
\hline 72 & Orange & 1.561 & .000 \\
\hline 73 & Owen & 1.290 & .000 \\
\hline 74 & Parke & 1.586 & .000 \\
\hline 75 & Perry & 1.806 & .000 \\
\hline 76 & Pike & 1.566 & .000 \\
\hline 77 & Porter & 15.737 & 8.851 \\
\hline 78 & Posey & 2.673 & 33.946 \\
\hline 79 & Pulaski & 3.111 & .000 \\
\hline 80 & Putnam & 1.575 & .000 \\
\hline 81 & Randolph & 1.842 & .000 \\
\hline 82 & Ripley & 2.432 & 1.187 \\
\hline 83 & Rush & 2.009 & .000 \\
\hline 84 & St. Joseph & 21.194 & 16.352 \\
\hline 85 & Scott & 1.298 & 3.336 \\
\hline 86 & Shelby & 2.652 & 3.415 \\
\hline 87 & Spencer & 2.785 & .169 \\
\hline 88 & Starke & 1.481 & .995 \\
\hline 89 & Steuben & 1.867 & .000 \\
\hline 90 & Sullivan & 2.181 & .189 \\
\hline 91 & Switzerland & 1.309 & .000 \\
\hline 92 & Tippecanoe & 8.823 & 39.006 \\
\hline 93 & Tipton & 2.135 & .170 \\
\hline 94 & Union & 1.606 & .791 \\
\hline 95 & Vanderburgh & 12.529 & 46.757 \\
\hline 96 & Vermillion & 1.640 & 27.257 \\
\hline 97 & Vigo & 6.283 & 18.903 \\
\hline 98 & Wabash & 2.400 & 6.051 \\
\hline 99 & Warren & 1.620 & .000 \\
\hline 100 & Warrick & 7.270 & 1.706 \\
\hline 101 & Washington & 1.302 & .000 \\
\hline 102 & Wayne & 4.296 & 2.097 \\
\hline
\end{tabular}

F31 


\begin{tabular}{|c|c|c|c|}
\hline & Locale & P10 30 & A10 30 \\
\hline 103 & Wells & 12.663 & .000 \\
\hline 104 & White & 3.247 & .000 \\
\hline 105 & Whitley & 2.653 & .211 \\
\hline 106 & lowa & 201.161 & 121.471 \\
\hline 107 & Kansas & 179.674 & 125.001 \\
\hline 108 & Kentucky_E & 142.215 & 166.161 \\
\hline 109 & Kentucky_W & 94.810 & 110.774 \\
\hline 110 & Louisiana & 219.577 & 512.493 \\
\hline 111 & Maine & 73.267. & 33.212 \\
\hline 112 & Maryland & 303.288 & 322.730 \\
\hline 113 & Massachusetts & 405.652 & 416.599 \\
\hline 114 & Michigan_E & 244.006 & 311.568 \\
\hline 115 & Michigan_W & 244.006 & 311.568 \\
\hline 116 & Minnesota & 388.850 & 196.040 \\
\hline 117 & Mississippi & 131.654 & 137.379 \\
\hline 118 & Missouri & 398.960 & 439.948 \\
\hline 119 & Montana & 51.969 & 10.446 \\
\hline 120 & Nebraska & 125.951 & 51.415 \\
\hline 121 & Nevada & 150.579 & 47.621 \\
\hline 122 & New Hampshire & 75.074 & 42.058 \\
\hline 123 & New Jersey & 938.986 & 1364.888 \\
\hline 124 & New Mexico & 66.825 & 27.175 \\
\hline 125 & New York & 1290.389 & 1103.931 \\
\hline 126 & North Carolina & 604.643 & 1144.881 \\
\hline 127 & North Dakota & 50.789 & .972 \\
\hline 128 & Ohio_N & 223.530 & 279.806 \\
\hline 129 & Ohio_M & 223.530 & 279.806 \\
\hline 130 & Ohio_s & 223.530 & 279.806 \\
\hline 131 & Oklahoma & 177.789 & 74.574 \\
\hline 132 & Oregon & 294.756 & 107.926 \\
\hline 133 & Pennsylvania & 651.282 & 603.889 \\
\hline 134 & Rhode Island & 49.464 & 49.276 \\
\hline 135 & South Carolina & 199.247 & 833.878 \\
\hline 136 & South Dakota & 52.132 & 13.128 \\
\hline 137 & \begin{tabular}{|l|} 
Tennessee \\
\end{tabular} & 439.662 & 765.675 \\
\hline 138 & Texas & 1564.253 & 2144.401 \\
\hline 139 & Utah & 172.190 & 151.845 \\
\hline 140 & Vermont & 43.508 & 7.518 \\
\hline 141 & Virginia & 363.502 & 468.383 \\
\hline 142 & Washington & 471.316 & 133.483 \\
\hline 143 & West Virginia & 58.581 & 228.144 \\
\hline 144 & \begin{tabular}{|l} 
Wisconsin \\
\end{tabular} & 358.949 & 251.373 \\
\hline 145 & Wyoming & 16.324 & 22.701 \\
\hline
\end{tabular}




\begin{tabular}{|c|c|c|c|}
\hline & Locale & P11 30 & A11 30 \\
\hline 1 & Alabama & 5292.841 & 5966.629 \\
\hline 2 & Arizona & 11154.713 & 12326.531 \\
\hline 3 & Arkansas & 2750.390 & 3929.044 \\
\hline 4 & California & 64686.806 & 55516.274 \\
\hline 5 & Colorado & 8011.632 & 6744.096 \\
\hline 6 & Connecticut & 4582.693 & 4521.253 \\
\hline 7 & Delaware & 884.912 & 1210.354 \\
\hline 8 & DC & .000 & .000 \\
\hline 9 & Florida & 31607.322 & 33970.331 \\
\hline 10 & Georgia & 18076.258 & 14060.561 \\
\hline 11 & Idaho & 1765.760 & 2330.610 \\
\hline 12 & Illinois_N & 14746.006 & 10951.030 \\
\hline 13 & Illinois_s & 7373.004 & 5475.515 \\
\hline 14 & Adams & 17.027 & 50.986 \\
\hline 15 & Allen & 969.684 & 526.494 \\
\hline 16 & Bartholomew & 84.214 & 112.025 \\
\hline 17 & Benton & 2.548 & 9.843 \\
\hline 18 & Blackford & 5.666 & 15.415 \\
\hline 19 & Boone & 63.076 & 96.035 \\
\hline 20 & Brown & 1.060 & 20.863 \\
\hline 21 & Carroll & 4.921 & 26.516 \\
\hline 22 & Cass & 28.005 & 51.864 \\
\hline 23 & Clark & 142.012 & 153.645 \\
\hline 24 & Clay & 2.992 & 34.697 \\
\hline 25 & Clinton & 16.703 & 46.756 \\
\hline 26 & Crawford & 1.394 & 16.965 \\
\hline 27 & Daviess & 11.539 & 40.768 \\
\hline 28 & Dearborn & 24.897 & 90.554 \\
\hline 29 & Decatur & 13.047 & 32.971 \\
\hline 30 & DeKalb & 39.337 & 65.765 \\
\hline 31 & Delaware & 115.822 & 163.105 \\
\hline 32 & Dubois & 135.648 & 58.095 \\
\hline 33 & Elkhart & 543.717 & 289.423 \\
\hline 34 & Fayette & 7.648 & 30.298 \\
\hline 35 & Floyd & 74.984 & 115.569 \\
\hline 36 & Fountain & 2.598 & 22.027 \\
\hline 37 & Franklin & 7.684 & 32.203 \\
\hline 38 & Fulton & 5.002 & 27.376 \\
\hline 39 & Gibson & 12.197 & 43.224 \\
\hline 40 & Grant & 39.737 & 79.878 \\
\hline 41 & Greene & 8.665 & 43.867 \\
\hline 42 & Hamilton & 559.825 & 514.552 \\
\hline 43 & Hancock & 28.974 & 115.015 \\
\hline 44 & Harrison & 27.796 & 62.615 \\
\hline 45 & Hendricks & 179.002 & 279.631 \\
\hline 46 & Henry & 19.115 & 55.698 \\
\hline 47 & Howard & 65.169 & 112.263 \\
\hline 48 & Huntington & 27.611 & 57.943 \\
\hline 49 & Jackson & 27.516 & 55.222 \\
\hline 50 & Jasper & 13.932 & 42.936 \\
\hline 51 & Jay & 5.805 & 25.281 \\
\hline
\end{tabular}




\begin{tabular}{|c|c|c|c|}
\hline & Locale & P11 30 & A11 30 \\
\hline 52 & Jefferson & 18.784 & 46.340 \\
\hline 53 & Jennings & 8.835 & 43.062 \\
\hline 54 & Johnson & 100.772 & 246.968 \\
\hline 55 & Knox & 33.092 & 47.419 \\
\hline 56 & Kosciusko & 58.157 & 105.128 \\
\hline 57 & LaGrange & 31.007 & 61.004 \\
\hline 58 & Lake & 544.355 & 623.629 \\
\hline 59 & La Porte & 85.656 & 140.750 \\
\hline 60 & Lawrence & 15.649 & 57.755 \\
\hline 61 & Madison & 61.748 & 181.759 \\
\hline 62 & Marion & 2428.141 & 1188.731 \\
\hline 63 & Marshall & 59.992 & 68.161 \\
\hline 64 & Martin & 2.008 & 12.133 \\
\hline 65 & Miami & 17.737 & 41.948 \\
\hline 66 & Monroe & 70.136 & 198.063 \\
\hline 67 & Montgomery & 17.916 & 51.972 \\
\hline 68 & Morgan & 47.018 & 120.389 \\
\hline 69 & Newton & 3.113 & 18.196 \\
\hline 70 & Noble & 23.899 & 67.045 \\
\hline 71 & Ohio & .000 & 7.559 \\
\hline 72 & Orange & 4.516 & 26.571 \\
\hline 73 & Owen & 7.080 & 32.253 \\
\hline 74 & Parke & 6.145 & 21.141 \\
\hline 75 & Perry & 5.361 & 22.656 \\
\hline 76 & Pike & 8.930 & 17.095 \\
\hline 77 & Porter & 152.430 & 204.583 \\
\hline 78 & Posey & 25.988 & 37.205 \\
\hline 79 & Pulaski & 11.358 & 17.697 \\
\hline 80 & Putnam & 5.632 & 52.556 \\
\hline 81 & Randolph & 10.362 & 32.356 \\
\hline 82 & Ripley & 43.599 & 38.205 \\
\hline 83 & Rush & 10.622 & 20.370 \\
\hline 84 & St. Joseph & 500.220 & 387.137 \\
\hline 85 & Scott & 7.058 & 33.515 \\
\hline 86 & Shelby & 74.405 & 57.641 \\
\hline 87 & Spencer & 6.964 & 25.568 \\
\hline 88 & Starke & 4.092 & 29.667 \\
\hline 89 & Steuben & 22.945 & 46.484 \\
\hline 90 & Sullivan & 6.342 & 29.213 \\
\hline 91 & Switzerland & 1.489 & 14.203 \\
\hline 92 & Tippecanoe & 96.597 & 253.622 \\
\hline 93 & Tipton & 7.184 & 20.072 \\
\hline 94 & Union & 6.482 & 9.210 \\
\hline 95 & Vanderburgh & 413.069 & 231.028 \\
\hline 96 & Vermillion & 1.007 & 19.471 \\
\hline 97 & Vigo & 100.398 & 130.164 \\
\hline 98 & Wabash & 15.361 & 43.000 \\
\hline 99 & Warren & 6.723 & 10.215 \\
\hline 100 & Warrick & 16.412 & 88.770 \\
\hline 101 & Washington & 9.715 & 40.082 \\
\hline 102 & Wayne & 92.626 & 79.460 \\
\hline
\end{tabular}




\begin{tabular}{|c|c|c|c|}
\hline & Locale & P11 30 & A11 30 \\
\hline 103 & Wells & 12.810 & 39.984 \\
\hline 104 & White & 14.287 & 32.715 \\
\hline 105 & Whitley & 21.936 & 47.700 \\
\hline 106 & lowa & 3332.142 & 3638.722 \\
\hline 107 & Kansas & 4100.933 & 3591.818 \\
\hline 108 & Kentucky_E & 2559.554 & 3331.115 \\
\hline 109 & Kentucky_W & 1706.370 & 2220.743 \\
\hline 110 & Louisiana & 5052.795 & 5921.360 \\
\hline 111 & Maine & 1138.392 & 1730.856 \\
\hline 112 & Maryland & 7380.459 & 8521.889 \\
\hline 113 & Massachusetts & 9750.390 & 8569.713 \\
\hline 114 & Michigan_E & 6628.601 & 6577.311 \\
\hline 115 & Michigan_W & 6628.601 & 6577.311 \\
\hline 116 & Minnesota & 10907.801 & 7609.963 \\
\hline 117 & Mississippi & 2192.195 & 3762.410 \\
\hline 118 & Missouri & 8241.493 & 7850.619 \\
\hline 119 & Montana & 924.690 & 1284.459 \\
\hline 120 & Nebraska & 2251.631 & 2235.638 \\
\hline 121 & Nevada & 4006.745 & 4711.427 \\
\hline 122 & New Hampshire & 1915.502 & 1975.015 \\
\hline 123 & New Jersey & 16814.718 & 11932.874 \\
\hline 124 & New Mexico & 1452.750 & 2553.270 \\
\hline 125 & New York & 21147.262 & 23865.489 \\
\hline 126 & North Carolina & 14780.986 & 14496.350 \\
\hline 127 & North Dakota & 787.411 & 764.811 \\
\hline 128 & Ohio_N & 538.893 & 4766.444 \\
\hline 129 & Ohio M & 538.893 & 4766.444 \\
\hline 130 & Ohio_s & 538.893 & 4766.444 \\
\hline 131 & Oklahoma & 4118.124 & 4767.213 \\
\hline 132 & Oregon & 6363.285 & 5818.338 \\
\hline 133 & Pennsylvania & 14783.030 & 15844.003 \\
\hline 134 & Rhode Island & 1349.374 & 1405.431 \\
\hline 135 & South Carolina & 4682.514 & 6173.053 \\
\hline 136 & South Dakota & 875.076 & 983.579 \\
\hline 137 & Tennessee & 9374.643 & 8891.210 \\
\hline 138 & Texas & 44733.818 & 39310.479 \\
\hline 139 & Utah & 4341.661 & 4123.264 \\
\hline 140 & Vermont & 566.550 & 870.445 \\
\hline 141 & Virginia & 8989.145 & 11813.609 \\
\hline 142 & Washington & 10148.722 & 10349.018 \\
\hline 143 & West Virginia & 1428.967 & 2156.350 \\
\hline 144 & Wisconsin & 7733.894 & 7526.406 \\
\hline 145 & Wyoming & 407.226 & 646.072 \\
\hline
\end{tabular}




\begin{tabular}{|c|c|c|c|}
\hline & Locale & P12 30 & A12 30 \\
\hline 1 & Alabama & 48241.179 & 56247.917 \\
\hline 2 & Arizona & 26274.679 & 30909.492 \\
\hline 3 & Arkansas & 45824.055 & 40954.961 \\
\hline 4 & California & 231877.087 & 233238.100 \\
\hline 5 & Colorado & 21243.102 & 34024.677 \\
\hline 6 & Connecticut & 16274.557 & 12737.083 \\
\hline 7 & Delaware & 11049.228 & 6972.599 \\
\hline 8 & $\mathrm{DC}$ & .000 & .000 \\
\hline 9 & Florida & 73692.553 & 77074.131 \\
\hline 10 & Georgia & 157813.322 & 159603.438 \\
\hline 11 & Idaho & 13755.883 & 11793.197 \\
\hline 12 & Illinois_N & 73966.400 & 59707.326 \\
\hline 13 & Illinois_S & 36983.200 & 29853.663 \\
\hline 14 & Adams & 1295.935 & 403.303 \\
\hline 15 & Allen & 5875.642 & 4323.453 \\
\hline 16 & Bartholomew & 1512.338 & 1499.242 \\
\hline 17 & Benton & 48.231 & 52.264 \\
\hline 18 & Blackford & 293.104 & 20.490 \\
\hline 19 & Boone & 22.227 & 6.884 \\
\hline 20 & Brown & 4.789 & 4.278 \\
\hline 21 & Carroll & 905.221 & 808.755 \\
\hline 22 & Cass & 1436.646 & 737.260 \\
\hline 23 & Clark & 651.735 & 392.890 \\
\hline 24 & Clay & 164.111 & 14.177 \\
\hline 25 & Clinton & 1242.140 & 1098.025 \\
\hline 26 & Crawford & .000 & .000 \\
\hline 27 & Daviess & 607.064 & 488.046 \\
\hline 28 & Dearborn & 85.498 & 2287.634 \\
\hline 29 & Decatur & 533.585 & 173.924 \\
\hline 30 & DeKalb & 2401.383 & 2916.141 \\
\hline 31 & Delaware & 311.303 & 840.703 \\
\hline 32 & Dubois & 1442.280 & 391.252 \\
\hline 33 & Elkhart & 7305.219 & 2606.307 \\
\hline 34 & Fayette & .000 & 50.430 \\
\hline 35 & Floyd & 1934.870 & 373.040 \\
\hline 36 & Fountain & 28.178 & 744.593 \\
\hline 37 & Franklin & 196.720 & 5.172 \\
\hline 38 & Fulton & 201.495 & 456.839 \\
\hline 39 & Gibson & 602.375 & 186.553 \\
\hline 40 & Grant & 530.354 & 282.187 \\
\hline 41 & Greene & 182.537 & 328.076 \\
\hline 42 & Hamilton & 2184.404 & 1229.404 \\
\hline 43 & Hancock & 33.250 & 22.222 \\
\hline 44 & Harrison & 724.140 & 444.663 \\
\hline 45 & Hendricks & 588.308 & 781.825 \\
\hline$\overline{46}$ & Henry & 218.969 & 396.766 \\
\hline 47 & Howard & 487.993 & 928.815 \\
\hline 48 & Huntington & 558.282 & 394.534 \\
\hline 49 & Jackson & 451.091 & 524.437 \\
\hline 50 & Jasper & 174.499 & 103.754 \\
\hline 51 & Jay & 332.832 & 287.527 \\
\hline
\end{tabular}




\begin{tabular}{|c|c|c|c|}
\hline & $\begin{array}{l}\text { Locale } \\
\end{array}$ & P12 30 & A12 30 \\
\hline 52 & Jefferson & 198.107 & 58.835 \\
\hline 53 & Jennings & 1298.005 & 668.418 \\
\hline 54 & Johnson & 1161.548 & 508.910 \\
\hline 55 & Knox & 61.810 & 362.159 \\
\hline 56 & Kosciusko & 1572.403 & 2363.713 \\
\hline 57 & LaGrange & 936.653 & 120.120 \\
\hline 58 & Lake & 1106.533 & 21563.769 \\
\hline 59 & La Porte & 972.044 & 1780.989 \\
\hline 60 & Lawrence & 228.743 & 1736.166 \\
\hline 61 & Madison & 694.019 & 582.376 \\
\hline 62 & Marion & 3941.623 & 8954.425 \\
\hline 63 & Marshall & 2794.420 & 1176.822 \\
\hline 64 & Martin & 24.752 & 169.569 \\
\hline 65 & Miami & 325.868 & 33.540 \\
\hline 66 & Monroe & 449.469 & 307.841 \\
\hline 67 & Montgomery & 905.211 & 944.800 \\
\hline 68 & Morgan & 135.589 & 104.264 \\
\hline 69 & Newton & 402.487 & 171.822 \\
\hline 70 & Noble & 2598.364 & 1428.843 \\
\hline 71 & Ohio & .000 & .000 \\
\hline 72 & Orange & 176.832 & 44.865 \\
\hline 73 & Owen & 98.093 & 94.297 \\
\hline 74 & Parke & 233.673 & 93.337 \\
\hline 75 & Perry & 68.965 & 393.843 \\
\hline 76 & Pike & .000 & 61.824 \\
\hline 77. & Porter & 473.232 & 8611.004 \\
\hline 78 & Posey & 350.633 & 114.096 \\
\hline 79 & Pulaski & 34.725 & 210.903 \\
\hline 80 & Putnam & 1132.472 & 4.548 \\
\hline 81 & Randolph & 81.673 & 297.208 \\
\hline 82 & Ripley & 15.159 & 28.501 \\
\hline 83 & Rush & 68.853 & 341.107 \\
\hline 84 & St. Joseph & 3260.103 & 2384.031 \\
\hline 85 & Scott & 1435.218 & 565.503 \\
\hline 86 & Shelby & 1878.223 & 589.929 \\
\hline 87 & Spencer & 344.811 & 184.826 \\
\hline 88 & Starke & 145.255 & .000 \\
\hline 89 & Steuben & 826.408 & 712.953 \\
\hline 90 & Sullivan & 165.874 & .000 \\
\hline 91 & Switzerland & 221.924 & .000 \\
\hline 92 & Tippecanoe & 1223.552 & 2723.868 \\
\hline 93 & Tipton & 78.916 & 125.769 \\
\hline 94 & Union & .000 & .000 \\
\hline 95 & Vanderburgh & 5498.630 & 2823.199 \\
\hline 96 & Vermillion & 3.309 & 2.957 \\
\hline 97 & Vigo & 1680.481 & 494.923 \\
\hline 98 & Wabash & 178.151 & 691.445 \\
\hline 99 & Warren & 14.609 & 4.524 \\
\hline 100 & Warrick & 25.307 & 4352.649 \\
\hline 101 & Washington & 258.209 & 63.900 \\
\hline 102 & Wayne & 1145.482 & 1020.774 \\
\hline
\end{tabular}




\begin{tabular}{|c|c|c|c|}
\hline & Locale & P12 30 & A12 30 \\
\hline 103 & Wells & 1096.891 & 473.744 \\
\hline 104 & White & 351.705 & 89.286 \\
\hline 105 & Whitley & 489.131 & $\overline{699.416}$ \\
\hline 106 & lowa & 36690.589 & 32013.268 \\
\hline 107 & Kansas & 29151.754 & 20487.157 \\
\hline 108 & Kentucky_E & 20897.923 & 29554.618 \\
\hline 109 & Kentucky W & 13931.948 & 19703.079 \\
\hline 110 & Louisiana & 14547.903 & 18152.616 \\
\hline 111 & Maine & 8168.298 & 7804.740 \\
\hline 112 & Maryland & 20447.909 & 24125.032 \\
\hline 113 & Massachusetts & 39176.107 & 28188.096 \\
\hline 114 & Michigan_E & 40702.055 & 31308.601 \\
\hline 115 & Michigan_W & 40702.055 & 31308.601 \\
\hline 116 & Minnesota & 49257.842 & 38064.294 \\
\hline 117 & Mississippi & 29307.412 & 22572.297 \\
\hline 118 & Missouri & 44712.008 & 44729.752 \\
\hline 119 & Montana & 1583.898 & 3426.003 \\
\hline 120 & Nebraska & 23049.702 & 17711.545 \\
\hline 121 & Nevada & 10879.465 & 9782.545 \\
\hline 122 & New Hampshire & 10854.205 & 9723.741 \\
\hline 123 & New Jersey & 54732.997 & 40903.837 \\
\hline 124 & New Mexico & 2779.713 & 4296.083 \\
\hline$\overline{125}$ & New York & 67088.497 & 64491.876 \\
\hline 126 & North Carolina & 136423.860 & $\overline{133606.931}$ \\
\hline 127 & North Dakota & 3290.400 & 2797.672 \\
\hline 128 & Ohio_N & 36983.693 & 36526.203 \\
\hline 129 & Ohio_M & 36983.693 & 36526.203 \\
\hline 130 & Ohio_s & 36983.693 & 36526.203 \\
\hline 131 & Oklahoma & 22691.012 & 18679.364 \\
\hline 132 & Oregon & 23009.424 & 35376.127 \\
\hline 133 & Pennsylvania & 89082.086 & 118588.784 \\
\hline 134 & Rhode Island & 8360.258 & 7262.780 \\
\hline 135 & South Carolina & 54362.592 & 39502.810 \\
\hline 136 & South Dakota & 6304.800 & 5478.241 \\
\hline 137 & Tennessee & 68897.068 & 57843.364 \\
\hline 138 & Texas & 154917.309 & 153834.394 \\
\hline 139 & Utah & 15995.531 & 21552.105 \\
\hline 140 & Vermont & 4224.332 & 3232.214 \\
\hline 141 & Virginia & 54913.392 & 70912.111 \\
\hline 142 & Washington & 40400.369 & 51194.323 \\
\hline 143 & West Virginia & 4758.703 & 13671.289 \\
\hline 144 & Wisconsin & 65846.329 & 65815.131 \\
\hline 145 & Wyoming & 723.793 & 909.671 \\
\hline
\end{tabular}




\begin{tabular}{|c|c|c|c|}
\hline & Locale & P13 30 & A13 30 \\
\hline 1 & Alabama & 5437.750 & 11620.474 \\
\hline 2 & Arizona & 4982.997 & 3969.422 \\
\hline 3 & Arkansas & 3121.382 & 8986.247 \\
\hline 4 & California & 31469.268 & 26751.935 \\
\hline 5 & Colorado & 3076.066 & 2375.128 \\
\hline 6 & Connecticut & 6148.923 & 3677.927 \\
\hline 7 & Delaware & 3321.310 & 1284.199 \\
\hline 8 & $\mathrm{DC}$ & .000 & .000 \\
\hline 9 & Florida & 12731.018 & 12515.536 \\
\hline 10 & Georgia & 12455.769 & 24155.997 \\
\hline 11. & Idaho & 1676.753 & 1683.758 \\
\hline 12 & Illinois_N & 13859.674 & 11815.675 \\
\hline 13 & Illinois_S & 6929.837 & 5907.838 \\
\hline 14 & Adams & 77.019 & .000 \\
\hline 15 & Allen & 163.421 & 579.880 \\
\hline 16 & Bartholomew & 75.852 & 469.046 \\
\hline 17 & Benton & .000 & 93.065 \\
\hline 18 & Blackford & .000 & 203.520 \\
\hline 19 & Boone & .000 & 9.607 \\
\hline 20 & Brown & 3.725 & .000 \\
\hline 21 & Carroll & .000 & .000 \\
\hline 22 & Cass & .000 & .000 \\
\hline 23 & Clark & 301.306 & 42.685 \\
\hline 24 & Clay & 22.536 & .000 \\
\hline 25 & Clinton & 69.899 & 38.421 \\
\hline 26 & Crawford & .000 & .000 \\
\hline 27 & Daviess & 25.092 & .000 \\
\hline 28 & Dearborn & 5.402 & .000 \\
\hline 29 & Decatur & .000 & .000 \\
\hline 30 & DeKalb & 104.173 & 129.320 \\
\hline 31 & Delaware & 33.698 & 290.459 \\
\hline 32 & Dubois & 24.643 & 140.910 \\
\hline 33 & Elkhart & 764.887 & 525.536 \\
\hline 34 & Fayette & .000 & .000 \\
\hline 35 & Floyd & 44.294 & 121.833 \\
\hline 36 & Fountain & 3.653 & .000 \\
\hline 37 & Franklin & .000 & .000 \\
\hline 38 & Fulton & 3.741 & 35.990 \\
\hline 39 & Gibson & .000 & .000 \\
\hline 40 & Grant & 27.362 & 192.085 \\
\hline 41 & Greene & .000 & .000 \\
\hline 42 & Hamilton & 37.260 & .000 \\
\hline 43 & Hancock & .000 & 170.837 \\
\hline 44 & Harrison & 26.164 & .000 \\
\hline 45 & Hendricks & 7.627 & .000 \\
\hline 46 & Henry & .000 & 30.064 \\
\hline 47 & Howard & .000 & .000 \\
\hline 48 & Huntington & 25.999 & 121.570 \\
\hline 49 & Jackson & 66.642 & 289.993 \\
\hline 50 & Jasper & .000 & .000 \\
\hline 51 & Jay & .000 & 32.179 \\
\hline
\end{tabular}




\begin{tabular}{|c|c|c|c|}
\hline & Locale & P13 30 & A13 30 \\
\hline 52 & Jefferson & 24.608 & .000 \\
\hline 53 & Jennings & 4.437 & 42.679 \\
\hline 54 & Johnson & 36.710 & 171.652 \\
\hline 55 & Knox & .000 & 30.657 \\
\hline 56 & Kosciusko & 133.349 & 6.439 \\
\hline 57 & LaGrange & 4.504 & .000 \\
\hline 58 & Lake & 548.419 & 241.607 \\
\hline 59 & La Porte & 120.226 & 283.584 \\
\hline 60 & Lawrence & .000 & .000 \\
\hline 61 & Madison & 141.810 & 106.095 \\
\hline 62 & Marion & 2355.294 & 1750.139 \\
\hline 63 & Marshall & 56.065 & 320.120 \\
\hline 64 & Martin & .000 & .000 \\
\hline 65 & Miami & 19.185 & 192.235 \\
\hline 66 & Monroe & 74.147 & 127.623 \\
\hline 67 & Montgomery & 68.886 & 110.437 \\
\hline 68 & Morgan & 190.713 & .000 \\
\hline 69 & Newton & 3.648 & 35.089 \\
\hline 70 & Noble & 89.392 & 116.108 \\
\hline 71 & Ohio & .000 & .000 \\
\hline 72 & Orange & .000 & .000 \\
\hline 73 & Owen & .000 & .000 \\
\hline 74 & Parke & .000 & .000 \\
\hline 75 & Perry & .000 & .000 \\
\hline 76 & Pike & .000 & .000 \\
\hline 77 & Porter & 182.826 & .000 \\
\hline 78 & Posey & 701.202 & .000 \\
\hline 79 & Pulaski & .000 & 6.275 \\
\hline 80 & Putnam & .000 & .000 \\
\hline 81 & Randolph & .000 & .000 \\
\hline 82 & Ripley & 24.522 & 114.666 \\
\hline 83 & Rush & .000 & 31.232 \\
\hline 84 & St. Joseph & 337.783 & 354.511 \\
\hline 85 & Scott & 68.916 & .000 \\
\hline 86 & Shelby & 70.532 & 5.889 \\
\hline 87 & Spencer & 3.497 & .000 \\
\hline 88 & Starke & 20.551 & 32.947 \\
\hline 89 & Steuben & .000 & 37.663 \\
\hline 90 & Sullivan & 3.911 & .000 \\
\hline 91 & Switzerland & .000 & 8.390 \\
\hline 92 & Tippecanoe & 805.738 & 47.201 \\
\hline 93 & Tipton & 3.508 & 33.739 \\
\hline 94 & Union & 16.329 & .000 \\
\hline 95 & Vanderburgh & 965.851 & 413.532 \\
\hline 96 & Vermillion & 563.043 & 90.266 \\
\hline 97 & Vigo & 390.462 & 317.852 \\
\hline 98 & Wabash & 125.000 & 200.398 \\
\hline 99 & Warren & .000 & .000 \\
\hline 100 & Warrick & 35.233 & 7.061 \\
\hline 101 & Washington & .000 & .000 \\
\hline 102 & Wayne & 43.311 & 118.889 \\
\hline
\end{tabular}




\begin{tabular}{|c|c|c|c|}
\hline & Locale & P13 30 & A13 30 \\
\hline 103 & Wells & .000 & 42.053 \\
\hline 104 & White & .000 & 35.523 \\
\hline 105 & Whitley & 4.357 & 122.227 \\
\hline 106 & lowa & 2509.180 & 3214.119 \\
\hline 107 & Kansas & 2582.094 & 2113.871 \\
\hline 108 & Kentucky_E & 3432.324 & 4021.670 \\
\hline 109 & Kentucky_W & 2288.216 & 2681.114 \\
\hline 110 & Louisiana & 10586.378 & 6859.656 \\
\hline 111 & Maine & 686.048 & 7399.222 \\
\hline 112 & Maryland & 6666.507 & 5166.881 \\
\hline 113 & Massachusetts & 8605.523 & 12059.680 \\
\hline 114 & Michigan_E & 6435.944 & 5814.152 \\
\hline 115 & Michigan_W & 6435.944 & 5814.152 \\
\hline 116 & Minnesota & 4049.522 & 11316.611 \\
\hline 117 & Mississippi & 2837.777 & 5392.075 \\
\hline 118 & Missouri & 9087.845 & 8514.833 \\
\hline 119 & Montana & 215.776 & 493.243 \\
\hline 120 & Nebraska & 1062.068 & 1011.102 \\
\hline 121 & Nevada & 983.681 & 732.535 \\
\hline 122 & New Hampshire & 868.777 & 2770.481 \\
\hline 123 & New Jersey & 28193.964 & 11493.821 \\
\hline 124 & New Mexico & 561.345 & 364.432 \\
\hline 125 & New York & 22803.472 & 15020.715 \\
\hline 126 & North Carolina & 23649.375 & 18230.351 \\
\hline 127 & North Dakota & 20.073 & 32.180 \\
\hline 128 & Ohio_N & 5779.842 & 5784.636 \\
\hline 129 & Ohio_M & 5779.842 & 5784.636 \\
\hline 130 & Ohio_s & 5779.842 & 5784.636 \\
\hline 131 & Oklahoma & 1540.442 & 2477.333 \\
\hline 132 & Oregon & 2229.395 & 6376.456 \\
\hline 133 & Pennsylvania & 12474.312 & 19105.342 \\
\hline 134 & Rhode Island & 1017.881 & 1234.032 \\
\hline 135 & South Carolina & 17225.090 & 10026.947 \\
\hline 136 & South Dakota & 271.184 & 396.219 \\
\hline 137 & Tennessee & 15816.246 & 12618.105 \\
\hline 138 & Texas & 44296.071 & 19150.676 \\
\hline 139 & Utah & 3136.607 & 2416.785 \\
\hline 140 & Vermont & 155.303 & 1161.901 \\
\hline 141 & Virginia & 9675.204 & 11954.101 \\
\hline 142 & Washington & 2757.309 & 12093.121 \\
\hline 143 & West Virginia & 4712.675 & 336.474 \\
\hline 144 & Wisconsin & 5192.508 & 27193.398 \\
\hline 145 & Wyoming & 468.924 & 6.014 \\
\hline
\end{tabular}




\begin{tabular}{|c|c|c|c|}
\hline & Locale & P14_30 & A14 30 \\
\hline 1 & Alabama & 936.462 & 8320.956 \\
\hline 2 & Arizona & 38375.446 & 4729.464 \\
\hline 3 & Arkansas & 1035.545 & 3721.714 \\
\hline 4 & California & 213.766 & 11663.046 \\
\hline 5 & Colorado & 185.403 & 982.142 \\
\hline 6 & Connecticut & 925.413 & 2073.754 \\
\hline 7 & Delaware & 1104.151 & 329.027 \\
\hline 8 & DC & .000 & .000 \\
\hline 9 & Florida & 1104.705 & 2893.953 \\
\hline 10 & Georgia & 1254.268 & 3191.679 \\
\hline 11 & Idaho & 591.716 & 94.012 \\
\hline 12 & Illinois_N & 363.520 & 9204.049 \\
\hline 13 & Illinois_s & 181.760 & 4602.025 \\
\hline 14 & Adams & .000 & 71.436 \\
\hline 15 & Allen & .000 & 1001.202 \\
\hline 16 & Bartholomew & .000 & 287.042 \\
\hline 17 & Benton & .000 & 18.460 \\
\hline 18 & Blackford & .000 & .000 \\
\hline 19 & Boone & .000 & .000 \\
\hline 20 & Brown & .000 & .000 \\
\hline 21 & Carroll & .000 & .000 \\
\hline 22 & Cass & .000 & .000 \\
\hline 23 & Clark & .000 & 53.094 \\
\hline 24 & Clay & .000 & 3.484 \\
\hline 25 & Clinton & .000 & .000 \\
\hline 26 & Crawford & .000 & .000 \\
\hline 27 & Daviess & .000 & .000 \\
\hline 28 & Dearborn & .000 & .000 \\
\hline 29 & Decatur & .000 & 3.575 \\
\hline 30 & DeKalb & .000 & 1018.798 \\
\hline 31 & Delaware & .000 & 263.648 \\
\hline 32 & Dubois & .000 & .000 \\
\hline 33 & Elkhart & .000 & 487.284 \\
\hline 34 & Fayette & .000 & 17.813 \\
\hline 35 & Floyd & .000 & 4.028 \\
\hline 36 & Fountain & .000 & 254.108 \\
\hline 37 & Franklin & .000 & .000 \\
\hline 38 & Fulton & .000 & 130.135 \\
\hline 39 & Gibson & .000 & .000 \\
\hline 40 & Grant & .000 & 46.749 \\
\hline 41 & Greene & .000 & 57.491 \\
\hline 42 & Hamilton & .000 & 262.651 \\
\hline 43 & Hancock & .000 & 5.461 \\
\hline 44 & Harrison & .000 & .000 \\
\hline 45 & Hendricks & .000 & 123.801 \\
\hline 46 & Henry & .000 & 108.708 \\
\hline 47 & Howard & .000 & 258.284 \\
\hline 48 & Huntington & .000 & 67.922 \\
\hline 49 & Jackson & .000 & 174.838 \\
\hline 50 & Jasper & .000 & .000 \\
\hline 51 & Jay & .000 & .000 \\
\hline
\end{tabular}




\begin{tabular}{|c|c|c|c|}
\hline & $\begin{array}{l}\text { Locale } \\
\end{array}$ & P14_30 & A14 30 \\
\hline 52 & Jefferson & .000 & 3.804 \\
\hline 53 & \begin{tabular}{|l} 
Jennings \\
\end{tabular} & .000 & 24.692 \\
\hline 54 & Johnson & .000 & 170.242 \\
\hline 55 & Knox & .000 & 110.851 \\
\hline 56 & Kosciusko & .000 & 651.941 \\
\hline 57 & LaGrange & .000 & 4.178 \\
\hline 58 & Lake & .000 & 7136.628 \\
\hline 59 & La Porte & .000 & 534.308 \\
\hline 60 & Lawrence & .000 & 558.058 \\
\hline 61 & Madison & .000 & 81.023 \\
\hline 62 & Marion & .000 & 1317.084 \\
\hline 63 & Marshall & .000 & 152.803 \\
\hline 64 & Martin & .000 & 52.083 \\
\hline 65 & Miami & .000 & .000 \\
\hline 66 & Monroe & .000 & .000 \\
\hline 67 & Montgomery & .000 & 273.826 \\
\hline 68 & Morgan & .000 & 28.302 \\
\hline 69 & Newton & .000 & 59.210 \\
\hline 70 & Noble & .000 & 286.736 \\
\hline 71 & \begin{tabular}{|l} 
Ohio \\
\end{tabular} & .000 & .000 \\
\hline 72 & Orange & .000 & .000 \\
\hline 73 & Owen & .000 & .000 \\
\hline 74 & Parke & .000 & .000 \\
\hline 75 & Perry & .000 & 113.020 \\
\hline 76 & Pike & .000 & 21.837 \\
\hline 77 & Porter & .000 & 2984.916 \\
\hline 78 & Posey & .000 & .000 \\
\hline 79 & Pulaski & .000 & 63.536 \\
\hline 80 & Putnam & .000 & .000 \\
\hline 81 & Randolph & .000 & 94.538 \\
\hline 82 & Ripley & .000 & .000 \\
\hline 83 & Rush & .000 & 112.933 \\
\hline 84 & St. Joseph & .000 & 636.065 \\
\hline 85 & Scott & .000 & 3.653 \\
\hline 86 & Shelby & .000 & 182.288 \\
\hline 87 & Spencer & .000 & 56.769 \\
\hline 88 & Starke & .000 & .000 \\
\hline 89 & Steuben & .000 & 181.943 \\
\hline 90 & Sullivan & .000 & .000 \\
\hline 91 & Switzerland & .000 & .000 \\
\hline 92 & Tippecanoe & .000 & 796.484 \\
\hline 93 & Tipton & .000 & 19.519 \\
\hline 94 & Union & .000 & .000 \\
\hline 95 & Vanderburgh & .000 & 66.882 \\
\hline 96 & Vermillion & .000 & .000 \\
\hline 97 & Vigo & .000 & 131.853 \\
\hline 98 & Wabash & .000 & 231.878 \\
\hline 99 & Warren & .000 & .000 \\
\hline 100 & Warrick & .000 & 1531.817 \\
\hline 101 & Washington & .000 & 3.863 \\
\hline 102 & Wayne & .000 & 265.015 \\
\hline
\end{tabular}




\begin{tabular}{|c|c|c|c|}
\hline & $\begin{array}{l}\text { Locale } \\
\end{array}$ & P14_30 & A14 30 \\
\hline 103 & Wells & .000 & 70.961 \\
\hline 104 & White & .000 & 20.552 \\
\hline 105 & Whitley & .000 & 245.275 \\
\hline 106 & lowa & 862.820 & 3274.789 \\
\hline 107 & Kansas & 934.379 & 992.672 \\
\hline 108 & Kentucky_E & 577.734 & 3436.974 \\
\hline 109 & Kentucky_W & 385.156 & 2291.316 \\
\hline 110 & Louisiana & 918.187 & 1380.063 \\
\hline 111 & Maine & 945.655 & 272.707 \\
\hline 112 & Maryland & 1132.787 & 3222.726 \\
\hline 113 & Massachusetts & 943.605 & 2163.501 \\
\hline 114 & Michigan_E & 16117.235 & 6582.011 \\
\hline 115 & Michigan_W & 16117.235 & 6582.011 \\
\hline 116 & Minnesota & 122984.529 & 3188.719 \\
\hline 117 & Mississippi & 928.810 & 1172.822 \\
\hline 118 & Missouri & 4846.664 & 4834.456 \\
\hline 119 & Montana & 1146.647 & 461.145 \\
\hline 120 & Nebraska & 908.811 & 410.595 \\
\hline 121 & Nevada & 238.625 & 1032.756 \\
\hline 122 & New Hampshire & .000 & 1564.326 \\
\hline 123 & New Jersey & 995.345 & 3894.507 \\
\hline 124 & New Mexico & 1145.140 & 663.613 \\
\hline 125 & New York & 2817.887 & 5952.465 \\
\hline 126 & North Carolina & 1297.918 & 3934.057 \\
\hline 127 & North Dakota & 806.989 & 38.786 \\
\hline 128 & Ohio_N & 289.751 & 8308.443 \\
\hline 129 & Ohio_M & 289.751 & 8308.443 \\
\hline 130 & Ohio_s & 289.751 & 8308.443 \\
\hline 131 & Oklahoma & 968.938 & 1996.930 \\
\hline 132 & Oregon & 2059.565 & 5040.658 \\
\hline 133 & Pennsylvania & 888.287 & 21294.668 \\
\hline 134 & Rhode Island & 311.738 & 1237.835 \\
\hline 135 & South Carolina & .000 & 2961.220 \\
\hline 136 & South Dakota & 906.032 & 214.947 \\
\hline 137 & Tennessee & 1108.399 & 7272.417 \\
\hline 138 & Texas & 3082.989 & 15311.927 \\
\hline 139 & Utah & 1333.481 & 3505.535 \\
\hline 140 & Vermont & 73.386 & 103.328 \\
\hline 141 & Virginia & 1185.908 & 3049.189 \\
\hline 142 & Washington & 1250.230 & 6291.658 \\
\hline 143 & West Virginia & 812.638 & 3525.522 \\
\hline 144 & Wisconsin & 979.775 & 10130.982 \\
\hline 145 & Wyoming & 904.917 & 43.493 \\
\hline
\end{tabular}




\begin{tabular}{|c|c|c|c|}
\hline & Locale & P15_30 & A15 30 \\
\hline 1 & Alabama & 74843.657 & 66589.907 \\
\hline 2 & Arizona & 270596.452 & 135341.495 \\
\hline 3 & Arkansas & 14285.132 & 61259.464 \\
\hline 4 & California & 97047.300 & 222254.635 \\
\hline 5 & Colorado & 57898.778 & 47110.401 \\
\hline 6 & Connecticut & 7841.458 & 11982.174 \\
\hline 7 & Delaware & 2183.062 & 11764.385 \\
\hline 8 & $\mathrm{DC}$ & .000 & .000 \\
\hline 9 & Florida & 134047.080 & 117353.114 \\
\hline 10 & Georgia & 88864.241 & 117244.908 \\
\hline 11 & Idaho & 34720.679 & 37068.021 \\
\hline 12 & Illinois_N & 75183.947 & 75253.146 \\
\hline 13 & Illinois_S & 25061.315 & 50122.630 \\
\hline 14 & Adams & 719.662 & 698.218 \\
\hline 15 & Allen & 1009.515 & 2263.210 \\
\hline 16 & Bartholomew & .000 & 1407.732 \\
\hline 17 & Benton & .000 & .000 \\
\hline 18 & Blackford & 81.024 & 80.222 \\
\hline 19 & Boone & 163.321 & 86.674 \\
\hline 20 & Brown & .000 & 9.327 \\
\hline 21 & Carroll & 657.894 & 2051.793 \\
\hline 22 & Cass & 572.883 & 1786.668 \\
\hline 23 & Clark & 2116.368 & 1248.713 \\
\hline 24 & Clay & 614.165 & 278.909 \\
\hline 25 & Clinton & .000 & 2089.402 \\
\hline 26 & Crawford & 2336.466 & 1025.272 \\
\hline 27 & Daviess & 4273.896 & 2939.392 \\
\hline 28 & Dearborn & 883.408 & 401.180 \\
\hline 29 & Decatur & 630.226 & 633.645 \\
\hline 30 & DeKalb & 577.856 & 322.826 \\
\hline 31 & Delaware & 4051.670 & 1902.020 \\
\hline 32 & Dubois & 671.604 & 1115.437 \\
\hline 33 & Elkhart & 714.704 & 1023.942 \\
\hline$\overline{34}$ & Fayette & 87.231 & 38.278 \\
\hline 35 & Floyd & .000 & 788.375 \\
\hline 36 & Fountain & .000 & 54.883 \\
\hline 37 & Franklin & 122.700 & 65.117 \\
\hline 38 & Fulton & 101.967 & 189.965 \\
\hline 39 & Gibson & 4980.912 & 2592.380 \\
\hline 40 & Grant & 470.960 & 397.787 \\
\hline 41 & Greene & 1689.265 & 1073.875 \\
\hline 42 & Hamilton & 2961.759 & 1506.482 \\
\hline 43 & Hancock & 160.449 & 85.150 \\
\hline 44 & Harrison & 1149.596 & 1473.831 \\
\hline 45 & Hendricks & 1247.202 & 1427.772 \\
\hline 46 & Henry & 85.178 & 84.336 \\
\hline 47 & Howard & 607.136 & 414.250 \\
\hline 48 & Huntington & 885.691 & 795.553 \\
\hline 49 & Jackson & 103.783 & 79.871 \\
\hline 50 & Jasper & 109.407 & 274.194 \\
\hline 51 & Jay & 547.024 & 611.981 \\
\hline
\end{tabular}




\begin{tabular}{|c|c|c|c|}
\hline & Locale & P15 30 & A15 30 \\
\hline 52 & Jefferson & .000 & 20.541 \\
\hline 53 & Jennings & 725.514 & 318.365 \\
\hline 54 & Johnson & 166.742 & 83.893 \\
\hline 55 & Knox & 4099.658 & 1904.330 \\
\hline 56 & Kosciusko & 109.463 & 969.328 \\
\hline 57 & LaGrange & 122.750 & 254.624 \\
\hline 58 & Lake & 601.050 & 1302.922 \\
\hline 59 & La Porte & 267.270 & 627.533 \\
\hline 60 & Lawrence & 590.309 & 310.692 \\
\hline 61 & Madison & 618.362 & 981.557 \\
\hline 62 & Marion & 1855.481 & 4573.691 \\
\hline 63 & Marshall & 117.535 & 512.711 \\
\hline 64 & Martin & .000 & 48.210 \\
\hline 65 & Miami & 522.860 & 277.480 \\
\hline 66 & Monroe & 1723.214 & 866.661 \\
\hline 67 & Montgomery & 107.278 & 416.713 \\
\hline 68 & Morgan & 831.605 & 377.655 \\
\hline 69 & Newton & 596.493 & 270.884 \\
\hline 70 & Noble & 112.787 & 826.732 \\
\hline 71 & Ohio & .000 & .000 \\
\hline 72 & Orange & 528.822 & 285.061 \\
\hline 73 & Owen & 113.052 & 59.996 \\
\hline 74 & Parke & .000 & 157.762 \\
\hline 75 & Perry & .000 & 8.137 \\
\hline 76 & Pike & 641.646 & 281.563 \\
\hline 77 & Porter & 107.615 & 365.615 \\
\hline 78 & Posey & 109.200 & 108.120 \\
\hline 79 & Pulaski & 640.071 & 348.505 \\
\hline 80 & Putnam & 669.006 & 303.483 \\
\hline 81 & Randolph & 91.076 & 104.401 \\
\hline 82 & Ripley & 668.314 & 303.499 \\
\hline 83 & Rush & 88.488 & 59.969 \\
\hline 84 & St. Joseph & 642.253 & 964.403 \\
\hline 85 & Scott & 107.324 & 416.892 \\
\hline 86 & Shelby & 1121.295 & 653.020 \\
\hline 87 & Spencer & 571.903 & 303.507 \\
\hline 88 & Starke & .000 & .000 \\
\hline 89 & Steuben & 106.708 & 293.901 \\
\hline 90 & Sullivan & 3997.401 & 1754.113 \\
\hline 91 & Switzerland & 855.704 & 375.494 \\
\hline 92 & Tippecanoe & 802.394 & 1143.431 \\
\hline 93 & Tipton & .000 & 153.704 \\
\hline 94 & Union & .000 & .000 \\
\hline 95 & Vanderburgh & 1965.198 & 4476.812 \\
\hline 96 & Vermillion & 87.684 & 44.922 \\
\hline 97 & Vigo & 3874.257 & 1935.496 \\
\hline 98 & Wabash & 90.844 & 89.945 \\
\hline 99 & Warren & 644.080 & 292.494 \\
\hline 100 & Warrick & 4500.955 & 1975.080 \\
\hline 101 & Washington & 113.507 & 112.384 \\
\hline 102 & Wayne & 84.918 & 587.340 \\
\hline
\end{tabular}




\begin{tabular}{|c|c|c|c|}
\hline & Locale & P15 30 & A15 30 \\
\hline 103 & Wells & 119.147 & 612.798 \\
\hline 104 & White & 100.646 & 83.005 \\
\hline 105 & Whitley & .000 & 10.909 \\
\hline 106 & lowa & 17059.177 & 48955.943 \\
\hline 107 & Kansas & 13617.968 & 35788.668 \\
\hline 108 & Kentucky_E & 124088.790 & 66661.908 \\
\hline 109 & Kentucky_W & 82725.864 & 44441.272 \\
\hline 110 & Louisiana & 17199.490 & 24210.503 \\
\hline 111 & Maine & 641.038 & 6499.217 \\
\hline 112 & Maryland & 23574.246 & 30388.482 \\
\hline 113 & Massachusetts & 18656.416 & 29334.099 \\
\hline 114 & Michigan_E & 20867.401 & 24686.627 \\
\hline 115 & Michigan_W & 20867.401 & 24686.627 \\
\hline 116 & Minnesota & 92803.581 & 89461.856 \\
\hline 117 & Mississippi & 11060.310 & 30605.881 \\
\hline 118 & Missouri & 47013.957 & 59687.748 \\
\hline 119 & Montana & 30867.364 & 15812.110 \\
\hline 120 & Nebraska & 6674.006 & 35290.039 \\
\hline 121 & Nevada & 241502.825 & 111769.729 \\
\hline 122 & New Hampshire & 4822.828 & 4727.865 \\
\hline 123 & New Jersey & 19679.376 & 39444.163 \\
\hline 124 & New Mexico & 61873.197 & 31319.647 \\
\hline 125 & New York & 31486.911 & 58254.033 \\
\hline 126 & North Carolina & 48493.240 & 92130.073 \\
\hline 127 & North Dakota & 14432.725 & 10867.046 \\
\hline 128 & Ohio_N & 25805.708 & 26260.363 \\
\hline 129 & Ohio_M & 25805.708 & 26260.363 \\
\hline 130 & Ohio_s & 25805.708 & 26260.363 \\
\hline 131 & Oklahoma & 22058.247 & 24326.552 \\
\hline 132 & Oregon & 26689.844 & 36444.528 \\
\hline 133 & Pennsylvania & 138002.653 & 131166.956 \\
\hline 134 & Rhode Island & 1157.177 & 3256.621 \\
\hline 135 & South Carolina & 21678.140 & 27447.275 \\
\hline 136 & South Dakota & 16204.069 & 14682.859 \\
\hline 137 & Tennessee & 45695.103 & 61800.571 \\
\hline 138 & Texas & 127308.015 & 178207.017 \\
\hline 139 & Utah & 74077.581 & 49153.480 \\
\hline 140 & Vermont & 8464.979 & 7629.405 \\
\hline 141 & Virginia & 140267.192 & 104349.542 \\
\hline 142 & Washington & 41132.118 & 61679.126 \\
\hline 143 & West Virginia & 154252.367 & 72125.289 \\
\hline 144 & Wisconsin & 19371.548 & 67092.196 \\
\hline 145 & Wyoming & 86165.455 & 38515.095 \\
\hline
\end{tabular}




\begin{tabular}{|c|c|c|c|}
\hline & $\begin{array}{l}\text { Locale } \\
\end{array}$ & P17 30 & A17 30 \\
\hline 1 & Alabama & 54904.282 & 46327.721 \\
\hline 2 & Arizona & 14733.882 & 44283.068 \\
\hline 3 & Arkansas & 24463.465 & 24415.066 \\
\hline 4 & California & 342562.328 & 343362.889 \\
\hline 5 & Colorado & 12549.172 & 26566.697 \\
\hline 6 & Connecticut & 9485.990 & 18369.464 \\
\hline 7 & Delaware & 20912.327 & 14491.072 \\
\hline 8 & DC & .000 & .000 \\
\hline 9 & Florida & 32965.315 & 118057.813 \\
\hline 10 & Georgia & 31702.409 & 58274.302 \\
\hline 11 & Idaho & 5150.049 & 9604.664 \\
\hline 12 & Illinois_N & 82237.200 & 75372.026 \\
\hline 13 & Illinois_S & 41118.600 & 37686.013 \\
\hline 14 & Adams & 240.230 & 276.588 \\
\hline 15 & Allen & 1474.804 & 2331.986 \\
\hline 16 & Bartholomew & .000 & 332.677 \\
\hline 17 & Benton & .000 & 29.231 \\
\hline 18 & Blackford & .000 & 45.779 \\
\hline 19 & Boone & .000 & 285.192 \\
\hline 20 & Brown & .000 & 61.956 \\
\hline 21 & Carroll & .000 & 78.745 \\
\hline 22 & Cass & .000 & 154.018 \\
\hline 23 & Clark & 2446.377 & 1731.001 \\
\hline 24 & Clay & .000 & 103.039 \\
\hline 25 & Clinton & .000 & 138.850 \\
\hline 26 & Crawford & .000 & 50.379 \\
\hline 27 & Daviess & 228.266 & 240.009 \\
\hline 28 & Dearborn & 294.889 & 422.572 \\
\hline 29 & Decatur & .000 & 97.913 \\
\hline 30 & DeKalb & .000 & 195.300 \\
\hline 31 & Delaware & .000 & 484.367 \\
\hline 32 & Dubois & .000 & 172.523 \\
\hline 33 & Elkhart & 238.574 & 983.805 \\
\hline 34 & Fayette & .000 & 89.974 \\
\hline 35 & Floyd & .000 & 343.203 \\
\hline 36 & Fountain & .000 & 65.413 \\
\hline 37 & Franklin & 1474.503 & 863.947 \\
\hline 38 & Fulton & .000 & 81.298 \\
\hline 39 & Gibson & .000 & 128.362 \\
\hline 40 & Grant & .000 & 237.211 \\
\hline 41 & Greene & .000 & 130.271 \\
\hline 42 & Hamilton & 338.969 & 1704.677 \\
\hline 43 & Hancock & .000 & 341.556 \\
\hline 44 & Harrison & 1748.713 & 1097.143 \\
\hline 45 & Hendricks & .000 & 830.412 \\
\hline 46 & Henry & .000 & 165.406 \\
\hline 47 & Howard & .000 & 333.384 \\
\hline 48 & Huntington & 236.521 & 295.315 \\
\hline 49 & Jackson & .000 & 163.992 \\
\hline 50 & Jasper & .000 & 127.507 \\
\hline 51 & Jay & 182.601 & 170.224 \\
\hline
\end{tabular}




\begin{tabular}{|c|c|c|c|}
\hline & Locale & P17 30 & A17 30 \\
\hline$\overline{52}$ & Jefferson & .000 & 137.614 \\
\hline 53 & Jennings & 1453.098 & 885.041 \\
\hline 54 & Johnson & .000 & 733.414 \\
\hline 55 & Knox & 173.962 & 231.465 \\
\hline 56 & Kosciusko & 219.239 & 426.433 \\
\hline 57 & LaGrange & .000 & 181.161 \\
\hline 58 & Lake & 43317.254 & 24423.155 \\
\hline 59 & La Porte & 198.260 & 521.287 \\
\hline 60 & Lawrence & 1126.002 & 758.235 \\
\hline 61 & Madison & .000 & 539.765 \\
\hline 62 & Marion & 2527.053 & 4846.902 \\
\hline 63 & Marshall & 1412.426 & 938.385 \\
\hline 64 & Martin & .000 & 36.030 \\
\hline 65 & Miami & .000 & 124.572 \\
\hline 66 & Monroe & 1489.788 & 1364.462 \\
\hline 67 & Montgomery & .000 & 154.340 \\
\hline 68 & Morgan & .000 & 357.515 \\
\hline 69 & Newton & .000 & 54.036 \\
\hline 70 & Noble & .000 & 199.101 \\
\hline 71 & Ohio & .000 & 22.447 \\
\hline 72 & Orange & .000 & 78.907 \\
\hline 73 & Owen & .000 & 95.781 \\
\hline 74 & Parke & .000 & 62.782 \\
\hline 75 & Perry & .000 & 67.282 \\
\hline 76 & Pike & .000 & 50.768 \\
\hline 77 & Porter & .000 & 607.543 \\
\hline$\overline{78}$ & Posey & 8201.645 & 4384.090 \\
\hline 79 & Pulaski & .000 & 52.556 \\
\hline 80 & Putnam & .000 & 156.075 \\
\hline 81 & Randolph & 182.411 & 191.136 \\
\hline 82 & Ripley & 223.089 & 229.701 \\
\hline 83 & Rush & .000 & 60.491 \\
\hline 84 & St. Joseph & 214.390 & 1261.380 \\
\hline 85 & Scott & 214.954 & 211.536 \\
\hline 86 & Shelby & .000 & 171.176 \\
\hline 87 & Spencer & .000 & 75.928 \\
\hline$\overline{88}$ & Starke & .000 & 88.101 \\
\hline 89 & Steuben & 213.720 & 249.406 \\
\hline 90 & Sullivan & .000 & 86.754 \\
\hline 91 & Switzerland & .000 & 42.178 \\
\hline$\overline{92}$ & Tippecanoe & 267.846 & 892.740 \\
\hline 93 & Tipton & .000 & 59.606 \\
\hline 94 & Union & .000 & 27.351 \\
\hline 95 & Vanderburgh & .000 & 686.077 \\
\hline$\overline{96}$ & Vermillion & .000 & 57.822 \\
\hline 97 & Vigo & .000 & 386.543 \\
\hline 98 & Wabash & 181.947 & 222.502 \\
\hline 99 & Warren & .000 & 30.334 \\
\hline 100 & Warrick & .000 & 263.618 \\
\hline 101 & Washington & .000 & 119.031 \\
\hline 102 & Wayne & .000 & 235.971 \\
\hline
\end{tabular}




\begin{tabular}{|c|c|c|c|}
\hline & Locale & P17 30 & A17 30 \\
\hline 103 & Wells & .000 & 118.739 \\
\hline 104 & White & .000 & 97.154 \\
\hline 105 & Whitley & .000 & 141.652 \\
\hline 106 & lowa & 3416.701 & 12586.135 \\
\hline 107 & Kansas & 58587.909 & 41194.729 \\
\hline 108 & Kentucky_E & 16655.048 & 18570.705 \\
\hline 109 & Kentucky_W & 11103.365 & 12380.470 \\
\hline 110 & Louisiana & 258194.033 & 152120.831 \\
\hline 111 & Maine & 3744.721 & 7091.322 \\
\hline 112 & Maryland & 27786.019 & 39785.572 \\
\hline 113 & Massachusetts & 22419.624 & 37131.343 \\
\hline 114 & Michigan_E & 21167.604 & 30562.180 \\
\hline 115 & Michigan_W & 21167.604 & 30562.180 \\
\hline 116 & Minnesota & 47831.020 & 47522.238 \\
\hline 117 & Mississippi & 40521.225 & 32287.382 \\
\hline 118 & Missouri & 31328.083 & 39637.773 \\
\hline 119 & Montana & 18181.849 & 13288.377 \\
\hline 120 & Nebraska & 205.647 & 6746.262 \\
\hline 121 & Nevada & 2485.796 & 15286.649 \\
\hline 122 & New Hampshire & 3966.798 & 7932.111 \\
\hline 123 & New Jersey & 79370.304 & 76793.932 \\
\hline 124 & New Mexico & 17317.511 & 16605.951 \\
\hline 125 & New York & 31670.718 & 87375.193 \\
\hline 126 & North Carolina & 20264.923 & 53608.758 \\
\hline 127 & North Dakota & 3195.614 & 3936.364 \\
\hline 128 & Ohio_N & 36952.523 & 33409.501 \\
\hline 129 & Ohio_M & 36952.523 & 33409.501 \\
\hline 130 & Ohio_s & 36952.523 & 33409.501 \\
\hline 131 & Oklahoma & 55777.870 & 43221.047 \\
\hline 132 & Oregon & 19584.985 & 27483.630 \\
\hline 133 & Pennsylvania & 145324.994 & 122775.496 \\
\hline 134 & Rhode Island & 212.629 & 4284.461 \\
\hline 135 & South Carolina & 9303.880 & 23179.875 \\
\hline 136 & South Dakota & 205.018 & 3027.733 \\
\hline 137 & Tennessee & 33683.768 & 43955.454 \\
\hline 138 & Texas & 680541.544 & 471346.779 \\
\hline 139 & Utah & 46226.841 & 36332.007 \\
\hline 140 & Vermont & 1356.328 & 3291.671 \\
\hline 141 & Virginia & 18033.044 & 44478.943 \\
\hline 142 & Washington & 57627.475 & 60760.935 \\
\hline 143 & West Virginia & 7263.445 & 10188.390 \\
\hline 144 & Wisconsin & 9267.261 & 27179.805 \\
\hline 145 & Wyoming & 17118.425 & 10838.463 \\
\hline
\end{tabular}




\begin{tabular}{|c|c|c|c|}
\hline & Locale & P18 30 & A18 30 \\
\hline 1 & Alabama & 25647.239 & 23830.274 \\
\hline 2 & Arizona & 6882.585 & 8791.603 \\
\hline 3 & Arkansas & 11427.530 & 11093.029 \\
\hline 4 & California & 160019.903 & 147011.416 \\
\hline 5 & Colorado & 5862.050 & 6693.566 \\
\hline 6 & Connecticut & 4431.156 & 7660.328 \\
\hline 7 & Delaware & 9768.700 & 9926.902 \\
\hline 8 & DC & .000 & .000 \\
\hline 9 & Florida & 15398.968 & 20746.241 \\
\hline 10 & Georgia & 14809.032 & 20095.963 \\
\hline 11 & Idaho & 2405.724 & 3028.794 \\
\hline 12 & Illinois_N & 38415.166 & 39580.518 \\
\hline 13 & Illinois_S & 19207.583 & 19790.259 \\
\hline 14 & Adams & 112.218 & 140.469 \\
\hline 15 & Allen & 688.920 & 651.918 \\
\hline 16 & Bartholomew & .000 & 51.589 \\
\hline 17 & Benton & .000 & .000 \\
\hline 18 & Blackford & .000 & .000 \\
\hline 19 & Boone & .000 & .000 \\
\hline 20 & Brown & .000 & 2.533 \\
\hline 21 & Carroll & .000 & .000 \\
\hline 22 & Cass & .000 & .000 \\
\hline 23 & Clark & 1142.767 & 1101.947 \\
\hline 24 & Clay & .000 & 15.327 \\
\hline 25 & Clinton & .000 & 47.541 \\
\hline 26 & Crawford & .000 & .000 \\
\hline 27 & Daviess & 106.629 & 100.764 \\
\hline 28 & Dearborn & 137.751 & 111.802 \\
\hline 29 & Decatur & .000 & .000 \\
\hline 30 & DeKalb & .000 & 70.851 \\
\hline 31 & Delaware & .000 & 22.919 \\
\hline 32 & Dubois & .000 & 16.761 \\
\hline 33 & Elkhart & 111.444 & 607.703 \\
\hline 34 & Fayette & .000 & .000 \\
\hline 35 & Floyd & .000 & 30.126 \\
\hline 36 & Fountain & .000 & 2.484 \\
\hline 37 & Franklin & 688.779 & 540.660 \\
\hline 38 & Fulton & .000 & 2.545 \\
\hline 39 & Gibson & .000 & .000 \\
\hline 40 & Grant & .000 & 18.609 \\
\hline 41 & Greene & .000 & .000 \\
\hline 42 & Hamilton & 158.342 & 149.633 \\
\hline 43 & Hancock & .000 & .000 \\
\hline 44 & Harrison & 816.870 & 658.999 \\
\hline 45 & Hendricks & .000 & 5.188 \\
\hline 46 & Henry & .000 & .000 \\
\hline 47 & Howard & .000 & .000 \\
\hline 48 & Huntington & 110.485 & 104.409 \\
\hline 49 & Jackson & .000 & 45.325 \\
\hline 50 & Jasper & .000 & .000 \\
\hline 51 & Jay & 85.298 & 66.955 \\
\hline
\end{tabular}




\begin{tabular}{|c|c|c|c|}
\hline & Locale & P18 30 & A18 30 \\
\hline 52 & Jefferson & .000 & 16.737 \\
\hline 53 & \begin{tabular}{|l|} 
Jennings \\
\end{tabular} & 678.780 & 535.829 \\
\hline 54 & Johnson & .000 & 24.967 \\
\hline 55 & Knox & 81.262 & 63.787 \\
\hline 56 & Kosciusko & 102.412 & 171.084 \\
\hline 57 & LaGrange & .000 & 3.063 \\
\hline 58 & Lake & 20234.632 & 16256.241 \\
\hline 59 & La Porte & 92.613 & 154.466 \\
\hline 60 & Lawrence & 525.985 & 412.874 \\
\hline 61 & Madison & .000 & 96.450 \\
\hline 62 & Marion & 1180.453 & 2528.511 \\
\hline 63 & Marshall & 659.781 & 556.029 \\
\hline 64 & Martin & .000 & .000 \\
\hline 65 & Miami & .000 & 13.049 \\
\hline 66 & Monroe & 695.919 & 596.694 \\
\hline 67 & Montgomery & .000 & 46.852 \\
\hline 68 & Morgan & .000 & 129.710 \\
\hline 69 & Newton & .000 & 2.481 \\
\hline 70 & Noble & .000 & 60.798 \\
\hline 71 & Ohio & .000 & .000 \\
\hline 72 & Orange & .000 & .000 \\
\hline 73 & Owen & .000 & .000 \\
\hline 74 & Parke & .000 & .000 \\
\hline 75 & Perry & .000 & .000 \\
\hline 76 & Pike & .000 & .000 \\
\hline 77 & Porter & .000 & 124.346 \\
\hline 78 & Posey & 3831.205 & 3484.227 \\
\hline 79 & Pulaski & .000 & .000 \\
\hline 80 & Putnam & .000 & .000 \\
\hline 81 & Randolph & 85.209 & 66.885 \\
\hline 82 & Ripley & 104.211 & 98.479 \\
\hline 83 & Rush & .000 & .000 \\
\hline 84 & St. Joseph & 100.147 & 308.348 \\
\hline 85 & Scott & 100.411 & 125.690 \\
\hline 86 & Shelby & .000 & 47.971 \\
\hline 87 & Spencer & .000 & 2.379 \\
\hline 88 & Starke & .000 & 13.977 \\
\hline 89 & Steuben & 99.834 & 78.365 \\
\hline 90 & Sullivan & .000 & 2.660 \\
\hline 91 & Switzerland & .000 & .000 \\
\hline 92 & Tippecanoe & 125.118 & 646.220 \\
\hline 93 & Tipton & .000 & 2.386 \\
\hline 94 & Union & .000 & 11.106 \\
\hline 95 & Vanderburgh & .000 & 656.906 \\
\hline 96 & Vermillion & .000 & 382.943 \\
\hline 97 & Vigo & .000 & 265.565 \\
\hline 98 & Wabash & 84.992 & 151.732 \\
\hline 99 & Warren & .000 & .000 \\
\hline 100 & Warrick & .000 & 23.963 \\
\hline 101 & Washington & .000 & .000 \\
\hline 102 & Wayne & .000 & 29.457 \\
\hline
\end{tabular}




\begin{tabular}{|c|c|c|c|}
\hline & Locale & P18_30 & A18 30 \\
\hline 103 & Wells & .000 & .000 \\
\hline 104 & White & .000 & .000 \\
\hline 105 & Whitley & .000 & 2.963 \\
\hline 106 & lowa & 1596.031 & 2959.383 \\
\hline 107 & Kansas & 27367.958 & 23238.736 \\
\hline 108 & Kentucky_E & 7780.013 & 8441.380 \\
\hline 109 & Kentucky_W & 5186.675 & 5627.586 \\
\hline 110 & Louisiana & 120609.246 & 101872.772 \\
\hline 111 & Maine & 1749.258 & 1839.689 \\
\hline 112 & Maryland & 12979.583 & 14722.470 \\
\hline 113 & Massachusetts & 10472.798 & 14073.547 \\
\hline 114 & Michigan_E & 9887.947 & 12138.866 \\
\hline 115 & Michigan_W & 9887.947 & 12138.866 \\
\hline 116 & Minnesota & 22343.132 & 20292.526 \\
\hline 117 & Mississippi & 18928.533 & 16788.079 \\
\hline 118 & Missouri & 14634.174 & 17668.077 \\
\hline 119 & Montana & 8493.221 & 6813.539 \\
\hline 120 & Nebraska & 96.063 & 797.751 \\
\hline 121 & Nevada & 1161.181 & 1580.506 \\
\hline 122 & New Hampshire & 1852.996 & 2045.398 \\
\hline 123 & New Jersey & 37075.963 & 48278.512 \\
\hline 124 & New Mexico & 8089.466 & 6731.642 \\
\hline 125 & New York & 14794.228 & 27122.143 \\
\hline 126 & North Carolina & 9466.280 & 23515.278 \\
\hline 127 & North Dakota & 1492.756 & 1185.396 \\
\hline 128 & Ohio_N & 17261.498 & 17480.526 \\
\hline 129 & Ohio_M & 17261.498 & 17480.526 \\
\hline 130 & Ohio_S & 17261.498 & 17480.526 \\
\hline 131 & Oklahoma & 26055.315 & 21499.912 \\
\hline 132 & Oregon & 9148.663 & 8697.557 \\
\hline 133 & Pennsylvania & 67885.139 & 61770.847 \\
\hline 134 & Rhode Island & 99.325 & 770.258 \\
\hline 135 & South Carolina & 4346.088 & 15126.806 \\
\hline 136 & South Dakota & 95.769 & 259.615 \\
\hline 137 & Tennessee & 15734.577 & 23108.040 \\
\hline 138 & Texas & 317898.913 & 279663.001 \\
\hline 139 & Utah & 21593.777 & 19083.415 \\
\hline 140 & Vermont & 633.577 & 602.955 \\
\hline 141 & Virginia & 8423.711 & 13192.633 \\
\hline 142 & Washington & 26919.314 & 23005.741 \\
\hline 143 & West Virginia & 3392.947 & 5868.545 \\
\hline 144 & Wisconsin & 4328.982 & 6929.639 \\
\hline 145 & Wyoming & 7996.468 & 6595.785 \\
\hline
\end{tabular}




\begin{tabular}{|c|c|c|c|}
\hline & Locale & P19 30 & A19 30 \\
\hline 1 & Alabama & 25845.283 & 24909.216 \\
\hline 2 & Arizona & 10029.538 & 11013.029 \\
\hline 3 & Arkansas & 12129.052 & 11956.724 \\
\hline 4 & California & 159097.201 & 152395.468 \\
\hline 5 & Colorado & 7516.834 & 7945.216 \\
\hline 6 & Connecticut & 9039.187 & 10702.797 \\
\hline 7 & Delaware & 10941.742 & 11023.245 \\
\hline 8 & DC & .000 & .000 \\
\hline 9 & Florida & 23829.014 & 26583.830 \\
\hline 10 & Georgia & 23102.675 & 25826.405 \\
\hline 11 & Idaho & 3448.640 & 3769.634 \\
\hline 12 & Illinois_N & 43729.378 & 44329.746 \\
\hline 13 & Illinois_S & 21864.689 & 22164.873 \\
\hline 14 & Adams & 159.817 & 174.371 \\
\hline 15 & Allen & 709.478 & 690.415 \\
\hline 16 & Bartholomew & 66.597 & 93.175 \\
\hline 17 & Benton & .000 & .000 \\
\hline 18 & Blackford & .000 & .000 \\
\hline 19 & Boone & .000 & .000 \\
\hline 20 & Brown & 3.270 & 4.575 \\
\hline 21 & Carroll & .000 & .000 \\
\hline 22 & Cass & .000 & .000 \\
\hline 23 & Clark & 1203.406 & 1182.376 \\
\hline 24 & Clay & 19.786 & 27.682 \\
\hline 25 & Clinton & 61.371 & 85.863 \\
\hline 26 & Crawford & .000 & .000 \\
\hline 27 & Daviess & 109.633 & 106.612 \\
\hline 28 & Dearborn & 117.915 & 104.547 \\
\hline 29 & Decatur & .000 & .000 \\
\hline 30 & DeKalb & 91.463 & 127.964 \\
\hline 31 & Delaware & 29.587 & 41.394 \\
\hline 32 & Dubois & 21.636 & 30.271 \\
\hline 33 & Elkhart & 763.121 & 1018.784 \\
\hline 34 & Fayette & .000 & .000 \\
\hline 35 & Floyd & 38.890 & 54.410 \\
\hline 36 & Fountain & 3.207 & 4.487 \\
\hline 37 & Franklin & 565.880 & 489.572 \\
\hline 38 & Fulton & 3.285 & 4.596 \\
\hline 39 & Gibson & .000 & .000 \\
\hline 40 & Grant & 24.023 & 33.610 \\
\hline 41 & Greene & .000 & .000 \\
\hline 42 & Hamilton & 162.803 & 158.316 \\
\hline 43 & Hancock & .000 & .000 \\
\hline 44 & Harrison & 694.087 & 612.755 \\
\hline 45 & Hendricks & 6.697 & 9.369 \\
\hline 46 & Henry & .000 & .000 \\
\hline 47 & Howard & .000 & .000 \\
\hline 48 & Huntington & 113.598 & 110.468 \\
\hline 49 & Jackson & 58.511 & 81.862 \\
\hline 50 & Jasper & .000 & .000 \\
\hline 51 & Jay & 70.078 & 60.628 \\
\hline
\end{tabular}




\begin{tabular}{|c|c|c|c|}
\hline & Locale & P19 30 & A19 30 \\
\hline 52 & Jefferson & 21.606 & 30.228 \\
\hline 53 & Jennings & 561.561 & 487.915 \\
\hline 54 & Johnson & 32.231 & 45.093 \\
\hline 55 & Knox & 66.763 & 57.760 \\
\hline 56 & Kosciusko & 201.218 & 236.596 \\
\hline 57 & LaGrange & 3.955 & 5.533 \\
\hline 58 & Lake & 17105.673 & 15056.079 \\
\hline 59 & La Porte & 181.645 & 213.511 \\
\hline 60 & Lawrence & 432.134 & 373.861 \\
\hline 61 & Madison & 124.508 & 174.197 \\
\hline 62 & Marion & 3037.745 & 3732.240 \\
\hline 63 & Marshall & 591.281 & 537.830 \\
\hline 64 & Martin & .000 & .000 \\
\hline 65 & Miami & 16.844 & 23.567 \\
\hline 66 & Monroe & 636.847 & 585.728 \\
\hline 67 & Montgomery & 60.481 & 84.619 \\
\hline 68 & Morgan & 167.444 & 234.269 \\
\hline 69 & Newton & 3.203 & 4.481 \\
\hline 70 & Noble & 78.485 & 109.807 \\
\hline 71 & Ohio & .000 & .000 \\
\hline 72 & Orange & .000 & .000 \\
\hline 73 & Owen & .000 & .000 \\
\hline 74 & Parke & .000 & .000 \\
\hline 75 & Perry & .000 & .000 \\
\hline 76 & Pike & .000 & .000 \\
\hline 77 & Porter & 160.519 & 224.579 \\
\hline 78 & Posey & 3763.250 & 3584.493 \\
\hline 79 & Pulaski & .000 & .000 \\
\hline 80 & Putnam & .000 & .000 \\
\hline 81 & Randolph & 70.005 & 60.565 \\
\hline 82 & Ripley & 107.147 & 104.194 \\
\hline 83 & Rush & .000 & .000 \\
\hline 84 & St. Joseph & 378.847 & 486.108 \\
\hline 85 & Scott & 143.002 & 156.025 \\
\hline 86 & Shelby & 61.926 & 86.640 \\
\hline 87 & Spencer & 3.071 & 4.296 \\
\hline 88 & Starke & 18.044 & 25.244 \\
\hline 89 & Steuben & 82.021 & 70.960 \\
\hline 90 & Sullivan & 3.434 & 4.805 \\
\hline 91 & Switzerland & .000 & .000 \\
\hline 92 & Tippecanoe & 810.222 & 1078.684 \\
\hline 93 & Tipton & 3.080 & 4.309 \\
\hline 94 & Union & 14.337 & 20.058 \\
\hline 95 & Vanderburgh & 848.006 & 1186.431 \\
\hline 96 & Vermillion & 494.345 & 691.630 \\
\hline 97 & Vigo & 342.821 & 479.635 \\
\hline 98 & Wabash & 179.576 & 213.959 \\
\hline 99 & Warren & .000 & .000 \\
\hline 100 & Warrick & 30.934 & 43.279 \\
\hline 101 & Washington & .000 & .000 \\
\hline 102 & Wayne & 38.027 & 53.202 \\
\hline
\end{tabular}




\begin{tabular}{|c|c|c|c|}
\hline & Locale & P19 30 & A19 30 \\
\hline 103 & Wells & .000 & .000 \\
\hline 104 & White & .000 & .000 \\
\hline 105 & Whitley & 3.825 & 5.352 \\
\hline 106 & lowa & 3514.282 & 4216.655 \\
\hline 107 & Kansas & 24751.742 & 22624.443 \\
\hline 108 & Kentucky_E & 9405.365 & 9746.089 \\
\hline 109 & Kentucky_W & 6270.243 & 6497.393 \\
\hline 110 & Louisiana & 108383.657 & 98730.969 \\
\hline 111 & Maine & 2039.480 & 2086.069 \\
\hline 112 & Maryland & 16516.750 & 17414.654 \\
\hline 113 & Massachusetts & 16159.684 & 18014.724 \\
\hline 114 & Michigan_E & 13774.322 & 14933.955 \\
\hline 115 & Michigan_W & 13774.322 & 14933.955 \\
\hline 116 & Minnesota & 21911.880 & 20855.445 \\
\hline 117 & Mississippi & 18042.651 & 16939.928 \\
\hline 118 & Missouri & 20002.019 & 21565.030 \\
\hline 119 & Montana & 7167.225 & 6301.883 \\
\hline 120 & Nebraska & 1011.406 & 1372.903 \\
\hline 121 & Nevada & 1817.652 & 2033.680 \\
\hline 122 & New Hampshire & 2285.142 & 2384.264 \\
\hline 123 & New Jersey & 55214.466 & 60985.814 \\
\hline 124 & New Mexico & 7138.917 & 6439.391 \\
\hline 125 & New York & 32175.673 & 38526.788 \\
\hline 126 & North Carolina & 28541.083 & 35778.869 \\
\hline 127 & North Dakota & 1244.027 & 1085.681 \\
\hline 128 & Ohio_N & 19256.162 & 19369.001 \\
\hline 129 & Ohio_M & 19256.162 & 19369.001 \\
\hline 130 & Ohio_s & 19256.162 & 19369.001 \\
\hline 131 & Oklahoma & 22758.756 & 20411.896 \\
\hline 132 & Oregon & 9473.650 & 9241.248 \\
\hline 133 & Pennsylvania & 66724.694 & 63574.723 \\
\hline 134 & Rhode Island & 975.289 & 1320.942 \\
\hline 135 & South Carolina & 18694.040 & 24248.068 \\
\hline 136 & South Dakota & 316.778 & 401.188 \\
\hline 137 & Tennessee & 26813.534 & 30612.207 \\
\hline 138 & Texas & 300067.646 & 280369.207 \\
\hline 139 & Utah & 20494.704 & 19201.411 \\
\hline 140 & Vermont & 656.882 & 641.106 \\
\hline 141 & Virginia & 15415.383 & 17872.244 \\
\hline 142 & Washington & 24536.987 & 22520.786 \\
\hline 143 & West Virginia & 6925.217 & 8200.599 \\
\hline 144 & Wisconsin & 8115.523 & 9455.333 \\
\hline 145 & Wyoming & 6981.368 & 6259.762 \\
\hline
\end{tabular}




\begin{tabular}{|c|c|c|c|}
\hline & Locale & P20 30 & A20 30 \\
\hline 1 & Alabama & 15301.789 & 13689.674 \\
\hline 2 & Arizona & 4106.324 & 9942.930 \\
\hline 3 & Arkansas & 6817.952 & 5838.935 \\
\hline 4 & California & 95471.905 & 112595.986 \\
\hline 5 & Colorado & 3497.446 & 6318.328 \\
\hline 6 & Connecticut & 2643.739 & 3796.081 \\
\hline 7 & Delaware & 5828.252 & 4846.844 \\
\hline 8 & $\overline{D C}$ & .000 & .000 \\
\hline 9 & Florida & 9187.413 & 14896.909 \\
\hline 10 & Georgia & 8835.442 & 8973.555 \\
\hline 11 & Idaho & 1435.316 & 2906.563 \\
\hline 12 & Illinois_N & 22919.456 & 22077.122 \\
\hline 13 & Illinois_s & 11459.728 & 11038.561 \\
\hline 14 & Adams & 66.952 & 116.006 \\
\hline 15 & Allen & 411.027 & 637.995 \\
\hline 16 & Bartholomew & .000 & 56.812 \\
\hline 17 & Benton & .000 & .000 \\
\hline 18 & Blackford & .000 & .000 \\
\hline 19 & Boone & .000 & 45.338 \\
\hline 20 & Brown & .000 & .704 \\
\hline 21 & Carroll & .000 & .000 \\
\hline 22 & Cass & .000 & 24.849 \\
\hline 23 & Clark & 681.804 & 553.130 \\
\hline 24 & Clay & .000 & .710 \\
\hline 25 & Clinton & .000 & 56.660 \\
\hline 26 & Crawford & .000 & .000 \\
\hline 27 & Daviess & 63.618 & 50.907 \\
\hline 28 & Dearborn & 82.185 & 66.786 \\
\hline 29 & Decatur & .000 & .000 \\
\hline 30 & DeKalb & .000 & 25.196 \\
\hline 31 & Delaware & .000 & .750 \\
\hline 32 & Dubois & .000 & 58.262 \\
\hline 33 & Elkhart & 66.491 & 236.729 \\
\hline 34 & Fayette & .000 & .000 \\
\hline 35 & Floyd & .000 & 49.938 \\
\hline 36 & Fountain & .000 & 4.145 \\
\hline 37 & Franklin & 410.943 & 328.835 \\
\hline 38 & Fulton & .000 & .708 \\
\hline 39 & Gibson & .000 & 18.185 \\
\hline 40 & Grant & .000 & .000 \\
\hline 41 & Greene & .000 & 4.020 \\
\hline 42 & Hamilton & 94.471 & 99.556 \\
\hline 43 & Hancock & .000 & 1.114 \\
\hline 44 & Harrison & 487.365 & 389.987 \\
\hline 45 & Hendricks & .000 & 25.246 \\
\hline 46 & Henry & .000 & 3.429 \\
\hline 47 & Howard & .000 & 4.705 \\
\hline 48 & Huntington & 65.918 & 177.896 \\
\hline 49 & Jackson & .000 & .000 \\
\hline 50 & Jasper & .000 & 13.288 \\
\hline 51 & Jay & 50.891 & 40.723 \\
\hline
\end{tabular}




\begin{tabular}{|c|c|c|c|}
\hline & Locale & P20 30 & A20 30 \\
\hline 52 & Jefferson & .000 & .000 \\
\hline 53 & Jennings & 404.977 & 324.061 \\
\hline 54 & Johnson & .000 & 66.539 \\
\hline 55 & Knox & 48.483 & 43.679 \\
\hline 56 & Kosciusko & 61.102 & 58.009 \\
\hline 57 & LaGrange & .000 & 5.111 \\
\hline 58 & Lake & 12072.491 & 9672.305 \\
\hline 59 & La Porte & 55.255 & 49.161 \\
\hline 60 & Lawrence & 313.816 & 255.016 \\
\hline 61 & Madison & .000 & .715 \\
\hline 62 & Marion & 704.288 & 700.581 \\
\hline 63 & Marshall & 393.642 & 315.806 \\
\hline 64 & Martin & .000 & .000 \\
\hline 65 & Miami & .000 & .605 \\
\hline 66 & Monroe & 415.203 & 419.486 \\
\hline 67 & Montgomery & .000 & .000 \\
\hline 68 & Morgan & .000 & 168.333 \\
\hline 69 & Newton & .000 & 12.074 \\
\hline 70 & Noble & .000 & 4.697 \\
\hline 71 & Ohio & .000 & .000 \\
\hline 72 & Orange & .000 & .000 \\
\hline 73 & Owen & .000 & .000 \\
\hline 74 & Parke & .000 & .000 \\
\hline 75 & Perry & .000 & .615 \\
\hline 76 & Pike & .000 & .000 \\
\hline 77 & Porter & .000 & 8.290 \\
\hline 78 & Posey & 2285.793 & 1829.080 \\
\hline 79 & Pulaski & .000 & .000 \\
\hline 80 & Putnam & .000 & 13.105 \\
\hline 81 & Randolph & 50.838 & 44.473 \\
\hline 82 & Ripley & 62.175 & 54.390 \\
\hline 83 & Rush & .000 & .614 \\
\hline 84 & St. Joseph & 59.750 & 116.008 \\
\hline 85 & Scott & 59.908 & 47.938 \\
\hline 86 & Shelby & .000 & .000 \\
\hline 87 & Spencer & .000 & .000 \\
\hline 88 & Starke & .000 & .000 \\
\hline 89 & Steuben & 59.564 & 52.106 \\
\hline 90 & Sullivan & .000 & .000 \\
\hline 91 & Switzerland & .000 & .000 \\
\hline 92 & Tippecanoe & 74.649 & 170.365 \\
\hline 93 & Tipton & .000 & .000 \\
\hline 94 & Union & .000 & .000 \\
\hline 95 & Vanderburgh & .000 & .779 \\
\hline 96 & Vermillion & .000 & .000 \\
\hline 97 & Vigo & .000 & 125.476 \\
\hline 98 & Wabash & 50.708 & 73.865 \\
\hline 99 & Warren & .000 & .000 \\
\hline 100 & Warrick & .000 & .000 \\
\hline 101 & Washington & .000 & .000 \\
\hline 102 & Wayne & .000 & 54.749 \\
\hline
\end{tabular}




\begin{tabular}{|c|c|c|c|}
\hline & Locale & P20 30 & A20 30 \\
\hline 103 & Wells & .000 & .827 \\
\hline 104 & White & .000 & 12.224 \\
\hline 105 & Whitley & .000 & 14.255 \\
\hline 106 & lowa & 952.232 & 1855.373 \\
\hline 107 & Kansas & 16328.413 & 13535.679 \\
\hline 108 & Kentucky_E & 4641.752 & 4234.615 \\
\hline 109 & Kentucky_W & 3094.501 & 2823.077 \\
\hline 110 & Louisiana & 71958.515 & 57736.205 \\
\hline 111 & Maine & 1043.651 & 1308.186 \\
\hline 112 & Maryland & 7743.946 & 8345.943 \\
\hline$\overline{113}$ & Massachusetts & 6248.335 & 12332.284 \\
\hline 114 & Michigan_E & 5899.398 & 5649.934 \\
\hline 115 & Michigan_W & 5899.398 & 5649.934 \\
\hline 116 & Minnesota & 13330.475 & 15839.075 \\
\hline 117 & Mississippi & 11293.240 & 9387.018 \\
\hline 118 & Missouri & 8731.117 & 8094.241 \\
\hline 119 & Montana & 5067.270 & 4092.046 \\
\hline 120 & Nebraska & 57.314 & 487.025 \\
\hline 121 & Nevada & 692.790 & 1055.818 \\
\hline 122 & New Hampshire & 1105.544 & 3109.256 \\
\hline 123 & New Jersey & 22120.454 & 20443.678 \\
\hline 124 & New Mexico & 4826.379 & 4727.505 \\
\hline 125 & New York & 8826.609 & 12855.623 \\
\hline 126 & North Carolina & 5647.821 & 9774.663 \\
\hline 127 & North Dakota & 890.616 & 806.376 \\
\hline 128 & Ohio_N & 10298.645 & 9072.984 \\
\hline 129 & Ohio_M & 10298.645 & 9072.984 \\
\hline 130 & Ohio_s & 10298.645 & 9072.984 \\
\hline 131 & Oklahoma & 15545.257 & 13414.125 \\
\hline 132 & Oregon & 5458.323 & 8159.490 \\
\hline 133 & Pennsylvania & 40501.984 & 36475.202 \\
\hline 134 & Rhode Island & 59.260 & 541.872 \\
\hline 135 & South Carolina & 2592.986 & 3545.932 \\
\hline 136 & South Dakota & 57.138 & 979.908 \\
\hline 137 & Tennessee & 9387.645 & 8690.508 \\
\hline 138 & Texas & 189666.500 & 166688.041 \\
\hline 139 & Utah & 12883.391 & 11989.031 \\
\hline 140 & Vermont & 378.008 & 1069.568 \\
\hline 141 & Virginia & 5025.798 & 6909.843 \\
\hline 142 & Washington & 16060.741 & 17529.666 \\
\hline 143 & West Virginia & 2024.318 & 1677.513 \\
\hline$\overline{144}$ & Wisconsin & 2582.780 & 3837.727 \\
\hline 145 & Wyoming & 4770.894 & 3849.433 \\
\hline
\end{tabular}




\begin{tabular}{|c|c|c|c|}
\hline & Locale & P21 30 & A21 30 \\
\hline 1 & Alabama & 635.350 & 274.543 \\
\hline 2 & Arizona & 345.927 & 370.046 \\
\hline 3 & Arkansas & 320.369 & 173.104 \\
\hline 4 & California & 2071.516 & 2325.045 \\
\hline 5 & Colorado & 142.572 & 245.451 \\
\hline 6 & Connecticut & 106.322 & 293.281 \\
\hline 7 & Delaware & 29.486 & 131.756 \\
\hline 8 & DC & .000 & .000 \\
\hline 9 & Florida & 726.752 & 1128.547 \\
\hline 10 & Georgia & 1387.781 & 716.788 \\
\hline 11 & Idaho & 54.344 & 110.910 \\
\hline 12 & Illinois_N & 299.240 & 716.700 \\
\hline 13 & Illinois_s & 149.620 & 358.350 \\
\hline 14 & Adams & 6.526 & 3.051 \\
\hline 15 & Allen & 24.612 & 21.254 \\
\hline 16 & Bartholomew & 18.189 & 3.089 \\
\hline 17 & Benton & 1.114 & .432 \\
\hline 18 & Blackford & .166 & .161 \\
\hline 19 & Boone & 2.012 & 1.464 \\
\hline 20 & Brown & .000 & .144 \\
\hline 21 & Carroll & 8.442 & .668 \\
\hline 22 & Cass & .196 & .619 \\
\hline 23 & Clark & 27.783 & 10.450 \\
\hline 24 & Clay & .210 & .903 \\
\hline 25 & Clinton & 1.341 & 2.588 \\
\hline 26 & Crawford & .000 & .000 \\
\hline 27 & Daviess & 1.404 & 1.361 \\
\hline 28 & Dearborn & .000 & .642 \\
\hline 29 & Decatur & .216 & 1.150 \\
\hline 30 & DeKalb & .258 & 3.587 \\
\hline 31 & Delaware & 3.128 & 2.791 \\
\hline 32 & Dubois & 151.443 & 2.316 \\
\hline 33 & Elkhart & 94.407 & 26.237 \\
\hline 34 & Fayette & 1.075 & .488 \\
\hline 35 & Floyd & 10.764 & 3.115 \\
\hline 36 & Fountain & .000 & .469 \\
\hline 37 & Franklin & .000 & .261 \\
\hline 38 & Fulton & .000 & .542 \\
\hline 39 & Gibson & .000 & .381 \\
\hline 40 & Grant & 2.820 & 1.496 \\
\hline 41 & Greene & .198 & .274 \\
\hline 42 & Hamilton & 9.290 & 10.326 \\
\hline 43 & Hancock & 1.976 & 2.206 \\
\hline 44 & Harrison & 19.389 & 1.196 \\
\hline 45 & Hendricks & .427 & 2.283 \\
\hline 46 & Henry & 3.060 & .990 \\
\hline 47 & Howard & 7.791 & .731 \\
\hline 48 & Huntington & .000 & 1.766 \\
\hline 49 & Jackson & 1.278 & 3.004 \\
\hline 50 & Jasper & .225 & .492 \\
\hline 51 & Jay & 1.123 & .604 \\
\hline
\end{tabular}




\begin{tabular}{|c|c|c|c|}
\hline & $\begin{array}{l}\text { Locale } \\
\end{array}$ & P21 30 & A21 30 \\
\hline 52 & Jefferson & .229 & .923 \\
\hline 53 & Jennings & .000 & .394 \\
\hline 54 & Johnson & 2.978 & 2.322 \\
\hline 55 & Knox & .178 & 1.259 \\
\hline 56 & Kosciusko & 7.020 & 4.755 \\
\hline 57 & LaGrange & 7.586 & 1.283 \\
\hline 58 & Lake & 5.203 & 22.739 \\
\hline 59 & La Porte & 4.333 & 6.148 \\
\hline 60 & Lawrence & .192 & .239 \\
\hline 61 & Madison & 8.831 & 5.645 \\
\hline 62 & Marion & 28.390 & 110.396 \\
\hline 63 & Marshall & 1.448 & 2.499 \\
\hline 64 & Martin & .000 & .189 \\
\hline 65 & Miami & 6.709 & 1.886 \\
\hline 66 & Monroe & 1.782 & 6.615 \\
\hline 67 & Montgomery & .000 & 2.417 \\
\hline 68 & Morgan & .285 & 5.863 \\
\hline 69 & Newton & 1.225 & .454 \\
\hline 70 & Noble & 1.389 & 3.540 \\
\hline 71 & Ohio & .000 & .000 \\
\hline 72 & Orange & 34.545 & .194 \\
\hline 73 & Owen & .000 & .040 \\
\hline 74 & Parke & .201 & .209 \\
\hline 75 & Perry & 5.327 & .138 \\
\hline 76 & Pike & .000 & .159 \\
\hline 77 & Porter & 1.970 & 8.392 \\
\hline 78 & Posey & .000 & 21.140 \\
\hline 79 & Pulaski & .219 & .968 \\
\hline 80 & Putnam & .000 & .203 \\
\hline 81 & Randolph & .901 & .190 \\
\hline 82 & Ripley & 4.002 & 1.401 \\
\hline 83 & Rush & .000 & .254 \\
\hline 84 & St. Joseph & 3.832 & 20.066 \\
\hline 85 & Scott & 1.322 & 2.034 \\
\hline 86 & Shelby & 6.824 & 2.543 \\
\hline 87 & Spencer & 14.677 & .895 \\
\hline 88 & Starke & .192 & .744 \\
\hline 89 & Steuben & .219 & .053 \\
\hline 90 & Sullivan & .000 & .661 \\
\hline 91 & Switzerland & .293 & .051 \\
\hline 92 & Tippecanoe & 20.592 & 26.292 \\
\hline 93 & Tipton & .000 & .589 \\
\hline 94 & Union & .000 & .693 \\
\hline 95 & Vanderburgh & 9.392 & 34.308 \\
\hline 96 & Vermillion & .000 & 16.549 \\
\hline 97 & Vigo & 1.273 & 13.968 \\
\hline 98 & Wabash & .187 & 3.891 \\
\hline 99 & Warren & .000 & .228 \\
\hline 100 & Warrick & .246 & 1.812 \\
\hline 101 & Washington & 40.781 & .040 \\
\hline 102 & Wayne & 12.448 & 2.548 \\
\hline
\end{tabular}




\begin{tabular}{|c|c|c|c|}
\hline & Locale & P21 30 & A21 30 \\
\hline 103 & Wells & .400 & 6.400 \\
\hline 104 & White & 5.917 & 1.120 \\
\hline 105 & Whitley & 1.073 & .517 \\
\hline 106 & lowa & 170.946 & 180.968 \\
\hline 107 & Kansas & 105.041 & 174.792 \\
\hline 108 & Kentucky_E & 92.225 & 174.106 \\
\hline 109 & Kentucky_W & 61.484 & 116.071 \\
\hline 110 & Louisiana & 28.475 & 428.725 \\
\hline 111 & Maine & 101.690 & 60.466 \\
\hline 112 & Maryland & 138.952 & 359.696 \\
\hline 113 & Massachusetts & 327.098 & 476.049 \\
\hline 114 & Michigan_E & 365.787 & 313.746 \\
\hline 115 & Michigan_W & 365.787 & 313.746 \\
\hline 116 & Minnesota & 307.601 & 332.719 \\
\hline 117 & Mississippi & 661.834 & 154.514 \\
\hline 118 & Missouri & 288.152 & 481.571 \\
\hline 119 & Montana & 19.422 & 34.377 \\
\hline 120 & \begin{tabular}{|l} 
Nebraska \\
\end{tabular} & 70.358 & 101.149 \\
\hline 121 & Nevada & 71.278 & 111.837 \\
\hline 122 & New Hampshire & 80.650 & 64.548 \\
\hline 123 & New Jersey & 330.415 & 1344.464 \\
\hline 124 & New Mexico & 23.435 & 52.504 \\
\hline 125 & New York & 593.662 & 1385.047 \\
\hline 126 & North Carolina & 4458.576 & 1022.177 \\
\hline 127 & North Dakota & 20.837 & 28.768 \\
\hline 128 & Ohio_N & 158.845 & 280.189 \\
\hline 129 & Ohio_M & 158.845 & 280.189 \\
\hline 130 & Ohio_s & 158.845 & 280.189 \\
\hline 131 & Oklahoma & 82.532 & 141.801 \\
\hline 132 & Oregon & 172.487 & 223.149 \\
\hline 133 & Pennsylvania & 685.805 & 694.931 \\
\hline 134 & Rhode Island & 129.210 & 54.836 \\
\hline 135 & South Carolina & 1126.352 & 606.697 \\
\hline 136 & South Dakota & 44.601 & 36.503 \\
\hline 137 & Tennessee & 837.811 & 695.443 \\
\hline 138 & Texas & 929.533 & 2147.486 \\
\hline 139 & Utah & 184.487 & 182.229 \\
\hline 140 & Vermont & 67.490 & 28.424 \\
\hline 141 & Virginia & 991.008 & 481.472 \\
\hline 142 & Washington & 229.975 & 336.909 \\
\hline 143 & West Virginia & 25.723 & 163.091 \\
\hline 144 & Wisconsin & 424.628 & 336.739 \\
\hline 145 & Wyoming & 4.709 & 22.117 \\
\hline
\end{tabular}




\begin{tabular}{|c|c|c|c|}
\hline & Locale & P22 30 & A22 30 \\
\hline 1 & Alabama & 5345.837 & 5345.837 \\
\hline 2 & Arizona & 11044.029 & 11044.029 \\
\hline 3 & Arkansas & 3520.251 & 3520.251 \\
\hline 4 & California & 49740.140 & 49740.140 \\
\hline 5 & Colorado & 6042.413 & 6042.413 \\
\hline 6 & Connecticut & 4050.844 & 4050.844 \\
\hline 7 & Delaware & 1084.424 & 1084.424 \\
\hline 8 & $\mathrm{DC}$ & .000 & .000 \\
\hline 9 & Florida & 30435.923 & 30435.923 \\
\hline 10 & Georgia & 12597.644 & 12597.644 \\
\hline 11 & Idaho & 2088.124 & 2088.124 \\
\hline 12 & Illinois_N & 9811.642 & 9811.642 \\
\hline 13 & Illinois_S & 4905.821 & 4905.821 \\
\hline 14 & Adams & 45.681 & 45.681 \\
\hline 15 & Allen & 471.716 & 471.716 \\
\hline 16 & Bartholomew & 100.369 & 100.369 \\
\hline 17 & Benton & 8.819 & 8.819 \\
\hline 18 & Blackford & 13.812 & 13.812 \\
\hline 19 & Boone & 86.043 & 86.043 \\
\hline 20 & Brown & 18.692 & 18.692 \\
\hline 21 & Carroll & 23.757 & 23.757 \\
\hline 22 & Cass & 46.467 & 46.467 \\
\hline 23 & Clark & 137.659 & 137.659 \\
\hline 24 & Clay & 31.087 & 31.087 \\
\hline 25 & Clinton & 41.891 & 41.891 \\
\hline 26 & Crawford & 15.200 & 15.200 \\
\hline 27 & Daviess & 36.526 & 36.526 \\
\hline 28 & Dearborn & 81.132 & 81.132 \\
\hline 29 & Decatur & 29.540 & 29.540 \\
\hline 30 & DeKalb & 58.922 & 58.922 \\
\hline 31 & Delaware & 146.134 & 146.134 \\
\hline 32 & Dubois & 52.051 & 52.051 \\
\hline 33 & Elkhart & 259.311 & 259.311 \\
\hline 34 & Fayette & 27.145 & 27.145 \\
\hline 35 & Floyd & 103.545 & 103.545 \\
\hline 36 & Fountain & 19.735 & 19.735 \\
\hline 37 & Franklin & 28.853 & 28.853 \\
\hline 38 & Fulton & 24.528 & 24.528 \\
\hline 39 & Gibson & 38.727 & 38.727 \\
\hline 40 & Grant & 71.567 & 71.567 \\
\hline 41 & Greene & 39.303 & 39.303 \\
\hline 42 & Hamilton & 461.016 & 461.016 \\
\hline 43 & Hancock & 103.048 & 103.048 \\
\hline 44 & Harrison & 56.101 & 56.101 \\
\hline 45 & Hendricks & 250.537 & 250.537 \\
\hline 46 & Henry & 49.903 & 49.903 \\
\hline 47 & Howard & 100.583 & 100.583 \\
\hline 48 & Huntington & 51.914 & 51.914 \\
\hline 49 & Jackson & 49.477 & 49.477 \\
\hline 50 & Jasper & 38.469 & 38.469 \\
\hline 51 & Jay & 22.651 & 22.651 \\
\hline
\end{tabular}




\begin{tabular}{|c|c|c|c|}
\hline & Locale & P22 30 & A22 30 \\
\hline 52 & Jefferson & 41.519 & 41.519 \\
\hline 53 & Jennings & 38.582 & 38.582 \\
\hline 54 & Johnson & 221.273 & 221.273 \\
\hline 55 & Knox & 42.486 & 42.486 \\
\hline 56 & Kosciusko & 94.190 & 94.190 \\
\hline 57 & LaGrange & 54.657 & 54.657 \\
\hline 58 & Lake & 558.744 & 558.744 \\
\hline 59 & La Porte & 126.105 & 126.105 \\
\hline 60 & Lawrence & 51.746 & 51.746 \\
\hline 61 & Madison & 162.848 & 162.848 \\
\hline 62 & Marion & 1065.051 & 1065.051 \\
\hline 63 & Marshall & 61.070 & 61.070 \\
\hline 64 & Martin & 10.870 & 10.870 \\
\hline 65 & Miami & 37.584 & 37.584 \\
\hline 66 & Monroe & 177.456 & 177.456 \\
\hline 67 & Montgomery & 46.565 & 46.565 \\
\hline 68 & Morgan & 107.863 & 107.863 \\
\hline 69 & Newton & 16.303 & 16.303 \\
\hline 70 & Noble & 60.069 & 60.069 \\
\hline 71 & Ohio & 6.772 & 6.772 \\
\hline 72 & Orange & 23.807 & 23.807 \\
\hline 73 & Owen & 28.897 & 28.897 \\
\hline 74 & Parke & 18.941 & 18.941 \\
\hline 75 & Perry & 20.299 & 20.299 \\
\hline 76 & Pike & 15.317 & 15.317 \\
\hline 77 & Porter & 183.297 & 183.297 \\
\hline 78 & Posey & 33.334 & 33.334 \\
\hline 79 & Pulaski & 15.856 & 15.856 \\
\hline 80 & Putnam & 47.088 & 47.088 \\
\hline 81 & Randolph & 28.990 & 28.990 \\
\hline 82 & Ripley & 34.230 & 34.230 \\
\hline 83 & Rush & 18.250 & 18.250 \\
\hline 84 & St. Joseph & 346.858 & 346.858 \\
\hline 85 & Scott & 30.028 & 30.028 \\
\hline 86 & Shelby & 51.644 & 51.644 \\
\hline 87 & Spencer & 22.908 & 22.908 \\
\hline 88 & Starke & 26.580 & 26.580 \\
\hline 89 & Steuben & 41.648 & 41.648 \\
\hline 90 & Sullivan & 26.174 & 26.174 \\
\hline 91 & Switzerland & 12.725 & 12.725 \\
\hline 92 & Tippecanoe & 227.234 & 227.234 \\
\hline 93 & Tipton & 17.983 & 17.983 \\
\hline 94 & Union & 8.252 & 8.252 \\
\hline 95 & Vanderburgh & 206.991 & 206.991 \\
\hline 96 & Vermillion & 17.445 & 17.445 \\
\hline 97 & Vigo & 116.621 & 116.621 \\
\hline 98 & Wabash & 38.526 & 38.526 \\
\hline 99 & Warren & 9.152 & 9.152 \\
\hline 100 & Warrick & 79.534 & 79.534 \\
\hline 101 & Washington & 35.912 & 35.912 \\
\hline 102 & Wayne & 71.193 & 71.193 \\
\hline
\end{tabular}




\begin{tabular}{|c|c|c|c|}
\hline & Locale & P22 30 & A22 30 \\
\hline 103 & Wells & 35.824 & 35.824 \\
\hline 104 & White & 29.312 & 29.312 \\
\hline 105 & Whitley & 42.737 & 42.737 \\
\hline 106 & lowa & 3260.135 & 3260.135 \\
\hline 107 & Kansas & 3218.111 & 3218.111 \\
\hline 108 & Kentucky_E & 2984.532 & 2984.532 \\
\hline 109 & Kentucky_W & 1989.688 & 1989.688 \\
\hline 110 & Louisiana & 5305.278 & 5305.278 \\
\hline 111 & Maine & 1550.771 & 1550.771 \\
\hline 112 & Maryland & 7635.238 & 7635.238 \\
\hline 113 & Massachusetts & 7678.086 & 7678.086 \\
\hline 114 & Michigan_E & 5892.981 & 5892.981 \\
\hline 115 & Michigan_W & 5892.981 & 5892.981 \\
\hline 116 & Minnesota & 6818.192 & 6818.192 \\
\hline 117 & Mississippi & 3370.954 & 3370.954 \\
\hline 118 & Missouri & 7033.809 & 7033.809 \\
\hline 119 & Montana & 1150.819 & 1150.819 \\
\hline 120 & Nebraska & 2003.033 & 2003.033 \\
\hline 121 & Nevada & 4221.231 & 4221.231 \\
\hline 122 & New Hampshire & 1769.527 & 1769.527 \\
\hline 123 & New Jersey & 10691.330 & 10691.330 \\
\hline 124 & New Mexico & 2287.618 & 2287.618 \\
\hline 125 & New York & 21382.429 & 21382.429 \\
\hline 126 & North Carolina & 12988.092 & 12988.092 \\
\hline 127 & North Dakota & 685.237 & 685.237 \\
\hline 128 & Ohio_N & 4270.524 & 4270.524 \\
\hline 129 & Ohio_M & 4270.524 & 4270.524 \\
\hline 130 & Ohio_s & 4270.524 & 4270.524 \\
\hline 131 & Oklahoma & 4271.213 & 4271.213 \\
\hline 132 & Oregon & 5212.975 & 5212.975 \\
\hline 133 & Pennsylvania & 14195.530 & 14195.530 \\
\hline 134 & Rhode Island & 1259.205 & 1259.205 \\
\hline 135 & South Carolina & 5530.784 & 5530.784 \\
\hline 136 & South Dakota & 881.243 & 881.243 \\
\hline 137 & Tennessee & 7966.133 & 7966.133 \\
\hline 138 & Texas & 35220.461 & 35220.461 \\
\hline 139 & Utah & 3694.263 & 3694.263 \\
\hline 140 & Vermont & 779.880 & 779.880 \\
\hline 141 & Virginia & 10584.474 & 10584.474 \\
\hline 142 & Washington & 9272.265 & 9272.265 \\
\hline 143 & West Virginia & 1931.995 & 1931.995 \\
\hline 144 & Wisconsin & 6743.329 & 6743.329 \\
\hline 145 & Wyoming & 578.852 & 578.852 \\
\hline
\end{tabular}




\begin{tabular}{|c|c|c|c|}
\hline & $\begin{array}{l}\text { Locale } \\
\end{array}$ & P23 30 & A23 30 \\
\hline 1 & Alabama & 2962.479 & 3955.848 \\
\hline 2 & Arizona & 3715.982 & 3061.754 \\
\hline 3 & Arkansas & 2271.974 & 2064.074 \\
\hline 4 & California & 22114.847 & 22948.660 \\
\hline 5 & Colorado & 1832.550 & 1876.553 \\
\hline 6 & Connecticut & 3942.493 & 1524.289 \\
\hline 7 & Delaware & 657.769 & 868.354 \\
\hline 8 & DC & .000 & .000 \\
\hline 9 & Florida & 6485.589 & 8300.292 \\
\hline 10 & Georgia & 4845.373 & 12466.741 \\
\hline 11 & Idaho & 631.713 & 589.825 \\
\hline 12 & Illinois_N & 8204.197 & 5667.725 \\
\hline 13 & Illinois_S & 4102.098 & 2833.863 \\
\hline 14 & Adams & 43.272 & 68.652 \\
\hline 15 & Allen & 269.197 & 379.305 \\
\hline 16 & Bartholomew & 95.969 & 57.540 \\
\hline 17 & Benton & 4.579 & 3.903 \\
\hline 18 & Blackford & 36.314 & 16.194 \\
\hline 19 & Boone & 34.446 & 14.456 \\
\hline 20 & Brown & 4.704 & 2.977 \\
\hline 21 & Carroll & 32.864 & 3.784 \\
\hline 22 & Cass & 47.099 & 41.517 \\
\hline 23 & Clark & 129.104 & 96.208 \\
\hline 24 & Clay & 10.709 & 13.202 \\
\hline 25 & Clinton & 28.094 & 15.446 \\
\hline 26 & Crawford & .000 & 2.421 \\
\hline 27 & Daviess & 15.561 & 13.166 \\
\hline 28 & Dearborn & 23.474 & 22.415 \\
\hline 29 & Decatur & 207.764 & 22.847 \\
\hline 30 & DeKalb & 294.293 & 131.930 \\
\hline 31 & Delaware & 144.958 & 36.699 \\
\hline 32 & Dubois & 14.973 & 61.792 \\
\hline 33 & Elkhart & 614.694 & 443.097 \\
\hline 34 & Fayette & 22.695 & 4.323 \\
\hline 35 & Floyd & 57.095 & 95.747 \\
\hline 36 & Fountain & 4.614 & 3.143 \\
\hline 37 & Franklin & .851 & 41.612 \\
\hline 38 & Fulton & 60.542 & 10.481 \\
\hline 39 & Gibson & 10.809 & 26.554 \\
\hline 40 & Grant & 12.939 & 36.402 \\
\hline 41 & Greene & 6.228 & 7.405 \\
\hline 42 & Hamilton & 143.345 & 203.755 \\
\hline 43 & Hancock & 51.095 & 17.742 \\
\hline 44 & Harrison & 10.835 & 52.835 \\
\hline 45 & Hendricks & 29.968 & 48.113 \\
\hline 46 & Henry & 20.683 & 20.879 \\
\hline 47 & Howard & 14.673 & 37.365 \\
\hline 48 & Huntington & 85.356 & 31.372 \\
\hline 49 & Jackson & 58.323 & 30.909 \\
\hline 50 & Jasper & 51.236 & 9.150 \\
\hline 51 & Jay & 59.901 & 14.315 \\
\hline
\end{tabular}




\begin{tabular}{|c|c|c|c|}
\hline & Locale & P23 30 & A23 30 \\
\hline 52 & Jefferson & 13.943 & 16.948 \\
\hline 53 & Jennings & 54.094 & 125.292 \\
\hline 54 & Johnson & 129.427 & 96.048 \\
\hline 55 & Knox & 7.050 & 10.367 \\
\hline 56 & Kosciusko & 126.201 & 79.993 \\
\hline 57 & LaGrange & 18.719 & 52.564 \\
\hline 58 & Lake & 246.279 & 918.921 \\
\hline 59 & La Porte & 165.312 & 61.062 \\
\hline 60 & Lawrence & 37.380 & 39.952 \\
\hline 61 & \begin{tabular}{|l} 
Madison \\
\end{tabular} & 93.453 & 44.142 \\
\hline 62 & Marion & 1045.425 & 344.326 \\
\hline 63 & Marshall & 90.791 & 172.109 \\
\hline 64 & Martin & .607 & 1.731 \\
\hline 65 & Miami & 18.428 & 22.071 \\
\hline 66 & Monroe & 42.820 & 80.144 \\
\hline 67 & Montgomery & 56.640 & 44.474 \\
\hline 68 & Morgan & 55.677 & 24.633 \\
\hline 69 & Newton & 4.607 & 23.202 \\
\hline 70 & Noble & 120.966 & 134.448 \\
\hline 71 & Ohio & .807 & 1.079 \\
\hline 72 & \begin{tabular}{|l|} 
Orange \\
\end{tabular} & 4.002 & 11.541 \\
\hline 73 & Owen & 1.020 & 13.174 \\
\hline 74 & Parke & .681 & 10.925 \\
\hline 75 & Perry & 3.686 & 9.539 \\
\hline 76 & Pike & 4.452 & 2.439 \\
\hline 77 & Porter & 139.158 & 45.835 \\
\hline 78 & Posey & 94.702 & 176.395 \\
\hline 79 & Pulaski & 12.952 & 2.525 \\
\hline 80 & Putnam & .749 & 65.896 \\
\hline 81 & Randolph & 7.014 & 10.490 \\
\hline 82 & Ripley & 19.381 & 10.069 \\
\hline 83 & Rush & 3.684 & 6.402 \\
\hline 84 & St. Joseph & 369.972 & 220.304 \\
\hline 85 & Scott & 15.413 & 83.674 \\
\hline 86 & Shelby & 34.560 & 101.233 \\
\hline 87 & Spencer & 4.417 & 20.112 \\
\hline 88 & Starke & 2.642 & 11.757 \\
\hline 89 & Steuben & 100.388 & 50.356 \\
\hline 90 & Sullivan & 1.242 & 12.761 \\
\hline 91 & Switzerland & .000 & 13.522 \\
\hline 92 & Tippecanoe & 160.101 & 87.907 \\
\hline 93 & Tipton & 8.675 & 2.864 \\
\hline 94 & Union & 2.818 & 1.314 \\
\hline 95 & Vanderburgh & 274.005 & 245.046 \\
\hline 96 & Vermillion & 83.038 & 2.778 \\
\hline 97 & Vigo & 98.227 & 99.932 \\
\hline 98 & Wabash & 80.044 & 17.884 \\
\hline 99 & Warren & 9.235 & 1.952 \\
\hline 100 & Warrick & 23.932 & 14.644 \\
\hline 101 & Washington & 47.959 & 17.430 \\
\hline 102 & Wayne & 43.039 & 56.043 \\
\hline
\end{tabular}




\begin{tabular}{|c|c|c|c|}
\hline & Locale & P23 30 & A23 30 \\
\hline 103 & Wells & 50.507 & 48.278 \\
\hline 104 & White & 65.637 & 22.412 \\
\hline 105 & Whitley & 58.304 & 31.852 \\
\hline 106 & lowa & 2044.371 & 1441.422 \\
\hline 107 & Kansas & 1391.117 & 2376.296 \\
\hline 108 & Kentucky_E & 1601.918 & 1699.337 \\
\hline 109 & Kentucky W & 1067.945 & 1132.892 \\
\hline 110 & Louisiana & 2850.286 & 5975.785 \\
\hline 111 & Maine & 454.456 & 677.443 \\
\hline 112 & Maryland & 2075.369 & 2303.709 \\
\hline 113 & Massachusetts & 4600.112 & 3387.976 \\
\hline 114 & Michigan_E & 4612.382 & 3102.193 \\
\hline 115 & Michigan_W & 4612.382 & 3102.193 \\
\hline 116 & Minnesota & 4755.723 & 3334.388 \\
\hline 117 & Mississippi & 1452.116 & 2366.994 \\
\hline 118 & Missouri & 4280.769 & 3134.415 \\
\hline 119 & Montana & 131.912 & 547.291 \\
\hline 120 & Nebraska & 856.111 & 679.243 \\
\hline 121 & Nevada & 814.049 & 1164.664 \\
\hline 122 & New Hampshire & 1268.624 & 934.136 \\
\hline 123 & New Jersey & 6660.179 & 5490.347 \\
\hline 124 & New Mexico & 303.468 & 719.758 \\
\hline 125 & New York & 7788.962 & 6782.021 \\
\hline 126 & North Carolina & 7489.704 & 9448.241 \\
\hline 127 & North Dakota & 126.244 & 229.028 \\
\hline 128 & Ohio_N & 4041.913 & 2955.615 \\
\hline 129 & Ohio_M & 4041.913 & 2955.615 \\
\hline 130 & Ohio_s & 4041.913 & 2955.615 \\
\hline 131 & Oklahoma & 2288.260 & 2578.918 \\
\hline 132 & Oregon & 2092.085 & 1805.757 \\
\hline 133 & Pennsylvania & 8806.344 & 8100.620 \\
\hline 134 & Rhode Island & 914.559 & 633.672 \\
\hline 135 & South Carolina & 4851.517 & 4278.881 \\
\hline 136 & South Dakota & 277.466 & 311.951 \\
\hline 137 & Tennessee & 5896.878 & 4737.816 \\
\hline 138 & Texas & 18576.582 & 23656.425 \\
\hline 139 & Utah & 1511.653 & 1917.488 \\
\hline 140 & Vermont & 302.181 & 271.694 \\
\hline 141 & Virginia & 3237.702 & 4013.206 \\
\hline 142 & Washington & 2567.802 & 3607.490 \\
\hline 143 & West Virginia & 1053.339 & 576.184 \\
\hline 144 & Wisconsin & 6633.188 & 3235.034 \\
\hline 145 & Wyoming & 123.349 & 429.282 \\
\hline
\end{tabular}




\begin{tabular}{|c|c|c|c|}
\hline & Locale & P24 30 & A24 30 \\
\hline 1 & Alabama & 7112.700 & 5879.311 \\
\hline 2 & Arizona & 1908.734 & 3445.193 \\
\hline 3 & Arkansas & 3169.175 & 3436.361 \\
\hline 4 & California & 44378.018 & 33302.282 \\
\hline 5 & Colorado & 1625.711 & 2794.075 \\
\hline 6 & Connecticut & 1228.884 & 3846.329 \\
\hline 7 & Delaware & 2709.135 & 1788.206 \\
\hline 8 & DC & .000 & .000 \\
\hline 9 & Florida & 4270.567 & 9191.812 \\
\hline 10 & Georgia & 4106.961 & 16965.167 \\
\hline 11 & Idaho & 667.175 & 981.325 \\
\hline 12 & Illinois_N & 10653.605 & 12107.819 \\
\hline 13 & Illinois_S & 5326.803 & 6053.910 \\
\hline 14 & Adams & 31.121 & 65.841 \\
\hline 15 & Allen & 191.057 & 546.943 \\
\hline 16 & Bartholomew & .000 & 287.347 \\
\hline 17 & Benton & .000 & 3.474 \\
\hline 18 & Blackford & .000 & 3.114 \\
\hline 19 & Boone & .000 & 46.552 \\
\hline 20 & Brown & .000 & .987 \\
\hline 21 & Carroll & .000 & 4.214 \\
\hline 22 & Cass & .000 & 13.330 \\
\hline 23 & Clark & 316.922 & 239.389 \\
\hline 24 & Clay & .000 & 12.202 \\
\hline 25 & Clinton & .000 & 33.450 \\
\hline 26 & Crawford & .000 & .000 \\
\hline 27 & Daviess & 29.571 & 27.403 \\
\hline 28 & Dearborn & 38.202 & 23.147 \\
\hline 29 & Decatur & .000 & 30.141 \\
\hline 30 & DeKalb & .000 & 79.031 \\
\hline 31 & Delaware & .000 & 82.496 \\
\hline 32 & Dubois & .000 & 16.639 \\
\hline 33 & Elkhart & 30.907 & 422.650 \\
\hline 34 & Fayette & .000 & 38.162 \\
\hline 35 & Floyd & .000 & 58.817 \\
\hline 36 & Fountain & .000 & .968 \\
\hline 37 & Franklin & 191.018 & 56.238 \\
\hline 38 & Fulton & .000 & 24.113 \\
\hline 39 & Gibson & .000 & 13.079 \\
\hline 40 & Grant & .000 & 46.257 \\
\hline 41 & Greene & .000 & 1.420 \\
\hline 42 & Hamilton & 43.913 & 95.421 \\
\hline 43 & Hancock & .000 & 23.946 \\
\hline 44 & Harrison & 226.541 & 73.632 \\
\hline 45 & Hendricks & .000 & 36.235 \\
\hline 46 & Henry & .000 & 27.977 \\
\hline 47 & Howard & .000 & 22.685 \\
\hline 48 & Huntington & 30.641 & 49.117 \\
\hline 49 & Jackson & .000 & 64.728 \\
\hline 50 & Jasper & .000 & .701 \\
\hline 51 & Jay & 23.656 & 18.235 \\
\hline
\end{tabular}




\begin{tabular}{|c|c|c|c|}
\hline & Locale & P24 30 & A24 30 \\
\hline 52 & Jefferson & .000 & 61.363 \\
\hline$\overline{53}$ & Jennings & 188.245 & 141.262 \\
\hline 54 & Johnson & .000 & 85.344 \\
\hline 55 & Knox & 22.536 & 10.919 \\
\hline 56 & Kosciusko & 28.402 & 115.125 \\
\hline 57 & LaGrange & .000 & 46.086 \\
\hline 58 & Lake & 5611.632 & 1753.664 \\
\hline 59 & La Porte & 25.684 & 161.611 \\
\hline 60 & Lawrence & 145.870 & 64.310 \\
\hline 61 & Madison & .000 & 67.094 \\
\hline 62 & Marion & 327.373 & 1350.139 \\
\hline 63 & Marshall & 182.976 & 86.181 \\
\hline 64 & Martin & .000 & .560 \\
\hline 65 & Miami & .000 & 17.698 \\
\hline 66 & Monroe & 192.998 & 221.616 \\
\hline 67 & Montgomery & .000 & 30.354 \\
\hline 68 & Morgan & .000 & 77.273 \\
\hline 69 & Newton & .000 & 6.252 \\
\hline 70 & Noble & .000 & 85.959 \\
\hline$\overline{71}$ & Ohio & .000 & .000 \\
\hline 72 & Orange & .000 & .616 \\
\hline 73 & Owen & .000 & 10.701 \\
\hline 74 & Parke & .000 & 3.771 \\
\hline 75 & Perry & .000 & 17.742 \\
\hline$\overline{76}$ & Pike & .000 & .000 \\
\hline 77 & Porter & .000 & 84.649 \\
\hline 78 & Posey & 1062.501 & 486.290 \\
\hline 79 & Pulaski & .000 & 32.799 \\
\hline 80 & Putnam & .000 & 12.095 \\
\hline 81 & Randolph & 23.631 & 37.992 \\
\hline 82 & Ripley & 28.901 & 14.296 \\
\hline 83 & Rush & .000 & 30.718 \\
\hline 84 & St. Joseph & 27.774 & 231.062 \\
\hline 85 & Scott & 27.847 & 57.529 \\
\hline 86 & Shelby & .000 & 43.899 \\
\hline 87 & Spencer & .000 & 11.611 \\
\hline 88 & Starke & .000 & 27.869 \\
\hline 89 & Steuben & 27.687 & 52.290 \\
\hline 90 & Sullivan & .000 & 1.037 \\
\hline 91 & Switzerland & .000 & .000 \\
\hline 92 & Tippecanoe & 34.699 & 464.999 \\
\hline 93 & Tipton & .000 & 11.645 \\
\hline 94 & Union & .000 & 4.991 \\
\hline 95 & Vanderburgh & .000 & 390.831 \\
\hline 96 & Vermillion & .000 & 149.251 \\
\hline 97 & Vigo & .000 & 134.206 \\
\hline 98 & Wabash & 23.571 & 68.817 \\
\hline 99 & Warren & .000 & 12.033 \\
\hline 100 & Warrick & .000 & 15.718 \\
\hline 101 & Washington & .000 & 54.528 \\
\hline 102 & Wayne & .000 & 31.334 \\
\hline
\end{tabular}




\begin{tabular}{|c|c|c|c|}
\hline & Locale & P24 30 & A24 30 \\
\hline 103 & Wells & .000 & 24.419 \\
\hline 104 & White & .000 & 20.756 \\
\hline 105 & Whitley & .000 & 10.813 \\
\hline 106 & lowa & 442.624 & 3357.762 \\
\hline 107 & Kansas & 7589.904 & 4377.841 \\
\hline 108 & Kentucky_E & 2157.616 & 2864.953 \\
\hline 109 & Kentucky_W & 1438.411 & 1909.968 \\
\hline 110 & Louisiana & 33448.335 & 12609.681 \\
\hline 111 & Maine & 485.118 & 783.225 \\
\hline 112 & Maryland & 3599.603 & 3726.529 \\
\hline 113 & Massachusetts & 2904.401 & 6082.250 \\
\hline$\overline{114}$ & Michigan_E & 2742.206 & 6212.660 \\
\hline 115 & Michigan_W & 2742.206 & 6212.660 \\
\hline 116 & Minnesota & 6196.379 & 6319.002 \\
\hline 117 & Mississippi & 5249.414 & 3942.509 \\
\hline 118 & Missouri & 4058.468 & 6369.468 \\
\hline 119 & Montana & 2355.409 & 838.382 \\
\hline 120 & Nebraska & 26.641 & 1142.600 \\
\hline 121 & Nevada & 322.028 & 656.065 \\
\hline 122 & New Hampshire & 513.888 & 1399.293 \\
\hline 123 & New Jersey & 10282.207 & 12937.660 \\
\hline 124 & New Mexico & 2243.436 & 854.883 \\
\hline 125 & New York & 4102.855 & 12890.642 \\
\hline 126 & North Carolina & 2625.266 & 15858.535 \\
\hline 127 & North Dakota & 413.983 & 457.145 \\
\hline 128 & Ohio_N & 4787.099 & 5327.102 \\
\hline 129 & Ohio_M & 4787.099 & 5327.102 \\
\hline 130 & Ohio_s & 4787.099 & 5327.102 \\
\hline 131 & Oklahoma & 7225.871 & 4315.163 \\
\hline 132 & Oregon & 2537.181 & 2655.218 \\
\hline 133 & Pennsylvania & 18826.458 & 14178.855 \\
\hline 134 & Rhode Island & 27.545 & 704.371 \\
\hline 135 & South Carolina & 1205.292 & 9310.475 \\
\hline 136 & South Dakota & 26.560 & 526.752 \\
\hline 137 & Tennessee & 4363.641 & 9806.166 \\
\hline 138 & Texas & 88162.306 & 46155.114 \\
\hline 139 & Utah & 5988.561 & 3354.860 \\
\hline 140 & Vermont & 175.709 & 368.848 \\
\hline$\overline{141}$ & Virginia & 2336.132 & 5726.842 \\
\hline 142 & Washington & 7465.483 & 4397.608 \\
\hline 143 & West Virginia & 940.960 & 1750.380 \\
\hline 144 & Wisconsin & 1200.549 & 8162.679 \\
\hline$\overline{145}$ & Wyoming & 2217.645 & 780.593 \\
\hline
\end{tabular}




\begin{tabular}{|c|c|c|c|}
\hline & Locale & P25 30 & A25 30 \\
\hline 1 & Alabama & 30974.940 & 30974.940 \\
\hline 2 & Arizona & 18759.537 & 18759.537 \\
\hline 3 & Arkansas & 20194.873 & 20194.873 \\
\hline 4 & California & 57407.081 & 57407.081 \\
\hline 5 & Colorado & 6072.004 & 6072.004 \\
\hline 6 & Connecticut & 1732.700 & 1732.700 \\
\hline 7 & Delaware & 1537.762 & 1537.762 \\
\hline 8 & DC & .000 & .000 \\
\hline 9 & Florida & 30754.472 & 30754.472 \\
\hline 10 & Georgia & 44236.378 & 44236.378 \\
\hline 11 & Idaho & 14508.106 & 14508.106 \\
\hline 12 & IIllinois_N & 6974.277 & 6974.277 \\
\hline 13 & Illinois_S & 3487.138 & 3487.138 \\
\hline 14 & Adams & 763.812 & 763.812 \\
\hline 15 & Allen & 246.853 & 246.853 \\
\hline 16 & Bartholomew & 78.358 & 78.358 \\
\hline 17 & Benton & 174.907 & 174.907 \\
\hline 18 & Blackford & 69.870 & 69.870 \\
\hline 19 & Boone & .000 & .000 \\
\hline 20 & Brown & 30.301 & 30.301 \\
\hline 21 & Carroll & .000 & .000 \\
\hline 22 & Cass & 177.341 & 177.341 \\
\hline 23 & Clark & 891.795 & 891.795 \\
\hline 24 & Clay & 67.900 & 67.900 \\
\hline 25 & Clinton & 39.715 & 39.715 \\
\hline 26 & Crawford & 88.564 & 88.564 \\
\hline 27 & Daviess & 283.505 & 283.505 \\
\hline 28 & Dearborn & 16.278 & 16.278 \\
\hline 29 & Decatur & 30.193 & 30.193 \\
\hline 30 & DeKalb & 927.737 & 927.737 \\
\hline 31 & Delaware & 71.670 & 71.670 \\
\hline 32 & Dubois & 2123.563 & 2123.563 \\
\hline 33 & Elkhart & 8401.986 & 8401.986 \\
\hline 34 & Fayette & .000 & .000 \\
\hline 35 & Floyd & 774.583 & 774.583 \\
\hline 36 & Fountain & 11.006 & 11.006 \\
\hline 37 & Franklin & 52.905 & 52.905 \\
\hline 38 & Fulton & 296.485 & 296.485 \\
\hline 39 & Gibson & .000 & .000 \\
\hline 40 & Grant & 52.068 & 52.068 \\
\hline 41 & Greene & 64.032 & 64.032 \\
\hline 42 & Hamilton & 235.759 & 235.759 \\
\hline 43 & Hancock & 49.668 & 49.668 \\
\hline 44 & Harrison & 273.498 & 273.498 \\
\hline 45 & Hendricks & 137.887 & 137.887 \\
\hline 46 & Henry & 56.502 & 56.502 \\
\hline 47 & Howard & 67.123 & 67.123 \\
\hline 48 & Huntington & 110.975 & 110.975 \\
\hline 49 & Jackson & 337.334 & 337.334 \\
\hline 50 & Jasper & 83.461 & 83.461 \\
\hline 51 & Jay & 60.477 & 60.477 \\
\hline
\end{tabular}




\begin{tabular}{|c|c|c|c|}
\hline & Locale & P25 30 & A25 30 \\
\hline 52 & Jefferson & 90.210 & 90.210 \\
\hline 53 & Jennings & 93.579 & 93.579 \\
\hline 54 & Johnson & 1882.158 & 1882.158 \\
\hline 55 & Knox & 72.980 & 72.980 \\
\hline 56 & Kosciusko & 377.580 & 377.580 \\
\hline 57 & LaGrange & 2551.323 & 2551.323 \\
\hline 58 & Lake & 262.479 & 262.479 \\
\hline 59 & La Porte & 228.728 & 228.728 \\
\hline 60 & Lawrence & 120.166 & 120.166 \\
\hline 61 & Madison & 203.953 & 203.953 \\
\hline 62 & Marion & 833.440 & 833.440 \\
\hline 63 & Marshall & 836.830 & 836.830 \\
\hline 64 & Martin & 9.668 & 9.668 \\
\hline 65 & Miami & 130.063 & 130.063 \\
\hline 66 & Monroe & 82.236 & 82.236 \\
\hline 67 & Montgomery & 49.813 & 49.813 \\
\hline 68 & Morgan & 280.416 & 280.416 \\
\hline 69 & Newton & .000 & .000 \\
\hline 70 & Noble & 74.816 & 74.816 \\
\hline 71 & Ohio & .000 & .000 \\
\hline 72 & Orange & 485.790 & 485.790 \\
\hline 73 & Owen & 154.984 & 154.984 \\
\hline 74 & Parke & 189.826 & 189.826 \\
\hline 75 & Perry & 58.744 & 58.744 \\
\hline 76 & Pike & 70.938 & 70.938 \\
\hline 77 & Porter & 128.494 & 128.494 \\
\hline 78 & Posey & 12.073 & 12.073 \\
\hline 79 & Pulaski & 11.794 & 11.794 \\
\hline 80 & Putnam & 71.577 & 71.577 \\
\hline 81 & Randolph & 379.603 & 379.603 \\
\hline 82 & Ripley & 150.236 & 150.236 \\
\hline 83 & Rush & 58.698 & 58.698 \\
\hline 84 & St. Joseph & 603.548 & 603.548 \\
\hline 85 & Scott & 71.193 & 71.193 \\
\hline 86 & Shelby & .000 & .000 \\
\hline 87 & Spencer & 159.124 & 159.124 \\
\hline 88 & Starke & 333.339 & 333.339 \\
\hline 89 & Steuben & 57.807 & 57.807 \\
\hline 90 & Sullivan & 104.887 & 104.887 \\
\hline 91 & Switzerland & .000 & .000 \\
\hline 92 & Tippecanoe & 75.404 & 75.404 \\
\hline 93 & Tipton & 63.409 & 63.409 \\
\hline 94 & Union & 11.442 & 11.442 \\
\hline 95 & Vanderburgh & 300.448 & 300.448 \\
\hline 96 & Vermillion & .000 & .000 \\
\hline 97 & Vigo & 133.638 & 133.638 \\
\hline 98 & Wabash & 175.760 & 175.760 \\
\hline 99 & Warren & 71.208 & 71.208 \\
\hline 100 & Warrick & 167.198 & 167.198 \\
\hline 101 & Washington & 283.606 & 283.606 \\
\hline 102 & Wayne & 56.330 & 56.330 \\
\hline
\end{tabular}




\begin{tabular}{|c|c|c|c|}
\hline & Locale & P25 30 & A25 30 \\
\hline 103 & Wells & 79.035 & 79.035 \\
\hline$\overline{104}$ & White & 28.930 & 28.930 \\
\hline 105 & Whitley & 229.714 & 229.714 \\
\hline 106 & lowa & 9284.563 & 9284.563 \\
\hline 107 & Kansas & 3584.170 & 3584.170 \\
\hline 108 & Kentucky_E & 8592.433 & 8592.433 \\
\hline 109 & Kentucky W & 5728.288 & 5728.288 \\
\hline 110 & Louisiana & 11067.372 & 11067.372 \\
\hline 111 & Maine & 9456.585 & 9456.585 \\
\hline 112 & Maryland & 5614.434 & 5614.434 \\
\hline 113 & Massachusetts & 3392.088 & 3392.088 \\
\hline 114 & Michigan_E & 7900.080 & 7900.080 \\
\hline 115 & Michigan W & 7900.080 & 7900.080 \\
\hline 116 & Minnesota & 20484.598 & 20484.598 \\
\hline 117 & Mississippi & 21680.817 & 21680.817 \\
\hline 118 & Missouri & 12588.345 & 12588.345 \\
\hline 119 & Montana & 6659.581 & 6659.581 \\
\hline 120 & Nebraska & 2876.511 & 2876.511 \\
\hline 121 & Nevada & 2357.817 & 2357.817 \\
\hline 122 & New Hampshire & 5293.582 & 5293.582 \\
\hline 123 & New Jersey & 3191.430 & 3191.430 \\
\hline 124 & New Mexico & 1895.831 & 1895.831 \\
\hline 125 & New York & 10996.457 & 10996.457 \\
\hline$\overline{126}$ & North Carolina & 55665.083 & 55665.083 \\
\hline 127 & North Dakota & 996.896 & 996.896 \\
\hline 128 & Ohio_N & 7594.491 & 7594.491 \\
\hline 129 & Ohio_M & 7594.491 & 7594.491 \\
\hline 130 & Ohio_s & 7594.491 & 7594.491 \\
\hline$\overline{131}$ & Oklahoma & 3790.564 & 3790.564 \\
\hline 132 & Oregon & 53276.315 & 53276.315 \\
\hline 133 & Pennsylvania & 27358.807 & 27358.807 \\
\hline 134 & Rhode Island & 598.590 & 598.590 \\
\hline 135 & South Carolina & 15342.794 & 15342.794 \\
\hline 136 & South Dakota & 1687.357 & 1687.357 \\
\hline 137 & Tennessee & 26311.755 & 26311.755 \\
\hline 138 & Texas & 50613.993 & 50613.993 \\
\hline 139 & Utah & 3695.984 & 3695.984 \\
\hline 140 & Vermont & 4151.486 & 4151.486 \\
\hline 141 & Virginia & 30680.264 & 30680.264 \\
\hline 142 & Washington & 33435.884 & 33435.884 \\
\hline 143 & West Virginia & 7918.322 & 7918.322 \\
\hline 144 & Wisconsin & 33649.758 & 33649.758 \\
\hline 145 & Wyoming & 1185.688 & 1185.688 \\
\hline
\end{tabular}




\begin{tabular}{|c|c|c|c|}
\hline & Locale & P26 30 & A26 30 \\
\hline 1 & Alabama & 26317.227 & 24717.580 \\
\hline 2 & Arizona & 15938.659 & 14370.636 \\
\hline 3 & Arkansas & 17158.162 & 15748.523 \\
\hline 4 & California & 48774.757 & 53602.187 \\
\hline 5 & Colorado & 5158.955 & 4903.179 \\
\hline 6 & Connecticut & 1472.153 & 1590.241 \\
\hline 7 & Delaware & 1306.528 & 1815.603 \\
\hline 8 & DC & .000 & .000 \\
\hline 9 & Florida & 26129.911 & 23844.383 \\
\hline 10 & Georgia & 37584.538 & 33788.617 \\
\hline 11 & Idaho & 12326.517 & 10911.782 \\
\hline 12 & Illinois $\mathbf{N}$ & 5925.553 & 7826.428 \\
\hline 13 & Illinois_s & 2962.776 & 3913.214 \\
\hline 14 & Adams & 648.957 & 573.475 \\
\hline 15 & Allen & 209.733 & 230.549 \\
\hline 16 & Bartholomew & 66.575 & 58.034 \\
\hline 17 & Benton & 148.606 & 129.541 \\
\hline 18 & Blackford & 59.364 & 51.748 \\
\hline 19 & Boone & .000 & .000 \\
\hline 20 & Brown & 25.744 & 22.442 \\
\hline 21 & Carroll & .000 & .000 \\
\hline 22 & Cass & 150.675 & 131.344 \\
\hline 23 & Clark & 757.696 & 739.651 \\
\hline 24 & Clay & 57.690 & 50.289 \\
\hline 25 & Clinton & 33.743 & 29.414 \\
\hline 26 & Crawford & 75.247 & 65.593 \\
\hline 27 & Daviess & 240.875 & 217.359 \\
\hline 28 & Dearborn & 13.830 & 21.598 \\
\hline 29 & Decatur & 25.653 & 22.362 \\
\hline 30 & DeKalb & 788.233 & 687.109 \\
\hline 31 & Delaware & 60.893 & 53.081 \\
\hline 32 & Dubois & 1804.242 & 1572.774 \\
\hline 33 & Elkhart & 7138.576 & 6230.480 \\
\hline 34 & Fayette & .000 & .000 \\
\hline 35 & Floyd & 658.109 & 573.679 \\
\hline 36 & Fountain & 9.351 & 8.152 \\
\hline 37 & Franklin & 44.950 & 86.896 \\
\hline 38 & Fulton & 251.902 & 219.585 \\
\hline 39 & Gibson & .000 & .000 \\
\hline 40 & Grant & 44.238 & 38.563 \\
\hline 41 & Greene & 54.403 & 47.424 \\
\hline 42 & Hamilton & 200.308 & 185.579 \\
\hline 43 & Hancock & 42.200 & 36.786 \\
\hline 44 & Harrison & 232.372 & 259.146 \\
\hline 45 & Hendricks & 117.153 & 102.123 \\
\hline 46 & Henry & 48.006 & 41.847 \\
\hline 47 & Howard & 57.030 & 49.713 \\
\hline 48 & Huntington & 94.288 & 89.845 \\
\hline 49 & Jackson & 286.609 & 249.840 \\
\hline 50 & Jasper & 70.911 & 61.813 \\
\hline 51 & Jay & 51.383 & 50.700 \\
\hline
\end{tabular}




\begin{tabular}{|c|c|c|c|}
\hline & Locale & P26 30 & A26 30 \\
\hline 52 & Jefferson & 76.645 & 66.812 \\
\hline 53 & Jennings & 79.508 & 116.328 \\
\hline 54 & Johnson & 1599.137 & 1393.982 \\
\hline 55 & Knox & 62.006 & 59.680 \\
\hline 56 & Kosciusko & 320.803 & 286.741 \\
\hline 57 & LaGrange & 2167.680 & 1889.586 \\
\hline 58 & Lake & 223.010 & 1596.080 \\
\hline 59 & La Porte & 194.334 & 175.818 \\
\hline 60 & Lawrence & 102.097 & 125.435 \\
\hline 61 & Madison & 173.285 & 151.054 \\
\hline 62 & Marion & 708.115 & 699.042 \\
\hline 63 & Marshall & 710.996 & 665.485 \\
\hline 64 & Martin & 8.214 & 7.160 \\
\hline 65 & Miami & 110.505 & 96.329 \\
\hline 66 & Monroe & 69.870 & 109.114 \\
\hline 67 & Montgomery & 42.323 & 36.893 \\
\hline 68 & Morgan & 238.250 & 207.685 \\
\hline 69 & Newton & .000 & .000 \\
\hline 70 & Noble & 63.566 & 55.411 \\
\hline 71 & Ohio & .000 & .000 \\
\hline 72 & Orange & 412.742 & 359.791 \\
\hline 73 & Owen & 131.679 & 114.786 \\
\hline 74 & Parke & 161.282 & 140.591 \\
\hline 75 & Perry & 49.910 & 43.507 \\
\hline 76 & Pike & 60.271 & 52.539 \\
\hline 77 & Porter & 109.172 & 95.166 \\
\hline 78 & Posey & 10.257 & 274.334 \\
\hline 79 & Pulaski & 10.021 & 8.735 \\
\hline 80 & Putnam & 60.814 & 53.012 \\
\hline 81 & Randolph & 322.522 & 287.048 \\
\hline 82 & Ripley & 127.645 & 118.488 \\
\hline 83 & Rush & 49.872 & 43.474 \\
\hline 84 & St. Joseph & 512.792 & 453.943 \\
\hline 85 & Scott & 60.487 & 59.683 \\
\hline 86 & Shelby & .000 & .000 \\
\hline 87 & Spencer & 135.196 & 117.852 \\
\hline 88 & Starke & 283.215 & 246.881 \\
\hline 89 & Steuben & 49.114 & 49.729 \\
\hline 90 & Sullivan & 89.115 & 77.683 \\
\hline 91 & Switzerland & .000 & .000 \\
\hline 92 & Tippecanoe & 64.065 & 64.513 \\
\hline 93 & Tipton & 53.874 & 46.963 \\
\hline 94 & Union & 9.721 & 8.474 \\
\hline 95 & Vanderburgh & 255.270 & 222.521 \\
\hline 96 & Vermillion & .000 & .000 \\
\hline 97 & Vigo & 113.543 & 98.976 \\
\hline 98 & Wabash & 149.331 & 136.061 \\
\hline 99 & Warren & 60.500 & 52.738 \\
\hline 100 & Warrick & 142.056 & 123.832 \\
\hline 101 & Washington & 240.960 & 210.047 \\
\hline 102 & Wayne & 47.859 & 41.719 \\
\hline
\end{tabular}




\begin{tabular}{|c|c|c|c|}
\hline & Locale & P26 30 & A26 30 \\
\hline 103 & Wells & 67.151 & 58.536 \\
\hline 104 & White & 24.580 & 21.427 \\
\hline 105 & Whitley & 195.172 & 170.133 \\
\hline 106 & lowa & 7888.440 & 6986.982 \\
\hline 107 & Kansas & 3045.217 & 4550.359 \\
\hline 108 & Kentucky_E & 7300.385 & 6902.743 \\
\hline 109 & Kentucky_W & 4866.924 & 4601.828 \\
\hline 110 & Louisiana & 9403.167 & 16551.593 \\
\hline 111 & Maine & 8034.595 & 7125.001 \\
\hline 112 & Maryland & 4770.190 & 5057.330 \\
\hline 113 & Massachusetts & 2882.018 & 3237.746 \\
\hline 114 & Michigan_E & 6712.142 & 6535.985 \\
\hline 115 & Michigan_W & 6712.142 & 6535.985 \\
\hline 116 & Minnesota & 17404.322 & 16719.241 \\
\hline 117 & Mississippi & 18420.665 & 17368.662 \\
\hline 118 & Missouri & 10695.431 & 10337.032 \\
\hline 119 & Montana & 5658.177 & 5520.620 \\
\hline 120 & Nebraska & 2443.969 & 2137.084 \\
\hline 121 & Nevada & 2003.271 & 1826.706 \\
\hline 122 & New Hampshire & 4497.584 & 4048.944 \\
\hline 123 & New Jersey & 2711.533 & 4931.971 \\
\hline 124 & New Mexico & 1610.754 & 1964.477 \\
\hline 125 & New York & 9342.916 & 9169.119 \\
\hline 126 & North Carolina & 47294.704 & 41882.955 \\
\hline 127 & North Dakota & 846.992 & 841.736 \\
\hline 128 & Ohio_N & 6452.505 & 6820.433 \\
\hline 129 & Ohio_M & 6452.505 & 6820.433 \\
\hline 130 & Ohio_S & 6452.505 & 6820.433 \\
\hline 131 & Oklahoma & 3220.576 & 4612.292 \\
\hline 132 & Oregon & 45265.136 & 40091.761 \\
\hline 133 & Pennsylvania & 23244.853 & 24965.242 \\
\hline 134 & Rhode Island & 508.580 & 450.214 \\
\hline 135 & South Carolina & 13035.693 & 11664.389 \\
\hline 136 & South Dakota & 1433.628 & 1256.341 \\
\hline 137 & Tennessee & 22355.247 & 20577.223 \\
\hline 138 & Texas & 43003.148 & 59507.520 \\
\hline 139 & Utah & 3140.217 & 4233.186 \\
\hline 140 & Vermont & 3527.226 & 3118.602 \\
\hline 141 & Virginia & 26066.862 & 23306.236 \\
\hline 142 & Washington & 28408.118 & 26628.346 \\
\hline 143 & West Virginia & 6727.641 & 6099.578 \\
\hline 144 & Wisconsin & 28589.832 & 25221.884 \\
\hline 145 & Wyoming & 1007.395 & 1432.082 \\
\hline
\end{tabular}




\begin{tabular}{|c|c|c|c|}
\hline & \begin{tabular}{|l} 
Locale \\
\end{tabular} & P27 30 & A27 30 \\
\hline 1 & Alabama & 8772.156 & 7559.497 \\
\hline 2 & Arizona & 2974.282 & 2952.751 \\
\hline 3 & Arkansas & 6684.315 & 8008.424 \\
\hline$\overline{4}$ & California & 21387.510 & 28000.572 \\
\hline 5 & Colorado & 1800.279 & 2765.090 \\
\hline$\overline{6}$ & Connecticut & 2732.878 & 2134.653 \\
\hline$\overline{7}$ & Delaware & 1051.340 & 1569.194 \\
\hline 8 & DC & .000 & .000 \\
\hline$\overline{9}$ & Florida & 9303.432 & 9671.250 \\
\hline 10 & Georgia & 17780.225 & 15499.942 \\
\hline 11 & Idaho & 1255.569 & 2445.521 \\
\hline 12 & Illinois_N & 9065.245 & 9509.454 \\
\hline$\overline{13}$ & Illinois_S & 4542.622 & 4754.727 \\
\hline 14 & Adams & 1.326 & 33.694 \\
\hline 15 & Allen & 430.764 & 374.911 \\
\hline$\overline{16}$ & Bartholomew & 341.849 & 285.346 \\
\hline 17 & Benton & 67.827 & 33.867 \\
\hline 18 & Blackford & 148.328 & 77.700 \\
\hline 19 & Boone & 7.002 & 4.718 \\
\hline 20 & Brown & .000 & .760 \\
\hline 21 & Carroll & .000 & 143.602 \\
\hline 22 & Cass & .000 & 125.046 \\
\hline 23 & Clark & 44.608 & 67.533 \\
\hline 24 & Clay & .000 & .766 \\
\hline 25 & Clinton & 28.002 & 184.160 \\
\hline 26 & Crawford & .000 & .000 \\
\hline 27 & Daviess & 1.260 & 89.077 \\
\hline 28 & Dearborn & 1.627 & 4.228 \\
\hline 29 & Decatur & .000 & 29.085 \\
\hline 30 & DeKalb & 94.251 & 52.701 \\
\hline 31 & Delaware & 211.692 & 115.807 \\
\hline 32 & Dubois & 102.698 & 118.125 \\
\hline 33 & Elkhart & 384.336 & 251.629 \\
\hline 34 & Fayette & .000 & .000 \\
\hline 35 & Floyd & 88.794 & 108.548 \\
\hline 36 & Fountain & .000 & 4.470 \\
\hline 37 & Franklin & 8.136 & 16.550 \\
\hline 38 & Fulton & 26.230 & 24.925 \\
\hline 39 & Gibson & .000 & 33.124 \\
\hline 40 & Grant & 139.994 & 85.467 \\
\hline 41 & Greene & .000 & 27.090 \\
\hline 42 & Hamilton & 1.870 & 20.439 \\
\hline 43 & Hancock & 124.509 & 63.369 \\
\hline 44 & Harrison & 9.649 & 97.493 \\
\hline 45 & Hendricks & .000 & 71.714 \\
\hline 46 & Henry & 21.911 & 14.765 \\
\hline 47 & Howard & .000 & 12.041 \\
\hline 48 & Huntington & 89.907 & 79.889 \\
\hline 49 & Jackson & 211.352 & 108.326 \\
\hline 50 & Jasper & .000 & 18.422 \\
\hline 51 & Jay & 24.460 & 43.940 \\
\hline
\end{tabular}




\begin{tabular}{|c|c|c|c|}
\hline & Locale & P27 30 & A27 30 \\
\hline 52 & Jefferson & .000 & 1.673 \\
\hline 53 & Jennings & 39.123 & 30.936 \\
\hline 54 & Johnson & 125.103 & 63.339 \\
\hline 55 & Knox & 23.303 & 21.581 \\
\hline 56 & Kosciusko & 5.903 & 79.706 \\
\hline 57 & LaGrange & .000 & 16.352 \\
\hline 58 & Lake & 415.112 & 631.782 \\
\hline 59 & La Porte & 207.775 & 146.859 \\
\hline 60 & Lawrence & 6.213 & 16.144 \\
\hline 61 & Madison & 77.324 & 96.454 \\
\hline 62 & Marion & 1289.475 & 969.879 \\
\hline 63 & Marshall & 241.102 & 169.026 \\
\hline 64 & Martin & .000 & 3.927 \\
\hline 65 & Miami & 140.104 & 73.868 \\
\hline 66 & Monroe & 101.234 & 71.236 \\
\hline 67 & Montgomery & 80.488 & 70.295 \\
\hline 68 & Morgan & .000 & 1.037 \\
\hline 69 & Newton & 25.573 & 13.513 \\
\hline 70 & \begin{tabular}{|l} 
Noble \\
\end{tabular} & 84.622 & 105.557 \\
\hline$\overline{71}$ & Ohio & .000 & .000 \\
\hline 72 & Orange & .000 & 4.317 \\
\hline 73 & Owen & .000 & .846 \\
\hline$\overline{74}$ & Parke & .000 & 12.850 \\
\hline 75 & Perry & .000 & .663 \\
\hline 76 & Pike & .000 & .000 \\
\hline 77 & Porter & .000 & 25.933 \\
\hline 78 & Posey & 45.257 & 91.852 \\
\hline 79 & Pulaski & 4.574 & 7.792 \\
\hline 80 & Putnam & .000 & .808 \\
\hline 81 & Randolph & 1.007 & 7.182 \\
\hline 82 & Ripley & 84.801 & 44.926 \\
\hline 83 & Rush & 22.763 & 13.087 \\
\hline 84 & St. Joseph & 259.557 & 186.876 \\
\hline 85 & Scott & 1.186 & 32.398 \\
\hline 86 & Shelby & 4.292 & 15.255 \\
\hline 87 & Spencer & .000 & 4.280 \\
\hline 88 & Starke & 24.012 & 11.990 \\
\hline 89 & Steuben & 28.629 & 36.095 \\
\hline 90 & Sullivan & .000 & .000 \\
\hline 91 & Switzerland & 6.114 & 3.053 \\
\hline 92 & Tippecanoe & 35.879 & 84.469 \\
\hline 93 & Tipton & 24.589 & 24.797 \\
\hline 94 & Union & .000 & .000 \\
\hline 95 & Vanderburgh & 301.389 & 444.879 \\
\hline 96 & Vermillion & 65.787 & 33.373 \\
\hline 97 & Vigo & 231.656 & 134.843 \\
\hline 98 & Wabash & 147.057 & 78.934 \\
\hline 99 & Warren & .000 & .803 \\
\hline 100 & Warrick & 5.146 & 2.569 \\
\hline 101 & Washington & .000 & 5.097 \\
\hline 102 & Wayne & 86.648 & 88.067 \\
\hline
\end{tabular}




\begin{tabular}{|c|c|c|c|}
\hline & Locale & P27 30 & A27 30 \\
\hline 103 & Wells & 30.649 & 60.957 \\
\hline 104 & White & 25.890 & 16.091 \\
\hline 105 & Whitley & 89.081 & 45.368 \\
\hline 106 & lowa & 2361.357 & 4583.541 \\
\hline 107 & Kansas & 1863.912 & 3818.579 \\
\hline 108 & Kentucky_E & 3022.963 & 2634.567 \\
\hline 109 & Kentucky_W & 2015.308 & 1756.378 \\
\hline 110 & Louisiana & 6424.144 & 6590.655 \\
\hline 111 & Maine & 5413.339 & 3238.756 \\
\hline 112 & Maryland & 3919.032 & 3807.362 \\
\hline 113 & Massachusetts & 8913.006 & 6348.686 \\
\hline 114 & Michigan_E & 4354.253 & 7210.160 \\
\hline 115 & Michigan_W & 4354.253 & 3605.080 \\
\hline 116 & Minnesota & 8511.665 & 8594.921 \\
\hline 117 & Mississippi & 4153.429 & 4489.286 \\
\hline 118 & Missouri & 6378.619 & 6611.882 \\
\hline 119 & Montana & 459.811 & 556.897 \\
\hline 120 & Nebraska & 738.043 & 3005.915 \\
\hline 121 & Nevada & 547.600 & 764.925 \\
\hline 122 & New Hampshire & 2041.061 & 1262.953 \\
\hline 123 & New Jersey & 8814.852 & 7533.409 \\
\hline 124 & New Mexico & 361.162 & 655.755 \\
\hline 125 & New York & 11122.105 & 9421.213 \\
\hline 126 & North Carolina & 13398.404 & 12619.637 \\
\hline 127 & North Dakota & 41.087 & 414.858 \\
\hline 128 & Ohio_N & 4419.842 & 3713.360 \\
\hline 129 & Ohio_M & 4419.842 & 3713.360 \\
\hline 130 & Ohio_s & 4419.842 & 3713.360 \\
\hline 131 & Oklahoma & 2113.304 & 2685.820 \\
\hline 132 & Oregon & 4755.337 & 4542.494 \\
\hline 133 & Pennsylvania & 14726.193 & 14244.222 \\
\hline 134 & Rhode Island & 900.557 & 675.214 \\
\hline 135 & South Carolina & 7359.144 & 5208.242 \\
\hline 136 & South Dakota & 289.902 & 763.112 \\
\hline 137 & Tennessee & 9382.151 & 8349.281 \\
\hline 138 & Texas & 17712.554 & 24148.309 \\
\hline 139 & Utah & 2016.472 & 2725.442 \\
\hline 140 & Vermont & 854.297 & 756.060 \\
\hline 141 & Virginia & 8811.853 & 8027.205 \\
\hline 142 & Washington & 9131.655 & 8565.264 \\
\hline 143 & West Virginia & 285.307 & 560.857 \\
\hline 144 & Wisconsin & 19870.136 & 14766.278 \\
\hline 145 & Wyoming & 98.843 & 241.050 \\
\hline
\end{tabular}




\begin{tabular}{|c|c|c|c|}
\hline & $\begin{aligned} \text { Locale } \\
\end{aligned}$ & P28 30 & A28 30 \\
\hline 1 & Alabama & 3189.271 & 2523.942 \\
\hline 2 & Arizona & 3572.065 & 4666.446 \\
\hline 3 & Arkansas & 3372.372 & 2723.102 \\
\hline 4 & California & 25745.578 & 27840.313 \\
\hline 5 & Colorado & 2758.763 & 3002.913 \\
\hline 6 & Connecticut & 1238.264 & 1484.656 \\
\hline 7 & Delaware & 699.675 & 622.609 \\
\hline 8 & DC & .000 & .000 \\
\hline 9 & Florida & 6967.342 & 8764.024 \\
\hline 10 & Georgia & 11204.008 & 5256.489 \\
\hline 11 & Idaho & 1895.250 & 1838.759 \\
\hline 12 & Illinois_N & 4586.557 & 4749.430 \\
\hline 13 & |llinois_s & 2293.279 & 2374.715 \\
\hline 14 & Adams & 44.116 & 46.659 \\
\hline 15 & Allen & 249.191 & 254.068 \\
\hline 16 & Bartholomew & 98.946 & 90.832 \\
\hline 17 & Benton & .000 & .933 \\
\hline 18 & Blackford & 2.433 & 3.238 \\
\hline 19 & Boone & 17.728 & 29.026 \\
\hline 20 & Brown & .771 & 2.648 \\
\hline 21 & Carroll & 96.031 & 72.644 \\
\hline 22 & Cass & 93.764 & 76.578 \\
\hline 23 & Clark & 26.885 & 30.510 \\
\hline 24 & Clay & .777 & 3.965 \\
\hline 25 & Clinton & 134.937 & 111.696 \\
\hline 26 & Crawford & .000 & 1.608 \\
\hline 27 & Daviess & 57.950 & 46.184 \\
\hline 28 & Dearborn & 1.118 & 9.555 \\
\hline 29 & Decatur & 19.450 & 17.329 \\
\hline 30 & DeKalb & 13.170 & 19.728 \\
\hline 31 & Delaware & 8.027 & 20.712 \\
\hline 32 & Dubois & 67.457 & 62.990 \\
\hline 33 & Elkhart & 169.569 & 133.921 \\
\hline 34 & Fayette & .000 & 2.871 \\
\hline 35 & Floyd & 61.567 & 63.600 \\
\hline 36 & Fountain & 4.536 & 6.038 \\
\hline 37 & Franklin & .614 & 3.500 \\
\hline 38 & Fulton & 8.174 & 8.672 \\
\hline 39 & Gibson & 28.934 & 28.026 \\
\hline 40 & Grant & .000 & .000 \\
\hline 41 & Greene & 20.498 & 19.101 \\
\hline 42 & Hamilton & 47.291 & 67.203 \\
\hline 43 & Hancock & 1.218 & 11.960 \\
\hline 44 & Harrison & 52.799 & 44.493 \\
\hline 45 & Hendricks & 59.275 & 72.285 \\
\hline 46 & Henry & 8.511 & 8.608 \\
\hline 47 & Howard & 9.807 & 18.525 \\
\hline 48 & Huntington & 69.922 & 75.029 \\
\hline 49 & Jackson & 2.819 & 6.599 \\
\hline 50 & Jasper & 17.276 & 18.731 \\
\hline 51 & Jay & 20.258 & 17.190 \\
\hline
\end{tabular}




\begin{tabular}{|c|c|c|c|}
\hline & $\begin{array}{l}\text { Locale } \\
\end{array}$ & P28 30 & A28 30 \\
\hline 52 & Jefferson & 2.141 & 5.208 \\
\hline 53 & Jennings & 41.474 & 4.081 \\
\hline 54 & Johnson & 26.928 & 52.197 \\
\hline 55 & Knox & 7.559 & 10.766 \\
\hline 56 & Kosciusko & 60.188 & 50.495 \\
\hline 57 & LaGrange & 13.964 & 15.946 \\
\hline 58 & Lake & 66.558 & 105.531 \\
\hline 59 & La Porte & 30.542 & 35.743 \\
\hline 60 & Lawrence & 5.126 & 9.191 \\
\hline 61 & Madison & 40.835 & 45.779 \\
\hline 62 & Marion & 295.438 & 320.602 \\
\hline 63 & Marshall & 31.871 & 25.150 \\
\hline 64 & Martin & 2.626 & 3.067 \\
\hline 65 & Miami & 3.639 & 6.144 \\
\hline 66 & Monroe & 45.362 & 60.357 \\
\hline 67 & Montgomery & 20.133 & 19.628 \\
\hline 68 & Morgan & 64.748 & 83.678 \\
\hline 69 & Newton & 5.001 & 7.235 \\
\hline 70 & Noble & 62.138 & 39.272 \\
\hline 71 & Ohio & .000 & .716 \\
\hline 72 & Orange & 2.887 & 4.626 \\
\hline 73 & Owen & 6.770 & 3.470 \\
\hline 74 & Parke & 8.593 & 8.279 \\
\hline 75 & Perry & 5.532 & 2.733 \\
\hline 76 & Pike & .000 & 1.620 \\
\hline 77 & Porter & 21.418 & 35.586 \\
\hline 78 & Posey & 9.272 & 5.920 \\
\hline 79 & Pulaski & 3.684 & 4.367 \\
\hline 80 & Putnam & 5.428 & 10.962 \\
\hline 81 & Randolph & 4.924 & 7.246 \\
\hline 82 & Ripley & 2.287 & 6.005 \\
\hline 83 & Rush & 2.190 & 3.033 \\
\hline 84 & St. Joseph & 79.747 & 92.911 \\
\hline 85 & Scott & 37.320 & 17.886 \\
\hline 86 & Shelby & 8.768 & 11.866 \\
\hline 87 & Spencer & 2.862 & 4.513 \\
\hline 88 & Starke & .000 & 2.811 \\
\hline 89 & Steuben & 20.971 & 16.127 \\
\hline 90 & Sullivan & .000 & 2.768 \\
\hline 91 & Switzerland & .000 & 1.346 \\
\hline 92 & Tippecanoe & 91.704 & 102.675 \\
\hline 93 & Tipton & 8.372 & 8.016 \\
\hline 94 & Union & .000 & .873 \\
\hline 95 & Vanderburgh & 235.675 & 165.998 \\
\hline 96 & Vermillion & .351 & 2.102 \\
\hline 97 & Vigo & 60.569 & 75.192 \\
\hline 98 & Wabash & 15.975 & 20.258 \\
\hline 99 & Warren & .537 & 1.360 \\
\hline 100 & Warrick & 1.098 & 8.412 \\
\hline 101 & Washington & 3.408 & 6.287 \\
\hline 102 & Wayne & 50.382 & 52.751 \\
\hline
\end{tabular}




\begin{tabular}{|c|c|c|c|}
\hline & Locale & P28 30 & A28 30 \\
\hline 103 & Wells & 31.928 & 26.437 \\
\hline 104 & White & 7.595 & 9.856 \\
\hline 105 & Whitley & 5.911 & 11.031 \\
\hline 106 & lowa & 2768.218 & 2460.523 \\
\hline 107 & Kansas & 1912.292 & 1726.518 \\
\hline 108 & Kentucky_E & 1122.391 & 1023.169 \\
\hline 109 & Kentucky_W & 748.260 & 682.112 \\
\hline 110 & Louisiana & 1081.482 & 1290.177 \\
\hline 111 & Maine & 686.125 & 613.029 \\
\hline 112 & Maryland & 1976.372 & 2521.127 \\
\hline 113 & Massachusetts & 4357.500 & 4779.217 \\
\hline 114 & Michigan_E & 1322.473 & 1637.183 \\
\hline 115 & Michigan_W & 1322.473 & 1637.183 \\
\hline 116 & Minnesota & 4770.275 & 4864.785 \\
\hline 117 & Mississippi & 1947.432 & 1530.208 \\
\hline 118 & Missouri & 2804.889 & 2769.757 \\
\hline 119 & Montana & 150.154 & 227.776 \\
\hline 120 & Nebraska & 1965.302 & 1687.179 \\
\hline 121 & Nevada & 563.973 & 890.761 \\
\hline 122 & New Hampshire & 1114.562 & 1239.429 \\
\hline 123 & New Jersey & 3214.498 & 3525.675 \\
\hline 124 & New Mexico & 555.987 & 776.744 \\
\hline 125 & New York & 5405.480 & 6498.665 \\
\hline 126 & North Carolina & 8824.398 & 6432.399 \\
\hline 127 & North Dakota & 300.406 & 292.768 \\
\hline 128 & Ohio_N & 1251.023 & 1400.539 \\
\hline 129 & Ohio_M & 1251.023 & 1400.539 \\
\hline 130 & Ohio_s & 1251.023 & 1400.539 \\
\hline 131 & Oklahoma & 1304.182 & 1449.992 \\
\hline 132 & Oregon & 2894.993 & 3151.663 \\
\hline 133 & Pennsylvania & 6070.147 & 6043.377 \\
\hline 134 & Rhode Island & 448.238 & 453.319 \\
\hline 135 & South Carolina & 2987.997 & 1925.501 \\
\hline 136 & South Dakota & 831.099 & 792.673 \\
\hline 137 & Tennessee & 3357.445 & 3005.663 \\
\hline 138 & Texas & 13473.326 & 14951.408 \\
\hline 139 & Utah & 1686.224 & 1769.048 \\
\hline 140 & Vermont & 512.249 & 565.232 \\
\hline 141 & Virginia & 3885.196 & 4053.206 \\
\hline 142 & Washington & 4348.836 & 4710.475 \\
\hline 143 & West Virginia & 273.690 & 405.430 \\
\hline 144 & Wisconsin & 4079.266 & 3798.854 \\
\hline 145 & Wyoming & 55.841 & 102.800 \\
\hline
\end{tabular}




\begin{tabular}{|c|c|c|c|}
\hline & Locale & P29 30 & A29 30 \\
\hline 1 & Alabama & 1994.154 & 1694.766 \\
\hline 2 & Arizona & 2266.166 & 3501.237 \\
\hline 3 & Arkansas & 1475.431 & 1116.009 \\
\hline 4 & California & 14354.101 & 15768.885 \\
\hline 5 & Colorado & 1537.702 & 1915.598 \\
\hline 6 & Connecticut & 1297.875 & 1284.220 \\
\hline 7 & Delaware & 413.370 & 343.790 \\
\hline 8 & DC & .000 & .000 \\
\hline 9 & Florida & 7555.896 & 9648.959 \\
\hline 10 & Georgia & 5107.194 & 3993.772 \\
\hline 11 & Idaho & 676.239 & 661.988 \\
\hline 12 & Illinois_N & 3702.284 & 3110.539 \\
\hline 13 & Illinois_S & 1851.142 & 1555.270 \\
\hline 14 & Adams & 6.999 & 14.482 \\
\hline 15 & Allen & 190.655 & 149.546 \\
\hline 16 & Bartholomew & 47.788 & 31.820 \\
\hline 17 & Benton & 11.662 & 2.796 \\
\hline 18 & Blackford & 18.806 & 4.379 \\
\hline 19 & Boone & 13.316 & 27.278 \\
\hline 20 & Brown & .307 & 5.926 \\
\hline 21 & Carroll & 5.702 & 7.532 \\
\hline 22 & Cass & 5.283 & 14.731 \\
\hline 23 & Clark & 18.356 & 43.641 \\
\hline 24 & Clay & 2.135 & 9.855 \\
\hline 25 & Clinton & 8.097 & 13.281 \\
\hline 26 & Crawford & .000 & 4.819 \\
\hline 27 & Daviess & 5.410 & 11.580 \\
\hline 28 & Dearborn & 4.140 & 25.721 \\
\hline 29 & Decatur & 9.813 & 9.365 \\
\hline 30 & DeKalb & 15.940 & 18.680 \\
\hline 31 & Delaware & 40.366 & 46.328 \\
\hline 32 & Dubois & 25.744 & 16.501 \\
\hline 33 & Elkhart & 79.846 & 82.208 \\
\hline 34 & Fayette & 4.167 & 8.606 \\
\hline 35 & Floyd & 26.074 & 32.826 \\
\hline 36 & Fountain & 3.100 & 6.257 \\
\hline 37 & Franklin & 2.226 & 9.147 \\
\hline 38 & Fulton & 6.783 & 7.776 \\
\hline 39 & Gibson & 3.248 & 12.277 \\
\hline 40 & Grant & 22.459 & 22.689 \\
\hline 41 & Greene & 2.335 & 12.460 \\
\hline 42 & Hamilton & 78.906 & 146.154 \\
\hline 43 & Hancock & 33.457 & 32.669 \\
\hline 44 & Harrison & 3.740 & 17.785 \\
\hline 45 & Hendricks & 17.598 & 79.427 \\
\hline 46 & Henry & 11.023 & 15.821 \\
\hline 47 & Howard & 6.241 & 31.887 \\
\hline 48 & Huntington & 19.055 & 16.458 \\
\hline 49 & Jackson & 34.005 & 15.685 \\
\hline 50 & Jasper & 4.201 & 12.196 \\
\hline 51 & Jay & 7.911 & 7.181 \\
\hline
\end{tabular}




\begin{tabular}{|c|c|c|c|}
\hline & $\begin{array}{r}\text { Locale } \\
\end{array}$ & P29 30 & A29 30 \\
\hline 52 & Jefferson & 1.791 & 13.162 \\
\hline 53 & Jennings & 5.923 & 12.231 \\
\hline 54 & Johnson & 25.443 & 70.149 \\
\hline 55 & Knox & 13.367 & 13.469 \\
\hline 56 & Kosciusko & 8.164 & 29.861 \\
\hline 57 & LaGrange & 9.835 & 17.328 \\
\hline 58 & Lake & 79.183 & 177.136 \\
\hline 59 & La Porte & 47.039 & 39.979 \\
\hline 60 & Lawrence & 2.040 & 16.405 \\
\hline 61 & Madison & 22.207 & 51.627 \\
\hline 62 & Marion & 509.803 & 337.648 \\
\hline 63 & Marshall & 34.885 & 19.361 \\
\hline 64 & Martin & 1.613 & 3.446 \\
\hline 65 & Miami & 27.823 & 11.915 \\
\hline 66 & Monroe & 49.053 & 56.258 \\
\hline 67 & Montgomery & 13.061 & 14.762 \\
\hline 68 & Morgan & 2.892 & 34.195 \\
\hline 69 & Newton & 5.982 & 5.168 \\
\hline 70 & Noble & 18.062 & 19.043 \\
\hline 71 & Ohio & .000 & 2.147 \\
\hline 72 & Orange & 1.657 & 7.547 \\
\hline$\overline{73}$ & Owen & .342 & 9.161 \\
\hline 74 & Parke & 1.780 & 6.005 \\
\hline 75 & Perry & 1.178 & 6.435 \\
\hline 76 & Pike & 1.358 & 4.856 \\
\hline 77 & Porter & 26.421 & 58.110 \\
\hline 78 & Posey & 7.066 & 10.568 \\
\hline 79 & Pulaski & 8.795 & 5.027 \\
\hline 80 & Putnam & 1.729 & 14.928 \\
\hline 81 & Randolph & 1.625 & 9.191 \\
\hline 82 & Ripley & 15.716 & 10.852 \\
\hline 83 & Rush & 4.843 & 5.786 \\
\hline 84 & St. Joseph & 118.109 & 109.963 \\
\hline 85 & Scott & .324 & 9.520 \\
\hline 86 & Shelby & 4.773 & 16.373 \\
\hline 87 & Spencer & 6.773 & 7.262 \\
\hline 88 & Starke & 4.092 & 8.427 \\
\hline 89 & Steuben & 3.678 & 13.203 \\
\hline 90 & Sullivan & 4.673 & 8.298 \\
\hline 91 & Switzerland & 1.150 & 4.034 \\
\hline 92 & Tippecanoe & 29.234 & 72.039 \\
\hline 93 & Tipton & 7.052 & 5.701 \\
\hline 94 & Union & 1.877 & 2.616 \\
\hline 95 & Vanderburgh & 89.444 & 65.621 \\
\hline 96 & Vermillion & 9.774 & 5.531 \\
\hline 97 & Vigo & 49.906 & 36.972 \\
\hline 98 & Wabash & 19.474 & 12.214 \\
\hline 99 & Warren & 1.947 & 2.901 \\
\hline 100 & Warrick & 7.355 & 25.214 \\
\hline 101 & Washington & .343 & 11.385 \\
\hline 102 & Wayne & 21.225 & 22.570 \\
\hline
\end{tabular}




\begin{tabular}{|c|c|c|c|}
\hline & Locale & P29 30 & A29 30 \\
\hline 103 & Wells & 58.215 & 11.357 \\
\hline 104 & White & 12.598 & 9.293 \\
\hline 105 & Whitley & 13.809 & 13.549 \\
\hline 106 & lowa & 1199.419 & 1033.545 \\
\hline 107 & Kansas & 1034.442 & 1020.223 \\
\hline 108 & Kentucky_E & 981.838 & 946.172 \\
\hline 109 & Kentucky_W & 654.559 & 630.782 \\
\hline 110 & Louisiana & 1629.346 & 1681.908 \\
\hline 111 & Maine & 980.263 & 491.634 \\
\hline 112 & Maryland & 1864.251 & 2420.564 \\
\hline 113 & Massachusetts & 2968.325 & 2434.148 \\
\hline 114 & Michigan_E & 1584.556 & 1868.225 \\
\hline 115 & Michigan_W & 1584.556 & 1868.225 \\
\hline 116 & Minnesota & 2807.760 & 2161.540 \\
\hline 117 & Mississippi & 1079.010 & 1068.678 \\
\hline 118 & Missouri & 2592.560 & 2229.896 \\
\hline 119 & Montana & 282.283 & 364.839 \\
\hline 120 & Nebraska & 687.266 & 635.012 \\
\hline 121 & Nevada & 773.991 & 1338.237 \\
\hline 122 & New Hampshire & 573.414 & 560.985 \\
\hline 123 & New Jersey & 5488.526 & 3389.423 \\
\hline 124 & New Mexico & 340.517 & 725.233 \\
\hline 125 & New York & 7469.519 & 6778.772 \\
\hline 126 & North Carolina & 4438.665 & 4117.554 \\
\hline 127 & North Dakota & 243.302 & 217.237 \\
\hline 128 & Ohio_N & 1457.854 & 1353.864 \\
\hline 129 & Ohio_M & 1457.854 & 1353.864 \\
\hline 130 & Ohio_s & 1457.854 & 1353.864 \\
\hline 131 & Oklahoma & 1041.535 & 1354.083 \\
\hline 132 & Oregon & 1899.447 & 1652.645 \\
\hline 133 & Pennsylvania & 4483.434 & 4500.343 \\
\hline 134 & Rhode Island & 322.056 & 399.200 \\
\hline 135 & South Carolina & 1778.363 & 1753.399 \\
\hline 136 & South Dakota & 278.413 & 279.376 \\
\hline 137 & Tennessee & 3105.949 & 2525.466 \\
\hline 138 & Texas & 9016.883 & 11165.778 \\
\hline 139 & Utah & 986.776 & 1171.175 \\
\hline 140 & Vermont & 303.705 & 247.242 \\
\hline 141 & Virginia & 2741.136 & 3355.546 \\
\hline 142 & Washington & 3229.477 & 2939.543 \\
\hline 143 & West Virginia & 255.646 & 612.491 \\
\hline 144 & Wisconsin & 3919.583 & 2137.806 \\
\hline 145 & Wyoming & 73.343 & 183.511 \\
\hline
\end{tabular}




\begin{tabular}{|c|c|c|c|}
\hline & Locale & $\mathrm{P} 30 \quad 30$ & $\mathrm{~A} 30 \quad 30$ \\
\hline 1 & Alabama & 4774.683 & 3931.264 \\
\hline 2 & Arizona & 647.390 & 1373.622 \\
\hline 3 & Arkansas & 915.743 & 1008.239 \\
\hline 4 & California & 9692.282 & 11821.894 \\
\hline 5 & Colorado & 744.970 & 1075.292 \\
\hline 6 & Connecticut & 740.913 & 868.196 \\
\hline 7 & Delaware & 328.210 & 360.895 \\
\hline 8 & DC & .000 & .000 \\
\hline 9 & Florida & 2970.682 & 4704.394 \\
\hline 10 & Georgia & 22581.486 & 17968.324 \\
\hline 11 & Idaho & 155.245 & 292.244 \\
\hline 12 & Illinois_N & 1263.429 & 1875.243 \\
\hline 13 & Illinois_s & 631.714 & 937.621 \\
\hline 14 & Adams & 1.468 & 5.036 \\
\hline 15 & Allen & 91.299 & 111.808 \\
\hline 16 & Bartholomew & 2.518 & 9.769 \\
\hline 17 & Benton & .000 & .680 \\
\hline 18 & Blackford & .000 & 1.065 \\
\hline 19 & Boone & .000 & 6.635 \\
\hline 20 & Brown & .000 & 1.441 \\
\hline 21 & Carroll & .000 & 1.832 \\
\hline 22 & Cass & 2.035 & 5.224 \\
\hline 23 & Clark & 30.417 & 38.497 \\
\hline 24 & Clay & .000 & 2.397 \\
\hline 25 & Clinton & .000 & 3.230 \\
\hline 26 & Crawford & .000 & 1.172 \\
\hline 27 & Daviess & 1.395 & 4.254 \\
\hline 28 & Dearborn & 1.802 & 8.114 \\
\hline 29 & Decatur & .000 & 2.278 \\
\hline 30 & DeKalb & .000 & 4.543 \\
\hline 31 & Delaware & 2.303 & 13.125 \\
\hline 32 & Dubois & 2.386 & 5.937 \\
\hline 33 & Elkhart & 148.222 & 139.632 \\
\hline 34 & Fayette & .000 & 2.093 \\
\hline 35 & Floyd & .000 & 7.984 \\
\hline 36 & Fountain & .000 & 1.522 \\
\hline 37 & Franklin & 9.011 & 11.514 \\
\hline 38 & Fulton & .000 & 1.891 \\
\hline 39 & Gibson & .000 & 2.986 \\
\hline 40 & Grant & 10.038 & 13.612 \\
\hline 41 & Greene & 2.057 & 4.690 \\
\hline 42 & Hamilton & 67.022 & 89.763 \\
\hline 43 & Hancock & .000 & 7.946 \\
\hline 44 & Harrison & 10.687 & 15.343 \\
\hline 45 & Hendricks & 4.431 & 22.891 \\
\hline 46 & Henry & 10.893 & 12.631 \\
\hline 47 & Howard & .000 & 7.756 \\
\hline 48 & Huntington & 3.963 & 7.523 \\
\hline 49 & Jackson & 2.212 & 5.599 \\
\hline 50 & Jasper & .000 & 2.966 \\
\hline 51 & Jay & 1.116 & 2.897 \\
\hline
\end{tabular}




\begin{tabular}{|c|c|c|c|}
\hline & Locale & P30 30 & A30 30 \\
\hline 52 & Jefferson & 2.382 & 5.122 \\
\hline 53 & Jennings & 105.531 & 90.061 \\
\hline 54 & Johnson & 3.554 & 19.927 \\
\hline 55 & Knox & 1.063 & 4.372 \\
\hline 56 & Kosciusko & 17.917 & 21.822 \\
\hline 57 & LaGrange & 2.616 & 6.324 \\
\hline 58 & Lake & 277.539 & 326.311 \\
\hline 59 & La Porte & 9.714 & 16.809 \\
\hline 60 & Lawrence & 8.879 & 12.694 \\
\hline 64 & Madison & 5.503 & 16.817 \\
\hline 62 & Marion & 114.497 & 176.819 \\
\hline 63 & Marshall & 23.663 & 25.727 \\
\hline 64 & Martin & .000 & .838 \\
\hline 65 & Miami & 1.857 & 4.396 \\
\hline 66 & Monroe & 24.959 & 35.853 \\
\hline 67 & Montgomery & .000 & 3.590 \\
\hline 68 & Morgan & 2.954 & 10.699 \\
\hline 69 & Newton & .000 & 1.257 \\
\hline 70 & Noble & 42.070 & 38.554 \\
\hline 71 & Ohio & .000 & .522 \\
\hline 72 & Orange & .000 & 1.836 \\
\hline 73 & Owen & 14.458 & 13.886 \\
\hline 74 & Parke & .000 & 1.461 \\
\hline 75 & Perry & 11.325 & 10.697 \\
\hline 76 & Pike & .000 & 1.181 \\
\hline 77 & Porter & 9.243 & 20.478 \\
\hline 78 & Posey & 64.089 & 65.501 \\
\hline 79 & Pulaski & .000 & 1.223 \\
\hline 80 & Putnam & .000 & 3.631 \\
\hline 81 & Randolph & 6.996 & 7.189 \\
\hline 82 & Ripley & 1.363 & 4.045 \\
\hline 83 & Rush & 1.886 & 2.928 \\
\hline 84 & St. Joseph & 42.390 & 61.035 \\
\hline 85 & Scott & 41.346 & 35.949 \\
\hline 86 & Shelby & .000 & 3.982 \\
\hline 87 & Spencer & .000 & 1.766 \\
\hline 88 & Starke & .000 & 2.050 \\
\hline 89 & Steuben & 14.953 & 15.561 \\
\hline 90 & Sullivan & .000 & 2.018 \\
\hline 91 & Switzerland & .000 & .981 \\
\hline 92 & Tippecanoe & 18.740 & 32.999 \\
\hline 93 & Tipton & .000 & 1.387 \\
\hline 94 & Union & .000 & .636 \\
\hline 95 & Vanderburgh & 90.968 & 89.117 \\
\hline 96 & Vermillion & .000 & 1.345 \\
\hline 97 & Vigo & 2.202 & 10.768 \\
\hline 98 & Wabash & 3.048 & 5.678 \\
\hline 99 & Warren & .000 & .706 \\
\hline 100 & Warrick & 2.558 & 8.195 \\
\hline 101 & Washington & .000 & 2.769 \\
\hline 102 & Wayne & .000 & 5.489 \\
\hline
\end{tabular}




\begin{tabular}{|c|c|c|c|}
\hline & Locale & P30 30 & A30 30 \\
\hline 103 & Wells & 3.822 & 5.639 \\
\hline 104 & White & 21.102 & 16.251 \\
\hline 105 & Whitley & .000 & 3.295 \\
\hline 106 & lowa & 270.863 & 472.378 \\
\hline 107 & Kansas & 636.191 & 839.245 \\
\hline 108 & Kentucky_E & 828.716 & 903.107 \\
\hline 109 & Kentucky_W & 552.477 & 602.072 \\
\hline 110 & Louisiana & 1861.624 & 2262.294 \\
\hline 111 & Maine & 942.542 & 801.581 \\
\hline 112 & Maryland & 593.804 & 1068.877 \\
\hline 113 & Massachusetts & 2661.701 & 2541.272 \\
\hline 114 & Michigan_E & 468.508 & 855.436 \\
\hline 115 & Michigan_W & 468.508 & 855.436 \\
\hline 116 & Minnesota & 826.847 & 1242.138 \\
\hline 117 & Mississippi & 1516.249 & 1489.705 \\
\hline 118 & Missouri & 885.861 & 1287.158 \\
\hline 119 & Montana & 148.122 & 231.969 \\
\hline 120 & Nebraska & 131.553 & 254.193 \\
\hline 121 & Nevada & 160.271 & 457.772 \\
\hline 122 & New Hampshire & 721.359 & 665.333 \\
\hline 123 & New Jersey & 2797.888 & 3011.194 \\
\hline 124 & New Mexico & 127.519 & 301.790 \\
\hline 125 & New York & 3471.934 & 4275.261 \\
\hline 126 & North Carolina & 24559.999 & 18051.900 \\
\hline 127 & North Dakota & 68.559 & 111.563 \\
\hline 128 & Ohio_N & 651.298 & 884.542 \\
\hline 129 & Ohio_M & 651.298 & 884.542 \\
\hline 130 & Ohio_s & 651.298 & 884.542 \\
\hline 131 & Oklahoma & 722.154 & 980.426 \\
\hline 132 & Oregon & 468.454 & 800.586 \\
\hline 133 & Pennsylvania & 4021.397 & 4299.858 \\
\hline 134 & Rhode Island & 945.214 & 751.381 \\
\hline 135 & South Carolina & 11549.495 & 8462.311 \\
\hline 136 & South Dakota & 247.520 & 254.632 \\
\hline 137 & Tennessee & 3288.639 & 3059.797 \\
\hline 138 & Texas & 8049.831 & 9982.735 \\
\hline 139 & Utah & 659.220 & 876.598 \\
\hline 140 & Vermont & 53.545 & 102.748 \\
\hline 141 & Virginia & 4181.499 & 3740.361 \\
\hline 142 & Washington & 996.690 & 1579.500 \\
\hline 143 & West Virginia & 105.907 & 238.422 \\
\hline 144 & Wisconsin & 877.024 & 1193.507 \\
\hline 145 & Wyoming & 118.792 & 163.733 \\
\hline
\end{tabular}




\begin{tabular}{|c|c|c|c|}
\hline & Locale & P31_30 & A31 30 \\
\hline 1 & Alabama & 28714.317 & 28802.841 \\
\hline 2 & Arizona & 36887.682 & 36664.313 \\
\hline 3 & Arkansas & 32747.258 & 33056.158 \\
\hline 4 & California & 210438.940 & 210235.237 \\
\hline 5 & Colorado & 25512.316 & 25486.634 \\
\hline 6 & Connecticut & 14565.783 & 14502.610 \\
\hline 7 & Delaware & 7497.477 & 7545.725 \\
\hline 8 & $\overline{D C}$ & .000 & .000 \\
\hline 9 & Florida & 107056.533 & 106538.725 \\
\hline 10 & Georgia & 67126.922 & 67325.765 \\
\hline 11 & Idaho & 14869.177 & 14969.910 \\
\hline 12 & Illinois_N & 49035.550 & 49131.634 \\
\hline 13 & Illinois_S & 24517.775 & 24565.817 \\
\hline 14 & Adams & 285.086 & 286.562 \\
\hline 15 & Allen & 2044.841 & 2043.799 \\
\hline 16 & Bartholomew & 866.046 & 873.629 \\
\hline 17 & Benton & 23.858 & 23.578 \\
\hline 18 & Blackford & 56.228 & 56.132 \\
\hline 19 & Boone & 239.107 & 236.492 \\
\hline 20 & Brown & 54.507 & 53.985 \\
\hline 21 & Carroll & 808.871 & 821.602 \\
\hline 22 & Cass & 774.093 & 784.361 \\
\hline 23 & Clark & 507.558 & 505.639 \\
\hline 24 & Clay & 88.071 & 87.156 \\
\hline 25 & Clinton & 995.734 & 1010.385 \\
\hline 26 & Crawford & 41.119 & 40.637 \\
\hline 27 & Daviess & 548.145 & 555.123 \\
\hline 28 & Dearborn & 225.197 & 222.727 \\
\hline 29 & Decatur & 230.724 & 232.518 \\
\hline 30 & DeKalb & 188.648 & 187.309 \\
\hline 31 & Delaware & 447.740 & 444.054 \\
\hline 32 & Dubois & 487.424 & 492.050 \\
\hline 33 & Elkhart & 1001.490 & 998.697 \\
\hline 34 & Fayette & 73.435 & 72.574 \\
\hline 35 & Floyd & 613.067 & 615.812 \\
\hline 36 & Fountain & 76.568 & 76.362 \\
\hline 37 & Franklin & 82.815 & 81.986 \\
\hline 38 & Fulton & 127.684 & 128.017 \\
\hline 39 & Gibson & 276.521 & 278.403 \\
\hline 40 & Grant & 274.324 & 273.515 \\
\hline 41 & Greene & 246.792 & 248.089 \\
\hline 42 & Hamilton & 1334.518 & 1321.476 \\
\hline 43 & Hancock & 284.999 & 281.843 \\
\hline 44 & Harrison & 561.157 & 566.791 \\
\hline 45 & Hendricks & 1049.619 & 1048.406 \\
\hline 46 & Henry & 154.833 & 153.610 \\
\hline 47 & Howard & 334.536 & 332.476 \\
\hline 48 & Huntington & 312.286 & 313.751 \\
\hline 49 & Jackson & 148.346 & 147.039 \\
\hline 50 & Jasper & 199.592 & 200.102 \\
\hline 51 & Jay & 218.356 & 220.482 \\
\hline
\end{tabular}




\begin{tabular}{|c|c|c|c|}
\hline & Locale & P31 30 & A31 30 \\
\hline 52 & Jefferson & 120.993 & 119.834 \\
\hline 53 & Jennings & 104.374 & 103.150 \\
\hline 54 & Johnson & 603.130 & 596.193 \\
\hline 55 & Knox & 159.424 & 158.882 \\
\hline 56 & Kosciusko & 643.894 & 647.953 \\
\hline 57 & LaGrange & 232.647 & 232.449 \\
\hline 58 & Lake & 1950.419 & 1940.642 \\
\hline 59 & La Porte & 556.641 & 556.543 \\
\hline 60 & Lawrence & 161.802 & 160.555 \\
\hline 61 & Madison & 740.488 & 740.753 \\
\hline 62 & Marion & 4468.967 & 4463.935 \\
\hline 63 & Marshall & 359.958 & 361.548 \\
\hline 64 & Martin & 49.768 & 49.792 \\
\hline 65 & Miami & 121.963 & 121.138 \\
\hline 66 & Monroe & 526.729 & 521.945 \\
\hline 67 & Montgomery & 282.077 & 283.427 \\
\hline 68 & Morgan & 297.177 & 293.853 \\
\hline 69 & Newton & 47.961 & 47.514 \\
\hline 70 & Noble & 490.750 & 494.789 \\
\hline 71 & Ohio & 18.321 & 18.106 \\
\hline 72 & Orange & 86.789 & 86.439 \\
\hline 73 & Owen & 82.562 & 81.725 \\
\hline 74 & Parke & 117.869 & 118.474 \\
\hline 75 & Perry & 58.351 & 57.769 \\
\hline 76 & Pike & 41.436 & 40.950 \\
\hline 77 & Porter & 630.332 & 626.953 \\
\hline 78 & Posey & 115.601 & 115.004 \\
\hline 79 & Pulaski & 71.458 & 71.473 \\
\hline 80 & Putnam & 131.573 & 130.156 \\
\hline 81 & Randolph & 105.638 & 105.212 \\
\hline 82 & Ripley & 96.924 & 95.917 \\
\hline 83 & Rush & 58.299 & 57.882 \\
\hline 84 & St. Joseph & 1226.610 & 1220.827 \\
\hline 85 & Scott & 237.409 & 239.285 \\
\hline 86 & Shelby & 207.697 & 207.290 \\
\hline 87 & Spencer & 84.164 & 83.839 \\
\hline 88 & Starke & 71.907 & 71.064 \\
\hline 89 & Steuben & 217.015 & 217.584 \\
\hline 90 & Sullivan & 70.807 & 69.977 \\
\hline 91 & Switzerland & 34.425 & 34.021 \\
\hline 92 & Tippecanoe & 948.927 & 947.771 \\
\hline 93 & Tipton & 113.563 & 114.168 \\
\hline 94 & Union & 22.324 & 22.062 \\
\hline 95 & Vanderburgh & 2086.440 & 2107.517 \\
\hline 96 & Vermillion & 49.916 & 49.412 \\
\hline 97 & Vigo & 414.914 & 413.015 \\
\hline 98 & Wabash & 125.374 & 124.535 \\
\hline 99 & Warren & 28.924 & 28.709 \\
\hline 100 & Warrick & 215.161 & 212.638 \\
\hline 101 & Washington & 123.579 & 122.918 \\
\hline 102 & Wayne & 424.907 & 426.855 \\
\hline
\end{tabular}




\begin{tabular}{|c|c|c|c|}
\hline & Locale & P31 30 & A31_30 \\
\hline 103 & Wells & 333.632 & 336.782 \\
\hline 104 & White & 95.699 & 95.066 \\
\hline 105 & Whitley & 120.222 & 118.949 \\
\hline 106 & lowa & 26333.403 & 26547.149 \\
\hline 107 & Kansas & 21296.579 & 21422.505 \\
\hline 108 & Kentucky_E & 13230.572 & 13229.282 \\
\hline 109 & Kentucky_W & 8820.382 & 8819.522 \\
\hline 110 & Louisiana & 21389.440 & 21348.591 \\
\hline 111 & Maine & 6821.230 & 6819.592 \\
\hline 112 & Maryland & 29120.332 & 29031.429 \\
\hline 113 & Massachusetts & 29702.336 & 29620.515 \\
\hline$\overline{114}$ & Michigan_E & 22500.668 & 22432.508 \\
\hline 115 & Michigan W & 22500.668 & 22432.508 \\
\hline 116 & Minnesota & 39028.454 & 39184.923 \\
\hline 117 & Mississippi & 19995.231 & 20085.254 \\
\hline 118 & Missouri & 35523.226 & 35598.815 \\
\hline 119 & Montana & 4070.721 & 4051.555 \\
\hline 120 & Nebraska & 19085.756 & 19269.715 \\
\hline 121 & Nevada & 13866.944 & 13777.364 \\
\hline 122 & New Hampshire & 5889.959 & 5853.802 \\
\hline 123 & New Jersey & 41934.116 & 41830.600 \\
\hline 124 & New Mexico & 7949.226 & 7908.544 \\
\hline 125 & New York & 76612.054 & 76273.639 \\
\hline 126 & North Carolina & 65058.195 & 65188.062 \\
\hline 127 & North Dakota & 3768.472 & 3781.410 \\
\hline 128 & Ohio_N & 17860.944 & 17839.713 \\
\hline 129 & Ohio_M & 17860.944 & 17839.713 \\
\hline 130 & Ohio_s & 17860.944 & 17839.713 \\
\hline 131 & Oklahoma & 17740.597 & 17717.130 \\
\hline 132 & Oregon & 24547.698 & 24571.492 \\
\hline 133 & Pennsylvania & 68222.835 & 68312.557 \\
\hline 134 & Rhode Island & 4567.384 & 4548.464 \\
\hline 135 & South Carolina & 22536.471 & 22498.193 \\
\hline 136 & South Dakota & 5581.966 & 5611.924 \\
\hline 137 & Tennessee & 39182.119 & 39248.719 \\
\hline 138 & Texas & 146948.984 & 146767.429 \\
\hline 139 & Utah & 17024.506 & 17034.638 \\
\hline 140 & Vermont & 3763.125 & 3768.327 \\
\hline 141 & Virginia & 46708.644 & 46700.215 \\
\hline 142 & Washington & 43509.883 & 43549.437 \\
\hline 143 & West Virginia & 7100.533 & 7073.185 \\
\hline 144 & Wisconsin & 42987.221 & 43221.422 \\
\hline 145 & Wyoming & 1863.492 & 1850.519 \\
\hline
\end{tabular}




\begin{tabular}{|c|c|c|c|}
\hline & Locale & P32 30 & A32 30 \\
\hline 1 & Alabama & 25837.337 & 19201.171 \\
\hline 2 & Arizona & 24167.692 & 15215.064 \\
\hline 3 & Arkansas & 12629.949 & 28212.225 \\
\hline 4 & California & 72870.091 & 111322.861 \\
\hline 5 & Colorado & 5635.999 & 12212.894 \\
\hline 6 & Connecticut & 14825.164 & 9738.753 \\
\hline 7 & Delaware & 2251.269 & 5986.773 \\
\hline 8 & DC & .000 & .000 \\
\hline 9 & Florida & 20413.646 & 35625.688 \\
\hline 10 & Georgia & 21394.000 & 45159.877 \\
\hline 11 & Idaho & 790.747 & 10722.273 \\
\hline 12 & Illinois_N & 30514.998 & 29953.204 \\
\hline 13 & Illinois_s & 15257.499 & 14976.602 \\
\hline 14 & Adams & 692.461 & 483.492 \\
\hline 15 & Allen & 4358.455 & 1972.386 \\
\hline 16 & Bartholomew & 1484.560 & 1124.380 \\
\hline 17 & Benton & 47.111 & .992 \\
\hline 18 & Blackford & 9.146 & 26.604 \\
\hline 19 & Boone & 94.327 & 62.163 \\
\hline 20 & Brown & 1.910 & 5.555 \\
\hline 21 & Carroll & 12.377 & 846.766 \\
\hline 22 & Cass & 550.017 & 1051.868 \\
\hline 23 & Clark & 144.256 & 160.349 \\
\hline 24 & Clay & 345.568 & 201.037 \\
\hline 25 & Clinton & 111.610 & 1060.022 \\
\hline 26 & Crawford & .000 & .000 \\
\hline 27 & Daviess & 16.939 & 516.507 \\
\hline 28 & Dearborn & .000 & 6.442 \\
\hline 29 & Decatur & 289.790 & 333.934 \\
\hline 30 & DeKalb & 2788.064 & 197.423 \\
\hline 31 & Delaware & 1318.294 & 449.731 \\
\hline 32 & Dubois & 368.516 & 605.752 \\
\hline 33 & Elkhart & 6087.954 & 3189.973 \\
\hline 34 & Fayette & 659.220 & 358.941 \\
\hline 35 & Floyd & 128.801 & 444.751 \\
\hline 36 & Fountain & 765.540 & 108.063 \\
\hline 37 & Franklin & 86.563 & 55.857 \\
\hline 38 & Fulton & 331.626 & 75.864 \\
\hline 39 & Gibson & 38.859 & 216.321 \\
\hline 40 & Grant & 598.170 & 372.824 \\
\hline 41 & Greene & 152.317 & 164.733 \\
\hline 42 & Hamilton & 953.979 & 278.064 \\
\hline 43 & Hancock & 449.909 & 261.600 \\
\hline 44 & Harrison & 205.322 & 581.349 \\
\hline 45 & Hendricks & 393.698 & 471.270 \\
\hline 46 & Henry & 547.838 & 185.924 \\
\hline 47 & Howard & 3966.762 & 2013.478 \\
\hline 48 & Huntington & 641.626 & 470.540 \\
\hline 49 & Jackson & 908.824 & 295.580 \\
\hline 50 & Jasper & 36.020 & 128.713 \\
\hline 51 & Jay & 64.319 & 214.624 \\
\hline
\end{tabular}




\begin{tabular}{|c|c|c|c|}
\hline & Locale & P32 30 & A32 30 \\
\hline 52 & Jefferson & 356.745 & 212.398 \\
\hline 53 & Jennings & 231.351 & 99.512 \\
\hline 54 & Johnson & 816.534 & 237.104 \\
\hline 55 & Knox & 274.314 & 51.116 \\
\hline 56 & Kosciusko & 1978.907 & 657.544 \\
\hline 57 & LaGrange & 301.015 & 265.174 \\
\hline 58 & Lake & 17929.531 & 713.132 \\
\hline 59 & La Porte & 1454.005 & 324.434 \\
\hline 60 & Lawrence & 1681.240 & 204.525 \\
\hline 61 & Madison & 1538.481 & 1119.275 \\
\hline 62 & Marion & 6330.638 & 3592.901 \\
\hline 63 & Marshall & 803.074 & 468.749 \\
\hline 64 & Martin & 128.117 & 22.957 \\
\hline 65 & Miami & 9.837 & 28.614 \\
\hline 66 & Monroe & 40.815 & 76.419 \\
\hline 67 & Montgomery & 951.688 & 338.224 \\
\hline 68 & Morgan & 115.251 & 32.679 \\
\hline 69 & Newton & 178.380 & 23.440 \\
\hline 70 & Noble & 1345.710 & 743.609 \\
\hline 71 & Ohio & .000 & .000 \\
\hline 72 & Orange & .000 & 25.241 \\
\hline 73 & Owen & .000 & 4.946 \\
\hline 74 & Parke & .000 & 75.123 \\
\hline 75 & Perry & 278.017 & 3.875 \\
\hline 76 & Pike & 53.717 & .000 \\
\hline 77 & Porter & 7344.561 & 152.792 \\
\hline 78 & Posey & .000 & 28.667 \\
\hline 79 & Pulaski & 158.297 & 33.376 \\
\hline 80 & Putnam & 152.249 & 93.522 \\
\hline 81 & Randolph & 361.056 & 105.635 \\
\hline 82 & Ripley & 2.095 & 6.096 \\
\hline 83 & Rush & 287.790 & 15.892 \\
\hline 84 & St. Joseph & 2397.744 & 810.940 \\
\hline 85 & Scott & 21.099 & 183.155 \\
\hline 86 & Shelby & 602.663 & 166.627 \\
\hline 87 & Spencer & 171.026 & 43.325 \\
\hline 88 & Starke & 51.630 & 30.114 \\
\hline 89 & Steuben & 980.144 & 428.288 \\
\hline 90 & Sullivan & 35.095 & 20.469 \\
\hline 91 & Switzerland & .000 & .000 \\
\hline 92 & Tippecanoe & 3435.583 & 1237.897 \\
\hline 93 & Tipton & 115.453 & 112.524 \\
\hline 94 & Union & .000 & .000 \\
\hline 95 & Vanderburgh & 181.634 & 1731.101 \\
\hline 96 & Vermillion & .000 & 3.069 \\
\hline 97 & Vigo & 397.229 & 154.613 \\
\hline 98 & Wabash & 643.026 & 66.213 \\
\hline 99 & Warren & 12.117 & 11.764 \\
\hline 100 & Warrick & 3807.602 & 23.048 \\
\hline 101 & Washington & 11.638 & 31.043 \\
\hline 102 & Wayne & 861.505 & 384.185 \\
\hline
\end{tabular}




\begin{tabular}{|c|c|c|c|}
\hline & Locale & P32 30 & A32 30 \\
\hline 103 & Wells & 259.734 & 316.584 \\
\hline 104 & White & 381.907 & 211.760 \\
\hline 105 & Whitley & 880.994 & 167.135 \\
\hline 106 & lowa & 11487.555 & 21748.845 \\
\hline 107 & Kansas & 12932.292 & 20314.879 \\
\hline 108 & Kentucky_E & 14080.438 & 9095.520 \\
\hline 109 & Kentucky_W & 9386.959 & 6063.680 \\
\hline 110 & Louisiana & 8188.075 & 10730.332 \\
\hline 111 & Maine & 4188.267 & 5012.415 \\
\hline 112 & Maryland & 10430.337 & 11004.177 \\
\hline 113 & Massachusetts & 7950.306 & 11602.918 \\
\hline 114 & Michigan_E & 41798.825 & 22330.964 \\
\hline 115 & Michigan_W & 41798.825 & 22330.964 \\
\hline 116 & Minnesota & 11313.566 & 25231.825 \\
\hline 117 & Mississippi & 8685.090 & 15645.689 \\
\hline 118 & Missouri & 26864.308 & 27330.873 \\
\hline 119 & Montana & 1292.106 & 1171.544 \\
\hline 120 & Nebraska & 2560.746 & 16314.221 \\
\hline 121 & Nevada & 3221.475 & 3156.680 \\
\hline 122 & New Hampshire & 4557.938 & 1657.603 \\
\hline 123 & New Jersey & 11880.505 & 16012.110 \\
\hline$\overline{124}$ & New Mexico & 2140.946 & 2281.723 \\
\hline 125 & New York & 23065.133 & 26072.612 \\
\hline 126 & North Carolina & 18997.000 & 39173.203 \\
\hline 127 & North Dakota & 610.838 & 2459.506 \\
\hline 128 & Ohio_N & 31109.239 & 13336.645 \\
\hline 129 & Ohio_M & 31109.239 & 13336.645 \\
\hline 130 & Ohio_s & 31109.239 & 13336.645 \\
\hline 131 & Oklahoma & 9259.718 & 9510.337 \\
\hline 132 & Oregon & 16624.665 & 14241.539 \\
\hline 133 & Pennsylvania & 62503.326 & 39525.786 \\
\hline 134 & Rhode Island & 3394.443 & 1512.791 \\
\hline 135 & South Carolina & 13060.039 & 11908.839 \\
\hline 136 & South Dakota & 980.331 & 3869.144 \\
\hline 137 & Tennessee & 31244.490 & 27669.446 \\
\hline 138 & Texas & 60039.973 & 71306.809 \\
\hline 139 & Utah & 13039.290 & 10502.762 \\
\hline 140 & Vermont & 786.498 & 2174.648 \\
\hline 141 & \begin{tabular}{|l} 
Virginia \\
\end{tabular} & 17116.296 & 25988.041 \\
\hline 142 & Washington & 41044.518 & 35688.185 \\
\hline 143 & West Virginia & 9294.343 & 2475.706 \\
\hline 144 & Wisconsin & 33987.116 & 33187.905 \\
\hline$\overline{145}$ & Wyoming & 179.115 & 377.553 \\
\hline
\end{tabular}




\begin{tabular}{|c|c|c|c|}
\hline & $\begin{array}{l}\text { Locale } \\
\end{array}$ & P33 30 & A33 30 \\
\hline 1 & Alabama & 12185.293 & 4595.451 \\
\hline 2 & Arizona & 4755.198 & 2582.174 \\
\hline 3 & Arkansas & 3122.543 & 3191.800 \\
\hline 4 & California & 33634.568 & 30679.023 \\
\hline 5 & Colorado & 2809.538 & 2197.012 \\
\hline 6 & Connecticut & 3719.152 & 2763.239 \\
\hline 7 & Delaware & 979.725 & 1266.384 \\
\hline 8 & DC & .000 & .000 \\
\hline 9 & Florida & 8871.160 & 6020.070 \\
\hline 10 & Georgia & 6354.687 & 5160.092 \\
\hline 11 & Idaho & 724.239 & 652.620 \\
\hline 12 & Illinois_N & 10097.971 & 11359.452 \\
\hline 13 & Illinois_S & 5048.986 & 5679.726 \\
\hline 14 & Adams & 59.865 & 63.484 \\
\hline 15 & Allen & 345.251 & 620.628 \\
\hline 16 & Bartholomew & 95.186 & 363.054 \\
\hline 17 & Benton & 5.357 & 4.751 \\
\hline 18 & Blackford & 56.166 & 4.258 \\
\hline 19 & Boone & 42.758 & 63.657 \\
\hline 20 & Brown & 5.085 & .000 \\
\hline 21 & Carroll & 36.311 & 5.762 \\
\hline 22 & Cass & 59.897 & 16.308 \\
\hline 23 & Clark & 195.509 & 217.472 \\
\hline 24 & Clay & 12.710 & 8.517 \\
\hline 25 & Clinton & 20.274 & 20.404 \\
\hline 26 & Crawford & .000 & .000 \\
\hline 27 & Daviess & 27.279 & 29.676 \\
\hline 28 & Dearborn & 66.994 & 31.374 \\
\hline 29 & Decatur & 225.531 & 41.216 \\
\hline 30 & DeKalb & 358.748 & 70.308 \\
\hline 31 & Delaware & 144.756 & 98.419 \\
\hline 32 & Dubois & 19.387 & 11.569 \\
\hline 33 & Elkhart & 697.046 & 164.770 \\
\hline 34 & Fayette & 23.755 & 52.184 \\
\hline 35 & Floyd & 68.339 & 64.372 \\
\hline 36 & Fountain & 4.987 & .000 \\
\hline 37 & Franklin & 52.630 & 85.301 \\
\hline 38 & Fulton & 64.318 & 31.616 \\
\hline 39 & Gibson & 15.755 & 17.885 \\
\hline 40 & Grant & 55.265 & 43.863 \\
\hline 41 & Greene & 19.218 & .000 \\
\hline 42 & Hamilton & 188.908 & 59.337 \\
\hline 43 & Hancock & 63.162 & 32.744 \\
\hline 44 & Harrison & 81.975 & 101.164 \\
\hline 45 & Hendricks & 31.089 & 42.603 \\
\hline 46 & Henry & 21.693 & 27.977 \\
\hline 47 & Howard & 35.729 & 31.020 \\
\hline 48 & Huntington & 102.696 & 56.711 \\
\hline 49 & Jackson & 51.962 & 62.267 \\
\hline 50 & Jasper & 57.194 & .958 \\
\hline 51 & Jay & 190.429 & 25.975 \\
\hline
\end{tabular}




\begin{tabular}{|c|c|c|c|}
\hline & Locale & P33 30 & A33 30 \\
\hline 52 & Jefferson & 11.828 & 72.741 \\
\hline 53 & Jennings & 100.747 & 108.633 \\
\hline 54 & Johnson & 131.856 & 100.041 \\
\hline 55 & Knox & 63.156 & 15.922 \\
\hline 56 & Kosciusko & 181.452 & 95.808 \\
\hline 57 & LaGrange & 19.398 & 58.917 \\
\hline 58 & Lake & 1615.958 & 2433.884 \\
\hline 59 & La Porte & 170.402 & 176.550 \\
\hline 60 & Lawrence & 115.860 & 92.469 \\
\hline 61 & Madison & 104.401 & 36.197 \\
\hline 62 & Marion & 932.035 & 919.865 \\
\hline 63 & Marshall & 136.128 & 91.386 \\
\hline 64 & Martin & 26.066 & .766 \\
\hline 65 & Miami & 17.650 & 15.494 \\
\hline 66 & Monroe & 116.454 & 269.692 \\
\hline 67 & Montgomery & 53.500 & 16.537 \\
\hline 68 & Morgan & 49.643 & 33.748 \\
\hline 69 & Newton & 4.208 & 7.227 \\
\hline 70 & Noble & 144.140 & 45.442 \\
\hline 71 & Ohio & .821 & .000 \\
\hline 72 & Orange & 4.816 & .842 \\
\hline 73 & Owen & 1.914 & .990 \\
\hline 74 & Parke & .692 & 5.156 \\
\hline 75 & Perry & 10.002 & 13.574 \\
\hline 76 & Pike & 5.357 & .000 \\
\hline 77 & Porter & 138.479 & 47.317 \\
\hline 78 & Posey & 261.623 & 444.340 \\
\hline 79 & Pulaski & 18.138 & 44.850 \\
\hline 80 & Putnam & 15.415 & 16.539 \\
\hline 81 & Randolph & 39.333 & 52.991 \\
\hline 82 & Ripley & 28.656 & 11.930 \\
\hline 83 & Rush & 7.866 & 40.225 \\
\hline 84 & St. Joseph & 367.816 & 157.067 \\
\hline 85 & Scott & 14.209 & 17.135 \\
\hline 86 & Shelby & 161.884 & 34.462 \\
\hline 87 & Spencer & 16.979 & 14.610 \\
\hline 88 & Starke & .724 & 30.660 \\
\hline 89 & Steuben & 152.313 & 59.843 \\
\hline 90 & Sullivan & 5.715 & .000 \\
\hline 91 & Switzerland & .000 & .000 \\
\hline 92 & Tippecanoe & 79.210 & 329.177 \\
\hline 93 & Tipton & 9.104 & 14.652 \\
\hline 94 & Union & .730 & .906 \\
\hline 95 & Vanderburgh & 207.772 & 99.637 \\
\hline 96 & Vermillion & 14.908 & .000 \\
\hline 97 & Vigo & 53.965 & 39.906 \\
\hline 98 & Wabash & 93.927 & 48.002 \\
\hline 99 & Warren & 9.391 & 16.454 \\
\hline 100 & Warrick & 20.661 & 6.308 \\
\hline 101 & Washington & 49.648 & 74.563 \\
\hline 102 & Wayne & 57.015 & 27.148 \\
\hline
\end{tabular}




\begin{tabular}{|c|c|c|c|}
\hline & $\begin{array}{l}\text { Locale } \\
\end{array}$ & P33 30 & A33 30 \\
\hline 103 & Wells & 51.358 & 30.994 \\
\hline 104 & White & 71.430 & 26.358 \\
\hline 105 & Whitley & 59.639 & 13.207 \\
\hline 106 & lowa & 2335.438 & 3477.917 \\
\hline 107 & Kansas & 3362.039 & 5135.113 \\
\hline 108 & Kentucky_E & 2253.429 & 2189.401 \\
\hline 109 & Kentucky_W & 1502.286 & 1459.601 \\
\hline 110 & Louisiana & 9997.498 & 14621.337 \\
\hline 111 & Maine & 594.259 & 467.610 \\
\hline 112 & Maryland & 2666.236 & 2655.081 \\
\hline 113 & Massachusetts & 4925.458 & 4290.224 \\
\hline 114 & Michigan_E & 5301.108 & 5997.150 \\
\hline 115 & Michigan_W & 5301.108 & 5997.150 \\
\hline 116 & Minnesota & 6865.992 & 7035.229 \\
\hline 117 & Mississippi & 2823.504 & 3682.580 \\
\hline 118 & Missouri & 5051.620 & 5012.651 \\
\hline$\overline{119}$ & Montana & 775.113 & 1143.677 \\
\hline 120 & Nebraska & 960.179 & 1094.771 \\
\hline 121 & Nevada & 1410.396 & 419.921 \\
\hline 122 & New Hampshire & 1514.196 & 1307.608 \\
\hline 123 & New Jersey & 6770.047 & 6794.941 \\
\hline 124 & New Mexico & 1001.820 & 1050.718 \\
\hline 125 & New York & 7481.254 & 7728.498 \\
\hline$\overline{126}$ & North Carolina & 7755.572 & 6619.976 \\
\hline 127 & North Dakota & 258.383 & 595.329 \\
\hline 128 & Ohio_N & 5473.943 & 5120.294 \\
\hline 129 & Ohio_M & 5473.943 & 5120.294 \\
\hline 130 & Ohio_s & 5473.943 & 5120.294 \\
\hline 131 & Oklahoma & 4519.495 & 5346.055 \\
\hline 132 & Oregon & 2927.947 & 2640.496 \\
\hline 133 & Pennsylvania & 14431.585 & 14138.270 \\
\hline 134 & Rhode Island & 890.538 & 344.538 \\
\hline 135 & South Carolina & 3894.998 & 3324.706 \\
\hline 136 & South Dakota & 333.720 & 468.754 \\
\hline 137 & Tennessee & 6409.584 & 6452.078 \\
\hline 138 & Texas & 38732.040 & 48195.221 \\
\hline 139 & Utah & 3006.076 & 3377.565 \\
\hline 140 & Vermont & 511.864 & 427.429 \\
\hline$\overline{141}$ & Virginia & 3801.231 & 3378.185 \\
\hline 142 & Washington & 4905.628 & 4841.642 \\
\hline 143 & West Virginia & 1105.808 & 703.577 \\
\hline 144 & Wisconsin & 7237.012 & 8832.422 \\
\hline 145 & Wyoming & 651.761 & 982.606 \\
\hline
\end{tabular}




\begin{tabular}{|c|c|c|c|}
\hline & Locale & P34 30 & A34 30 \\
\hline 1 & Alabama & 2429.183 & 2006.512 \\
\hline 2 & Arizona & 2144.232 & 2963.091 \\
\hline 3 & Arkansas & 2132.485 & 1639.316 \\
\hline 4 & California & 14888.014 & 14618.647 \\
\hline 5 & Colorado & 1342.352 & 1434.059 \\
\hline 6 & Connecticut & 2000.160 & 2924.690 \\
\hline 7 & Delaware & 452.036 & 579.817 \\
\hline 8 & DC & .000 & .000 \\
\hline 9 & Florida & 4675.566 & 4966.992 \\
\hline 10 & Georgia & 4518.346 & 4601.608 \\
\hline 11 & Idaho & 350.397 & 412.347 \\
\hline 12 & Illinois_N & 7076.340 & 5633.821 \\
\hline 13 & Illinois_s & 3538.170 & 2816.911 \\
\hline 14 & Adams & 110.612 & 98.788 \\
\hline 15 & Allen & 637.083 & 517.598 \\
\hline 16 & Bartholomew & 260.069 & 279.248 \\
\hline 17 & Benton & 6.259 & 2.448 \\
\hline 18 & Blackford & 22.625 & 3.191 \\
\hline 19 & Boone & 36.498 & 42.157 \\
\hline 20 & Brown & .000 & .611 \\
\hline 21 & Carroll & 3.205 & 4.318 \\
\hline 22 & Cass & 56.814 & 79.601 \\
\hline 23 & Clark & 78.110 & 71.564 \\
\hline 24 & Clay & 16.680 & 50.265 \\
\hline 25 & Clinton & 24.050 & 30.932 \\
\hline 26 & Crawford & .000 & .000 \\
\hline 27 & Daviess & 14.276 & 12.830 \\
\hline 28 & Dearborn & 14.569 & 7.832 \\
\hline 29 & Decatur & 49.187 & 56.069 \\
\hline 30 & DeKalb & 216.494 & 79.913 \\
\hline 31 & Delaware & 72.321 & 136.990 \\
\hline 32 & Dubois & 81.957 & 56.022 \\
\hline 33 & Elkhart & 542.546 & 785.013 \\
\hline 34 & Fayette & 29.027 & 104.959 \\
\hline 35 & Floyd & 150.531 & 49.998 \\
\hline 36 & Fountain & .000 & 18.732 \\
\hline 37 & Franklin & 17.903 & 14.346 \\
\hline 38 & Fulton & 27.104 & 16.677 \\
\hline 39 & Gibson & 39.458 & 13.461 \\
\hline 40 & Grant & 52.501 & 86.412 \\
\hline 41 & Greene & .000 & 1.426 \\
\hline 42 & Hamilton & 151.667 & 63.195 \\
\hline 43 & Hancock & 20.139 & 72.444 \\
\hline 44 & Harrison & 21.232 & 32.981 \\
\hline 45 & Hendricks & 32.013 & 32.359 \\
\hline 46 & Henry & 25.500 & 49.794 \\
\hline 47 & Howard & 48.153 & 450.401 \\
\hline 48 & Huntington & 49.625 & 85.245 \\
\hline 49 & Jackson & 66.188 & 98.259 \\
\hline 50 & Jasper & 4.910 & 5.162 \\
\hline 51 & Jay & 19.654 & 16.008 \\
\hline
\end{tabular}




\begin{tabular}{|c|c|c|c|}
\hline & Locale & P34 30 & A34 30 \\
\hline 52 & Jefferson & 53.503 & 81.905 \\
\hline 53 & Jennings & 73.070 & 37.237 \\
\hline 54 & Johnson & 140.801 & 102.455 \\
\hline 55 & Knox & 4.261 & 3.314 \\
\hline 56 & Kosciusko & 122.597 & 101.395 \\
\hline 57 & LaGrange & 94.151 & 66.071 \\
\hline 58 & Lake & 102.769 & 157.095 \\
\hline 59 & La Porte & 144.643 & 107.649 \\
\hline 60 & Lawrence & 32.246 & 55.468 \\
\hline 61 & Madison & 42.947 & 205.908 \\
\hline 62 & Marion & 547.765 & 1000.149 \\
\hline 63 & Marshall & 194.141 & 68.751 \\
\hline 64 & Martin & .426 & .359 \\
\hline 65 & Miami & 30.406 & 10.403 \\
\hline 66 & Monroe & 128.346 & 101.526 \\
\hline 67 & Montgomery & 62.841 & 50.807 \\
\hline 68 & Morgan & 27.181 & 40.256 \\
\hline 69 & Newton & 33.846 & 8.021 \\
\hline 70 & Noble & 172.133 & 113.730 \\
\hline 71 & Ohio & .000 & .000 \\
\hline 72 & Orange & 11.686 & .394 \\
\hline 73 & Owen & 1.305 & .464 \\
\hline 74 & Parke & 14.315 & 2.415 \\
\hline 75 & Perry & 7.550 & 6.358 \\
\hline 76 & Pike & .000 & .000 \\
\hline 77 & Porter & 48.562 & 40.142 \\
\hline 78 & Posey & 21.175 & 70.631 \\
\hline 79 & Pulaski & 24.948 & 21.269 \\
\hline 80 & Putnam & 93.729 & 27.666 \\
\hline 81 & Randolph & 27.693 & 37.064 \\
\hline 82 & Ripley & .743 & 2.650 \\
\hline 83 & Rush & 25.915 & 20.147 \\
\hline 84 & St. Joseph & 282.026 & 209.926 \\
\hline 85 & Scott & 79.341 & 10.904 \\
\hline 86 & Shelby & 153.798 & 43.158 \\
\hline 87 & Spencer & 31.957 & 11.288 \\
\hline 88 & Starke & 27.945 & 23.107 \\
\hline 89 & Steuben & 73.528 & 92.358 \\
\hline 90 & Sullivan & 12.437 & 4.971 \\
\hline 91 & Switzerland & 16.640 & .000 \\
\hline 92 & Tippecanoe & 229.078 & 418.700 \\
\hline 93 & Tipton & 8.150 & 16.026 \\
\hline 94 & Union & .504 & 2.007 \\
\hline 95 & Vanderburgh & 290.060 & 142.493 \\
\hline 96 & Vermillion & .000 & 54.556 \\
\hline 97 & \begin{tabular}{|l} 
Vigo \\
\end{tabular} & 138.190 & 66.061 \\
\hline 98 & Wabash & 31.888 & 39.541 \\
\hline 99 & Warren & 9.868 & 9.292 \\
\hline 100 & Warrick & 4.309 & 11.538 \\
\hline 101 & Washington & 58.427 & 35.203 \\
\hline 102 & Wayne & 79.812 & 44.335 \\
\hline
\end{tabular}




\begin{tabular}{|c|c|c|c|}
\hline & Locale & P34 30 & A34 30 \\
\hline 103 & Wells & 76.817 & 25.661 \\
\hline 104 & White & 38.616 & 55.698 \\
\hline 105 & Whitley & 43.601 & 42.934 \\
\hline 106 & lowa & 2885.927 & 2235.545 \\
\hline 107 & Kansas & 2038.477 & 2560.414 \\
\hline 108 & Kentucky_E & 1556.116 & 1676.947 \\
\hline 109 & Kentucky_W & 1037.411 & 1117.964 \\
\hline 110 & Louisiana & 786.119 & 2034.090 \\
\hline 111 & Maine & 350.865 & 651.908 \\
\hline 112 & Maryland & 1328.937 & 1521.021 \\
\hline 113 & Massachusetts & 3372.242 & 2625.601 \\
\hline 114 & Michigan_E & 5030.229 & 6252.790 \\
\hline 115 & Michigan_W & 5030.229 & 6252.790 \\
\hline 116 & Minnesota & 4121.141 & 2943.426 \\
\hline 117 & Mississippi & 1635.159 & 1743.711 \\
\hline 118 & Missouri & 3440.964 & 4402.591 \\
\hline 119 & Montana & 114.028 & 121.808 \\
\hline 120 & Nebraska & 1047.187 & 813.415 \\
\hline 121 & Nevada & 691.003 & 318.834 \\
\hline 122 & New Hampshire & 1180.150 & 690.152 \\
\hline 123 & New Jersey & 3823.353 & 4227.415 \\
\hline 124 & New Mexico & 111.146 & 179.299 \\
\hline 125 & New York & 5852.230 & 6138.129 \\
\hline 126 & North Carolina & 7577.055 & 6103.923 \\
\hline 127 & North Dakota & 289.765 & 268.177 \\
\hline 128 & Ohio $N$ & 3819.852 & 3428.914 \\
\hline 129 & Ohio M & 3819.852 & 3428.914 \\
\hline 130 & Ohio_s & 3819.852 & 3428.914 \\
\hline 131 & Oklahoma & 2309.299 & 1824.944 \\
\hline 132 & Oregon & 1525.754 & 1515.026 \\
\hline 133 & Pennsylvania & 6795.036 & 5514.900 \\
\hline 134 & Rhode Island & 592.302 & 300.405 \\
\hline 135 & South Carolina & 3496.396 & 3748.891 \\
\hline 136 & South Dakota & 365.680 & 299.778 \\
\hline 137 & Tennessee & 5501.976 & 5458.178 \\
\hline 138 & Texas & 12225.510 & 12747.132 \\
\hline 139 & Utah & 909.258 & 1305.816 \\
\hline 140 & Vermont & 350.540 & 250.920 \\
\hline 141 & Virginia & 3335.833 & 3326.143 \\
\hline 142 & Washington & 2100.987 & 4436.669 \\
\hline 143 & West Virginia & 350.791 & 685.622 \\
\hline 144 & Wisconsin & 7091.569 & 5594.075 \\
\hline 145 & Wyoming & 58.897 & 86.330 \\
\hline
\end{tabular}




\begin{tabular}{|c|c|c|c|}
\hline & Locale & P35 30 & A35 30 \\
\hline 1 & Alabama & 12384.474 & 12514.963 \\
\hline 2 & Arizona & 13210.819 & 15032.339 \\
\hline 3 & Arkansas & 8972.317 & 7791.525 \\
\hline 4 & California & 93084.008 & 91219.377 \\
\hline 5 & Colorado & 6515.426 & 8147.020 \\
\hline 6 & Connecticut & 13200.693 & 6583.764 \\
\hline 7 & Delaware & 2187.730 & 3277.077 \\
\hline 8 & $\overline{D C}$ & .000 & .000 \\
\hline 9 & Florida & 21417.114 & 38803.102 \\
\hline 10 & Georgia & 14911.318 & 21302.591 \\
\hline 11 & Idaho & 1978.265 & 2786.594 \\
\hline 12 & Illinois_N & 30697.608 & 23003.648 \\
\hline 13 & Illinois_s & 15348.804 & 11501.824 \\
\hline 14 & Adams & 148.202 & 271.274 \\
\hline 15 & Allen & 1083.412 & 1424.550 \\
\hline 16 & Bartholomew & 345.413 & 250.645 \\
\hline 17 & Benton & 18.344 & 18.214 \\
\hline 18 & Blackford & 145.486 & 65.816 \\
\hline 19 & Boone & 138.004 & 90.333 \\
\hline 20 & Brown & 16.929 & 19.020 \\
\hline 21 & Carroll & 131.663 & 24.174 \\
\hline 22 & Cass & 188.695 & 169.279 \\
\hline 23 & Clark & 509.817 & 360.279 \\
\hline 24 & Clay & 31.297 & 62.149 \\
\hline 25 & Clinton & 76.550 & 75.079 \\
\hline 26 & Crawford & .000 & 15.466 \\
\hline 27 & Daviess & 63.209 & 62.231 \\
\hline 28 & Dearborn & 109.077 & 114.934 \\
\hline 29 & Decatur & 832.375 & 97.163 \\
\hline 30 & DeKalb & 1125.380 & 513.231 \\
\hline 31 & Delaware & 563.394 & 193.608 \\
\hline 32 & Dubois & 47.291 & 245.945 \\
\hline 33 & Elkhart & 2083.091 & 1448.181 \\
\hline 34 & Fayette & 90.924 & 27.621 \\
\hline 35 & Floyd & 205.926 & 398.516 \\
\hline 36 & Fountain & 16.603 & 20.081 \\
\hline 37 & Franklin & 92.486 & 152.594 \\
\hline 38 & Fulton & 240.627 & 49.278 \\
\hline 39 & Gibson & 43.306 & 114.811 \\
\hline 40 & Grant & 37.745 & 144.631 \\
\hline 41 & Greene & 24.953 & 39.992 \\
\hline 42 & Hamilton & 575.576 & 817.998 \\
\hline 43 & Hancock & 204.704 & 109.774 \\
\hline 44 & Harrison & 135.572 & 203.238 \\
\hline$\overline{45}$ & Hendricks & 116.134 & 276.176 \\
\hline 46 & Henry & 82.865 & 76.172 \\
\hline 47 & Howard & 58.784 & 181.299 \\
\hline 48 & Huntington & 342.861 & 130.902 \\
\hline 49 & Jackson & 199.334 & 130.968 \\
\hline 50 & Jasper & 205.271 & 50.327 \\
\hline 51 & Jay & 251.015 & 60.960 \\
\hline
\end{tabular}




\begin{tabular}{|c|c|c|c|}
\hline & Locale & P35 30 & A35 30 \\
\hline 52 & Jefferson & 43.186 & 75.570 \\
\hline 53 & Jennings & 302.215 & 267.413 \\
\hline 54 & Johnson & 499.620 & 442.745 \\
\hline 55 & Knox & 38.756 & 54.933 \\
\hline 56 & Kosciusko & 450.162 & 302.482 \\
\hline 57 & LaGrange & 72.674 & 212.455 \\
\hline 58 & Lake & 3320.990 & 3209.977 \\
\hline 59 & La Porte & 612.346 & 273.699 \\
\hline 60 & Lawrence & 217.778 & 155.381 \\
\hline 61 & Madison & 301.359 & 223.994 \\
\hline 62 & Marion & 3127.761 & 1516.542 \\
\hline 63 & Marshall & 420.187 & 618.702 \\
\hline 64 & Martin & 2.431 & 11.061 \\
\hline 65 & Miami & 63.946 & 93.915 \\
\hline 66 & Monroe & 223.354 & 325.988 \\
\hline 67 & Montgomery & 191.436 & 184.452 \\
\hline 68 & Morgan & 124.821 & 131.241 \\
\hline 69 & Newton & 16.580 & 92.804 \\
\hline 70 & Noble & 438.585 & 436.385 \\
\hline 71 & \begin{tabular}{|l|} 
Ohio \\
\end{tabular} & 3.235 & 6.891 \\
\hline 72 & Orange & 16.035 & 52.889 \\
\hline 73 & Owen & 4.085 & 31.330 \\
\hline 74 & Parke & 2.727 & 48.525 \\
\hline 75 & Perry & 14.769 & 20.655 \\
\hline 76 & Pike & 17.835 & 15.585 \\
\hline 77 & Porter & 463.341 & 243.344 \\
\hline 78 & Posey & 513.675 & 561.869 \\
\hline 79 & Pulaski & 51.891 & 16.134 \\
\hline 80 & Putnam & 2.999 & 263.912 \\
\hline 81 & Randolph & 39.119 & 49.528 \\
\hline 82 & Ripley & 78.494 & 49.838 \\
\hline 83 & Rush & 14.758 & 27.615 \\
\hline 84 & St. Joseph & 1321.195 & 879.244 \\
\hline 85 & Scott & 39.237 & 237.912 \\
\hline 86 & Shelby & 102.128 & 396.568 \\
\hline 87 & Spencer & 15.896 & 84.203 \\
\hline 88 & Starke & '.000 & 54.876 \\
\hline 89 & Steuben & 415.102 & 174.010 \\
\hline 90 & Sullivan & 2.963 & 58.413 \\
\hline 91 & Switzerland & .000 & 55.467 \\
\hline 92 & Tippecanoe & 242.557 & 384.795 \\
\hline 93 & Tipton & 32.947 & 18.299 \\
\hline 94 & Union & 2.877 & 8.397 \\
\hline 95 & Vanderburgh & 600.239 & 810.191 \\
\hline 96 & Vermillion & 42.651 & 17.751 \\
\hline 97 & Vigo & 192.402 & 415.061 \\
\hline 98 & Wabash & 267.286 & 76.978 \\
\hline 99 & Warren & 36.999 & 11.141 \\
\hline 100 & Warrick & 77.733 & 82.973 \\
\hline 101 & Washington & 192.139 & 79.857 \\
\hline 102 & Wayne & 150.118 & 237.797 \\
\hline
\end{tabular}




\begin{tabular}{|c|c|c|c|}
\hline & Locale & P35 30 & A35 30 \\
\hline 103 & Wells & 202.349 & 188.688 \\
\hline 104 & White & 262.967 & 91.038 \\
\hline 105 & Whitley & 231.344 & 136.127 \\
\hline 106 & lowa & 7104.372 & 6208.752 \\
\hline 107 & Kansas & 7782.554 & 9080.982 \\
\hline 108 & Kentucky_E & 5655.956 & 6145.990 \\
\hline 109 & Kentucky_W & 3770.638 & 4097.327 \\
\hline 110 & Louisiana & 21563.658 & 21423.998 \\
\hline 111 & Maine & 1693.538 & 2314.580 \\
\hline 112 & Maryland & 6559.228 & 11136.019 \\
\hline 113 & Massachusetts & 15351.247 & 13353.441 \\
\hline 114 & Michigan_E & 16442.345 & 13178.773 \\
\hline 115 & Michigan_W & 16442.345 & 13178.773 \\
\hline 116 & Minnesota & 19856.642 & 13915.396 \\
\hline 117 & Mississippi & 6803.816 & 7835.477 \\
\hline 118 & Missouri & 14361.561 & 13047.317 \\
\hline 119 & Montana & 1515.707 & 2287.318 \\
\hline 120 & Nebraska & 2895.223 & 3185.650 \\
\hline 121 & Nevada & 2904.830 & 5799.108 \\
\hline 122 & New Hampshire & 4874.674 & 3492.220 \\
\hline 123 & New Jersey & 16954.773 & 21687.897 \\
\hline 124 & New Mexico & 1972.801 & 3452.309 \\
\hline 125 & New York & 21372.238 & 29994.857 \\
\hline 126 & North Carolina & 19048.543 & 25899.225 \\
\hline 127 & North Dakota & 688.488 & 1022.200 \\
\hline 128 & Ohio__N & 15448.382 & 11808.843 \\
\hline 129 & Ohio_M & 15448.382 & 11808.843 \\
\hline 130 & Ohio_s & 15448.382 & 11808.843 \\
\hline 131 & Oklahoma & 11743.635 & 10166.009 \\
\hline 132 & Oregon & 8416.380 & 8089.622 \\
\hline 133 & Pennsylvania & 37634.792 & 31298.785 \\
\hline 134 & Rhode Island & 3152.571 & 2333.726 \\
\hline 135 & South Carolina & 11126.098 & 11090.385 \\
\hline 136 & South Dakota & 984.322 & 1192.466 \\
\hline 137 & Tennessee & 17512.691 & 17534.026 \\
\hline 138 & Texas & 92718.639 & 90297.437 \\
\hline 139 & Utah & 7233.090 & 7512.100 \\
\hline 140 & Vermont & 1212.581 & 1264.552 \\
\hline 141 & Virginia & 9076.950 & 16922.866 \\
\hline 142 & Washington & 12348.495 & 15688.663 \\
\hline 143 & West Virginia & 2231.282 & 2841.153 \\
\hline 144 & Wisconsin & 24460.019 & 13677.435 \\
\hline 145 & Wyoming & 1286.760 & 1650.064 \\
\hline
\end{tabular}




\begin{tabular}{|c|c|c|c|}
\hline & Locale & P36 30 & A36 30 \\
\hline 1 & Alabama & 5108.025 & 5108.025 \\
\hline 2 & Arizona & 11925.032 & 11925.032 \\
\hline 3 & Arkansas & 3306.203 & 3306.203 \\
\hline 4 & California & 42034.625 & 42034.625 \\
\hline 5 & Colorado & 3063.655 & 3063.655 \\
\hline 6 & Connecticut & 9251.699 & 9251.699 \\
\hline 7 & Delaware & 1371.870 & 1371.870 \\
\hline 8 & DC & .000 & .000 \\
\hline 9 & Florida & 12649.158 & 12649.158 \\
\hline 10 & Georgia & 12885.099 & 12885.099 \\
\hline 11 & Idaho & 532.315 & 532.315 \\
\hline 12 & Illinois $\mathbf{N}$ & 7491.702 & 7491.702 \\
\hline 13 & Illinois_s & 3745.851 & 3745.851 \\
\hline 14 & Adams & 491.640 & 491.640 \\
\hline 15 & Allen & 1803.550 & 1803.550 \\
\hline 16 & Bartholomew & 740.661 & 740.661 \\
\hline 17 & Benton & 1.618 & 1.618 \\
\hline 18 & Blackford & 8.702 & 8.702 \\
\hline 19 & Boone & 89.746 & 89.746 \\
\hline 20 & Brown & 1.817 & 1.817 \\
\hline 21 & Carroll & 11.776 & 11.776 \\
\hline 22 & Cass & 523.304 & 523.304 \\
\hline 23 & Clark & 12.988 & 12.988 \\
\hline 24 & Clay & 320.631 & 320.631 \\
\hline 25 & Clinton & 106.189 & 106.189 \\
\hline 26 & Crawford & .000 & .000 \\
\hline 27 & Daviess & 16.116 & 16.116 \\
\hline 28 & Dearborn & .000 & .000 \\
\hline 29 & Decatur & 267.349 & 267.349 \\
\hline 30 & DeKalb & 268.248 & 268.248 \\
\hline 31 & Delaware & 637.224 & 637.224 \\
\hline 32 & Dubois & 350.618 & 350.618 \\
\hline 33 & Elkhart & 4651.830 & 4651.830 \\
\hline 34 & Fayette & 585.514 & 585.514 \\
\hline 35 & Floyd & 113.119 & 113.119 \\
\hline 36 & Fountain & 133.644 & 133.644 \\
\hline 37 & Franklin & 82.359 & 82.359 \\
\hline 38 & Fulton & 10.951 & 10.951 \\
\hline 39 & Gibson & 36.972 & 36.972 \\
\hline 40 & Grant & 459.706 & 459.706 \\
\hline 41 & Greene & 10.367 & 10.367 \\
\hline 42 & Hamilton & 292.935 & 292.935 \\
\hline 43 & Hancock & 415.278 & 415.278 \\
\hline 44 & Harrison & 195.350 & 195.350 \\
\hline 45 & Hendricks & 84.831 & 84.831 \\
\hline 46 & Henry & 266.809 & 266.809 \\
\hline 47 & Howard & 3169.613 & 3169.613 \\
\hline 48 & Huntington & 451.498 & 451.498 \\
\hline 49 & Jackson & 455.493 & 455.493 \\
\hline 50 & Jasper & 34.270 & 34.270 \\
\hline 51 & Jay & 61.196 & 61.196 \\
\hline
\end{tabular}




\begin{tabular}{|c|c|c|c|}
\hline & Locale & P36 30 & A36 30 \\
\hline 52 & Jefferson & 330.515 & 330.515 \\
\hline 53 & Jennings & 162.327 & 162.327 \\
\hline 54 & Johnson & 378.440 & 378.440 \\
\hline 55 & Knox & 1.555 & 1.555 \\
\hline 56 & Kosciusko & 356.986 & 356.986 \\
\hline 57 & LaGrange & 276.618 & 276.618 \\
\hline 58 & Lake & 356.100 & 356.100 \\
\hline 59 & La Porte & 132.887 & 132.887 \\
\hline 60 & Lawrence & 293.502 & 293.502 \\
\hline 61 & Madison & 1274.134 & 1274.134 \\
\hline 62 & Marion & 2940.654 & 2940.654 \\
\hline 63 & Marshall & 406.449 & 406.449 \\
\hline 64 & Martin & .000 & .000 \\
\hline 65 & Miami & 9.359 & 9.359 \\
\hline 66 & Monroe & 38.833 & 38.833 \\
\hline 67 & Montgomery & 264.602 & 264.602 \\
\hline 68 & Morgan & 43.415 & 43.415 \\
\hline 69 & Newton & 31.141 & 31.141 \\
\hline 70 & Noble & 609.273 & 609.273 \\
\hline 71 & Ohio & .000 & .000 \\
\hline 72 & Orange & .000 & .000 \\
\hline 73 & Owen & .000 & .000 \\
\hline 74 & Parke & .000 & .000 \\
\hline 75 & Perry & .000 & .000 \\
\hline 76 & Pike & .000 & .000 \\
\hline 77 & Porter & 1.926 & 1.926 \\
\hline 78 & Posey & .000 & .000 \\
\hline 79 & Pulaski & 1.909 & 1.909 \\
\hline 80 & Putnam & 144.855 & 144.855 \\
\hline 81 & Randolph & 122.264 & 122.264 \\
\hline 82 & Ripley & 1.994 & 1.994 \\
\hline 83 & Rush & 9.503 & 9.503 \\
\hline 84 & St. Joseph & 792.637 & 792.637 \\
\hline 85 & Scott & 11.526 & 11.526 \\
\hline 86 & Shelby & 146.764 & 146.764 \\
\hline 87 & Spencer & 29.857 & 29.857 \\
\hline 88 & Starke & 49.122 & 49.122 \\
\hline 89 & Steuben & 506.719 & 506.719 \\
\hline 90 & Sullivan & 33.390 & 33.390 \\
\hline 91 & Switzerland & .000 & .000 \\
\hline 92 & Tippecanoe & 1404.626 & 1404.626 \\
\hline 93 & Tipton & 64.162 & 64.162 \\
\hline 94 & Union & .000 & .000 \\
\hline 95 & Vanderburgh & 16.281 & 16.281 \\
\hline 96 & Vermillion & .000 & .000 \\
\hline 97 & Vigo & 69.346 & 69.346 \\
\hline 98 & Wabash & 69.106 & 69.106 \\
\hline 99 & Warren & 11.529 & 11.529 \\
\hline 100 & Warrick & 37.596 & 37.596 \\
\hline 101 & Washington & 2.032 & 2.032 \\
\hline 102 & Wayne & 199.419 & 199.419 \\
\hline
\end{tabular}




\begin{tabular}{|c|c|c|c|}
\hline & Locale & P36 30 & A36 30 \\
\hline 103 & Wells & 81.040 & 81.040 \\
\hline 104 & White & 315.259 & 315.259 \\
\hline 105 & Whitley & 264.161 & 264.161 \\
\hline 106 & lowa & 3265.280 & 3265.280 \\
\hline 107 & Kansas & 9980.936 & 9980.936 \\
\hline 108 & Kentucky_E & 5352.653 & 5352.653 \\
\hline 109 & Kentucky W & 3568.036 & 3568.036 \\
\hline 110 & Louisiana & 4560.484 & 4560.484 \\
\hline 111 & Maine & 3346.605 & 3346.605 \\
\hline 112 & Maryland & 2381.258 & 2381.258 \\
\hline 113 & Massachusetts & 2500.697 & 2500.697 \\
\hline 114 & Michigan_E & 24364.134 & 24364.134 \\
\hline 115 & Michigan_W & 24364.134 & 24364.134 \\
\hline 116 & Minnesota & 3301.180 & 3301.180 \\
\hline 117 & Mississippi & 5518.388 & 5518.388 \\
\hline 118 & Missouri & 14244.953 & 14244.953 \\
\hline 119 & Montana & 150.082 & 150.082 \\
\hline 120 & Nebraska & 1475.416 & 1475.416 \\
\hline 121 & Nevada & 647.944 & 647.944 \\
\hline 122 & New Hampshire & 675.405 & 675.405 \\
\hline 123 & New Jersey & 2188.751 & 2188.751 \\
\hline 124 & New Mexico & 483.837 & 483.837 \\
\hline 125 & New York & 8013.700 & 8013.700 \\
\hline 126 & North Carolina & 8867.047 & 8867.047 \\
\hline 127 & North Dakota & 490.395 & 490.395 \\
\hline 128 & Ohio $\mathbf{N}$ & 10153.166 & 10153.166 \\
\hline 129 & Ohio M & 10153.166 & 10153.166 \\
\hline 130 & Ohio_s & 10153.166 & 10153.166 \\
\hline 131 & Oklahoma & 4136.357 & 4136.357 \\
\hline 132 & Oregon & 4020.035 & 4020.035 \\
\hline 133 & Pennsylvania & 9629.425 & 9629.425 \\
\hline 134 & Rhode Island & 332.541 & 332.541 \\
\hline 135 & South Carolina & 5495.272 & 5495.272 \\
\hline 136 & South Dakota & 429.655 & 429.655 \\
\hline 137 & Tennessee & 12706.572 & 12706.572 \\
\hline 138 & Texas & 21287.770 & 21287.770 \\
\hline 139 & Utah & 4201.610 & 4201.610 \\
\hline 140 & Vermont & 506.469 & 506.469 \\
\hline 141 & Virginia & 9148.639 & 9148.639 \\
\hline 142 & Washington & 24326.006 & 24326.006 \\
\hline 143 & West Virginia & 591.771 & 591.771 \\
\hline 144 & Wisconsin & 8625.785 & 8625.785 \\
\hline 145 & Wyoming & 68.624 & 68.624 \\
\hline
\end{tabular}




\begin{tabular}{|c|c|c|c|}
\hline & Locale & P37 30 & A37 30 \\
\hline 1 & Alabama & 365.453 & 214.694 \\
\hline 2 & Arizona & 207.716 & 57.614 \\
\hline 3 & Arkansas & 163.456 & 95.660 \\
\hline 4 & California & 512.236 & 1339.531 \\
\hline 5 & Colorado & 43.135 & 49.071 \\
\hline 6 & Connecticut & 91.078 & 37.093 \\
\hline 7 & Delaware & 14.451 & 81.774 \\
\hline 8 & $\mathrm{DC}$ & .000 & .000 \\
\hline 9 & Florida & 127.101 & 128.905 \\
\hline 10 & Georgia & 140.177 & 123.967 \\
\hline 11 & Idaho & 4.129 & 20.138 \\
\hline 12 & Illinois $\mathbf{N}$ & 404.237 & 321.575 \\
\hline 13 & Illinois_S & 202.119 & 160.787 \\
\hline 14 & Adams & 3.137 & .939 \\
\hline 15 & Allen & 43.972 & 5.767 \\
\hline 16 & \begin{tabular}{|l|} 
Bartholomew \\
\end{tabular} & 12.607 & .000 \\
\hline 17 & Benton & .811 & .000 \\
\hline 18 & Blackford & .000 & .000 \\
\hline 19 & Boone & .000 & .000 \\
\hline 20 & Brown & .000 & .000 \\
\hline 21 & Carroll & .000 & .000 \\
\hline 22 & Cass & .000 & .000 \\
\hline 23 & Clark & 2.332 & 9.566 \\
\hline 24 & Clay & .153 & .000 \\
\hline 25 & Clinton & .000 & .000 \\
\hline 26 & Crawford & .000 & .000 \\
\hline 27 & Daviess & .000 & .893 \\
\hline 28 & Dearborn & .000 & 1.153 \\
\hline 29 & Decatur & .157 & .000 \\
\hline 30 & DeKalb & 44.745 & .000 \\
\hline 31 & Delaware & 11.579 & .000 \\
\hline 32 & Dubois & .000 & .000 \\
\hline 33 & Elkhart & 21.401 & .933 \\
\hline 34 & Fayette & .782 & .000 \\
\hline 35 & Floyd & .177 & .000 \\
\hline 36 & Fountain & 11.160 & .000 \\
\hline 37 & Franklin & .000 & 5.766 \\
\hline 38 & Fulton & 5.715 & .000 \\
\hline 39 & Gibson & .000 & .000 \\
\hline 40 & Grant & 2.053 & .000 \\
\hline 41 & Greene & 2.525 & .000 \\
\hline 42 & Hamilton & 11.535 & 1.325 \\
\hline 43 & Hancock & .240 & .000 \\
\hline 44 & Harrison & .000 & 6.838 \\
\hline 45 & Hendricks & 5.437 & .000 \\
\hline 46 & Henry & 4.774 & .000 \\
\hline 47 & Howard & 11.344 & .000 \\
\hline 48 & Huntington & 2.983 & .925 \\
\hline 49 & Jackson & 7.679 & .000 \\
\hline 50 & Jasper & .000 & .000 \\
\hline 51 & Jay & .000 & .714 \\
\hline
\end{tabular}




\begin{tabular}{|c|c|c|c|}
\hline & $\begin{array}{l}\text { Locale } \\
\end{array}$ & P37 30 & A37 30 \\
\hline 52 & Jefferson & .167 & .000 \\
\hline 53 & Jennings & 1.084 & 5.682 \\
\hline 54 & Johnson & 7.477 & .000 \\
\hline 55 & Knox & 4.869 & .680 \\
\hline 56 & Kosciusko & 28.633 & .857 \\
\hline 57 & LaGrange & .183 & .000 \\
\hline 58 & Lake & 313.437 & 169.385 \\
\hline 59 & La Porte & 23.467 & .775 \\
\hline 60 & Lawrence & 24.510 & 4.403 \\
\hline 61 & Madison & 3.558 & .000 \\
\hline 62 & Marion & 57.846 & 9.882 \\
\hline 63 & Marshall & 6.711 & 5.523 \\
\hline 64 & Martin & 2.287 & .000 \\
\hline 65 & Miami & .000 & .000 \\
\hline 66 & Monroe & .000 & 5.826 \\
\hline 67 & Montgomery & 12.026 & .000 \\
\hline 68 & Morgan & 1.243 & .000 \\
\hline 69 & Newton & 2.600 & .000 \\
\hline 70 & Noble & 12.593 & .000 \\
\hline 71 & Ohio & .000 & .000 \\
\hline 72 & Orange & .000 & .000 \\
\hline 73 & Owen & .000 & .000 \\
\hline 74 & Parke & .000 & .000 \\
\hline 75 & Perry & 4.964 & .000 \\
\hline 76 & Pike & .959 & .000 \\
\hline 77 & Porter & 131.096 & .000 \\
\hline 78 & Posey & .000 & 32.071 \\
\hline 79 & Pulaski & 2.790 & .000 \\
\hline 80 & Putnam & .000 & .000 \\
\hline 81 & Randolph & 4.152 & .713 \\
\hline 82 & Ripley & .000 & .872 \\
\hline 83 & Rush & 4.960 & .000 \\
\hline 84 & St. Joseph & 27.936 & .838 \\
\hline 85 & Scott & .160 & .841 \\
\hline 86 & Shelby & 8.006 & .000 \\
\hline 87. & Spencer & 2.493 & .000 \\
\hline 88 & Starke & .000 & .000 \\
\hline 89 & Steuben & 7.991 & .836 \\
\hline 90 & Sullivan & .000 & .000 \\
\hline 91 & Switzerland & .000 & .000 \\
\hline 92 & Tippecanoe & 34.981 & 1.047 \\
\hline 93 & Tipton & .857 & .000 \\
\hline 94 & Union & .000 & .000 \\
\hline 95 & Vanderburgh & 2.937 & .000 \\
\hline 96 & Vermillion & .000 & .000 \\
\hline 97 & Vigo & 5.791 & .000 \\
\hline 98 & Wabash & 10.184 & .711 \\
\hline 99 & Warren & .000 & .000 \\
\hline 100 & Warrick & 67.277 & .000 \\
\hline 101 & Washington & .170 & .000 \\
\hline 102 & Wayne & 11.639 & .000 \\
\hline
\end{tabular}




\begin{tabular}{|c|c|c|c|}
\hline & Locale & P37 30 & A37 30 \\
\hline 103 & Wells & 3.117 & .000 \\
\hline 104 & White & .903 & .000 \\
\hline 105 & Whitley & 10.772 & .000 \\
\hline 106 & lowa & 143.827 & 13.360 \\
\hline 107 & Kansas & 43.598 & 229.098 \\
\hline 108 & Kentucky E & 150.950 & 65.127 \\
\hline 109 & Kentucky_W & 100.634 & 43.418 \\
\hline 110 & Louisiana & 60.612 & 1009.624 \\
\hline 111 & Maine & 11.977 & 14.643 \\
\hline 112 & Maryland & 141.541 & 108.652 \\
\hline 113 & Massachusetts & 95.020 & 87.668 \\
\hline 114 & Michigan_E & 289.079 & 82.773 \\
\hline 115 & Michigan_W & 289.079 & 82.773 \\
\hline 116 & Minnesota & 140.047 & 187.035 \\
\hline 117 & Mississippi & 51.510 & 158.451 \\
\hline 118 & Missouri & 212.327 & 122.503 \\
\hline 119 & Montana & 20.253 & 71.097 \\
\hline 120 & Nebraska & 18.033 & .804 \\
\hline 121 & Nevada & 45.358 & 9.720 \\
\hline 122 & New Hampshire & 68.704 & 15.511 \\
\hline 123 & New Jersey & 171.045 & 310.364 \\
\hline 124 & New Mexico & 29.146 & 67.717 \\
\hline 125 & New York & 261.429 & 123.843 \\
\hline 126 & North Carolina & 172.782 & 79.243 \\
\hline 127 & North Dakota & 1.703 & 12.496 \\
\hline 128 & Ohio $N$ & 364.903 & 144.496 \\
\hline 129 & Ohio_M & 364.903 & 144.496 \\
\hline 130 & Ohio_s & 364.903 & 144.496 \\
\hline 131 & Oklahoma & 87.704 & 218.110 \\
\hline 132 & Oregon & 221.383 & 76.584 \\
\hline 133 & Pennsylvania & 935.252 & 568.269 \\
\hline 134 & Rhode Island & 54.365 & .831 \\
\hline 135 & South Carolina & 130.055 & 36.381 \\
\hline 136 & South Dakota & 9.440 & .802 \\
\hline 137 & Tennessee & 319.401 & 131.715 \\
\hline 138 & Texas & 672.493 & 2661.141 \\
\hline 139 & Utah & 153.961 & 180.762 \\
\hline 140 & Vermont & 4.538 & 5.304 \\
\hline 141 & Virginia & 133.919 & 70.515 \\
\hline 142 & Washington & 276.327 & 225.342 \\
\hline 143 & West Virginia & 154.839 & 28.402 \\
\hline 144 & Wisconsin & 444.948 & 36.238 \\
\hline 145 & Wyoming & 1.910 & 66.939 \\
\hline
\end{tabular}




\begin{tabular}{|c|c|c|c|}
\hline & Locale & P38 30 & A38 30 \\
\hline 1 & Alabama & 88.197 & 106.228 \\
\hline 2 & Arizoná & 185.876 & 155.598 \\
\hline 3 & Arkansas & 45.831 & 53.028 \\
\hline$\overline{4}$ & California & 1077.907 & 986.075 \\
\hline 5 & Colorado & 133.502 & 115.692 \\
\hline$\overline{6}$ & Connecticut & 76.364 & 72.535 \\
\hline$\overline{7}$ & Delaware & 14.746 & 17.995 \\
\hline$\overline{8}$ & DC & .000 & .000 \\
\hline 9 & Florida & 526.688 & 489.694 \\
\hline 10 & Georgia & 301.213 & 494.279 \\
\hline 11 & Idaho & 29.424 & 32.277 \\
\hline 12 & Illinois_N & 245.720 & 211.941 \\
\hline 13 & Illinois_S & 122.860 & 105.970 \\
\hline 14 & Adams & .284 & .347 \\
\hline 15 & Allen & 16.158 & 13.507 \\
\hline 16 & Bartholomew & 1.403 & .955 \\
\hline 17 & Benton & .042 & .131 \\
\hline 18 & Blackford & .094 & .087 \\
\hline 19 & Boone & 1.051 & .885 \\
\hline 20 & Brown & .018 & .018 \\
\hline 21 & Carroll & .082 & .210 \\
\hline 22 & Cass & .467 & .421 \\
\hline 23 & Clark & 2.366 & 1.864 \\
\hline 24 & Clay & .050 & .088 \\
\hline 25 & Clinton & .278 & .279 \\
\hline 26 & Crawford & .023 & .011 \\
\hline 27 & Daviess & .192 & .255 \\
\hline 28 & Dearborn & .415 & .326 \\
\hline 29 & Decatur & .217 & .399 \\
\hline 30 & DeKalb & .655 & .464 \\
\hline 31 & Delaware & 1.930 & 1.442 \\
\hline 32 & Dubois & 2.260 & 1.551 \\
\hline 33 & Elkhart & 9.060 & 8.025 \\
\hline 34 & Fayette & .127 & .186 \\
\hline 35 & Floyd & 1.249 & 1.075 \\
\hline 36 & Fountain & .043 & .114 \\
\hline 37 & Franklin & .128 & .129 \\
\hline 38 & Fulton & .083 & .151 \\
\hline 39 & Gibson & .203 & .196 \\
\hline 40 & Grant & .662 & .679 \\
\hline 41 & Greene & .144 & .176 \\
\hline 42 & Hamilton & 9.329 & 8.014 \\
\hline 43 & Hancock & .483 & .797 \\
\hline 44 & Harrison & .463 & .337 \\
\hline 45 & Hendricks & 2.983 & 2.056 \\
\hline 46 & Henry & .319 & .600 \\
\hline 47 & Howard & 1.086 & .715 \\
\hline 48 & Huntington & .460 & .526 \\
\hline 49 & Jackson & .459 & .536 \\
\hline 50 & Jasper & .232 & .238 \\
\hline 51 & Jay & .097 & .201 \\
\hline
\end{tabular}

F111 


\begin{tabular}{|c|c|c|c|}
\hline & $\begin{array}{l}\text { Locale } \\
\end{array}$ & P38 30 & A38 30 \\
\hline 52 & Jefferson & .313 & .248 \\
\hline 53 & Jennings & .147 & 1.849 \\
\hline 54 & Johnson & 1.679 & 1.201 \\
\hline 55 & Knox & .551 & .589 \\
\hline 56 & Kosciusko & .969 & .971 \\
\hline 57 & LaGrange & .517 & .591 \\
\hline 58 & Lake & 9.071 & 6.388 \\
\hline 59 & La Porte & 1.427 & 1.411 \\
\hline 60 & Lawrence & .261 & .223 \\
\hline 61 & Madison & 1.029 & .970 \\
\hline 62 & Marion & 40.461 & 32.070 \\
\hline 63 & Marshall & 1.000 & .975 \\
\hline 64 & Martin & .033 & .064 \\
\hline 65 & Miami & .296 & .516 \\
\hline 66 & Monroe & 1.169 & 1.987 \\
\hline 67 & Montgomery & .299 & .253 \\
\hline 68 & Morgan & .783 & .520 \\
\hline 69 & Newton & .052 & .114 \\
\hline 70 & Noble & .398 & 1.180 \\
\hline 71 & Ohio & .000 & .000 \\
\hline 72 & Orange & .075 & .086 \\
\hline 73 & Owen & .118 & .323 \\
\hline 74 & Parke & .102 & .103 \\
\hline 75 & Perry & .089 & .279 \\
\hline 76 & Pike & .149 & .113 \\
\hline 77 & Porter & 2.540 & 2.066 \\
\hline 78 & Posey & .433 & .669 \\
\hline 79 & Pulaski & .189 & .339 \\
\hline 80 & Putnam & .094 & .097 \\
\hline 81 & Randolph & .173 & .133 \\
\hline 82 & Ripley & .727 & .530 \\
\hline 83 & Rush & .177 & .184 \\
\hline 84 & St. Joseph & 8.335 & 7.383 \\
\hline 85 & Scott & .118 & .775 \\
\hline 86 & Shelby & 1.240 & .731 \\
\hline 87 & Spencer & .116 & .259 \\
\hline 88 & Starke & .068 & .071 \\
\hline 89 & Steuben & .382 & .441 \\
\hline 90 & Sullivan & .106 & .191 \\
\hline 91 & Switzerland & .025 & .025 \\
\hline 92 & Tippecanoe & 1.610 & 1.838 \\
\hline 93 & Tipton & .120 & .182 \\
\hline 94 & Union & .108 & .109 \\
\hline 95 & Vanderburgh & 6.883 & 6.552 \\
\hline 96 & Vermillion & .017 & .069 \\
\hline 97 & Vigo & 1.673 & 1.530 \\
\hline 98 & Wabash & .256 & .228 \\
\hline 99 & Warren & .112 & .113 \\
\hline 100 & Warrick & .273 & .380 \\
\hline 101 & Washington & .162 & .089 \\
\hline 102 & Wayne & 1.543 & 1.081 \\
\hline
\end{tabular}

F112 


\begin{tabular}{|c|c|c|c|}
\hline & Locale & P38 30 & A38 30 \\
\hline 103 & Wells & .213 & 1.778 \\
\hline 104 & White & .238 & .439 \\
\hline 105 & Whitley & .366 & .278 \\
\hline 106 & lowa & 55.525 & 58.798 \\
\hline 107 & Kansas & 68.336 & 63.405 \\
\hline 108 & |Kentucky_E & 42.651 & 50.631 \\
\hline 109 & Kentucky_W & 28.434 & 33.759 \\
\hline 110 & Louisiana & 84.197 & 76.858 \\
\hline 111 & Maine & 18.970 & 26.624 \\
\hline 112 & Maryland & 122.984 & 105.721 \\
\hline$\overline{113}$ & Massachusetts & 162.475 & 156.263 \\
\hline 114 & Michigan_E & 110.456 & 91.561 \\
\hline 115 & Michigan W & 110.456 & 91.561 \\
\hline 116 & Minnesota & 181.762 & 151.042 \\
\hline 117 & Mississippi & 36.530 & 53.276 \\
\hline 118 & Missouri & 137.332 & 133.360 \\
\hline 119 & Montana & 15.409 & 15.191 \\
\hline 120 & Nebraska & 37.520 & 37.762 \\
\hline 121 & Nevada & 66.766 & 56.258 \\
\hline 122 & New Hampshire & 31.919 & 31.448 \\
\hline 123 & New Jersey & 280.192 & 291.974 \\
\hline 124 & New Mexico & 24.208 & 21.267 \\
\hline 125 & New York & 352.387 & 390.048 \\
\hline 126 & North Carolina & 246.303 & 329.685 \\
\hline 127 & North Dakota & 13.121 & 14.329 \\
\hline 128 & Ohio_N & 8.980 & 38.325 \\
\hline 129 & Ohio_M & 8.980 & 38.325 \\
\hline 130 & Ohio_s & 8.980 & 38.325 \\
\hline 131 & Oklahoma & 68.622 & 64.044 \\
\hline 132 & Oregon & 106.034 & 97.513 \\
\hline 133 & Pennsylvania & 246.337 & 234.068 \\
\hline 134 & Rhode Island & 22.485 & 22.108 \\
\hline 135 & South Carolina & 78.027 & 125.729 \\
\hline 136 & South Dakota & 14.582 & 17.289 \\
\hline 137 & Tennessee & 156.214 & 163.033 \\
\hline 138 & Texas & 745.421 & 634.398 \\
\hline 139 & Utah & 72.347 & 64.798 \\
\hline 140 & Vermont & 9.441 & 11.220 \\
\hline 141 & Virginia & 149.790 & 143.828 \\
\hline 142 & Washington & 169.113 & 157.176 \\
\hline 143 & West Virginia & 23.812 & 18.795 \\
\hline 144 & Wisconsin & 128.874 & 119.569 \\
\hline 145 & Wyoming & 6.786 & 5.708 \\
\hline
\end{tabular}




\begin{tabular}{|c|c|c|c|}
\hline & Locale & P39 30 & A39 30 \\
\hline 1 & Alabama & 970.998 & 746.278 \\
\hline 2 & Arizona & 1323.014 & 1541.745 \\
\hline 3 & Arkansas & 809.096 & 491.427 \\
\hline 4 & California & 6150.541 & 6943.717 \\
\hline 5 & Colorado & 451.922 & 843.520 \\
\hline 6 & Connecticut & 606.648 & 565.497 \\
\hline 7 & Delaware & 106.721 & 151.385 \\
\hline 8 & DC & .000 & .000 \\
\hline 9 & Florida & 2088.686 & 4248.851 \\
\hline 10 & Georgia & 1595.791 & 1758.629 \\
\hline 11 & Idaho & 144.069 & 291.502 \\
\hline 12 & Illinois_N & 1010.206 & 1369.704 \\
\hline 13 & Illinois_S & 505.103 & 684.852 \\
\hline 14 & Adams & 38.989 & 6.377 \\
\hline 15 & Allen & 144.163 & 65.851 \\
\hline 16 & Bartholomew & 77.041 & $14: 012$ \\
\hline 17 & Benton & 2.520 & 1.231 \\
\hline 18 & Blackford & .801 & 1.928 \\
\hline 19 & Boone & 8.914 & 12.012 \\
\hline 20 & Brown & .091 & 2.609 \\
\hline 21 & Carroll & 19.079 & 3.317 \\
\hline 22 & Cass & 26.719 & 6.487 \\
\hline 23 & Clark & 61.496 & 19.217 \\
\hline 24 & Clay & 16.568 & 4.340 \\
\hline 25 & Clinton & 8.271 & 5.848 \\
\hline 26 & Crawford & .000 & 2.122 \\
\hline 27 & Daviess & 3.884 & 5.099 \\
\hline 28 & Dearborn & .000 & 11.326 \\
\hline 29 & Decatur & 13.903 & 4.124 \\
\hline 30 & DeKalb & 14.041 & 8.226 \\
\hline 31 & Delaware & 38.862 & 20.400 \\
\hline 32 & Dubois & 349.262 & 7.266 \\
\hline 33 & Elkhart & 440.099 & 36.200 \\
\hline 34 & Fayette & 31.768 & 3.789 \\
\hline 35 & Floyd & 29.255 & 14.455 \\
\hline 36 & Fountain & 6.714 & 2.755 \\
\hline 37 & Franklin & 4.137 & 4.028 \\
\hline 38 & Fulton & .550 & 3.424 \\
\hline 39 & Gibson & 1.857 & 5.406 \\
\hline 40 & Grant & 29.270 & 9.991 \\
\hline 41 & Greene & .955 & 5.487 \\
\hline 42 & Hamilton & 34.576 & 64.358 \\
\hline 43 & Hancock & 25.191 & 14.386 \\
\hline 44 & Harrison & 52.275 & 7.832 \\
\hline 45 & Hendricks & 5.196 & 34.975 \\
\hline 46 & Henry & 20.106 & 6.966 \\
\hline 47 & Howard & 176.293 & 14.041 \\
\hline 48 & Huntington & 22.682 & 7.247 \\
\hline 49 & Jackson & 25.682 & 6.907 \\
\hline 50 & Jasper & 2.214 & 5.370 \\
\hline 51 & Jay & 5.534 & 3.162 \\
\hline
\end{tabular}

F114 


\begin{tabular}{|c|c|c|c|}
\hline & Locale & P39 30 & A39 30 \\
\hline 52 & Jefferson & 17.107 & 5.796 \\
\hline 53 & Jennings & 8.155 & 5.386 \\
\hline 54 & Johnson & 25.534 & 30.890 \\
\hline 55 & Knox & .469 & 5.931 \\
\hline 56 & Kosciusko & 32.994 & 13.149 \\
\hline 57 & LaGrange & 30.508 & 7.630 \\
\hline 58 & Lake & 29.284 & 78.001 \\
\hline 59 & La Porte & 14.464 & 17.604 \\
\hline 60 & Lawrence & 15.166 & 7.224 \\
\hline 61 & Madison & 83.053 & 22.734 \\
\hline 62 & Marion & 208.081 & 148.681 \\
\hline 63 & Marshall & 23.590 & 8.525 \\
\hline 64 & Martin & .000 & 1.518 \\
\hline 65 & Miami & 15.163 & 5.247 \\
\hline 66 & Monroe & 5.853 & 24.773 \\
\hline 67 & Montgomery & 13.293 & 6.500 \\
\hline 68 & Morgan & 2.804 & 15.058 \\
\hline 69 & Newton & 4.246 & 2.276 \\
\hline 70 & Noble & 33.651 & 8.386 \\
\hline$\overline{71}$ & Ohio & .000 & .945 \\
\hline 72 & Orange & 75.651 & 3.323 \\
\hline 73 & Owen & .000 & 4.034 \\
\hline$\overline{74}$ & Parke & .441 & 2.644 \\
\hline 75 & Perry & 11.666 & 2.834 \\
\hline$\overline{76}$ & Pike & .000 & 2.138 \\
\hline 77 & Porter & 2.564 & 25.588 \\
\hline$\overline{78}$ & Posey & .000 & 4.653 \\
\hline 79 & Pulaski & .576 & 2.214 \\
\hline 80 & Putnam & 7.277 & 6.574 \\
\hline 81 & Randolph & 6.552 & 4.047 \\
\hline 82 & Ripley & 8.864 & 4.779 \\
\hline 83 & Rush & .477 & 2.548 \\
\hline 84 & St. Joseph & 47.905 & 48.421 \\
\hline 85 & Scott & 3.474 & 4.192 \\
\hline 86 & Shelby & 22.317 & 7.210 \\
\hline 87 & Spencer & 33.641 & 3.198 \\
\hline 88 & Starke & 2.887 & 3.711 \\
\hline 89 & Steuben & 25.936 & 5.814 \\
\hline 90 & Sullivan & 1.677 & 3.654 \\
\hline 91 & Switzerland & .641 & 1.776 \\
\hline 92 & Tippecanoe & 115.660 & 31.722 \\
\hline 93 & Tipton & 3.223 & 2.510 \\
\hline 94 & Union & .000 & 1.152 \\
\hline 95 & Vanderburgh & 21.064 & 28.896 \\
\hline 96 & Vermillion & .000 & 2.435 \\
\hline 97 & Vigo & 6.271 & 16.280 \\
\hline 98 & Wabash & 3.880 & 5.378 \\
\hline 99 & Warren & .579 & 1.278 \\
\hline 100 & Warrick & 2.428 & 11.103 \\
\hline 101 & Washington & 89.410 & 5.013 \\
\hline 102 & Wayne & 37.278 & 9.939 \\
\hline
\end{tabular}




\begin{tabular}{|c|c|c|c|}
\hline & Locale & P39 30 & A39 30 \\
\hline 103 & Wells & 4.607 & 5.001 \\
\hline 104 & White & 23.757 & 4.092 \\
\hline 105 & Whitley & 15.620 & 5.966 \\
\hline 106 & lowa & 534.910 & 455.114 \\
\hline 107 & Kansas & 727.670 & 449.248 \\
\hline 108 & Kentucky_E & 440.727 & 416.640 \\
\hline 109 & Kentucky_W & 293.818 & 277.760 \\
\hline 110 & Louisiana & 287.873 & 740.616 \\
\hline 111 & Maine & 252.283 & 216.487 \\
\hline 112 & Maryland & 362.624 & 1065.878 \\
\hline 113 & Massachusetts & 462.442 & 1071.860 \\
\hline 114 & Michigan_E & 2015.416 & 822.600 \\
\hline 115 & Michigan_W & 2015.416 & 822.660 \\
\hline 116 & Minnesota & 812.911 & 951.819 \\
\hline 117 & Mississippi & 1645.917 & 470.585 \\
\hline 118 & Missouri & 1325.867 & 981.919 \\
\hline 119 & Montana & 48.154 & 160.654 \\
\hline 120 & Nebraska & 217.183 & 279.623 \\
\hline 121 & Nevada & 188.051 & 589.283 \\
\hline 122 & New Hampshire & 113.553 & 247.026 \\
\hline 123 & New Jersey & 536.730 & 1492.508 \\
\hline 124 & New Mexico & 73.651 & 319.351 \\
\hline 125 & New York & 1341.967 & 2984.984 \\
\hline 126 & North Carolina & 5575.201 & 1813.136 \\
\hline 127 & North Dakota & 68.702 & 95.659 \\
\hline 128 & Ohio_N & 823.580 & 596.165 \\
\hline 129 & Ohio_M & 823.580 & 596.165 \\
\hline 130 & Ohio_s & 823.580 & 596.165 \\
\hline 131 & Oklahoma & 375.634 & 596.261 \\
\hline 132 & Oregon & 569.739 & 727.731 \\
\hline 133 & Pennsylvania & 1591.172 & 1981.694 \\
\hline 134 & Rhode Island & 119.424 & 175.785 \\
\hline 135 & South Carolina & 593.583 & 772.097 \\
\hline 136 & South Dakota & 97.292 & 123.021 \\
\hline 137 & Tennessee & 2052.488 & 1112.071 \\
\hline 138 & Texas & 2842.340 & 4916.771 \\
\hline 139 & Utah & 609.701 & 515.719 \\
\hline 140 & Vermont & 169.205 & 108.871 \\
\hline 141 & Virginia & 1842.942 & 1477.591 \\
\hline 142 & Washington & 1695.388 & 1294.407 \\
\hline 143 & West Virginia & 76.209 & 269.706 \\
\hline 144 & Wisconsin & 1286.023 & 941.368 \\
\hline 145 & Wyoming & 13.468 & 80.808 \\
\hline
\end{tabular}




\begin{tabular}{|c|c|c|c|}
\hline & Locale & P40 30 & A40 30 \\
\hline 1 & Alabama & 4570.922 & 5098.789 \\
\hline 2 & Arizona & 4649.071 & 6214.697 \\
\hline 3 & Arkansas & 4168.602 & 2922.279 \\
\hline 4 & California & 28457.311 & 26673.752 \\
\hline 5 & Colorado & 3399.898 & 3133.440 \\
\hline 6 & Connecticut & 1940.526 & 2043.325 \\
\hline 7 & Delaware & 941.174 & 616.812 \\
\hline 8 & DC & .000 & .000 \\
\hline 9 & Florida & 14107.711 & 15866.733 \\
\hline 10 & Georgia & 17068.546 & 10757.449 \\
\hline 11 & Idaho & 1815.198 & 1855.513 \\
\hline 12 & Illinois $\mathbf{N}$ & 6199.978 & 4905.824 \\
\hline 13 & Illinois_S & 3099.988 & 2452.912 \\
\hline 14 & Adams & 33.435 & 68.475 \\
\hline 15 & Allen & 292.432 & 229.571 \\
\hline 16 & Bartholomew & 102.784 & 50.384 \\
\hline 17 & Benton & 2.847 & 14.936 \\
\hline 18 & Blackford & 6.642 & 10.629 \\
\hline 19 & Boone & 28.513 & 38.991 \\
\hline 20 & Brown & 6.491 & 10.366 \\
\hline 21 & Carroll & 93.823 & 10.766 \\
\hline 22 & Cass & 91.309 & 32.149 \\
\hline 23 & Clark & 69.855 & 118.160 \\
\hline 24 & Clay & 10.496 & 18.334 \\
\hline 25 & Clinton & 115.623 & 21.467 \\
\hline 26 & Crawford & 4.907 & 12.427 \\
\hline 27 & Daviess & 63.782 & 34.284 \\
\hline 28 & Dearborn & 26.855 & 37.784 \\
\hline 29 & Decatur & 26.987 & 15.275 \\
\hline 30 & DeKalb & 22.407 & 84.728 \\
\hline 31 & Delaware & 54.699 & 70.704 \\
\hline 32 & Dubois & 58.417 & 156.409 \\
\hline 33 & Elkhart & 210.364 & 643.396 \\
\hline 34 & Fayette & 8.764 & 12.301 \\
\hline 35 & Floyd & 71.954 & 95.370 \\
\hline 36 & Fountain & 9.054 & 9.632 \\
\hline 37 & Franklin & 9.866 & 16.384 \\
\hline 38 & Fulton & 15.015 & 29.659 \\
\hline 39 & Gibson & 32.376 & 17.549 \\
\hline 40 & Grant & 38.789 & 35.688 \\
\hline 41 & Greene & 30.242 & 21.815 \\
\hline 42 & Hamilton & 198.841 & 224.186 \\
\hline 43 & Hancock & 33.990 & 49.803 \\
\hline 44 & Harrison & 65.480 & 42.529 \\
\hline 45 & Hendricks & 126.712 & 122.157 \\
\hline 46 & Henry & 25.290 & 26.148 \\
\hline 47 & Howard & 39.697 & 49.778 \\
\hline 48 & Huntington & 38.234 & 30.466 \\
\hline 49 & Jackson & 19.049 & 43.520 \\
\hline 50 & Jasper & 23.472 & 22.653 \\
\hline 51 & Jay & 25.488 & 14.047 \\
\hline
\end{tabular}




\begin{tabular}{|c|c|c|c|}
\hline & Locale & P40 30 & $A 40 \quad 30$ \\
\hline 52 & Jefferson & 15.914 & 24.457 \\
\hline 53 & Jennings & 73.533 & 23.337 \\
\hline 54 & Johnson & 74.209 & 217.993 \\
\hline 55 & Knox & 18.864 & 23.817 \\
\hline 56 & Kosciusko & 85.160 & 66.641 \\
\hline 57 & LaGrange & 29.110 & 184.345 \\
\hline 58 & Lake & 239.268 & 269.615 \\
\hline 59 & La Porte & 66.980 & 73.306 \\
\hline 60 & Lawrence & 20.493 & 30.965 \\
\hline 61 & Madison & 90.057 & 86.874 \\
\hline 62 & Marion & 585.831 & 536.749 \\
\hline 63 & Marshall & 51.749 & 80.015 \\
\hline 64 & Martin & 5.865 & 5.531 \\
\hline 65 & Miami & 15.655 & 25.166 \\
\hline 66 & Monroe & 72.711 & 85.559 \\
\hline 67 & Montgomery & 33.096 & 24.217 \\
\hline 68 & Morgan & 37.313 & 66.418 \\
\hline 69 & Newton & 5.710 & 7.388 \\
\hline 70 & Noble & 83.959 & 31.900 \\
\hline 71 & Ohio & 2.186 & 3.069 \\
\hline 72 & Orange & 10.276 & 41.173 \\
\hline 73 & Owen & 18.974 & 22.789 \\
\hline 74 & Parke & 13.824 & 20.456 \\
\hline 75 & Perry & 14.108 & 12.873 \\
\hline 76 & Pike & 4.945 & 11.378 \\
\hline 77 & Porter & 76.186 & 93.115 \\
\hline 78 & Posey & 22.529 & 15.860 \\
\hline 79 & Pulaski & 8.424 & 7.923 \\
\hline 80 & Putnam & 15.687 & 25.815 \\
\hline 81 & Randolph & 12.508 & 38.586 \\
\hline 82 & Ripley & 11.552 & 24.908 \\
\hline 83 & Rush & 8.117 & 11.942 \\
\hline 84 & St. Joseph & 170.570 & 195.264 \\
\hline 85 & Scott & 53.063 & 18.060 \\
\hline 86 & Shelby & 24.540 & 23.403 \\
\hline 87 & Spencer & 9.964 & 20.333 \\
\hline 88 & Starke & 8.582 & 32.894 \\
\hline 89 & Steuben & 34.143 & 22.489 \\
\hline 90 & Sullivan & 8.450 & 18.421 \\
\hline 91 & Switzerland & 4.108 & 5.766 \\
\hline 92 & Tippecanoe & 122.840 & 107.689 \\
\hline 93 & Tipton & 13.317 & 12.115 \\
\hline 94 & Union & 2.664 & 4.455 \\
\hline 95 & Vanderburgh & 300.169 & 112.942 \\
\hline 96 & Vermillion & 5.947 & 7.905 \\
\hline 97 & Vigo & 50.547 & 61.206 \\
\hline 98 & Wabash & 16.109 & 28.451 \\
\hline 99 & Warren & 3.437 & 8.601 \\
\hline 100 & Warrick & 27.295 & 46.499 \\
\hline 101 & Washington & 14.652 & 34.012 \\
\hline 102 & Wayne & 49.864 & 35.785 \\
\hline
\end{tabular}

F118 


\begin{tabular}{|c|c|c|c|}
\hline & Locale & P40 30 & A40 30 \\
\hline 103 & Wells & 40.560 & 21.549 \\
\hline 104 & White & 12.717 & 20.593 \\
\hline 105 & Whitley & 14.331 & 33.734 \\
\hline 106 & lowa & 3228.660 & 2061.870 \\
\hline 107 & Kansas & 2662.572 & 1686.601 \\
\hline 108 & Kentucky_E & 1947.932 & 1922.783 \\
\hline 109 & Kentucky_W & 1298.622 & 1281.747 \\
\hline 110 & Louisiana & 2697.823 & 3100.260 \\
\hline 111 & Maine & 1056.349 & 1445.427 \\
\hline 112 & Maryland & 3566.731 & 3878.015 \\
\hline 113 & Massachusetts & 4205.551 & 4105.752 \\
\hline 114 & Michigan E & 2852.898 & 3175.051 \\
\hline 115 & Michigan W & 2852.898 & 3175.051 \\
\hline 116 & Minnesota & 4857.544 & 4399.922 \\
\hline 117 & Mississippi & 2956.615 & 2971.681 \\
\hline 118 & Missouri & 4568.824 & 3997.456 \\
\hline 119 & Montana & 501.152 & 940.128 \\
\hline 120 & Nebraska & 2284.168 & 1099.625 \\
\hline 121 & Nevada & 1736.294 & 2060.991 \\
\hline 122 & New Hampshire & 908.867 & 1238.836 \\
\hline 123 & New Jersey & 5713.186 & 5368.406 \\
\hline 124 & New Mexico & 951.284 & 1157.381 \\
\hline 125 & New York & 10289.122 & 10771.037 \\
\hline 126 & North Carolina & 12081.056 & 14425.446 \\
\hline 127 & North Dakota & 470.036 & 374.579 \\
\hline 128 & Ohio_N & 2295.848 & 2447.707 \\
\hline 129 & Ohio_M & 2295.848 & 2447.707 \\
\hline 130 & Ohio_s & 2295.848 & 2447.707 \\
\hline 131 & Oklahoma & 2304.974 & 2186.693 \\
\hline 132 & Oregon & 3088.331 & 5705.401 \\
\hline 133 & Pennsylvania & 9075.765 & 8574.504 \\
\hline 134 & Rhode Island & 708.882 & 804.788 \\
\hline 135 & South Carolina & 4815.753 & 5811.674 \\
\hline 136 & South Dakota & 757.940 & 528.853 \\
\hline 137 & Tennessee & 5560.237 & 5714.679 \\
\hline 138 & Texas & 19183.567 & 19412.799 \\
\hline 139 & Utah & 2231.422 & 1911.126 \\
\hline 140 & Vermont & 462.090 & 617.474 \\
\hline 141 & Virginia & 6210.786 & 7574.235 \\
\hline 142 & Washington & 5460.787 & 6326.158 \\
\hline 143 & West Virginia & 856.039 & 1381.509 \\
\hline 144 & Wisconsin & 5375.060 & 5244.731 \\
\hline 145 & Wyoming & 229.575 & 336.790 \\
\hline
\end{tabular}




\begin{tabular}{|c|c|c|c|}
\hline & Locale & P41 30 & A41 30 \\
\hline 1 & Alabama & 6833.510 & 9462.089 \\
\hline 2 & Arizona & 9285.044 & 7849.592 \\
\hline 3 & Arkansas & 5647.915 & 8798.541 \\
\hline 4 & California & 54553.679 & 46488.103 \\
\hline 5 & Colorado & 4338.784 & 4328.204 \\
\hline 6 & Connecticut & 9516.327 & 6251.157 \\
\hline 7 & Delaware & 696.646 & 1364.459 \\
\hline 8 & DC & .000 & .000 \\
\hline 9 & Florida & 14639.395 & 13229.596 \\
\hline 10 & Georgia & 9794.052 & 12560.373 \\
\hline 11 & Idaho & 1256.381 & 2455.675 \\
\hline 12 & Illinois_N & 19390.133 & 17654.202 \\
\hline 13 & Illinois_S & 9695.067 & 8827.101 \\
\hline 14 & Adams & 100.750 & 109.761 \\
\hline 15 & Allen & 749.329 & 918.167 \\
\hline 16 & Bartholomew & 260.307 & 358.635 \\
\hline 17 & Benton & 13.824 & 14.175 \\
\hline 18 & Blackford & 109.640 & 58.149 \\
\hline 19 & Boone & 104.001 & 52.910 \\
\hline 20 & Brown & 12.758 & 7.106 \\
\hline 21 & Carroll & 99.223 & 194.196 \\
\hline 22 & Cass & 142.203 & 196.829 \\
\hline 23 & Clark & 272.831 & 182.878 \\
\hline 24 & Clay & 23.586 & 13.871 \\
\hline 25 & Clinton & 57.689 & 200.375 \\
\hline 26 & Crawford & .000 & .000 \\
\hline 27 & Daviess & 37.243 & 105.942 \\
\hline 28 & Dearborn & 68.776 & 35.285 \\
\hline 29 & Decatur & 627.287 & 342.448 \\
\hline 30 & DeKalb & 848.099 & 830.311 \\
\hline 31 & Delaware & 424.580 & 325.509 \\
\hline 32 & Dubois & 35.639 & 85.157 \\
\hline 33 & Elkhart & 1558.979 & 1025.857 \\
\hline 34 & Fayette & 68.522 & 41.097 \\
\hline 35 & Floyd & 155.188 & 143.494 \\
\hline 36 & Fountain & 12.512 & 111.297 \\
\hline 37 & Franklin & 2.570 & 2.204 \\
\hline 38 & \begin{tabular}{|l} 
Fulton \\
\end{tabular} & 181.339 & 153.543 \\
\hline 39 & Gibson & 32.636 & 49.638 \\
\hline 40 & Grant & 28.445 & 48.343 \\
\hline 41 & Greene & 18.805 & 59.431 \\
\hline 42 & Hamilton & 418.329 & 328.807 \\
\hline 43 & Hancock & 154.267 & 80.025 \\
\hline 44 & Harrison & 22.557 & 90.872 \\
\hline 45 & Hendricks & 87.520 & 164.844 \\
\hline 46 & Henry & 62.448 & 77.912 \\
\hline 47 & Howard & 44.300 & 136.383 \\
\hline 48 & Huntington & 247.616 & 183.357 \\
\hline 49 & Jackson & 150.221 & 146.659 \\
\hline 50 & Jasper & 154.694 & 95.453 \\
\hline 51 & Jay & 180.854 & 120.430 \\
\hline
\end{tabular}




\begin{tabular}{|c|c|c|c|}
\hline & Locale & P41 30 & A41 30 \\
\hline 52 & Jefferson & 32.545 & 19.365 \\
\hline 53 & Jennings & 161.599 & 90.068 \\
\hline 54 & Johnson & 376.519 & 255.344 \\
\hline 55 & Knox & 21.287 & 63.107 \\
\hline 56 & Kosciusko & 329.266 & 497.342 \\
\hline 57 & LaGrange & 54.768 & 45.365 \\
\hline 58 & Lake & 530.679 & 3173.584 \\
\hline 59 & La Porte & 452.445 & 478.210 \\
\hline 60 & Lawrence & 112.858 & 281.187 \\
\hline 61 & Madison & 227.108 & 203.278 \\
\hline 62 & Marion & 2242.069 & 1944.271 \\
\hline 63 & Marshall & 252.356 & 223.763 \\
\hline 64 & Martin & 1.832 & 25.485 \\
\hline 65 & Miami & 48.191 & 27.893 \\
\hline 66 & Monroe & 100.498 & 59.016 \\
\hline 67 & Montgomery & 144.268 & 210.435 \\
\hline 68 & Morgan & 94.067 & 58.988 \\
\hline 69 & Newton & 12.495 & 30.393 \\
\hline 70 & Noble & 330.522 & 341.588 \\
\hline 71 & Ohio & 2.438 & 1.211 \\
\hline 72 & Orange & 12.084 & 10.361 \\
\hline 73 & Owen & 3.079 & 2.383 \\
\hline 74 & Parke & 2.055 & 13.986 \\
\hline 75 & Perry & 11.130 & 50.930 \\
\hline 76 & Pike & 13.441 & 15.321 \\
\hline 77 & Porter & 349.179 & 1381.021 \\
\hline 78 & Posey & 13.724 & 11.767 \\
\hline 79 & Pulaski & 39.106 & 50.135 \\
\hline 80 & Putnam & 2.260 & 1.938 \\
\hline 81 & Randolph & 21.176 & 53.233 \\
\hline 82 & Ripley & 48.997 & 25.187 \\
\hline 83 & Rush & 11.121 & 51.959 \\
\hline 84 & St. Joseph & 985.906 & 797.712 \\
\hline 85 & Scott & 19.784 & 41.666 \\
\hline 86 & Shelby & 76.965 & 123.617 \\
\hline 87 & Spencer & 11.980 & 32.739 \\
\hline 88 & Starke & .000 & .000 \\
\hline 89 & Steuben & 303.095 & 242.916 \\
\hline 90 & Sullivan & 2.233 & 1.109 \\
\hline 91 & Switzerland & .000 & .000 \\
\hline 92 & Tippecanoe & 170.600 & 465.029 \\
\hline 93 & Tipton & 24.829 & 32.694 \\
\hline 94 & Union & 2.168 & 1.077 \\
\hline 95 & Vanderburgh & 452.347 & 548.274 \\
\hline 96 & Vermillion & 32.143 & 16.501 \\
\hline 97 & Vigo & 144.996 & 143.577 \\
\hline 98 & Wabash & 193.146 & 191.858 \\
\hline 99 & Warren & 27.883 & 14.665 \\
\hline 100 & Warrick & 58.581 & 635.362 \\
\hline 101 & Washington & 144.798 & 78.619 \\
\hline 102 & Wayne & 113.131 & 206.305 \\
\hline
\end{tabular}




\begin{tabular}{|c|c|c|c|}
\hline & Locale & P41_30 & A41 30 \\
\hline 103 & Wells & 152.492 & 149.919 \\
\hline 104 & White & 198.175 & 109.795 \\
\hline 105 & Whitley & 174.343 & 184.598 \\
\hline 106 & lowa & 5198.384 & 7287.136 \\
\hline 107 & Kansas & 3197.753 & 4431.847 \\
\hline 108 & Kentucky_E & 3504.155 & 4104.860 \\
\hline 109 & Kentucky_W & 2336.103 & 2736.573 \\
\hline 110 & Louisiana & 4496.106 & 4149.626 \\
\hline 111 & Maine & 1105.787 & 1168.373 \\
\hline 112 & Maryland & 3678.124 & 4750.292 \\
\hline 113 & Massachusetts & 10548.194 & 7835.386 \\
\hline 114 & Michigan_E & 11427.456 & 9559.341 \\
\hline 115 & Michigan_W & 11427.456 & 9559.341 \\
\hline 116 & Minnesota & 12786.633 & 11620.847 \\
\hline 117 & Mississippi & 3282.667 & 4211.644 \\
\hline 118 & Missouri & 9396.790 & 9792.239 \\
\hline 119 & Montana & 314.509 & 525.097 \\
\hline 120 & Nebraska & 2172.509 & 3901.488 \\
\hline 121 & Nevada & 2075.943 & 1916.482 \\
\hline 122 & New Hampshire & 3493.015 & 2569.356 \\
\hline 123 & New Jersey & 9163.901 & 8626.614 \\
\hline 124 & New Mexico & 698.330 & 952.231 \\
\hline 125 & New York & 14664.514 & 13294.270 \\
\hline 126 & North Carolina & 13432.611 & 14054.019 \\
\hline 127 & North Dakota & 373.369 & 573.463 \\
\hline 128 & Ohio_N & 9959.773 & 9464.601 \\
\hline 129 & Ohio. M & 9959.773 & 9464.601 \\
\hline 130 & Ohio_s & 9959.773 & 9464.601 \\
\hline 131 & Oklahoma & 6310.797 & 5129.772 \\
\hline 132 & Oregon & 5451.053 & 6736.059 \\
\hline 133 & Pennsylvania & 21745.945 & 25035.845 \\
\hline 134 & Rhode Island & 2366.132 & 1891.494 \\
\hline 135 & South Carolina & 7961.181 & 6601.632 \\
\hline 136 & South Dakota & 732.462 & 1071.319 \\
\hline 137 & Tennessee & 11664.272 & 12104.989 \\
\hline 138 & Texas & 38891.546 & 35438.849 \\
\hline 139 & Utah & 3346.419 & 4418.271 \\
\hline 140 & Vermont & 852.066 & 785.999 \\
\hline 141 & Virginia & 6019.518 & 7715.015 \\
\hline 142 & Washington & 6682.421 & 9396.011 \\
\hline 143 & West Virginia & 1350.844 & 2431.185 \\
\hline 144 & Wisconsin & 18011.435 & 17774.286 \\
\hline 145 & Wyoming & 190.386 & 169.713 \\
\hline
\end{tabular}




\begin{tabular}{|c|c|c|c|}
\hline & $\begin{array}{r}\text { Locale } \\
\end{array}$ & P43 30 & A43 30 \\
\hline 1 & Alabama & 3638.355 & 4009.877 \\
\hline 2 & Arizona & 7516.521 & 7084.926 \\
\hline 3 & Arkansas & 2395.868 & 2399.117 \\
\hline 4 & California & 33852.933 & 33214.198 \\
\hline 5 & Colorado & 4112.441 & 4006.975 \\
\hline 6 & Connecticut & 2756.987 & 2661.657 \\
\hline 7 & Delaware & 738.055 & 715.462 \\
\hline 8 & DC & .000 & .000 \\
\hline 9 & Florida & 20714.563 & 19891.036 \\
\hline 10 & Georgia & 8573.904 & 12918.182 \\
\hline 11 & Idaho & 1421.169 & 1351.967 \\
\hline 12 & Illinois_N & 6677.763 & 6396.802 \\
\hline 13 & Illinois_s & 3338.881 & 3198.401 \\
\hline 14 & Adams & 31.091 & 28.691 \\
\hline 15 & Allen & 321.048 & 324.192 \\
\hline 16 & Bartholomew & 68.311 & 63.907 \\
\hline 17 & Benton & 6.002 & 5.539 \\
\hline 18 & Blackford & 9.400 & 8.675 \\
\hline 19 & Boone & 58.561 & 54.041 \\
\hline 20 & Brown & 12.722 & 11.740 \\
\hline 21 & Carroll & 16.169 & 14.921 \\
\hline 22 & Cass & 31.626 & 29.886 \\
\hline 23 & Clark & 93.690 & 91.790 \\
\hline 24 & Clay & 21.158 & 19.525 \\
\hline 25 & Clinton & 28.511 & 26.311 \\
\hline 26 & Crawford & 10.345 & 9.546 \\
\hline 27 & Daviess & 24.860 & 22.941 \\
\hline 28 & Dearborn & 55.218 & 50.957 \\
\hline 29 & Decatur & 20.105 & 18.553 \\
\hline 30 & DeKalb & 40.102 & 37.007 \\
\hline 31 & Delaware & 99.459 & 92.576 \\
\hline 32 & Dubois & 35.425 & 33.514 \\
\hline 33 & Elkhart & 176.486 & 213.011 \\
\hline 34 & Fayette & 18.475 & 17.049 \\
\hline 35 & Floyd & 70.472 & 65.033 \\
\hline 36 & Fountain & 13.432 & 12.395 \\
\hline 37 & Franklin & 19.637 & 18.121 \\
\hline 38 & Fulton & 16.694 & 15.405 \\
\hline 39 & Gibson & 26.357 & 24.323 \\
\hline 40 & Grant & 48.708 & 48.409 \\
\hline 41 & Greene & 26.750 & 25.394 \\
\hline 42 & Hamilton & 313.766 & 311.310 \\
\hline 43 & Hancock & 70.134 & 64.721 \\
\hline 44 & Harrison & 38.182 & 35.235 \\
\hline 45 & Hendricks & 170.515 & 158.882 \\
\hline 46 & Henry & 33.964 & 35.098 \\
\hline 47 & Howard & 68.456 & 63.173 \\
\hline 48 & Huntington & 35.333 & 33.473 \\
\hline 49 & Jackson & 33.674 & 31.837 \\
\hline 50 & Jasper & 26.182 & 24.161 \\
\hline 51 & Jay & 15.416 & 14.226 \\
\hline
\end{tabular}




\begin{tabular}{|c|c|c|c|}
\hline & Locale & P43 30 & A43 30 \\
\hline 52 & Jefferson & 28.257 & 26.898 \\
\hline 53 & Jennings & 26.259 & 57.547 \\
\hline 54 & Johnson & 150.597 & 140.199 \\
\hline 55 & Knox & 28.915 & 26.684 \\
\hline 56 & Kosciusko & 64.105 & 64.466 \\
\hline 57 & LaGrange & 37.199 & 35.230 \\
\hline 58 & Lake & 380.279 & 355.345 \\
\hline 59 & La Porte & 85.827 & 79.930 \\
\hline 60 & Lawrence & 35.218 & 33.188 \\
\hline 61 & Madison & 110.834 & 103.794 \\
\hline 62 & Marion & 724.869 & 700.707 \\
\hline 63 & Marshall & 41.564 & 43.537 \\
\hline 64 & Martin & 7.398 & 6.827 \\
\hline 65 & Miami & 25.579 & 24.245 \\
\hline 66 & Monroe & 120.776 & 116.919 \\
\hline 67 & Montgomery & 31.692 & 29.246 \\
\hline 68 & Morgan & 73.411 & 68.764 \\
\hline 69 & Newton & 11.096 & 10.239 \\
\hline 70 & Noble & 40.883 & 52.229 \\
\hline 71 & Ohio & 4.609 & 4.254 \\
\hline 72 & Orange & 16.203 & 14.952 \\
\hline 73 & Owen & 19.667 & 23.133 \\
\hline 74 & Parke & 12.891 & 11.896 \\
\hline 75 & Perry & 13.816 & 16.653 \\
\hline 76 & Pike & 10.425 & 9.620 \\
\hline 77 & Porter & 124.751 & 115.914 \\
\hline 78 & Posey & 22.687 & 25.750 \\
\hline 79 & Pulaski & 10.792 & 9.959 \\
\hline 80 & Putnam & 32.048 & 29.575 \\
\hline 81 & Randolph & 19.730 & 18.208 \\
\hline 82 & Ripley & 23.297 & 21.499 \\
\hline 83 & Rush & 12.421 & 12.112 \\
\hline 84 & St. Joseph & 236.070 & 231.613 \\
\hline 85 & Scott & 20.437 & 32.659 \\
\hline 86 & Shelby & 35.149 & 32.436 \\
\hline 87 & Spencer & 15.591 & 14.388 \\
\hline 88 & Starke & 18.090 & 16.694 \\
\hline 89 & Steuben & 28.346 & 30.862 \\
\hline 90 & Sullivan & 17.814 & 16.439 \\
\hline 91 & Switzerland & 8.661 & 7.992 \\
\hline 92 & Tippecanoe & 154.655 & 148.614 \\
\hline 93 & Tipton & 12.239 & 11.295 \\
\hline 94 & Union & 5.616 & 5.183 \\
\hline 95 & Vanderburgh & 140.877 & 160.944 \\
\hline 96 & Vermillion & 11.873 & 10.957 \\
\hline 97 & Vigo & 79.372 & 74.005 \\
\hline 98 & Wabash & 26.221 & 24.864 \\
\hline 99 & Warren & 6.229 & 5.748 \\
\hline 100 & Warrick & 54.131 & 50.835 \\
\hline 101 & Washington & 24.442 & 22.555 \\
\hline 102 & Wayne & 48.454 & 44.714 \\
\hline
\end{tabular}




\begin{tabular}{|c|c|c|c|}
\hline & Locale & P43 30 & A43 30 \\
\hline 103 & Wells & 24.381 & 23.375 \\
\hline 104 & White & 19.949 & 19.149 \\
\hline 105 & Whitley & 29.086 & 26.842 \\
\hline 106 & lowa & 2218.835 & 2129.234 \\
\hline 107 & Kansas & 2190.233 & 2112.170 \\
\hline 108 & Kentucky_E & 2031.260 & 2085.973 \\
\hline 109 & Kentucky_W & 1354.174 & 1390.649 \\
\hline 110 & Louisiana & 3610.750 & 3425.212 \\
\hline 111 & Maine & 1055.448 & 1111.356 \\
\hline 112 & Maryland & 5196.511 & 4862.117 \\
\hline 113 & Massachusetts & 5225.673 & 5200.515 \\
\hline 114 & Michigan E & 4010.738 & 3805.630 \\
\hline 115 & Michigan W & 4010.738 & 3805.630 \\
\hline 116 & Minnesota & 4640.433 & 4432.111 \\
\hline 117 & Mississippi & 2294.257 & 2449.862 \\
\hline 118 & Missouri & 4787.181 & 4630.111 \\
\hline 119 & Montana & 783.242 & 733.060 \\
\hline 120 & Nebraska & 1363.256 & 1288.669 \\
\hline 121 & Nevada & 2872.952 & 2700.462 \\
\hline 122 & Now Hampshire & 1204.333 & 1225.904 \\
\hline 123 & New Jersey & 7276.475 & 7127.240 \\
\hline 124 & New Mexico & 1556.943 & 1441.692 \\
\hline 125 & New York & 14552.792 & 14091.964 \\
\hline 126 & North Carolina & 8839.642 & 10571.424 \\
\hline 127 & North Dakota & 466.369 & 445.246 \\
\hline 128 & Ohio N & 2906.501 & 2784.302 \\
\hline 129 & Ohio M & 2906.501 & 2784.302 \\
\hline 130 & Ohio_s & 2906.501 & 2784.302 \\
\hline 131 & Oklahoma & 2906.970 & 2797.304 \\
\hline 132 & Oregon & 3547.929 & 3381.418 \\
\hline 133 & Pennsylvania & 9661.419 & 9484.319 \\
\hline 134 & Rhode Island & 857.009 & 882.514 \\
\hline 135 & South Carolina & 3764.229 & 4648.478 \\
\hline 136 & South Dakota & 599.771 & 609.886 \\
\hline 137 & Tennessee & 5421.717 & 5520.520 \\
\hline 138 & Texas & 23970.900 & 23121.346 \\
\hline 139 & Utah & 2514.300 & 2443.112 \\
\hline 140 & Vermont & 530.783 & 500.183 \\
\hline 141 & Virginia & 7203.749 & 7030.793 \\
\hline 142 & Washington & 6310.665 & 6006.471 \\
\hline 143 & West Virginia & 1314.908 & 1221.857 \\
\hline 144 & Wisconsin & 4589.481 & 4417.932 \\
\hline 145 & Wyoming & 393.964 & 368.065 \\
\hline
\end{tabular}




\section{Table of Figures}

$\begin{array}{lll}\text { Figure } \quad \text { Title } & \text { Page }\end{array}$

1-1 The Counties of Indiana 2

1-2 Highway Network Utilized 4

1-3 Indiana Portion of the Highway Network 5

1-4 Interconnections of the State and Interstate Systems 6

1-5 Study Rail Network $\quad 10$

1-6 Indiana Portion of Rail Network $\quad 11$

1-7 Nodes of the Indiana Rail Network 15

4-1 Relationship of Total Productions and Total Attractions 62

4-2 Evaluation of the 1997 Models with 2002 Estimates 69

6-1 Market Area for General Air Freight of Indiana 90

6-2 Market Area for FedEx Parcels Moving by Air $\quad 91$

6-3 Market Area for UPS Parcels Moving by Air 92

6-4 Market Area for USPS Parcels Moving by Air 93

6-5 Market Area for DHL Parcels Moving by Air $\quad 94$

7-1 US Daily Truck Volumes for $1997 \quad 98$

7-2 Indiana Daily Truck Volumes for $1997 \quad 99$

7-3 Map of Sampled Locations 102

7-4 Relationship of Counts and Model Estimates 103

7-5 US Daily Rail Volumes for $1997 \quad 107$

7-6 Indiana Rail Flows $1997 \quad 108$

8-1 Indiana Daily Truckload Freight Volumes for 2015119

8-2 Indiana Daily Truckload Freight Volumes for $2030 \quad 120$

8-3 Indiana Daily Railcar Freight Volumes for $2015 \quad 121$

8-4 Indiana Daily Railcar Freight Volumes for 2030122 


\section{Table of Tables}

\section{Table Title}

Page

1-1

1-2

2-1

3-1

3-2

4-1

4-1a

4-2

4-3

4-3a

4-4

4-5

5-1

5-2

5-3

5-4

5-5

7-1

8-1

8-2
Nodes of the Highway Network and Coordinates

Nodes of the Rail Network and Coordinates

Major Commodity Groups Included in the Study

Value and Tons of Commodities for Indiana, 1997

Value and Tons of Commodities for Indiana, 2002

Production Models Developed

Production Models Developed (ver. 2)

Employment Variables Used in Modeling

Attraction Models Developed

Attraction Models Developed (ver. 2)

Adjusted Explanation for Zero Intercept - Production

Adjusted Explanation for Zero Intercept - Attraction

Production Constrained Model Attributes

Fully-Constrained Gravity Model Exponential Values

Actual and Modeled Average Distances

IU Density Factors vs. Cambridge Payload Factors

Sampling Locations for Model Evaluation

Proportional Increases in Employment by Area

Labor Productivity Growth Factors for Indiana, 2002-2030
Commodity Density Values for Railcars and Trucks
7

12

19

23

24

58

59

60

64

65

66

67

75

78

79

82

85

101

111

117 
